NUREG / CR -4913

SAND87-0891

R1 and RD

Printed May 1987

\title{
Round-Robin Pretest Analyses of a 1:6-Scale Reinforced Concrete Containment Model Subject to Static Internal Pressurization
}

David B. Clauss

Prepared by

Sandia National Laboratories

Albuquerque, New Mexico 87185 and Livermore, California 94550

for the United States Department of Energy

under Contract DE-AC04-76DP00789

\section{When printing a copy of any digitized SAND}

Report, you are required to update the Prepared for markings to current standards. U. S. NUCLEAR REGULATORY COMMISSION 


\section{NOTICE}

This report was prepared as an account of work sponsored by an agency of the United States Government. Neither the United States Government nor any agency thereof, or any of their employees, makes any warranty, expressed or implied, or assumes any legal liability or responsibility for any third party's use, or the results of such use, of any information, apparatus product or process disclosed in this report, or represents that its use by such third party would not infringe privately owned rights.

Available from

Superintendent of Documents

U.S. Government Printing Office

Post Office Box 37082

Washington, D.C. 20013-7082

and

National Technical Information Service Springfield, VA 22161 


\section{ABSTRACT}

Analyses of a 1:6-scale reinforced concrete containment model that will be tested to failure at Sandia National Laboratories in the spring of 1987 were conducted by the following organizations in the United States and Europe:

Sandia National Laboratories (USA)

Argonne National Laboratory (USA)

Electric Power Research Institute (USA)

Commissariat a L'Energie Atomique (France)

HM Nuclear Installations Irispectorate (U.K.)

Comitato Nazionale per la ricerca e per lo sviluppo dell'Energia Nucleare e delle Energie Alternative (Italy)

U.K. Atomic Energy Authority, Safety and Reliability Directorate (U.K.)

Gesellschaft fuer Reaktorsicherheit (FRG)

Brookhaven National Laboratory (USA)

Central Electricity Generating Board (U.K.)

Each organization was supplied with a standard information package, which included construction drawings and actual material properties for most of the materials used in the model. Each organization worked independently using their own analytical methods.

This report includes descriptions of the various analytical approaches and pretest predictions submitted by each organization. Significant milestones that occur with increasing pressure, such as damage to the concrete (cracking and crushing) and yielding of the steel components, and the failure pressure (capacity) and failure mechanism are described. Analytical predictions for pressure histories of strain in the liner and rebar and displacements are compared at locations where experimental results will be available after the test. Thus, these predictions can be compared to one another and to experimental results after the test. 


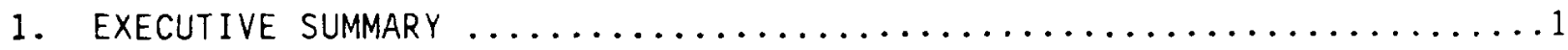

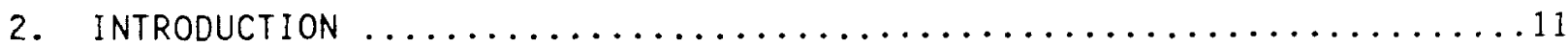

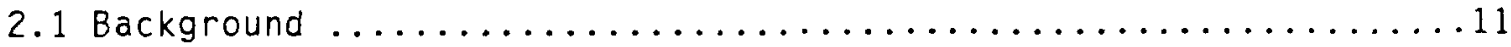

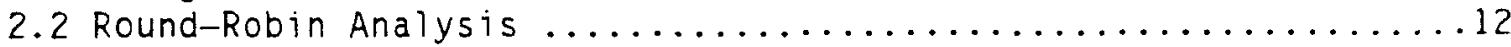

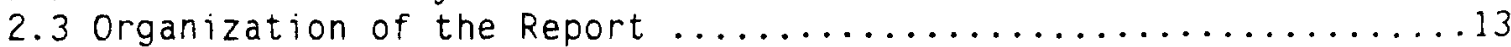

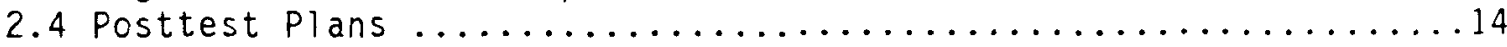

3. DESCRIPTION OF THE $1: 6$-SCALE REINFORCED CONCRETE CONTAINMENT MODEL . .15

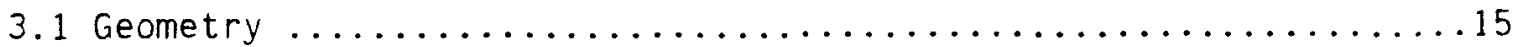

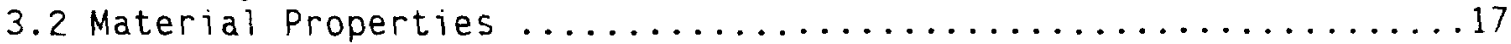

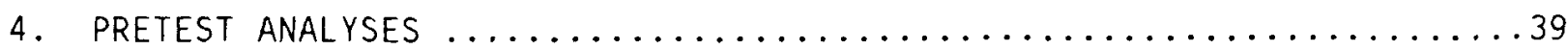

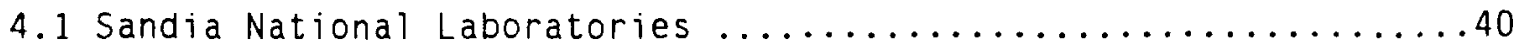

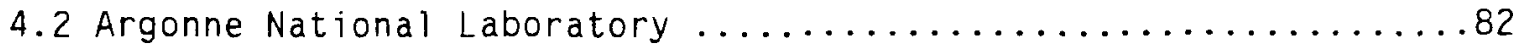

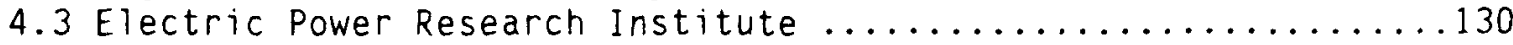

4.4 Commissariat a L'Energie Atomique .......................

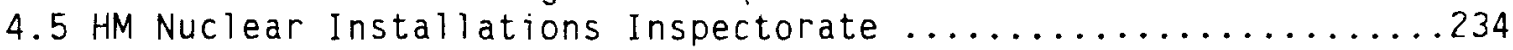

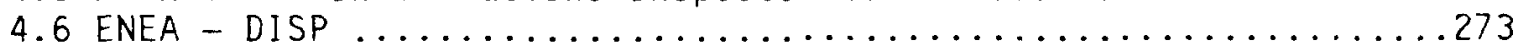

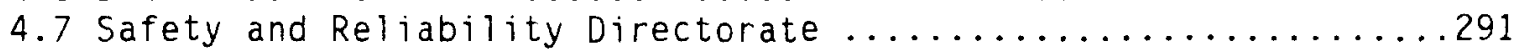

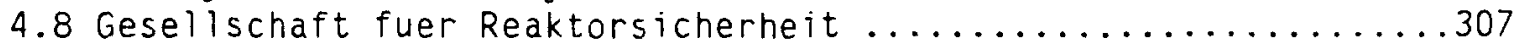

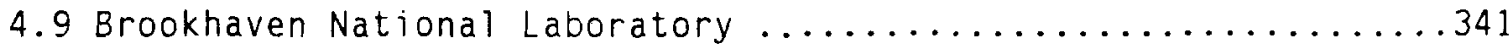

4.10 Central Electricity Generating Board ................... 374

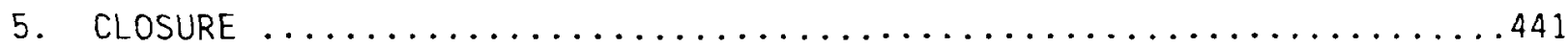

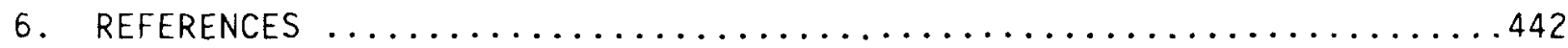

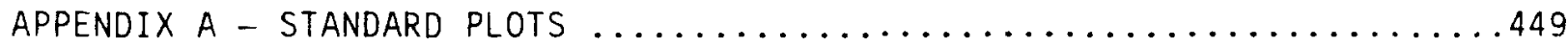

APPENDIX B - FORCE BALANCING EQUATIONS (CEGB) $\ldots \ldots \ldots \ldots \ldots \ldots \ldots \ldots \ldots$ 
1.1 Schematic of 1:6-Scale Containment Model

$1.2 \mathrm{a}$

$1.2 \mathrm{~b}$

$1.3 \mathrm{a}$

$1.3 b$

3.1

Radial Displacement at Cylinder Midheight (Low Pressure)

Radial Displacement at Cylinder Midheight

Vertical Displacement at Cylinder Midheight (Low Pressure)

Vertical Displacement at Cylinder Midheight

Schematic of the 1:6-Scale Reinforced Concrete

Containment Model - Elevation View

3.2

3.3

3.4

3.5

3.6

3.7

3.8

Reinforcement in the Basemat and Cylinder Basemat Junction

Photograph During Construction of Basemat Cylinder Junction

Reinforcement in the Cylinder (Free-field)

Photograph During Construction of Reinforcement in the Dome 25

Photograph of Reinforcement Around Equipment Hatch A 26

Photograph of Reinforcement Around Equipment Hatch B 27

Cylinder Stretchout - Reinforcement Layers 1 and $3 \quad 28$

3.9

Cylinder Stretchout - Reinforcement Layer 2

3.10

3.11

3.12

3.13

3.14

Cylinder Stretchout - Reinforcement Layer 4 and 6

Cylinder Stretchout - Reinforcement Layer 5

Cylinder Stretchout - Reinforcement Layer 7

Cylinder Stretchout - Reinforcement Layer 8

Additional Reinforcement in Bosses of Selected Penetrations 34

3.15

Details of Liner Knuckle

Cylinder Stretchout - Liner Details

3.17

Stud Pattern in Lower Cylinder

3.18

Stud Pattern Around Personnel Airlock A

Uniaxial stress-strain curves used for the concrete

4.1 .1

Nonlinear membrane idealization of the cylinder wall

4.1 .3

Membrane theory results for stresses in the rebar and liner at the midheight of the cylinder

60

4.1 .4

Outline of the axisymmetric finite element shell model

4.1 .5

Deformed shape plot of Model 1 at $191 \mathrm{psi}(1.32 \mathrm{MPa})$

4.1 .6

Deformed shape plot of Model 2 at 168 psi (1.16 MPa)

64

4.1 .7

Radial displacement at the cylinder midheight vs. pressure

64

4.1 .8

Basemat uplift at the basemat cylinder junction vs. pressure

65

4.1 .9

4.1 .10

Vertical displacement at the dome apex vs. pressure

65

Hoop strain at the shell midthickness

in the dome and cylinder (Model 1)

66

4.1 .11

Meridional strain at the shell midthickness

66

in the dome and cylinder (Model 1)

4.1 .12

Hoop strains along the lower surface of the basemat (Model 1)

4.1 .13

Meridional strains along the lower surface of the basemat

(Model 1)

4.1.14 Meridional strains at the midthickness of the containment wall in the dome and cylinder (Model 1)

4.1 .15

Hoop strain at the midthickness of

in the dome and cylinder (Model 1)

4.1 .16

4.1 .17

Stresses in the Layer 1 hoop reinforcement (Model 2)

Stresses in the Layer 2 hoop reinforcement (Model 2)

Stresses in the Layer 5 meridional reinforcement (Model 2) 70

4.1 .18

4.1 .19

Stresses in the Layer 7 seismic reinforcement (Model 2)

70

4.1 .20

Model 1 results for rebar strains vs pressure at EL 13' -9 "

71

4.1 .21

Model 2 results for rebar strains vs pressure at EL 13'-9"

72 
4.1.22 Equivalent plastic strain in the liner vs. pressure at the cylinder midheight

4.1 .23

Transverse (radial) shear force at $140 \mathrm{psi}(0.965 \mathrm{MPa})$

72

4.1 .24

4.1 .25

4.1 .26

Meridional bending moment at $140 \mathrm{psi}(0.965 \mathrm{MPa})$

Hoop membrane force at 140 psi $(0.965 \mathrm{MPa})$

Radial shear force 3 inches $(80 \mathrm{~mm})$ above the

base of the cylinder vs. pressure

Page

4.1 .27

Radial shear force 3 inches $(80 \mathrm{~mm})$ below the

springline vs. pressure

4.1 .28

Radial shear force 3 inches $(80 \mathrm{~mm})$ from the edge

of the dome plates vs. pressure

4.1.29 Meridional bending moment 3 inches $(80 \mathrm{~mm})$ above the

base of the cylinder vs. pressure

4.1.30 Crushing parameter on the outside of the cylinder wall 3 inches $(80 \mathrm{~mm})$ above the top of the basemat vs. pressure

4.1 .31

Model 1 results for rebar strains vs. pressure 3 inches

$(80 \mathrm{~mm})$ above the base of the cylinder

4.1 .32

Model 2 results for rebar strains vs.

$(80 \mathrm{~mm})$ above the base of the cylinder

pressure 3 inches

Finite element mesh used for continuum/shell Model 3 and 4 7 78

4.1 .33

4.1 .34

Radial displacement of the cylinder at EL $6^{\prime}-3^{\prime \prime}$

4.1 .35

Basemat uplift at the basemat cylinder junction vs. pressure

from results of continuum/shell Models 3 and 4

4.1 .36

Radial shear force 1 inch $(25 \mathrm{~mm})$ above the base of the

cylinder from results of continuum/shell Models 3 and 4

4.1 .37

Model 3 results for rebar strains vs. pressure 1 inch $(25 \mathrm{~mm})$

above the base of the cylinder

4.1 .38

Model 4 results for rebar strains vs. pressure 1 inch $(25 \mathrm{~mm})$

above the base of the cylinder

4.1 .39

Crushing parameter on the outside of the cylinder wall 3 inches

$(76 \mathrm{~mm})$ above the basemat from Models 3 and 4

4.2 .1

Loading Method for Dynamic Relaxation

4.2 .2

Sequence of Calculations Using Dynamic Relaxation

4.2 .3

Representation of Reinforcement in Concrete of the

Axisymmetric Continuum Element

4.2 .4

Representation of Reinforcement in Concrete of the

Axisymmetric Shell Element

4.2 .5

Discretization of Shell Model

4.2 .6

$4 \cdot 2.7$

Deformation of Vessel at Impending Failure

Radial Displacement of Liner and Vertical Displacement

Relative to Cylinder Base at EL 6'-0"

4.2.8 Radial Displacement of Liner and Vertical Displacement Relative to Cylinder Base at EL 11'-0"

4.2.9 Radial Displacement of Liner and Vertical Displacement Relative to Cylinder Base at EL 18'-0"

4.2.10 Radial Displacement of Liner and Vertical Displacement

Relative to Cylinder Base at EL 24'-0"

4.2.11 Maximum Principal Strain on Inside Liner Surface,

Axial Strain in Layer 5 Meridional Rebar,

and Axial Strain in Layer 6 Hoop Rebar at EL 6'-9" 
4.2.12 Maximum Principal Strain on Inside Liner Surface, Axial Strain in Layer 5 Meridional Rebar, and Axial Strain in Layer 6 Hoop Rebar at EL 13'-9"

4.2.13 Maximum Principal Strain on Inside Liner Surface, Axial Strain in Layer 5 Meridional Rebar, Axial Strain in Layer 6 Hoop Rebar, and Axial Strain in Layer 8 Seismic Rebar at EL 20'-0"

4.2.14 Maximum Principal Strain on Inside Liner Surface, Axial Strain in Layer 2 Meridional Rebar, and Axial Strain in Layer 5 Meridional Rebar at EL 2'-0.8"

4.2.15 Maximum Principal Strain on Inside Liner Surface, Axial Strain in Layer 2 Meridional Rebar, and Axial Strain in Layer 5 Meridional Rebar at EL 23'-11" 113

4.2 .16 Vertical Displacement of Liner at Dome Apex Vertical Displacement of Liner at $\phi=70 £$ Relative to Springline and Horizontal Displacement of Liner

4.2.18 Maximum Principal Strain on Inside Liner Surface, Axial Strain in Layer 2 Meridional Rebar, and Axial Strain in Layer 5 Meridional Rebar at $\phi=72^{\circ}$

4.2.19 Maximum Principal Strain on Inside Liner Surface, Axial Strain in Layer 4 Hoop Rebar, and Axial Strain in Layer 5 Meridional Rebar at $\phi=37^{\circ}$ Maximum Principal Strain on Inside Surface of Liner in Springline and Dome Region Discretization of Shell and Slab Model Radial Displacement of Liner at EL $2^{\prime}-2^{\prime \prime}$

4.2 .23 Radial Displacement of Liner at EL 4'-1"

4.2 .24 Vertical Displacement (Uplift) Relative to Center of Basemat at EL 2'-0"

4.2.25 Discretization of Refined Shell and Slab Model

4.2.26 Finite Element Mesh of 50 Degree Segment Model of 1/6th Scale Concrete Containment Test

4.2.27 Radial Displacement of Liner and Vertical Displacement

Relative to Cylinder Base at EL $24^{\prime}-0^{\prime \prime}$ and $\theta=180^{\circ}$ Change in Sleeve Horizontal and Vertical Diameters of Equipment Hatch $B$

4.2.31 Maximum Principal Strain on Inside Liner Surface at EL 13'-0" Adjacent to Equipment Hatch

4.3 .1 Compressive Uniaxial Stress-Strain

4.3 .2

Analys is C-3 Deformed Grid at 100 psi (0.69 $\mathrm{MPa}$ )

4.3 .3

C-3 Analysis Liner Meridional Strain at 60,100 , and 140 psi $(0.41,0.69$, and $0.97 \mathrm{MPa}$ )

4.3.4 C-3 Analysis Liner Hoop Strain at 50, 60, and 80 psi $(0.34,0.41$, and $0.55 \mathrm{MPa})$ 
4.3.8 C4B Analysis Computational Grid

Page

4.3.9 C4B Analysis - Deformed Shape and Meridional Strain Profile

160

4.3 .10

Outside and Inside Hidden Line View of Global Quarter Model

Model Includes $270^{\circ}$ to $0^{\circ}$ and $E L=3^{\prime}-3^{\prime \prime}$ to the Dome Apex

161

4.3.11 Outside View of Quarter Model Grid With Hoop, Meridional, Seismic and Extra Rebar Layers

162

Global Quarter Model Deformed Shapes

4.3 .12

Deformed Concrete Outlines for $\theta=0^{\circ}, 315^{\circ}$, and $270^{\circ}$

4.3 .13

at $136 \mathrm{psi}(0.94 \mathrm{MPa})$

4.3.14 Diametral Length Change Histories for Hatch-A and Personnel Airlock Sleeves

4.3.15 Computational Grids for Equipment Hatches $A$ and $B$ Local Analyses

4.3 .16

Local Hatch Maximum Principal Liner Strain Profiles at 150 psi ( $1.03 \mathrm{MPa}$ )

4.3.17 Pressure Nozzle and Constrained Pipe Penetration Local

Analysis Deformed Grids at 104 psi (0.72 MPa)

4.3 .18

4" Pressure Nozzle and 8" Penetration Local Analyses Maximum

Principal Liner Strain Profiles at $150 \mathrm{psi}$ (1.03 MPa)

163

164

165

C4B Analysis Deformed Grid (Basemat) at $140 \mathrm{psi}(0.97 \mathrm{MPa})$

4.3 .19

C4B Analysis Progression of Basemat Liftoff from 0 to 140 psi (0 to $0.97 \mathrm{MPa}$ )

4.3 .20

4.4 .1

Mesh for calculation 1 (rigid soil)

4.4 .2

Mesh for calculation 2 (elastic soil)

4.4 .3

4.4.4A

$4.4 .4 \mathrm{~B}$

4.4 .5

4.4 .6

Mesh of the rebars modelization

167

Comparison between real and modeled uniaxial concrete curves

168

Location of nodes for radial and axial displacements

Deformed structure at $29 \mathrm{psi}(0.2 \mathrm{MPa})$ (calculation 1)

169

4.4 .7

Deformed structure at 43.5 psi $(0.3 \mathrm{MPa})$ (calculation 1)

170

171

4.4 .8

Deformed structure at 58 psi (0.4 MPa) (calculation 1)

171

181

182

183

184

185

186

187

Deformed structure at 72.5 psi $(0.5 \mathrm{MPa})$ (calculation 1)

188

4.4 .9

Deformed structure at $79.8 \mathrm{psi}(0.55 \mathrm{MPa})$ (calculation 1 )

189

4.4 .10

4.4 .11

4.4 .12

Deformed structure at $116 \mathrm{psi}(0.8 \mathrm{MPa})$ (calculation 1)

190

Deformed structure at 130.5 psi $(0.9 \mathrm{MPa})$ (calculation 1 )

191

4.4 .13

Radial displacement versus pressure (calculation 1)

193

4.4 .14

Axial displacements versus pressure (calculation 1 )

194

195

4.4 .15

4.4 .16

4.4 .17

Meridional crack pattern at $29 \mathrm{psi}(0.2 \mathrm{MPa}$ ) (calculation 1)

196

Meridional crack pattern at 43.5 psi (0.3 MPa) (calculation 1) 197

Meridional crack pattern at $72.5 \mathrm{psi}(0.5 \mathrm{MPa})$ (calculation 1) 198

4.4 .18

Meridional

crack pattern at 79.8 psi (0.55 MPa)

(calculation 1)

4.4.19 Meridional crack pattern at 92.8 psi (0.64 MPa)

(calculation 1)

199

4.4 .20

Meridional crack pattern at

$142 \mathrm{psi}(0.98 \mathrm{MPa})$

(calculation 1)

4.4 .21

4.4 .22

4.4 .23

4.4 .24

4.4.25A

Hoop crack pattern at 43.5

5 psi (0.3 MPa) (calculation 1)

Hoop crack pattern at 72.5 psi $(0.5 \mathrm{MPa})$ (calculation 1 )

Hoop crack pattern at 79.8 psi $(0.55 \mathrm{MPa})$ (calculation 1 )

Hoop crack pattern at 92.8 psi $(0.64 \mathrm{MPa})$ (calculation 1 )

Hoop crack pattern at 142 psi $(0.98 \mathrm{MPa})$ (calculation 1)

Shear crack pattern at 142 psi ( $0.98 \mathrm{MPa})$ (calculation 1) 
4.4.25C Plastic strains in the liner (calculation 1)

4.4 .26

Deformed structure under dead weight (calculation 2)

4.4 .27

Deformed structure at 29 psi $(0.2 \mathrm{MPa}$ ) (calculation 2)

4.4 .28

Deformed structure at $43.5 \mathrm{ps}$ i $(0.3 \mathrm{MPa}$ ) (calculation 2)

210

4.4 .29

Deformed structure at 58 psi $(0.4 \mathrm{MPa})$ (calculation 2)

211

4.4 .30

Deformed structure at 72.5 psi $(0.5 \mathrm{MPa})$ (calculation 2)

212

4.4 .31

Deformed structure at $79.8 \mathrm{psi}(0.55 \mathrm{MPa})$ (calculation 2)

213

4.4 .32

Deformed structure at 116 psi (0.8 MPa) (calculation 2)

214

4.4 .33

Deformed structure at 130.5 psi $(0.9 \mathrm{MPa})$ (calculation 2)

215

4.4 .34

4.4 .35

Deformed structure at $142 \mathrm{psi}(0.98 \mathrm{MPa})$ (calculation 2)

216

4.4 .36

4.4 .37

218

Meridional crack pattern at 43.5 psi (0.3 MPa) (calculation 2) 219

Meridional crack pattern at 58 psi $(0.4 \mathrm{MPa})$ (calculation 2) 220

4.4 .38

Meridional crack pattern at $72.5 \mathrm{psi}(0.5 \mathrm{MPa})$ (calculation 2) 221

4.4 .39

4.4 .40

Meridional crack pattern at $87 \mathrm{psi}(0.6 \mathrm{MPa})$ (calculation 2)

222

Meridional crack pattern at $142 \mathrm{psi}(0.98 \mathrm{MPa})$ (calculation 2) 223

4.4 .41

4.4 .42

4.4 .43

4.4 .44

Hoop crack pattern at $43.5 \mathrm{psi}(0.3 \mathrm{MPa})$ (calculation 2)

224

Hoop crack pattern at 58 psi $(0.4 \mathrm{MPa})$ (calculation 2) 225

Hoop crack pattern at 72.5 psi (0.5 MPa) (calculation 2) 226

4.4 .45

4.4 .46

Hoop crack pattern at $92.8 \mathrm{psi}(0.64 \mathrm{MPa}$ ) (calculation 2)

227

Hoop crack pattern at 142 psi $(0.98 \mathrm{MPa})$ (calculation 2) 228

Shear crack pattern at $116 \mathrm{psi}(0.8 \mathrm{MPa})$ (calculation 2) 229

4.4 .47

Shear crack pattern at 142 psi $(0.98 \mathrm{MPa}$ ) (calculation 2 )

230

4.4 .48

Radial displacements versus pressure (calculation 2)

231

4.4 .49

4.4 .50

4.5 .1

Axial displacements versus pressure (calculation 2)

$23 \hat{2}$

Plastic strains in the liner (calculation 2)

233

4.5 .2

Axisymmetric and 3-D Quarter Models

252

4.5 .3

Nonl inear Analysis

253

4.5 .4

Steel and Concrete Stress-strain Relationships

254

Moment Curvature Program Calibration

255

4.5 .5

Flowchart : Main Program

256

Softening Elements for Meridional Bending

257

4.5 .7

Flowchart : Softening of the Meridional Moment

258

4.5 .8

Softening Elements for Meridional Strain

259

4.5 .9

Flowchart : Softening of the Meridional Strain

260

4.5.10

Softening Elements for Hoop Stress

261

4.5 .11

Flowchart : Softening of the Hoop Forces

262

Flowchart : Liner Softening

263

4.5 .12

Meridional Bending Moments

264

4.5 .14

Meridional Curvature

265

4.5 .15

Radial Shear Force

266

4.5 .16

4.5 .17

Hoop Stress

267

4.5 .18

Comparison of Meridional Moments - Axisymmetric vs 3-D Models

268

Displaced Shape

4.5 .19

Displaced Shape

269

Displaced Shape

270

4.5 .20

Wall Base Shear Force

271

4.5 .21

4.6 .1

Concrete elements mesh

272

4.6 .2

Truss elements mesh (rebars and liner)

283

4.6 .3

Detail of truss elements at the cylinder wall base

284

Displacements at $p=3 P d$

285

4.6 .4

Displacement at $p=4 \mathrm{Pd}$

286

4.6 .5

287 
Cylinder displacements at EL 11'-0" (detail)

Uniaxial Steel Properties

4.8.8:

4.8.9:

Rebar \#4 Modified Stress-Strain Curve (Analysis)

thar H4 Seismic Reinforcement

4.8.11:

Modified Stress-Strain Curve (Analysis)

General Configuration of Containment

Finite Element Configuration Used in Dome Section 
4.9.19

4.9.20

4.9 .21

4.9 .22

4.9 .23

4.9 .24

4.9 .25

4.9 .26

4.10 .1

4.10 .2

4.10 .3

4. 10.4

4.10 .5

4.10.6

4.10 .7

4.10 .8

4.10 .9

4.10 .10

4.10 .11

4.10 .12

4.10 .13

4.10 .14

4.10 .15

4.10 .16

4.10 .17

4.10 .18

4.10 .19

4.10 .20

4.10 .21

4.10 .22

4.10 .23

4.10 .24

4.10 .25

4.10 .26

4.10 .27

4.10 .28

4.10 .29

4.10 .30

4.10 .31

4.10 .32

4.10 .33

4.10 .34

4.10 .35

4.10 .36

4.10 .37

4.10 .38

Deformed Configuration at 104 psi $(0.717 \mathrm{MPa})$

Page

Deformed Configuration at $131 \mathrm{ps} i(0.903 \mathrm{MPa})$

Computed Vertical Displacements Along Structure

368

Radial Displacements Along Cylinder Wall

369

Hoop Strains Developed in Hoop Rebar

Strains in Meridional Steel in Layer 5 (Outer Layer)

370

370

371

Strains in Meridional Steel in Layer 2 (Inner Layer) 372

Comparison of Strains in Meridional Steel

373

Summary of 1/6th Scale Model Ultimate Load Analysis 388

ADINA-TW Axisymmetric Model

Wall Reinforcement and Liner -

Actual and as Modelled in ADINA-TW

389

Axisymmetric Model with Penetration Thickening (Hatch A)

390

Axisymmetric Model with Penetration Thickening (Hatch B)

392

393

3-D Linear Elastic Model

394

395

Triaxial Compressive Failure Envelopes

396

Triaxial Tensile Failure Envelope

Shear Retention Factor

Effective Tensile Stiffening

397

Uniaxial Stress-strain Relationship for Concrete

Modification of Uniaxial Stress-strain Relationship for

Multiaxial Conditions

Derivation of $\gamma_{1}$ from the Triaxial Compressive

Failure Envelope

398

399

Poisson's Ratio

Stress-strain Relationship for Reinforcement

400

Stress-strain Relationship for Liner

Soil Stiffness

Force Balancing Method, Internal Pressure

vs Radial Displacement

Nonlinear Axisymmetric Analysis Displacement Results in Cylinder Wall

Nonlinear Axisymmetric Analysis Displacement Results in Dome

Displaced Shape at $0.345 \mathrm{MPa}$ (50 psi)

Displaced Shape at $0.366 \mathrm{MPa}$ (53 psi)

Displaced Shape at $0.517 \mathrm{MPa}$ (75 psi)

Displaced Shape at $0.690 \mathrm{MPa}$ (100 psi)

Displaced Shape at $0.897 \mathrm{MPa}$ (130 psi)

Displaced Shape at $0.959 \mathrm{MPa}$ (139 psi)

Displaced Shape at $1.103 \mathrm{MPa}$ (160 psi)

Wall/Base Junction Crack Pattern at $0.317 \mathrm{MPa}$ (46 psi)

Wall/Base Junction Crack Pattern at $0.366 \mathrm{MPa}$ (53 psi)

Wall/Base Junction Crack Pattern at $0.421 \mathrm{MPa}$ (61 psi)

Wall/Base Junction Crack Pattern at $0.566 \mathrm{MPa}$ (82 psi)

Wall/Base Junction Crack Pattern at $0.690 \mathrm{MPa}$ (100 psi)

Wall/Base Junction Crack Pattern at $1.000 \mathrm{MPa}$ (145 psi)

Wall/Base Junction Crack Pattern at $1.100 \mathrm{MPa}$ (159.5 psi)

401

402

403

404

405

Wall/Base Junction Crack Pattern at $1.103 \mathrm{MPa}$ (160 psi)

406

Progressive Vertical Crack Pattern in Cylindrical Wall

Progressive Vertical Crack Pattern in Dome

407

408

409

410

411

412

413

414

415

416

417

418

419

420

421

422

423

424

425 
4.10.39 Progressive Horizontal Crack Pattern $2.5 \mathrm{~m}$ Above Base 426

4.10.40 Wall Liner Hoop Stresses vs Pressure 427

4.10.41 Wall Liner Vertical Stresses vs Pressure 428

4.10.42 Dome Liner Hoop and Radial Stresses vs Pressure 429

4.10.43 Rebar Hoop Stresses in Wall vs Pressure 430

4.10.44 Hoop Reinforcement in Dome Stress vs Pressure 431

4.10.45 Base Liner Plate Radial Strain at Wall/Base Junction vs Pressure

4.10.46 Displaced Shape of Barrel Thickened Section (Hatch A) at $0.1 \mathrm{MPa}$ ( $14.5 \mathrm{psi}$ )

4.10.47 Displaced Shape of Barrel Thickened Section (Hatch B) at $0.1 \mathrm{MPa}$ (14.5 psi)

4.10.48 Comparison of Displacements from an Unthickened Model to Those with Penetration Thickenings

4.10.49 Linear Elastic 2-D Analysis Displacement Results

4.10.50 Linear Elastic 3-D Analysis Displacement Results at 4.44 m Elevation

4.10.51 Linear Elastic 3-D Analysis Displacement Results

Along Vertical Sections of Model
4.10 .52 Comparison of 3-D Displacements with

434

435

436

437

Linear Elastic 2-D Displacements

4.10.53 Comparison of 3-D Displacements with

Linear Elastic Axisymmetric Displacements

A.1 Vertical displacement (uplift) relative to center of basemat EL $2^{\prime}-0 ", r=10^{\prime}-9 ", \theta=85$

A.2 Radial displacement of liner at EL $2^{\prime}-2^{\prime \prime}, \theta=180$

A.3 Radial displacement of liner at $E L 4^{\prime}-1^{\prime \prime}, \theta=180$

A. 4

A. 5

Radial displacement of liner at EL $6^{\prime}-0^{\prime \prime}, \theta=225$

Vertical displacement relative to cylinder base at $E L 6^{\prime}-0^{\prime \prime}, \theta=225$

A.6 Radial displacement of liner at EL 11'-0", $\theta=225$

A.7 Vertical displacement relative to cylinder base at $\mathrm{EL} 11^{\prime}-0^{\prime \prime}, \theta=225$

A.8 Radial displacement of 1 iner at EL $18^{\prime}-0^{\prime \prime}, \theta=225$

A.9 Vertical displacement relative to cylinder base at $E L 18^{\prime}-0^{\prime \prime}, \theta=225$

A.10 Radial displacement of 1 iner at EL $24^{\prime}-0$ ",$\theta=180$

A.11 Vertical displacement relative to cylinder base

A.12 Maximum principal strain on inside liner surface at $\mathrm{EL} 6^{\prime}-9^{\prime \prime}, \theta=45$

A. 13

A. 14

A. 15

Axial strain in layer 5 meridional rebar at EL $6^{\prime}-9 ", \theta=45$ Maximum principal strain on inside liner surface at EL $20^{\prime}-0^{\prime \prime}, \theta=45$ 
A.22 Maximum principal strain on inside liner surface

Page

A. 23 Axial strain in layer 2 meridional rebar
A 23 (jol

at EL 2'-0.8", $\theta=90$ (just above knuckle)

A. 24

Axial strain in layer 5 meridional rebar

at EL 2'-0.8", $\theta=90$ (just above knuckle)

A. 25

Maximum principal strain on inside liner surface

at $E L 23^{\prime}-11^{\prime \prime}, \theta=90$

A.26 Axial strain in layer 2 meridional rebar

at EL $23^{\prime}-11^{\prime \prime}, \theta=90$

A.27 Axial strain in layer 5 meridional rebar

at EL $23^{\prime}-11^{\prime \prime}, \theta=90$

A. 28

Vertical displacement of liner at dome apex, $\phi=90$

A.29 Vertical displacement of liner relative to springline, $\phi=70, \theta=90$

A. 30

Horizontal displacement of liner, $\phi=70, \theta=90$

A. 31

Maximum principal strain on inside liner surface, $\phi=72, \theta=90$

A.32 Axial strain in layer 2 meridional rebar, $\phi=72, \theta=90$

474

475

476

477

478

479

480

481

482

483

484

485

A. 34

Axial strain in layer 5 meridional rebar, $\phi=72, \theta=90$

Maximum principal strain on inside liner surface, $\phi=37, \theta=90$

A.35 Axial strain in layer 4 hoop rebar, $\phi=37, \theta=90$

A. 36

A. 37

Axial strain in layer 5 meridional rebar, $\phi=37, \theta=90$

Horizontal displacement of inside edge of equipment hatch $B$

sleeve at midthickness (top) at EL $14^{\prime}-7^{\prime \prime}, \theta=180$

A. 38 Radial displacement of liner at EL $20^{\prime}-1.5^{\prime \prime}, \theta=312.5$ (constrained pipe)

A.39 Radial displacement of liner at EL 20'-1.5", $\theta=305$ (constrained pipe)

A.40 Radial displacement of liner at EL 20'-1.5", $\theta=290$ (constrained pipe)

A.41 Maximum principal strain on inside liner surface at EL $13^{\prime}-0^{\prime \prime}, \theta=158$

A.42 Axial strain in layer 2 meridional rebar at EL 13'-0", $\theta=158$

A.43 Axial strain in layer 3 hoop rebar at $E L 13^{\prime}-0^{\prime \prime}, \theta=158$

486

487

488

489

489

490

490

491

492

492

A.44 Change in sleeve horizontal diameter (Equipment hatch $B$ at mid-thickness of cylinder wall)

A.45 Change in sleeve vertical diameter (Equipment hatch $B$ at mid-thickness of cylinder wall)

493

493

A.46 Maximum principal strain on inside surface of liner vs position (knuckle region) at $100 \mathrm{psi}(0.689 \mathrm{MPa}$ )

494

A.47 Maximum principal strain on inside surface of liner vs position (knuckle region) at $150 \mathrm{psi}(1.034 \mathrm{MPa}$ )

494

Maximum principal strain on inside surface of liner vs position (springline and dome region) at $100 \mathrm{psi}(0.689 \mathrm{MPa})$

A. 50

Maximum principal strain on inside surface of liner vs position (springline and dome region) at $150 \mathrm{psi}$ (1.034 MPa)

A. 52 of layer 11 rebar) at 100 psi (0.689 MPa) 
A.53 Radial shear vs pressure (cylinder from basemat to termination of layer 11 rebar) at 150 psi ( $1.034 \mathrm{MPa}$ )

A.55 Meridional moment vs pressure (cylinder from basemat to termination of layer 11 rebar) at $100 \mathrm{psi}(0.689 \mathrm{MPa})$

A.56 Meridional moment vs pressure (cylinder from basemat to termination of layer 11 rebar) at $150 \mathrm{psi}(1.034 \mathrm{MPa})$

A.58 Axial force vs pressure (cy)inder from basemat to termination of layer 11 rebar) at $100 \mathrm{psi}(0.689 \mathrm{MPa})$

A.59 Axial force vs pressure (cylinder from basemat to termination of layer 11 rebar) at 150 psi ( $1.034 \mathrm{MPa}$ )

A.61 Hoop force vs pressure (cylinder from basemat to termination of layer 11 rebar) at $100 \mathrm{psi}(0.689 \mathrm{MPa})$

A.62 Hoop force vs pressure (cylinder from basemat to termination of layer 11 rebar) at $150 \mathrm{psi}(1.034 \mathrm{MPa})$

Radial shear vs position (springline region) at $100 \mathrm{psi}(0.689 \mathrm{MPa})$

A.65 Radial shear vs position (springline region) at $150 \mathrm{psi}(1.034 \mathrm{MPa})$

A.67 Meridional moment vs position (springline region) at 100 psi $(0.689 \mathrm{MPa})$

A.68 Meridional moment vs position (springline region) at $150 \mathrm{psi}(1.034 \mathrm{MPa})$

Axial force vs position (springline region)

A.71 Axial force vs position (springline region) at $150 \mathrm{psi}(1.034 \mathrm{MPa})$

A.73 Hoop force vs position (springline region) at 100 psi $(0.689 \mathrm{MPa})$

A.74 Hoop force vs position (springline region)

A.76 Maximum principal strain on inside liner surface at $E L 13^{\prime}-0^{\prime \prime}$ vs $\theta$ (adjacent to equipment hatch $B$ ) at $100 \mathrm{psi}(0.689 \mathrm{MPa}$ )

A.77 Maximum principal strain on inside liner surface at EL 13'-0" vs $\theta$ (adjacent to equipment hatch B) at $150 \mathrm{psi}(1.034 \mathrm{MPa}$ ) 503

A.79 Maximum principal strain on inside liner surface at EL 29'-1.5" vs $\theta$ (adjacent to constrained pipe) at $100 \mathrm{psi}(0.689 \mathrm{MPa}$ ) 504 A.80 Maximum principal strain on inside liner surface at EL $29^{\prime}-1.5^{\prime \prime}$ vs $\theta$ (adjacent to constrained pipe) at $150 \mathrm{psi}$ (1.034 MPa) 505

B.1 Assumed Stress-Strain Relationships

\section{Table}

1.1

1.2

1.3

3.1

3.2

3.3

3.4

3.5

3.6

4.1.1
Pressure History Milestones (Global Response)

Milestones in Local Response at Basemat Cylinder Junction

$\underline{\text { Page }}$

Failure Predictions for the 1:6-Scale Containment Model

Concrete Material Properties

Cylinder and Basemat Liner Properties

Dome Liner Properties

Rebar Material Properties

Liner Thicknesses

Mill Properties for Reinforcing Bar

Analys is Summary
3

5 
4.1 .2

4.1 .3

4.1 .4

4.1 .5

4.1 .6

4.1 .7

4.1 .8

4.1 .9

$4 \cdot 1 \cdot 10$

4.1 .11

4.2.1

4.2 .2

4.2 .3

4.2 .4

4.2 .5

4.2 .6

4.2 .7

4.2 .8

4.3.1

4.3.2

4.3 .3

4.3 .4

4.5 .1

4.5 .2

4.7 .1

4.7 .2

4.8 .1

4.8 .2

4.8 .3

4.8 .4

4.8 .5

4.8 .6

4.8 .7

4.8 .8

4.10 .1

4.10 .2

Material Properties for Cylinder and Basemat Liner

Material Properties for Dome Liner

Rebar Properties Based on Nominal Cross-Sectional Areas

Material Parameters used with the Concrete Constitutive Mode 1 in ABAQUS

Nominal Cross-sectional Areas of Reinforcing Bars

in the Containment Model

Lengths and Thicknesses of Shell Elements Used in Each Region of the Finite Element Mesh in Models 1 and 2

Maximum and Minimum Step Sizes and Convergence Tolerance

Specified for the Model 1 and 2 Analyses
Pressures at which Yielding Occurs in the Liner and Rebars at the Midheight of the Cylinder

Comparison of Shear Stress from Model 2 Analysis to Shear Strength at $170 \mathrm{ps}$ i (1.2 MPa) Internal Pressure

Maximum and Minimum Step Sizes and Convergence Tolerances Specified for the Model 3 and 4 Analyses

Concrete Material Properties

56

Rebar Material Properties

Cylinder and Basemat Liner Material Properties

Dome Liner Material Properties

Parameter Defining the Failure Surface of Concrete

84

85

Response of Shell to Pressurization

85

Response of Refined Model of Shell/Slab Junction to Pressurization

Response of Three-Dimensional Model to Pressurization

Compression Failure Envelopes

Special Concrete Parameters Needed for ADINA-Concrete Model 317

Rebar Material Properties 
This report would not have been possible without the cooperation and efforts of a large number of individuals and groups. Sandia and the USNRC are extremely grateful to the following individuals and their organizations, whose contributions were made in a spirit of volunteerism and engineering advancement:

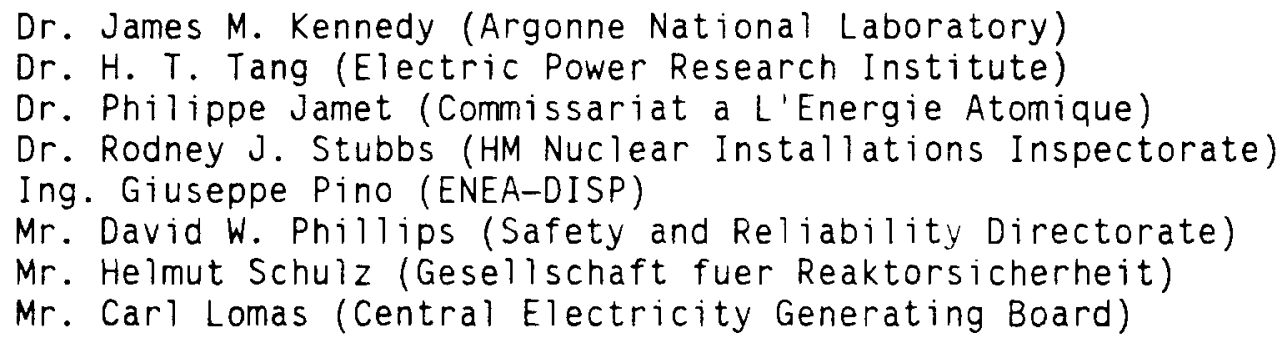

These organizations received no financial support from Sandia or the USNRC, and their efforts are gratefully acknowledged.

Acknowledgment is also made to $\mathrm{Dr}$. J. Randy Weatherby, who conducted the Sandia analysis with little additional assistance. His tireless efforts in developing models, running calculations, interpreting results and investigating failure criteria are much appreciated. Mr. Dan Horschel deserves a large share of the credit for successful construction and instrumentation of the model and material properties testing.

Thanks are also due to Mr. Douglas Brinson, for his assistance in the preparation of figures and the plots in Appendix A, and to Mrs. Ruby Cochrell, for her assistance in obtaining typing services and editing the report.

Finally, thanks are extended to Dr. Walter von Riesemann and Dr. James Costello who spent many days and weeks "on the beat" soliciting and encouraging organizations to participate in this round-robin exercise. 


\section{EXECUTIVE SUMMARY}

Testing and analysis of scale models of containment buildings that are pressurized to failure are being conducted at Sandia National Laboratories as part of the Containment Integrity Programs sponsored by the U.S. Nuclear Regulatory Commission (NRC). The overall objective of the programs is to develop test validated methods that can be used to predict the performance of light water reactor containment buildings subject to loads beyond the design basis. Five scale steel containment models have already been tested; as a result, analytical methods appropriate to steel containment buildings were validated. Currently, final preparations for testing a 1:6-scale reinforced concrete containment model are being completed.

Pretest analyses of the 1:6-scale reinforced concrete model containment model have been conducted by a number of organizations in the United States and Europe in order to predict the response and failure of the model caused by static internal pressurization. The organizations that have participated in the pretest analysis and have submitted descriptions of their analysis and results are listed below:

Sandia National Laboratories (SNL), United States

Argonne National Laboratory (ANL), United States

Electric Power Research Institute (EPRI), United States

Commissariat a L'Energie Atomique (CEA), France

HM Nuclear Installations Inspectorate (NII), United Kingdom

Comitato Nazionale per la ricerca e per lo sviluppo dell'Energia

Nucleare e delle Energie Alternative (ENEA-DISP), Italy

U.K. Atomic Energy Authority - Safety and Reliability Directorate (SRD)

Gesellschaft fuer Reaktorsicherheit (GRS), Federal Republic of Germany

Brookhaven National Laboratory (BNL), United States

Central Electricity Generating Board (CEGB), United Kingdom

The model will be pressurized to failure using nitrogen gas at ambient temperature in the spring of 1987 at SNL. Figure 1.1 is a photograph of the model and the test site.

SNL and NRC invited organizations to participate in the pretest analysis beginning in early 1986; the analytical effort was coordinated by SNL. SNL has attempted to encourage independent modelling efforts while trying to maintain consistent input. Each organization was supplied with the same basic information, which included construction drawings, specifications, and as-measured material properties.

Several benefits were expected from the round-robin analysis. First, because of the large number of analytical approaches and interpretations, a greater number of potential limit states have been recognized. As a result, the instrumentation plan for the model is more thorough and SNL's ability to anticipate and respond to events during the high pressure test has been enhanced. Second, a measure of the uncertainty in pretest predictions will be obtained by comparisons of different analyses as well as comparisons between analytical and experimental results. Third, a iarge number of state-of-the-art finite element codes have been applied to the probiem, including ABAQUS, ADINA, CASTEM, NEPTUNE, NFAP, PAFEC, and TEMP-STRESS, which will make it possible to evaluate the suitability of these codes for 
nonlinear analysis of reinforced concrete structures. Finally, the roundrobin exercise has led to greater recognition of the importance of containment performance and reliable prediction techniques; it has also facilitated the exchange of information on these and other related topics.

This report describes the pretest analyses and compares the results produced by the participating organizations. Background information on the containment integrity programs and on the scale model analyses is given in Section 2. The geometry and material properties of the reinforced concrete containment model are discussed in Section 3. The heart of the report is contained in Section 4, which consists of subsections written by each organization participating in the round robin. These subsections describe the analytical approach, results, and conclusions reached by each group regarding the behavior of the model.

In addition, each organization was asked to submit results at certain locations for response measures, such as strain and displacement, as a function of pressure. These are referred to as the standard plots, and they appear in Appendix $A$. The locations specified in the standard plots correspond to locations where instrumentation was placed to enable comparisons of different analyses with each other and directly with the experimental data.

At this time, comments on the comparison between different analyses are limited primarily to observations. A true evaluation of the accuracy of the analyses can be made only in light of the experimental results. As discussed later in this report, SNL and, no doubt, many or most of the other organizations will follow through with posttest analyses and assessments of the strengths and weaknesses of the different analytical approaches that have been taken.

The predicted pressure levels at which certain damage mechanisms initiate in areas characterized by membrane behavior (global response) are shown in Table 1.1. Hoop cracking refers to cracks extending in the meridional direction, i.e., cracks that result from circumferential (hoop) stress. Similarly, meridional cracking implies cracks that lie in a plane perpendicular to the surface, i.e., cracks that are due to meridional stress. Although there are differences in the predicted pressure levels at which cracking initiates, all of the analyses predict widespread cracking before the attainment of any limit states. Also, all organizations expect hoop cracking in the cylinder to begin below or near the model design pressure of $46 \mathrm{psig}(0.317 \mathrm{MPa})$. It should be noted that there are significant variations in the concrete constitutive models used by different organizations; in a few cases, cracking was not explicitly modelled.

The initiation of general yielding of the steel liner is predicted over a fairly wide range of pressure, from 82 to 115 psig (.565 to .793 MPa). The variation is at least partly attributable to differences in the analytical models of the liner; some of the models do not treat the biaxial state of stress in the liner because the hoop and meridional stiffness are represented with separate elements. The yield pressure calculated based on a uniaxial state of stress may be $15 \%$ lower than that calculated using the von Mises yield criterion for a biaxial state of stress in which the principal stresses are both tensile. A second difficulty is introduced by the concrete, which has cracked but probably still retains some tensile load 
carrying capability in the pressure range over which liner yielding is predicted. There is great uncertainty and thus wide differences in the analytical modelling of how the tensile capability of the concrete decreases after cracking. Clearly, the calculated liner yield pressure will depend on how much tensile load carrying capability the concrete is allowed to retain in the analytical model after cracking, and the value of strain at which the tensile load carrying capability of the concrete is assumed to go to zero.

Table 1.1

Pressure History Milestones (Global Response)

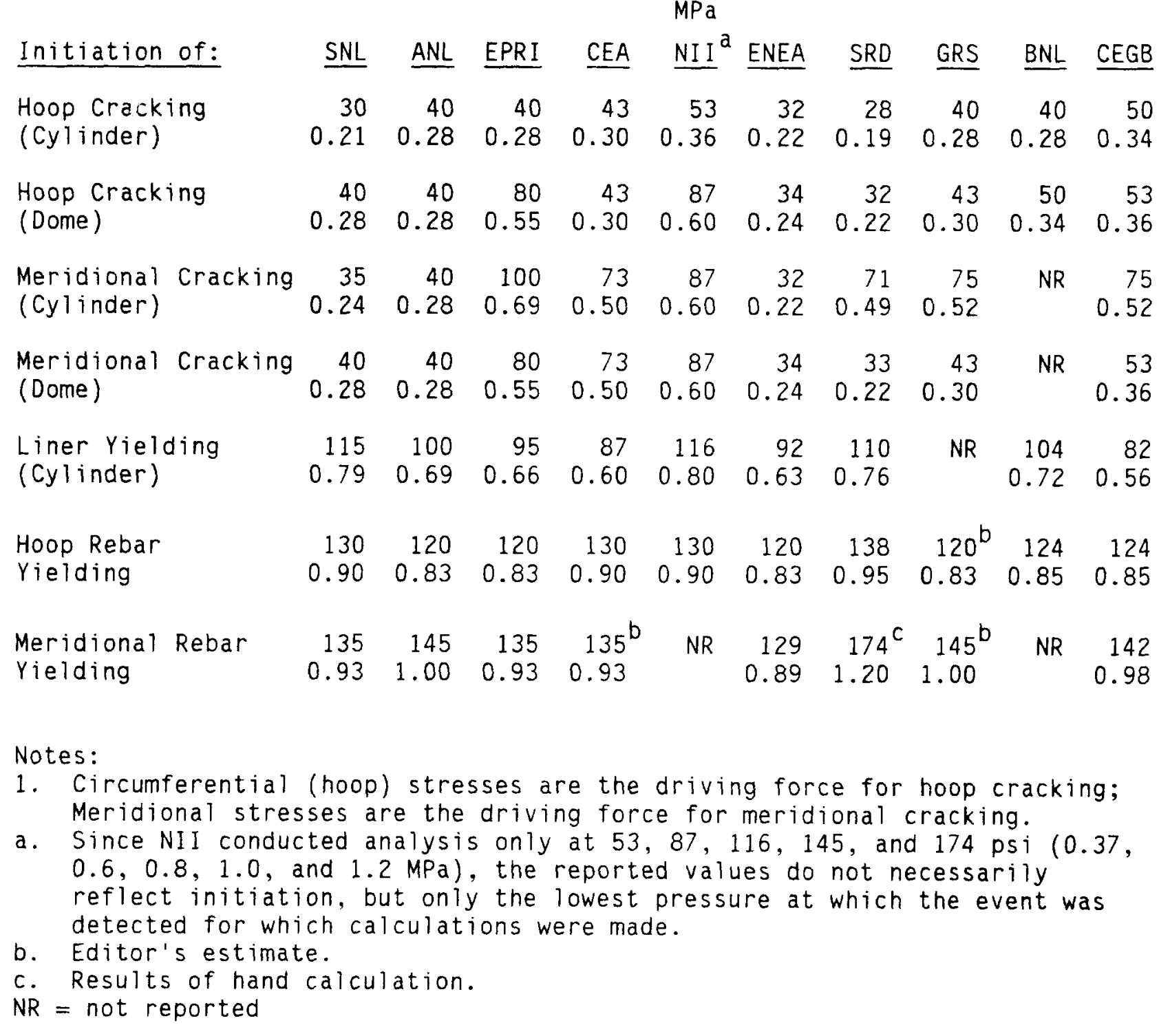


In contrast to those for the liner, the calculations for initiation of general yielding in the hoop rebar (deformed reinforcing steel) produce quite similar results, with most predictions falling in a range between 120 and $130 \mathrm{psig}(0.827$ and $0.896 \mathrm{MPa})$. With the exception of SRD, which reported results from a hand calculation, the results for meridional rebar yielding are also quite similar. (A number of organizations did not calculate a pressure at which meridional rebars would begin to yield because they predicted failure at a lower pressure.) Since the rebars are essentially uniaxial load carrying members, they are much simpler to model analytically. Furthermore, in the pressure ranges for which general yielding of the rebars begins, nearly all of the analytical models assume that the concrete cannot carry any tensile load. From this standpoint, the agreement is not surprising.

The radial displacement of the cylinder near midheight away from penetrations is shown in Figure 1.2 as a function of pressure. Figure $1.2 a$ shows the details of the response at low pressure; hoop cracking in the concrete causes a sudden increase in displacement with little or no increase in pressure. Some of the calculations did not explicitly model concrete cracking (e.g., SNL and NII) and thus a sudden increase in displacement is not observed. The onset of rapidly increasing radial displacement seen in Figure 1.2b corresponds to general yielding of the wall, including both hoop and seismic rebars. The vertical displacement of the cylinder at midheight relative to the base of the cylinder (Figure 1.3) does not exhibit the single sudden increase that characterizes the radial displacement. The difference is due to the fact that meridional cracks develop over a relatively wide pressure range, whereas the hoop cracks occur almost simultaneously throughout the cylinder. Note also that the magnitude of the radial displacement is significantly greater than the vertical displacement, even after general yielding.

While global response is a useful and interesting measure of behavior, there is the possibility that local response may govern failure. For instance, the liner is subject to local strain concentrations at discontinuities, which may result in small tears in the liner and loss of integrity. This hypothesis has been considered in some depth by EPRI and to a lesser extent by several other organizations including SNL. As another example, the shear strength of the wall may be exceeded due to localized shear forces and bending moments, which predominantly occur at the basemat cylinder wall junction.

Milestones in the local response at the basemat cylinder junction are recorded in Table 1.2. Meridional cracking of the concrete on the inside face is expected at very low pressure and should be the first part of the model to experience damage. The meridional rebar at the junction yields before general yielding of the hoop rebar. There was considerable difference in the predictions with regard to basemat uplift. About half the analysts calculated uplift in excess of $1 / 2$ inch $(12.7 \mathrm{~mm})$ as indicated in Table 1.2; the remainder did not predict significant uplift (see also Figure A.1). Many analysts predicted crushing of the concrete on the outer face of the wall, which significantly reduces the shear and moment resistance.

The "best estimate" capacity of the model and the limit state mechanism predicted by each organization are compared in Table 1.3. The predicted capacity varies from 128 to 190 psig ( 0.800 to $1.310 \mathrm{MPa})$, which is 
Table 1.2

Milestones in Local Response at Basemat Cylinder Junction

\begin{tabular}{|c|c|c|c|c|c|c|c|c|c|c|}
\hline & & & & & $\begin{array}{c}\text { Pressu } \\
\text { psig } \\
\text { MPa }\end{array}$ & & & & & \\
\hline Initiation of: & $\underline{S N L}$ & ANL & EPRI & CEA & ${ }^{a}$ NII & ENEA & SRD & GRS & BNL & $\underline{\text { CEGB }}$ \\
\hline $\begin{array}{l}\text { Meridional } \\
\text { Cracking }\end{array}$ & NR & $\begin{array}{r}25 \\
0.17\end{array}$ & $\begin{array}{r}30 \\
0.21\end{array}$ & $\begin{array}{r}29 \\
0.20\end{array}$ & $\begin{array}{r}53 \\
0.37\end{array}$ & $\begin{array}{r}18 \\
0.12\end{array}$ & NR & $\begin{array}{r}29 \\
0.20\end{array}$ & $\begin{array}{r}30 \\
0.21\end{array}$ & $\begin{array}{r}20 \\
0.14\end{array}$ \\
\hline $\begin{array}{l}\text { Liner } \\
\text { Yielding }\end{array}$ & NR & $\begin{array}{r}105 \\
0.72\end{array}$ & $\begin{array}{c}60 \\
0.41\end{array}$ & NR & $\begin{array}{l}110^{b} \\
0.76\end{array}$ & $\begin{array}{r}92 \\
0.63\end{array}$ & NR & NR & NR & $\begin{array}{r}127 \\
0.88\end{array}$ \\
\hline $\begin{array}{l}\text { Meridional Rebar } \\
\text { Yielding }\end{array}$ & $\begin{array}{l}127 \\
0.88\end{array}$ & $\begin{array}{r}135 \\
0.93\end{array}$ & $\begin{array}{r}130 \\
0.90\end{array}$ & $N R$ & $\begin{array}{r}92 \\
0.63\end{array}$ & $\begin{array}{r}133 \\
0.92\end{array}$ & NR & $\begin{array}{r}130 \\
0.90\end{array}$ & $\begin{array}{r}104 \\
0.72\end{array}$ & $\begin{array}{r}140 \\
0.97\end{array}$ \\
\hline $\begin{aligned} & \text { Upl ift }= 0.5 \text { inch } \\
&(12.7 \mathrm{~mm})\end{aligned}$ & NE & $\begin{array}{r}157 \\
1.08\end{array}$ & $\begin{array}{r}129 \\
0.89\end{array}$ & $\begin{array}{r}111 \\
0.77\end{array}$ & NE & NR & $\begin{array}{r}132 \\
0.91\end{array}$ & $\begin{array}{l}117 \\
0.81\end{array}$ & NE & NE \\
\hline Crushing & $\begin{array}{r}145 \\
1.00\end{array}$ & $\begin{array}{r}140 \\
0.97\end{array}$ & $\begin{array}{r}140 \\
0.97\end{array}$ & NR & NR & $\begin{array}{r}138 \\
0.95\end{array}$ & NR & $\begin{array}{r}116 \\
0.80\end{array}$ & NR & $\begin{array}{r}159 \\
1.10\end{array}$ \\
\hline
\end{tabular}

Notes:

a. Since NII conducted analysis only at $53,87,116,145$, and 174 psi $(0.37$, $0.6,0.8,1.0$, and $1.2 \mathrm{MPa})$, the reported values do not necessarily reflect initiation, but only the lowest pressure at which the event was detected for which calculations were made.

b. NII predicts that the restraint to the liner at the wall base is insufficient to achieve the full yield stress and thus the liner is expected to detach itself at this point below $110 \mathrm{psi}(0.76 \mathrm{MPa})$. $N R=$ not reported

$\mathrm{NE}=$ uplift was less than 0.5 inches over the pressure range analyzed

approximately 2.8 to 4.1 times the design pressure. (As described on page 251, NII's prediction is conservative and perhaps should not be viewed in the same light as the other calculations.) Seven of the ten organizations identified the basemat cylinder junction as a location where failure was likely to occur, but even among those, there were wide variations in the predicted capacity and limit mechanism. Failure at the basemat cylinder junction was ascribed to shear, flexure, or liner strain limits. Several groups also calculated the pressure at which the hoop rebar would fail; BNL and CEA defined this as the point at which gross yielding or rapidly increasing displacements began; SNL, ANL, and GRS correlated failure with the ultimate strength of a rebar splice. Clearly, there are significant differences in the failure criteria that were applied; a consensus approach to the use and interpretation of calculated response measures (displacement, strain, force, moment, stress) has not emerged.

Each organization was also asked to indicate the maximum pressure at which they had high confidence the containment model would not fail (in contrast, 
Table 1.3 represents "best estimate" values for failure). Of the seven organizations that responded to this inquiry, the values ranged from 92 to 160 psig ( 0.634 to $1.103 \mathrm{MPa})$ or 2.0 to about 3.5 times design pressure. (The reported values were $92,100,105,127,135,138$, and 160 psig $(0.63,0.69,0.72,0.88,0.93,0.95$, and $1.10 \mathrm{MPa}))$. It is interesting that some organizations had high confidence that the containment model would not fail at pressure levels greater than the best estimate failure capacity made by others. Also, judging from the range of values reported, the uncertainty in the maximum pressure at which the model is not expected to fail is nearly the same as the uncertainty in the "best estimate" for capacity.

A definitive assessment of state-of-the-art computational methods for predicting performance of LWR reinforced concrete containment buildings loaded to failure is premature. Nevertheless, a few tentative observations may be made at this time. Although existing approaches may not adequately model tensile cracking, this is not likely to be critical as the limit state is approached. Near the limit state, tensile loads are carried predominantly by steel elements, which by consequence govern the global response. However, given the apparent importance of the basemat cylinder junction, it may be critical to accurately model shear and compression resistance of the concrete, particularly after damage has occurred. The possibility of local liner failure is recognized as a major uncertainty in the analysis by nearly everyone. There is a strong need for test validated failure criteria. To be of general use, these criteria must be explicit expressions in terms of parameters that can be calculated with state-of-theart computational tools. Criteria that are based on the analyst's

experience or, worse still, lack of convergence in a finite element program should not be accepted.

A more thorough evaluation of analytical methods will be made after testing of the model is completed and the pretest predictions and experimental results can be compared. SNL plans to publish a second NUREG report that will conclude the work on the reinforced concrete scale model. The organizations participating in the round-robin analysis will again be invited to write sections for this report, which would describe any additional analyses they might choose to conduct and their assessment of analytical methods, based on comparisons with the experimental results. It is expected that many of the organizations will conduct posttest analyses to gain additional insight into the actual fajlure mechanism realized from high pressure testing of the scale model.

Research into the behavior of reinforced concrete containment structures should not end with the scale model. Variations in the designs of actual reinforced concrete containment buildings implies that variations in the behavior and the realization of the other potential limit states are to be expected. The need for developing test validated methods that can be used to predict containment performance on a case-by-case basis is founded on this idea. At a minimum, it would appear to be necessary to conduct separate effects tests to study potential limit states other than the one realized in the testing of the scale model and to propose applicable, test validated failure criteria. In addition, the effects of elevated temperature should be investigated by testing and/or analysis. 
Table 1.3

Failure Predictions for the 1:6-Scale Containment Model

\begin{tabular}{|c|c|c|}
\hline $\begin{array}{l}\text { Org. } \\
\text { Code }\end{array}$ & $\begin{array}{l}\text { Cap } \\
\text { psig }\end{array}$ & $\begin{array}{l}\text { acity } \\
\text { (MPa) }\end{array}$ \\
\hline SNL & 168 & $(1.16)$ \\
\hline ANL & $180-190$ & $(1.24-1.31)$ \\
\hline EPRI & $140-150$ & $(0.97-1.03)$ \\
\hline CEA & 138 & $(0.95)$ \\
\hline $\mathrm{NII}$ * & 130 & $(0.90)$ \\
\hline ENEA & $161-184$ & $(1.11-1.27)$ \\
\hline SRD & 164 & $(1.13)$ \\
\hline GRS & $\begin{array}{l}167-174 \\
174-189\end{array}$ & $\begin{array}{l}(1.15-1.20) \\
(1.20-1.30)\end{array}$ \\
\hline BNL & 128 & $(0.88)$ \\
\hline CEGB & 160 & $(1.10)$ \\
\hline
\end{tabular}

Limit Mechanism

Flexural failure at the cylinder-basemat juncture brought on by extensive crushing of concrete.

Either (1) failure of a hoop rebar splice near midheight, (2) failure of a weld in the liner near the basemat, or (3) failure of the liner just above the knuckle.

Liner tearing at the connection of the liner to the wall-basemat juncture knuckle, triggered by basemat bending failure.

Plasticity of rebars corresponding to rapidly increasing displacements of the structure.

Transverse shear failure of cylinder just above the shear reinforcement (some probability for local tearing of liner around studs).

Failure at the base of the cylinder caused by combined effect of bending, tension, and shear.

Rebar at the pole of the basemat exceeds its ultimate strength.

Failure at cylinder-basemat intersection. Failure of hoop reinforcement or tearing of liner.

Flexural/shear failure at the wall-basemat junction or gross yielding of hoop rebars.

Flexural failure at the wall-basemat junction.

* Lowest bound of estimates (see page 251) 


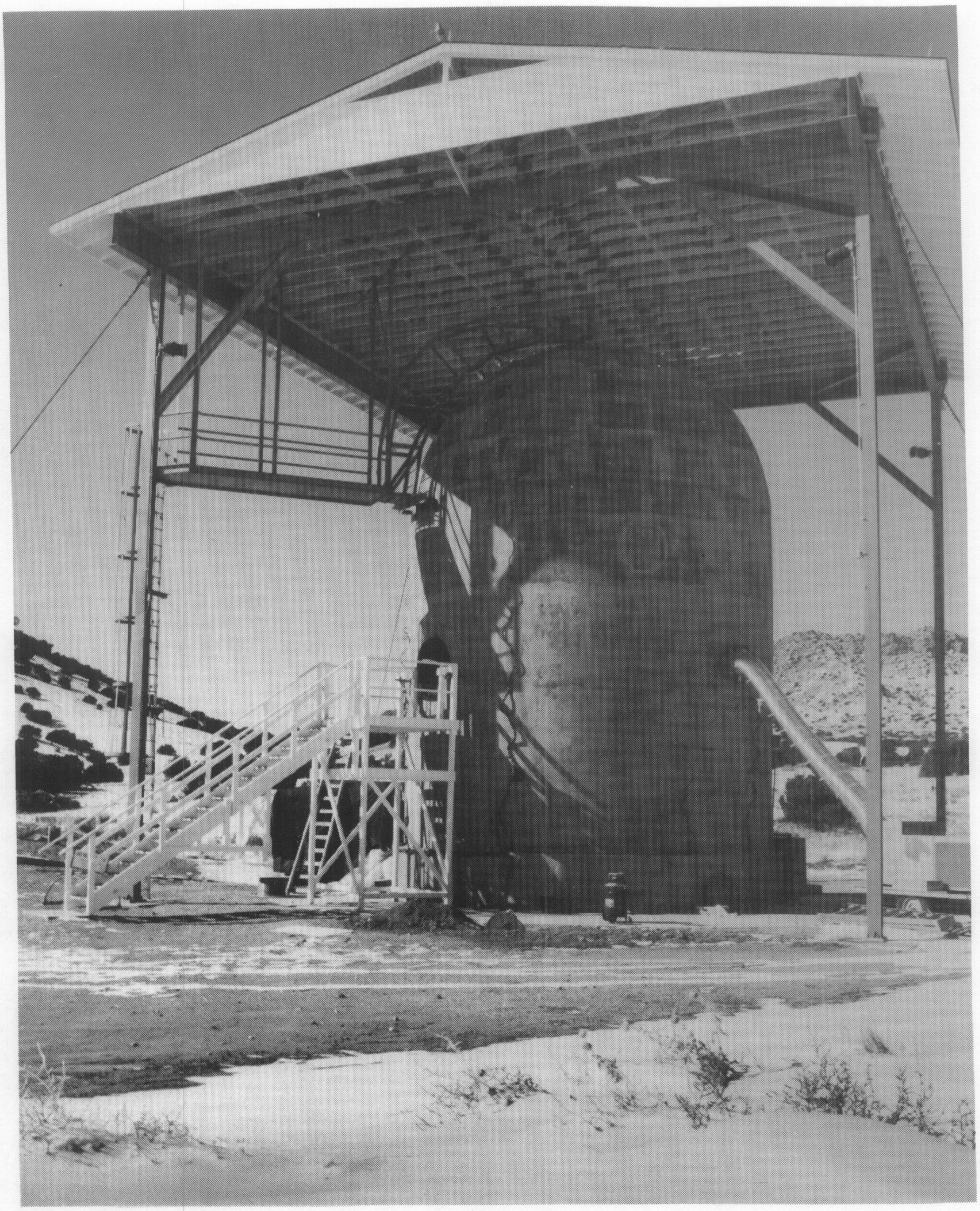

Figure 1.1 1:6-Scale Reinforced Concrete Containment Model 


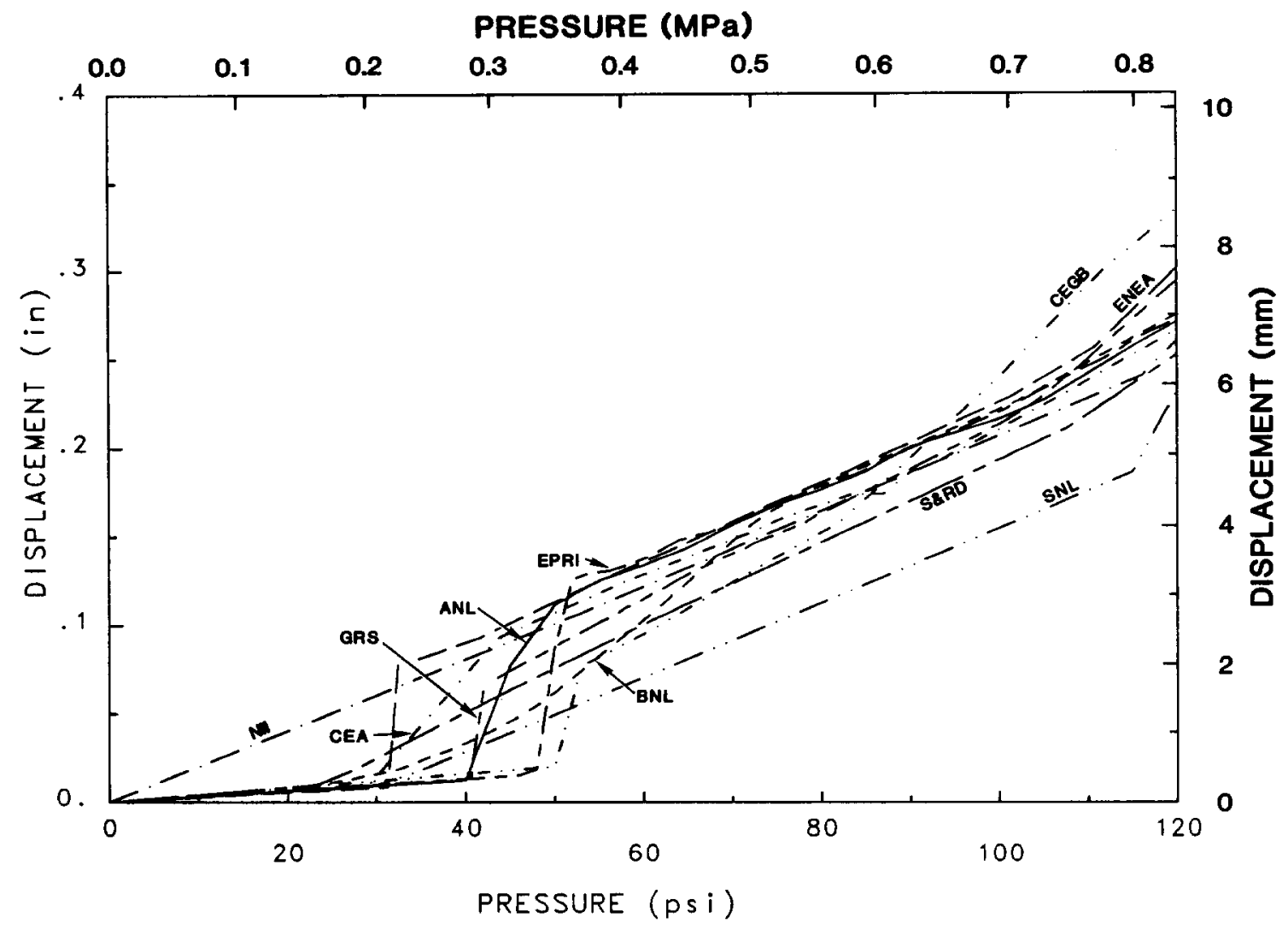

Figure 1.2a Radial Displacement at Cylinder Midheight (Low Pressure)

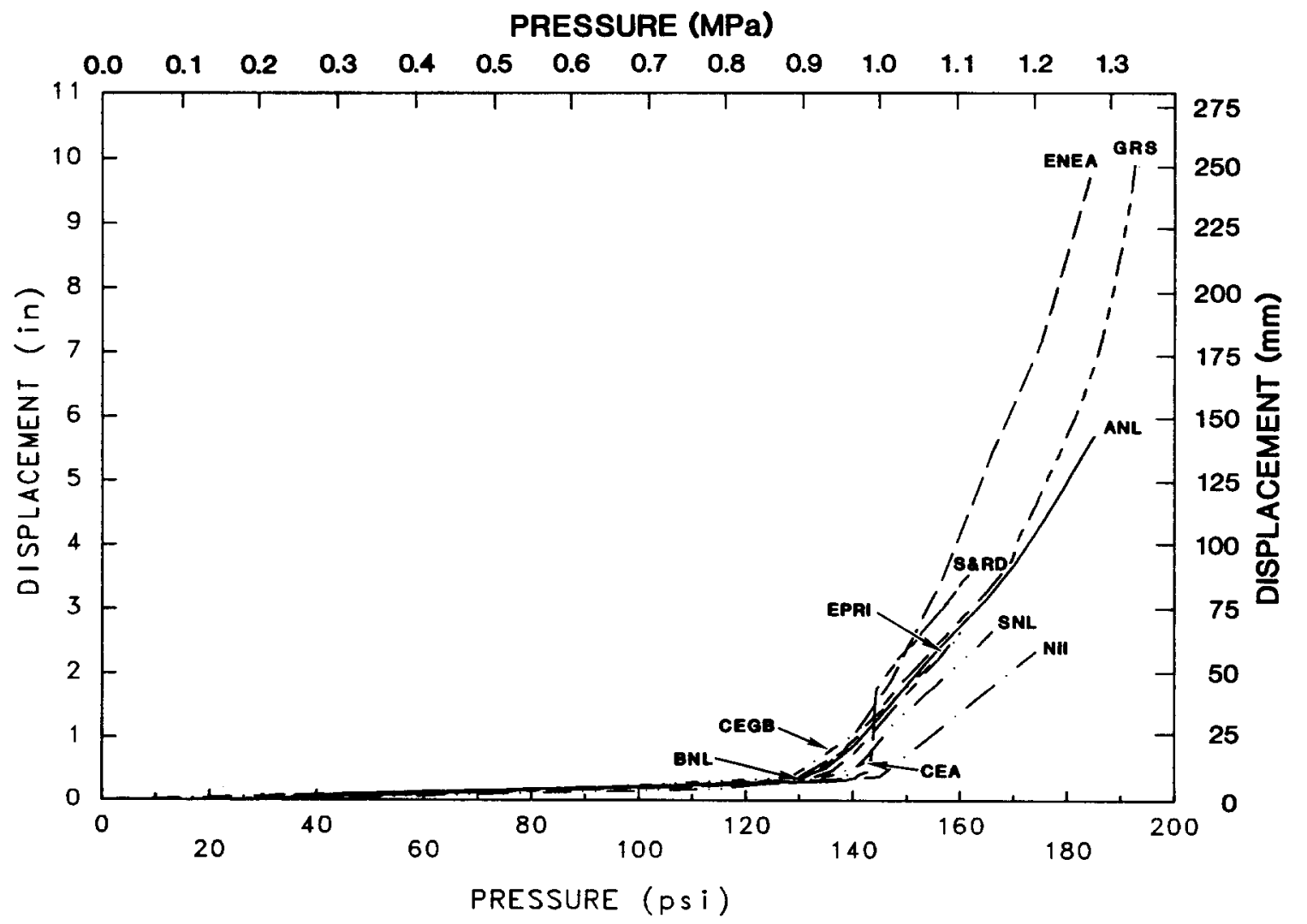

Figure 1.2b Radial Displacement at Cylinder Midheight 


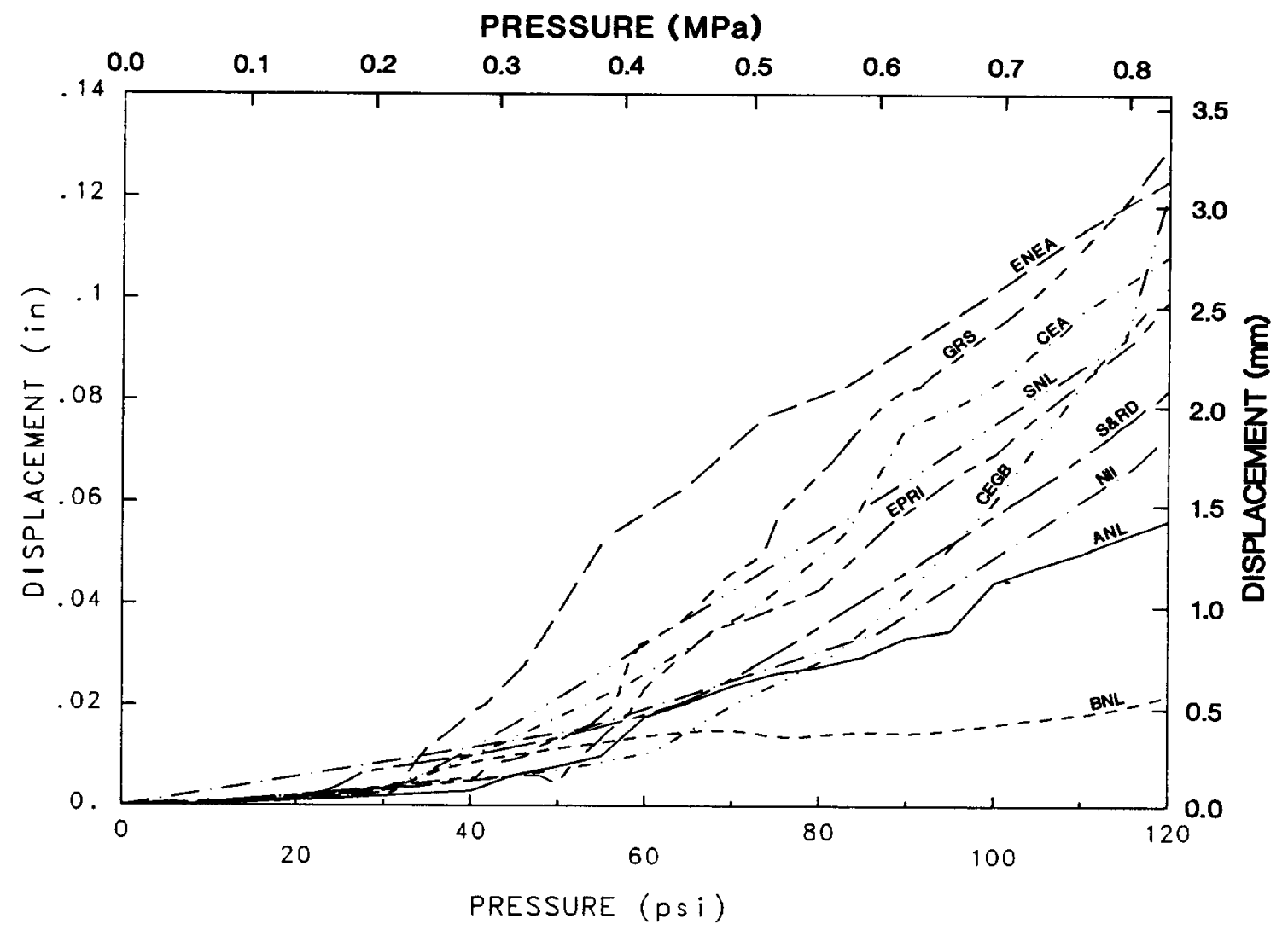

Figure 1.3a Vertical Displacement at Cylinder Midheight (Low Pressure)

PRESSURE (MPa)

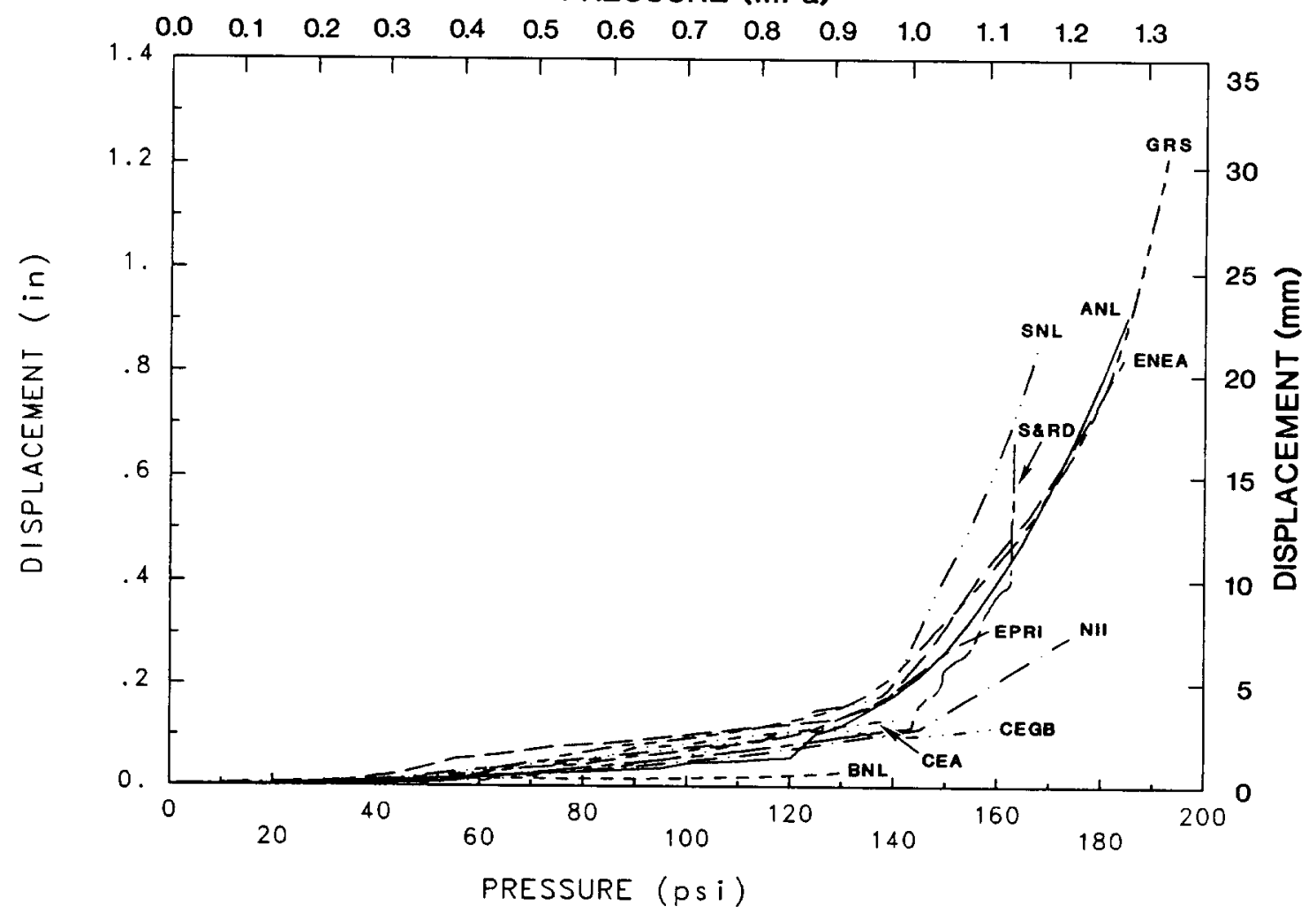

Figure 1.3b Vertical Displacement at Cylinder Midheight 


\section{INTRODUCTION}

\subsection{Background}

In a light water reactor (LWR) nuclear power plant, the containment building is the last engineered barrier to the release of radioactivity. Thus, the performance of the containment building significantly affects the safety of a nuclear power plant. In the event of a severe or degraded core (Class 9) accident, the containment building may be subject to internal pressure and temperature levels much greater than the design basis. Knowledge of the performance of the containment building under these conditions is crucial to reliable emergency preparedness, accident mitigation, and risk assessment.

The U.S. Nuclear Regulatory Commission (NRC) has established a set of programs with the ultimate objective of identifying methods that can be used to predict the performance of LWR containment buildings subject to loads beyond the design basis. The programs are known collectively as the Containment Integrity Programs; the objectives and organization of the individual programs are described in [2.1]. The programs are managed by the Containment Integrity Division at Sandia National Laboratories (SNL).

One indicator of containment building performance is damage, such as cracking or crushing of concrete and yielding of steel. However, the final measure of performance is failure, which is most logically defined on a functional basis. On this basis, failure must be defined as any event that leads to a significant release of radioactive material to outside the containment boundary. A release may be considered significant when it has a measurable effect on safety, which is generally considered to occur if leakage is on the order of $10 \%$ volume per day or greater. A reliable methodology for predicting containment performance must consider all potential failure mechanisms, including, for example, failure to isolate, leakage past the sealing surface of penetrations, and tearing or other material failures of the containment shell. Furthermore, analytical methods should be validated by relevant experimental data.

Thus, an important part of the Containment Integrity Programs is to conduct experiments that can be used to validate analytical methods. In fact there is a symbiotic relationship between testing and analysis in these programs: testing is used to validate analytical methods and analysis is used to help plan the tests. An experimental data base that can be used to validate methods for predicting containment performance up to and including failure is being developed through tests conducted on scale models of containment buildings, penetrations, and seals and gaskets.

Five steel containment scale models have already been pressurized to failure, four of $1: 32-5 c a l e[2.2]$ and one of $1: 8$-scale [2.3,2.4]. Methods for predicting the behavior of steel containment buildings have been identified and validated using this experimental data base $[2.5,2.6]$. The tests and analysis indicate that the capacity of steel containments is significantly greater than their design pressure, but that catastrophic rupture is possible unless preceded by leakage from penetrations or valves [2.6]. The leakage potential of penetrations is being investigated through tests on seals and gaskets, penetrations, and valves [2.7-2.9]. Detailed representations of equipment hatches, including pressure seating and pressure unseating designs, have been included in the scale models. A full 
size personnel airlock will be tested under internal pressure at elevated temperatures in mid-1987 [2.10].

Currently, final preparations are being made to test a $1: 6$-scale model of a reinforced concrete containment building by statically pressurizing the model to failure with nitrogen gas. The experiment is expected to provide a large data base that can be be used to validate analytical models. SNL's previous experience has been that pretest analyses are useful in that (1) they enable insights that can be used to help plan instrumentation and to help run the test, and (2) "blind" predictions are generated for the model behavior, which can be used to assess the uncertainties in the analytical methods.

Two points need to be made concerning the conduct of the test. First, it must be noted that the containment model may be subject to several cycles of loading from ambient to low pressure (up to $1.15 \times$ design pressure, $P_{d}$ ) in order to perform a Structural Integrity Test (SIT), an Integrated Leak Rate Test (ILRT), and other system checkouts. Second, the high pressure test (test to failure) will occur over a two to three day period. During the high pressure test, pressure may be held constant for up to several hours in order to conduct ILRTs. In general, the analyses do not account for the effects of low pressure cycling or time dependent effects during high pressure testing.

\subsection{Round-Robin Analysis}

A significant effort was made to identify organizations that would collaborate with SNL to make pretest predictions. Although the absence of funding would appear to have been a major obstacle to this pursuit, the response was enthusiastic. A number of organizations welcomed the invitation to participate in the analysis of the model as a unique opportunity to evaluate their analytical methods. The organizations participating in the analysis round robin are listed below:

Sandia National Laboratories (SNL), USA

Argonne National Laboratory (ANL), USA

Electric Power Research Institute (EPRI), USA

Commissariat a L'Energie Atomique (CEA), France

HM Nuclear Installations Inspectorate (NII), UK

Comitato Nazionale per la ricerca e per lo sviluppo dell'Energia

Nucleare e delle Energie Alternative (ENEA-DISP), Italy

UK Atomic Energy Authority, Safety and Reliability Directorate (SRD), UK Gesellschaft fuer Reaktorsicherheit (GRS), FRG

Brookhaven National Laboratory (BNL), USA

Central Electricity Generating Board (CEGB), UK

These organizations represent a diverse range of interests, from regulators to industry. Except for BNL and SNL, none of the organizations received funding from the NRC. An exception was made for BNL because of interest in assessing the NFAP code, which has been used frequently by NRC to calculate the capability of concrete containment buildings.

Each participant was supplied with the same information for analyzing the model, which included construction drawings by United Engineers and 
Constructors and Chicago Bridge and Iron (for the liner and penetrations), specifications, as-measured material properties, and photographs of the model in various stages of construction. SNL attempted to encourage independent modelling and analysis efforts; however, consistency in the basic inputs was desirable. For this reason, standard material properties were supplied as given in Section 3.2. Of course, each organization has a unique perspective; as a result, objectives were not always alike and different analytical approaches were adopted.

SNL made two important requests of the participating organizations. First, each group was asked to write a section for this pretest analysis report describing their analytical methods, assumptions, material models, results, and predictions for the model behavior. A limit of forty pages was suggested and drafts were requested by February 1, 1987. Second, each group was asked to generate "standard" plots of response quantities such as strain or displacement as a function of pressure or position. The standard plots were chosen to correspond to instrumented locations, such that comparisons between the different analyses as well as with measured response would be facilitated. These plots, which appear in Appendix A, were also due February 1. Other coordination activities are also planned, including a posttest meeting of analysts at SNL, special sessions at the 9 th

International Conference on Structural Mechanics in Reactor Technology, and a final NUREG report, which will include comparisons of analytical and experimental results, posttest analyses, and evaluations of analytical methods.

It should be noted that some organizations became involved after the initial information package was sent out, and thus they had less calendar time to complete their analyses. The order in which the organizations appear in tables and other lists represents the order in which they joined the roundrobin analysis effort. Also, the resources, budget, and manpower available to this task appear to have varied widely among the participating organizations.

\subsection{Organization of the Report}

This report consists of four major sections. The Executive Summary is a brief synopsis of the entire report, including observations on the comparisons between the different analyses. No attempt has been made to judge the analyses at this point; this will be done at a later stage when the experimental results can be used as a yardstick. In the second section of this report, background information on containment integrity research and the coordination of the round-robin analysis is presented. The geometry of the 1:6-scale reinforced concrete model and as-measured material properties are described in Section 3. These three sections were written by the editor of this report and may not reflect the opinions or beliefs of other participants in the round-robin analysis.

Section 4 is composed of subsections submitted by each organization participating in the analysis. The authors are listed at the beginning of each major subsection. Each subsection begins with a brief one-page synopsis, followed by an in-depth discussion of the analytical approach, modelling considerations, failure criteria, results and predictions for failure. 
The standard plots referred to in Section 2.2 appear in Appendix A. Each organization supplied SNL with a magnetic computer tape with their plot data in a standard format. SNL converted these tapes into a neutral file format that could be used by the GRAFAID code [2.11] so that the results from each organization for a given curve could be combined, thereby simplifying comparisons of the different analyses. Most of the organizations did not provide data for all plot quantitities; some of the requested data was beyond the scope of the analyses. It is left to the reader to note differences in the analytical results and to draw whatever inferences are to be made.

The remaining appendices provide supplementary information for the analyses described in Section 4.

Throughout this report, gage pressure is used for internal pressure in the containment model unless specifically noted otherwise.

\subsection{Posttest Plans}

While much has been learned from the pretest analyses of the scale model, there will undoubtedly be some unexpected local behaviors observed during the course of the test. Posttest analyses may be needed to understand such behaviors and to provide additional insight into the realized limit state of the containment model. The strengths and weaknesses of analytical methods can then be assessed in light of the experimental data. SNL intends to prepare a NUREG report that will describe the posttest investigations; it is hoped that the organizations that have participated in the pretest analysis will also provide input for this report.

The NRC's ultimate goal in the Containment Integrity Programs is to have validated methods that can be used to reliably predict the performance of containment buildings subject to loads beyond the design basis. Thus, it will be necessary to provide guidelines for the application of the methods validated for the scale model to actual reinforced concrete containment structures. Most likely, this will require the study of other potential limit states; additional criteria for evaluating limit states not realized in the scale model test will have to be proposed and validated. The effects of elevated temperature in conjunction with internal pressure must also be considered. 


\section{DESCRIPTION OF THE 1:6-SCALE REINFORCED CONCRETE CONTAINMENT MODEL}

This section describes the geometry and material properties of the 1:6-scale model. It is not intended that all of the details of the model be described here, rather, the intent is to give the flavor of the features that are significant to a structural analysis. For a description of the design and construction of the model, the reader is referred to [3.1,3.2]. The actual construction drawings [3.3,3.4] are available from SNL and will appear later in $[3.5]$.

\subsection{Geometry}

A schematic of the 1:6-scale model, which was fabricated by United Engineers and Constructors, Inc. to a design pressure of $46 \mathrm{psi}(0.317 \mathrm{MPa})$, is shown in elevation view in Figure 3.1. The model consists of a basemat that is 300 inches $(7620 \mathrm{~mm})$ in diameter and 40 inches $(1016 \mathrm{~mm})$ thick and a cylinder and dome, both with an inside diameter of 264 inches $(6706 \mathrm{~mm})$ and having thicknesses of $9-3 / 4$ and 7 inches (248 and $178 \mathrm{~mm}$ ), respectively. A number of penetrations are found in the cylinder, including equipment hatches, personnel airlock representations, and piping clusters. A steel liner is used to provide a leak-tight pressure boundary; the liner thickness is $1 / 16$ inch $(1.59 \mathrm{~mm})$ along the basemat and cylinder and $1 / 12$ inch $(2.11$ $\mathrm{mm}$ ) along the dome. Before the basemat liner was installed, a 3 inch (76 $\mathrm{mm}$ ) thick concrete leveling course was placed on top of the basemat from the center out to the cylinder wall. (Effectively, the basemat is 43 inches $(1092 \mathrm{~mm})$ thick, except for that portion outside of the cylinder wall, which is 40 inches $(1016 \mathrm{~mm})$ thick). The model sits atop a "mudmat", which is used to provide a moisture barrier between the soil and the model basemat, that is 492 inches (12497 $\mathrm{mm}$ ) in diameter and has a minimum thickness of 6 inches $(152 \mathrm{~mm})$. The soil is described as silty clay with sand. Based on bearing plate tests, an average soil modulus of $390 \mathrm{ksf} / \mathrm{ft} \star(61.26 \mathrm{MPa} / \mathrm{m})$ was recommended by Western Technologies. It should be noted that this recommended value includes typical soil factors of safety that may not be appropriate for analytical models used to predict failure; for details of the soil tests the reader is referred to [3.6].

The reinforcing steel in the basemat consists of the following: (1) on the lower face, a rectangular grid of \#6 rebar** on 4 inch $(102 \mathrm{~mm})$ centers, (2) on the upper face, a rectangular grid of \#5 rebar on 6 inch (152 m) centers, \#5 rebars in the radial direction, and \#6 rebars in the circumferential direction, and (3) shear ties through the thickness of the basemat of \#3 rebar. A typical cross section is shown in Figure 3.2. At the basemat cylinder junction, additional layers of reinforcement are used, including shear ties, diagonal bars (layer 11), and an additional layer of meridional bars (layer 10), to carry the high shear forces and bending moments. Figure 3.3 is a photograph of the model taken during construction that shows some of the reinforcement in the basemat and at the cylinder basemat junction.

$\star 1 \mathrm{ksf}=1000 \mathrm{ib} / \mathrm{ft}^{2}$

$\star \star A ~ \# 6$ rebar has a nominal diameter of $6 / 8$ inch $(19 \mathrm{~mm})$; the size designation indicates the nominal diameter in eighths of an inch 
A typical cross section of the cylinder wall in the free-field (away from penetrations) is depicted in Figure 3.4. There are eight layers of reinforcement steel, including four layers of \#4 rebar spaced at 4.5 inches $(114 \mathrm{~mm})$ in the hoop direction, two layers of \#4 rebar spaced at 4.5 inches $(114 \mathrm{~mm})$ in the meridional direction, and two layers of orthogonal \#4 rebar spaced at 6.25 inches $(159 \mathrm{~mm})$ and inclined at 45 degrees.

The wall thickness tapers from 9-3/4 to 7 inches (248 to $174 \mathrm{~mm}$ ) beginning from the springline over an arc length of roughly 26 inches $(660 \mathrm{~mm})$. The diagonal (seismic) bars are terminated in the dome at an elevation of $30^{\prime}-$ 10". Half of the meridional bars are terminated at elevation $34^{\prime}-10^{\prime \prime}$ as shown in Figure 3.5. The dome apex plates can also be seen in Figure 3.5. Half of the meridional rebars above elevation 34'-10" are tied into the dome apex plates.

The reinforcement patterns around the major penetrations are very complex and can be fully described only by reference to the construction drawings. Figures 3.6 and 3.7 are photographs of the reinforcement around equipment hatches $A$ and $B$, which are typical of most penetrations. Reinforcement layers 1 through 8 are 'bent' around the major penetrations, as shown in Figures 3.8 through 3.13. Additional layers of reinforcement around penetrations, such as 'sun ray' (projecting radially outward from penetration) bars, circular bars around the penetration, and shear ties, can be seen in Figures $3.6,3.7$, and 3.14 .

The reinforcement in the mudmat consists of a rectangular pattern of \#5 bars on 12 inch ( $305 \mathrm{~mm}$ ) centers located approximately 2.5 inches $(64 \mathrm{~mm}$ ) below the top of the mudmat. A parafin based curing compound was sprayed on the mudmat, which was finished with a steel trowel, before the concrete for the basemat was placed. As a result, the bond between the concrete in the mudmat and basemat is expected to be negligible. Within a radius of 54 inches $(1.37 \mathrm{~m})$ of the center of the mudmat, a number of keyways were formed with matching keys in the basemat. This was intended to prevent the basemat from sliding relative to the mudmat. Although some analysts included the mudmat in their finite element models, SNL considers the structural coupling between the basemat and the mudmat to be insignificant.

The basemat liner is covered by a circular slab of concrete consisting of a 3 inch $(7.6 \mathrm{~mm})$ protective layer and a 12 inch $(30.5 \mathrm{~mm})$ pad for the model internal structure that is roughly 230 inches $(5840 \mathrm{~mm})$ in diameter. The basemat liner is not anchored to the concrete in the basemat or to the protective course above it. The basemat liner is welded to a quarter pipe section, referred to hereafter as the knuckle. The details of the attachment of the liner to the knuckle are shown in Figure 3.15. Note that the diameter of the top protective course and pad of concrete is less than that of the cylinder, and thus the inside surface of the knuckle is not supported by concrete.

A stretch out of the cylinder liner is shown in Figure 3.16 , which also shows the layout of the penetrations in the model. The cylinder liner is anchored to the concrete by means of headed studs; the type and spacing of these studs varies. The first row of studs is approximately 0.8 inches $(20.3 \mathrm{~mm})$ above the top of the knuckle, and for elevations up to $6^{\prime}-6^{\prime \prime}, 1 / 2$ inch $(12.7 \mathrm{~mm})$ long headed studs are used with a spacing of 2 by 2 inches ( 51 by $51 \mathrm{~mm}$ ) up to elevation $5^{\prime}-2^{\prime \prime}$ and 4 by 4 inches (102 by $102 \mathrm{~mm}$ ) 
between elevation $5^{\prime}-2^{\prime \prime}$ and $6^{\prime}-6^{\prime \prime}$. Above elevation $6^{\prime}-6^{\prime \prime}, 3 / 4$ inch (19 mm) long headed studs were used with 2 by 2 inch ( 51 by $51 \mathrm{~mm}$ ) spacing near penetrations and 6 by 6 inch $(152$ by $152 \mathrm{~mm})$ spacing elsewhere. Figures 3.17 and 3.18 are photographs of the liner with studs attached near the base of the cylinder (including the transition regions at elevations 5'-2" and $\left.6^{\prime}-6^{\prime \prime}\right)$ and near personnel airlock $A$, respectively. Around penetrations, thickened sections of the liner $(3 / 16$ inch, $4.76 \mathrm{~mm}$ ) were used (see Figure 3.16 ). In the dome, the studs were uniformly spaced at 8 by 8 inches (203 by $203 \mathrm{~mm}$ ). It should be noted that the cylinder liner was elastically formed whereas the dome section of the liner was dished by a pressing operation.

\subsection{As-Measured Material Properties}

All materials used in constructing the model have mechanical properties that are the same, or as nearly the same as possible as those used in actual reinforced concrete containment buildings. Reference stress-strain curves were derived based on actual measurements of the properties of the most widely used materials in the model, including the $1 / 16$ and $1 / 12$ inch ( 1.59 and $2.11 \mathrm{~mm}$ ) thick liner material, the \#4 rebar, and various lifts of the concrete.

The concrete used in the scale model was composed of well-rounded coarse aggregate with a nominal maximum size of $3 / 8$ inch $(9.5 \mathrm{~mm})$. High-range water reducers (superplasticizers) were used with a water/cement ratio of 0.53. The concrete was centrally batched and hauled into the test site, which resulted in a consistent, high quality mix. In order to test the properties, 15 concrete cylinders were poured from each 1 ift of the model (there were a total of 11 lifts; 1 for the basemat, 6 for the cylinder, and 4 for the dome). Direct tension, split cylinder, and compression tests have been run on specimens from each lift aged for 28 days, 4 months, or 6 months. Material properties of the concrete will also be measured at the time of high pressure testing. Standard cylinders were used in the split cylinder and compression tests; the direct tension tests were conducted with a dog-bone specimen developed by United Engineers. Based on the tests, it was felt that the variation in the properties between the different lifts was negligible (the data were within normal statistical limits fro a single 1 ift of concrete). Thus, SNL has recommended a single stress-strain curve, as given in Table 3.1 , to represent the concrete for the entire model. The recommended curve is based on extrapolation of strength values out to the expected time of high pressure testing. The compressive strength is estimated at $6.8 \mathrm{ksi}(46.9 \mathrm{MPa})$. The tensile response is assumed to be linear up to the ultimate tensile strength, which was estimated at $0.5 \mathrm{ks} i$ * (3.45 MPa).

Uniaxial tensile tests were conducted on the liner materials and the \#4 rebar. Average results of these tests (the recommended values) are presented in Tables 3.2 through 3.4. For a given material, the measured properties were very consistent for the number of tensile coupons tested. The stress-strain curve for the \#4 rebar is based on the nominal crosssectional area, which is $0.2 \mathrm{in}^{2}\left(129 \mathrm{~mm}^{2}\right)$. For the liner materials, the

$\overline{\star 1 \mathrm{ks} i}=1000 \mathrm{psi}$ 
stress-strain curve is based on the actual cross-sectional area of the tensile coupons; thus, the actual thickness of the liner should be used in an analytical model. However, the difference between the nominal thicknesses and the average actual thicknesses of the liner materials is small, as indicated by Table 3.5. Therefore, the analytical results should not be particularly sensitive to this factor, especially since the liner represents less than $20 \%$ of the total strength of the containment model wall. Because the \#4 rebar is predominant in the model, it was the only size rebar for which a complete stress-strain curve was made available. It was recommended that this stress-strain curve (see Table 3.4) be used for all the reinforcing steel in the model; however, mill properties for each size of rebar used in the model were also made available to the analysts. The mill properties and nominal cross-sectional areas for the rebars used in the model are presented in Table 3.6. Note that the properties in Table 3.4 reflect a reduction in the ultimate strength caused by splices. 
Table 3.1 - Concrete Material Properties

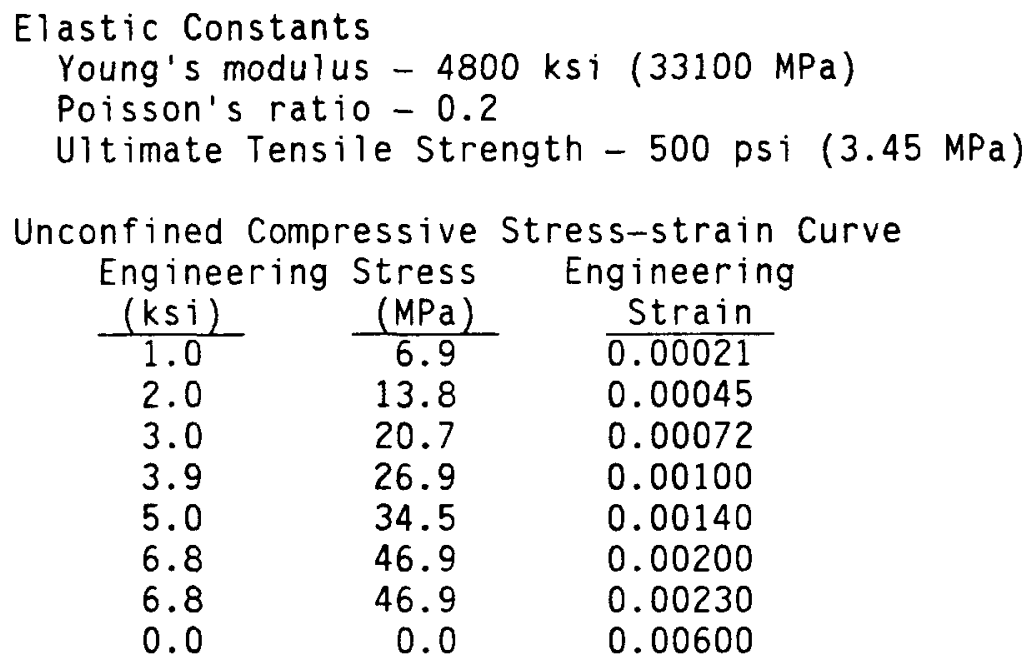

Table 3.2 - Cylinder and Basemat Liner Properties

Elastic Constants:

Modulus - $30000 \mathrm{ksi}(207000 \mathrm{MPa})$

Poisson's ratio - 0.3

Yield stress - $50.2 \mathrm{ksi}$ (346 $\mathrm{MPa}$ )

Engineering Stress-strain curve

\begin{tabular}{|c|c|c|}
\hline $\begin{array}{l}\text { Engineerin } \\
\text { (ksi) }\end{array}$ & $\begin{array}{r}\text { Stress } \\
(\mathrm{MPa})\end{array}$ & $\begin{array}{l}\text { Plastic } \\
\text { Strain }\end{array}$ \\
\hline 50.2 & 346 & 0 \\
\hline 50.2 & 346 & 0.0157 \\
\hline 59.0 & 407 & 0.0308 \\
\hline 66.0 & 455 & 0.0696 \\
\hline 68.0 & 469 & 0.0937 \\
\hline & 482 & 620 \\
\hline
\end{tabular}

Table 3.3 - Dome Liner Properties

Elastic Constants:

Modulus - $30000 \mathrm{ksi}$ (207000 MPa)

Poisson's ratio - 0.3

Yield stress - $51.4 \mathrm{ksi}(354 \mathrm{MPa}$ )

Engineering Stress-strain curve

Engineering Stress Plastic

$\frac{(\mathrm{ksi})}{51.4} \quad \frac{(\mathrm{MPa})}{354} \quad \frac{\text { strain }}{0}$

$\begin{array}{lll}61.1 & 421 & 0.0230\end{array}$

$\begin{array}{lll}66.9 & 461 & 0.0478\end{array}$

$\begin{array}{lll}70.5 & 486 & 0.0977\end{array}$

$\begin{array}{lll}71.0 & 489 & 0.1476\end{array}$ 
Elastic Constants:

Modulus - $31000 \mathrm{ksi}(214000 \mathrm{MPa})$

Poisson's ratio - 0.3

Yield stress - $66.6 \mathrm{ksi}$ (459 $\mathrm{MPa}$ )

Engineering Stress-strain curve

\begin{tabular}{|c|c|c|}
\hline $\begin{array}{l}\text { Eng ineering } \\
(\mathrm{ksi})\end{array}$ & $\begin{array}{r}\text { Stress } \\
(\mathrm{MPa})\end{array}$ & $\begin{array}{l}\text { Plastic } \\
\text { Strain }\end{array}$ \\
\hline 66.6 & 459 & 0 . \\
\hline 73.3 & 505 & 0.0094 \\
\hline 85.6 & 590 & 0.0200 \\
\hline 99.0 & 682 & 0.0430 \\
\hline
\end{tabular}

Table 3.5-Liner Thicknesses

Cylinder and Basemat Liner:

Nominal Thickness - $1 / 16$ inch $(1.59 \mathrm{~mm})$

Actual Thickness - 0.068 inch $(1.73 \mathrm{~mm})$

Dome Liner:

Nominal Thickness - $1 / 12$ inch $(2.12 \mathrm{~mm})$

Actual Thickness - 0.090 inch $(2.29 \mathrm{~mm})$

$\frac{\text { Table } 3.6 \text { - Mill Properties for Reinforcing Bar }}{\text { Nominal }}$

Bar Area

Yield Strength Ultimate Strength

Elongation

$\frac{\text { Size }}{6 \mathrm{~mm}}$

$\frac{\left(i n^{2}\right)}{0.04}$

$\frac{\left(\mathrm{mm}^{2}\right)}{28}$

$\frac{(\mathrm{ksi})}{72.2} \frac{(\mathrm{MPa})}{497}$

$\frac{(\mathrm{ksi})}{100.9} \frac{\text { (MPa) }}{695}$

$\frac{(\mathrm{in} / \mathrm{in})}{0.27}$

$6 \mathrm{~mm}$

0.04

$69.9 \quad 482$

$68.0 \quad 469$

99.0682

68.0469

$66.5 \quad 458$

$\# 4$

0.11

71

0.20

129

0.31

198

$64.8 \quad 447$

103.0710

105.0723

$94.0 \quad 648$

0.23

0.15

0.14

0.15

\#6

$0.44 \quad 285$

103.0

710

0.14 


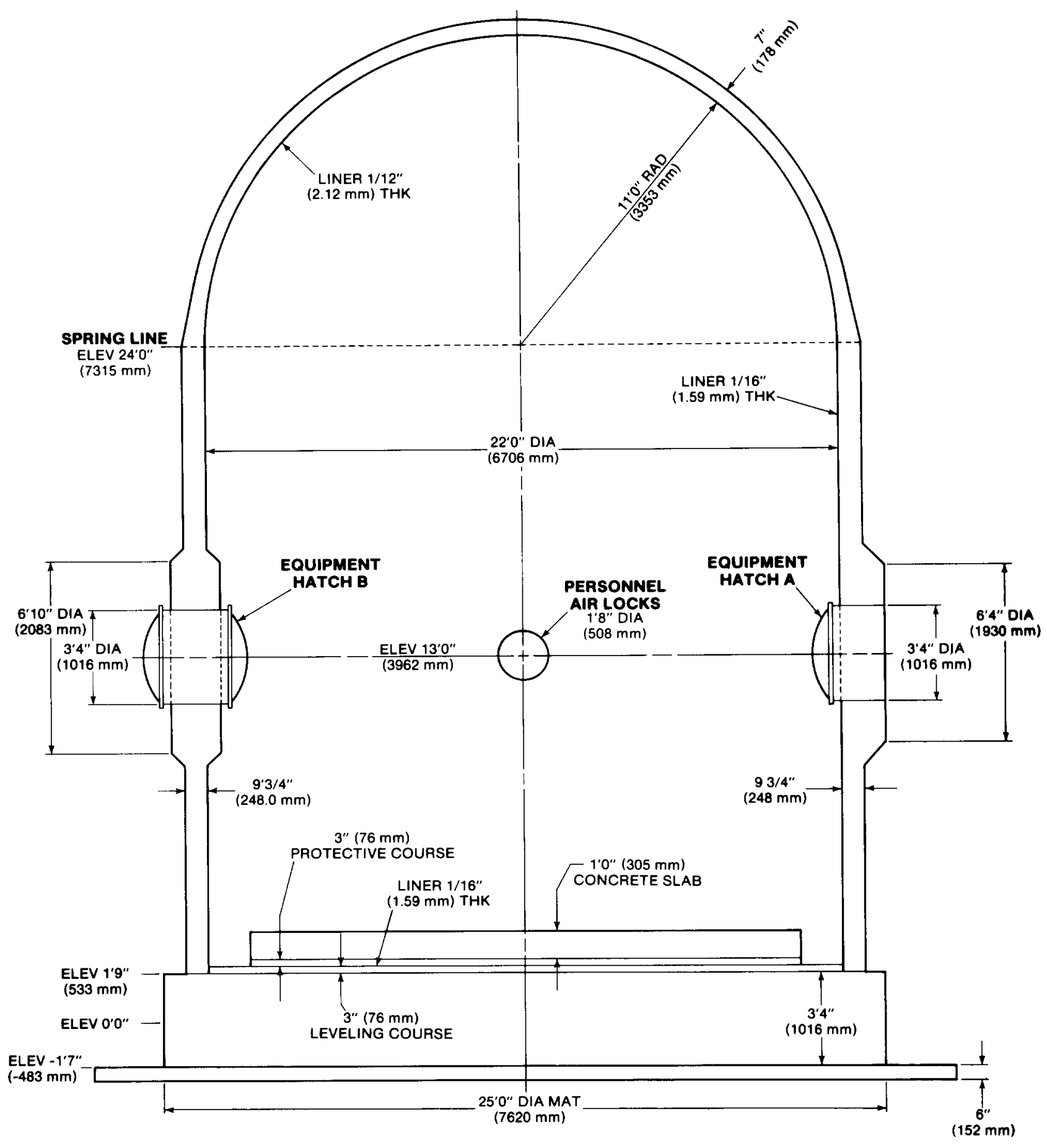

Figure 3.1 Schematic of the 1:6-Scale Reinforced Concrete Containment Model - Elevation View 


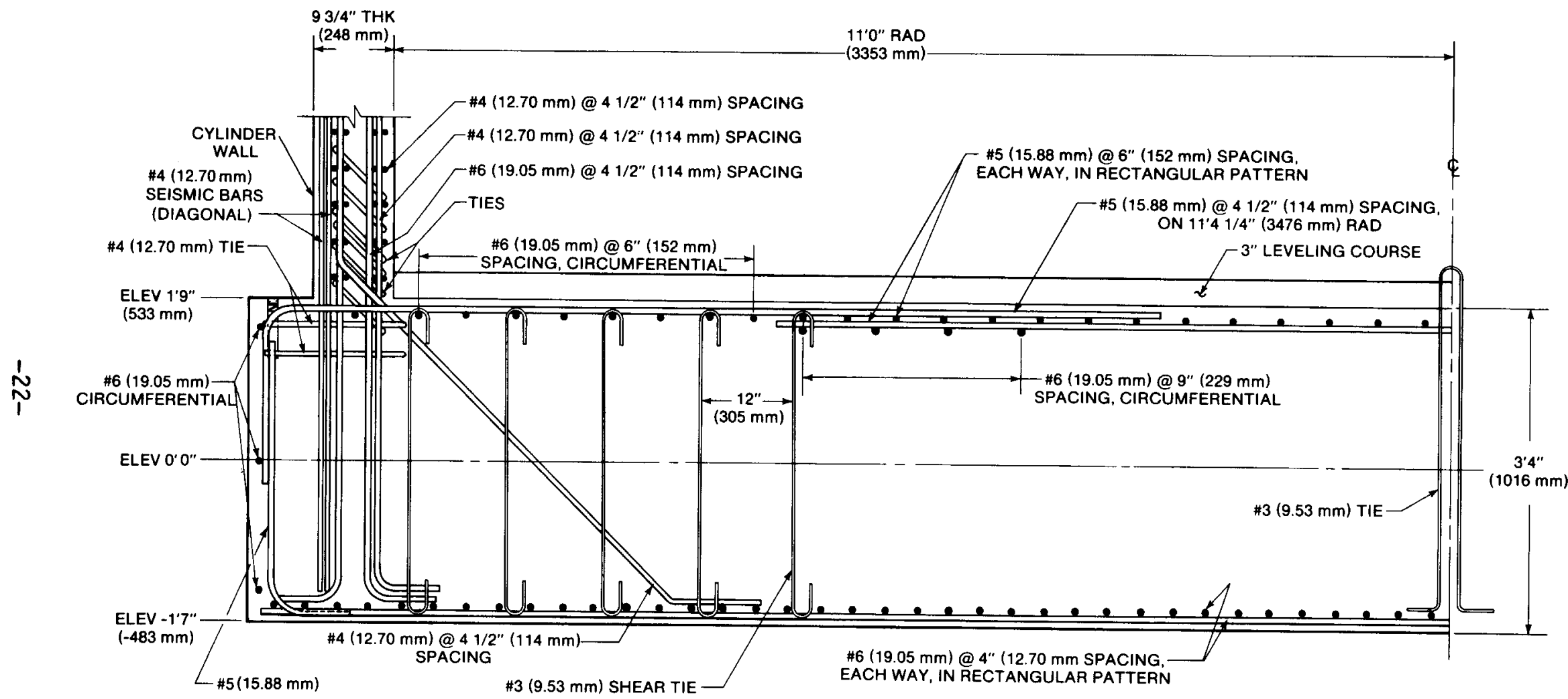

Figure 3.2 Reinforcement in the Basemat and Cylinder Basemat Junction 


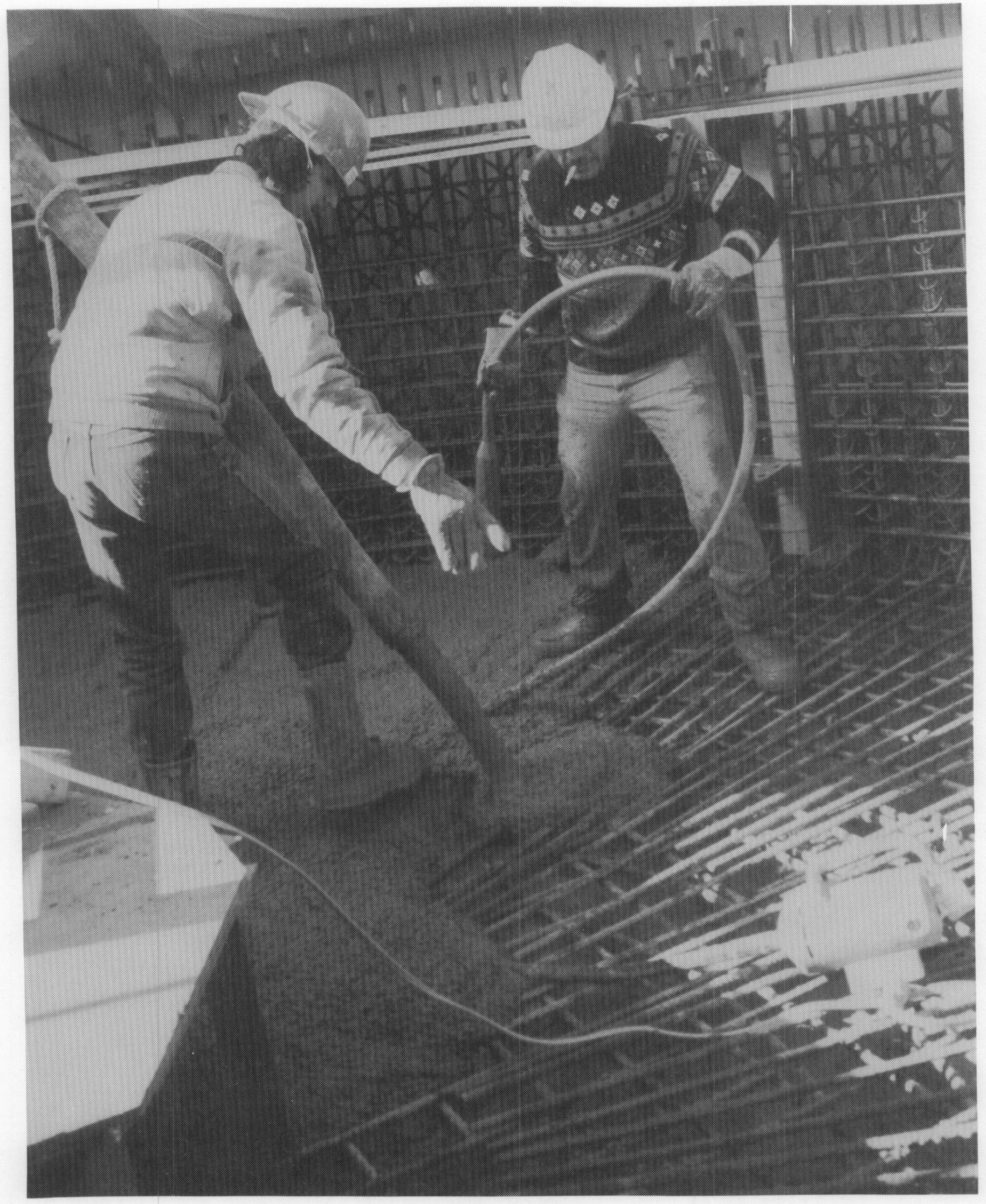

Figure 3.3 Photograph During Construction of Basemat Cylinder Junction 
REINFORCING BAR DETAILS

\begin{tabular}{|c|c|c|}
\hline LAYER & TYPE & SIZE \\
\hline 1 & HOOP & \#4 \\
\hline 2 & MERIDIONAL & $\# 4$ \\
\hline 3 & HOOP & \#4 \\
\hline 4 & HOOP & \#4 \\
\hline 5 & MERIDIONAL & $\# 4$ \\
\hline 6 & HOOP & $\# 4$ \\
\hline 7 & SEISMIC ( $45^{\circ}$ DIAG) & \#4 \\
\hline 8 & SEISMIC ( $45^{\circ}$ DIAG) & $\# 4$ \\
\hline
\end{tabular}

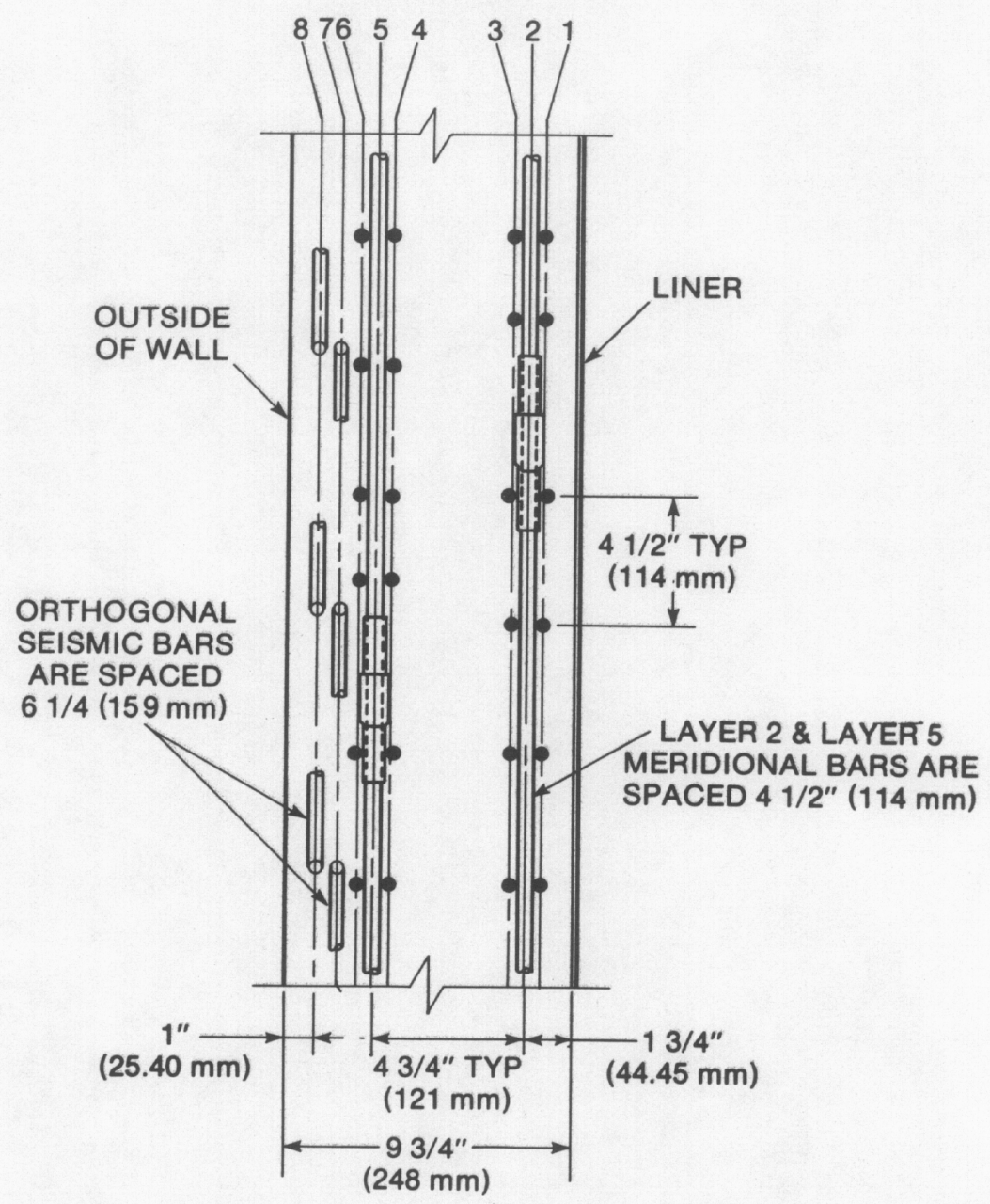

Figure 3.4 Reinforcement in the Cylinder (Free-field) 


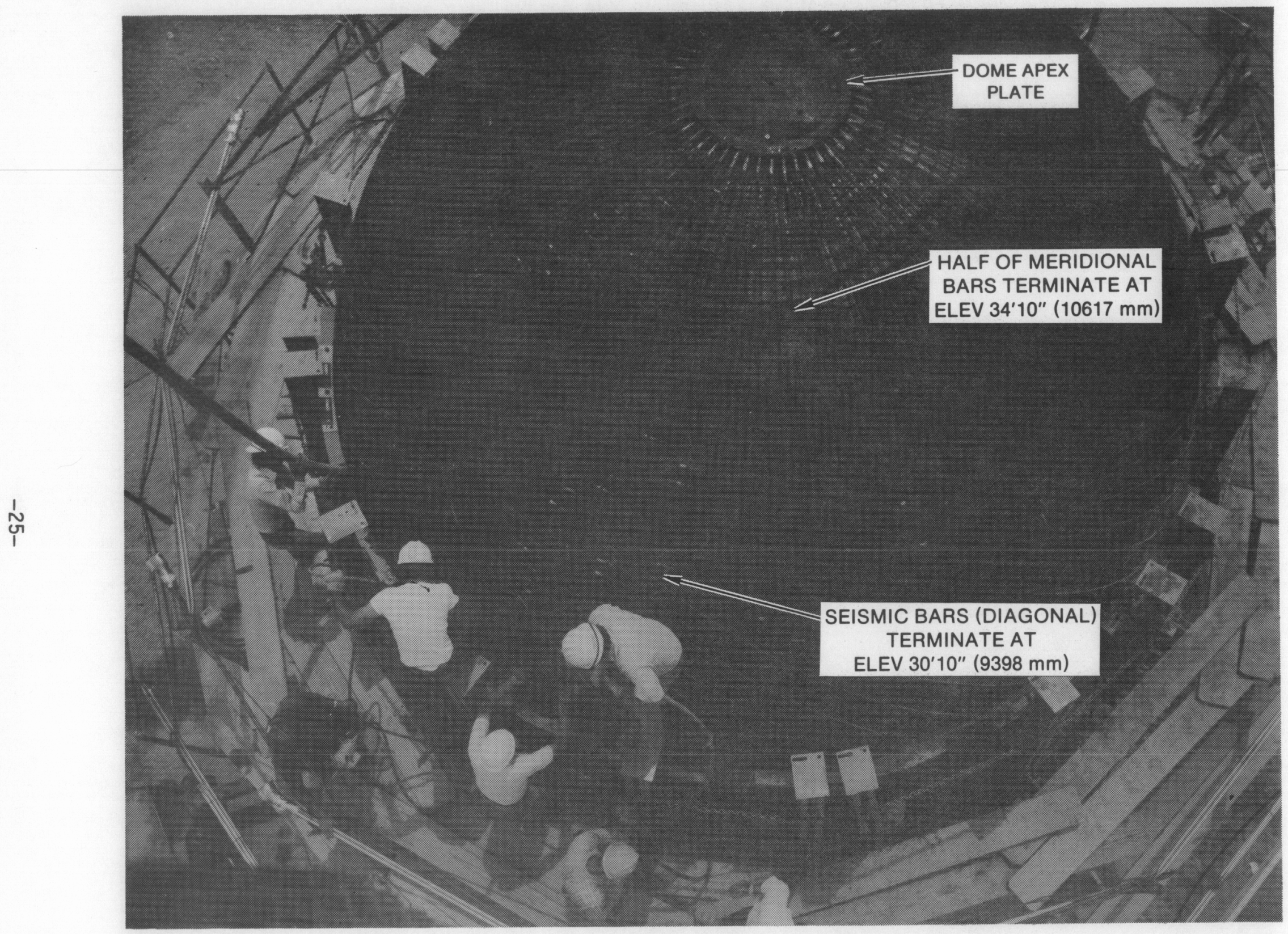

Figure 3.5 Photograph During Construction of Reinforcement in the Dome 


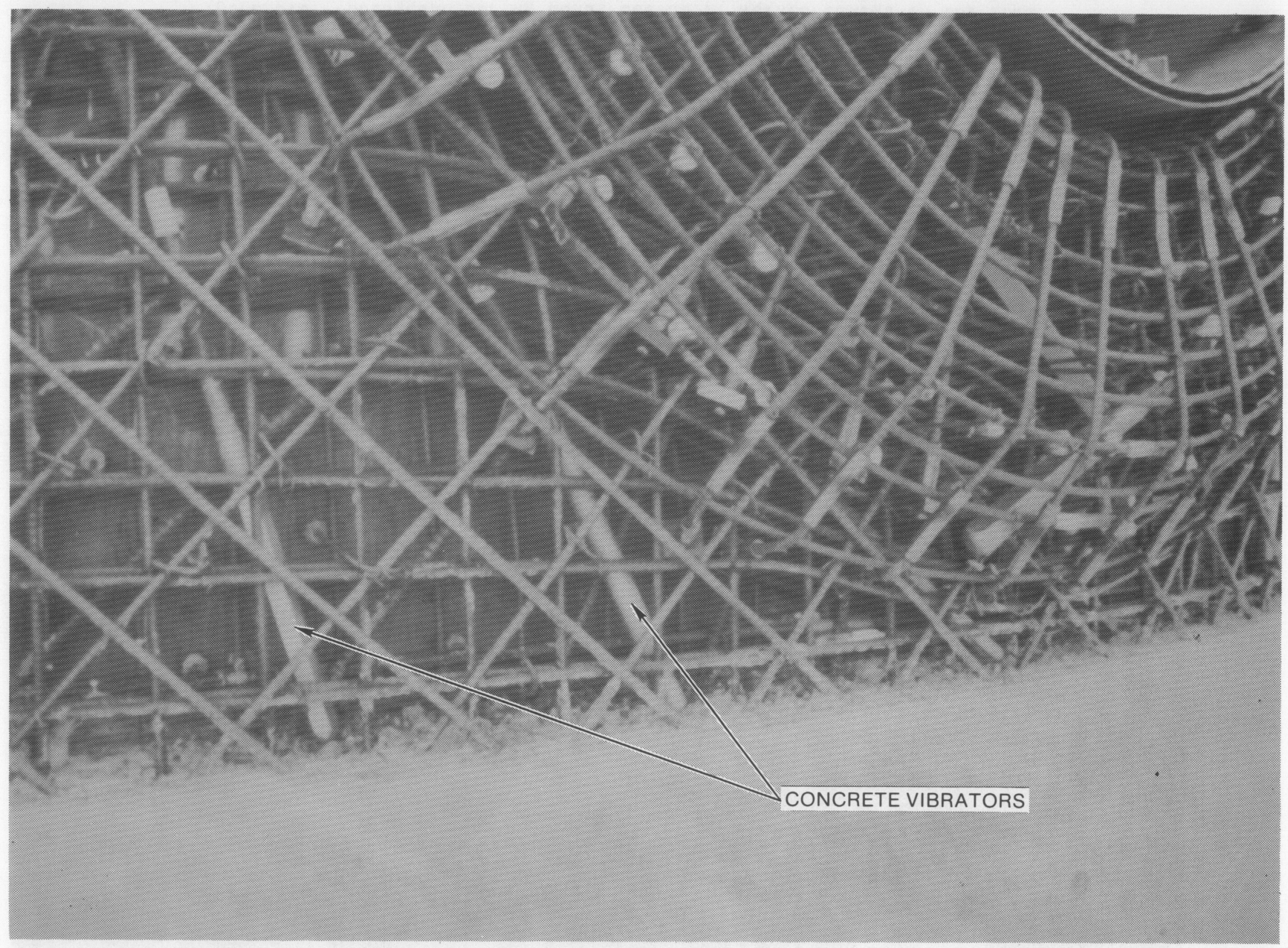

Figure 3.6 Photograph of Reinforcement Around Equipment Hatch A 


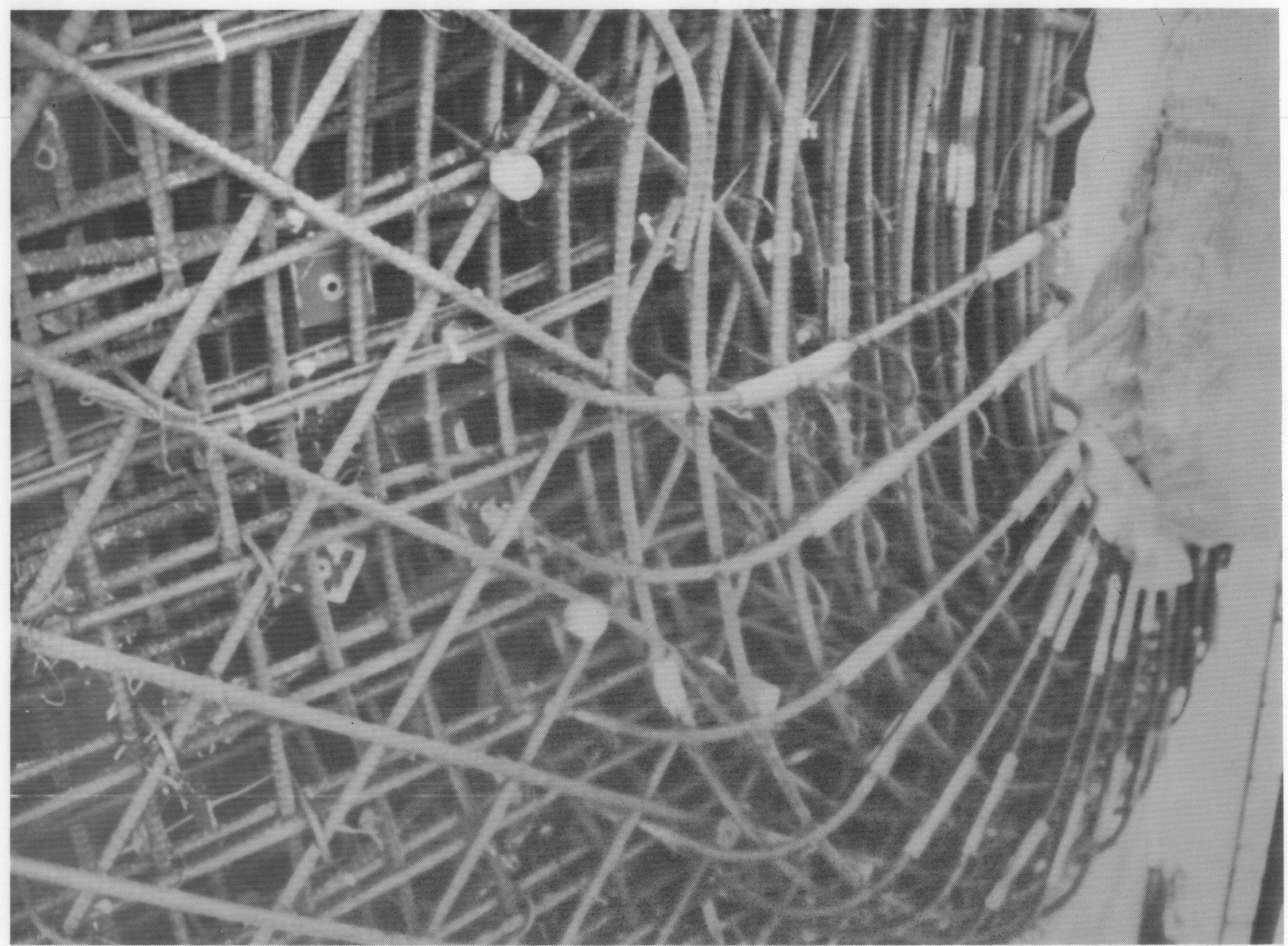

Figure 3.7 Photograph of Reinforcement Around Equipment Hatch B 


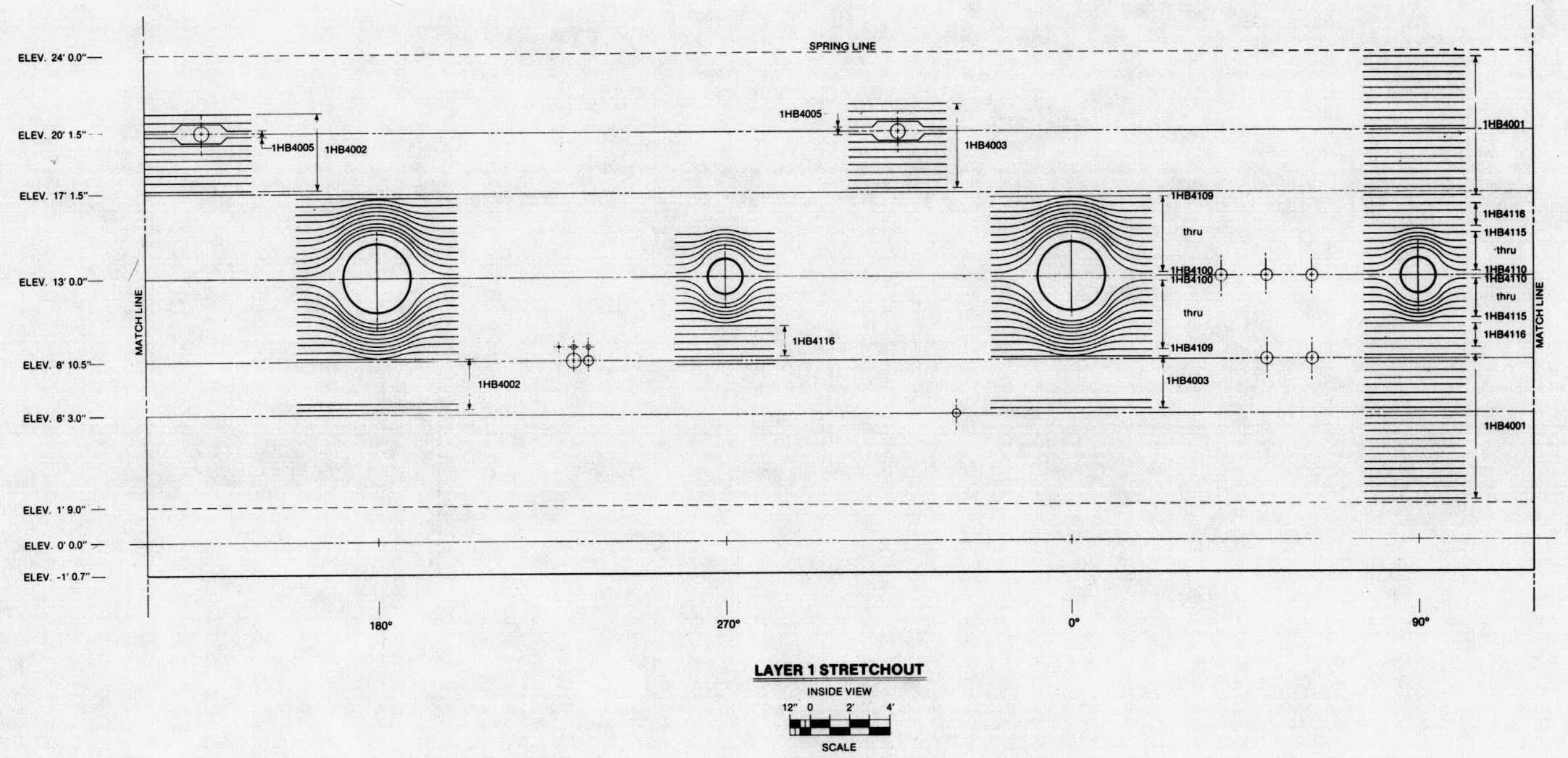

Figure 3.8 Cylinder Stretchout - Reinforcement Layers 1 and 3 


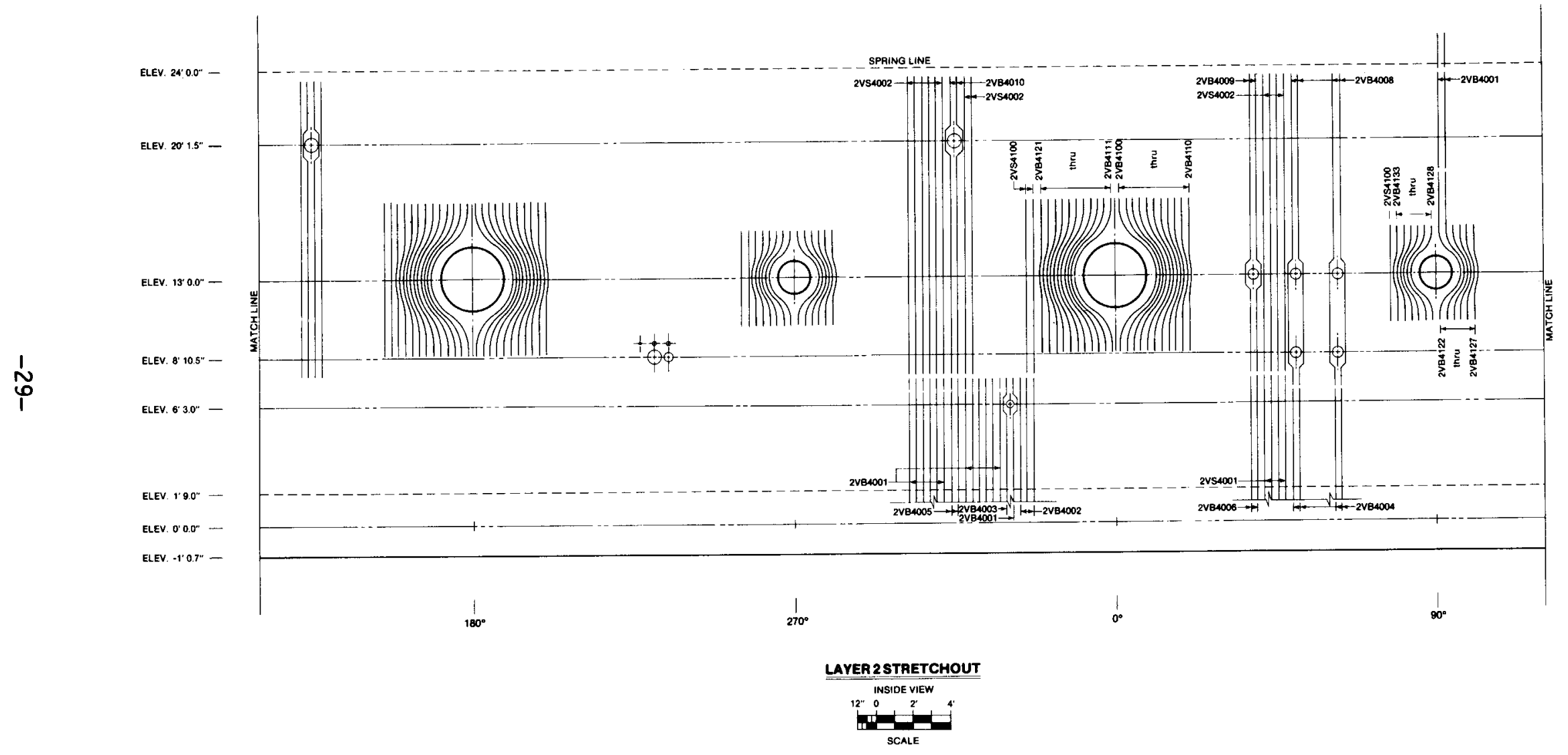

Figure 3.9 Cylinder Stretchout - Reinforcement Layer 2 


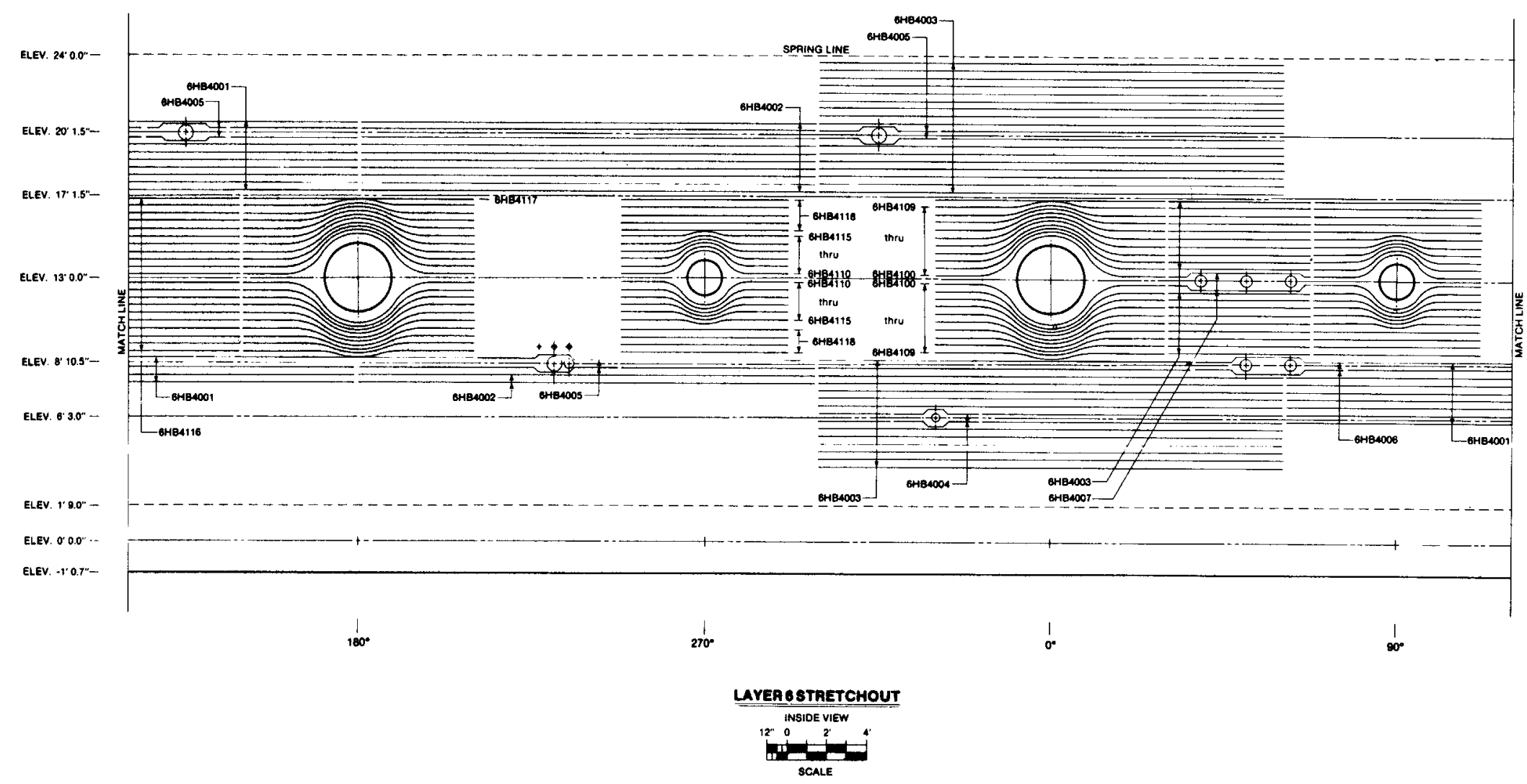

Figure 3.10 Cylinder Stretchout - Reinforcement Layers 4 and 6 


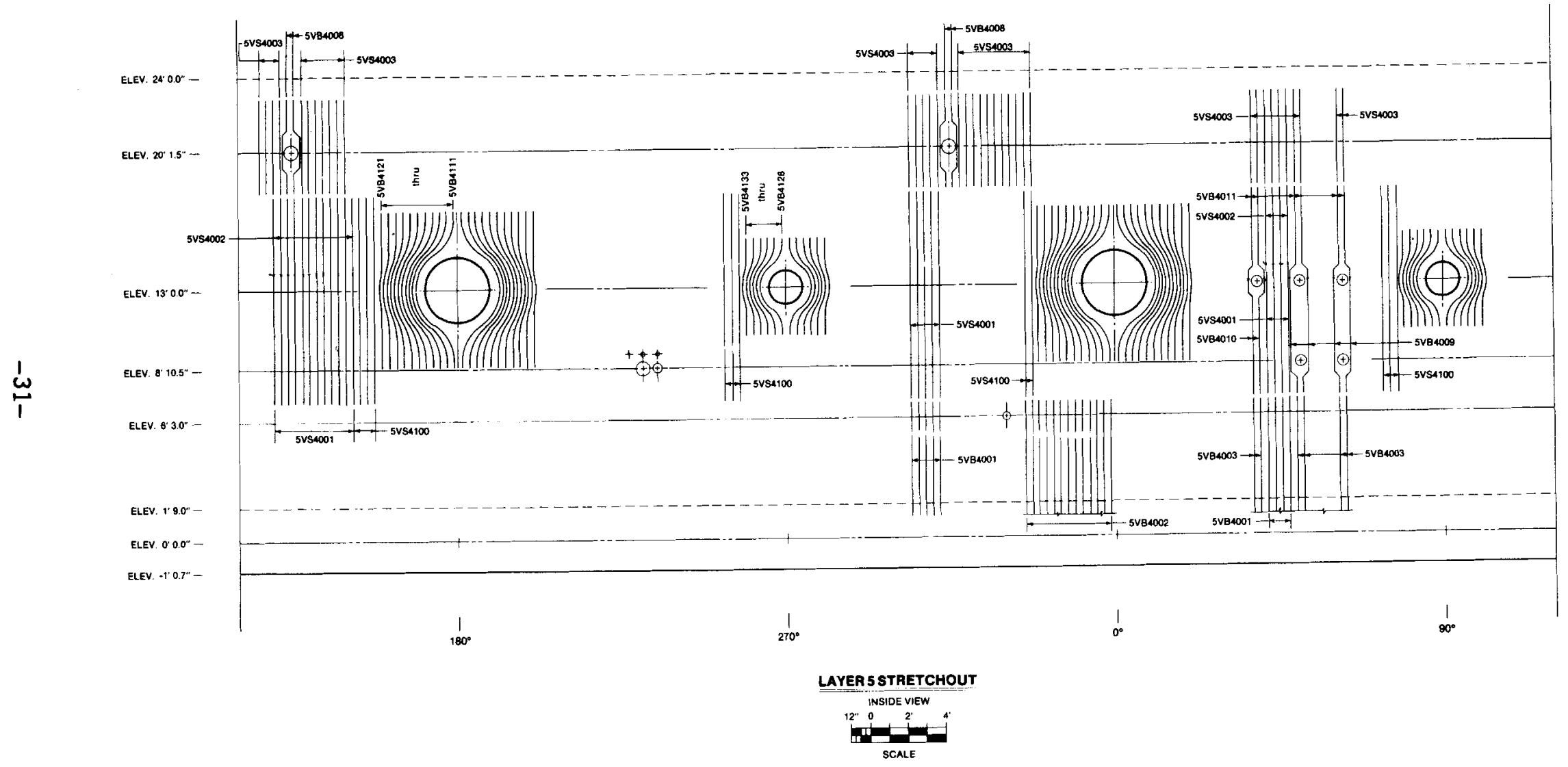

Figure 3.11 Cylinder Stretchout - Reinforcement Layer 5 


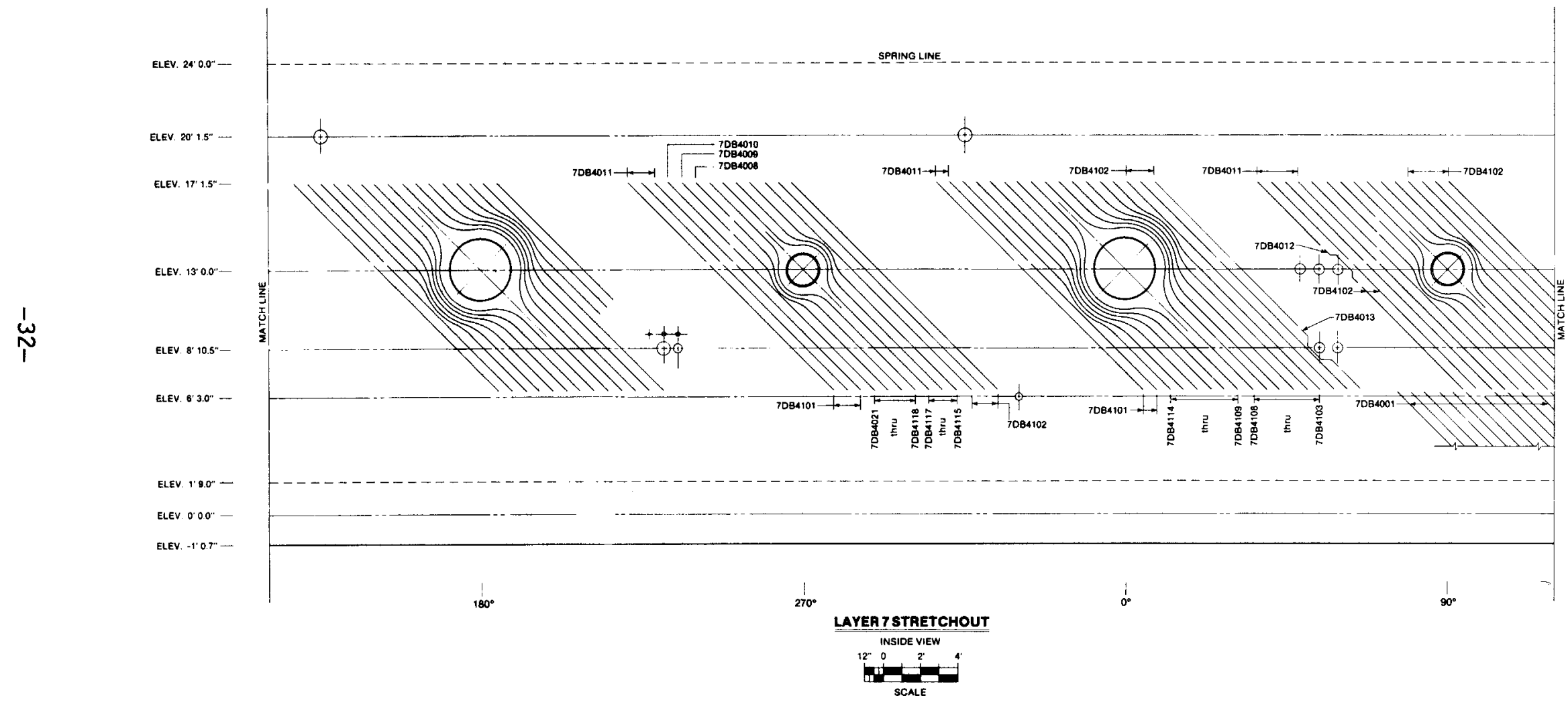

Figure 3.12 Cylinder Stretchout - Reinforcement Layer 7 


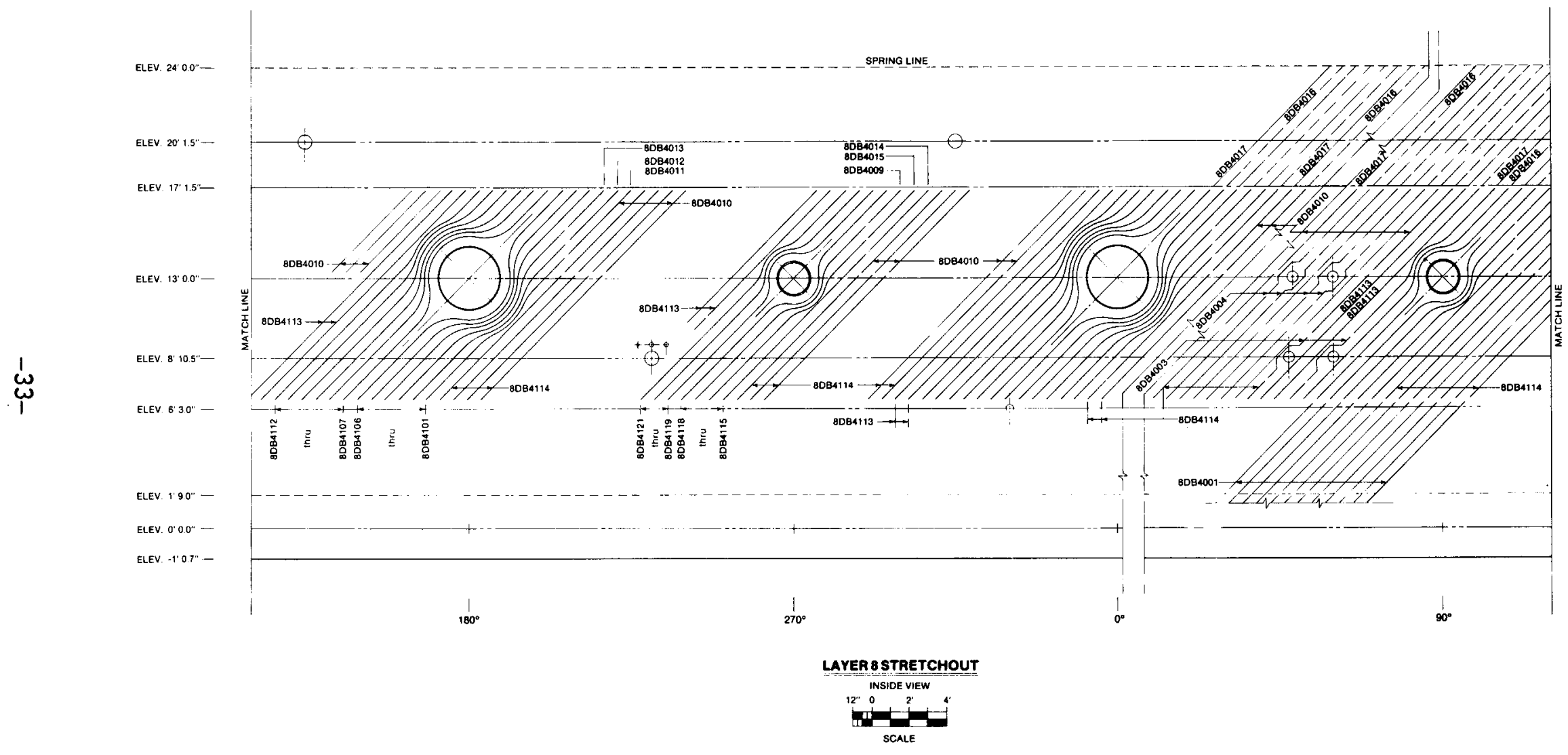

Figure 3.13 Cylinder Stretchout - Reinforcement Layer 8 


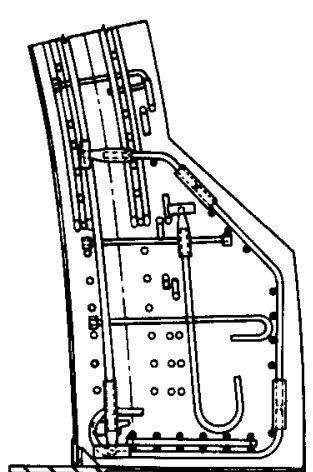

SECTION AT HORIZONTAL $\&$ (3 O'CLOCK)

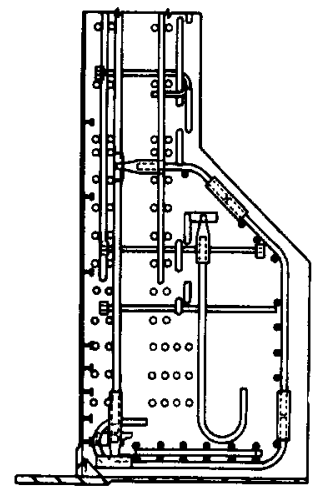

SECTION AT VERTICAL $\Phi(12$ O'CLOCK)

BOSS@Az 0

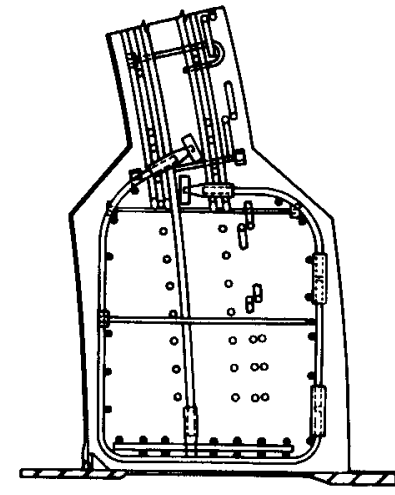

SECTION AT HORIZONTAL \& (3 O'CLOCK)

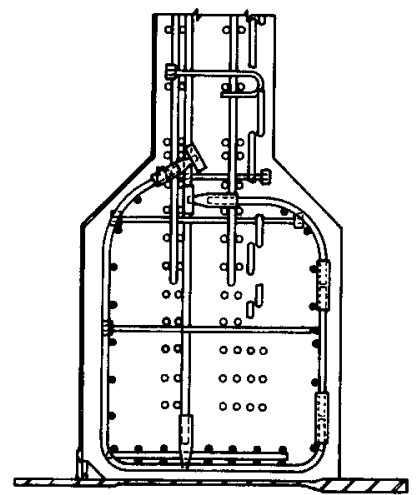

SECTION AT VERTICAL $\&$ (12 O'CLOCK)

BOSS@Az 180 12" 9" 6" 3" 0 anim

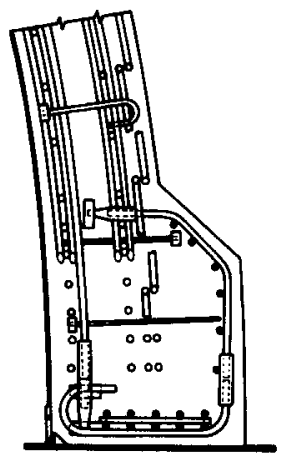

SECTION AT HORIZONTAL $\&$ (3 O'CLOCK)

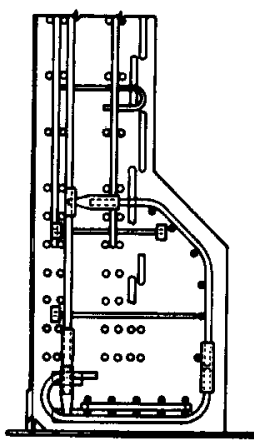

SECTION AT VERTICAL $\&$ (12 O'CLOCK)

PL-A@Az 90 \& 270

Figure 3.14 Additional Reinforcement in Bosses of Selected Penetrations 


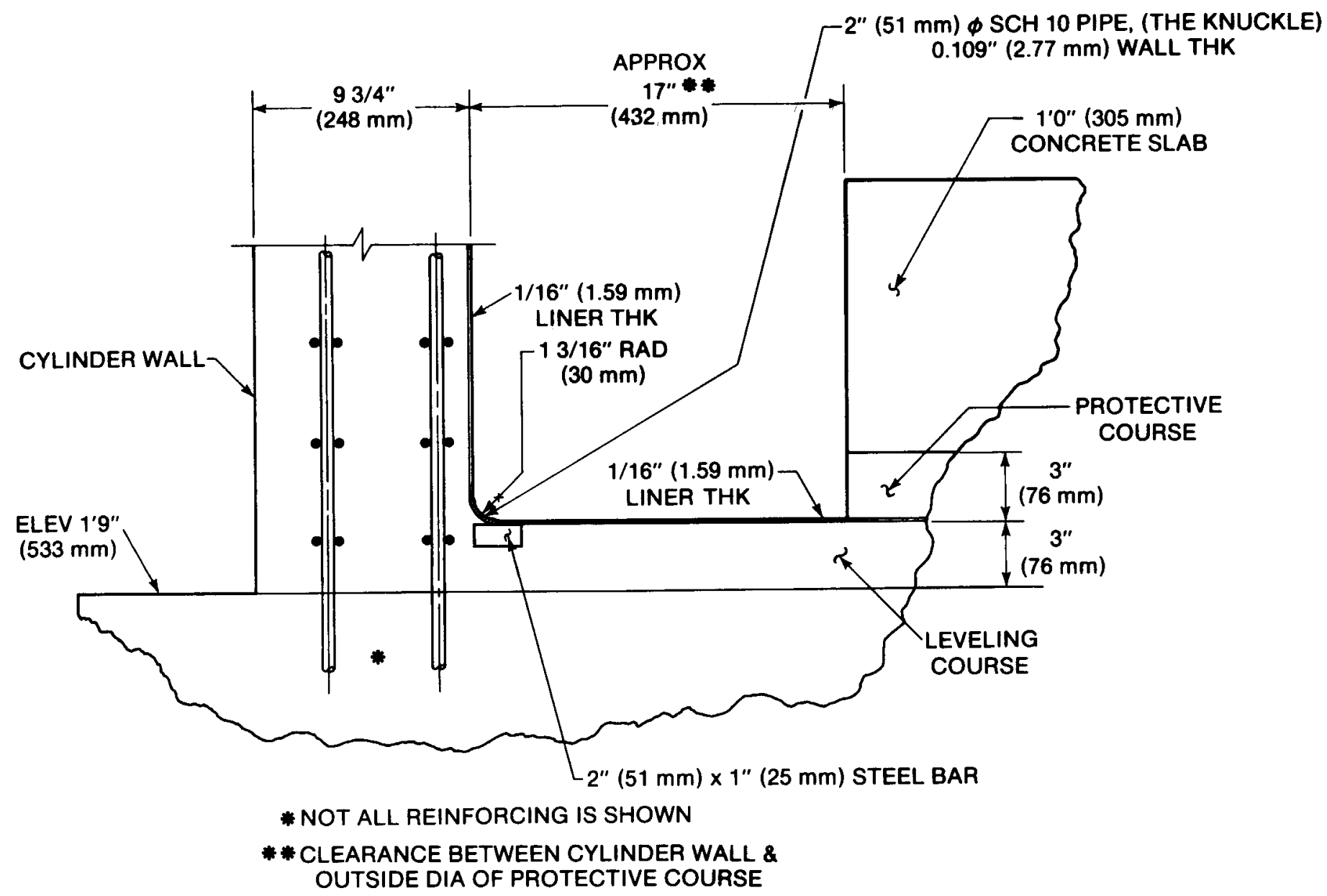

Figure 3.15 Details of Liner Knuckle 


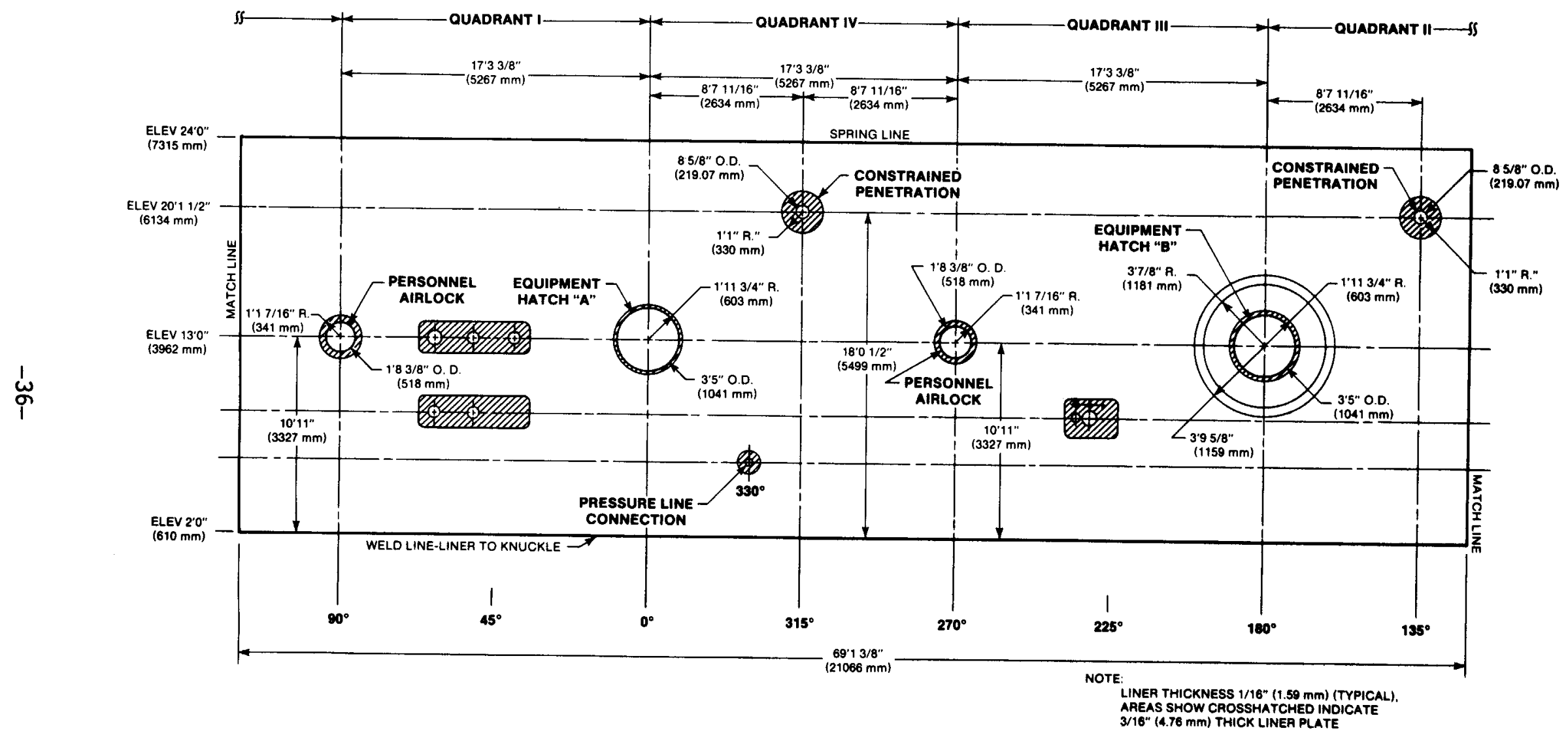

LINER STRETCHOUT

VIEWED FROM OUTSIDE

Figure 3.16 Cylinder Stretchout - Liner Details 


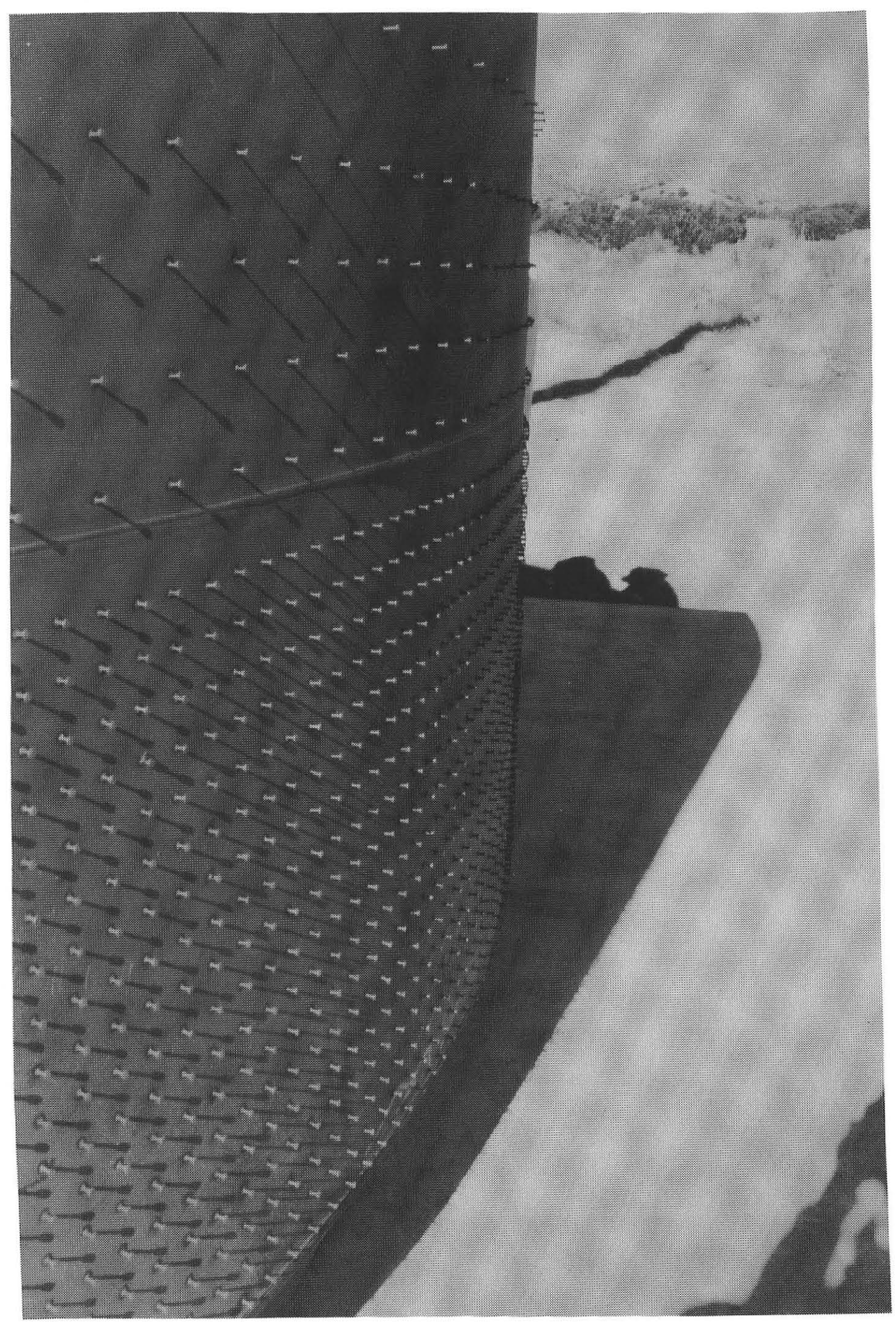

Figure 3.17 Stud Pattern in Lower Cylinder 


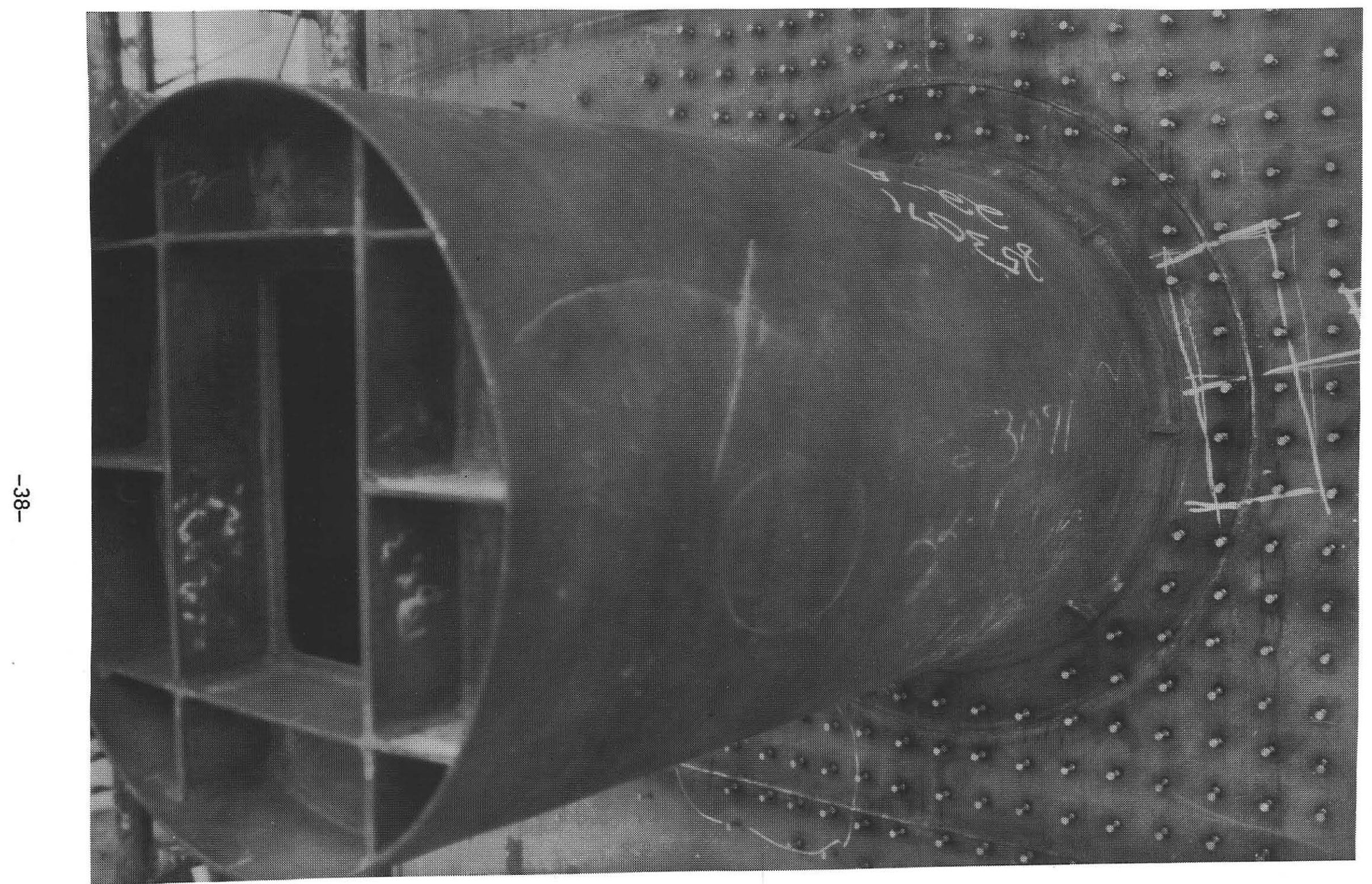

Figure 3.18 Stud Pattern Around Personnel Airlock A 


\section{PRETEST ANALYSES}

Each organization participating in the round-robin analysis contributed a section to this report; the order of presentation corresponds to the order in which the organizations formally joined into a cooperative agreement to conduct analyses and exchange information with the NRC. It is noted again that, with the exception of SNL and BNL, these analyses were not funded by the NRC. It is to be expected that the manpower, time, and resources brought to bear on this problem by each organization were not identical and may have differed considerably. SNL and NRC are grateful for the enthusiasm and cooperation demonstrated by the organizations that have volunteered their manpower and resources for the round-robin analysis.

In general, finite element approaches were used to calculate the model response, although some organizations supplement their finite element analyses with hand calculations or other simplified approaches. As part of their section, each organization was asked to prepare a brief summary of their analytical approach. In general, this summary appears at the beginning of each subsection. A list of the primary analytical codes used by each organization follows:

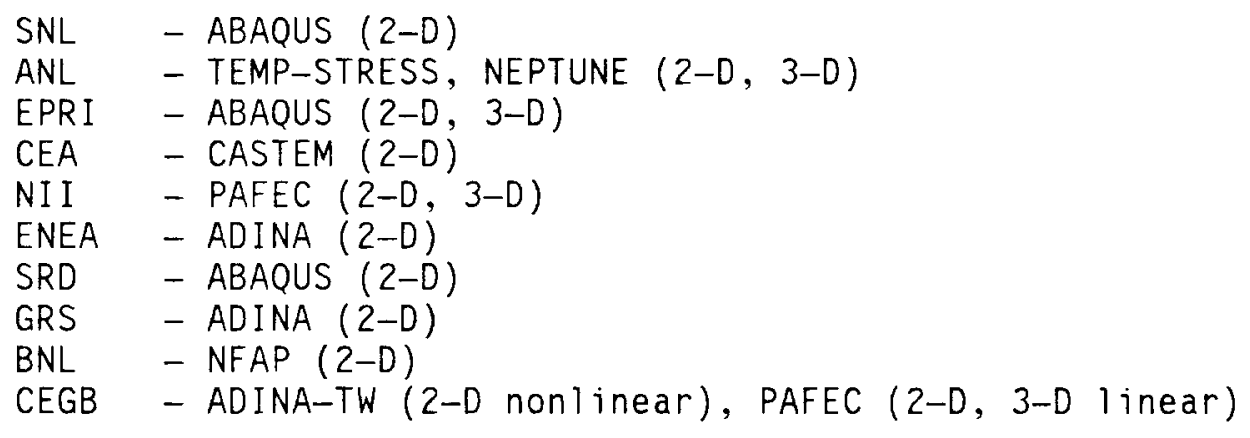

Results of the analyses are described in the following sections and are compared in the Executive Summary and Appendix A. 


\subsection{Sandia National Laboratories}

This section was written by $J$. R. Weatherby of the Engineering Analysis Department at Sandia National Laboratories (SNL).

\subsubsection{Summary}

A series of structural analyses was conducted at SNL to predict the structural response, failure pressure, and failure mode of the $1: 6$-scale containment model. This section of the report summarizes the methods, results, and conclusions of the SNL structural study.

The major objective of SNL's in-house pretest analysis effort was to evaluate the ability of existing, commercially available finite element codes to model the structural response of a reinforced concrete containment building subject to high internal pressure. For this reason, no attempts were made to develop new capabilities in this area. The ABAQUS general purpose finite element code [4.1.1], version 4-5-171 was used in all numerical calculations. This particular code was chosen because it is representative of the state of the art in commercially available finite element programs.

The scope of the analytical effort consisted of nonlinear axisymmetric finite element shell and continuum analyses of the containment structure. A nonlinear membrane analysis of the cylinder wall was also conducted and compared with the finite element model predictions. Table 4.1 .1 contains a synopsis of the important results from these analyses.

All of the models considered are limited in that they do not provide information about the state of stress and deformation in the vicinity of equipment hatches and other penetrations that are present in the actual 1:6scale model. While some of these penetrations are considered potential failure sites, there was not sufficient time to examine these regions.

\subsubsection{Material Models}

In order to predict the response of the containment building at high internal pressures, material models were introduced to describe the behavior of the liner, concrete, and steel reinforcement well beyond the elastic limit. Because geometric nonlinear effects are not considered in any of the analyses to be presented, engineering stress and strain measures are used exclusively in the definition of material properties. In the finite element calculations presented here, the post-yield stress-strain behavior of the liner and reinforcing steel have been represented through the use of a von Mises yield function together with isotropic hardening and an associated plastic flow rule. Elastic constants and points from the piecewise linear stress-plastic strain curves used in the analyses are listed in Tables 4.1 .2 through 4.1.4 for the iner and rebar. These values were obtained by averaging results from a number of uniaxial tensile tests that were conducted on samples of the liner material and \#4 rebar.

The standard concrete constitutive model supplied with the ABAQUS code was used in all finite element analyses. A complete description of the model is given in the ABAQUS Theoretical Manual [4.1.1]. This material model is 


\section{Table 4.1.1 Analys is Summary}

Analytical Methods

Constitutive Models

Failure Criterion

Predicted Failure Mode

Predicted Capacity nonlinear axisymmetric finite element shell and continuum models; nonlinear membrane analysis.

Concrete: elastic/plastic material with Chen and Chen yield surface and associated flow rule. Liner: elastic/plastic material with von Mises yield surface, associated flow rule, and isotropic hardening.

Rebar: piecewise linear uniaxial stress-strain curves.

Lack of convergence in finite element solution and evidence of concrete crushing imply structural instability.

Structural instability due to crushing failure of concrete on the outside of the cylinder wall near the basemat cylinder junction.

$170 \mathrm{psig}(1.17 \mathrm{MPa})$

\section{Pressure Event}

$30-35$ psi

$(.21-.24 \mathrm{MPa})$

$35-40$ psi (.24-.28 MPa)

40-45 psi (.28-.31 MPa)

100-120 psi (.69-.83 MPa)

115 psi

(.79 MPa)

130 psi

$(.90 \mathrm{MPa})$

135-140 psi (.93-.97 MPa)

170 psi

(1.17 MPa)

190 psi

(1.31 MPa)
Hoop strain at the midthickness of the cylinder exceeds the cracking strain* of the concrete.

Meridional strain at the midthickness of the cylinder exceeds the cracking strain* of the concrete.

Hoop and meridional strains at the midthickness of the dome exceed the cracking strain* of the concrete.

Hoop and meridional strains along the lower surface of the basemat exceed the cracking strain* of the concrete.

Liner yields in the cylinder midsection.

Hoop bars yield in the cylinder midsection.

Seismic and meridional bars yield in the cylinder midsection.

Concrete above the cylinder basemat junction crushes leading to numerical instability in the finite element model.

Hoop bars reach ultimate stress at the cylinder midheight.

* The cracking strain for the concrete is taken to be the strain level at maximum load in a uniaxial tensile test. 
Table 4.1.2

Material Properties for Cylinder and Basemat Liner

$\begin{array}{cl}\text { Engineering stress } & \text { Plastic } \\ \text { (ksi) } & \text { strain }\end{array}$

$\begin{array}{rll}50.2 & 346 & 0 . \\ 50.2 & 346 & .0157 \\ 59.0 & 407 & .0308 \\ 66.0 & 455 & .0696 \\ 68.0 & 469 & .0937 \\ 70.0 & 483 & .1620 \\ & & \\ \text { Young's modulus } & =30000 \mathrm{ksi}(207000 \mathrm{MPa}) \\ \text { Poisson's ratio } & =0.3 \mathrm{ks}(346 \mathrm{MPa}) \\ \text { Yield strength } & =50.2 \mathrm{ksi} \\ \text { Thickness } & =.0625 \text { inches }(1.59 \mathrm{~mm})\end{array}$

Table 4.1.3

Material Properties for Dome Liner
$\begin{array}{ccc}\text { Engineering stress } & \text { Table 4.1.3 } \\ \text { (ksi) } & \text { (MPa) } & \begin{array}{c}\text { Plastic } \\ \text { strain }\end{array} \\ 51.4 & 354 & 0 . \\ 61.1 & 421 & .0230 \\ 66.9 & 461 & .0478 \\ 70.5 & 486 & .0977 \\ 71.0 & 490 & .1476\end{array}$

Young's modulus $=30000 \mathrm{ksi}(207000 \mathrm{MPa})$

Poisson's ratio $=0.3$

Yield strength $=51.4 \mathrm{ksi}(354 \mathrm{MPa})$

Thickness $=.0833$ inches $(2.12 \mathrm{~mm})$

Rebar Material Properties Based on Nominal Cross-sectional Areas
$\begin{array}{rll}\text { Engineering stress } & \text { Plastic } \\ \text { (ksi) } & \text { (MPa) } & \text { strain } \\ 66.6 & 459 & 0 . \\ 73.3 & 505 & .0094 \\ 85.6 & 590 & .0200 \\ 99.0 & 683 & .0430 \\ \text { Young's modulus } & =31000 \mathrm{ksi}(214000 \mathrm{MPa}) \\ \text { Poisson's ratio } & =0.3 \\ \text { Yield strength } & =66.6 \mathrm{ksi}(459 \mathrm{MPa}) \\ & \end{array}$


capable of representing yielding, crushing, and cracking of the concrete and is an extension of the constitutive theory developed by Chen and Chen [4.1.2]. The theory defines two surfaces in stress space: a yield surface that surrounds the region of linearly elastic response, and a failure surface where cracking or crushing occurs. Between the yield and failure surfaces, the material is assumed to deform according to an associated plastic flow rule with isotropic strain hardening. After cracking or crushing, the concrete softens with increasing deformation finally losing all strength once a critical strain level is reached.

Numerical problems frequently arise once cracking takes place in a finite element solution. Previous work [4.1.3] indicated that after cracking occurs there are certain pressure ranges where the iterative procedure in the ABAQUS program fails to converge, leaving unacceptably large force imbalances in the structural model. To avoid these numerical difficulties, cracking was prevented by supplying the uniaxial stress-strain curves shown in Figure 4.1 .1 as input to the concrete model. Here, the softening behavior caused by cracking and crushing has been eliminated by using a very large value for the plastic strains corresponding to the ultimate tensile and ultimate compressive stresses. Two stress-strain curves have been defined in order to model the response at both low strain levels (Curve A) and high strain levels (Curve $B$ ). One set of finite element analyses was conducted using Curve $A$ for the concrete in the dome and cylinder; a second set of analyses was conducted using Curve $B$ for the concrete in the dome and cylinder. The reasoning behind this approach will be discussed later. Table 4.1.5 lists the remaining material constants required by the ABAQUS constitutive routine for concrete models $A$ and $B$. No biaxial compression data was available so the default values supplied in the ABAQUS code were used for those parameters related to the biaxial compressive strength of the concrete.

\subsubsection{Failure Criteria}

Realistic failure criteria must be adopted for all potential failure mechanisms in order to predict the ultimate pressure capacity of the containment. Failure is defined as any event that results in significant leakage. A high degree of uncertainty exists in selecting these criteria since, in many cases, the criteria are based on failure points observed in simple uniaxial tension and compression tests and applied to locations in the structure that are subject to more complex multiaxial loading conditions.

Except for regions where a large transverse shear force is present, the steel reinforcing bars are primarily in a state of uniaxial tension. Under these loading conditions, the reinforcing bars are assumed to fail at a tensile stress level of $99 \mathrm{ksi}(680 \mathrm{MPa})$. This value is based on results of tensile tests on spliced rebar specimens similar to those used in the construction of the containment model. The nominal cross-sectional area of the rebar specimens was used to convert from the failure load to the ultimate stress. Nominal cross-sectional areas for each type of rebar in the containment model are listed in Table 4.1.6. These areas were used in all of the analyses to be presented. 
Table 4.1.5

Material Parameters Used with the Concrete Constitutive Model in ABAQUS

\section{Parameter Description}

Young's modulus

Poisson's ratio

Ratio of each non-zero stress component at failure under biaxial compression to the stress magnitude at failure under uniaxial compression.

Ratio of the stress at failure under uniaxial tension to the stress at failure under uniaxial compression.

Ratio of the magnitude of a component of plastic strain at failure under biaxial compression to the plastic strain at failure under uniaxial compression.

Ratio of the magnitude of plastic strain at failure under uniaxial tension to the plastic strain at failure under uniaxial compression.

Notes:

1 corresponds to Curve $A$ in Figure 4.1 .1

2 corresponds to Curve $B$ in Figure 4.1.1

* default value in ABAQUS

$$
\begin{array}{ll}
\text { Model } A^{1} & \text { Model } B^{2} \\
4800 \mathrm{ksi} & 4800 \mathrm{ksi} \\
33000 \mathrm{MPa} & 33000 \mathrm{MPa}
\end{array}
$$
0.2
0.2

$1.16^{\star} \quad 1.16^{\star}$ $.0735 \quad .00147$

$1.28^{\star} \quad 1.28^{\star}$

1. 1.

Table 4.1 .6

\begin{tabular}{|c|c|c|}
\hline 3ar size & $i n^{2}$ & $\mathrm{~mm}^{2}$ \\
\hline$\# 2$ & .05 & 32 \\
\hline$\# 3$ & .11 & 71 \\
\hline \#4 & .20 & 129 \\
\hline$\# 5$ & .31 & 200 \\
\hline$\# 6$ & .44 & 280 \\
\hline
\end{tabular}

Nominal Cross-sectional Areas of Reinforcing Bars in the Containment Model

Area 
The failure criterion used for the steel liner is based on equivalent plastic strain. The equivalent plastic strain, $\bar{\epsilon}^{p}$, is defined as

$$
\bar{\epsilon}^{p}=\int_{0}^{t}\left(\frac{2}{3} \sum_{i, j} \dot{\epsilon}_{i j}^{p} \dot{\epsilon}_{i j}^{p}\right)^{\frac{1}{2}} d t
$$

where

$$
\dot{\epsilon}_{i j}^{p}=\text { plastic strain rate. }
$$

Failure is assumed to occur when the equivalent plastic strain reaches the level of equivalent plastic strain at maximum load in a uniaxial tensile test. This value is approximately $15 \%$ for both the $1 / 12 \mathrm{th}$-inch and $1 / 16 \mathrm{th}-$ inch thick liners.

Another possible type of material failure is concrete crushing, which can occur in regions of high compressive stresses brought about by bending. As discussed previously, the response of the concrete is assumed to be almost perfectly plastic after the compressive yield stress is reached so that the compressive yield and failure surfaces virtually coincide in stress space. In order to determine if the stress state lies on or near the compressive yield or failure surface, the crushing parameter, $\psi$, is introduced. This parameter was derived from the description of the concrete constitutive model given in the ABAQUS Theoretical Manual and is based on the assumption that the compressive yield strength is equal to the compressive ultimate strength. The crushing parameter, $\psi$, is defined by the relation

$$
\psi=\frac{\frac{1}{3} q^{2}-\beta_{c} \bar{p}}{\tau_{c}^{2}+\beta_{c} \bar{p}}
$$

where

$$
\begin{gathered}
\beta_{c}=\frac{f_{c}^{\prime 2}-f_{b c}^{2}}{2 f_{c}^{\prime}-4 f_{b c}} \\
\tau_{c}^{2}=\frac{f_{c}^{\prime} f_{b c}\left(2 f_{c}^{\prime}-f_{b c}\right)}{3\left(2 f_{b c}-f_{c}^{\prime}\right)} \\
q=\text { von Mises stress }=\frac{1}{\sqrt{2}}\left(\left(\sigma_{1}-\sigma_{2}\right)^{2}+\left(\sigma_{2}-\sigma_{3}\right)^{2}+\left(\sigma_{3}-\sigma_{1}\right)^{2}\right)^{\frac{1}{2}} \\
\bar{p}=\text { equivalent pressure }=-\frac{1}{3}\left(\sigma_{1}+\sigma_{2}+\sigma_{3}\right) \\
f_{c}^{\prime}=\text { uniaxial compressive strength } \\
f_{b c}=\text { biaxial compressive strength } \\
\sigma_{1}, \sigma_{2}, \sigma_{3}=\text { principal stresses }
\end{gathered}
$$

When $\psi<1$, the stress state lies inside of the compressive failure envelope; and when $\psi=1$, the stress state lies on the compressive failure surface and crushing is said to occur.

The first incidence of crushing is not considered to indicate failure of the containment structure; however, because of crushing, a limit point may exist 
beyond which deformations become very large with little or no increase in internal pressure. When such a condition develops, it is considered to be an indication that the containment structure is very near failure. Many times numerical solutions cannot be obtained beyond such a limit point so that other factors must be considered to help determine if instability in a numerical analysis is caused by the existence of a true limit point.

One of the most difficult modes of failure to evaluate in a reinforced concrete structure is failure brought about by shearing forces acting perpendicular to the reinforcement direction. In the cylinder and dome, these shearing forces act perpendicular to the surface of the shell in the radial direction and, hence, are referred to as radial shear forces. When a crack develops through the concrete wall, the shear load is carried by friction and aggregate interlock across the crack surfaces, and by dowel action of the reinforcing bars that span the crack plane. The models used to represent the rebar and concrete are too simplified to directly evaluate the potential for shear failure based solely on the failure criteria for the concrete and steel discussed previously. For this reason, empirical criteria were adopted to estimate the shear strength of a section under the combined action of radial shear and membrane tension. Results of "push-off" tests presented in [4.1.4] indicate that the ultimate shear strength of a reinforced concrete section falls within the range

$$
1.9 \sqrt{f_{c}^{\prime}}<\tau_{u}<17 \sqrt{f_{c}^{\prime}}
$$

where

$$
\begin{aligned}
& \tau_{u}=\text { ultimate shear strength, psi. } \\
& f_{c}^{1}=\text { ultimate compressive strength of concrete, psi. }
\end{aligned}
$$

Between these limits, the ultimate shear strength is assumed to be given by the relation

$$
\tau_{u}=\left[.013\left(\rho f_{y}-o_{v}\right)+1.9\right] \sqrt{f_{c}^{\prime}}
$$

where

$$
\begin{aligned}
& \rho=\text { meridional reinforcement ratio }=A_{s} / A_{g} \\
& f_{y}=\text { tensile yield strength of steel, } p s i \\
& \sigma_{v}=P_{m} / A_{g} \\
& A_{s}=\text { area of steel in meridional direction, in } \\
& A_{g}=\text { gross area of containment wall in meridional direction, } i^{2} \\
& P_{m}=\text { force in meridional direction, lb. }
\end{aligned}
$$

The area of the liner is not included in calculating $A_{s}$ or $A_{g}$; likewise, the meridional force carried by the liner is not included in $P_{m}$. A second criterion based on the shear-friction model [4.1.5] was also used to estimate the ultimate shear strength between the limits in Equation (4.1.3). The shear-friction model assumes that the shear strength varies according to 


$$
\tau_{u}=\rho f_{y}-\sigma_{v}
$$

Shear failure is assumed to occur when the applied shear force, $V$, is such that

$$
V / A_{g}>\tau_{u}
$$

\subsubsection{Nonlinear Membrane Analysis}

Before proceeding into a discussion of the finite element results, a simplified nonlinear membrane analysis of the cylinder wall will be presented. The results of this analysis serve as a check on the finite element calculations and also provide an upper bound on the strength of the containment structure. Figure 4.1.2 illustrates the idealized membrane model, which is used to represent a section of the cylinder wall. In this analysis, the hoop and meridional strains are assumed to be uniform through the thickness of the cylinder wall. Bending resulting from the shear forces and moments at the springline and basemat cylinder junction are ignored so that the analysis is expected to be most accurate near the midheight of the cylinder, far away from penetrations and other discontinuities. Small stress and strain measures are used in the analysis.

The loading on the cylinder consists of an internal pressure, $p$, and a vertical load, $W$, caused by the weight of the structure. To simulate conditions at the midheight of the cylinder, $W$ is set equal to the weight of the dome plus one-half the weight of the cylinder. The internal pressure generates a force per unit length of $p R$ in the hoop direction and $p R / 2$ in the meridional direction ( $R=$ the cylinder radius); the weight contributes a force per unit length of $W /(2 \pi R)$ in the meridional direction.

Because of the low tensile strength of the concrete (500 psi), the tension carrying capacity of the concrete is ignored, and the applied forces are assumed to be carried in the liner and rebar. The reinforcement in the wall of the cylinder is composed of \#4 steel bars running in the hoop and meridional directions and \#4 seismic bars that run at angles of \pm 45 degrees to the meridional direction. Each layer of reinforcement is assumed to carry load only along the direction of reinforcement. The liner is in a state of biaxial stress and carries a share of the applied load in both the meridional and hoop directions.

By transforming the uniaxial stress state in each reinforcement layer into components in the hoop and meridional directions, the equilibrium equations for the membrane analysis can be written as

$$
\begin{aligned}
& \sigma_{n} s_{n} n_{h} A+0.5 \sigma_{s} s_{s} n_{s} A+t_{1} \sigma_{1}^{h}=p R, \\
& \sigma_{m} s_{m} n_{m} A+0.5 \sigma_{s} s_{s} n_{s} A+t_{1} \sigma_{1}^{m}=p R / 2-W / 2 \pi R,
\end{aligned}
$$

where

$\sigma_{h}, \sigma_{s}, \sigma_{m}=$ axial stresses in the hoop, seismic, and meridional bars, $n_{h}, n_{s}, n_{m}=$ number of layers of hoop, seismic, and meridional bars, 


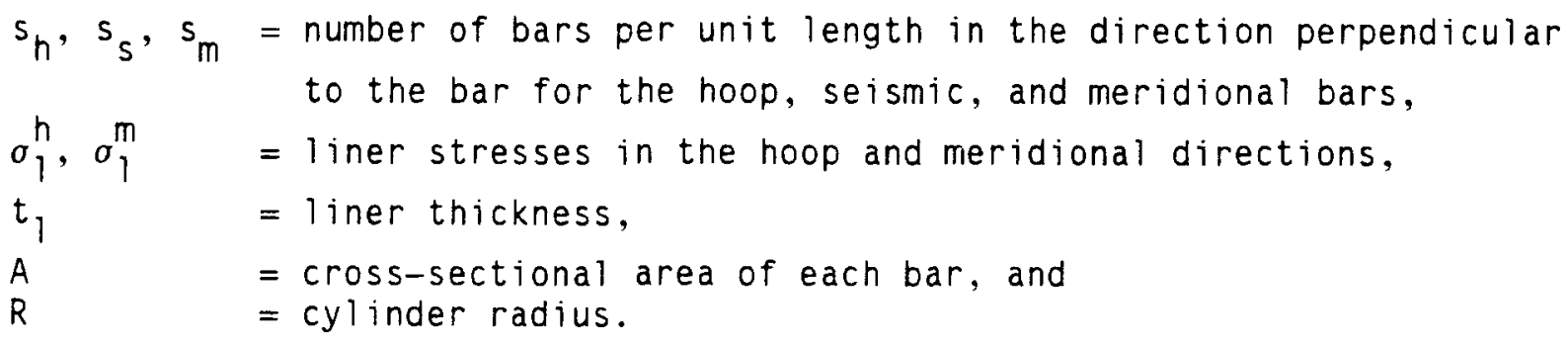

Stress-strain equations must be introduced to solve Equations (4.1.7) and (4.1.8). The stress in each rebar layer is expressed in terms of the hoop and meridional strains through the relations

where

$$
\begin{aligned}
& \sigma_{h}=f \operatorname{cn}\left(\epsilon_{h}\right), \\
& \sigma_{m}=\operatorname{fcn}\left(\epsilon_{m}\right), \\
& \sigma_{s}=\operatorname{fcn}\left(\epsilon_{s}\right),
\end{aligned}
$$

$$
\begin{gathered}
\epsilon_{h}, \epsilon_{m}=\text { hoop and meridional strains, respectively, } \\
\epsilon_{s}=\left(\epsilon_{h}+\epsilon_{m}\right) / 2=\text { strain in the direction of the seismic bars, and } \\
f c n=\text { uniaxial stress-strain curve for a single rebar. }
\end{gathered}
$$

Since the liner is in a state of biaxial tension, the stress-strain equations with plastic deformation are more complicated than those for the uniaxial stress state existing in the rebar layers. To simplify the analysis, the liner was assumed to be a linearly hardening material, and a radial return algorithm [4.1.6] was used to integrate the rate constitutive equations for the liner.

The equilibrium equations represent a nonlinear system of equations with two unknowns: $\epsilon_{h}$ and $\epsilon_{m}$. A short computer program was written to solve the system of equations using a modified Newton method. The solution was obtained over a range of internal pressure varying from 5 to 191 psig (.034$1.32 \mathrm{MPa})$ by incrementally increasing the pressure in steps of 2 psig (.014 $\mathrm{MPa})$. Equilibrium iterations were continued during each pressure step until the norm of the out-of-balance forces was less than $1 \times 10^{-5}$ of the norm of the applied forces.

Material properties used for the rebar are the same as those listed in Table 4.1.4. The yield and ultimate strength of the reinforcing steel were taken to be $66.6 \mathrm{ksi}(459 \mathrm{MPa})$ and $99 \mathrm{ksi}(683 \mathrm{MPa}$ ) based on a nominal crosssectional area of $.20 \mathrm{in}^{2}\left(129 \mathrm{~mm}^{2}\right)$. Between yield and ultimate, a piecewise-linear stress-strain curve was used for the rebar. The liner was treated as a linearly hardening material with an initial modulus of 30000 ksi $(210000 \mathrm{MPa})$, a yield strength of $50.2 \mathrm{ksi}(346 \mathrm{MPa})$, a hardening modulus of $256 \mathrm{ksi}(1770 \mathrm{MPa})$, and a thickness of .0625 inches $(1.59 \mathrm{~mm})$. Figure 4.1 .3 shows a plot of the stresses in the liner and reinforcement as a function of pressure as predicted by the membrane analysis.

These results were generated with a deadweight load of $.221 \times 10^{6}$ ib applied in the meridional direction to account for the weight of the containment 
structure above the midheight of the cylinder. The analysis predicts that liner yielding occurs at a pressure of $95 \mathrm{psig}(.66 \mathrm{MPa})$. Yielding of the hoop bars occurs at an internal pressure of 129 psig (.89 MPa), followed by yielding of the seismic reinforcement at $135 \mathrm{psig}$ (.93 MPa), and yielding of the meridional bars at $149 \mathrm{psig}(1.03 \mathrm{MPa})$. The ultimate strength of the hoop bars is reached at a pressure of $191 \mathrm{psig}(1.32 \mathrm{MPa})$. These results are relatively insensitive to vertical position in the cylinder since the weight of the structure above the cylinder midheight accounts for only $2 \%$ of the total meridional force at $190 \mathrm{psig}(1.3 \mathrm{MPa}$ ) internal pressure.

\subsubsection{Finite Element Shell Analyses}

The nonlinear membrane analysis presented in the previous section provides considerable insight into the containment response at the midheight of the cylinder; however, it gives no information about stresses in the dome or in the region near the base of the cylinder where a significant meridional bending moment and radial shear force exist. To evaluate the containment response in these locations, nonlinear finite element shell analyses were conducted using the ABAQUS finite element code. Only material nonlinear effects were considered in these analyses; geometric nonlinearities were neglected.

An axisymmetric finite element model of the containment structure was constructed using two-noded, thick-shell elements for the basemat, dome, and cylinder. The concrete, liner, rebar, and dome plates are all represented. The generalized force-moment strain-curvature relations for the shell are obtained by using simpson's rule to integrate the stresses through the thickness of the shell. In these analyses, nine integration points were used through the thickness of the concrete layer, and five integration points were used through the liner thickness. Single-point integration was used along the length of each shell element to evaluate the element's contribution to nodal forces and moments. A model for the soil that supports the basemat was not included in the analyses; instead, the basemat is assumed to rest on a rigid surface. Gap elements are used between the basemat and the rigid surface to allow for basemat uplift.

The layered shell and rebar options in the ABAQUS code were used to place the liner and rebar at the appropriate locations through the thickness of the shell. Each layer of steel reinforcement is represented by an orthotropic layer that carries load only in the direction of the reinforcement. The rebar option was used to represent the seismic as well as the hoop and meridional reinforcement. Problems were encountered when the rebar, liner, and concrete were all placed in a single layered-shell element. These problems were circumvented by overlaying three separate shell elements at each location in the model. One of these elements contained the rebar layers; a second element contained the steel liner; and a third element was used for the concrete. Nominal values were used for the thickness of the dome, cylinder, and basemat liners. These values are listed in Tables 4.1 .2 and 4.1.3. The cross-sectional areas used for the reinforcing bars are listed in Table 4.1.6. To account for the reduced shear stiffness of the concrete after cracking, the transverse shear stiffness of the concrete shell elements was set equal to $G t / 6$, where $G$ and $t$ are the shear modulus and concrete thickness, respectively. This value is equal to $20 \%$ of the default value used in ABAQUS. The liner and rebar elements were given a very low transverse shear stiffness $(.01 \mathrm{lb} / \mathrm{in})$. 
Figure 4.1.4 shows an outline of the shell model used in the analyses. This model contains a total of 92 nodes. The base of the cylinder has been offset from the centerline of the basemat by 20 inches to account for the thickness of the basemat. A multipoint constraint is used to force slope and displacement continuity at the cylinder basemat junction. Table 4.1.7 lists the element length and containment thickness in each region of the model. Given that the element length is in all cases less than the shell thickness, further mesh refinement would not be expected to yield a more accurate prediction for the response of the structure.

Two separate analyses were conducted using the finite element model shown in Figure 4.1.4. In the first analysis, Curve A of Figure 4.1.1 (500 psi tensile yield strength) was used as the uniaxial stress-strain curve for the concrete at all locations in the containment structure. This analysis will be referred to as Model 1. In Model 2 the uniaxial stress-strain curve

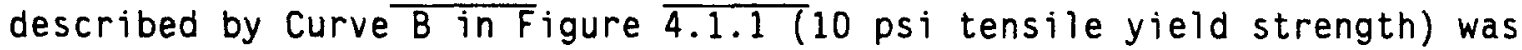
used for the concrete in the dome and cylinder, and Curve $A$ was again used for the concrete in the basemat.

Two analyses were used rather than a single analysis in order to provide a bound on the response of the containment structure and to obtain more realistic results at both low and high pressures. At very low pressures, before concrete cracking occurs in the actual containment, Model 1 will give a more accurate prediction of structural response than Model 2. After cracking occurs, panel tests suggest that the concrete will continue to contribute significantly to the overall stiffness of the structure [4.1.7]. These tests indicate that the stiffening effect of the cracked concrete decreases with increasing strain and finally vanishes as the strains approach the yield strain of the rebar. For this reason, the Mode 1 results are expected to remain the most accurate of the two sets of results until both the meridional and hoop strains reach a level equal to half of the rebar yield strain over a significantly large region in the cylinder and dome. Model 2 will become more accurate as the reinforcement approaches yield in the dome and cylinder.

In both finite element analyses, the dead load caused by the weight of the structure was applied in the first step. The internal pressure was then increased using automatic load incrementation. Equilibrium iterations were continued within each load step until the force imbalance at every node was less than the convergence tolerance. Table 4.1 .8 shows the convergence tolerance and maximum pressure step size allowed within each pressure range. Equilibrium iterations were continued within each load step until the maximum force residual was less than the specified convergence tolerance. Convergence was achieved in each step of the analysis up to pressures of 190 psig (1.3 MPa) and $168 \mathrm{psig}(1.16 \mathrm{MPa}$ ) for Model 1 and Model 2, respectively. Convergence could not be obtained beyond these pressures in either model, and in both cases the node with the largest force imbalance was the first node above the cylinder basemat junction. Further investigation revealed that in each case the concrete had crushed on the outside of the cylinder in the first element above the basemat. This is believed to be the event that prevented convergence at higher pressures.

Deformed shape plots at maximum pressure are shown in Figures 4.1 .5 and 4.1 .6 for Models 1 and 2, respectively. Note that the presence of meridional curvature in the midsection of the cylinder violates the no- 
bending assumption made in the nonlinear membrane analysis. Figure 4.1 .7 shows the radial displacement at the midheight of the cylinder as predicted by the membrane and finite element analyses. As expected, the membrane analysis closely agrees with the results from Model 2, which uses a tensile yield strength of $10 \mathrm{psi}(.07 \mathrm{MPa})$ for the concrete in the dome and cylinder. The stiffer response of Model 2 is probably attributable to meridional bending, which increases the stress in the seismic bars above the values predicted by the membrane analysis. In Figure 4.1 .8 basemat uplift is plotted as a function of pressure for a point directly beneath the cylinder wall. The displacement predictions from Models 1 and 2 are very close at this location because both analyses use the same concrete model (Curve $A$ ) in the basemat. Figure 4.1 .9 shows the vertical displacement of the dome apex as a function of pressure. Both models predict that the apex of the dome moves continuously upward in contrast to results from one of the earlier analyses reported in [4.1.3] where the dome apex moved downward with increasing pressure. Concrete cracking was not suppressed in this earlier analysis, and, as a result, a convergent numerical solution was not obtained in most load steps. Given the results from Models 1 and 2 , it appears that the downward displacement reported in [4.1.3] was caused by large residual (out-of-balance) forces in the finite element model and does not represent a physically real phenomenon.

Even though cracking was suppressed in the analysis, concrete cracking can be traced approximately by examining the strains in Model 1 and comparing them to the strain level at which cracking occurs in direct tension tests (approximately .01\%). This approach is very approximate and is not expected to yield results that are as accurate as those obtained by a true cracking analysis. Figures 4.1.10 through 4.1 .13 show the hoop and meridional strains at the centerline of the cylinder and dome and along the lower surface of the basemat. In these Figures, the coordinate $s$, defined in Figure 4.1.4, has been used to identify position within the model; $s=s_{0}$

corresponds to the basemat cylinder junction. This data was used to determine the cracking sequence, which is listed in Table 4.1.1.

The midthickness strains can also be examined to estimate the pressure level at which the results from Model 2 become more representative of the actual containment response than the results from Model 1 . It is assumed that the load carried by the concrete decreases linearly with increasing strain after cracking and finally vanishes when the hoop and meridional strains exceed a value of . $2 \%$, which is the yield strain of the reinforcing steel. Figures 4.1.14 and 4.1.15 show the meridional and hoop strains in the dome and cylinder wall for the pressure range 80-140 psig (.55-.97 MPa). From these Figures it can be seen that by $120 \mathrm{psig}(.83 \mathrm{MPa})$ the strain in both the meridional and hoop directions has reached a level close to .1\% over much of the dome and cylinder. By the time this strain level is reached, the average stress carried by the concrete in both directions will have dropped to a value equal to half of the concrete tensile strength (provided the average stress in the concrete decreases linearly as assumed). For this reason, 120 psig (.83 $\mathrm{MPa}$ ) is chosen as the cutoff above which the results from Model 2 are thought to best approximate the structural response.

Figures 4.1.16 through 4.1.19 show the stresses in reinforcement layers 1 , 2 , 5, and 8 as a function of position in the cylinder and dome regions for internal pressures ranging from $130-168 \mathrm{psig}(.90-1.16 \mathrm{MPa})$. These plots 
were made from the results of the Model 2 analysis, which is believed to be the most accurate of the two analyses in this pressure range. The sudden stress jumps seen in these plots are caused by rebar terminations in the structure. The actual stress distribution would be expected to be smoother as it takes a finite distance to transfer load into a bar. At $168 \mathrm{psig}$ (1.16 MPa) the most highly stressed bars in the structure are the hoop bars in the cylinder midsection. Bending at the basemat cylinder junction also produces high stresses in the Layer 2 meridional bars, and until $140 \mathrm{psig}$ (.96 $\mathrm{MPa}$ ) the maximum stress in the meridional reinforcement occurs at this location. The termination of Layer 2 and Layer 5 meridional bars adjacent to the dome plates was not included in the finite element model making the reinforcement at this point in the model twice as dense as that in the actual structure. The stresses in the meridional bars at this location can be estimated by multiplying the predicted stresses by a factor of two. Performing this calculation shows the inside meridional bars near the dome plates are at a stress level of approximately $86 \mathrm{ksi}$ (593 MPa) at $168 \mathrm{psig}$ (1.16 MPa) internal pressure.

The highest strains in the liner and reinforcement are found at the midsection of the cylinder. Yielding of the liner and reinforcement at this location is summarized in Table 4.1.9 for the two shell models and for the membrane analysis. In Model 2 the liner, hoop bars, and seismic bars yield at pressures very close to those predicted by the membrane analysis. There is a slightly larger discrepancy (12 psi (.08 $\mathrm{MPa})$ or $9 \%$ ) between the two analyses with regard to yielding of the meridional reinforcement. Figures 4.1.20 and 4.1 .21 contain plots of the strains in rebar layers $2,3,5$, and 8 as a function of pressure at an elevation of $13^{\prime}-9^{\prime \prime}, 12$ inches $(300 \mathrm{~mm})$ above the cylinder midheight. Note that bending develops after yielding of the meridional bars as evidenced by the different strain levels in the Layer 2 and Layer 5 meridional reinforcement. The strain level in all rebar layers remain well below the value of $4.6 \%$ necessary to cause failure of the rebar splices $(4.6 \%$ is the strain that corresponds to the $99 \mathrm{ksi}$ (683 $\mathrm{MPa}$ ) strength of the spliced bars).

Figure 4.1 .22 contains history plots of the equivalent plastic strain in the liner as a function of pressure at the midheight of the cylinder. Each point in this figure should be interpreted as an average value of plastic strain since strain concentrations brought on by cracks in the concrete wall are not accounted for in the analysis. Uniaxial tensile tests of the liner material show that the equivalent plastic strain reaches $15 \%$ at the ultimate stress. The average value of plastic strain in the liner remains well below this level; however, this does not rule out the possibility of liner tearing caused by strain concentrations in the vicinity of cracks in the concrete wall. In addition, there is reason to question the use of a maximum equivalent plastic strain obtained from uniaxial tensile tests as a criterion for predicting liner tearing under biaxial loading conditions.

Figures 4.1.23, 4.1.24, and 4.1.25 show the distribution of the radial shear force, the meridional moment, and hoop membrane force at 140 psig (.97 MPa) internal pressure. As expected, the largest value of the hoop membrane force is reached in the central region of the cylinder where it is approximately equal to $\mathrm{PR}$. The largest value of the meridional moment occurs at the intersection of the basemat and cylinder. The average transverse shear stress, obtained by dividing the shear force in Figure 4.1 .23 by the shell thickness, also reaches a maximum value just above the 
Table 4.1 .7

Lengths and Thicknesses of Shell Elements Used

in Each Region of the Finite Element Mesh in Models 1 and 2

\begin{tabular}{lcc} 
location & Element Length & Shell Thicknes \\
\cline { 3 - 4 } & $\begin{array}{c}6.84 \text { inches } \\
(174 \mathrm{~mm})\end{array}$ & $\begin{array}{c}43 \text { inches } \\
(1090 \mathrm{~mm})\end{array}$ \\
cylinder & $\begin{array}{c}6.68 \text { inches } \\
(461 \mathrm{~mm})\end{array}$ & $\begin{array}{c}9.75 \text { inches } \\
(248 \mathrm{~mm})\end{array}$ \\
dome & $\begin{array}{c}6.91 \text { inches } \\
(176 \mathrm{~mm})\end{array}$ & 7 inches \\
& &
\end{tabular}

Table 4.1.8

Maximum and Minimum Step Sizes and

Convergence Tolerances Specified for the Model 1 and 2 Analyses

\begin{tabular}{|c|c|c|c|}
\hline $\begin{array}{c}\text { Pressure } \\
\text { Range } \\
\end{array}$ & $\begin{array}{c}\text { Maximum } \\
\text { Pressure Step }\end{array}$ & $\begin{array}{l}\text { Minimum } \\
\text { Pressure Step }\end{array}$ & $\begin{array}{c}\text { Convergence } \\
\text { Tolerance }\end{array}$ \\
\hline $\begin{array}{c}0-50 \mathrm{psi} \\
(0-.345 \mathrm{MPa})\end{array}$ & $\begin{array}{c}5 . \mathrm{psi} \\
(.0345 \mathrm{MPa})\end{array}$ & $\begin{array}{c}.5 \mathrm{psi} \\
(.00345 \mathrm{MPa})\end{array}$ & $\begin{array}{l}625 \mathrm{lb} \\
(2780 \mathrm{~N})\end{array}$ \\
\hline $\begin{array}{c}50-100 \mathrm{psi} \\
(.345-.689 \mathrm{MPa})\end{array}$ & $\begin{array}{c}5 . \quad \mathrm{psi} \\
\left(.0345^{\mathrm{MPa}}\right)\end{array}$ & $\begin{array}{c}.5 \mathrm{psi} \\
(.00345 \mathrm{MPa})\end{array}$ & $\begin{array}{l}1250 \mathrm{lb} \\
(5560 \mathrm{~N})\end{array}$ \\
\hline $\begin{array}{c}100-150 \mathrm{psi} \\
(.689-1.03 \mathrm{MPa})\end{array}$ & $\begin{array}{c}5 . \mathrm{psi} \\
(.0345 \mathrm{MPa})\end{array}$ & $\begin{array}{c}.5 \mathrm{psi} \\
(.00345 \mathrm{MPa})\end{array}$ & $\begin{array}{l}1875 \mathrm{lb} \\
(8340 \mathrm{~N})\end{array}$ \\
\hline $\begin{array}{c}>150 \mathrm{psi} \\
(>1.03 \mathrm{MPa})\end{array}$ & $\begin{array}{c}.5 \mathrm{psi} \\
(.00345 \mathrm{MPa})\end{array}$ & 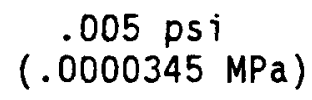 & $\begin{array}{l}2500 \mathrm{lb} \\
(11100 \mathrm{~N})\end{array}$ \\
\hline
\end{tabular}

Table 4.1 .9

Pressures at Which Yielding Occurs in the Liner and Reinforcement at the Midheight of the Cylinder in the Model 1, Model 2, and Membrane Analyses

\begin{tabular}{|c|c|c|c|}
\hline Event & Model 1 & Mode 12 & Membrane Anal \\
\hline liner yields & $\begin{array}{l}115 \mathrm{psi} \\
(.793 \mathrm{MPa})\end{array}$ & $\begin{array}{l}90 \mathrm{psi} \\
(.621 \mathrm{MPa})\end{array}$ & $\begin{array}{c}95 \mathrm{psi} \\
(.655 \mathrm{MPa})\end{array}$ \\
\hline hoop bars yield & $\begin{array}{c}152 \mathrm{psi} \\
(1.05 \mathrm{MPa})\end{array}$ & $\begin{array}{c}130 \mathrm{psi} \\
(.896 \mathrm{MPa})\end{array}$ & $\begin{array}{c}129 \mathrm{psi} \\
(.889 \mathrm{MPa})\end{array}$ \\
\hline seismic bars yield & $\begin{array}{c}158 \mathrm{psi} \\
(1.09 \mathrm{MPa})\end{array}$ & $\begin{array}{l}135 \mathrm{psi} \\
(.931 \mathrm{MPa})\end{array}$ & $\begin{array}{l}135 \mathrm{psi} \\
(.931 \mathrm{MPa})\end{array}$ \\
\hline meridional bars yield & $\begin{array}{c}162 \mathrm{psi} \\
(1.12 \mathrm{MPa})\end{array}$ & $\begin{array}{l}137 \mathrm{ps} i \\
(.945 \mathrm{MPa} .)\end{array}$ & $\begin{array}{c}149 \mathrm{psi} \\
(1.03 \mathrm{MPa})\end{array}$ \\
\hline
\end{tabular}


basemat cylinder junction. Other peaks in the shear stress are present at the springline and near the dome apex, adjacent to the dome plates.

Figures 4.1.26 through 4.1 .28 show plots of the radial shear force as a function of pressure at three critical locations: the cylinder basemat junction, the springline, and the edge of the dome plates. Equations (4.1.4) and (4.1.5) were used to determine the potential for shear failure in these regions at $168 \mathrm{psig}(1.16 \mathrm{MPa}$ ) internal pressure. At this pressure, all meridional bars near the dome plates and all meridional and seismic bars at the springline are above yield, so that the shear strength at these locations corresponds to the lower extreme $\left(1.9 \sqrt{f_{c}^{1}}\right)$ of the range in Equation (4.1.3). The results of the strength calculations are listed in Table 4.1.10 and compared to the predicted levels of shear stress from the finite element results of Model 2. Based on this comparison, it appears that a shear failure is not likely to occur at either the top or the base of the cylinder. There appears to be a much higher probability of shear failure at the edge of the dome plate at this pressure.

As mentioned earlier, the final failure or lack of convergence beyond the loads reported here is believed to be caused by crushing of the concrete on the outside of the cylinder combined with yielding of the meridional reinforcement in the first element above the basemat. Crushing is brought about by the large meridional bending moment that develops at the cylinder basemat junction. Figure 4.1.29 shows the bending moment at this location as a function of pressure for both shell models. In Figure 4.1.30, the crushing parameter, $\psi$, (see Equation (4.1.2)) at the base of the cylinder is plotted as a function of pressure for Models 1 and 2 . Crushing begins in Model 1 at a pressure of $167 \mathrm{psig}(1.15 \mathrm{MPa}$ ) and in Model 2 at a pressure of $145 \mathrm{psig}$ ( $1 \mathrm{MPa}$ ). In both models, the pressure level where a convergent solution can no longer be obtained is approximately $25 \mathrm{psi}$ (.17 MPa) higher than the point at which crushing is initiated. The strains in the meridional and seismic bars at the cylinder/basemat junction are plotted as a function of pressure in Figures 4.1 .31 and 4.1 .32 for Models 1 and 2 , respectively. The inside meridional reinforcement at this location reaches yield at an internal pressure of $147 \mathrm{psig}(1.01 \mathrm{MPa})$ in Model 1 and 127 psig (.875 MPa) in Model 2.

\subsubsection{Finite Element Analyses with a Continuum Basemat}

Because the thickness of the basemat is large relative to its diameter, there is reason to question the use of shell theory in this region of the structure. To investigate this question, a second set of finite element analyses was made using a continuum representation for the basemat and lower cylinder wall regions. As will be seen, the results of these analyses are in reasonable agreement with results from the shell models discussed in the previous section.

The finite element mesh that was used in this set of axisymmetric analyses is shown in Figure 4.1.33. This model contains a total of 461 nodes. Eight-noded, quadrilateral continuum elements were used to model the basemat and cylinder wall up to an elevation of 75 inches where the Layer 10 meridional reinforcement is terminated. In this region, the steel liner is modelled with three-noded axisymmetric shell elements that are attached along the inside surface of the containment model. At elevations above the 
point where the Layer 10 reinforcement is terminated, the containment wall and liner are modelled with two-noded, thick-shell elements. As in the previous shell analyses, three separate shell elements are used at each location in the upper cylinder and dome regions: one for the liner, one for the concrete, and one that contains the rebar layers. The rebar option in the ABAQUS computer code was again used to represent the reinforcement in both the continuum and she 11 sections of the model. The \#2 and \#3 shear ties below elevation 75 inches $(191 \mathrm{~cm}$ ) are also included in the model. Gap elements were placed along the lower surface of the basemat to allow for uplift of the basemat from a rigid foundation.

Four-point Gaussian quadrature was used to integrate the continuum elements. The two-noded shell elements were integrated using five integration points through the thickness and one point along the length of the shell. Threenoded shell elements were integrated with five integration points through the thickness and two integration points along the length of the shell.

The shell and continuum regions of the finite element model are joined together through the use of multipoint constraints in the ABAQUS program. Nodes along the upper edge of the continuum region are constrained to move so that the deformed section remains $p l a n e$ and does not warp. The rotation of this plane is forced to remain equal to the rotation of the first node at the lower edge of the shell region. Also, the displacement of the first node at the base of the shell section is tied to the displacement of the center node on the upper edge of the continuum region.

As in the previous section, two separate analyses were conducted using the same finite element mesh (Figure 4.1.33) but with different constitutive models for the concrete. In one analysis, Curve A of Figure 4.1.1 (500 psi tensile yield strength) was used as the uniaxial stress-strain curve for the concrete at all locations in the structure. This analysis is referred to as Model 3. In Mode 1 4, Curve B of Figure 4.1 .1 was used for the concrete in the dome and cylinder, and Curve $A$ was again used for the concrete in the basemat. As before, the shear rigidity of the concrete shell elements was reduced to $20 \%$ of the default value to account for loss of shear stiffness due to cracking. To determine the effects of the finite element modelling assumptions (shell versus continuum), the results from Model 3 should be compared to the results from Model 1 and, likewise, the Model 4 results should be compared against the results from Model 2.

The loading procedure described for Models 1 and 2 was also followed for applying loads in Models 3 and 4 . Table 4.1.11 lists the convergence tolerances along with the maximum and minimum pressure step sizes allowed within each pressure range. Convergence was achieved in each step of the analysis up to pressures of $188 \mathrm{psig}(1.3 \mathrm{MPa}$ ) and $166 \mathrm{psig}$ (1.14 MPa) for Models 3 and 4 , respectively. The Model 3 analysis was not terminated because of lack of convergence; however, convergence could not be obtained beyond an internal pressure of $166 \mathrm{psig}(1.14 \mathrm{MPa})$ in Model 4 . At this pressure, the largest force residuals in Model 4 occurred at nodes located near the base of the cylinder, on the outer surface of the containment wall. Further examination revealed that the concrete at this location was rapidiy approaching the crushing point just prior to $166 \mathrm{psig}(1.14 \mathrm{MPa})$. As in Models 1 and 2 , it is believed that the lack of convergence at higher pressures was caused by crushing of the concrete just above the basemat cylinder junction. 


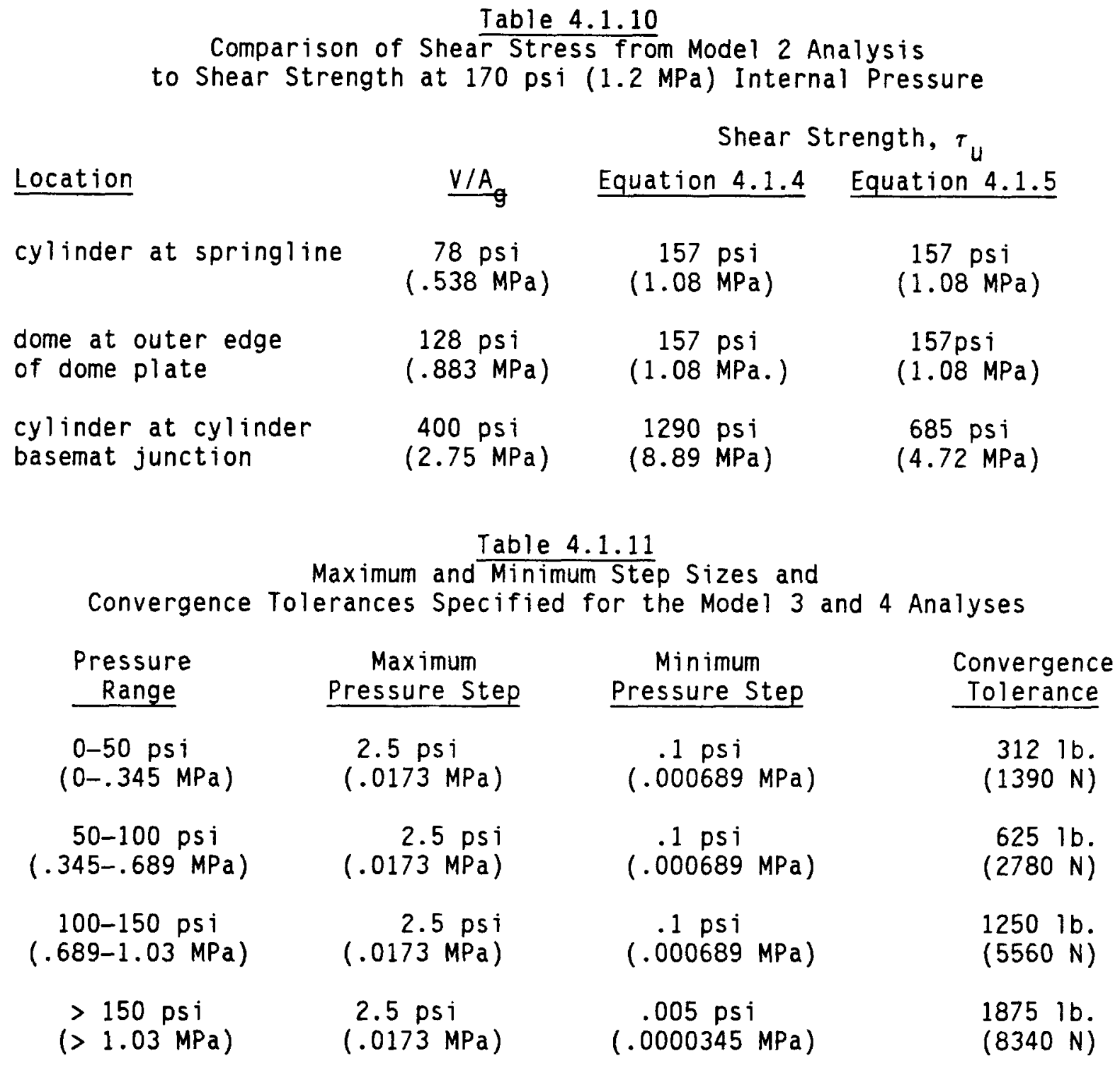

$-56-$ 
Before comparing the results from Models 3 and 4 to those obtained from Models 1 and 2 , one fact should be noted. The equivalent nodal forces for axisymetric shell elements subject to a pressure loading are evaluated based on the radial position of the centerline of the shell, whereas for continuum elements the equivalent nodal forces are calculated based on the radial position of the element face to which the pressure is applied. The latter method is the correct way to calculate nodal forces. As a consequence of this difference, a pressure $p$ applied to the inside of the 9.75 inch thick continuum cylinder wall is equivalent to a pressure of .96p applied to the inside of the shell cylinder of the same thickness. In Models 3 and 4 , the pressure applied in the shell region was reduced by $4 \%$ relative to that applied in the continuum region to account for this discrepancy; however, no adjustment was made in the Model 1 and 2 analyses. This discrepancy accounts for much of the difference in predicted response between the full shell analyses and the corresponding shell/continuum analyses.

Figure 4.1.34 shows the history of radial displacement at the upper termination of the Layer 10 reinforcement (elevation 75 inches $(190 \mathrm{~cm}$.$) ) as$ predicted by Models 1 through 4. In comparing these curves, recall that the same concrete properties are used in Models 1 and 3, and that a second set of concrete properties are used in Models 2 and 4 . The curves from the continuum/shell analyses are shifted to the right of the curves from the corresponding full shell analyses. As discussed previously, a $4 \%$ pressure shift can be accounted for by the different methods used to calculate equivalent nodal forces from the applied pressure loading. This shift accounts for most of the difference in predicted displacement.

The basemat uplift beneath the cylinder wall is plotted as a function of pressure in Figure 4.1 .35 for Models 3 and 4 . These predictions are in close agreement with results from the corresponding full shell analyses presented in Figure 4.1.8. Figure 4.1 .36 shows the radial shear force as a function of pressure at a location just above the cylinder basemat junction. These results are also in good agreement with the shear forces calculated at this location in the full shell models (see Figure 4.1.26). Figures 4.1.37 and 4.1.38 contain history plots of the strain in the meridional and seismic reinforcement at the base of the cylinder. In general, the strains in these layers are somewhat lower than the strains at the same location in the full shell models (see Figures 4.1 .31 and 4.1.32). This is because a rigid connection is assumed to exist between the centerline of the basemat and the edge of the cylinder in the full shell models. In contrast, this connection is not perfectly rigid in the continuum analyses of this region. The end result is that less bending is induced at the base of the cylinder in Models 3 and 4 leading to lower strains in the meridional and seismic reinforcement. Also, the shear ties which are included in the Model 3 and Model 4 analyses carry part of the meridional load, thus reducing the strain levels in the meridional and seismic reinforcement below the levels seen in the Model 1 and Model 2 analyses where the shear ties were not modelled.

Crushing above the cylinder basemat junction is believed to be the cause for the lack of convergence seen in the Model 4 analysis above 166 psig ( 1.14 $\mathrm{MPa}$ ) internal pressure. Figure 4.1.39 shows the crushing parameter (Equation (4.1.2)) at this location as a function of internal pressure for both Models 3 and 4 . The value of the crushing parameter at a given pressure level is consistently lower in each continuum/shell analysis as 
compared to the corresponding full shell analysis (see Figure 4.1.30). The reduction in the crushing loads is again brought about by the more flexible connection between the basemat and cylinder in the continuum models as compared to the rigid basemat-to-cylinder connection used in the shell models of this area. Even though the history of the crushing parameter is considerably different, Models 2 and 4 become numericaliy unstable at virtually the same pressure level.

\subsubsection{Standard Plots of the Structural Response}

One of the objectives in the pretest analysis phase of this project was to provide strain and displacement predictions for instrumented locations throughout the containment structure. These predictions are contained in Appendix $A$ of this report. Since analyses were conducted using a variety of models, a choice had to be made as to which set of results was most representative of the actual containment response. For the pressure range from $0-120$ psig $(0-.83 \mathrm{MPa})$, all of the predictions reported in Appendix $A$ were taken from results of the Model 1 analysis (shell model) with the exception of plots 2 and 11 , which were taken from results generated by the Model 3 analysis (shell/continuum model). Plots 2 and 11 contain history plots of the strains and deformations in the region near the basemat cylinder junction. Above $120 \mathrm{psig}(.83 \mathrm{MPa})$, the predictions reported in Appendix $A$ were taken from the Model 2 analysis for all plots except plots 2 and 11 , which were taken from the results of Model 4. The 120 psig (.83 MPa) cutoff was established based on observations of the strain levels in the dome and cylinder regions (see Figures 4.1.14 and 4.1.15). At this pressure level, both the meridional and hoop strains in the dome and cylinder regions are large enough so that the contribution of the concrete to the total tension stiffness in these regions is considered to be negligible.

\subsubsection{Conclusion}

Nonlinear axisymmetric membrane, shell, and continuum analyses of the 1:6scale containment model have been presented along with descriptions of the material models and failure criteria used for the different elements of the structure. Table 4.1.1 summarizes the expected history of events based on the analytical results.

The nonlinear membrane analysis gives an upper bound of 192 psig (1.32 MPa) for the failure pressure of the containment structure. Results from the membrane analysis are in close agreement with finite element predictions with regard to radial expansion, rebar yielding, and liner yielding near the midheight of the cylinder.

Separate finite element analyses were conducted using two sets of concrete material properties to obtain results both at lower pressures, where the concrete contributes significantly to the overall stiffness of the structure under tensile loading, and at higher pressures, where the concrete in the dome and cylinder is so heavily damaged that it contributes little to the overall structural stiffness in tension. On the basis of these calculations, strain and displacement predictions were made for points corresponding to instrumented locations in the 1:6-scale model (see Appendix A). 
The finite element results from Models 2 and 4 suggest that a limit point is reached at an internal pressure of approximately $170 \mathrm{psig}(1.17 \mathrm{MPa})$ due to concrete crushing at the base of the cylinder. Convergent numerical solutions could not be obtained for higher internal pressures. It is speculated that beyond this point, the base of the cylinder will undergo large rotations resulting in failure of the meridional bars or leakage because of liner tearing at this location. The possibility exists, however, that a plastic hinge forms at this location, and the section continues to carry load beyond the 170 psig pressure level. In this event, the next most probable failure modes appear to be failure of the hoop reinforcement near the midheight of the containment at approximately 190 psig or a shear failure adjacent to the dome plates. Further analysis is necessary to evaluate the potential for failure around the equipment hatches and constrained penetrations.

\subsubsection{Acknowledgments}

Sincere appreciation is expressed to J. Jung for his early contributions to the pretest analysis effort. Special thanks are also extended to $C$. $H$. Conley and to members of the Peer Review Panel on the NRC Containment Integrity Program for providing valuable suggestions and discussions related to the analytical work. 


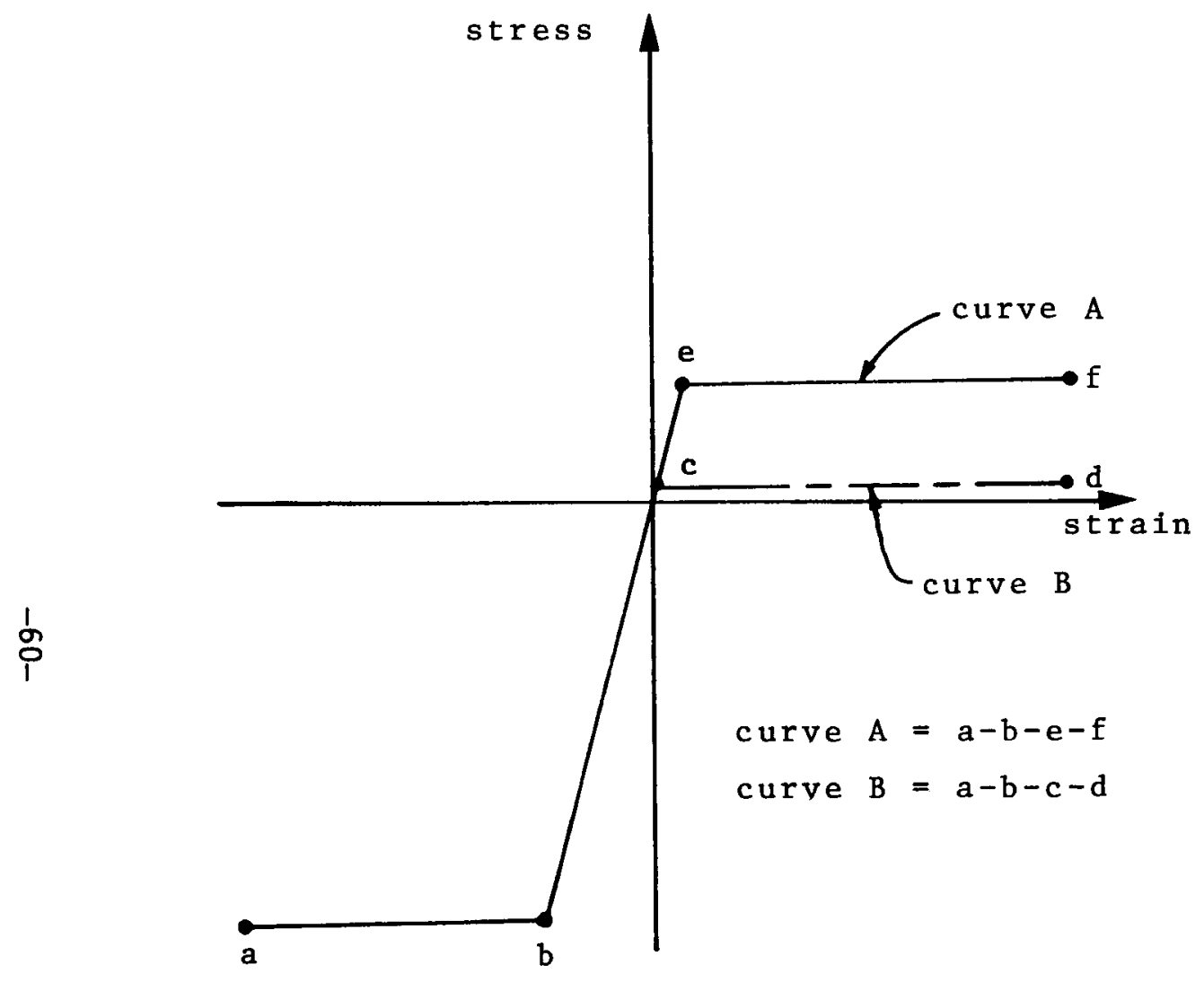

\begin{tabular}{|c|c|c|}
\hline pcint & stress & strain \\
\hline$a$ & $\begin{array}{c}-6800 \mathrm{psi} \\
(46.9 \mathrm{MPa} .)\end{array}$ & -2 \\
\hline$b$ & $\begin{array}{c}-6790 \mathrm{psi} \\
(46.8 \mathrm{MPa} .)\end{array}$ & $-1.42 \cdot 10^{-3}$ \\
\hline C & $\begin{array}{c}9.99 \mathrm{ps} i \\
(.0688 \mathrm{MPa} .)\end{array}$ & $2.08 \cdot 10^{-6}$ \\
\hline$d$ & $\begin{array}{l}10.0 \mathrm{psi} \\
(.0689 \mathrm{MPa} .)\end{array}$ & 2. \\
\hline $\mathrm{e}$ & $\begin{array}{c}499 \mathrm{psi} \\
(3.44 \mathrm{MPa} .)\end{array}$ & $1.04 \cdot 10^{-4}$ \\
\hline$f$ & $\begin{array}{c}500 \mathrm{psi} \\
(3.45 \mathrm{MPa} .)\end{array}$ & 2. \\
\hline
\end{tabular}

Figure 4.1.1 Uniaxial Stress-strain Curves Used for the Concrete 


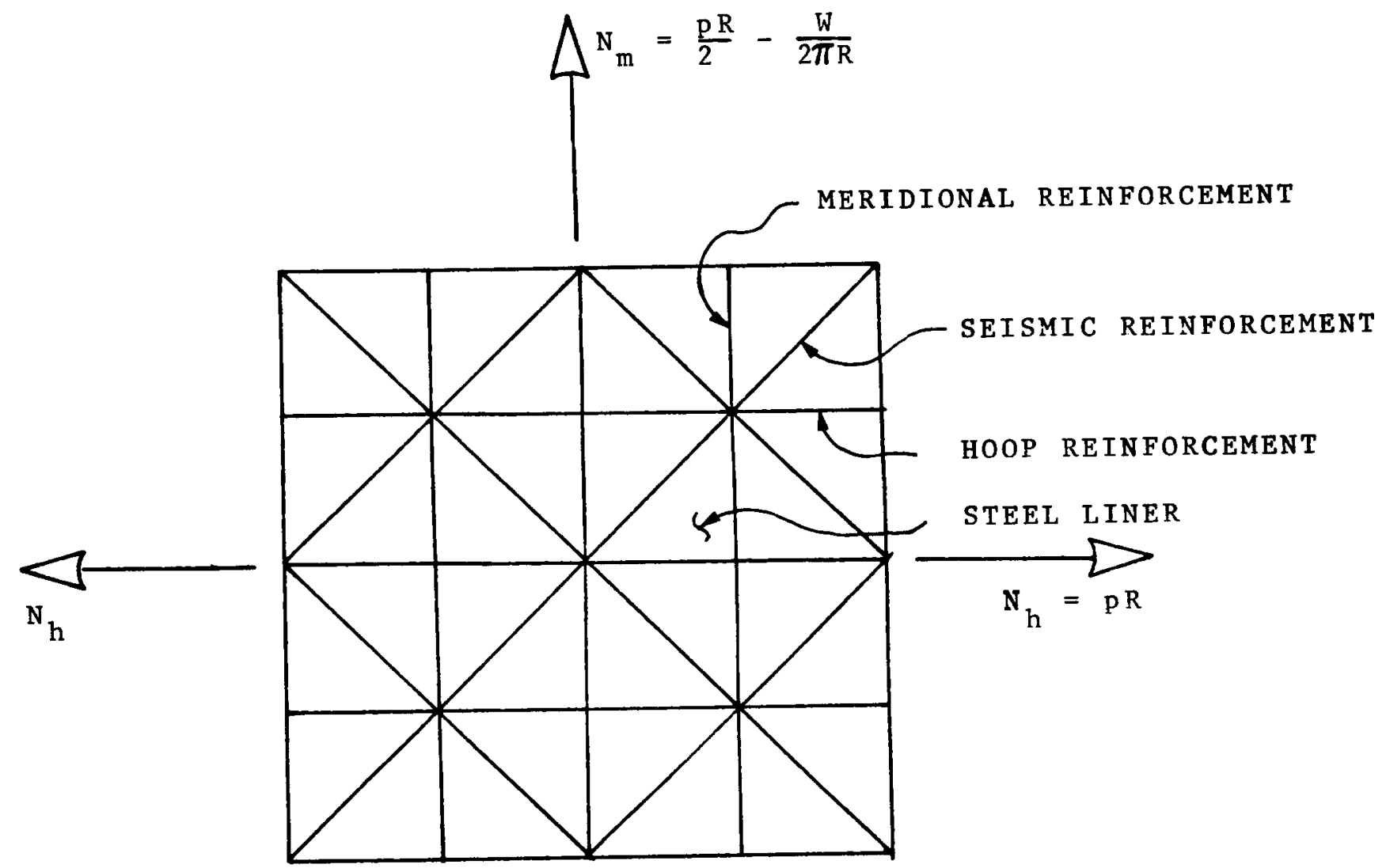

$N_{h}=$ hoop membrane force

$N_{m}=$ meridional membrane force

$W=$ weight of the overburden at the midheight of the cylinder

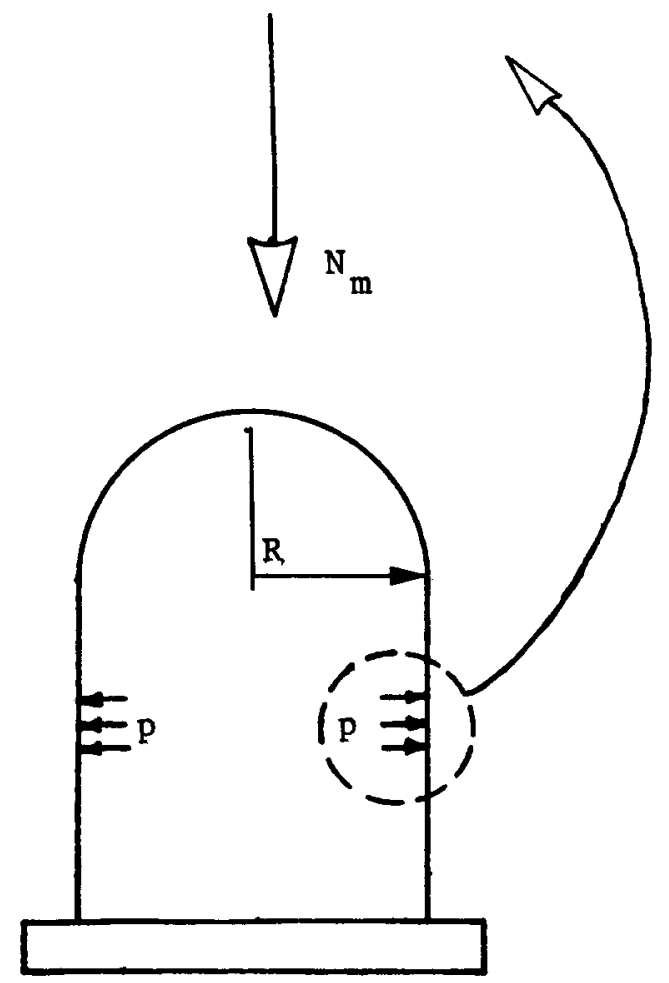

Figure 4.1.2 Nonlinear membrane idealization of the cylinder wall 


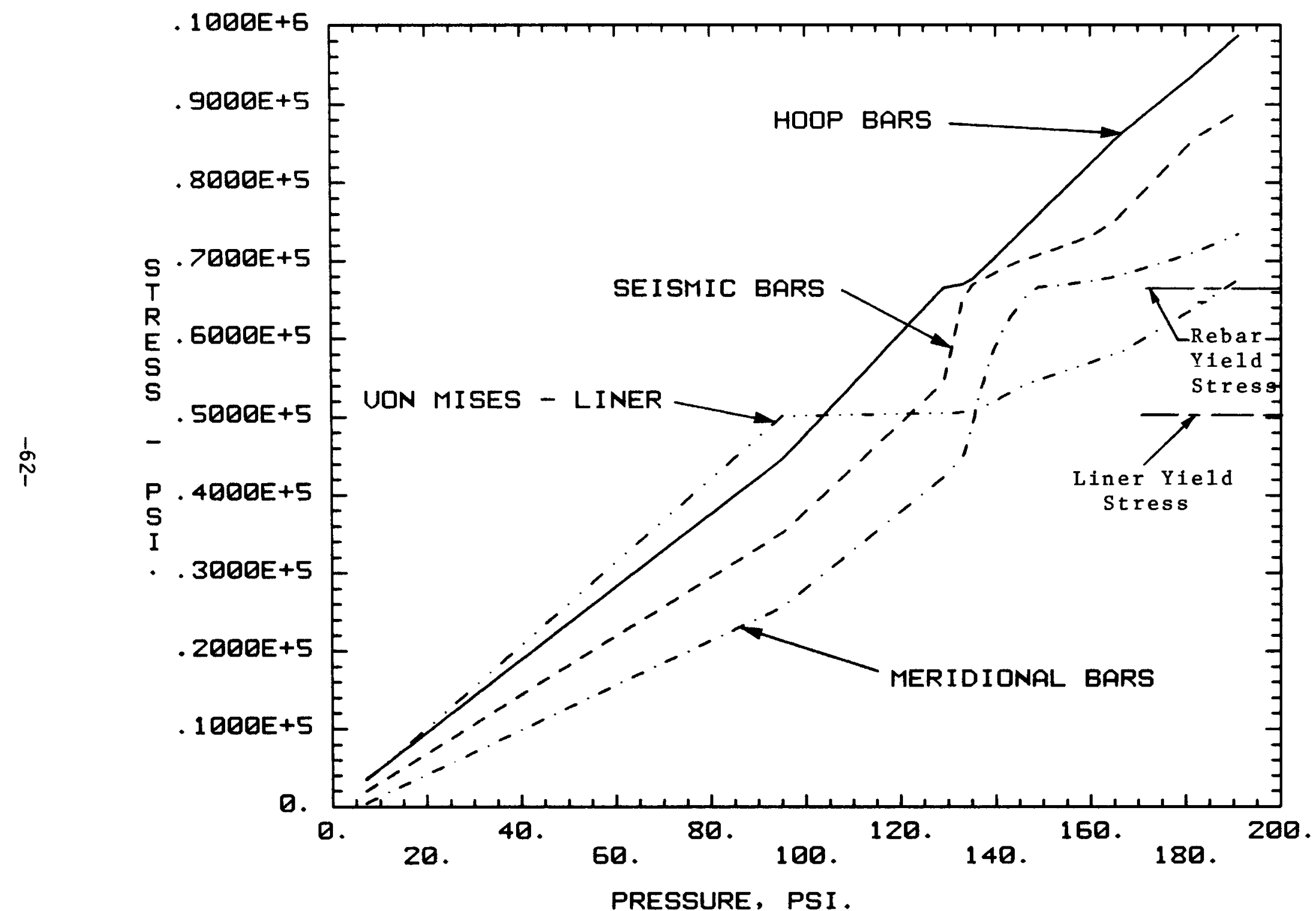

Figure 4.1.3 Membrane Theory Results for Stresses in the Rebar and Liner at the Midheight of the Cylinder 


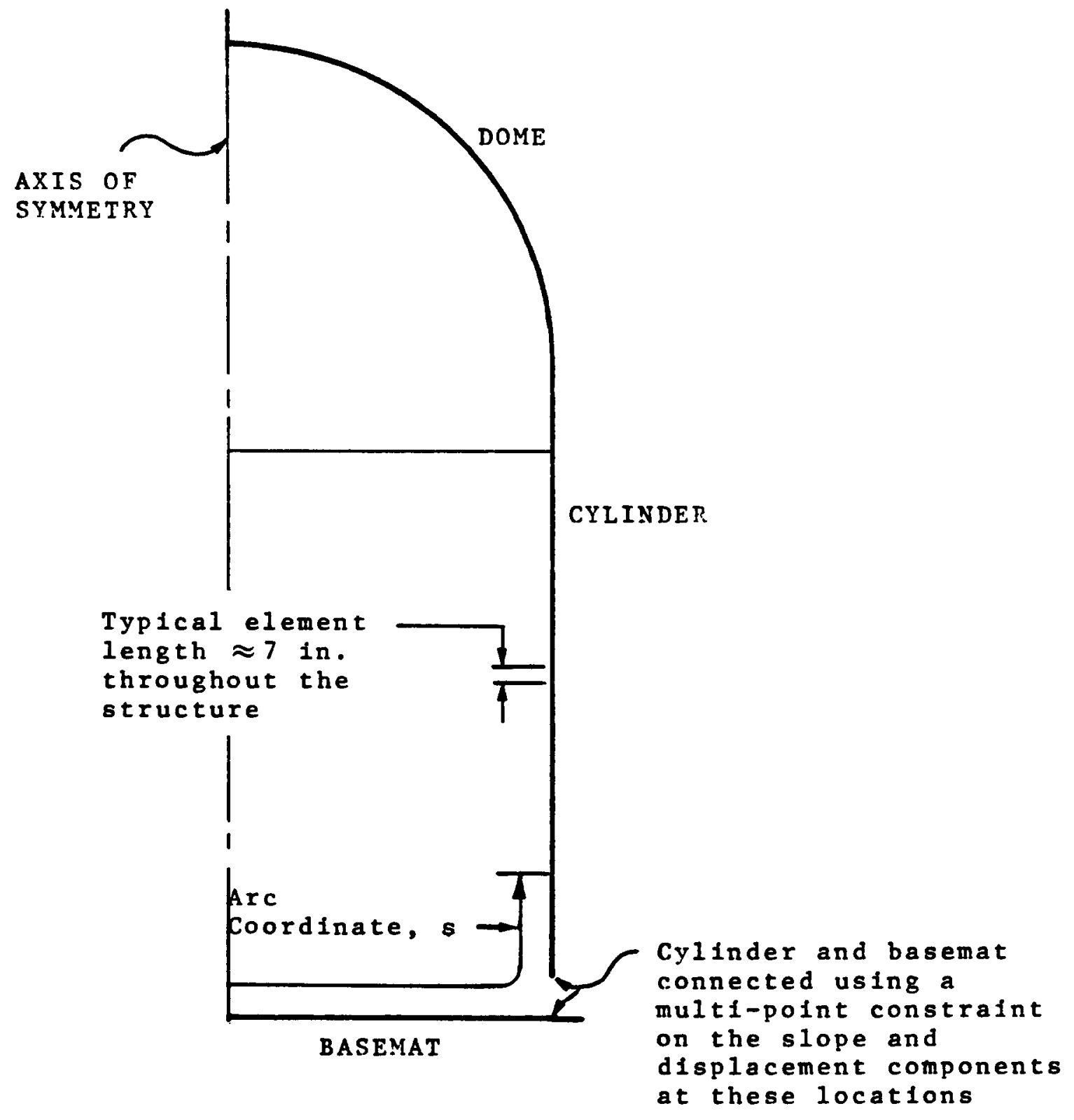

Figure 4.1.4 Outline of the axisymmetric finite element shell model 


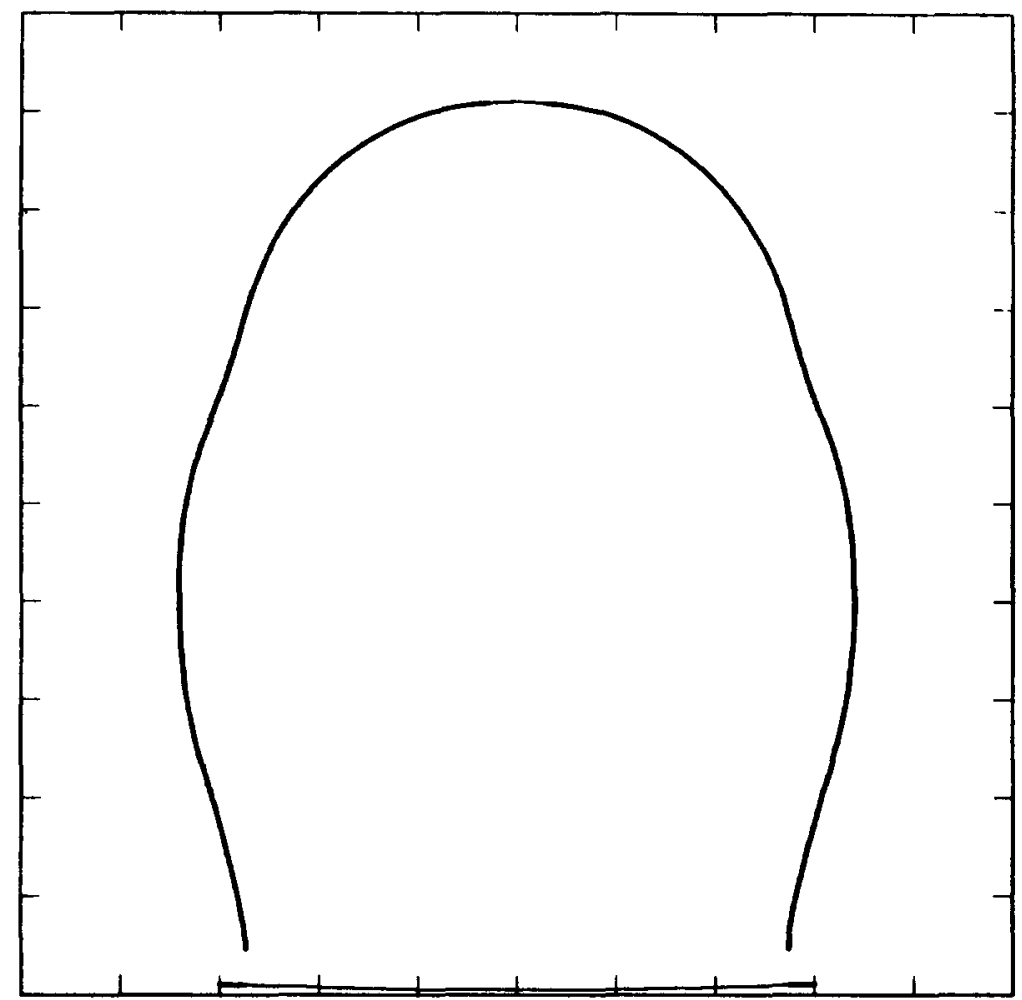

Figure 4.1.5 Deformed shape plot of Model 1 at 191 psi (1.32 MPa) (displacements multiplied by a factor of 10)

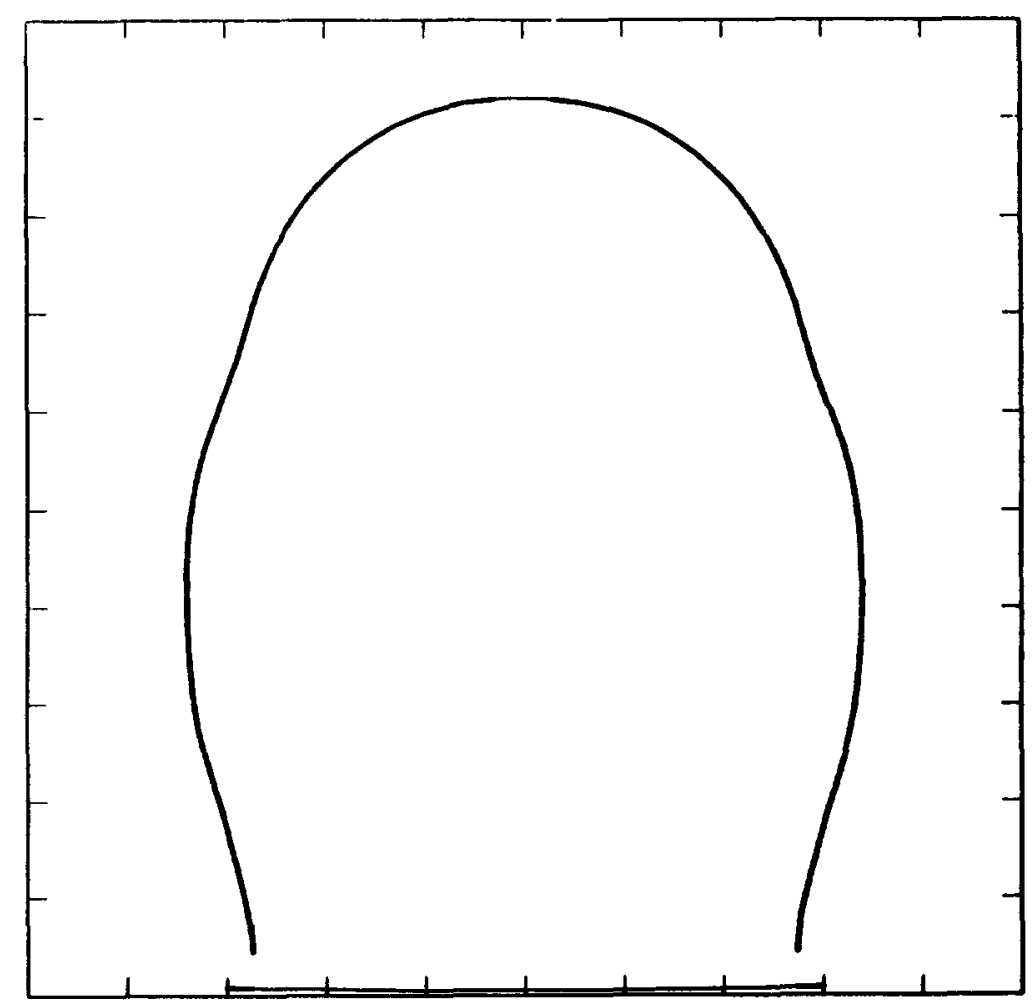

Figure 4.1.6 Deformed shape plot of Model 2 at $168 \mathrm{psi}$ (1.16 MPa) (displacements multiplied by a factor of 10) 


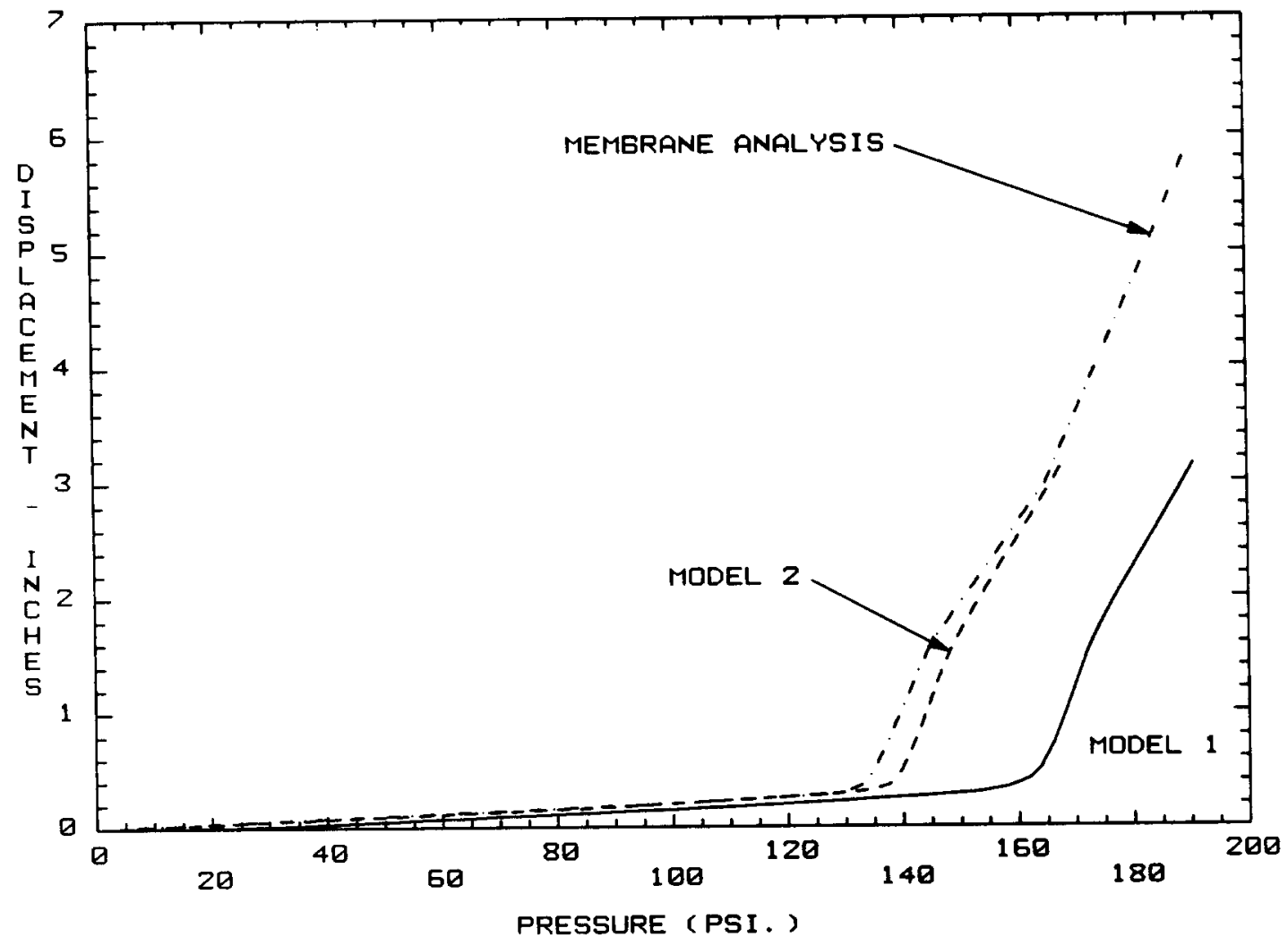

Figure 4.1.7 Radial displacement at the cylinder midheight vs. pressure

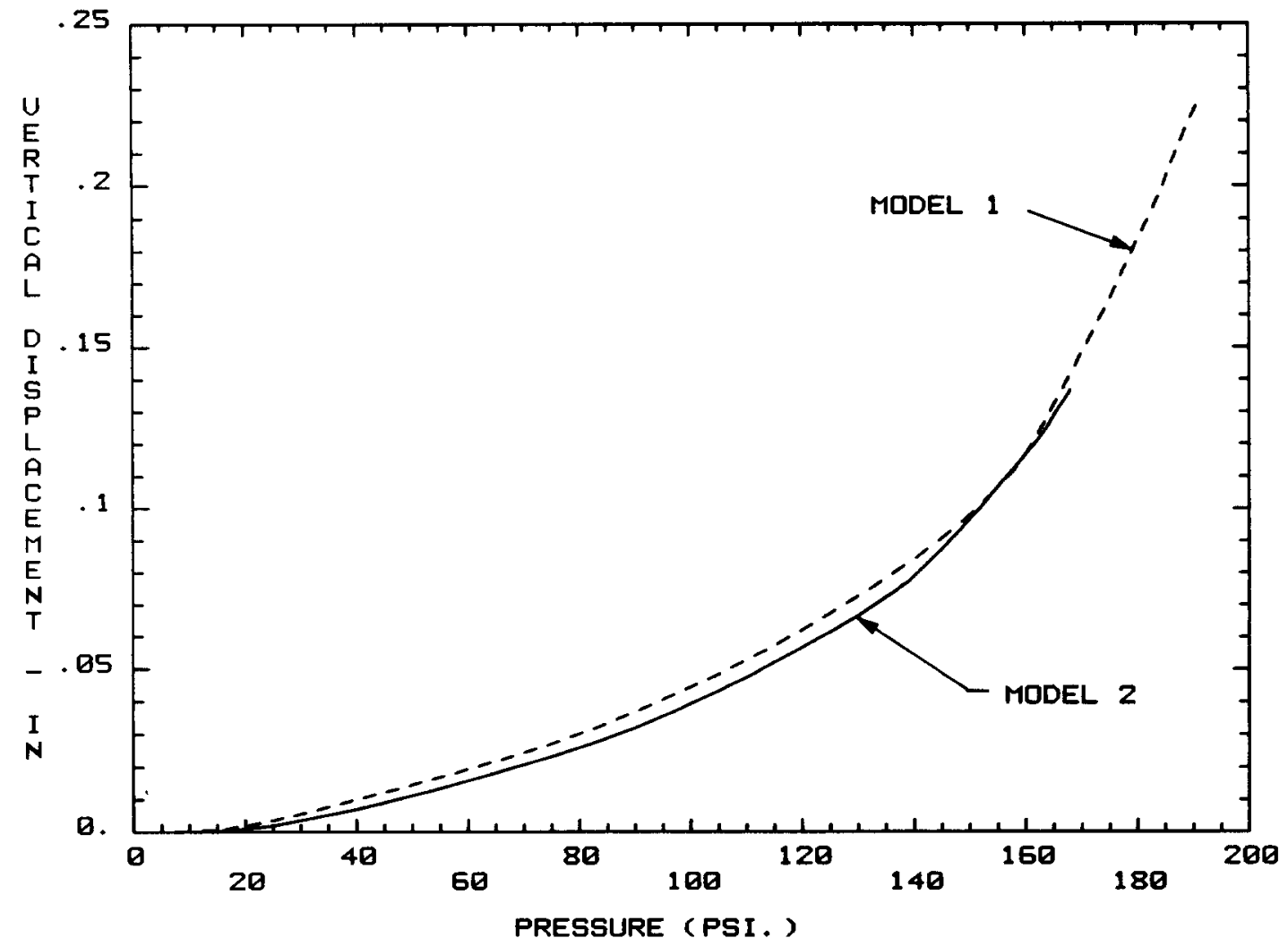

Figure 4.1.8 Basemat uplift at the basemat cylinder junction vs. pressure $-65-$ 


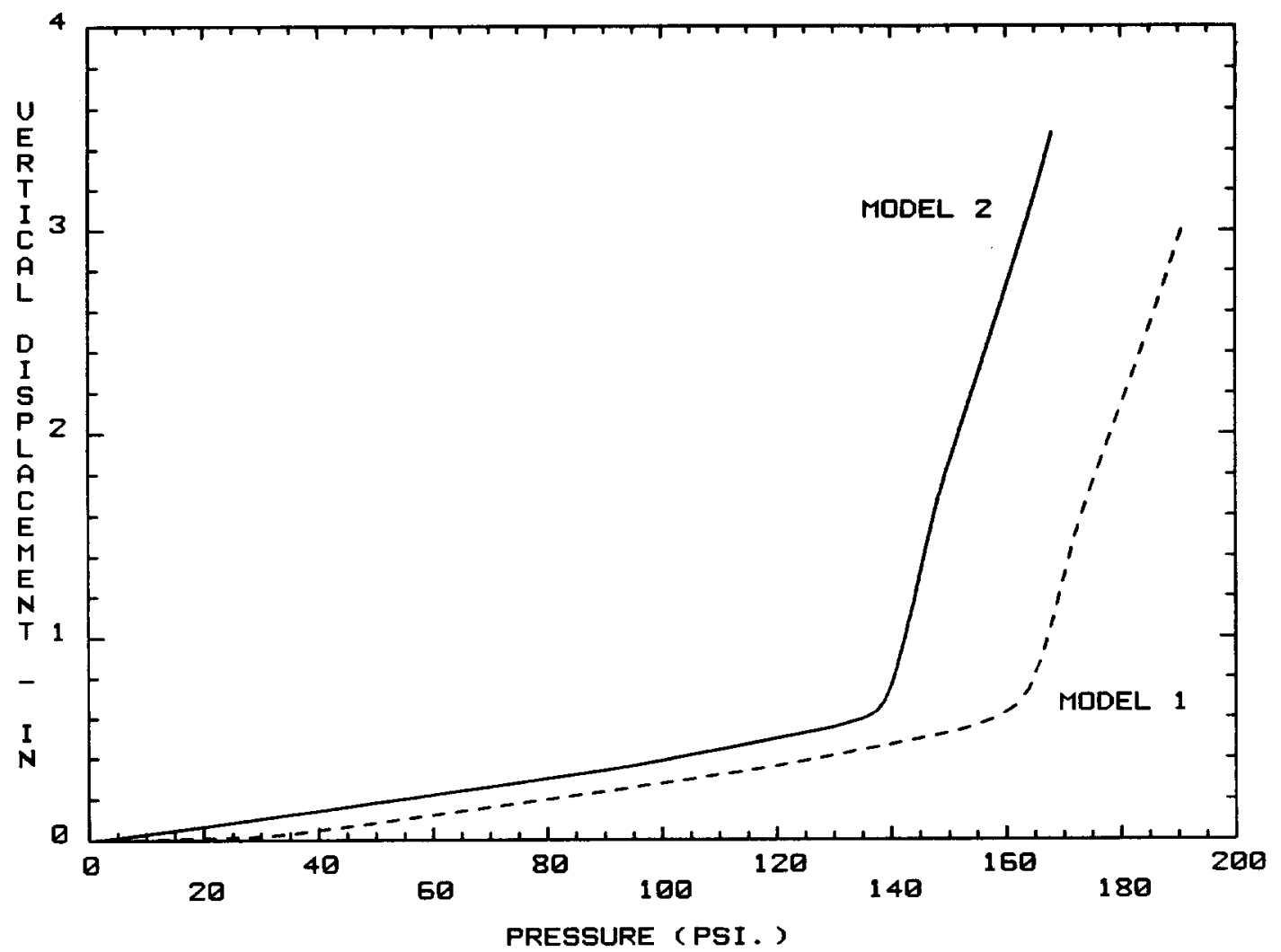

Figure 4.1.9 Vertical displacement at the dome apex vs. pressure

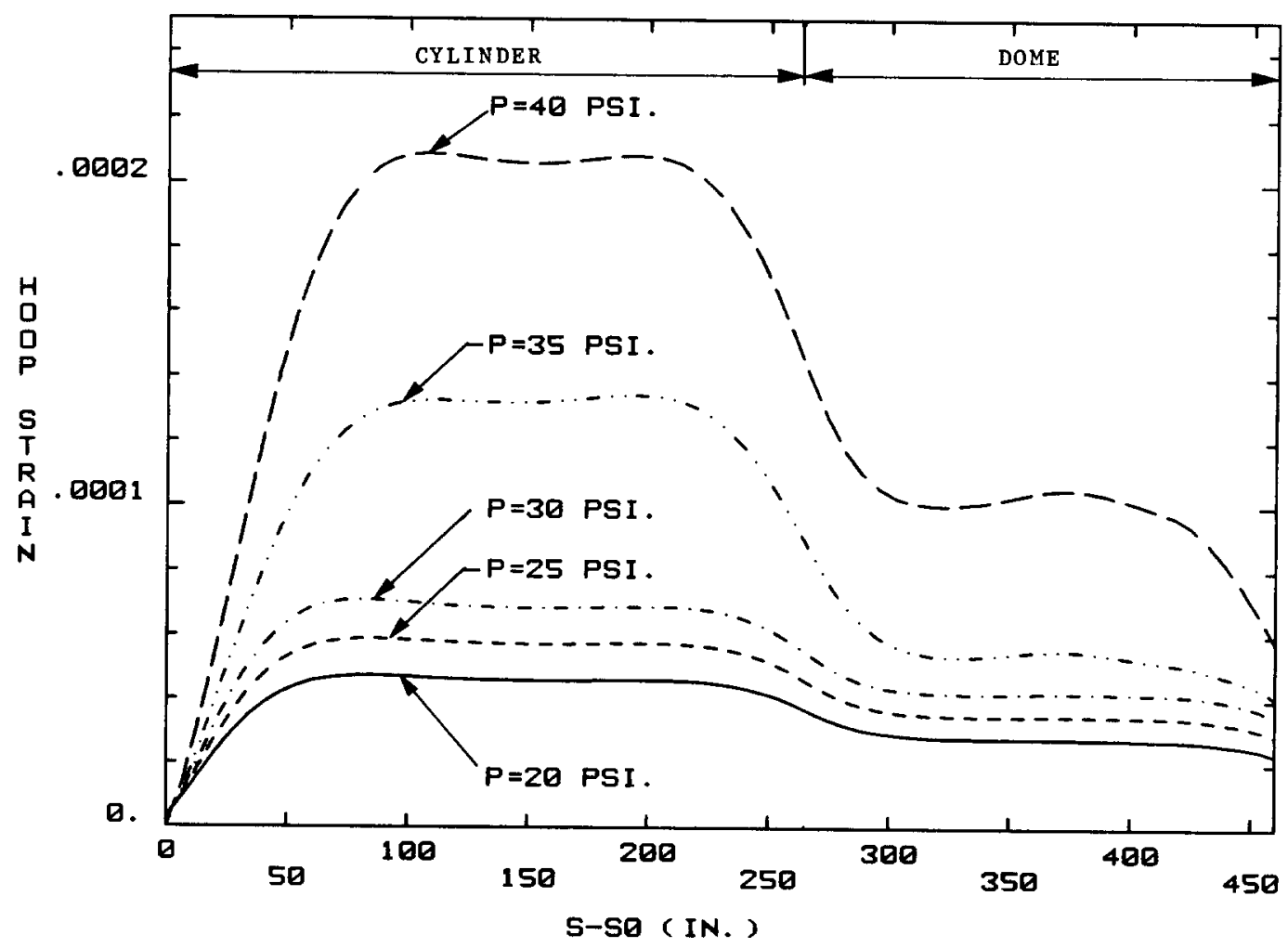

Figure 4.1.10 Hoop strain at the shell midthickness in the dome and cylinder (Model 1) 


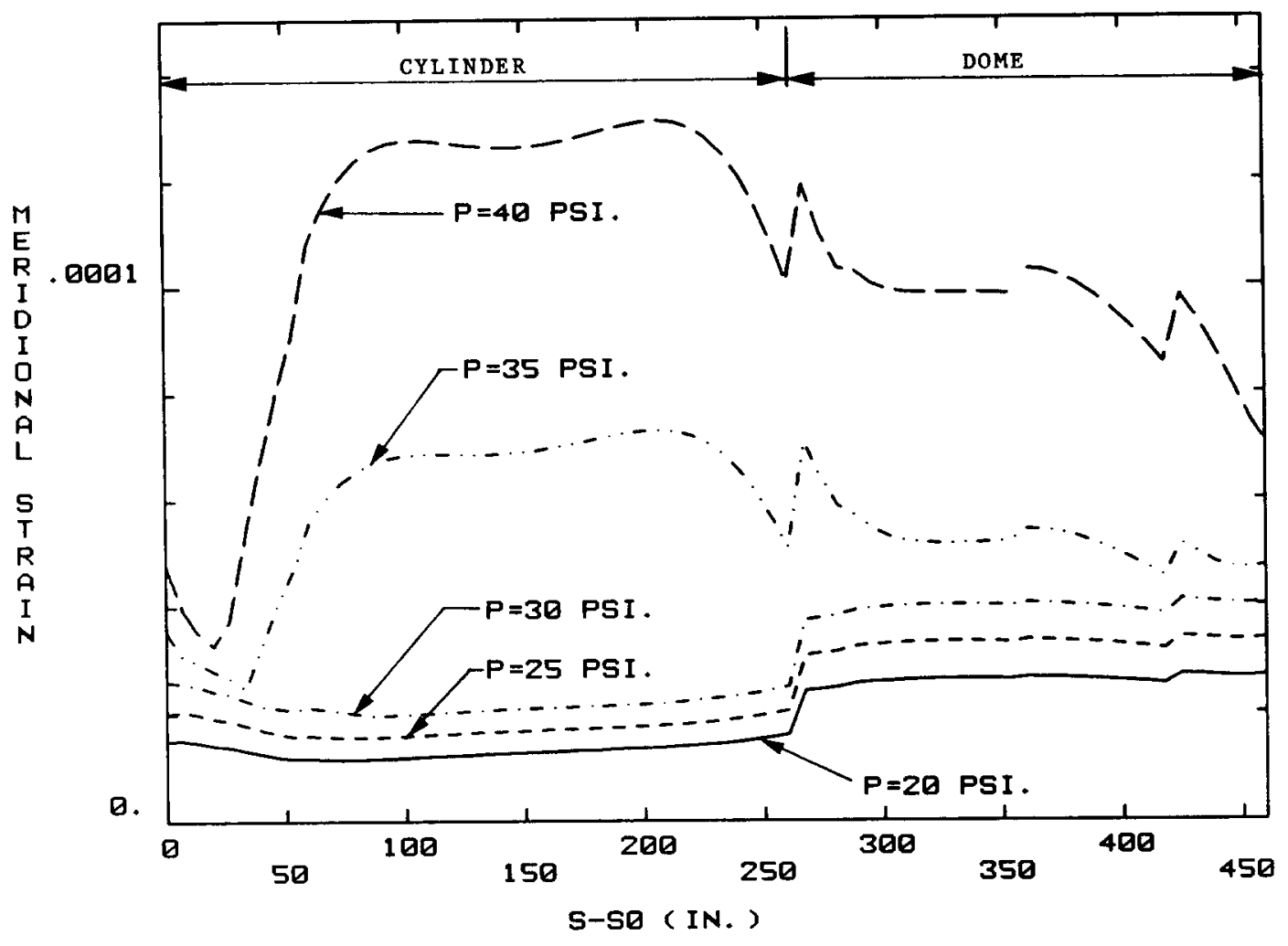

Figure 4.1.11 Meridional strain at the shell midthickness in the dome and cylinder (Model 1)

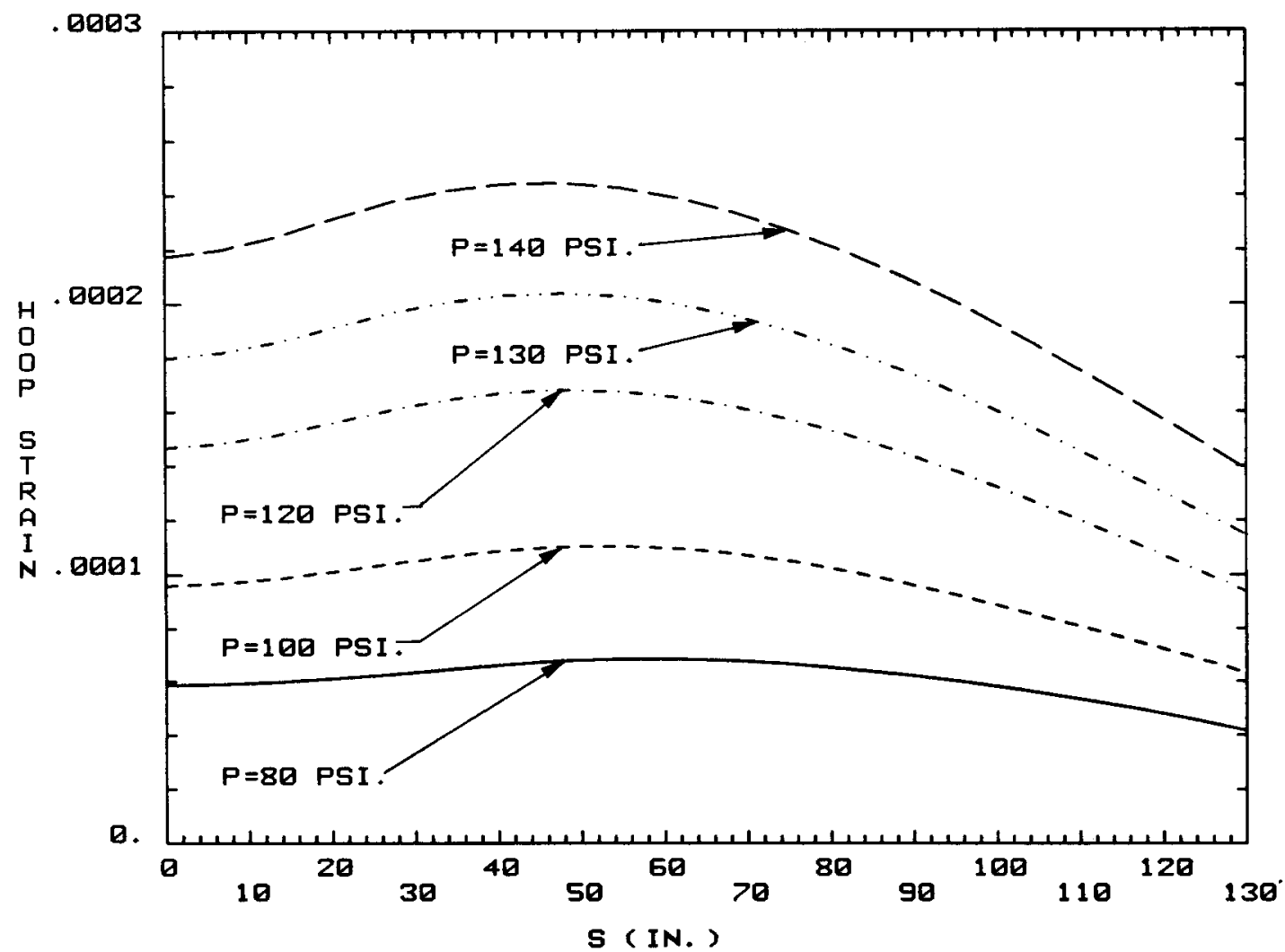

Figure 4.1.12 Hoop strains along the lower surface of the basemat (Model 1) 


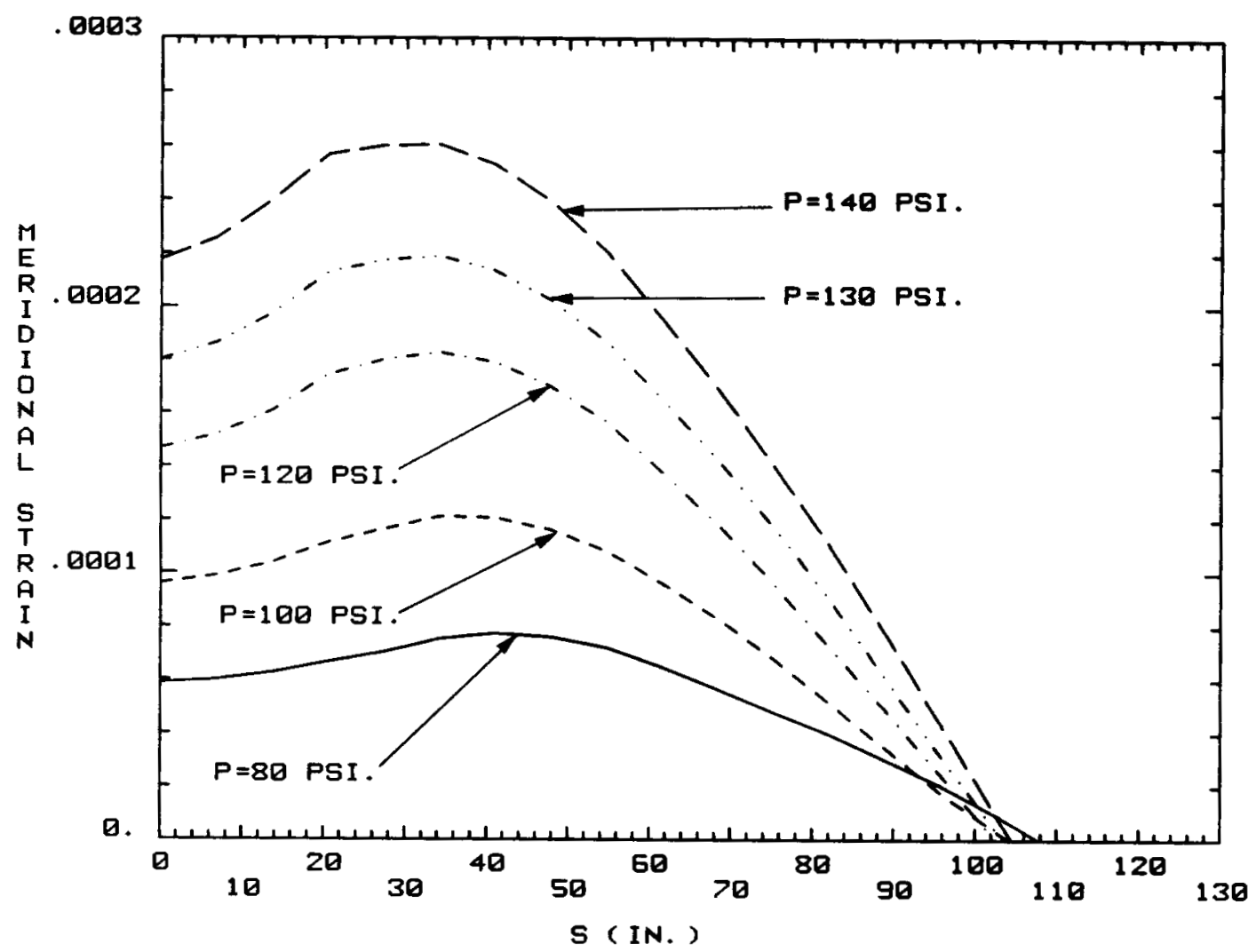

Figure 4.1.13 Meridional strains along the lower surface of the basemat (Model 1)

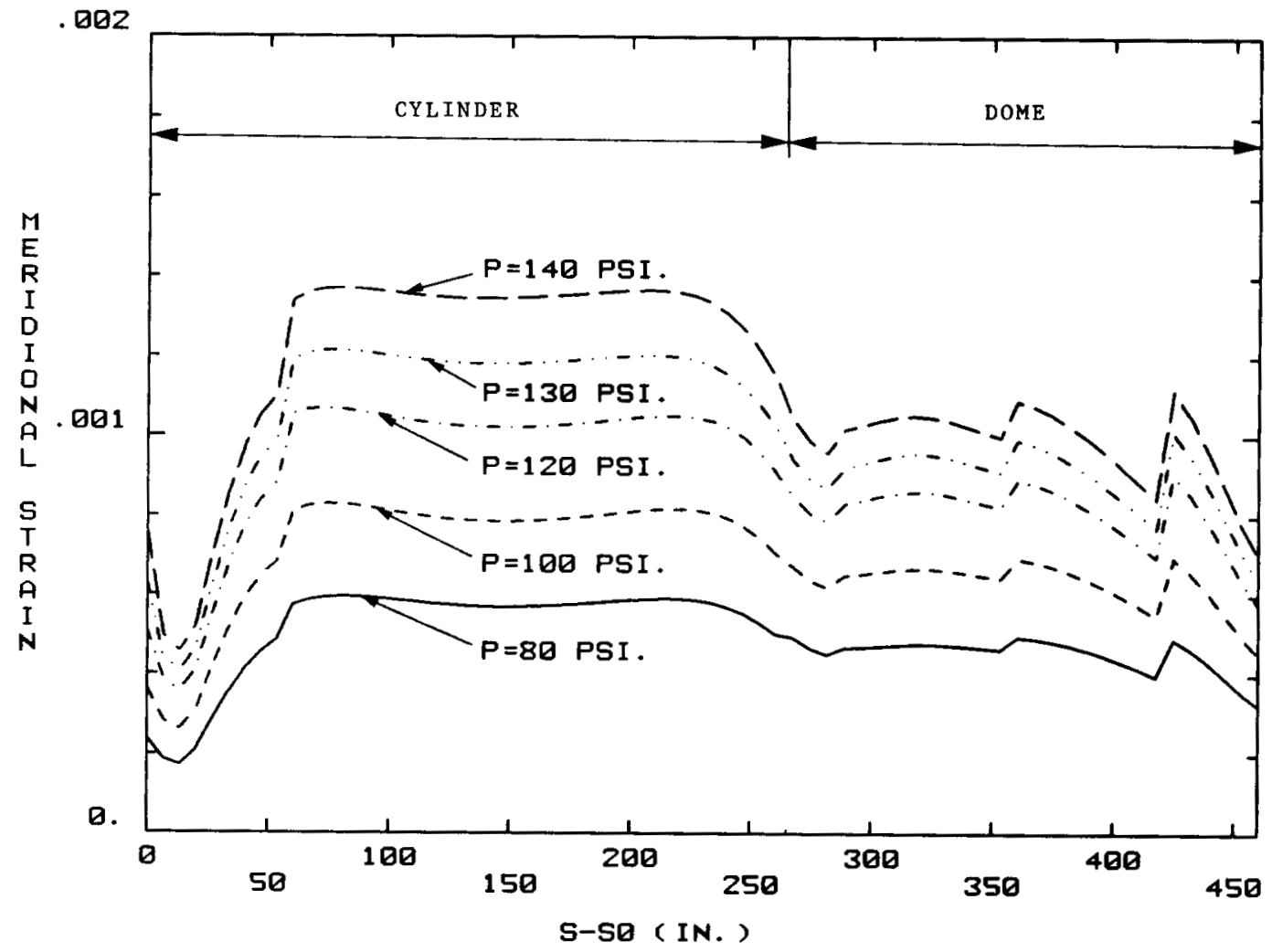

Figure 4.1.14 Meridional strains at the midthickness of the containment wall in the dome and cylinder (Model 1) 


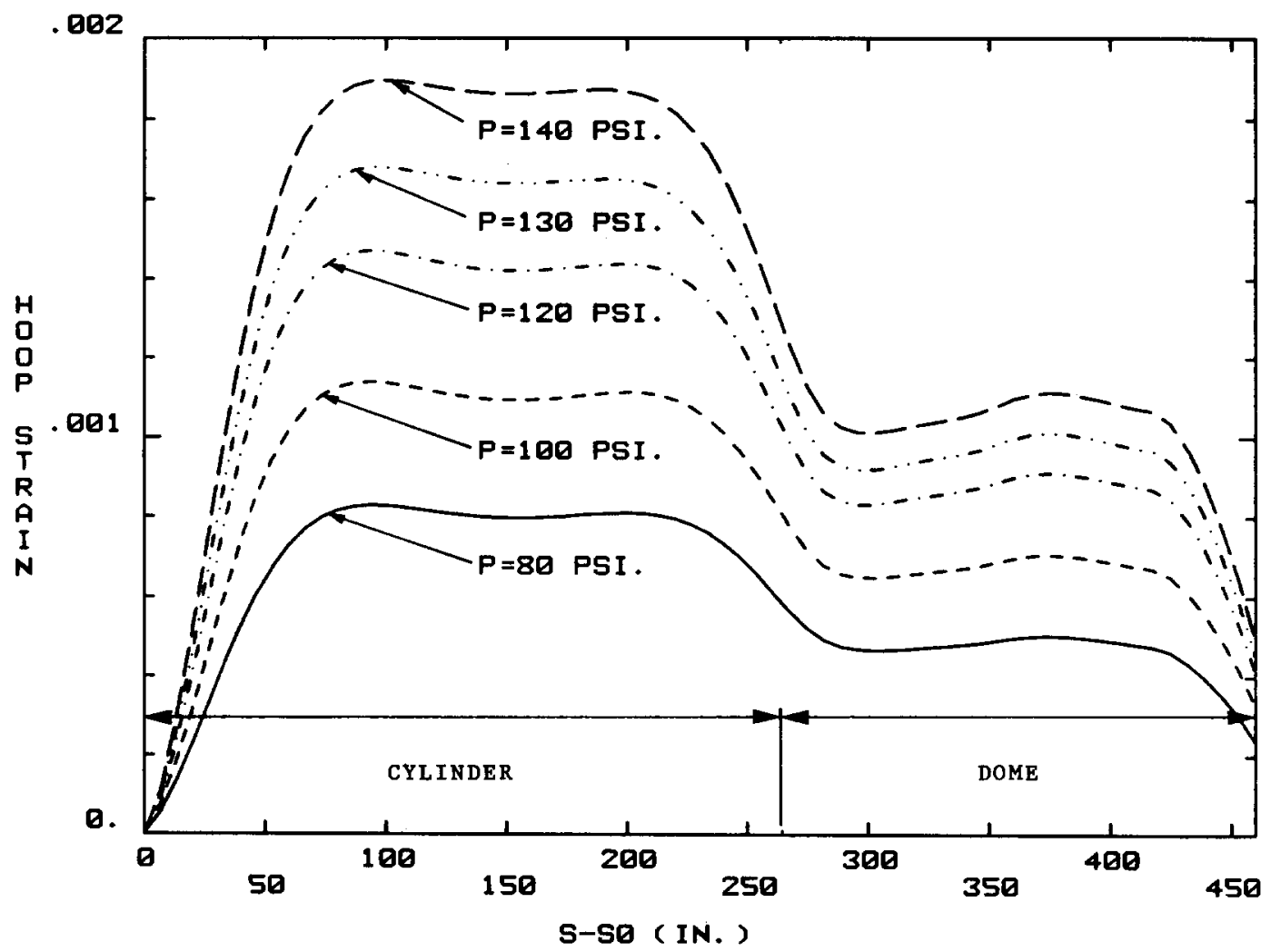

Figure 4.1.15 Hoop strain at the midthickness of the containment wall in the dome and cylinder (Model 1)

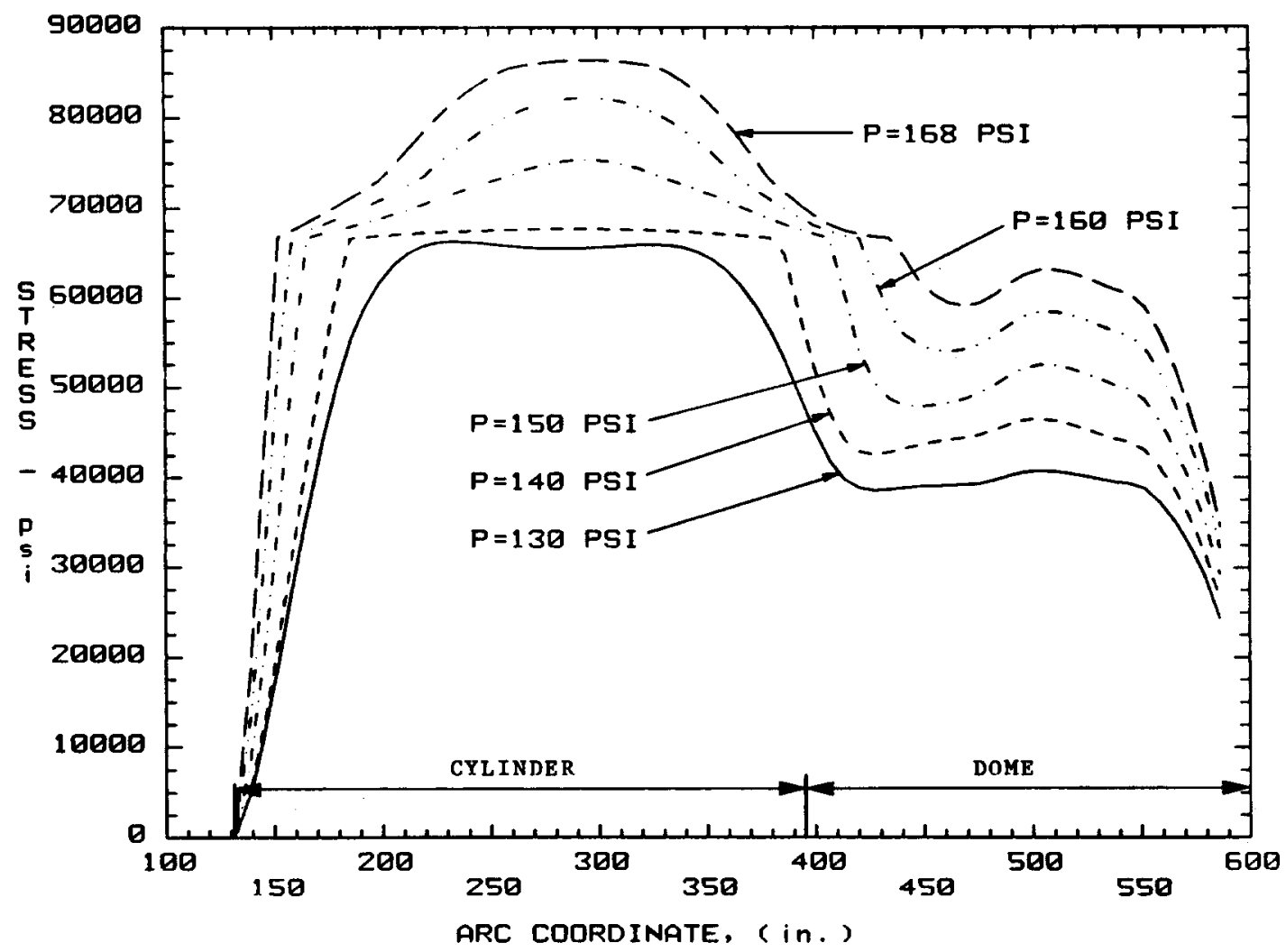

Figure 4.1.16 Stresses in the Layer 1 hoop reinforcement (Model 2) 


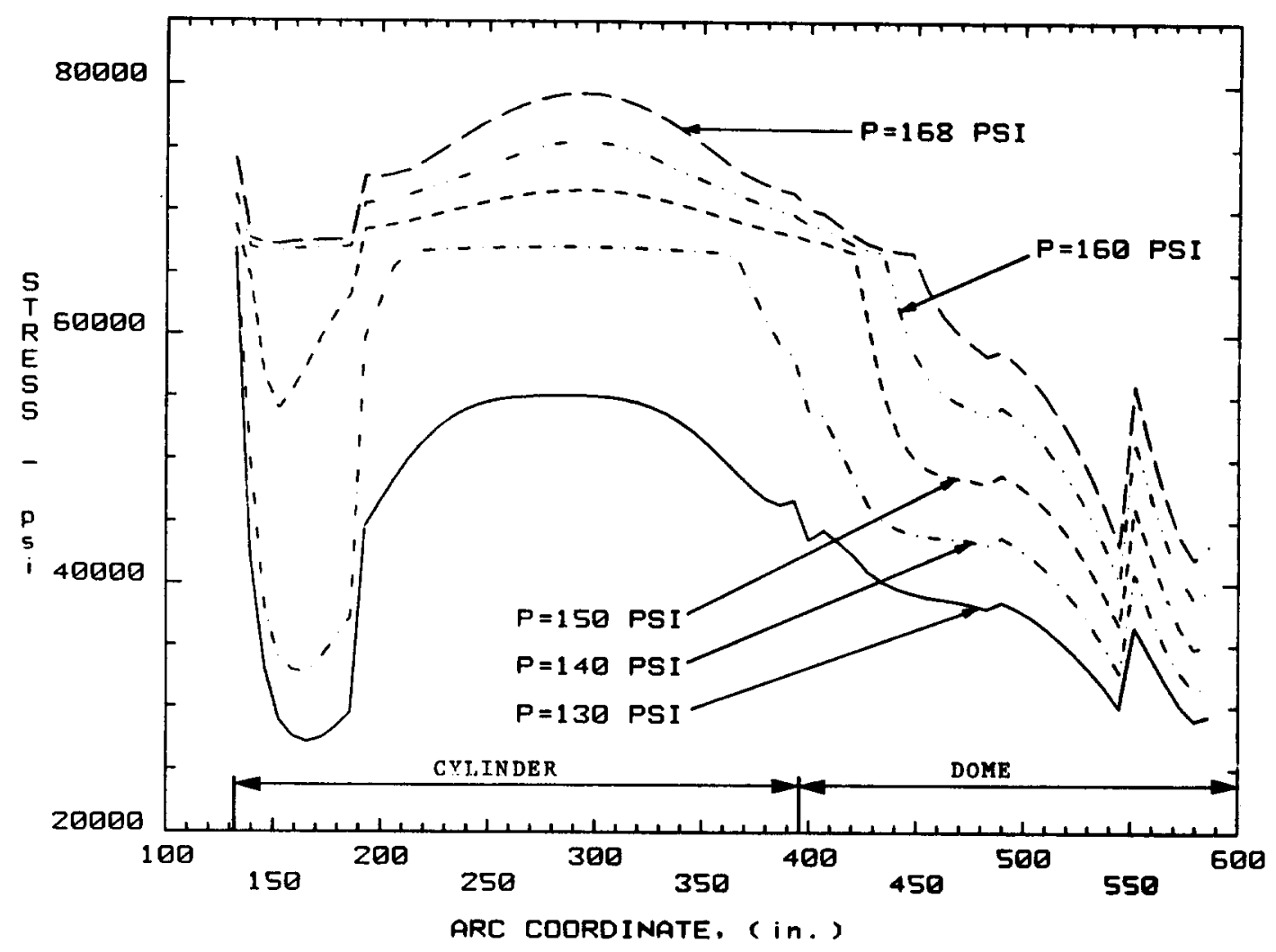

Figure 4.1.17 Stresses in the Layer 2 meridional reinforcement (Model 2)

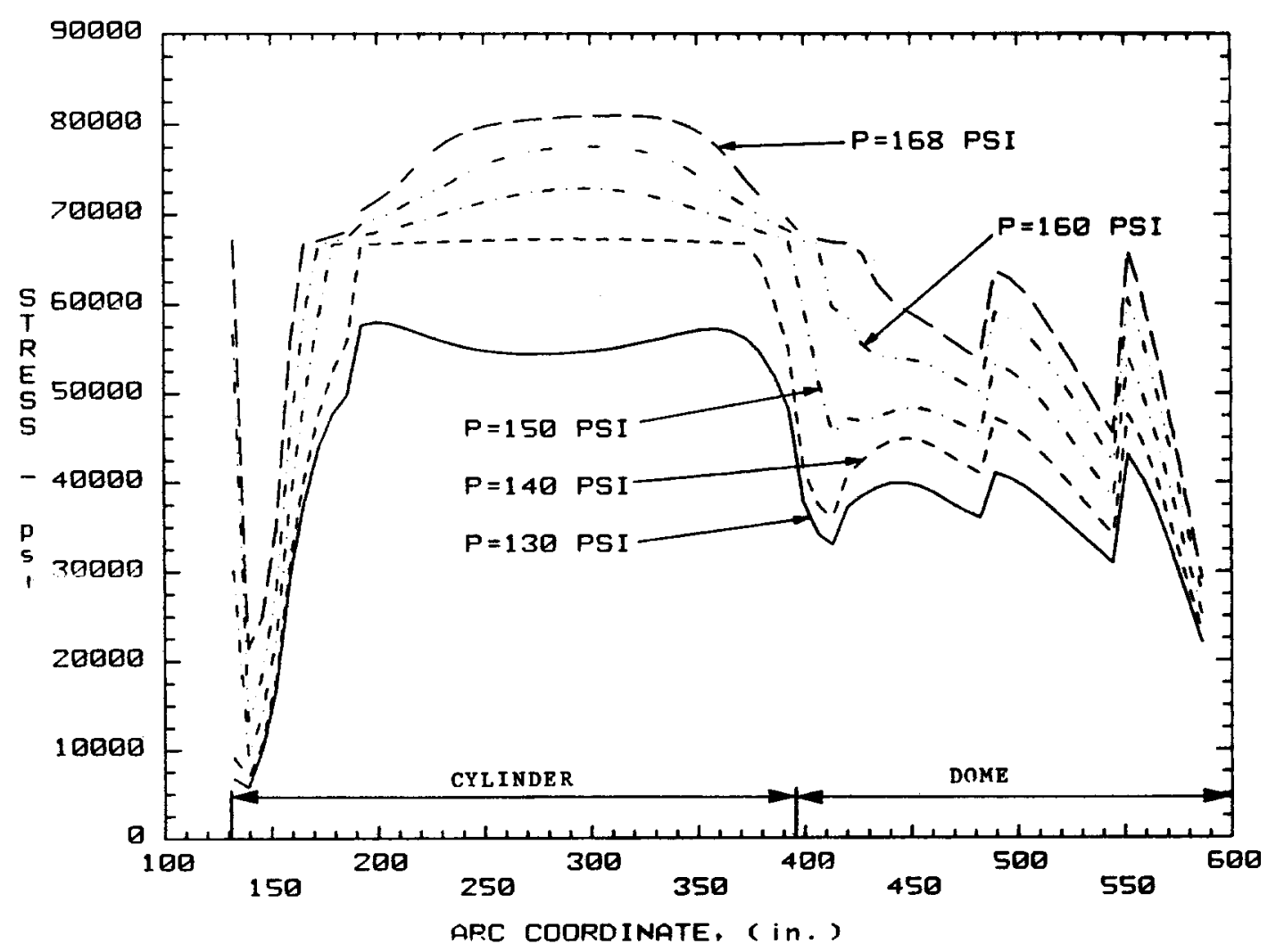

Figure 4.1.18 Stresses in the Layer 5 meridional reinforcement (Model 2) $-70$ 


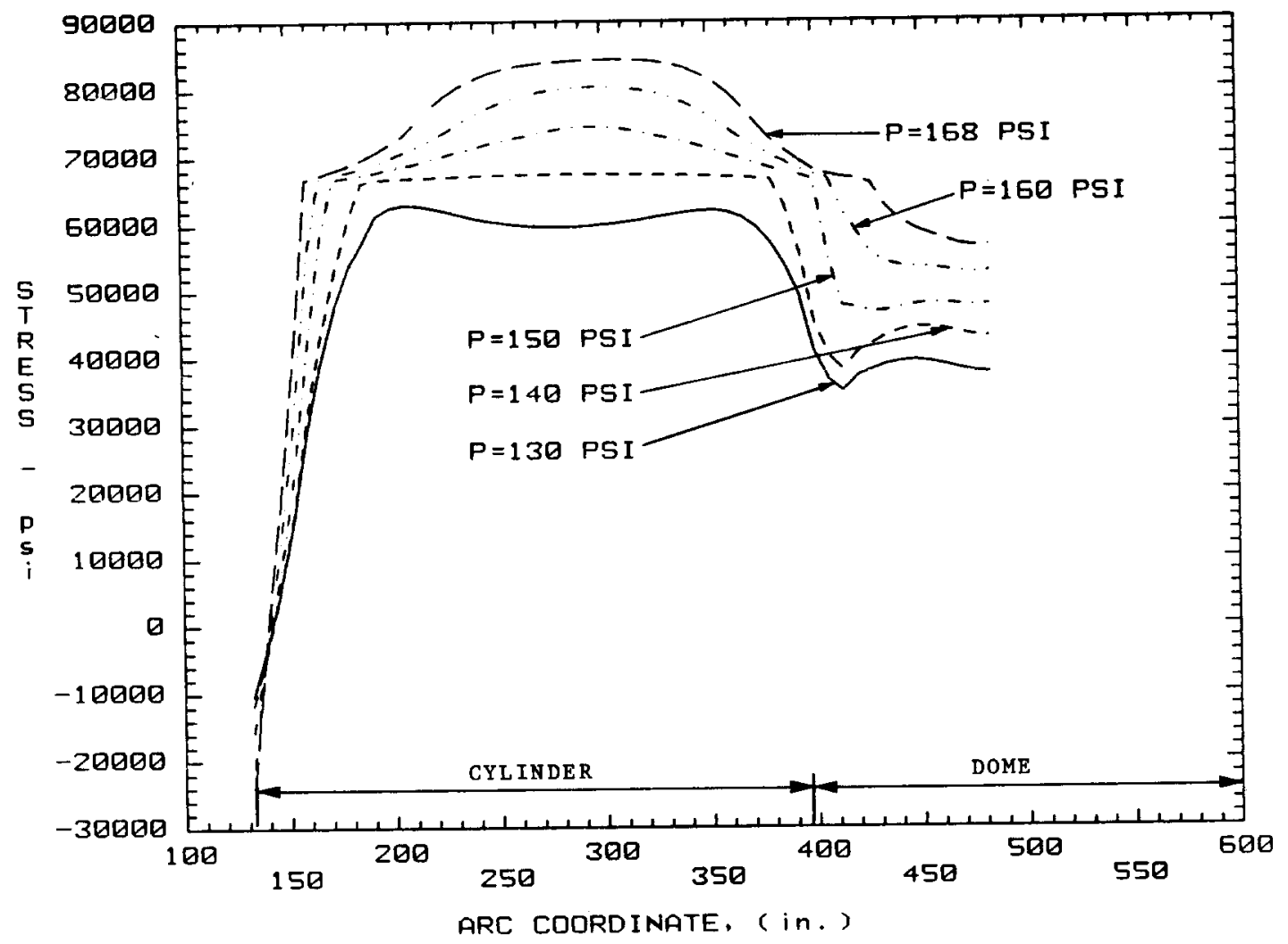

Figure 4.1.19 Stresses in the Layer 8 seismic reinforcement (Model 2)

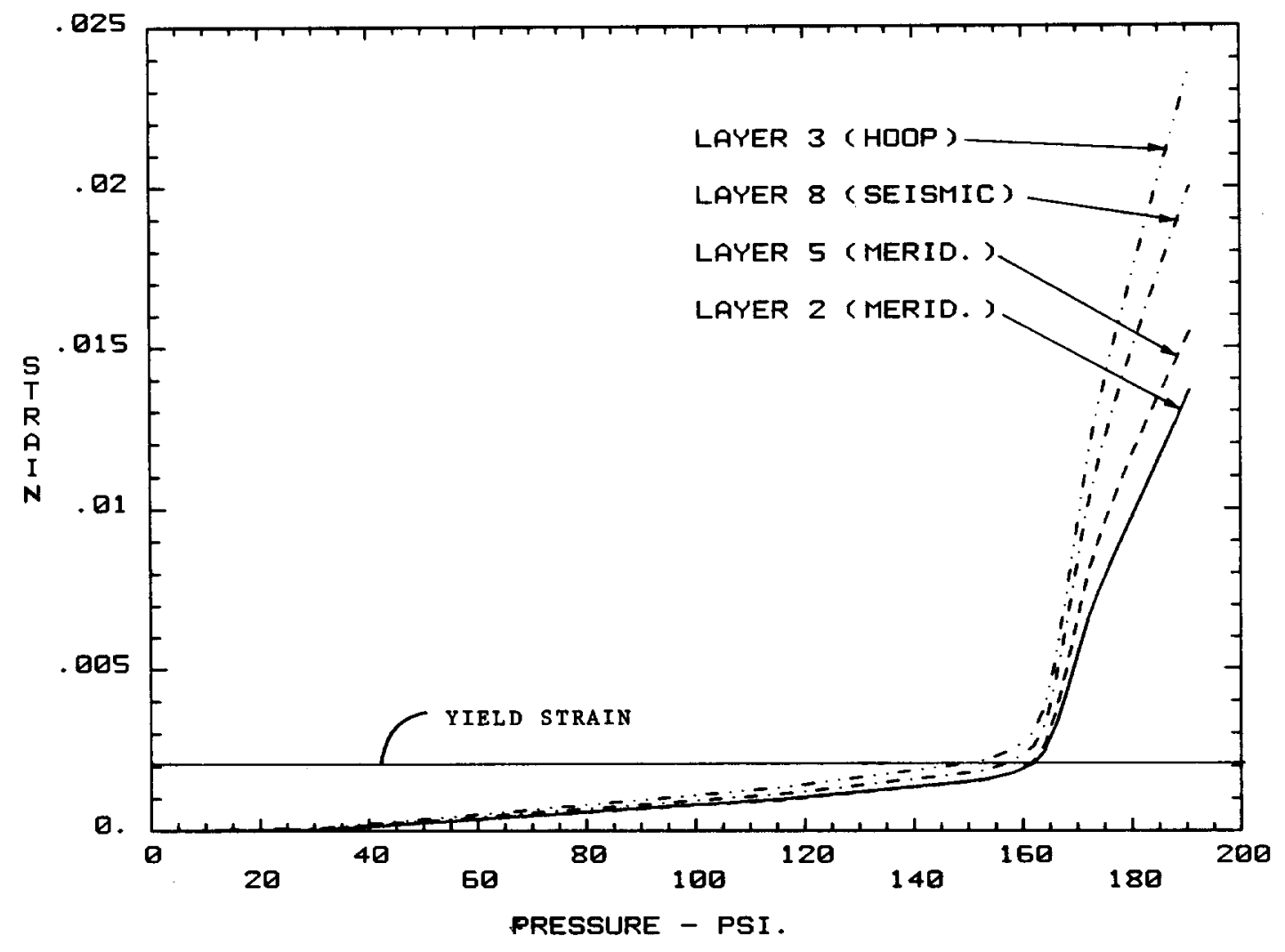

Figure 4.1.20 Model 1 results for rebar strains vs pressure at EL 13'-9" 


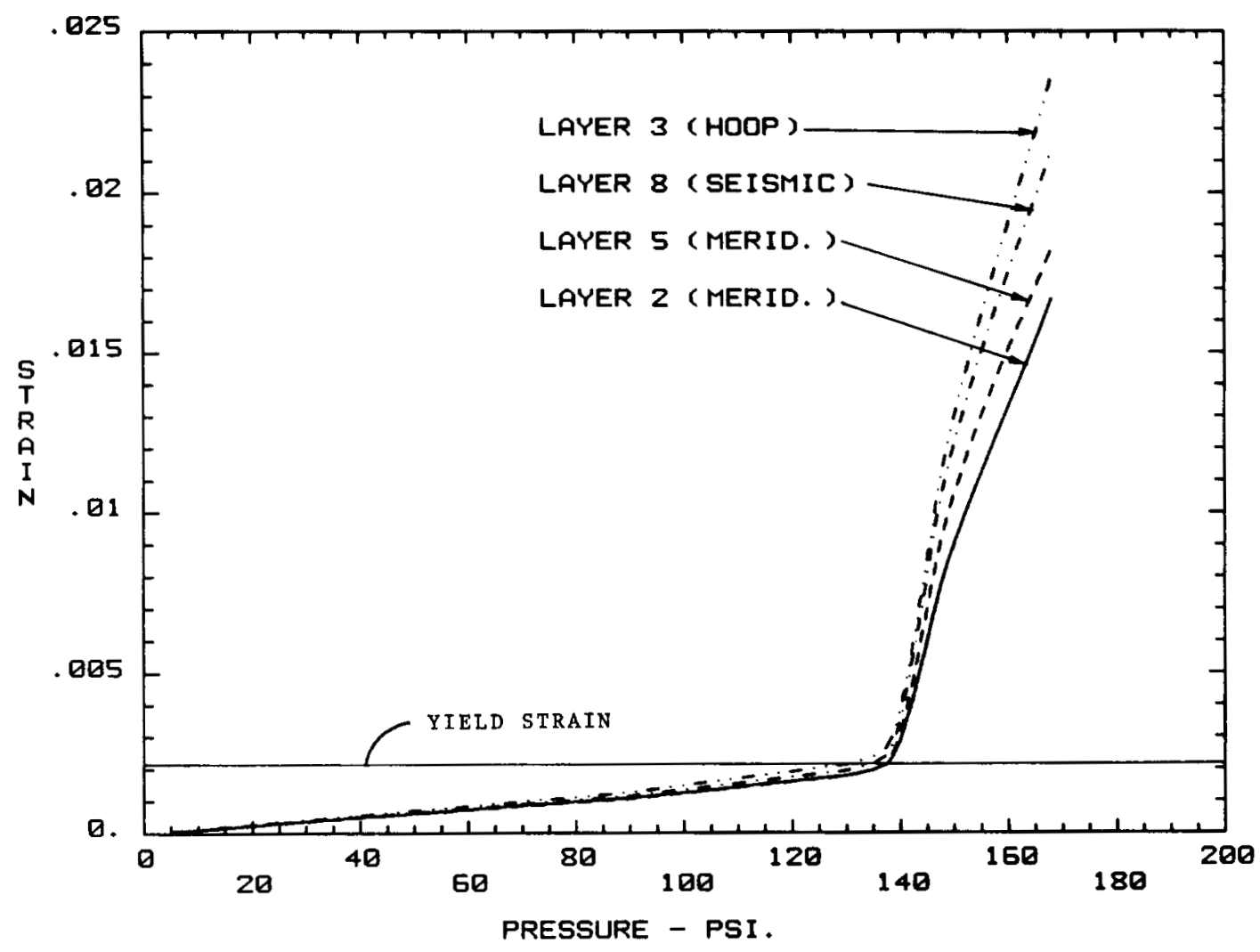

Figure 4.1.21 Model 2 results for rebar strains vs pressure at EL 13'-9"

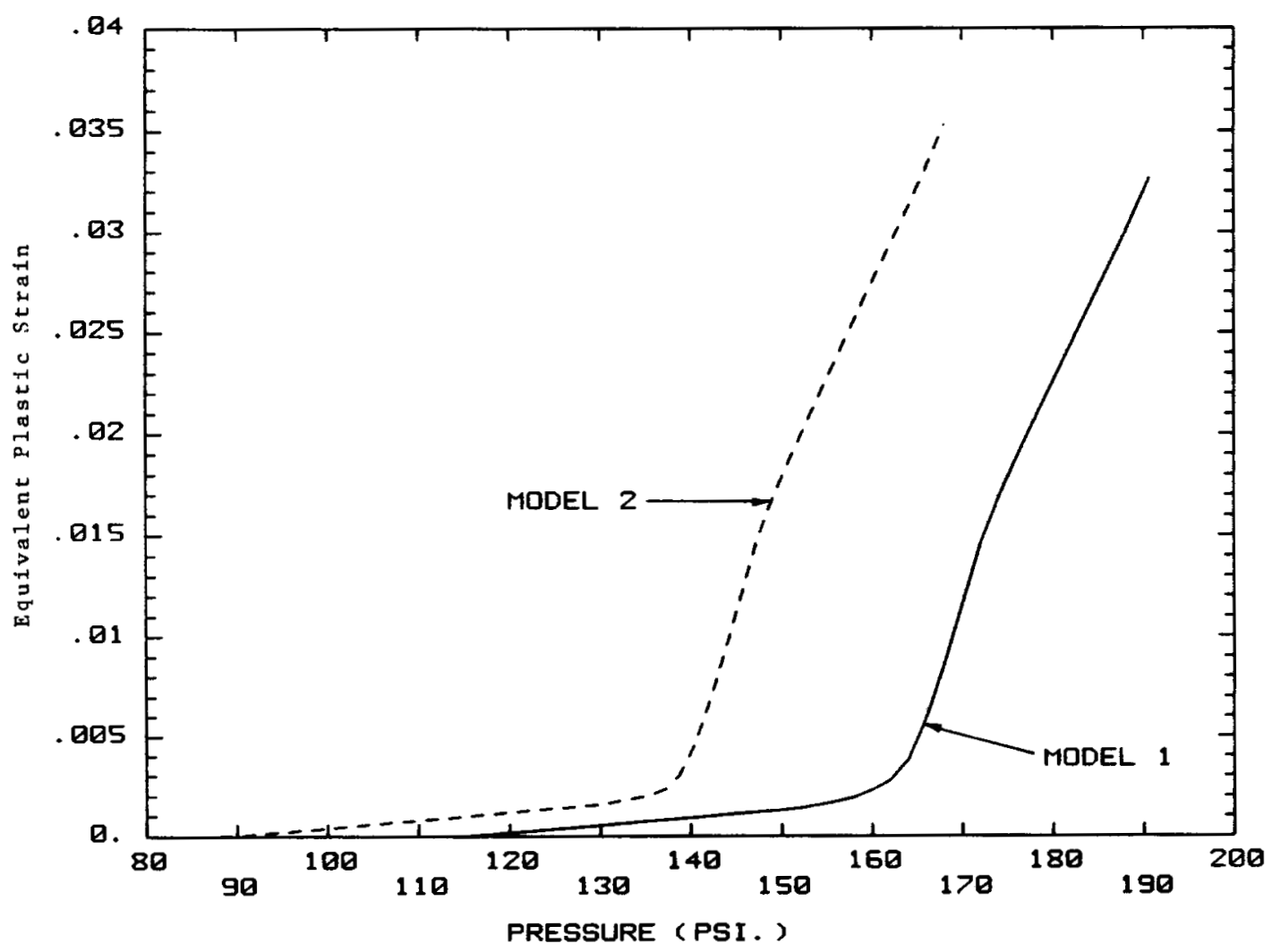

Figure 4.1.22 Equivalent plastic strain in the liner vs. pressure at the cylinder midheight 


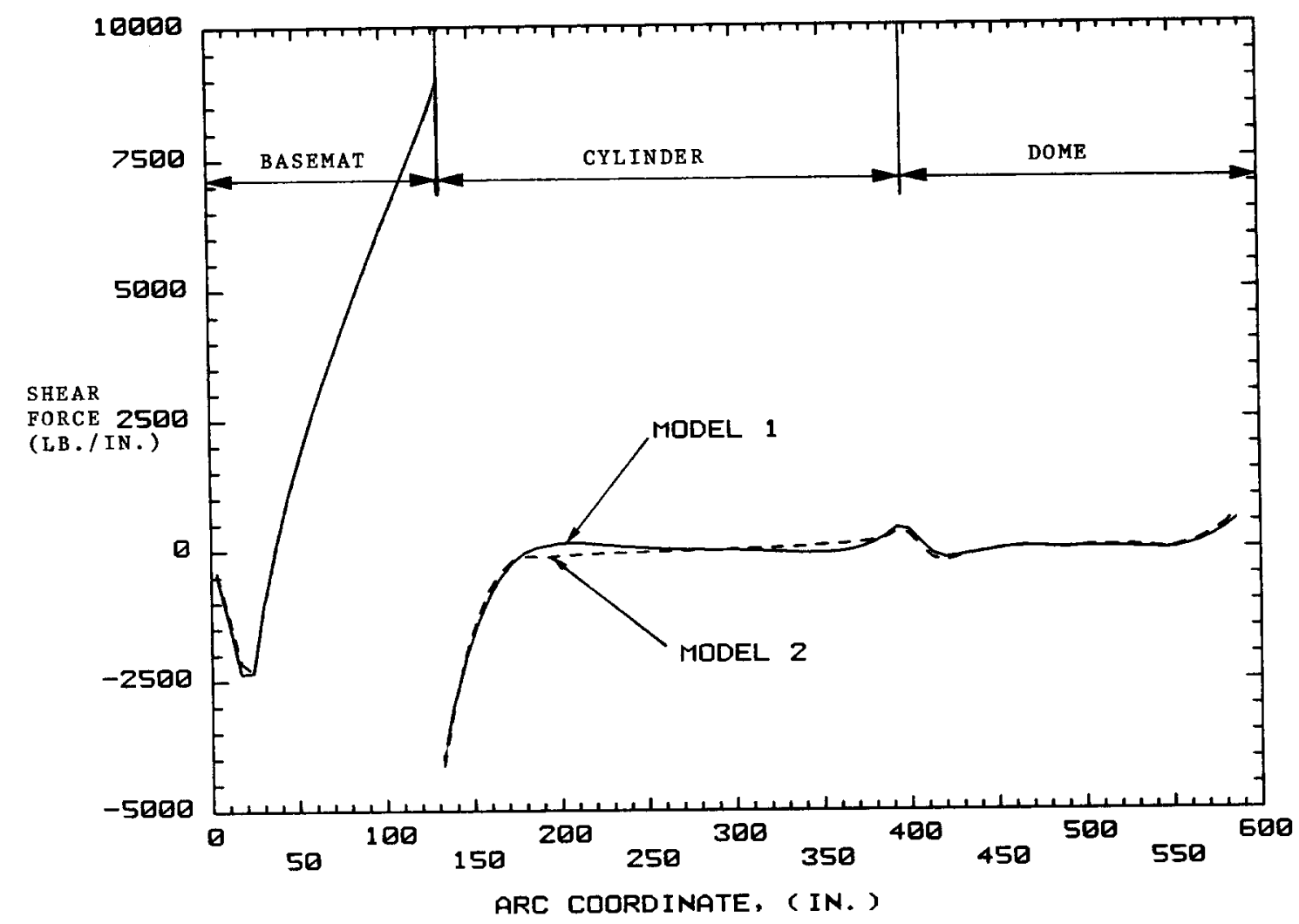

Figure 4.1.23 Transverse (radial) shear force at $140 \mathrm{psi}$ (0.965 MPa)

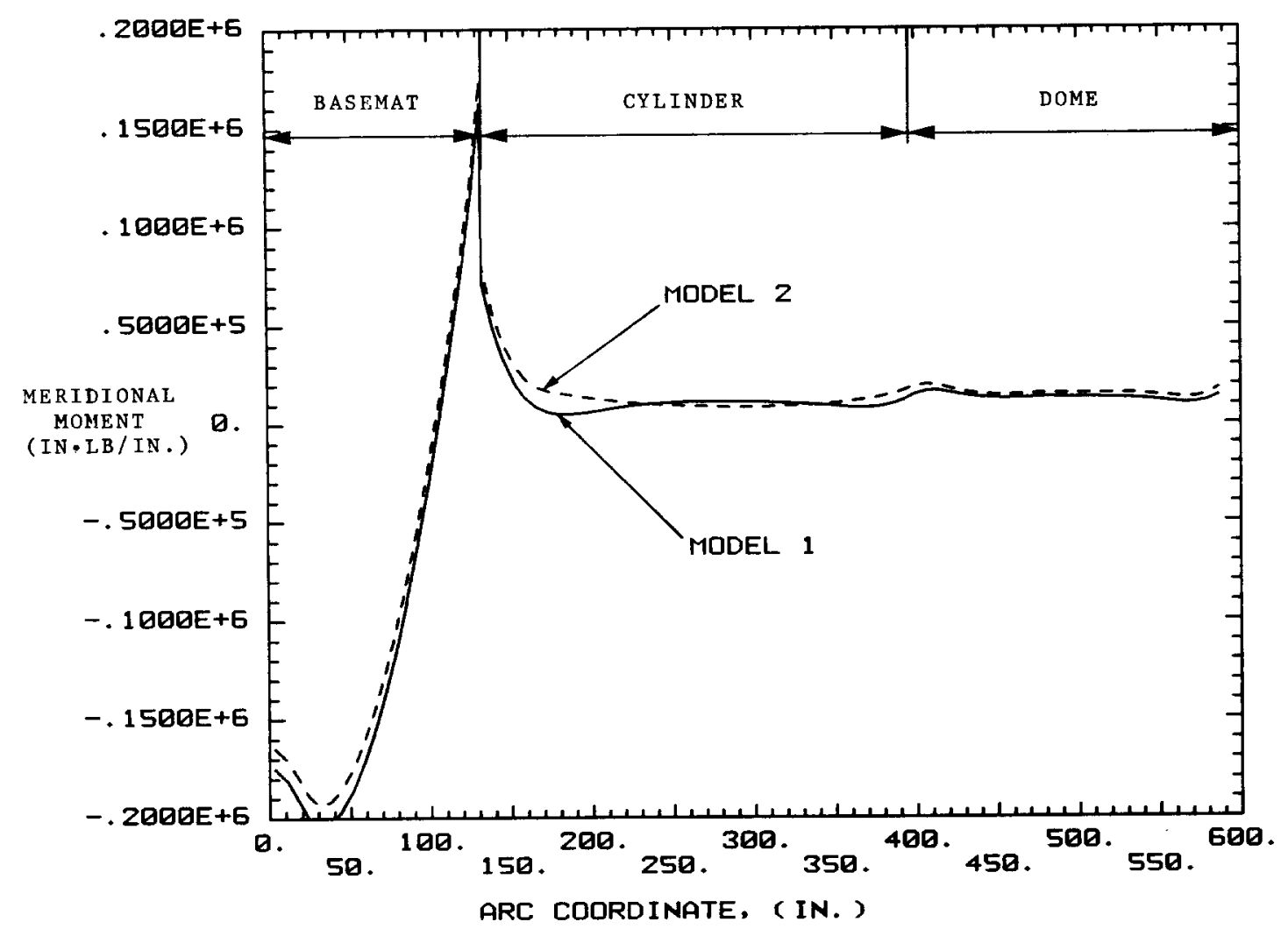

Figure 4.1.24 Meridional bending moment at $140 \mathrm{psi}(0.965 \mathrm{MPa})$ 


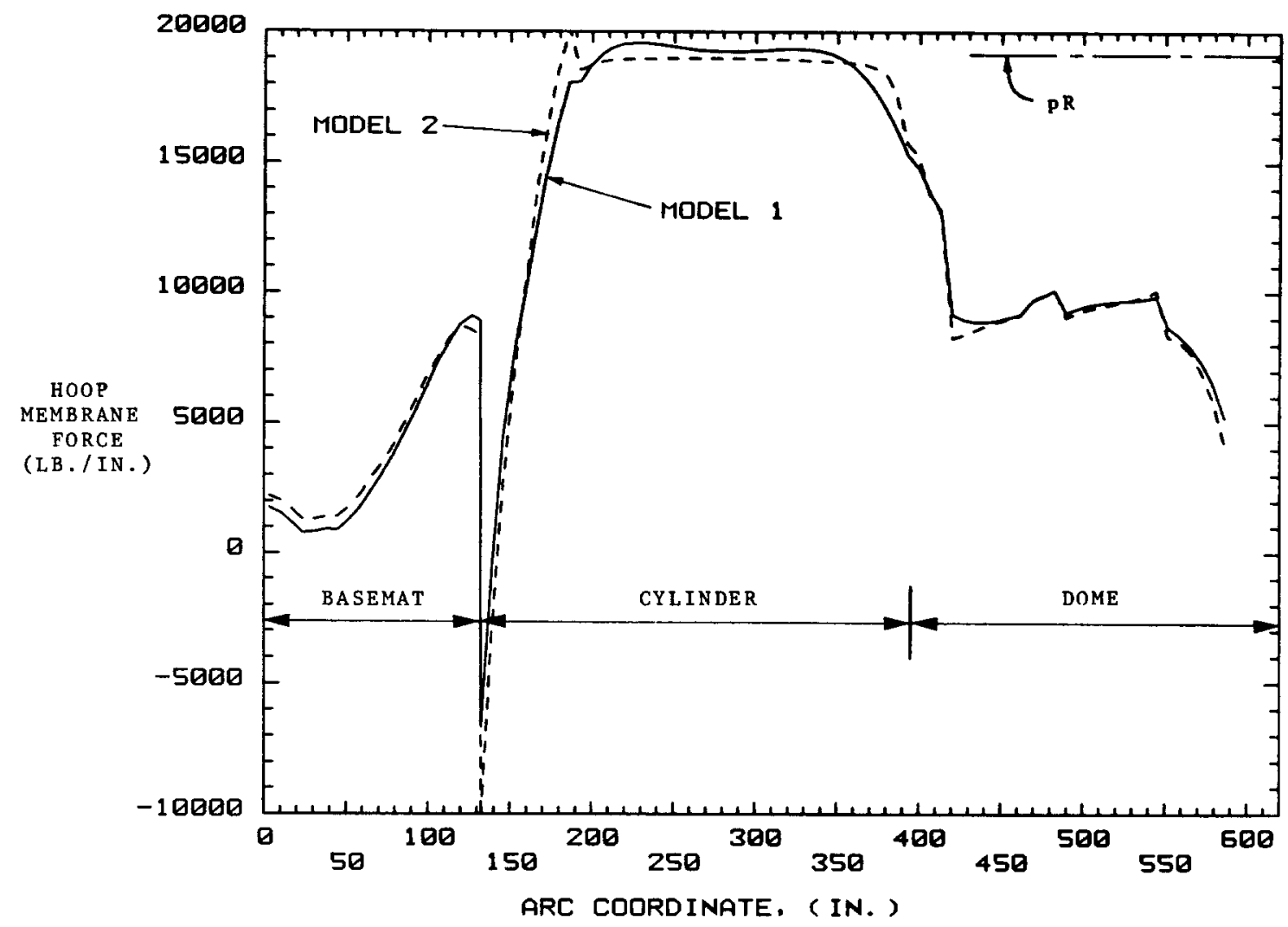

Figure 4.1.25 Hoop membrane force at 140 psi (0.965 MPa)

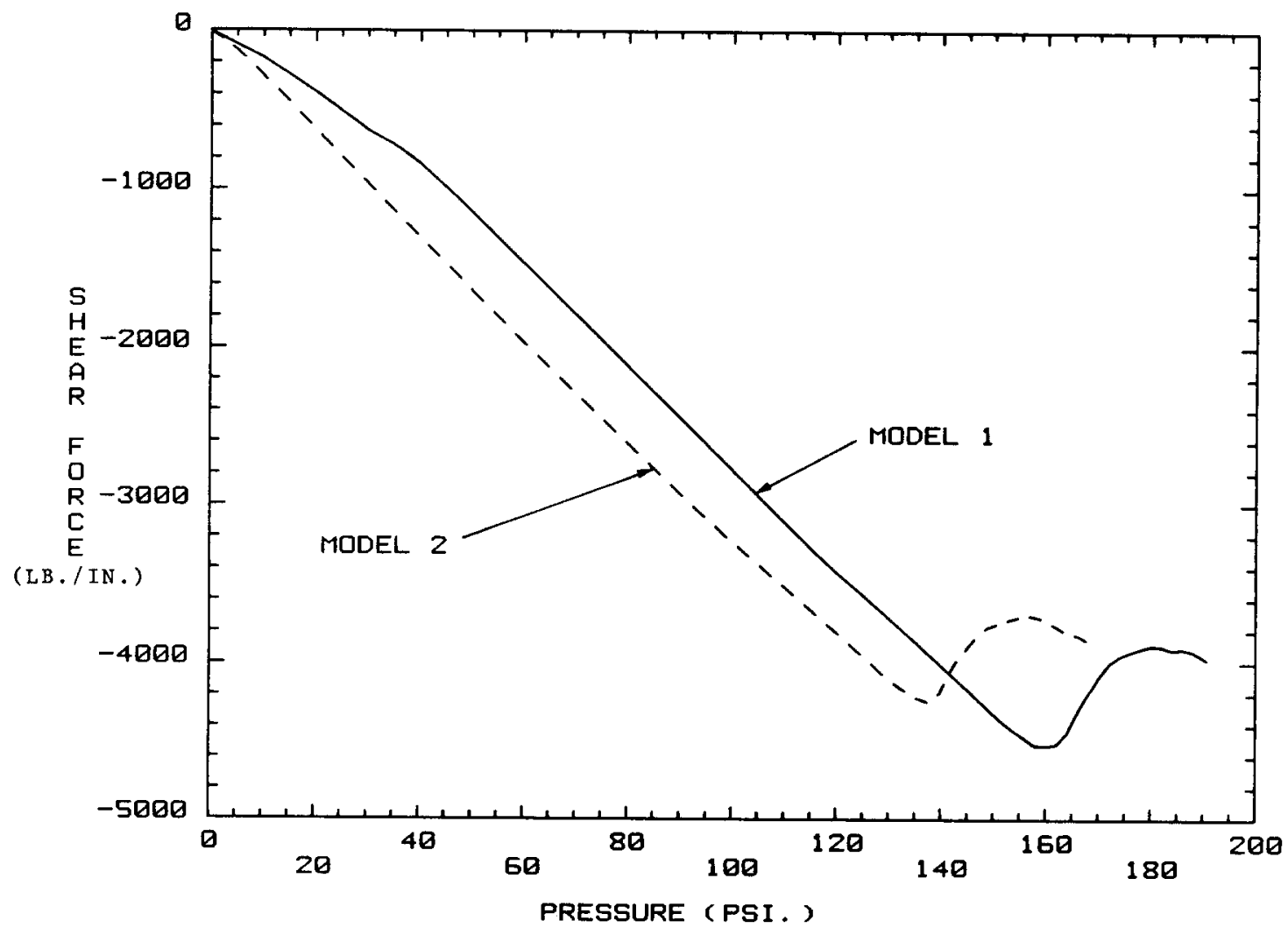

Figure 4.1 .26 Radial shear force 3 inches $(80 \mathrm{~mm})$ above the base of the cylinder vs. pressure 


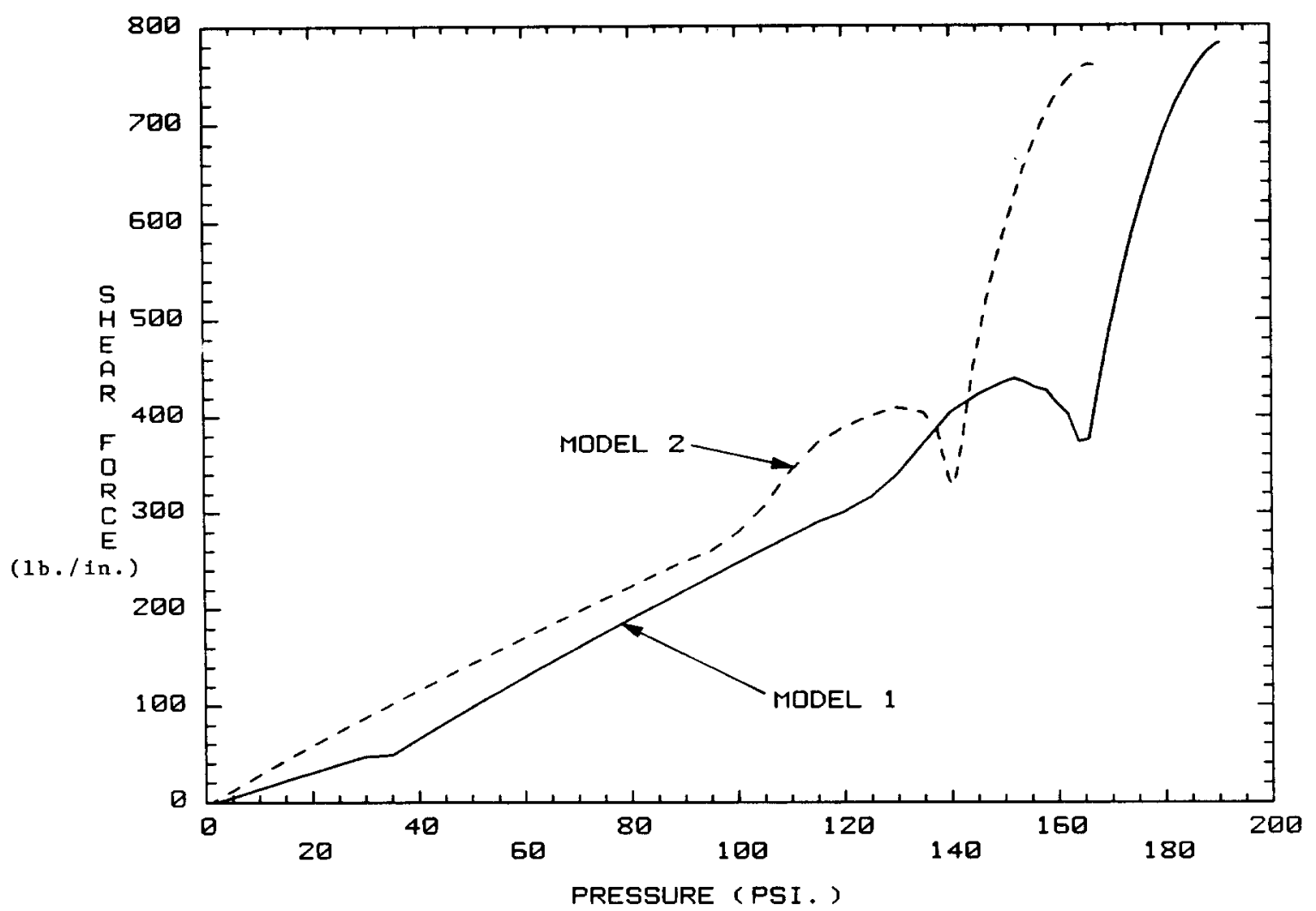

Figure 4.1.27 Radial shear force 3 inches $(80 \mathrm{~mm})$ below the springline vs. pressure

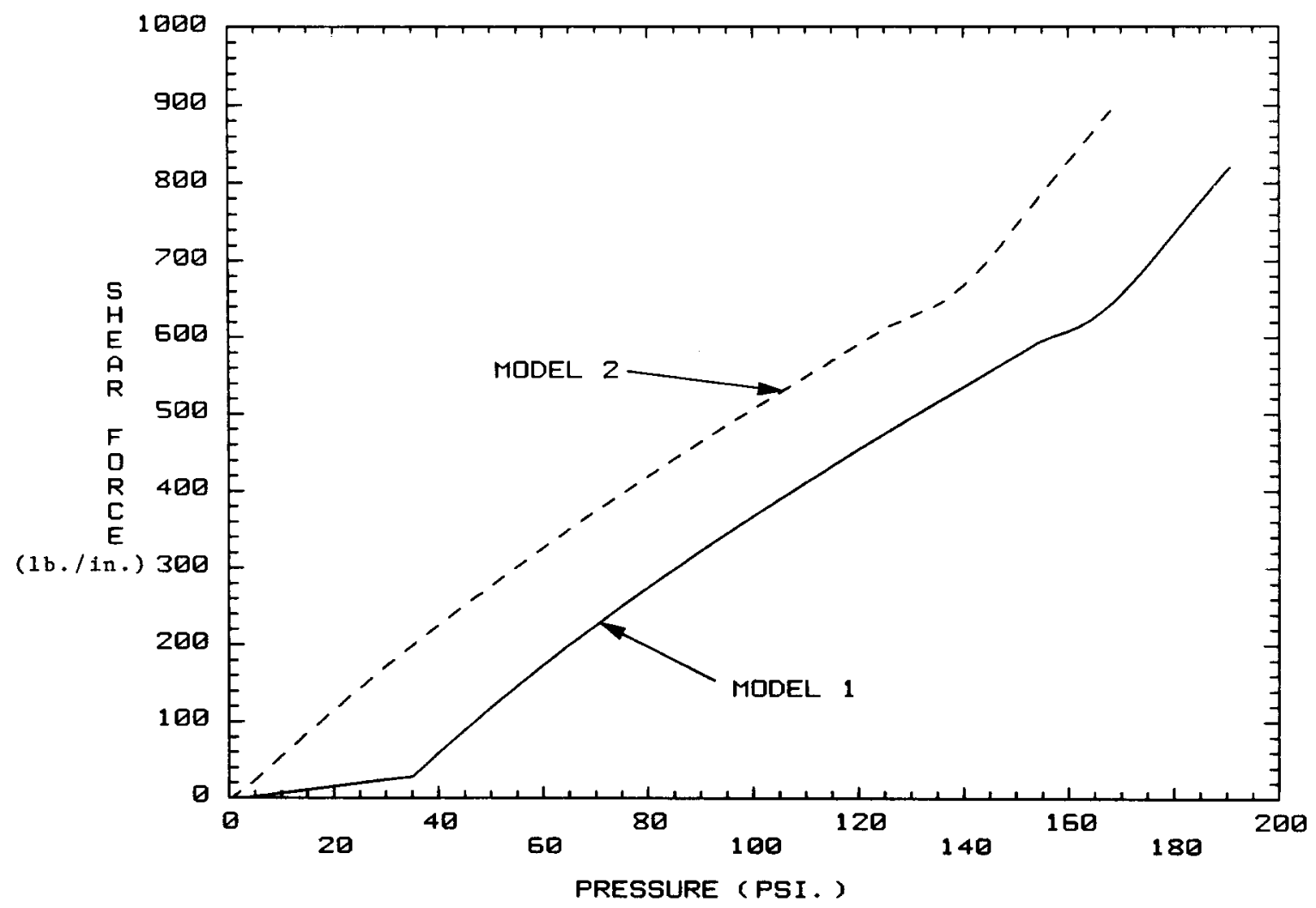

Figure 4.1.28 Radial shear force 3 inches $(80 \mathrm{~mm})$ from the edge of the dome plates vs. pressure 


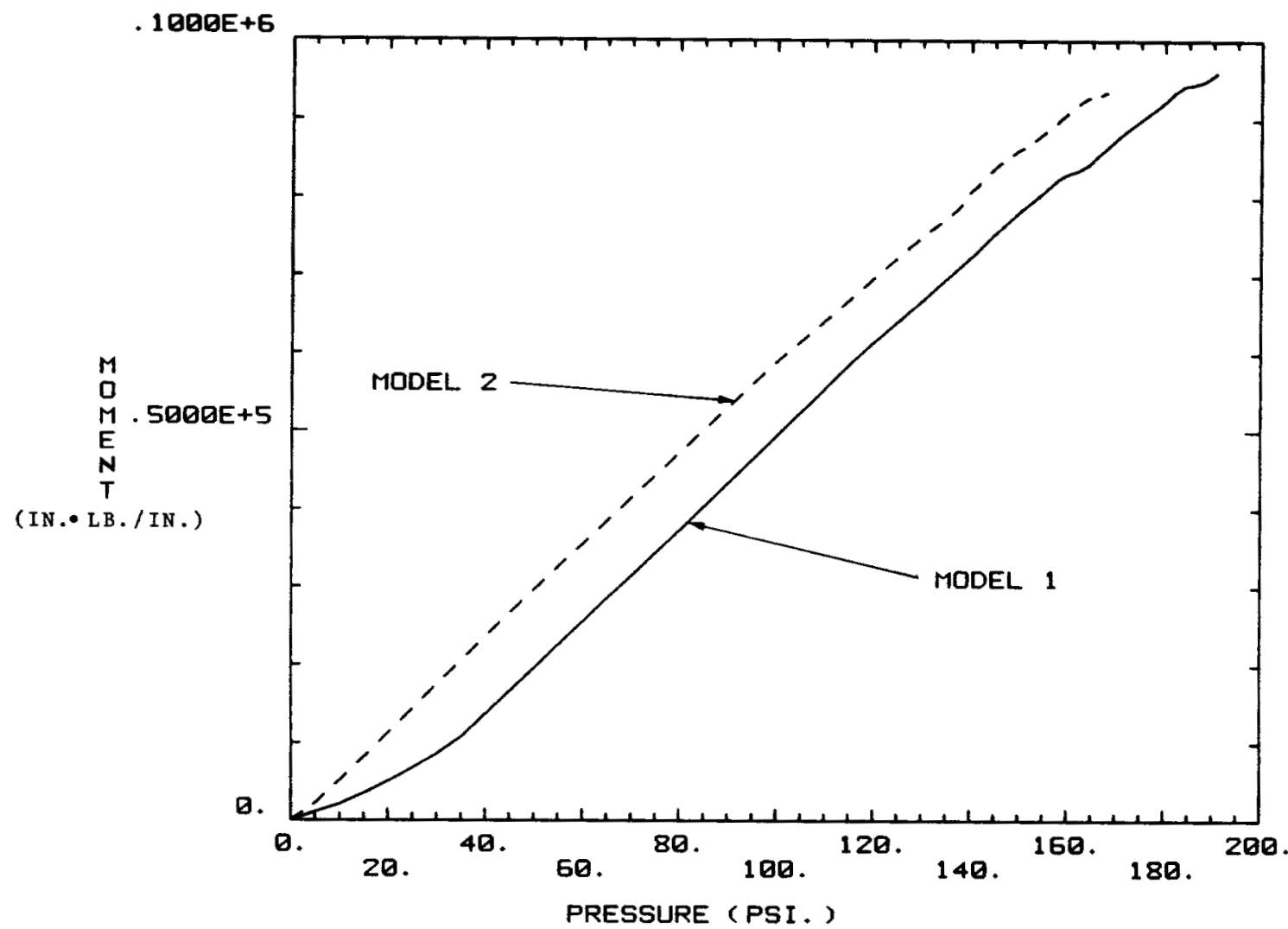

Figure 4.1.29 Meridional bending moment 3 inches $(80 \mathrm{~mm}$ ) above the base of the cylinder vs. pressure

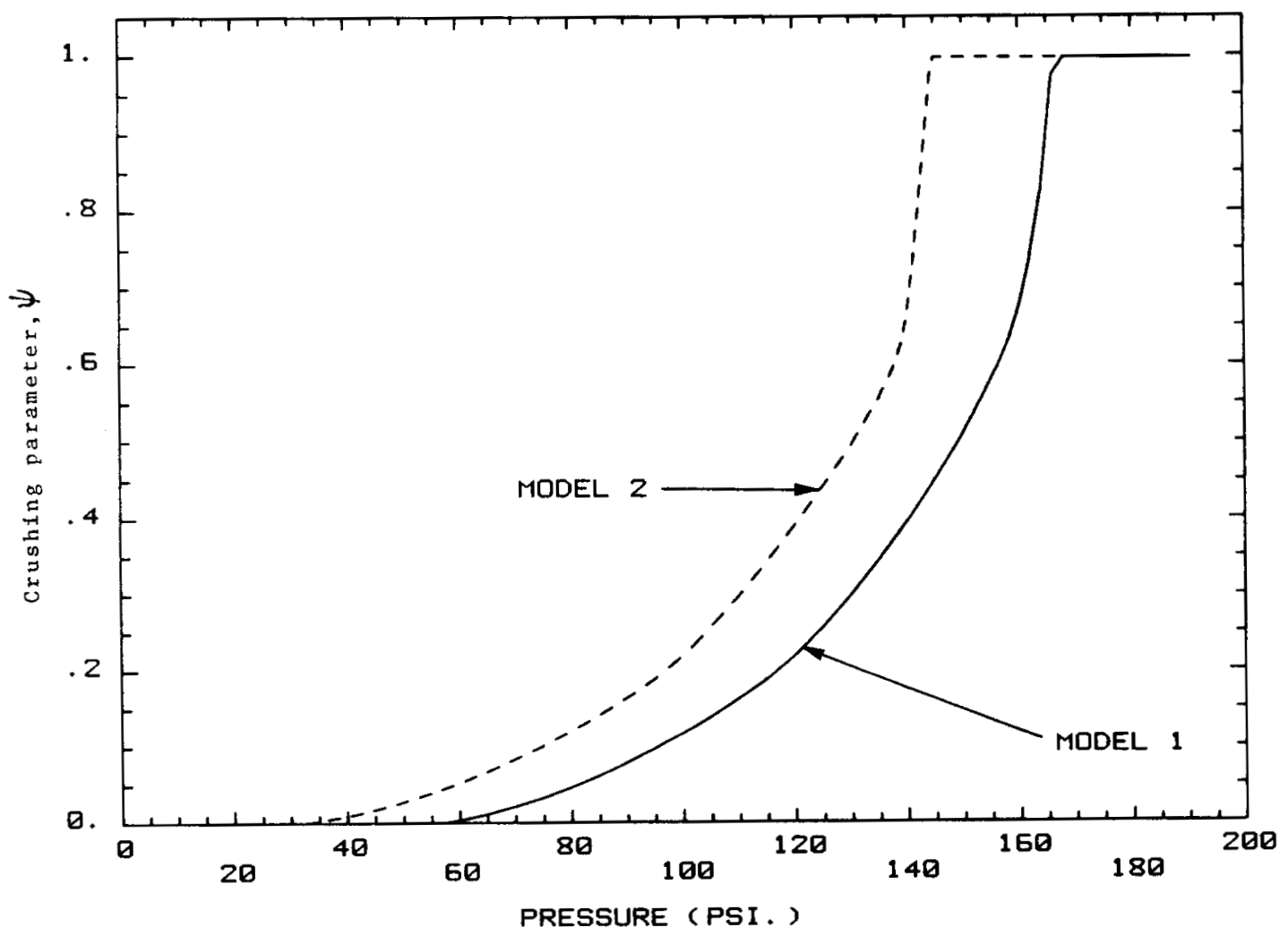

Figure 4.1.30 Crushing parameter on the outside of the cylinder wall 3 inches $(80 \mathrm{~mm})$ above the top of the basemat $v s$. pressure 


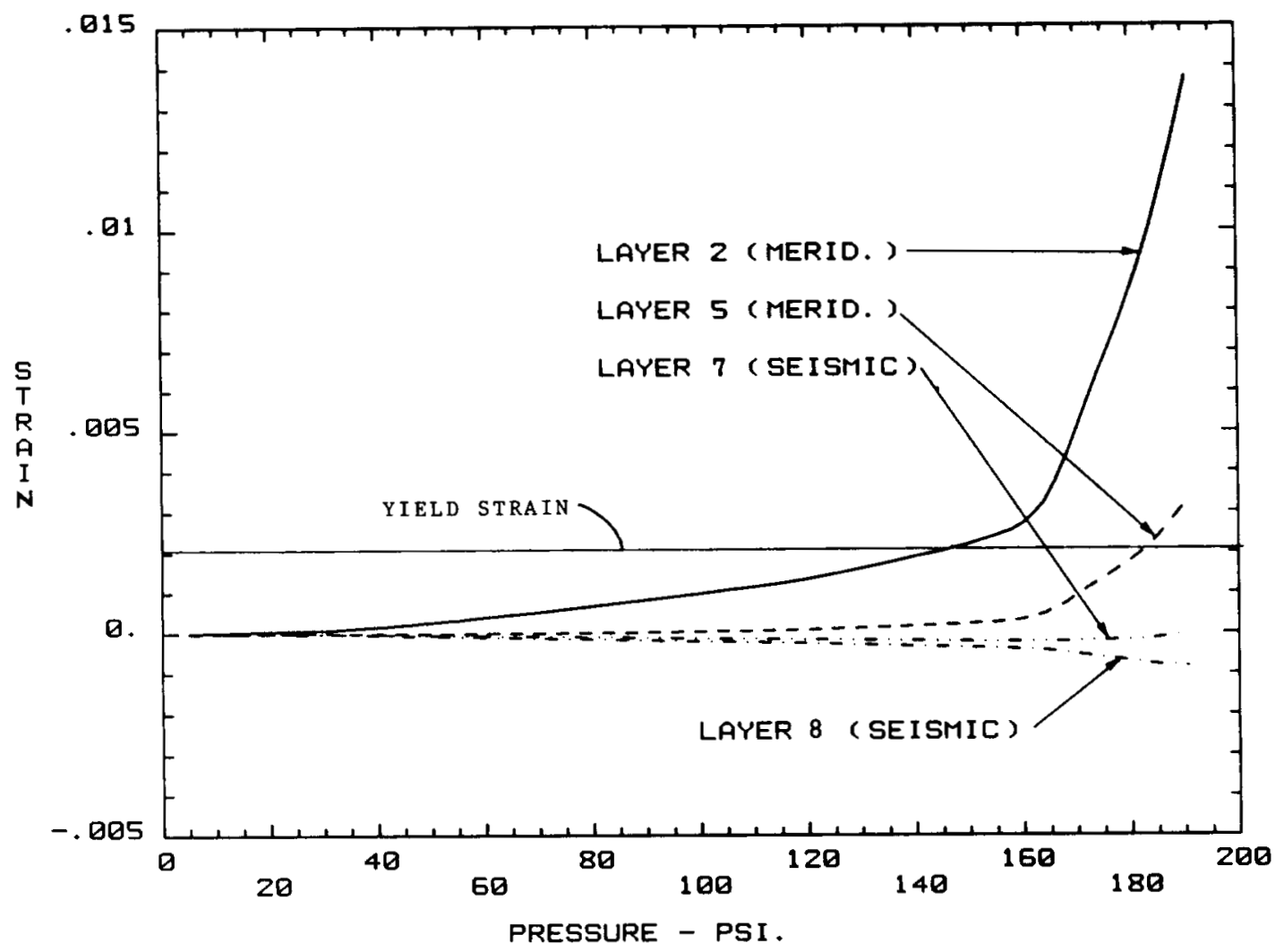

Figure 4.1.31 Model 1 results for rebar strains vs. pressure 3 inches $(80 \mathrm{~mm})$ above the base of the cylinder

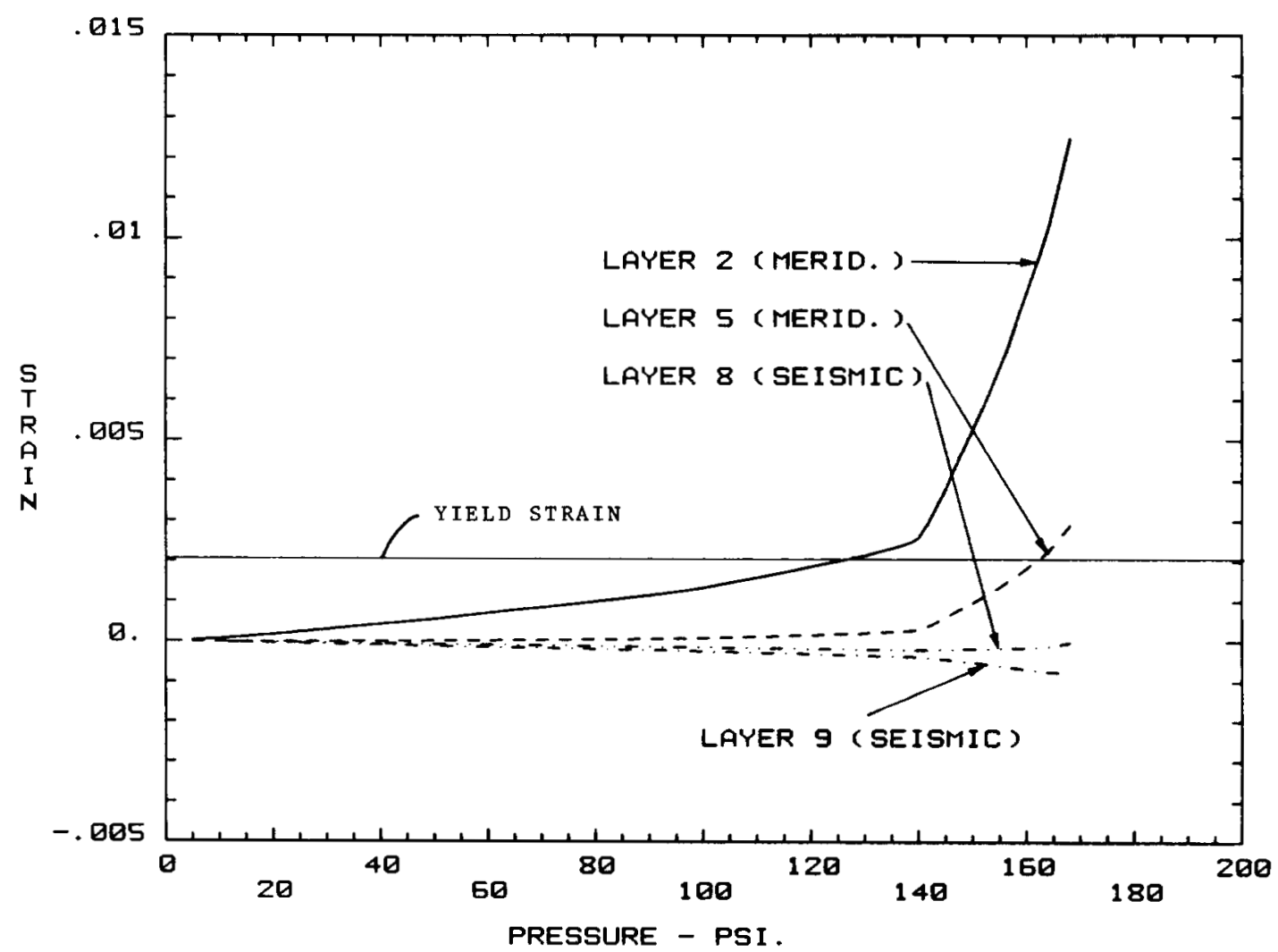

Figure 4.1 .32 Model 2 results for rebar strains vs. pressure 3 inches $(80 \mathrm{~mm})$ above the base of the cylinder 


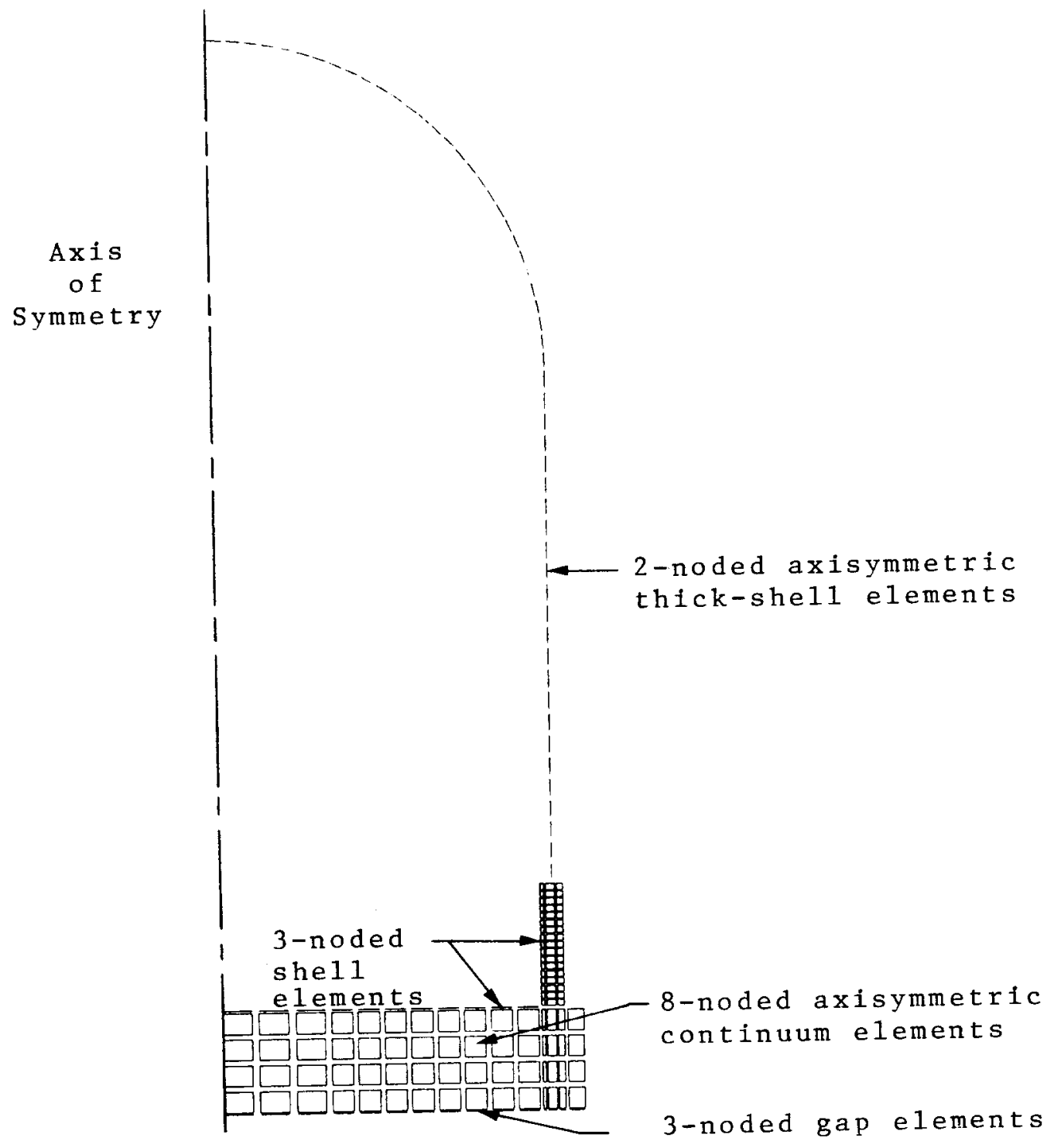

Figure 4.1.33 Finite element mesh used for continuum/shell Model 3 and 4 


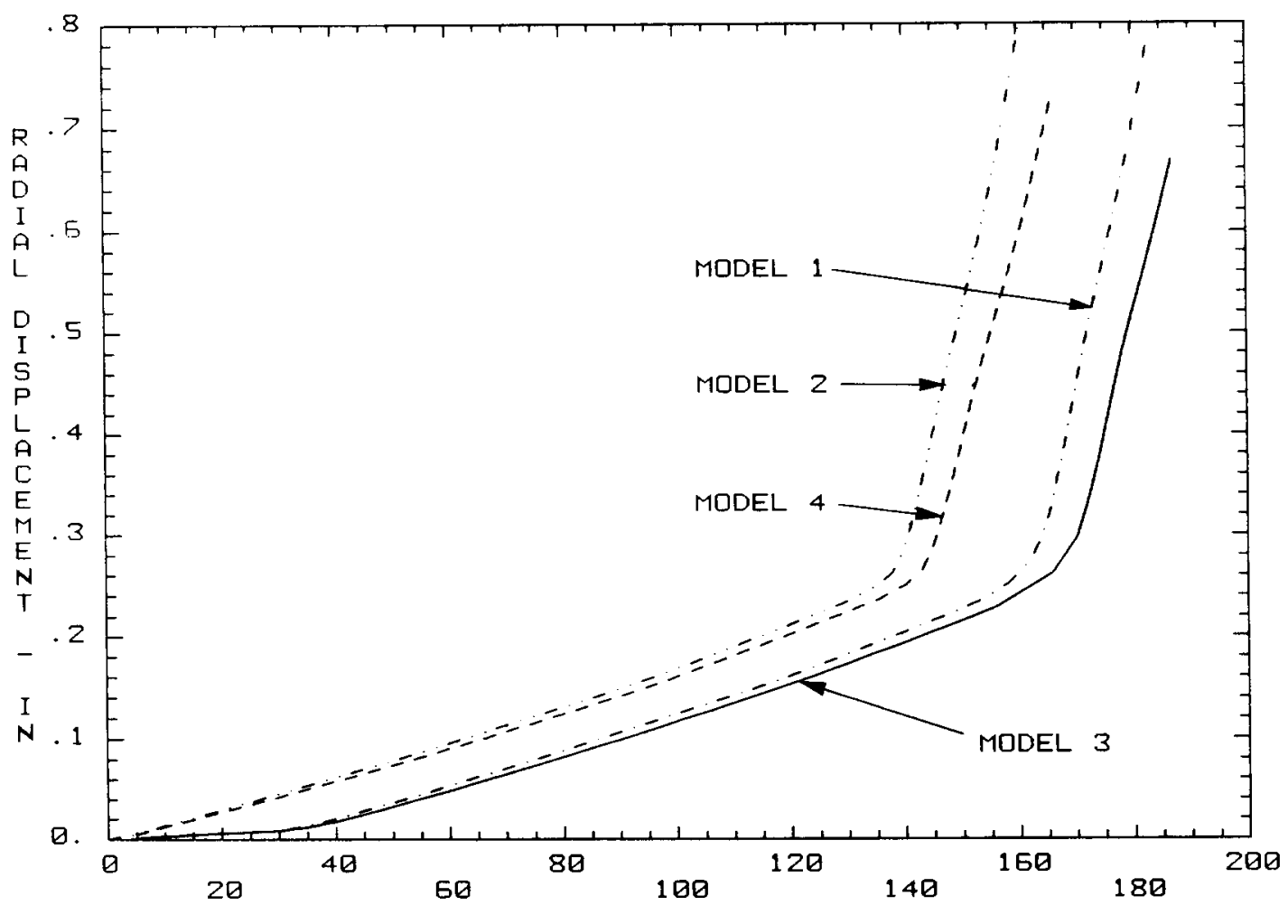

PRESSURE ( PSI)

Figure 4.1.34 Radial displacement of the cylinder at EL 6'-3"

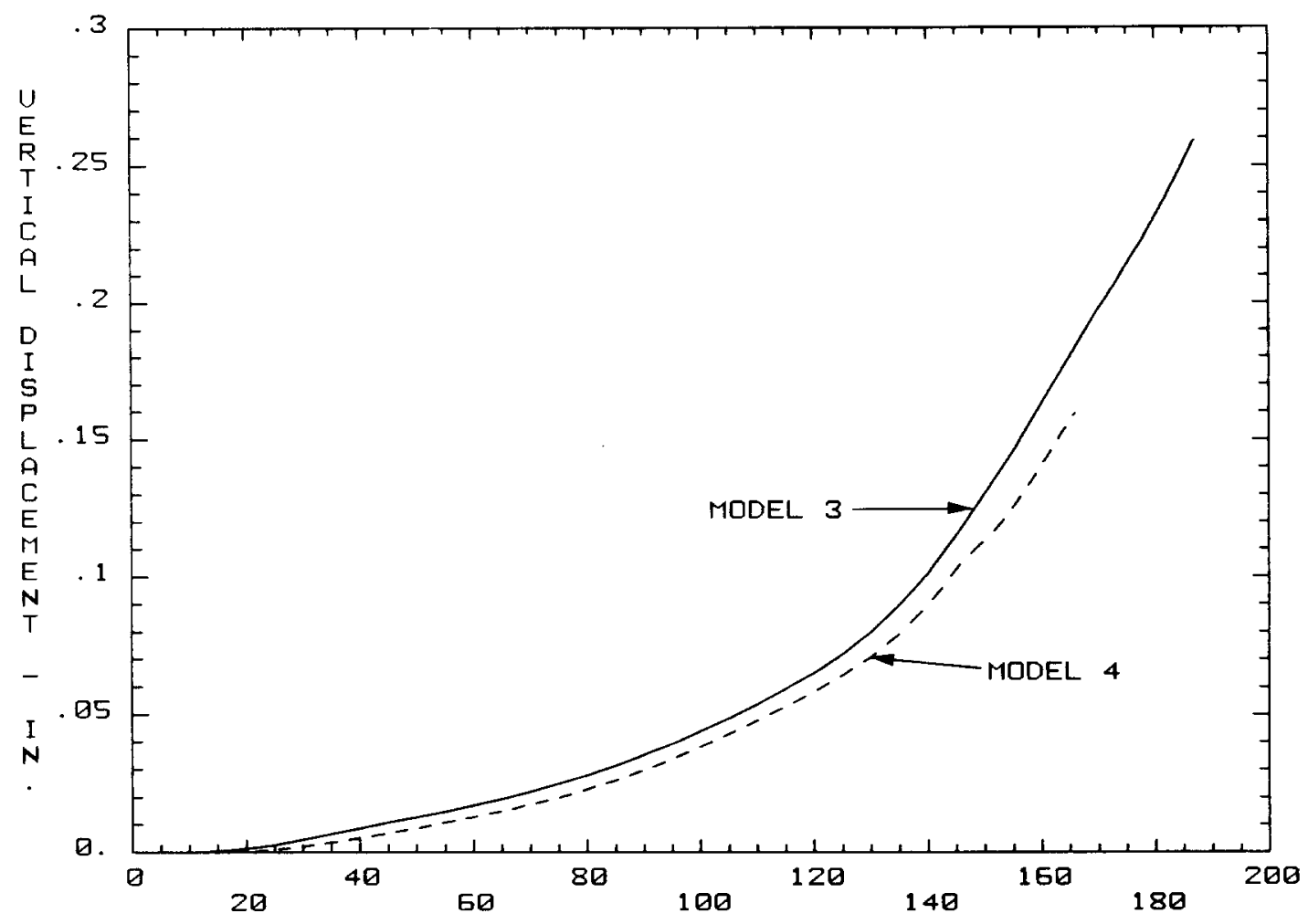

Figure 4.1.35 Basemat uplift at the basemat cylinder junction vs. pressure from results of continuum/shell Models 3 and 4 


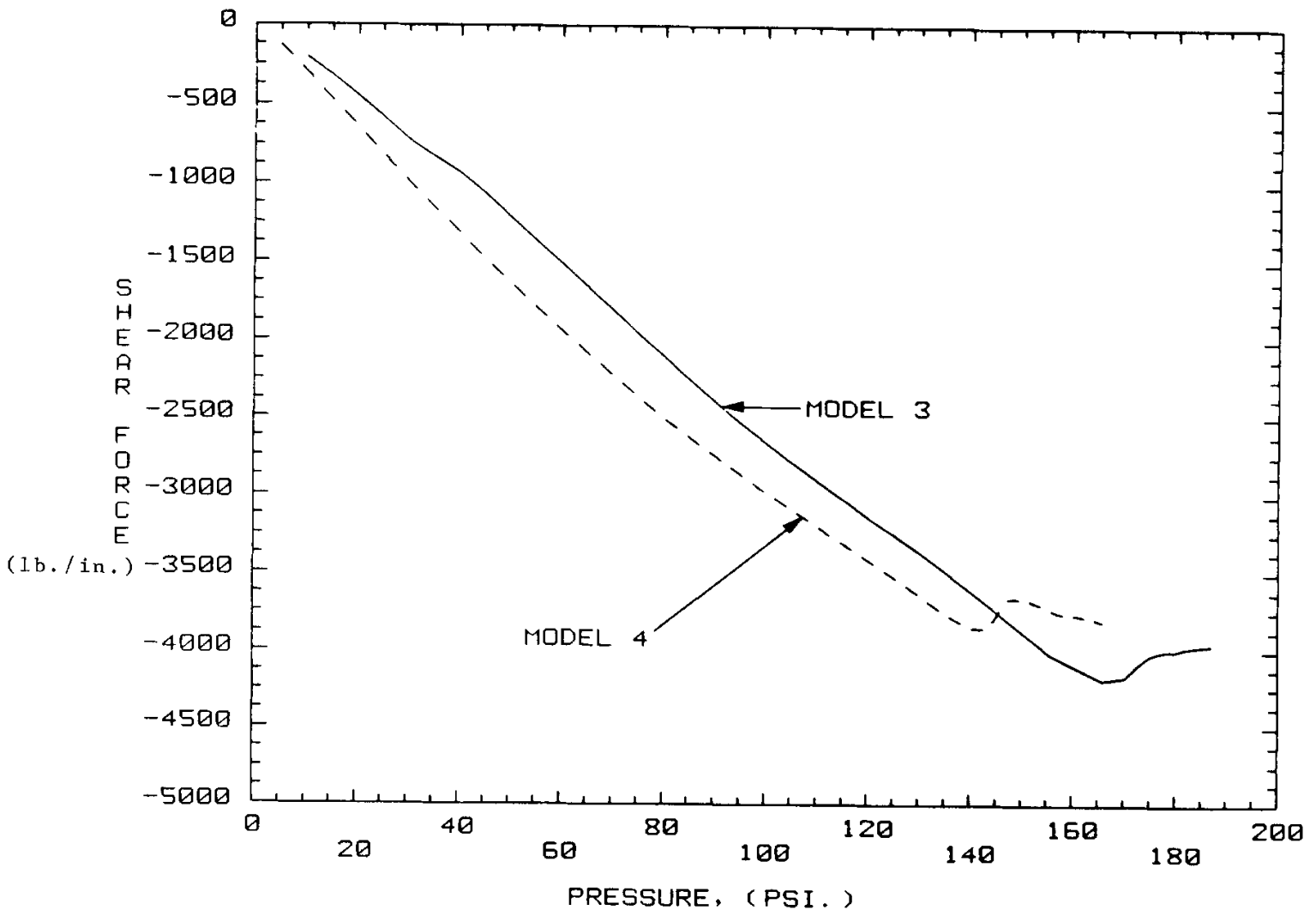

Figure 4.1.36 Radial shear force 1 inch $(25 \mathrm{~mm})$ above the base of the cylinder from results of continuum/shell Models 3 and 4

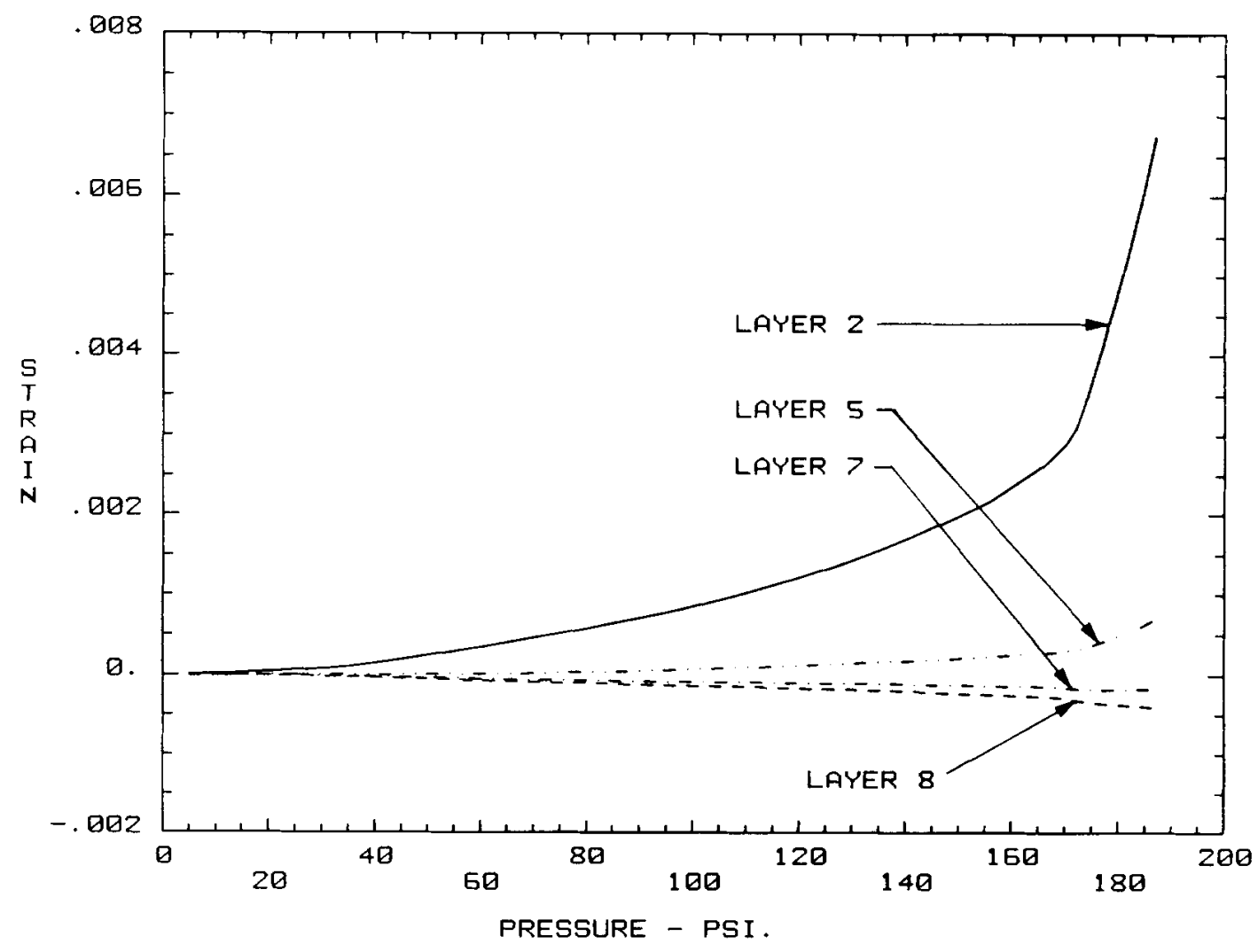

Figure 4.1.37 Model 3 results for rebar strains $v s$. pressure 1 inch $(25 \mathrm{~mm})$ above the base of the cylinder 


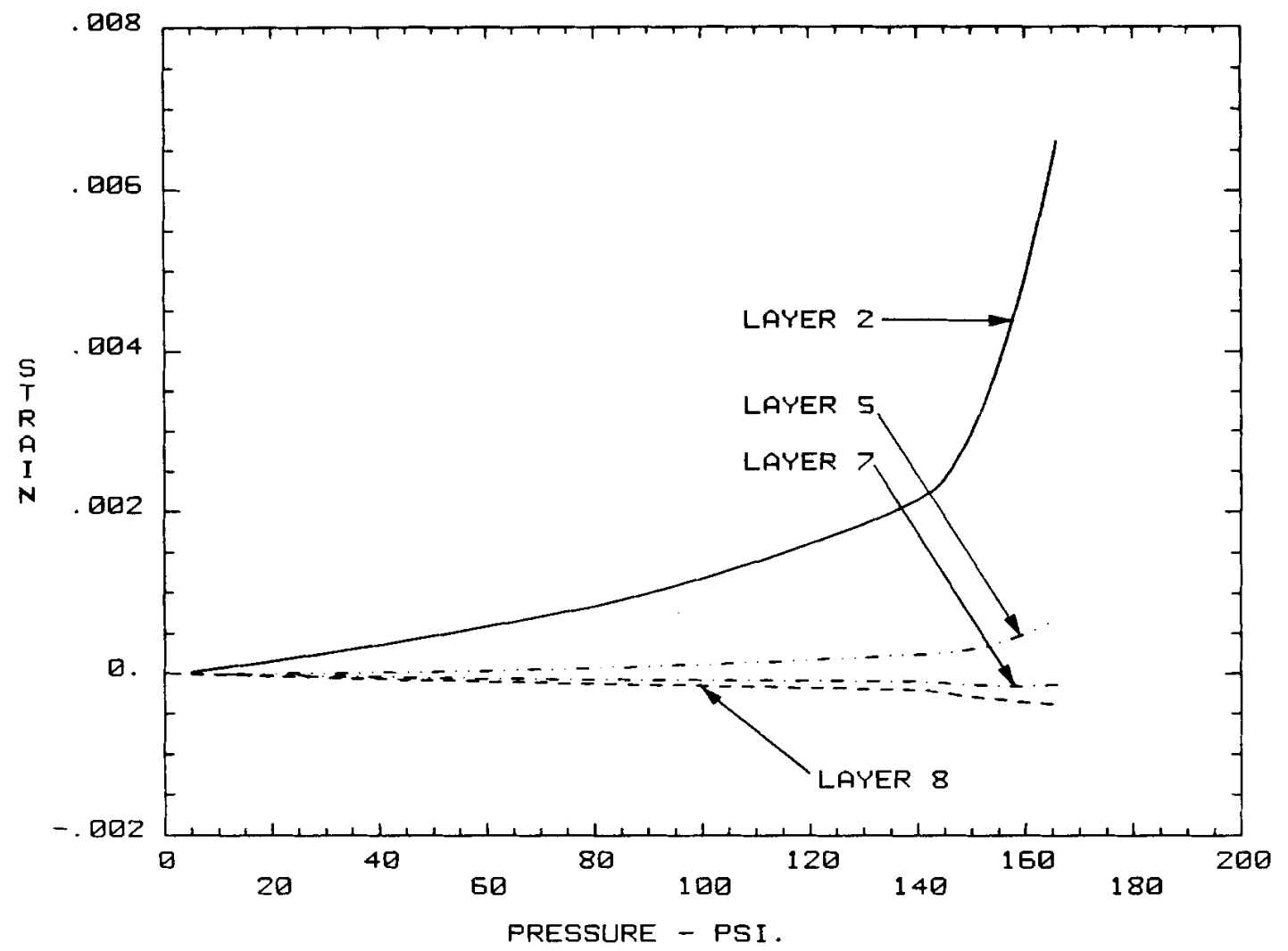

Figure 4.1 .38 Model 4 results for rebar strains vs. pressure 1 inch (25 mm) above the base of the cylinder

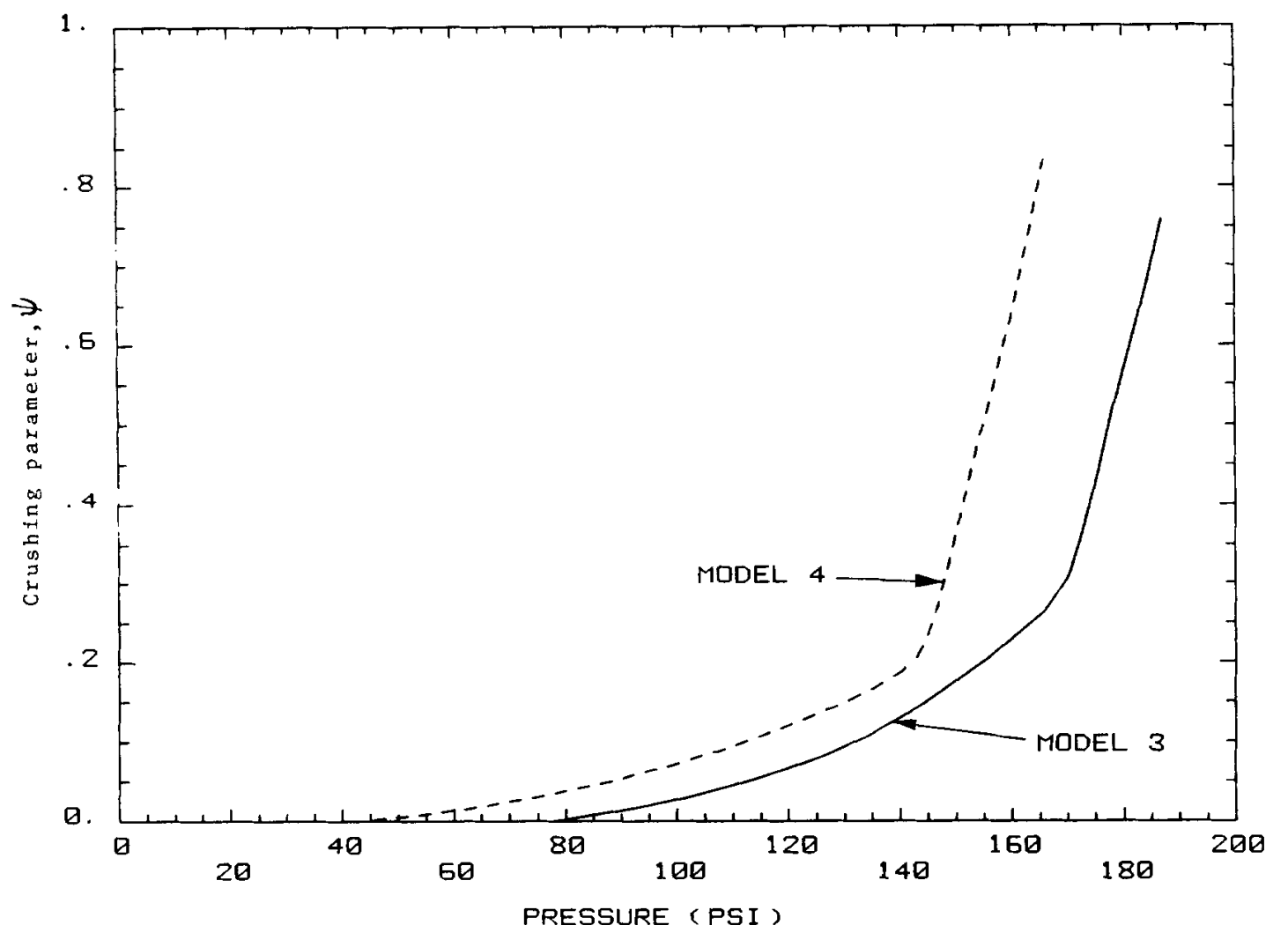

Figure 4.1.39 Crushing parameter on the outside of the cylinder wall 3 inches (76 $\mathrm{mm}$ ) above the basemat from Models 3 and 4 


\subsection{Argonne National Laboratory}

The authors of this section are P. A. Pfeiffer, R. F. Kulak, J. M. Kennedy, A. H. Marchertas, and C. Fiala of the Reactor Analysis and Safety Division at Argonne National Laboratory.

\subsubsection{Summary}

Pretest predictions were made by the Reactor Analysis and Safety Division of Argonne National Laboratory for the response of the 1:6-scale reinforced concrete model to be tested by SNL. For this purpose a series of axisymmetric models were studied with the two-dimensional computer program TEMP-STRESS and a three-dimensional circumferential segment model with the program NEPTUNE.

In both computer programs, both geometric and material nonlinearities were fully treated. The rebars were included in the models by a homogenization approach which accounts for their area, direction and elastic-plastic properties. The concrete constitutive model is based on a von Mises yield condition and an associated flow law; cracking is treated. The strength capacity is characterized by a four-parameter failure surface, and after failure an element-size-independent approach is used.

Solutions to the nonlinear equations which govern the systems in both TEMPSTRESS and NEPTUNE are obtained by dynamic relaxation. The load is applied incrementally, and iterations are continued until equilibrium is reached within a specified tolerance before proceeding to the next load increment.

Three axisymmetric models were analyzed. The first was a simplified model, which only represented the cylindrical and spherical containment shell and omitted the basemat. The latter was included in the two more complex models. The complex models also included representations of the foundation and sliding interfaces, which permitted separation and sliding between components of the basemat and the basemat and foundation.

The 1:6-scale concrete containment model contains several large penetrations, such as an equipment hatch and personnel airlock, that will cause deviations from a pure axisymmetric response and may decrease the capacity of the containment. In order to predict the behavior near these regions, a nominal 50 degree circumferential segment of the model was developed. The segment includes the cylinder from the basemat to the springline and dome. The cylinder portion contains one-half of the penetration opening for Equipment Hatch $B$. The basemat was not included in this model; thus, its effects on the cylinder at the cylinder-basemat junction are not taken into account.

The two-dimensional models all predict similar sequences of initial damage: meridional cracking commences at base of vessel at 25 psig $(0.172 \mathrm{MPa})$; yielding of the liner begins at the midpoint of the cylinder at 100 psig ( $0.690 \mathrm{MPa})$ and propagates up and down; yielding of the hoop reinforcement starts at $120-125 \mathrm{psig}(0.827-0.862 \mathrm{MPa})$. The sequence of damage milestones and pressure levels predicted by the three-dimensional analysis agrees with the two-dimensional results. A dimple deformation pattern occurred in the region of the boss, indicating additional bending strains. 
The three two-dimensional models all indicate failure at 185-190 psig $(1.276-1.310 \mathrm{MPa})$. However, the three models predict three different failure mechanisms: (1) hoop failure of the vessel at midheight following failure of a splice in this area, (2) failure of a weld in the liner near the basemat due to excessive strains, and (3) failure of the liner just above the knuckle due to compression failure of the concrete. In reality, simultaneous failure in more than one mechanism is unlikely since the onset of failure in any one mechanism will reduce the pressure and therefore reduce the stress on other possible sites of failure.

The three-dimensional model predicts failure at an internal pressure of 180185 psig (1.241-1.276 $\mathrm{MPa}$ ) when the splices of the hoop rebars fail just above the cylinder midheight in a region away from the equipment hatch opening.

\subsubsection{Introduction}

Prediction of the response of the 1:6-scale reinforced concrete containment model in this section was prepared by the staff of the RAS Engineering Mechanics Program of Argonne National Laboratory (ANL). The computer programs used to arrive at the final results have been fully developed at ANL. The two-dimensional code TEMP-STRESS [4.2.1-4.2.5] has been developed at ANL for stress analysis of plane and axisymmetric 2-D reinforced structures under various thermal conditions. The NEPTUNE [4.2.6-4.2.8] code was primarily intended for 3-D fluid structure interaction problems. Both programs are applicable to a wide variety of nonlinear problems, and are utilized in the present study to predict the response of the reinforced containment model to internal pressurization. The comparison of these pretest computations with test data on the containment model will provide a unique opportunity to test the validity of the respective codes to predict failure modes.

The analytical approaches in TEMP-STRESS and NEPTUNE are similar. Both codes are based on the explicit temporal integration coupled with dynamic relaxation. This enables the codes to be used for static as well as dynamic problems. The choice of the integration scheme was primarily dictated by the highly nonlinear concrete behavior and the relative ease of accounting for nonlinearity in this type of algorithm, and the fact that, al though within each load step this procedure introduces some dynamic response,

\subsubsection{Numerical Method}

\subsubsection{General Finite Element Equations}

The explicit time integration scheme is used in the TEMP-STRESS and NEPTUNE codes. The numerical algorithm for the explicit time integration is based on the solution of the following equation of motion:

$$
[M]\{\ddot{d}\}=\left\{F^{e x t}\right\}-\left\{F^{i n t}\right\}-\left\{F^{\text {damp }}\right\}+\left\{F^{g r a v}\right\},
$$

where $[M]$ is the lumped mass matrix, $[d]$ is the column matrix of nodal displacements (where the superposed dot indicates time derivatives), $\{\mathrm{F}\}$ is the column matrix of mechanical forces (internal, external, damping, or 
gravity). The internal forces are element-dependent and may be expressed as follows:

$$
\left\{F^{i n t}\right\}=\int_{V}[B]^{\top}\{\sigma\} d V,
$$

where $V$ is the volume of the element and $\{\sigma\}$ is the element stress matrix. The $[B]$ matrix relates the velocity strain $\{\dot{\epsilon}\}$ of the element to the nodal velocities $\{\dot{d}\}$ as

$$
[\dot{\epsilon}]=[B]\{\dot{d}\}
$$

The damping force is introduced into the equations of motion to facilitate the dynamic relaxation. The particular form used is given by

$$
\left\{F^{\text {damp }}\right\}=\left(\frac{2 \beta_{1}}{\omega_{\max }}[K]+2 \beta_{2} \omega_{\min }[M]\right)[\dot{d}],
$$

where the first term pertains to the stiffness-proportional damping and the second term refers to the mass-proportional damping; $\beta_{1}$ and $\beta_{2}$ are the damping ratios for the highest and lowest mesh frequency, respectively; $\omega_{\max }$ and $\omega_{\min }$ are the maximum and minimum circular frequencies of the mesh, respectively. The stiffness matrix [K] approximates the model stiffnesses; i.e., nodal stiffness of the $j$-th degree of freedom, $K_{j}$, is given as

$$
K_{j}=\left|\Delta F_{j}^{i n t} / \Delta d j\right|
$$

The minimum circular frequency $\omega_{\text {min }}$ is computed from an approximate Rayleigh quotient, and the maximum frequency $\omega_{\max }$ is related to the critical time step for the mesh as follows:

$$
\omega_{\min } \approx \sqrt{\sum_{I=1}^{N} k_{I} d_{I}^{2} / \sum_{I=1}^{N} M_{I} d_{I}^{2}}, \quad \omega_{\max } \approx \frac{2}{\Delta t},
$$

where $N$ is the total number of degrees of freedom and $\Delta t$ is the critical time step of the mesh.

\subsubsection{Explicit Temporal Integration}

In order to take advantage of the explicit formulation, the following difference form of Eq. (4.2.1) is utilized: 


$$
\begin{aligned}
{[M][\ddot{d}]^{i}=} & \left\{F^{e x t}\right\}^{i}-\left\{F^{i n t}\right\}^{i}+\left\{F^{g r a v}\right\}^{i}-2 \beta_{1} / \omega_{\max }[K][\ddot{d}\}^{i-1 / 2} \\
& -2 \beta_{2} \omega_{\min }[M]\{\dot{d}\}^{i},
\end{aligned}
$$

where

$$
\{\dot{d}\}=\frac{1}{2 \Delta t}\left(\{d\}^{i+1}-\{d\}^{i-1}\right)=\{\dot{d}\}^{i-1 / 2}+\frac{\Delta t}{2}\{\ddot{d}\}^{i} .
$$

By substituting Eq. (4.2.8) into Eq. (4.2.7) and solving for acceleration, we get

$$
\begin{aligned}
\ddot{d}\}^{j} & =\frac{[M]^{-1}}{1+\Delta t \beta_{2} \omega_{\min }}\left(\left\{F^{e x t}\right\}^{i}-\left\{F^{i n t}\right\}^{i}+\left\{F^{g r a v}\right\}^{j}-\left(2 \beta_{1} / \omega_{\max }[K]\right.\right. \\
& \left.\left.-2 \beta_{2} \omega_{\min }[M]\right)\{\dot{d}\}^{i-1 / 2}\right) .
\end{aligned}
$$

The velocities and displacements are integrated by the central difference equations as follows:

$$
\begin{aligned}
\{\dot{d}\}^{j+1 / 2} & =\{\dot{d}\}^{j-1 / 2}+\Delta t\{\ddot{d}\}^{i}, \\
\{d\}^{j+1} & =\{d\}^{i}+\Delta t\{\dot{d}\}^{i+1 / 2} .
\end{aligned}
$$

\subsubsection{Dynamic Relaxation}

In the computer code the external load $\left\{\mathrm{F}^{\mathrm{ext}}\right\}$ in Eq. (4.2.1) is prescribed by input. To facilitate the dynamic relaxation procedure, this loading is applied incrementally, i.e. in load steps of specified magnitude. Hence, in the dynamic relaxation procedure, for each of the load increments the structure responds dynamically and then approaches static equilibrium asymptotically, as shown in Figure 4.2.1. Thus, there may be some slight deviations between a purely static solution in path dependent materials, but there is no consistent drift from equilibrium. We have chosen load increments which are sufficiently small so that the effects of the intermediate dynamic responses appear to be negligible.

Equilibrium of the system is checked by means of two criteria. One of the criteria makes a comparison of the unbalanced force with the internal force, while in the other criterion the displacements are compared at successive iteration steps. The criteria are expressed in mathematical terms as follows:

$$
100 \sqrt{\sum_{I=1}^{N}\left(F_{I}^{e x t}-F_{I}^{i n t}\right)^{2} / \sum_{I=1}^{N}\left(F_{I}^{e x t}\right)^{2}} \leq \epsilon_{F}
$$


and

$$
100\left(\sum_{I=1}^{N} \dot{d}_{I} \Delta t / \sum_{I=1}^{N} d_{I}\right) \leq \epsilon_{d},
$$

where $\epsilon_{F}$ and $\epsilon_{d}$ are the force and displacement tolerances in percent. For this calculation, $\epsilon_{F}=2$ and $\epsilon_{d}=0.1$ with $\beta_{1}=0$ and $\beta_{2}=90$ percent. The sequence of computations using dynamic relaxation is shown in figure 4.2.2.

\subsubsection{Material Models}

Both the steel and the concrete are modeled as nonlinear materials. The constitutive equations for these materials are based on an elastic-plastic law with initial yielding and the subsequent loading surface described by the von Mises condition. An associated flow rule is used.

The uniaxial strength data for the materials is given in Tables 4.2.1-4.2.4; except for minor alterations, it corresponds to that recommended by SNL. The rebar material properties are modified to take into account the strength of the splices. Wherever mechanical splices are located, the strength of the rebar is assumed to be the average holding strength of the splice. The average strength capacity of the splice is taken to be $99 \mathrm{ksi}$ (683 MPa).

The stress-strain data for the rebar differs slightly from the data recommended by SNL. Instead of $73.3 \mathrm{ksi}$ ( $505 \mathrm{MPa}$ ) for the second value in Table 4.2.2, we use $75.0 \mathrm{ksi}$ ( $517 \mathrm{MPa}$ ), and $50.25 \mathrm{ksi}$ (306 MPa) is used instead of $50.0 \mathrm{ksi}(346 \mathrm{MPa})$ in Table 4.2.3. This is done to assure that the hardening slopes in the plastic region increase monotonically with strain. The constitutive equations use isotropic hardening.

The strength capacity of the concrete in multiaxial stress space is characterized by the so-called Hsieh-Ting-Chen [4.2.9] four parameter failure surface. The concrete response after failure is simulated using the element-size-independent cracking criterion established by Bazant and oh $[4.2 .10]$. In the uniaxial stress-strain relationship, a linear reduction of strength is specified from the ultimate stress down to zero. The maximum strain in tension (where the stress is specified as zero) for the solutions described in this report is $0.0007 \mathrm{in} / \mathrm{in}$. The input data required to define the failure surface is given in Table 4.2.5.

\subsubsection{Axisymmetric Simulation}

TEMP-STRESS is a 2-D code for the stress analysis of plane and axisymmetric reinforced/prestressed concrete problems. Elements available for representing the materials are a quadrilateral continuum element with one point integration and a flexural element with two-point integration along its length and an arbitrary number of integration points through the depth.

The rebars are modeled by what is known as "homogenization": the stressstrain law of the rebars is embedded in the stress-strain law of the elements. This approach can account for the direction, the position, and the percentage of reinforcement. The rebars are assumed to remain rigidly bonded to the concrete; debonding of rebars and concrete is not considered. 
Table 4.2.1 Concrete Material Properties

Elastic Constants

Young's Modulus - $4800 \mathrm{ksi}$ ( $33100 \mathrm{MPa})$

Poisson's ratio - 0.2

Ultimate Tensile Strength - 500 psi ( $3.45 \mathrm{MPa})$

Engineering Stress

(ksi) (MPa)

$\begin{array}{rrr}1.0 & 6.9 & 0.00021 \\ 2.0 & 13.8 & 0.00045 \\ 3.0 & 20.7 & 0.00072 \\ 4.0 & 26.9 & 0.00100 \\ 5.0 & 34.5 & 0.00140 \\ 6.8 & 46.9 & 0.00200 \\ 6.8 & 46.9 & 0.00230 \\ 0.0 & 0.0 & 0.00600\end{array}$

Table 4.2.2 Rebar Material Properties

Elastic Constants

Modulus - $31000 \mathrm{ksi}(214000 \mathrm{MPa})$

Poisson's ratio -0.3

Engineering Stress

(ksi) (MPa)

Engineering Strain

$\begin{array}{cl}66.6 & 459 \\ 76.0 \star & 524 \\ 85.6 & 590 \\ 99.0 & 682 \\ 105.0 \star \star & 724 \star \star \\ 107.0 \star \star & 738 \star \star\end{array}$

0.00215

0.01176

0.02276

0.04619

$0.0650 \star \star$

$0.0950 * \star$

Table 4.2.3 Cylinder and Basemat Liner Material Properties

Elastic Constants

Modulus - $30000 \mathrm{ksi}(207000 \mathrm{MPa})$

Poisson's ratio -0.3

Engineering Stress

$\begin{array}{lll}(\mathrm{ksi}) & (\mathrm{MPa}) & \text { Engineering St } \\ 50.2 & 346 & 0.00167 \\ 50.25^{\star} & 346 & 0.01738 \\ 59.0 & 407 & 0.03277 \\ 66.0 & 455 & 0.07180 \\ 68.0 & 469 & 0.09597 \\ 70.0 & 482 & 0.16433\end{array}$

*Vaive changed from that supplied by SNL.

**Estimated from Stress-Strain Data Supplied by SNL. 
Table 4.2.4 Dome Liner Material Properties

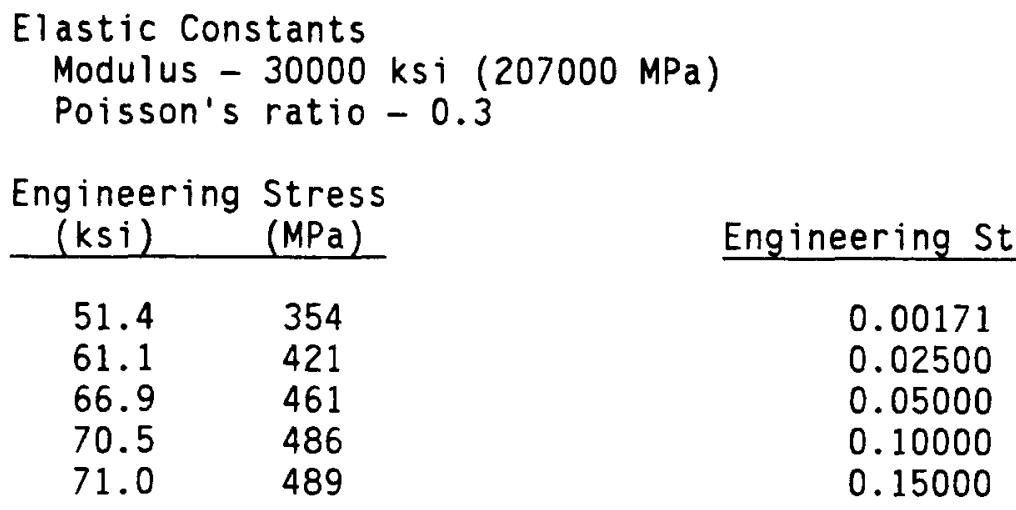

Table 4.2.5 Parameters Defining the Failure Surface of Concrete

Definition

1. Ultimate tensile strength, $f_{t}$

2. Equal biaxial compression, $f_{b c}$

384. Confined triaxial compression, $f_{p c}^{\prime}$ $\left(f_{C C}^{\prime}>f_{p c}^{\prime}\right)$
, $f_{C C}^{\prime}$
Value

$0.07535 f_{c}^{\prime *}$

$1.15 \mathrm{f}_{\mathrm{C}}^{\prime}$

$0.8 \mathrm{f}^{\prime} \mathrm{c}$

$4.2 f^{\prime} c$

${ }^{*} f_{c}^{\prime}$ is the maximum uniaxial strength of concrete in compression.

Reinforcement in the continuum element can be specified in the hoop direction and arbitrary orthogonal directions in the $r-z$ or the $x-y$ plane. The reinforcement arrangement in the continuum element is shown in Figure 4.2.3. In the flexural element, reinforcement can be specified at arbitrary layers measured from the neutral axis and spanning from the axial (meridional) direction through the hoop direction. Inclined reinforcement through the depth of the cross-section, representing the connecting ties, can also be treated. Reinforcement options in the flexural element are shown in Figure 4.2.4.

The elements account for the cracking in the concrete. A total of three orthogonal cracks may coexist at the centroid of the axisymetric continuum elements: one crack in the hoop direction and two orthogonal cracks in the $r-z$ plane. Similarly, two orthogonal cracks may occur in the axisymetric shell element at each integration point: one crack in the hoop direction and one crack in the meridional direction. 
In addition to that specified within the concrete elements, reinforcement can also be modeled by means of discrete rod and ring elements. The combination of homogenized and discrete elements can thus be used to represent the details of reinforcement in the containment test structure.

\subsubsection{Finite Element Models and Results}

The finite element models, which were utilized to predict the response of the test structure under internal pressurization, varied in complexity. Generally, the first model was simple, simulating the general global behavior of the containment shell. The subsequent models, which increased in complexity, revealed additional information.

The first finite element model consists only of the axisymetric cylindrical vessel and a spherical dome. This model is intended to provide an understanding of the global deformation of the containment wall during pressurization. The next finite element model also includes a simple model of the concrete slab to which the vessel is attached. This model provides an estimate of the up-lift during the test. Details of the junction between the test vessel and the slab are represented by still another finite element model. This model gives a refined picture of the liner response at the vessel base.

\subsection{Reinforced Shel1 Model}

The purpose of the simplified reinforced shell model, which is composed only of axisymetric reinforced shell elements, is to portray the global response of the vessel. It is shown in Figure 4.2.5. This model consists of 31 reinforced concrete shell elements: eleven of them representing the 7 inch $(178 \mathrm{~mm})$ thick spherical dome, one element representing the transition from the dome to the cylinder, and eighteen elements representing the 9-3/4 inch $(248 \mathrm{~mm})$ thick cylinder. A liner on the inside surface of the vessel is made up of steel shell elements. The liner elements, which are offset from the reinforced concrete elements, have different thicknesses and material properties in the dome and in the cylinder. The thickness of the liner in the dome (used in the finite element model) is 0.09 inch $(2.29 \mathrm{~mm})$ and that in the cylinder and base is 0.068 inch $(1.73 \mathrm{~mm})$.

The modeling of the reinforcement closely corresponds to the structural specifications of the containment structure. The number of reinforcement layers ranges from four in the spherical dome to ten layers at the base of the cylinder. Fourteen different combinations of reinforcements are used within the flexural elements. The nominal cross-sectional dimensions of the reinforcement bars are used in the model.

Pressurization of the shell model is imposed by monotonically increasing the internal loading in $5 \mathrm{psig}(0.0345 \mathrm{MPa})$ increments. The important

milestones in the response of the model are shown in Table 4.2.6. It is observed that up to $25 \mathrm{psig}(0.172 \mathrm{MPa})$ the behavior of the vessel is entirely elastic. At internal pressures 25-40 psig (0.172-0.276 MPa) meridional cracking at the base of the vessel propagates from the inside of the vessel to the neutral surface. The onset of hoop and meridional cracking in the cylinder above 40 psig $(0.276 \mathrm{MPa})$ is followed by hoop and meridional cracking in the dome. Hoop and meridional cracking are basically completed at 90 and $115 \mathrm{psig}(0.621$ and $0.793 \mathrm{MPa})$, respectively. 
Yielding of the liner is observed to start at about $100 \mathrm{psig}(0.689 \mathrm{MPa})$ at mid-cylinder height, propagates along the cylinder and, finally reaches the base at about 105 psig ( $0.724 \mathrm{MPa})$. The yielding of hoop reinforcement on the inside of the concrete vessel begins at about $120-125$ psig $(0.827-0.862$ $\mathrm{MPa}$ ). Failure of hoop reinforcement is governed by the strength of the splices; this stress limit of the splices of $99 \mathrm{ksi}$ (683 MPa) is reached at an internal pressure of about 185-190 psig (1.276-1.310 MPa). Failure of the hoop reinforcement was found to cause catastrophic failure of the liner and rupture; in subsequent analyses the failure was not modeled, but instead the attainment of the ultimate stress for the splices was considered to constitute failure of the vessel. The deformed configuration of the vessel, before impending failure, is shown in Figure 4.2 .6 with no magnification.

This model provides information on the response of the containment vessel relative to the vertical displacement of the cylinder base. Deformation of the vessel, as well as the strains in the liner and the rebars are obtained for internal pressures ranging from 5 psig $(0.0345 \mathrm{MPa})$ up to about $185 \mathrm{psig}$ $(1.276 \mathrm{MPa})$. Maximum vertical displacement at the dome apex before impending failure of the hoop splices is 2.2 inches $(56 \mathrm{~mm})$; the corresponding vertical and horizontal displacements at the vessel springline are 2.6 inches $(66 \mathrm{~mm})$ and 2.4 inches $(61 \mathrm{~mm})$. The maximum radial deformation at midheight of the cylinder at the same instant is 5.7 inches $(145 \mathrm{~mm})$ and the respective strain in the 1 iner is $0.043 \mathrm{in} / \mathrm{in}$. Displacements and strains for the model are given in Figures 4.2.7 through 4.2.20.

\section{Tabie 4.2.6 Response of Shell to Pressurization}

\begin{tabular}{cc}
\multicolumn{2}{c}{ Pressure } \\
psig & $\underline{\mathrm{MPa}}$ \\
$0-25$ & $0-0.172$ \\
$25-40$ & $0.172-0.276$ \\
$40-90$ & $0.276-0.621$ \\
$40-115$ & $0.276-0.793$ \\
$95-110$ & $0.655-0.758$ \\
$100-105$ & $0.689-0.724$ \\
$120-125$ & $0.827-0.862$ \\
$185-190$ & $1.276-1.310$
\end{tabular}

\section{Response}

Elastic behavior.

Meridional cracking on inside base of vessel.

Hoop cracking in cylinder and hoop/ meridional cracking of the dome.

Meridional cracking in the cylinder.

Yielding of liner begins and spreads along cylinder.

Yielding of liner at the base.

Yielding of hoop reinforcement starts.

The ultimate strength of the splice is reached in the cylinder (at point $A$ in Figure 4.2.6) leading to failure of the liner. 


\subsection{Reinforced Shell with Slab Model}

The purpose of this finite element model is to estimate the possible vertical uplift of the base of the vessel during internal pressurization and to provide information on the effect of the slab on the failure mode. This model is an extension of that described in Section 4.2.5.1.1. The previously described vessel model is connected to a model of the slab consisting of reinforced axisymmetric continuum elements. The finite element model is shown in Figure 4.2.21. Note that the gap in the basemat at the line of symmetry represents the void that was indicated in drawing No. 7847-F-1204. The bottom of the slab rests on a soil foundation that is modeled by a layer of elastic continuum elements for which an elastic modulus of $350 \mathrm{psi}(2.41 \mathrm{MPa})$ and a depth of 10 inches $(254 \mathrm{~mm})$ were used. A frictionless sliding interface is used between the elastic foundation and the slab so that uplift of the slab can be modeled. Also, a no-tension constitutive model was used for the soil, so it does not resist the slab from uplifting.

The model of the slab also includes a frictionless interface between the embedded liner and the concrete above the liner. This is done to simulate the effect of steel/concrete interaction during the flexural deformation of the slab. It was felt that the slider would simulate the actual role of the concrete above the liner better than a rigid connection. With a rigid connection, the flexural stiffness of the bottom slab is very large whereas the absence of a shear transfer mechanism between the liner and the superposed concrete prevents it from contributing substantially to the flexural capacity of the slab.

Reinforcement of the slab was estimated from the supplied structural drawings. Very little reinforcement was placed in the concrete of the slab above the liner, so it was neglected in the analytical model. In the vessel portion of this model the splices are used to connect individual lengths of reinforcing bars. The strength of the rebars in that part of the model is, therefore, limited by the ultimate strength of the splices. In the slab portion of this model splices are not used so that the full strength of the rebars should be utilized. The strength of the rebars in the slab is specified at their ultimate limit, as indicated in Table 4.2.2. This also enables us to observe other probable failure mechanisms of this model. Of particular concern are the meridional reinforcing bars and the liner on the bottom corner of the vessel. Because of the possibility of the slab to move upward, the angular deformation at the cylinder-slab intersection could be greater than in the previous shell model with fixed support. This would increase the stress at the junction over what is predicted by the simplified model.

Because of the elastic support below the bottom slab, the effect of the weight of the structure had to be accounted for first. This is accomplished by subjecting the finite element model to the weight of the structure prior to initiating the internal pressure. This solution provides the settlement (approximately a 0.26 inch $(6.6 \mathrm{~mm})$ translation downward), i.e. the original vertical displacement, before internal pressure is applied. As before, internal pressurization is applied in $5 \mathrm{psig}(0.0345 \mathrm{MPa})$ load increments.

The response of the shell portion of this model to internal pressurization is similar to the results obtained with the previous model. Stress and 
strain results for both models are similar in the shell, except near the bottom support. The sequence of cracking of the concrete, the yielding of the liner and reinforcement in the shell for this model is nearly the same as the information depicted in Table 4.2.6. Radial deformation of the shell is different from previous results near the junction of the cylinder and the slab (see figures 4.2.22 and 4.2.23). The vertical displacement of the dome is larger than before, reflecting the uplift of the slab which was 1.2 inches $(31 \mathrm{~mm})$ before impending failure (see Figure 4.2.24). Maximum vertical displacement at the dome apex before impending failure of the hoop splices is 2.4 inches $(61 \mathrm{~mm})$, the corresponding vertical and horizontal displacements at the vessel spring 1 ine are 2.8 inches $(72 \mathrm{~mm})$ and 2.5 inches $(64 \mathrm{~mm})$. The maximum radial deformation at midheight of the cylinder at the same instant is 6.0 inches $(152 \mathrm{~mm})$ and the respective strain in the liner is $0.045 \mathrm{in} / \mathrm{in}$.

The mode of failure predicted by this model is also similar to that of the previous model. We considered failure to have occurred when the stresses in the rebars exceeded the nominal strength of the splices. Failure occurred in the hoop rebars at the midheight of the cylinder, leading to rupture of the liner at 185-190 psig (1.276-1.310 MPa).

Since modeling the curvature of the liner at the corner would entail a prohibitively refined model, hand calculations were made to estimate the strains in the corner using the relative rotations between the horizontal and vertical section of the liner and data on its radius of curvature. These hand calculations indicate that maximum strains would be on the order of 3.6 percent when failure occurs at the midheight of the cylinder, no failure would occur due to the rotation of the liner at the corner. While an unwelded corner should experience no difficulties at these nominal strains, because of the presence of difficult welds and inherent stress concentrations in this area, failure could possibly occur there.

\subsection{Detailed Refined Model of the Shell/Slab Junction}

This finite element model was used to reveal more detailed information on the behavior of shell/slab junction during pressurization. Reinforced axisymmetric continuum elements in this model represent not only the slab, but also part of the shell, as shown in Figure 4.2.25. As was the case in the preceding model, the slab rests on an elastic foundation (soil). Again, frictionless sliding is provided between the nodes of the elastic foundation and the bottom of the slab and also between the embedded liner and the surrounding concrete.

As displayed in Figure 4.2.25, a rigid interconnection is used to model the interaction between large and small elements. The joint between the continuum and flexural elements in the wall of the shell assumes that plane sections remain plane.

The reinforcement in these additional continuum elements is modeled by smearing the respective steel rebars over the continuum elements. The reinforcement in the hoop, radial and axial directions is estimated from the supplied structural drawings.

The settlement of the model into the elastic foundation is first obtained when the weight of the structure is imposed on the finite element model. 
Subsequent internal pressurization is obtained by load increments as in the previous models.

The refined finite element model of the shell/slab junction indicates a different mode of failure, which is near the junction. Because of the detailed modeling of concrete, reinforcement and the liner at the junction, the model was able to capture the high shear force that develops between the basemat and the wall. The previous models could not capture this behavior because the shell element that was utilized is formulated under the assumption that through-the-thickness stresses are neglected.

The start of failure at the junction takes place at about 140 psig $(0.965$ $\mathrm{MPa}$ ) internal pressure; at this pressure, two to three rows of concrete fail in compression in the radial direction. These elements are located between the knuckle of the liner (inside of vessel) and the top of the outer basemat, see Figure 4.2.25. After the concrete fails in compression, the rebar shear ties at these locations fail in tension, and about 40 percent of the meridional reinforcement also fail in tension. As the internal pressure increases, the meridional reinforcement layer nearest the liner is stressed the highest, and at around 185 psig ( $1.276 \mathrm{MPa})$ this layer fails in tension, and then the liner rips open just above the knuckle due to the meridional tension. At this pressure, the failure mode observed in the previous models at the midheight cylinder could also occur in this model.

The important milestones of the response of this model with respect to internal pressure are outlined in Table 4.2.7. It is observed that the initial response regarding the cracking of concrete and the initiation of yielding do not differ appreciably from the results shown in Table 4.2.6.

\subsubsection{Three-Dimensional Simulation}

NEPTUNE is a three-dimensional finite element program that was developed to simulate the response of reactor components in three-dimensional space to design basis and beyond-design-basis loads. The code has evolved over the years to address the then current safety issues. Since the code was developed to solve a variety of problems, the current version is a general purpose three-dimensional finite element code primarily suited for nonlinear problems. The code is capable of treating problems that involve: (1) plate and/or shell structures, (2) fluids, (3) continua, (4) fluid-structure interaction, (5) media-structure interaction, (6) contact mechanics, and (7) silent boundaries. An important feature of NEPTUNE is its ability to handle nonlinear problems, which often occur during beyond-design basis loads. The element formulations can properly treat large deformations (geometric nonlinearities), and the rate-type material models can handle large material strains (material nonlinearities). Explicit solution algorithms are used to economically solve short duration transient problems. An extensive element library is provided to model bars, plates, shells, solids, fluids, rigid bodies, rigid links, interfaces, and silent boundaries.

In order to use the code for the simulation of the containment model, it was necessary to develop: (1) a static analysis capability, and (2) a shell element that can model reinforced concrete. The dynamic relaxation (DR) method was chosen to perform static analysis because of its (1) architecture, which is similar to the existing explicit time integration 


\begin{tabular}{|c|c|c|}
\hline \multicolumn{2}{|c|}{ Pressure } & \multirow[b]{2}{*}{ Response } \\
\hline psig & $\underline{\mathrm{MPa}}$ & \\
\hline $0-25$ & $0-0.172$ & Elastic behavior. \\
\hline $25-40$ & $0.172-0.276$ & $\begin{array}{l}\text { Meridional cracking on inside base of } \\
\text { vessel. }\end{array}$ \\
\hline $40-90$ & $0.276-0.621$ & $\begin{array}{l}\text { Hoop cracking in cylinder and hoop/ } \\
\text { meridional cracking of the dome. }\end{array}$ \\
\hline $40-115$ & $0.276-0.793$ & Meridional cracking in the cylinder. \\
\hline $95-110$ & $0.655-0.758$ & $\begin{array}{l}\text { Yielding of liner begins and spreads } \\
\text { along cylinder. }\end{array}$ \\
\hline $100-105$ & $0.689-0.724$ & Yielding of liner at the base. \\
\hline $120-125$ & $0.827-0.862$ & Yielding of hoop reinforcement starts. \\
\hline $135-140$ & $0.931-0.965$ & $\begin{array}{l}\text { Radial compressive crushing of concrete } \\
\text { at junction of wall and basemat. Partial } \\
\text { fracture of some meridional and shear tie } \\
\text { rebar. }\end{array}$ \\
\hline $185-190$ & $1.276-1.310$ & $\begin{array}{l}\text { Complete junction failure, liner rips } \\
\text { just above knuckle location. }\end{array}$ \\
\hline
\end{tabular}

scheme, (2) robustness, and (3) small core requirements (i.e. relative to implicit solvers).

To simulate the response of the 1:6-scale concrete containment model, it was necessary to use a plate element that would represent the behavior of reinforced concrete with a steel liner on one of its outer surfaces. A thin plate element, which existed in NEPTUNE, has some of the features needed to properly simulate the mechanics of the response of the containment during pressurization. The plate element is a bilinear four-node quadrilateral [4.2.11] that is based upon a velocity-strain formulation and a one-point quadrature rule in the plane of the element and a five-point quadrature rule through the depth. It can treat problems characterized by large deformations and finite strains. For this analysis, this plate element was enhanced with a material model for concrete, a representation for reinforcing bars, and a liner model that accounts for its membrane response.

The material model for concrete is identical to the model used in TEMPSTRESS. Some changes to the TEMP-STRESS coding were required, however, in order to make the TEMP-STRESS concrete module compatible with the NEPTUNE coding. Within each of the five through-the-depth layers of the element, cracking is simulated by a smeared cracking model that allows two orthogonal cracks perpendicular to the depth direction to develop. The remaining 
details of the concrete model were previously given in Section 4.2 .4 and will not be repeated here.

The rebars are represented in the element by discrete layers of smeared reinforcing steel. Within the element, individual layers are used to represent corresponding layers of actual reinforcement. The following geometric information is required for each layer of rebars: location in the depth of the cross-section relative to the centroidal plane, orientation of the bars relative to side one of the element, true cross-sectional area of the bars, and spacing between bars. The stress state in the bars is assumed to be uniaxial. The uniaxial stress state is rotated into a two dimensional stress state in the quadrilateral element coordinate system; bending of the bars is not considered. The reinforcement is assumed to lie along a straight line within each layer of an element. Regions in which the reinforcement follows a curved line are approximated by specifying different bar orientations from element to element. The reinforcement is taken to be rigidly bonded to the concrete throughout the simulation, thus debonding of rebars and concrete cannot occur. Reinforcements through the depth of the cross-section, such as connecting ties, cannot be treated. A uniaxial elasto-plastic strain hardening material model is used to represent the response of the rebars. The rebars are assumed to rupture when the predicted axial strain exceeds the ultimate strain value, as given in Table 4.2.2.

The final ingredient that was added to the original plate element was an additional layer positioned outside of the existing inside surface layer of concrete for capturing the membrane response of the liner. A twodimensional elasto-plastic strain hardening material model with the von Mises yield criterion is used to represent the liner steel. Failure of the liner is assumed to occur when the calculated equivalent true-strain exceeds the value for the ultimate strain given in Tables 4.2.3 and 4.2.4.

A small set of test problems were successfully run to verify the developed methodology and its implementation in the code. However, a larger problem set needs to be exercised before complete confidence is gained. Also, longer than expected CPU times were noted when large parts of the structure behave plastically. Changes in the method that should reduce CPU times are being implemented into the code.

\subsubsection{Finite Element Model}

The 1:6-scale concrete containment model contains several large penetrations, such as an equipment hatch and personnel lock, that will cause deviations from a pure axisymmetric response and may decrease the capacity of the containment. In order to predict the behavior near these regions, a nominal 50 degree circumferential segment of the model was developed (Figure 4.2.26). The segment includes the cylinder from the basemat to the springline and the dome. The cylinder portion contains one-half of the penetration opening for Equipment Hatch $B$. The basemat was not included in this model; thus, its effects on the cylinder at the cylinder-basemat junction are not taken into account.

The model consists of 266 quadrilateral plate elements and 305 nodes with 1830 degrees of freedom. The elements are 7 inches $(178 \mathrm{~mm})$ thick in the spherical dome with a $1 / 12$ inch $(2.12 \mathrm{~mm})$ thick liner added to the inside $-95-$ 
surface and $9-3 / 4$ inch $(248 \mathrm{~mm})$ thick in the cylinder with a 0.068 inch $(1.73 \mathrm{~mm})$ thick liner. The elements in the region of the boss are 21 inches $(533 \mathrm{~mm})$ thick, and those in the transition region are 15-3/8 inches (391 $\mathrm{mm})$; both have a 0.068 inch $(1.73 \mathrm{~mm})$ liner on the inside surface.

The topology of the reinforcement was specified for each element by prescribing the location of each layer in the depth direction and the orientation relative to side one of the element. The circumferential, meridional, and seismic rebar topology is captured fairly accurately in regions remote from the penetration. The curved shape of the rebars near the penetration is approximated by straight bars within each element, but the orientation of the bars from small groups of contiguous elements to small groups of contiguous elements is varied to globally capture the curved shape. The number of layers of bars in each element correspond to the specifications. The extra reinforcement that forms a cage around the penetration opening is also taken into account as are the sun-ray bars. The ties in the depth direction are not treated. Rebar layers 10 and 11 , which are located at the cylinder-basemat juncture, are accounted for in the model. Nominal values of the rebar areas were used in the analysis. It is assumed that the development length of the rebars is maintained throughout the loading. The apex plates, which anchor the rebars in the dome, are modelled by overlaying two quadrilateral plate elements, which have only steel material properties specified, onto another quadrilateral plate element, which has only concrete material properties specified.

The model is subjected to a fixed boundary condition (no translations or rotations allowed) at the base of the cylinder. Symmetry conditions are enforced on the nodes that lie in each of the two vertical symmetry planes; the first plane contains the meridional edge of the model with the penetration opening, and the second plane contains the other meridional edge. The symmetry boundary condition is a roller condition in which motion (translational and rotational) is only allowed in the symmetry plane. The apex node is only permitted to move in the vertical direction; no rotations are allowed.

The load is a pressure incrementally applied to the inside surface of the containment and a line load, which is equivalent to the pressure load on the hatch cover, applied in the radial direction to the edge of the penetration opening in the updated geometry. A pressure of 20 psig $(0.138 \mathrm{MPa})$ is applied in the first load step, and 5 psig (0.034 MPa) increments are used thereafter.

The numerical simulation was performed on an IBM 3033 computer, which is not a supercomputer. The containment model required 2.2 megabytes of core and about 6 hours of CPU to reach failure. Note, a recent computer benchmark study conducted by ANL's Computer Service Division indicated that the NEPTUNE code runs eight times faster on a CRAY. Thus, this problem would require about 45 minutes on a CRAY. The 6 hour CPU requirement imposes severe limitations on turnaround because of the pricing factor that is applied to computer cost. Since limited in-house funds were used, most of the computations were performed during weekends at which highly reduced rates were in effect. Therefore, a considerable amount of real time was required to perform the tasks of debugging, model evolution, model verification, and postprocessing. 


\subsubsection{Results}

The model was pressurized incrementally up to failure, which occurred at 180 psig (1.241 MPa). The response milestones are listed in Table 4.2.8. All materials in the model behaved elastically up to $25 \mathrm{psig}(0.172 \mathrm{MPa})$. Cracking at the inner diameter of the boss at the 6 and $120^{\prime}$ clock positions began at the pressure of $30 \mathrm{psig}(0.207 \mathrm{MPa})$. Meridional cracks also formed at the base of the cylinder. It was noted that the radial displacements of the cylinder near the region of the boss and including the boss were less than those further away. This deformation pattern formed an inward dimple. Beginning at 40 psig ( $0.276 \mathrm{MPa})$, extensive cracking occurred in the boss, cylinder, and dome. The liner material begins to behave plastically at 85 psig $(0.586 \mathrm{MPa})$. Stress in the hoop reinforcement reached the yield value at 125 psig $(0.862 \mathrm{MPa})$, and the meridional rebars were stressed to yield at 145 psig ( $1.000 \mathrm{MPa})$. Displacements and strains for the model are given in Figures 4.2.27 through 4.2.31.

Table 4.2.9 Response of Three-Dimensional Model to Pressurization

\begin{tabular}{|c|c|}
\hline \multicolumn{2}{|c|}{ Pressure } \\
\hline psig & $\mathrm{MPa}$ \\
\hline 25 & 0.172 \\
\hline 30 & 0.207 \\
\hline 40 & 0.276 \\
\hline 85 & 0.586 \\
\hline 125 & 0.862 \\
\hline 145 & 1.000 \\
\hline 180 & 1.241 \\
\hline
\end{tabular}

\section{Response}

Elastic behavior.

Cracking at 6 and $120^{\prime c l o c k}$ positions of boss; meridional cracking at base of cylinder.

Extensive cracking in boss, cylinder, and dome.

Liner material begins to behave plastically.

Hoop rebars behave plastically.

Meridional rebars behave plastically.

Final pressure at which equilibrium was obtained. A splice in a layer 6 (hoop) rebar failed at point $A$ shown in Figure 4.2.26 when 185 psig of pressure was applied. 


\subsubsection{Conclusions}

The three TEMP-STRESS models indicate rather similar levels of failure pressure, all of which lie in the range of 185-190 psi (1.276-1.310 MPa). However, the three models predict distinctly different failure mechanisms at these pressures. It appears from these studies that the failure modes in an analytical model would occur almost simultaneously. However, in reality a simultaneous occurrence of several failure mechanisms is not likely since the onset of failure will reduce the pressure and therefore reduce the stress on the other possible sites of failures. Therefore, it is impossible to predict which failure mechanism will occur; we predict one of the following three mechanisms:

1) Hoop failure of the vessel at the midheight, point $A$ in Figure 4.2.6, following the failure of the splice in this area.

2) Failure of a weld in the liner near the basemat due to excessive strain.

3) Failure of the liner just above the knuckle due to compressive failure of the concrete.

The response of the three-dimensional model was qualitatively similar to the two-dimensional shell model. Furthermore, the sequence of damage milestones occurred at pressures that agreed qualitatively with the two-dimensional results. However, a dimple deformation pattern occurred in the region of the boss indicating bending deformations. The three-dimensional model predicts failure at an internal pressure between 180-185 psig (1.241-1.276 $\mathrm{MPa}$ ) when the splices in layer 6 fail just above cylinder midheight in a region away from the equipment hatch opening. Note, if the development length of either the sun ray rebars in the boss area or the layer 10 and 11 rebars near the basemat is not maintained, then these areas could weaken and become potential failure sites.

It is our conclusion that the test model has been properly designed, with full understanding of basic design requirements and it possesses no weak components that would fail prematurely. Thus, the test model is equally strong in all of its weakest links and the failure mode is difficult to predict.

\subsubsection{Acknowledgments}

Acknowledgment is extended to Mr. Ralph Seidensticker, Manager of the Applied Mechanics Program, Argonne National Laboratory, for his support, encouragement, and suggestions. To Professor Ted Belytschko, Northwestern University, goes our appreciation for help and suggestions during the course of this effort. Thanks are also due to Professor Daniel Schoeberle, University of Illinois, Chicago, for his help in retrieving needed data from the numerical models for the preparation of the report. This work was performed under the auspices of the U.S. Department of Energy, Office of Technology Support Program, under Contract W-31-109-Eng-38. 

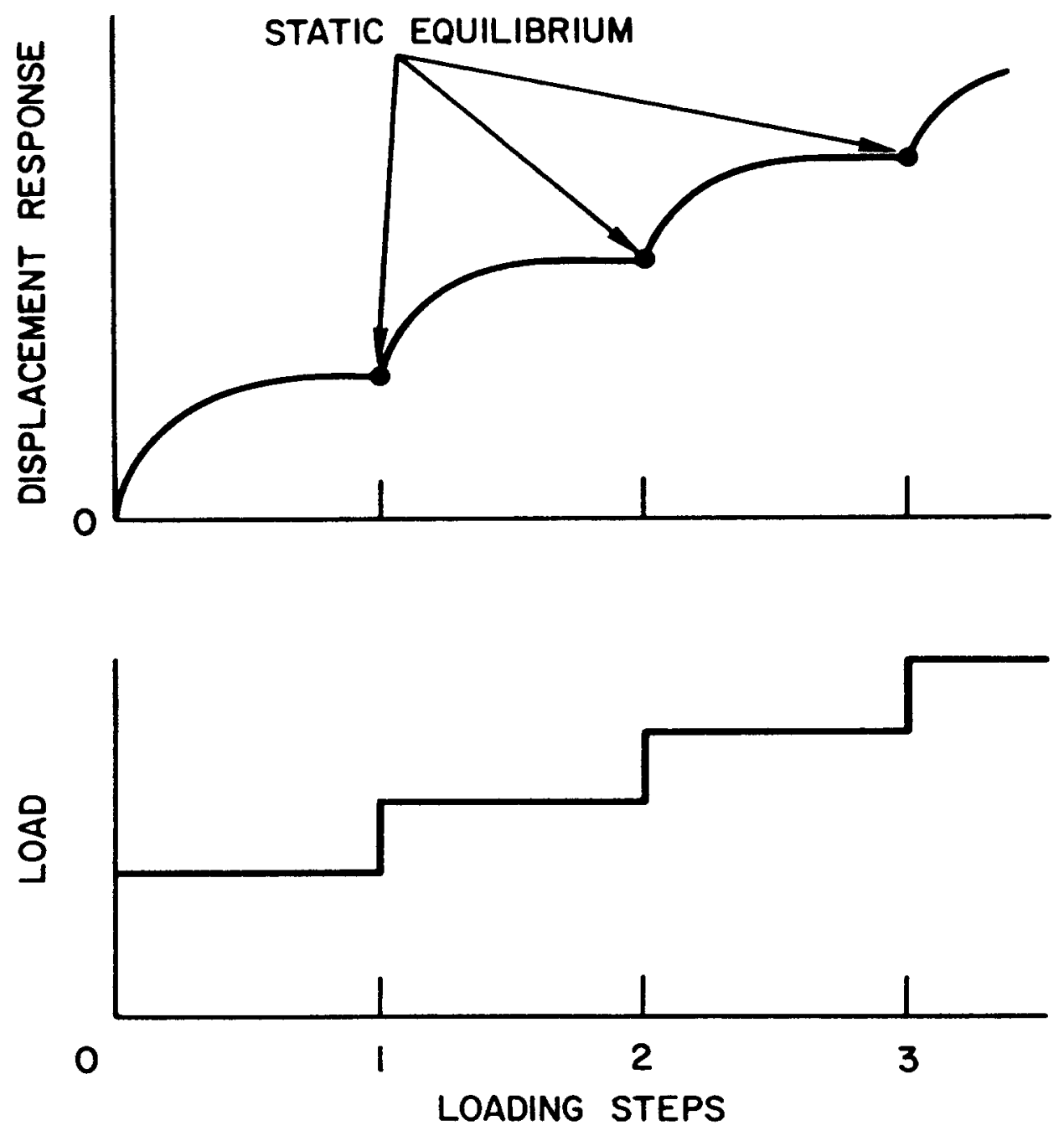

Figure 4.2.1 Loading Method for Dynamic Relaxation 


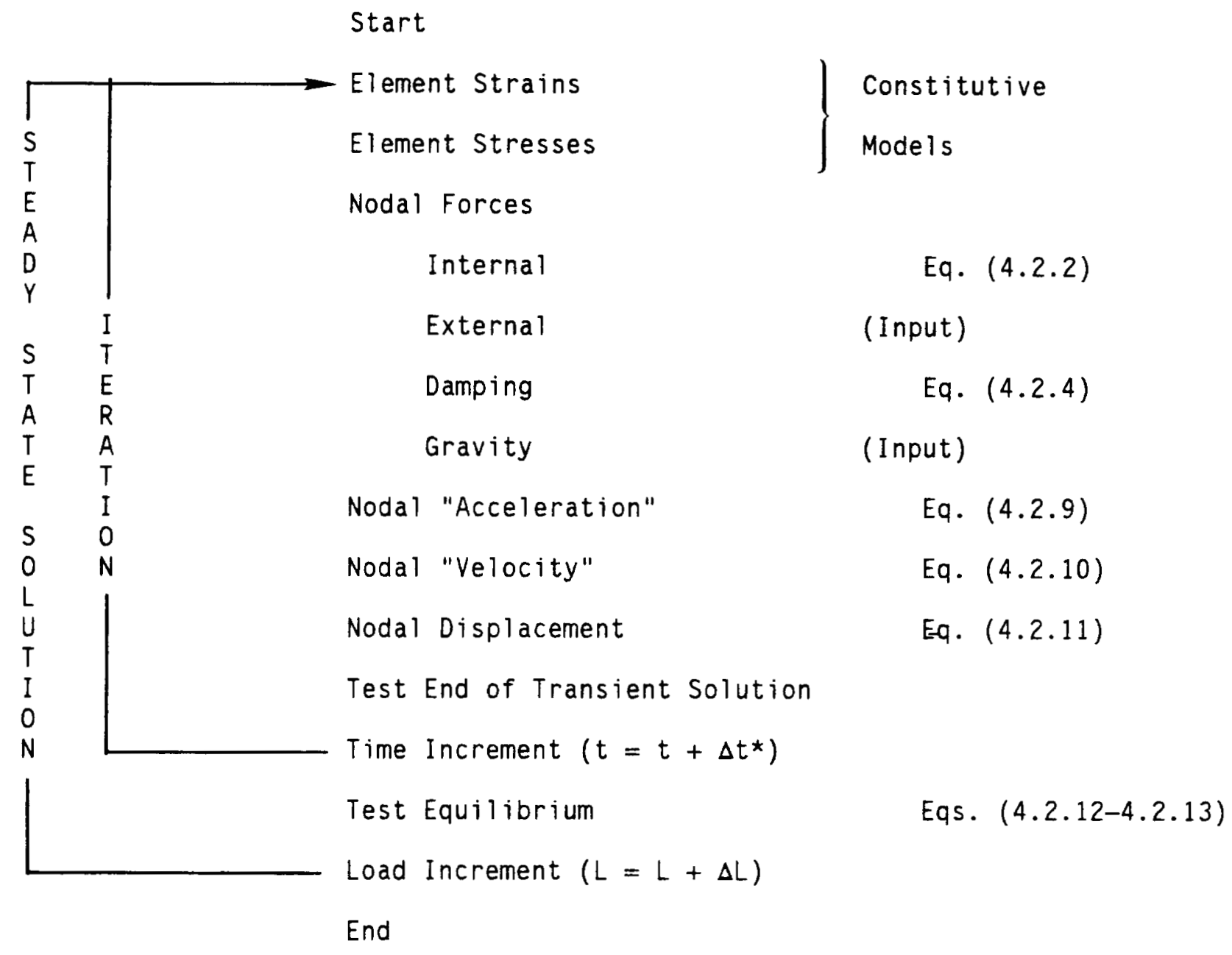

*For problems involving dynamic relaxation $\Delta t$ is used as an iteration parameter, not a time increment.

Figure 4.2.2 Sequence of Calculations Using Dynamic Relaxation 


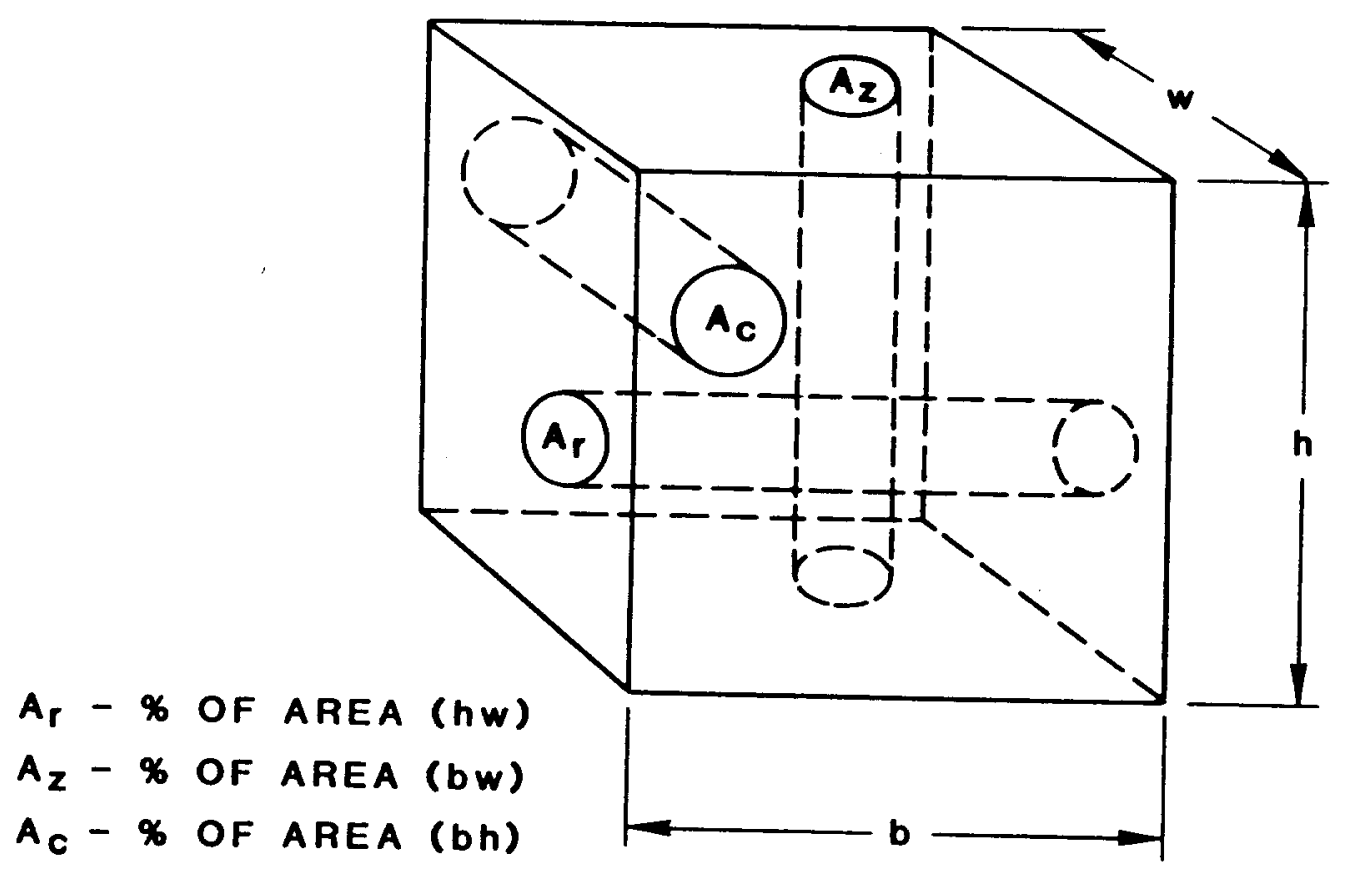

Figure 4.2.3 Representation of Reinforcement in Concrete of the Axisymmetric Continuum Element 


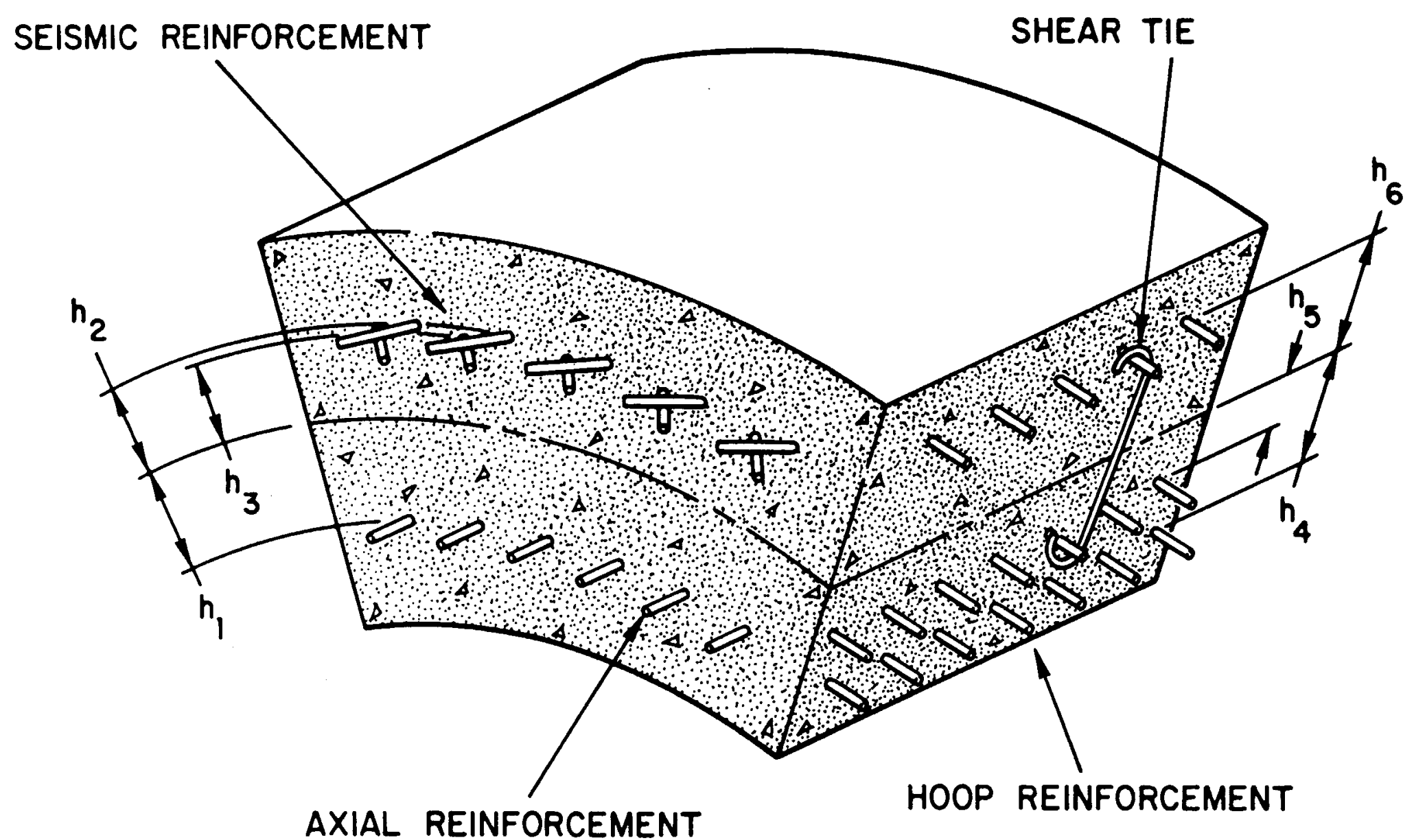

Figure 4.2.4 Representation of Reinforcement in Concrete of the Axisymmetric Shell Element 


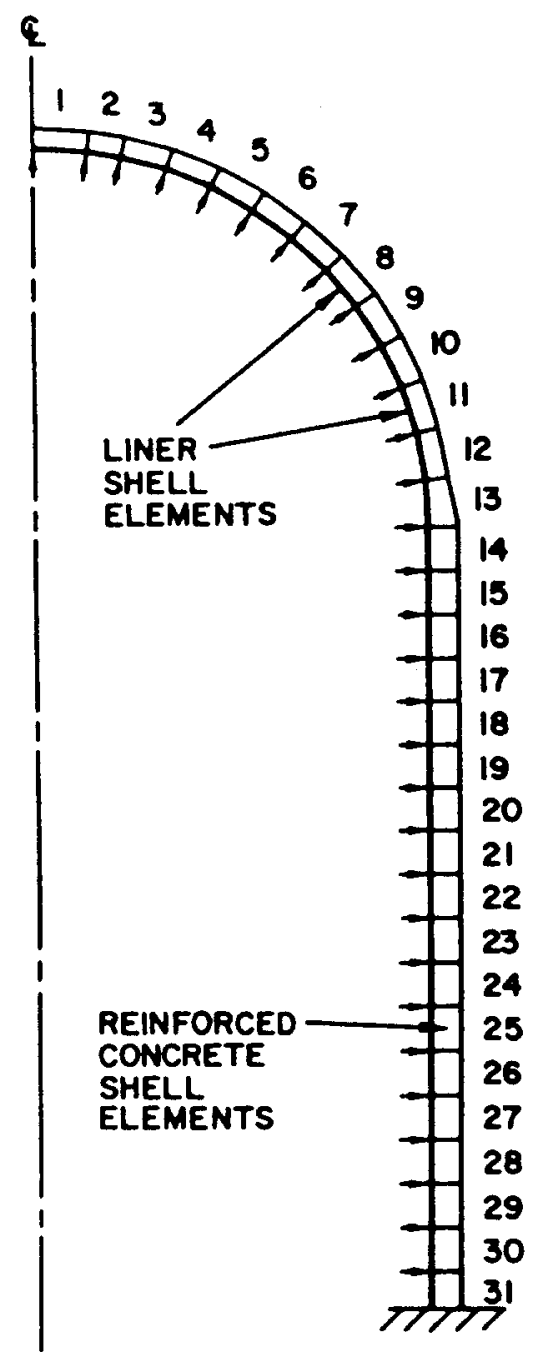

ELEMENT NUMBERS

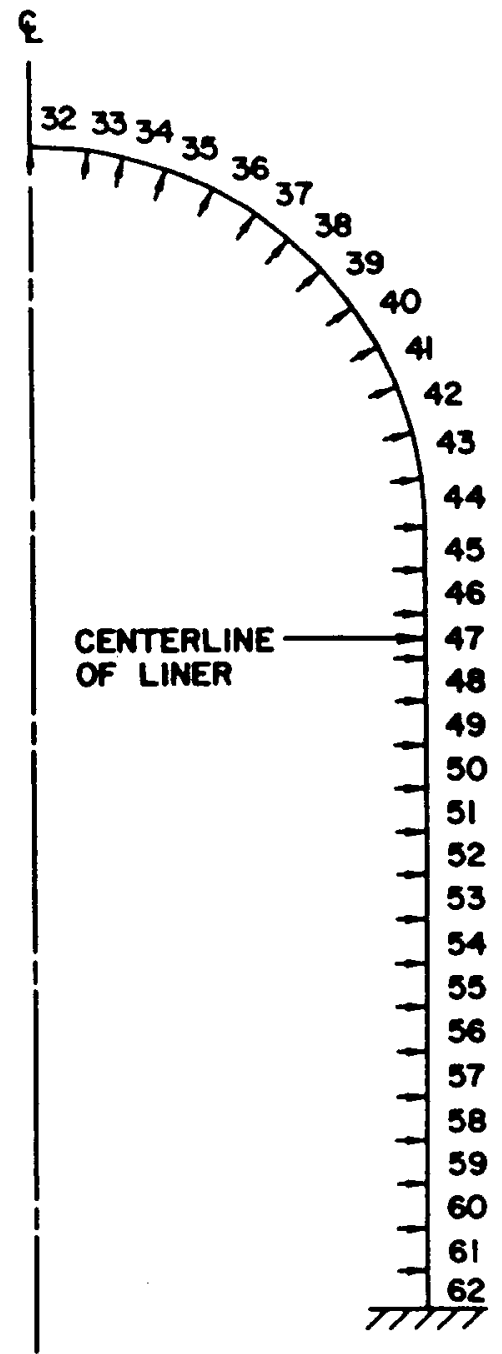

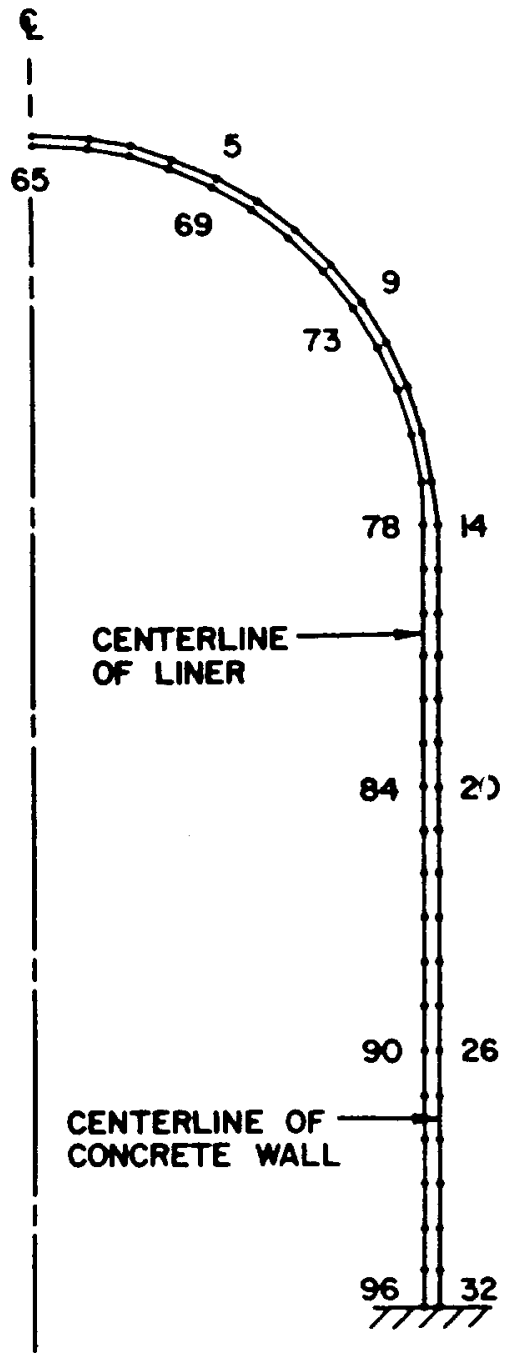

NODE NUMBERS

Figure 4.2.5 Discretization of Shell Model 


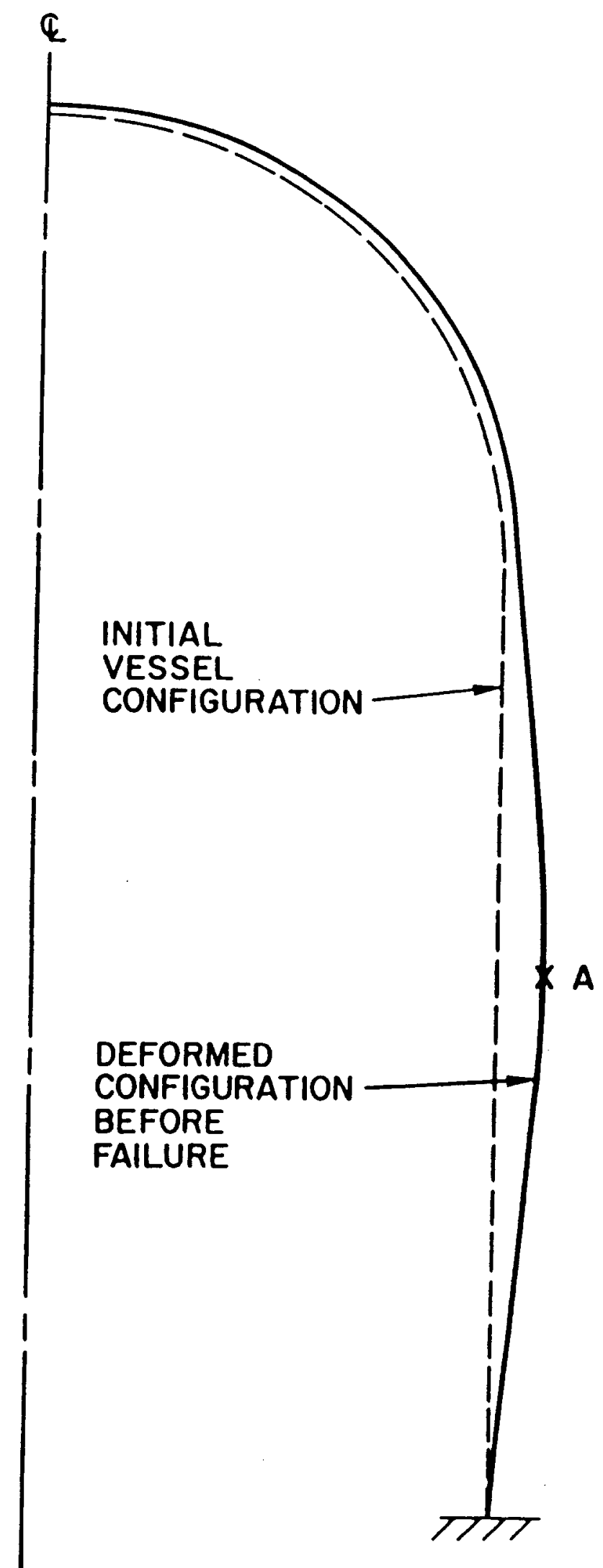

Figure 4.2.6 Deformation of Vessel at Impending Failure

$-104-$ 


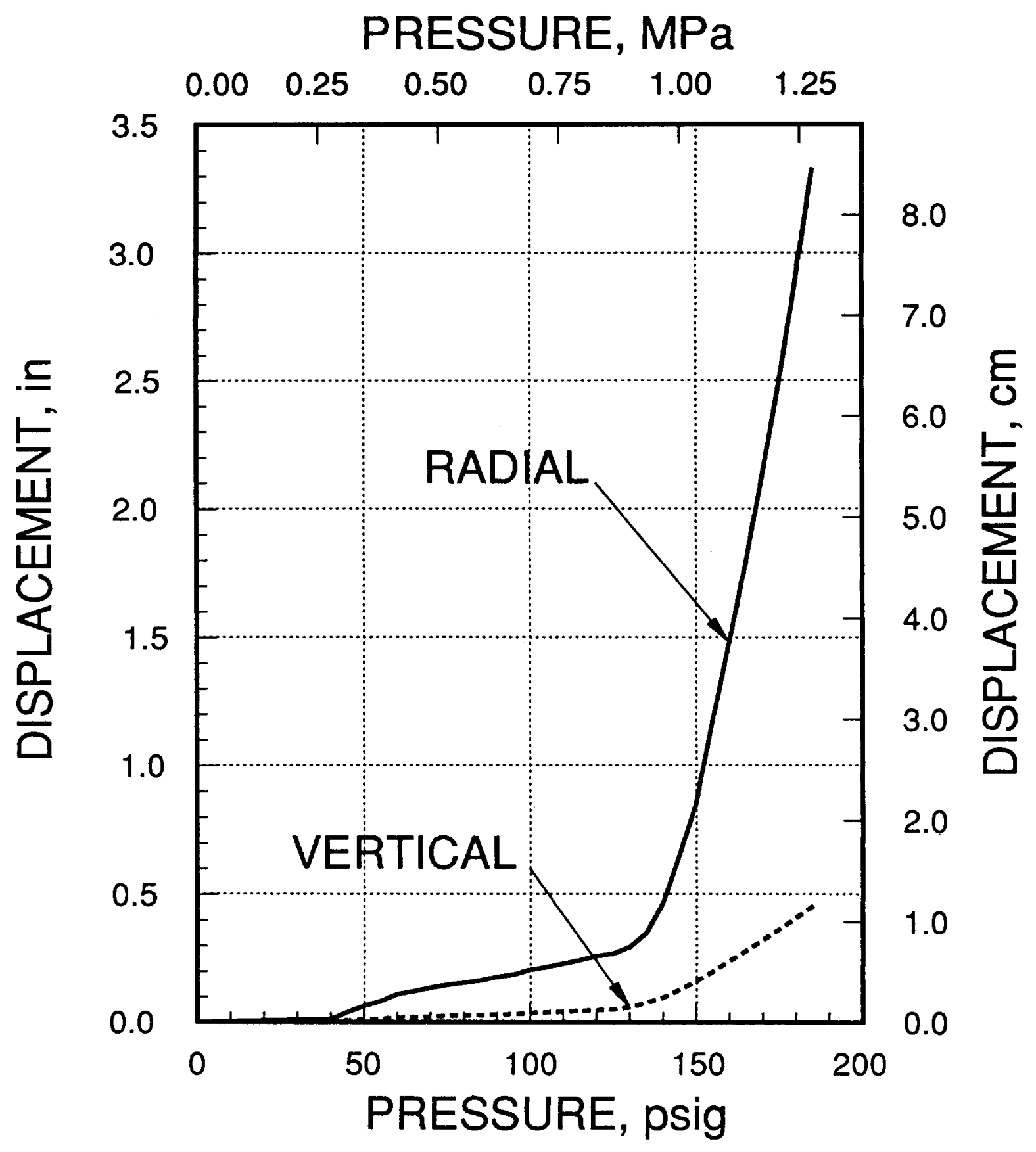

Figure 4.2.7 Radial Displacement of Liner and Vertical Displacement Relative to Cylinder Base at EL 6'-0" 


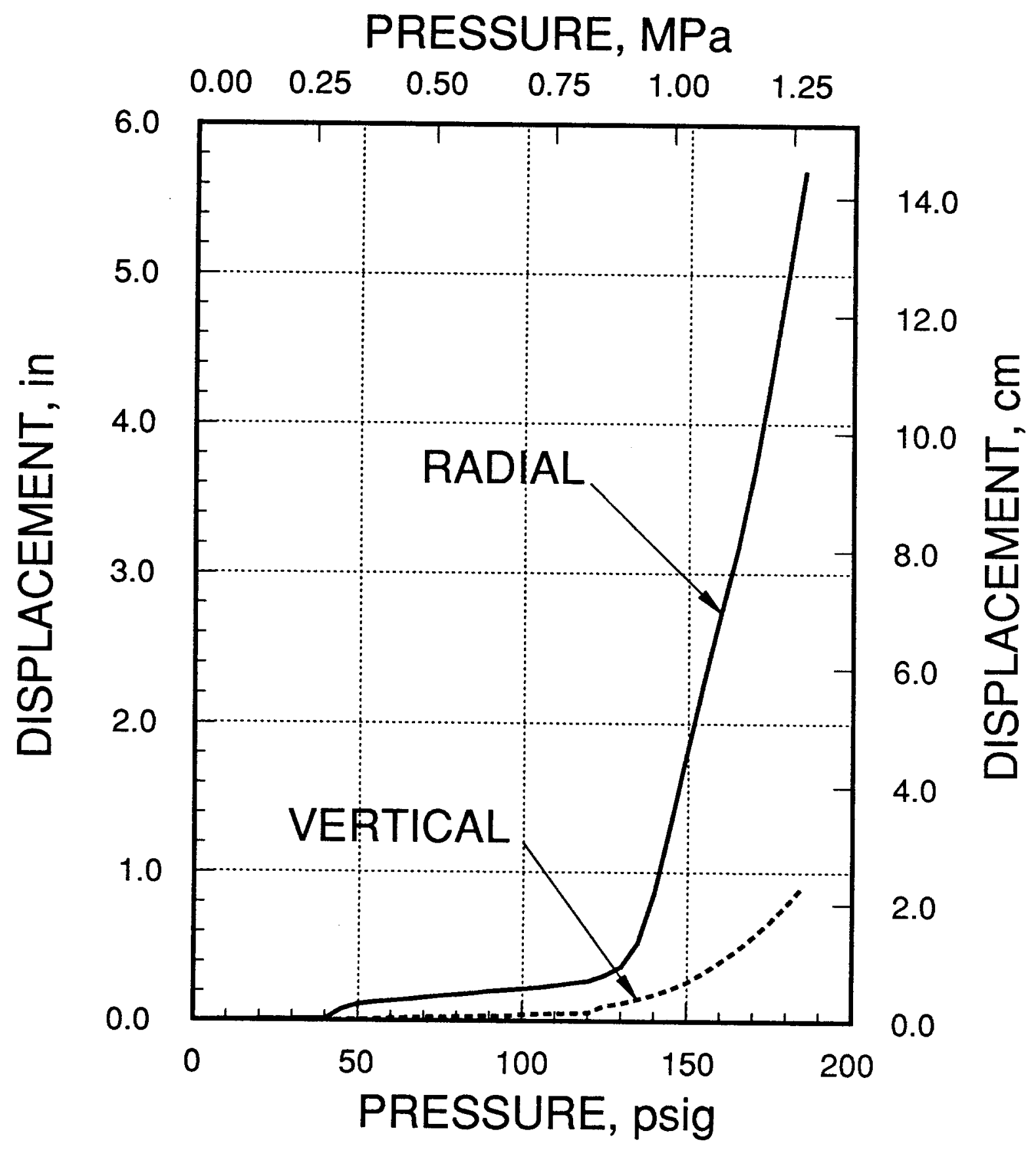

Figure 4.2.8 Radial Displacement of Liner and Vertical Displacement Relative to Cylinder Base at EL 11'-0" 


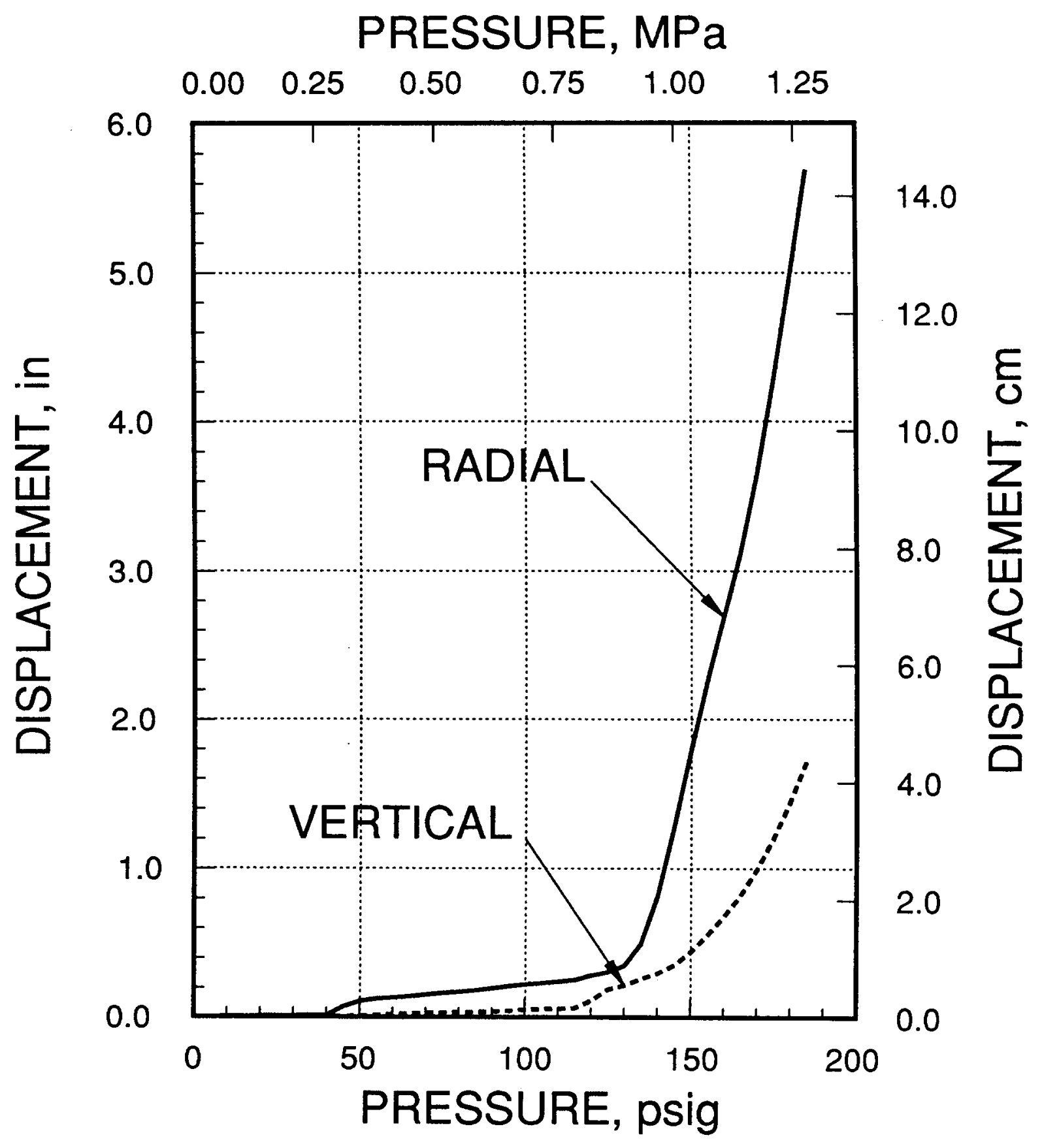

Figure 4.2.9 Radial Displacement of Liner and Vertical Displacement Relative to Cylinder Base at EL 18'-0" 


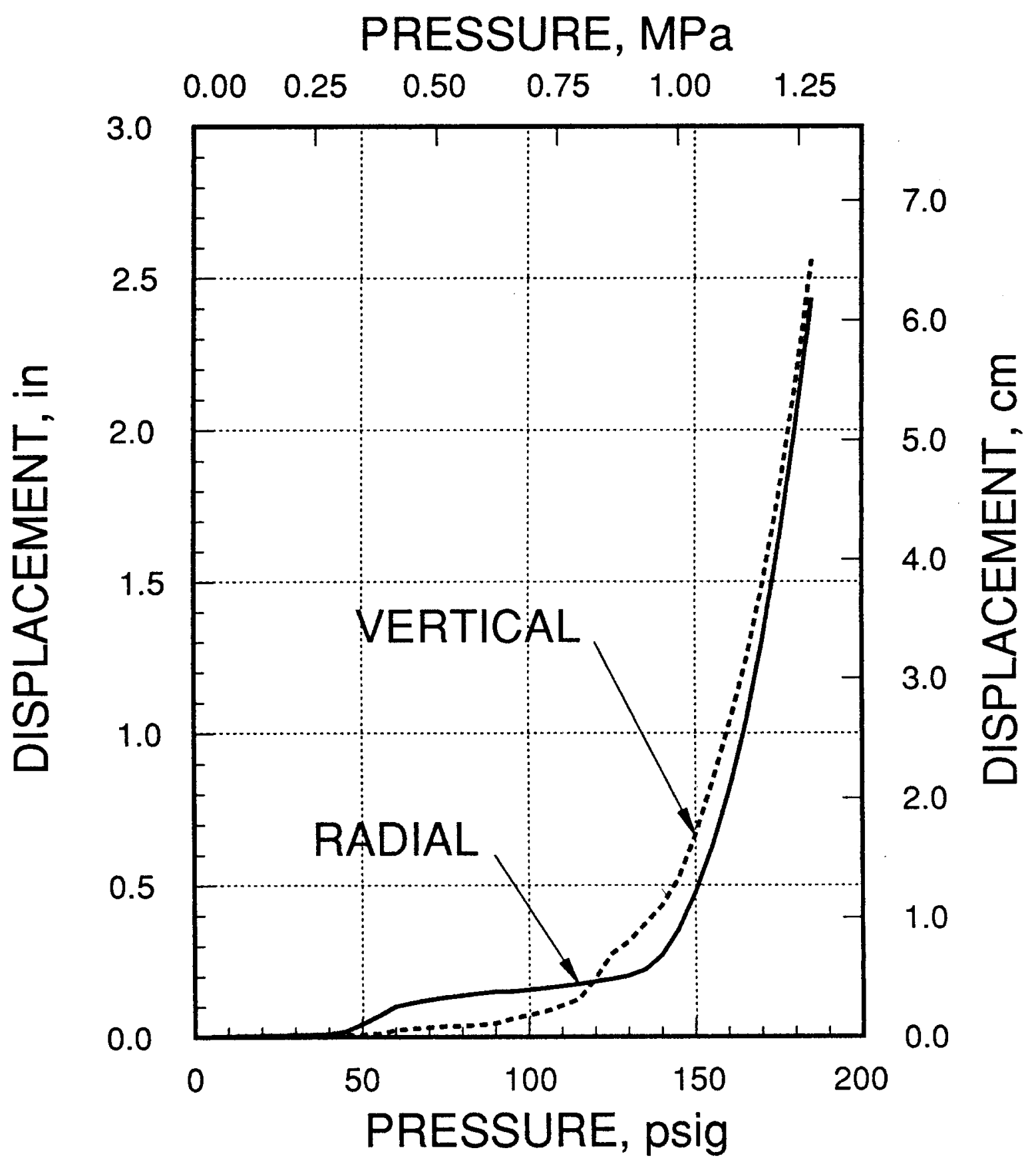

Figure 4.2.10 Radial Displacement of Liner and Vertical Displacement Relative to Cylinder Base at EL 24'-0" 


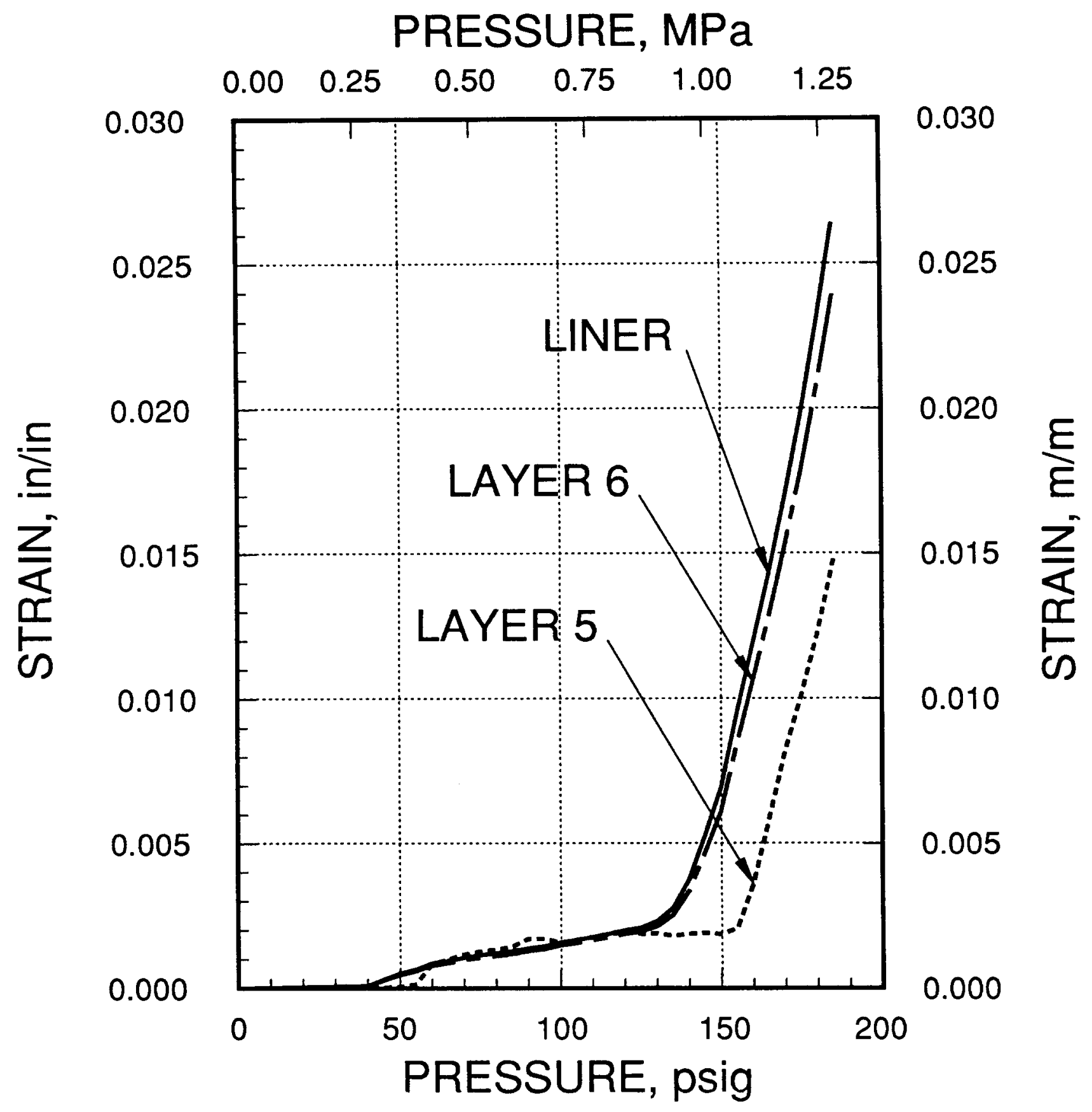

Figure 4.2.11 Maximum Principal Strain on Inside Liner Surface, Axial Strain in Layer 5 Meridional Rebar, and Axial Strain in Layer 6 Hoop Rebar at EL 6'-9" 


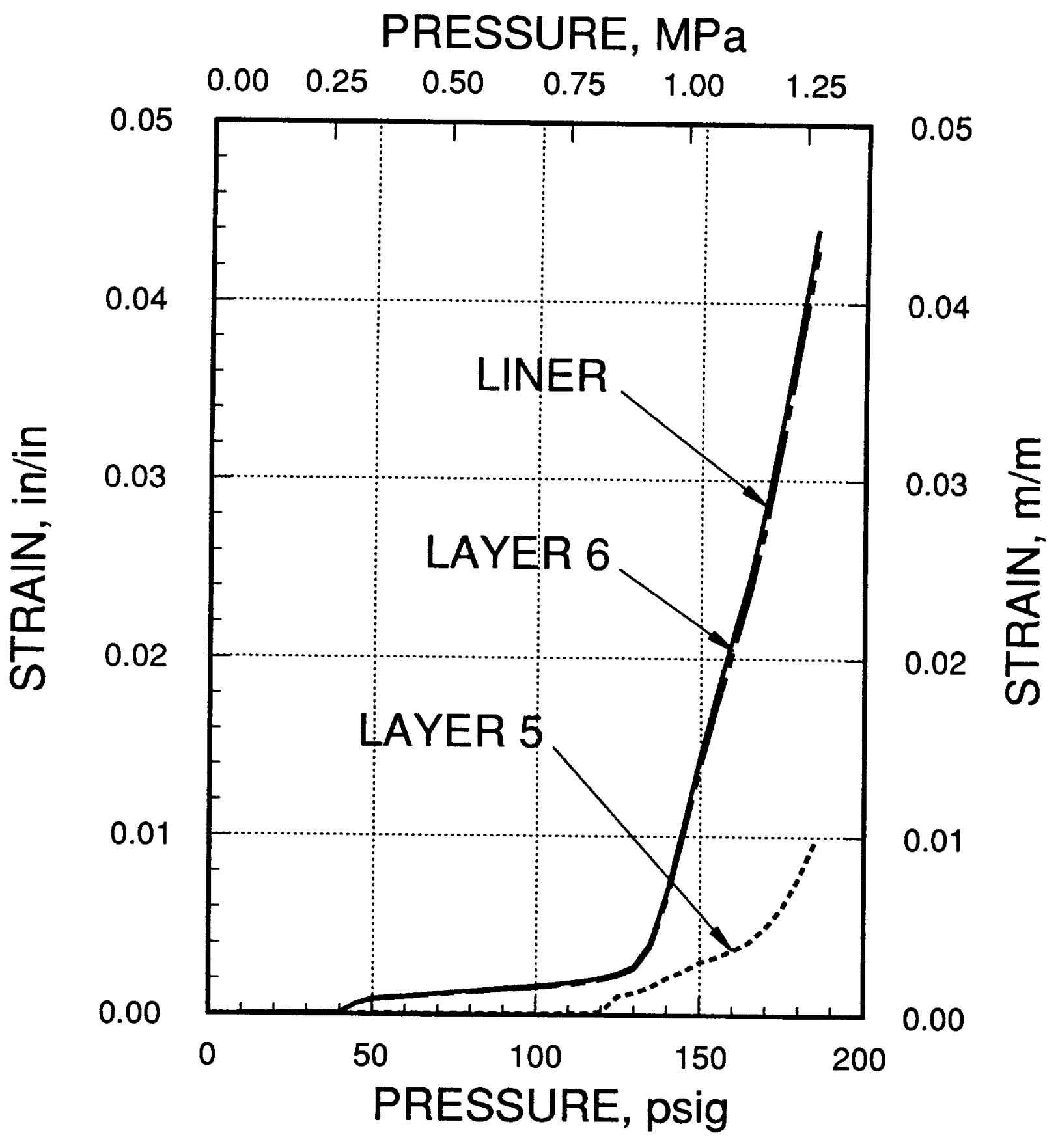

Figure 4.2.12 Maximum Principal Strain on Inside Liner Surface, Axial Strain in Layer 5 Meridional Rebar, and Axial Strain in Layer 6 Hoop Rebar at EL 13'-9" 


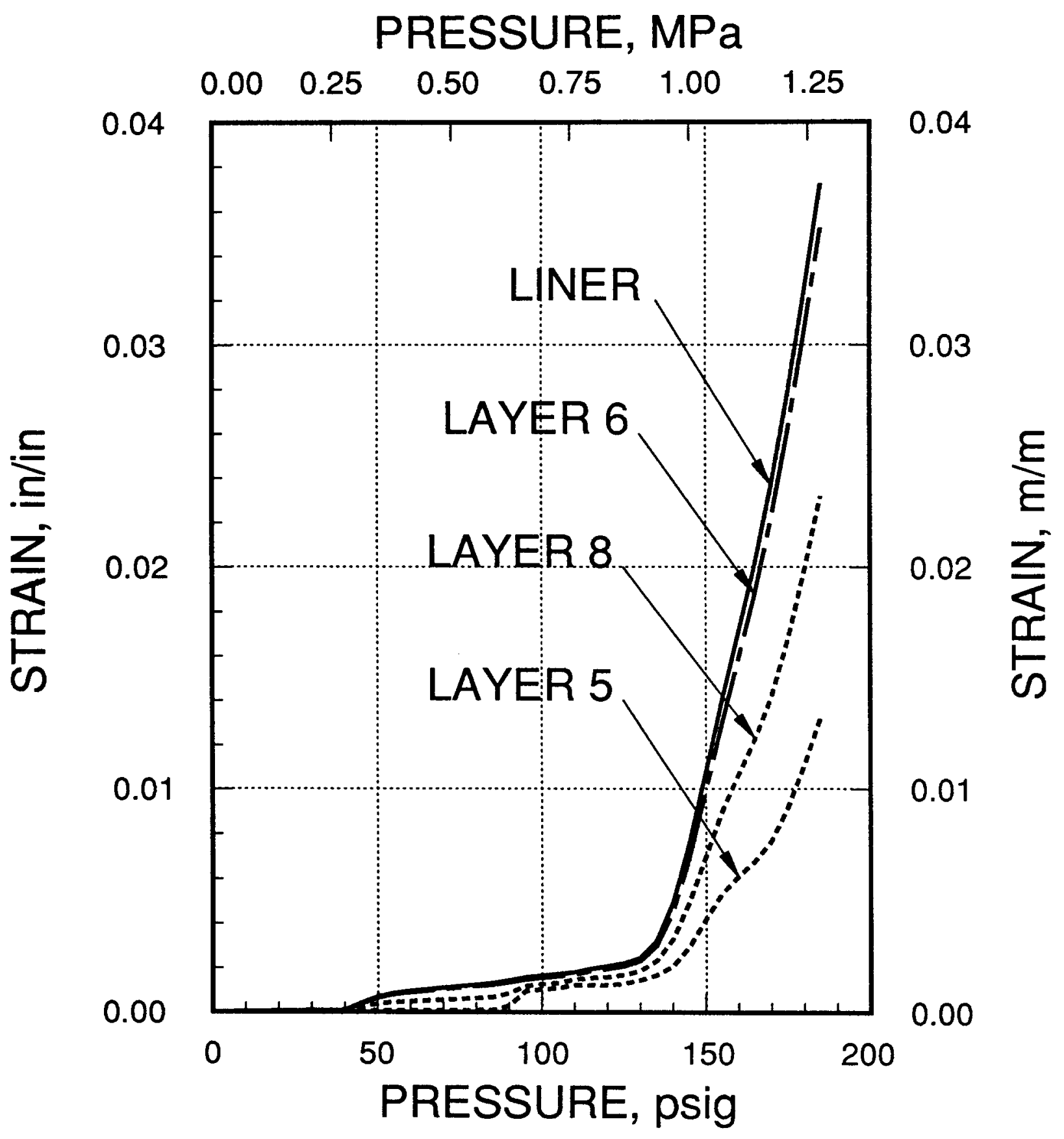

Figure 4.2.13 Maximum Principal Strain on Inside Liner Surface, Axial Strain in Layer 5 Meridional Rebar, Axial Strain in Layer 6 Hoop Rebar,

and Axial Strain in Layer 8 Seismic Rebar at EL 20'-0" 


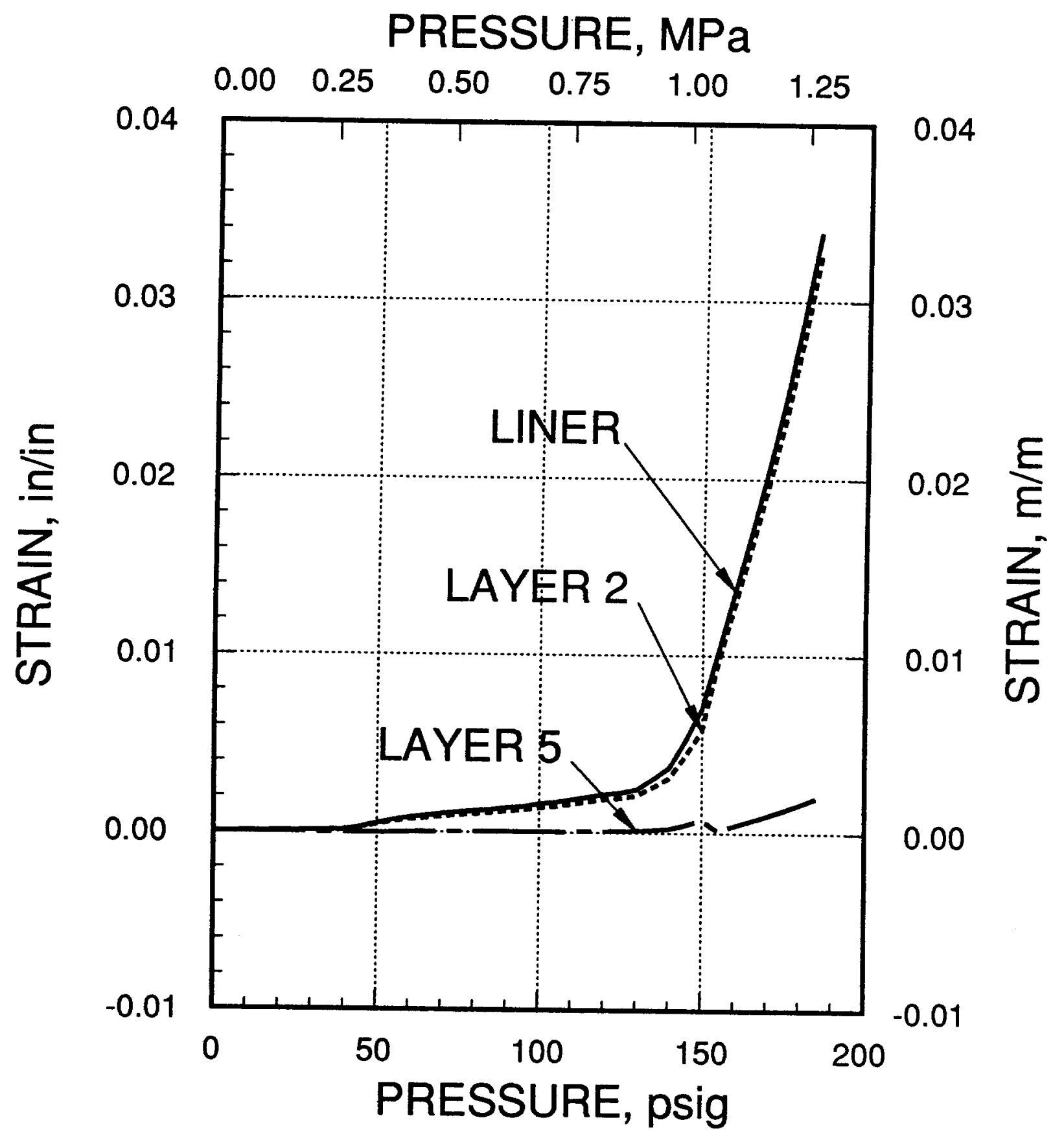

Figure 4.2.14 Maximum Principal Strain on Inside Liner Surface, Axial Strain in Layer 2 Meridional Rebar, and Axial Strain in Layer 5 Meridional Rebar at EL 2'-0.8" 


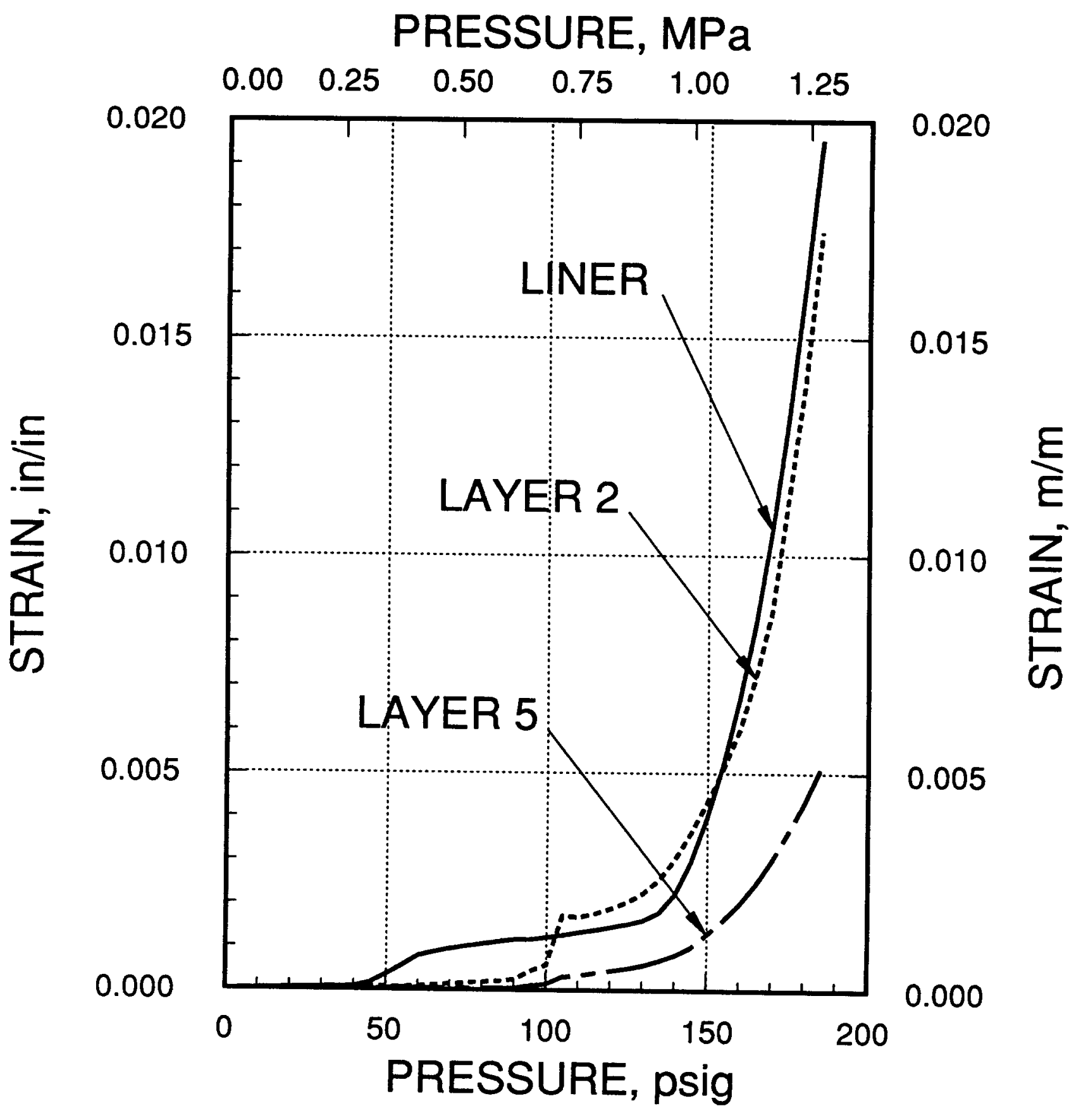

Figure 4.2.15 Maximum Principal Strain on Inside Liner Surface, Axial Strain in Layer 2 Meridional Rebar, and Axial Strain in Layer 5 Meridional Rebar at EL 23'-11" 


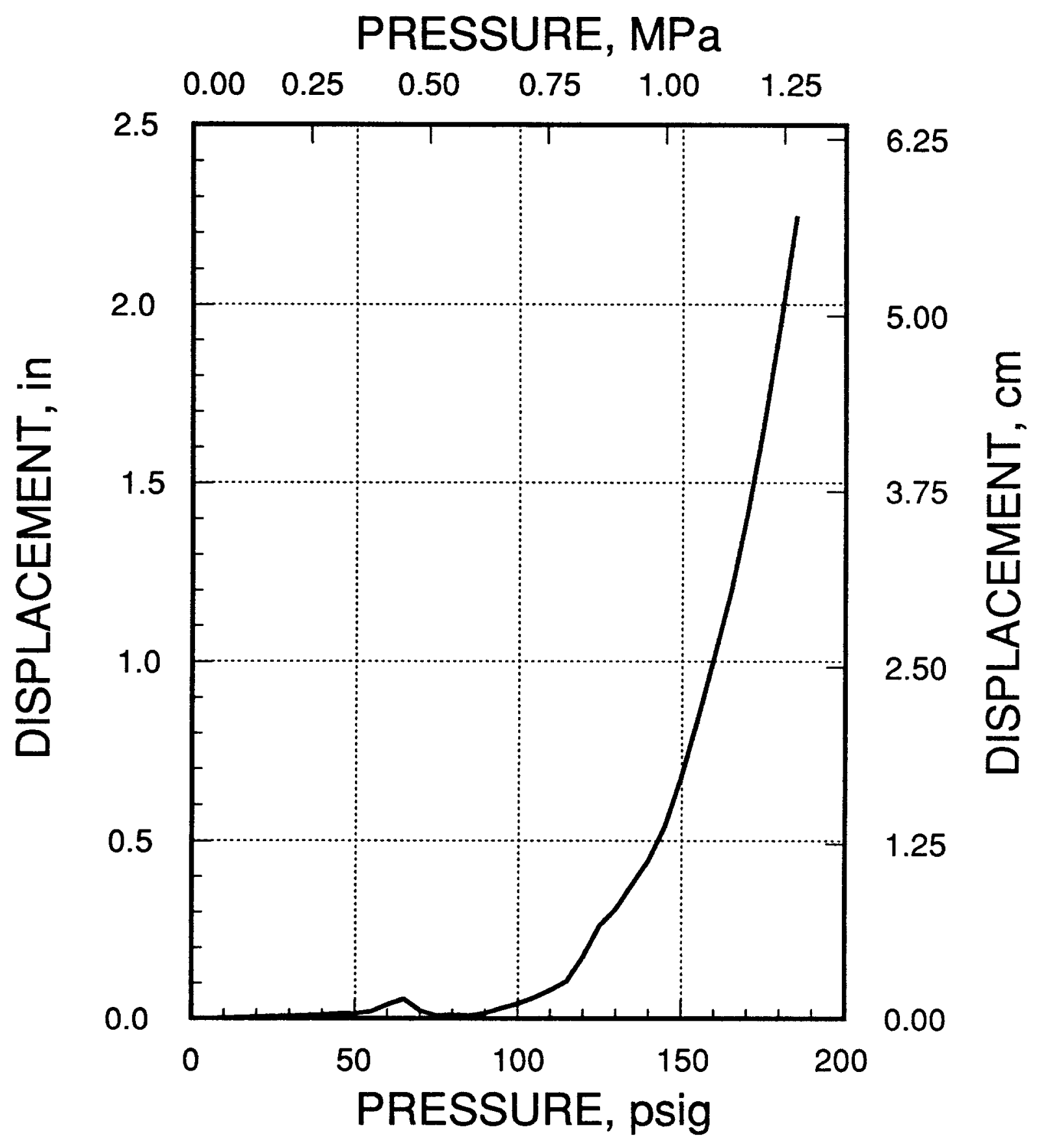

Figure 4.2.16 Vertical Displacement of Liner at Dome Apex 


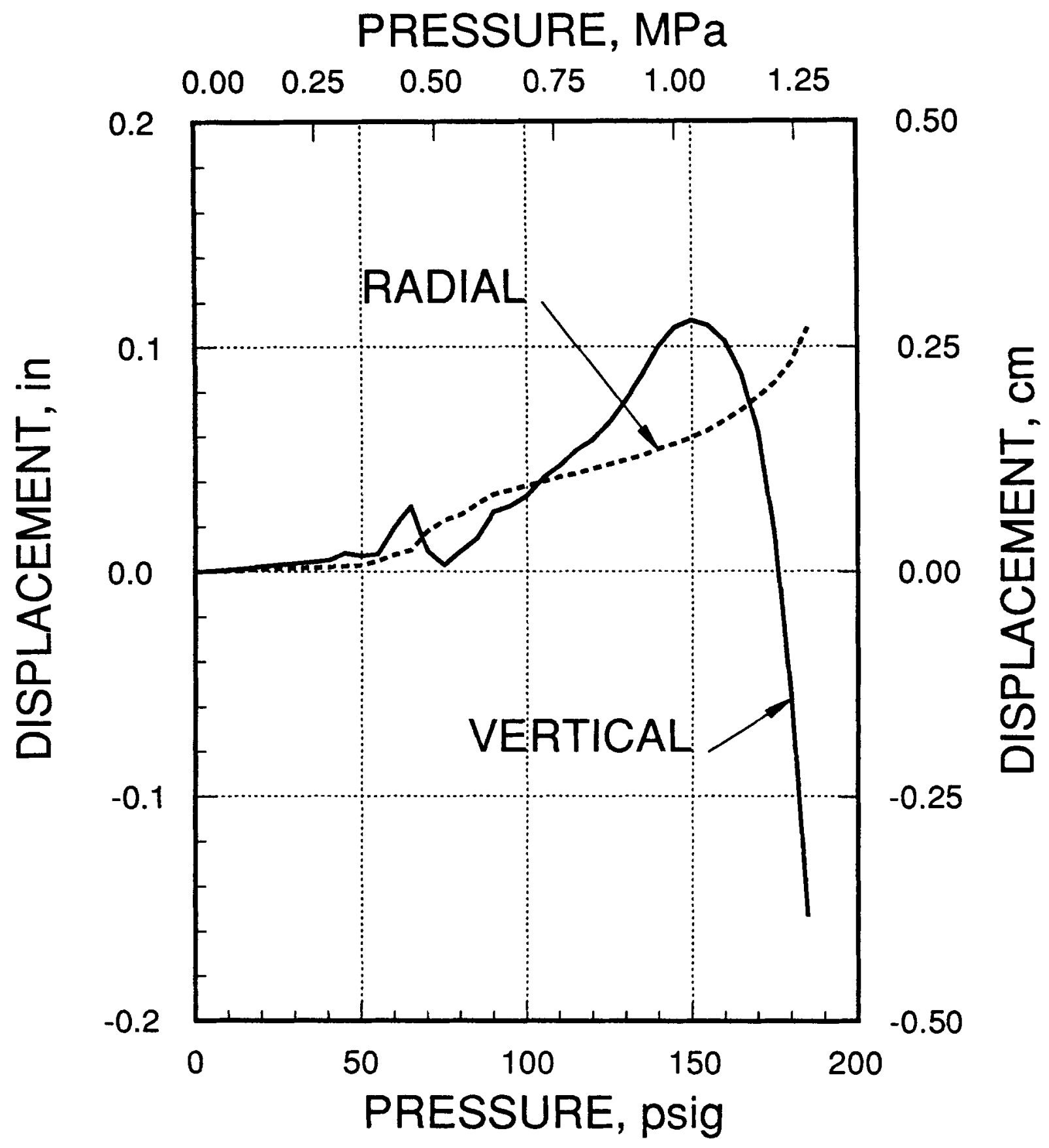

Figure 4.2.17 Vertical Displacement of Liner at $\phi=70^{\circ}$ Relative to Springline and Horizontal Displacement of Liner 


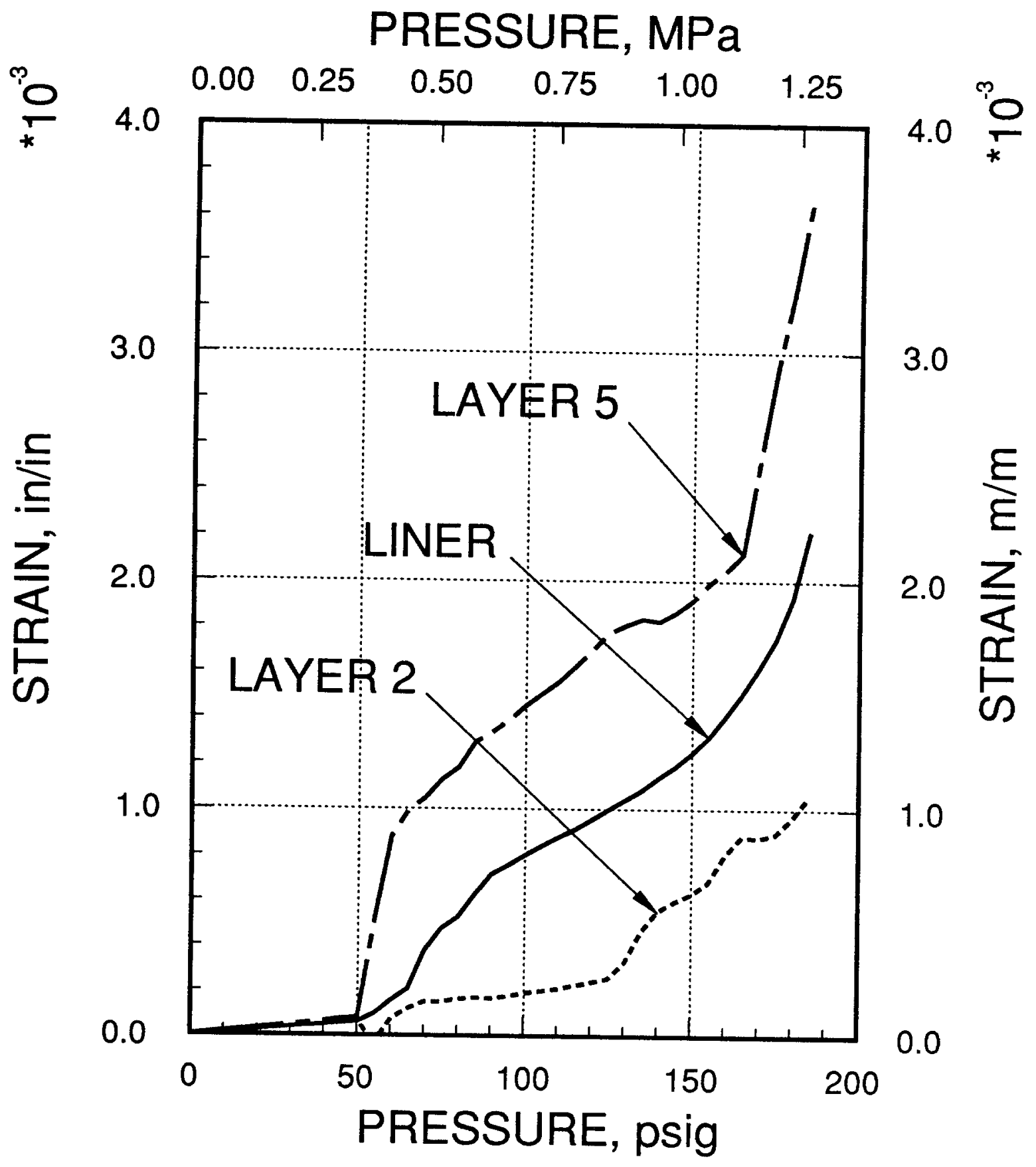

Figure 4.2.18 Maximum Principal Strain on Inside Liner Surface, Axial Strain in Layer 2 Meridional Rebar, and Axial Strain in Layer 5 Meridional Rebar at $\phi=72^{\circ}$ 


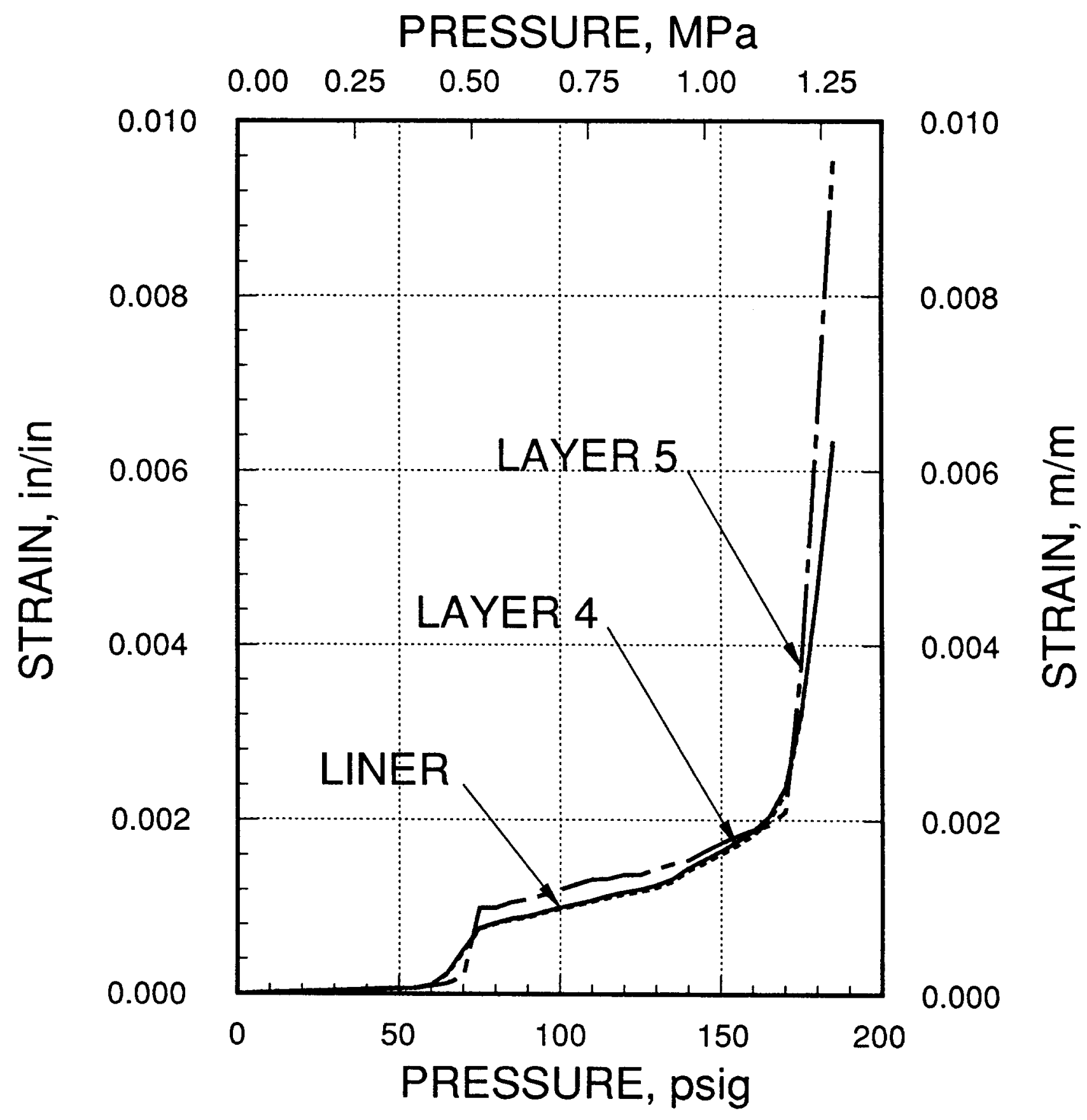

Figure 4.2.19 Maximum Principal Strain on Inside Liner Surface, Axial Strain in Layer 4 Hoop Rebar, and Axial Strain in Layer 5 Meridional Rebar at $\phi=37^{\circ}$ 


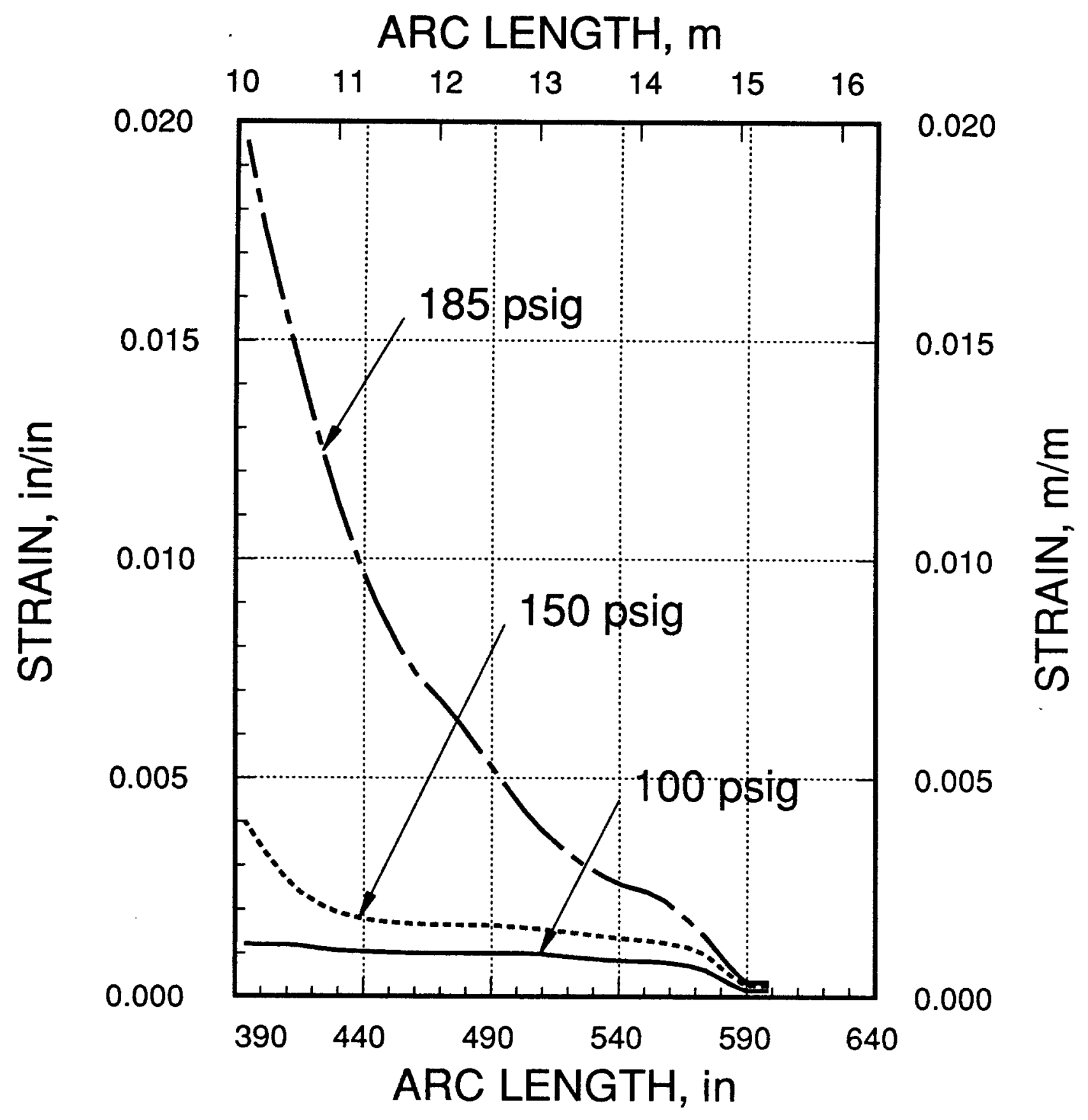

Figure 4.2.20 Maximum Principal Strain on Inside Surface of Liner in Springline and Dome Region 


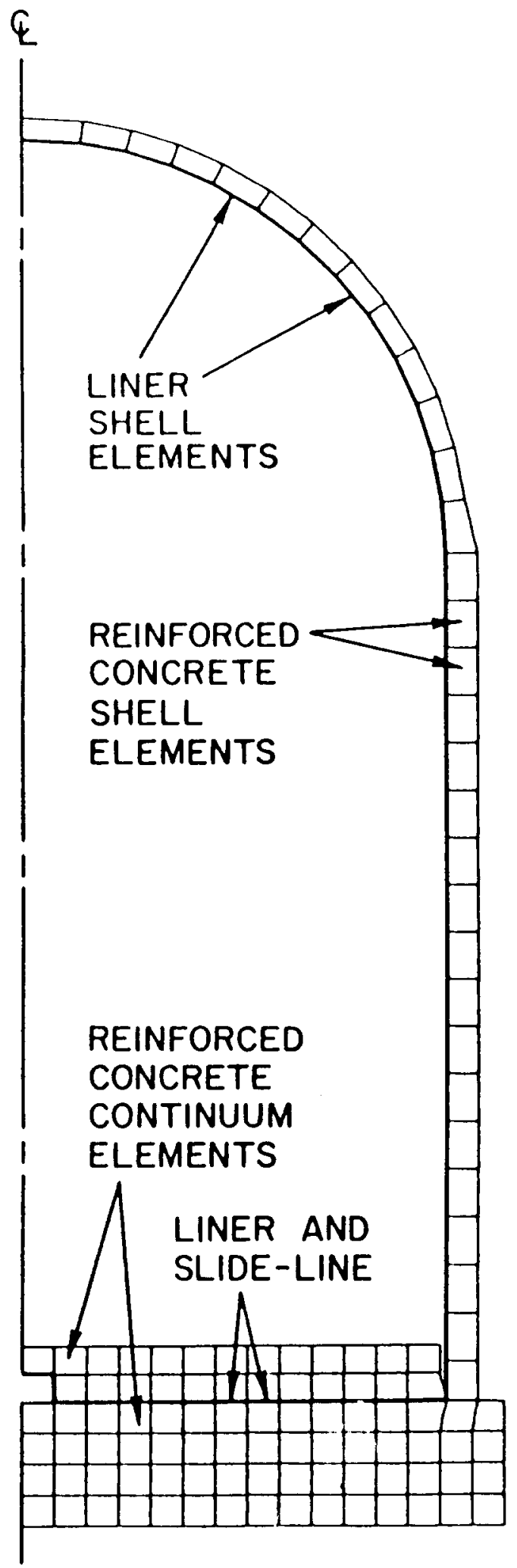

Figure 4.2.21 Discretization of Shell and Slab Model 


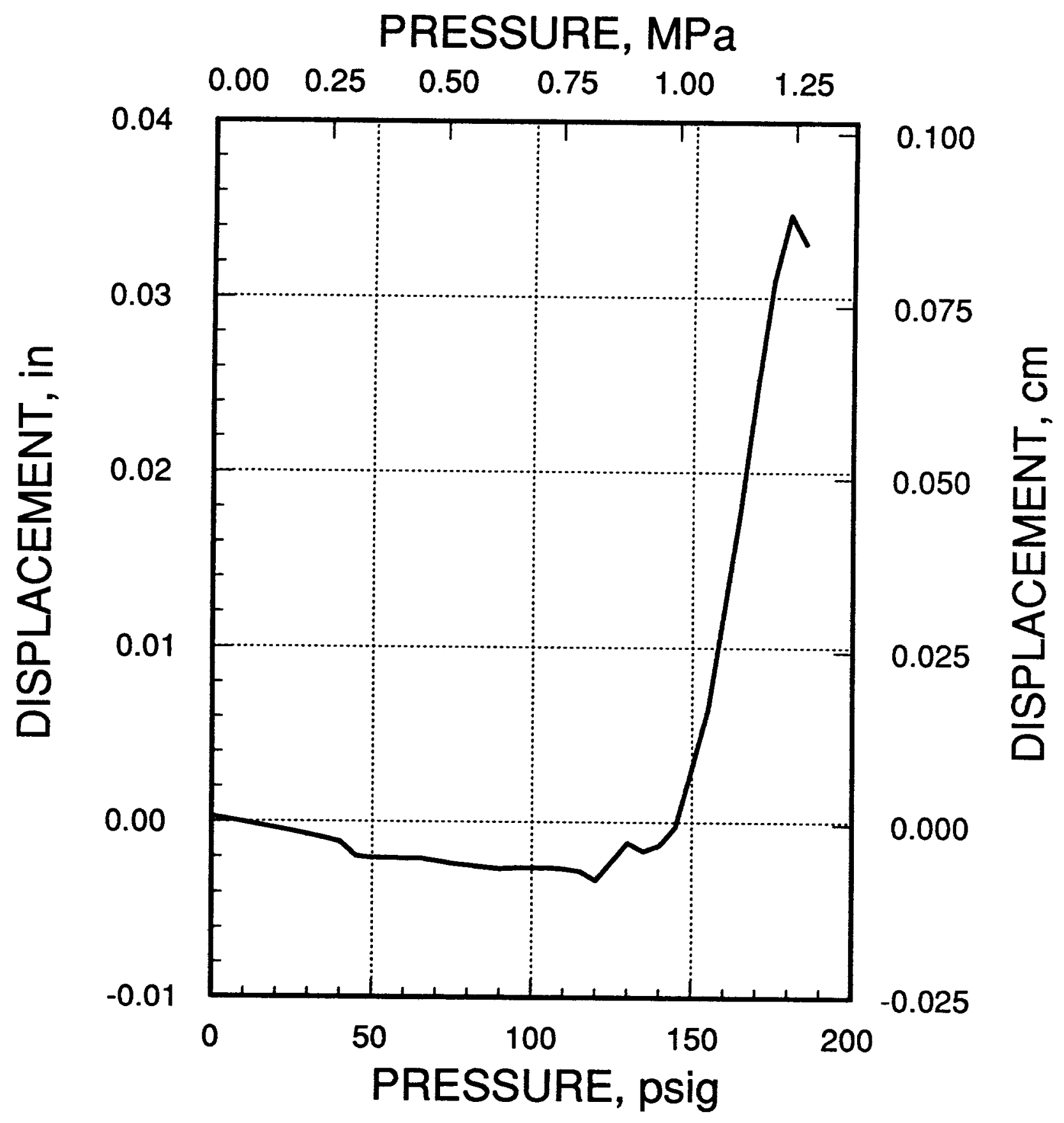

Figure 4.2.22 Radial Displacement of Liner at EL $2^{\prime}-2$ " 


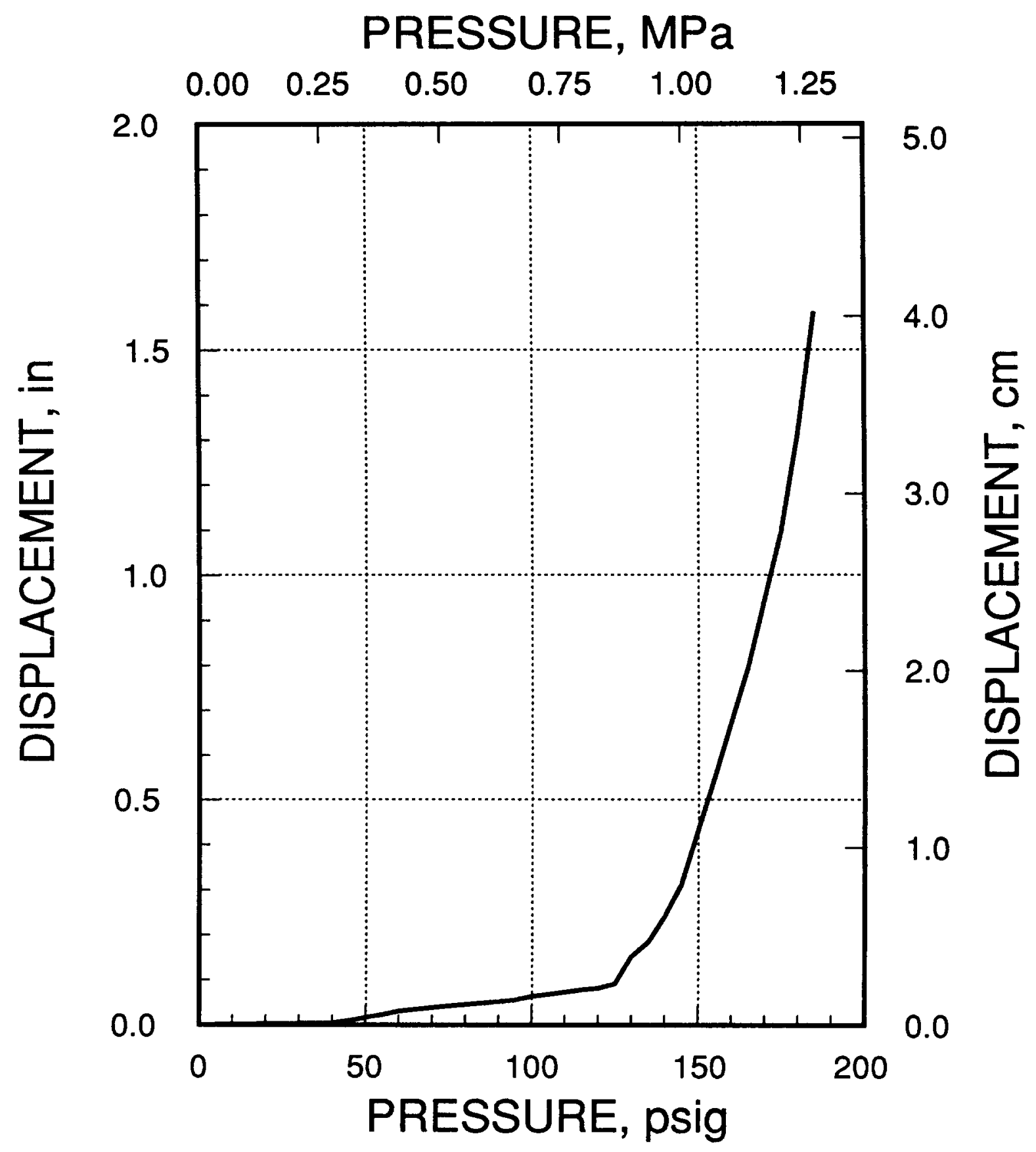

Figure 4.2.23 Radial Displacement of Liner at EL $4^{\prime}-1$ " 


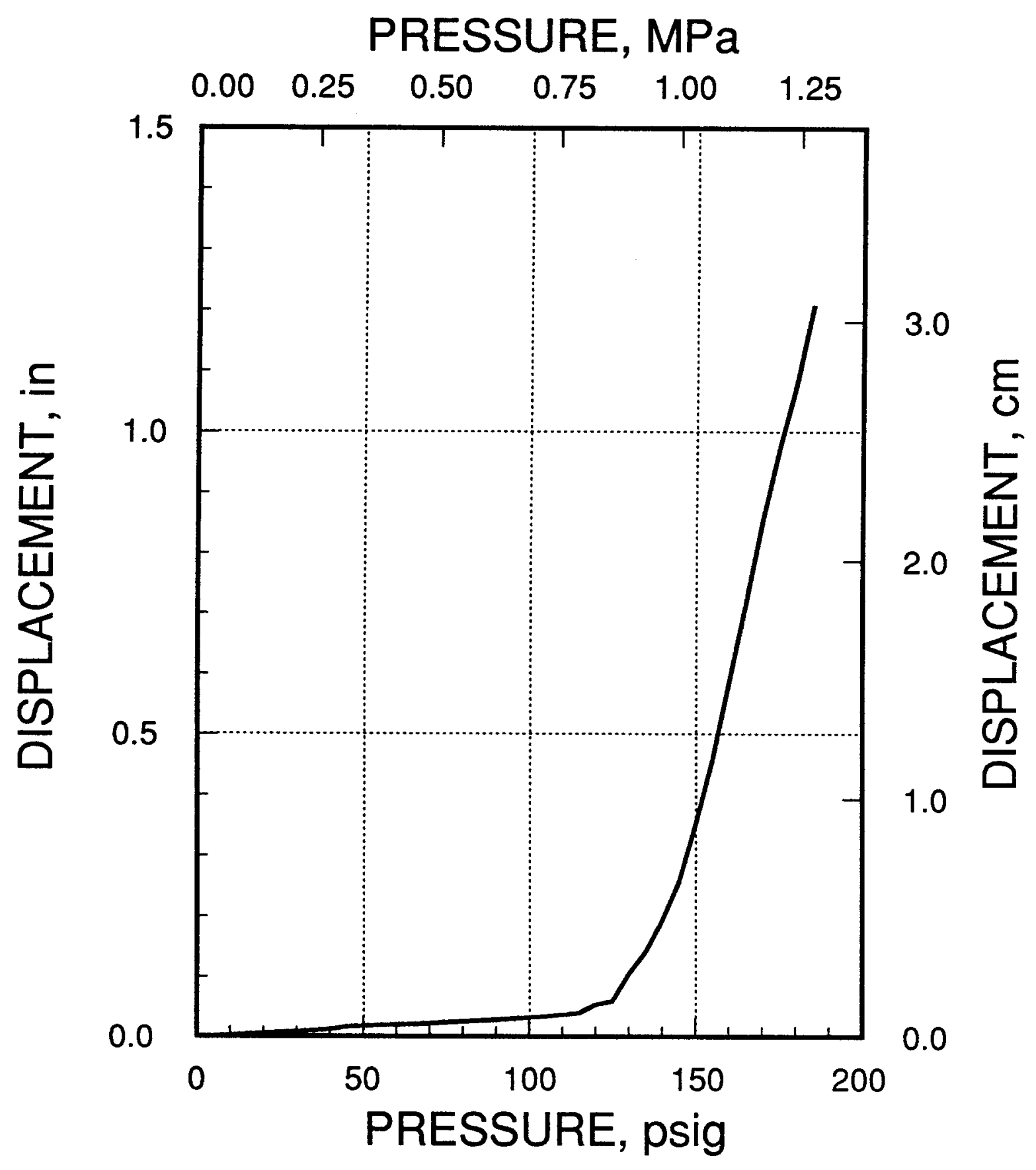

Figure 4.2.24 Vertical Displacement (Uplift) Relative to Center of Basemat at EL 2'-0" 


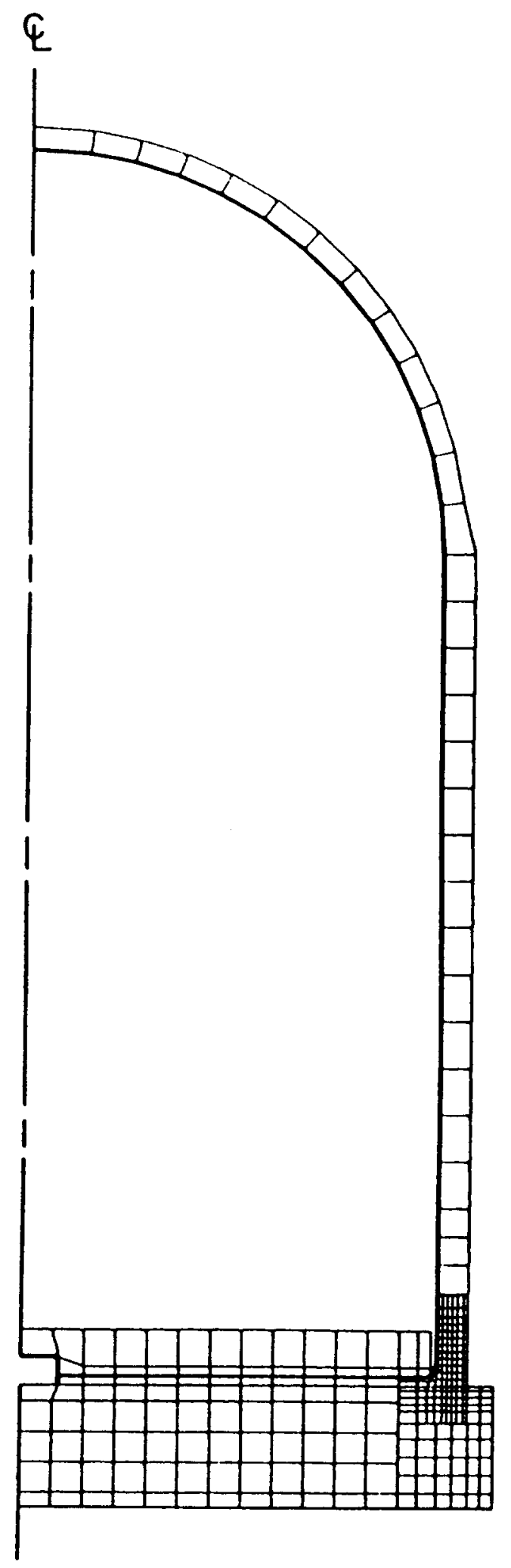

Figure 4.2.25 Discretization of Refined Shell and Slab Model 


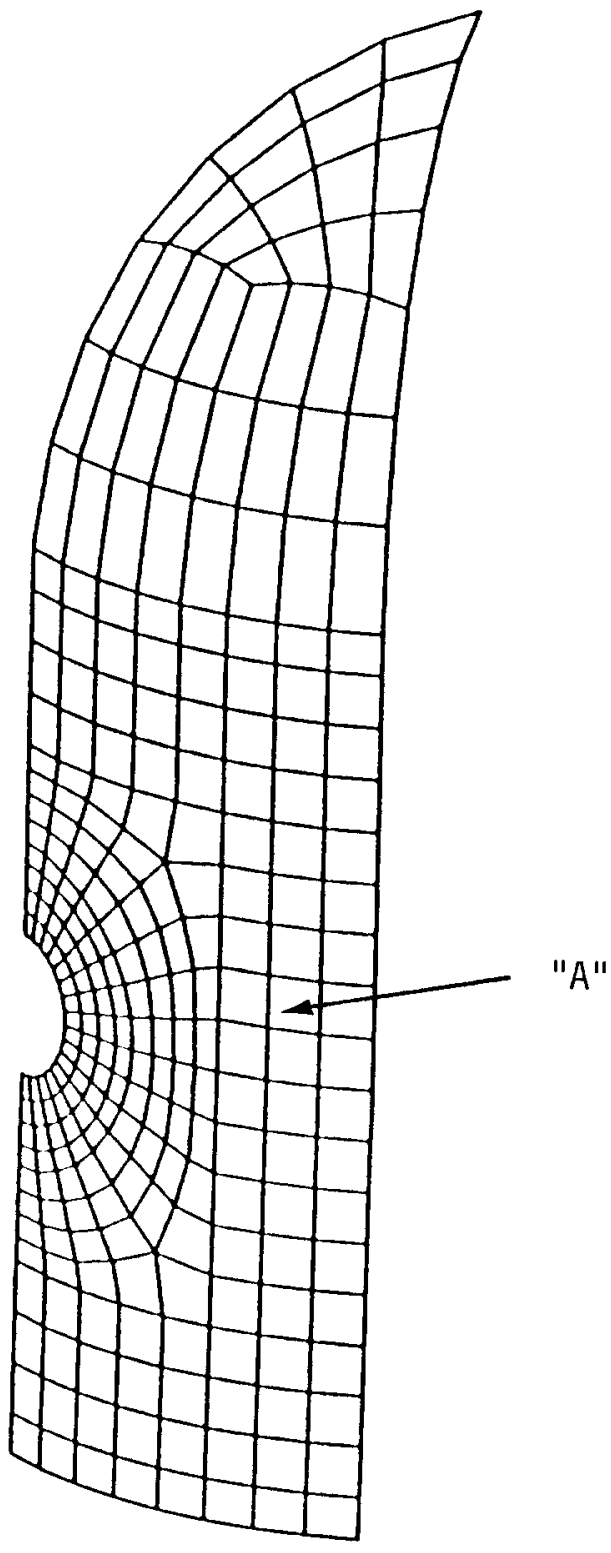

Figure 4.2.26 Finite Element Mesh of 50 Degree Segment Model of $1 / 6 \mathrm{th}$ Scale Concrete Containment Test

$-124-$ 


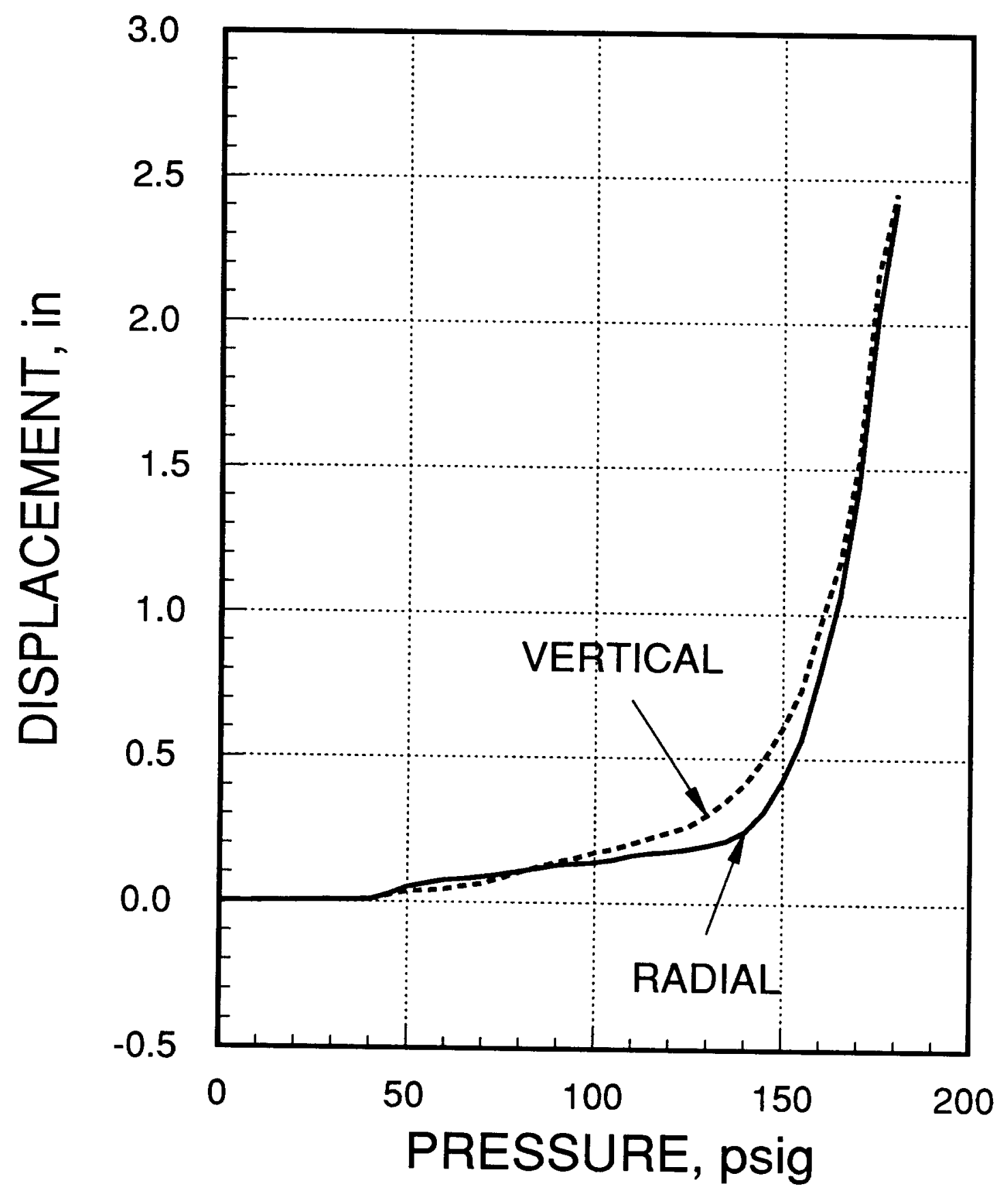

Figure 4.2.27 Radial Displacement of Liner and Vertical Displacement Relative to Cylinder Base at $E L 24^{\prime}-0^{\prime \prime}$ and $\theta=180^{\circ}$ 


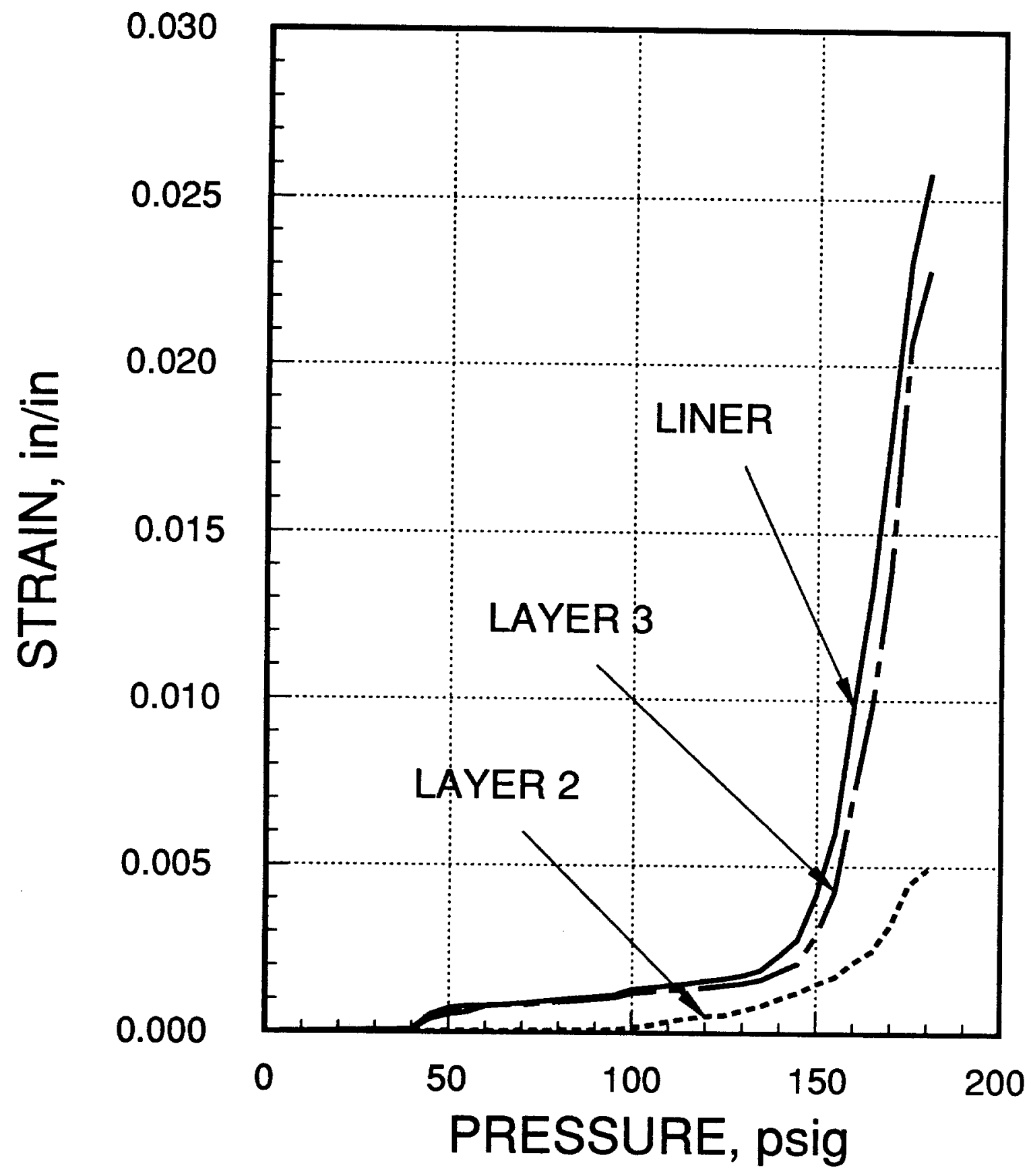

Figure 4.2.28 Maximum Principal Strain on Inside Liner Surface and Axial Strains of Rebar Layers 2 and 3 at EL $13^{\prime}-0^{\prime \prime}$ and $\theta=158^{\circ}$ 


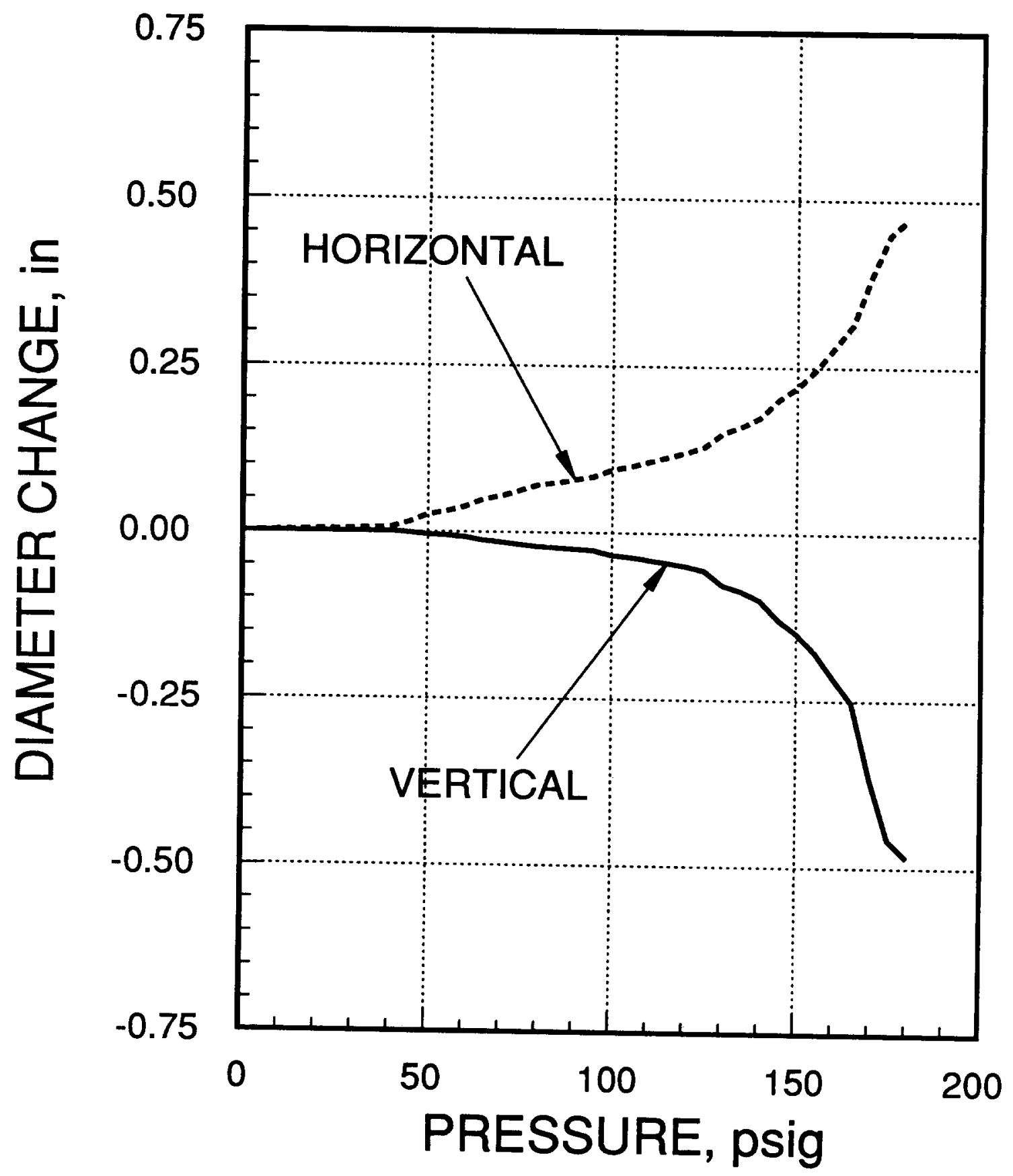

Figure 4.2.29 Change in Sleeve Horizontal and Vertical Diameters of Equipment Hatch B 


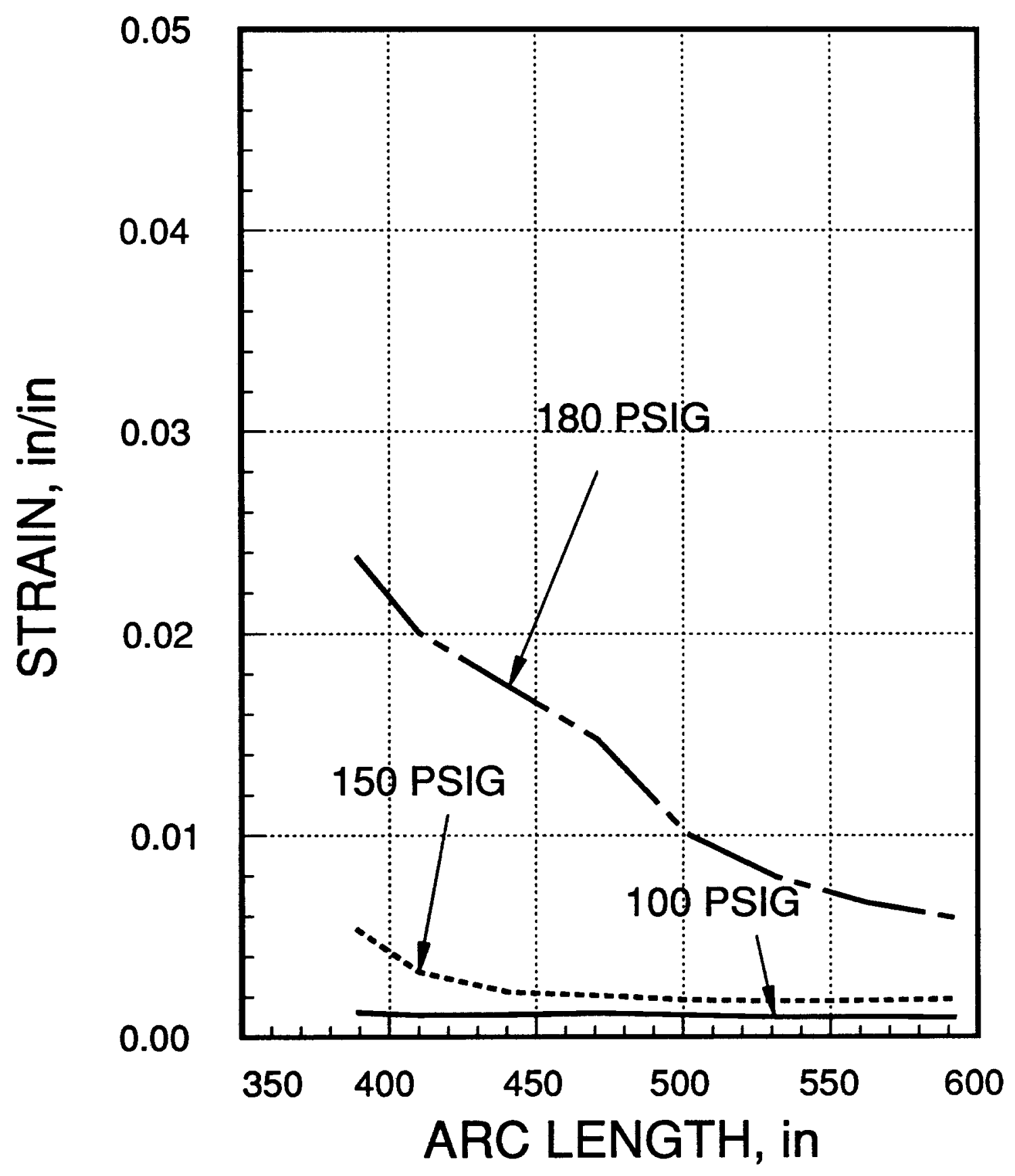

Figure 4.2.30 Maximum Principal Strain on Inside Surface of Liner in Springline and Dome Region 


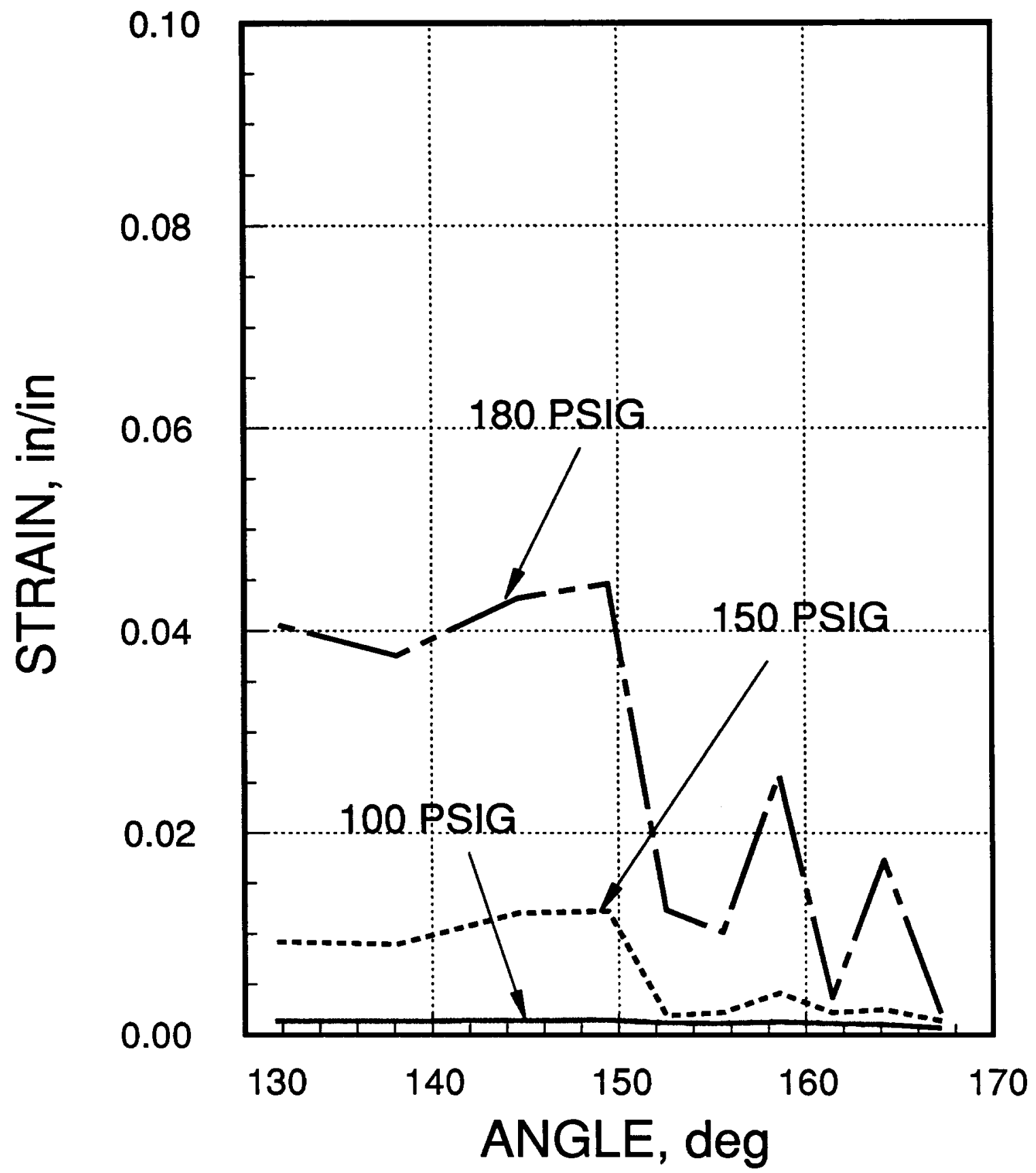

Figure 4.2.31 Maximum Principal Strain on Inside Liner Surface at EL 13'-0" Adjacent to Equipment Hatch 


\subsection{Electric Power Research Institute}

Section 4.3 was prepared by ANATECH International Corporation. The principal investigators were R. A. Dameron, R. S. Dunham, Y. R. Rashid, and M. F. Sullaway. The work was conducted for the Nuclear Pawer Division of the Electric Power Research Institute, where the project manager was $H$. T. Tang.

\subsubsection{Summary}

This report presents the results of pretest analyses of the structural behavior of the Sandia 1:6 Scale Reinforced Concrete Containment Model. This work is part of EPRI's ongoing research to develop test-validated methodology for the prediction of the overpressure behavior of concrete containments. Table 4.3.1 is a summary outlining the predictions, which was requested by SNL.

The approach taken for the 1:6 scale model was first to conduct global axisymmetric analyses to identify critical regions, then to analyze these regions separately in detailed 2-D axisymetric and $r-\theta$ geometries, and finally to select the most likely failure locations and perform detailed local 3-D analyses. In extending the current analysis capabilities to 3-D, many valuable automated procedures were developed for grid generation, postprocessing of results, automated rebar generation, and application of large sets of displacement boundary conditions to the edges of local models.

The 2-D analysis series show that 1 iner strain concentrations are located at the wall basemat juncture, at the springline where liner thickness transition occurs, and near each penetration where the rigid-inclusion effect of the over-reinforced penetration causes severe localized liner strain. The axisymmetric analyses also show that large bending moments are induced in the basemat and that at high pressures a plastic moment hinge forms followed by significant basemat liftoff. The 2-D analysis series thus enabled a preliminary ranking of these potential failure locations and established the framework for the 3-D analysis series. The 3-D series focussed on three-dimensional effects which could not be simulated through axisymmetric or plane analysis, particularly the interaction of hoop and meridional deformations around penetrations. In the local 3-D models hatch deformations and displacement gradients were calculated, and detailed liner strain profiles were developed, thus forming a basis for failure predictions.

The structural failure mode that is predicted to occur first is the rupture of the liner seam at the wall-basemat juncture. This failure mode is driven in part by the failure of the basemat in bending due to the yielding of the basemat bottom bars at $135 \mathrm{ps} i(.93 \mathrm{MPa})$. At $140 \mathrm{psi}(.97 \mathrm{MPa})$, the effective liner strains at the juncture reach 6 percent. Between 140 and $160 \mathrm{psi}$ (.97 and $1.10 \mathrm{MPa}$ ), the effective strain at this location grows to 16 percent, which is the ductility limit of the material. Over the same pressure range, basemat edge liftoff exceeds one inch, which is probably larger than is possible without depressurization. Thus, failure is expected to occur between 140 and $150 \mathrm{psi}(.97$ and $1.03 \mathrm{MPa})$ at the basemat juncture. Failure at this location is also linked to the behavior of the liner anchorage, and this is discussed in the report. While the basemat juncture is predicted to be the first leakage location, at $150 \mathrm{psi}$ ( $1.03 \mathrm{MPa}$ ) many 
Table 4.3.1 Summary Table

Item

Analytical Method

Constitutive Models

Mode 1

Geometries

Failure Criterion

Predicted Failure Mode

Predicted Failure Pressure
Description

ABAQUS [4.3.3] with small displacement theory.

Steel: ABAQUS nonlinear isotropic material with stressstrain curves as provided by SNL.

Concrete: Subroutine UMAT $[4.3 .2,4.3 .4]$ with integration point tensile cracking and shear retention. Compressive plasticity with no strain softening for 2-D analysis but with strain softening for 3-D analyses.

Series of 2-D axisymmetric and $r-\theta$ analyses for locating and ranking failure locations. Detailed axisymetric analysis for final wall-basemat juncture prediction. Global and local 3-D analyses of wall, dome and major penetrations. Rebar modeled as tension-compression subelements. Concrete was modeled with quadratic continuum elements. The liner was modeled with quadratic continuum elements in areas of local interest and with shell elements elsewhere.

Large localized liner strain causing liner rupture and leakage. Effective (von Mises) strain of 15 percent was reached at one location at $160 \mathrm{psi}(1.10 \mathrm{MPa})$ which is the material ductility limit. However, correlation with previous tests [4.3.2] shows that at stiffness discontinuities strains may be locally amplified and the ductility at welded seams is probably reduced [4.3.9], thus liner rupture is predicted at calculated effective strains of 6 percent to 8 percent.

Liner tearing at the connection of the liner to the wallbasemat juncture knuckle. Liner tearing mechanism is triggered by basemat bending failure.

Between 140 and 150 psi (0.9 to $1.03 \mathrm{MPa})$.

local areas near the hatches and the penetrations sustain maximum principal strains ranging from 3 percent to 6 percent. If leakage is delayed at the basemat juncture, failure is predicted at the $30^{\prime}$ clock or $90^{\prime}$ clock position in the $1 / 16^{\prime \prime}(1.59 \mathrm{~mm})$ liner material next to the $3 / 16^{\prime \prime}(4.76 \mathrm{~mm})$ reinforced liner around a penetration. The penetrations are ranked from highest failure potential to lowest as follows: the 8" constrained penetrations, 4" pressure nozzle connection, Equipment Hatch A, Equipment Hatch $B$, personnel airlocks at $90^{\circ}$ and $270^{\circ}$, respectively, and finally, the small penetrations.

\subsubsection{Introduction and Scope of Work}

This report describes analyses performed for the SNL 1:6 Scale Reinforced Concrete Containment Model, using methods developed by ANATECH as part of EPRI sponsored research to predict the overpressure behavior of concrete 
containments. As one of the many pretest analysis teams, we have followed guidelines set forth by SNL regarding the content and organization of this report. Thus, we begin by describing in Section 4.3.3 the analytical approach, including finite element modeling, material representation, loading and boundary conditions. In Sections 4.3.4 and 4.3.5 we present the results of two- and three-dimensional analyses, respectively. The 2-D analyses were carried out in $r-z$ and $r-\theta$ geometries to make specific predictions of the parts of the response that are predominantly twodimensional. Detailed discussion of the results are presented in Section 4.3.6, and conclusions are given in Section 4.3.7. Many specific illustrations are included in the main text as supporting material, but the bulk of the analysis results are in the form of standardized plots, as requested by SNL, and are included in Appendix $A$.

The scope of this phase of the current research encompasses the application of analysis tools that have been developed in the past few years [4.3.1] to the response analysis and failure prediction of the $1: 6$ Scale Model. Prior to the present work, these tools have been used for the analysis of prototypical reinforced and prestressed concrete containments [4.3.2] and structural specimens. The present analysis is the first application of these techniques to an as-built containment model structure.

Pretest predictions of containment structural response require very detailed calculations for many discontinuity regions. However, because of computational limitations, it is impossible to calculate an accurate response at all of the points of strain concentration in a single model. Therefore, it was necessary to perform scoping analyses to identify high strain locations which were then analyzed in greater detail in separate 3-D models. This required the development of a 3-D concrete constitutive model, the implementation of this model in the ABAQUS code, and the development of extensive pre- and postprocessing capabilities to handle the large volume of output data generated. The computations were carried out on a VAX 11/750. It is significant to point out that the computer run times were measured in days and weeks, which was important in choosing grid sizes, optimizing solution wave fronts and selecting load steps.

The familiar notion that concrete containments leak before they burst catastrophically under excess pressure will soon be put to the test in the 1:6 scale model. This failure mode is caused by localized dislocation motion near stiffness discontinuities and at major cracks. Liner concrete interaction in the presence of positive anchorage is the primary mechanism of this failure mode. As will be shown in this report, the present analysis identifies a number of such mechanisms in the 1:6 scale model, particularly in the wall-basemat juncture area where liner connection, liner-welded stud anchorage design, and basemat bottom reinforcing design play major roles in the failure of the structure.

\subsubsection{Analytical Approach}

Under extreme loading conditions resulting from overpressure, concrete containment buildings become tension structures and develop numerous discontinuities that are potential leakage locations. To accurately predict this type of behavior and related failure modes it is essential that concrete constitutive behavior be accurately modeled and that an analysis methodology is used which has been experimentally verified for similar 
engineering structures. This section describes the analysis approach used for the $1: 6$ scale model.

\subsubsection{Computer Code and Analysis Options Invoked}

ABAQUS-EPGEN, version 4-5-171 released in JuTy 1985 [4.3.3], was used for all of the analyses described herein. ABAQUS-EPGEN is a well-established general purpose finite element code and provides extensive modeling capabilities for a very large class of engineering structures subjected to static or dynamic loadings. The code can treat material nonlinearities and large displacement/ large strain geometric nonlinearities. A constitutive model for concrete was developed and implemented for use with the code [4.3.2] as part of EPRI's containment research program. The code and the constitutive model have been applied extensively to reinforced and prestressed concrete containment structures and full scale structural specimen tests. As a part of its concrete analysis capabilities, the code includes an extensive user-oriented input scheme to permit the convenient utilization of concrete structural modeling. This is described in detail in Reference [4.3.3]. The two most helpful user features for these problems are the option to call a user written SUBROUTINE UMAT for a user supplied general constitutive model and the provision for a rebar sub-element, which allows the definition of a concrete element with rebars positioned anywhere in the element. Small displacement theory was used throughout the analyses, i.e., no geometric nonlinearities were included. The UMAT constitutive model developed by ANATECH is described later in this section.

Varying loads and varying displacement boundary conditions were applied. Load increments are fixed by the user and can be changed in a RESTART run. The number of iteration cycles performed at each load increment to handle material nonlinearities is preset by the user. For the current analyses, the number of cycles was set by trial-and-error based on experience in performing dozens of analyses with similar geometries. Selecting this parameter is very important for this type of analysis as a means of conserving computation time.

Solution convergence measure in ABAQUS is a force-based criterion and is controlled by a user specified residual force tolerance. It should be noted, however, that in concrete analysis, failure to meet the ABAQUS force convergence criterion does not necessarily imply inaccurate solution. Large force residuals are attributed to the fact that in partially cracked elements the stresses are discontinuous as are the nodal point forces which are computed from the element stresses; however, the displacement field is continuous. Thus a more appropriate convergence measure for concrete analysis would be a displacement-based criterion which is not available in ABAQUS rather than a force-based criterion. Therefore, we relied on experience and other checks which were routinely made to ensure solution quality. For example, overall equilibrium must be satisfied by ensuring that the sum of the external loads and reaction forces on the structure is zero or small. Another check which becomes intuitive after performing many such analyses is that if load increments are too large or the solution is divergent, force residuals grow without bound on subsequent iterations within the same load increment. If this situation occurs it shows up immediately and can usually be traced to a grid error or excessively large load steps. 
The ABAQUS solution algorithm to solve the nonlinear equations is a full Newton method where the stiffness matrix and the unbalanced forces are recalculated at the end of every iteration. Generally, a minimum of three iterations were used in each load step.

\subsubsection{Material Properties and Constitutive Modeling}

Material properties for all the analyses are consistent with the standardized properties provided by SNL. Steel properties were modeled with ABAQUS isotropic material with the stress-strain data points identical to the tables provided by SNL in [4.3.10]; \#4 bar properties reported by SNL were used for all rebars. Nominal rebar sizes and liner thicknesses were used as follows.

$\begin{array}{lll}\text { \#3 bars: } & \text { Area } & =0.110 \mathrm{in}^{2}\left(71 \mathrm{~mm}^{2}\right) \\ \text { \#4 bars: } & \text { Area } & =0.200 \mathrm{in}^{2}\left(129 \mathrm{~mm}^{2}\right) \\ \text { \#5 bars: } & \text { Area } & =0.310 \mathrm{in}^{2}\left(200 \mathrm{~mm}^{2}\right) \\ \text { \#6 bars: } & \text { Area } & =0.440 \mathrm{in}^{2}\left(284 \mathrm{~mm}^{2}\right) \\ \text { 1/16" Liner: } & \text { Thickness }=0.0625 \mathrm{in}(1.59 \mathrm{~mm}) \\ 3 / 16 " \text { Liner: } & \text { Thickness }=0.188 \mathrm{in}(4.78 \mathrm{~mm}) \\ 1 / 12 " \text { Liner: } & \text { Thickness }=0.0833 \text { in }(2.12 \mathrm{~mm})\end{array}$

The same concrete cracking model and cracking properties used in previous containment analyses were used in the $1: 6$ scale model analysis. A concrete constitutive model with modified Drucker-Prager yield surface was incorporated in the latest version of the user routine UMAT developed by ANATECH. Detailed descriptions of earlier versions of UMAT may be found in [4.3.2] and [4.3.4]. The cracking algorithms of the current UMAT are the same as in earlier versions. The newer version includes compressive plasticity as well as creep and aging capabilities; however, no creep or aging was used for the $1: 6$ scale model. Detailed description of UMAT is beyond the scope of this report, but it is appropriate to describe a few of its main features as they pertain to the present analysis. The plain concrete model accounts for compressive plasticity and tensile cracking (i.e., cracking smeared over the integration points) with post-cracking shear retention [4.3.5]. For the $1: 6$ scale model the shear retention term is quite important, especially in the local region of the base of the cylinder where large shear transfer occurs coupled with major cracking. However, tension stiffening which is included in some cracking models [4.3.5] seems to be of little importance because containment structures are practically pure tension structures; after cracking, the effective tension carrying area in a typical wall section immediately drops to the area of the steel elements. Consequently, the strain in the wall across a crack immediately jumps by an order of magnitude, making any reasonable level of tension stiffening negligible. The ABAQUS-EPGEN code interfaces to UMAT in the following manner. During any load increment (i.e., an equilibrium iteration), the code passes to the subroutine: the total stresses at the end of the previous increment, $a_{n-1}$; the total strains at the end of the previous increment, $\epsilon_{n-1}$; the incremental strains in the current increment, $\Delta \epsilon_{n}$ (these are always zero at the start of an increment); the cracking status variables at the end of the previous increment; and general control information such as time, temperature, material properties, etc. The 
subroutine then computes and passes back to the main code: the total stresses at the current state, $\sigma_{n}$, and the current cracking status variables.

For the 1:6 scale model the concrete tensile properties input to UMAT are:

$$
\begin{aligned}
& E=4800 \mathrm{ksi}(33100 \mathrm{MPa}) \\
& \text { Poisson's Ratio }=0.2 \\
& \text { Ultimate Tensile Strength }=500 \mathrm{psi}(3.45 \mathrm{MPa}) \\
& \text { Ultimate Cracking Strain }=104 \mathrm{microns}
\end{aligned}
$$

Two distinct models for compressive plasticity were used. A compressive plasticity model with no strain softening was used for all of the 2-D scoping studies and the final axisymmetric and $r-\theta$ analyses. When the 3-D analysis series was started a stress-strain curve with strain softening was introduced. In the non-softening model, the rising portion of the SNL standard curve was matched, but the stress remained at ultimate for larger strains. In the second model, the total curve was matched fairly well except that the UMAT curve is a parametric equation rather than a sequence of line segments; thus, the SNL curve could not be matched precisely. However, the differences between the two curves are small, and they occur at very high strain levels which are of little interest in the present analysis. Compressive stress strain plots of the various curves mentioned above are shown in Figure 4.3.1. The curve labeled "6-MONTH UNIAX TEST" is taken from one of the typical uniaxial tests sent out by SNL in October. The curve marked "UMAT OUTPUT" is stress results for a strain controlled 3-D continuum element test problem.

The differences in the compressive modeling have little or no effect on the model response except for a small region at the base of the cylinder and at the center of the basemat where concrete crushing occurred. However, at these locations, concrete crushing affects the model's response at high pressures where the formation of plastic moment hinges at these two locations triggered significant basemat liftoff. This phenomenon will be addressed in detail in later sections.

\subsubsection{Rebar Modeling}

As mentioned earlier, all reinforcement was modeled using the ABAQUS REBAR sub-element, which superimposes rebar stiffness onto an existing concrete element, while taking into account the rebar position and orientation within the element. The advantage of these sub-elements is that user input is relatively straightforward (although rather labor intensive in 3-D elements). The resulting stiffness contribution of the bar is similar to that of adding a truss element. To facilitate generation of rebars in 3-D elements, a FORTRAN program called REGEN was developed. The program, with relatively simple free format input, allows the spatial generation of arbitrary rebar lines and areas or surfaces, then plots the bars as they are generated, and searches an ABAQUS input deck with nodal point coordinates and element connectivities to find which elements contain bar segments. By calculating element face intersection points and angles, it generates ABAQUS *REBAR input. 


\subsubsection{Analysis Series Outline}

This subsection outlines the analysis series used to develop comprehensive pretest predictions for the $1: 6$ scale model. The analysis strategy is described as follows: (a) conduct global axisymetric analyses to identify points of liner strain concentration and to develop a parent grid to be used for later local effects analyses; (b) select which local effects are most critical and analyze separately with a series of two-dimensional detailed analyses in $r-z$ and $r-\theta$ geometries; (c) select the most likely failure locations from (b) and analyze in 3-D. The analyses which were conducted are listed in the Table 4.3 .2 in chronological order of their completion.

The 2-D analysis series was aimed at identifying and ranking possible failure modes and leakage locations, estimating failure pressure levels, and preparing for the final 3-D analysis series. Many factors influenced the planning of the 3-D series. First, it was felt that the basemat and wallbasemat juncture were modeled very accurately with the axisymetric analyses. The main response component that had not been determined with certainty by the 2-D analyses was the interaction between hoop and meridional deformations around the hatches. For these reasons, the basemat was left out of the 3-D series. Grid size was determined in a trade-off between cost and accuracy. A $90^{\circ}$ quarter model geometry was chosen. The $1: 6$ scale model quadrant which contained the highest failure potential penetrations based on the 2-D analyses was from $270^{\circ}$ to $0^{\circ}$. This segment contained Equipment Hatch $A$, an $8^{\prime \prime}$ constrained penetration, the 4" pressure nozzle and the $270^{\circ}$ personnel airlock. The $90^{\circ}$ global model was planned to be relatively coarse to provide displacement boundary conditions for the more refined models of the hatch and penetration regions.

\subsubsection{2-D Analyses}

\subsubsection{Axisymetric Analysis}

The current analysis series was begun with a brief global axisymetric grid convergence study to investigate the tradeoffs between accuracy, modeling complexity and computation time. This study produced what is deemed to be a reliable global grid that could be used as a parent grid in which to embed various local effects grid refinements; however, study of grid convergence was not exhaustive. The third grid of this study, $\mathrm{C} 3$, is shown in a deformed grid plot at $p=100$ psi $(.69 \mathrm{MPa})$ in Figure 4.3.2. The analysis was carried out to $p=140$ psi (.97 MPa). The evolution of the model behavior as pressure increases is shown graphically in Figures 4.3.3 through 4.3.5, which display liner strain profiles meridionally at increasing pressures. In these figures, strains are plotted versus position with positive and negative values shown on either side of the dashed line (dashed line is zero). No scale is given, but the highest strain on the plot is given so that other strains may be relatively scaled. In the present discussion, the terms "hoop cracking" and "meridional cracking" are used to refer respectively to cracks caused by the hoop strain and the principal strain in the $r-z$ plane.

Meridional bending locations are very pronounced in Figure 4.3.3. At 60 psi $(0.41 \mathrm{MPa})$, after hoop cracking but before general meridional cracking, most of the meridional liner strains are small while the strain just above the wall-basemat juncture is already half of the uniaxial yield strain. 


\section{Table 4.3.2 Outline of Analysis Series}

Code

C1

$\mathrm{C} 2$

C3

C3HA

$\mathrm{C} 3 \mathrm{HB}$

C4

C4B

$\mathrm{C} 4 \mathrm{C}$

C5

C3P

CRTA

CRTPA

C3-D

C3-DHA

C3-DPN

C3-DPN

C3-DHB

\section{Description}

First of global axisymmetric grid convergence study. Very coarse grid, 40 concrete 8-node continuum elements, 21 liner 3-node shell elements.

Second of global axisymmetric grid convergence study. 86 concrete 8 -node continuum elements, 46 liner 3 -node shell elements.

Third of global axisymetric grid convergence study. Grid deemed accurate for global results. Still needs refinement at wallbasemat juncture. 200 concrete 8 -node continuum elements, 58 liner 3-node shell elements.

C3 global grid with Equipment Hatch A added. Axisymetric shell wall is continuous through hatch. Thickened shell and hatch elements modeled with plane stress elements and beam elements.

Same as C3HA but with hatch B geometry.

C3 global grid but greatly refined at wall-basemat juncture. Total of approximately 450 8-node continuum concrete elements, 50 3-node liner shell elements, and 50 8-node liner continuum elements at juncture to better resolve liner bending.

Same as $\mathrm{C} 4$ with added refinement at liner knuckle including "singularity" element at liner-knuckle seam weld.

Restart of $\mathrm{C} 4 \mathrm{~B}$ at 140 psi allowing knuckle to tear off of backing bar.

C3 global grid with refinement at springline to study liner deformation there.

C3 global grid with 8" constrained penetration plus 4" pressure nozzle connection.

Containment $r-\theta$ Run $A ; 180^{\circ} r-\theta$ slice modeling equipment hatch $A$, $B$, and the constrained penetration. Model has plane stress geometry with $264 "(6.71 \mathrm{~m})$ out-of-plane thickness.

r- $\theta$ analysis with both personnel airlocks; plane stress geometry.

3-D analysis of $90^{\circ}$ segment of wall/dome.

3-D analysis of equipment Hatch $A$.

3-D analysis of constrained pipe penetration.

3-D analysis of pressure nozzle connection.

3-D analysis of Equipment Hatch B. 
Significant bending also occurs a few feet above the basemat juncture and at the springline. At $100 \mathrm{psi}(.69 \mathrm{MPa})$, the cylinder wall is cracked meridionally over most of its length. At $140 \mathrm{psi}(.97 \mathrm{MPa}$ ) when significant basemat liftoff begins to develop, a sharp peak in strain occurs at the wall/basemat juncture. The basemat liftoff phenomenon is discussed in detail in Section 4.3.6.2. Figure 4.3.4 and 4.3.5 show similar progression of hoop strain. Initial hoop cracking develops in the model between 45 and $50 \mathrm{psi}(.31$ and $.34 \mathrm{MPa})$, and it occurs at the cylinder midheight. This is illustrated in Figure 4.3 .4 at $50 \mathrm{psi}$ (.34 MPa) where the larger liner hoop strains occur where the concrete is cracked. At $60 \mathrm{psi}(.41 \mathrm{MPa})$ the cylinder is entirely hoop-cracked and liner hoop strain up the wall is relatively constant. At $80 \mathrm{psi}(.55 \mathrm{MPa})$ the dome begins to show hoop cracking starting at the springline and moving up, and at $100 \mathrm{psi}(.69 \mathrm{MPa})$, the entire model superstructure is cracked in the hoop and meridional directions. At 140 psi $(.97 \mathrm{MPa})$, liner hoop strain again increases disproportionately at the cylinder midheight marking the onset of hoop rebar yielding there.

It was observed from these and similar analyses from previous research that the foundation pressure distribution on the bottom of the basemat plays a significant role in driving the section moments in the basemat and in the wall at the wall-basemat juncture. The basemat-soil interface was modeled using nonlinear no-tension springs. Relatively stiff spring constants were calculated in proportion to the tributary area at each node: approximately $7780 \mathrm{lbs} / \mathrm{in}^{2} /$ in $\left(2110 \mathrm{mN} / \mathrm{m}^{3}\right)$. This represents a foundation material directly under the basemat that displaces 0.001 inches $(.025 \mathrm{~mm})$ under the entire structure dead load of approximately 550,000 lbs (2.45 MN). This spring stiffness was chosen somewhat arbitrarily and is significantly stiffer than the soil spring constant field measurements provided by SNL. However, since the foundation support seen by the containment basemat is actually the lower concrete foundation mat which in turn interacts with the soil, the foundation stiffness seen by the containment basemat would be stiffer than is indicated by the field soil measurements, thus the stiffspring approximation was made. The stiffness is zero in the tensile direction, which is a reasonable approximation since the drawings show that the lower foundation mat is painted with bond breaker. From past experience, the value of the compression spring constant has little or no effect on the contact pattern of the bottom of the basemat as long as the springs are distributed in proportion to area. The important feature is to allow liftoff to occur so that foundation pressures are redistributed according to the contact pattern.

\subsubsection{Penetration Analyses}

Once a framework for the containment response was established by the $\mathrm{C} 3$ analysis, penetrations were added to the $C 3$ grid as plane stress elements superimposed on the axisymetric grid. Thus, the plane stress substructure offered no hoop constraints to the axisymmetric wall. However, the resistance to local punching shear is not correctly modeled. This type of behavior can be studied only in a 3-D analysis.

Separate axisymmetric studies of this type were performed for Equipment Hatch A, Equipment Hatch B, the personnel airlocks, an 8" constrained penetration, and the $4 "$ pressure nozzle. The concrete was modeled with 
axisymmetric 8-node quadrilaterals, and plane stress quads were used for the embossed regions. The liner was modeled with 2- and 3-node axisymetric shells, and the local steel shell portions of each penetration were modeled with beam elements. A deformed grid plot at $120 \mathrm{psi}(.83 \mathrm{MPa}$ ) is shown for Equipment Hatch $A$ in Figure 4.3.6. In this model the hatch moves along with the shell wall and does not significantly affect the global structural response. The hatch moves out only slightly less than the rest of the wall. Local liner strain concentrations exist near the hatch, and the concrete at the wall-boss boundary is subjected to more shearing stresses than the rest of the cylinder shell. The behavior of Hatch $B$ is similar except that the peak liner strains were higher for Hatch $A$ than for Hatch $B$. The primary difference in behavior between the two hatches is the fact that for Hatch $B$ there is less bending across the wall thickness because the "wall-boss" consists of added material inside and outside of the cylinder wall. In Hatch $A$, the cylinder wall has extra stiffness on the outside, thus causing higher bending at points labeled 1 and 2 in Figure 4.3.6, and consequently higher failure potential because of the higher liner strains. Similar results were obtained for the personnel airlocks embedded in the axisymmetric grid. The $90^{\circ}$ personnel airlock caused higher strain concentrations then the $270^{\circ}$ airlock, because the $270^{\circ}$ airlock is much more flexible. However, the $270^{\circ}$ airlock is subject to greater ovalization, which will be addressed in the 3-D discussion.

For the 8" diameter constrained penetration and the 4" diameter test pressure nozzle, the purpose of the analysis was to evaluate the effects of the penetration discontinuity on the axisymmetric behavior. The tie rod for the 8 " constrained penetration was modelled using a single 2-node beam element with the area of a \#2 bar that extended from the penetration over to the symmetry axis, where it was restrained with a roller boundary condition against horizontal motion. In 2-D, the results of these analyses showed the disturbance to the structural system caused by these penetrations to be minimal. However, when 3-D effects are included, high local liner strains develop next to the thickened portion of the liner around these penetrations, as previous studies [4.3.7] have shown, which makes these location candidates for premature liner tearing. These penetrations are reexamined in the 3-D analysis discussion.

All of the axisymmetric analyses showed high concentrations of strain at the springline juncture. This concentration was generally not as high as that at the wall-basemat juncture, but nearly so, thus warranting further investigation. In addition, a liner rupture at the springline leads to a more direct leakage than a rupture at the basemat juncture because all of the concrete wall is in tension at the springline, whereas at the juncture, a small compression exists on the outside of the wall. Therefore, the C5 grid, which includes additional refinement at the springline juncture, was developed. Structural features at the springline which may cause large local strains include: transition from the cylindrical stiffness to the higher dome stiffness, a gradual reduction in concrete thickness by $2-3 / 4$ " $(69.9 \mathrm{~mm})$, and an abrupt change in liner thickness from 1/16" (1.59 mm) in the cylinder to $1 / 12^{\prime \prime}(2.12 \mathrm{~mm})$ in the dome. Moreover, this abrupt thickness change is accomplished with a field welded seam at the springline causing a possible crack initiation location at high strains. Refinements of the previous $\mathrm{C} 3$ grid include the addition of 22 extra rows of concrete elements near the springline and the use of 8-node continuum elements to model the liner in this region. These changes improved the modeling of the 
liner's bending behavior and showed more severe strain concentrations than those predicted when the liner was modeled with shell elements.

It is convenient to classify structural response and modes of deformation as primarily related to either meridional action or hoop action. For the wall basemat juncture or the springline strain concentration, hoop response is of secondary interest. However, for most of the penetrations, hoop response is the most important, especially at the belt line of the cylinder wall. Here hoop strains are roughly twice the meridional strains, and strain concentrations develop earlier because of much earlier hoop cracking. Ovalization of the penetrations, which affects seal tightness, is influenced by the relative magnitudes of hoop and meridional cracking. These effects require modeling of the interaction between meridional and hoop deformations which is impossible to do in a two-dimensional analysis. However, by examining the meridional ( $r-z$ geometry) behavior and the hoop ( $r-\theta$ geometry) behavior separately, significant insight was gained into the final failure analysis of the hatch and penetration local effects.

Several plane stress $r-\theta$ slice models were analyzed in which plane stress and beam elements were used. The hatch elements out of plane thicknesses ranged from 30" to 60" (762 to $1524 \mathrm{~mm}$ ), while the remaining elements were $264^{\prime \prime}(6.71 \mathrm{~m})$ thick which is the height of the $1: 6$ scale model cylinder. A deformed grid plot at $100 \mathrm{psi}(.69 \mathrm{MPa})$ is shown in Figure 4.3 .7 with enlarged views of the hatch regions. Earlier in the analysis, at 40 psi (.28 $\mathrm{MPa})$, both hatches bulged out more than the rest of the shell, acting as rigid "plugs", but after all hoop cracking occurred, the shell moved out further. Note that the 8 " constrained penetration and the tie rod have little effect on the overall response. At $100 \mathrm{psi}(.69 \mathrm{MPa})$, significant local distortion occurs around the hatches. The hatch covers appear to be severely deformed, but this has no effect on the response quantities of interest; plane stress representation of the hatch covers greatly underestimates their stiffness. The maximum liner hoop strain at the end of the analysis ( $120 \mathrm{psi}, .83 \mathrm{MPa}$ ) was about 0.9 percent next to Hatch $A$ and 0.7 percent next to Hatch $B$. A similar $180^{\circ} \mathrm{r}-\theta$ grid was analyzed with the $90^{\circ}$ and $270^{\circ}$ personnel airlocks in place of the hatches, and this produced similar results. The peak strains next to the airlocks were generally lower than next to the hatches; the $90^{\circ}$ airlock, with two heavily stiffened interior plates, showed the highest strains of the two airlocks.

\subsubsection{Detailed Results of Final Axisymmetric Analysis}

Two axisymmetric analyses were performed to focus on the behavior at the wall-basemat juncture region. The reasons for this further study were to achieve better modeling of the severe strain concentrations at the liner knuckle welds in an attempt to establish failure criteria for these points and to get a more accurate representation of the liner-concrete interface. A closeup view of the $C A B$ juncture region containing the grid refinements is shown in Figure 4.3.8. These grid refinements are further illustrated in the schematic which shows the contact springs. The surfaces marked "contact springs" have ABAQUS nonlinear springs between nodes perpendicular to the surface with large spring constants for relative movement of the surfaces toward each other and zero spring constants for movement of the surfaces away from each other. The surfaces marked "stud interface springs" have nonlinear springs parallel to the surface for shear resistance and perpendicular to the surface for pullout resistance. The spacing and 
magnitude of these springs accurately model the studs in the $1: 6$ scale model. Stud spring constants are representative of the stud shear and pullout test data provided by SNL [4.3.8]. Other forms of interface models were attempted based on previous research [4.3.1], but the modeling scheme arrived at here produced the most consistent results.

Figure 4.3.8 shows the added grid refinement near the knuckle-liner welds for predicting possible tearing. The construction configuration at the weld on the lower edge of the knuckle to the basemat liner is such that a sharp notch exists at the corner of the knuckle and the $2 " \times 1 "$ (51 $\mathrm{mm} \times 25 \mathrm{~mm}$ ) steel backing bar. This, combined with the fact that field weld uniformity is more difficult to control make this point a candidate for tearing. The upper weld, on the other hand, is a shop weld. Treating the notch as a flaw, singularity elements were placed as shown in the figure, with all 5 adjacent midside nodes moved to the element side quarter points.

A closeup of the deformed juncture region at $140 \mathrm{psi}(.97 \mathrm{MPa})$ with displacements magnified by 5 is shown at the top of Figure 4.3.9. At this stage, the mechanism for eventual failure has begun, and at 150 psi (1.03 $\mathrm{MPa})$ the deformation pattern looks similar but larger in magnitude. The liner peeled away from the concrete wall for the first one inch above the knuckle (as shown in the figure), which indicates a possible pullout of the first row of welded studs above the knuckle.

The high strains at the edges of the knuckle prompted two different treatments of the analysis in the pressure range of 140 to 160 psi (.97 to $1.10 \mathrm{MPa})$. In the first, the analysis was restarted without change and continued to $160 \mathrm{psi}(1.10 \mathrm{MPa})$. At $140 \mathrm{psi}(.97 \mathrm{MPa})$, the strains near the singularity node indicate the possibility of liner failure either by tearing off the backing bar or by rupture in the liner material. At the same pressure, strains at the top of the knuckle were of similar magnitude. In order to further investigate the bottom of the knuckle, the analysis was restarted for the second time from $140 \mathrm{psi}(.97 \mathrm{MPa})$ using the ABAQUS *MODEL CHANGE command to detach the liner from the backing bar and let the knuckle lift up by removing three small elements on the top of the backing bar. This was named analysis C4C. *MODEL CHANGE was invoked gradually between 140 and $152 \mathrm{psi}(.97$ and $1.05 \mathrm{MPa})$ and then remained in effect until $160 \mathrm{psi}$ $(1.10 \mathrm{MPa})$. This simulates the $1: 6 \mathrm{scale}$ model liner knuckle breaking off of the backing bar. The effect on displacements of tearing away from the backing bar is to allow a net upward movement of the cylinder liner, thus relaxing the stress at the liner-knuckle juncture. When this occurred, liner strains near the weld leveled off; whereas when the knuckle stayed in contact (C4B), strains continued to rise sharply. It should be noted that failure in the manner of $C 4 C$ without at least partial tearing of the linerknuckle weld is unlikely. Figure 4.3.9 also shows the liner strain profile for C4B. The dashed line is bottom or "concrete-side" integration point strain and the solid line is top or "pressure-side" integration point strain. Strains elsewhere in the structure are similar to previous analyses. Between 140 and $160 \mathrm{psi}(0.97$ and $1.10 \mathrm{MPa})$, C4B strains at the bottom knuckle weld grow very rapidly, probably representing a failure mechanism - the meridional strains grow from 3.6 percent to 9.0 percent (Von Mises effective strains of 6 percent to 16 percent). However, in $\mathrm{C} 4 \mathrm{C}$, once the liner is released from the backing bar, strains level off. The rationale for allowing the knuckle to tear away from the backing bar at a Von Mises strain of 6 percent is supported by literature [4.3.9], which 
shows that in the embrittled zone around a steel weldment, ductility is sometimes significantly reduced. As mentioned before, it is also likely that tearing through the liner at the top or bottom of the knuckle would occur at this load level instead of only tearing off of the backing bar, but since this constitutes failure of the pressure retaining system, no posttearing analysis was necessary for that scenario.

The conclusions which can be drawn from these analyses are: (1) if premature liner failure does not occur near a penetration (discussed later in this report) or at a weak seam elsewhere in the structure, liner failure will first occur at the edge of the knuckle between a pressure load of 140 and 150 psi $(0.97$ and $1.03 \mathrm{MPa})$; and (2) failure of this type will be by tearing at the weld between the knuckle and the basemat liner or at the weld between the top of the knuckle and the cylinder liner.

\subsubsection{3-D Analyses}

As discussed in the preceding section, the main response component that is lacking in the 2-D analyses is the interaction between hoop and meridional deformations around the hatches and penetrations. The basemat and wallbasemat juncture were modeled reasonably accurately by axisymmetric analysis and were excluded from further 3-D analysis. The following analysis was implemented:

1. Analyze a $90^{\circ}$ wall/dome model which contains portions of the structure that are of most interest and highest failure potential. The cut at the base of the wall is driven by displacement boundary conditions generated in the most detailed of the axisymmetric analyses (C4B).

2. Extract nodal displacements along faces and cuts around penetrations and hatches to develop displacement boundary conditions for local 3-D runs with smaller grids.

3. Analyze local models with more refinement to determine maximum liner strains and failure mechanisms.

From the 2-D analyses, the 1:6 scale model quadrant which contained the most critical penetrations is from $270^{\circ}$ to $0^{\circ}$. The model contains equipment hatch $A$, an $8^{\prime \prime}$ constrained penetration, the $4^{\prime \prime}$ pressure nozzle, and the $270^{\circ}$ personnel airlock. Strain concentrations will probably be higher next to the $90^{\circ}$ airlock, but the $270^{\circ}$ airlock is most susceptible to ovalization and is therefore included in the model. The local models originally planned were equipment hatch $A$, the 8 " constrained penetration and the 4 " pressure nozzle because these rank highest in liner failure potential. However, based on the request for standardized predictions from SNL, detailed information at hatch $B$ is requested, so a local model of hatch $B$ was also developed and analyzed for the same boundary conditions as hatch $A$.

The results of the 3-D analyses provide much needed 3-D information such as displacement gradients and severe dimpling near the hatches, hatch ovalization, effect of local details in rebar patterns which go around the hatches and penetrations, and triaxial liner strains and strain gradients. All of the results in the area of hatches and penetrations which are presented in this report, including those listed in the SNL set of standardized predictions, are taken from the 3-D analyses. 


\subsubsection{Global 3-D Quarter-Model Analysis}

The computational grid for the $90^{\circ}$ wall/dome quarter model is illustrated in Figure 4.3.10. The grid in the global regions away from the hatches is relatively coarse but is considered adequate for capturing the displacement behavior of the $1: 6$ scale model. Twenty-node continuum elements with reduced $(2 \times 2 \times 2)$ integration were used for all of the concrete, and 8 -node shell elements with 5 point integration through the thickness were used for all of the liner plate and hatch steel. Discretization of the grid is actually better than it appears in Figure 4.3.10 because this figure does not plot the midside nodes which allow each element to have curved sides (midside nodes are not plotted with the current "hidden line" plotter). One concrete element is used through the thickness, because in the past, the 20node elements have demonstrated excellent bending capability, and the results along the meridional plane $\theta=315^{\circ}$ compare well with axisymmetric analysis where three second order elements through the thickness were used. A total of 218 C3D20R concrete elements and 428 S8R liner plate and steel hatch elements were used. A total of 2365 nodes were used, with 14,190 degrees of freedom.

Figure 4.3.10 shows a grid refinement transition of $2: 1$ at the rectilinear boundaries of the hatch regions. Full model compatibility was achieved at these boundaries through the use of ABAQUS multipoint constraint or *MPC commands. The dome apex was not collapsed to a single point but to a small radius because a 15-node wedge was not available. Along $\theta-270^{\circ}$ and $\theta=$ $0^{\circ}$, symmetry boundary conditions were imposed.

The model is cut near the bottom of the wall at the $1: 6$ scale model elevation of $z=3^{\prime} 3^{\prime \prime}$, which is 13 inches $(.33 \mathrm{~m})$ above the top of the wallbasemat knuckle. This cut excludes the critical shear and bending moment zone at the juncture but includes the significant sloping of the shell wall. Investigation of an axisymmetric run without hatches (C4B) and axisymmetric runs with a hatch (C3HA, etc.) revealed that the boundary displacements at this elevation varied between runs by no more than 2 percent. Therefore, it was deemed sufficient to apply bottom face boundary conditions that were constant with $\theta$. The boundary conditions vary significantly at the three nodes through the thickness, as is evident in the deformed shape plots presented later in this section. The $x$ and $y$ components of displacements were generated so that their vector sum always added up to the same radial component. Pressure was applied to all inside surfaces including hatch sleeves and end caps. Some, but not all, rebar layers in the model are shown in Figure 4.3.11. All rebars were modeled using ABAQUS «REBAR subelements and automatic generation using the FORTRAN program described in Section 4.3.3. For the 3-D quarter model, 3168 rebar sub-elements were generated.

The load stepping history was made coarser then the typical axisymetric analyses by approximately a factor of 2 . This load stepping was selected by trial and error within reasonable computation time limits, giving careful consideration to solution accuracy, concrete cracking, steel plasticity, and force residuals. Based on the axisymmetric analyses, between 140 and 150 psi (.97 and $1.03 \mathrm{MPa}$ ) a deformation mechanism will be reached involving basemat liftoff and liner tearing, with almost certain test depressurization; thus, the analysis was stopped at $148 \mathrm{psi}(1.02 \mathrm{MPa})$. Many of SNL's requested standardized plots are freeze-in-time profiles, 
requested at 100,150 , and $200 \mathrm{psi}(.69,1.03$ and $1.38 \mathrm{MPa})$. Therefore, in general, ANATECH will be reporting the first two of these requested curves.

In addition to providing displacement boundary conditions for the finer local 3-D analyses, the 3-D quarter model shows interesting global results. Several deformed grid plots are shown in Figures 4.3 .12 and 4.3.13.

Displacement magnifications are given in the captions for each figure. The left plot of Figure 4.3.12 shows the liner and hatches, without concrete, for clarity. The shell wall rotation at the base is similar to the axisymmetric analysis because of the 2-D imposed boundary conditions at the bottom edge. Further up, however, both hatches behave as rigid inclusions in the shell wall. The dimpling which can be observed around the hatches intensifies liner strain concentrations near the hatches, resulting in much higher strain in 3-D than in 2-D. The radial displacement pattern at the beltline of the cylinder wall (in line with the hatches) is much different than that at the springline. This is shown in the right plot of figure 4.3.13 with only a few elements around the beltline plotted. Figure 4.3.13 shows three deformed slices of the $3-D$ grid at $p=136 \mathrm{psi}(.94 \mathrm{MPa})$ for $270^{\circ}, 315^{\circ}$, and $0^{\circ}$. These plots clearly show the difference in cylinder radial displacement at the hatches versus the clean wall section $\left(\theta=315^{\circ}\right)$. Both hatches behave as rigid inclusions because of the increased concrete wall thickness and because of the high rebar density around the hatches.

Another aspect of the containment response that was impossible to capture in 2-D analysis is hatch ovalization. Figure 4.3.14 shows diametral length change histories for the hatch $A$ and the personnel airlock sleeves. The diameter changes are measured across the opening at the attachment to the liner. The curves in each plot are labeled as the diameter change from 3 to $90^{\prime}$ clock and from 6 to $120^{\prime}$ clock. At the end of the analysis, the ratio between horizontal and vertical extension is 4.6 for hatch $A$ and 2.8 for the personnel airlock. The airlock actually expands more in proportion to its diameter than the equipment hatch. This was anticipated due to the construction detail used, i.e., the airlock has no stiffening plate or hatch cover in the plane of the liner. The hatch sleeve diameter is $40^{\prime \prime}(1.02 \mathrm{~m})$, giving a maximum percentage hoop diametral change of 4.6 percent. The airlock diameter is $20 "(.51 \mathrm{~m})$, giving a maximum hoop diametral change of 7 percent. This ovalization further intensifies the liner strain concentrations near the hatches, and may provide useful data for future evaluation of hatch covers and seals.

\subsubsection{Local 3-D Hatch Analyses}

The computational grids for the local 3-D analysis of Equipment Hatches $A$ and $B$ are illustrated in Figure 4.3.15. The liner thickness has been exaggerated for clarity. Each plot shows a "hidden line" plot of the grid combined with two layers of rebar. Not all layers of bars are shown, but in the analyses each bar from the construction drawings was modeled, including the multiple layers of hoop and meridional bars, seismic, "sun-ray", and extra shear bars. In Section 4.3.4, it was shown that liner strain concentrations were larger for the hatch $A$ geometry than for hatch $B$; therefore, hatch $A$ was modeled in the global analysis. Because of time limitation, a hatch $B$ version of the global run was not analyzed. Instead, displacement boundary conditions from hatch $A$ were used to drive a local model of hatch $B$ under the assumptions that away from the hatch the displacement fields are not affected by hatch details. Near the edges, the 
two local grids are identical, so it is felt that this simulation of the displacement field was good.

The primary differences in the local hatch grids and the global grid are extra refinement near the hatch (i.e., near the liner strain concentration) and the use of multiple layers of continuum elements to model the liner instead of the single layer shell element which was used in the global analysis. It was shown in Section 4.3.4 that typical liner strain concentrations were larger and the strain gradients more refined when 2 layers of continuum elements were used to model the liner instead of a single layer of shell elements. For the hatch $A$ local analysis, two extra element rows were added, which enabled the calculation of a refined strain profile in the radially outward direction. The hatch sleeve and hatch cover are still modeled with shell elements. For the hatch A local model, $8020-$ node continuum concrete elements, 80 20-node continuum liner elements, and 72 8-node steel shell elements were used; for hatch B, 144 20-node continuum concrete elements, 152 20-node continuum steel liner elements, and 112 8node shell hatch elements were used. A quarter symmetry was selected for the hatches to keep the problem of manageable size. From examining the 3-D global model results, the hatch response is nearly symmetric about the 3 o'clock line of the hatch, so the quarter-symmetry model approximation is justified.

Results of the local Equipment Hatch analyses provide detailed information about the strain field around the hatches which could not be accomplished with the coarse global model. Due to space limitations, deformed grid plots and other graphical results are not included here. Examination of deformed grids and other data gives evidence of the cause of the high liner strains in the first 20 inches $(.51 \mathrm{~m}$ ) out from the hatches, particularly Hatch $A$. Not only does the liner change thickness from $3 / 16^{\prime \prime}$ to $1 / 16^{\prime \prime}(4.76$ to 1.59 $\mathrm{mm})$, but the hatch inclusion causes a reverse in curvature of the shell wall. This accentuates the severe liner tension and also induces small amounts of bending which would enhance the potential for failure. Figure 4.3.16 illustrates the liner strain concentration mentioned above by showing strain profiles moving from the edge of the hatch sleeve outward along a 3 o'clock line, a 1:30 line and a 12 o'clock line for both hatches. Figure 4.3.16 is taken at $150 \mathrm{psi}(1.03 \mathrm{MPa})$. All strains are maximum principal strains, but investigation of the individual directional strain components shows that hoop strain dominates the principal strain calculation. For Hatch $A$, the maximum principal strain is 3.5 percent along the 3:00 line, 7 inches $(178 \mathrm{~mm})$ out, which is in the first element of the $1 / 16^{\prime \prime}(1.59 \mathrm{~mm})$ material. In the $1: 6$ scale model, this is near a seam weld between the two material thicknesses. For Hatch $B$, the maximum principal strain at the end of the analysis (150 psi, $1.03 \mathrm{MPa}$ ) is 1.2 percent which occurs at the edge of the embossed concrete $\left(\theta=158^{\circ}\right)$.

\subsubsection{Local 3-D Penetration Analyses}

The computational grids for the local 3-D analysis of the 4 "diameter pressure nozzle region and the 8 " constrained penetration region are illustrated in the deformed structure plots of Figure 4.3 .17 (concrete elements not shown). Both grids contain 4 layers of hoop bars, 2 layers of meridional bars, and 2 layers of seismic bars similar to the local hatch analyses, and the 4 inch nozzle region contains \#6 shear reinforcement extending from the basemat up several feet into the wall. Unilke the hatch 
analyses, quarter symmetry could not be used because the global quarter model grid could not be made to line up with each penetration along vertical and horizontal axes. In addition, the penetration response varies significantly in the axial direction because one penetration is located near the bottom of the wall where significant curvature and displacement gradient take place. The response is vertically asymmetric for the pressure nozzle because of the presence of the \#6 bars mentioned above which are terminated near the same elevation as the nozzle.

Another difference in the small penetration analyses is in the modeling of the transition in liner thickness from the $3 / 16^{\prime \prime}(4.76 \mathrm{~mm})$ thick portion near the penetration to the global liner thickness of $1 / 16 "(1.59 \mathrm{~mm})$. For the hatches this element was relatively close to the hatch, and the transition was made by changing from 3 liner continuum elements to 2 . For the penetrations more rows of elements were used moving radially out from the penetrations, thus enabling the modeling of the exact thickness transition shown on the construction drawings, i.e., changing from $3 / 16^{\prime \prime}$ to $1 / 16 "$ ( 4.76 to $1.59 \mathrm{~mm}$ ) at a slope of 4 in 12 or over a lateral distance of $3 / 8 "(9.53 \mathrm{~mm})$. Two liner continuum elements are used through the thickness throughout, and the thickness transition is introduced across one element row. Both penetration analyses use the same grid pattern and number of elements but with different dimensions, namely, 80 20-node concrete elements, 160 20-node liner elements, and approximately 60 8-node shell penetration elements. Displacement boundary conditions and pressure were applied in a manner consistent with the discussions in the previous subsections. At the edges of the grids, displacements at nodes without oneto-one correspondence with global grid nodes were interpolated using second order interpolation.

Figure 4.3.18 shows strain profiles at $150 \mathrm{psi}(1.03 \mathrm{MPa})$ for the two penetration grids. The top plot of each figure is a profile moving out from the edge of the nozzle pipe along the 3:00 position, and the bottom plot is a profile moving in the meridional direction from the bottom of the grid up to the pipe, then with a gap across the pipe and on up to the top of the grid. All strains are maximum principal strains, which are generally dominated by the hoop component. These plots show the severe strain concentrations near the reinforced portion of the liner at about 4 inches $(102 \mathrm{~mm})$ out from the nozzle pipe and 9 inches $(229 \mathrm{~mm})$ out from the 8 " penetration. The maximum strain at $150 \mathrm{psi}(1.03 \mathrm{MPa})$ is 5 percent at the 3:00 position for the 4" nozzle and 6 percent for the constrained penetration. These strains are significantly higher than what was predicted in the 2-D analysis. The strain concentration for the 8 " penetration in the shell wall is larger than for the nozzle. This is attributed to a higher global hoop strain in the 8 " penetration region than in the 4 " nozzle region. In the latter case the global hoop strains are partially confined by the proximity of the nozzle to the basemat.

\subsubsection{Discussion of Results}

\subsubsection{Comparison of 2-D and 3-D Analyses}

The parts of the 1:6 scale model that are predominantly axisymmetric are the basemat and wall-basemat juncture and the dome from springline to apex. It is shown in Section 4.3.4 and 4.3.5 that axisymmetric modeling of the cylinder wall with embedded hatches and penetrations leads to very different 
results than 3-D modeling. Therefore, the 2-D results were used only to rank possible failure locations rather than compute detailed strain information. Table 4.3.3 gives comparisons of key response quantities in the $2-D$ and $3-D$ analyses.

Table 4.3.3. Comparison of 2-D and 3-D Key Response Quantities

Quantity or Milestone

Pressure

2-D Result

3-D Result

psi (MPa)

Onset of 1 st Hoop Cracking

$\begin{array}{cc}\text { Radial } & \text { Displ. } \\ " 1 & " \\ " & "\end{array}$

- Cyl. Midhgt
- Cyl. Midhgt
- Hatch-A
- Hatch-B

Vertical Displ. - Dome Apex

Max Pr. Strain - Springline

- Hatch-A

- Hatch-B

- 4" Nozzle

- 8" Penetr.

- Hatch-A

- Hatch-B

- 4" Nozzle

- 8" Penetr.

$150(1.03)$
$120(0.83)$
$120(0.83)$
$120(0.83)$

$150(1.03)$

$150(1.03)$

$100(0.69)$

$100(0.69)$

$100(0.69)$

$100(0.69)$

$150(1.03)$

$150(1.03)$

$150(1.03)$

$150(1.03)$
40 psi $(0.28 \mathrm{MPa}), \quad z=13^{\prime}$

45 psi $(0.31 \mathrm{MPa})$, $z=13^{\prime}, \theta=315^{\circ}$

$$
\begin{array}{ccc}
r-z & 1.8^{\prime \prime} & (46 \mathrm{~mm}) \\
r-z & 0.3^{\prime \prime} & (7.6 \mathrm{~mm}) \\
r-z & 0.28^{\prime \prime}, & r-\theta \quad 0.0^{\prime \prime} \\
r-z & 0.29^{\prime \prime}, & r-\theta-0.5^{\prime \prime}
\end{array}
$$

1.3" (32 mm)

$0.26^{\prime \prime}(66 \mathrm{~mm})$

$0.07^{\prime \prime}(1.8 \mathrm{~mm})$

$0.08 "(2.0 \mathrm{~mm})$

$$
\begin{gathered}
r-z 1.6 "(41 \mathrm{~mm}) \\
r-z \quad 0.9 \% \\
0.6 \% \\
0.25 \% \\
0.3 \% \\
0.3 \%
\end{gathered}
$$

$1.2 "(30 \mathrm{~mm})$

$0.4 \%$ (Coarse Grid)

$0.9 \%$

$0.4 \%$

$1.6 \%$

$2.2 \%$

$3.4 \%$

$1.2 \%$

$5.0 \%$

$6.0 \%$

\subsubsection{Basemat Liftoff Phenomenon}

One of the dominant features of the $1: 6$ scale model response at high pressure (>120 psi, $0.83 \mathrm{MPa}$ ) is basemat liftoff. The liner strain concentration at the wall-basemat juncture (which we regard as the most critical failure location) seems to be partially driven by the basemat liftoff mechanism. The liftoff behavior was predicted in all of the global axisymmetric analyses, and it was discussed briefly in Section 4.3.4. The most refined basemat model is the C4B grid which shows liftoff at 140 psi (.97 MPa) in Figure 4.3.19. The progression of predicted basemat liftoff, from zero liftoff at less than $20 \mathrm{psi}(.14 \mathrm{MPa})$ to 1 inch $(25 \mathrm{~mm})$ at 140 psi (.97 MPa), is summarized in Figure 4.3.20. The figure shows vertical upward displacement profiles along the bottom of the basemat from the center to the outer edge. For better visual comparisons, the logarithms of displacements are plotted; therefore, points with negative (compressive) or zero

displacement fall on the bottom axis $\left(10^{-4}\right)$ of the plot. Moving out toward the edge, as soon as the basemat is no longer in contact (i.e., liftoff greater than $10^{-4}$ ), positive displacements are plotted. The intersection of 
each curve with the $10^{-4}$ displacement axis shows the edge of the basemat contact pattern.

The large liftoff beginning at approximately $130 \mathrm{psi}$ (.90 MPa) could actually be called failure of the basemat since such large displacements (> 0.5 inches, $13 \mathrm{~mm}$ ) for a model of $1: 6$ scale would represent displacements in an actual containment that are probably larger than would be allowed for in the engineering of the containment system. It should be noted, however, that this is an unsupported opinion of the authors of this section. For the $1: 6$ scale model with no pipe or electrical systems running in and out of the containment, it is possible for the model to survive to pressure levels 10 or $20 \mathrm{psi}$ (.07 to .14 MPa) higher. However, loss of pressure to liner rupture at the liner/knuckle connection is predicted to precede excessively large liftoff displacement.

Examination of the section stresses and strains across the first and second vertical rows of elements next to the symmetry axis of the C4B analysis reveals that large liftoff is triggered by the yielding of the basemat bottom reinforcement. The basemat acts as a deep slab built in at the axis of symmetry and is acted on by distributed foundation force on the bottom which resists the dead load, distributed downward force on the top (pressure) and a very large concentrated upward force where the wall is attached. The distance between this upward force and the line of action of the pressure on the basemat causes a large moment couple. The bottom of the basemat will be in high tension and the top will be in high compression.

The basemat bottom reinforcement consists of only \#6 bars at 4 inches (102 $\mathrm{mm}$ ) on center each way in a rectangular pattern. Not including the top reinforcing, this represents a percentage of tension steel of only 0.24 percent on a typical radial line, which is a small tensile reinforcement ratio. This is less than the minimum required by the ACI code for flexural members. ACI 318-83 Section 10.5 requires $\rho_{\min }=200 / \mathrm{fy}=0.33$ percent. Comparison can be made to basemats of prototypical reinforced containments analyzed in [4.3.2] where a typical full scale containment basemat had a radius of approximately $75 \mathrm{ft}(22.9 \mathrm{~m})$ and a thickness of nine $\mathrm{ft}(2.7 \mathrm{~m})$ with a three $\mathrm{ft}(0.9 \mathrm{~m})$ overlay on top of the liner for a total thickness of $12 \mathrm{ft}(3.7 \mathrm{~m})$. This basemat had bottom reinforcement as follows. At a radius greater than $30 \mathrm{ft}(9.1 \mathrm{~m})$ : three radial layers of \#18 bars with 12 in $(.30 \mathrm{~m})$ spacing and 1 hoop layer of \#18 bars with 8 in $(0.20 \mathrm{~m})$ spacing. Inside $30 \mathrm{ft}(9.1 \mathrm{~m})$ radius: one layer each way in a rectangular pattern at 8 in $(0.20 \mathrm{~m})$ spacing. This gives reinforcement ratios of 0.37 percent with a radius less than $30 \mathrm{ft}(9.1 \mathrm{~m})$ and 0.74 percent elsewhere. The geometry of the prototype [4.3.2] is representative of many existing containments but is not intended to be exactly patterned after any one containment. Thus, the 1:6 scale model is highly under-reinforced as a flexural member, yet its flexural response may help trigger the governing failure mode.

Additional contributions to the liftoff phenomena, although to a lesser extent, come from the moment formation at the base of the wall, and the compressive crushing of the concrete at the top of the basemat in the section near the symmetry line. Neither of these effects is as great as the loss of tensile strength in the bottom of the basemat at the symetry line. With regards to the first effect, at pressures greater than 130 ps $i \quad(.90$ $\mathrm{MPa}$ ) as the basemat starts to lift up significantly, higher bending moment 
occurs across the base of the wall, and as the moment carrying capacity at this location decreases (through concrete crushing or yielding rebar), the structure offers less resistance to uplift. In the second effect, concrete crushing at the top directly affects the moment carrying capacity of the bending section at the symetry line. In the $C 4 B$ analysis, the top surface concrete, or in beam bending terms, the extreme fiber concrete, reaches ultimate effective stress between 135 and $140 \mathrm{psi}$ (.93 and .97 MPa).

As mentioned in Section 4.3.3, all of the axisymmetric analyses utilized a concrete compressive plasticity model with no strain softening. This allowed the concrete to maintain its compressive strength regardless of how much the symetry axis bending section rotates as a plastic hinge. Consequently, after $140 \mathrm{psi}(.97 \mathrm{MPa})$ the liftoff solution was still tractable up to 160 psi ( $1.10 \mathrm{MPa})$, although by this point liftoff reached 2.5 inches $(64 \mathrm{~mm})$ which is an unrealistic situation to occur without loss of test pressure. The solution was stopped there because the liner reached its ductility limit next to the basemat juncture knuckle. More recently C4B was re-analyzed with the compressive strain softening model; the analysis could not be continued past $140 \mathrm{psi}(0.97 \mathrm{MPa})$ because of a divergent solution even though load increment sizes were reduced. The source of the divergence was loss of moment capacity next to the symetry line as the compressive concrete strain softened. Even though the analysis with the strain softening model failed between 135 and $140 \mathrm{psi}(.93$ and $.97 \mathrm{MPa}$ ), the other results are still useful for ranking failure locations. The strain softening model gives even stronger evidence that basemat failure may dominate the model failure.

\subsubsection{Liner Anchorage}

Based on the detailed analyses described herein, the mechanism that will most likely lead to depressurization is liner tearing at the liner-knuckle connection of the wall-basemat juncture. This failure mode, discussed in detail in Section 4.3.4.3, is linked to basemat liftoff, discussed in Section 4.3.6.2. However, a structural component that controls when and where the liner will fail in this region is liner anchorage. The effect of mechanical bonding of the liner to the concrete has been the subject of much analytical and experimental research [4.3.1, 4.3.2], which has shown that substantial local liner strains can occur across a dislocation crack because of the effectiveness of liner anchorage. For example, for a containment wall specimen prototype in [4.3.2], it was shown that a $0.4 "(10 \mathrm{~mm})$ dislocation motion in the region of a stiffness discontinuity caused a liner strain well above the liner rupture limit, while the same dislocation produced a maximum rebar strain of only 0.75 percent. Much of the research of the effects of liner anchorage has been for prestressed containments which utilize welded angles and channels for anchorage. However, a full scale specimen of a section of reinforced concrete containment wall with large pipe penetration was recently tested [4.3.11], which demonstrated the effectiveness of welded stud anchorage. Effective stud anchorage in typical containments can be partially attributed to the cormon design practice of extending the studs beyond the innermost layers of main wall reinforcing. In the case of the $1: 6$ scale model at the juncture region, anchorage is provided by 0.148 " diameter $\times 0.5^{\prime \prime}$ long $(3.8 \times 12.7 \mathrm{~mm})$ welded studs spaced in a 2 " $\times 2$ " $(51 \mathrm{~mm} \times 51 \mathrm{~mm})$ grid pattern starting at elevation $z=2 ' 2^{\prime \prime}$ or 0.81 inches $(20.6 \mathrm{~mm})$ above the top of the knuckle. The first layer of rebars is located $1-1 / 2$ inches $(38.1 \mathrm{~mm})$ in from the liner so the $1: 6$ scale model 
studs do not penetrate the rebar cage. Figure 4.3 .9 showed that this first 0.81 inches $(20.6 \mathrm{~mm})$ of liner is subjected to severe bending and stretching and that the liner tends to peel away from the cylinder wall starting at the knuckle and moving up. The peak strain next to the knuckle is greatly affected by the anchorage effectiveness just above the knuckle. The other liner-knuckle strain concentration is at the bottom of the knuckle, but since there are no studs in the basemat liner, the stud design has little effect on the strains at that location.

As a result of the relatively weak liner stud anchorage, high liner-knuckle strains in the 1:6 scale model occur, as reported. relatively late in the test. With stronger liner anchorage, liner strains would be more localized and cause higher strain concentration factors. It is hoped that this critical failure mode will not be eliminated from the test by the stud design details. In order to evaluate this effect, the as-built welded stud modeling of the CAB model in Section 4.3.4 was replaced with beam elements with the same cross-sectional properties as the as-built studs, but extending 2 inches $(51 \mathrm{~mm})$ into the containment wall, past the first layer of hoop and meridional reinforcing. This quick and rough analys is is not sufficientiy detailed for a definitive evaluation of stud anchorage effects, but it is adequate for the present purposes. This revised model was analyzed with the same load history as C4B and produced virtually the same displacement and strain histories everywhere in the model except for the first inch above the liner knuckle connection. At that location, liner effective strain from $C 4 B$ at 140 psi (0.96 MPa) was 6 percent; for the revised analysis the strain increased by 50 percent to 9 percent. A larger increase would probably be observed if the elevation of the lowest stud were made closer to the top of the knuckle, thus further concentrating the strain. The strains at the bottom of the knuckle near the weld singularity discussed in Section 4.3.4 were unchanged. Lowering the elevation of the lowest stud close to the top of the knuckle could also reduce the strain at the bottom of the knuckle by decreasing the pivoting of the knuckle about its base, thus making the knuckle top strain concentration the controlling rupture location.

\subsubsection{Pretest Prediction Conclusions}

This section is a brief summary of the expected behavior during the 1:6 scale model test. The key features of the structure response are outlined in Table 4.3.4 in order of milestones reached with increasing pressure. Following the table is the statement of the most likely cause of pressure loss (or failure) in the test. Also included with this is a brief ranking of less likely failure modes.

Failure Prediction

1 - Local Liner Rupture. This is the primary focus of this research effort. There are so many large strain concentration locations that the probability of reaching rebar failure without a liner tear is extremely low, i.e., while the probability of depressurization by $180 \mathrm{psi}(1.24 \mathrm{MPa})$ is approximately 1.0 , the probability at $140 \mathrm{psi}(.97 \mathrm{MPa})$ is also very high (perhaps 0.95$)$. In a comprehensive probabilistic failure study such factors as construction defects and material flaws should be taken into account, but that is beyond the scope of the current research. This is especially pertinent because the structure's most severe liner strain concentrations occur at liner seams, 
and often at field welded seams, which significantly increases the chance of premature leakage by the presence of weldment flaws or reduced ductility along welds. Nevertheless, short of a flaw occurring at a secondary strain concentration location (listed in item 4 below), initial liner rupture is most likely to occur at the wall basemat juncture between 140 and 150 psi (.97 and 1.03 MPa). (See Section 4.3.4 for more detailed discussion of this mechanism.) Liner rupture is predicted to be localized at first, but widespread in $\theta$ soon thereafter (within a few psi), so that leakage will be larger than the test pressurization capability. However, no leak rate estimate has been made. This failure is linked to the failure of the inner

\section{Table 4.3.4 Summary of Key Features of Expected Behavior}

Pressure

psi (MPa) Milestone Description

$30(.21)$ Local concrete cracking near the wall-basemat juncture begins due to shear and bending there.

$40(.28)$ Concrete cylinder hoop cracking begins at cylinder mid-height.

50 (.34) Full length of cylinder wall is cracked in hoop direction.

$60(.41)$ Onset of local liner yielding next to knuckle (primary strain component is meridional).

$70(.48)$ Onset of local liner yielding nest to $8^{\prime \prime}$ penetration and 4" pressure nozzle (hoop).

$80(.55)$ Full length of dome cracks in hoop direction.

85 (.59) Onset of local liner yielding next to equipment hatches (hoop).

$90(.62)$ Onset of local liner yielding next to personnel airlock at $90^{\circ}$.

$95(.66)$ Global cylinder liner yielding.

$100(.69)$ Global meridional concrete cracking.

$120(.83)$ Onset of hoop rebar yielding starting at cylinder midheight, midway between hatches.

130 (.90) Basemat bottom bars yield, triggering large basemat liftoff.

$135(.93)$ Onset of global meridional rebar yielding.

140 (.97) High probability of liner rupture next to liner/knuckle connection accompanied by knuck.le tearing off backing bar and local welded stud failure at juncture.

150-160 Possibility of liner ruptures developing in order of list in Conclusion \#4 below, but there is a low probability of reaching this pressure. 
basemat in bending and to the construction details of the welded studs, which are very short and do not penetrate the main reinforcement cage. It is also linked to the construction details of the liner/knuckle connection.

2 - Basemat Failure. The bottom bars in the basemat yield between 130 and $135 \mathrm{psi}(.90$ and $.93 \mathrm{MPa})$. This causes substantial increase in the incremental basemat liftoff so that at the basemat outer edge liftoff reaches $1 "(25 \mathrm{~mm})$ at $140 \mathrm{psi}(.97 \mathrm{MPa})$. Large basemat motion contributes heavily to liner failure potential at the wall-base juncture.

3 - Global Rebar Failure. As is the case with a typical containment, the 1:6 scale model is a complex structure where many different local response mechanisms act together to produce the total response. The "ultimate failure pressure" or "ultimate failure mode" cannot be predicted by analyzing a clean reinforced cylinder and dome cap and reporting the global rebar failure point. However, since the inclusion of other response components seems to cause local liner strain concentrations which act to lower the ultimate pressure that could be reached by a clean shell, global rebar failure serves as a good upper bound on containment overpressure or a point where the probability of pressure 1055 approaches 1.0 . The $1: 6$ scale model approaches this point at $180 \mathrm{psi}(1.24 \mathrm{MPa})$. At $160 \mathrm{ps} i(1.10 \mathrm{MPa})$, the hoop bars at cylinder mid-height reach a state where incremental pressure increases cause large radial displacement increases.

4 - Ranking of Liner Rupture Locations. Again, these predictions are predicated on the assumptions of perfect seams and materials, especially around hatches and penetrations where seams are complicated and include $1 / 16^{\prime \prime}(1.59 \mathrm{~mm})$ material welded to $3 / 16^{\prime \prime}(4.76 \mathrm{~mm})$ material. Liner rupture locations are ranked from high to low probability of rupture as follows:

1. Wall liner to knuckle seam

2. Knuckle to basemat liner seam

3. Next to $1 / 16 "$ to $3 / 16 "(1.59$ to $4.76 \mathrm{~mm})$ seam at $30^{\prime}$ clock or 9 $0^{\prime c l o c k}$ position of the 8 " penetrations

4. Same for 4" pressure nozzle connection

5. Same for equipment hatch $A$

6. Same for equipment hatch $B$

7. Same for personnel airlock at $90^{\circ}$

8. Same for personnel airlock at $270^{\circ}$

9 . Springline seam of $1 / 16^{\prime \prime}\left(1.59 \mathrm{~mm}\right.$ material to $1 / 12^{\prime \prime}(2.12 \mathrm{~mm})$ material

10. $1 / 16^{\prime \prime}$ to $3 / 16^{\prime \prime}(1.59 \mathrm{~mm})$ seam next to miscellaneous small penetrations

11. Vertical liner seam near cylinder mid-height (hoop failure)

12. Liner fracture next to a welded stud in first $1^{\prime}(30 \mathrm{~m})$ above wallbasemat juncture

13. Liner failure at the dome apex

14. Any liner seam in the dome

15. General liner failure away from a seam 


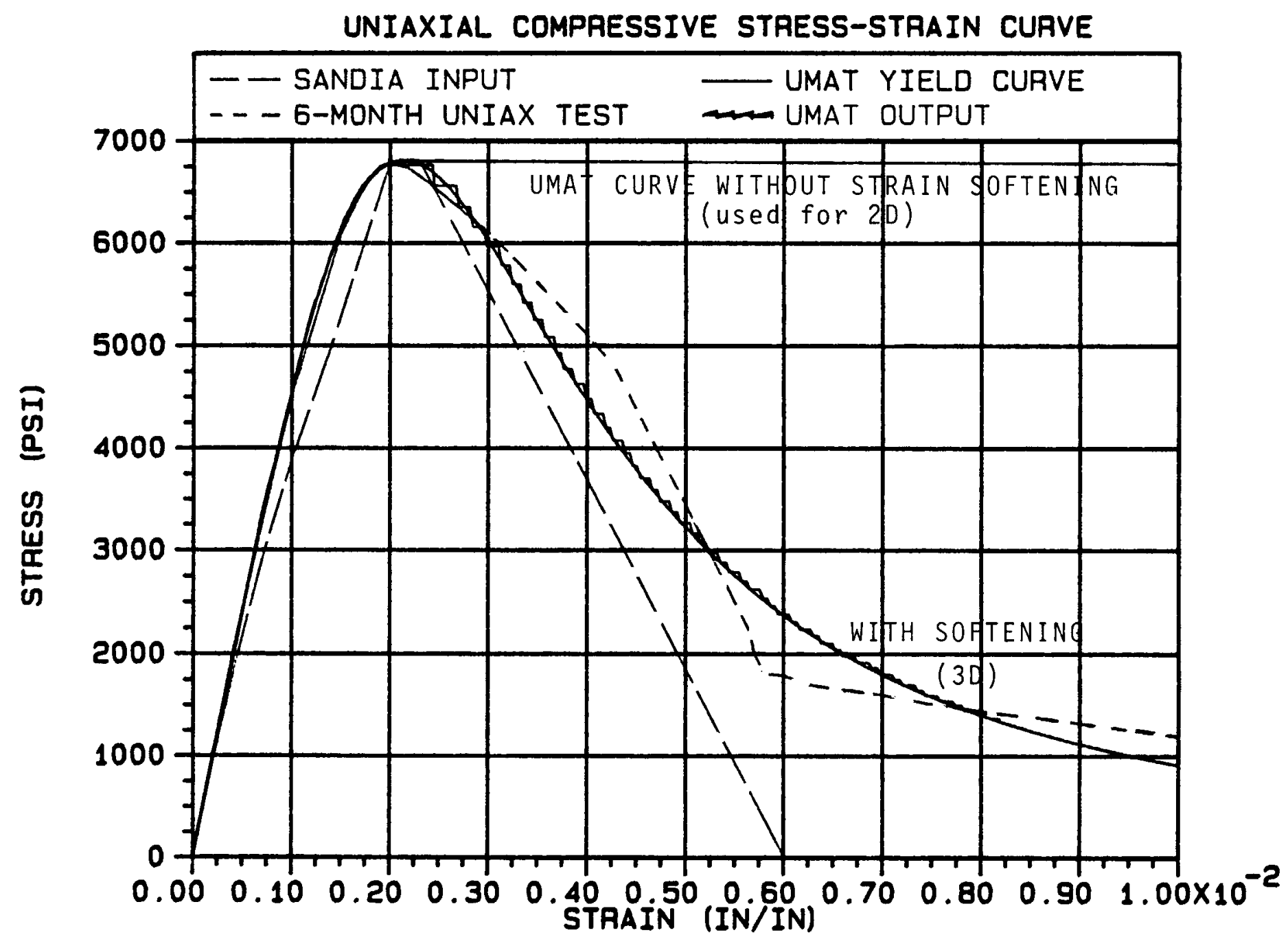

Figure 4.3.1 Compressive Uniaxial Stress-Strain 

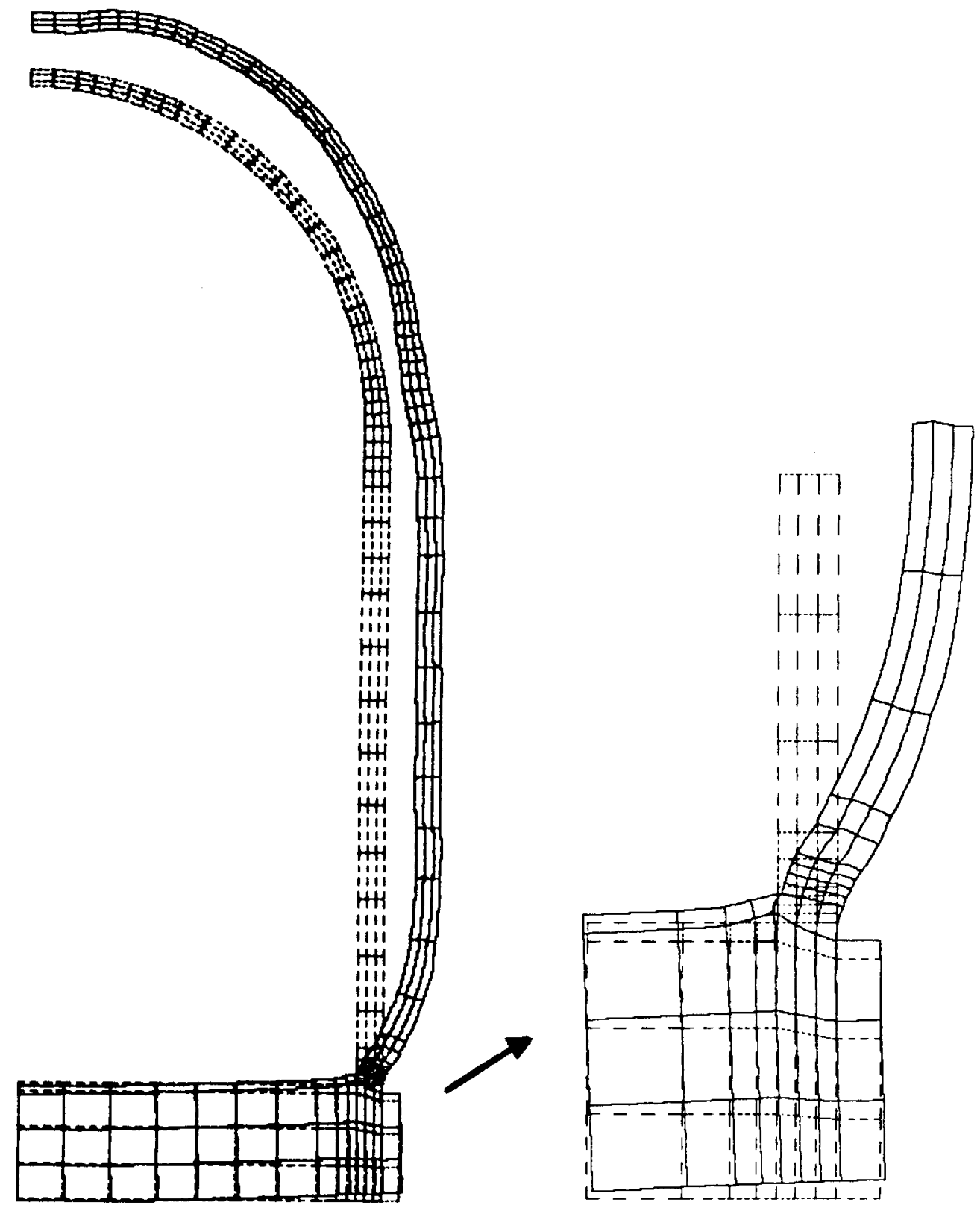

Figure 4.3.2 Analysis C-3 Deformed Grid at $100 \mathrm{psi}(0.69 \mathrm{MPa})$ 


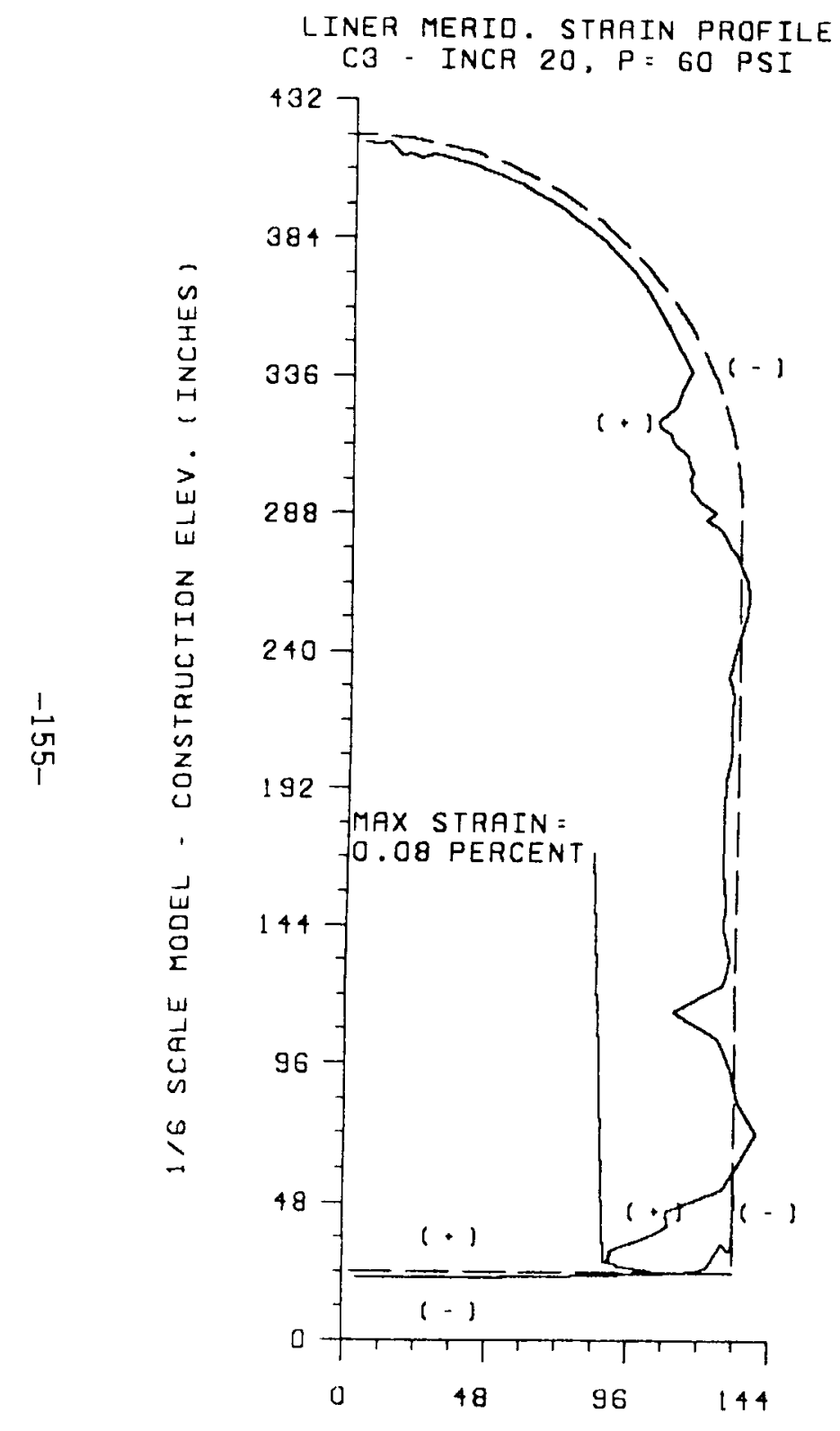
LINER MERID. STRAIN PROFILE
C3 - INCR $30, P=100$ PSI

LINER MERID. STRAIN PROFILE

C3 - INCR 40, $P=140$ PSI
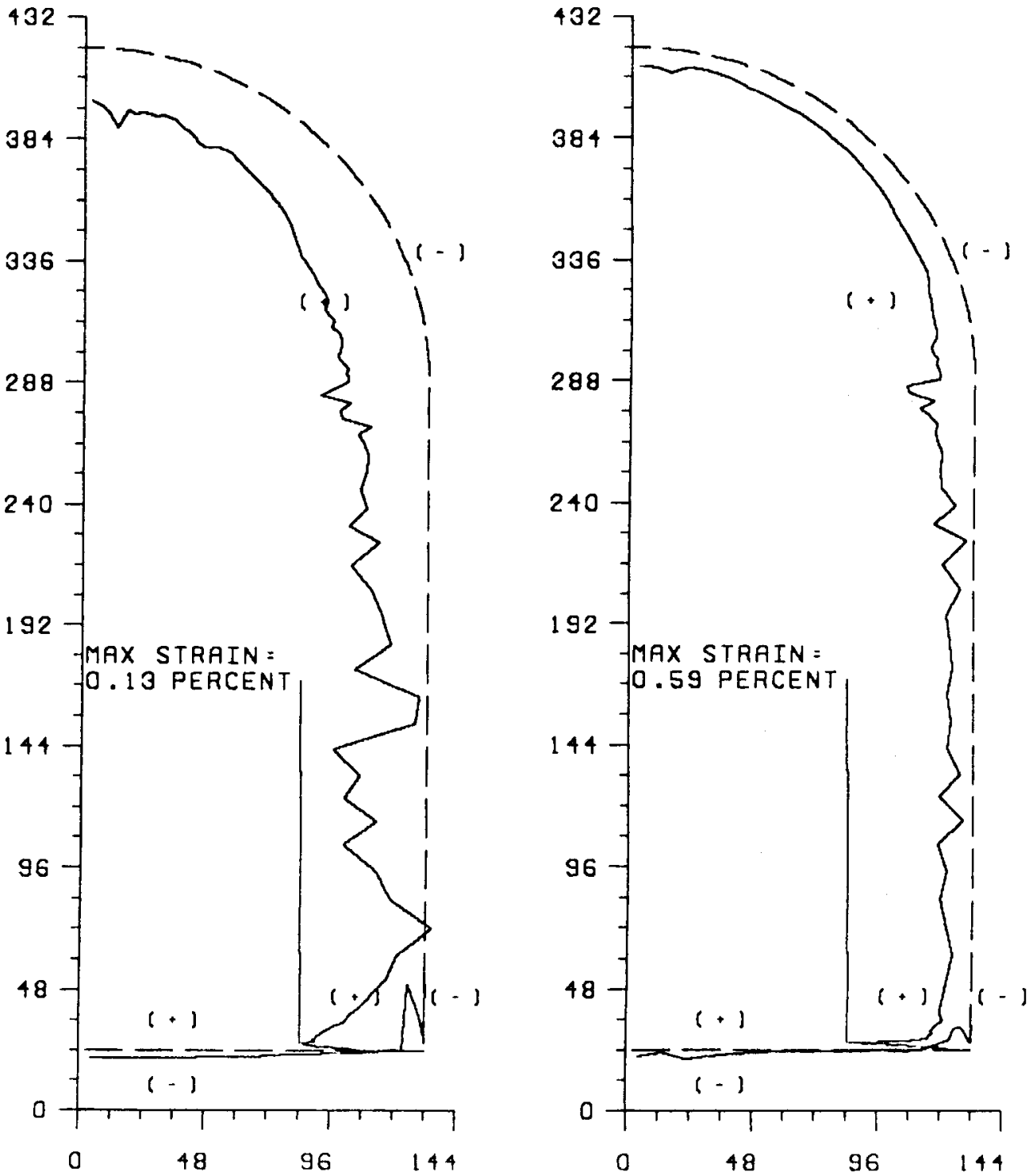

Figure 4.3.3 C-3 Analysis Liner Meridional Strain at 60, 100 , and 140 psi $(0.41,0.69$, and $0.97 \mathrm{MPa}$ ) 

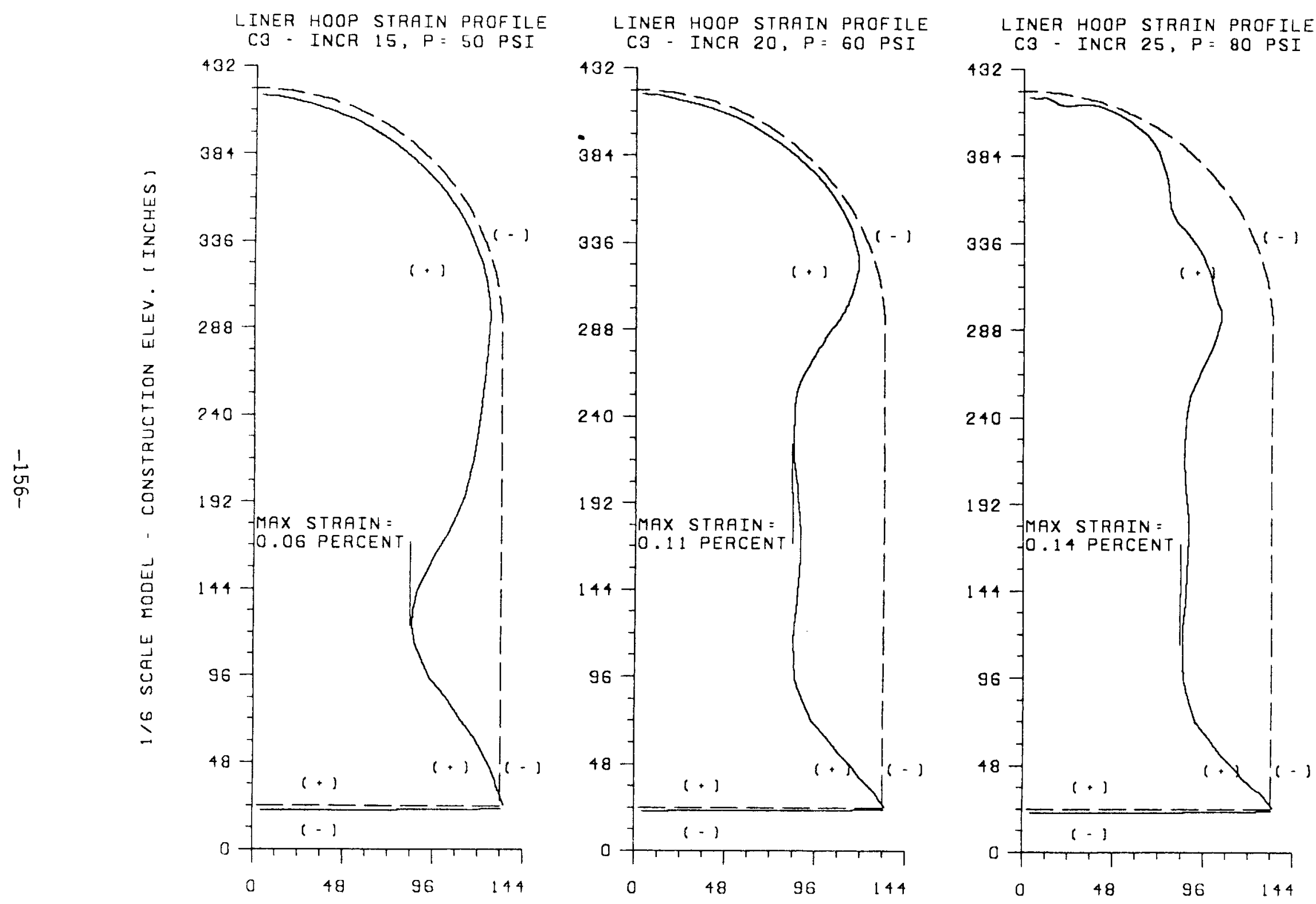

Figure 4.3.4 C-3 Analysis Liner Hoop Strain at 50, 60, and 80 psi $(0.34,0.41$, and $0.55 \mathrm{MPa})$ 

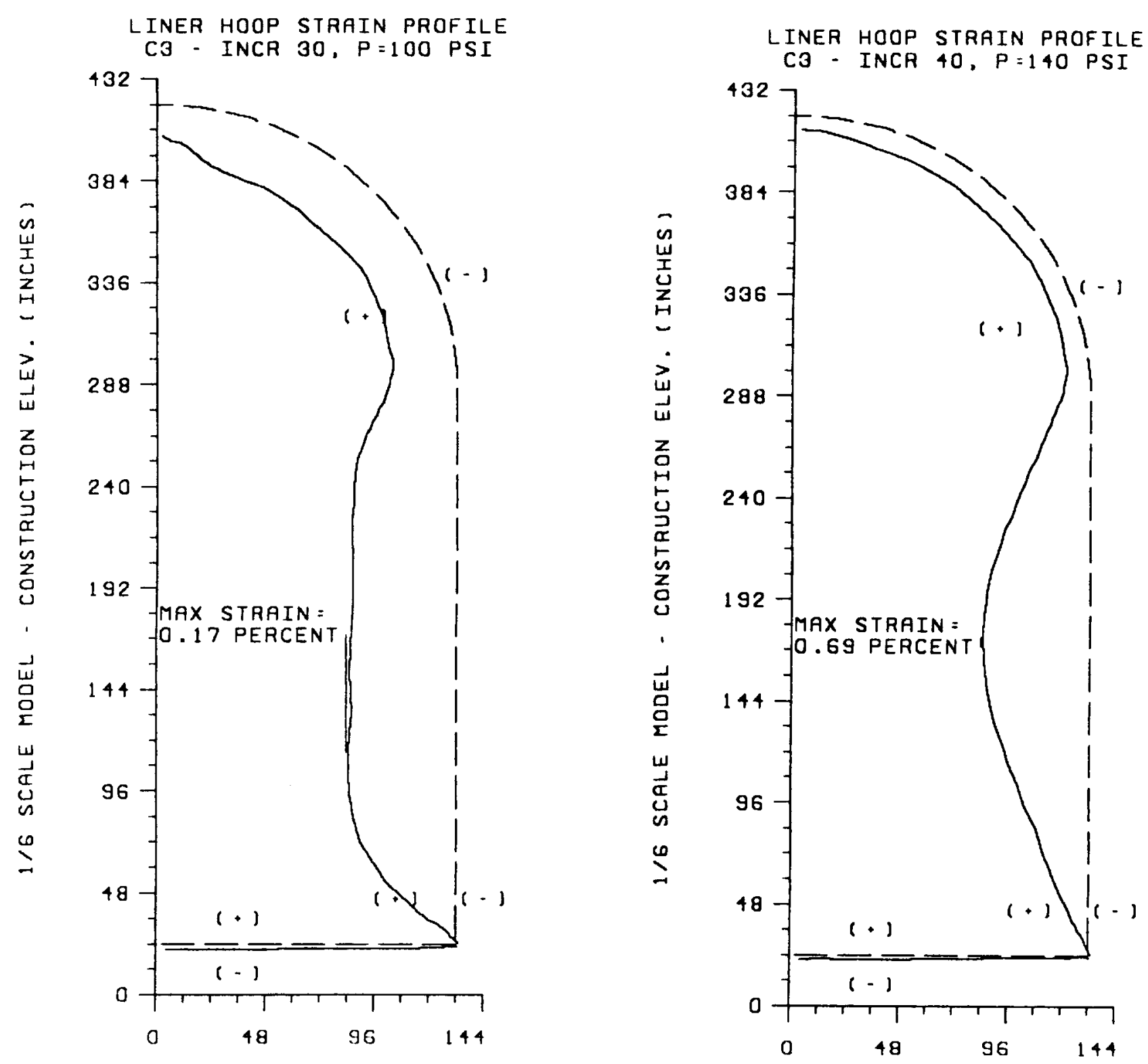

Figure 4.3.5 C-3 Analysis Liner Hoop Strain at 100 and 140 psi $(0.69$ and $0.97 \mathrm{MPa})$ 


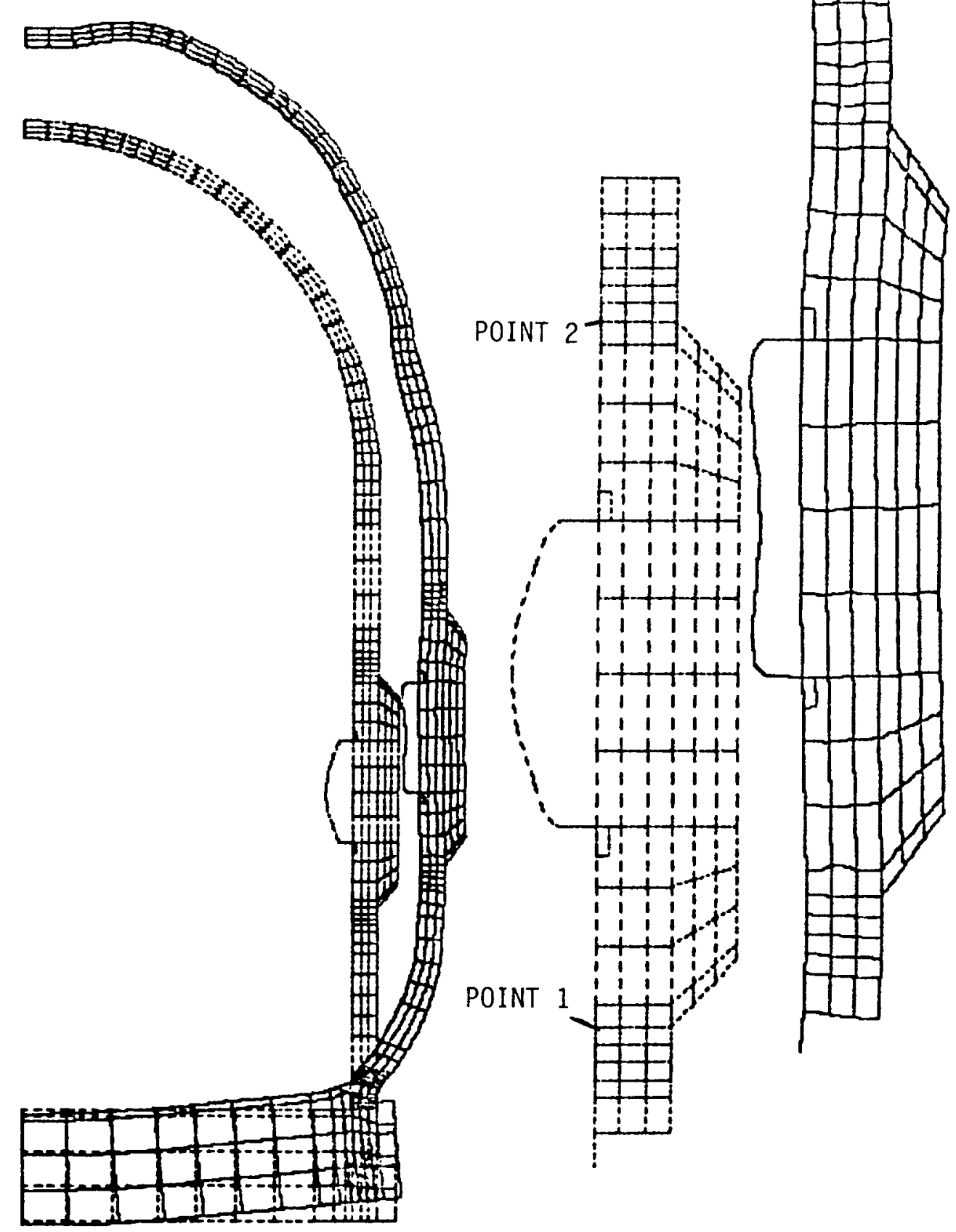

Figure 4.3.6 C3HA Analysis Deformed Grid at 120 psi (0.83 $\mathrm{MPa}$ ) 


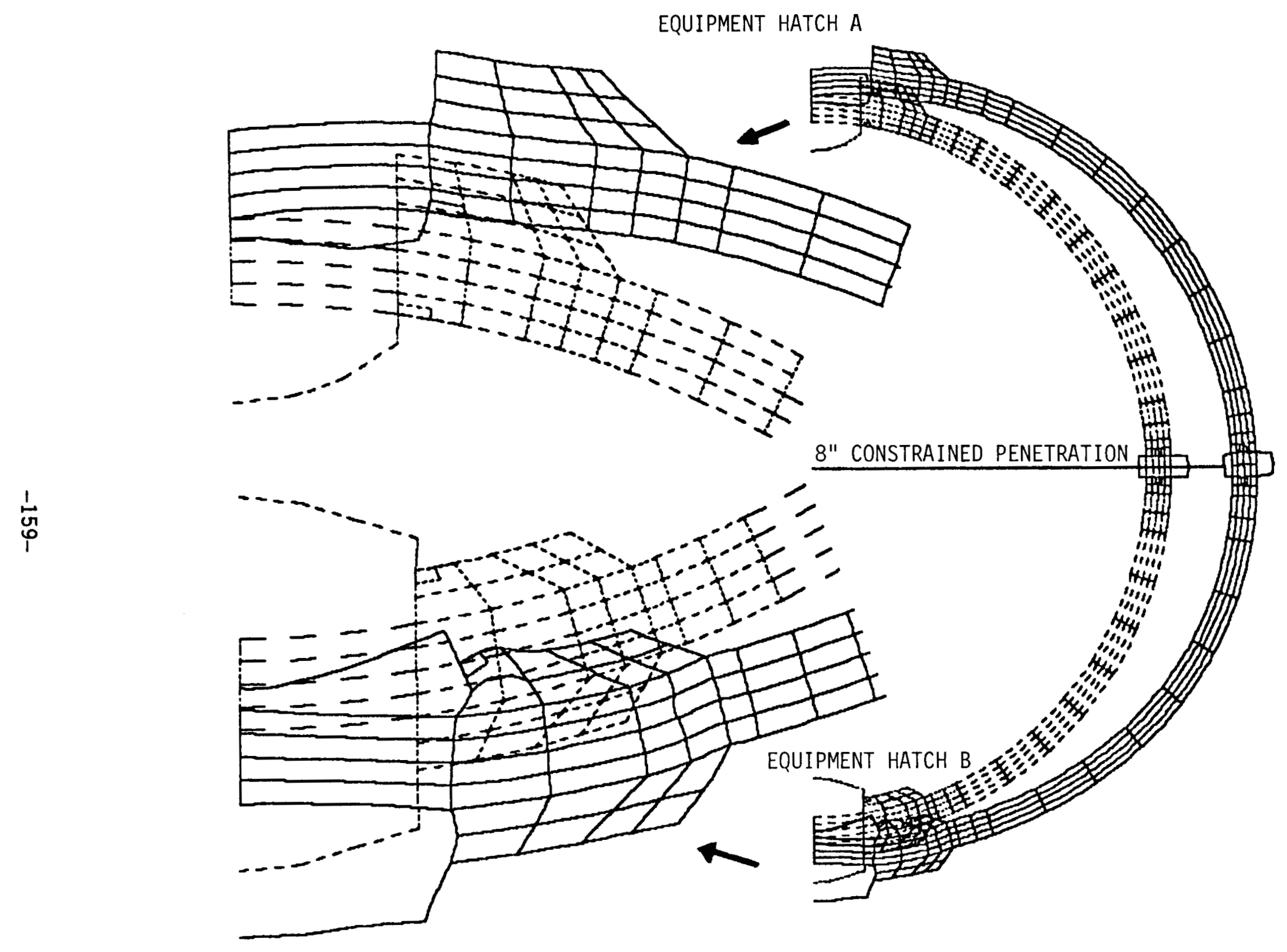

Figure 4.3.7 CRTA Analysis Deformed Grid at 100 psi (0.69 MPa) 

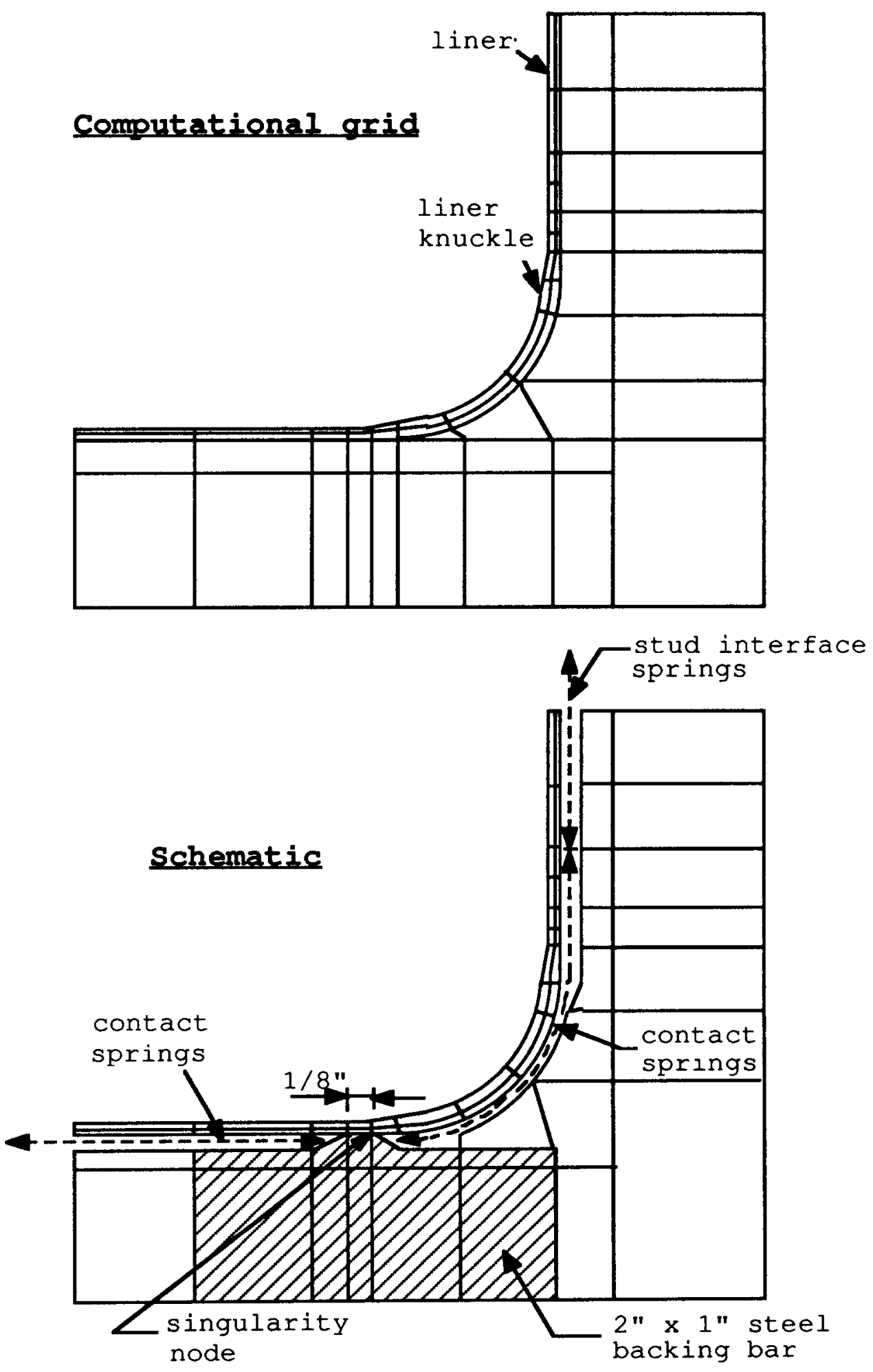

Figure 4.3.8 C4B Analysis Computational Grid 

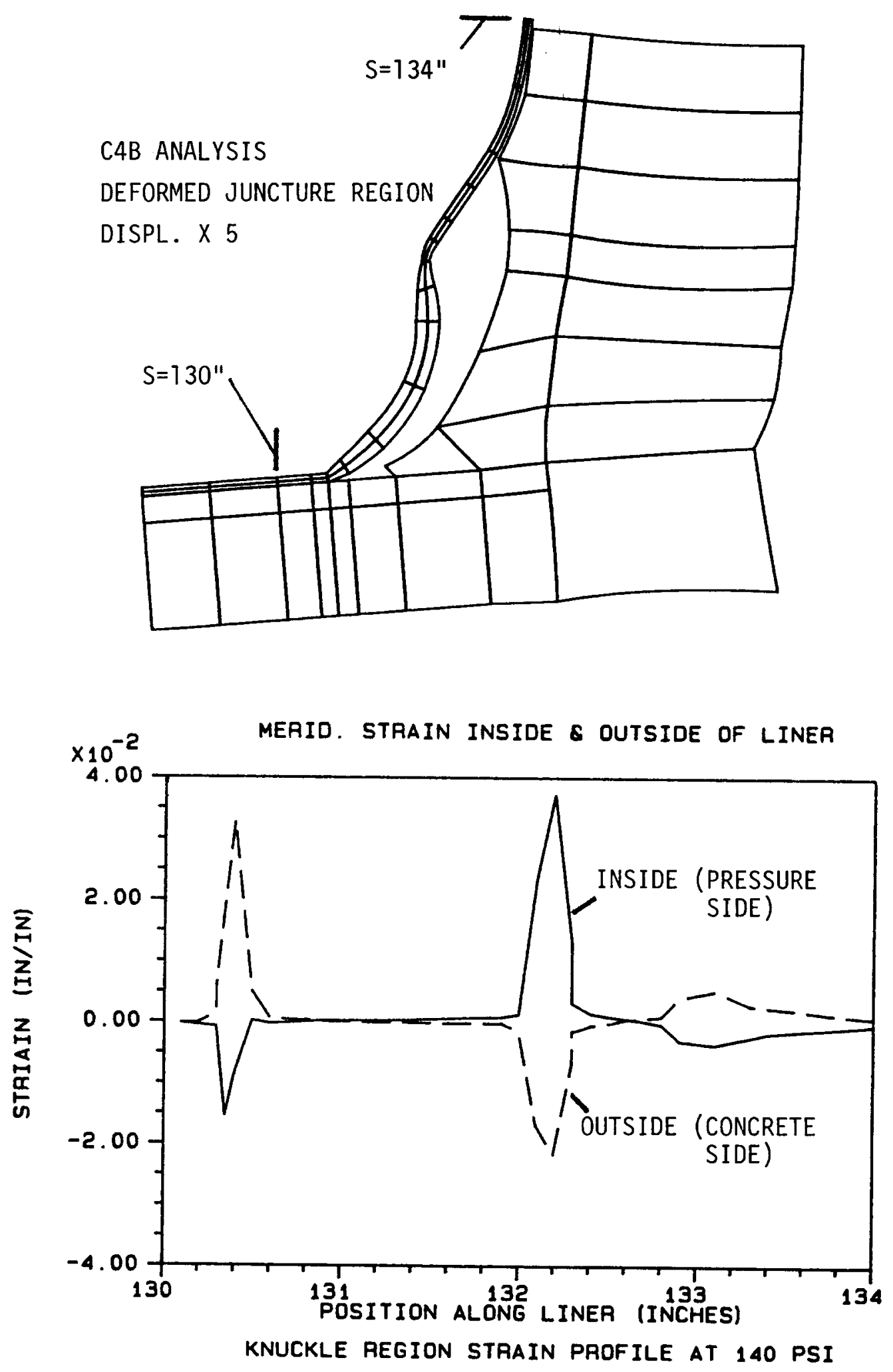

Figure 4.3.9 C4B Analysis - Deformed Shape and Meridional Strain Profile 

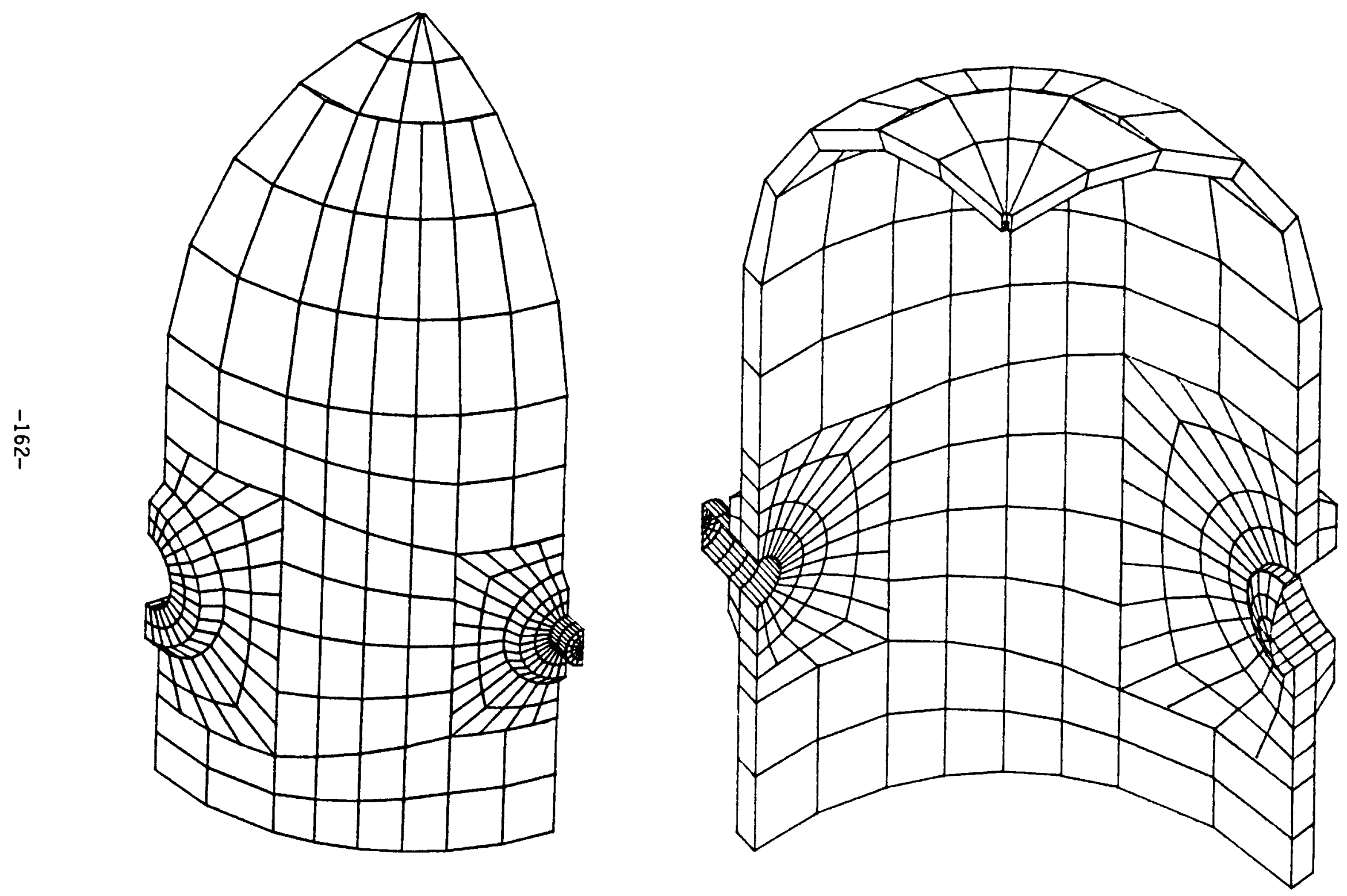

Figure 4.3.10 Outside and Inside Hidden Line View of Global Quarter Model Model Includes $270^{\circ}$ to $0^{\circ}$ and $E L=3^{\prime}-3^{\prime \prime}$ to the Dome Apex 


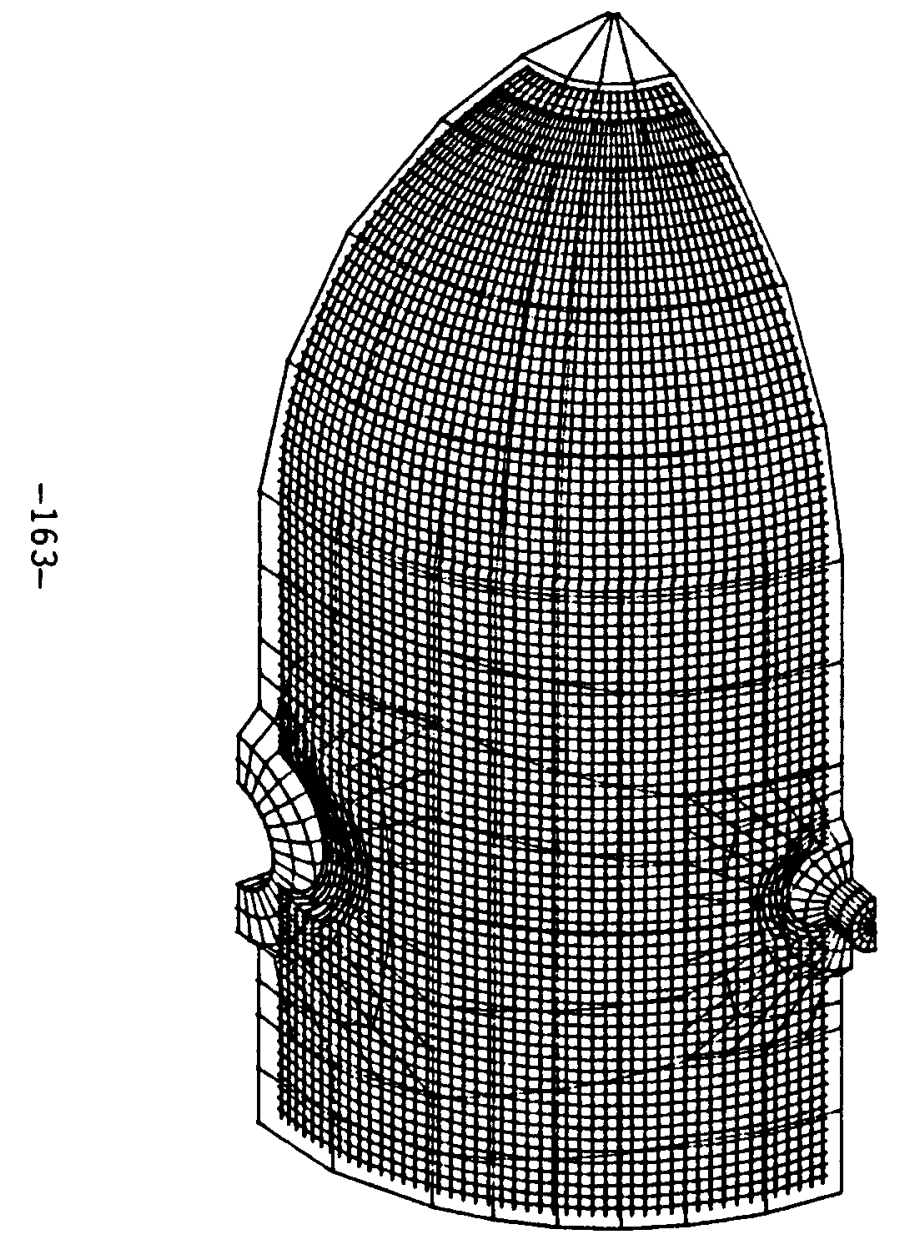

Inner Layer of Hoop \& Merid.

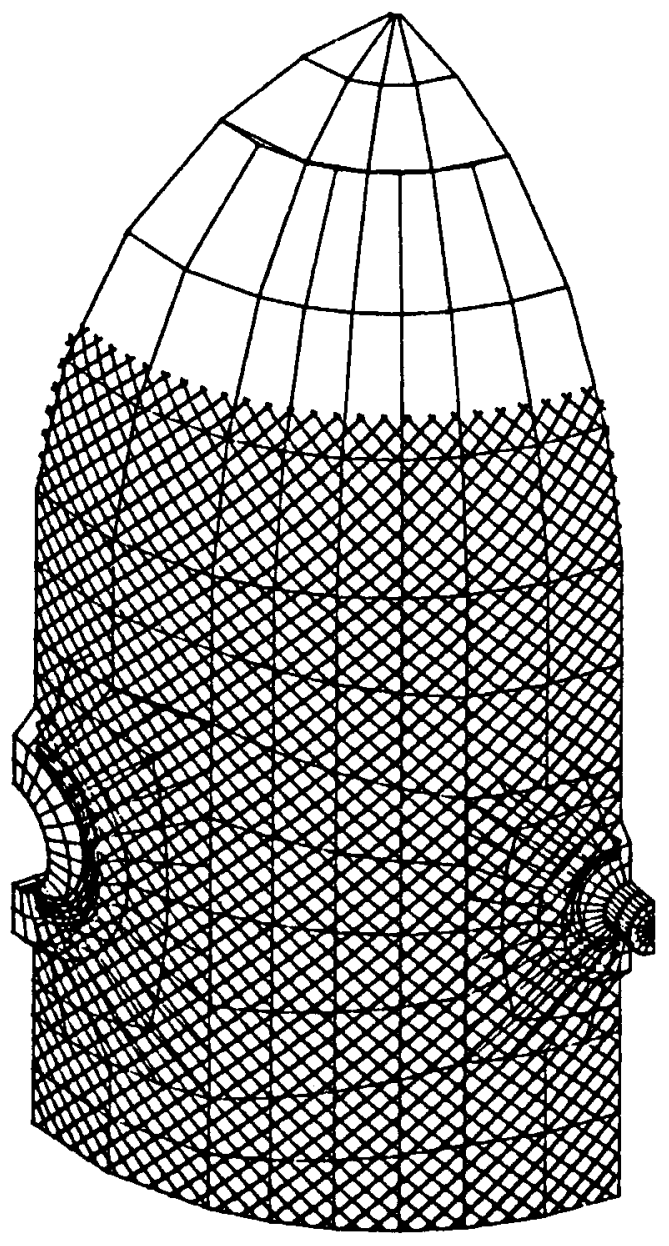

Seismic Layers

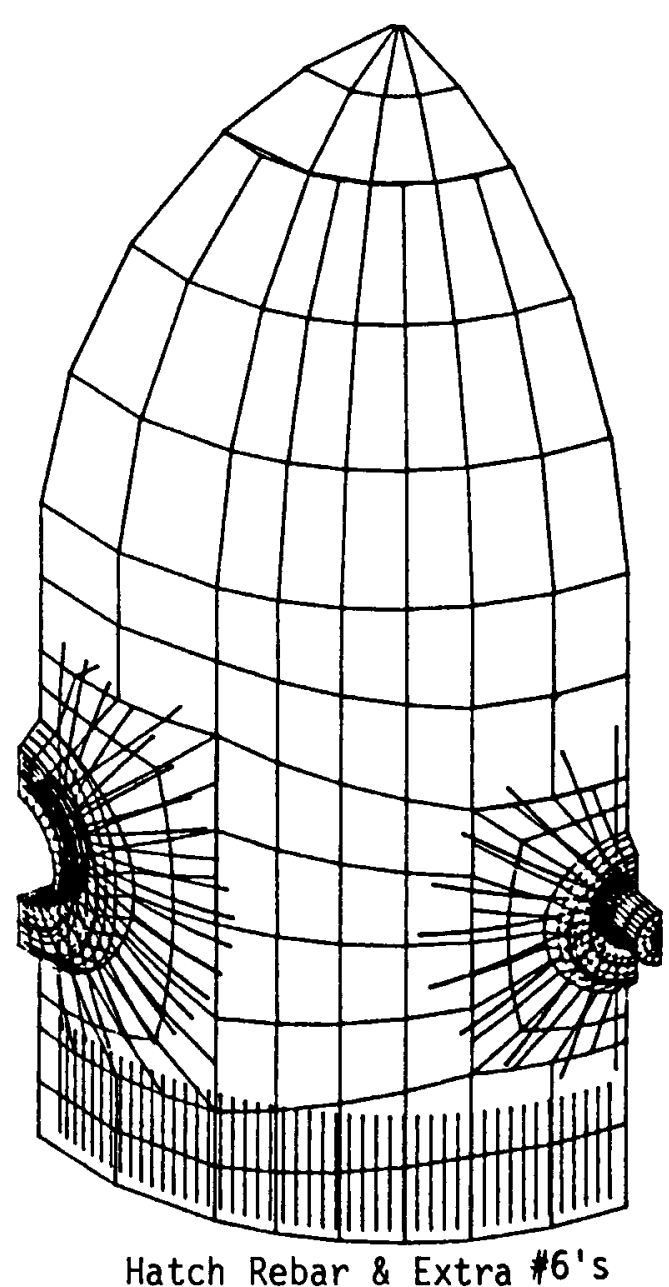

Hatch Rebar \& Extra "6's

Figure 4.3.11 Outside View of Quarter Model Grid With Hoop, Meridional, Seismic and Extra Rebar Layers 


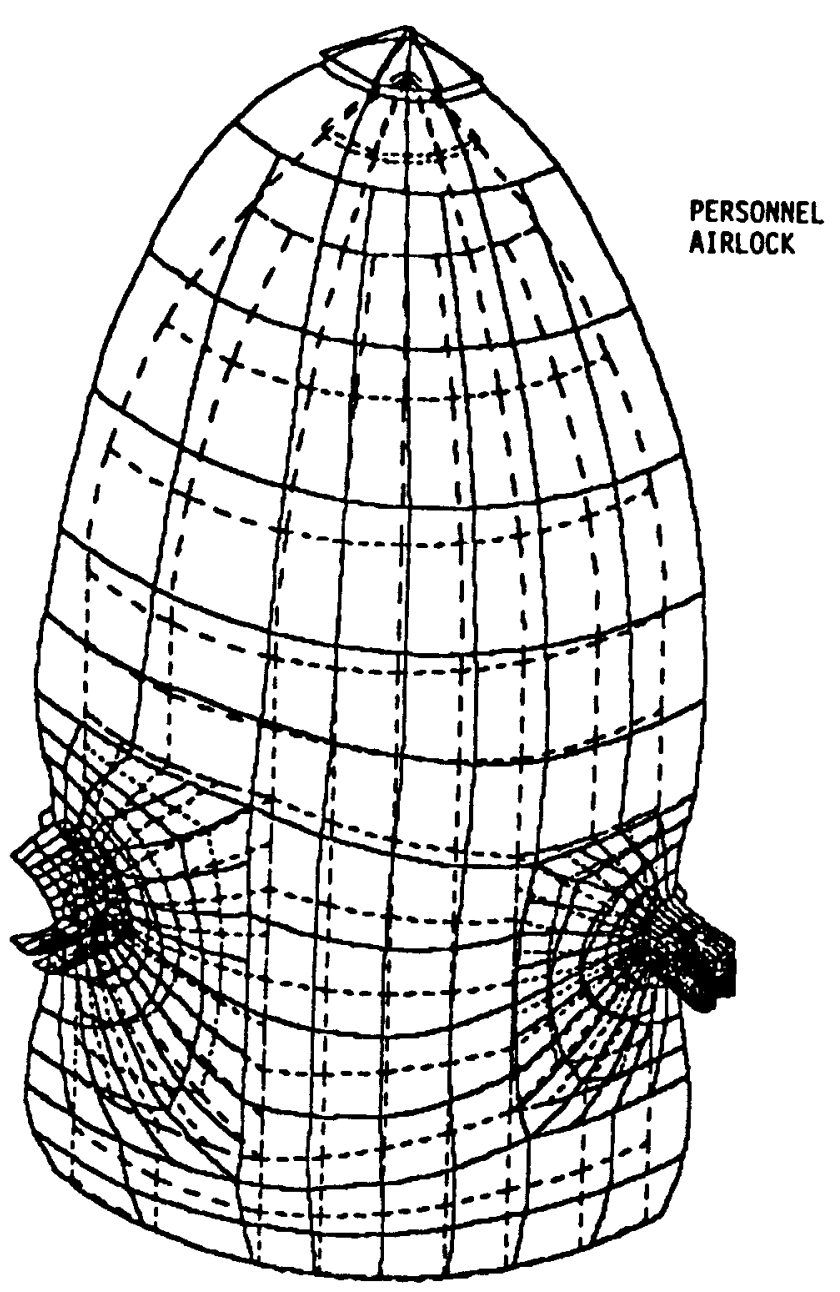

96 psi
Displ. X 100 Liner only

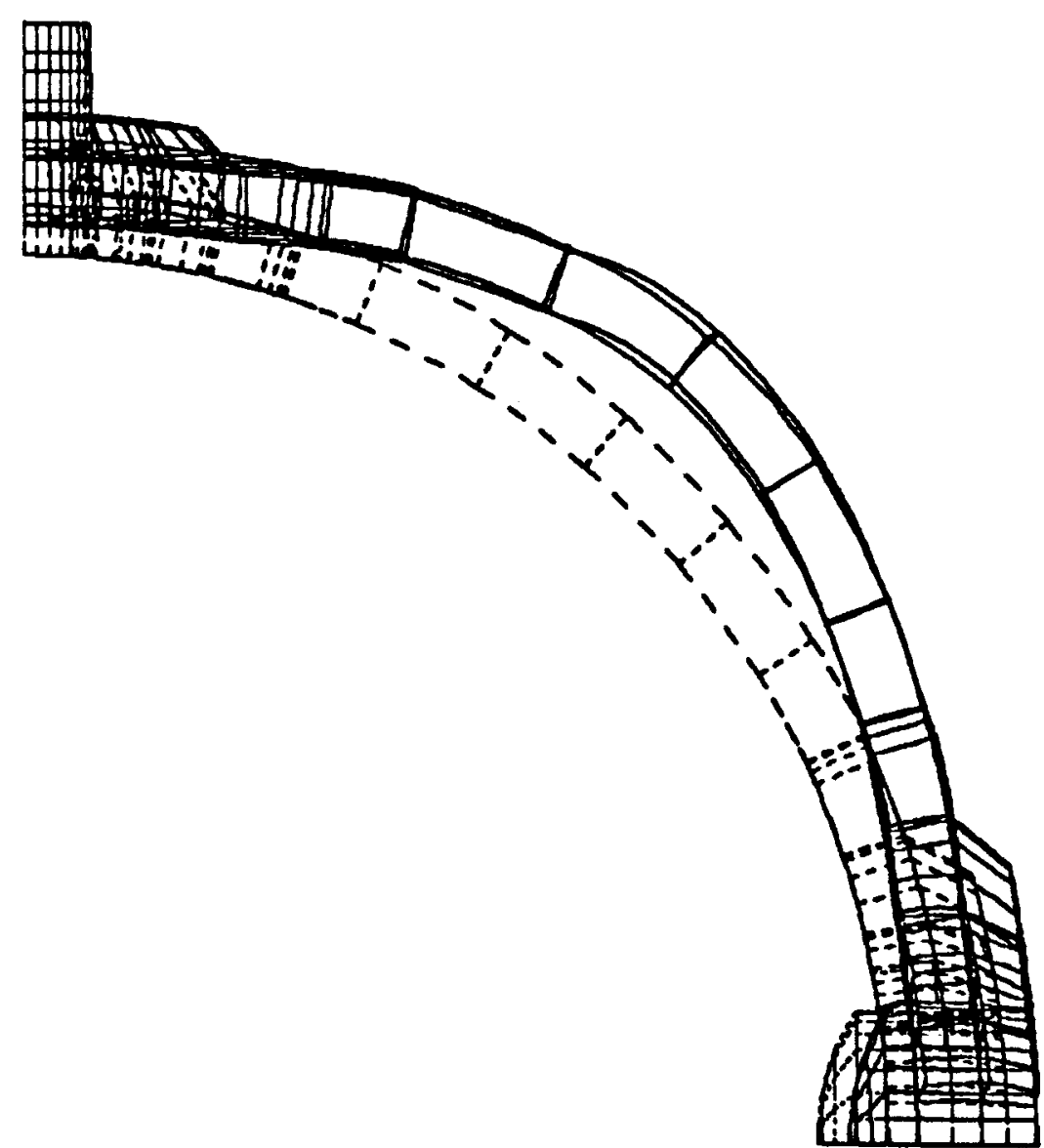

EQUIPMENT HATCH A

96 psi Displ. $\times 100$

Plan View $z=13^{\prime}-2^{\prime \prime}$

Figure 4.3.12 Global Quarter Model Deformed Shapes 


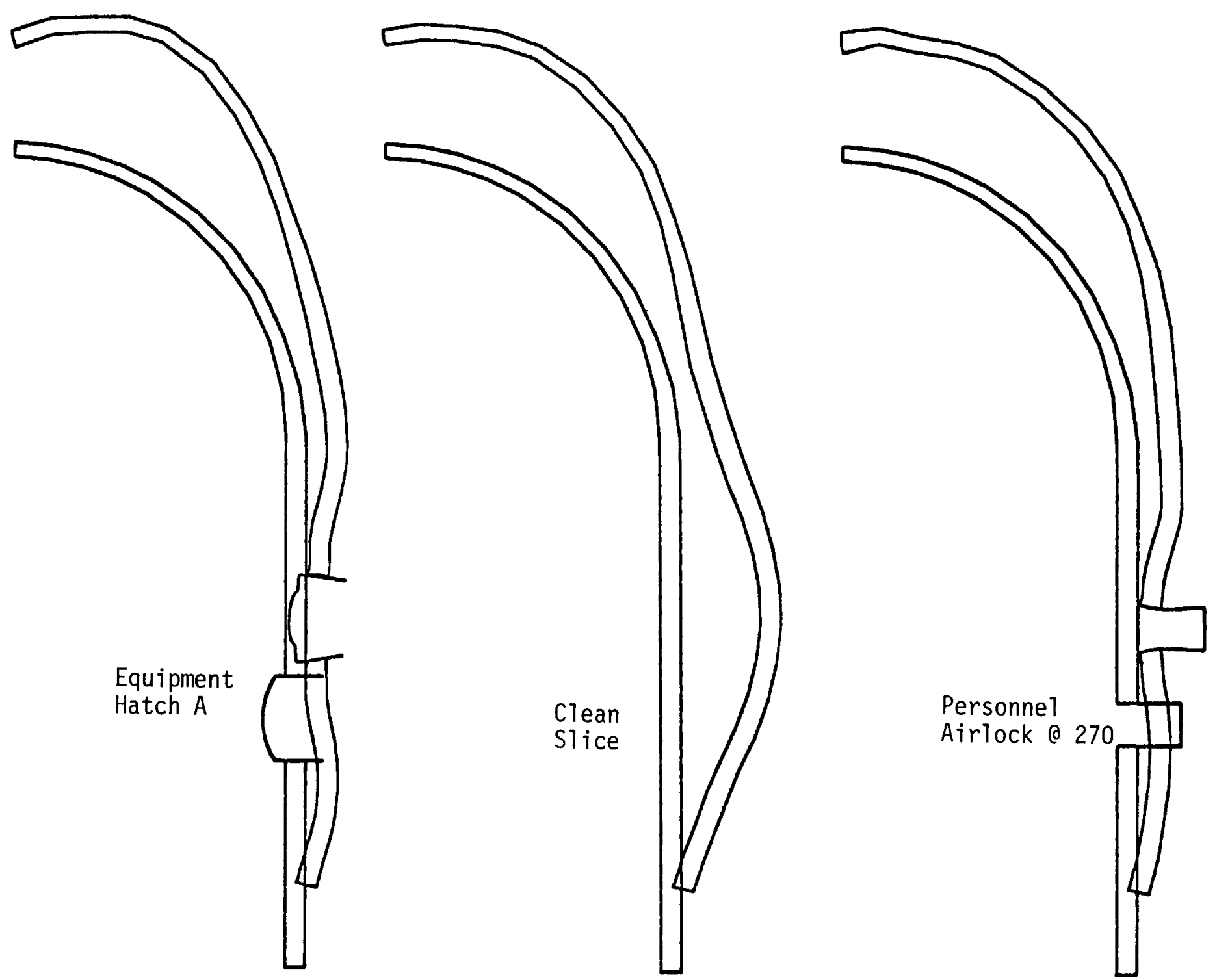

Figure 4.3.13 Deformed Concrete Outlines for $\theta=0^{\circ}, 315^{\circ}$, and $270^{\circ}$ at $136 \mathrm{psi}(0.94 \mathrm{MPa})$ 

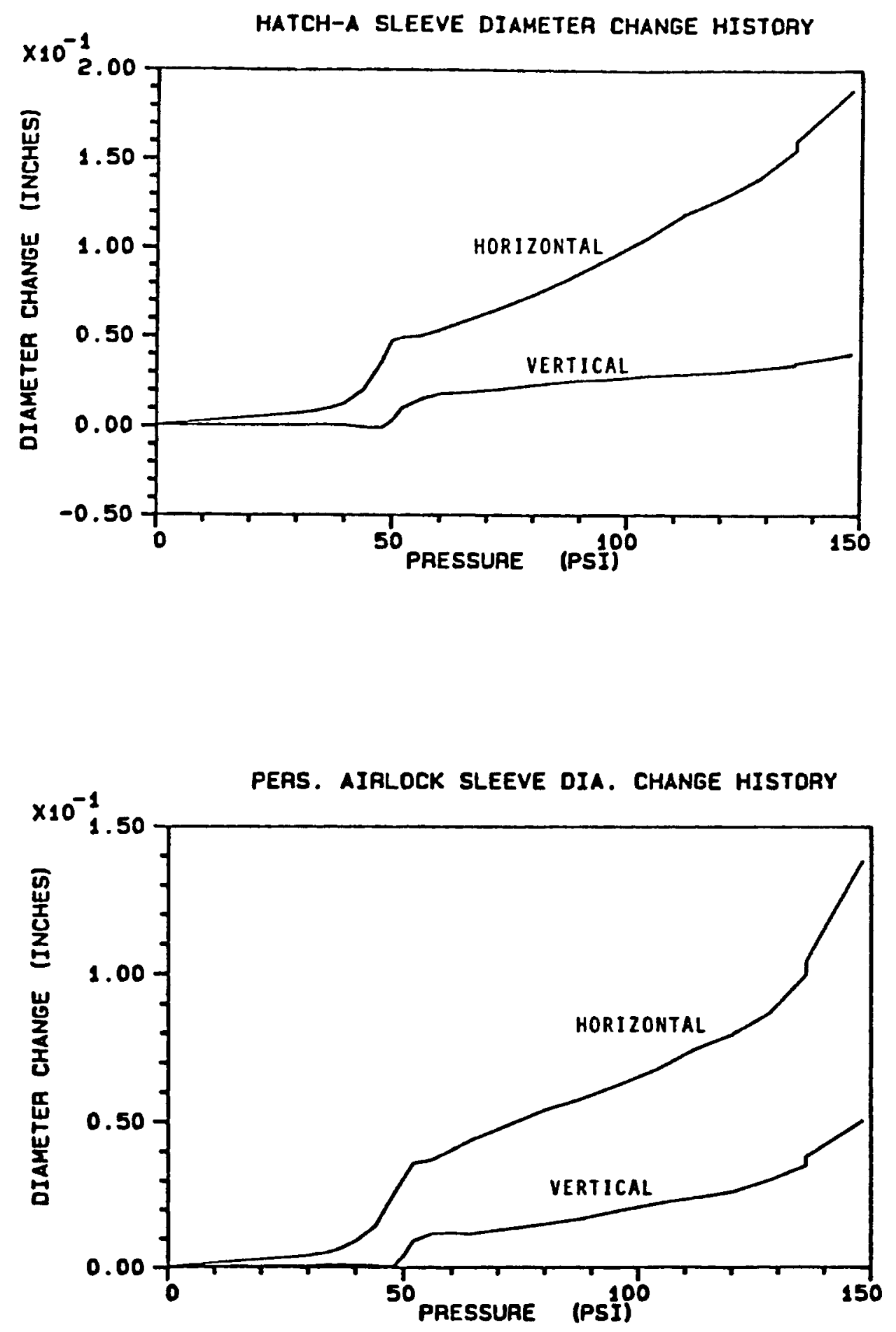

Figure 4.3.14 Diametral Length Change Histories for Hatch-A and Personnel Airlock Sleeves

$$
-166-
$$




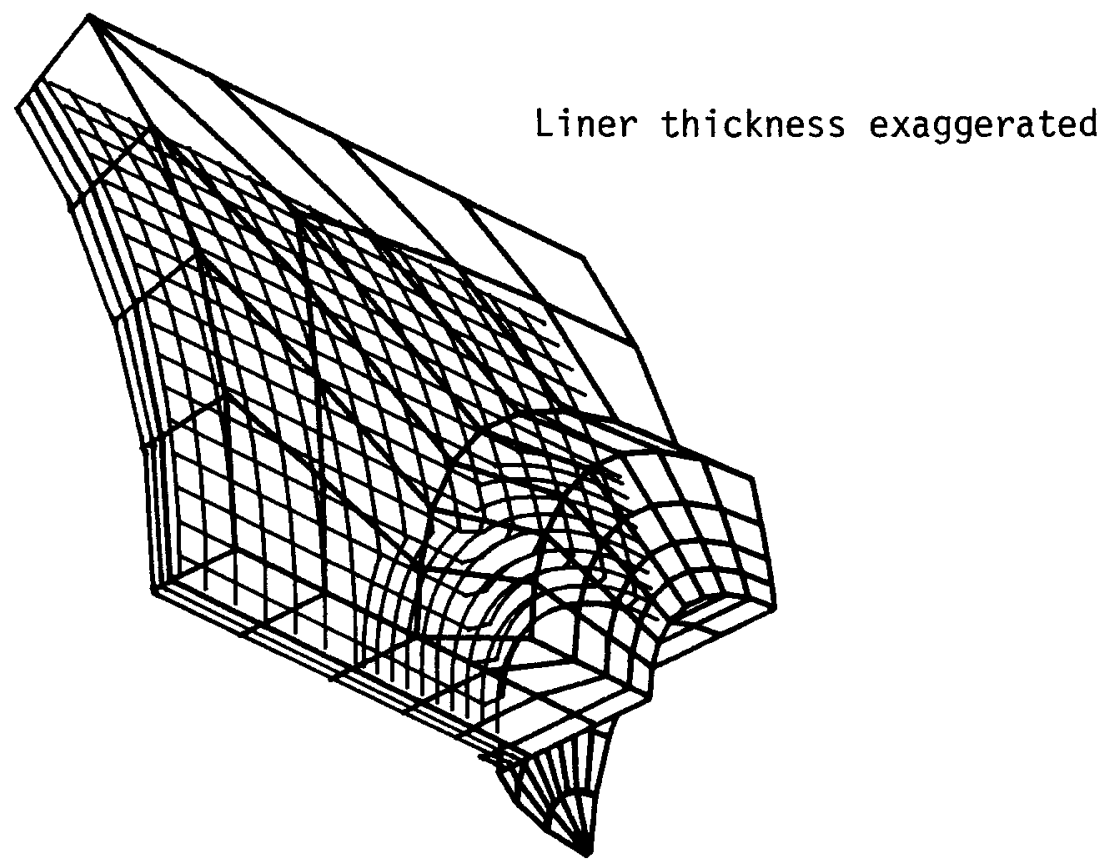

Hatch A with a hoop \& merid. rebar layer shown

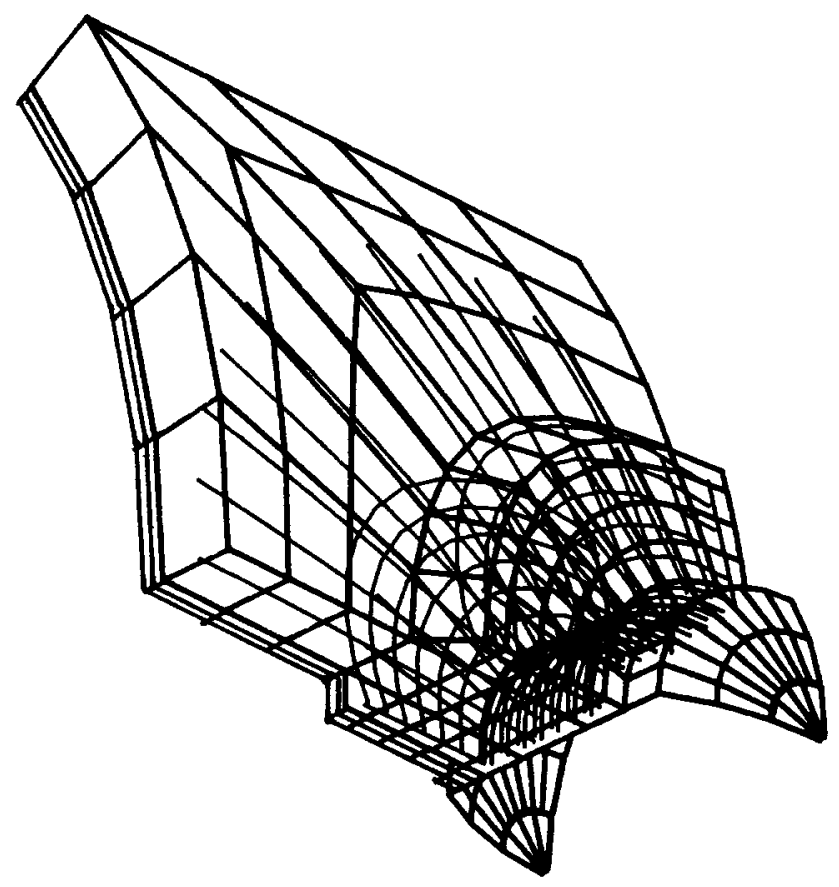

Hatch B with extra hatch reinforcement shown

Figure 4.3.15 Computational Grids for Equipment Hatches $A$ and $B$ Local Analyses 

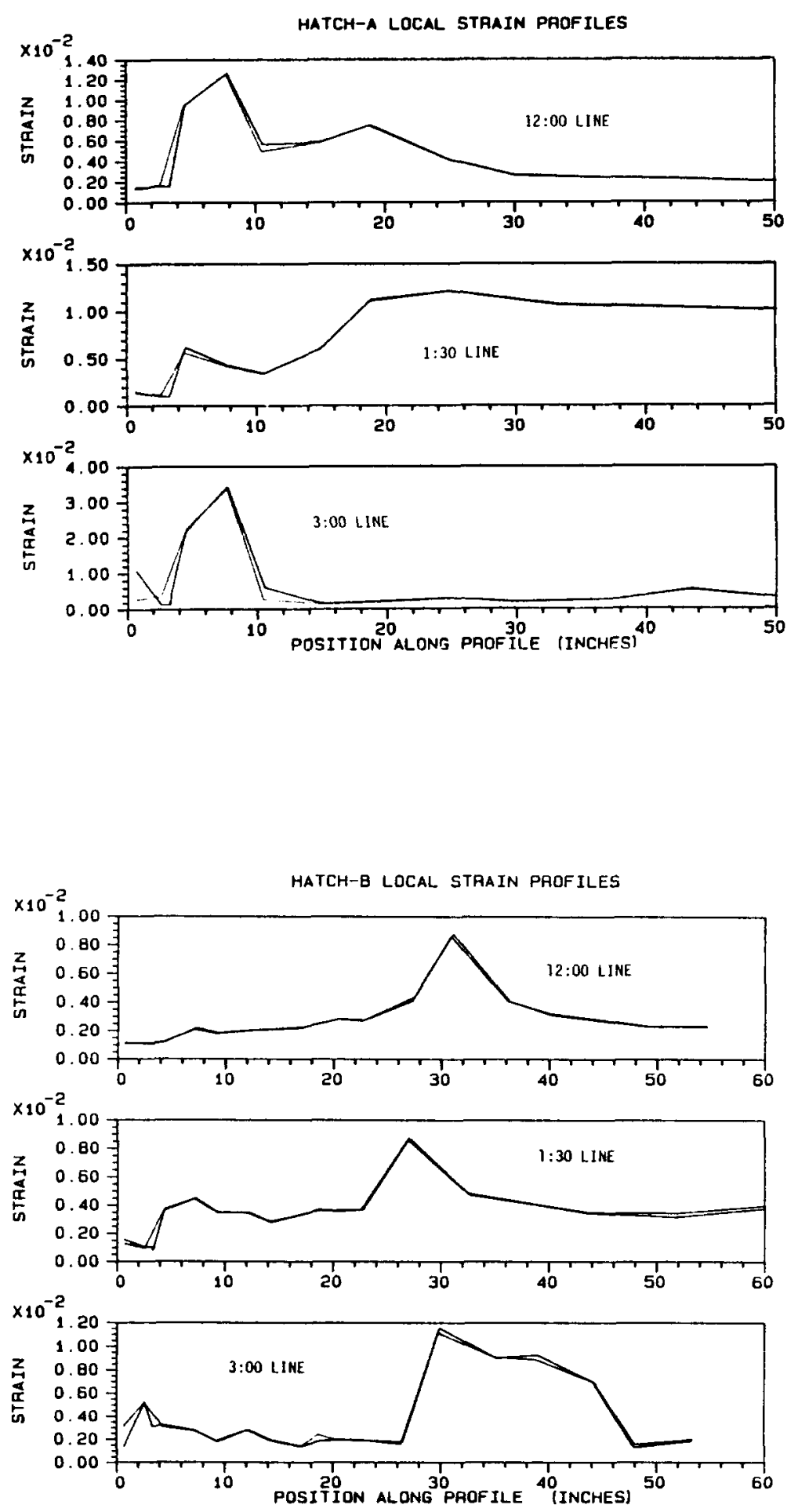

Figure 4.3.16 Local Hatch Maximum Principal Liner Strain Profiles at $150 \mathrm{psi}(1.03 \mathrm{MPa})$ 

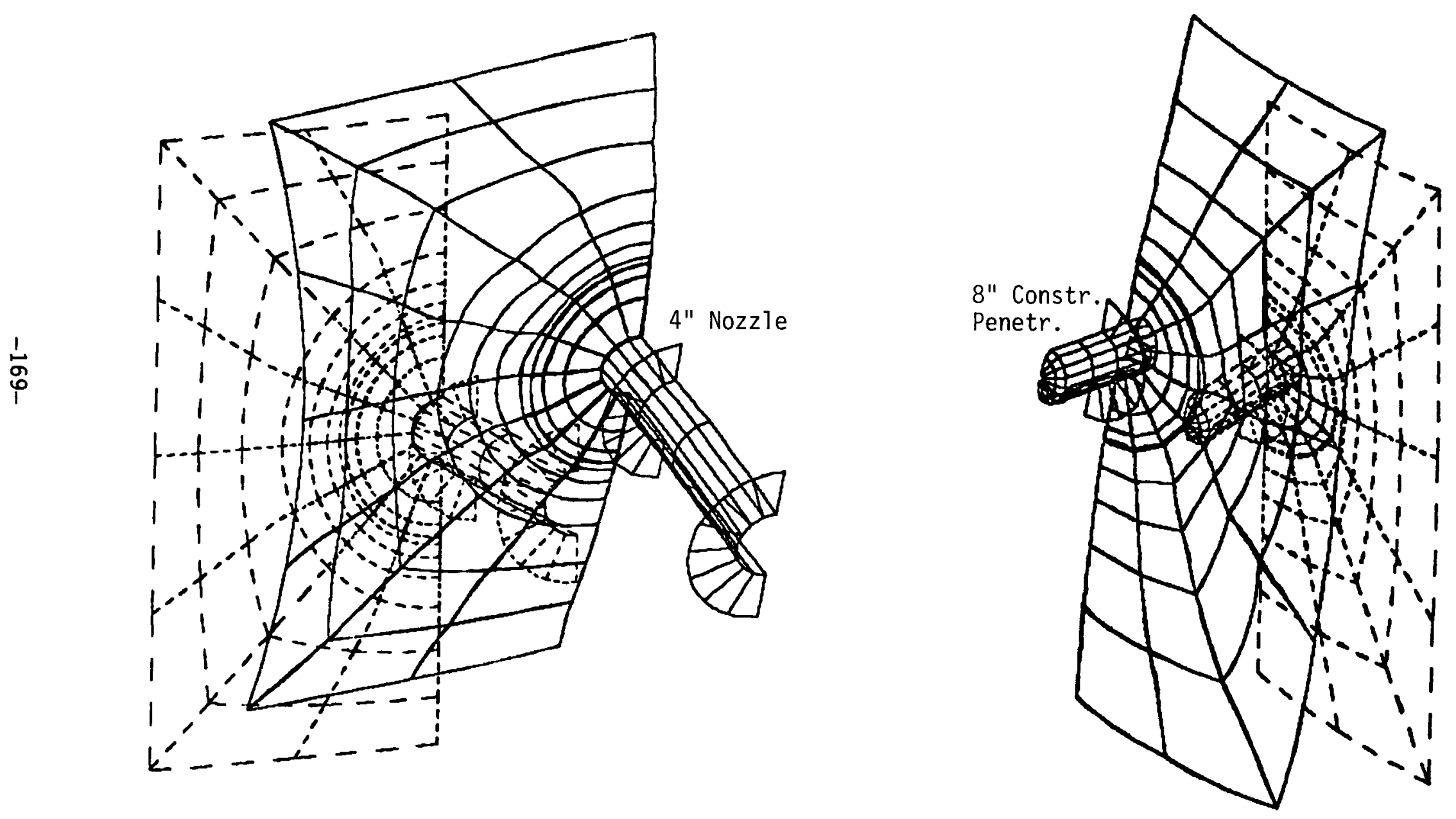

Figure 4.3.17 Pressure Nozzle and Constrained Pipe Penetration Local Analysis Deformed Grids at $104 \mathrm{psi}$ (0.72 MPa) 

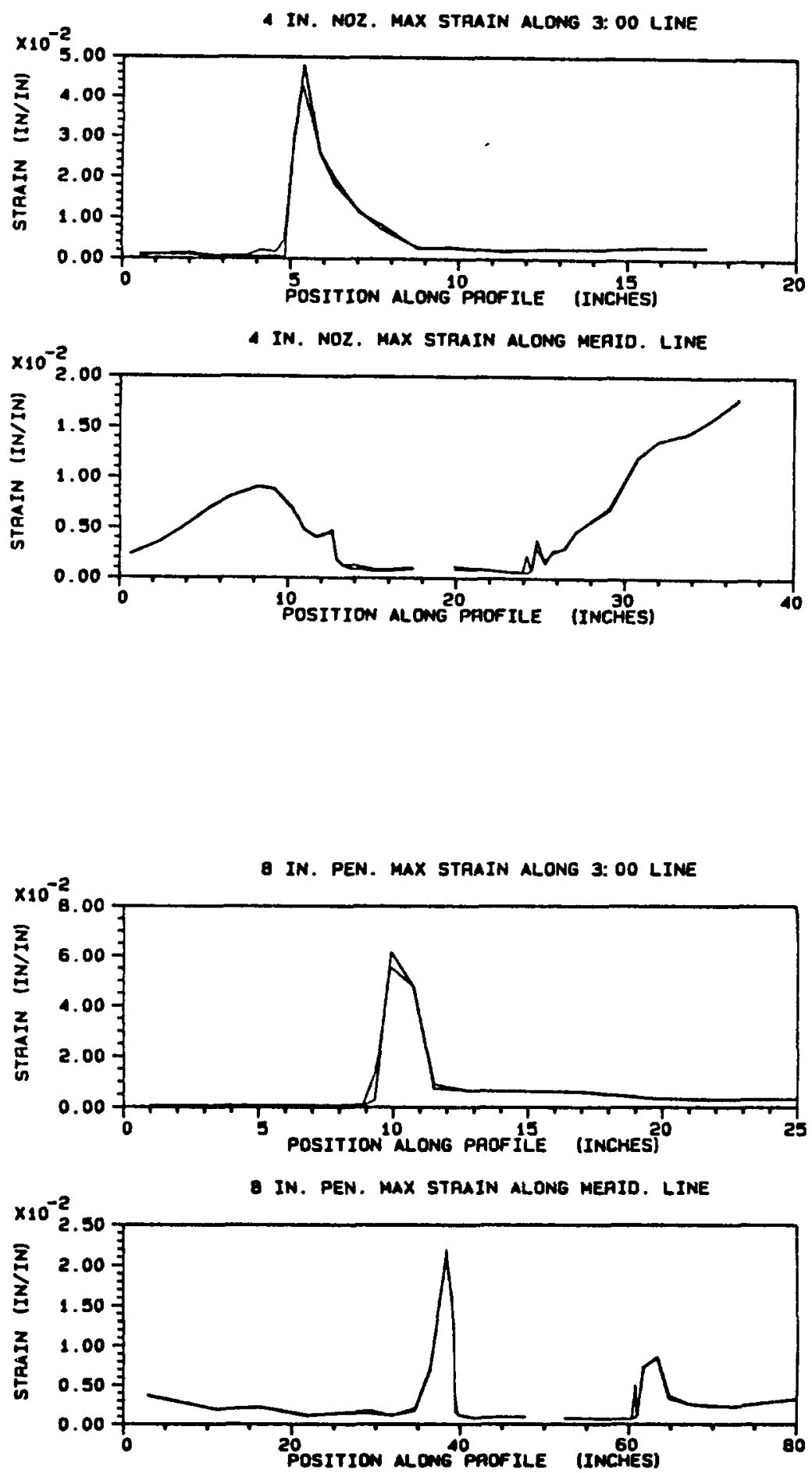

Figure 4.3.18 4" Pressure Nozzle and 8" Penetration Local Analyses Maximum Principal Liner Strain Profiles at $150 \mathrm{psi}$ ( $1.03 \mathrm{MPa}$ ) 


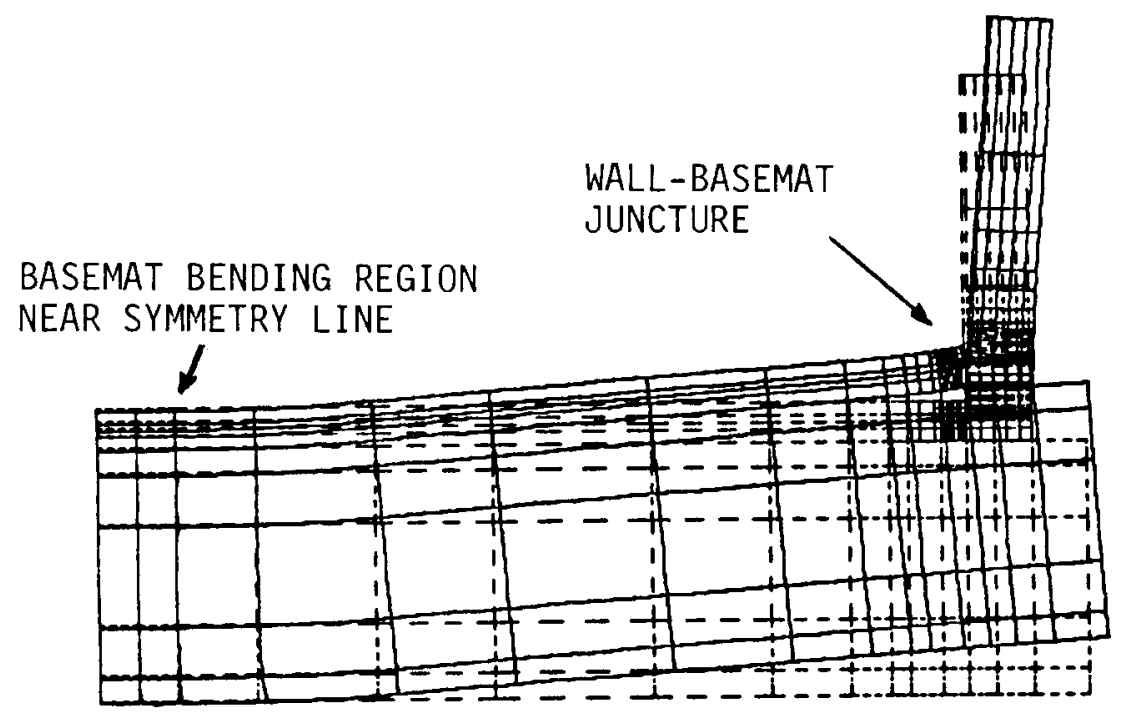

Figure 4.3.19 C4B Analysis Deformed Grid (Basemat) at 140 psi (0.97 MPa)

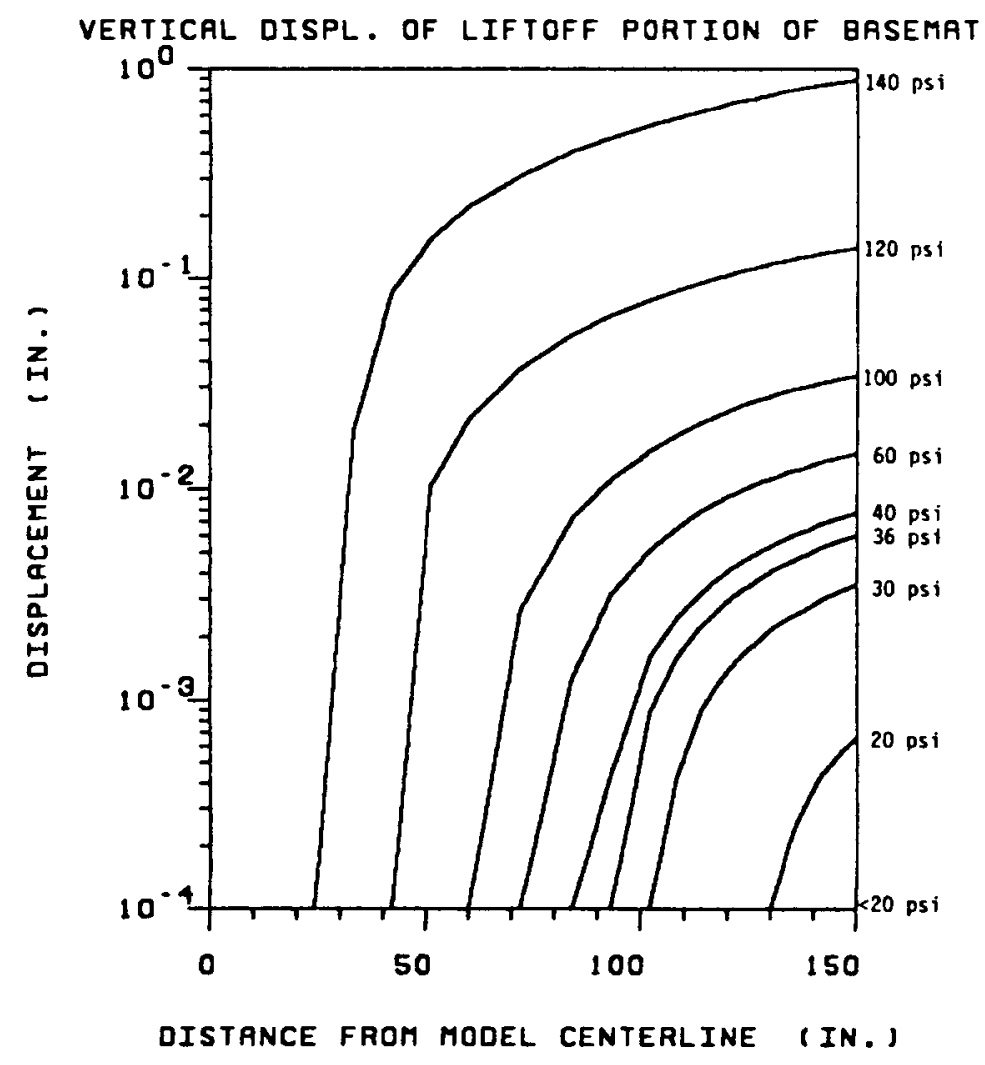

Figure 4.3.20 C4B Analysis Progression of Basemat Liftoff from 0 to 140 psi (0 to $0.97 \mathrm{MPa}$ ) 


\subsection{Comnissariat a L'Energie Atomique}

This section was authored by A. Millard*, G. Nahas**, Ph. Jamet* and B. Barbe***.

\subsubsection{Summary}

- Finite element analysis using the CASTEM system.

- Multi-criteria concrete model, including traction damage, shear damage, confining pressure damage.

- Isotropic hardening plastic model for the rebars and the liner.

- Axisymetric modelization.

- Failure criterion: rapidly increasing displacement of the structure.

- Predicted failure mode: plasticity of rebars after development of meridional and hoop cracks in the concrete.

- Predicted failure pressure: $138 \mathrm{psi}$ (0.95 MPa).

\subsubsection{General Characteristics of the Calculations}

The main goal of the computation performed by C.E.A./D.E.M.T./L.A.M.S. is to try to predict the overall behavior of the 1:6-scale reinforced concrete containment model, subjected to an internal increasing pressure.

More precisely, the failure mode as well as the failure pressure will be determined. Therefore, an axisymmetrical analysis was decided, without accounting for the various penetrations of the model.

The calculations have been performed on one hand with an infinitely rigid soil and on the other hand with an elastic soil.

The CASTEM system has been used: the code GIBI for the mesh generation; the code INCA for the mechanical calculation; the code ALICE for the postprocessing of the results.

We have used the standard versions of these programs, dated January 1986, without special modification.

The only modeled nonlinearity was material, displacements being assumed to be small.

$\overline{\star C E A ~-~ D E M T / S M T S / L A M S ~-~ F r a n c e ~}$

$\star \star$ SOCOTEC Industries - France

$\star \star \star$ CEA - IPSN/DAS/SAM - France 


\subsubsection{Description of the Structure}

\subsubsection{The Mesh}

The structure has been modeled using various axisymmetric finite elements:

- 1913 linear triangular elements for the concrete;

- 657 two nodes thin shell elements for the meridional rebars, for the seismic rebars, for the basemat rebars, for the layer 11 rebar and for the liner;

- 419 one node truss elements for the hoop rebars.

The linear triangular elements have three nodes with two degrees of freedom per node. The thin shell elements are flat, with two nodes and three degrees of freedom per node. The truss elements have one node with two degrees of freedom per node.

In the second calculation, the soil has been modeled using 31 flat thin shell elements. All these elements are analytically integrated. Figures 4.4.1 and 4.4.2 show the whole mesh for the calculations without and with soil modelization.

Figure 4.4.3 shows the rebars and the liner modelization.

The shear ties and the termination of bars have not been modeled in this analysis.

The concrete located under the basemat rebars has not been modeled since it has no resistance. The total number of degrees of freedom is 3564 .

\subsubsection{Characteristics of the Steel Elements}

Basement Rectangular Rebars:

The basemat rectangular rebars have been modeled on one hand by an equivalent axisymmetric shell, the thickness of which is determined on the basis of an equivalent ultimate load, and on the other hand by truss elements, the cross section of which are proportional to the radius.

The results obtained are the following:

- for the lower rebars, the equivalent shell thickness is $e=0.110$ in $(.0028 \mathrm{~m})$

- for the upper rebars, the equivalent shell thickness is $e=0.051$ in $(.0013 \mathrm{~m})$

Basemat Radial Rebars:

The basemat radial rebars have been modeled using a shell with varying thickness, depending on the radius $r$ :

$$
t=3.02410^{-3} / r \quad \text { (in meters) }
$$

Basemat Hoop Rebars:

The cross sections of the basemat hoop rebars have been calculated in accordance with the nominal values and the location of the truss elements. 
Basemat Layer 11 Rebars:

The thickness of the equivalent shell varies with the radius, $r$, to the center of the element:

$$
e=3.87110^{-3} / \mathrm{r} \quad \text { (in meters) }
$$

Meridional Rebars:

The thickness of the equivalent shell is calculated from the number of rebars, their cross-section and their location.

Seismic Rebars:

The seismic rebars are replaced by meridional and hoop rebars on the basis of an equivalent shell and truss elements using the same approach as before.

Steel Plates at the Top of the Dome:

They have been modeled using shell elements, with thicknesses according to the plans.

Liner:

The liner has been modeled using shell elements with thicknesses according to the plans. Note that for all elements, we have used the nominal thicknesses given by SNL on the plans.

\subsubsection{Constitutive Models and Properties}

\subsubsection{Concrete}

The model used for the concrete has already been presented (see References [4.4.3] and [4.4.4]). In this model, three principal damage modes are represented: (1) shear damage, (2) traction damage, and (3) confining pressure damage.

For the shear damage, two types of behavior have been identified from experiments [4.4.2]:

- a brittle behavior for low confining pressures. This behavior is modeled by two Drucker-Prager criteria, one with strain-softening, limited by a second fixed Drucker-Prager criterion.

- A ductile behavior, for high confining pressures. This behavior is modeled by a Von Mises criterion with strain hardening and limited by a fixed Drucker-Prager criterion.

For the traction damage, a maximum principal stress criterion is assumed. When the tensile strength is reached, the resistance in the corresponding direction is immediately set to zero. The model enables the representation of the anisotropy induced by the cracks. The direction of the cracks is kept in memory.

For the confining pressure damage, the criterion is based on the first invariant of the stress tensor. Linear strain hardening has been assumed.

For all criteria, the plastic strains are obtained through the normality principle. 
The following properties have been adopted for the calculation:

$$
\begin{array}{ll}
\text { Young's modulus } & =4800 \mathrm{ksi}(33100 \mathrm{MPa}) \\
\text { Poisson's ratio } & =0.2 \\
\text { Specific weight } & =144 \mathrm{lb} / \mathrm{ft}^{3}\left(2300 \mathrm{~kg} / \mathrm{m}^{3}\right) \\
\text { Limit in traction } & =0.5 \mathrm{ksi}(3.45 \mathrm{MPa}) \\
\text { Limit in compression } & =6.8 \mathrm{ksi}(46.9 \mathrm{MPa})
\end{array}
$$

Figure 4.4.4A shows the comparison between the experimental compression curve and the one introduced in the computation.

\subsubsection{Rebars}

For the rebars, a classical elasto-plastic model based on a Von Mises criterion with isotropic strain hardening is used.

The following properties have been adopted for the calculation:

$$
\begin{aligned}
\text { Young's modulus } & =31000 \mathrm{ksi}(21400 \mathrm{MPa}) \\
\text { Poisson's ratio } & =0 \\
\text { Density } & =486 \mathrm{lb} / \mathrm{ft}^{3}\left(7800 \mathrm{~kg} / \mathrm{m}^{3}\right) \\
\text { Yield stress } & =66.6 \mathrm{ksi}(459 \mathrm{MPa}) \\
\text { Ultimate stress } & =98.9 \mathrm{ksi}(682 \mathrm{MPa}) \\
\text { Ultimate strain } & =.0451
\end{aligned}
$$

Note that the Poisson's ratio has been assumed to be zero in order to have only a uniaxial behavior of the rebars.

\subsubsection{Liner and Steel Plates}

The same model has been used for the liner and the steel plates, with the following properties:

$$
\begin{array}{ll}
\text { Young's modulus } & =30000 \mathrm{ksi}(20700 \mathrm{MP}) \\
\text { Poisson's ratio } & =0.3 \\
\text { Density } & =486 \mathrm{lb} / \mathrm{ft}^{3}(7800 \mathrm{~kg} \\
\text { Yield stress (basemat } & \\
\text { and cylinder dome) } & =50.2 \mathrm{ksi}(346 \mathrm{MPa}) \\
\text { Yield stress (dome) } & =51.3 \mathrm{ksi}(354 \mathrm{MPa}) \\
\text { Ultimate stress (basemat } & \\
\quad \text { and cylinder) } & =69.9 \mathrm{ksi}(482 \mathrm{MPa}) \\
\text { Ultimate stress (dome) } & =70.9 \mathrm{ksi}(489 \mathrm{MPa}) \\
\text { Ultimate strain (basemat } & \\
\text { and cylinder) } & =0.164 \\
\text { Ultimate strain (dome) } & =0.149
\end{array}
$$

\subsubsection{Soi1}

For the computation including soil modelization, some elastic vertical thin shell elements have been introduced under the basemat. Their thickness depends on the radial distance. 
Their stiffness has been calculated according to measurements performed by Western Technologies Inc. (see Reference [4.4.7]).

$$
K_{s}=390 \mathrm{ksf} / \mathrm{ft}(61.26 \mathrm{MPa} / \mathrm{m})
$$

\subsubsection{Interaction Between Concrete and Reinforcement}

In our calculation, we assumed a perfect connection between the concrete and the steel.

\subsubsection{Computational Strategy}

\subsubsection{Boundary Conditions}

In our two computations, unilateral constraints have been introduced at the bottom of the basemat in order to allow uplift of the basemat under internal pressure.

Symmetry conditions have been assumed on the axis.

\subsubsection{Load History}

The structure is subjected to its dead weight, which is maintained constant during the calculation, and to an increasing pressure.

\subsubsection{Solution Algorithm}

The algorithm used is the classical initial stress method (see Reference $[4.4 .6])$. The convergence criteria are based:

- on one hand, on the relative difference between plastic energy increments:

$$
\operatorname{Max} \frac{\left|\Delta w_{(n+1)}^{p}-\Delta w_{(n)}^{p}\right|}{\left|\Delta w_{(n+1)}^{p}\right|}<\epsilon
$$

where $\Delta W_{(n)}^{p}$ is the plastic energy increment in the step at iteration n.

- on the other hand, on the relative difference between displacements increments:

$$
\frac{\|\Delta q(n+1)-\Delta q(n)\|}{\| \Delta q(n+1)} \| \epsilon
$$

where $\Delta q(n)$ is the displacements increment at iteration $n$.

The tolerance $\epsilon$ has been set to 0.01 in the calculation. The pressure has been applied in 28 steps. The computation has been stopped due to a convergence failure for a pressure $p=124 \mathrm{psi}(0.98 \mathrm{MPa})$. For this 
pressure, the concrete is extensively cracked and the plastic strain of the rebars increases very rapidly, meaning that the structural limit pressure is nearly reached.

\subsubsection{Failure Mechanisms}

\subsubsection{Case of an Infinitely Rigid Soil}

Displacements History:

Figures 4.4 .5 to 4.4 .12 show the deformations of the containment for various pressure levels. The displacements have been amplified by a factor 30 . The main events which can be observed are:

- at $p=43 \mathrm{psi}(0.3 \mathrm{MPa})$, a sudden increase of the radial displacement of the cylinder.

- at $p=80 \mathrm{psi}(0.55 \mathrm{MPa})$, an important uplift movement of the bas emat.

- at $p=142 \mathrm{psi}(0.98 \mathrm{MPa})$, the radial and vertical displacements increase significantly.

Figures 4.4.13 and 4.4.14 show the radial and vertical displacements at the locations shown on Figure 4.4.4B. It can be noted that the structure behaves linearly elastically up to a $43 \mathrm{psi}(0.3 \mathrm{MPa})$ pressure. Then a first nonlinearity due to cracks in the concrete occurs (see Figure 4.4.13). A second discontinuity is observed on Figure 4.4 .14 , corresponding to a sudden increase of the dome apex displacement at $p=73 \mathrm{psi}(0.5 \mathrm{MPa})$. Finally, at $p 131 \mathrm{psi}(0.9 \mathrm{MPa})$, the displacements begin to increase rapidly (Figure 4.4.13).

Meridional Cracks:

The first failure mechanism to occur is the development of meridional cracks for a pressure of $29 \mathrm{psi}(0.2 \mathrm{MPa})$ at the junction between the basemat and the cylinder as shown on Figure 4.4.15.

These cracks then develop in the cylinder where some rebars terminate, at the bottom of the cylinder and also in the dome, near the steel plates, at a pressure $p=43 \mathrm{ps} i(0.3 \mathrm{MPa})$. The radial direction of these cracks can be clearly observed on Figure 4.4.16.

Figure 4.4.17 shows that for a pressure $p=73 \mathrm{psi}(0.5 \mathrm{MPa})$, the dome is completely cracked, and the first cracks occur in the basemat. They develop rapidly for a small increment (see Figure 4.4.18).

Then for a $93 \mathrm{psi}(0.64 \mathrm{MPa})$ pressure, the cylinder and the dome are completely cracked as well as an important part of the basemat, as seen on Figure 4.4.19. Afterwards, the cracks pattern does not change very much (see Figure 4.4.20).

Hoop Cracks:

The second important failure mechanism to occur is the development of hoop cracks, for a pressure of $43 \mathrm{psi}(0.3 \mathrm{MPa})$, in the whole cylinder and the dome (see Figure 4.4.21). It causes a sudden increase in the radial displacement of the cylinder, as can be seen on Figure 4.4.6. 
For a $73 \mathrm{psi}(0.5 \mathrm{MPa})$ pressure, the first hoop cracks happen at the bottom of the basemat (see Figure 4.4.22).

Then, for an $80 \mathrm{psi}(0.55 \mathrm{MPa})$ pressure, the lower half part of the basemat is completely cracked (see Figure 4.4.23) which results in an uplift movement of the basemat (see Figure 4.4.9). Afterwards, for a greater pressure, the hoop cracks do not develop much as can be seen on Figures 4.4.24 and 4.4.25A.

Rebars and Liner Plasticity:

WARNING: In the analysis, concrete is represented as an equivalent homogeneous material. On the other hand, stresses and strains in rebars and in the liner are probably greatly influenced by the specific location of real cracks. Therefore, the computations only give "average" values for these quantities, and direct comparison cannot be made between experimental and numerical results.

The evolution of radial and vertical displacements of some points chosen in the structure shows that up to $131 \mathrm{psi}(0.9 \mathrm{MPa})$ pressure the behavior of the rebars is purely elastic "in average." Then, the displacements increase rapidly as the loading approaches the ultimate load. Figure 4.4.25C shows the "average" plastic strain along the curvilinear abscissa of the liner, for various values of the pressure. The liner is elastic "in average," up to $87 \mathrm{psi}$ (0.6 MPa) pressure. The maximum "average" equivalent plastic strain obtained is .00584 located close to the dome apex, near the steel plates.

Shear Failure:

This failure mechanism is very limited in the computation: it is noticeable only at $142 \mathrm{psi}(0.98 \mathrm{MPa})$ pressure at the inner part of the junction between the cylinder and the basemat (see Figure 4.4.25B).

Discussion:

The above discussion clearly indicates that the major failure mechanisms which are likely to occur are the meridional cracking, the hoop cracking and finally the rebars plasticity.

\subsubsection{Case of an Elastic Soil}

In this paragraph, the emphasis is laid mainly upon the results that significantly differ from the previous ones.

Displacement History:

In this case, the gravity load has been applied first in order to evaluate the corresponding displacements of the soil, shown in Figure 4.4.26.

Then the internal pressure is gradually increased. Figures 4.4 .27 to 4.4.34 show the increments of displacements of the structure, due to pressure only, amplified by a factor 30 .

The main events which can be observed are:

- at $p=43 \mathrm{psi}(0.3 \mathrm{MPa})$, an increase in the radial displacement, as for the case of an infinitely rigid soil. 
- at $p=80 \mathrm{psi}(0.55 \mathrm{MPa})$, contrary to the previous case, the movement of the basemat is more a rotation, due to the deformability of the soil, together with a downwards translation.

Finally, at $142 \mathrm{psi}(0.98 \mathrm{MPa})$ again the displacements increase rapidly. Figures 4.4.48 and 4.4.49 show the evolution of radial and axial displacements at the location shown by Figure 4.4.4B.

The curves are very similar to those obtained with calculation 1. The main difference can be seen by comparison of Figures 4.4 .14 and 4.4 .49 between pressures $73 \mathrm{psi}(0.5 \mathrm{MPa})$ and $87 \mathrm{psi}(0.6 \mathrm{MPa})$.

Meridional Cracks:

This is again the first failure mechanism to occur in the structure. As seen on Figures 4.4.35 to 4.4.40, the difference with the previous case is the initiation of vertical cracks, near the axis, in the basemat. This is due to the fact that the soil deformability authorizes the behavior of the basemat as a plate under uniform pressure. Thus, for the same pressure $p=$ $73 \mathrm{psi}(0.5 \mathrm{MPa})$, the basemat is much more cracked in the present case (see Figure 4.4.38). Another consequence is that the cylinder and the dome are completely cracked for $p=87 \mathrm{psi}(0.6 \mathrm{MPa})$ (instead of $94 \mathrm{psi}(0.65 \mathrm{MPa}$ ) in the previous case).

Hoop Cracks:

Again, in this case, hoop cracks develop in the basemat, according to a plate beñर्ng behavior, for a lower pressure ( $p=58 \mathrm{ps} i(0.4 \mathrm{MPa})$ than in the previous case. Note that the basemat hoop cracks pattern is completely different due to the soil elasticity (Figure 4.4 .41 to 4.4 .45 ).

Rebars and Liner Plasticity:

As in the previous calculation, the displacements increase rapidly, for a $131 \mathrm{psi}(0.9 \mathrm{MPa})$ pressure, when the rebars are plastic. The maximum equivalent "average" plastic strain in the liner is .00614 (Figure 4.4.50).

Shear Failure:

In this case, the failure mechanism occurs at $116 \mathrm{ps} i$ ( $0.8 \mathrm{MPa}$ ) (Figure 4.4.46), but remains very limited (Figure 4.4.47) as in the previous case.

\subsubsection{Comparison Between the Two Cases}

The overall behavior of the containment predicted in the two cases is very similar. The main difference which can be drawn from the comparison is that the soil elasticity is responsible for a different development of the cracks, principally in the basemat:

- meridional cracks are initiated for a lower pressure and are localized near the axis, in the second case.

- the hoop cracks develop more progressively and start at a lower pressure, in the second case.

Therefore, only one discontinuity is visible on the dome apex displacement, at $p=80 \mathrm{ps} i(0.55 \mathrm{MPa})$ due to the final meridional cracking of the cylinder, instead of two for calculation 1 , due to the sudden hoop cracking of the basemat at $p=73 \mathrm{psi}(0.5 \mathrm{MPa})$ and then to the final meridional 
cracking of the cylinder at $p=87 \mathrm{psi}(0.6 \mathrm{MPa})$. However, the final crack patterns are identical, as well as the limit load.

\subsubsection{Miscellaneous}

Using the standard version of some CASTEM programs, it has been possible to calculate the SNL 1:6-scale concrete containment model.

For computer cost, and period of time, these analyses are comparable to studies performed with classical elasto-plastic materials (i.e. metals with Von Mises behavior), on meshes with the same number of degrees of freedom.

Concerning the general results of the analysis, several comments must be made:

- only 2-D computations have been carried out, therefore, the analyses only apply if the actual failure mechanism is an overall failure of the structure. If major damages appear due to penetrations or if the liner undergoes severe leaking, it is obvious that no comparison between experimental and numerical results can be made.

- the concrete is treated as an equivalent homogeneous material. In the test, cracks will appear at discrete locations. Therefore local stresses or deformations given by the analyses can only be considered as averaged quantities, which are not directly comparable to experimental data. Direct comparison can be carried out on pressuredisplacement curves, and values of pressure corresponding to specific phenomena such as radial cracking, hoop cracking basemat uplift, ultimate behavior.

- tension-stiffening has not been taken into account in the analyses. It is not believed to have a significant influence on the ultimate behavior of the structure. However, it might affect the shape of some of the pressure-displacement curves.

REMARK: Knowing a posteriori the behavior of the structure, it is possible to calculate the ultimate pressure load, assuming that the ultimate strength is due to the hoop rebars only. The corresponding pressure value is found equal to $P_{u}=131$ psi $(0.90 \mathrm{MPa})$, which is in good agreement with the calculation prediction. 


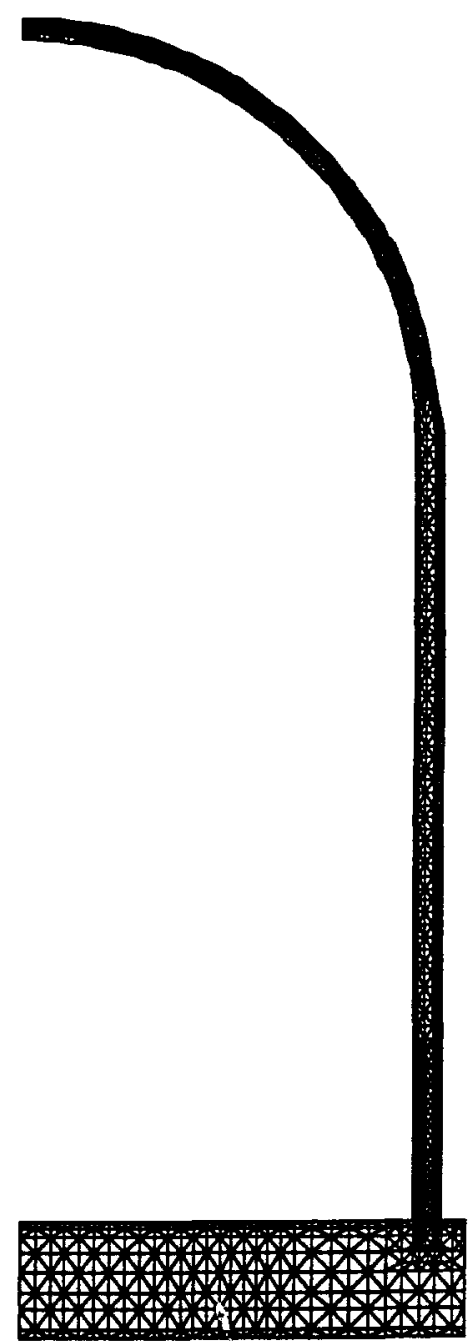

PRESSURISATION D UNE ENCEINTE DE CONFINEMENT

Figure 4.4.1 Mesh for calculation 1 (rigid soil) 


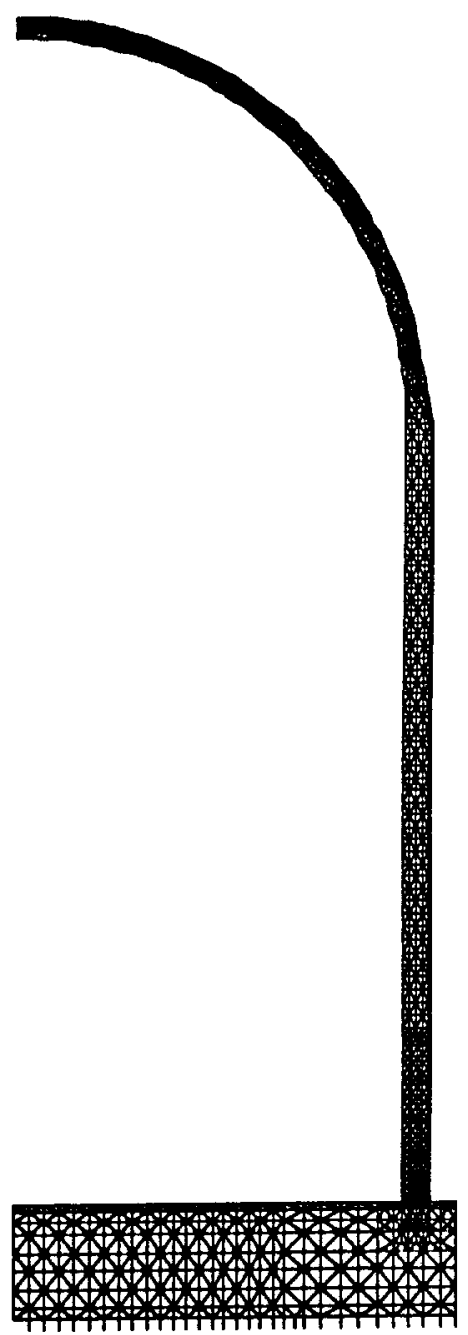

PRESSURISATION D UNE ENCEINTE DE CONFINEMENT

Figure 4.4.2 Mesh for calculation 2 (elastic soil) 


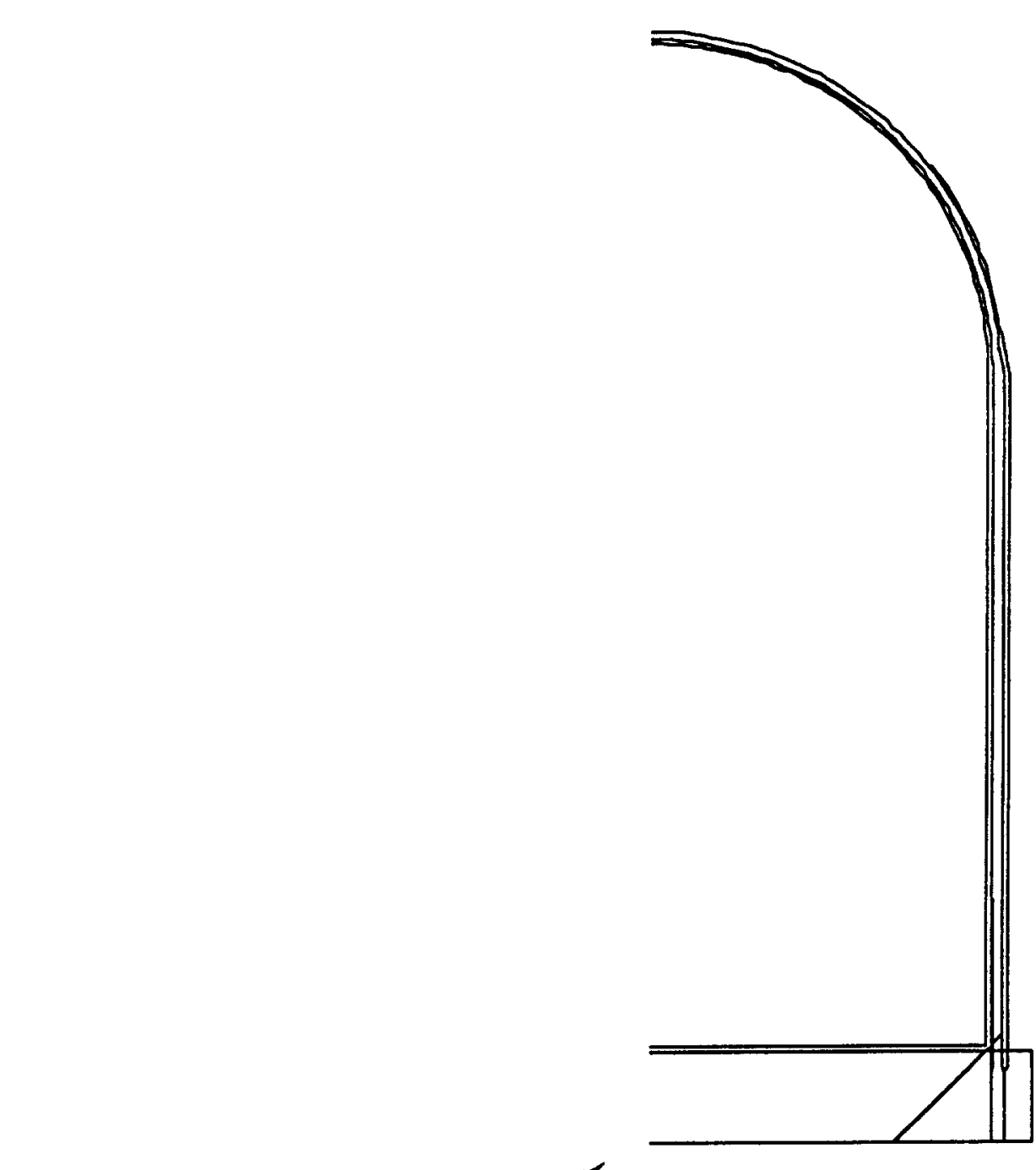

PRESSURISATION D UNE ENCEINTE DE CONNFINEMENT

Figure 4.4.3 Mesh of the rebars modelization 


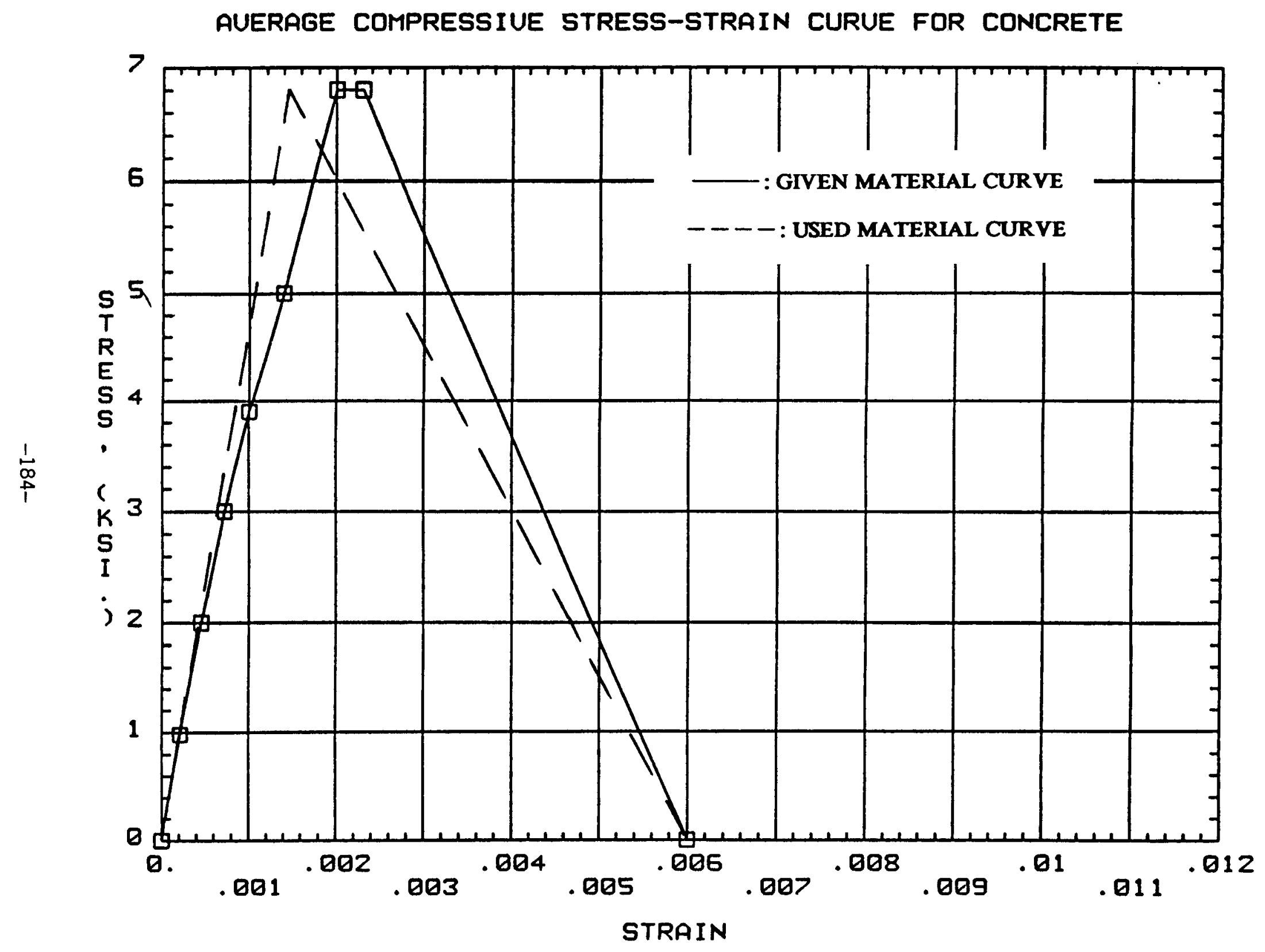

Figure 4.4.4A Comparison between real and modeled uniaxial concrete curves 


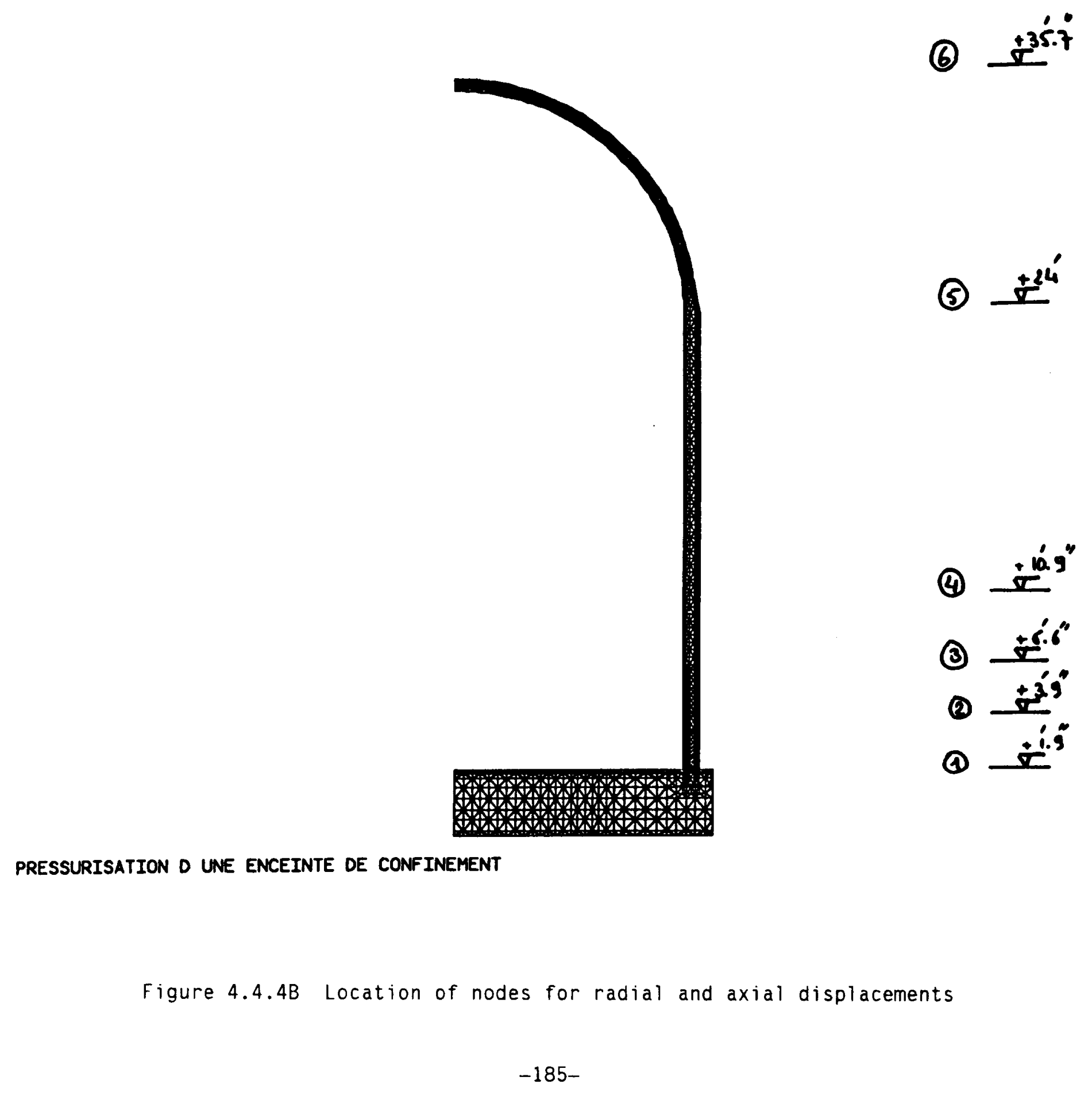




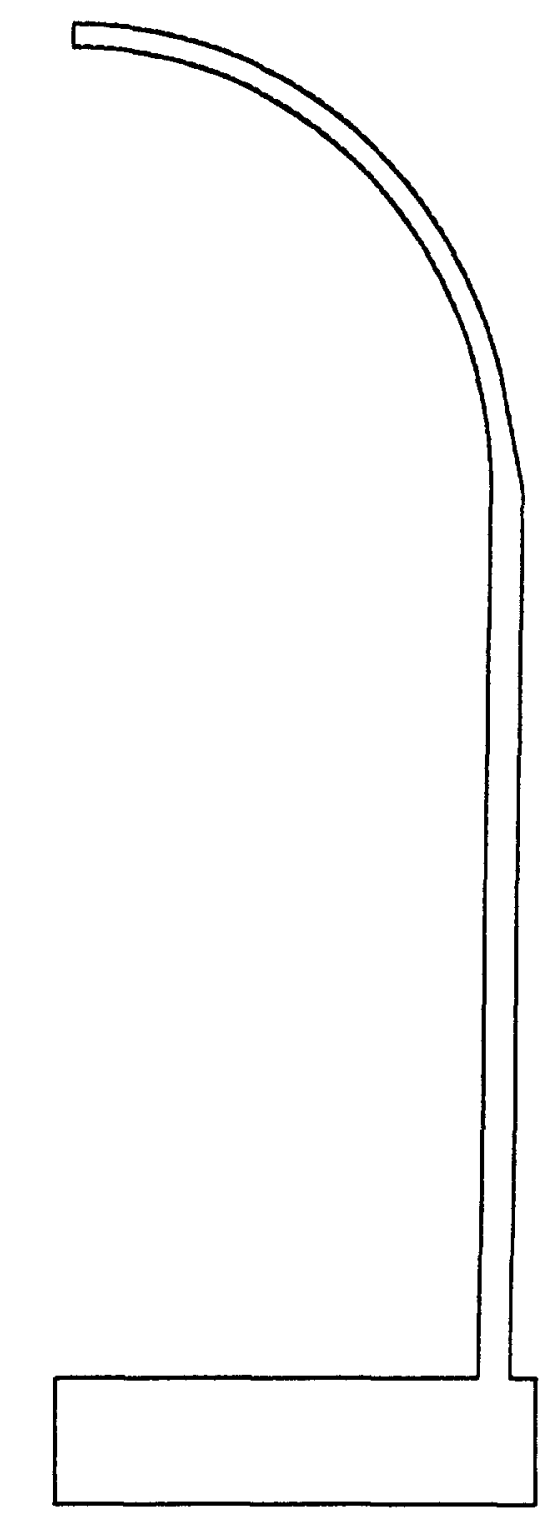

DEFORMEE PRESSION 0.2 MPA

Figure 4.4.5 Deformed structure at $29 \mathrm{psi}(0.2 \mathrm{MPa})$ (calculation 1) 


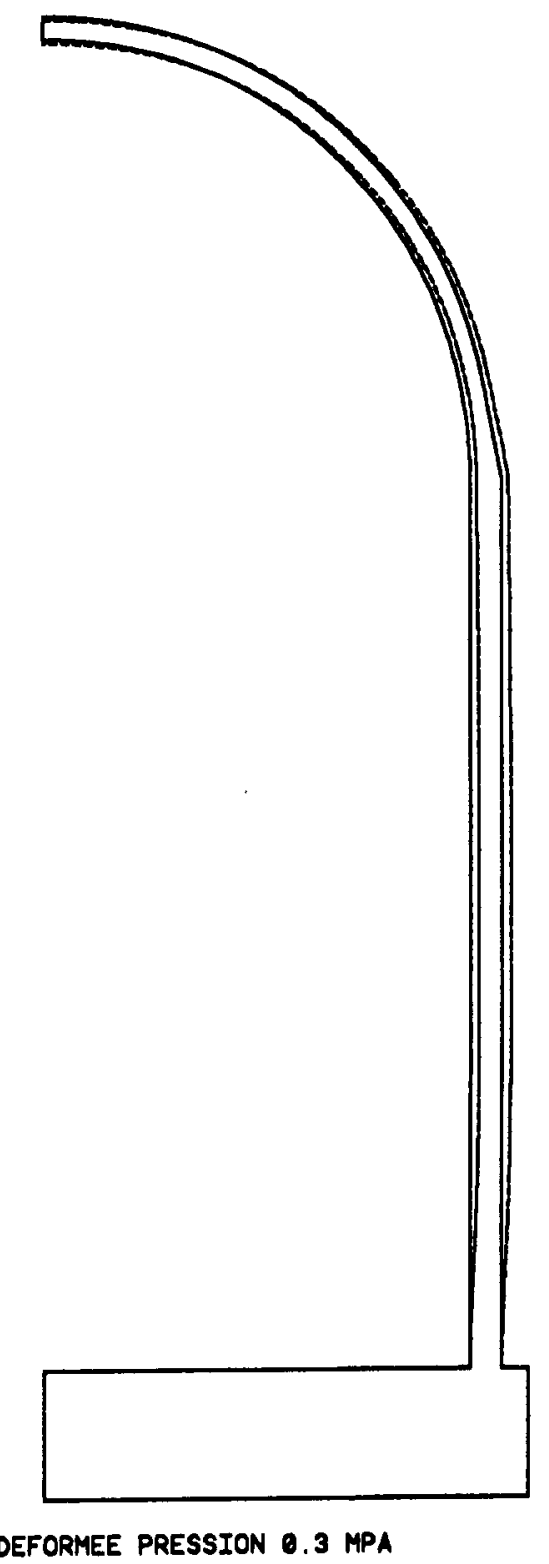

Figure 4.4.6 Deformed structure at $43.5 \mathrm{psi}(0.3 \mathrm{MPa})$ (calculation 1 )

$-187-$ 


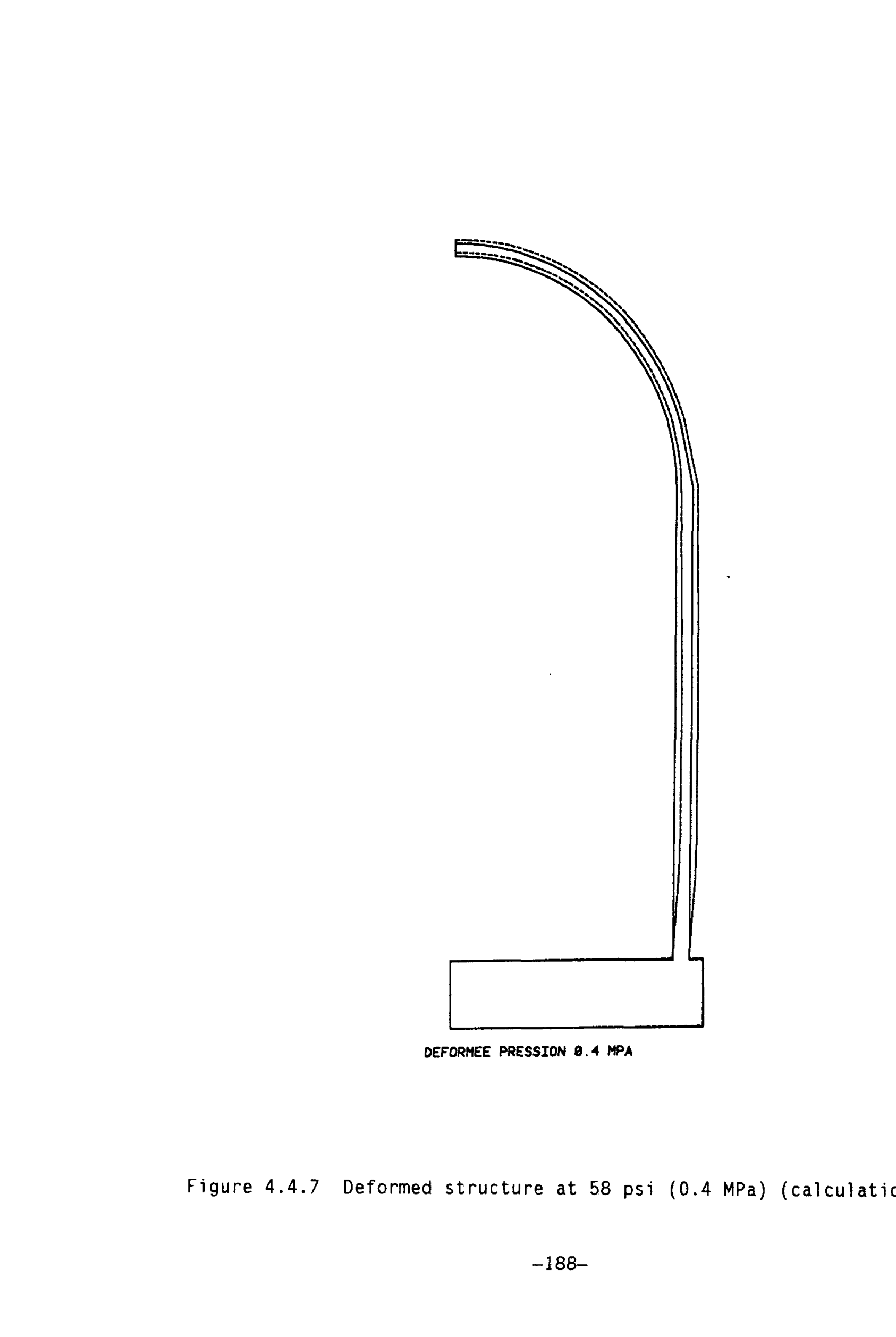




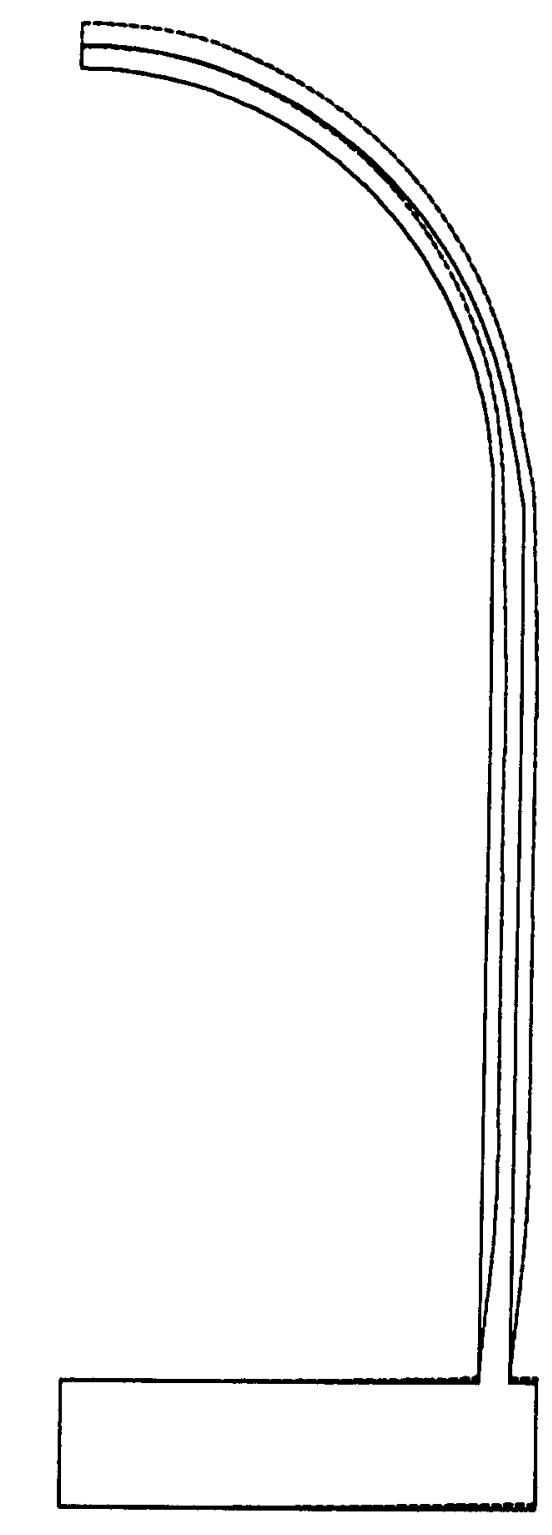

DEFORMEE PRESSION 0.5 MPA

Figure 4.4.8 Deformed structure at $72.5 \mathrm{psi}(0.5 \mathrm{MPa})$ (calculation 1 ) 


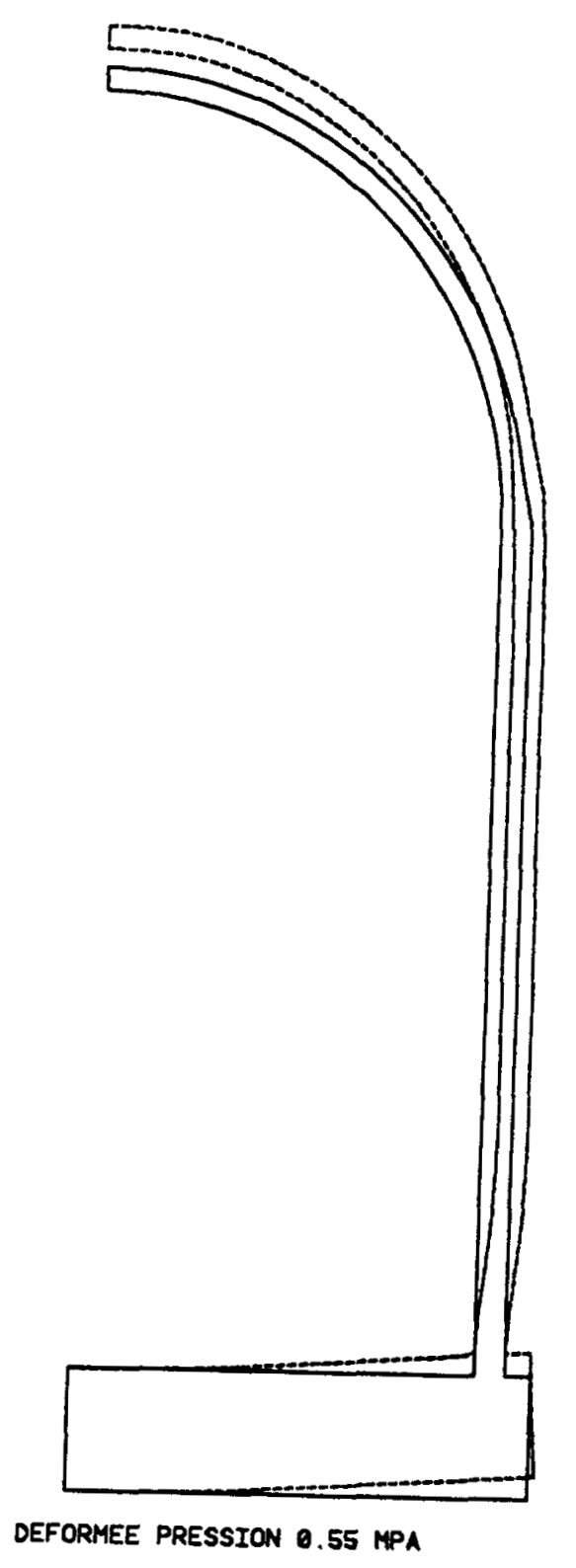

Figure 4.4.9 Deformed structure at $79.8 \mathrm{psi}(0.55 \mathrm{MPa}$ ) (calculation 1) 


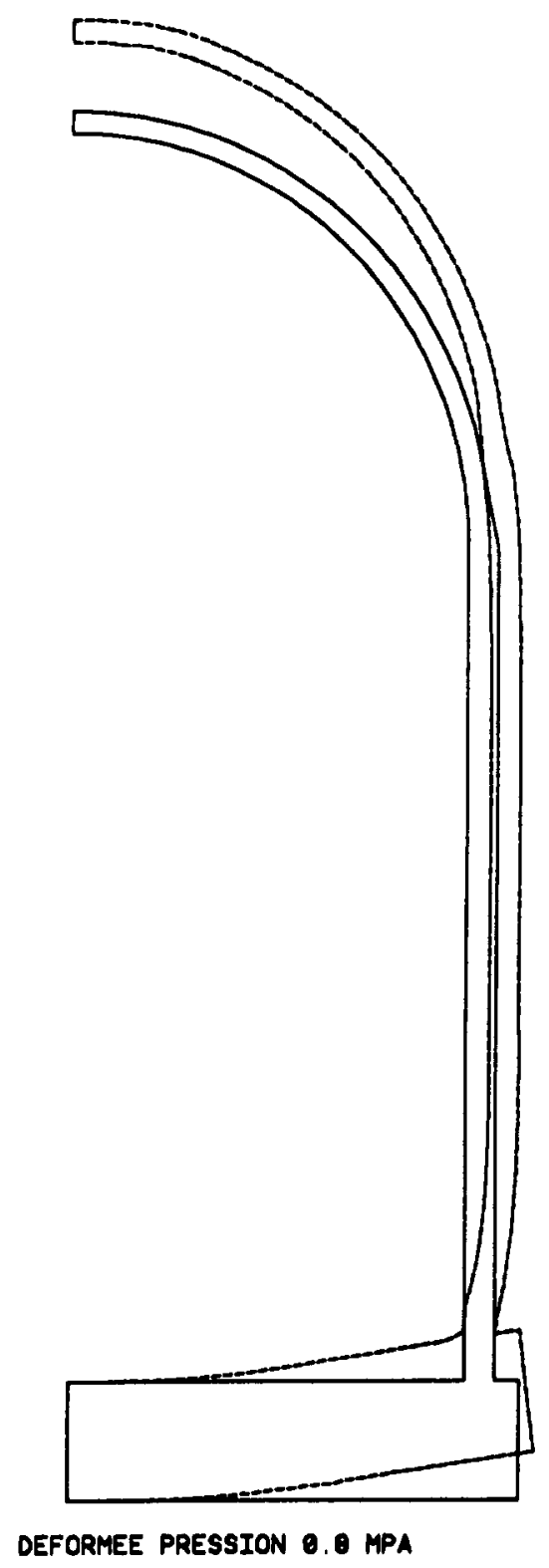

Figure 4.4.10 Deformed structure at $116 \mathrm{psi}(0.8 \mathrm{MPa})$ (calculation 1 ) 


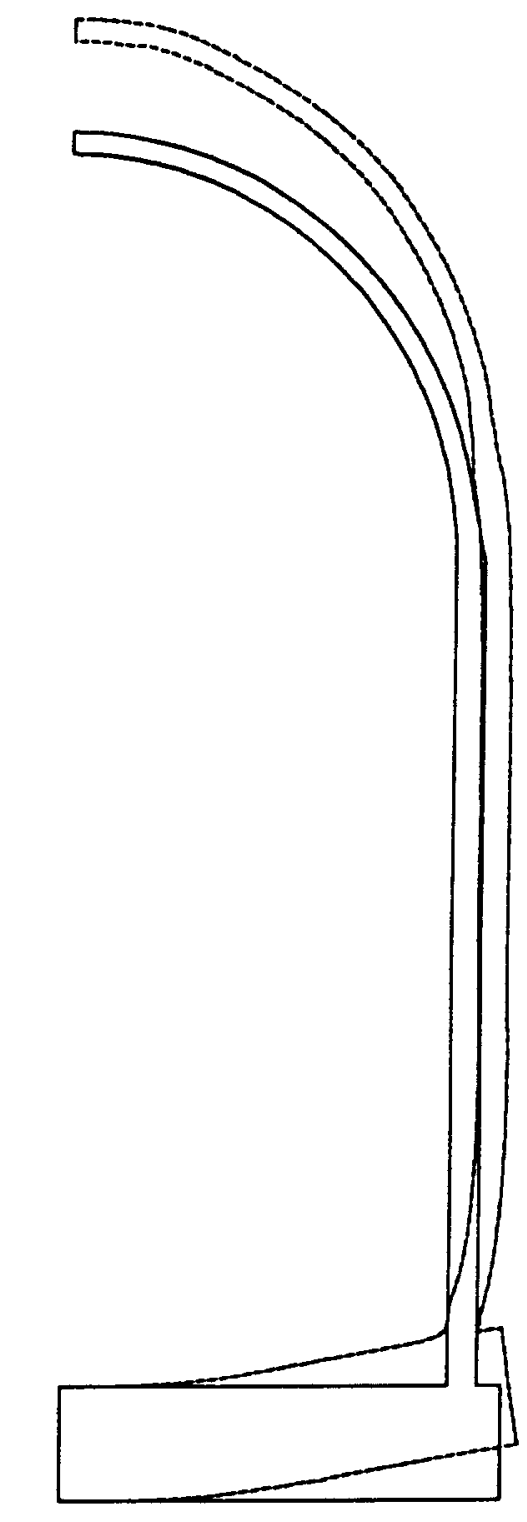

DEFORHEE PRESSION 0.0 MPA

Figure 4.4.11 Deformed structure at 130.5 psi (0.9 MPa) (calculation 1) 


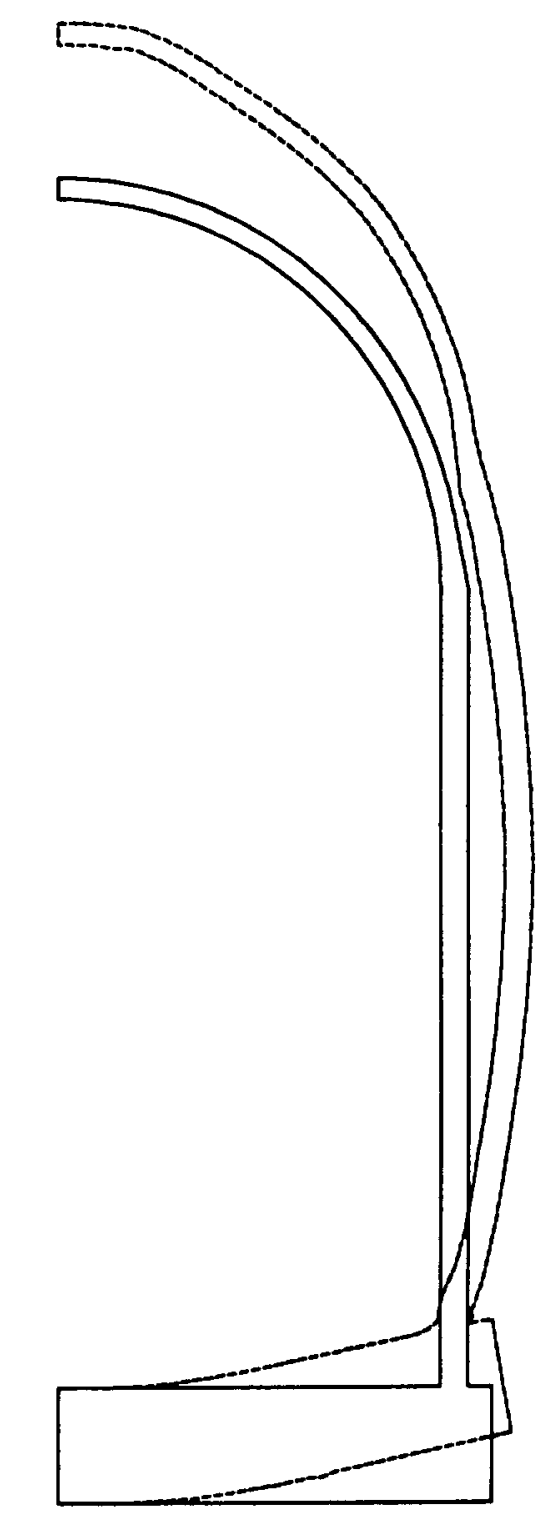

DEFORMEE PRESSION 0.08 MPA

Figure 4.4.12 Deformed structure at $142 \mathrm{psi}$ (0.98 MPa) (calculation 1) 


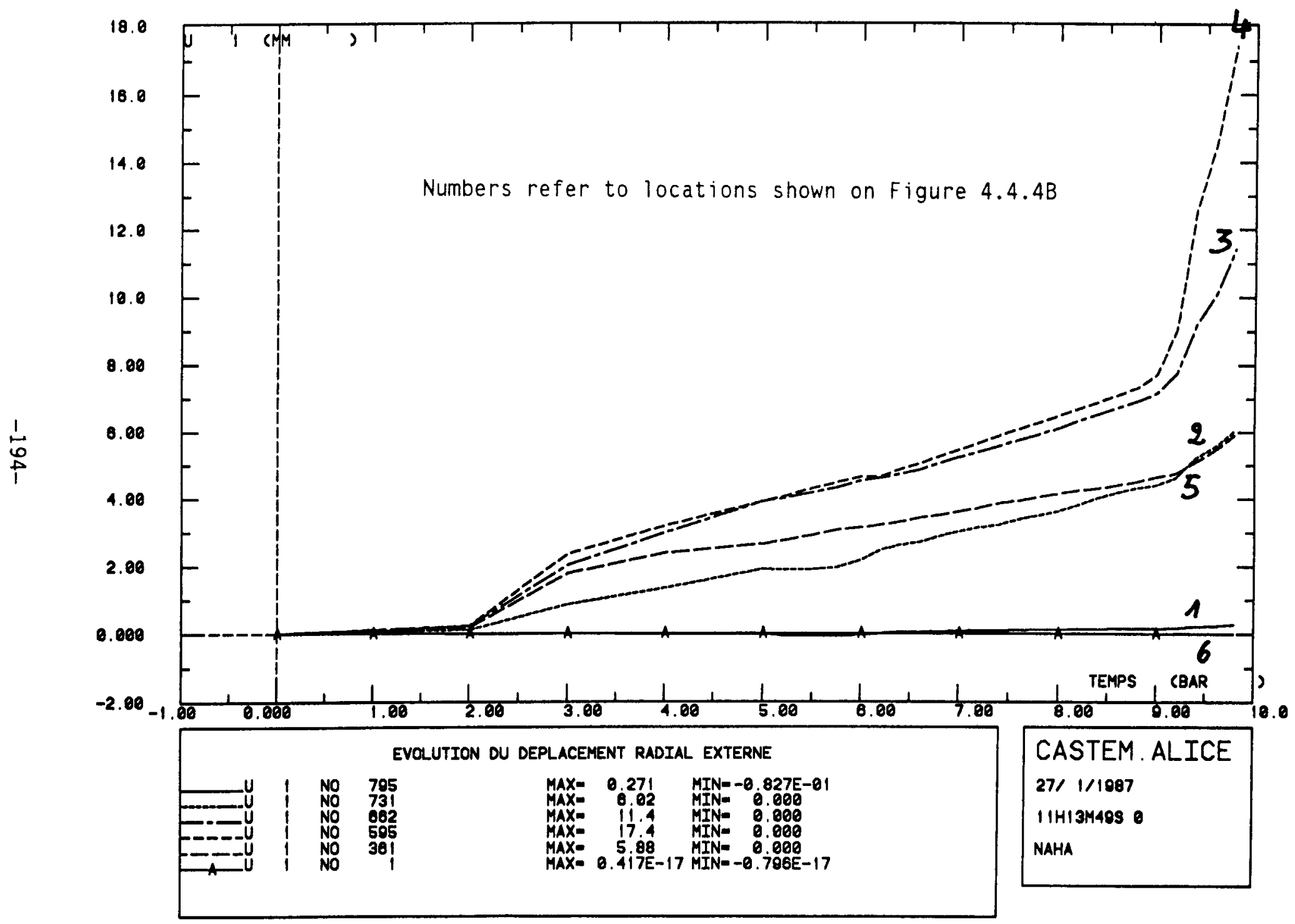

Figure 4.4.13 Radial displacement versus pressure (calculation 1) 


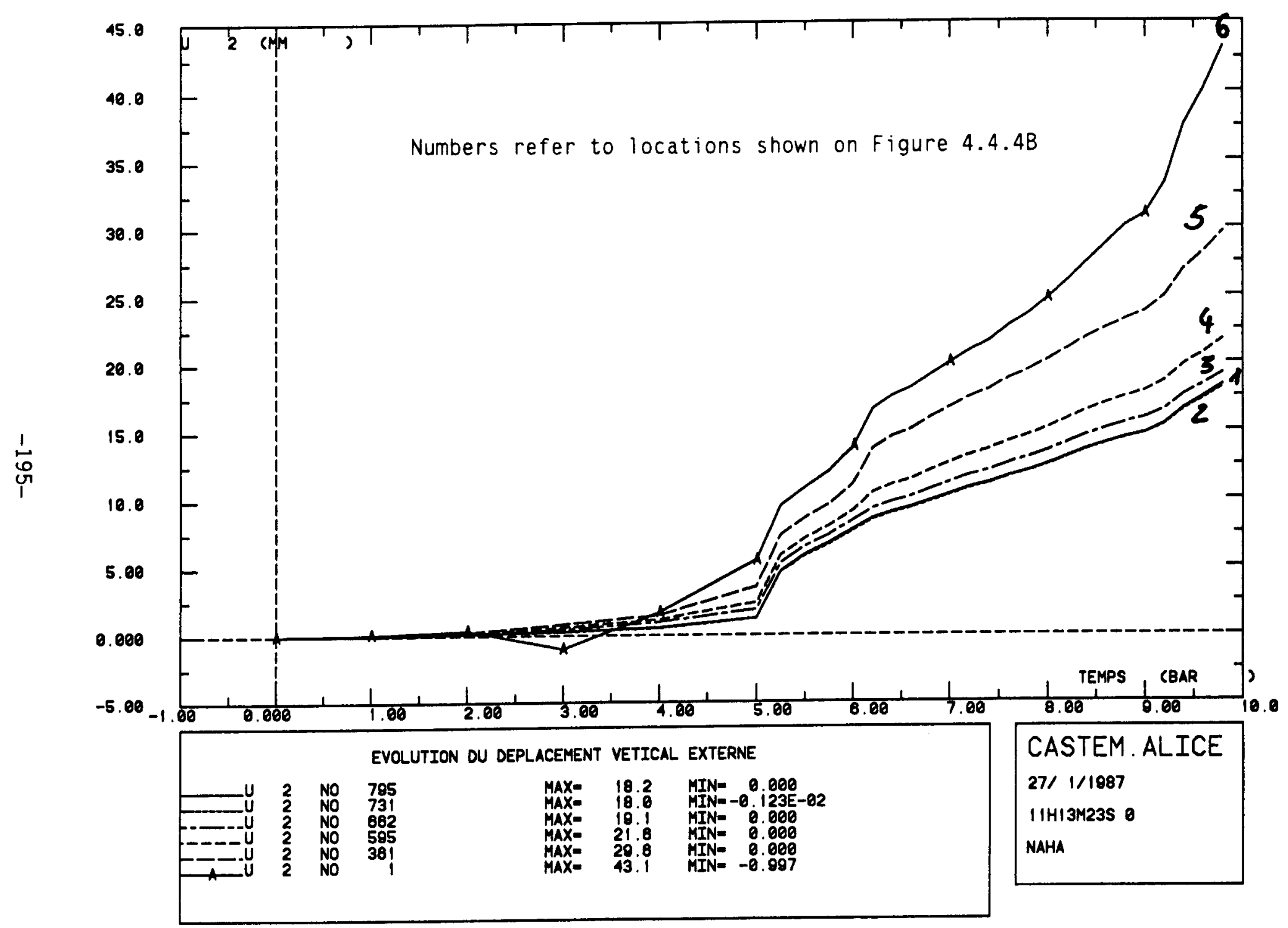

Figure 4.4.14 Axial displacements versus pressure (calculation 1) 


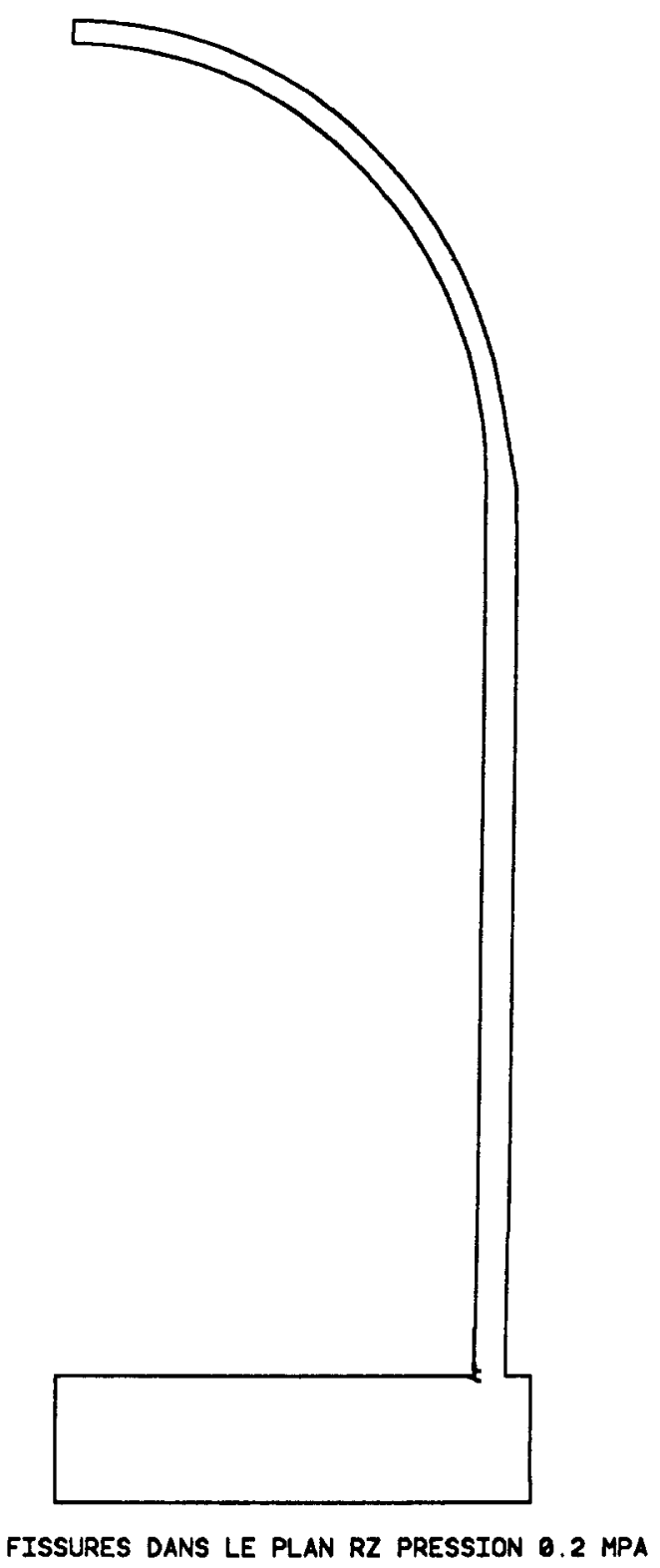

Figure 4.4.15 Meridional crack pattern at $29 \mathrm{psi}$ (0.2 MPa) (calculation 1) 


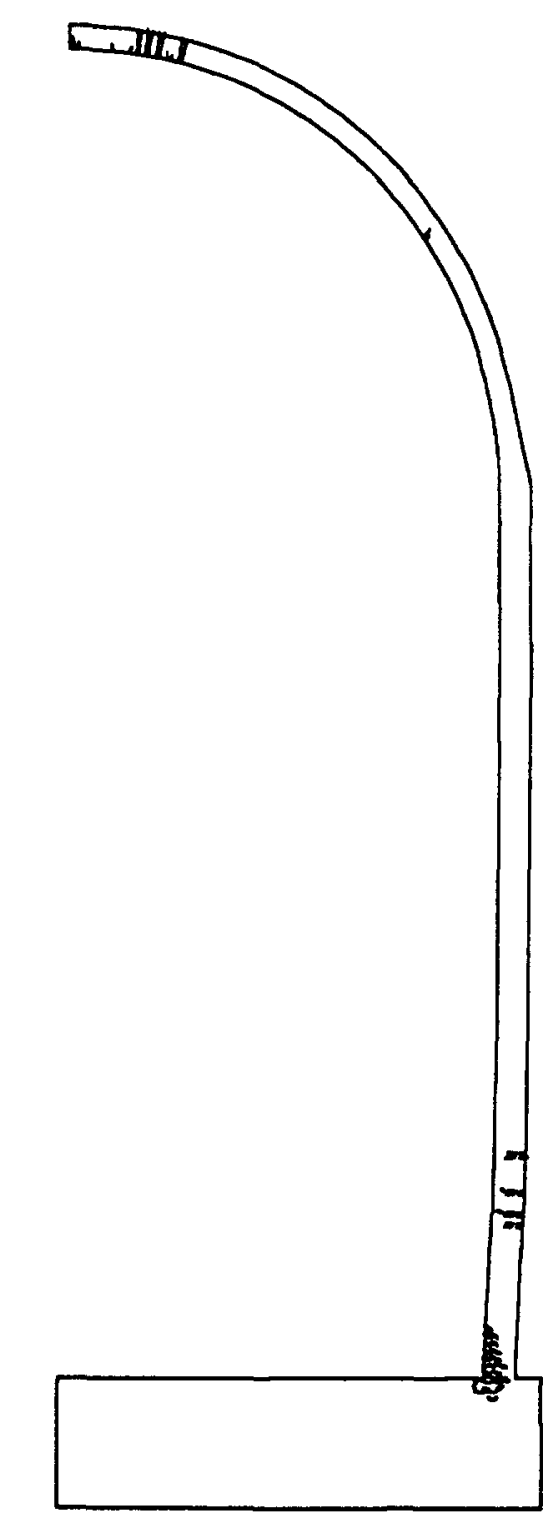

FISSURES DANS LE PLAN RZ PRESSION O.3 MPA

Figure 4.4 .16

Meridional crack pattern at $43.5 \mathrm{psi}(0.3 \mathrm{MPa})$ (calculation 1 ) 


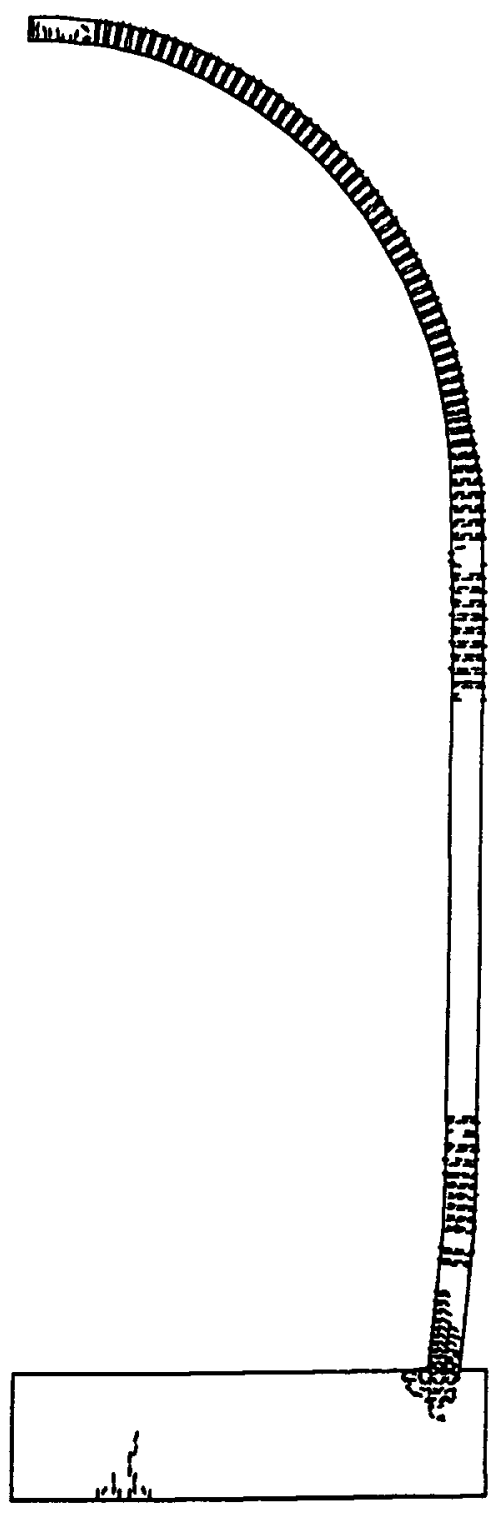

FISSURES DANG LE PLAN RI PRESSION 0.5 MA

Figure 4.4 .17

Meridional crack pattern at 72.5 psi ( $0.5 \mathrm{MPa}$ ) (calculation 1 )

$-198-$ 


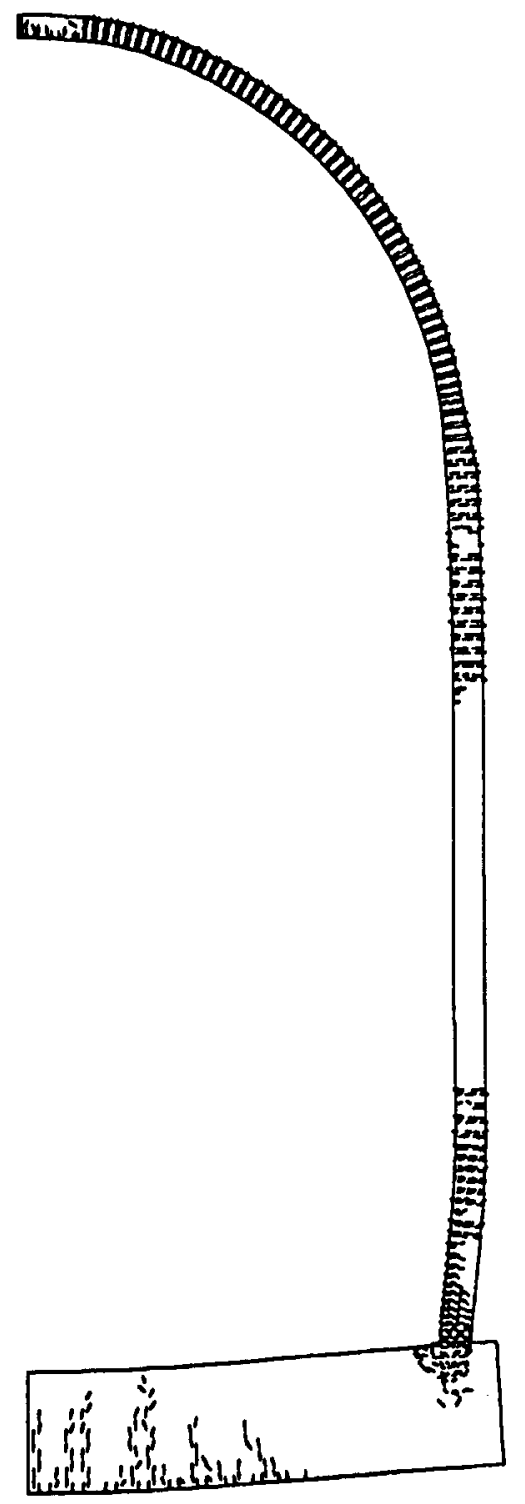

FISSURES DANS LE PLAN RZ PRESSION 0.55 MPA

Figure 4.4 .18

Meridional crack pattern at 79.8 psi ( $0.55 \mathrm{MPa})$ (calculation 1)

-199- 


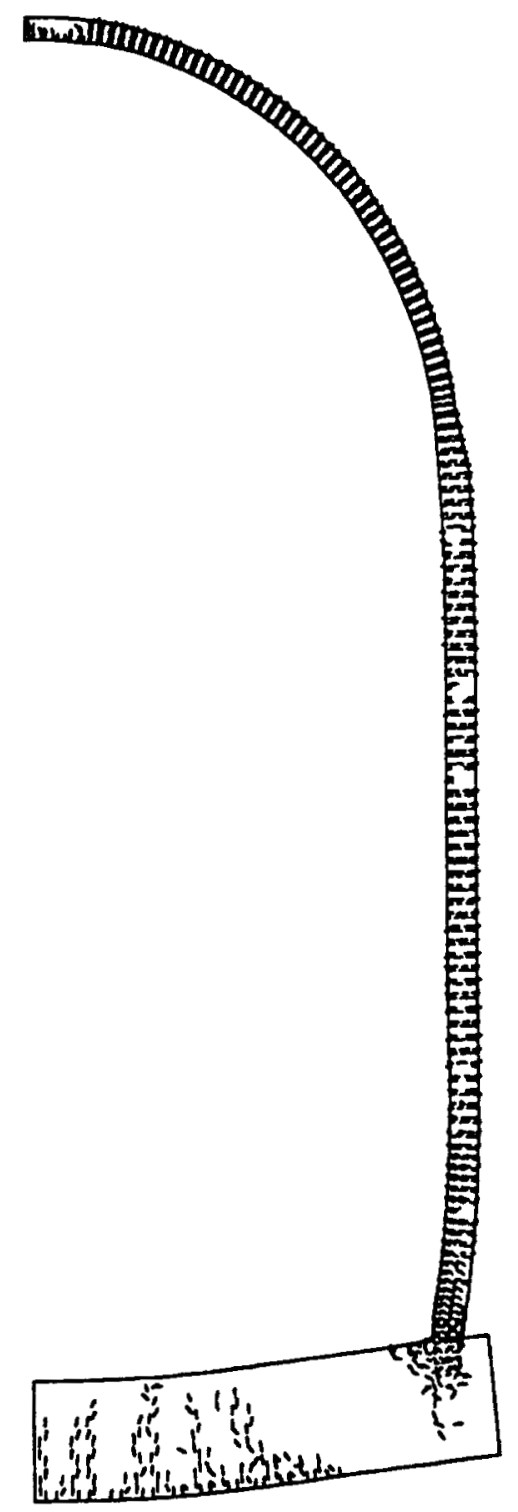

FISSURES DANG LE PLAN RI PRESSION $0.64 \mathrm{MPA}$

Figure 4.4 .19

Meridional crack pattern at 92.8 psi $(0.64 \mathrm{MPa})$ (calculation 1)

$-200-$ 


$$
2
$$




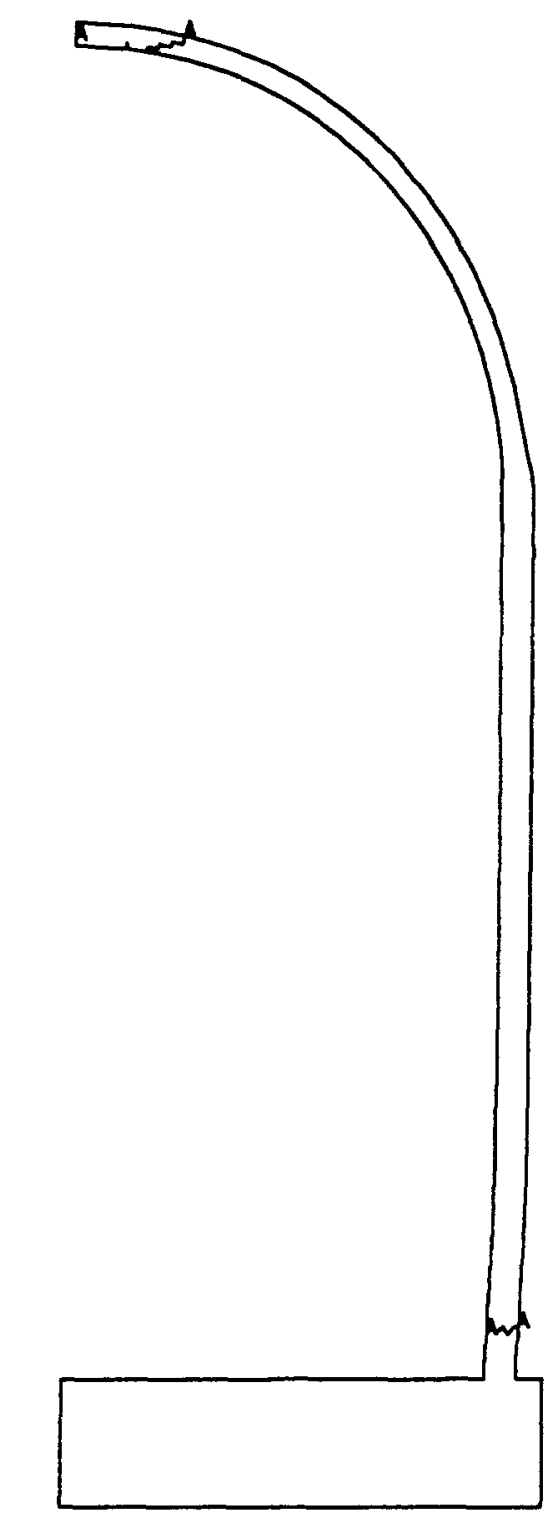

ZONES ENDOMMAGEES EN TETA PRESSION 0.3 MPA

Figure 4.4.21 Hoop crack pattern at $43.5 \mathrm{psi}(0.3 \mathrm{MPa})$ (calculation 1 ) 


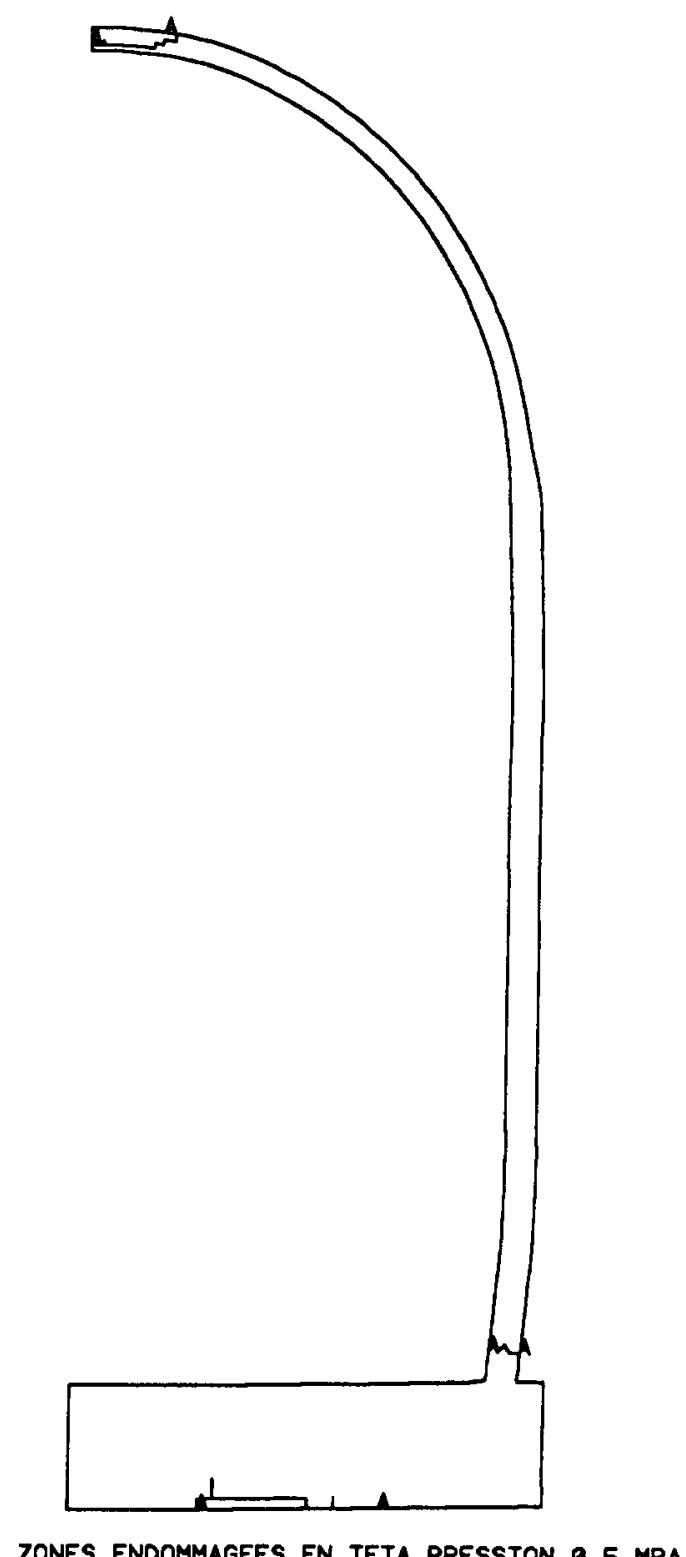

Figure 4.4.22 Hoop crack pattern at $72.5 \mathrm{psi}(0.5 \mathrm{MPa})$ (calculation 1 ) 


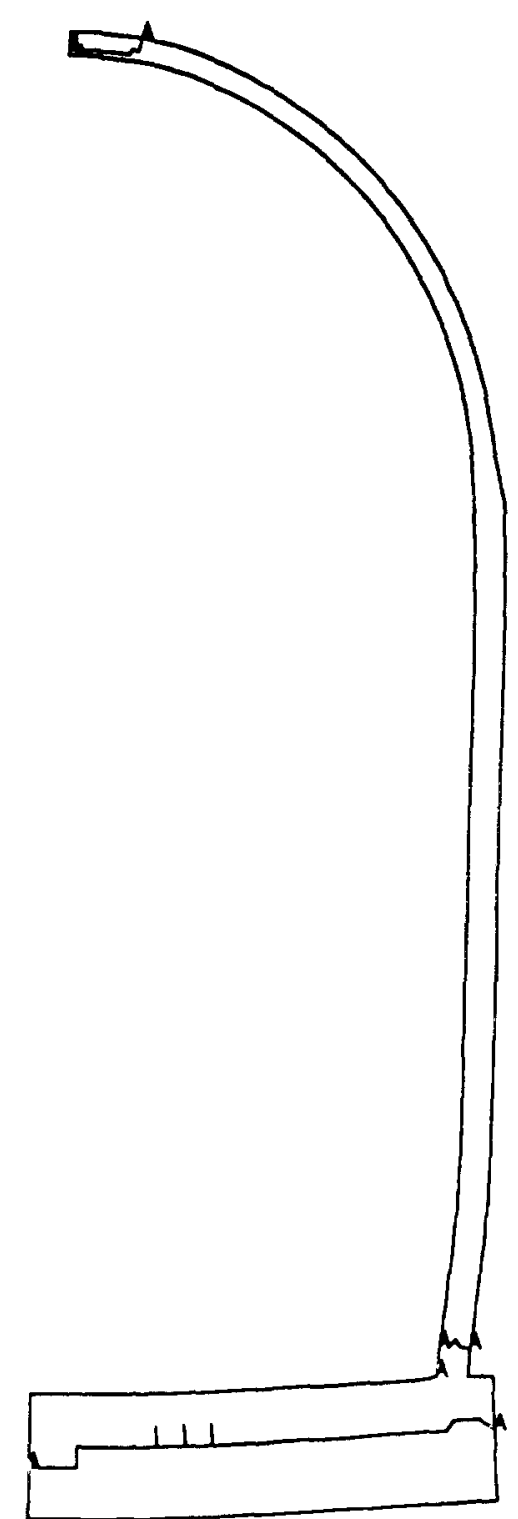

IONES ENOOMMAGEES EN TETA PRESSION 0.55 MPA

Figure 4.4.23 Hoop crack pattern at 79.8 psi (0.55 MPa) (calculation 1) 


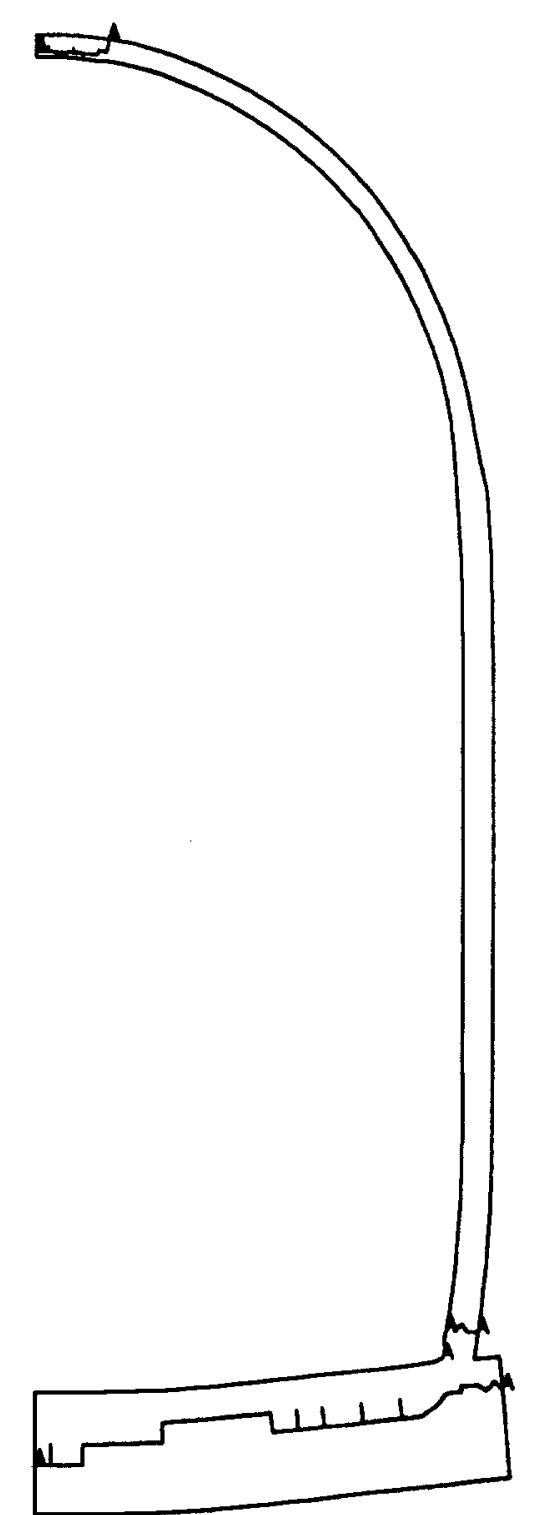

ZONES ENDOMMAGEES EN TETA PRESSION $0.04 \mathrm{MPA}$

Figure 4.4.24 Hoop crack pattern at $92.8 \mathrm{psi}(0.64 \mathrm{MPa})$ (calculation 1 ) 


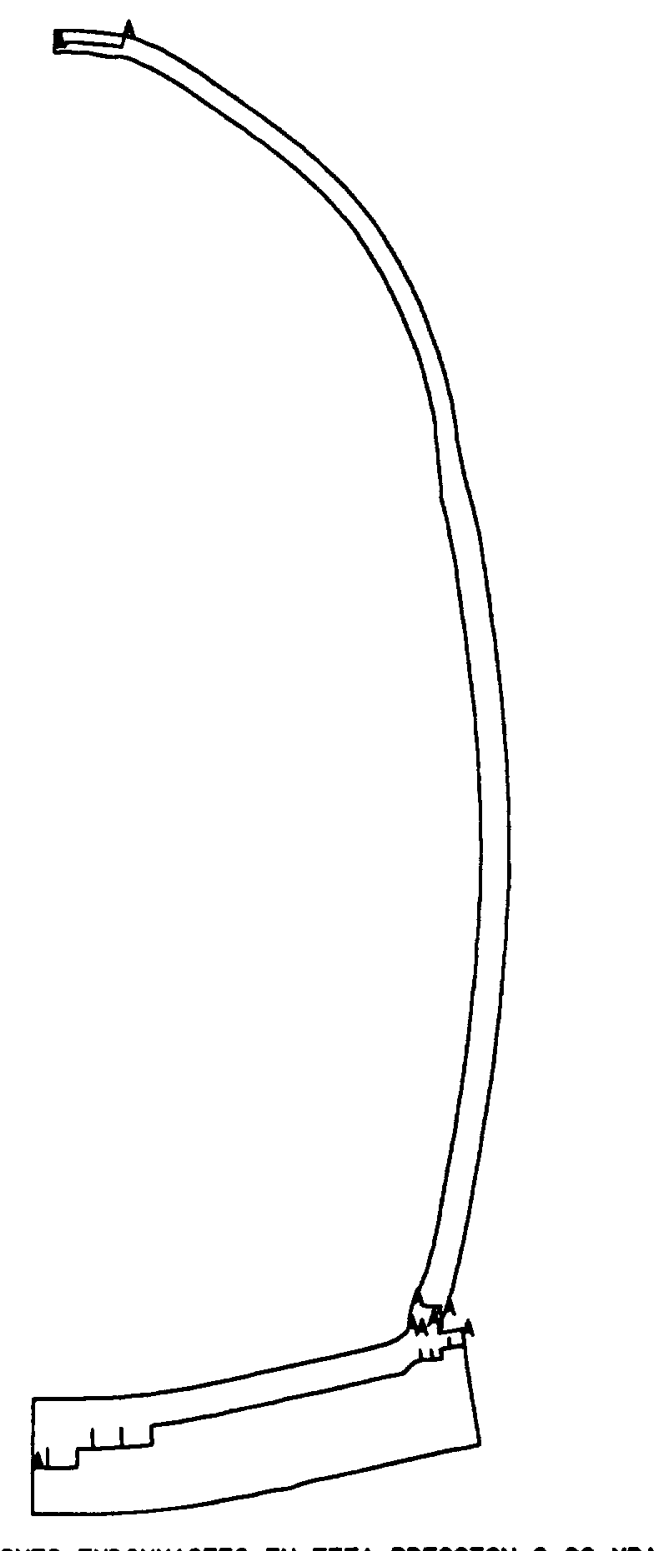

Figure 4.4.25A Hoop crack pattern at $142 \mathrm{psi}(0.98 \mathrm{MPa})$ (calculation 1 ) 


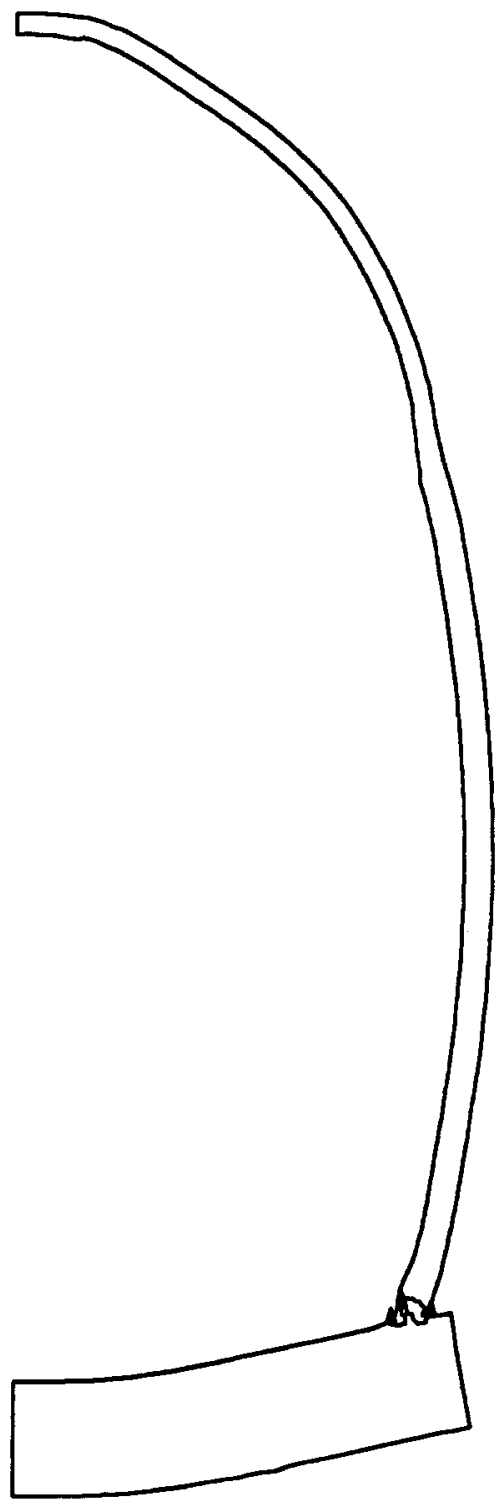

ZONES ENDOMMAGEES EN CISAILLEMENT PRESSION 0.08 MPA

Figure $4.4 .25 \mathrm{~B}$

Shear crack pattern at 142 psi ( $0.98 \mathrm{MPa}$ ) (calculation 1)

$-207-$ 


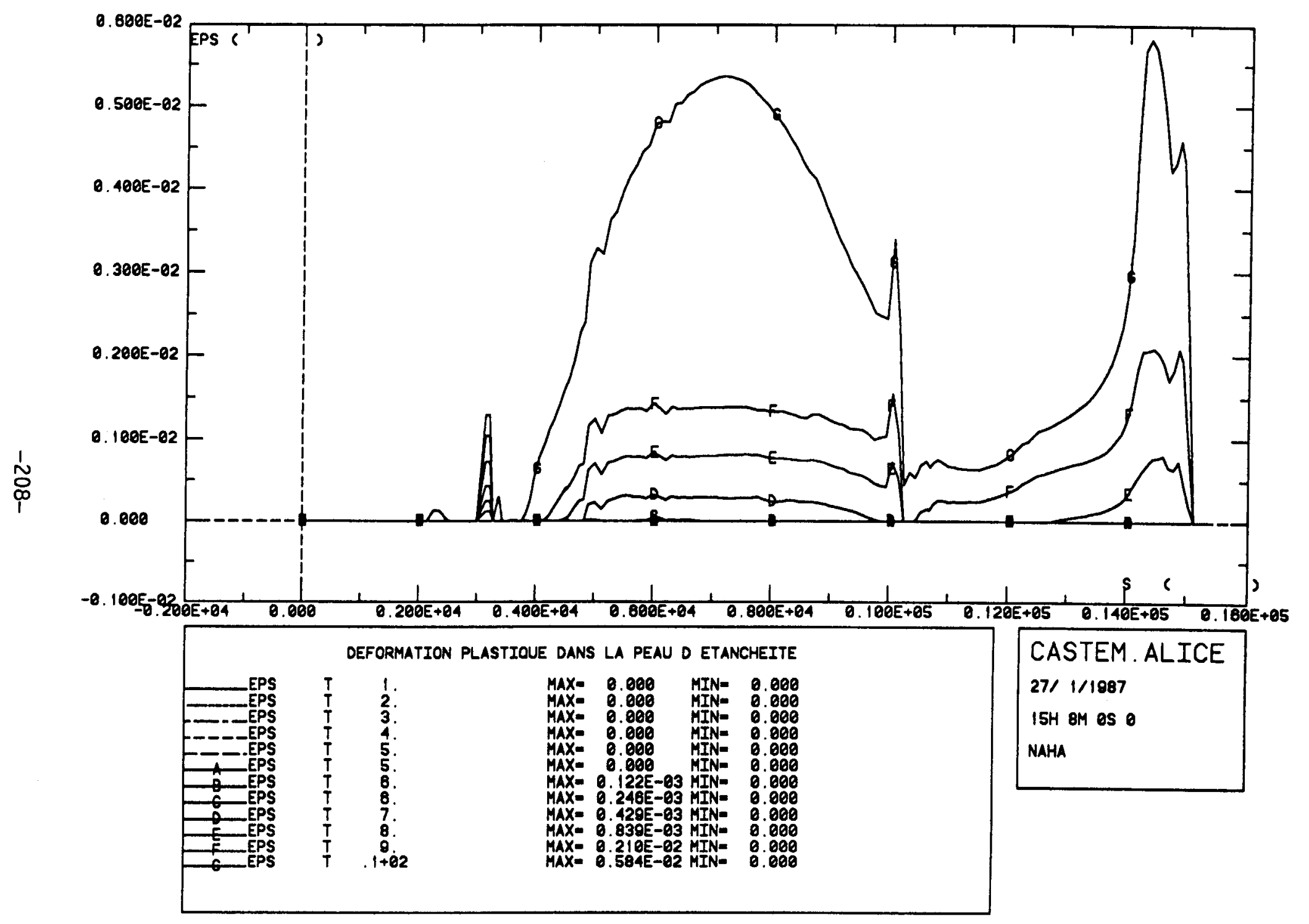

Figure 4.4.25C Plastic strains in the liner (calculation 1) 


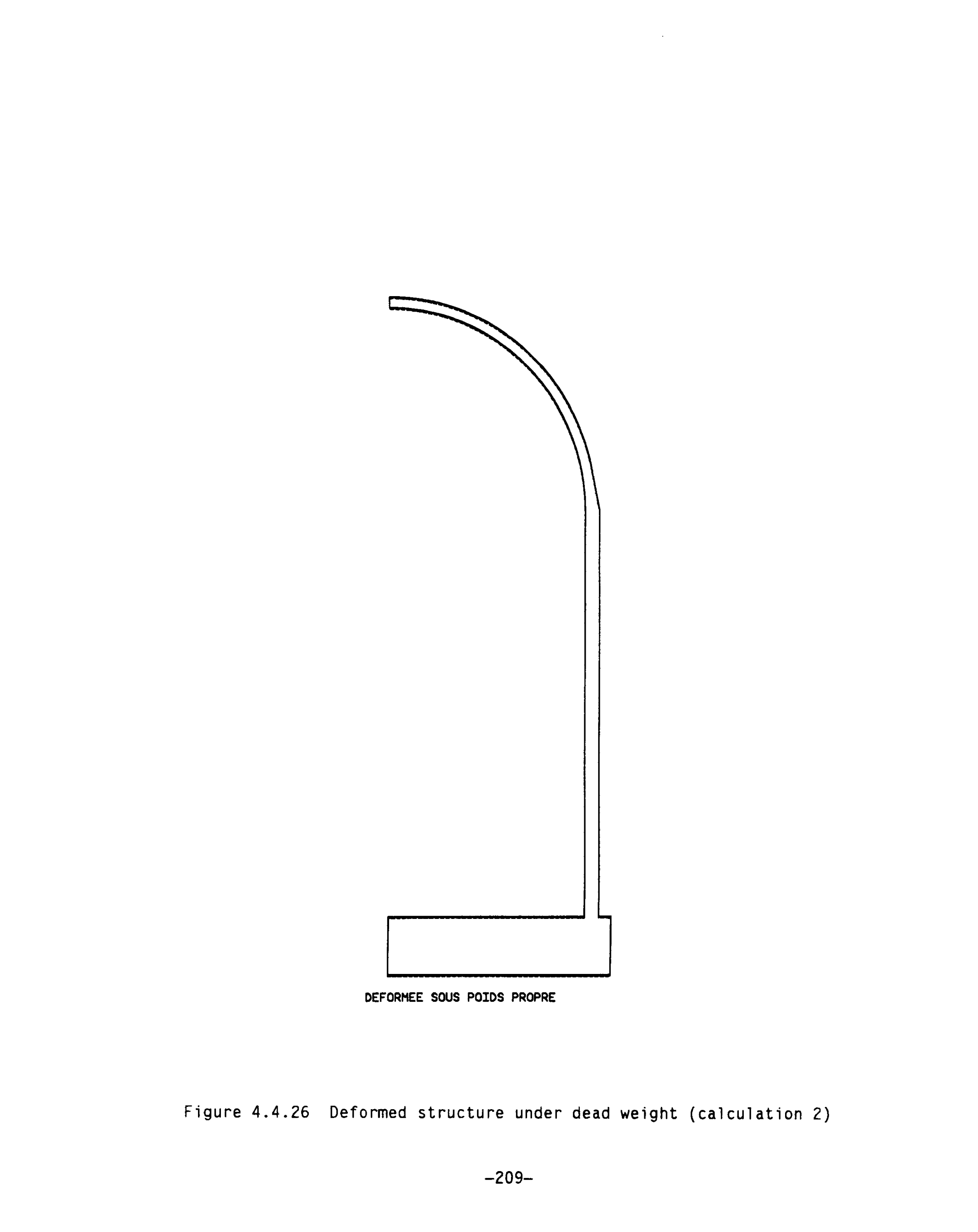




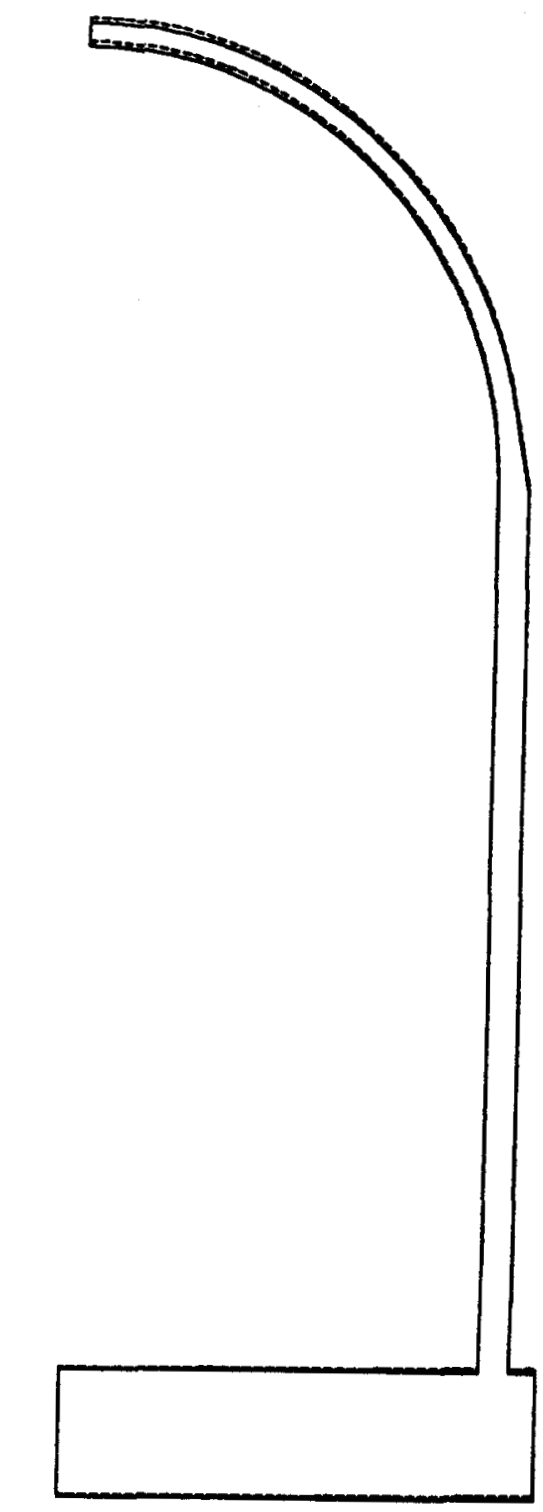

DEFORMEE PRESSION $0.2 \mathrm{MPA}$

Figure 4.4.27 Deformed structure at 29 psi (0.2 MPa) (calculation 2) 


$$
\underline{\partial}
$$




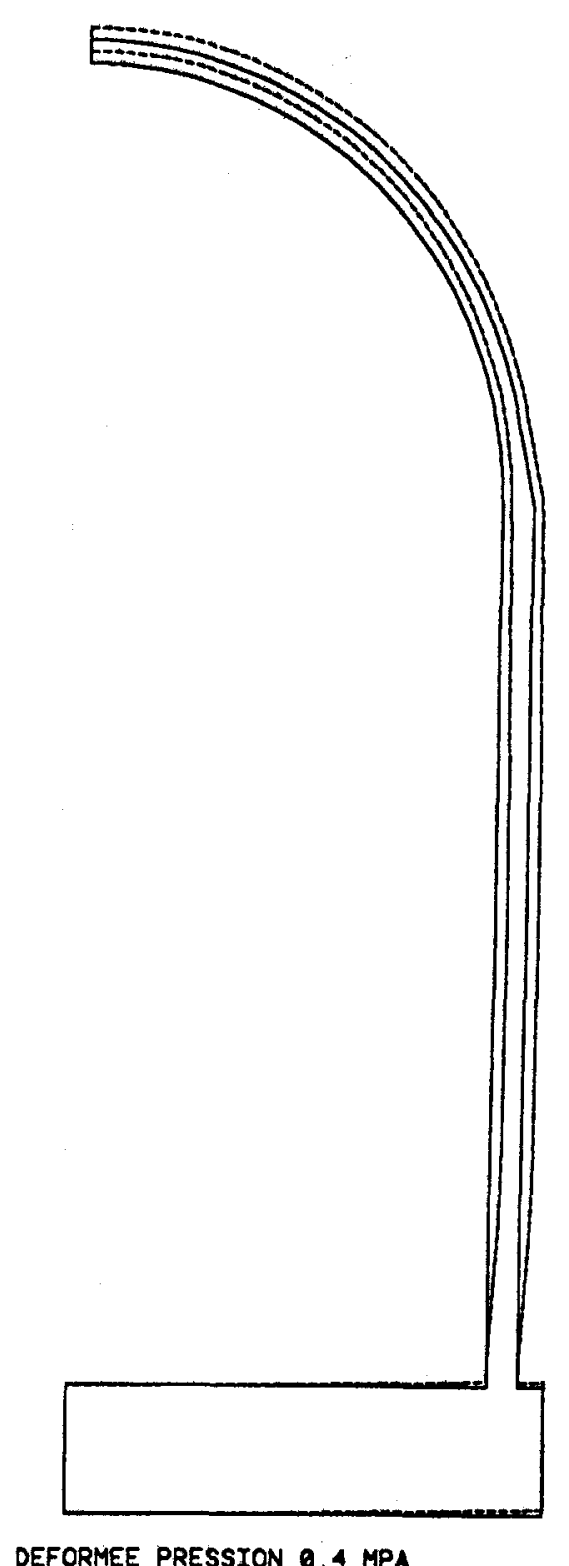

Figure 4.4.29 Deformed structure at 58 psi (0.4 MPa) (calculation 2) 


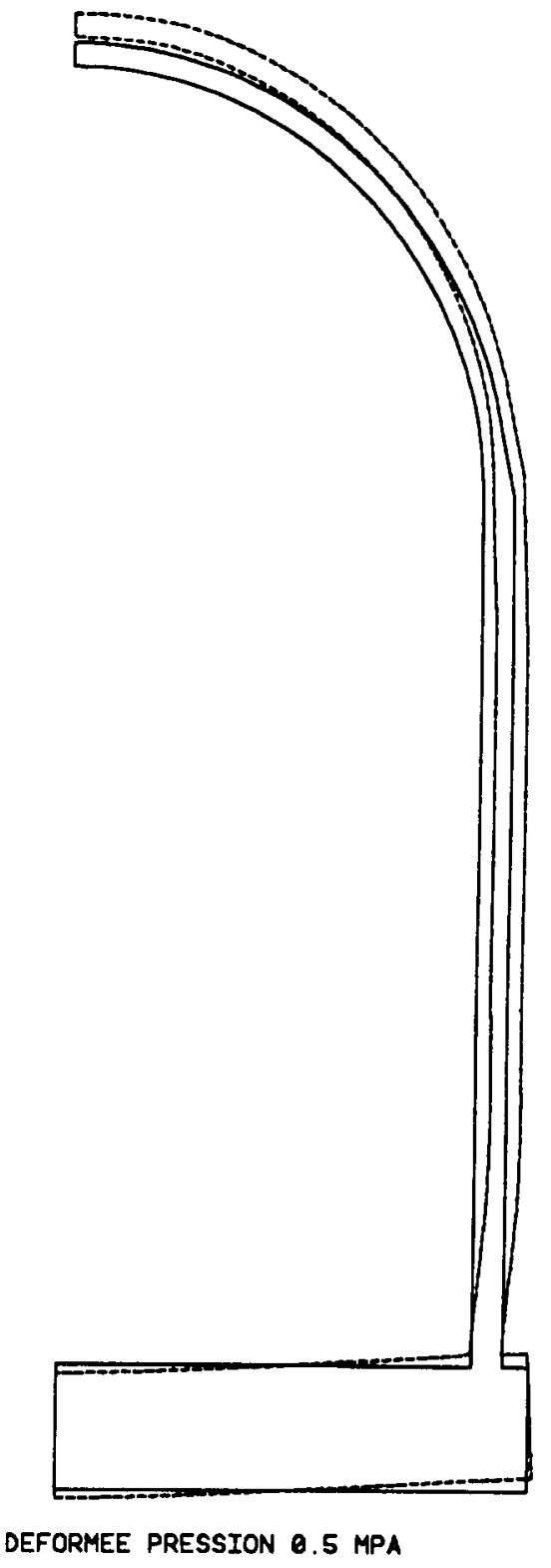

Figure 4.4.30 Deformed structure at 72.5 psi (0.5 MPa) (calculation 2) 


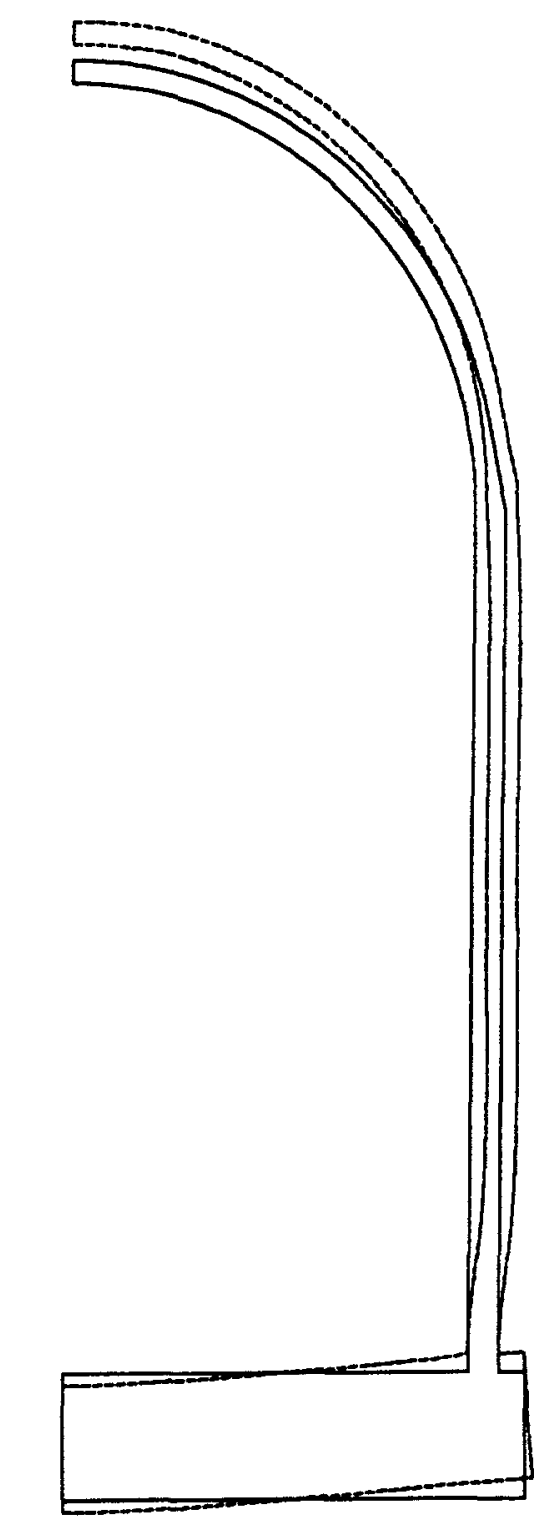

DEFORMEE PRESSION 0.55 MPA

Figure 4.4.31 Deformed structure at $79.8 \mathrm{psi}(0.55 \mathrm{MPa})$ (calculation 2) 


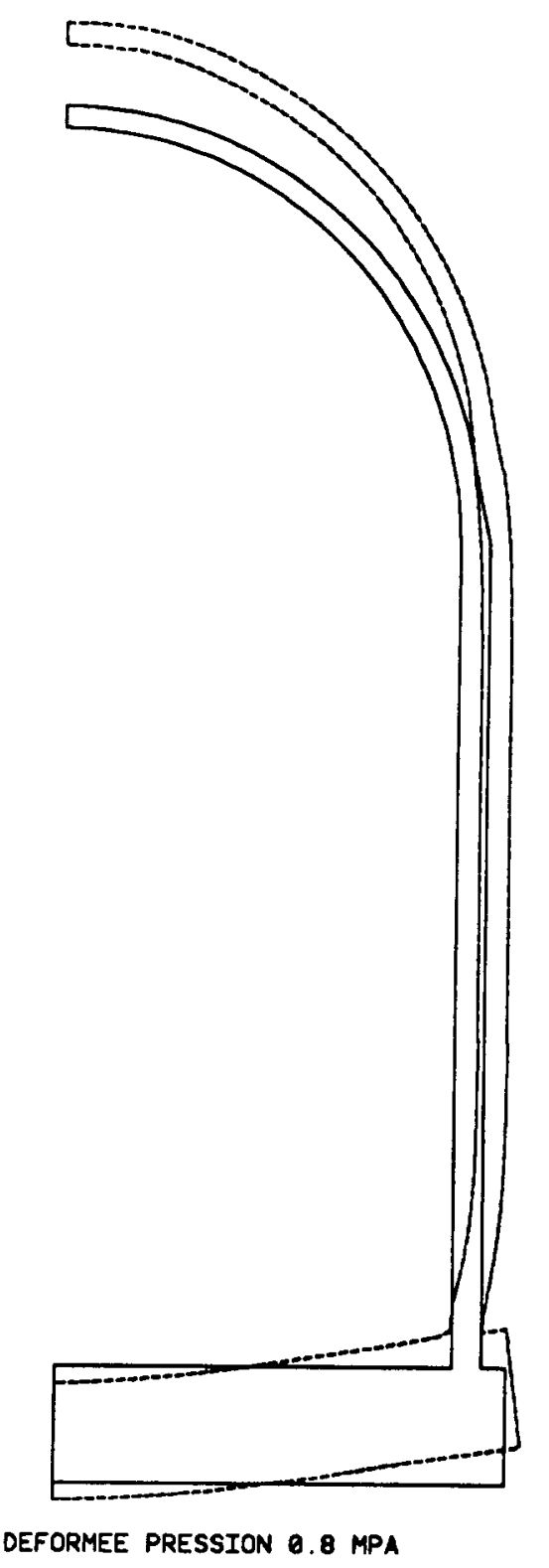

Figure 4.4.32 Deformed structure at $116 \mathrm{psi}(0.8 \mathrm{MPa})$ (calculation 2) 


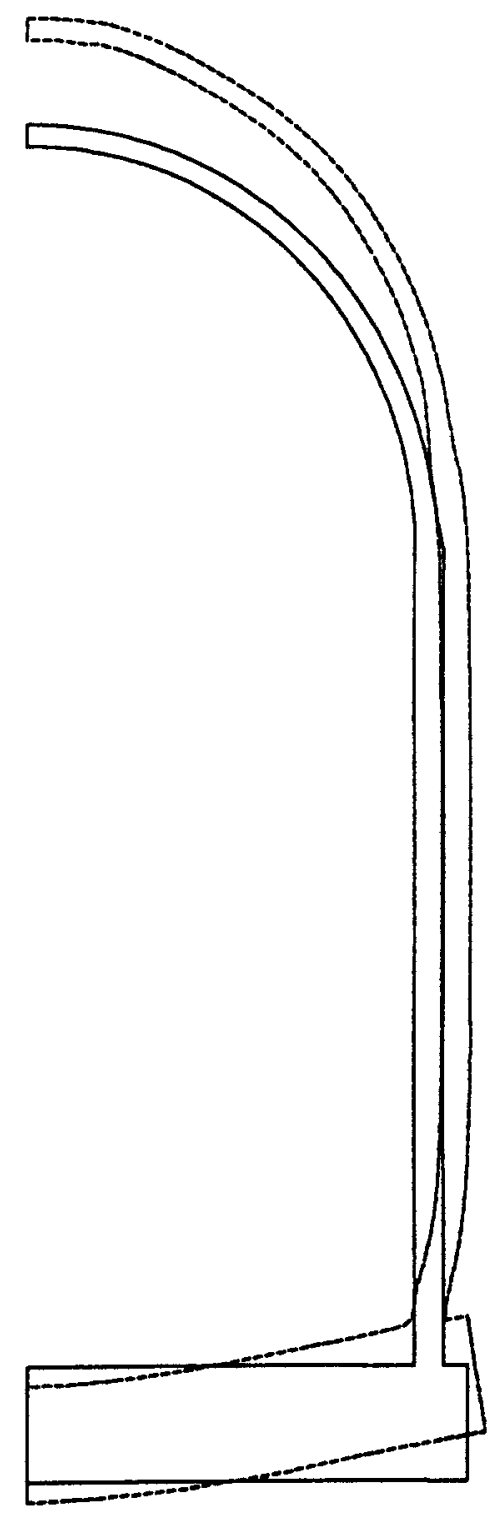

DEFORMEE PRESSION 0.0 MPA

Figure 4.4.33 Deformed structure at $130.5 \mathrm{psi}(0.9 \mathrm{MPa})$ (calculation 2)

$-216-$ 


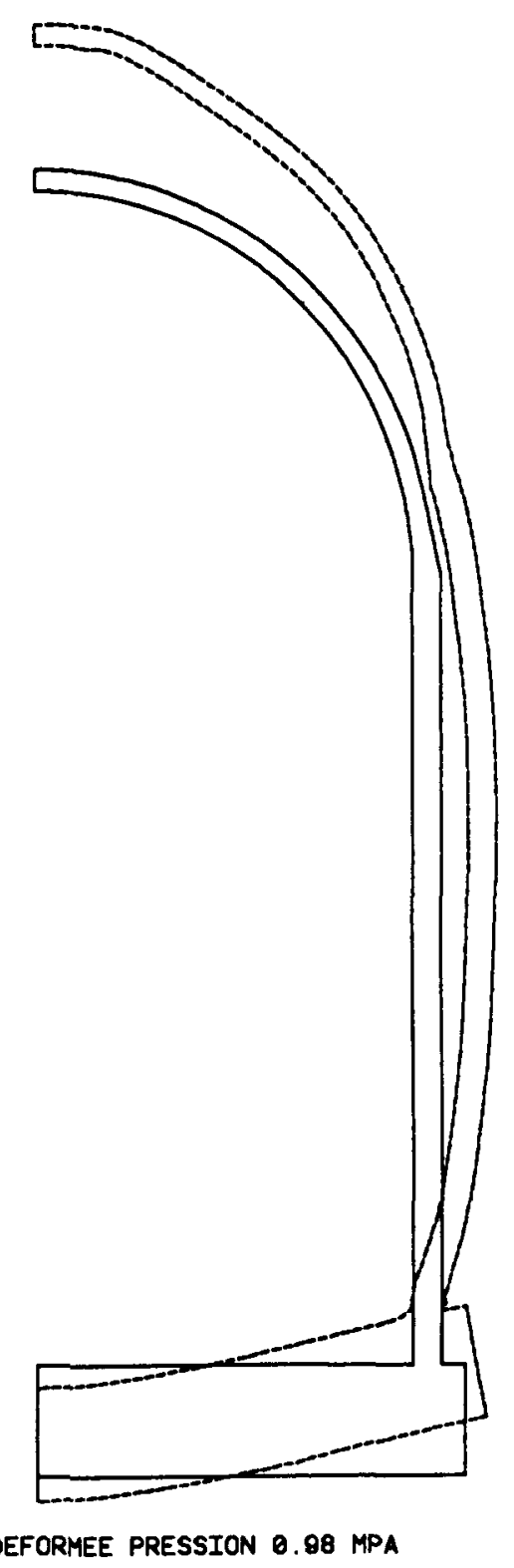

Figure 4.4.34 Deformed structure at $142 \mathrm{psi}(0.98 \mathrm{MPa})$ (calculation 2) 


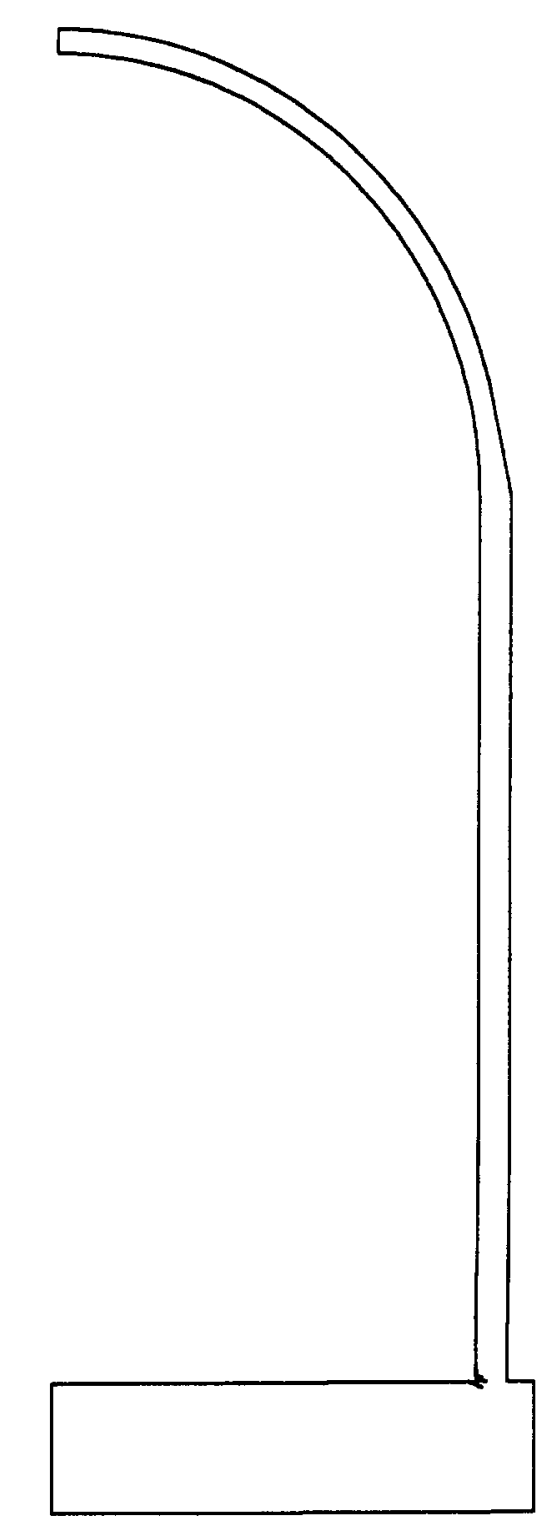

FISSURES DANS LE PLAN RZ PRESSION 0.2 MPA

Figure 4.4.35 Meridional crack pattern at 29 psi (0.2 MPa) (calculation 2) 


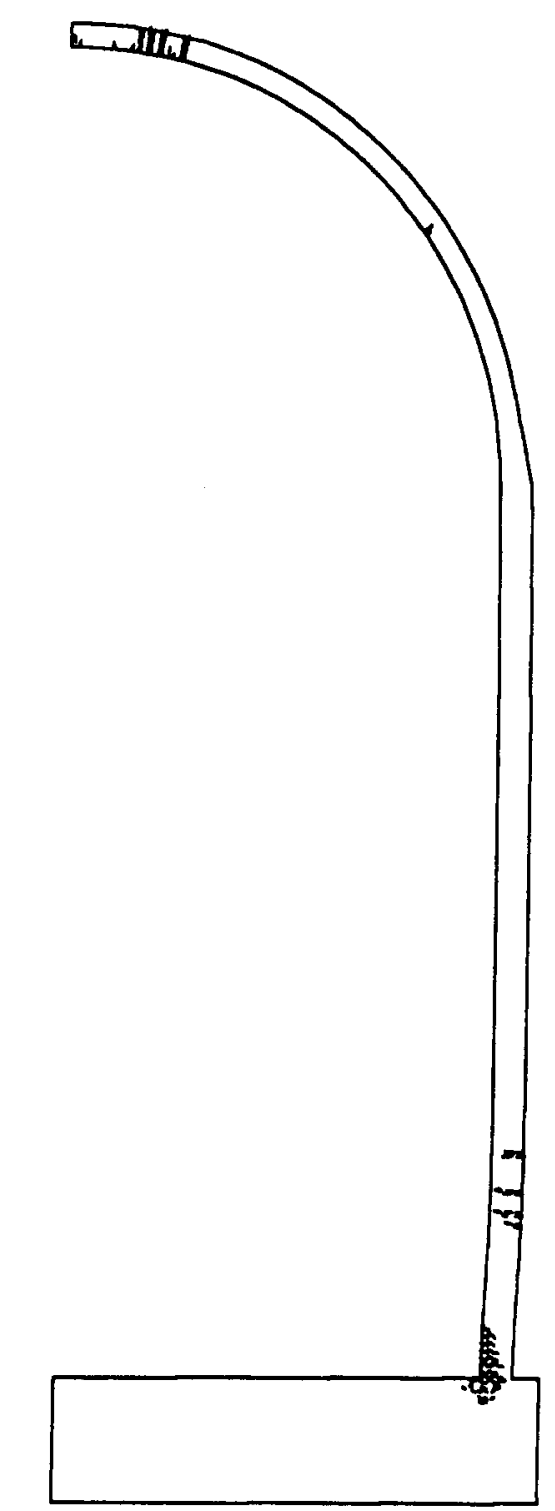

FISSURES DANS LE PLAN RZ PRESSION 0.3 MPA

Figure 4.4 .36

Meridional crack pattern at $43.5 \mathrm{psi}(0.3 \mathrm{MPa})$ (calculation 2) 


$$
2
$$




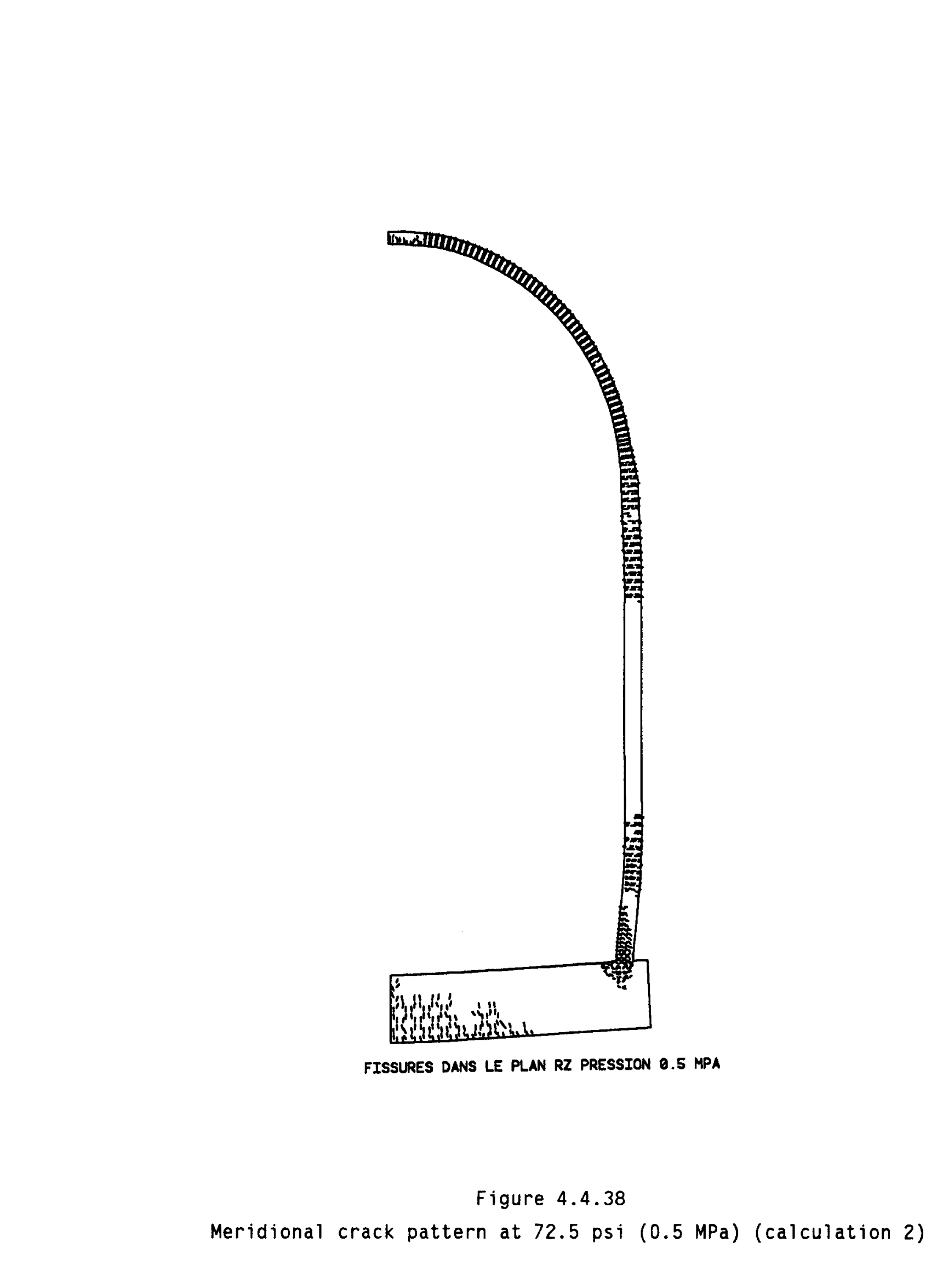




$$
2
$$




$$
2
$$




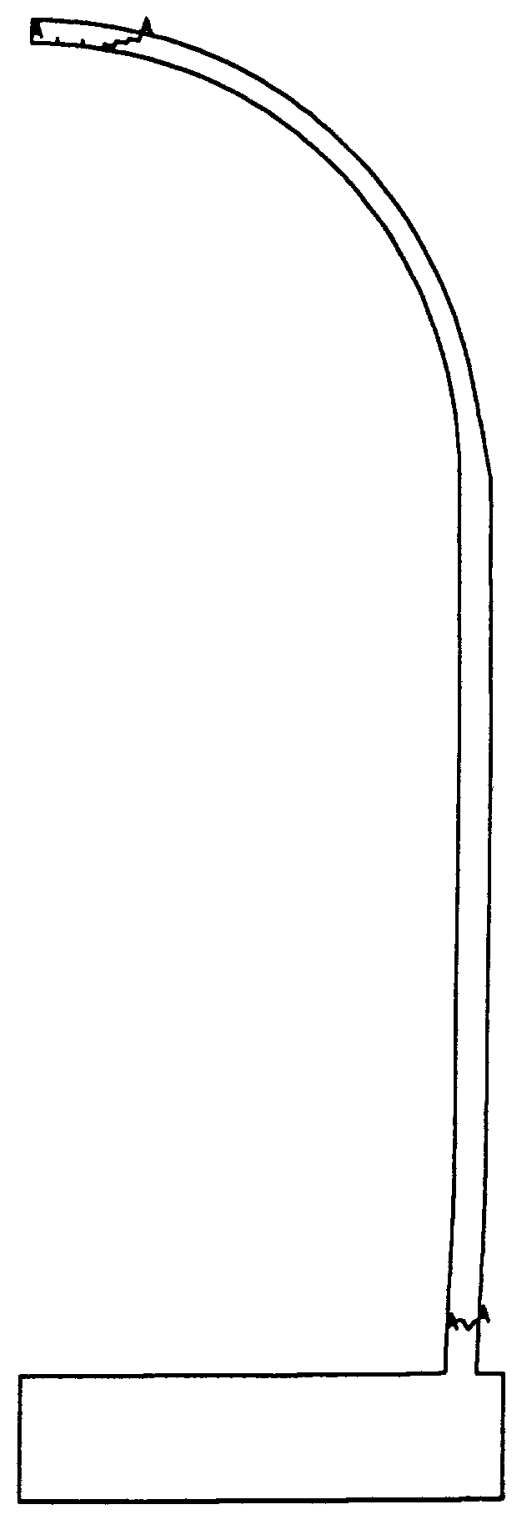

ZONES ENDOMMAGEES EN TETA PRESSION 0.3 MPA

Figure 4.4.41 Hoop crack pattern at 43.5 psi (0.3 MPa) (calculation 2)

$-224-$ 


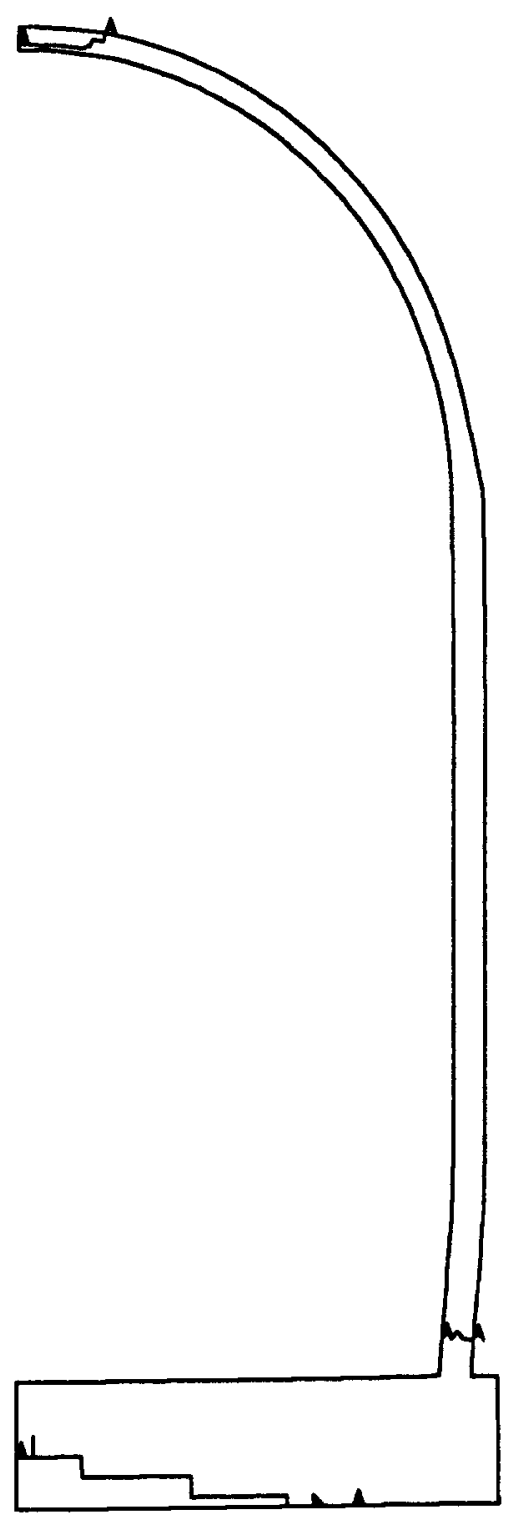

ZONES ENDOMMAGEES EN TETA PRESSION 0.4 MPA

Figure 4.4 .42 Hoop crack pattern at 58 psi (0.4 MPa) (calculation 2)

$-225-$ 


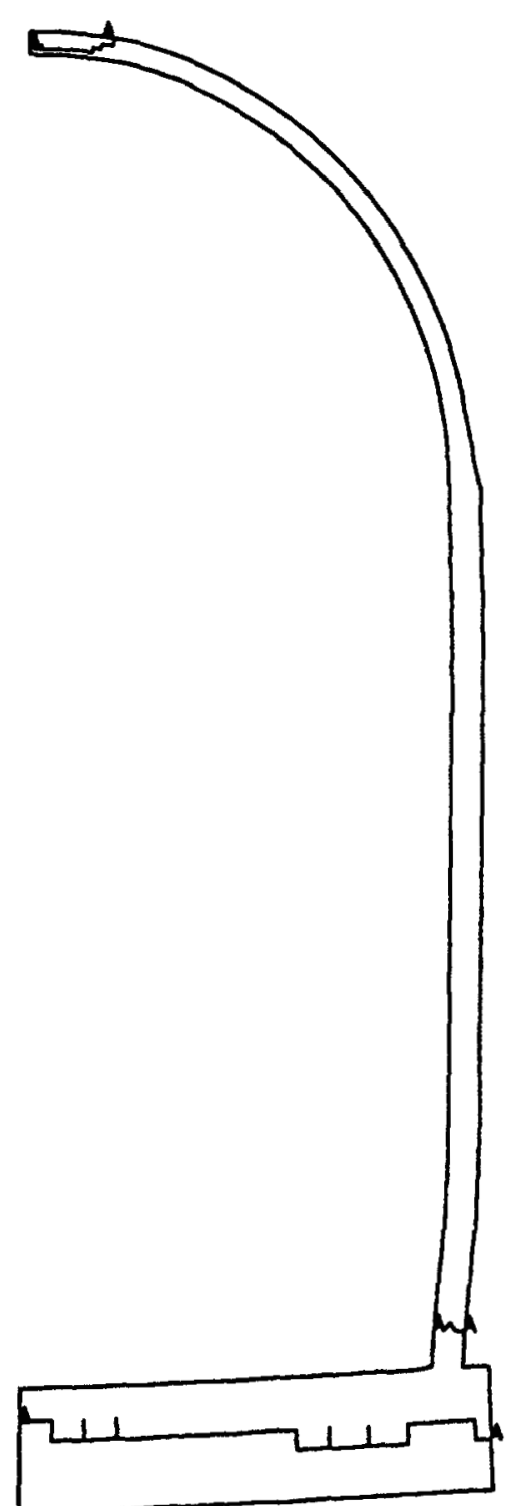

ZONES ENDOMMAGEES EN TETA PRESSION 0.5 MPA

Figure 4.4 .43 Hoop crack pattern at $72.5 \mathrm{psi}(0.5 \mathrm{MPa})$ (calculation 2) 


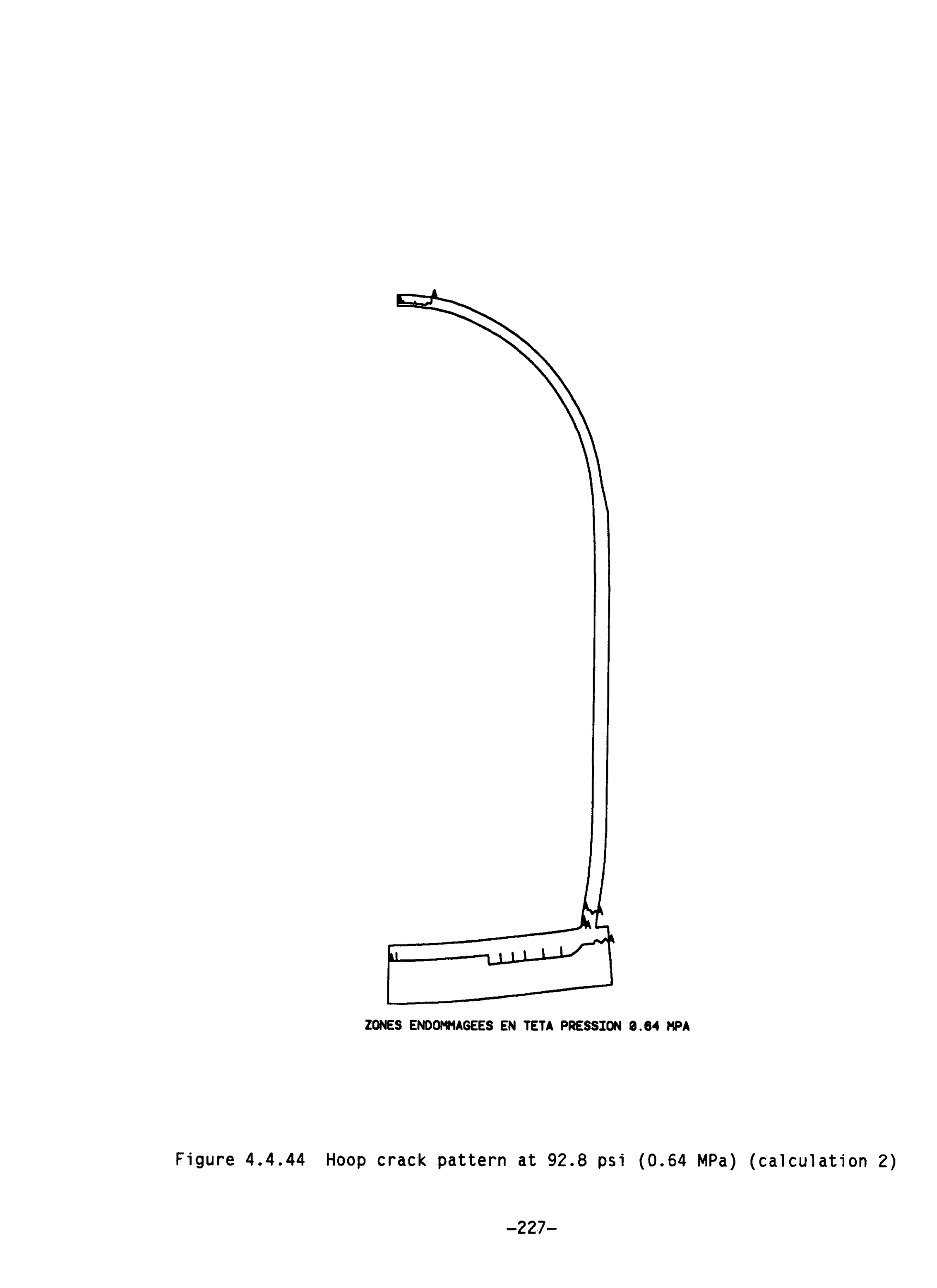




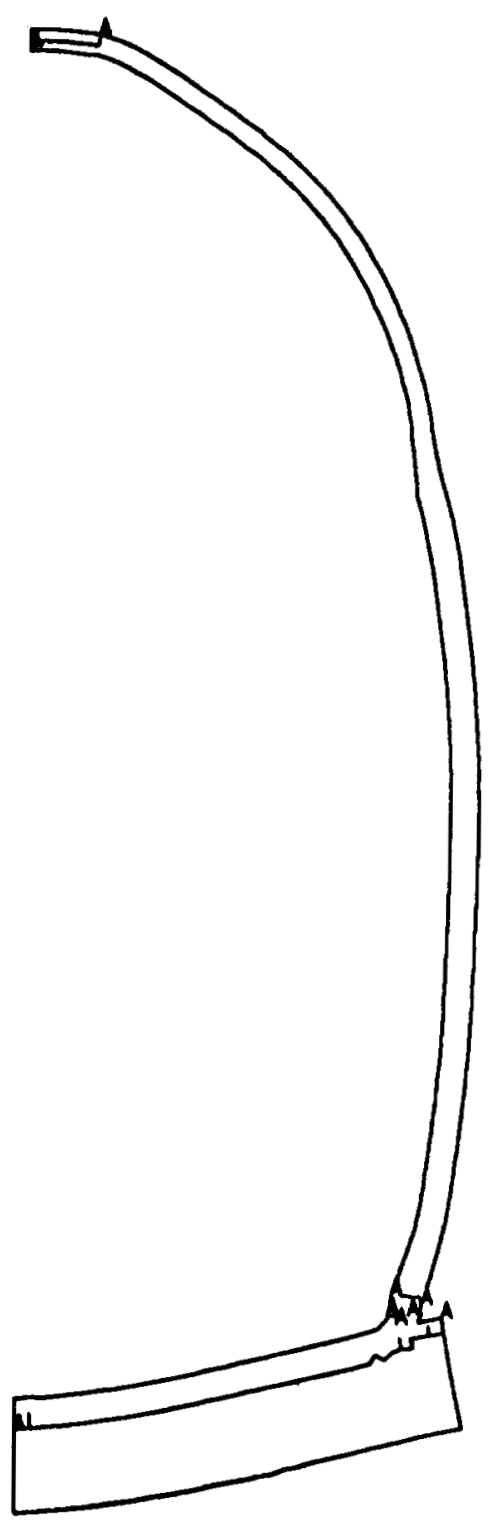

ZONES ENDOMMAGEES EN TETA PRESSION 0.98 MPA

Figure 4.4.45 Hoop crack pattern at $142 \mathrm{psi}(0.98 \mathrm{MPa}$ ) (calculation 2)

$-228-$ 


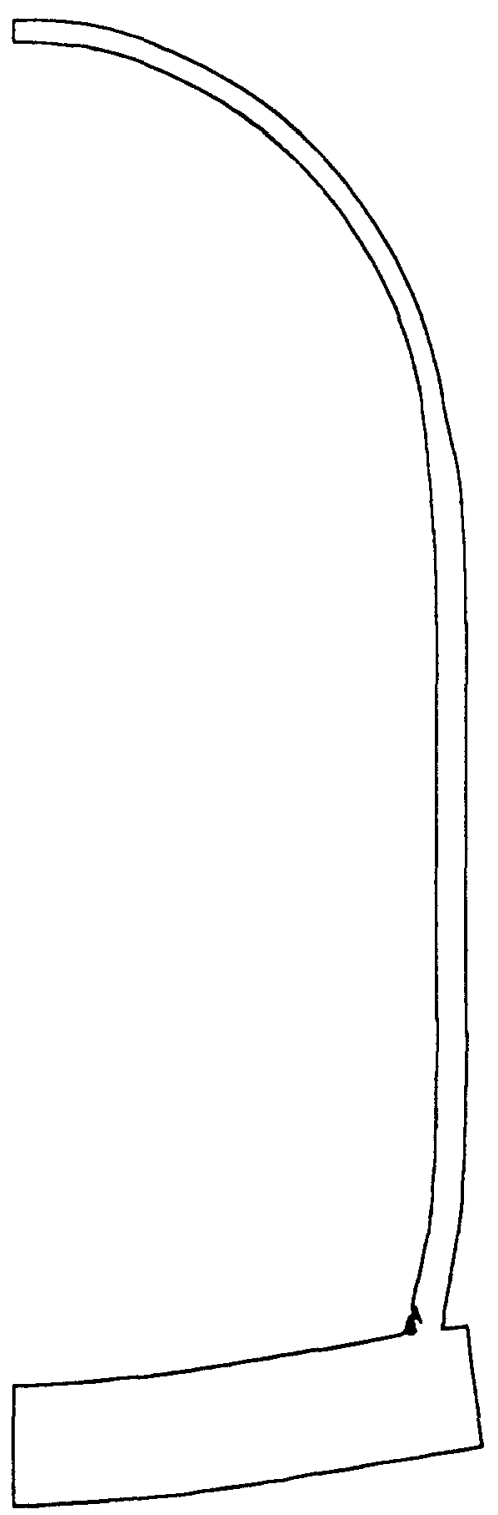

ZONES ENDOMMAGEES EN CISAILLEMENT PRESSION $6.8 \mathrm{MPA}$

Figure 4.4.46 Shear crack pattern at $116 \mathrm{psi}(0.8 \mathrm{MPa})$ (calculation 2)

$-229-$ 


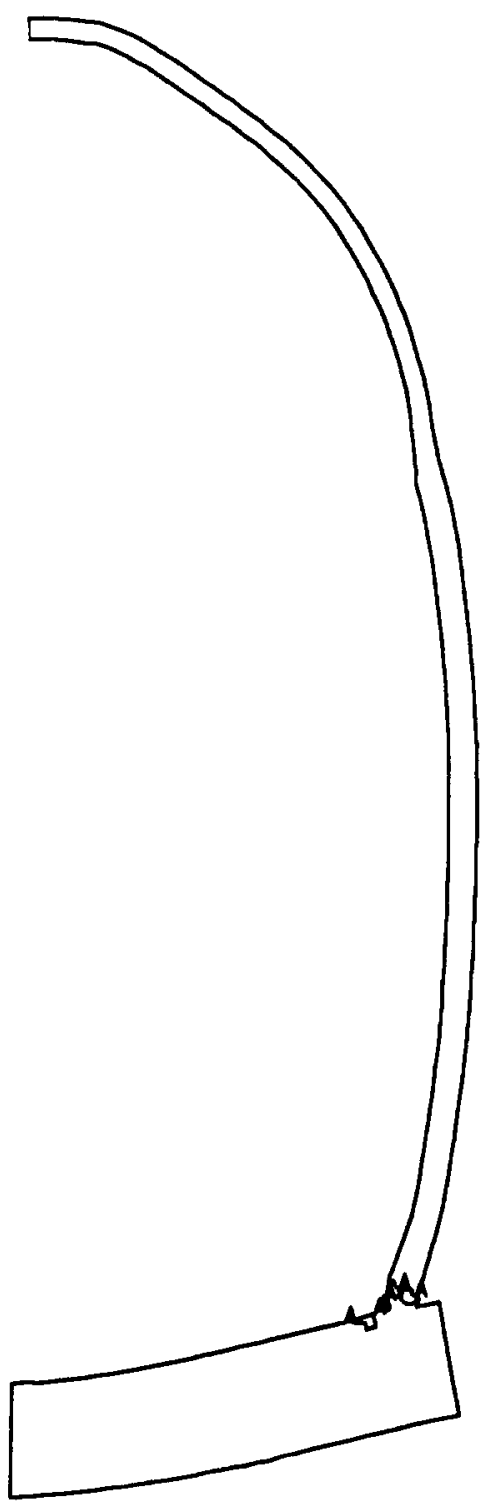

ZONES ENDOMMAGEES EN CISATLLEMENT PRESSION $0.08 \mathrm{MPA}$

Figure 4.4.47 Shear crack pattern at $142 \mathrm{psi}(0.98 \mathrm{MPa})$ (calculation 2)

$-230-$ 


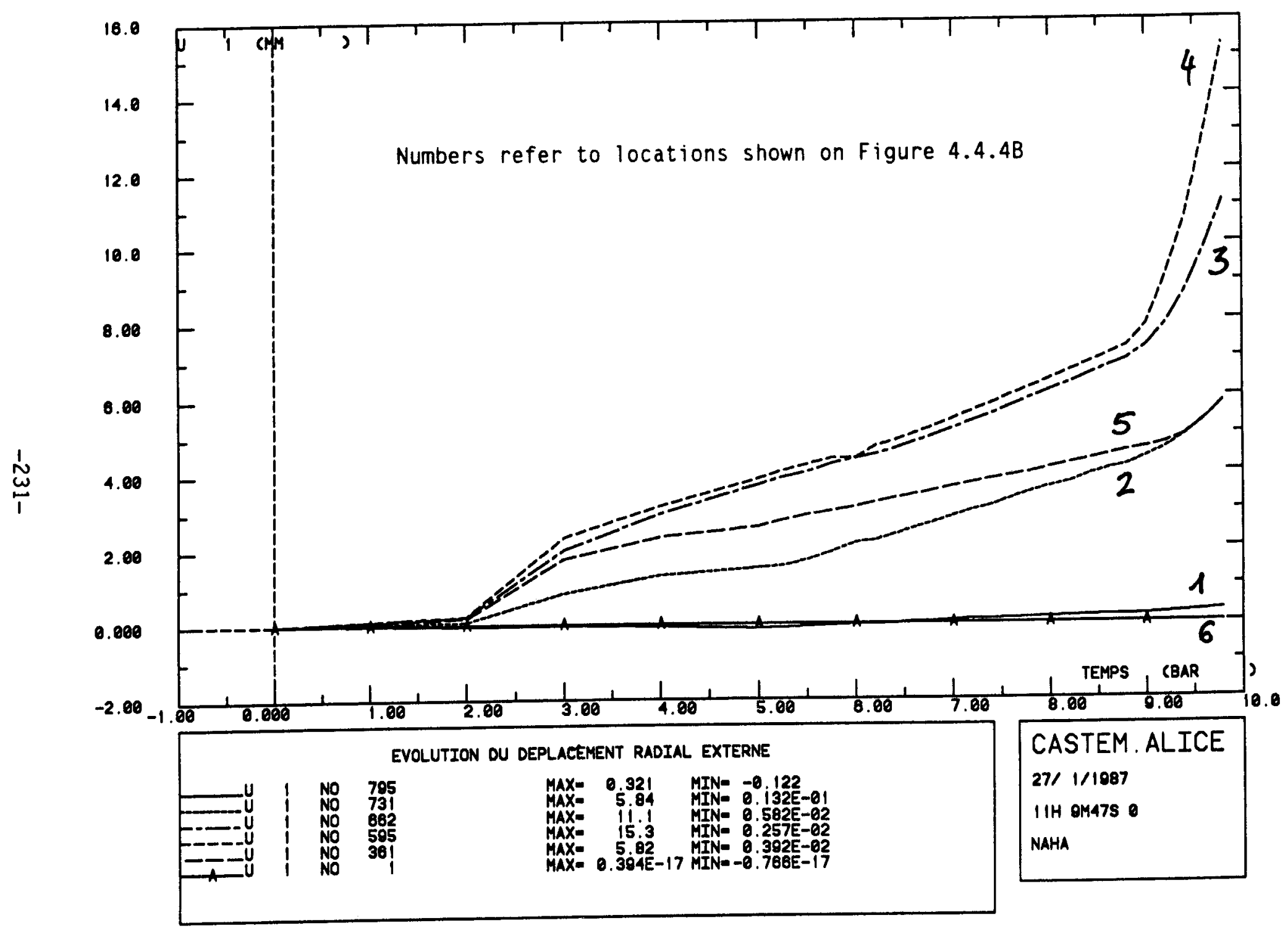

Figure 4.4.48 Radial displacements versus pressure (calculation 2) 


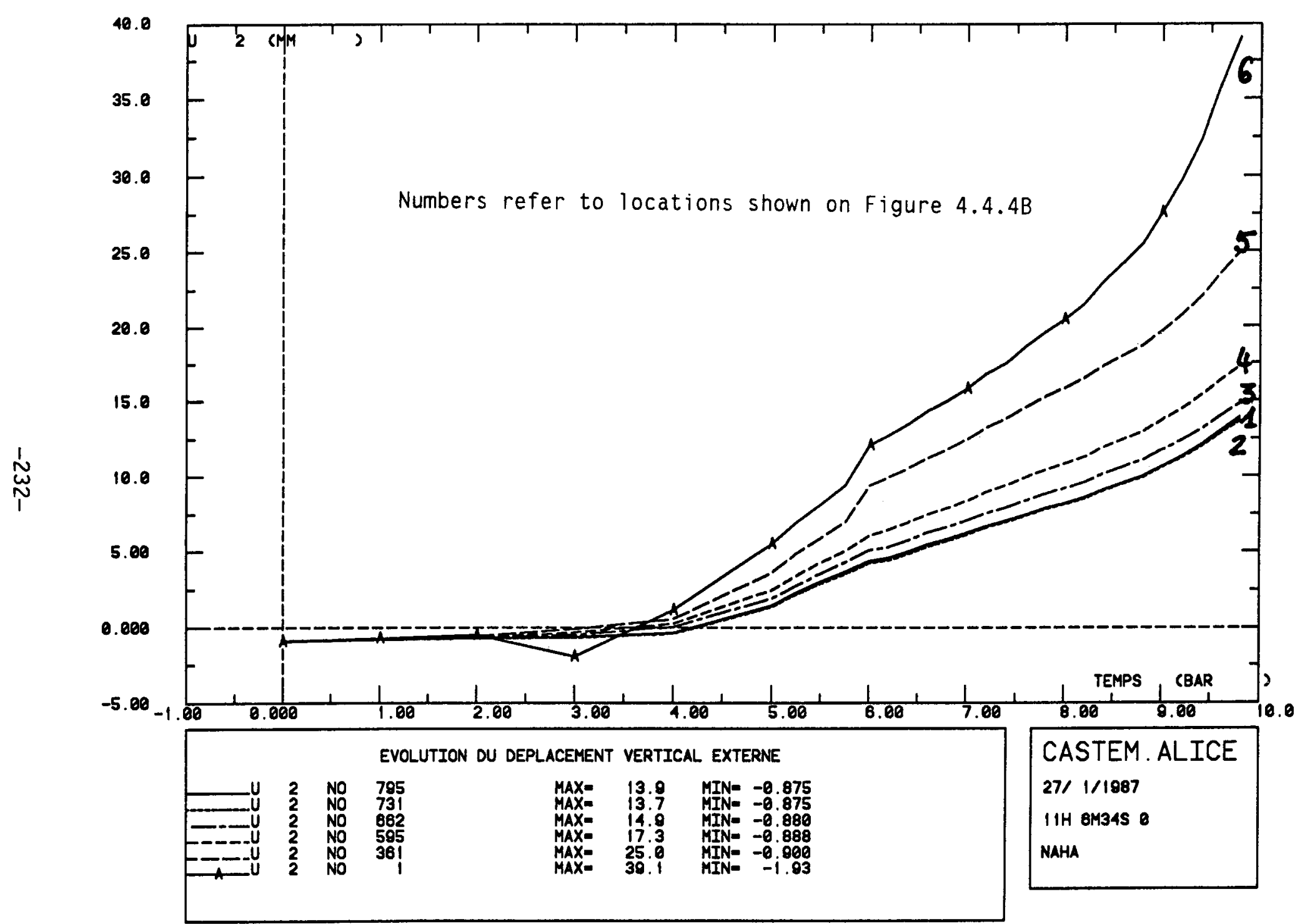

Figure 4.4.49 Axial displacements versus pressure (calculation 2) 


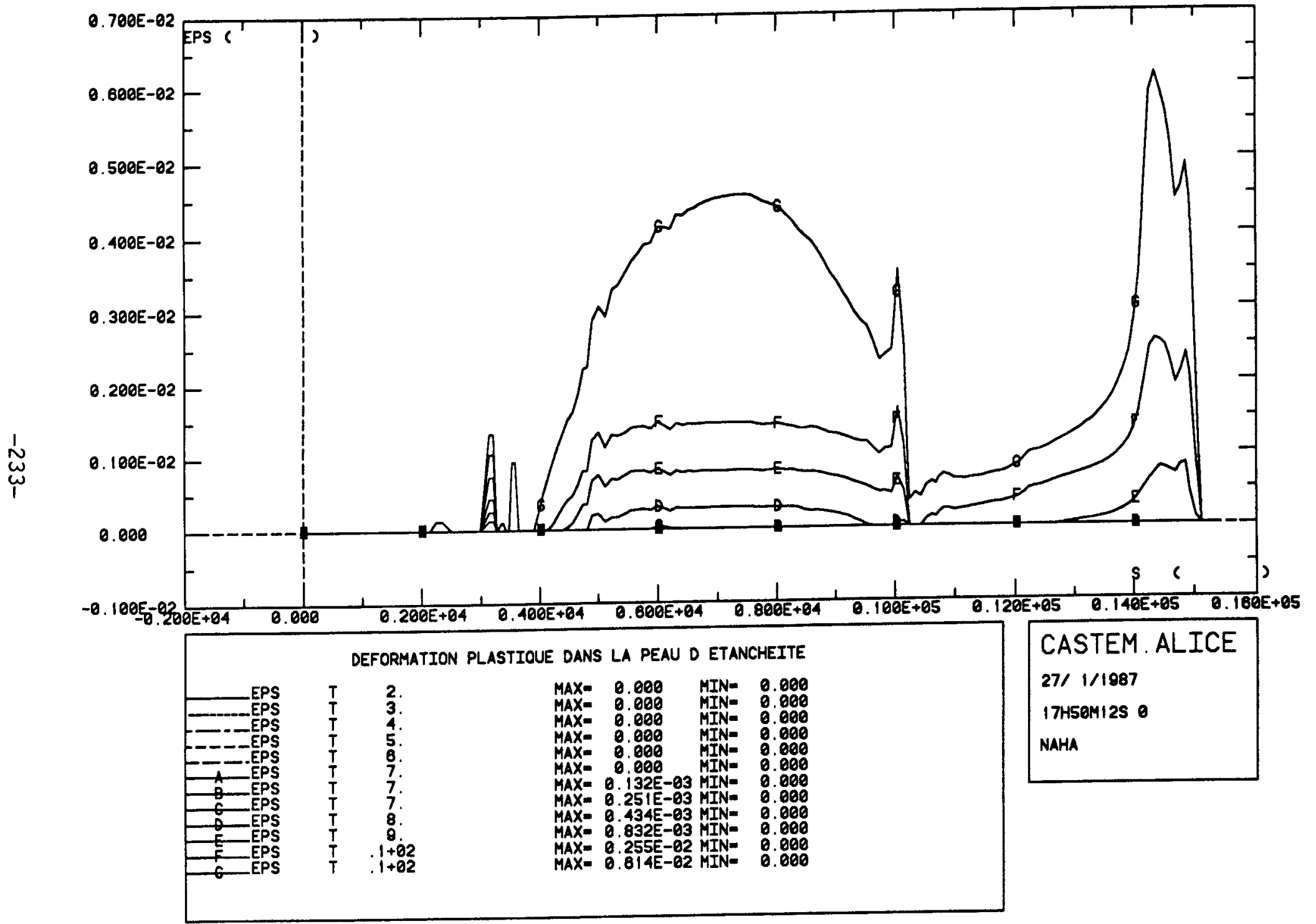

Figure 4.4.50 Plastic strains in the liner (calculation 2) 


\subsection{HM Nuclear Installations Inspectorate}

The authors of this section are R. J. Stubbs and I. W. Todd of HM Nuclear Installations Inspectorate and D. Collier and B. Walker of Ove Arup \& Partners, United Kingdom.

\subsubsection{Summary}

Analytical Method

The analysis was performed by running linear-elastic analyses at discrete pressure steps. At each pressure step an initial linear elastic analysis was carried out using best estimate element stiffnesses. The analysis results were compared with calculated nonlinear relationships and the elements were softened using nodal loads. The analysis was re-submitted with the softening loads. This procedure was repeated until convergence was achieved.

Constitutive Models

The concrete compressive stress-strain model used was the modified Kent Park. All other relationships were based on the material properties supplied.

Model Geometry

The majority of the analysis was carried out using an axisymetric orthotropic finite element computer model. A 3-D linear elastic analysis of a quarter model of the containment was carried out to assess the influence of the non-axisymmetric details.

Failure Criterion

The concrete reinforcement and liner had a finite strain to failure built into the softening routine. However shear failure had to be assessed externally to the computer program.

Predicted Failure Mode

The analysis was carried out only to predict the global behavior of the containment structure, therefore detailed assessment of possible failure mechanisms due to the penetrations were not assessed.

The most likely global mode of failure of the structure is a shear failure of the cylinder above the shear reinforcement. However, as the restraint to the liner at the wall base is insufficient to achieve yielding in the vertical direction, the local deformation may cause local tearing around the studs.

Predicted Failure Pressure

The minimum pressure at which the containment will fail in shear is 116 psi (0.8 $\mathrm{MPa}$ ), in accordance with the ASME code. Taking into consideration that the code is conservative, failure will probably occur between $2.8 \mathrm{P}_{\mathrm{d}}$ and 3.8 $P_{d}$. However, it is possible that the liner will tear at the wall base at a lower pressure.

\section{5 .2 Introduction}


The regulation of nuclear installations in the United Kingdom is achieved by means of a licensing system administered by HM Nuclear Installations Inspectorate. For some years, the Inspectorate has been assessing a modified SNUPPS design for the introduction of a PWR system to the UK. Part of this work has involved the analysis of the prestressed containment structure proposed for the Sizewell B site; this and the present study have been carried out on behalf of the inspectorate by consultants ove Arup and Partners (OAP).

The requirements of the Inspectorate with regard to structural analysis are rather different to those of the licensee. The licensee is responsible for ensuring that the structure complies with statutory requirements, and is also economical. The regulator, on the other hand, is concerned less with the detail of the design than with the adequacy of its approach. Such analysis therefore tends to concentrate upon sensitivity studies, and to take a somewhat broader view. For this reason, the approach adopted for the Sizewell structure was not a full nonlinear analysis, but a more simplified approach. Basically, a linear analysis is performed, to which nonlinearity is added only at those locations and at those load levels at which it is required. The method is therefore highly economical, requires a greater level of engineering judgement, and in addition, provides a diverse check on the more sophisticated analyses submitted by the licensee.

It is this technique which has been used for the pre-test predictive calculations of the behavior of the Sandia model containment. Some improvements have been made since the sizewell work. For example, nonlinearity is now introduced by the use of pseudo-loads rather than softening of the element stiffness; this is more economical in terms of computer time and allows a greater number of variables to be modified. The technique is, however, fundamentally the same, and the Inspectorate is interested to see how its predictions compare with those of more advanced techniques.

The computer modelling of the structure is described in more detail in the following section, but in outline was based upon the PAFEC suite [4.5.1] of finite elements, and has been carried out on a VAX 11/785 computer. The analysis was performed in two stages. First, the global behavior was determined by means of an axisymmetric analysis of the finite element idealization. The second stage considered the effects of the penetrations on the axisymmetric behavior, using a three-dimensional model of a quarter of the containment, and a local sub-model of an equipment hatch. The finite element representations used in both steps are illustrated in figure 4.5.1.

It is necessary to state at the outset the limitations of this technique. Firstly, it is not the intention of this study to investigate the detailed failure mechanism but to assess the structure's behavior up to the range of pressures at which failure might be expected to initiate. Estimates of this range of pressure appear in the findings of this study. Secondly, the technique is not capable of providing detailed predictions in highly localized areas, and for this reason no plots are given of, for example, the liner strain on the knuckle at the wall-base connection. (Special analytical techniques had to be introduced at this location due to the prediction of limited restraint to the liner at the wall base. This is described in detail in the text.) 


\subsubsection{Axisymmetric Analysis}

\subsubsection{Introduction}

For the first stage of the analysis the containment was assumed to be axisymetric for the finite element idealization. The effects of the nonaxisymmetric elements, such as the penetrations, were ignored since the analysis was primarily to investigate the global structural response. The analysis was carried out twice. The first analysis was carried out using the preliminary material properties and the results are reported in [4.5.2]. The axisymmetric model of the containment was modified to incorporate additional features not used in the initial analysis, and the model was rerun with the final material properties given by Sandia Laboratories (Table 4.5.1).

The approach was a development of the method used to analyze the sizewell ' $B$ ' containment. Rather than using a specialized nonlinear finite element code it was decided to account for the nonlinear behavior of the reinforced concrete and elastic plastic behavior of the liner using a quasi-linear approach. For the Sizewell 'B' analysis, the nonlinear softening was carried by modifying the stiffness of the elements and carrying out iterative linear elastic analyses until convergence was achieved. Although this method was successful it was limited by the number of variables that could be modified. For this study it was decided to employ a load softening technique for the wall section. This had advantages over the stiffness softening technique previously employed:

i) The inverted structure stiffness matrix could be saved and used for each iteration, which gave economies in computer time and cost.

ii) Certain features that cannot be modelled using stiffness softening, such as a moment with zero curvature, could be modelled using load softening.

iii) More parameters could be independently softened using load softening.

Previously thin shell axisymmetric elements were used. For this study, the axisymmetric thick shell element was selected as it has the capability for specifying orthotropic material properties. Before using the orthotropic thick shell elements to model the containment structure, the element was tested for accuracy and to assess the required mesh density.

A model representative of the lower cylindrical part of the Sandia containment was generated using one element through the thickness. The model, subjected to an internal pressure load, was analyzed twice, first using isotropic elements and secondly using orthotropic elements with material properties representing the isotropic elements. Only minor differences were found between the models.

In addition, the same structure was analyzed to assess how many elements are required through the wall thickness to achieve realistic results. Models were analyzed with one, two and four elements through the thickness. The results were compared with the closed form solution and in all cases very good agreement was found. In terms of base shear the four element thick 
Table 4.5.1 Recommended Material Properties

Concrete Material Properties

Elastic Constants

Young!s modulus - $4800 \mathrm{ksi}(33100 \mathrm{MPa})$

Poisson's ratio - 0.2

Ultimate Tensile Strength - $500 \mathrm{psi}$ ( $3.45 \mathrm{MPa})$

Unconfined Compressive Stress-strain Curve

Engineering Stress Engineering

\begin{tabular}{|c|c|c|}
\hline (ksi) & $(\mathrm{MPa})$ & Strain \\
\hline $\begin{array}{l}1.0 \\
2.0 \\
3.0 \\
3.9 \\
5.0 \\
6.8 \\
6.8 \\
0.0\end{array}$ & $\begin{array}{r}6.9 \\
13.8 \\
20.7 \\
26.9 \\
34.5 \\
46.9 \\
46.9 \\
0.0\end{array}$ & $\begin{array}{l}0.00021 \\
0.00045 \\
0.00072 \\
0.00100 \\
0.00140 \\
0.00200 \\
0.00230 \\
0.00600\end{array}$ \\
\hline
\end{tabular}

\section{Rebar Material Properties}

Elastic Constants:

Modulus - $31000 \mathrm{ksi}(214000 \mathrm{MPa})$

Poisson's ratio -0.3

Yield stress - $66.6 \mathrm{ksi}$ (459 MPa)

Engineering Stress-strain curve

Engineering Stress

Plastic

(ksi) (MPa) Strain

$\begin{array}{lll}66.6 & 459 & 0 . \\ 73.3 & 505 & 0.0094 \\ 85.6 & 590 & 0.0200 \\ 99.0 & 682 & 0.0430\end{array}$

Cylinder and Basemat Liner

Elastic Constants:

Modulus - $30000 \mathrm{ksi}(207000 \mathrm{MPa})$

Poisson's ratio -0.3

Yield stress - $50.2 \mathrm{ksi}$ (346 MPa)

Ultimate stress - $69.9 \mathrm{ksi}$ (482 MPa)

Dome Liner

Elastic Constants:

Modulus - $30000 \mathrm{ksi}(207000 \mathrm{MPa})$

Poisson's ratio - 0.3

Yield stress - $51.4 \mathrm{ksi}$ ( $354 \mathrm{MPa}$ )

Ultimate stress - $70.9 \mathrm{ksi}$ (489 MPa) 
model was in error by 0.5 percent, the one element thick model in error by 1.2 percent. Therefore, for the analysis a model with one element through the cylindrical section and the dome was adopted.

\subsubsection{Computer Model}

The computer model used for the axisymmetric analysis is shown in Figure 4.5.1. The reinforced concrete cylinder and dome were represented by thick shell elements, one element through the wall thickness. The reinforced concrete base was modelled with four elements through the thickness.

In order to assess the biaxial stress state in the liner independently of the concrete it was decided to model the liner using thin shell elements with the nodes coupled to the inside face of the thick shell elements using repeat freedoms. Poisson's ratio of the liner was 0.3 while the liner was elastic. As soon as the liner was predicted to have yielded, Poisson's ratio was reduced to zero to simplify the softening procedure.

The foundation stiffness was represented by springs to ground that were taken out if they went into tension.

\subsubsection{Site Characteristics}

The ground at the test site is described as loose gravel and decomposed granite fines. It is also described as silty clay with sand in the laboratory report of the physical properties of the soil. Some soil has been imported to the site, which is described as silty sand.

Three plate bearing tests have been performed on the site in accordance with ASTM D-1194. Plate sizes of 12,18 and 30 inches $(305,457$, and $762 \mathrm{~mm}$ ) were used. A value of subgrade reaction modulus of $390 \mathrm{ksf} / \mathrm{ft}\left(61 \mathrm{MN} / \mathrm{m}^{3}\right)$ was recommended by the American consultant.

Using the results of the plate bearing tests OAP calculate an average elastic stiffness of $128 \mathrm{ksf} / \mathrm{ft}\left(20 \mathrm{MN} / \mathrm{m}^{3}\right)$. Assuming a rigid base with a radius of 142 in $(3.8 \mathrm{~m})$ the lower bound modulus of subgrade reaction is $26.8 \mathrm{ksf} / \mathrm{ft}\left(4.2 \mathrm{MN} / \mathrm{m}^{3}\right)$. As an extreme upper bound, assuming rock below the level of influence of the plate bearing tests, the modulus of subgrade reaction is $320 \mathrm{ksf} / \mathrm{ft}\left(50 \mathrm{MN} / \mathrm{m}^{3}\right)$. For the following analyses, $255 \mathrm{ksf} / \mathrm{ft}$ $\left(40 \mathrm{MN} / \mathrm{m}^{3}\right.$ ) was used as a reasonable upper bound stiffness. The lower bound of $25.5 \mathrm{ksf} / \mathrm{ft}\left(4 \mathrm{MN} / \mathrm{m}^{3}\right)$ was also used to assess the influence of the foundation stiffness.

\subsubsection{Quasi-linear Analysis}

The containment being constructed from reinforced concrete behaves nonlinearly when pressurized internally. Even with orthotropic materials it was not possible without altering the geometry to correctly model the axial, bending and shear stiffness for all three orthogonal directions

simultaneously. Therefore a decision of first order material parameters was taken, and these parameters were accurately represented in the model. For each section the chosen parameters were as follows: 
(i) Cylindrical section

Meridional, Radial \& Hoop Stiffness

Meridional moment/curvature

Poisson's ratio effects

Radial and Hoop Shear Stiffness

(ii) Dome section

Meridional, Radial \& Hoop stiffness

Poisson's ratio effects

Radial \& Hoop shear stiffness

(iii) Base section

Meridional, Radial \& Hoop stiffness

Radial moment curvature

Poisson's ratio effects

Radial \& Hoop shear stiffness

(iv) Liner

Meridional Radial \& Hoop stiffness

The analysis was performed by running linear-elastic analyses at discrete pressure steps. At each pressure step the stiffness of each element was calculated for the first order material parameter given above. These stiffnesses were either based on the determinate stresses and strains (such as in the dome) or were extrapolated from the previous pressure increment. It can be seen in (i) above that it was not possible without changing the element geometry to represent both the correct cylinder axial and bending stiffness in the meridional direction with only one stiffness. Therefore, at each pressure increment the meridional moment/curvature was compared with the nonlinear relationship and the elements were softened if necessary using nodal loads. However, modification to the moment curvature relationship caused both redistribution of loads in the hoop direction and variations to meridional strains and liner forces, invalidating the original material properties assumptions. Therefore, at each iteration the concrete cylinder elements and the linear were also softened in the meridional and hoop directions.

The calculated.softening forces were combined with the pressure and gravity forces and the model reanalyzed. This procedure was repeated until convergence was achieved (Figure 4.5.2). The base and the dome stiffnesses were not adjusted between each pressure step. For the base a multi-element approach was adopted whereby each layer of elements was independently adjusted to model the correct radial and bending stiffness. In the dome only the membrane stiffness was correctly represented.

It should be noted that although the nonlinear moments and forces have ultimate values beyond which convergence would not be possible, there is no such modelled criterion for the shear force. (Unlike the large 3-D nonlinear programs). Therefore the prediction of ultimate shear failure is carried out external to the computer analysis. 


\subsubsection{Material Modelling}

Introduction:

The model was constructed such that one element represented the composite behavior of the concrete and the reinforcement, in all three orthogonal directions. Therefore, in this analysis for the calculation of the material models, all the triaxial stresses were assumed decoupled. However this simplification was not considered to produce significant inaccuracies as the containment in general is in biaxial tensile stress state.

Moment-curvature:

A program was written to calculate the moment curvature of any section for a given axial load. The basis of the calculation was that the strain remains linear even at large curvatures.

The program divides the section into a series of slices of either confined or unconfined concrete (depending on the presence of confining links) and areas of reinforcement. The curvature was fixed and the axial strain iterated until the axial force on the section was that prescribed. The curvature was then incremented for another solution until failure was predicted. The concrete compressive stress-strain model used was the modified Kent Park [4.5.3]. For concrete confined by links the stressstrain relationship was effectively stretched and the concrete assumed to have a small resistance at high strains. In contrast, the unconfined concrete was assumed to have no residual strength at high strains. The concrete was assumed to have a small allowable tensile strength before cracking, and to take into account the influence of bond and tensile concrete stresses between cracks, a modified stress-strain relationship as indicated by a dashed line on Figure 4.5.3 has been used. The otherwise assumed abrupt loss of tensile strength for concrete is shown by a solid line. A study by Halvorsen reported by Chen [4.5.4] demonstrates that this method gives very good results. For the final analysis the tensile stressstrain relationship was further modified, increasing $\epsilon_{\max }$ to 0.0015 to ensure a positive axial wall stiffness throughout the range.

The assumed stress-strain relationship for the reinforcement is shown on Figure 4.5.3.

Moment-curvature calibration:

The moment-curvature program was first calibrated using the results of a series of tests carried out by Goodsir [4.5.5] in New Zealand. These results are particularly useful as the structure, a shear wall, was tested well into the nonlinear range. The comparison between the measured results and those produced by the moment-curvature program are shown on Figure 4.5.4. It can be seen that very good agreement is achieved for two tests with different curvature directions and axial loads.

\subsubsection{Element Softening Program}

Introduction:

Previously the element softening using the stiffness softening approach was carried out by hand. For this study softening by hand was considered to be too time consuming and restrictive, therefore an automated approach was adopted. 
On completion of the PAFEC analysis the specially written program calculated the softening moments and forces for each element, which were then compared with those from the previous iteration. If the moments and forces had not converged a revised PAFEC input data deck was automatically re-written containing all the softening nodal loads and the analysis was restarted. When all the elements had converged the analysis stopped and a universal file was written for post processing. See Figure 4.5 .5 for the flowchart.

Meridional Moment-curvature softening in cylinder:

The meridional moment of each cylindrical element was calculated from the stresses taking into consideration the wall geometry. The top and bottom moments were averaged, from which the curvature was calculated knowing the input meridional stiffness. In addition the element curvature was calculated directly from the nodal displacements of the wall faces for direct comparison.

Also the axial force on the section was calculated directly from knowledge of the internal pressure and the liner force. The hoop strain was calculated in order to determine the strains in the seismic reinforcement at $45^{\circ}$. Then the moment curvature program was accessed directly with the curvature, hoop strain and axial force.

For each element the nonlinear moment is calculated for quantity of reinforcement and concrete confinement at that section (the change in vertical reinforcement is modelled by gradually increasing the area of reinforcement over the anchorage length). For later calculation the axial strain is also calculated for the particular axial force and curvature given. The difference between the analysis moment and the non-linear moment was denoted the pseudo moment and was degraded to four equivalent axial loads to be applied to the corner nodes (Figure 4.5.6). A flowchart for the subroutine is shown (Figure 4.5.7).

Meridional softening in cylinder:

For each element the determinate meridional stress was converted to an equivalent strain, and compared with the axial strain from the momentcurvature calculation. The two strains were converted into equivalent forces for the complete circumference and the difference between the two forces denoted as the meridional pseudo force. The pseudo force was converted to six equivalent nodal forces to soften (or stiffen) the element (Figure 4.5.8). A flowchart of the subroutine is shown (Figure 4.5.9).

Hoop softening in cylinder:

For each element the average hoop strain was calculated and converted to a hoop force. A modified version of the moment curvature program was used to calculate the nonlinear hoop force for the calculated hoop strain (and zero curvature). In this case the meridional strain was also input into the moment curvature program and the strain in the seismic reinforcement was assumed to be the average of the hoop and meridional strains, the component of hoop force was calculated accordingly. The difference between the hoop and nonlinear hoop force denoted the pseudo hoop force.

The pseudo hoop force was converted into an equivalent internal pressure and then converted to an equivalent total force over the element height and applied as radial nodal loads (Figure 4.5.10). A flowchart of the

subroutine is shown (Figure 4.5.11). 
The radial shear stiffness for each element was modified at each pressure increment based on the average of the current axial and radial stiffnesses.

Poisson's ratio:

The Poisson's ratio was reduced to effectively zero in each element if the concrete represented has cracked.

Liner:

In the analysis the liner is modelled separately but it is assumed to act compositely with the reinforced concrete. For the liner attached to the cylindrical section, the meridional and hoop stresses were calculated from the nodal deformations. The stresses were combined using the von Mises criteria to assess if the liner has yielded. Where the liner stress is found to exceed the von Mises yield criterion the equivalent orthogonal plastic stresses at the yield point using a radial return criterion were calculated and the difference between the linear-elastic liner stress and the stress at the yield point was denoted the pseudo stress. Nodal loads were used to soften the liner in a similar manner to those described earlier. When the converged solution was reached the plastic strains in each direction and the total plastic strain in the liner for each element were stored in a file. For the next pressure increment the plastic stresses were calculated from the previous plastic strain and the yield stress enhanced depending on the total plastic strain. The liner was computed to fail when the total plastic strain exceeds the calculated maximum elongation for biaxial tension using the material tests results. A flowchart of the subroutine is shown (Figure 4.5.12).

Hand calculations indicated that the restraint to the liner at the wall base was insufficient to achieve the full yield stress. Therefore for the wall base the assumption that the liner was composite with the concrete would have been conservative. To model the reduction in liner meridional stiffness in this region the bottom three liner elements were decoupled from the wall elements and the repeat freedoms were replaced by springs. The actual value of the spring stiffness was difficult to determine. A value equivalent to the elastic stiffness of the bottom three liner elements was used. The spring forces were monitored during each iteration and if they exceeded a value representative of the ultimate strength of the restraint (including the stud connectors), the springs were softened until convergence was achieved.

This modification resulted in a greater meridional tensile force in the concrete, thus reducing the radial shear capacity.

\subsubsection{Analysis Pressures}

Initial hand calculations indicated that based on thin shell theory there was sufficient resistance in the reinforcement and the liner to potentially withstand over four times the design pressure, although the deformation to achieve this pressure would be considerable. Therefore pressure increments of approximately $29 \mathrm{psi}(0.2 \mathrm{MPa})$ were adopted for the analysis commencing at 15 percent over the design pressure. The five pressure increments analyzed were chosen to highlight the changes in the modes of behavior of the containment. The following pressures were analyzed: $52.9,87,116$, 145 , and 174 psi $(0.365,0.6,0.8,1.0$, and $1.2 \mathrm{MPa})$. Milestones in the structural response at these pressures are given in Section 4.5.4. 


\subsubsection{Convergence}

The convergence of the analysis was found to be reasonably stable except for the meridional stiffness of the cylinder. There were a number of reasons why the meridional stiffness caused problems. For example, the axial strain was not only directly influenced by the axial force but also it was significantly affected by changes in the meridional bending moment, hoop strain and liner forces. In addition over a range of meridional strains between 0.00005 and 0.0007 the cylinder had an effective negative stiffness, caused by the rate of reduction in the concrete tensile capacity exceeding the rate of increase in force in the reinforcement. This problem was overcome by increasing the strain over which the concrete was assumed to act in tension. To damp the numerical oscillations between iterations the pseudo softening forces input into next iteration were the average of the stored pseudo forces from the current and five preceeding iterations. As the pseudo forces are initially set at zero this also had the effect of ramping up the softening forces.

The actual convergence criteria was based on the change in pseudo softening forces between each iteration. When the change in pseudo force was small in comparison with the linear elastic analysis force, the element was deemed to have converged. The total number of iterations required was found to increase with the internal pressure. At $52.9 \mathrm{psi}(0.365 \mathrm{MPa})$ only eight iterations were required whereas at $174 \mathrm{psi}(1.2 \mathrm{MPa})$ the structure was so nonlinear over 30 iterations were necessary. Each iteration took approximately 2.5 minutes c.p.u. time.

\subsubsection{Results}

\section{Cylindrical Section:}

In the hoop direction the containment can resist about three times the design pressure before yielding. At this point the displacement would still be small. However, to sustain pressures over about $145 \mathrm{psi}$ ( $1.0 \mathrm{MPa}$ ) large deformations are required as the additional strength is provided by work hardening of the reinforcement and the liner. These deformations would be accompanied by a large amount of vertical cracking in the concrete cylinder.

For comparison the meridional bending moment, curvature, radial shear forces and hoop stresses at each pressure increment are shown on Figures 4.5.13 4.5 .16 respectively.

The moment is calculated about the mid depth of the concrete cylindrical section. It does not include the liner meridional force. It can be seen that, even though at the higher pressures the curvature at the wall base is rapidly increasing, the moment is only increasing slowly. This is caused by the reduction in moment capacity due to the high axial tension force.

The effect in coupling the hoop and meridional strain to calculate the force in the $45^{\circ}$ seismic bars is to increase the negative bending moments in the areas of high hoop strain, and effectively prestress down the cylinder due to the high hoop strains.

The significant change in wall reinforcement occurs in the wall at the top of the eighth element from the base. This reduction in bending stiffness does not appear to have any noticeable effect. 
Dome:

The dome was modelled to represent only the correct membrane stiffness, therefore the bending and shear forces were not assessed. From the plots of the displaced shape it can be seen that the dome, although it generally follows the radial growth of the cylinder, is slightly stiffer creating some discontinuity effects especially at the higher pressures.

Base:

Once flexural cracking has occurred the moment capacity drops and the stiffness is reduced by an order of magnitude. The geometry of the elements in the base were adjusted such that the bending stiffness could be reduced whilst maintaining the correct axial stiffness. The radial forces in the base are caused by shear in the walls, resisted by the hoop tension and also by the concrete in tension. If the concrete is cracked due to bending or shrinkage the radial stiffness will be overestimated. However this may not have a large effect as the force will also be resisted by the hoop tension.

Foundations:

At high pressure lift off occurs under the edge of the slab under the wall when assuming the upper bound foundation stiffness of $255 \mathrm{ksf} / \mathrm{ft}\left(40 \mathrm{MN} / \mathrm{m}^{3}\right)$. At $25.6 \mathrm{ksf} / \mathrm{ft}\left(4 \mathrm{MN} / \mathrm{m}^{3}\right)$ no lift off occurs. The analys is of $P=1.2 \mathrm{MPa}$ was carried out for both bounds of the foundation stiffness to assess its effect on the cylinder moments and forces. The results of the two runs showed no significant differences.

Liner:

The liner is softened for the membrane forces only, which is justified by the fact that globally the bending stresses are very small.

The initial analysis was also carried out without the liner artificially softened at the wall base in the meridional direction. In these runs the stress in the liner was found to be highest at the wall base due to the combination of axial tension and high curvature. However, in the latest analyses the maximum stress in the liner at the wall base is limited to 7.2 ksi (50 MPa) in the meridional direction, (it is assumed fully effective in the hoop direction).

In general the highest hoop liner stresses occur in the center of the wall section and the highest meridional stresses occur where the combination of meridional strain plus curvature is at a maximum, now to be found near the top of the cylindrical section. The deformation in the spring connecting the bottom of the cylinder liner to the concrete at the wall base is equivalent to slippage of the liner up the wall due to insufficient restraint. This value was compared with the results of the preliminary stud embedment tests (supplied by Sandia) to assess the possibility of stud failure. The slippage values for the five pressures cases considered are $0.01,0.021,0.042,0.075,0.2$ inches $(0.26 \mathrm{~mm}, 0.55 \mathrm{~mm}, 1.07 \mathrm{~mm}, 1.91 \mathrm{~mm}$ and $5.17 \mathrm{~mm}$ ). 


\subsubsection{Three-Dimensional Analysis}

\subsubsection{Introduction}

The purpose of carrying out the three-dimensional analysis was to study how the non-axisymmetric features of the containment modified the global structural response predicted using the axisymmetric model.

As described previously, simplified techniques can provide information adequate for the Inspectorate's requirements, and such an approach was adopted for this work. A fully nonlinear analysis was, as previously, considered inappropriate, and even the pseudo-load approach described in the preceding section would have required excessive development to accommodate the level of complexity around the penetrations. An alternative approach was therefore adopted.

By postulating that the penetrations and the other non-axisymetric features had no effect on the overall structural response of the containment, it was possible to take advantage of the results from the nonlinear axisymetric analysis. Thus, the nonlinear analysis of the containment including penetrations could be reduced to a 3-0 linear elastic analysis of the containment using material properties calculated from the final converged solution of the axisymmetric models.

Such an approach therefore presumes the result that the presence of penetrations in the three-dimensional model would have no effect upon the global behavior. However, it is possible to check this assumption by comparing the displacements and bending moments predicted by each of these two techniques, i.e.

i) two-dimensional axisymmetric model, analyzed as nonlinear using the pseudo-load method.

ii) three-dimensional model, analyzed as linear but using "softened" material properties as predicted by the axisymmetric analysis.

The comparison of the results appears in Section 4.5.3.4, following more detailed descriptions of the modelling techniques and material properties used.

\subsubsection{Computer Model}

The three-dimensional finite element model of the containment is comprised of three basic elements; solid elements for the concrete walls and base, shell elements for the liner, and ground springs to represent the foundation stiffness. It was required that both the solid and shell elements be able to model orthotropic material properties.

For the concrete walls and base, 20 node isoparametric brick elements were used, except at the center of the base and top of the dome, where 15 node wedge elements were used. A discretization of one element through the thickness of the wall was adopted, as this had been found to give acceptable results. The only suitable PAFEC elements for the liner were the 8-node quadrilateral and 6-node triangular semi-loof shell elements. 
Due to the overall size of the problem the discretization of the model was approximately half as fine as that used by the axisymmetric model, with elements being placed every $15^{\circ}$ around the circumference.

Initially a half model of the containment was set up but this was abandoned in favor of a quarter model to reduce the computer C.P.U. time. The quarter model included half of one personnel airlock and half of equipment hatch $B$. The appropriate boundary conditions were applied to the structure.

\subsubsection{Material Properties}

The orthotropic material properties of the concrete and liner elements were calculated from the results of the axisymmetric analysis. Properties were calculated for each horizontal layer of elements.

For the concrete elements a secant modulus was calculated in the hoop direction from the hoop stress and strain. Unfortunately, in the meridional direction using this secant modulus technique only the bending stiffness or the axial stiffness could be modelled in any one element. The compromise adopted was to model the bending stiffness at the wall base and the axial stiffness higher up the wall. Therefore, the secant moduli were calculated from the axial stresses and strains for all but the bottom three element layers at the wall base. In these layers the meridional stiffness was calculated from the bending moment and curvature, as this was shown to be dominant in this area. In the through thickness direction the modulus of uncracked concrete was used. Poisson's ratio was taken to be 0.01 throughout, representing cracked concrete. The orthotropic properties of the base and dome elements were the same as those used in the axisymmetric analyses. Around the hatches the orthotropic properties were calculated assuming the concrete to be in tension and only the reinforcement to be effective.

Isotropic properties were used for the liner elements unless they had yielded in the axisymetric analysis. For the yielding elements, secant moduli were calculated in the hoop and meridional directions. Around the hatches the liner elements were initially assumed to be linear elastic. At the end of each run the liner stresses around the penetrations were inspected. If in any element the von Mises yield criterion was exceeded a more realistic secant modulus for the liner was calculated in the orthotropic directions and the analysis repeated. A similar procedure was adopted for checking the solid elements around the penetrations to assess if the reinforcement had yielded.

\subsubsection{Results}

The quarter model described above has been run for the 52.9 psi, 87 psi, 116 psi, 145 psi $(0.365,0.600,0.800$ and $1.00 \mathrm{MPa})$, load cases. For all four cases reasonable comparison can be made with the results from the axisymetric analyses. In order to make comparisons between the two models the values given for the three-dimensional model have been taken from a vertical cross section midway between the two hatches.

Good agreement was found between the displacements shown by both models (see Table 4.5.2). The meridional bending moments shown in Figure 4.5.17 at the wall base are also in good agreement. However, further up the wall, where 
the stiffness is derived from the meridional rather than bending stiffness, as would be expected the moments do not show the same agreement. Also using the quasi-linear method it was possible to achieve a negative moment for a positive curvature. Using a stiffness softening approach this was not possible. The meridional moment at the wall base away from the central position were found to vary by no more than three percent from the central value.

The hoop stresses in the concrete also show that the effect of the penetration is localized as the hoop stresses change very little over the central portion of the wall.

\subsubsection{Discussion}

It was found from the comparison of bending moments and shear forces at the wall base between the axisymmetric model and the 3-D model that the penetrations have a negligible effect on the critical forces at the wall base. Also the overall displacements are similar between the two models. There were some differences, some of which were caused by the fact that the

\section{TABLE 4.5.2 Comparison of Displacements (mm)}

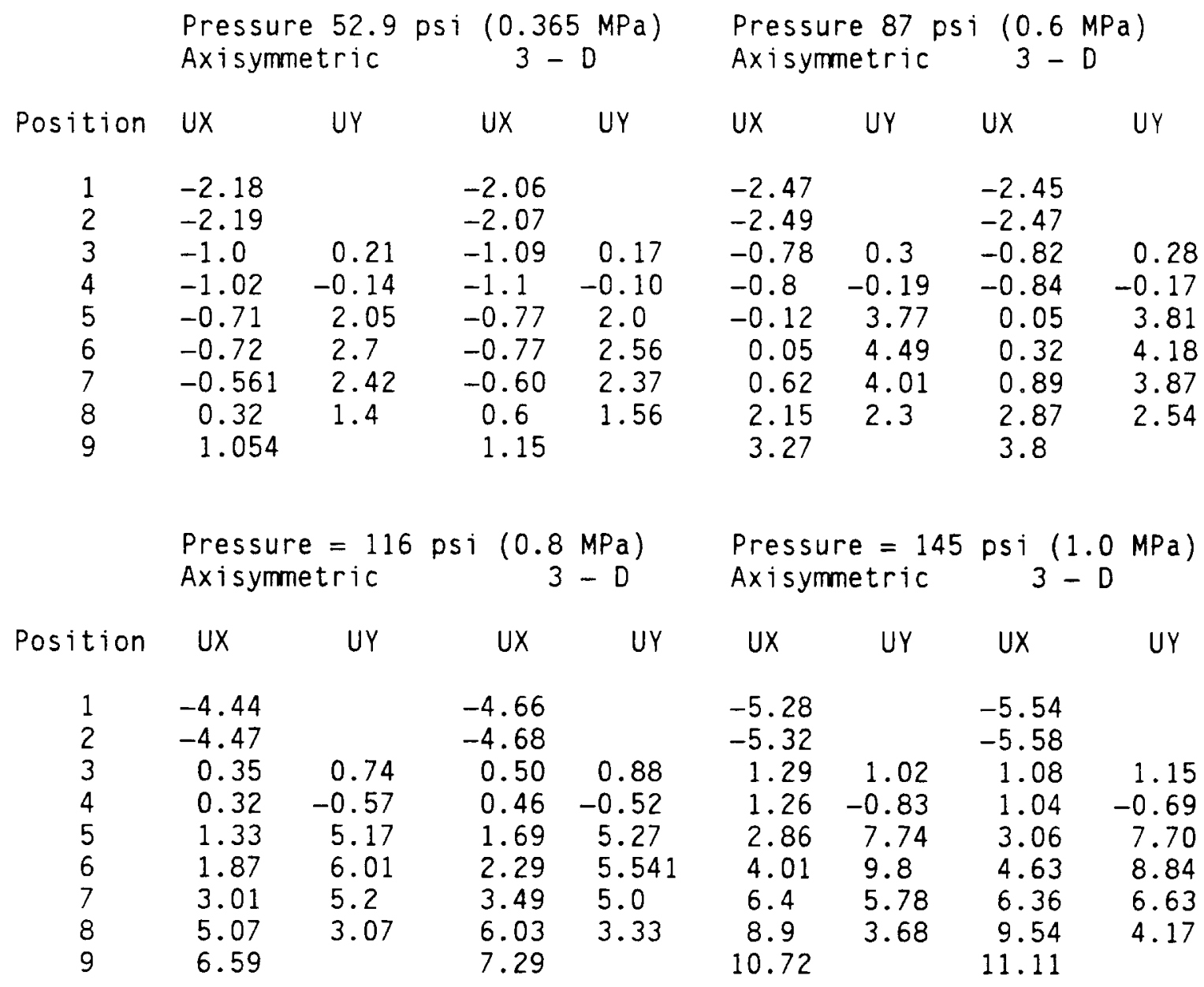

Note: for positions refer to Figure 4.5 .18 
3-D model had a coarser mesh than the axisymetric models. However, in general the results showed that the global effects are adequately predicted using the axisymetric analysis.

An important result of the above conclusion is that investigations into the behavior of the area of the structure around the penetration can be carried out using a local submodel, with boundary conditions from the axisymetric models rather than refining the mesh in a large 3-D model.

As discussed above, the containment was analyzed as a quarter model, therefore the effect of the restraining bar at high level could not be assessed. The effect of the bar was investigated by hand calculation and it was concluded that it would not effect the global response.

\subsubsection{Standard Results Plots}

A number of standard plots have been produced from the results of the axisymmetric analysis and are reproduced in Appendix $A$.

\subsubsection{Milestones in Structural Response}

Pressure $=52.9 \mathrm{psi}(0.365 \mathrm{MPa})$

Displacements: Figure 4.5 .18

Cylinder:

Meridional flexural cracks at base on inside

Hoop

Liner partially cracked

max. axial stress $=8.7 \mathrm{ksi}(60 \mathrm{MPa})$ (mid height)

max. hoop stress $=27 \mathrm{ksi}(186 \mathrm{MPa})$ (mid height)

Dome :

Concrete

uncracked

Liner

biaxial stress $=23.8 \mathrm{ksi}(164 \mathrm{MPa})$

Base:

Concrete uncracked

Liner $\quad \max$. stress $=4.5 \mathrm{ksi}(31 \mathrm{MPa})$ (comp)

Pressure $=87 \mathrm{psi}(0.6 \mathrm{MPa})$

Displacements: Figure 4.5.18

Cylinder:

Meridional flexural cracks at base on inside

Hoop

Liner top $3 / 4$ cracked

max. axial stress $=17 \mathrm{ksi}(117 \mathrm{MPa})$ (elastic)

max. hoop stress $=45.7 \mathrm{ksi}(315 \mathrm{MPa})$ (elastic) (mid height)

Dome:

Concrete

cracked

Liner

biaxial stress $=39.2 \mathrm{ksi}(270 \mathrm{MPa})$ (elastic)

Base:

Concrete

cracked

Liner

max. stress $=5.9 \mathrm{ksi}(41 \mathrm{MPa})(\mathrm{comp})$ 


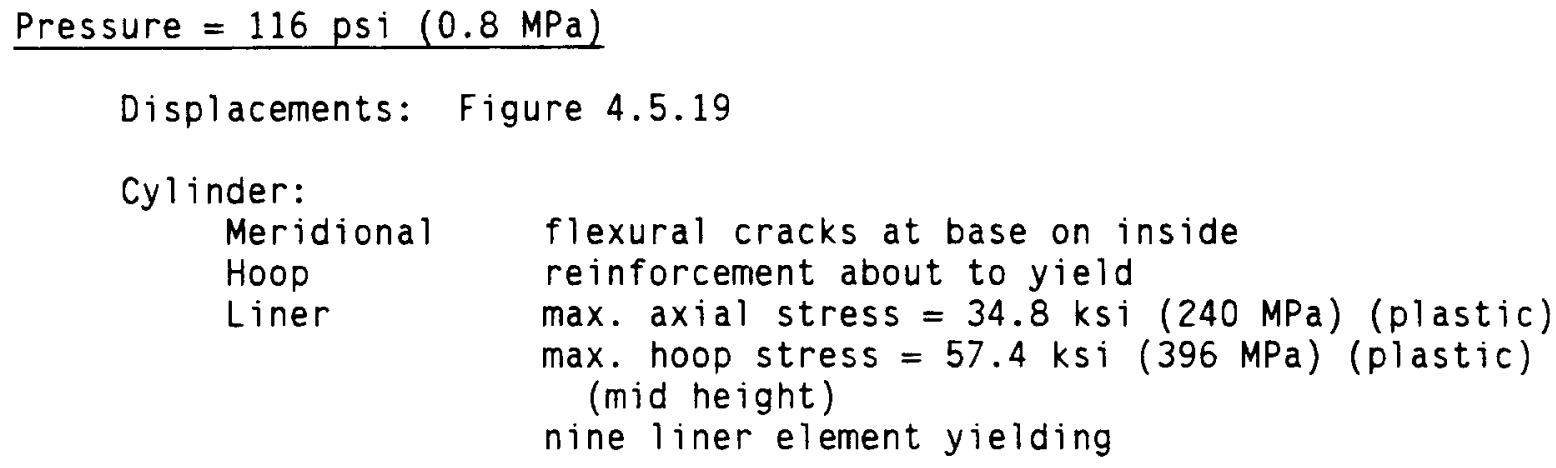

Displacements: Figure 4.5.19

Cylinder:

Meridional flexural cracks at base on inside

Hoop reinforcement about to yield

Liner $\quad \max$. axial stress $=34.8 \mathrm{ksi}(240 \mathrm{MPa})$ (plastic)

$\max$. hoop stress $=57.4 \mathrm{ksi}(396 \mathrm{MPa})$ (plastic)

(mid height)

nine liner element yielding

Dome :
Concrete
cracked
Liner
biaxial stress $=51.6 \mathrm{ksi}(356 \mathrm{MPa})$ (elastic)

Base :

Concrete cracked

Liner $\quad \max$. stress $=24.6 \mathrm{ksi}(170 \mathrm{MPa})$ (comp)

Pressure $=145 \mathrm{psi}(1.0 \mathrm{MPa})$

Displacements: Figure 4.5.19

Cylinder:

Meridional flexural cracks at base on inside and lower mid $1 / 4$ of wall outside (bottom element yielding is

Hoop bending)

Liner

reinforcement yielding many vertical cracks nearly full height

max. axial stress $=35.1 \mathrm{ksi}(242 \mathrm{MPa})$ (plastic) $\max$. hoop stress $=58.4 \mathrm{ksi}(403 \mathrm{MPa})$ (plastic) (mid height)

All liner elements plastic

Dome :

Concrete

reinforced about to yield

Liner

biaxial stress $=51.6 \mathrm{ksi}(356 \mathrm{MPa})$ (elastic)

Base:

Concrete

cracked

Liner

$\max$. stress $=33.3 \mathrm{ksi}(230 \mathrm{MPa})(\mathrm{comp})$ 
Pressure $=174$ psi $(1.2 \mathrm{MPa})$

Displacements: Figure 4.5.20

Cylinder:

Meridional flexural cracks $2 / 3$ of outer wall above base (bottom element yielding is bending)

Hoop reinforcement yielding - large deformations and cracking

Liner

max. axial stress $=27.4 \mathrm{ksi}(189 \mathrm{MPa})$ (plastic)

$\max$. hoop stress $=62.8 \mathrm{ksi}(433 \mathrm{MPa})$ (plastic)

(mid height)

Dome :

Concrete

reinforcement yielding, cracking

Liner

biaxial stress $=63.5 \mathrm{ksi}(438 \mathrm{MPa})(\mathrm{plastic})$

Base:

Concrete

cracked

Liner

$\max$. stress $=23.6 \mathrm{ksi}(163 \mathrm{MPa})($ comp $)$

\subsubsection{Discussion}

If the cylindrical wall of the containment were free to radially deform due to the internal pressure and not be restrained by the base (or the dome) radial shear forces would not be generated.

Although it is straightforward to calculate the ultimate pressure at which the containment will fail by barrel bursting or dome bursting, there are no simple methods of assessing the ultimate shear resistance. In general, the radial shear forces peak at the wall base intersection. However, they also exist around equipment hatches and personnel locks. Although there are a number of methods of assessing shear resistance of a beam, there is little guidance if the beam is also subject to a large axial tension and there is almost no information if in addition the beam is a cylinder with hoop reinforcement and internal pressure. In [4.5.6], the recent research in this area is reviewed and a number of formulas are presented. However, it was found that not only did the contribution of, for example, axial tension to reducing the shear resistance as described by Beeby and Rangan differ by a factor of 8 , but all the formulas were found to be unsuitable for all sections apart from the wall base. Figure 4.5 .21 shows the comparison of the shear force calculated at the wall base with a number of different formulas. It can be seen that for all cases the resistance is calculated to exceed the shear force applied.

In addition to the wall base three other sections were assessed where the quantity of shear reinforcement changes. Level 2 is $1^{\prime}-0 "$ (300 mm) above the base. Level 3 is $2^{\prime}-0^{\prime \prime}(600 \mathrm{~mm})$ above the base and Level 4 is $4^{\prime}-0^{\prime \prime}$ $(1200 \mathrm{~mm})$ above the base and is the position where the shear reinforcement is discontinued. In each case the applied shear force is compared with the ultimate shear resistance calculated using the ASME Section III Div. 2 formula. This indicates that there is a potential for a shear failure at Level 4 at a pressure of $116 \mathrm{psi}(0.8 \mathrm{MPa})$, or $2.5 \mathrm{P}$. Also at the point where the cylinder meets the dome the calculated shear force exceeds the 
shear resistance calculated using the ASME formula at the same pressure. However, it is generally recognized that the ASME formula is conservative (although it is not known by how much) and it is also conceptually difficult to visualize a radial shear failure in the wall because as soon as the shear-induced displacements occur the hoop reinforcement will pick up more load reducing the shear force.

Therefore, on the basis of the analysis to date, there is no indication that the containment will fail at pressure less than 116 psi (.8 MPa) or $2.5 \mathrm{P}$ with an upper bound of $174 \mathrm{psi}(1.2 \mathrm{MPa})$ or $3.8 \mathrm{P}$. To narrow the bounds ${ }^{\mathrm{d}}$ it is necessary not only to establish the level of conservatism in the radial shear formulas but also to inspect the details of the containment in much greater depth. It is most likely that failure will initiate at a local stress concentration caused by perhaps the penetration, liner details or even poor workmanship during construction. Due to the lack of restraint to the liner in the vertical direction at the wall base, it is probable that the meridional stress in the liner caused by the axial tension and high curvature will cause some liner studs to fail, probably by pulling out, but possibly by tearing the liner, causing premature leakage.

If the ASME shear formula is conservative and local effects do not precipitate an early failure, it is probably that the lower failure bound will be at the point where plastic deformation commences (about 130 psi (0.9 $\mathrm{MPa}$ ) or $\left.2.8 \mathrm{P}_{\mathrm{D}}\right)$.

\subsubsection{Summary of Conclusions}

(i) A 3-D computer model has been generated and run to assess the effects of the large penetrations on the overall structural behavior. The results indicate that the penetrations only have a localized effect.

(ii) The concrete cylinder reinforcement yielded in the hoop direction at the wall mid height at $2.8 \mathrm{P}$. It is calculated to reach its ultimate strength at $4.6 \mathrm{P}$. The concrete dome reinforcement is calculated to yield at $3.9 \mathrm{P}$ and reach its ultimate strength at $5.6 \mathrm{P}$. The first plastic moments occurred at the wall base at 2.4 $P_{P}$. The cylinder liner yielded at $2.5 \mathrm{P}_{\mathrm{p}}$ and the dome liner ylelded at $3.2 P_{D}$. The liner on the base remained elastic up to $3.8 \mathrm{P}_{\mathrm{D}}$.

(iii) The shear reinforcement at the wall base is sufficient to resist the generated shear forces up to $3.8 \mathrm{P}_{D}$, however the shear forces in the unreinforced section exceed the ASME 1 imit at $2.5 \mathrm{P}_{D}$.

(iv) Taking into consideration that the ASME code is conservative the failure of the containment will probably occur between $2.8 \mathrm{Pp}_{\mathrm{p}}$ and $3.8 P_{D}$, unless it is initiated at a local stress concentration. 


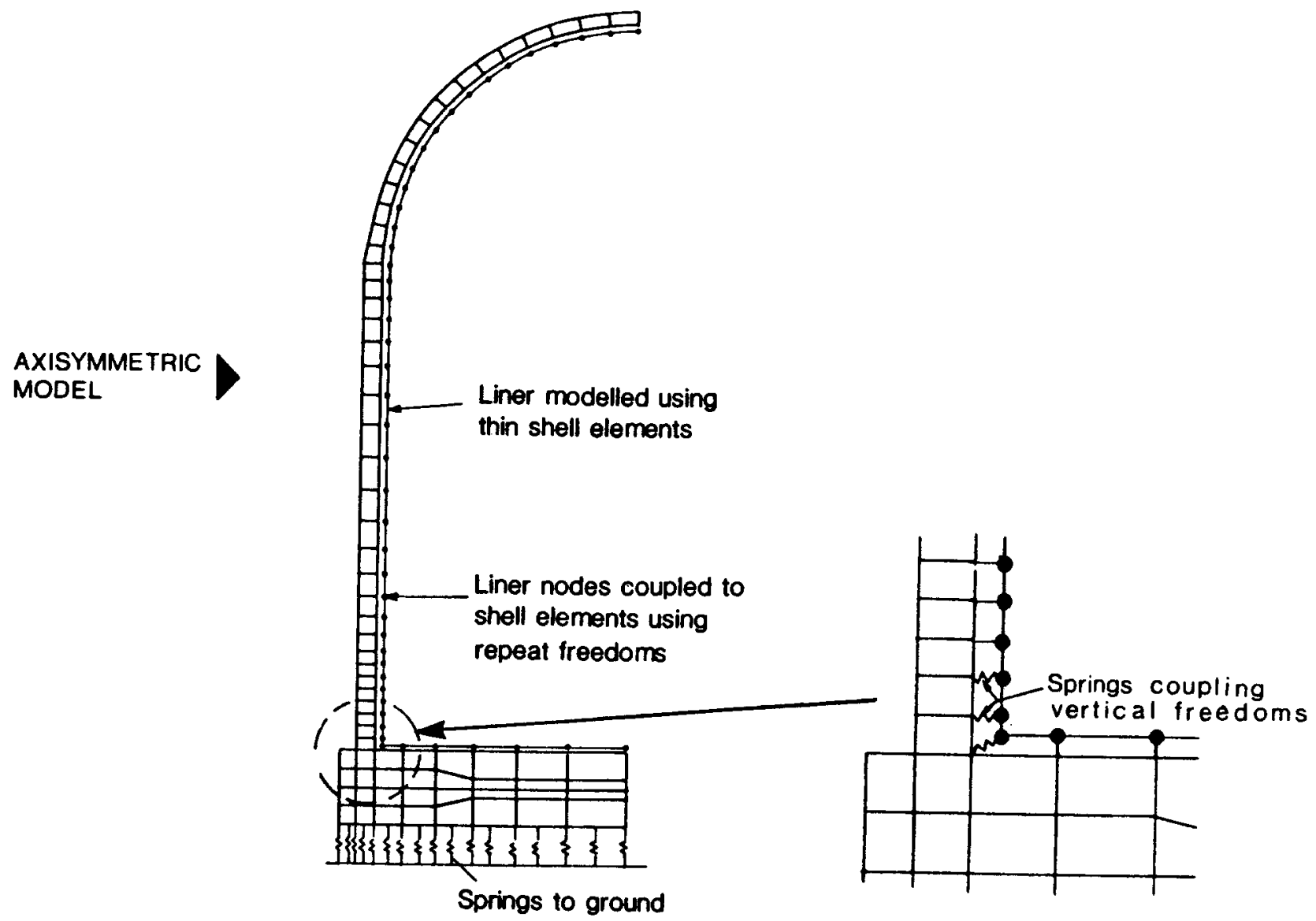

3D-QUARTER MODEL

20 noded orthotropic solid elements representing the reinforced concrete
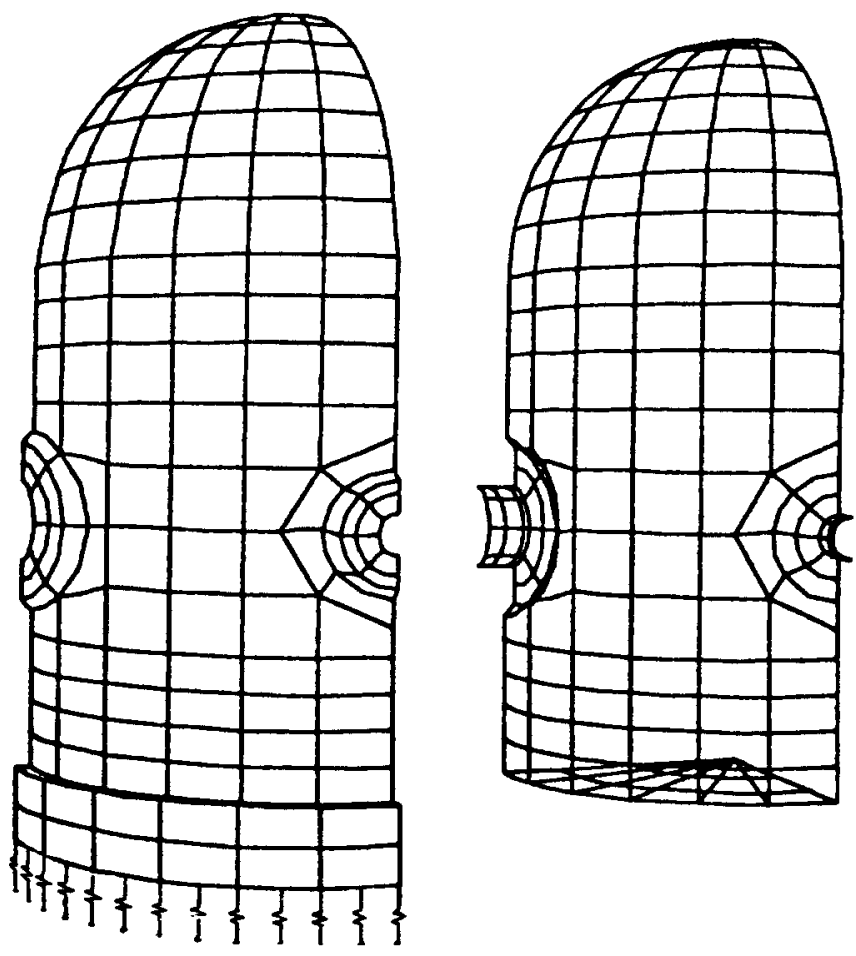

Semi- loof thin shell elements representing the liner

Figure 4.5.1 Axisymetric and 3-D Quarter Models 

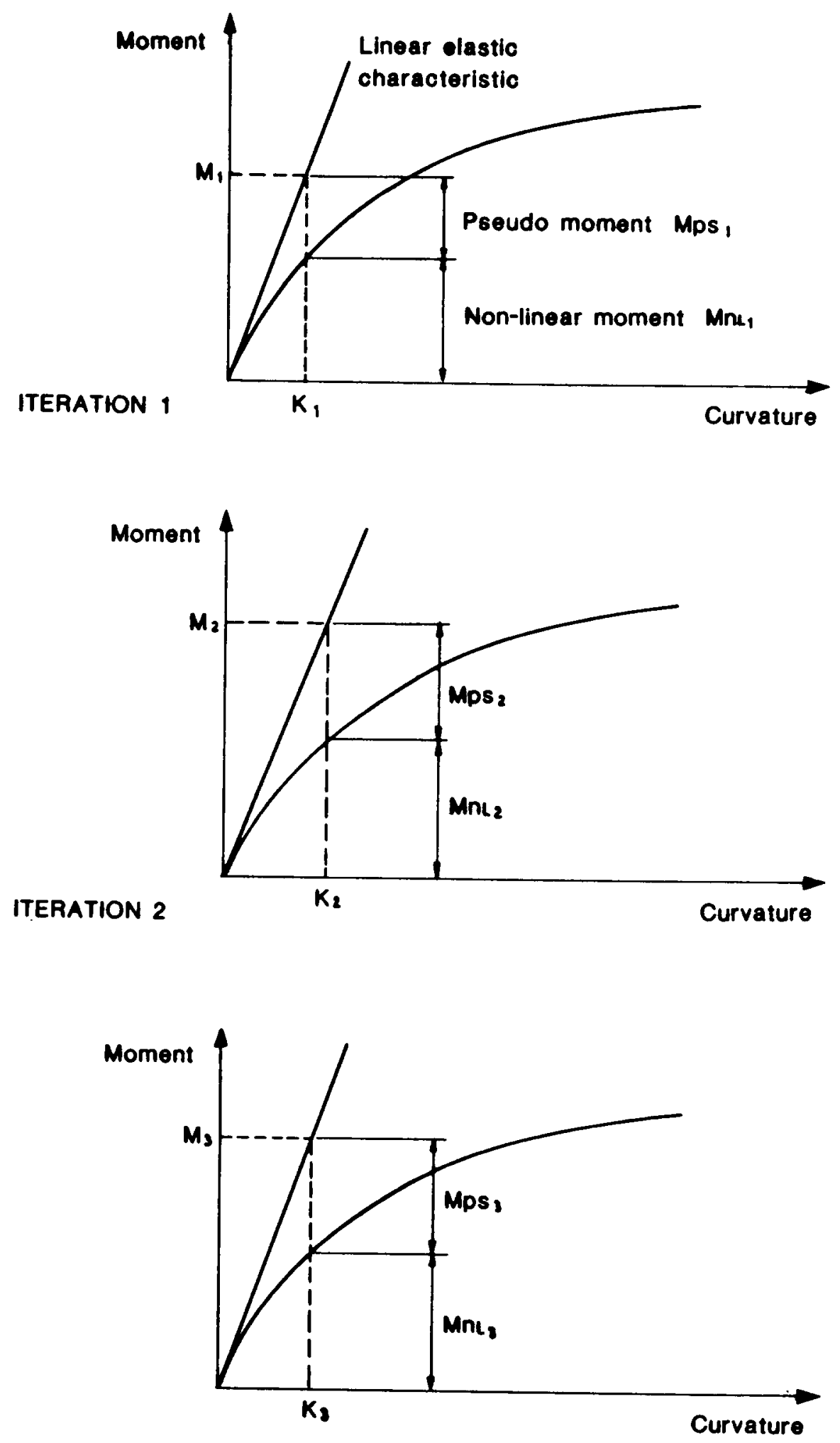

Iterations cease when $\frac{\text { Mpsin+1 }}{M p s n^{-}}<0.05 \mathrm{MnLa}$

Figure 4.5.2 Nonlinear analys is 


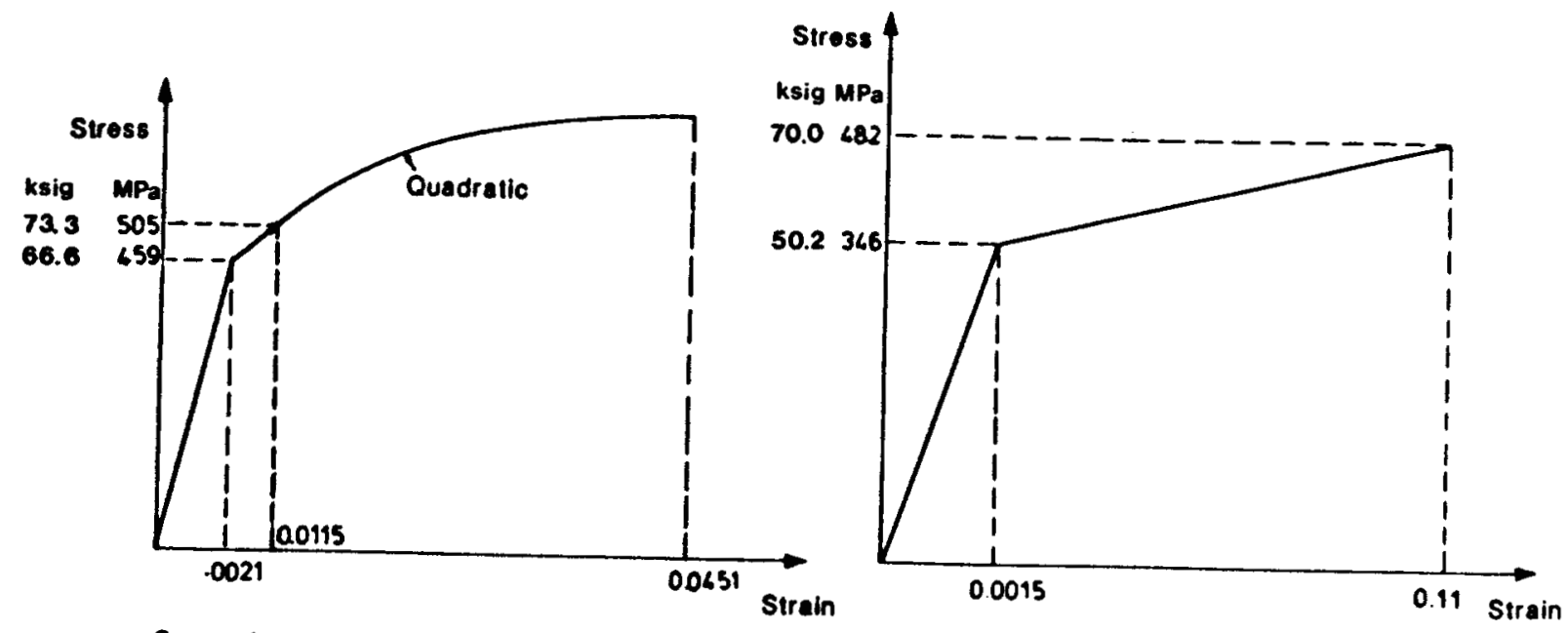

Same shape for tension and compression

Reinforcement

STEEL STRESS/STRAIN RELATIONSHIPS

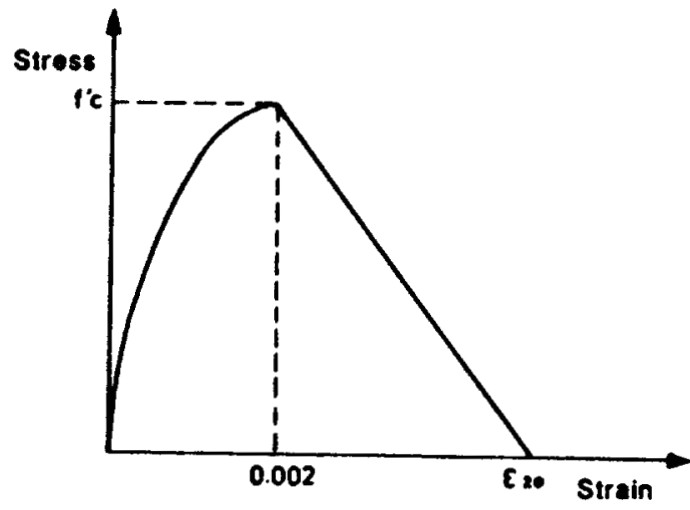

Uncorr fined concrete in compression

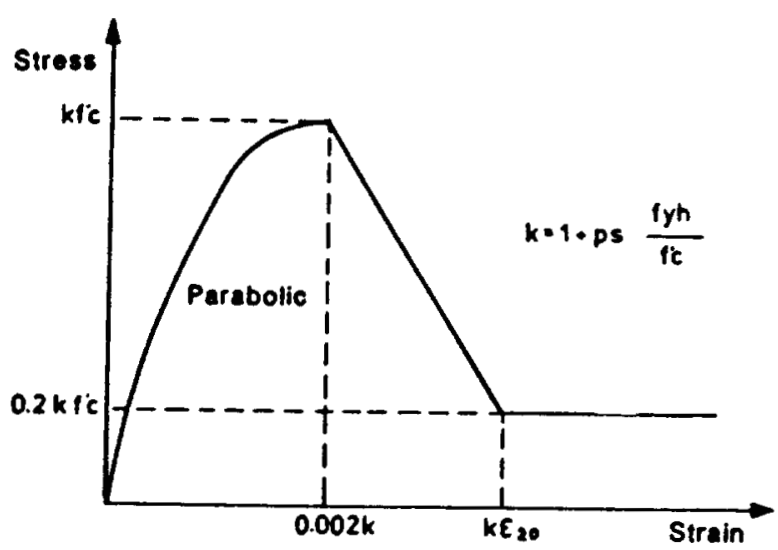

Contined concrete in compression

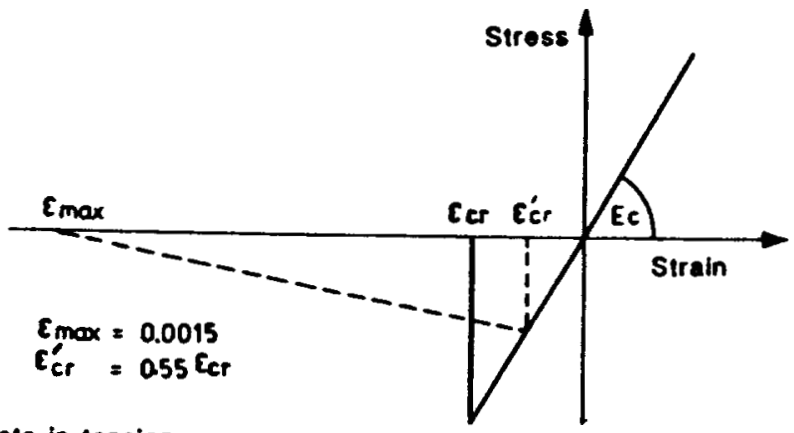

Concrete in tension

CONCRETE STRESS/STRAIN RELATIONSHIPS

Figure 4.5.3 Steel and Concrete Stress-strain Relationships 

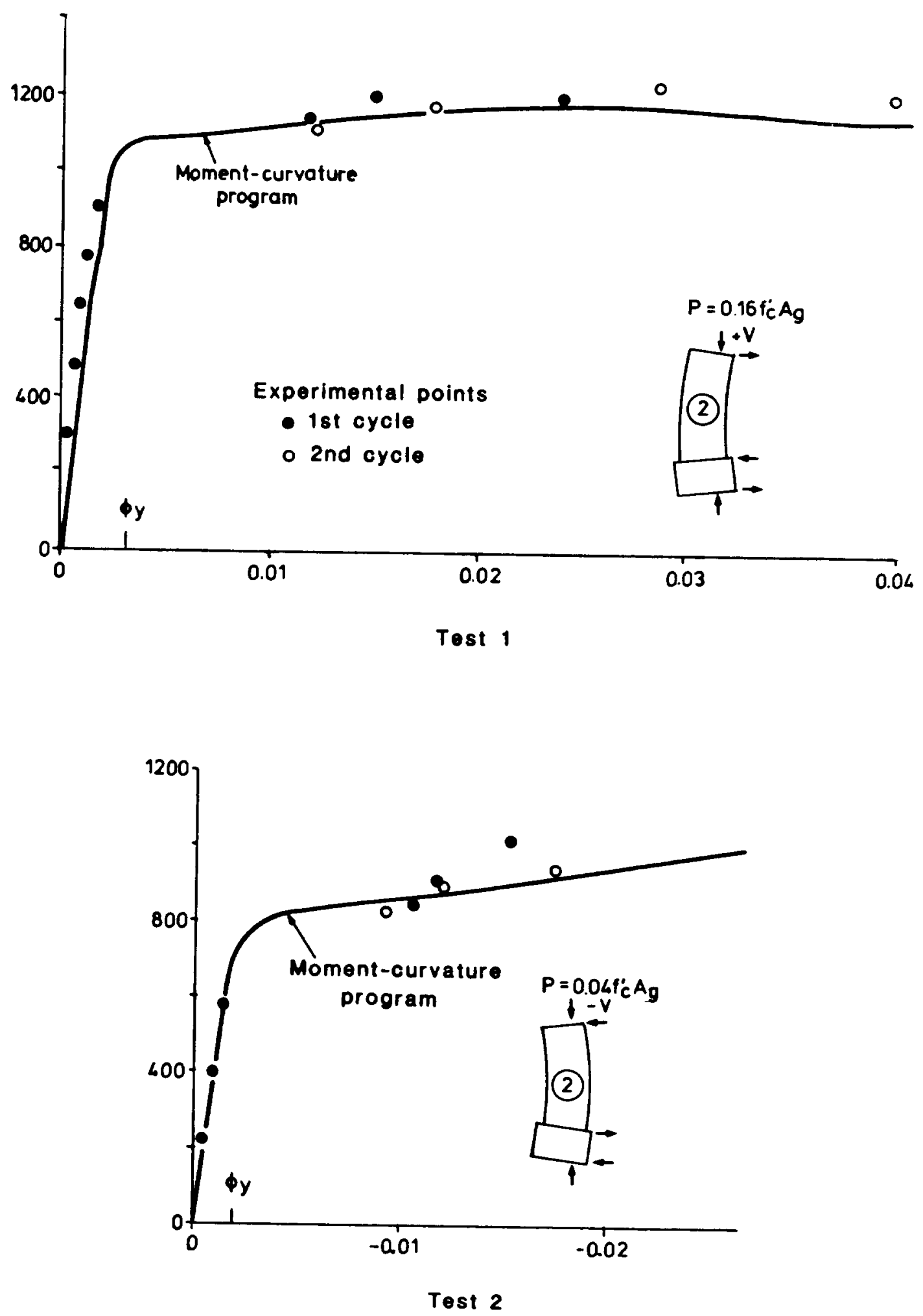

Figure 4.5.4 Moment Curvature Program Calibration 


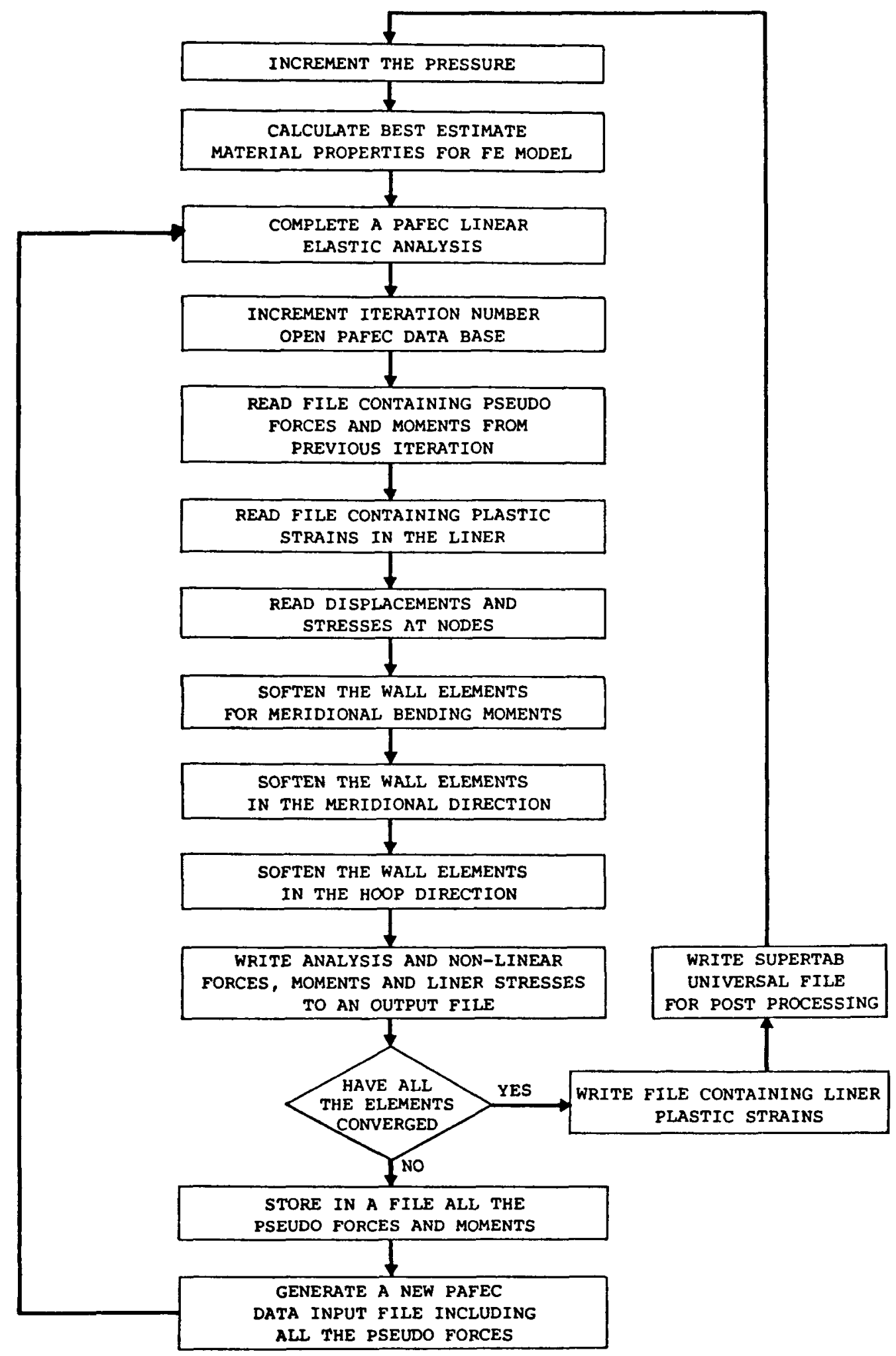

Figure 4.5.5 Flowchart : Main Program 


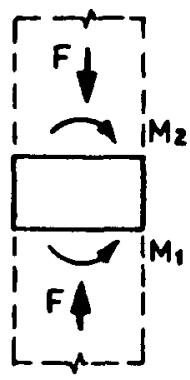

$$
\begin{aligned}
& M_{i}=\frac{M_{1}+M_{2}}{2} \\
& k_{i}=\frac{M_{i}}{E I}
\end{aligned}
$$

Stage 1 - Forces and moments from linear-elastic analysis

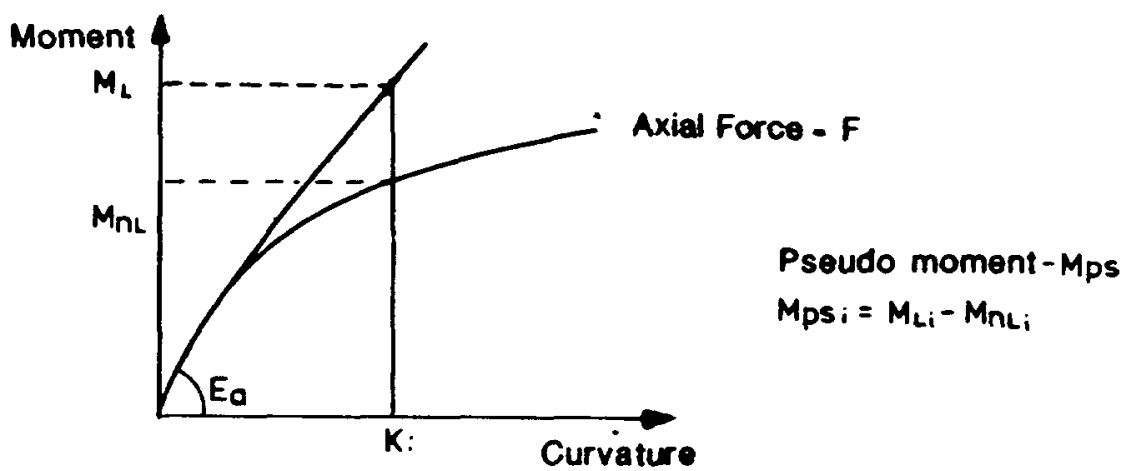

Stage 2 - Calculation of non-linear moment

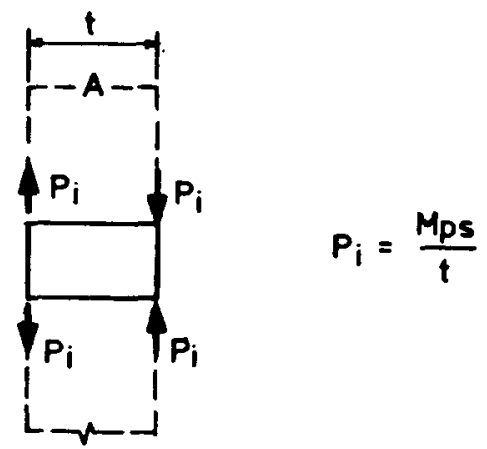

Stage 3 - Pseudo moments converted to node loads

Figure 4.5.6 Softening Elements for Meridional Bending $-257-$ 


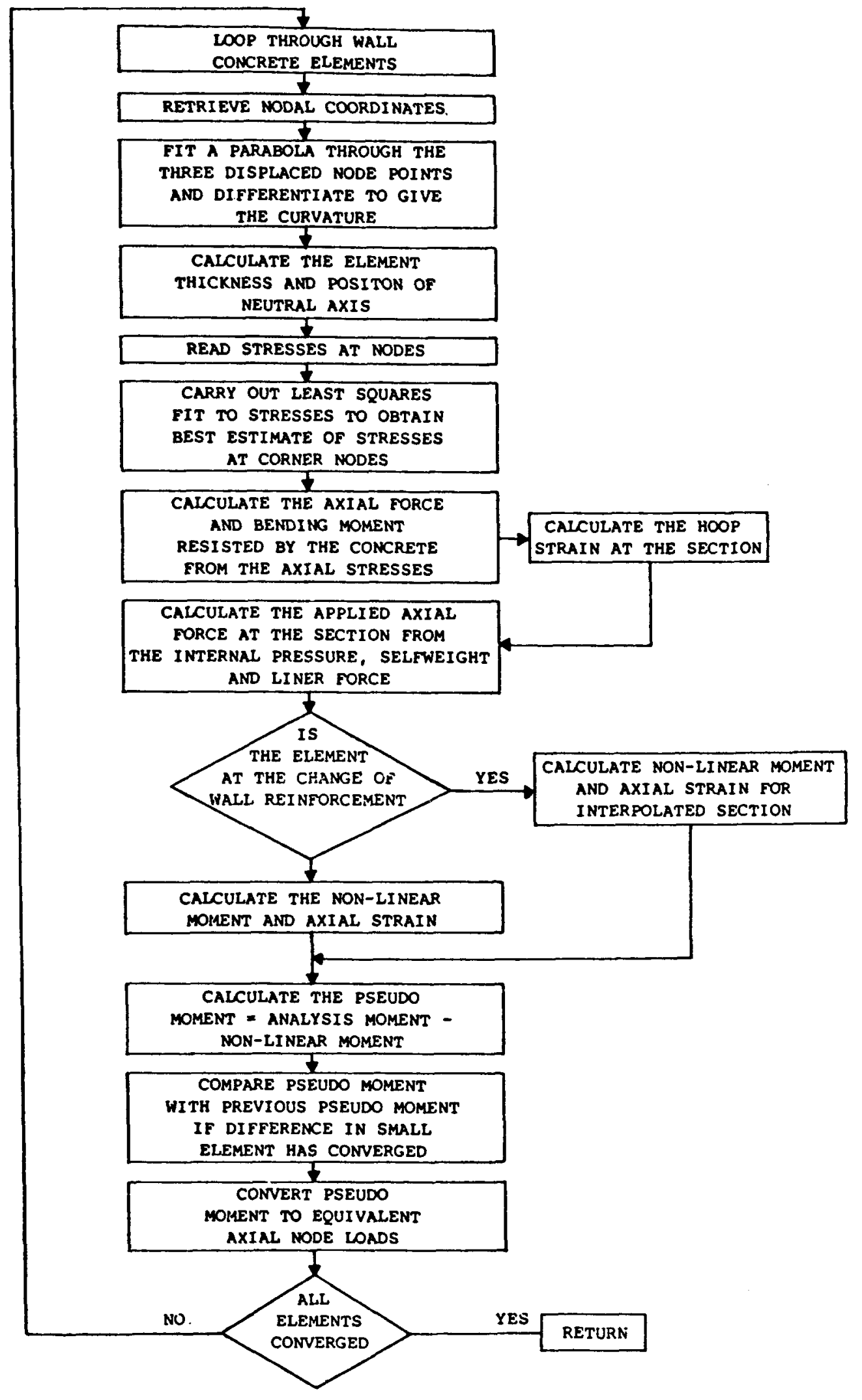

Figure 4.5.7 Flowchart : Softening of the Meridional Moment 


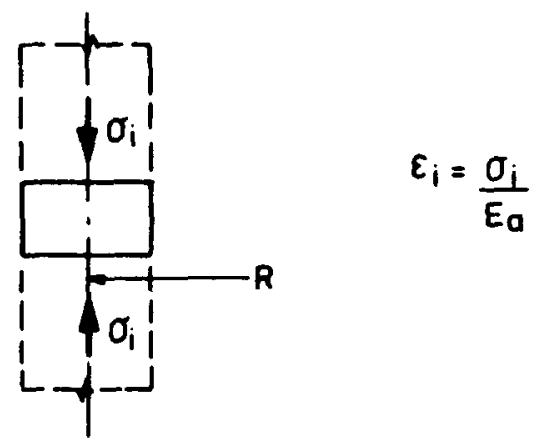

Stage 1 - Calculation of element axial strain

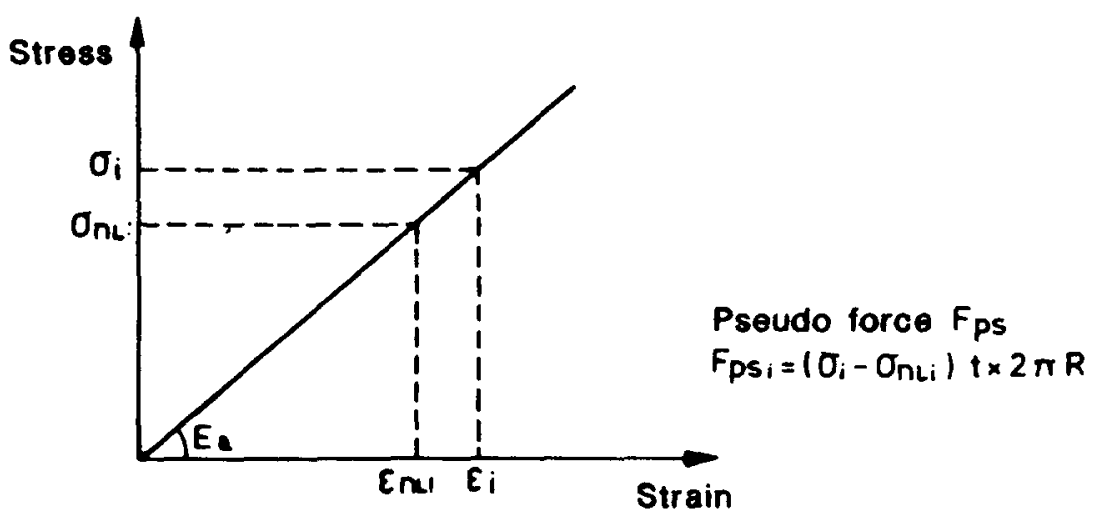

Stage 2 - Calculation of non-linear torce

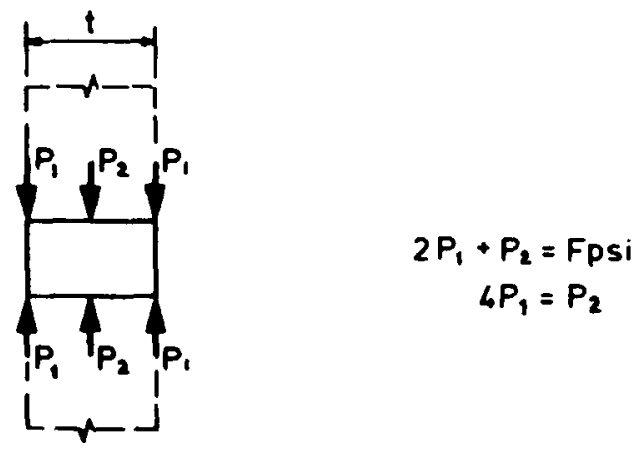

Stage 3 - Pseudo force converted to node loads

Figure 4.5.8 Softening Elements for Meridional Strain $-259-$ 


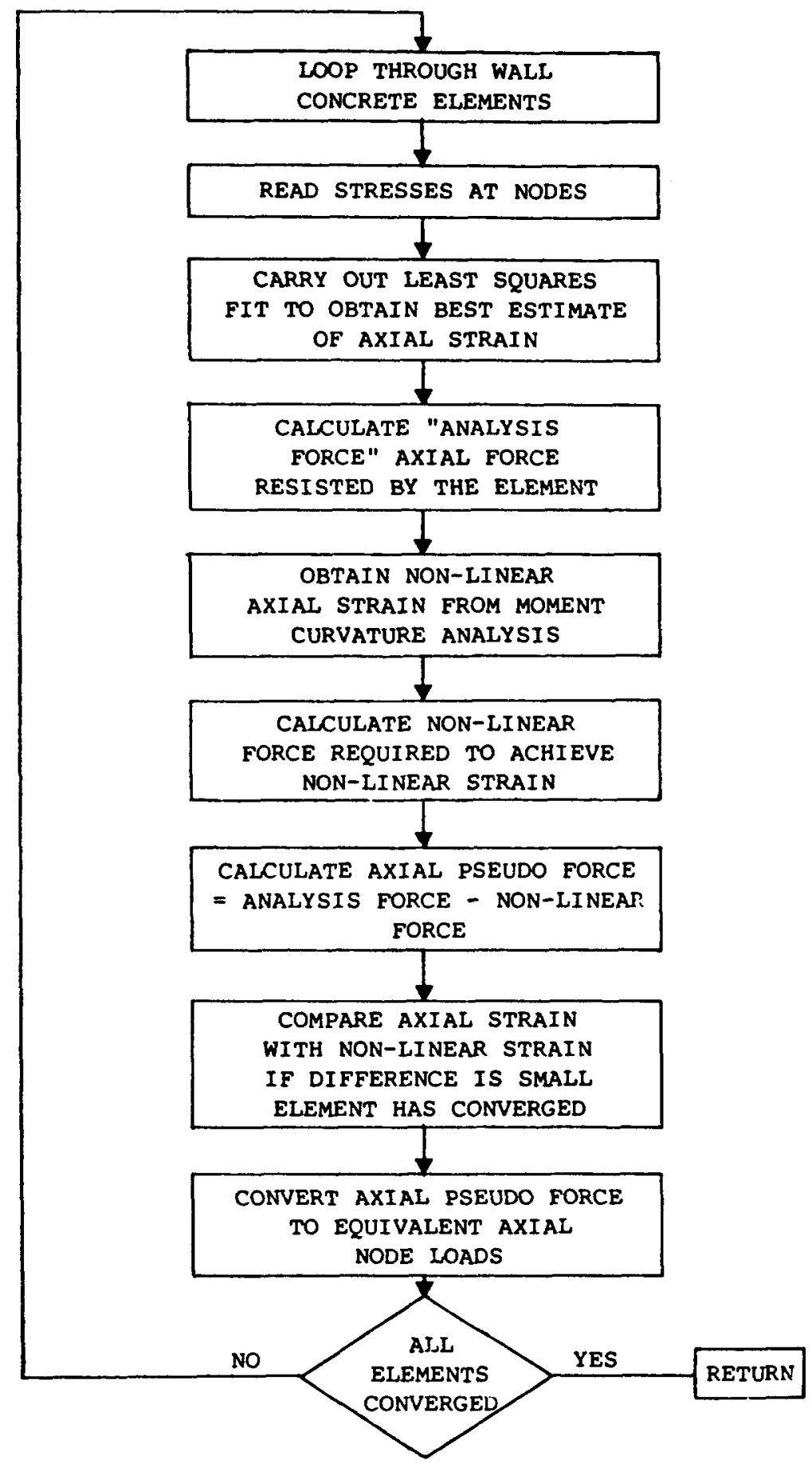

Figure 4.5.9 Flowchart : Softening of the Meridional Strain 


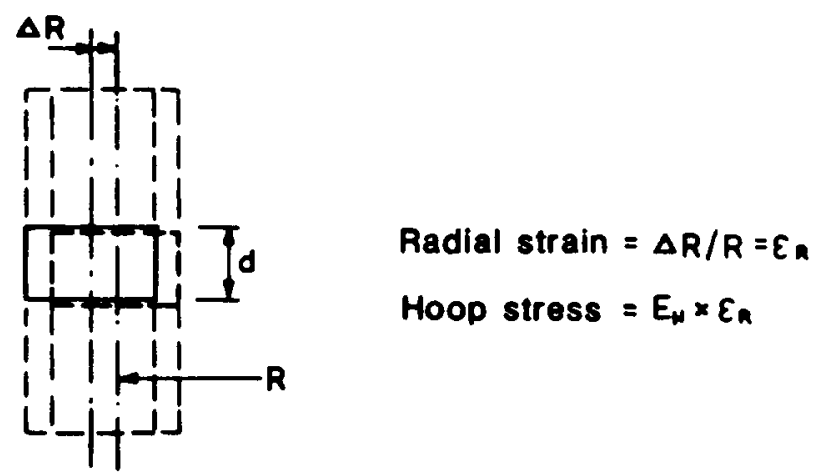

Stage 1 - Calculation of element hoop strain

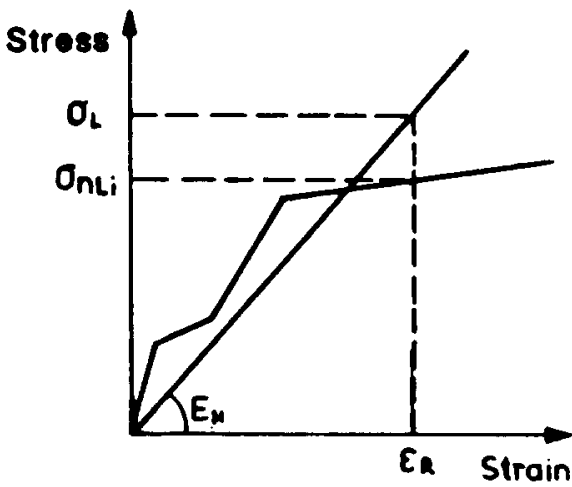

$$
\begin{aligned}
& \quad \frac{\operatorname{Pr}}{t}=0 \\
& \text { Equivalent pseudo } \\
& \text { pressure }=\frac{\left(\sigma_{i}-\sigma_{n L i} \mid \times t\right.}{R} \\
& \text { Pseudo force Pps. } \\
& \text { Fps: }=\left(\sigma_{i}-\sigma_{n L i}\right) \times t \times 2 \pi \times d
\end{aligned}
$$

Stage 2 - Calculation of non-linear force

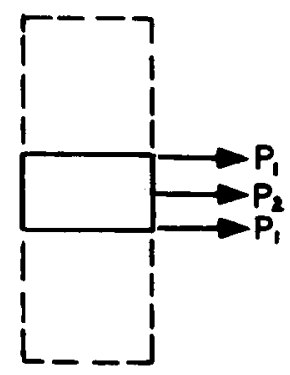

$$
\begin{aligned}
2 P_{1}+P_{2} & =F p s i \\
4 P_{1} & =P_{2}
\end{aligned}
$$

Stage 3 - Pseudo force converted to node loads

Figure 4.5.10 Softening Elements for Hoop Stress 


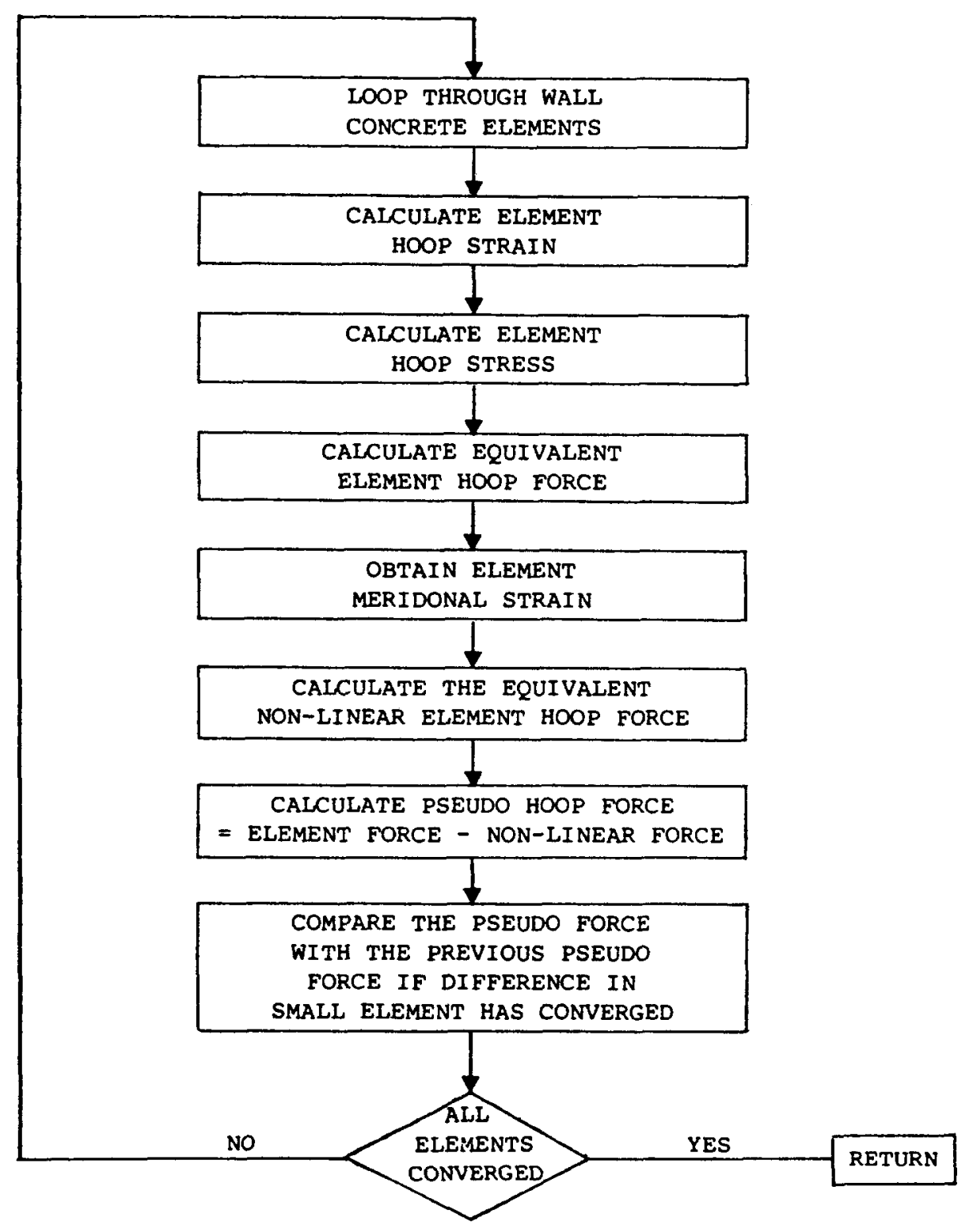

Figure 4.5.11 Flowchart : Softening of the Hoop Forces 


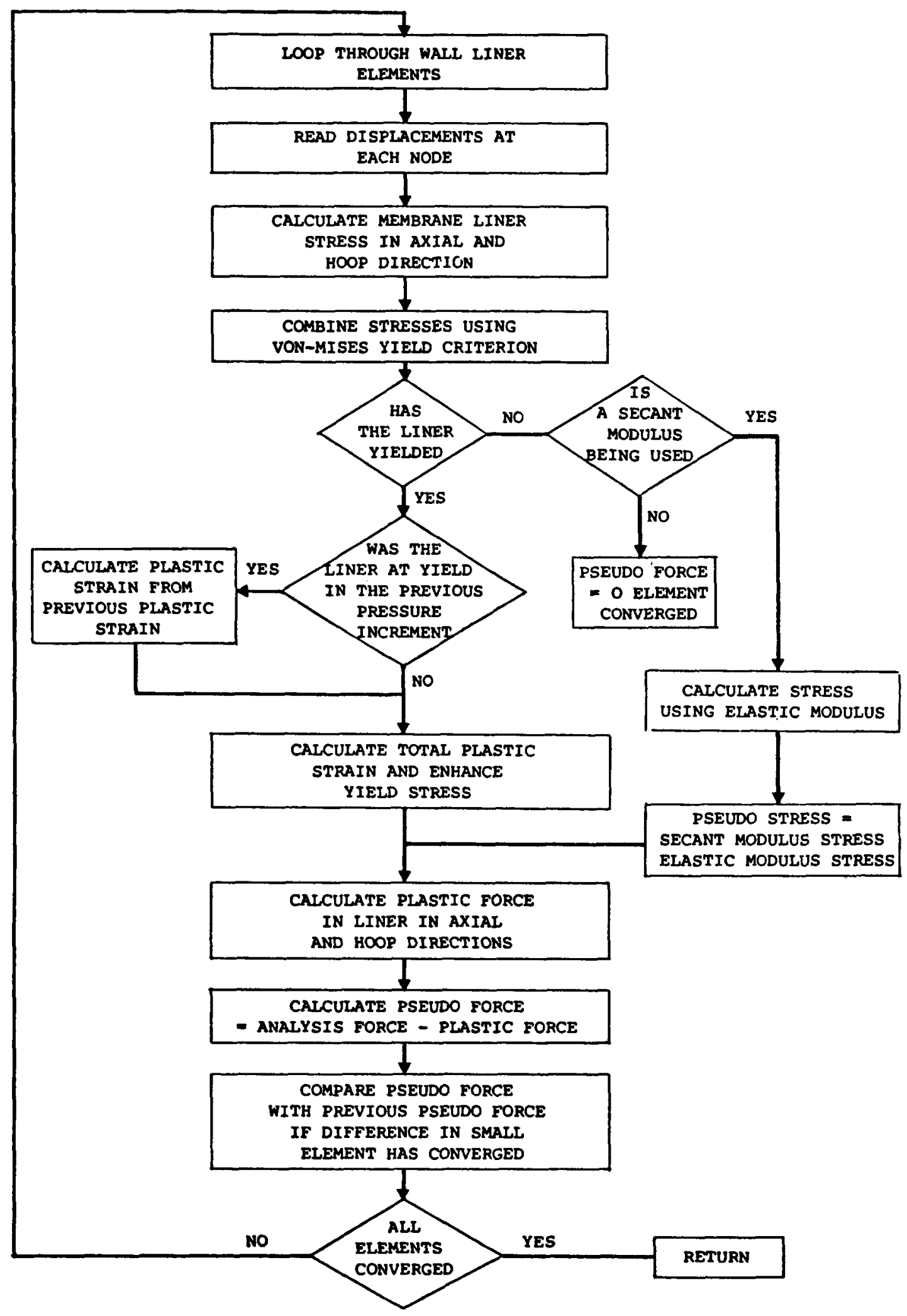

Figure 4.5.12 Flowchart : Liner Softening 


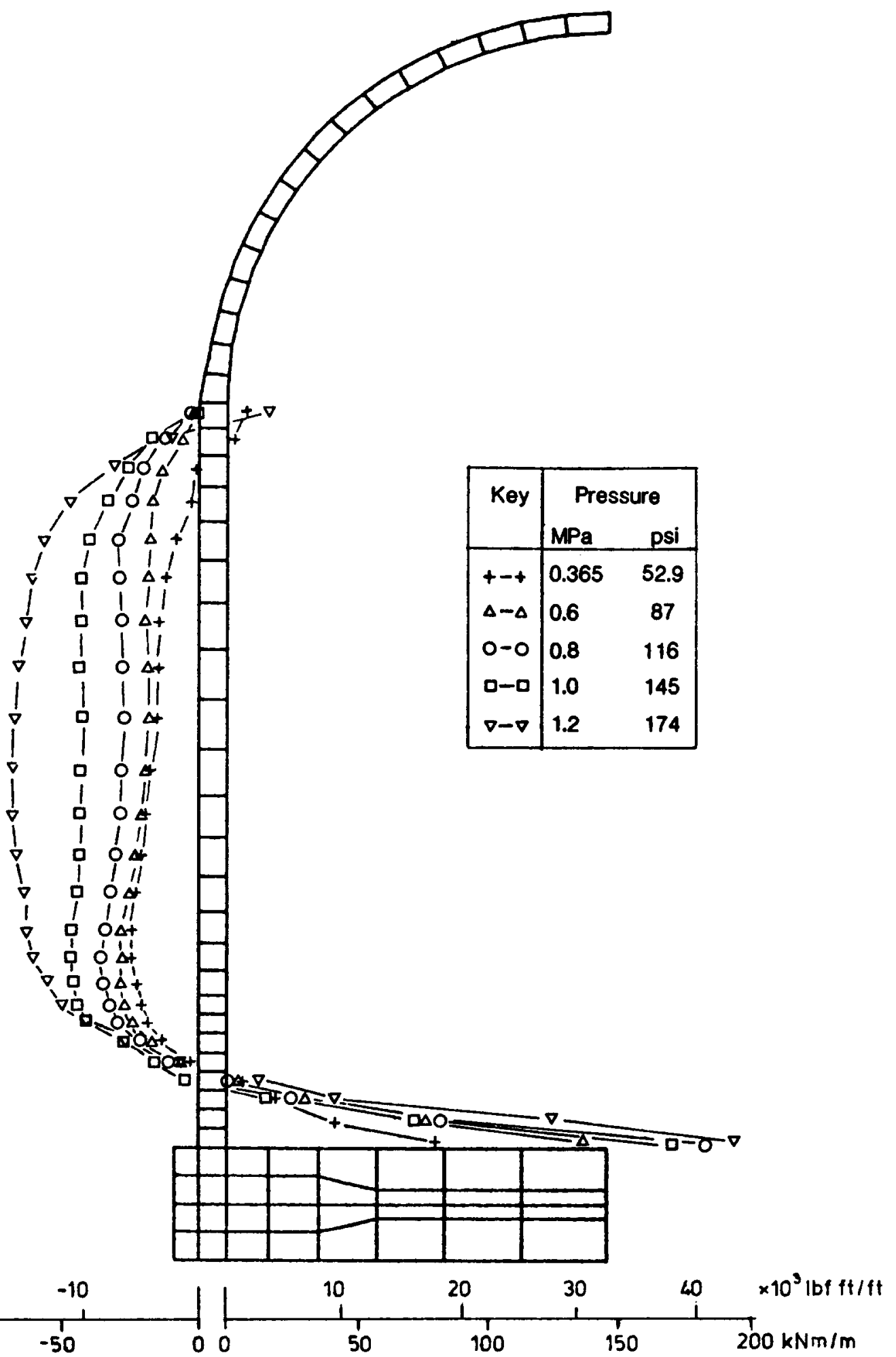

Figure 4.5.13 Meridional Bending Moments 


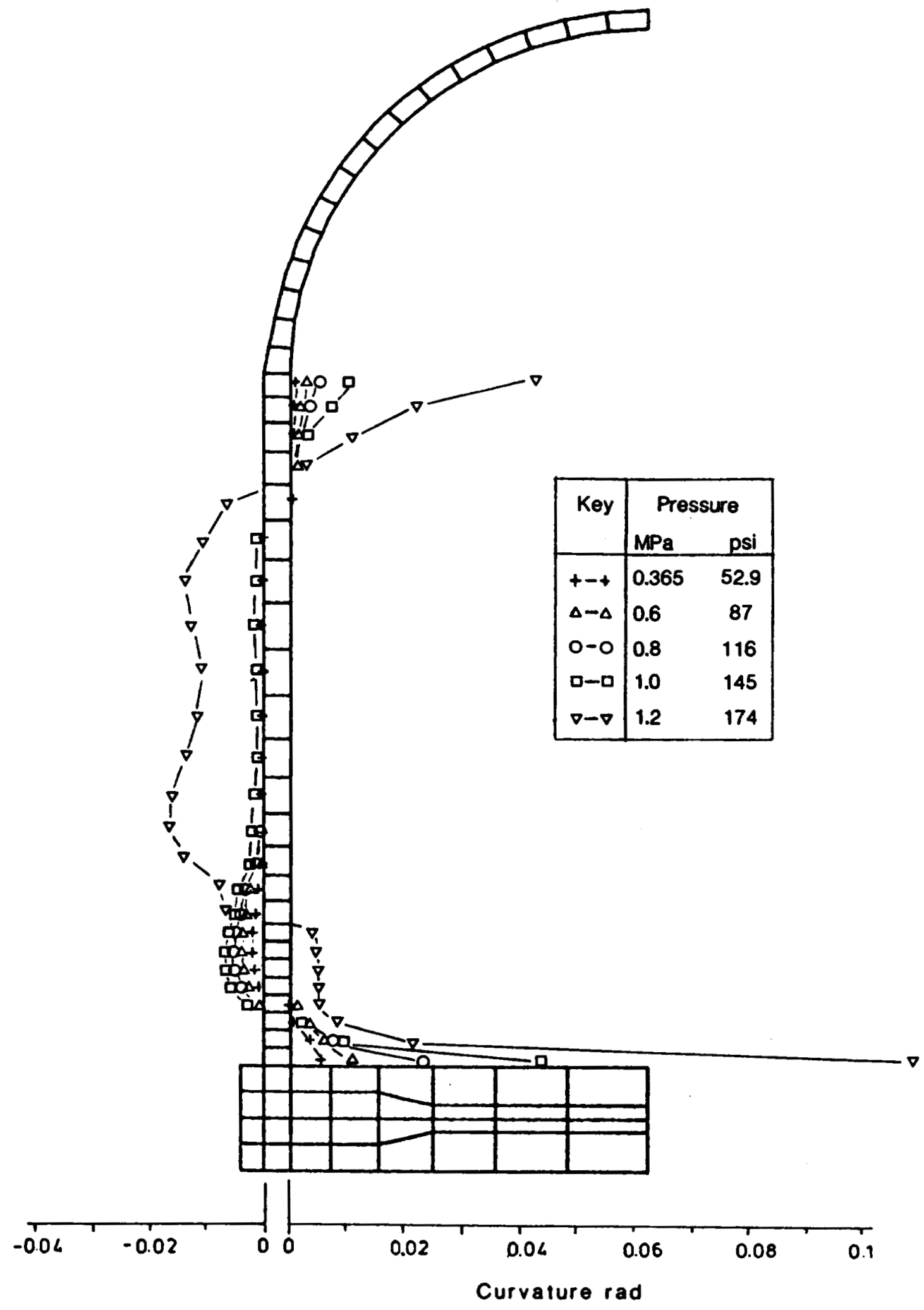

Figure 4.5.14 Meridional Curvature 


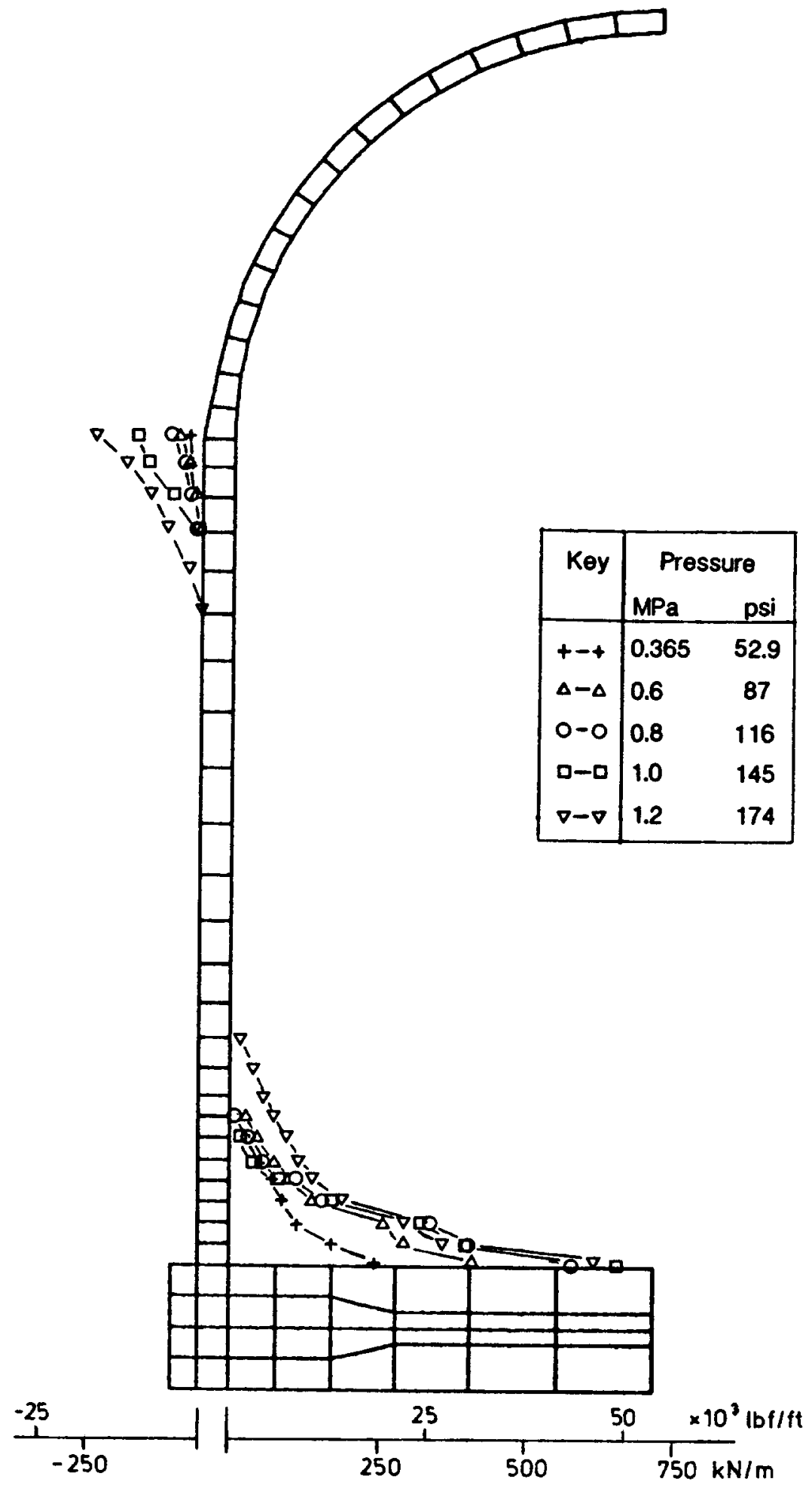

Figure 4.5.15 Radial Shear Force 


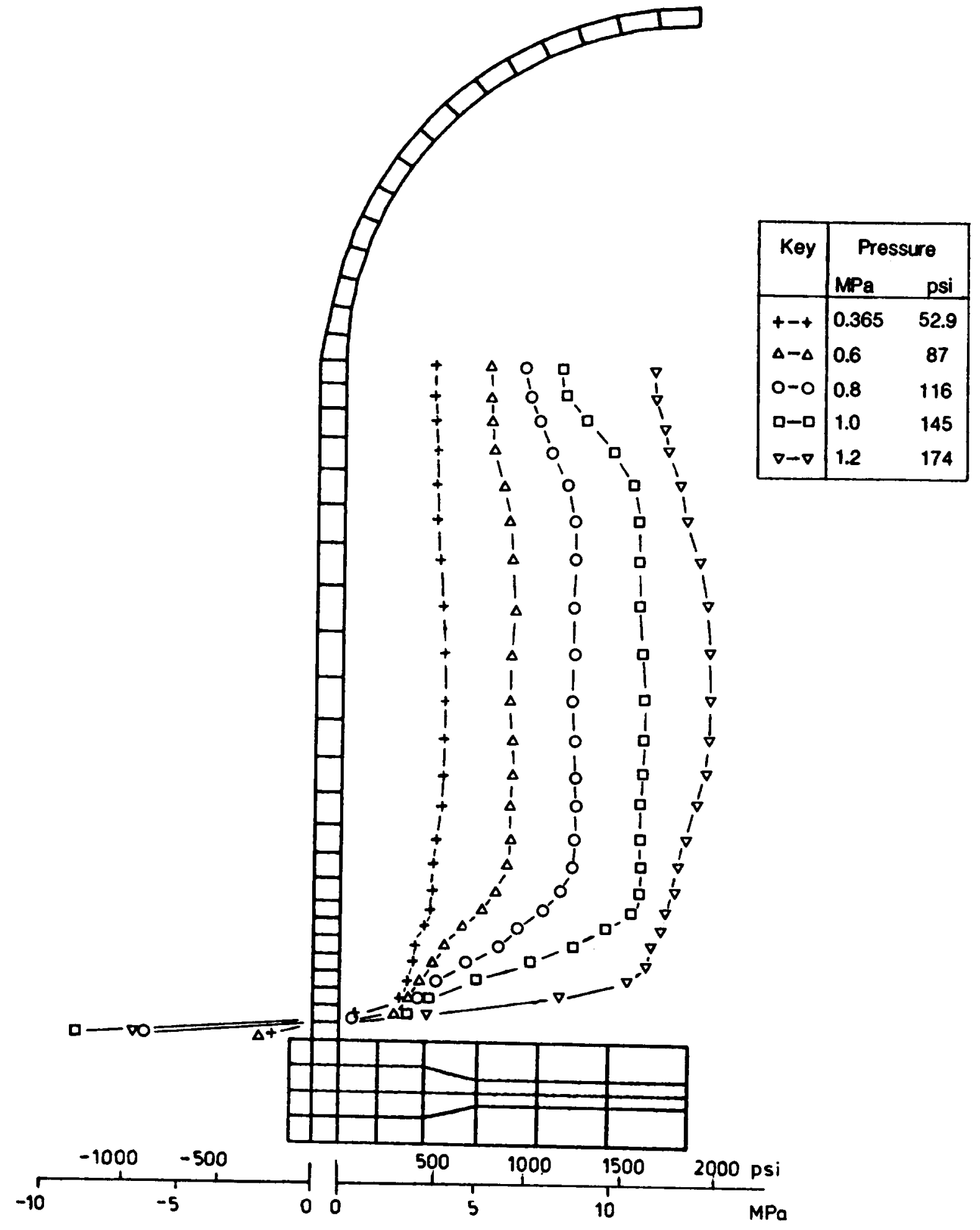

Figure 4.5.16 Hoop Stress 

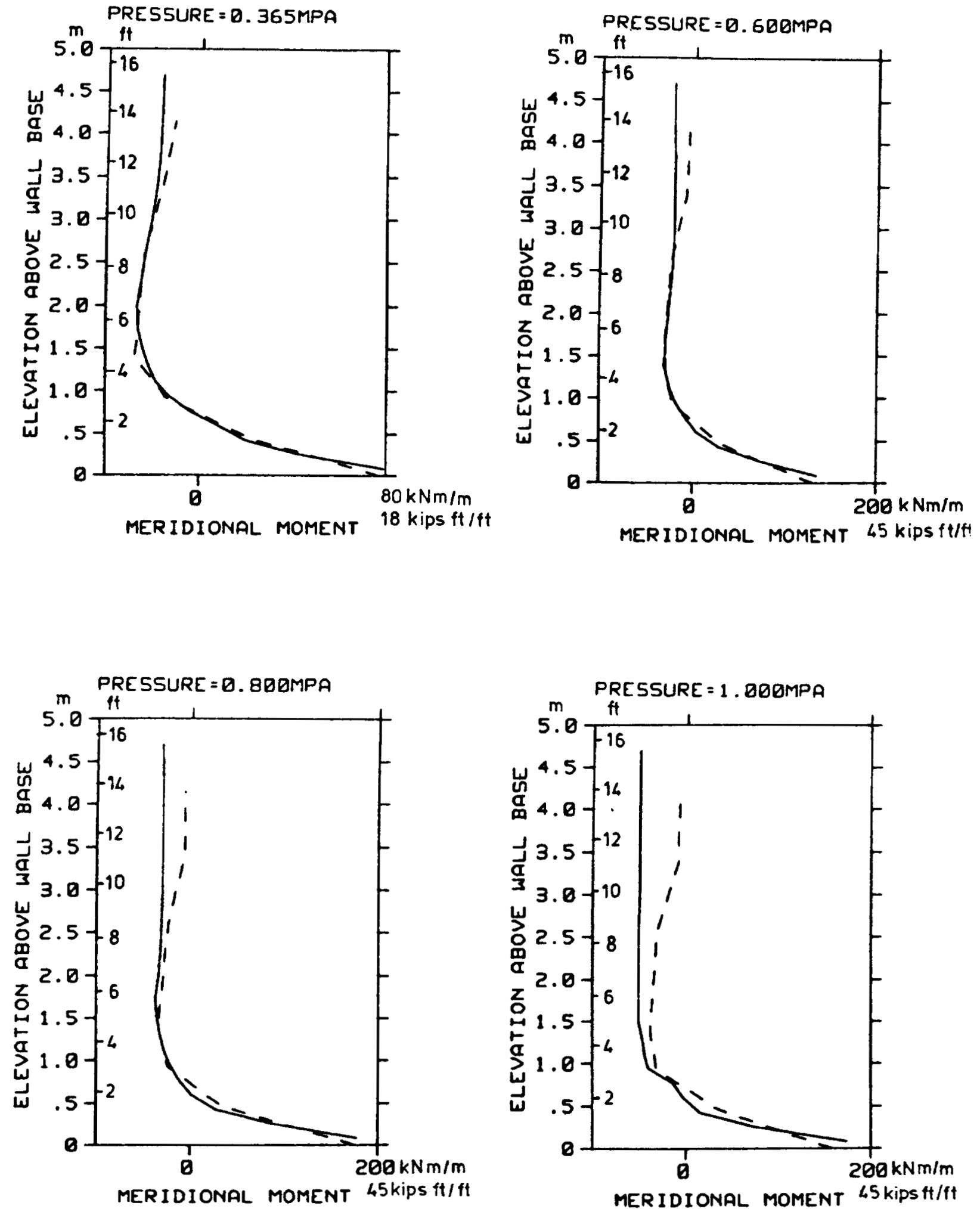

AXISYMMETRIC

- - - 3 DIMENSIONAL

Figure 4.5.17 Comparison of Meridional Moment - Axisymetric vs. 3-D Models 

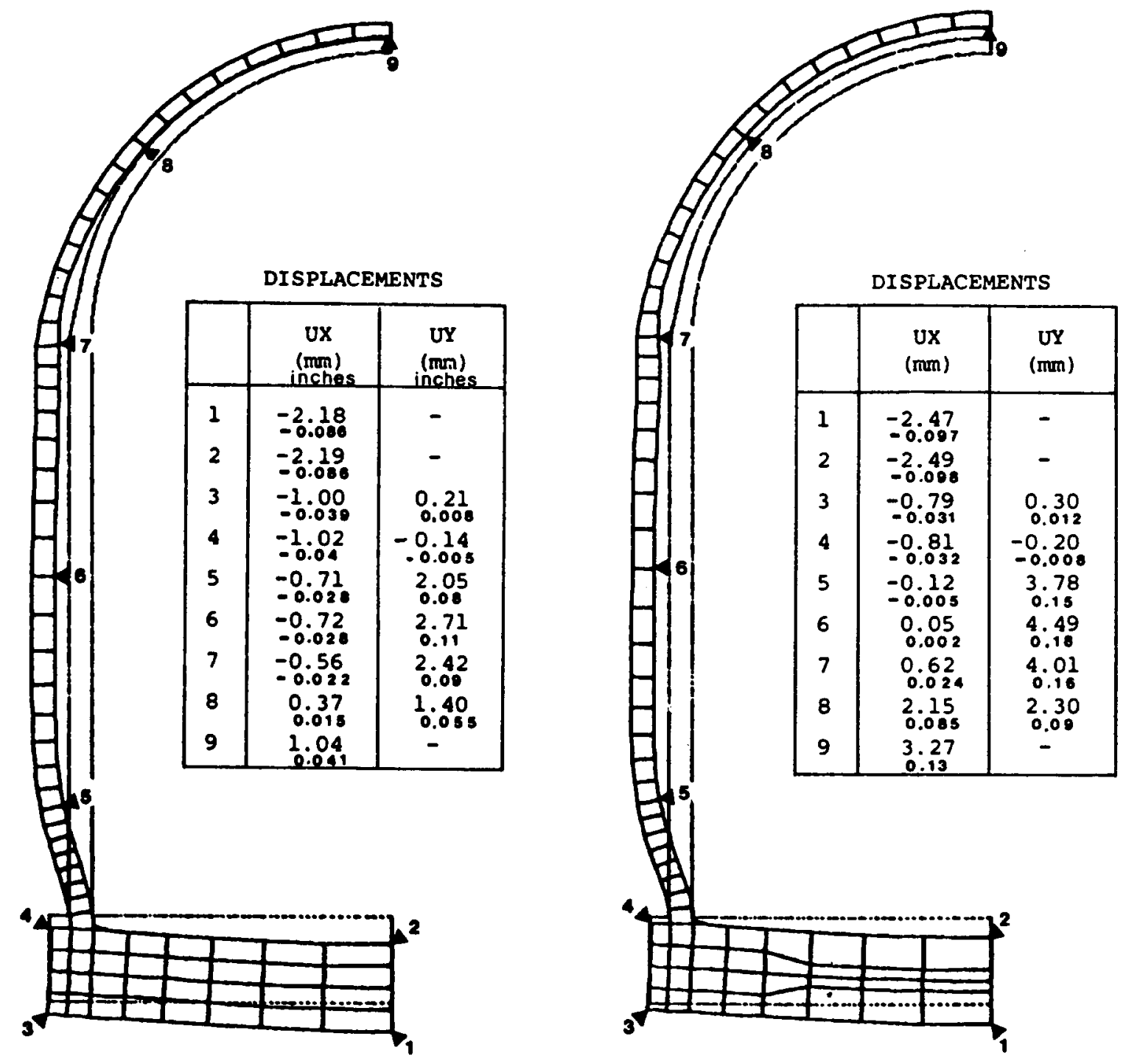

Pressure $=0.365 \mathrm{MPa}(52.9 \mathrm{psi})$ $-\frac{1}{-1}$

Pressure $=0.6 \mathrm{MPa}(87 \mathrm{psi})$

Figure 4.5.18 Displaced Shape 

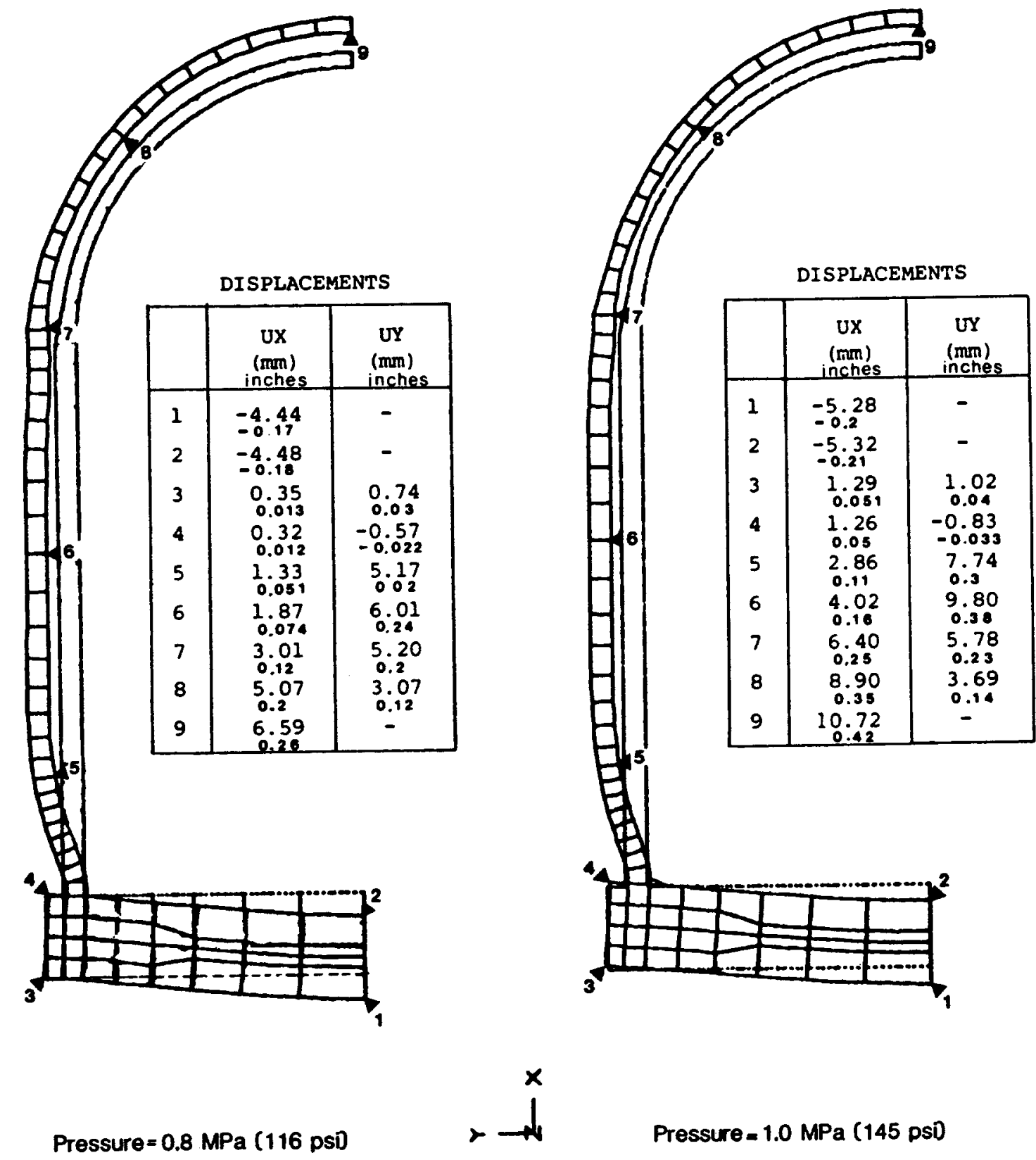

Figure 4.5.19 Displaced Shape 


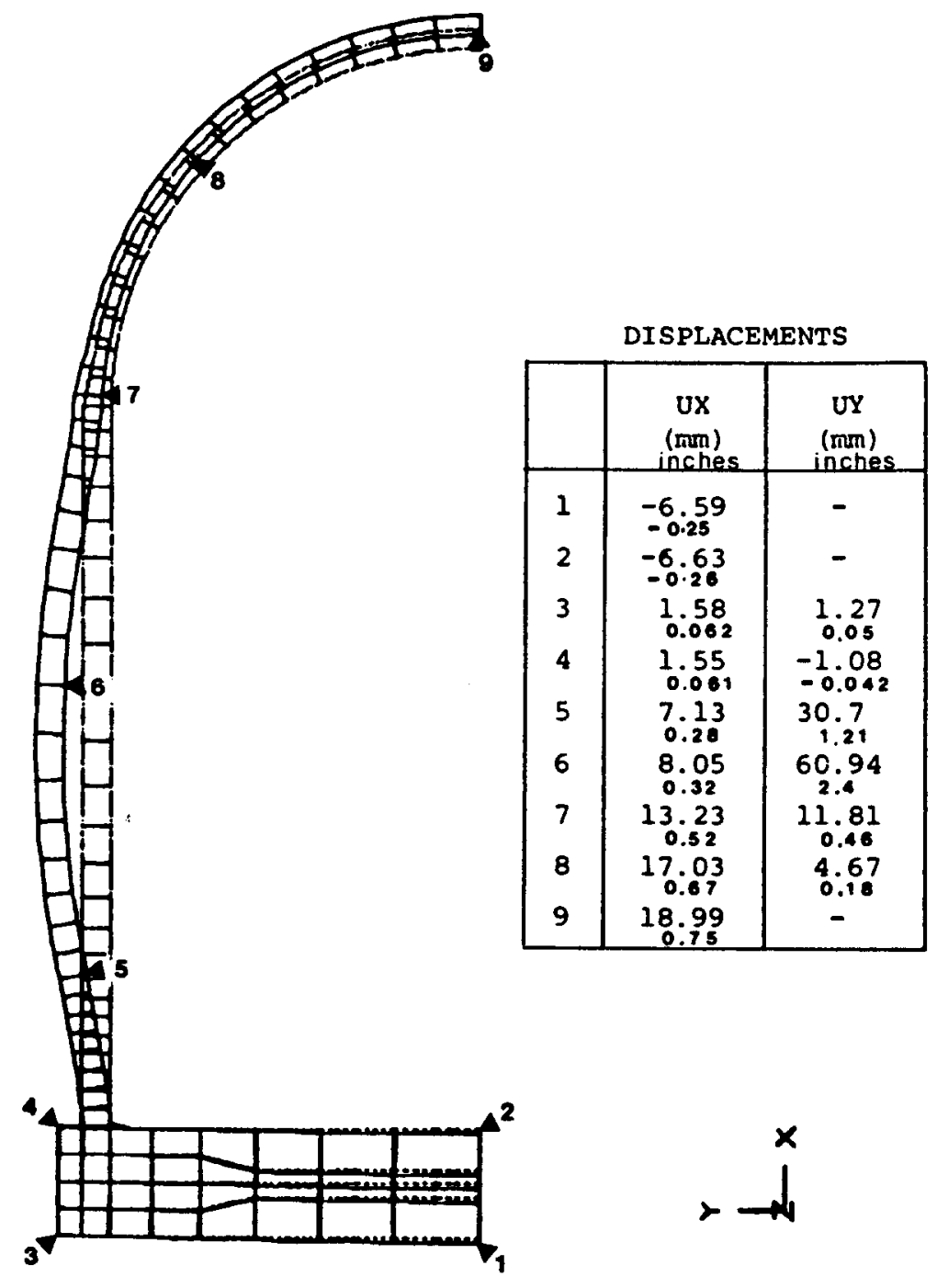

Pressure $=1.2 \mathrm{MPa}(174 \mathrm{psi})$

Figure 4.5.20 Displaced Shape 


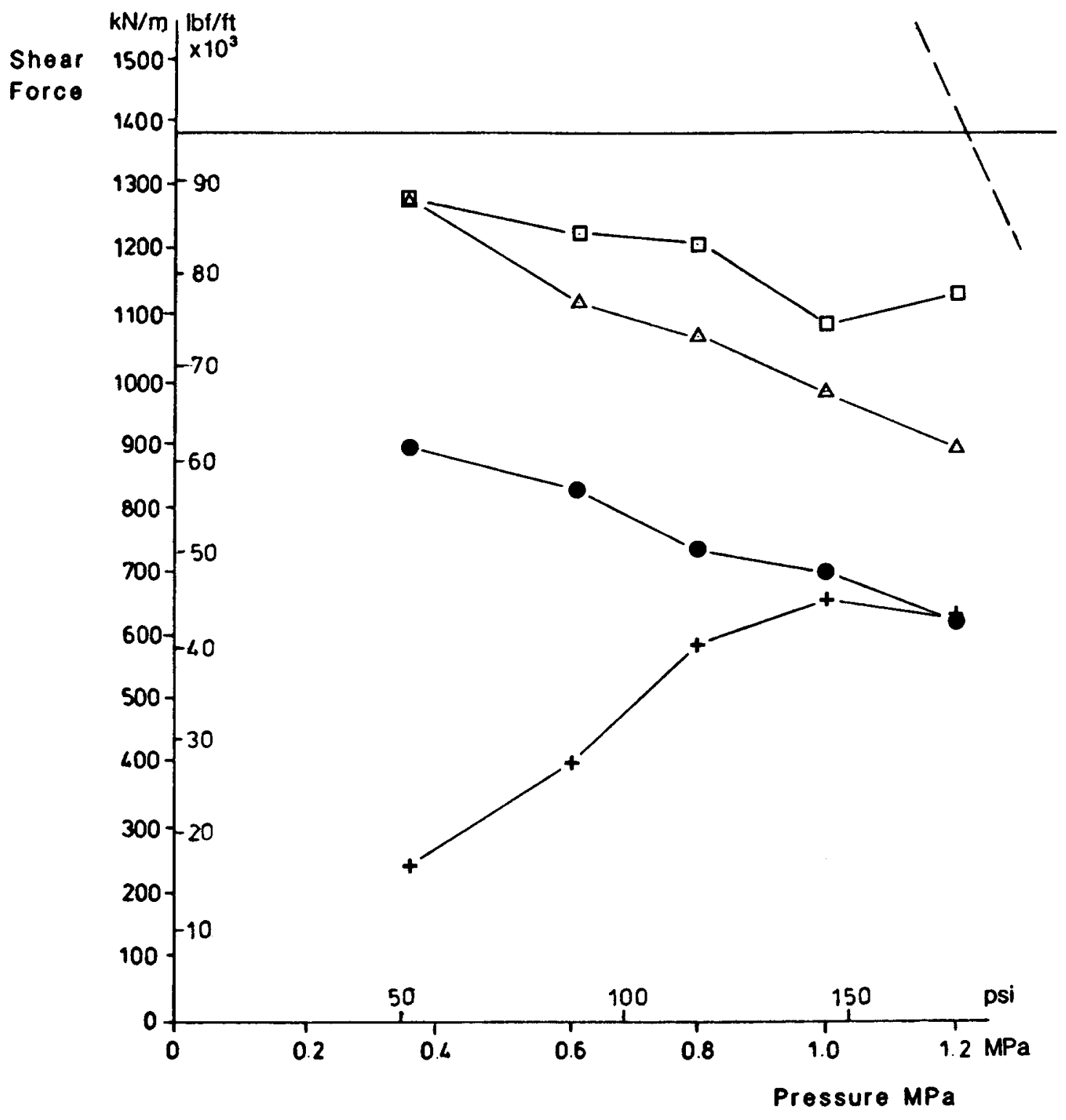

+ Predicted Shear Force

- ASME Section III Div 2

$\triangle$ NZS 3101 Part 1

- Modified Rangan Formula

- - Uli Dowel Force

Park \& Paulay Friction Formula

Figure 4.5.21 Wall Base Shear Force 


\subsection{ENEA-DISP}

This section was written by Giampiero Orsini and Giuseppe Pino of ENEA-DISP, Nuclear Safety and Health Protection Directorate, Rome, Italy.

\subsubsection{Summary}

The containment has been analyzed using the ADINA code (Dec.1978 version), considering an axisymetric continuum model clamped at the cylinder wall bottom. It consists of 300 eight-noded isoparametric concrete elements, 252 ring and 283 truss elements representing hoop, meridional, seismic rebars with their nominal area, 99 ring and 100 meridional truss elements representing a lattice equivalent to the liner nominal thickness. Ring and truss elements have been placed at the actual rebar positions. The number of the model nodes is 1109 with 2197 DOF. The concrete material model takes into account the multiaxial stress-strain behavior by considering a triaxial failure envelope, described in terms of discrete principal stress ratios. When crushing occurs, the material has no more stiffness. If the computed principal stress exceeds the uniaxial cut-off tensile stress, a crack is formed perpendicular to this stress and both the normal and shear stiffnesses are decreased, the former to $0.1 \mathrm{E}-3$ and the latter to 0.5 times the initial stiffness.

The steel material model follows the elastic-plastic stress-strain law with isotropic hardening.

The F.E. system has been solved using an incremental solution of the equations, performing equilibrium iterations (BFGS method) up to $0.67 \mathrm{P}_{d}$. Sixteen integration points in the concrete elements were considered.

Analyzing the stresses and strains that were computed, the most likely failure is that of the bottom wall due to the combined effect of bending, tension and shear at a pressure value between 3.5 and $4 P_{d}$. Since penetrations were not considered, the prediction assumes their integrity up to the wall failure.

\subsubsection{Analytical Model}

\subsubsection{Finite Element Model}

The containment structure has been analyzed using the ADINA finite element code version Dec.1978 [4.6.1]. The finite element model is an axisymetric continuum model, consisting of 300 eight-noded isoparametric elements representing the concrete, 252 ring elements representing the hoop rebars, and 283 two-noded truss elements representing the meridional rebars and the shear resisting rebars (11 elements) at the cylinder-basemat junction.

The liner has also been modeled by truss elements (99 ring elements and 100 two-noded meridional elements). The two dome cap steel plates in the apex region and the grout in between have been modeled by linear elastic eightnoded isoparametric elements.

Three elements have been used through the thickness of both the cylinder and dome walls (Figure 4.6.1). The truss elements representing the rebars have 
been placed in the actual position shown in the design drawings, and the diagonal rebars have been modeled with equivalent hoop and meridional trusses (Figure 4.6.2). In Figure 4.6.3 the rebar modeling at the cylinder wall base is shown.

The total number of the model nodes is 1109 with 2197 D.0.F. The present model does not include the basemat and the nodes at the base of the cylinder are restrained in every direction. In the dome apex, the nodes on the axis of revolution are allowed to displace along the vertical axis only.

\subsubsection{Material Model for Concrete}

The concrete behavior has been represented by the model implemented in the ADINA code [4.6.2]. The basic principle is to treat the general multiaxial stress-strain behavior as an equivalent uniaxial relation. To this purpose twenty-four input points are required, covering all the expected principal stress values, to model the triaxial concrete compressive failure envelope. The basic features of this model include (1) a nonlinear stress-strain relation allowing for the weakening of the material, (2) stress induced orthotropy, (3) failure envelopes defining tension and compression failures, (4) post-cracking and crushing modeling ability, including strain softening, and (5) loading and unloading conditions. The concrete uniaxial parameters, used in the analysis, are:

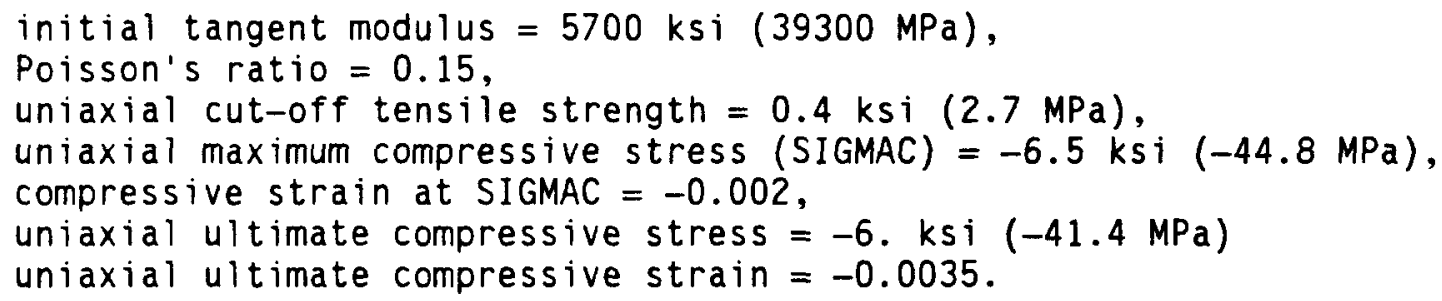

The following parameters, in terms of discrete principal stress ratios to describe the triaxial failure envelope, are required:

\begin{tabular}{|c|c|c|c|c|c|c|}
\hline $\begin{array}{l}\text { principal } \\
\text { stress ratios }\end{array}$ & $I=1$ & 2 & 3 & 4 & 5 & 6 \\
\hline SP1(I) & 0.0 & 0.25 & 0.5 & 0.75 & 1.0 & 3. \\
\hline $\begin{array}{l}S P 3(I, 1) \\
\text { (at } S P 2=S P 1)\end{array}$ & 1.0 & 1.4 & 1.7 & 2.2 & 2.5 & 2.8 \\
\hline $\begin{array}{l}\mathrm{SP3}(I, 2) \\
\text { (at } \mathrm{SPZ}=.75 \mathrm{SP} 3)\end{array}$ & 1.3 & 1.5 & 2.0 & 2.3 & 2.7 & 3.2 \\
\hline $\begin{array}{l}\mathrm{SP} 3(\mathrm{I}, 3) \\
\text { (at } \mathrm{SP} 2=\mathrm{SP} 3)\end{array}$ & 1.25 & 1.45 & 1.95 & 2.25 & 2.65 & 3.15 \\
\hline
\end{tabular}


If the maximum principal stress exceeds the uniaxial cut-off tensile stress, a tensile failure plane is defined perpendicular to this stress. Once a tensile failure plane has occurred, the normal stiffness across this plane is decreased to the original stiffness times the normal stiffness reduction factor (STIFAC), and similarly, the shear stiffness is decreased to the original shear stiffness times the shear stiffness reduction factor (SHEFAC).

In the present analysis, these parameters assume the following values: STIFAC $=0.1 E-3$.

SHEFAC $=0.5$.

\subsubsection{Material Model for Steel}

The elastic plastic stress-strain law, with isotropic hardening, has been used to represent the behavior of the rebars and the liner. The parameters for the rebars are:

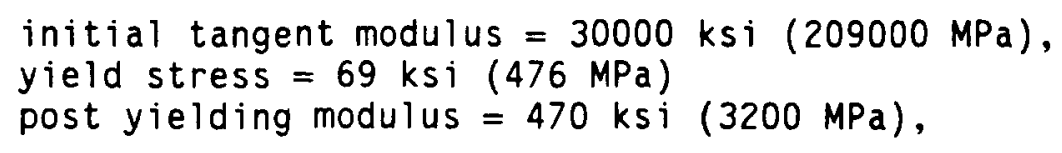

For the liner:

$$
\begin{aligned}
& \text { initial tangent modulus }=30000 \mathrm{ksi}(209000 \mathrm{MPa}) \text {, } \\
& \text { yield stress }=51.5 \mathrm{ks} i(355 \mathrm{MPa}), \\
& \text { post-yielding modulus }=110 \mathrm{ksi}(700 \mathrm{MPa}) .
\end{aligned}
$$

\subsubsection{Method of Analytical Solution}

The finite element system has been evaluated using an incremental solution of the equations of equilibrium:

$$
t_{K U}=t^{t+d t_{R}-t_{F}}
$$

where ${ }^{t_{K}}=$ tangent stiffness matrix corresponding to the configuration of the system at time $t, U=$ vector of nodal point incremental displacements (i.e., $\left.\mathbf{U}={ }^{t+d t} \mathbf{U}-{ }^{t} \mathbf{U}\right),{ }^{t+d t_{R}}=$ vector of externally applied nodal point loads corresponding to time $t+d t$, and ${ }^{t} F=$ vector of nodal point forces corresponding to the internal stresses at time $t$.

The solution of the previous equation yields, in general, an approximate displacement increment $U$. To improve the solution accuracy and in some cases to prevent the development of instabilities it may be necessary to use equilibrium iteration in each or preselected time steps. In this case the BFGS (Broyden-Fletcher-Goldfarb-Shanno) matrix updated method has been considered. The algorithm used is:

$$
\begin{aligned}
& t_{K^{+} d U^{(i)}}={ }^{t+d t_{R}-}{ }^{t+d t_{F}}(i-1) \\
& t+d t_{U^{(i)}}=t+d t_{U^{(i-1)}}+\operatorname{beta} d U^{(i)}
\end{aligned}
$$


where ${ }^{t} K^{+}$is an updated stiffness matrix (based on the iteration history) and 'beta' is an accelerating factor determined by a line search in the direction of $\mathrm{dU}^{(i)}[4.6 .1]$

The applied loads are: dead load, as a load proportional to the lumped mass, with its whole value from the beginning of the analysis and internal pressure, as concentrated loads acting at the inner nodes of the structure, with values increasing step by step.

The analyses have been performed with the following load steps (the internal pressure values are referred to the design pressure, $P_{d}$, equal to $46 \mathrm{psi}$ $(0.317 \mathrm{MPa}))$ :

\begin{tabular}{lclll}
\hline LOAD PATH & $\begin{array}{l}\text { LOAD STEP } \\
\text { INCREMENT } \\
\left(\% P_{d}\right)\end{array}$ & $\left.\begin{array}{l}\text { STIFFNESS } \\
\text { REFORMATION }\end{array}\right)$ & $\begin{array}{l}\text { EQUILIBRIUM } \\
\text { ITERATIONS }\end{array}$ & NOTES \\
\hline $0-50$ & 5 & $\begin{array}{l}\text { at every } \\
\text { step }\end{array}$ & $\begin{array}{l}\text { at every } \\
\text { step }\end{array}$ & $\begin{array}{l}\text { Maximum number (90) } \\
\text { of eq. it. } \\
\text { reached }\end{array}$ \\
\hline $50-67.5$ & 0.5 & $\begin{array}{l}\text { at every } \\
\text { step }\end{array}$ & $\begin{array}{l}\text { at every } \\
\text { step }\end{array}$ & $\begin{array}{l}\text { Out of balance } \\
\text { load larger } \\
\text { load incremental }\end{array}$ \\
\hline $67.5-401$ & 0.5 & $\begin{array}{l}\text { load every } \\
\text { step }\end{array}$ & no & $\begin{array}{l}\text { Negative } \\
\text { pivot. }\end{array}$ \\
\hline
\end{tabular}

In this analysis sixteen integration points are considered for the concrete 2-D elements. In previous analyses four integration points were used for the same elements in order to reduce the computer time. In that case when the pressure load reached about $65 \% \mathrm{P}_{d}$ some instabilities developed and the run stopped, having found a negative pivot for the equation corresponding to a node located at $1 / 4$ cylinder height. Close to that region, with the sixteen integration points model, at $300 \% \mathrm{P}_{\mathrm{d}}$ pressure level, an abnormal

displacement distribution of the outer nodes may be observed (Figure 4.6.4). Nevertheless the calculation went on without instabilities and in the following steps the displacement of the whole cylinder wall absorbed that local protuberance (Figure 4.6.5). 


\subsubsection{Analytical Model Approximations}

In this subsection the main approximations of the analyzed model are pointed out in order to keep them in mind when studying the calculational results.

a) The analyses performed are axisymetric analyses, therefore no penetrations were considered (e.g., the equipment hatches, the personnel airlocks and pipes). Thus, stress concentrations in these regions are disregarded and failures initiating in these locations cannot be predicted. On the other hand, such a model enables calculations within the capacities of present computers. After the test, local analyses around the penetrations may be necessary to complete the study of the mode 1 .

b) The basemat is not included in the model and soil-structure interaction is disregarded. The possible increasing of the bending moment and shear force at the basemat-wall junction is not evaluated.

c) the liner is modeled as a rebar lattice along the principal (hoop and meridional) directions. The biaxial stress state behavior is neglected.

d) In the apex of the dome, the steel cap plates and the grout in between are modeled with linear elastic elements.

e) After the start of concrete cracking, equilibrium iterations were discontinued in order to avoid lack of convergence. However, in this part of the analysis the load steps were chosen to be quite small $(0.5 \%$ of $P_{d}$ ) to obtain a reliable solution.

\subsubsection{Analyses Results}

\subsubsection{Main Steps During Internal Pressure Rising}

Before describing the analyses results it is useful to show significant points of structural behavior during loading. a) The first cracks in concrete happen at the cylinder wall base at $40 \% P_{d}$.
These cracks are caused by tension and bending moment and their plane s almost horizontal.

b) The extensive cracking of concrete due to hoop forces, begins at $68 \% P_{d}$

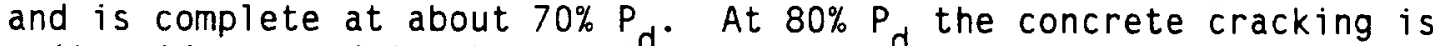
quite wide-spread in the meridional direction as well.

c) The yielding of the liner begins at $200 \% P_{d}$.

d) The yielding of the hoop rebars begins at $258 \% \mathrm{P}_{d}$ at the cylinder midheight.

e) The yielding of the meridional rebars begins at $285 \% P_{d}$ at the cylinder midheight.

f) General yielding is reached at about $300 \% P_{d}$ in the cylinder wall, both along meridional and hoop directions. 


\subsubsection{Concrete Cracking}

The containment structure is divided into four regions for a detailed description of the development of concrete cracks:

- region 1: cylinder wall-basemat junction;

- region 2: cylinder wall;

- region 3: dome-cylinder wall junction (springline);

- region 4: dome.

Region 1: cylinder wall-basemat junction

As could reasonably be anticipated, cracking of the concrete appears first at the inner side of the cylinder wall base due to meridional tension stresses caused by the bending moment. The bending moment effects are relevant up to 70 inches $(178 \mathrm{~cm}$ ) from the basemat top (15 concrete elements). At the inner layer of elements, the concrete begins to crack at $40 \% \mathrm{P}_{\mathrm{d}}$ and the crack planes have inclination, with respect to the vertical plane, ranging from 79 to 83 degrees.

In the intermediate layer of elements, the concrete begins to crack at $45 \%$ $P_{d}$ and the crack planes have inclination, with respect to the vertical plane, ranging from 76 to 83 degrees.

In the outer layer of elements, the concrete begins to crack at $55 \% \mathrm{P}_{d}$ and cracks planes have inclination, with respect to the horizontal plane, ranging from 68 to 83 degrees. These cracks appear at the inner side of the elements, at the locations of the first 5 integration points. At the other integration points, at the outer side, the concrete never cracks due to the meridional compression stresses.

At high values of the internal pressure concrete begins to crush. Crushing first occurred at $3 P_{d}$ in the outer layer of elements and, at $4 P_{d}$, where the computation stopped owing to numerical instability, concrete crushing had spread along the wall thickness at the bottom. It must be noted that the reference crushing stress attains values of 6.7 to $8.9 \mathrm{ksi}$ (47 to 62 $\mathrm{MPa}$ ), because of the effect of confinement due to the two other principal stresses.

Hand calculations have been performed to check the previous results, using well known formulas for an internally loaded cylinder restrained at the base. The following formulas has been derived to calculate the internal pressure at which concrete begins to crack:

$p_{c}=\operatorname{sigma}_{c} /\left(A / A_{c}+1 /\left(2 L^{2} W\right)\right)-G / A_{C}$

where $p_{C}$ is the internal pressure at which concrete begins to crack; sigma ${ }_{C}$ is the concrete tensile strength; $A$ is the cylinder internal area; $A_{C}$ is the concrete cylinder wall horizontal section area; $L$ is the cylinder characteristic length; $W$ is the strength modulus of the concrete section; and $G$ is the dead load. The result obtained is: 
$p_{c}=14 \mathrm{psi}(0.1 \mathrm{MPa})$, or $0.31 \mathrm{P}_{\mathrm{d}}$

This pressure value must be compared with the $40 \% \mathrm{P}_{\mathrm{d}}$ value relative to the inner concrete elements cracking. The hand calculated value does not take into account the contributions of the rebars and of the liner.

Region 2: cylinder wall

When the first cracks appear at the wall-base junction, the maximum tensile stresses in the cylinder wall region are hoop stresses and their values range from $0.06 \mathrm{ksi}(0.4 \mathrm{MPa})$ up to $0.1 \mathrm{ksi}(0.7 \mathrm{MPa})$. In this region, concrete cracking begins at $68 \% \mathrm{P}_{d}$ and the cracks planes are vertical. At $70 \% \mathrm{P}_{d}$, all the three concrete elements layers, through the wall thickness are cracked due to hoop stresses. The cracks appear at the same time in the three layers and at this time almost all the cylinder wall is cracked.

At the same value of the internal pressure, $70 \% \mathrm{P}_{d}$, cracks along horizontal planes caused by meridional stresses appear about 60 inches $(160 \mathrm{~cm})$ from the base. Increasing the internal pressure, the horizontal cracking develops along the wall height and is quite widespread at $73 \% \mathrm{P}_{d}$.

Also for this region, hand calculations have been performed to check the results obtained with the ADINA Code. A simple concrete ring has been analyzed to find the internal pressure level at which the hoop stresses reach the tensile strength of the concrete. Using the formula for membrane stress in a cylinder subjected to internal pressure:

$p_{c}=\operatorname{sigma}{ }_{c} A_{r} / R$

where $p_{C}$ is the internal pressure at which concrete begins to crack; sigma is the concrete tensile strength; $A_{r}$ is the concrete ring area (for unit height); and $R$ is the containment internal radius. The result obtained is $p_{c}=30 \mathrm{psi}(0.21 \mathrm{MPa})\left(65 \% \mathrm{P}_{d}\right)$

A similar evaluation for the horizontal cracks leads to the following value of the internal pressure:

$P_{c}=\operatorname{sigma}_{C} / A / A_{C}-G / A_{C}$

where the symbols have the same meaning as above. The result obtained is $P_{c}=64$ psi $(0.45 \mathrm{MPa})\left(139 \% \mathrm{P}_{d}\right)$

Region 3: dome-cylinder wall junction (springline) The concrete cracking begins in all element layers at $73 \% \mathrm{P}_{d}$, with most crack planes that have inclination, with respect to the vertical plane, equal to about 85 degrees.

At the same pressure meridional crack planes appear as well. 
Region 4: dome

Increasing the internal pressure from $73 \% \mathrm{P}_{d}$, the cracking of the concrete spreads quite soon to the dome, and at $75 \% \mathrm{P}_{d}$ almost all the dome elements are cracked in both meridional and hoop directions.

\subsubsection{Rebar Yielding}

The same subdivision of the containment in regions specified in the previous subsection will be adopted to describe the rebar behavior.

Region 1: cylinder wall-basemat junction

a) Meridional rebars: The inner layer of \#4 and \#6 rebars begins to yield at $2.9 \mathrm{P}_{\mathrm{d}}$ (1 truss element at the bottom). When the computed containment failure is reached, at $4 P_{d}$, the strain value of these rebars is $7.2 \%$

With hand calculations, the pressure at which the inner layer of rebars yields has been evaluated and its value is $3.5 \mathrm{P}_{\mathrm{d}}$. At this pressure level, the compression stress in the outer layer of concrete elements is $3.1 \mathrm{ksi}(22 \mathrm{MPa})$.

b) Hoop rebars: In this region, the first two hoop rebars are still elastic at $3.6 \mathrm{P}_{d}$. At ultimate, $4 \mathrm{P}_{d}$, all the hoop rebars have yielded.

c) Diagonal seismic rebars: As already said, diagonal seismic rebars have been modeled with equivalent meridional and hoop rebars. In region 1 , they behave as the analogous ones described above.

d) Shear rebars: The yielding of the shear rebars occurs at $3.2 P_{d}$ and the strain at $4 \mathrm{P}_{\mathrm{d}}$ is $1.9 \%$.

Region 2: cylinder wall

a) Meridional rebars: The outer layer of rebars begins to yield at $2.8 P_{d}$. At $3.2 P_{d}$, almost all the elements are yielded. When the estimated containment failure is reached, at $4 P_{d}$, the strain value of these rebars is about $2 \%$. The inner layer of rebars begins to yield at 2.9 $P_{d}$. At $4 P_{d}$, about $50 \%$ of the elements have been yielded. When the estimated containment failure is reached, at $4 \mathrm{P}_{d}$, the strain value of the inner rebars is about $1 \%$.

b) Hoop rebars: The outer layer of hoop rebars begins to yield at $2.6 P_{d}$. At $2.9 P_{d}$, almost all the elements are yielded. At the computed ultimate pressure the strain is about $6.5 \%$. 
The inner layer of hoop rebars begins to yield at $2.6 \mathrm{P}_{\mathrm{g}}$. At $2.8 \mathrm{P}_{\mathrm{d}}$, almost all the elements are yielded. At the estimated ditimate pressure, the computed strain is about $7 \%$.

Also these results have been checked performing hand calculations. The formulas for membrane stress have been used, considering the liner too, and the following value for the internal pressure, at yielding of the steel, has been found:

$p=2.9 P_{d}$

c) Diagonal seismic rebars: The equivalent seismic hoop rebars begin to yield at $2.5 \mathrm{P}_{\mathrm{f}}$ and at $2.9 \mathrm{P}_{\mathrm{d}}$ all the elements are yielded. The equivalent merfdional rebars begin to yield at $2.5 \mathrm{P}_{d}$ and at $3.3 \mathrm{P}_{d}$ the yielding is reached in almost all rebars.

\section{Region 3: dome-cylinder wall junction (springline)}

a) Meridional rebars: The outer layer of rebars begins to yield at $3 P_{d}$. At $3.4 \mathrm{P}$, almost all the elements are yielded. When the estimated containment failure is reached, at $4 \mathrm{P}_{\mathrm{d}}$, the strain value of this layer is $1.1 \%$. The inner layer of rebars begins to yield at $2.9 \mathrm{P}_{\text {. At }} 3.4$

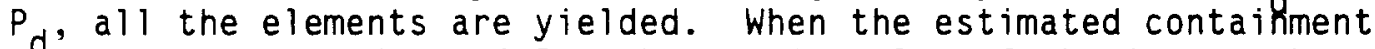
failure is reached, at $4 P_{d}$, the strain value of the inner rebars is $6.3 \%$.

b) Hoop rebars: The outer layer of hoop rebars begins to yield at $3.2 \mathrm{P}_{\mathrm{d}}$. At $3.4 \mathrm{P}_{d}$, all the elements are yielded. At the estimated ultimate pressure, the strain is $1 \%$.

Region 4: dome

a) Meridional rebars: In this region the meridional rebars do not reach yielding.

b) Hoop rebars: The hoop rebars also do not reach yielding, except for the first 10 elements near the springline, as said before.

\subsubsection{Liner}

The yielding of the equivalent rebars, used to represent the liner in the F.E. model, begins when the internal pressure reaches the value of $2 P_{d}$, both at the wall base and midheight. At ultimate $\left(4 P_{f}\right)$, the computed maximum strain is $11 \%$ at the base, $9.2 \%$ at the springline and $7 \%$ in the cylinder wall.

\subsubsection{Displacements}

In Figure 4.6.6, the point at which concrete cracks is clearly detectable. The structure softens and a sharp increase of the radial displacements occurs $\left(d=0.08\right.$ inches $(2.0 \mathrm{~mm})$ at $\left.75 \% \mathrm{P}_{\mathrm{g}}\right)$. After this point, the displacement increases and the curve slope is different from that of the initial segment, related to homogeneous resistant sections with uncracked concrete. Extending this section of the curve it nearly meets the axis origin. 
At another significant point of the curve, about $300 \% \mathrm{P}_{\text {, the }}$ beginning of the containment strength loss is shown: the displacemeht increases rapidly with relatively small increases of the internal pressure. The development of a general yield state in the cylinder wall is evident. The maximum radial displacement occurs at midheight of the cylinder and its value is nearly 10 inches $(25.4 \mathrm{~cm})$ at $4 \mathrm{P}$. The springline displacement (Figure 4.6.7) shows a stiffer behavior, along the radial direction, whereas the vertical displacement is greater than the radial one.

Looking at the vertical displacement of the dome apex (Figure 4.6.8) the same significant points of the previous chart are evident, e.g., concrete cracking and the beginning of yielding, which occur at approximately the same pressure levels. Figure 4.6 .8 shows vertical displacement values similar to those obtained at the spriigline. After the start of concrete cracking, the springline vertical displacement is slightly greater than that at the apex at the same load. The value computed at 4 P is 2.2 inches (5.6 $\mathrm{cm}$ ) at the springline and 2.0 inches $(5.1 \mathrm{~cm})$ at the dome apex.

\subsubsection{Pressure History Plots}

Addition plots of displacament and strains as requested by Sandia are given in Appendix A.

\subsubsection{Conclusions}

The stop of computation by the ADINA code occurred when extensive crushing of the concrete at the basemat-wall junction took place due to the combined effects of meridional tension and bending moment at the clamped wall bottom. At this time the internal pressure has reached the value of 184 psi $(1.27$ $\mathrm{MPa}$ ), four times the design pressure load. That mode of failure could be deemed quite reasonable, considering moreover that the bending effect would have increased if the foundation and the soil-structure interaction had been taken into account in the present analysis.

Looking at the rest of the structure, it appears that a large strain of the hoop rebars in the cylinder wall is required, about $7 \%$, in order to carry such pressure load. Considering that the hardening modulus of the rebars has been taken as a secant modulus, the effective deformation of the hoop rebars might be less than $7 \%$ at the limit state evaluated. On the other hand, the ADINA Code takes into account the triaxial stress state of the concrete elements to decide if crushing occurs. Doing so, the confinement effect of the base restraint allows a large increase of the ultimate compression stress of the concrete, which in this specific case reaches the value of $8.9 \mathrm{ksi}(62 \mathrm{MPa})$. In our opinion, it is more reasonable to rely on the large strain capability of the steel rebars instead of such increases in the concrete strength. Therefore the containment failure mode should be the rupture of the cylinder wall base due to the combined effect of meridional tension and bending moment, at a pressure value lying in the range $3.5-4 \mathrm{P}_{d}$.

This assessment is an upper bound of the containment strength, whose occurrence assumes that penetrations and the wall region around them (not considered in this analysis), maintain their structural integrity up to the ultimate pressure load. 
SANDIA CONTAI MMENT MODEL

FRFGGIIRF LEVEL $=n$ ESh

$Y$ IIF, MAX, DISF, =

$Z$ DIR. MAX. DISF, =

SCALE FACTOR=
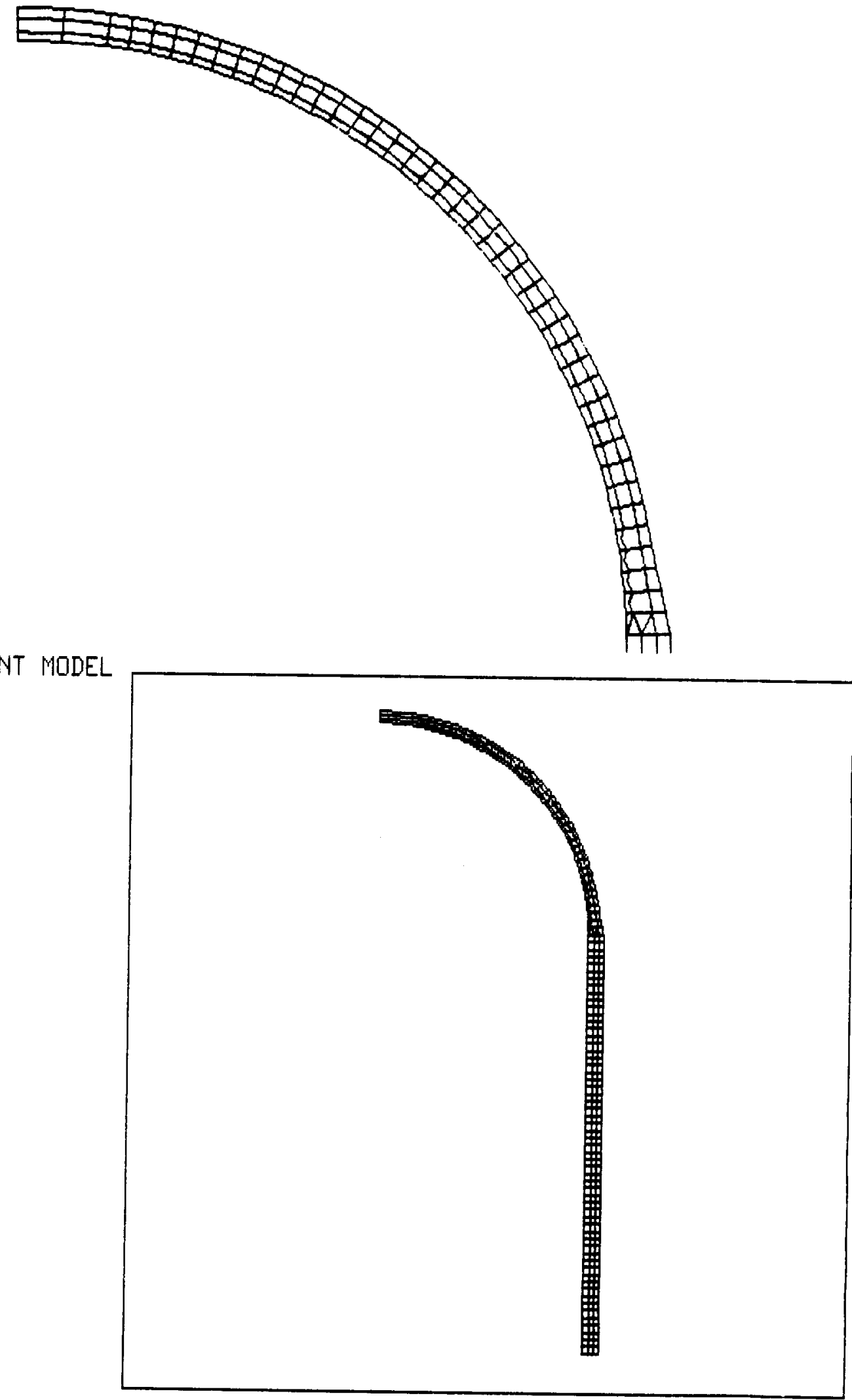

Figure 4.6.1 Concrete elements mesh 


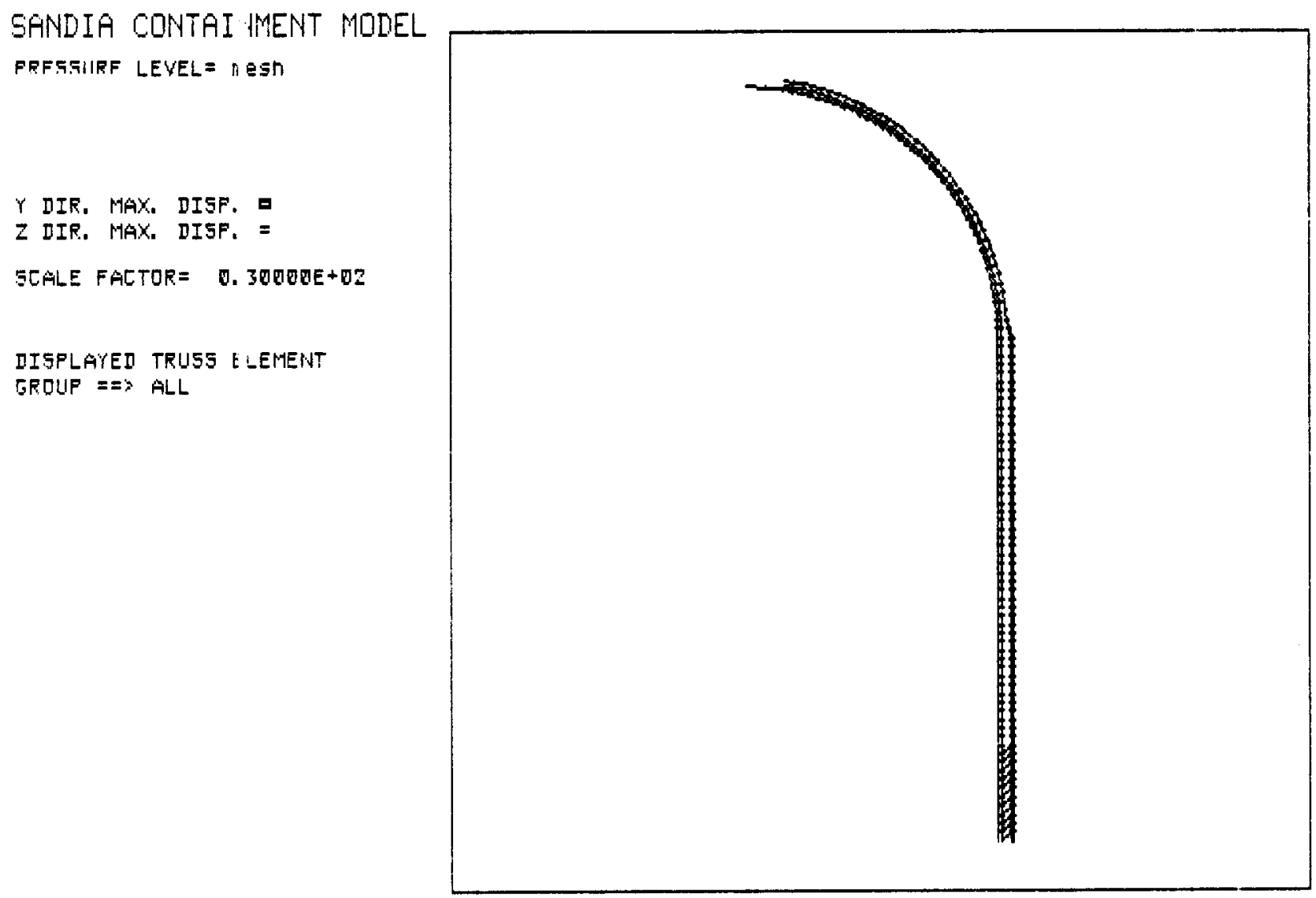

Figure 4.6.2 Truss elements mesh (rebars and liner) 


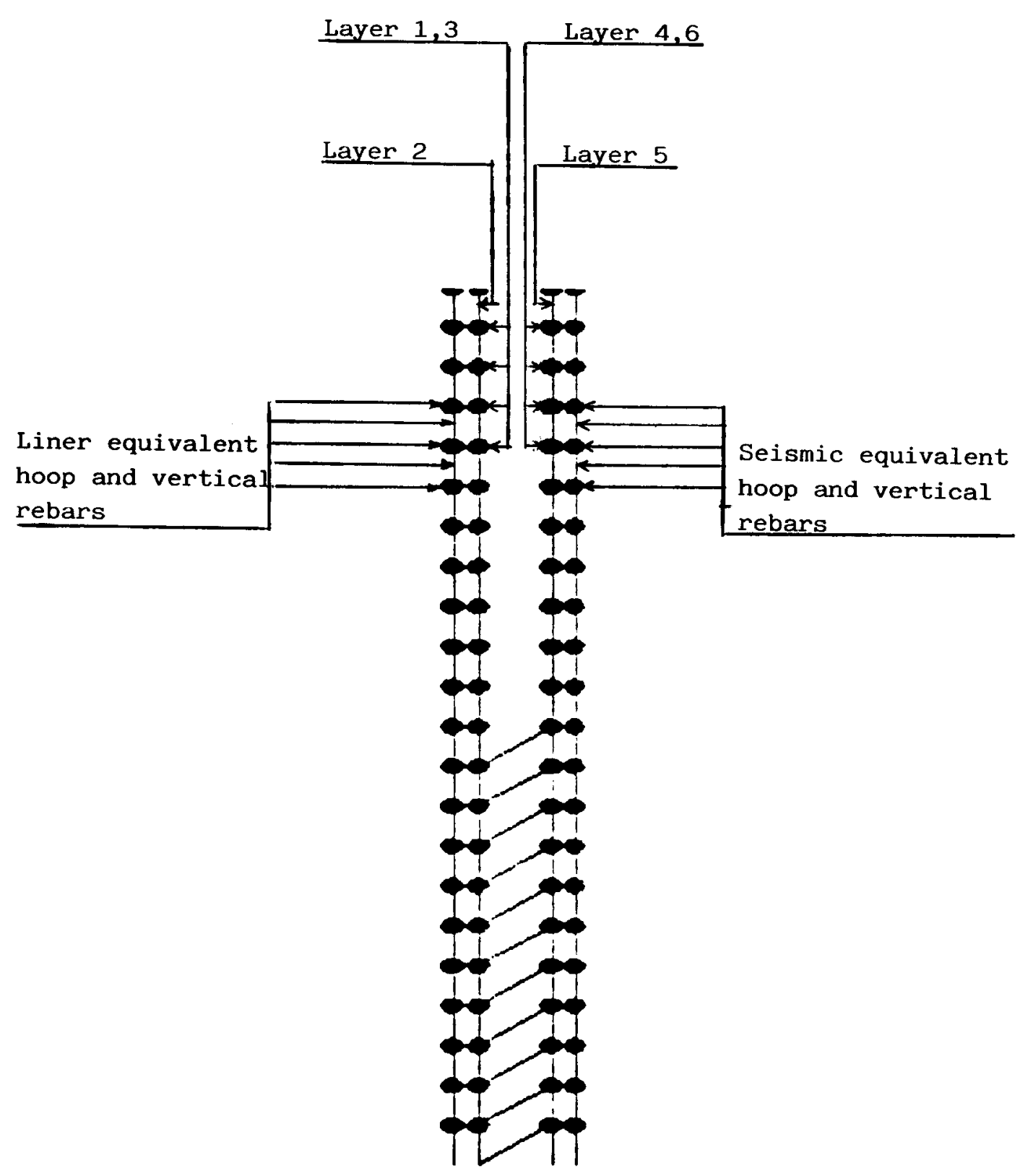

Figure 4.6.3 Detail of truss elements at the cylinder wall base 


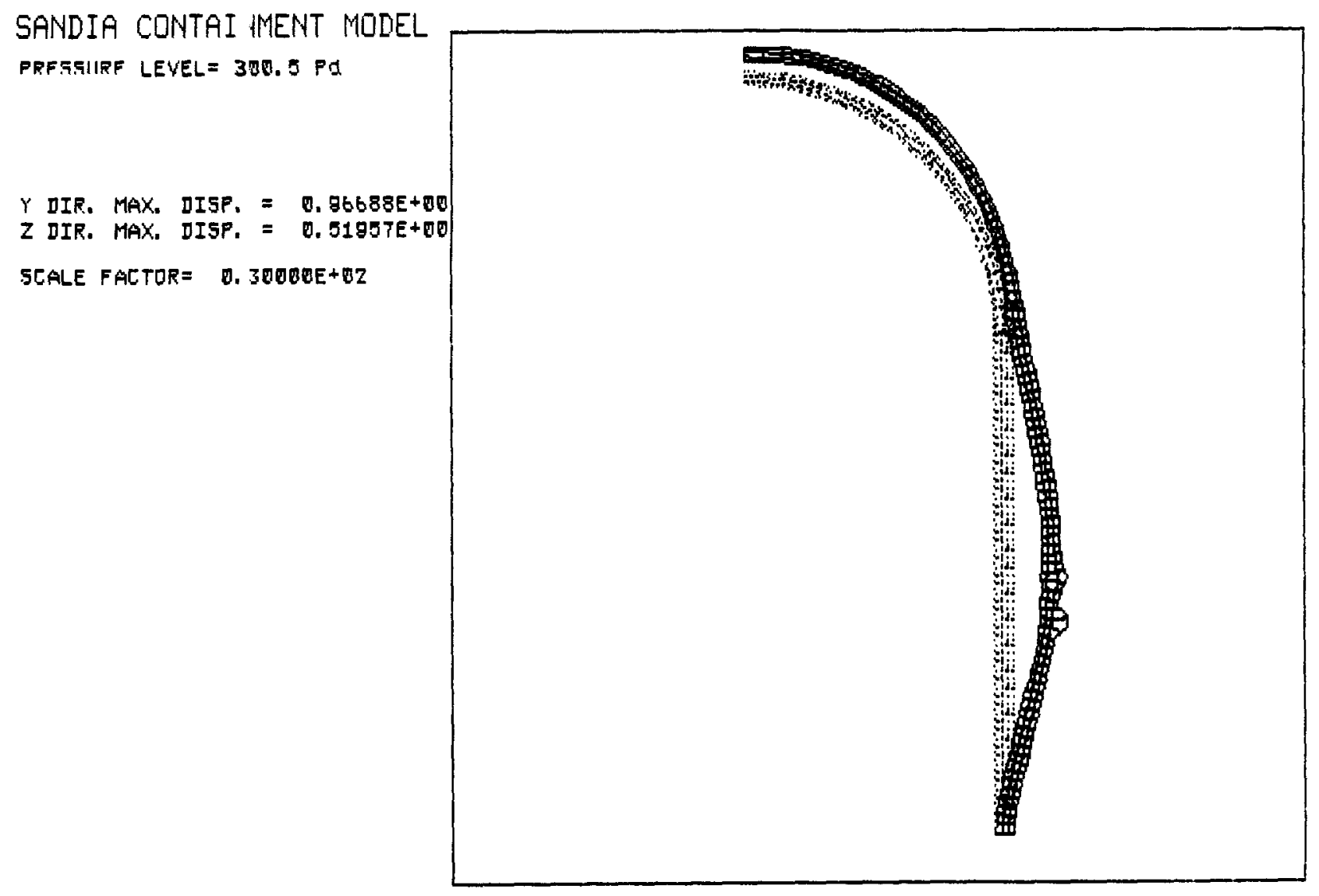

Figure 4.6.4 Displacements at $p=3 P_{d}$

$-286-$ 
SANDIA CONTAI MENT MODEL PFFGSIIRF LEVEL $=400.5 \mathrm{Pd}$

$Y$ DIR. MAX, DISF, - $0.10038 E+02$

$Z$ DIR. MAX. DISP. $=0.25822 E+01$

SLALE FACTOR= $0.30000 E+01$

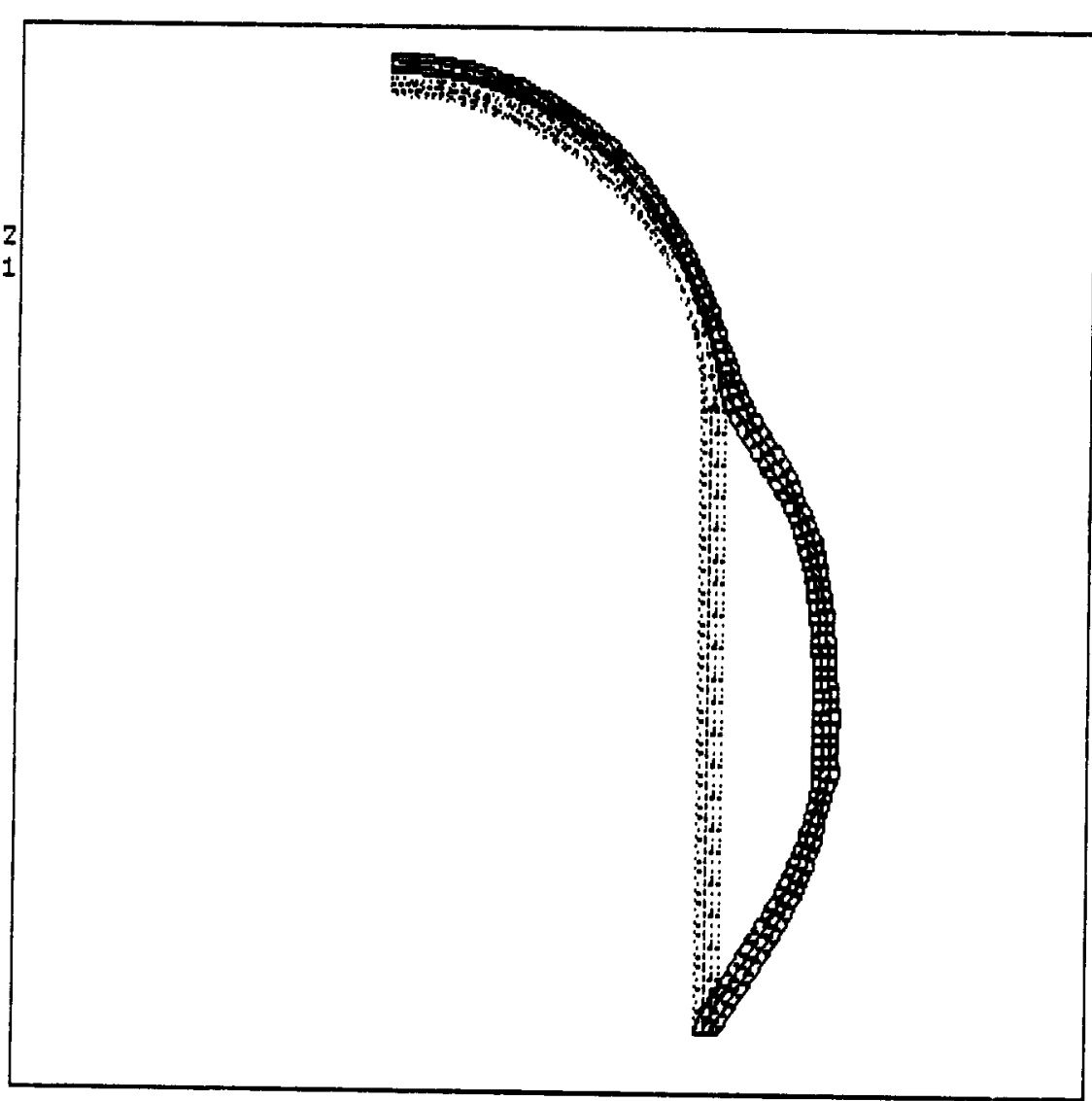

Figure 4.6.5 Displacement at $p=4 P_{d}$ 


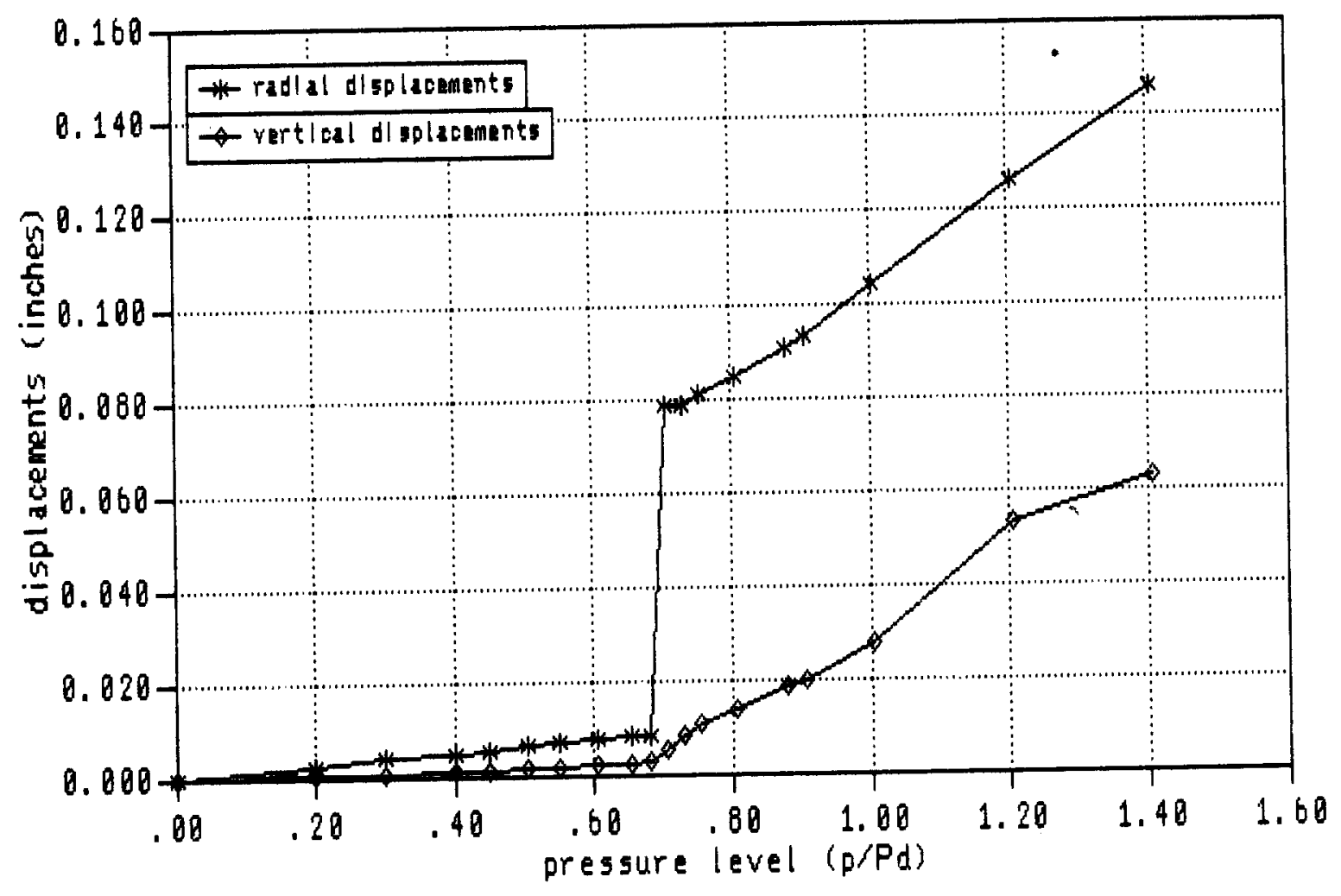

Figure 4.6.6a Cylinder displacements at EL 11'-0" (detail)

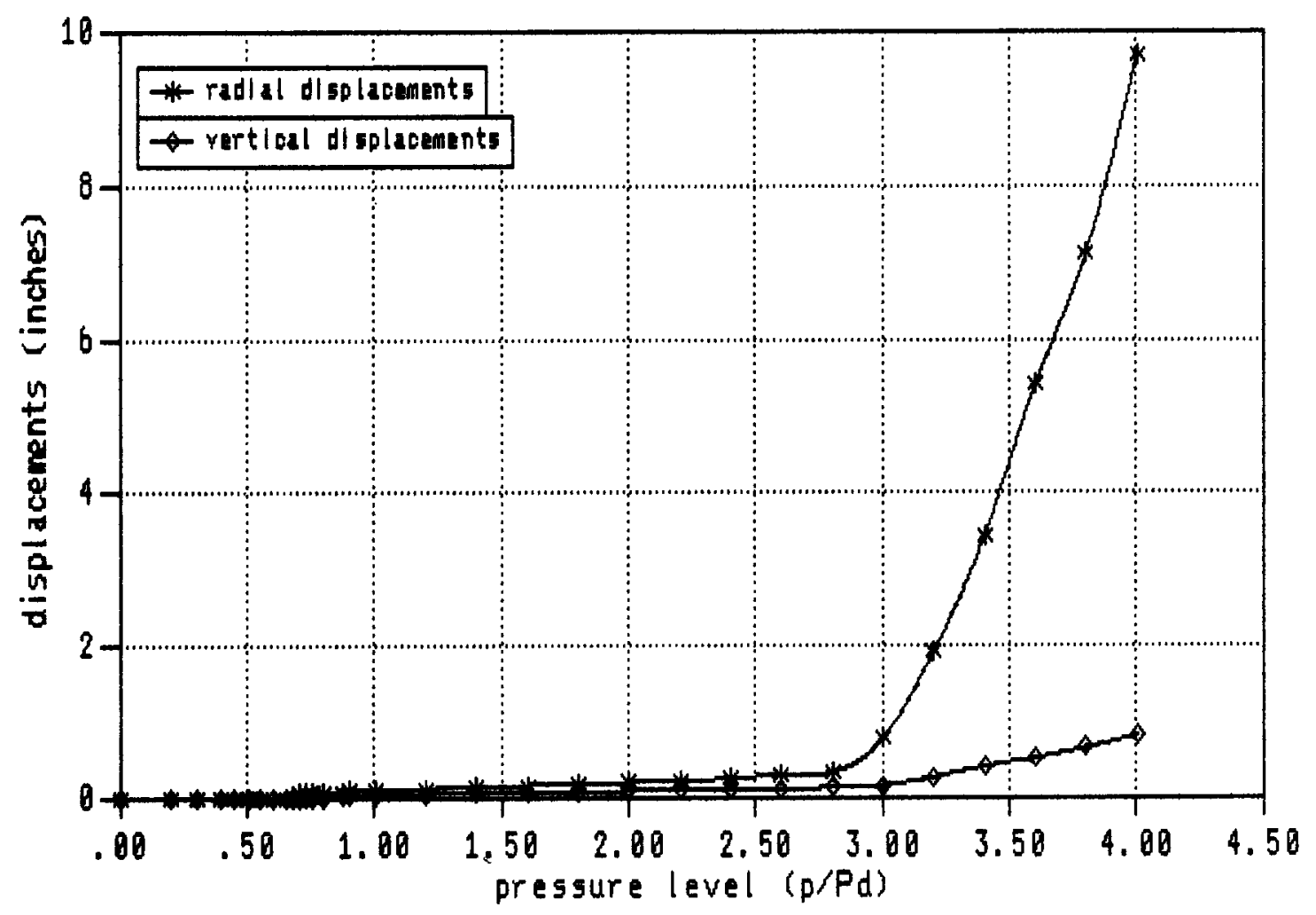

Figure 4.6.6b Cylinder displacements at EL 11'-0" 


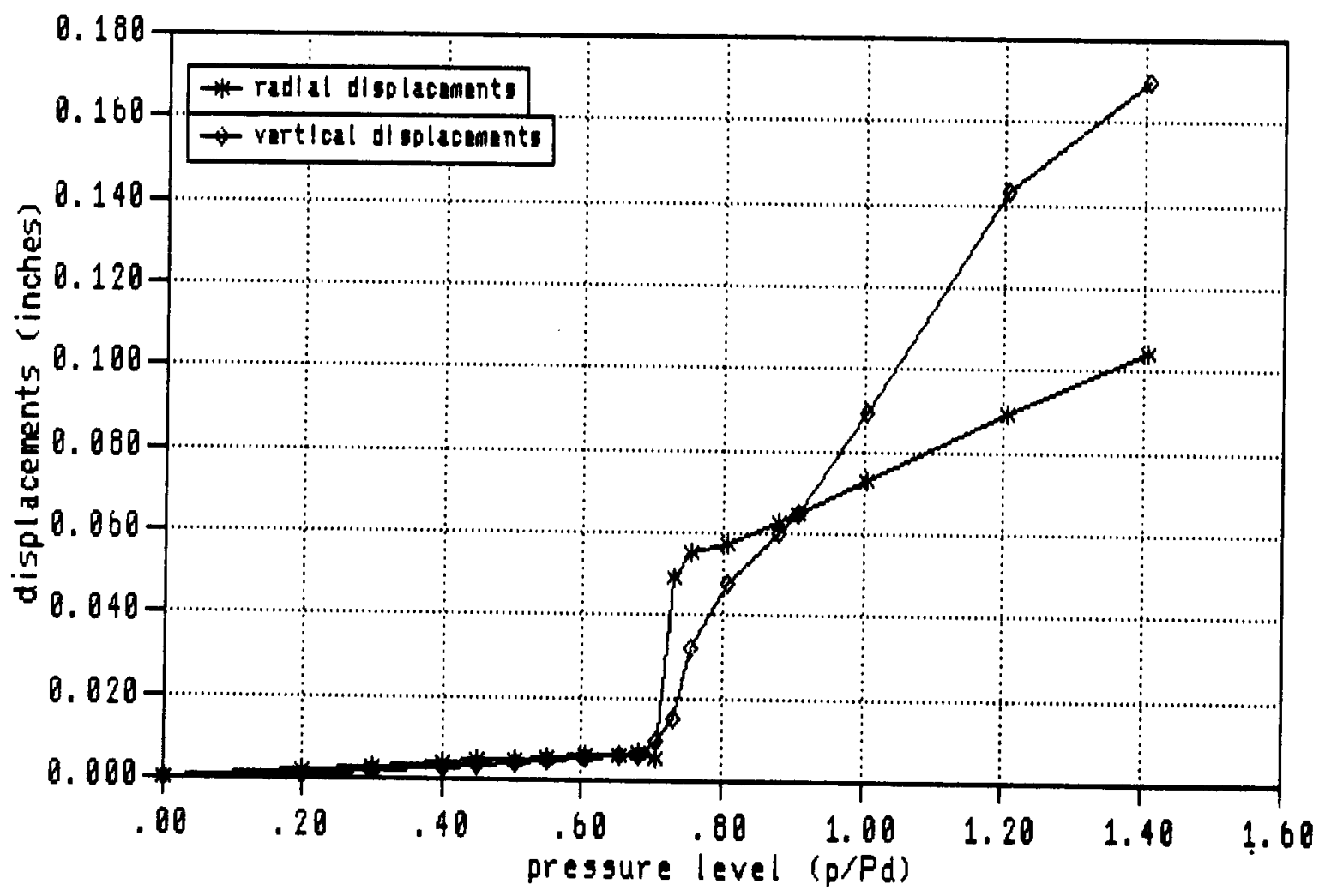

Figure 4.6.7a Cylinder displacement at EL 24'-0" (detail)

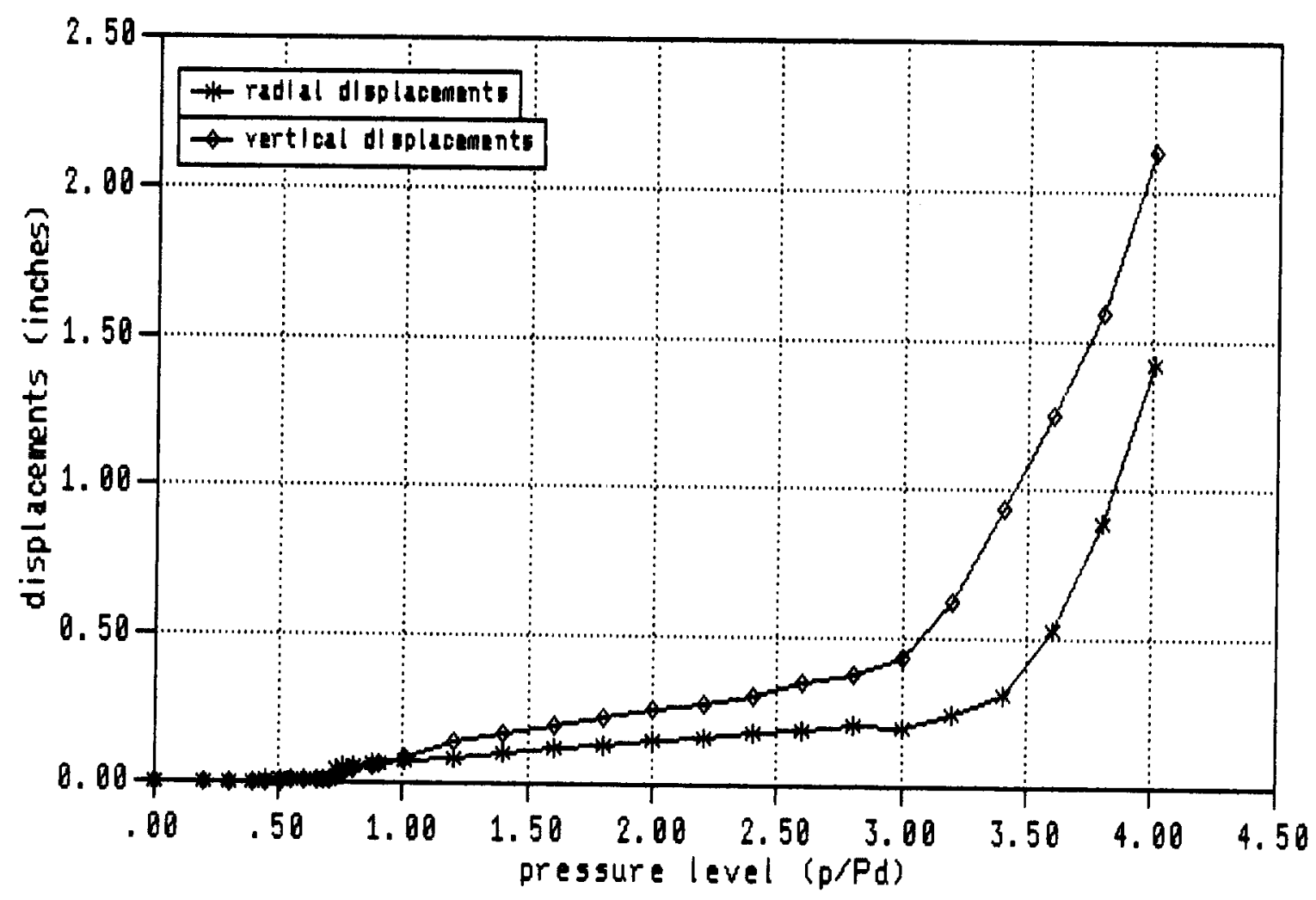

Figure 4.6.7b Cylinder displacement at EL 24'-0" 


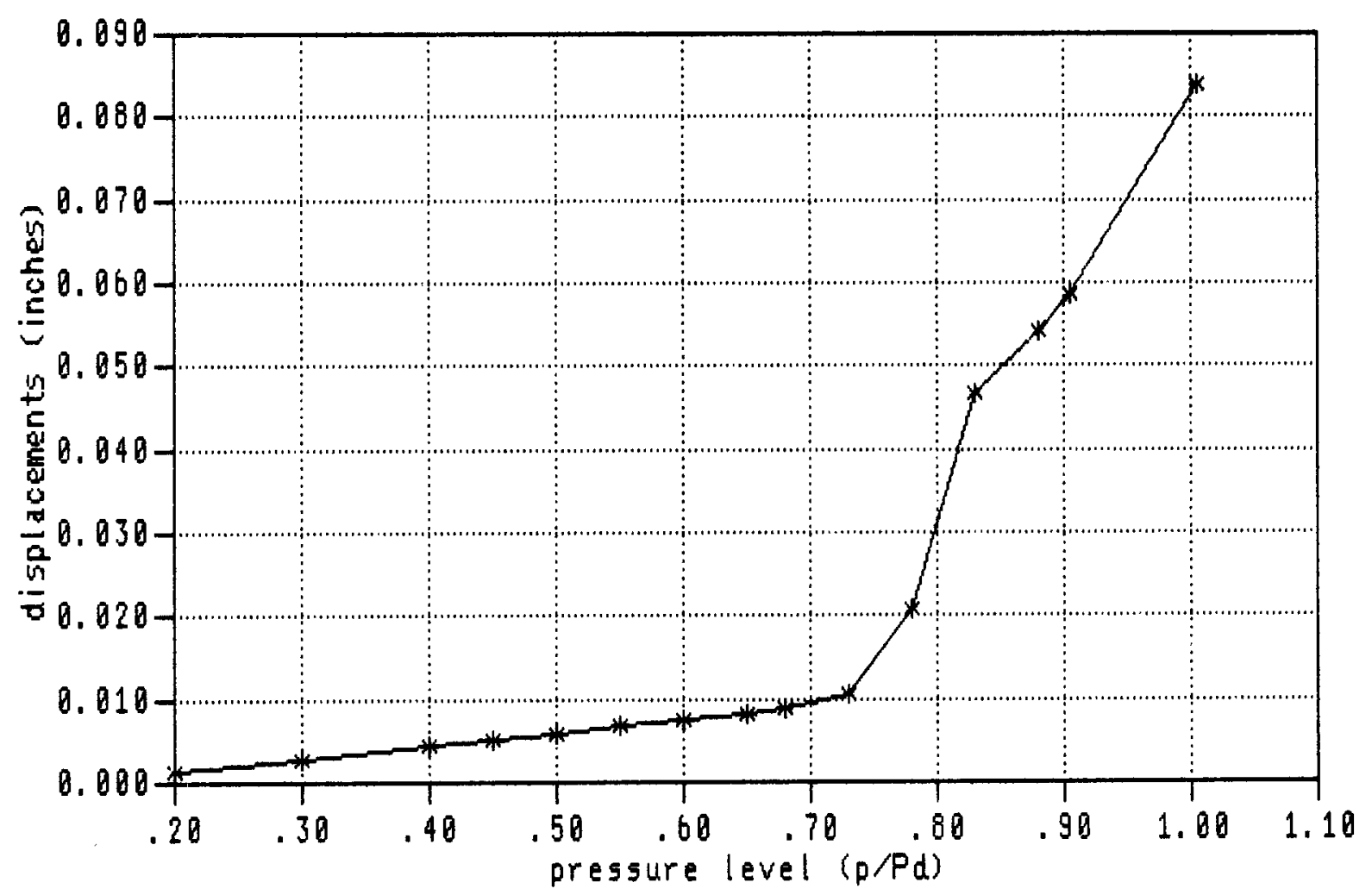

Figure 4.6.8a Dome displacement at apex (detail)

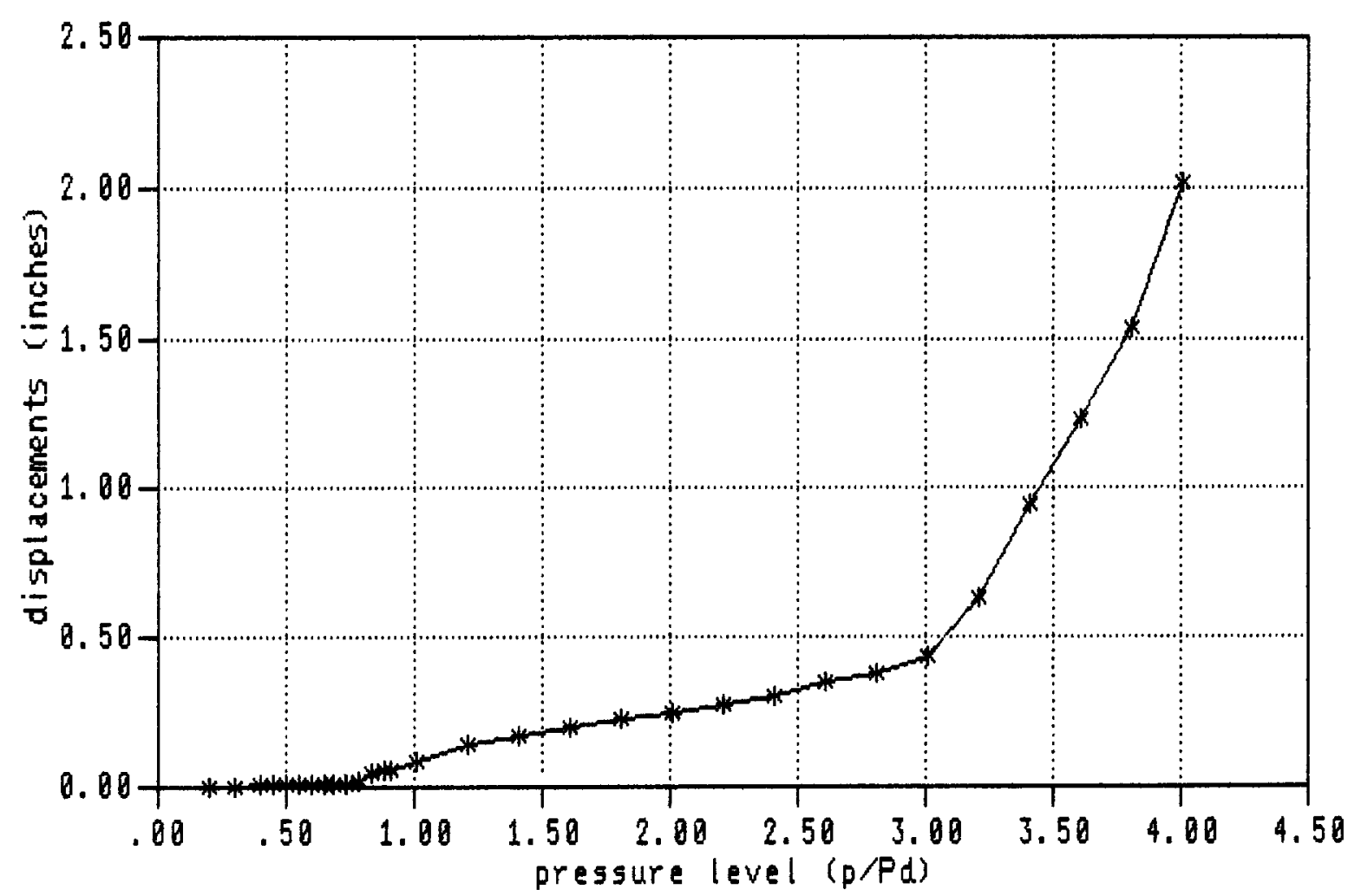

Figure 4.6.8b Dome displacement at dome apex 


\subsection{Safety and Reliability Directorate}

This section was authored by M. H. Bleackley, J. A. Hargreaves, L. P. Harrop, D. W. Phillips, J. Jowett \& P. Barr from the Safety and Reliability Directorate of the U.K. Atomic Energy Authority.

\subsubsection{Surmary}

Finite element axisymmetric studies have been undertaken pertaining to the $1 / 6$ th scale reinforced concrete containment building. Results from these studies will be compared to other similar studies in different organizations to predict the response to internal static overpressurization.

The main predictions are that the hoop rebar will yield at 138 psi 10.95 $\mathrm{MPa}$ ) and that the periphery of the basemat will be subject to excessive uplift at $164 \mathrm{psi}(1.13 \mathrm{MPa})$ when the tensile rebar at the pole of the basemat reaches its ultimate tensile strength (UTS).

\section{Synopsis of Results}

Organization:

Analytical Method:

Constitutive Model:

Model Geometry:

Failure Criterion:

Predicted Failure Mode: Tensile failure of the rebar due to bending at the pole of the basemat.

\subsubsection{Introduction}

This paper discusses a non-linear finite element axisymetric analysis of a $1 / 6$ th scale model reinforced concrete PWR containment to predict the performance of the containment when the design pressure is exceeded.

The actual concrete containment is being constructed at Sandia National Laboratories, New Mexico for the USNRC. It is intended that the containment will be tested to failure to determine its response to internal static overpressurization. As a precursor to the experimental test, a number of organizations are participating in an international effort to predict the outcome of the test by analytical methods. The results from the heavily instrumented test will then be compared with the analytical predictions. This paper represents one contribution based on finite element calculations using the non-linear finite element code ABAQUS mounted on the UKAEA CRAYXMP $/ 24$. 
Three distinct numerical axisymetric analyses have been undertaken: firstly a typical section of containment wall was modelled using a ring-beam representation, secondly a full shell analysis was performed and finally a mixed shell/continuum model was undertaken with the dome and the upper part of the cylinder wall modelled by shells and the remaining lower parts of the containment modelled by continuum elements. Only a limited analys is was undertaken on the latter model. These three analyses provided a natural progression along a learning curve from a well defined ring-beam model with simple boundary conditions to a fully fledged geometry with a complicated response.

\subsubsection{Numerical Model}

The finite element code ABAQUS 4-5-171 [4.7.1] was used in this analysis. This code was chosen because of its non-linear capabilities (small strain/displacement theory) and constitutive concrete model. This version of ABAQUS incorporates a modified version of the Chen-chen concrete constitutive model.

The Chen-Chen model treats concrete as an elastic-plastic strain hardening material which fractures in tension. Elastic response is assumed whenever a stress point lies within a yield surface and an elastic-plastic behavior whenever the yield surface is reached. Also defined is a failure surface beyond which stress states cannot be reached.

The yield and failure surfaces of the chen-Chen model are both defined by a combination of parabolic and hyperbolic functions. The parabolic function is used to represent the triaxial compression region, and the hyperbolic function the tension/compression region. If the compression failure surface is reached, the concrete is assumed to crush with all strength lost $(\sigma=0)$. If the tension surface is reached, cracking normal to the most tensile principal strain is flagged. To limit the excessive degree of tensile straining before cracking which existed in Chen and Chen's original model, Hibbitt et al., [4.7.1] have added a limiting tensile strain criterion for cracking.

Whilst the ABAQUS implementation of the Chen-Chen model provides a good representation of concrete behavior in biaxial or triaxial compression, its performance in the tension region is not so good. For a stress state of equal biaxial tension, the model underpredicts the failure strength by 40 percent. As both the walls and roof of a containment structure experience a biaxial tensile stress field, the calculation is likely to underpredict the pressure at which initial cracking occurs.

For the present application, a more serious shortcoming in the ABAQUS model is its use of the smeared cracking approximation. With this approximation no attempt is made to simulate the formation and development of discrete cracks, rather the displacements associated with crack opening are smeared uniformiy across an element and the stiffness of that element is correspondingly reduced. This approximation greatly simplifies the calculations but for the present application it means that possible local liner failure modes cannot be examined directly using ABAQUS.

When the smeared cracking approximation is employed, some means of accounting for the tension stiffening effect is needed. In the ABAQUS model 
the artifice of giving the concrete a decaying post-tensile failure stress/strain curve is used. In the current calculations a linear decay function which reaches zero at a strain of one percent has been used. Such a treatment also has the benefit of improving code convergence. However it should be noted that in a review conducted by Gilbert [4.7.2], the above method was revealed as being less stable than a method based on the adjustment of the rebar stress/strain curve.

The reinforcing bars within the concrete can be arbitrarily specified because the rebar is not represented by separate elements. The model sees the rebar in an element as a sheet with equivalent cross-sectional area, axial stiffness and orientation as the specified rebar. Numerically, the rebar will exhibit only axial stiffness e.g. no shear stiffness. The rectangular reinforcement met at the base of the model has been replicated as specified by ABAQUS input procedures, but it is not clear how this reinforcement is treated at the pole of the basemat within ABAQUS.

The weight of the containment has been modelled by assuming the density of concrete with no rebar.

The containment building is not truly axisymmetric by virtue of penetrations such as personnel airlocks and equipment hatches. Although it is an obvious over-simplification, we chose to model the containment as an axisymmetric structure because of its near symmetry and the pressure loading is naturally axisymmetric. Such a model is practicable given the size requirements for running a non-linear analysis.

Two types of element are used in this analysis: the eight-noded axisymmetric quadrilateral (CAX8R) and the three-noded quadrilateral shell element (SAX2). The former are integrated by $2 \times 2$ Gauss integration and the latter by two integration stations along the shell length and five through each layer of shell.

\subsubsection{Material Properties}

All the material properties used in this analysis are those supplied by SNL and are given in Figure 4.7.1 and Table 4.7.1: the engineering uniaxial stress/plastic strain curves for the \#4 rebar, concrete, basemat/ cylinder and dome liner. Various curves were supplied by SNL for each type of uniaxial test from data available in March 1986. A representative uniaxial stress/plastic strain relationship was chosen for each material by the authors. Subsequently, recommended material properties for each tested type of material were supplied by SNL [4.7.3] in October 1986 but only those pertaining to the dome were used by the authors. The steel used in the rebar and the basemat/cylinder wall liner exhibited a distinct yield plateau with no significant work-hardening in evidence until a plastic strain of 0.5 percent to 1.5 percent. The stress values of the uniaxial stress/strain curve for the \#4 are based on a nominal diameter of $0.5^{\prime \prime}(0.1963 \mathrm{sq}$. in., $126.6 \mathrm{sq}$. $\mathrm{mm}$ cross-sectional area). The material properties of the \#4 rebar are also taken as representative of the \#2, \#3, \#5 and \#6 rebar since the \#4 rebar is by far the most common rebar in the containment.

The concrete compressive stress/strain data are based on a 90 day test. The slope of the curve is taken as linear up to $5.9 \mathrm{ksi}$ (40.48 MPa) and using a 
Table 4.7.1: List of Material Properties

Steel

\begin{tabular}{|c|c|c|c|c|c|c|c|c|}
\hline & \multicolumn{2}{|c|}{ Rebar } & \multicolumn{3}{|c|}{$\begin{array}{l}\text { Basemat/Wall } \\
\text { Liner }\end{array}$} & \multicolumn{3}{|c|}{ Dome Liner } \\
\hline \multicolumn{9}{|c|}{$E=30000 \mathrm{ksi}$} \\
\hline ksi & $\begin{array}{c}\bar{\sigma} \\
\mathrm{MPa}\end{array}$ & $\begin{array}{l}\bar{\epsilon} \rho \\
\%\end{array}$ & ksi & $\begin{array}{c}\bar{\sigma} \\
\mathrm{MPa}\end{array}$ & $\begin{array}{l}\bar{\epsilon} \rho \\
\%\end{array}$ & ksi & $\begin{array}{c}\bar{\sigma} \\
\mathrm{MPa}\end{array}$ & $\begin{array}{l}\bar{\epsilon} \rho \\
\%\end{array}$ \\
\hline $\begin{array}{r}68.1 \\
68.1 \\
82.4 \\
93.7 \\
100.4 \\
105.0 \\
107.8 \\
108.3 \\
108.7\end{array}$ & $\begin{array}{l}469.87 \\
469.87 \\
567.95 \\
645.75 \\
692.43 \\
723.75 \\
743.00 \\
746.9 \\
749.31\end{array}$ & $\begin{array}{l}0.0 \\
0.5168 \\
1.4942 \\
2.661 \\
3.8556 \\
5.0544 \\
6.2601 \\
7.4748 \\
8.6904\end{array}$ & $\begin{array}{l}49.4 \\
49.4 \\
55.3 \\
62.9 \\
65.6 \\
66.9 \\
68.2 \\
68.5 \\
69.4\end{array}$ & $\begin{array}{l}340.72 \\
340.72 \\
401.82 \\
433.79 \\
452.05 \\
461.18 \\
470.31 \\
472.60 \\
478.48\end{array}$ & $\begin{array}{l}0.0 \\
1.5080 \\
3.1519 \\
4.8094 \\
6.4736 \\
8.1422 \\
9.98108 \\
11.4827 \\
16.1643\end{array}$ & $\begin{array}{l}51.3 \\
61.1 \\
66.9 \\
70.5 \\
70.9\end{array}$ & $\begin{array}{l}354.0 \\
421.0 \\
461.0 \\
486.0 \\
489.0\end{array}$ & $\begin{array}{l}0.0 \\
2.300 \\
4.78 \\
9.77 \\
14.76\end{array}$ \\
\hline
\end{tabular}

Concrete

$\begin{array}{clc}\sigma & \epsilon \rho & \\ M P a & \% & \\ 40.48 & 0.0 & \\ 40.79 & 0.00342 & E=3600 \mathrm{ksi} \quad(24800 \mathrm{MPa}) \\ 41.08 & 0.00655 & \nu=0.15 \\ 41.61 & 0.01980 & \rho g=23.544 \mathrm{kN} / \mathrm{m}^{3}\end{array}$

Ratio of the stress components under biaxial compression $\left(\sigma_{1}=\sigma_{2}\right)$ at failure to the failure stress in uniaxial compression $=1.16$ (ABAQUS default).

Ratio of the plastic strain components under biaxial compression at failure to the plastic strain at failure in uniaxial compression $=1.28$ (ABAQUS default).

Ratio of the uniaxial tension failure stress to the uniaxial compressive failure stress $=0.06$.

Ratio of the uniaxial tensile failure plastic strain to the uniaxial compressive failure plastic strain $=0.09$ (ABAQUS default). 
Young's modulus of $3600 \mathrm{ksi}(24800 \mathrm{MPa})$, which is $1 / 3$ less than recommended now [4.7.3]. A value of $363 \mathrm{psi}(2.5 \mathrm{MPa})$ is obtained from a SNL direct tension test at 120 days. This is compared to $500 \mathrm{psi}(3.45 \mathrm{MPa}$ ) recommended by [4.7.3], which originates from a split tension test. The former value is more appropriate for the input of ABAQUS [4.7.1]. The uniaxial stress/strain curve in tension is assumed to be linear up to the failure tensile strength. It is also assumed to loose its tensile strength linearly from $363 \mathrm{psi}(2.5 \mathrm{MPa})$ until one percent strain is reached when the concrete is specified to have zero strength.

\subsubsection{Finite Element Models}

\subsubsection{Ring-beam Model}

The mesh and rebar positioning for the ring-beam, which is representative of the containment wall above the shear reinforcement level (>3.6 ft (1100 mm) above the top of the basemat), are shown in Figure 4.7.2. The mesh representation is of a square cross-section. It consists of six elements: two shell elements representing the liner and four continuum elements the concrete. The section is rigidly fixed in the meridional position but is free to move radially. This representation approximates to a cross-section at mid-cylinder height above the shear reinforcement. This model incorporates the eight layers of primary reinforcement: four layers of circumferential (12 rebars), two layers of meridional and two of diagonal.

The ring is loaded by static load increments of uniform pressure along the liner face at the nodes.

\subsubsection{Shell Model}

An axisymmetric shell model provides a crude but economical representation of the entire containment.

Forty-six three-noded axisymetric shell elements (11 representing the basemat, 20 the wall and 15 the dome) are used with a single element representing the containment wall thickness. Each element is in turn divided into two layers so as to represent the reinforced concrete and steel liner.

The model is fixed kinematically at two nodes: in the radial direction at the apex and both radially and vertically at the centre of the basemat.

The inside of the containment is loaded by uniform pressure, applied in increments as a static load.

This model may lack refinement (e.g. these shell elements have a pseudo thickness) but it provides the global response at minimal cost.

Most of the major reinforcement has been modelled with the exception of the shear reinforcement in the wall and the vertical rebar in the basemat.

\subsubsection{She11/Continuum Mode 1}

The next level of complexity in this finite element analysis is an axisymmetric model incorporating solid elements. The main additional 
capability of this model is to represent transverse shear, especially at the cylinder wall/basemat junction.

In the interests of economy, as shown in Figure 4.7.3, the containment above the shear reinforcement level (>4.92 ft $(1.5 \mathrm{~m}$ ) above the basemat) is made up of 30 shell elements from the previous model. Below this level the containment is modelled with three hundred and fifty 2-D axisymmetric eightnoded elements and the $1 / 16 "(1.6 \mathrm{~mm})$ liner by fifty-four three-noded shelis (SAX2). The concrete wall thickness consists of two eight-noded elements and the entire wall has $2 \times 32=642-0$ elements. The basemat is eleven elements thick and consists of $11 \times 26=286$ eight-noded elements. In total, the model possesses 434 elements 1250 nodes.

A detail of the mesh and rebar for the continuum section of the model is shown in Figure 4.7.4. The \#2 and \#3 shear ties in the wall are clearly visible as is the inclined \#4 rebar.

\section{7 .6 Results}

\subsubsection{Results for the Ring-beam Model}

The ring-beam is loaded by uniform pressure loading using ABAQUS's automatic incremental loader.

Figure 4.7.5 shows the pressure/displacement curve for this ring-beam model. The curve has four distinct liner siopes.

The first slope is linear up to $0.85 x$ design pressure (39 psi, $0.27 \mathrm{MPa}$ ); at this point the concrete cracks on a plane normal to the hoop rebar. The model now loses stiffness to account for the concrete cracking. This modified stiffness response now continues up to $3.15 x$ design pressure (145 psi, $100 \mathrm{MPa}$ ), at which point the liner yields plastically. The liner yielding reduces the stiffness response of the beam further, and the curve follows a new linear slope to 3.88 x design pressure (178 psi, $1.23 \mathrm{MPa})$. The hoop rebar yields plastically at this pressure. The response is now essentially horizontal with no increase in load, as the 12 hoop rebars in the model yield virtually simultaneously leaving only the seismic rebar with any load carrying capacity and able to sustain only one-half of the stress of the hoop rebar. The seismic rebar is in the plane of maximum shear ( 45 degrees to the hoop and meridional direction) and as the meridional stress is approximately zero in this model, it is only to be expected the seismic rebar sustains this stress.

The hoop rebar is unable to exhibit a further increase in load owing to the shape of the uniaxial stress/strain curve. To obtain maximum load, 15 load increments were required, the short horizontal region required an equal number of increments. The little progress of the latter load steps is due to the horizontal (Luder's) portion of the uniaxial stress-strain curve. The Luders band of the \#4 rebar extends to 0.5 percent strain which is equal to a hoop strain of $\mathrm{dr} / \mathrm{r}$, and hence is equivalent to a deformation of 0.66 inches $(16.75 \mathrm{~mm})$.

The response of the ring-beam model is summarized in Table 4.7.2. 


\section{Table 4.7.2 Prediction for the Response of the Containment Model}

\begin{tabular}{|c|c|c|c|c|}
\hline Shel1 & $\begin{array}{l}\text { umerical } \\
\text { Shell/ } \\
2-D\end{array}$ & $\begin{array}{l}\text { Ring- } \\
\text { beam }\end{array}$ & $\begin{array}{l}\text { Analytical } \\
\quad[4.7 .5]\end{array}$ & Comments \\
\hline \multicolumn{5}{|c|}{ Internal Pressure psi (MPa) } \\
\hline $\begin{array}{c}28 \\
(0.19)\end{array}$ & $\begin{array}{c}28 \\
(0.19)\end{array}$ & $\begin{array}{c}39 \\
(0.27)\end{array}$ & $\begin{array}{c}30 \\
(0.21)\end{array}$ & $\begin{array}{l}\text { Meridional cracks through concrete in } \\
\text { undisturbed regions of the cylinder. }\end{array}$ \\
\hline $\begin{array}{c}32 \\
(0.22)\end{array}$ & $\begin{array}{c}41 \\
(0.28)\end{array}$ & - & $\begin{array}{c}38 \\
(0.26)\end{array}$ & $\begin{array}{l}\text { Meridional cracks through concrete in } \\
\text { dome. }\end{array}$ \\
\hline $\begin{array}{c}32 \\
(0.22)\end{array}$ & & - & $(0.33)$ & $\begin{array}{l}\text { Circumferential cracks in cylinder just } \\
\text { below the spring line. }\end{array}$ \\
\hline $\begin{array}{c}33 \\
(0.23)\end{array}$ & & - & & Circumferential cracking in dome. \\
\hline$(0.41)$ & & - & & $\begin{array}{l}\text { Dome entirely cracked in orthogonal } \\
\text { planes. }\end{array}$ \\
\hline $\begin{array}{c}71 \\
(0.49)\end{array}$ & & - & $(0.43)$ & $\begin{array}{l}\text { Horizontal cracks through concrete in } \\
\text { undisturbed regions of the cylinder. }\end{array}$ \\
\hline $\begin{array}{l}110 \\
(0.76)\end{array}$ & & $\begin{array}{l}145 \\
(1.00)\end{array}$ & $\begin{array}{c}86 \\
(0.59)\end{array}$ & $\begin{array}{l}\text { Liner on cylinder wall yields in } \\
\text { undisturbed regions of cylinder. }\end{array}$ \\
\hline$(0.91)$ & & - & & $\begin{array}{l}\text { Hoop tension rebar yields at the pole of } \\
\text { the basemat. }\end{array}$ \\
\hline $\begin{array}{l}138 \\
(0.95)\end{array}$ & & - & & $\begin{array}{l}\text { Concrete crushed at basemat/cylinder wall } \\
\text { intersection. }\end{array}$ \\
\hline $\begin{array}{l}138 \\
(0.95)\end{array}$ & & $\begin{array}{l}178 \\
(1.23)\end{array}$ & $\begin{array}{l}129 \\
(0.89)\end{array}$ & $\begin{array}{l}\text { Hoop rebar yields in cylinder away from } \\
\text { discontinuities. }\end{array}$ \\
\hline $\begin{array}{l}145 \\
(1.00)\end{array}$ & & - & & $\begin{array}{l}\text { Longitudinal tensile rebars yields at the } \\
\text { pole of the basemat. }\end{array}$ \\
\hline $\begin{array}{l}155 \\
(1.07\end{array}$ & & - & & $\begin{array}{l}\text { Hoop compression rebar yields at the pole } \\
\text { of the basemat. }\end{array}$ \\
\hline \multirow[t]{4}{*}{$\begin{array}{l}164 \\
(1.13)\end{array}$} & & - & & $\begin{array}{l}\text { All tensile rebar at pole of basemat } \\
\text { reaches UTS. }\end{array}$ \\
\hline & & - & $\begin{array}{l}167 \\
(1.15)\end{array}$ & Hoop rebar in dome yields. \\
\hline & & - & $\begin{array}{l}174 \\
(1.20)\end{array}$ & $\begin{array}{l}\text { Axial rebar yields in undisturbed regions } \\
\text { of the cylinder. }\end{array}$ \\
\hline & & & $\begin{array}{l}229 \\
>(1.58)\end{array}$ & $\begin{array}{l}\text { Failure in bending of basemat/cylinder } \\
\text { wall junction. }\end{array}$ \\
\hline
\end{tabular}




\subsubsection{Results for the Shell Model}

The shell model was loaded by 133 load increments to a maximum load of 164 psi $(1.13 \mathrm{MPa})$. The first one hundred increments were applied in fixed steps of $9.5 \mathrm{kPa}$ up to $0.95 \mathrm{MPa}$.

The remaining thirty-three increments were applied using the ABAQUS automatic incremental loader. For these remaining increments, if convergence was not achieved within the first eight iterations of a load increment, convergence was abandoned and the residual forces transferred to the next load increment. This can cause problems if the residual forces are of the same order as the applied forces. The uniaxial stress/strain curves for the steel has been modified slightly to aid convergence by removing the Luders band.

As the basemat is subject to uplift, it has been judged unnecessary to model basemat/soil interaction. Indeed the recommended soil compliance [4.7.3] of $390 \mathrm{ksf} / \mathrm{ft}(61.3 \mathrm{MPa} / \mathrm{m})$ is low enough for it to be unlikely for it to impose any significant vertical or shear loading on the stiff basemat.

The pressure/displacement response curve for the containment at mid-cylinder height is shown in Figure 4.7.5. It has the same form as the ring-beam model, with the concrete cracking, liner and hoop rebar yielding at roughly the same displacements. For a given displacement, the containment at this height supports a lower pressure, for unlike the ring-beam model, the cylinder wall is subject to bending. Initially both models have the same compliance. The concrete cracks at $22 \mathrm{psi}(0.15 \mathrm{MPa})$ in the cylinder wall and the response is linear to $109 \mathrm{psi}(0.75 \mathrm{MPa})$ when the liner yields. Further loading yields the hoop rebar at $138 \mathrm{psi}(0.95 \mathrm{MPa})$.

Up to this load the residual forces (the out-of-balance forces produced by the difference in applied loading and internal stresses) are kept to the order of $1 / 100$ th of the applied load. The moment tolerance is simply the maximum residual force specified multiplied by a typical element size: 11.8 inches $(0.3 \mathrm{~m})$ in this case. For higher loads, this criterion is relaxed so that the residual forces and moments can increase by a factor of 10 . For loads greater than $144 \mathrm{psi}(0.99 \mathrm{MPa}),(>0.5 \mathrm{in.}(12 \mathrm{~mm})$ radial displacement), convergence is never again achieved.

Figure 4.7.6 shows the state of the containment at the maximum load achieved of $164 \mathrm{psi}(1.13 \mathrm{MPa})$. Excessive displacements are occurring at the periphery of the basemat. The maximum effective strain in the cylinder wall is approximately three percent ( 4 inches $(100 \mathrm{~mm}$ ) radial displacement). This analysis though cannot model accurately the strains at the cylinder wall/basemat junction. The largest residual forces occur in the basemat and the lower section of the cylinder wall.

At maximum load, due to bending, all the tensile rebar at the pole of the basemat reach their UTS. The liner at the pole position also attains its UTS, but in compression. The centre of the basemat essentially becomes a hinge at this point as the basemat loses its bending stiffness. At this load the ABAQUS computation stops.

The global response of the shell model is outlined in Table 4.7.2. It should be noted that the numbers obtained from Figure 4.7 .5 and Table 4.7.2 
may not correspond, as the former represents a local response and the latter a global response.

\subsubsection{Results for the She 11/2-D Model}

When loading this model, even at very low loads ( 7 psi, <0.05 MPa), two problems became apparent: (1) the junction of the shell and 2-D elements in the cylinder wall and (2) the boundary conditions pertaining to the basemat.

The former condition allowed excessive relative rotation and a 'kink' developed along the interface line between the shell and 2-D elements. This is not surprising as the shell has a rotational DoF which is unrestrained at the interface and so the shell can rotate as a hinge (albeit in axisymmetrical mode). The problem was rectified by applying a rigid constraint from seven inches $(178 \mathrm{~mm})$ above the interface in the shell element to three inches $(76 \mathrm{~mm})$ below the interface in the centre of the wall thickness. This 10 inch $(0.25 \mathrm{~m})$ high restrain is equivalent to applying a rigid ring through the centre of the wall.

Initially, the constraint on the lower face of the basemat was modelled as in the shell model, with the basemat restrained radially and meridionally along the axis of symmetry. This produced excessive concrete cracking and corresponding poor convergence. The lower face of the basemat was then modelled using the RIGID SURFACE option in ABAQUS but the cost incurred was twice that of modelling the structure with the boundary of the entire lower face of the basemat fixed vertically.

The few results presented in Table 4.7 .2 for this model have been obtained by making the first three elements of the bottom set of elements of the basemat rigid (see Figure 4.7.4). The innermost two of the three are constrained vertically. This fixes the basemat against vertical motion within a radius of 1.59 inches $(40 \mathrm{~mm})$ of the centre line of the model.

Figure 4.7.5 shows the pressure/radial displacement response to $1.1 x$ design pressure (51 psi, $0.35 \mathrm{MPa}$ ). The analysis was terminated at this load after 45 increments owing to cost: 13 minutes of CRAY CPU time had been consumed. After the initial meridional cracking in the cylinder wall the numerical response became very sluggish with smaller load increments and more iterations.

The comparison in Figure 4.7 .5 with this model and the shell model is very close. This is not surprising as the displacements are taken at the same distance above the centre of the basemat $11.5 \mathrm{ft}, 3.5 \mathrm{~m}$ ). This is above the continuum elements and hence both model responses is produced by shell elements at this height. It is to be expected the results pertaining to the dome in Table 4.7.2 for the shell model would be applicable to this model.

At the maximum loading of this model, the uplift on the periphery of the basemat is approximately three-quarters that of the wholly shell model. This analysis has not detected the phenomenon of the dome displacing downwards after $25 \mathrm{psi}(0.17 \mathrm{MPa})$ as had [4.7.4]. The latter result was achieved using a wholly $2-D$ continuum analysis. 


\subsubsection{Discussion and Conclusion}

The response of the ring-beam and containment together with the analytical [4.7.5] results are compared in Table 4.7.2. The plots required by SNL [4.7.3] are given in Appendix A.

The results obtained by [4.7.5] for the various stages of damage show good agreement with the numerical model predictions, particularly at the lower pressures. The differences are partly due to biaxial effects which are not accounted for in the analytical and, to some extent, the ring-beam model.

The simple axisymmetric shell model is more numerically stable than the continuum element model when heavy cracking has occurred. Most of the numerical problems pertain to the cracking of the concrete, and if the stiffness is reduced sufficiently to represent the cracking, numerical problems may ensue.

Although this analysis has been taken to a pressure of $3.6 x$ design pressure (164 psi, $1.13 \mathrm{MPa}$ ) it is difficult to predict when failure of the vessel will occur i.e. When the liner is ruptured and the gas leakage rate is in excess of the rate of gas input and hence internal pressure of the containment cannot be sustained. This failure may manifest itself as a gross and rapid structural failure or a local rupture.

At the maximum load attained, the maximum effective strain $\bar{\epsilon} \rho$ in tension is only about three percent. Using the simple premise, and without recourse to any ductile mechanics theories, that the liner will tear on reaching its ultimate tensile strength in uniaxial elongation at 16 percent [4.7.5], leakage will therefore not occur. As this finite element study has precluded penetrations that give rise to stress concentrations, it is not possible to predict when rupture of the liner will occur.

The liner at the pole of the basemat in the shell model does attain its UTS in compression at maximum loading which would indicate buckling of the liner.

The overall conclusion about the overpressure behavior of the model containment is that it should survive without gross distortion of the pressure boundary to $138 \mathrm{psi}(0.95 \mathrm{MPa})$ when the hoop rebar in the cylinder wall yields.

The mode of failure predicted is tensile failure of the rebar due to bending at the pole of the basemat which occurs at $164 \mathrm{psi}(1.13 \mathrm{MPa})$. This results in excessive uplift of the basemat periphery. However, the ABAQUS modeling of the rebar in this region may not be representative in the axisymmetric shell model and hence this mode of failure may not be realistic. A likely mode of failure would then be hoop deformation of the cylinder wall at a pressure above $138 \mathrm{psi}(0.95 \mathrm{MPa})$.

It would seem that the liner will fail from a stress concentration, given the amount of deformation the liner must be subject to in attaining an effective strain of 16 percent. This could be a known concentration as the basemat/cylinder wall junction or an unknown concentration such as a weld defect. 
Finally we would like to point out that the finite element study reported here proceeded in parallel with, and to some extent guided by hand calculations [4.7.5]. We are of the opinion that these two approaches are complementary with the cost advantages of hand calculations balanced against the greater versatility to model detailed behavior with the finite element method.

\subsubsection{Ackowledgments}

The authors are grateful to SNL for the opportunity to undertake this analysis by supplying the construction details and material properties. Thanks also to P. Bainbridge and I. R. Brearley for help in preparing the text and innumerable drawings. 
๑

a.

2

$\rightarrow$

(2)

c)

a)

o

5

$\boldsymbol{\omega}$

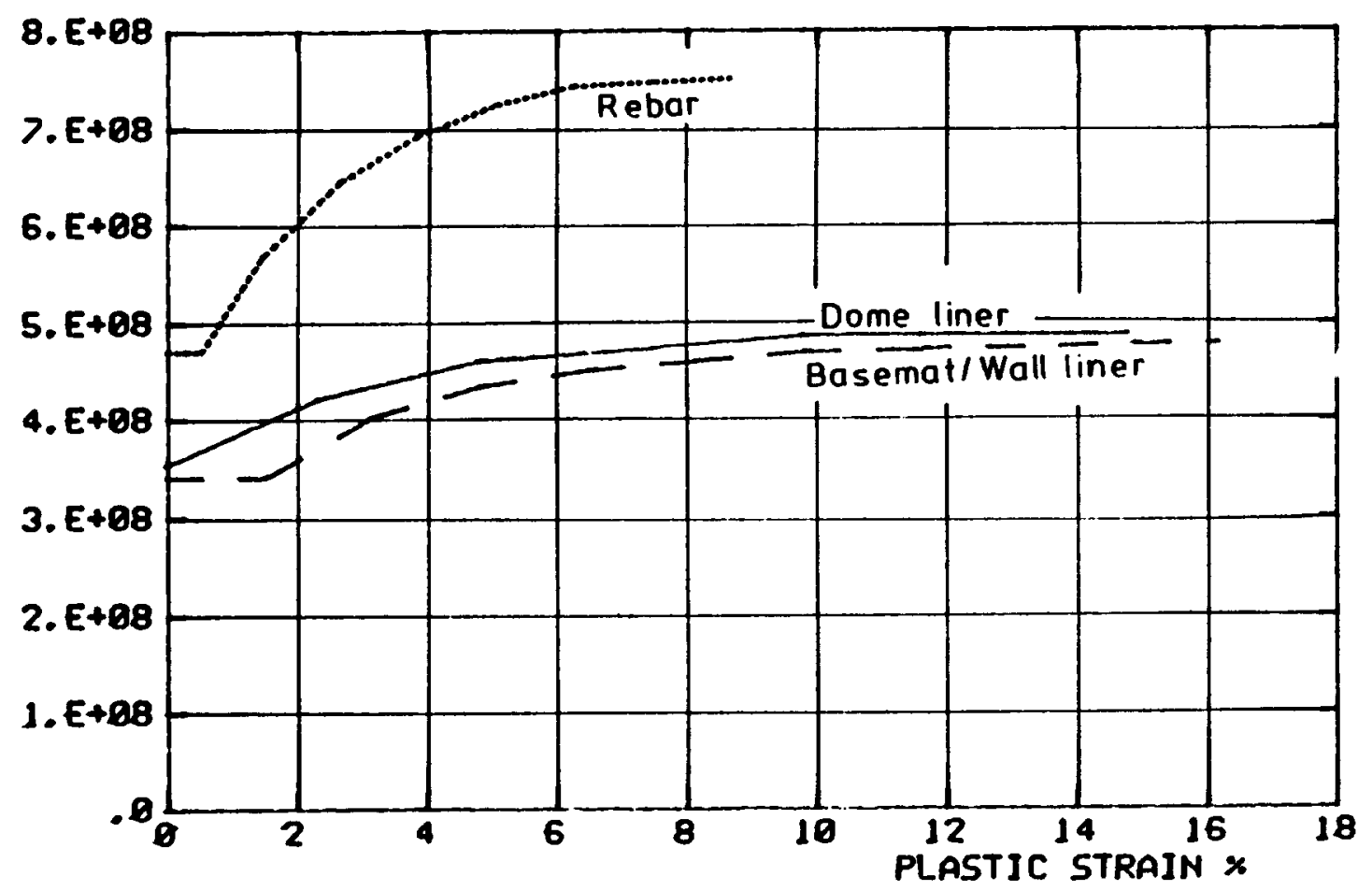

Figure 4.7.1 Uniaxial Steel Properties 


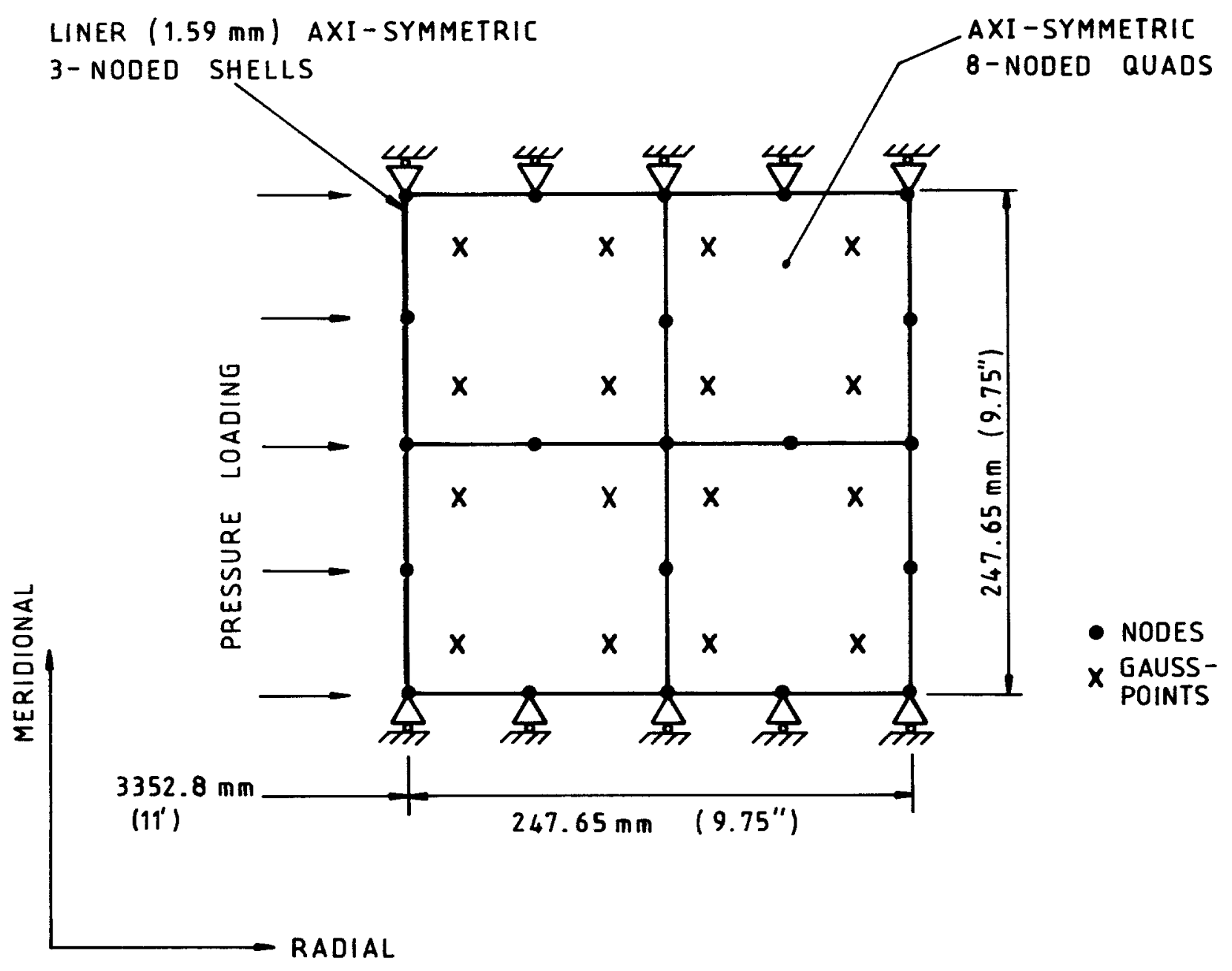

Figure 4.7:2 Cross-section Through Ring-Beam Model 


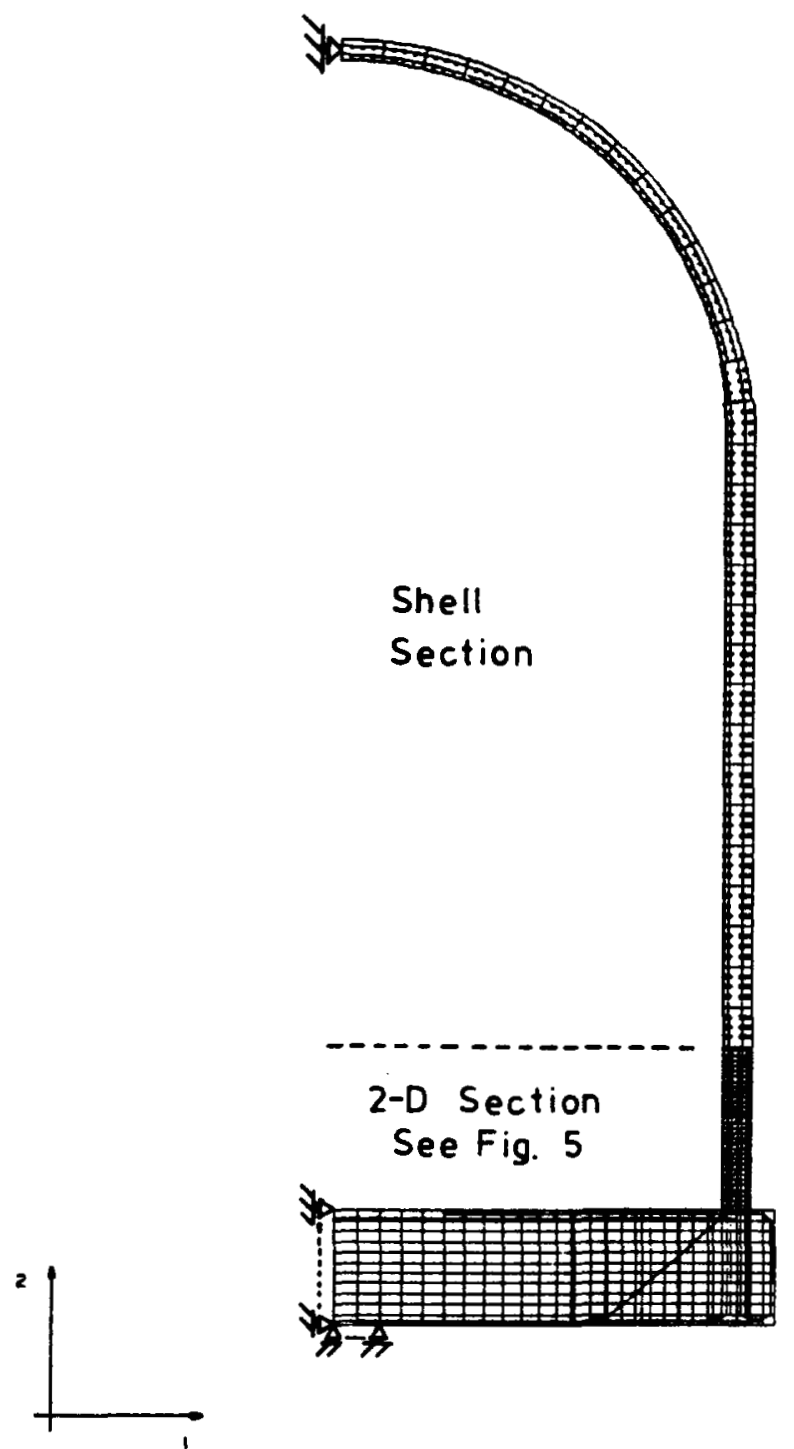

Figure 4.7.3 Display of Rebar in the 2-D Shell Model
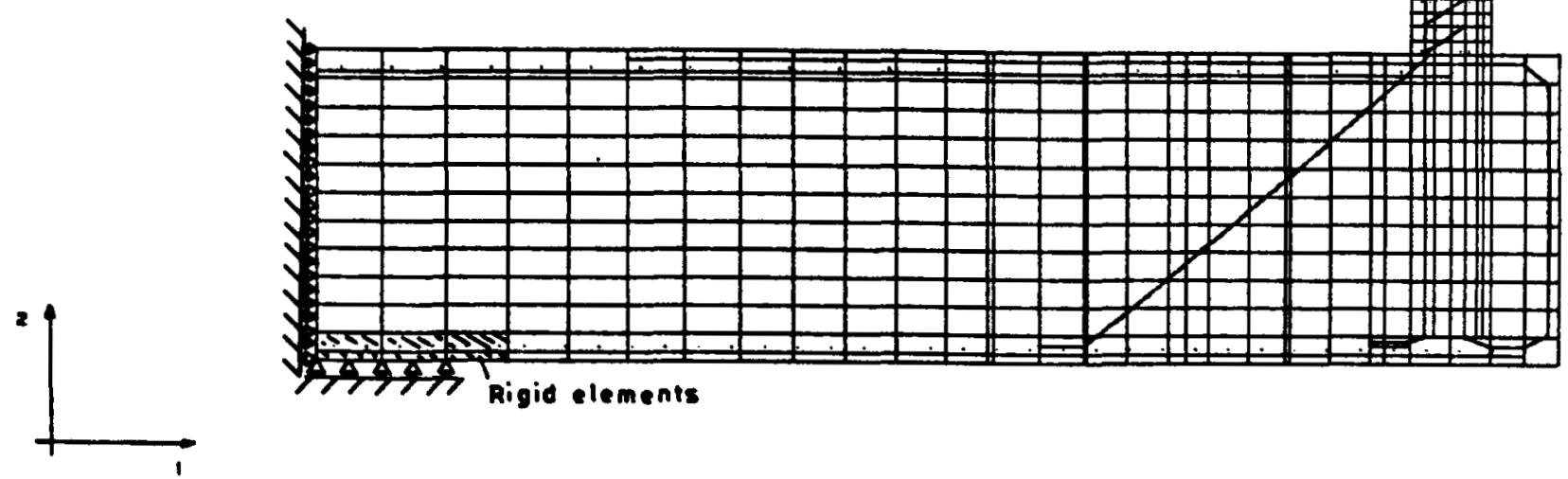

Figure 4.7.4 Display of Rebar in the 2-D Elements 


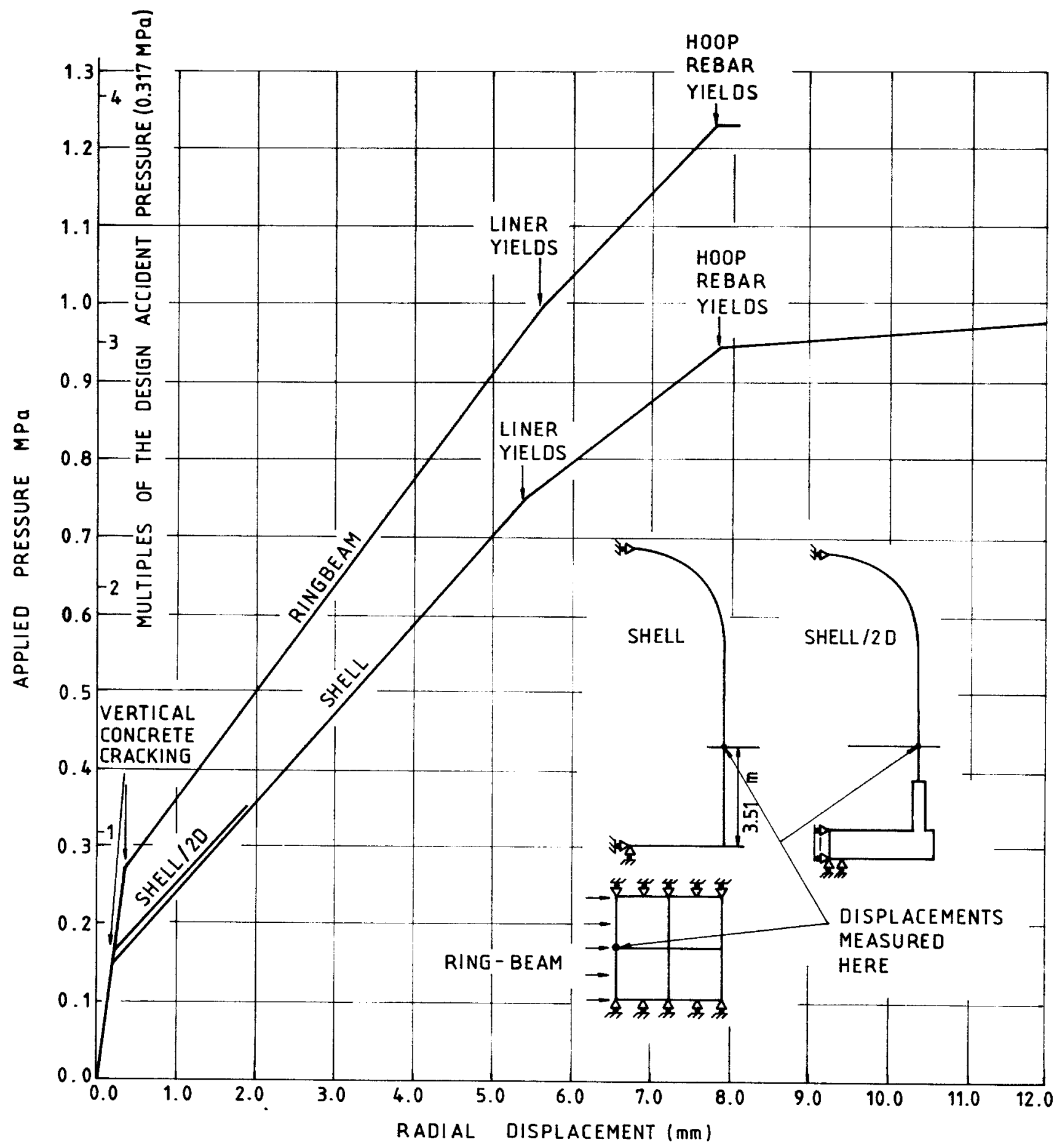

Figure 4.7.5 Overall Pressure Displacement Response 
DISPL.

MAG. FACTOR - + 1.5

SOLID LINES - DISPLACED MESH

DASHED LINES - ORIGINAL MESH

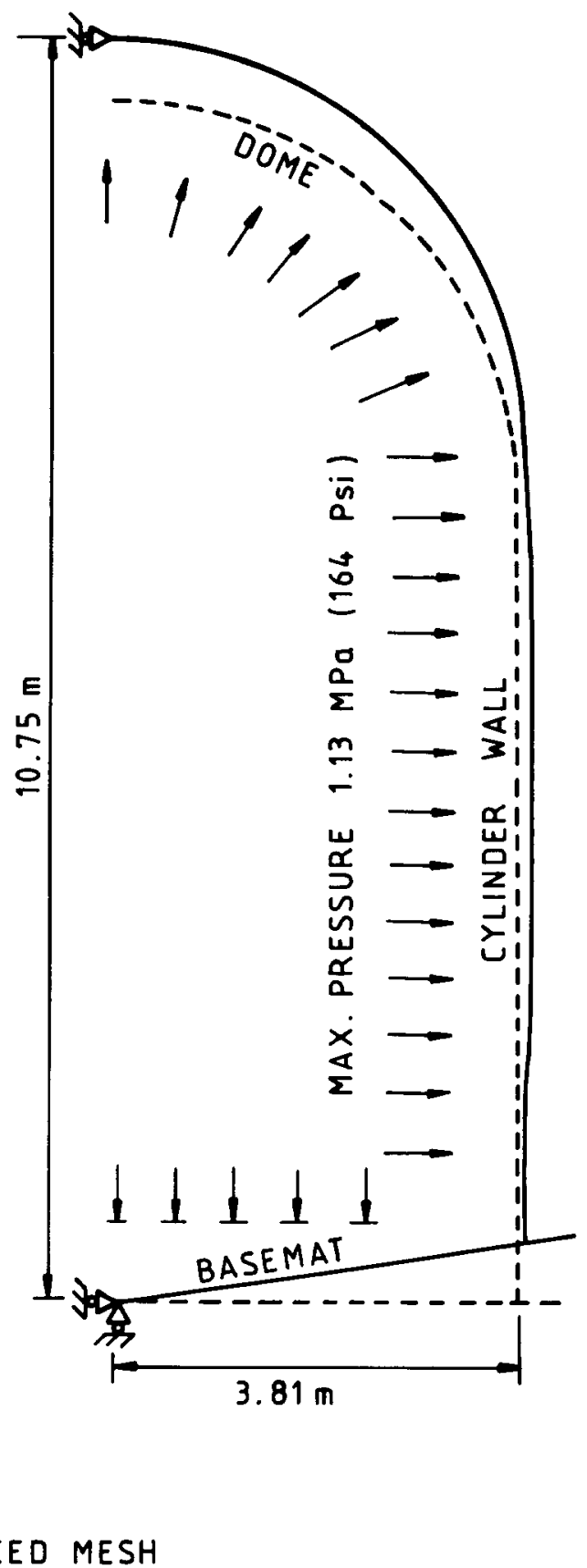

ABAQUS VERSION 4-5-171

Figure 4.7.6 Axisymmetric Containment 


\subsection{Gesellschaft fuer Reaktorsicherheit}

The authors of this section are P. Bachmann, P. Eisert, P. Gruner, W. Kuntze, and H. Schulz of Gesellschaft fuer Reaktorsicherheit (GRS), and J. Eibl and F.-H. Schlueter of the Institut fuer Massivbau and Baustofftechnologie at the Universtitat Karlsruhe.

\subsubsection{Summary}

A pre-test analysis of a pressurized 1:6-scale 1 ined and reinforced concrete containment model is presented. The high pressure experiment will be conducted by Sandia National Laboratories (SNL).

Based upon material properties of concrete, liner steel and reinforcement steel as obtained by SNL from in-situ experiments, the numerical analysis was performed with an axisymmetric finite element model. The non-linear analysis incorporates the interaction between concrete and reinforcement which was determined by supplementary tests at the University of Karlsruhe. Following the evaluation of damage formation in concrete and steel during pressurization, the investigations identify potential mechanisms of structural failure. For a quick-look reference, model characteristics, calculation procedures, and results are compacted in the "Table Summary."

The numerical analysis and the supplementary tests were sponsored by the Bundesminister fuer Forschung und Technologie (BMFT) to whom we express our thanks.

\section{Table Summary}

Analytical Methods

Constitutive Mode is

Mode 1 Geometry

Failure Criterion

Potential

Failure Modes and Failure Pressures
Incremental non-linear finite element analysis, Updated Lagrangian formulation (liner, reinforcement), total Lagrangian formulation (concrete).

Static step-by-step integration without equilibrium iteration (steps of $0.001 \mathrm{MPa}$ above $0.2 \mathrm{MPa}$, dead load considered).

Nonlinear elastic (reinforcement), Multilinear elastic-plastic, isotropic hardening (liner) Bilinear elastic-plastic, isotropic hardening (knuckle, support frame, dome plates) ADINA-concrete mode?

Axisymmetric, 2-D isoparametric finite elements (1 iner, dome plates, concrete); ring trusses (hoop reinforcement); and two-node trusses (other reinforcement, support frame)

Liner: Strain criterion considering multiaxial stress conditions and reduction factors for welds and undetected faults; Reinforcement: strain criterion considering partial weakening of load carrying capacity of rebars.

Leakage of liner in cylinder mid-section at $1.2-1.3 \mathrm{MPa}$ (174-189 psi)

Hoop reinforcement in cylinder mid-section at $1.2-1.3 \mathrm{MPa}$ (174-189 psi)

Cylinder basemat intersection at 1.15-1.2 MPa (167-174 psi) 


\subsubsection{Introduction}

The high pressure test to be performed on behalf of USNRC at Sandia National Laboratories with a 1:6-scale lined and reinforced concrete containment offers a unique opportunity to validate or discard material models, structural models and numerical methods.

The calculations will be performed by several analysts employing a wide scope of computational procedures. Thus, the analysis results obtained by distinct methods may be compared.

The analyses will be performed by two major steps. In the first phase, the response of the structure due to high pressure loading will be calculated prior to the experiments. These pre-test results will then be compared to the experimental data. Depending on the outcome of this comparison, the idealization and the material properties used might be subject to modification. In the second phase, these modifications will be incorporated in further calculations to improve the results of the pre-test computations.

In Section 4.8.3, the finite element model of the 1:6-scale containment is described. Boundary conditions are discussed in Section 4.8.4. Material properties of concrete, steel reinforcement and the 1 iner are presented in section 4.8.5. This chapter also contains a summary of experimental investigations of the bond transfer mechanism between reinforcement and concrete which were carried out at the Institut fuer Massivbau and Baustofftechnologie of the University of Karlsruhe. Following a discussion of the employed numerical procedures in Section 4.8 .6 , the results of the pre-test computations are presented in Section 4.8.7.

\subsubsection{Finite Element Model of the Structure}

\subsubsection{General features}

Within the scope of the pre-test calculations, the general behavior of the structure will be analyzed with an axisymmetric model. In the idealization, local geometric variations will be neglected. This simplification is justified if localized irregularities in the geometry of the structure do not significantly influence the dominant failure modes. This assumption seems to be reasonable on account of the strongly reinforced areas around hatches and airlocks. Final justifications of these arguments are to be expected from the test results. If the test results should disprove the simplifying assumptions, further improvements of the idealization will have to be incorporated in the post-test analysis.

In addition, it must be mentioned that the axisymmetric idealization does not distinguish between local stiffness variations due to the discrete arrangement of the meridional bars. Since, however, the spacings between these bars are rather small, the error made by smearing the meridional reinforcement in the circumferential direction is not expected to be significant.

The finite element mesh used in the pre-test calculations is shown in Figure 4.8.1. The concrete shell and basemat is modeled by 2-D isoparametric elements. The cross-sectional areas of these elements range from $4 \mathrm{in}^{2}$ 
$\left(25.8 \mathrm{~cm}^{2}\right)$ in the dome, the cylinder and the outer basemat parts to some $23 \mathrm{in}^{2}\left(148.4 \mathrm{~cm}^{2}\right)$ in the basemat at the inner positions. 2-D isoparametric elements are also used for the liner. The element lengths range from some $2-1 / 4$ inches $(57.2 \mathrm{~mm})$ to some six inches $(152.4 \mathrm{~mm})$.

Meridional reinforcement, shear ties and the $45^{\circ}$ bars in the basemat are modeled by two-node trusses. For the hoop reinforcement, ring trusses are used. The bending reinforcement near the top and the bottom of the basemat is replaced by equivalent ring and two-node trusses. The procedure to obtain the equivalent trusses is described later. Two-node trusses are also used for the support frame in the basemat, whereas the steel plates at the dome top is modeled by $2-D$ isoparametric elements.

The model contains 3187 Elements connected at 2335 nodal points. The number of degrees of freedom is 4567 .

\subsubsection{Features related to main structural parts}

\subsection{Containment she 11}

Aside from the concrete element where the seismic reinforcement ends in the dome, all concrete elements are four-node elements with four integration points. The cylindrical section and parts of the dome between the spring line and the position where the seismic reinforcement ends are modeled by six elements across the wall thickness. The rest of the dome sections contain five concrete elements across the wall.

Pre-calculations showed that the discretization is sufficiently fine to produce rather smooth stress distributions across the containment shell. In case that the test results require an even finer resolution at the transition areas between cylinder and basemat and between cylinder and dome, respectively, the modeling of these sections will be improved by using elements with a higher number of nodal points in the post-test calculations.

To guarantee proper bending behavior of the shell, the meridional reinforcement and the seismic reinforcement as well is positioned as in the structure. The locations of ring trusses in the model, however, slightly deviate from the positions of the hoop reinforcement in the structure. Yet, the contents of steel is preserved.

The tapering off of meridional reinforcement is taken care of by respective reductions of cross-section areas of the trusses.

As al ready mentioned, the seismic reinforcement has to be transformed into an axisymmetric representation. The orthogonal inclined mesh of the seismic reinforcement of spacing $D$ is first replaced by an orthogonal upright mesh with spacing $D$ times $\sqrt{ } 2$. Assuming that the steel contents remains unchanged the cross-section areas of the replacement trusses have to be increased by a factor of $\sqrt{ } 2$ as compared to the cross-sections of the \#4 bars of the inclined seismic reinforcement. This result is compatible with the outcome of a simple equilibrium consideration assuming equal stretching of both meshes. 
Reinforcement bars which do not run along the circumferential direction are to be smeared in the axisymmetric case. They are modeled by two-node trusses. The cross-section areas of these trusses have to be referred to one radiant. This holds for the meridional reinforcement, the shear ties in the lower cylinder and the meridional replacement bars of the seismic reinforcement.

In the upper dome area, the meridional reinforcement is connected to the four-node isoparametric elements representing the steel plates by degenerated four-node elements of triangular shape (Figure 4.8.2).

Variations of contents of steel in lower cylinder (ending bars \#6 at EL $6^{\prime}-6^{\prime \prime}(1.98 \mathrm{~m})$ and the ending diagonal bars which come up from the basemat and end at EL $3^{\prime}-9^{\prime \prime}(1.14 \mathrm{~m})$ ) are considered by variations of crosssection areas of the lower meridional truss layers ( 2 and 5 ).

\subsection{Basemat}

The basemat is modeled by fourteen layers of concrete. Figure 4.8 .1 shows a fine mesh in the vicinity of the interface between basemat and cylinder. The mesh is becoming more coarse with decreasing height and radius. In Figure 4.8.3 an enlarged view of the cylinder-basemat transition area is shown.

Hoop and vertical reinforcement is modeled by ring trusses and two-node trusses, respectively. The seismic reinforcement, which is, according to the drawings, not connected to other reinforcement, ends at the top of the second concrete layer above ground.

The diagonal bars running under forty-five degrees and connecting the basemat with the lower cylinder are again modeled by two-node trusses.

Since the support frame in the basemat will carry some amount of the shear loads, this steel structure was modeled, too, by smearing its steel contents on radial and vertical two-node trusses.

To determine a reinforcement pattern which fits into the axisymmetric model and which is equivalent to the rectangular mesh of the upper and lower bending reinforcement, the steel is assumed to be continuousiy distributed with constant density in both orthogonal directions. Assuming further that stretching in the respective mesh planes has central symmetry, the densities of equivalent radial and ring trusses, respectively, can be determined by simple equilibrium considerations. From the latter densities, the crosssection areas of radial and ring trusses were obtained.

The mud mat was not modeled since it does not contribute to the loadcarrying capacity in any significant way.

\subsection{Steel liner}

Two-D isoparametric elements are also employed to form the liner. In general, these elements contain four nodal points with the exception of the liner knuckle where six-node elements are used. Figure 4.8 .4 depicts this latter section. If bending stresses in the knuckle turn out to be of major importance this section of the liner will be replaced by higher valued shape 
functions for the post-test calculations. On the basis of the construction drawings it was concluded that the space between the liner Knuckle and the surrounding concrete structure was empty. Latest information, however, showed that this space is filled with concrete. The remodeling of this area will be postponed until the test results are available.

This radius of the knuckle amounts to 2.25 inches $(57.2 \mathrm{~mm})$ in the computer model whereas, in the structure, this radius is 1.2 inches $(30.5 \mathrm{~mm})$. This variation was necessary for reasons of the mesh size in the adjacent concrete structures. As the knuckle is stiffer than the attached liner shell, yielding should occur first near this junction in the liner shell. With respect to possible failure of this junction its actual position does not seem to be of great importance. The width of the knuckle model is roughly 0.15 inch $(3.81 \mathrm{~mm})$ and tapers off at the ends to $1 / 16$ inch $(1.59$ $\mathrm{mm})$.

The width of the dome section of the liner is $1 / 12$ inch $(2.11 \mathrm{~mm})$ and of the cylindrical and base parts $1 / 16$ inch $(1.59 \mathrm{~mm})$.

Studs were not explicitly modeled. The liner-concrete connections are dealt with in the next section.

The fill slab above the liner bottom was not modeled explicitly. To account for the weight of this slab its mass is considered by an increased mass density of the elements forming the liner bottom.

\subsubsection{Boundary Conditions and Steel-Concrete Connections}

\subsubsection{Reinforcement-Concrete Connections}

In general, ring and meridional trusses are fixed to nodal points of the concrete element mesh in the dome and in the cylinder. Aside from transition regions where bending occurs, there is no major relative motion between meridional and hoop bars to be expected at crossing points of the bars under the symmetric loading by internal pressure. In addition, after cracks have formed the concrete pieces should generally follow the motion of the net of reinforcement bars.

Ending trusses in the lower section of the cylinder are again assumed to be fixed at concrete mesh points. The same assumption holds also for the seismic reinforcement modeled by meridional trusses which end above the spring line in the dome. If concrete cracks in these areas the ending bars gradually lose their ability to carry part of the load which is a desired result. However, for the ring trusses which replace part of the seismic reinforcement in the model, this effect will not occur. Therefore, other measures should be taken into account to model the post-cracking states of this type of reinforcement. Also, the reduction of load-carrying amount of those meridional bars of the main reinforcement which end in the dome should be considered. At present, a gradual weakening of the reinforcement under consideration (either by reduction of cross-section areas or by changing respective stress-strain diagrams) cannot be carried out with ADINA. On account of these restrictions, the interaction between steel and concrete cannot be described as a function of loading history. Possible impacts of the discussed reduction of load-carrying capability on failure modes have 
therefore to be evaluated after the calculations on grounds of the computed load-deformation history.

The forty-five degree trusses which model the diagonal bars in the basemat are anchored to the meridional trusses in the lower cylindrical part and to the lower bending reinforcement of the mat. They do not directly couple with other steel reinforcement. There is, however, a connection with the concrete mesh points. Vertical reinforcement bars which run from the cylinder into the basemat and traverse the upper bending reinforcement are connected to concrete mesh points and anchored at the lower bending reinforcement. The vertical trusses which are used to simulate part of the seismic reinforcement end within the basemat. They are not coupled to any other type of reinforcement.

Hoop reinforcement and vertical reinforcement in the basemat is modeled by ring and two-node trusses, respectively, which are both fixed at concrete mesh points.

The truss model of the support frame is attached to the concrete mesh points but has no direct connections with other reinforcement.

\subsubsection{Liner-Concrete Connections}

In the axisymmetric model the nodal points of the outer liner surface are fixed to the nodal points of the inner concrete surface. Studs are not modeled explicitly.

This simplification seems to be reasonable for areas which are predominantly loaded by membrane stresses.

In other areas where stretching and bending occurs, there might be slight relative motion of stud ends anchored within the concrete layer and the ends welded to the liner shell. Since, however, concrete cracks at rather low strain levels and since microcracks develop predominantly at faults, it may be argued that the bonds between concrete and studs gradually become weaker as deformation goes on. Hence, it seems that bending strains transmitted into the liner by studs are not of great importance at higb strain levels where cracks in concrete open up and the liner begins to yield due to membrane strains. If, however, bending strains due to stud action may be neglected, then the interaction between the liner and the concrete as modeled should approximately be correct.

Yet, an overestimation of the stresses in the liner knuckle caused by "nailing" the ends of the bend onto the adjacent concrete structure as it is done in the model cannot be excluded.

If this simplified approach to the liner-concrete interaction is to be reconsidered in view of the experimental results, then realistic force deformation characteristics of the studs should be made available.

The nodal points of the lower surface of the liner bottom are rigidly connected to the mesh points of the upper surface of the basemat. This idealization does not quite match the anchorage in the structure where only the circumference of the liner bottom and its center are fixed to the basemat. Since partial sliding of the bottom of the liner with respect to 
the basemat should be very small, the approach taken in the model should be acceptable.

\subsubsection{Basemat - soil interaction}

The modulus of subgrade reaction as given by 4.8 .1 seems to be obtained by averaging the initial slopes of the pressure path measurements for the 12 , 18, and 30 inch disks and extrapolating the mean value linearly to the cross-section area of the basemat. The modulus obtained in this way amounts to $1.16 \times 10^{10} \mathrm{~N} / \mathrm{m}(390 \mathrm{ksf} / \mathrm{ft})$. According to the theory of elastic halfspace, however, the soil spring constant should vary with the square root of the cross-section area. It was thought that extrapolation according to half-space theory is preferable. The value of the soil spring constant obtained according to the latter procedure amount to $\bar{K}=25.2 \mathrm{ksf} / \mathrm{ft}(7.5 \mathrm{x}$ $\left.10^{8} \mathrm{~N} / \mathrm{m}\right)$. This value was used in the model calculations. The total spring force was assumed to be distributed into single forces under the assumption that equal areas carry same weights. This leads to spring constants varying linearly with their radial position under the basemat as

$K(r)=\frac{\bar{K}}{r_{a}^{2} \pi} \cdot r$

$r_{a}$ : outer radius of basemat

The soil springs were modeled as linear compression/no-tension trusses

transmitting forces only in the vertical direction.

\subsubsection{Material Properties}

\subsubsection{Concrete}

4.8.5.1.1 Summary of main features of the ADINA concrete model

The material behavior of concrete is described in the finite element code ADINA [4.8.2] by three basic features:

(1) The constitutive equations are formulated by incremental elastic nonlinear stress-strain relations to allow for weakening of the material under increasing compressive stresses.

(2) The ultimate load-carrying capacity is described by failure surfaces in stress space for the compression and tension.

(3) To model post-cracking and post-crushing behavior of concrete, special strategies are postulated.

To model stress-induced anisotropy different stress-strain relations are used for material loading and unloading, respectively. To decide whether the material is loading or unloading in the stress step, a loading function is defined which depends on the second invariant of the deviatoric stresses and on the hydrostatic stress. The respective largest value ever encountered during the loading history, $F_{\text {max }}$, is stored. Unloading occurs if the loading function is smaller than or equal to $F_{\max }$. 
In this case, the material is supposed to be isotropic elastic and the initial Young's modulus is used to formulate the incremental stress-strain relation. On the other hand, if the loading function is larger than $F_{\text {max }}$, i.e., the material is loading, different stress-strain matrices are used depending on the stress state of the material prior to this step. If the material was in tension or low compression the material is still assumed to be isotropic. However, the Young's modulus is obtained in this case by an averaging scheme which employs the principal stresses and tension moduli which are derived from the uniaxial stress-strain curve for each principal direction. Under high compression, on the other hand, an orthotropic stress-strain matrix with the directions of orthotropy defined by the principal stresses is used. The elements of this anisotropic matrix again are derived from the uniaxial stress-strain curve employing the principal stresses and the tangent moduli which are referred to the three directions of principal stresses. The boundary between low and high compression states is monitored by a user-defined parameter. If the smallest principal stress component is larger than the product of this parameter and the compressive strength of the material under multiaxial conditions, isotropic behavior is assumed. If the smallest principal stress component is smaller than this product orthotropic stress-strain relations are employed. In any case, Poisson's ratio is regarded as being constant.

The uniaxial stress-strain curve of concrete is, in ADINA, approximated by an analytical function. This function is to be determined by the following input quantities: initial tangent modulus, maximum compressive stress and its related strain, ultimate compressive stress and its related strain, and tensile strength.

To model cracking and crushing of concrete, failure surfaces are used for the compression-compression region, the compression-tensile region, and the tensile-tensile region. In addition, the failure surfaces are employed to define uniaxial stress-strain behavior under multiaxial stress conditions. The tensile failure surface is composed of three planes each of which is perpendicular to the principal stress directions. The positions of these planes in stress space are fixed by the uniaxial tensile strength. To adapt the failure surface in the compression-compression region, 24 input parameters have to be provided by the user. They determine a set of curves in the plane of two principal stress directions. Each curve belongs to a certain value of the third principal stress. These curves can be thought of as being traces of cuts perpendicular to the third principal stress through the compression-compression failure surface. The transition failure states between the tensile-tensile region and the compression-compression region are determined by linear interpolation.

Cracking occurs if the largest principal stress is larger than the tensile failure stress. In this case, it is assumed that a plane of failure develops perpendicular to the principal stress direction, the normal and shear stiffness are reduced by user-provided factors and the normal stress is released.

If the stress state 1 ies on or outside of the compression-compression failure surface the material is assumed to be crushed. 
After a tensile failure plane has developed further cracking may occur. It is, however, assumed that a maximum of three tensile failure planes can be formed, each being orthogonal on the two other planes. Whether a tensile failure plane is active or inactive is controlled by the normal strain across the plane.

The material is assumed to strain-soften if it is crushed and subjected to further compressive stresses. After the ultimate compressive strain has been reached the material has no further stiffness and all stresses will be released.

\subsection{Preparation of input data for the ADINA concrete model}

Table 4.8.1 lists the parameters to be used to define the uniaxial stressstrain curve of concrete. The values listed there are the same as those proposed by Sandia.

\section{Table 4.8.1: Uniaxial Concrete Properties}

Young's modulus

Poisson's ratio

Tensile strength

Compressive strength

Strain at compressive strength

Uitimate compressive strength

Strain at ultimate compressive strength

$$
\begin{aligned}
& E=4800 \mathrm{ks})(33000 \mathrm{MPa}) \\
& \nu=0.2 \\
& \sigma_{t}=0.5 \mathrm{ksi}(3.45 \mathrm{MPa}) \\
& \sigma_{c}=-6.8 \mathrm{ksi}(-47.0 \mathrm{MPa}) \\
& { }_{c}=-0.0021 \\
& { }_{c}=-6.8 \mathrm{ksi} \quad(-47.0 \mathrm{MPa}) \\
& { }_{u}=-0.003
\end{aligned}
$$

To generate the 24 input parameters which define the failure surface for stress states in the compression-compression region, the following method was applied. According to ottosen [4.8.3], a stress state on the failure surface is most conveniently described by the octahedral normal stress, the octahedral shear stress and an angle in the deviatoric plane, the latter quantity being a function of the third invariant of stress. In the deviatoric plane, the trace of this failure surface is of triangular shape with convex curved edges and corners. Proceeding along the hydrostatic axis (i.e. along the direction of negative octahedral normal stress), the traced area in the deviatoric plane becomes larger, and the trace itself more and more approximates the shape of a circle. There are two distinct meridians on that surface. They pass through the deviatoric plane at an angle of $\theta=$ 0 (tensile meridian) and at an angle $\theta=60^{\circ}$ (compressive meridian).

Ottosen showed that if failure stresses are scaled to the compressive strength of concrete, the available experimental data fit very well the two meridional traces. This shows that failure of concrete in the compressioncompression range may to a very good approximation be described by a single failure surface if stresses are referred to the compressive strength. To determine this surface analytically the following quantities must be known: the uniaxial compressive strength, the uniaxial tensile strength, the biaxial compressive strength and the stress coordinates of one point on the compressive meridian. The first two quantities are given in Table 4.8.1. Supported by experience, the value for the biaxial compressive strength is taken as 1.16 times the uniaxial compressive strength. Concerning the point 
on the compressive meridian, the scaled octahedral normal stress and the scaled octahedral shear stress are 2.89 and 2.31 times the uniaxial compressive strength, respectively.

To define failure curves as needed for the ADINA concrete model, the ottosen surface is cut with planes perpendicular to the axis of the first principal stress. Thus, a set of failure curves in the planes spanned by the second and third principal stresses is obtained. Only three points on each of these failure curves can be used for the ADINA input. Failure states in between these points are linearly interpolated by the program. Numerical values for the input parameters as obtained in this way are listed in Table 4.8.2. Further information on the parameters listed in this table may be obtained from the ADINA manual [4.8.2]. The linearized failure curves as used in the program are shown in Figure 4.8.5. Table 4.8.3 contains additional special concrete parameters which are needed for the ADINA concrete model. The uniaxial strains corresponding to the compressive strength under uniaxial conditions and the compressive strength under multiaxial conditions, respectively are supposed to be equal (GAMMA $=1.0$ ). Depending on the value of KAPPA, either isotropic behavior or anisotropic behavior is used (KAPPA $=0.5$ ). This means if the minimum principal stress is smaller than 50 percent of the current uniaxial maximum compressive strength, an orthotropic stress-strain matrix is used. Otherwise, isotropic behavior is assumed. The constant CLFN is put equal to zero which means that the loading function depends only on the second invariant of the deviatoric stresses.

After cracking has occurred, the normal stiffness in a concrete element is reduced by a factor STIFAC. In order to avoid numerical difficulties this factor is taken to be somewhat larger than zero (STIFAC $=0.0001$ ). A gradual reduction of the shear stiffness after cracking has occurred is not possible in the present version of ADINA, as was pointed out earlier. Therefore, a constant shear stiffness reduction factor had to be used (SHEFAC $=0.2)$. Comparisons between finite element analyses and tests of shear panels seem to support this value of reduction [4.8.4].

\subsubsection{Steel Reinforcement}

In the model, concrete and steel elements are only connected at the nodal points. Hence, there is a priori no steel concrete interaction between the nodal points. This means, if a crack plane forms in concrete perpendicular to the steel element, the concrete loses immediately its stiffness and the load carried before cracking by the concrete is now carried over the steel element. This would be a proper description if simultaneously a continuum of parallel crack planes would form in the concrete element. Tests show that cracks are usually initiated at discrete positions. Although the bond between concrete and steel is lost at the location where the crack plane crosses the steel, the bond is gradually increasing with increasing distances from the crack plane. Hence, an increasing amount of load is again transmitted to concrete. Therefore, the strain in the steel due to a certain longitudinal load is smaller than the steel strain obtained if there would be complete bond breakdown. This change of straining of the composite element that contains a single crack plane reflects itself in a modified stress-strain diagram for the steel if the applied force is referred to the cross-section area of the steel. In this way, the bond action of uncracked zones in a region containing a single crack can be projected onto the stress-strain behavior of the steel. To obtain this modified material 
Table 4.8.2: Compression Failure Envelopes

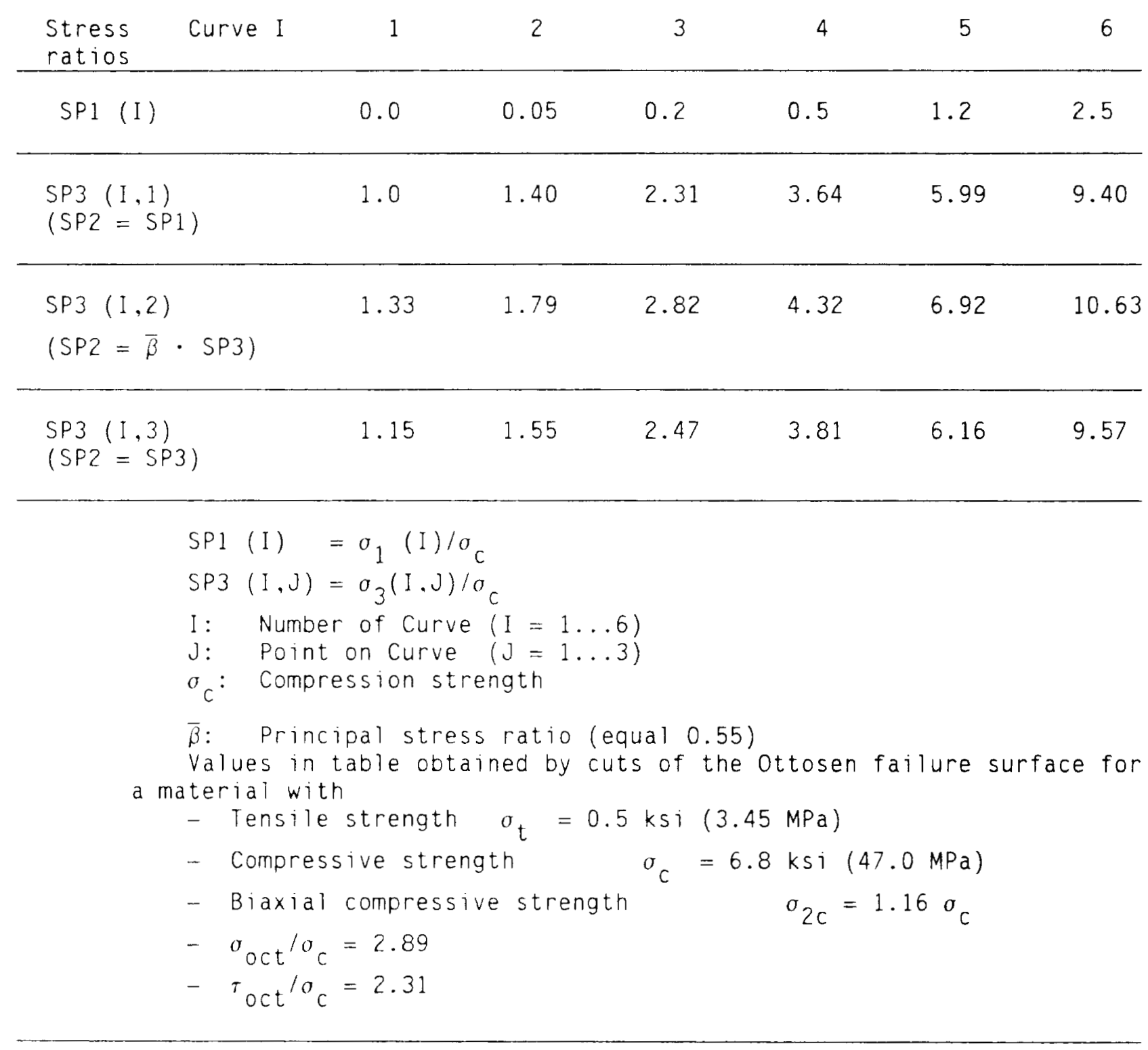

Iable 4.8.3: Special Concrete Parameters Needed for ADINA-Concrete Model

Uniaxial critical strain scaling factor

Control parameter for changing material law

Constant used in loading function

Normal stiffness reduction factor

Shear stiffness reduction factor

$$
\begin{aligned}
& \text { GAMA }=1.0 \\
& \text { KAPA }=0.5 \\
& \text { CLFN }=0.0 \\
& \text { STIFAC }=0.0001 \\
& \text { SHEFAC }=0.2
\end{aligned}
$$


behavior of reinforcing steel that reflects the steel-concrete interaction in certain sections of the containment, respective experiments were conducted at the University of Karlsruhe.

\subsection{Modified stress-strain behavior for hoop and seismic reinforcement in the cylindrical section of the 1:6-scale containment}

To obtain the modified stress-strain behavior for the reinforcing steel, two groups of tests were performed in Karlsruhe [4.8.5]. In the first group, specimens representing a typical wall element of the concrete containment subjected to circumferential loading were tensioned uniaxially. The segments were reinforced with four bars in longitudinal direction representing the hoop reinforcement. Dividing the total applied load by the cross-sections of the reinforcing bars and measuring the deformation, the modified stress-strain relations were obtained. The mean stress-strain curve of all tests for rebar \#4 is presented in Figure 4.8.6. The results shown are referred to the nominal bar cross-section of 0.2 in $^{2}\left(129 \mathrm{~mm}^{2}\right)$.

The second group of tests were conducted to study the bond transfer mechanism for the diagonal seismic reinforcement under biaxial tensile loading. As to the test setup, we may refer to [4.8.5]. The mean stressstrain curve obtained for these tests for rebar \#4 is shown in Figure 4.8.7. Again, the results are based on the nominal bar cross-section of 0.2 in $^{2}$ $\left(129 \mathrm{~mm}^{2}\right)$.

\subsection{Stress-strain relations used in the analysis}

To incorporate in the analysis the steel-concrete interaction, the stressstrain diagrams obtained at the University of Karlsruhe will be used for the hoop reinforcement and the seismic reinforcement in the cylinder.

The tests have shown that the deviations of the modified stress-strain curves from the material behavior of the bare steel are confined to strains well below the yield strain. The amount of the deviations is a function of steel content. For low steel contents, one obtains larger deviations than for high steel contents.

For the meridional reinforcement in the cylinder, we apply the same modified stress-strain relations as for the hoop reinforcement. This is justified for the following reasons. The steel content in the meridional direction is approximately 50 percent of the reinforcement in hoop direction. Therefore, the deviations of the modified stress-strain behavior from the behavior of the bare steel as obtained for the hoop reinforcement should be approximately twice as large for the meridional reinforcement. On the other hand, due to circumferential stresses, the bonds between meridional rebars and concrete will be weakened. This reduction in bond strength may be assumed to amount to some 50 percent [4.8.6]. The modified stress-strain behavior is also employed in those sections of the meridional rebars \#4 which extend into the basemat.

Although there were no tests conducted for the dome section, it will be assumed that the modified stress-strain behavior found for the cylindrical 
parts may, to a good degree of approximation, also be employed for the dome reinforcement.

For all other reinforcement steel which has not been explicitly addressed above we use the steel stress-strain behavior as obtained by Sandia. The latter material curves will in all cases be based on the nominal crosssections of the respective bars.

In the low strain range, where concrete has not yet cracked, the load is carried both by the uncracked concrete and the steel. Hence, there is some amount in the modified stress-strain curves of the tests which is to be attributed to the concrete. Since, in the analysis, the tensile behavior of concrete is modeled up to its maximum tensile strength, the material characteristic of the bare steel is used until cracking of concrete is initiated. The material behavior of steel used in the analys is is exhibited in Figures 4.8.8, 4.8.9 and 4.8.10. Figures 4.8 .8 and 4.8.9 refer to rebar \#4 of the main reinforcement. Figure 4.8.10 shows the steel behavior for the seismic reinforcement. The slopes chosen in these figures to connect the steel stress stages just before concrete cracks and after cracking has occurred, respectively, are to some extent arbitrary. They depend on the rapidity of stress redistribution following crack initiation in the concrete. In the concrete model presently available in the ADINA program, the stresses carried by the concrete are immediately released when the tensile strength of concrete is passed. For this reason, the slopes in the steel stress-strain curves should be as steep as possible. The slopes were adapted according to the requirement that stiffening of the compound material normal to a crack plane must not occur after cracking has taken place.

It is to be noted that possible slight changes in the modified stress-strain curves for the main reinforcement and for the seismic reinforcement, respectively, due to the mutual interaction of both types of reinforcement, were disregarded.

For a comparison, Figure 4.8.11 shows the material behavior of the bare steel which is used for those reinforcing bars for which steel-concrete interaction is not considered. The shown curve for rebar \#4 is taken from [4.8.5]. It is essentially the same as that obtained by the Sandia measurements. Young's modulus and yield strength for rebars \#4 are listed in Table 4.8.4.

The material behavior of the reinforcing steel is modeled as being nonlinear elastic. There is no major unloading to be expected in regions of large straining so that use of elasto-plastic material behavior should not be relevant.

\subsubsection{Steel Plates in Dome, Support Frame in Basemat and Liner Knuckle}

The material properties of these structural parts have not been measured. The respective data have been taken from ASME Tables [4.8.7, 4.8.8, 4.8.9]. The material data used in the analys is for the steel plates (ASTM A 516 Grade 60), the support frame (ASTM A 36) and the knuckle (ASME SA 106 Grade B) are listed in Table 4.8.5. Bilinear elasto-plastic behavior with isotropic hardening is assumed for these structural parts. 
Table 4.8.4: Rebar Material Properties

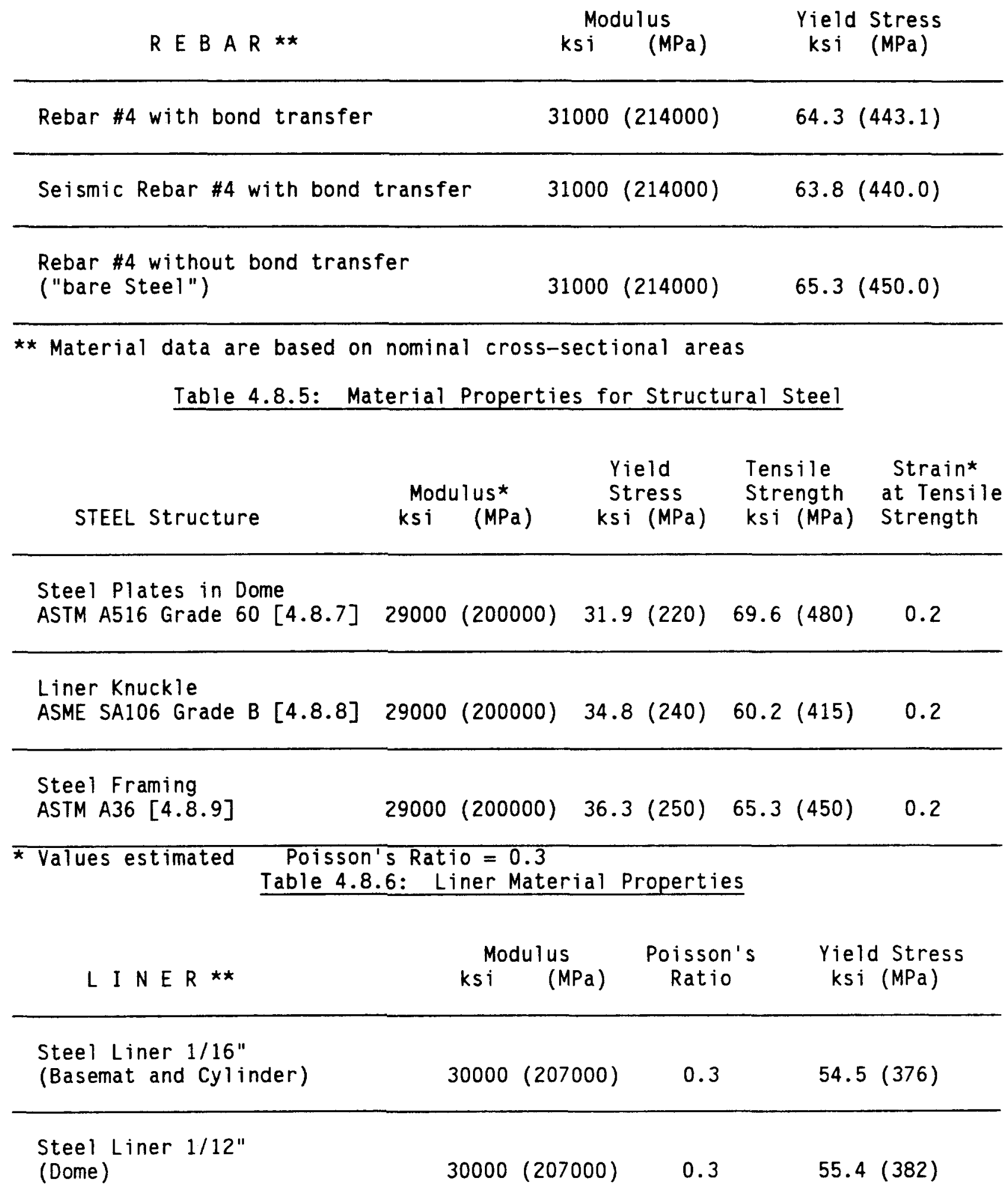

** Material data are based on nominal cross-sectional areas 


\subsubsection{Liner}

For the cylindrical and bottom sections of the liner, as well as for the dome section, the respective material curves as obtained by Sandia are used. They are shown in Figures 4.8.12 and 4.8.13. The stresses are in each case based upon the respective nominal liner thicknesses. To describe the stress-strain behavior in the analysis, the multilinear elasto-plastic model of ADINA is employed. Table 4.8 .6 contains additional material properties of the liner.

\subsubsection{Numerical Procedure}

The numerical step by step computation of the structural response will be conducted first without equilibrium iterations. A variety of investigations of simple reinforced concrete structures under monotonically increasing quasi-static loading (e.g., [4.8.10]) seems to indicate that the deviations of results obtained with iteration and without iteration, respectively, do not affect significantly the load-deformation behavior after concrete has cracked and the load is redistributed to the reinforcing steel, provided sufficiently small load increments are used in the computation. Deviations become, however, somewhat larger during the loading phase when concrete cracking takes place.

Very little respective experience is available for more complex structures. Therefore, the containment response as obtained by the straight-forward method must be confirmed by a further investigation that takes into account the step-by-step balancing of residual forces. The results of this extended study will be presented as soon as they become available.

In the first step of the static analysis without equilibrium iteration the structure will be loaded by $14.5 \mathrm{psig}(0.1 \mathrm{MPa})$ internal pressure and by dead load. In further loading up to $29 \mathrm{psig}(0.2 \mathrm{MPa})$ a load increment of $1.45 \mathrm{psig}(0.01 \mathrm{MPa})$ is used. Above $29 \mathrm{psig}(0.2 \mathrm{MPa})$ a constant increment of $0.145 \mathrm{psig}(0.001 \mathrm{MPa})$ is maintained. Stiffness reformation is applied in each step. To incorporate large displacements and large strains, as well, the updated Lagrangian formulation is used for liner and reinforcement elements; for the concrete elements we apply the total Lagrangian formulation which is suited for large displacements and small strains. Deformation-dependent update of pressure loading is considered.

\section{8 .7 Results}

\subsubsection{Standard plot quantities}

Plots of the quantities that were requested for comparison with experimental data ([4.8.11]) are presented in Appendix A. Aside from quantities which cannot be obtained with the axisymmetric model (e.g., data at penetrations) and from the section forces of the liner which cannot be derived with sufficient precision during yielding from the output results at integration points of the liner 2-D elements, all data listed in [4.8.11] are presented.

The computed results may be compared with experimental data measured in regions which are sufficientiy far away from penetrations and other 
irregularities of the structure. On account of the axial symmetry of the analysis model there is no angular dependence of the computed data.

\subsubsection{Evaluation of analysis results at special structural positions in view of potential failure mechanisms}

To follow the evolution of damage in concrete and straining in steel at higher pressure levels at positions of the structure where deformations are expected to be severe, the following sections denoted by elevations (EL) levels, were selected:

a) Positions of selected concrete and reinforcement sections

BLC: basemat lower center region (radius 0 to $4^{\prime}, E L-\left(1^{\prime}-7^{\prime \prime}\right)$ and $0^{\prime}$ )

CBI: cylinder basemat intersection (between EL $0^{\prime}$ and $5^{\prime}-6^{\prime \prime}$ )

CTL10: cylinder near terminating layer 10 (between EL $5^{\prime}-6^{\prime \prime}$ and $7^{\prime}-7^{\prime \prime}$ )

CC: cylinder center section (between EL $12^{\prime}-0^{\prime \prime}$ and $14^{\prime}-0^{\prime \prime}$ )

CSL: cylinder near springline (between EL $22^{\prime}-0^{\prime \prime}$ and $24^{\prime}-0^{\prime \prime}$ )

DSL: dome near springline (between $0^{\circ}$ and $11.3^{\circ}$ )

DTL8: dome near terminating layer 8 (between $32^{\circ}$ and $42^{\circ}$ )

DC: dome center (between $50^{\circ}$ and $60^{\circ}$ )

DTO: dome top (between $70^{\circ}$ and $90^{\circ}$ )

BH: hoop reinforcement in basemat near lower surface

BL11: layer 11 reinforcement near basemat/cylinder intersection

b) Liner positions

LK: $\quad$ liner knuckle

LLC: liner lower cylinder (between EL $2^{\prime}-0^{\prime \prime}$ and $5^{\prime}-0^{\prime \prime}$ )

LCC: liner center cylinder (between EL 12'-0" and $14^{\prime}-0^{\prime \prime}$ )

\subsection{Evolution of damage in concrete}

Table 4.8 .7 exhibits the progress of crack formation in the various structural regions. Figure 4.8 .14 shows the evolution of crack patterns at the cylinder basemat intersection for various selected values of internal pressure. The following notations for the identification of crack planes is used:

hoop cracks: normal of crack plane points in circumferential direction 
meridional cracks: normal of crack plane parallel to meridional reinforcement. (definition holds for cylindrical and dome sections as well)

According to Table 4.8 .7 hoop cracks begin generally to form at about $P_{I}=$ $40.6-42.1$ psi $(0.28-0.29 \mathrm{MPa})$ in the cylindrical regions and are fully developed (through cracks) at about $P_{T}=42.1 \mathrm{psi}(0.29 \mathrm{MPa})$. Through-wall hoop cracks at the springline are developed at the same pressure. In the lower cylindrical region where strong bending behavior is dominant the hoop cracks are fully developed at about $49.3 \mathrm{psi}(0.34 \mathrm{MPa})$. Due to bending in the lower basemat hoop cracks form between 53.7 and 56.6 psi $(0.37$ and 0.39 $\mathrm{MPa})$.

In the dome, hoop and meridional cracks begin to form at about $P_{I}=43.5$ psi (0.3 $\mathrm{MPa}$ ) due to equivalent straining in both directions. On account of increasing and varying amounts of bending in the dome and the transition region of the springline the planes of the meridional cracks will be fully developed at higher pressures, ranging from $50.8 \mathrm{psi}(0.35 \mathrm{MPa})$ to $76.8 \mathrm{ps} i$ $(0.53 \mathrm{MPa})$.

Meridional cracks in the cylinder center begin to form at 75.4 psi $(0.52$ $\mathrm{MPa})$. They penetrate the wall at about $81.2 \mathrm{psi}(0.56 \mathrm{MPa})$. Due to bending in the lower cylinder meridional cracks form first at inner sections of the cylindrical wall at lower pressure values $(46.4-47.9$ psi, $0.32-0.33$ MPa). Through-wall meridional cracks appear in this section between 84.1 psi $(0.58 \mathrm{MPa})$ and $87 \mathrm{psi}(0.60 \mathrm{MPa})$.

The pressure values obtained by the numerical analysis for cracking in the cylinder and dome center sections agree well with results of simple hand calculations.

There are several regions where also third crack planes developed. This was observed in the lower basemat center at about $56.6 \mathrm{psi}(0.39 \mathrm{MPa})$ and in regions where the geometry and the contents of steel varies at pressure values ranging from roughly $87 \mathrm{psi}(0.6 \mathrm{MPa})$ through $130 \mathrm{psi}(0.9 \mathrm{MPa})$. A thorough identification of the reasons for these latter crack patterns is, however, impeded by numerical errors which occur in the stress calculations.

Crushing of concrete was only observed at the cylinder/basemat intersection. The evolution of compression and cracking damage in this area is shown in Figure 4.8.14. At an internal pressure of $116 \mathrm{psi}(0.8 \mathrm{MPa})$ crushing of the concrete begins at the corner between cylinder and basemat. A section of $4 \mathrm{in}^{2}\left(25.8 \mathrm{~cm}^{2}\right)$ has failed completely at about $145 \mathrm{psi}(1.0 \mathrm{MPa})$. At $174 \mathrm{psi}(1.2 \mathrm{MPa})$, an area of some $30 \mathrm{in}^{2}\left(193.5 \mathrm{~cm}^{2}\right)$ extending from the cylinder surface to the position of layer 11 reinforcement is crushed. The crushed area amounts to some $45 \mathrm{in}^{2}\left(290.3 \mathrm{~cm}^{2}\right)$ at the pressure of $193 \mathrm{psi}$ (1.33 MPa) where the calculation failed for reasons to be discussed in the next subsection. At this pressure the compression area extends in depth to about two-thirds of the wall thickness and joins the inner zone which is cracked due to bending. There is no appreciable capacity left to carry shear stresses by the concrete at this pressure. 
Table 4.8.7 Development of Crack Formation in Concrete

\begin{tabular}{|c|c|c|c|c|c|c|c|}
\hline CONCRETE - Region & $\underset{(\mathrm{MPa})}{\mathrm{P}_{\mathbf{I}}}$ & $\begin{array}{c}\text { Crack } \\
\mathrm{P}_{\mathrm{T}} \\
(\mathrm{MPa})\end{array}$ & Angle & $\underset{(\mathrm{MPa})}{\mathrm{P}_{\mathrm{I}}}$ & $\begin{array}{c}\text { Crack } \\
\mathrm{P}_{\mathrm{T}} \\
\text { (MPa) }\end{array}$ & Angle & Comments \\
\hline B L C & 0.37 & 0.39 & hoop & 0.37 & 0.41 & $0^{\circ}$ & $\begin{array}{l}\text { Initiation of Crack } 1 \text { and } 2 \text { at } \\
\text { lower Center of Basemat }\end{array}$ \\
\hline C B I & 0.29 & 0.34 & hoop & 0.33 & 0.60 & $\sim 90^{\circ}$ & Initiation of Crack 2 from Inside \\
\hline C T L 10 & 0.28 & 0.29 & hoop & 0.32 & 0.58 & $\sim 90^{\circ}$ & Initiation of Crack 2 from outside \\
\hline C C & 0.28 & 0.29 & hoop & 0.52 & 0.56 & $\begin{array}{r}60^{\circ} \\
\text { to } \\
120^{\circ}\end{array}$ & Initiation of Crack 2 from Inside \\
\hline C S L & 0.29 & 0.29 & hoop & 0.37 & 0.53 & $-93^{0}$ & Initiation of Crack 2 from Inside \\
\hline D S L & 0.29 & 0.29 & hoop & 0.29 & 0.48 & $\sim 100^{\circ}$ & Initiation of Crack 2 from Inside \\
\hline D T L 8 & 0.30 & 0.30 & hoop & 0.30 & 0.37 & $\sim 130^{\circ}$ & $\begin{array}{l}\text { Crack } 2 \text { starting from Termination } \\
\text { of Seismic Reinforcement }\end{array}$ \\
\hline D C & 0.30 & 0.30 & hoop & 0.30 & 0.42 & $\sim 145^{\circ}$ & Initiation of Crack 2 from Inside \\
\hline D T 0 & 0.30 & & hoop & 0.30 & & $-170^{\circ}$ & $\begin{array}{l}\text { Initiation of crack } 1 \text { and } 2 \text { from } \\
\text { Inside }\end{array}$ \\
\hline
\end{tabular}




\subsection{Strains in the reinforcement and the liner}

For various pressure levels the maxima of strains in the regions defined above are listed in Table 4.8.8. The data exhibited are total strains for the reinforcement and accumulated effective plastic strains obtained at integration points for the 2-D-liner elements. The upper left figure in a section of the table denotes meridional strains of the inner reinforcement, the upper right figure the meridional strains in the outer reinforcement layer, and the lower figure refers to hoop strains. The respective maximum value of strains of outer and inner hoop bars is exhibited.

The numerical calculations failed at $193 \mathrm{psi}$ (1.331 MPa) for the following reason. To compute strains at the inner surface of the liner 2-D elements, truss elements with very low stiffness were connected to inner surface nodes of the liner at positions where comparison with measured strains is requested. The stress-strain curves used for these trusses were cut off at a strain of 0.2 . As may be seen from Table 4.8 .8 (line LK (knuckle) in column 7) the accumulated effective plastic strain at respective integration points of the 2-D element exceeds by 4.5 percentage points the cut-off strain of the connected truss element. (The deviation is due to the multiaxial stress state in the knuckle as opposed to the uniaxial stress state in the connected truss elements.)

The high strains developed according to the analysis seem to indicate that the liner knuckle is one of the most vulnerable links in the structure. However, a more thorough assessment of this finding is indispensable for the following reasons. Firstly, the knuckle section was not modeled quite accurately. The change in geometry of the knuckle to fit the ends of the bend to the pattern of the adjacent concrete mesh, as described in section 4.8.3.2.3 (the radius of the knuckle is in the model roughly twice as large as in the structure), should have been considered too by an increased thickness of the bend. Employing a properly scaled thickness would reduce the strains in this section roughly by approximately a factor of two. The moment at the knuckle-liner bottom-junction resulting from membrane stresses in the lower cylindrical part of the liner will become smaller by a factor of two by reducing the knuckle radius by the same amount. Secondly, the material behavior of the knuckle was not determined experimentally. Hence, the missing material data had to be taken from charts [4.8.8]. Generally the strength values in these charts represent lower bounds of the respective data. According to Table 4.8.5 the yield strength and the tensile strength were taken to be $38.8 \mathrm{ksi}(240 \mathrm{MPa}$ ) and $60.2 \mathrm{ksi}(415 \mathrm{MPa})$, respectively.

Taking credit of the above geometric effect and of somewhat more realistic strength values should reduce the maximum strains in the knuckle by a factor of about 2.5. The corresponding estimated strains for various pressures are marked by "*)" in row LK of Table 4.8.8.

A proper remodeling of the knuckle and the surrounding concrete area could not be performed prior to the deadline of this report. The improvements will however be considered in the computations which take into account equilibrium iterations.

From the last column of Table 4.8.8 it is seen that strains in the dome sections (DSL, DTL8, DC and DTO) remain moderate even at the rather high pressure load of $1.33 \mathrm{MPa}(193 \mathrm{ps} \mathrm{i})$. A dominant potential for failure is 
Table 4.8.8 Strains (in Percent) in Reinforcement and Liner

Rebar Legend: Layer 2/Layer 5

Layer $1,3,4,6$

PRESSURE

130 (psi) 145 (psi) 160 (psi) 174 (psi) 189 (psi) 193 (psi)

Position $0.9(\mathrm{MPa}) 1.0(\mathrm{MPa}) 1.1(\mathrm{MPa}) 1.2(\mathrm{MPa}) 1.3(\mathrm{MPa}) 1.33(\mathrm{MPa})$

\begin{tabular}{|c|c|c|c|c|c|c|}
\hline$B L C$ & 0.19 & 0.52 & 1.39 & 2.31 & 2.67 & 2.77 \\
\hline CBI & $\begin{array}{c}0.61 / 0.10 \\
0.06\end{array}$ & $\begin{array}{c}1.35 / 0.11 \\
0.12\end{array}$ & $\begin{array}{c}2.26 / 0.23 \\
0.24\end{array}$ & $\begin{array}{c}3.59 / 1.43 \\
0.50\end{array}$ & $\begin{array}{c}5.59 / 2.00 \\
0.91\end{array}$ & $\begin{array}{c}6.54 / 2.45 \\
1.15\end{array}$ \\
\hline CTL10 & $\begin{array}{l}10.10 \\
0.22\end{array}$ & $\begin{array}{l}10.06 \\
0.55\end{array}$ & $\begin{array}{l}10.06 \\
1.06\end{array}$ & $\begin{array}{l}10.13 \\
1.97\end{array}$ & $\begin{array}{l}10.15 \\
3.36\end{array}$ & $\begin{array}{l}10.15 \\
4.24\end{array}$ \\
\hline $\mathrm{CC}$ & $\begin{array}{c}0.12 / 0.11 \\
0.27\end{array}$ & $\begin{array}{c}0.13 / 0.23 \\
1.2\end{array}$ & $\begin{array}{c}0.18 / 0.24 \\
2.29\end{array}$ & $\begin{array}{c}0.33 / 0.25 \\
3.59\end{array}$ & $\begin{array}{c}1.38 / 1.85 \\
6.18\end{array}$ & $\begin{array}{c}2.16 / 2.78 \\
7.80\end{array}$ \\
\hline CSL & $\begin{array}{c}0.15 / 0.09 \\
0.18\end{array}$ & $\begin{array}{c}0.33 / 0.11 \\
0.38\end{array}$ & $\begin{array}{c}0.62 / 0.21 \\
0.82\end{array}$ & $\begin{array}{c}0.96 / 0.38 \\
1.50\end{array}$ & $\begin{array}{c}1.56 / 0.51 \\
2.92\end{array}$ & $\begin{array}{c}1.97 / 0.71 \\
3.77\end{array}$ \\
\hline DSL & $\begin{array}{c}0.14 / 0.07 \\
0.14\end{array}$ & $\begin{array}{c}0.25 / 0.11 \\
0.26\end{array}$ & $\begin{array}{c}0.58 / 0.19 \\
0.54\end{array}$ & $\begin{array}{c}0.89 / 0.29 \\
1.04\end{array}$ & $\begin{array}{c}1.50 / 0.39 \\
2.12\end{array}$ & $\begin{array}{c}1.91 / 0.81 \\
2.87\end{array}$ \\
\hline DTL8 & 0.12 & 0.14 & 0.18 & 0.24 & 0.70 & 0.96 \\
\hline$D C$ & $\begin{array}{c}0.10 / 0.09 \\
0.14\end{array}$ & $\begin{array}{c}0.12 / 0.11 \\
0.16\end{array}$ & $\begin{array}{c}0.15 / 0.14 \\
0.20\end{array}$ & $\begin{array}{c}0.18 / 0.16 \\
0.25\end{array}$ & $\begin{array}{c}0.28 / 0.20 \\
0.59\end{array}$ & $\begin{array}{c}0.36 / 0.21 \\
0.78\end{array}$ \\
\hline DTO & $\begin{array}{c}0.21 / 0.13 \\
0.13\end{array}$ & $\begin{array}{c}0.23 / 0.15 \\
0.15\end{array}$ & $\begin{array}{c}0.37 / 0.19 \\
0.18\end{array}$ & $\begin{array}{c}0.58 / 0.27 \\
0.22\end{array}$ & $\begin{array}{c}1.22 / 0.51 \\
0.35\end{array}$ & $\begin{array}{c}1.61 / 0.65 \\
0.52\end{array}$ \\
\hline LK & $\begin{array}{l}0.21 \\
\left.0.08^{\star}\right)\end{array}$ & $\begin{array}{l}0.51 \\
0.20 *)\end{array}$ & $\begin{array}{l}2.19 \\
\left.0.90^{*}\right)\end{array}$ & $\begin{array}{l}9.28 \\
\left.3.70^{\star}\right)\end{array}$ & $\begin{array}{l}20.0 \\
\left.8.00^{*}\right)\end{array}$ & $\begin{array}{l}24.5 \\
\left.9.80^{\star}\right)\end{array}$ \\
\hline LLC & 0.00 & 0.23 & 1.35 & 2.54 & 4.08 & 4.89 \\
\hline LCC & 0.16 & 1.26 & 2.54 & 4.17 & 7.98 & 10.5 \\
\hline $\mathrm{BH}$ & 0.13 & 0.21 & 0.36 & 0.81 & 1.03 & 1.08 \\
\hline BL 11 & 0.19 & 0.45 & 0.82 & 0.99 & 1.40 & 1.57 \\
\hline
\end{tabular}


therefore not seen in these areas, although a slight loss of the amount of load carried by terminating meridional reinforcement may occur due to circumferential strains in the dome shell (DC). Also for the basemat bending reinforcement $(B L C)$ and the basemat hoop reinforcement (BH) failure is not expected.

Strongest straining arises according to the present analysis in the following structural parts:

liner mid-section (LCC)

liner knuckle (LK)

hoop reinforcement in cylinder mid-section (CC)

although the strains in the hoop bars at the lower cylinder (CBI) remain below some 1.15 percent during loading up to the highest pressure reached, a gradual weakening of the bonds between concrete and layer 11 bars cannot be excluded above some 0.5 percent strain.

\subsection{Potential Failure Mechanisms}

\subsection{Liner leakage}

Taking into account that ultimate strains will be reduced under multiaxial conditions (triaxiality factor [4.8.12]) by a factor between $\sqrt{ } 3$ and 2 for cylindrical and spherical symmetry of shells respectively, and assuming further a reduction factor for welds of 0.9 and for possible undetected faults of 0.9 , about seven percent is reasonable in the failure strain of the liner. (The uniaxial ultimate strain is 16.3 percent according to tests.) Excluding leakage due to low weld quality, which in general is a) ready detected at strains of some two percent, the liner may be assumed with high confidence to be leak proof up to strains of five percent. According to this argumentation, which is largely based upon experience, and due to the computed strains of Table 4.8.8, failure of the liner mid-section seems to be likely to occur between $174 \mathrm{psi}(1.2 \mathrm{MPa})$ and $189 \mathrm{psi}$ (1.3 MPa).

\subsection{Liner knuckle fracture}

The reasoning of section 4.8.7.2.3.1, though applicable to multiaxial membrane stress states, cannot be employed for the knuckle, where predominantly bending occurs. Here, a stress criterion seems to be better suited.

Choosing a best estimate value of the tensile strength that is 20 percent higher than the lower bound value of $60 \mathrm{ksi}$ (415 MPa) according to Table 4.8.5 and covering possible enhancement of stresses due to stress concentrations by a factor of two-thirds, yields an effective ultimate tensile strength of some $48 \mathrm{ksi}(330 \mathrm{MPa})$. Comparing this value with the calculated effective stress by taking into consideration a stress reduction factor of about two, which is due to the geometric inconsistency between the model and the structure, leads at $189 \mathrm{psi}(1.3 \mathrm{MPa})$ internal pressure to an effective stress of about $44 \mathrm{ksi}(300 \mathrm{MPa})$. Hence, fracture of the knuckle does not seem to be likely to occur up to internal pressures of some 189 psi (1.3 $\mathrm{MPa})$. It is, however, to be noted that this conclusion which is based 
upon the present model of the knuckle might be subject to revision in the refined investigation.

\subsection{Hoop reinforcement in the mid-section of the concrete cylinder}

The present results show that failure of the structure due to rapid extension of yielding of hoop reinforcement between $181 \mathrm{psi}(1.25 \mathrm{MPa})$ and $188 \mathrm{ps} i$ (1.3 MPa) cannot be excluded. There is always a chance for failure of a few bars at lower strains (e.g., due to improper splice connections). If, say, one rebar out of 20 in this middle section would fail at 188 psi (1.3 MPa), then the stress in each of the remaining hoops, would be larger than the tensile strength and failure would be unavoidable (as may be concluded from Table 4.8.8 and Figure 4.8.6). On the other hand, total failure of the hoop reinforcement may be excluded if the internal pressure does not exceed 174 psi (1.2 MPa), provided at least 95 percent of the hoop rebars remain active in this section.

\subsection{Cylinder/basemat intersection}

As pointed out in section 4.8.7.2.1 and Figure 4.8.14 the zone crushed concrete is rather confined up to the maximum pressure loading reached in the analysis. Hence, in this pressure range a complete loss of bond between concrete and layer 11 bars on account of crushing does not occur. On the other hand, the strains in the hoop reinforcement in the lower cylinder reach 0.5 percent at $74 \mathrm{psi}(1.2 \mathrm{MPa})$. This circumferential strain may cause local loss of bond between the meridional rebars and the concrete. The gap widths between \# 4 rebars of 1 ayer 11 and concrete are at this strain roughly 0.01 in $(0.25 \mathrm{~mm})$. Hence, partial loss of shear and tensile load carrying capacity of the layer 11 rebars cannot be excluded. The shear loads carried by concrete at the intersection must according to the damage exhibited in Figure 4.8 .14 be assumed to be negligible at $174 \mathrm{psi}$ (1.2 MPa). If weakening of the steel-concrete bond is considered, also \# 6 rebars of layer 10 might lose their load carrying capacity in meridional direction. Assuming, for example, a loss of load carrying capacity of 40 percent (due to weakening of the steel-concrete bonds at a circumferential strain of 0.5 percent) for bars of layers 10 and 11 , the stresses in the meridional reinforcement would be increased by a factor of 1.26. The maximum strain in the meridional bars at the intersection (CBI) amounts to 3.59 percent at a pressure of $174 \mathrm{psi}(1.2 \mathrm{MPa}$ ). The corresponding stress (Figure 4.8.6) is roughly $93 \mathrm{ksi}(641 \mathrm{MPa})$. In view of the tensile strength of some $107 \mathrm{ks} i$ (740 MPa), it is obvious that an increase in stress by 26 percent cannot be carried by the meridional reinforcement. Under these conditions a combined shear and tensile failure at the intersection is very likely to happen at pressures of about $174 \mathrm{psi}(1.2 \mathrm{MPa})$.

Following the same reasoning at a pressure of $160 \mathrm{psi}$ ( $1.1 \mathrm{MPa}$ ), where maximum circumferential strains amount to 0.24 percent in the CBI-region, a load carrying reduction for layer 10 and 11 rebars of 20 percent would increase the stresses in the meridional bars from $84 \mathrm{ksi}$ ( $580 \mathrm{MPa}$ ) to some $94.3 \mathrm{ksi}(650 \mathrm{MPa})$. Under these conditions the meridional and the shear loads at the cylinder/basemat intersection should still be carried by the reinforcement. In addition, as bending will lock to some extent the reinforcement that terminates in the lower section of the concrete cylinder, there is a high confidence that the intersection does not fail in the pressure range up to $160 \mathrm{psi}(1.1 \mathrm{MPa})$.

$$
-328-
$$




\subsubsection{Deformation History}

A sequence of pressure-deformation states is shown in Figure $4.8 .15 \mathrm{a}$ and Figure 4.8.15b. The drawings are self-explanatory and need not be commented in detail. It is, however, pointed out that the deformations are rather moderate up to pressures of some $130.5 \mathrm{psi}(0.9 \mathrm{MPa})$, whereas above pressure loads of some $145 \mathrm{psi}(1.0 \mathrm{MPa})$, displacements increase very quickly.

\subsubsection{Concluding Remarks}

The discussion of potential failure mechanisms as identified with the axisymmetric model using straight-forward numerical computations without iteration shows that there is no unique distinction of failure modes with respect to the pressure scale. Any one of the mechanisms elaborated upon may be the first in causing failure of the structure. At about 174 psi (1.2 $\mathrm{MPa}$ ) failure due to one or the other of the discussed mechanisms cannot be excluded. The probability for structural failure seems, however, to be low in the pressure range up to $160 \mathrm{psi}$ (1.1 MPa). This evaluation does not seem to become subject to revision if equilibrium iteration is incorporated in the numerical computations, as calculated stress-strain values fit to a high degree of approximation the stress-strain curves for the reinforcement and the liner, thus indicating that equilibrium has been reached at least for stresses above the yield stress. It must, however, be noted that other failure mechanisms may be activated prior to those which could be identified within the axisymmetric model used here. For example, there might occur leakage in seals or fracture at liner/penetration junctions. 


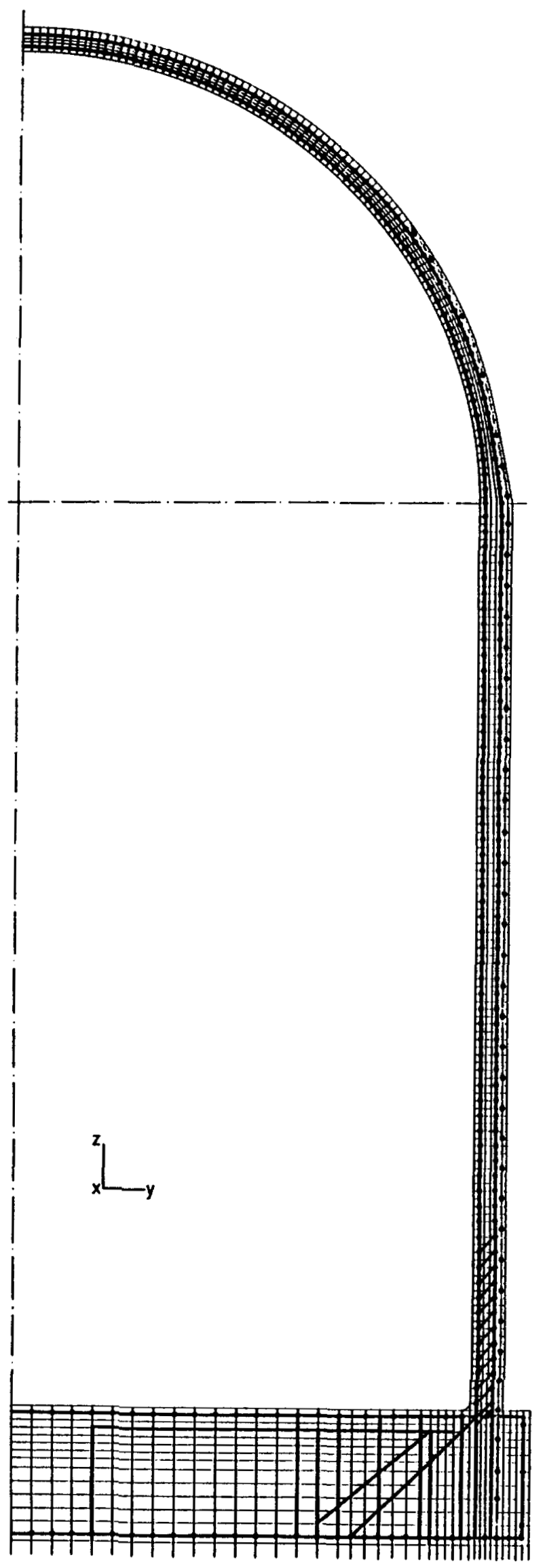

Figure 4.8.1: F E - Mesh of Containment Model $-330$ 


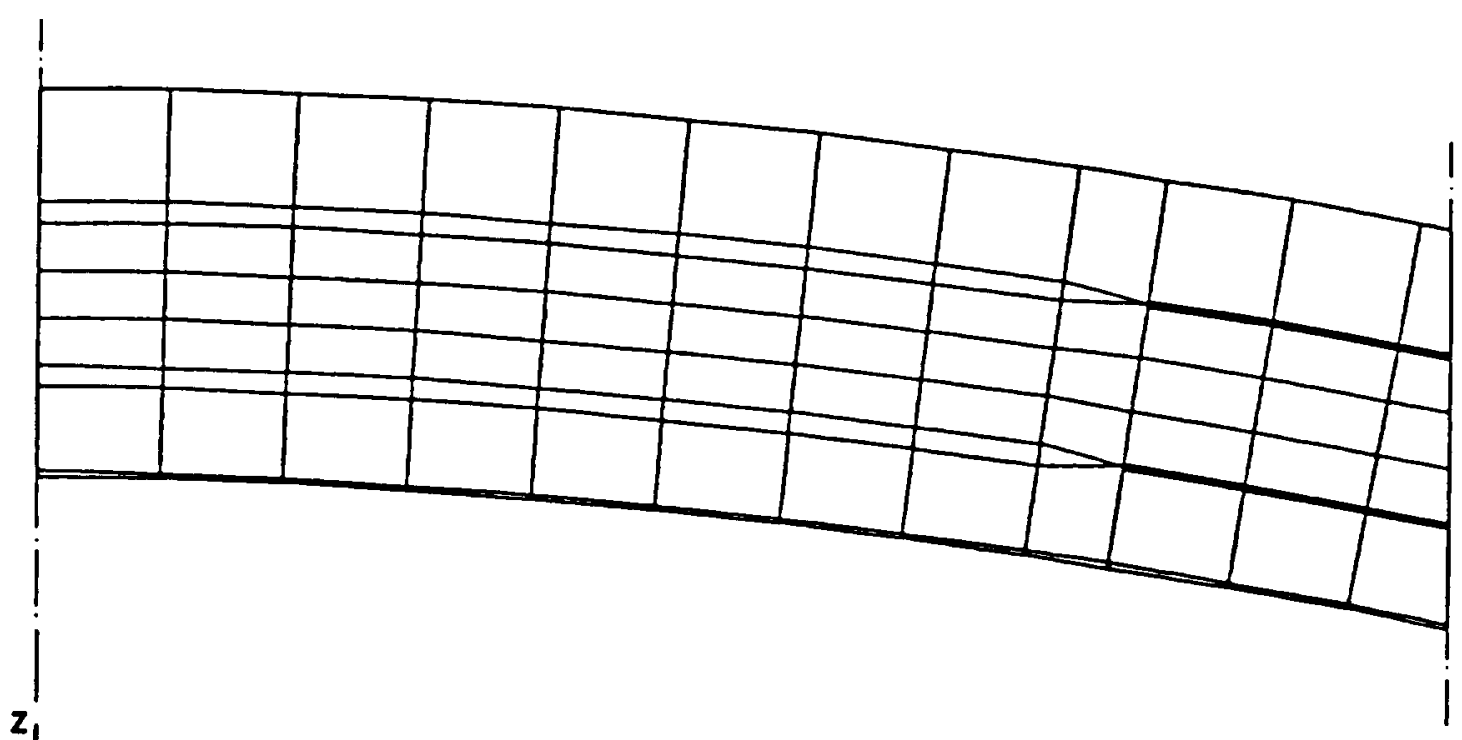

$x$

Figure 4.8.2: Top of Dome - Steel Plates

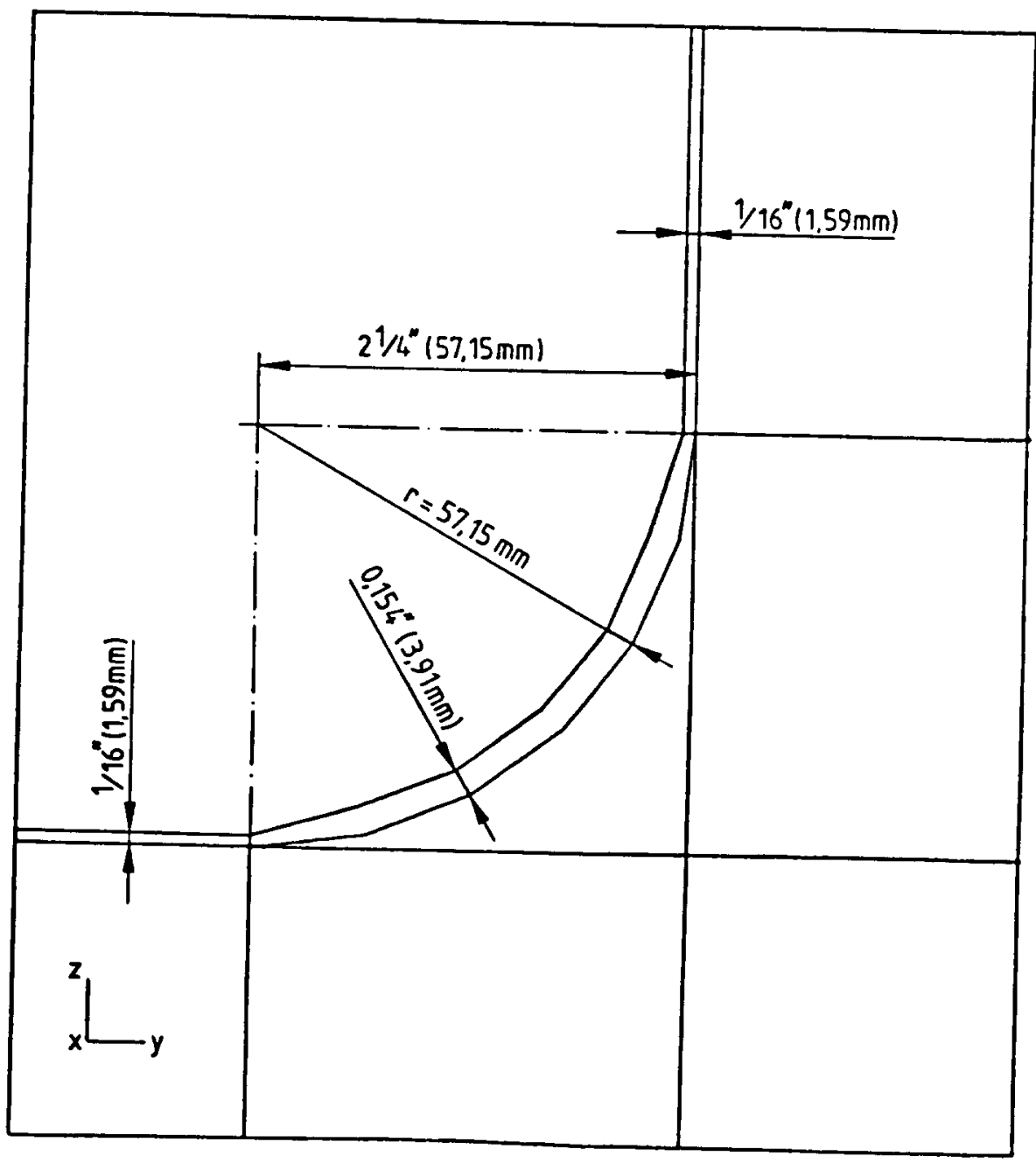

Figure 4.8.4: Knuckle Region at Cylinder - Basemat Intersection $-331-$ 


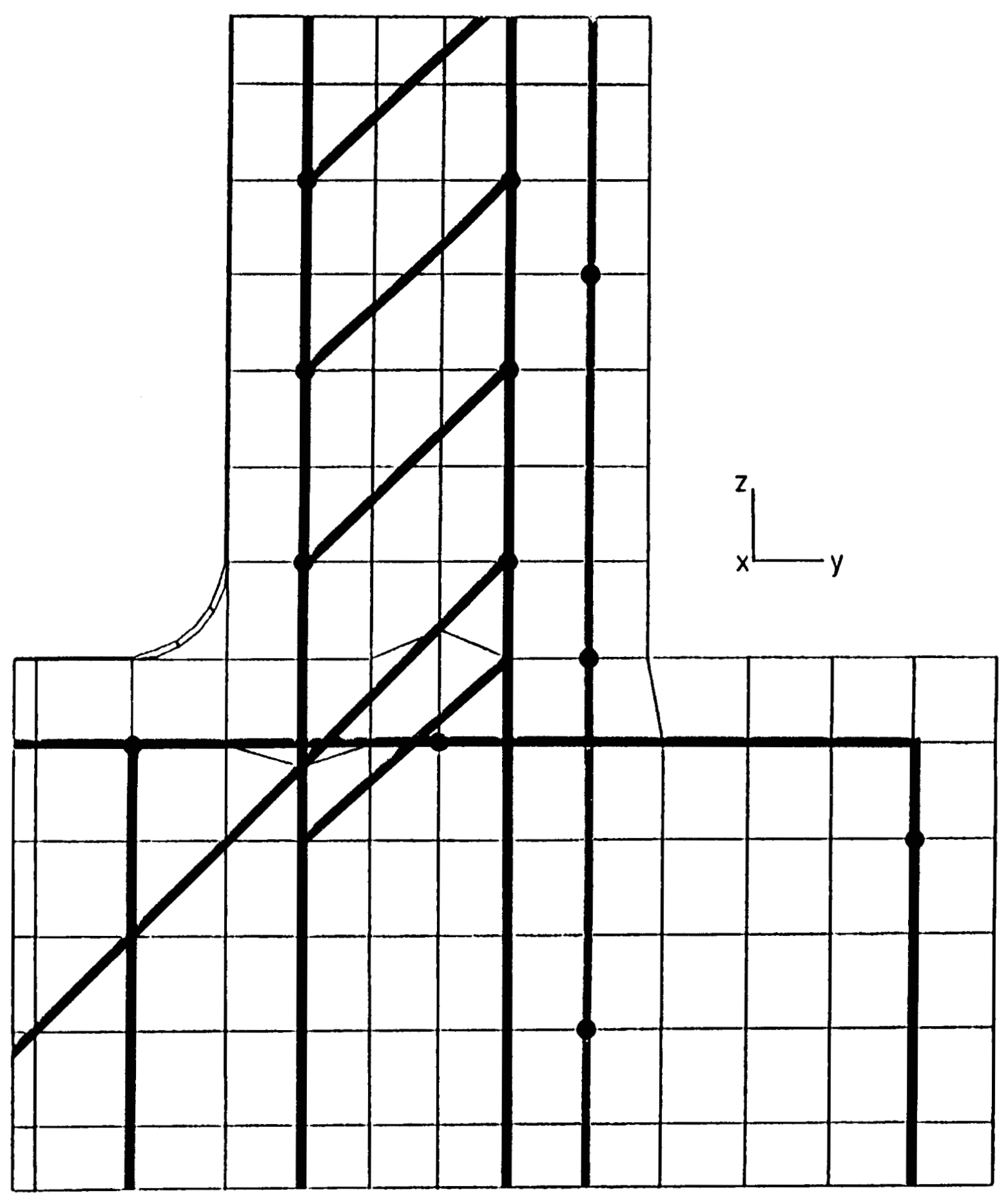

Figure 4.8.3: Cylinder - Basemat Intersection 


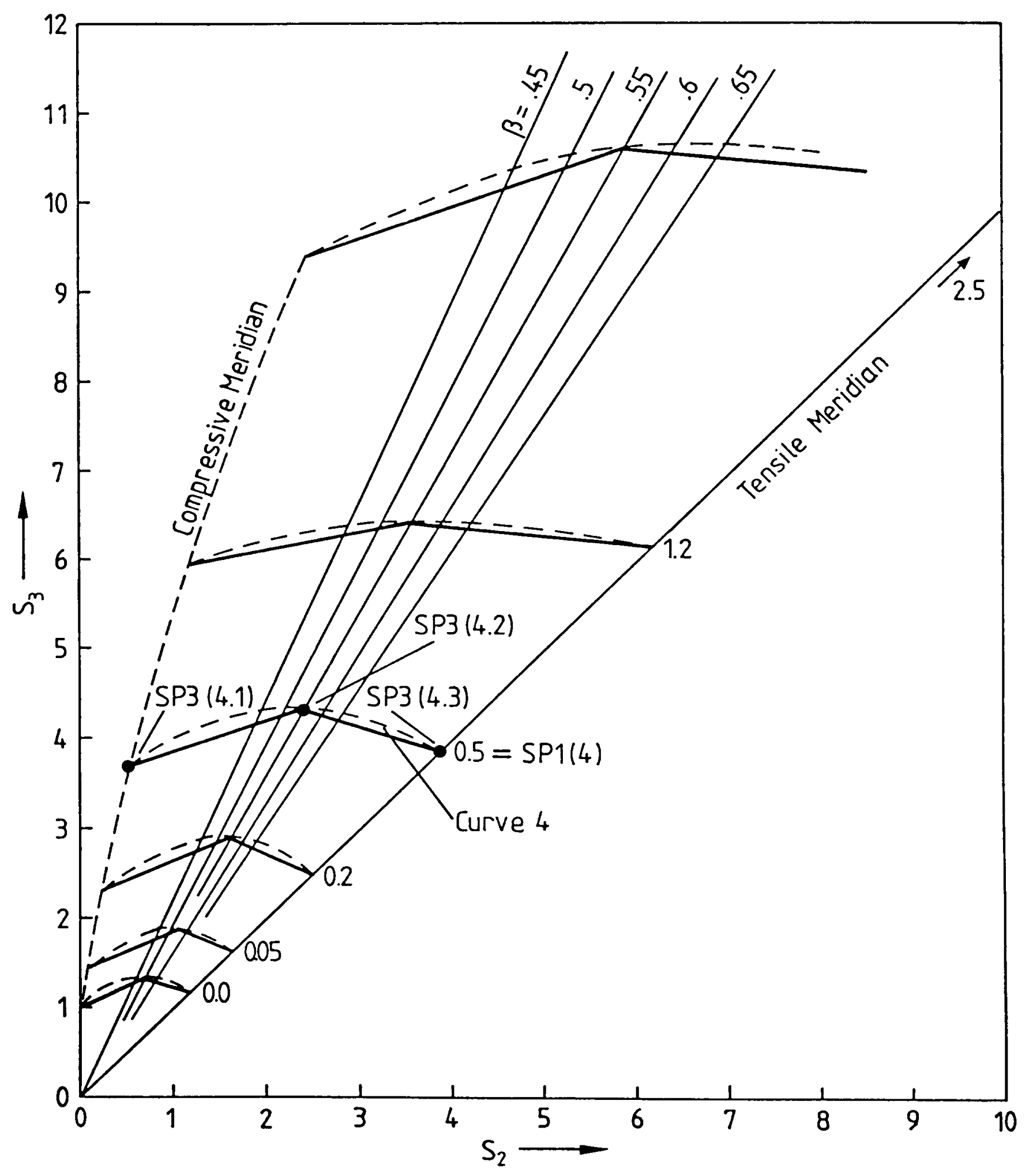

Figure 4.8.5: ADINA Compression Failure Envelopes (Concrete)

$$
S_{i}=\sigma_{i} / \sigma_{c} ; \sigma^{t} / \sigma_{c}=0.073 ; \bar{\beta}=0.55
$$

Data according to OTIOSEN - Charts by University of Karlsruhe 


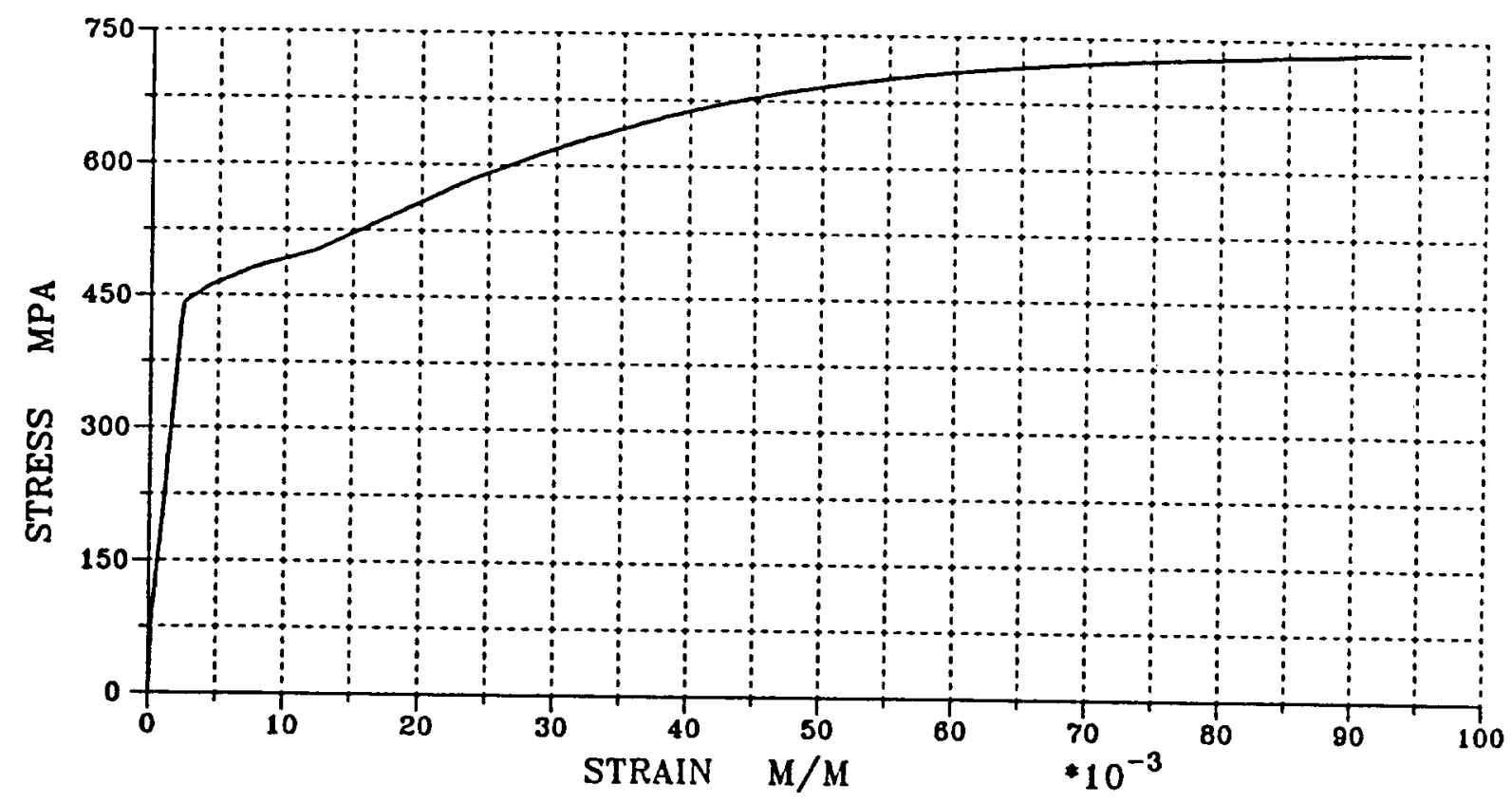

Figure 4.8.6: Rebar \#4 Modified mean Stress-Strain Curve (Tests by University of Karlsruhe)

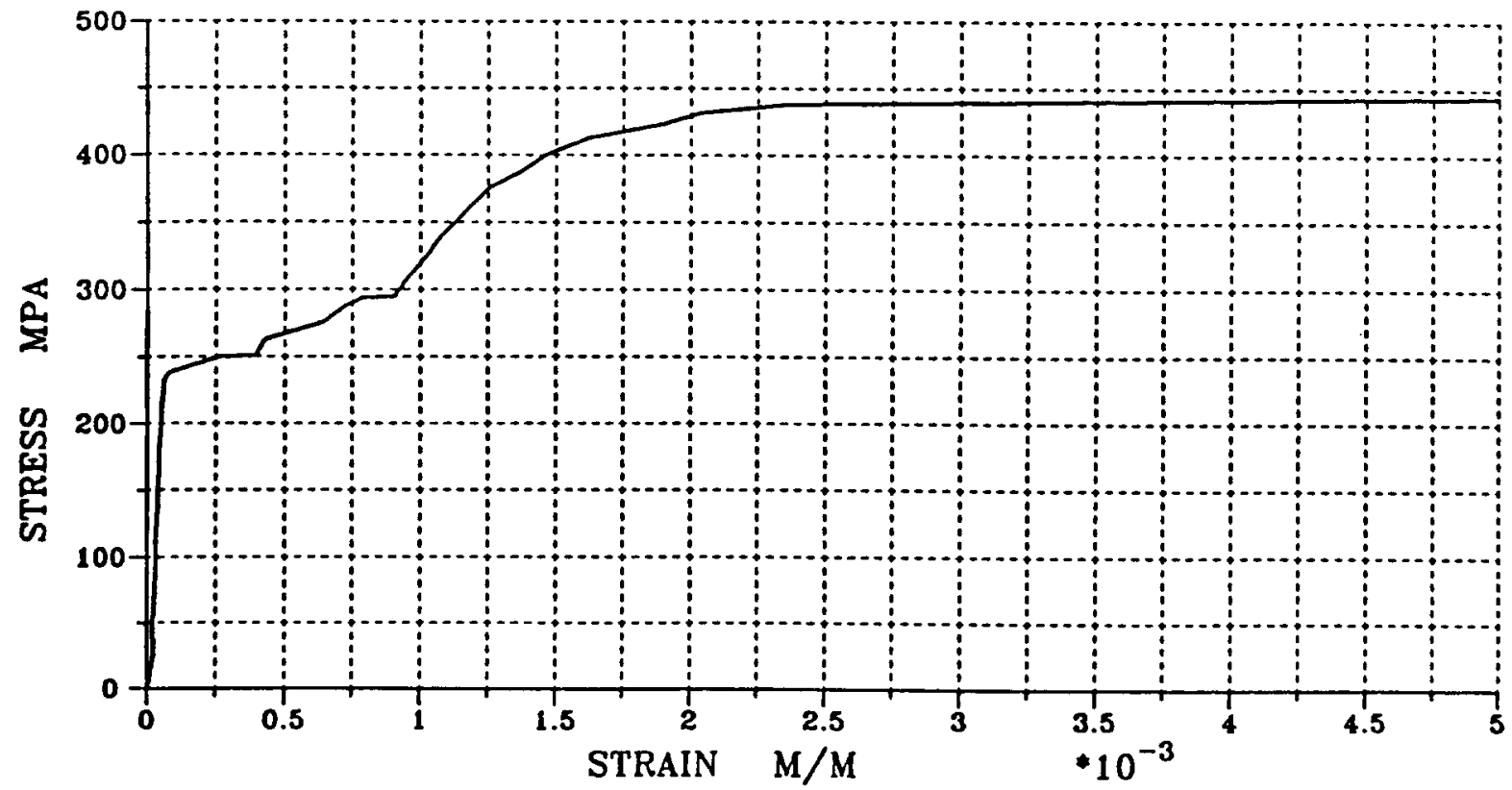

Figure 4.8.7: Rebar \#4 Seismic Reinforcement Modified mean Stress-Strain Curve (Tests by University of Karlsruhe) 


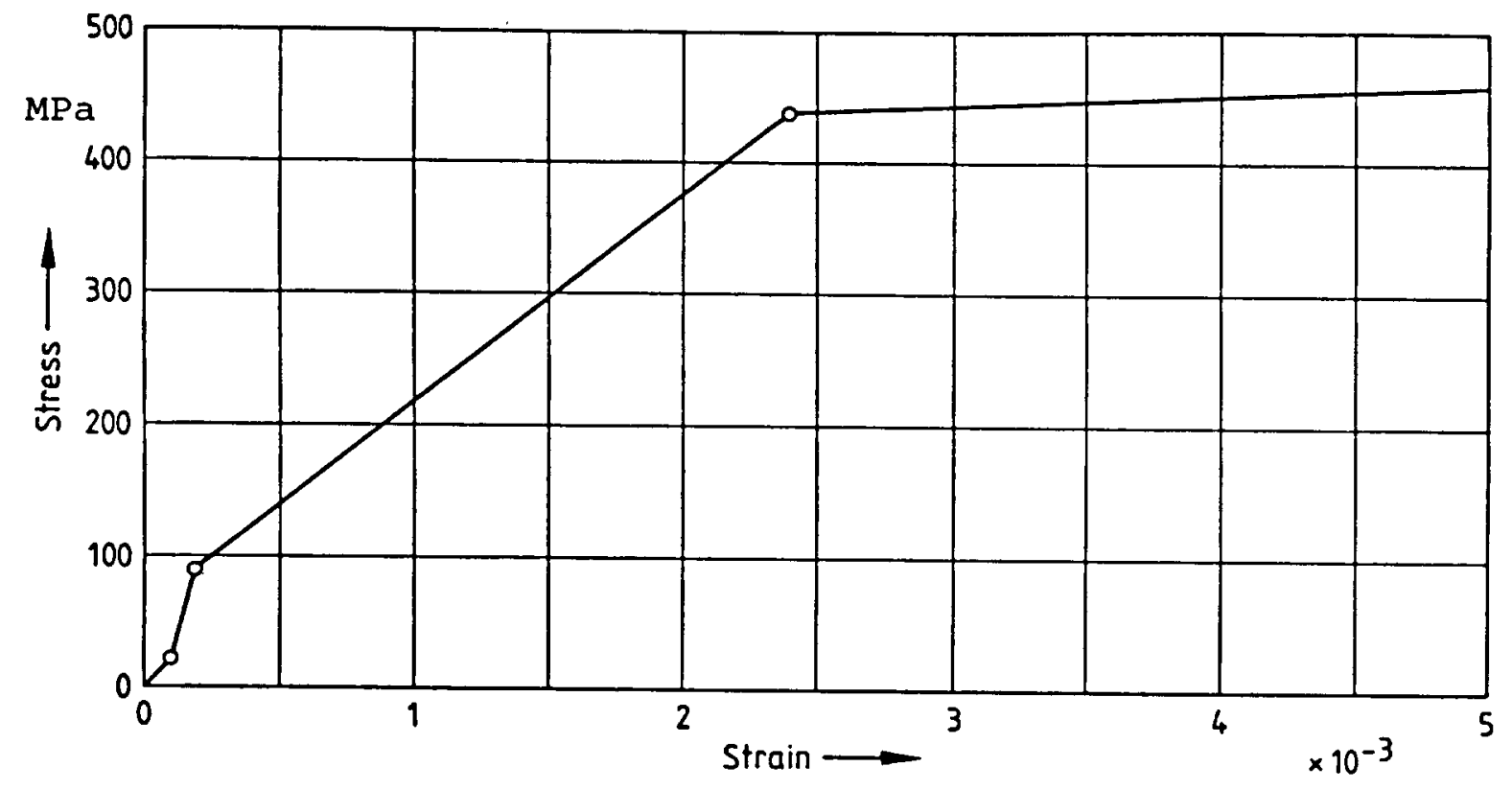

Figure 4.8.8: Rebar \#4 Modified Stress-Strain Curve (Analysis)

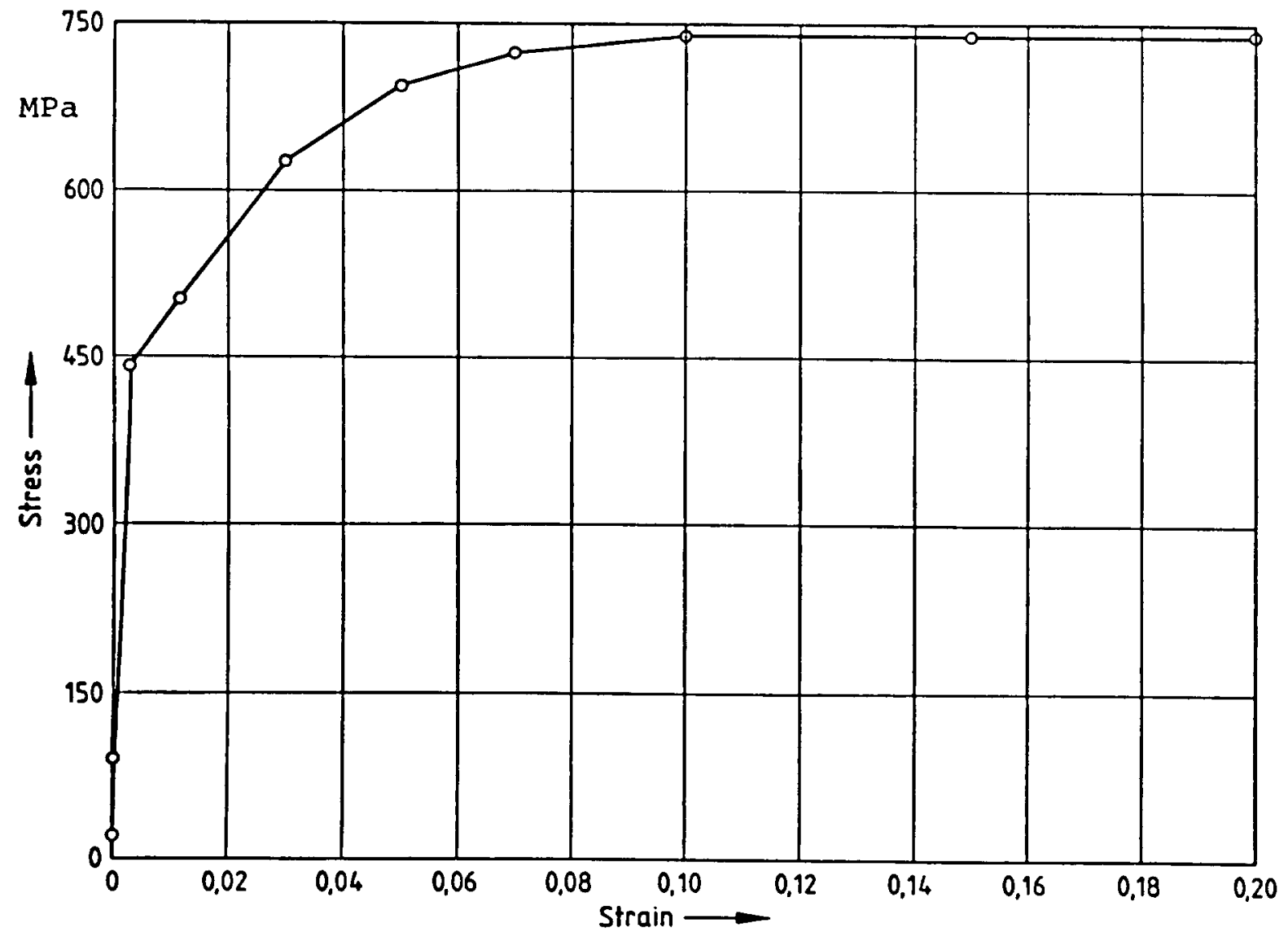

Figure 4.8.9: Rebar \#4 Modified Stress-Strain Curve (Analysis) 


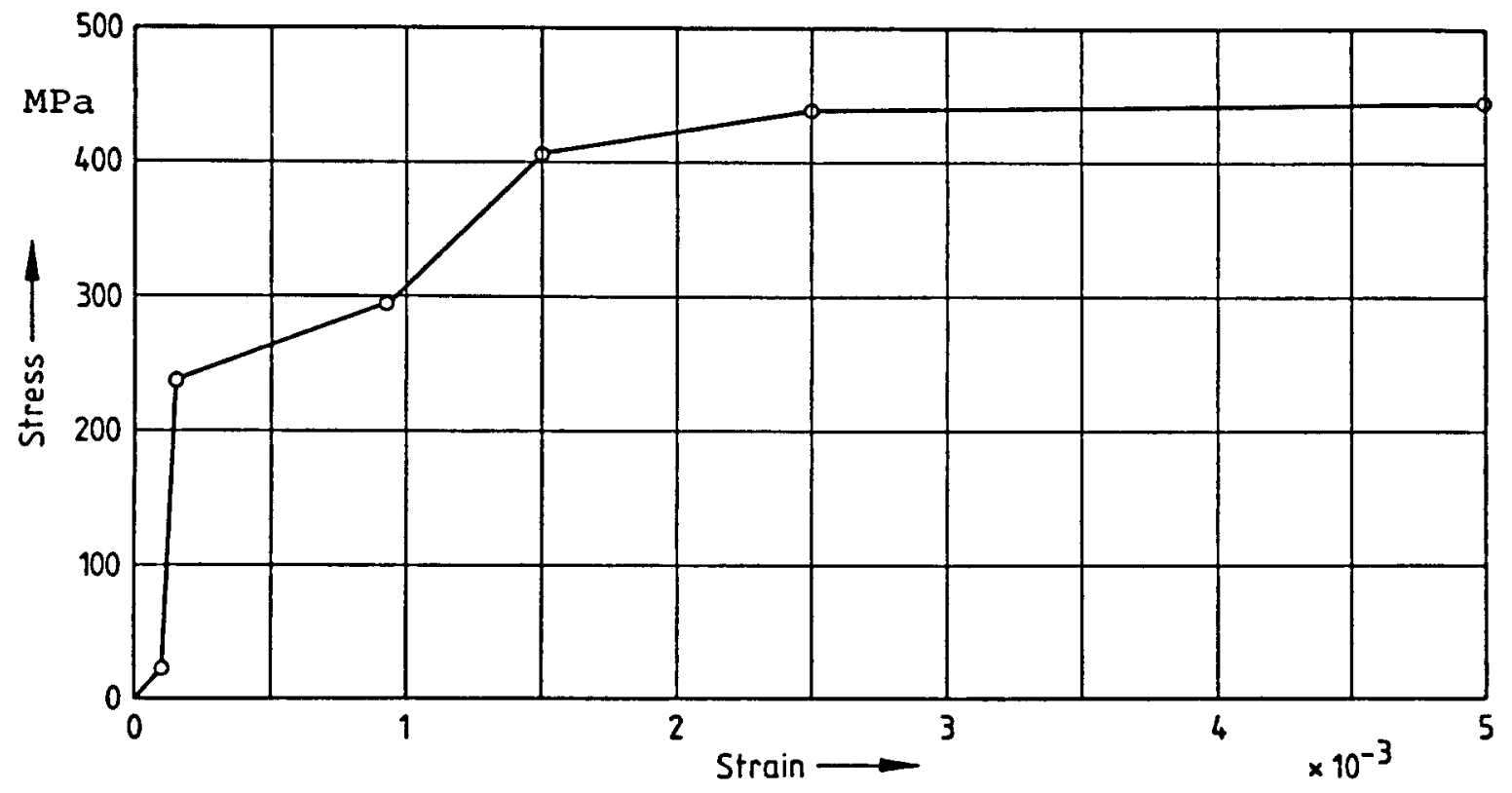

Figure 4.8.10: Rebar \#4 Seismic Reinforcement Modified STRESS - STRAIN - Curve (Analysis)

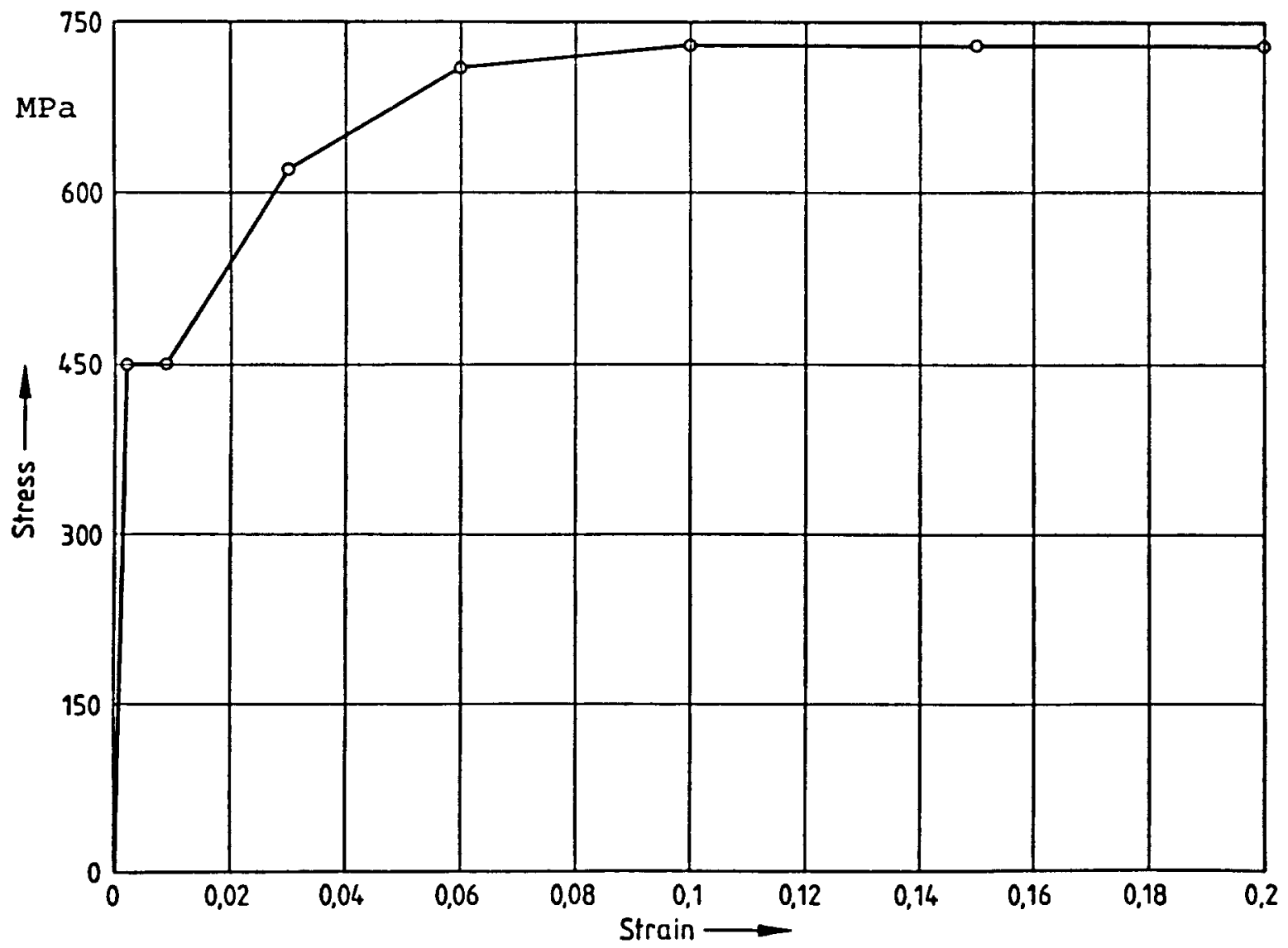

Figure 4.8.11: Rebar \#4 Stress-Strain Curve for bare Steel (Tests by University of Karlsruhe) 


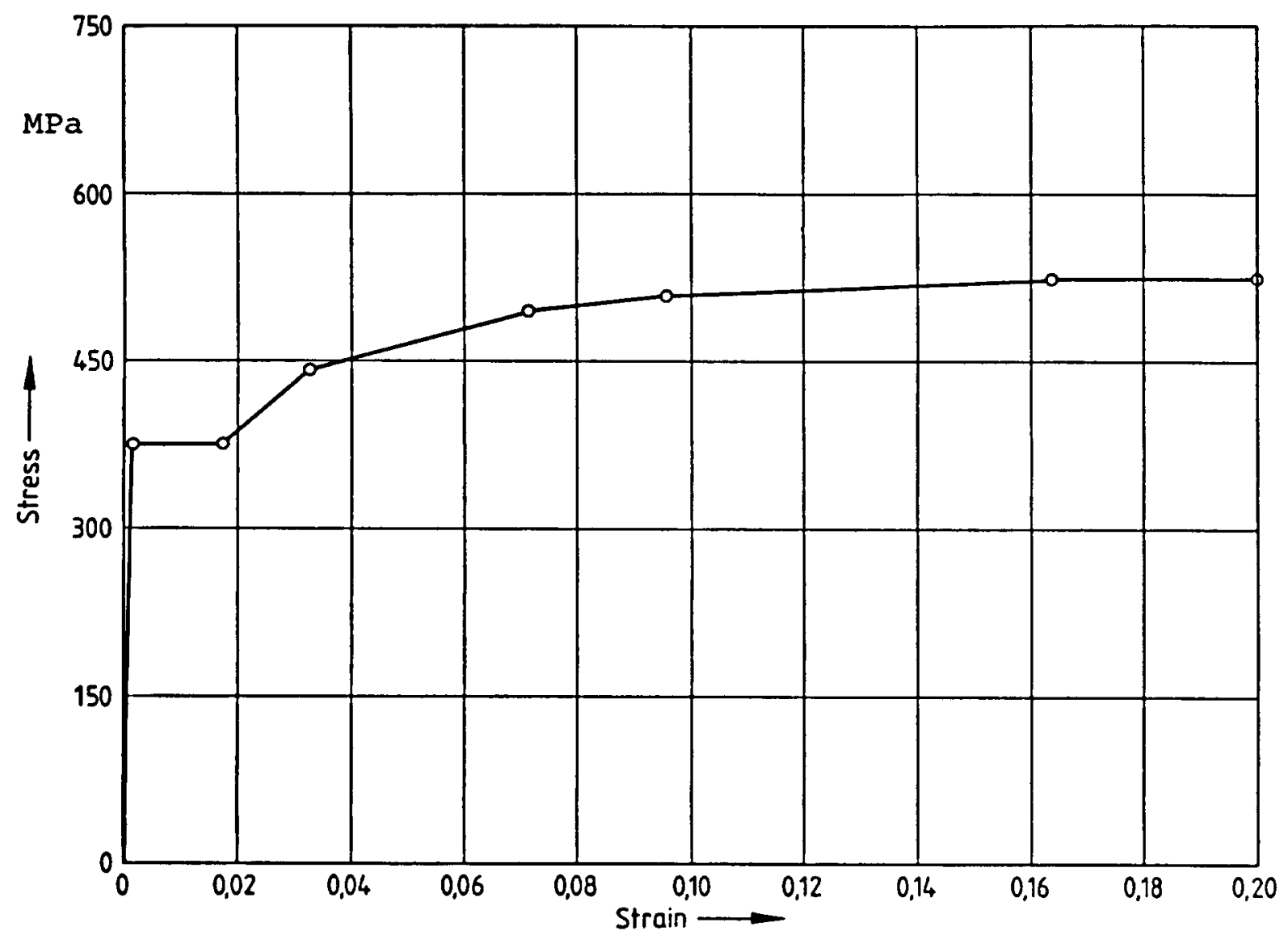

Figure 4.8.12: Steel-Liner 1/16" Stress-Strain Curve

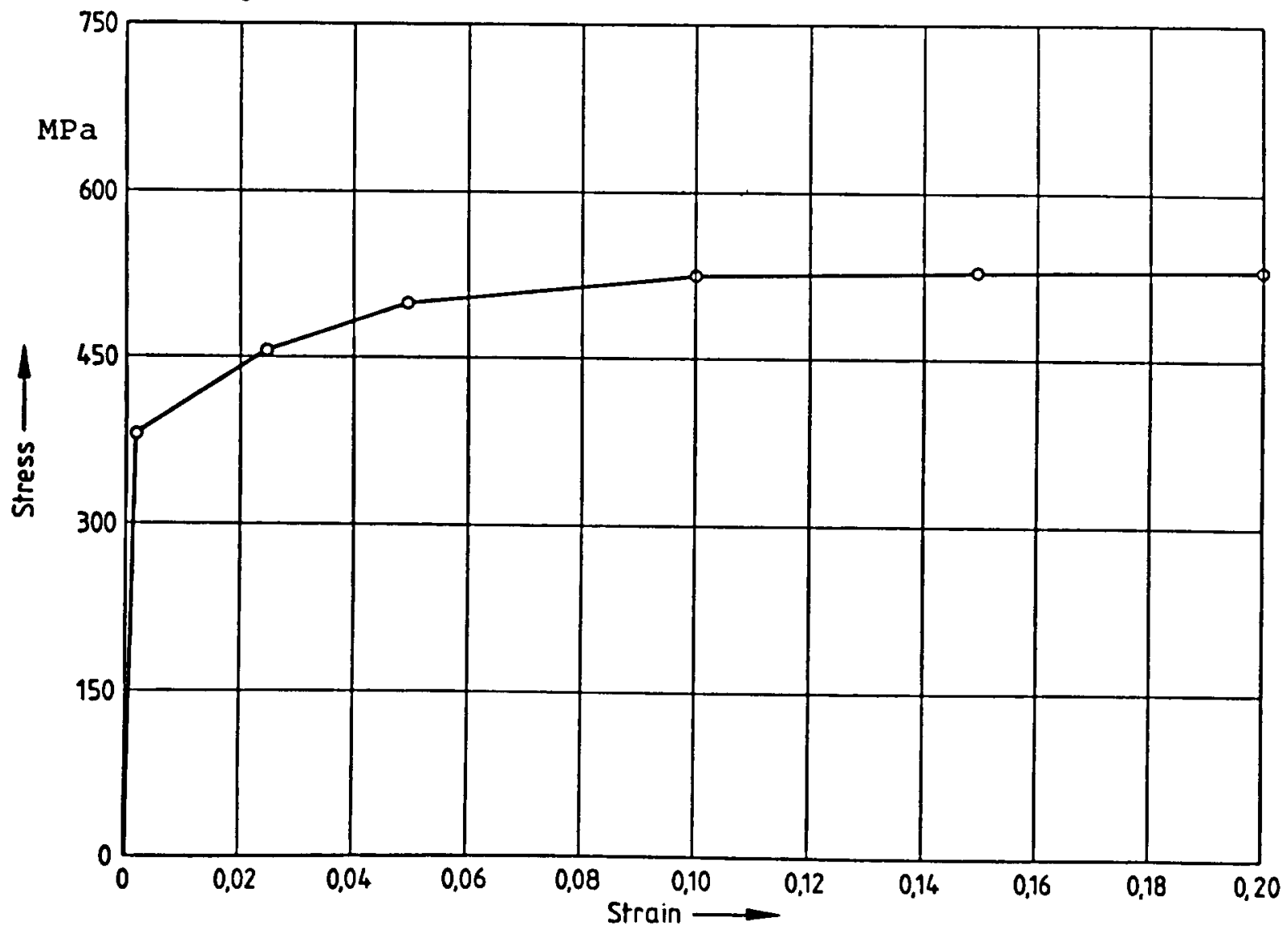

Figure 4.8.13: Steel-Liner 1/12" Stress-Strain Curve 


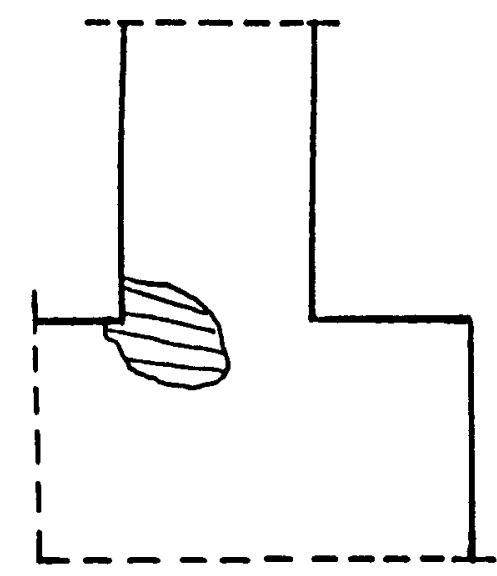

$0.2 \mathrm{MPa}$

$\stackrel{1}{\omega}$

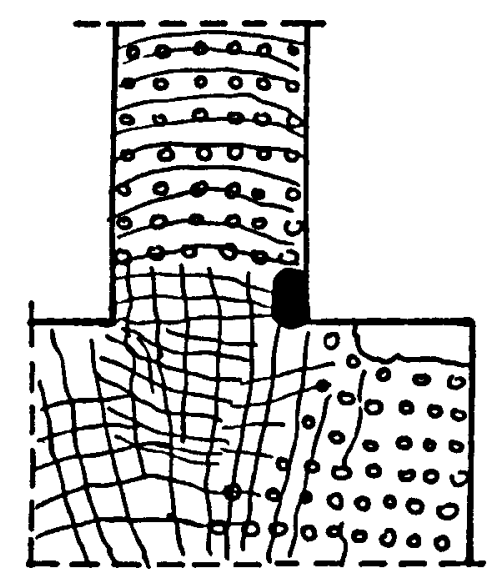

$1,0 \mathrm{MPa}$

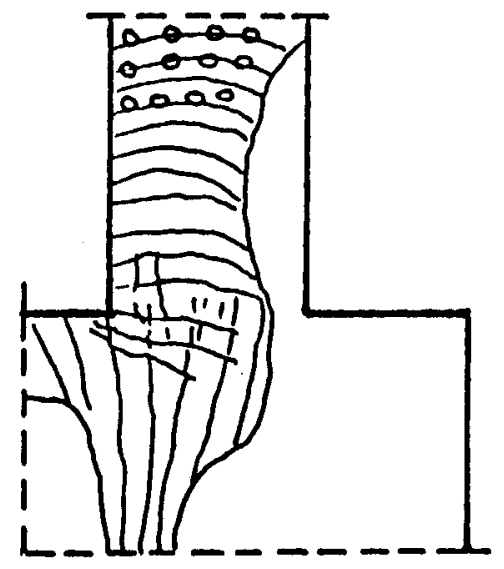

$0.4 \mathrm{MPa}$

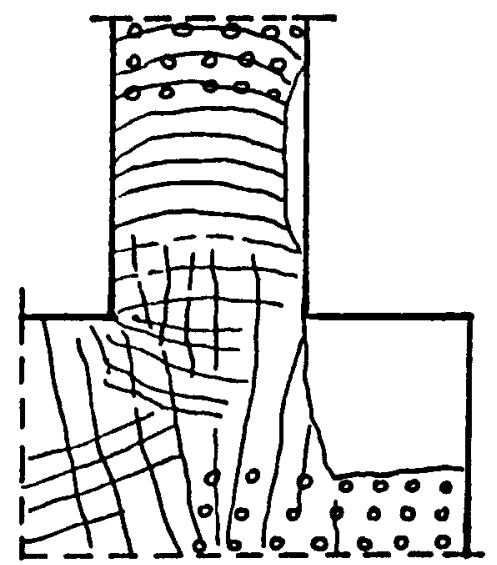

$0.6 \mathrm{MPa}$

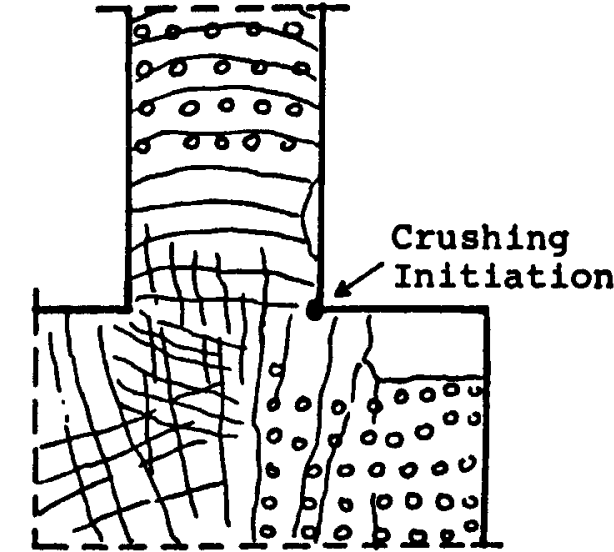

$0.8 \mathrm{MPa}$

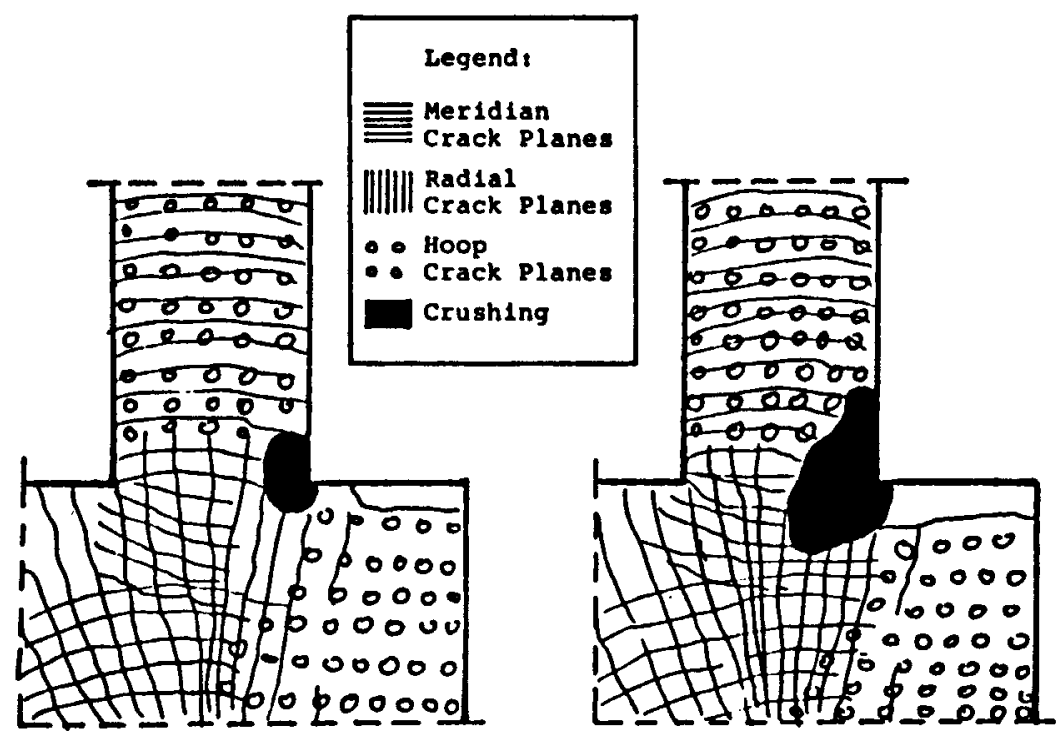

$1,1 \mathrm{MPa}$
$1,2 \mathrm{MPa}$

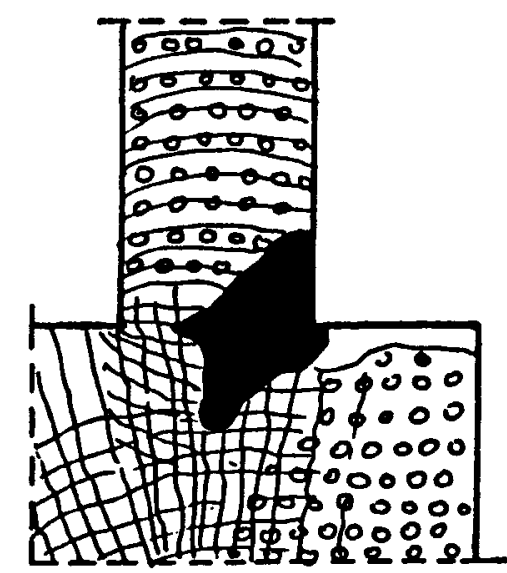

$1,3 \mathrm{MPa}$

Figure 4.8.14: Sketches of Crack Patterns at Cylinder Basemat Intersection 


$$
222
$$




$$
2022
$$




\subsection{Brookhaven National Laboratory}

This section was prepared by $C$. J. Costantino of the City College of New York and J. Pires and S. Pepper of the Structural Analysis Division of the Department of Nuclear Energy at Brookhaven National Laboratory.

\subsubsection{Introduction}

Brookhaven National Laboratory (BNL), along with a number of other organizations, undertook in the Fall of 1986 to perform a detailed response analysis of the $1 / 6$ th scale model containment structure. This reinforced concrete facility, designed as a typical containment structure for an actual nuclear power plant, is to be pressurized to failure in the Spring of 1987. Each of the organizations involved have been given the task of predicting the response and mode of failure of the vessel. The goal of the effort is obviously to improve the analysis capability available to the engineering community, particularly with regard to the ability to forecast behavior at and near failure of the various structural materials involved.

The failure of concrete structures and in particular containment facilities is controlled by a number of complex interacting mechanisms, such as the development of cracks under multiaxial tensile stress states, yielding and crushing under corresponding compressive states, shear transfer across cracked zones, and the interaction of steel rebar with the concrete. To be able to predict with reasonable confidence the response near failure of these reinforced structures, these various complex mechanisms must be simulated within the context of detailed analytic models for each individual mechanism, these then being combined to yield the overall material model required for the prediction. Numerous constitutive models for both concrete and steel materials have been proposed in the literature over the years, each having the objective of forecasting the behavior of the materials at and near failure. One of the primary objectives then of this exercise is to try to improve the modeling capability, particularly with regard to the failure modes associated with containment structures.

\subsubsection{BNL Predictional Model - The NFAP Code}

BNL has developed and exercised a large scale computer program to study the nonlinear behavior of reinforced concrete structures near failure. This effort has been aimed at developing complete analytic material models which have applicability to all sorts of concrete structures, including containment facilities. The analysis tool bears the acronym NFAP, standing for the Nonlinear Finite Element Analysis Program. Detailed descriptions of the Code are presented in [4.9.1 and 4.9.2], with only summary descriptions provided herein for completeness of this report.

\subsubsection{General Solution Procedures}

The NFAP Code is based on a finite element formulation of the equilibrium equations for the structure/loading system, and includes the capability to treat nonlinear constitutive material behavior, large displacement/strain problems as well as static or dynamic load conditions. For the containment facility of interest to this study, the structure is simulated as a twodimensional axisymmetric finite element model, subjected to the effects of both gravity loadings as well as statically applied internal pressures. 
Effects of penetrations have been neglected as having small influences on the global pressure response of the structure. Large displacement effects have been included in this calculation, using the total Lagrangian formulation described in [4.9.3].

As can be expected for this highly nonlinear calculation, the numerical solution proceeds in a standard incremental fashion. In order to develop the iterative solution procedure, the nodal equilibrium equations are rewritten in terms of incremental displacement as

$$
[K(U)]\{\Delta U\}=\left\{\Delta F_{e}\right\}-\left\{\Delta F_{i}\right\}
$$

where solutions are obtained at each load step for the vector of incremental nodal displacements, $\{\Delta U\}$. The stiffness matrix [K] of Equation (4.9.1) is typically a function of the total displacements, $\{U\}$, and the load increments shown are the externally applied loadings, $\left\{\mathrm{F}_{\mathrm{e}}\right\}$, (which may be a function of geometry) and the internal correction forces, $\left\{F_{j}\right\}$, which account for the nonlinear effects which occur during the load step.

In the standard calculation conducted with NFAP, iterations are conducted at each load step to ensure that acceptable convergence occurs. At the discretion of the user, the stiffness matrix can be updated at each iteration within a load step (full Newton-Raphson procedure) or only at the end of each load increment (modified Newton-Raphson). The second method (used for this calculation) requires more iterations to convergence per load increment but saves calculation time required in the $K$-matrix update as compared with the first method. Iterations are carried out until the norm of the increment of the displacement vector at a given iteration is found to be small as compared to the previous iteration solution, or

$$
\left(\left|\Delta U_{i+1}\right|-\left|\Delta U_{j}\right|\right) /\left|\Delta U_{i+1}\right| \leq \epsilon
$$

where the subscript $i$ refers to the iteration number. For this calculation, a value of 0.001 was used for the convergence parameter, $\epsilon$.

\subsubsection{Modeling of Materials}

The specific details of the material models used in this calculation are presented in [4.9.1 and 4.9.2], with only a short summary contained in the following. The interaction between steel and concrete is handled in one of two ways in this calculation. Some steel reinforcement, primarily the shear steel (stirrups) located in the lower section of the cylinder wall, is specifically modeled in the containment by means of axial or truss elements. These bars are connected to the nodes of the surrounding concrete elements, and are therefore subjected to the same displacement field as the concrete elements.

The vast majority of steel reinforcement is modeled in the analysis using the distributed or smeared concept typically used for reinforced concrete structures. This is done to limit the amount of computation required as well as to limit data preparation requirements. In this method, the steel is smeared into the concrete element, resulting in a locally homogeneous continuum. The separate constitutive properties of each material component 
are tracked throughout the calculation to ensure that individual stress paths are properly modeled. Specific smearing and desmearing procedures, as described in [4.9.2], are then used to ensure that node point loads satisfy the required equilibrium equations.

\subsection{Uniaxial Steel Rebar}

The stress-strain data provided for the rebar essentially consists of three sections, namely, an initial elastic zone, a short perfectly plastic zone, and a hardening section which is approximately parabolic in shape. For this calculation, the stress-strain data above the yield point has been represented as a simple linear hardening material. A hardening modulus of $786 \mathrm{ksi}(5420 \mathrm{MPa})$ for stresses above the yield point was used, which was obtained by a linear regression calculation.

\subsection{Multiaxial Steel Models}

The constitutive model for the multiaxial steel, such as the liner materials, is based on the standard incremental theory of plasticity, using the von Mises yield criterion and an associated flow rule. Hardening is included by considering an isotropic hardening mechanism. A linear hardening rule was used for both liner materials, with a value of $152 \mathrm{ksi}$ (1050 MPa) used for the $1 / 16(1.59 \mathrm{~mm})$ liner used along the basemat/cylinder wall. For the dome liner, a larger hardening modulus of $516 \mathrm{ksi}$ (3560 MPa) was used to fit the initial slope of the stress-plastic stain data provided.

\subsection{Concrete Stress-Strain Mode1}

The elastic-plastic material behavior proposed by Chen and Chen [4.9.5] is used in NFAP to predict the nonlinear behavior of the concrete. This model has been found to yield reasonable agreement with experimental data and has been employed for other failure evaluations of concrete containments. The model is based on separate assumed failure surfaces in the tension and compression zones in stress space. The yield criteria are developed in terms of the stress invariants, and include both an associated flow rule, as well as a strain hardening parameter to track changes in the yield surface with strain. The specific values for the concrete parameters used for this calculation are:

$$
\begin{array}{rlrl}
E & =\text { Young's modulus } & & =4800 \mathrm{ksi}(33100 \mathrm{MPa}) \\
\nu & =\text { Poisson's ratio } & & =0.2 \mathrm{ks}(3.10 \mathrm{MPa}) \\
f_{t} & =\text { Tensile yield stress } & & =0.45 \mathrm{ks}(3 \mathrm{MPa}) \\
f^{\prime}{ }_{t} & =\text { Tensile fracture stress } & & =0.5 \mathrm{ksi}(3.45 \mathrm{ks} \\
f_{c} & =\text { Compressive yield stress } & =3.9 \mathrm{ks}(26.9 \mathrm{MPa}) \\
f^{\prime}{ }_{c} & =\text { Compressive fracture stress } & =6.8 \mathrm{ksi}(46.9 \mathrm{MPa})
\end{array}
$$

\subsection{Fracture and Crushing of Concrete}

The ability of the concrete to fracture in both tension (cracking) and compression (crushing) have been included in the analysis. A dual stress and strain criterion is used to define the initiation of cracking, which are based on the stress and strain invariant for multiaxial states, and are correlated with available experimental data for cracking strains in uniaxial 
compression and tension. When the concrete crushes, the stiffness of the concrete element is assumed to be zero for additional loading of the material. For the case of tension cracking, however, the stiffnesses associated with strains normal to the crack direction are reduced to a small fracture of their initial value. The shear stiffness reduction factor is initially set to a value of 0.5 , which is gradually reduced as the width of the crack increases. The specific fracture strains assumed for this calculation are

$$
\begin{aligned}
& \epsilon^{\prime}{ }_{t}=\text { Fracture strain in tension }=0.00021 \\
& \epsilon^{\prime}{ }_{c}=\text { Fracture strain in compression }=0.0023
\end{aligned}
$$

\subsection{Soil Foundation Model}

Based on previous experiences with containment calculations, especially at the higher pressure regions of interest near failure, relative basemat movements can have a significant effect on the calculation of stresses at the basemat-cylinder wall intersection, a potential failure point in the structure [4.9.4]. Therefore, in our NFAP calculation, it was felt important to include the soil flexibility in the model. The soil is represented as a bilinear elastic winkler foundation, that is, a one dimensional soil element with a relatively high stiffness under compressive loading and a zero stiffness under tensile loading.

The data provided by Sandia included a recommended value of $390 \mathrm{ks} / \mathrm{ft}$

$(61.2 \mathrm{MPa} / \mathrm{m})$ for the subgrade modulus. However, by examining the plate load test data provided, and using standard extrapolation techniques typically used for such data, it was found that this recommended value was probably much too high. For our NFAP calculation, therefore, a value of about $70 \mathrm{ksf} / \mathrm{ft}(11.0 \mathrm{MPa} / \mathrm{m})$ was used for the coefficient of subgrade reaction. As will be discussed later, it was found that the dead load calculation yielded a vertical settlement of the structure of 0.17 inches $(4.32 \mathrm{~mm})$, a value that can reasonably be expected for this soil under these dead load stresses.

\subsubsection{Finite Element Modeling}

The general configuration of the finite element model used in this calculation is shown in Figures 4.9.1 and 4.9.2. As mentioned above, the model is an axisymmetric one, and includes both the mudmat and foundation soil in the structural model. The complete finite element model, shown in Figure 4.9.2, consists of 1549 node points and 539 elements. Of the 539 elements, 459 are two dimensional, 8-noded isoparametric elements, while 80 are one-dimensional axisymmetric truss elements. The truss elements are used to represent the winkler soil springs and some shear steel (stirrups) in the lower cylindrical wall. Detailed sketches of the finite element models used in the various section of the structure are shown in figures 4.9.3, 4.9.4 and 4.9.5. Generally, the mudmat is simulated by means of three elements through its thickness, the basemat by seven elements through the thickness, and the cylindrical wall and dome by seven elements through the thickness. In addition, separate elements are used to represent the steel liner plate in the various sections, with the liner considered attached to the concrete at its node points. Thus, a total of eight 
elements are used to represent the through-thickness properties of the structural wall, dome and basemat.

Detailed sketches of the finite element mode used through the cylindrical wall section are shown in Figures 4.9.6 and 4.9.7. As can be seen, two of the elements are modeled as plain concrete, two have smeared hoop steel, two have smeared meridional (longitudinal) steel, and the outside element includes the smeared seismic steel. The steel liner element is then added to the inside face of the wall. By comparing Figures 4.9 .6 and 4.9 .7 , the only difference between the models above and below the elevation of $6^{\prime} 6^{\prime \prime}$ is the amount of meridional stee included in the third concrete element (counting from the inside face). To simplify some of the modeling requirements, the inner and outer hoop steel was adjusted slightly so as to reside in only two elements, with these elements being separate from those containing the meridional steel. As mentioned previously, specific truss elements were then used in the wall below the elevation of $6^{\prime} 6$ " to represent the shear steel (Figure 4.9.3).

A typical model used through the thickness of the dome is shown in Figure 4.9.8, where again a seven element cluster is used to represent the wall action. The transition elements used from the springline to the standard dome elements are presented in Figure 4.9.9. The details of the mudmat and basemat elements are shown in Figures 4.9.10 and 4.9.11.

\subsubsection{Results of Calculations}

As mentioned previously, this axisymmetric model was subjected to gravity and internal pressure loadings. The large displacement formulation was included in the calculation and a modified Newton-Raphson incremental solution scheme was used. Gravity loads were first turned on, followed by incremental pressure loadings of $5 \mathrm{psi}(.034 \mathrm{MPa})$ until a pressure of 30 psi (.207 MPa) was reached. Beyond this pressure, the incremental loadings were reduced to $2 \mathrm{psi}(.014 \mathrm{MPa}$ ) to reduce convergence problems due to nonlinear material effects as well as improving the tracking of concrete cracking. From $126 \mathrm{psi}(0.869 \mathrm{MPa})$ to the end of the calculations at $131 \mathrm{psi}(.903$ $\mathrm{MPa})$, pressure increments of $1 / 4 \mathrm{psi}(1.72 \mathrm{kPa}$ ) were used as extensive yielding occurs in this pressure range.

\subsubsection{Structural Deformations}

Plots of the deformed configuration as a function of load are shown in Figures 4.9 .12 to 4.9 .20 , the purpose of which are to indicate the primary characteristics of the computed response of the containment structure to the applied loads. The deformations shown in these figures are magnified 100 times. The effect of the dead load, Figure 4.9 .12 , is to essentially develop a vertical rigid body movement of the structure into the foundation soil of about 0.17 inches $(4.32 \mathrm{~mm})$. The only significant deformation under dead load is found in the mudmat extending beyond the structure. As internal pressure is applied, the containment vessel responds primarily elastically (Figure 4.9.13) until the pressure reaches a value of about 30 psi (.207 MPa) (Figure 4.9.14). At this pressure, some nonlinear concrete strains develop in the outer elements of the cylindrical wall due to shell bending. Of more importance, however, concrete flexural cracking begins at the inside face of the cylinder at its intersection with the basemat. The dome remains essentially elastic. 
As the applied pressure reaches $40 \mathrm{psi}(.276 \mathrm{MPa})$, hoop cracking begins in the outer elements of the cylinder wall near mid-height. As can be seen in Figure 4.9.15, the deformations now begin to significantly increase, as the cracking at the basemat/cylinder wall junction extends into the wall. At 50 psi (.245 MPa), the hoop cracking increases along the length of the wall and cracking of the concrete develops in the dome area. At 70 psi, (.483 MPa), bending cracks begin to form at the center of the basemat, as the basemat curls up due to the pressure loads. Shear cracks now develop in the cylinder wall above the zone with shear steel (stirrups) at an elevation of about $6^{\prime}$. This in turn leads to an increase in the extent of the hoop cracks as the shear stiffness near the bottom of the cylinder decreases. In addition, shear cracks develop in the dome, immediately above the top of the seismic steel area. At about $80 \mathrm{psi}(.552 \mathrm{MPa}$ ) (Figure 4.9 .17 ), multiple cracking develops in the wall/basemat junction as the cracked zone in this area increases. The stresses in the meridional steel dowels embedded into the basemat become larger as the zone of cracked concrete in this area increases.

At about $90 \mathrm{psi}(.621 \mathrm{MPa})$, the cracked zone at the wall/basemat junction extends through the entire wall thickness, and hoop cracking extends throughout most of the superstructure. The area of bending cracks in the basemat extends over a larger depth, but the stresses in the steel in this area are still small. The steel liner is still elastic, but peak stresses are of the order of $50 \mathrm{ksi}(.345 \mathrm{MPa})$ in the center of the cylinder wall. At about $104 \mathrm{psi}(.717 \mathrm{MPa})$, yielding begins to develop in the steel rebar dowels which extend into the basemat below the cylinder/wall junction. In addition, some yielding of the liner occurs at midheight of the fylinder. The stresses in the dome reinforcing are still reasonably small, reacting a value of about 25 to $30 \mathrm{ksi}(.172$ to $.207 \mathrm{MPa}$ ).

At about 124 psi (.855 MPa), yielding of the hoop steel beings in the cylinder wall in the area above the stirrups, and yielding of the dowel steel becomes more extensive. Strains in the basemat dowels reach a value of about 0.9 percent, or about four times yield. The plastically deformed area of the steel liner also spreads upwards towards the springline, while liner stresses in the dome still remain below yield. Total yielding of the hoop steel occurs at about $128 \mathrm{psi}(.883 \mathrm{MPa})$, above which the calculations indicate a rather general ballooning of the superstructure. The hoop steel continues to deform plastically, although hoop strains are still only about two times the yield strain for the rebar. The calculations were discontinued at $131 \mathrm{psi}(.903 \mathrm{MPa})$, with the final deformed configuration shown in Figure 4.9.20.

\subsubsection{Specific Responses of Interest}

In addition to the structural profiles discussed above, some plots of specific parameters of interest as a function of applied pressure are presented in the following. A detailed set of the individual plots requested by Sandia are included in Appendix $A$.

The computed vertical displacements at various locations on the structure are shown in Figure 4.9.21. As can be seen from the plots of total displacement, the center of the basemat, after the initial dead load solution, is pushed down into the foundation soils by the applied internal pressures, while the edge of the basemat curls up. This effect is, of 
course, as would be anticipated. However, a question that must still be resolved is the importance of this behavior on the overall response of the structure. If the foundation soils were significantly stiffer than indicated by the data presented by Sandia, it can be anticipated that the shear forces developed at the wall/basemat junction would be higher than those in the current calculation. This would in turn cause cracking to develop sooner (or at lower applied pressures) than calculated. The impact of this factor has not been evaluated in this calculation.

Plots of the relative displacements shown in Figure 4.9.21 are of some interest. The solid curve of Figure 4.9.21B shows the displacement of the edge of the basemat relate to the basemat centerline. As can be seen, this relative displacement increases monotonically with pressure and is essentially uncoupled from any changes that are taking place in the superstructure. On the other hand, the vertical displacement of the springline measured with respect to the edge of the basemat shows significant changes which are associated with the changes in characteristics of the cylinder wall as the concrete cracking occurs. At about 30 psi (.207 $\mathrm{MPa}$ ), the concrete begins to show significant nonlinear behavior, reducing the effective stiffness of the wall. At about $40 \mathrm{psi}(.276 \mathrm{MPa})$, hoop cracking develops in the wall, beyond which the longitudinal steel essentially controls the vertical stiffness of the wall. Above 100 psi (.690 MPa), yield begins to develop in the steel dowels, again softening the apparent vertical stiffness of the wall at the springline. Similar comparisons can be made from the plot of the crown displacement measured with respect to the springline. Concrete cracking in the dome area, which occurs at a higher pressure than in the wall, leads to a relate response which is similar to that at the springline.

Figure 4.9 .22 presents a comparison of hoop displacements at the center of the wall and at the springline. As is expected, the displacements at the center of the wall are about twice those at the springline. As cracking in the hoop direction extends in the cylinder wall, the displacements gradually increase until hoop yielding occurs, at which time the radial displacements increase rapidly. The strains developed in the hoop rebars are shown in Figure 4.9.23 and show the increase in the hoop steel strains as cracking develops in the concrete. The strains shown are relatively constant along the length of the wall. Near the springline $\left(z=24^{\prime}\right)$ and basemat, smaller hoop strains are calculated.

The strains developed in the vertical meridional steel are shown in Figure 4.9.24 for the case of the outer layer of steel (layer 5). In Figure 4.9.24A, it may be noted that the strains developed in these bars are greatest at the junction of the cylinder wall with the basemat, and decrease as you progress up the length of the wall. The relationship of the meridional strains near the base of the wall to the gross response of the superstructure is shown in Figure 4.9.24B. Similar data is shown in Figure 4.9.25 for the inner layer of the meridional steel, from which a similar general behavior can be noted. A comparison of strains developed in the inner and outer meridional steel is shown in Figure 4.9.26. As can be seen, significant differences in behavior can be noted at the top and bottom of the wall. At the top, near the springline, the differences in strains can be attributed to the bending that is developed by the action of the flexible dome. At the bottom, however, the larger moments developed and the extensive cracking of the wall led to the generation of large plastic 
strains in the inner steel, as well as tensile strains in the outer steel. Plastic tensile strains develop in the outer steel layer at higher pressures. This complex behavior is different than would normally be anticipated from simpler analyses.

\subsubsection{CONCLUSIONS}

On the basis of this complex calculation, several conclusions can be reached. First, two primary modes of potential failure of the containment develop as internal pressure is applied. At a pressure of about 128 psi (.883 MPa), total yielding of the hoop steel occurs which extends well into the dome area. At this pressure, the calculations indicate a general ballooning of the containment structure begins as additional pressure is applied. The computed pressure-hoop displacement relationship will then track the rebar stress-strain curve, which shows higher capacity at much larger steel strains of 2 to 3 percent, presuming of course that failure does not occur from a different mechanism.

The calculations also indicate a second potential failure mode developing at the base of the cylinder wall. The cracked zone of concrete extends through the thickness of the cylinder wall at about $90 \mathrm{psi}$ (.621 MPa). Additional pressure then causes this zone to extend into the basemat and at the same time causes yielding of the dowel steel in both the inner and outer faces of the containment wall. The calculated strains in the dowels are about 4 times yield at pressures of about $124 \mathrm{psi}(.855 \mathrm{MPa})$. Continued pressure can then be applied to the structure until either pullout of the dowels occurs or the cracking of the concrete reaches the outer edge of the basemat, which is unreinforced. It is this latter behavior which will most probably lead to a localized failure and therefore an inability to support additional load.

Two primary areas of uncertainty exist in the calculation. The first reflects the uncertainty in the effect of the foundation soil stiffness on the calculated behavior in the vicinity of the basemat/wall junction. As mentioned previously, other calculations have indicated that this effect may significantly effect the overall behavior of the basemat/cylinder wall junction near failure. Instrumentation placed in the soil under the basemat would have allowed an independent evaluation of this behavior. In addition, the calculated behavior in the vicinity of the basemat/wall junction indicates an extremely complex set of responses which directly influences the growth of the cracked zone in this area. These calculations, in turn, are directly influenced by the fineness of the finite element mesh used in this vicinity. 


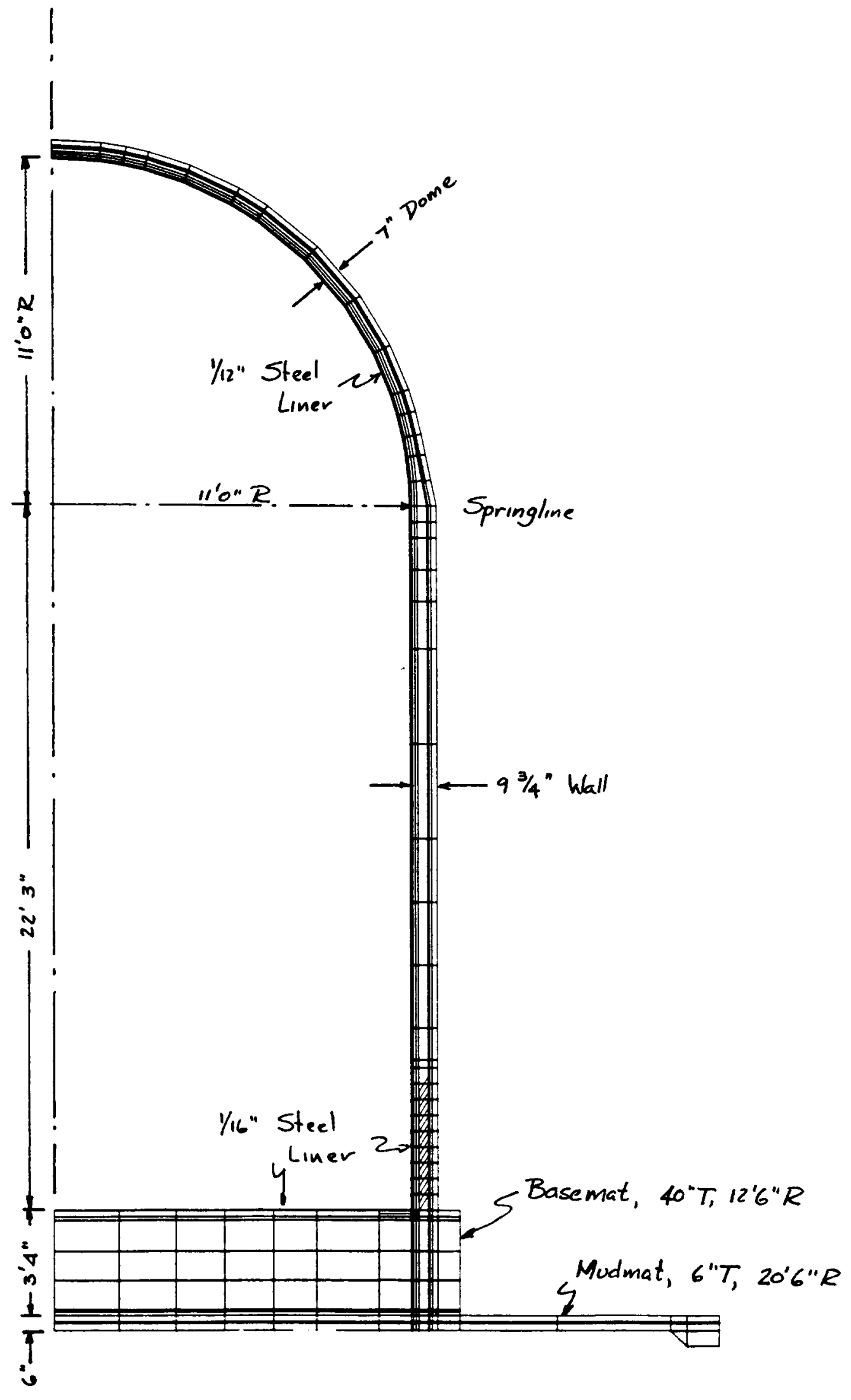

Figure 4.9.1 General Configuration of Containment 


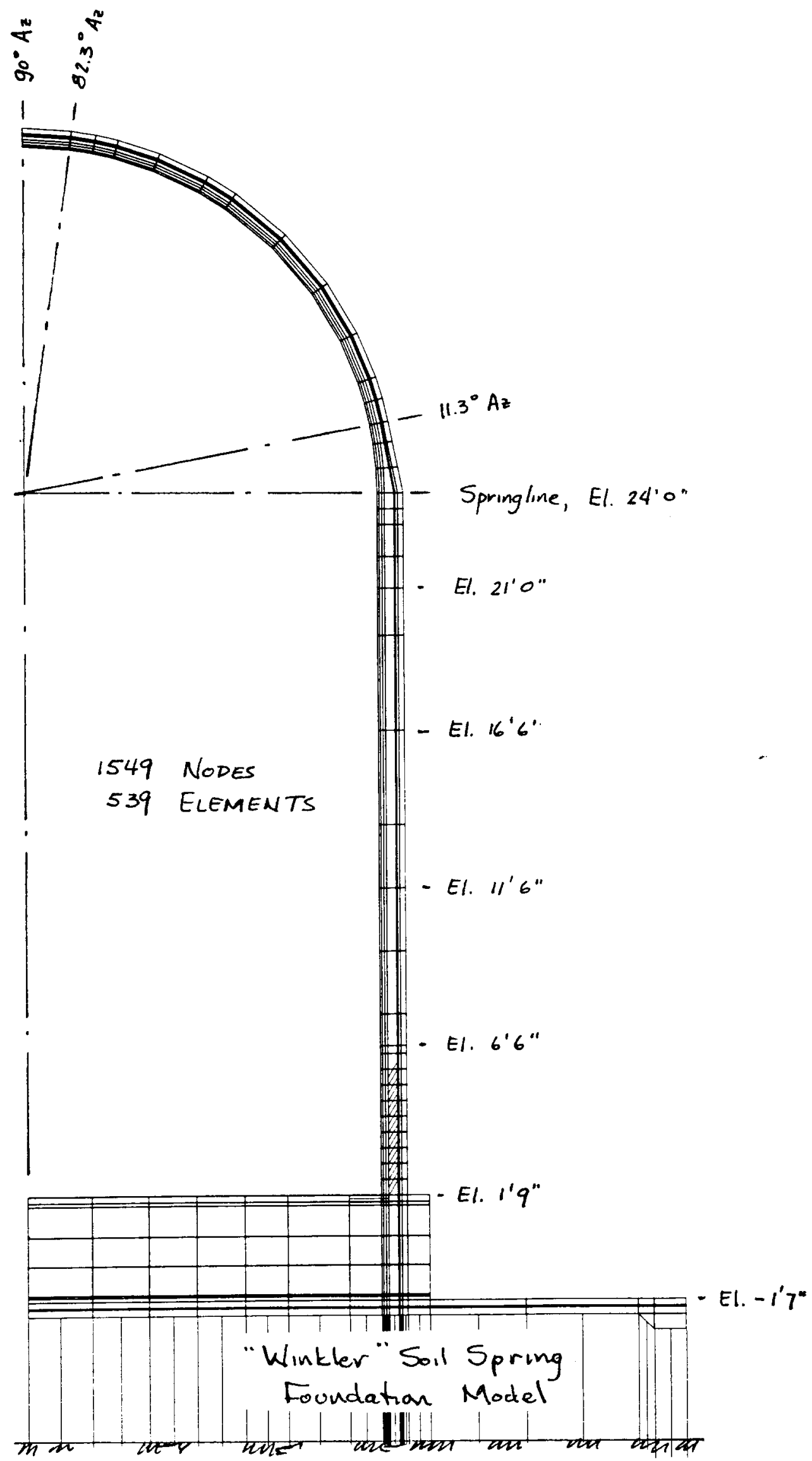

Figure 4.9.2 Winkler Soil Spring Foundation Model 


$$
\text { i }
$$

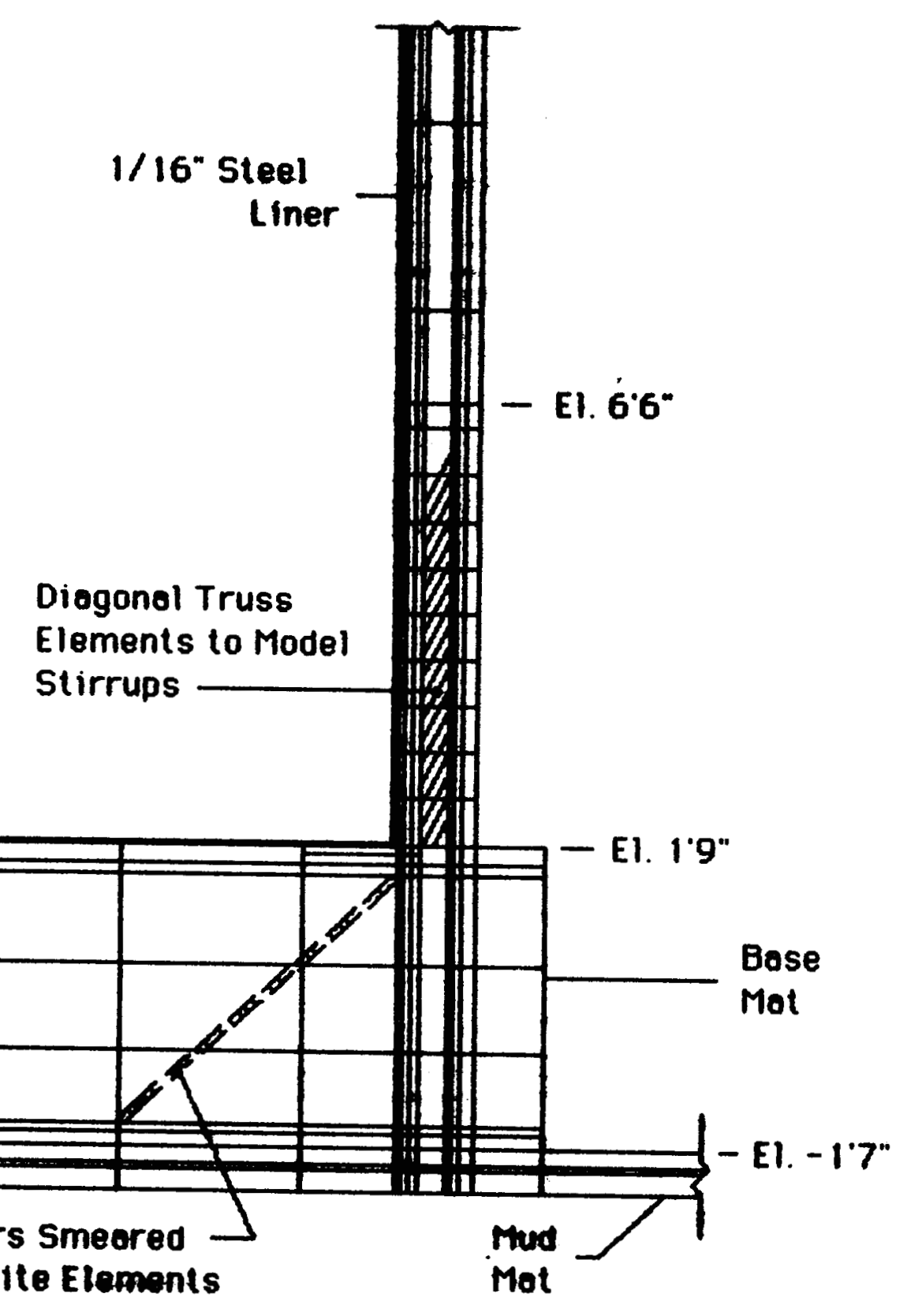

Figure 4.9 .3

Finite Element Configuration Used for Basemat/Mudmat/Cylinder Wall 


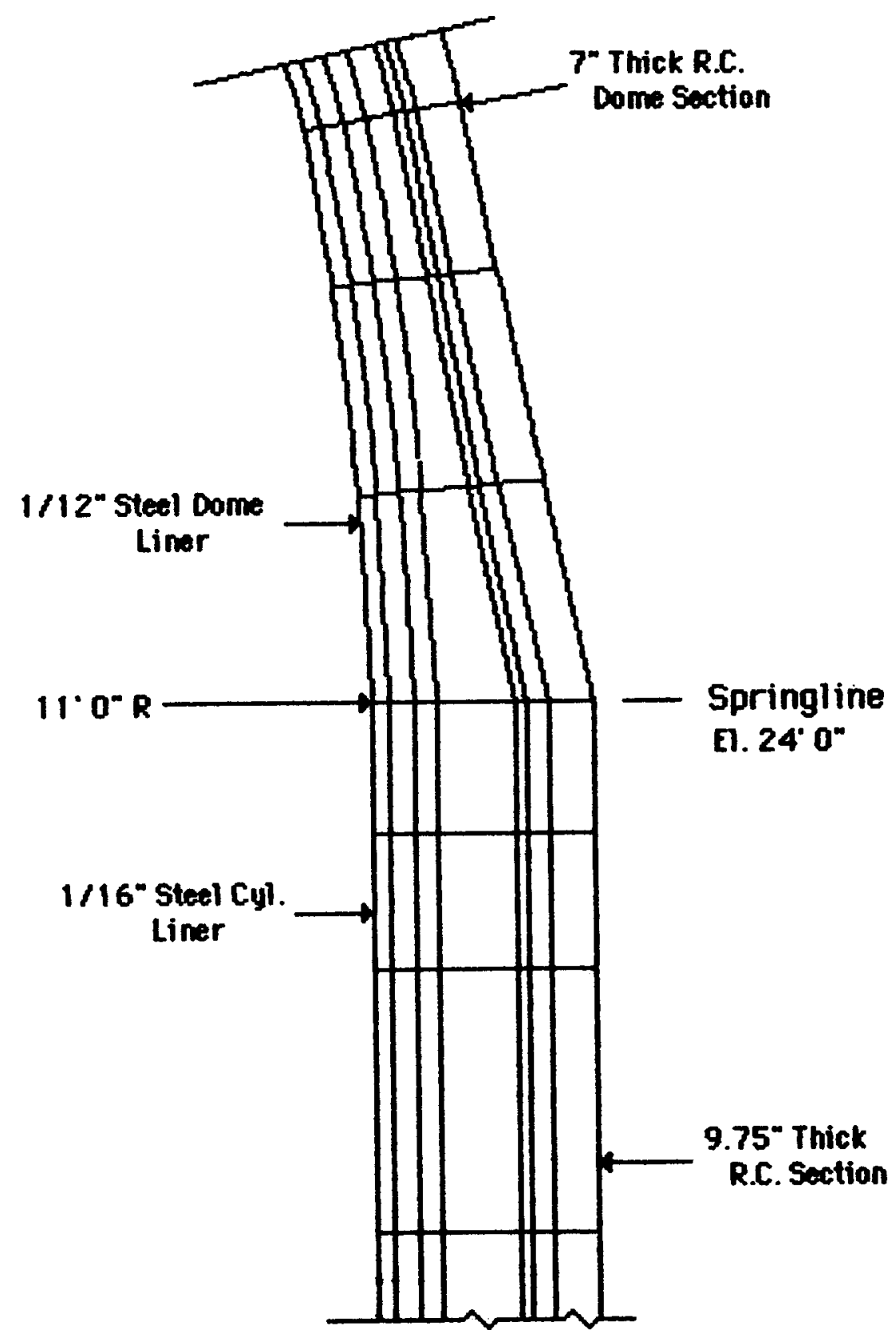

Figure 4.9 .4

Finite Element Configuration Used in Wall and Springline Section 


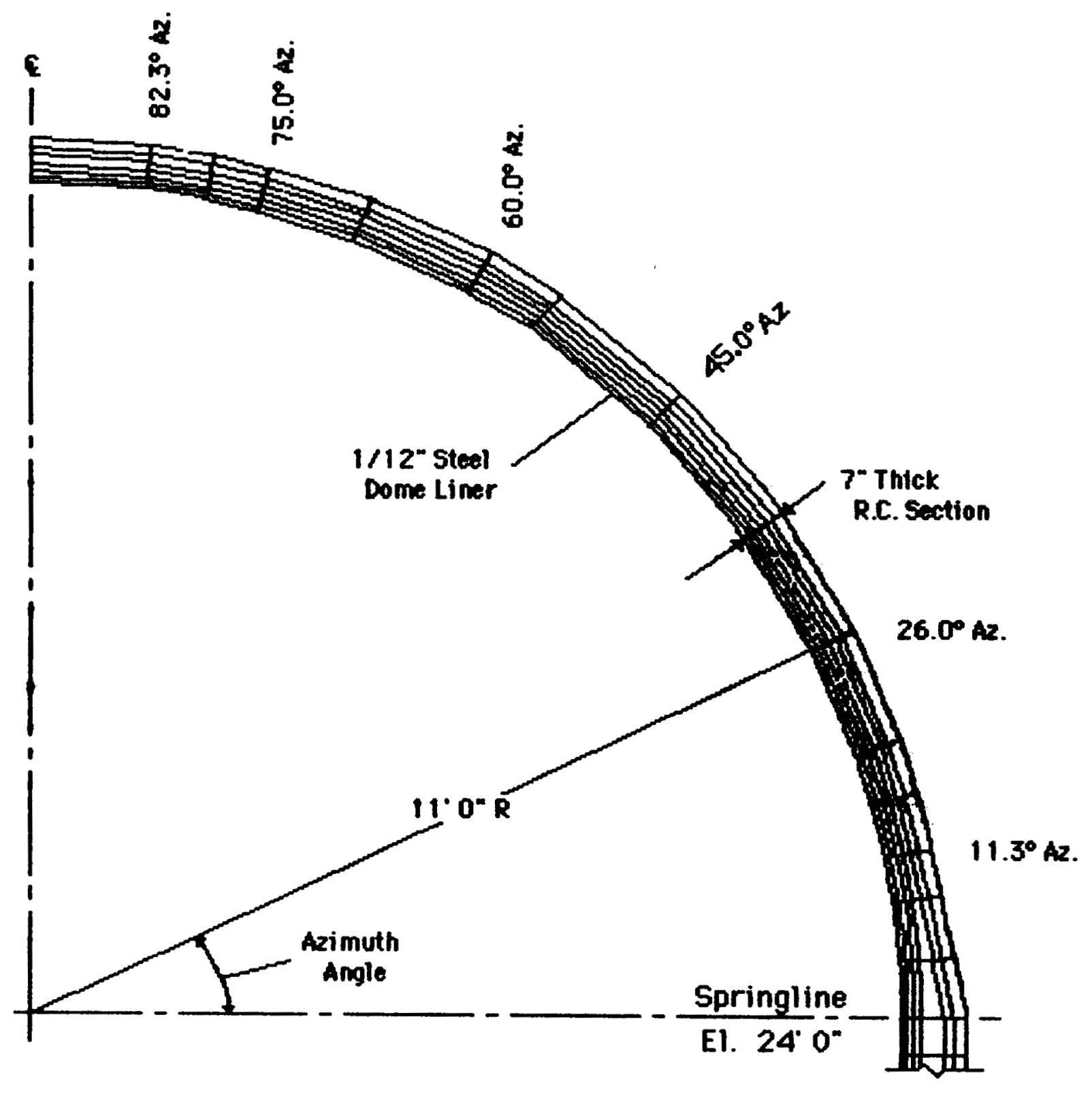

Figure 4.9 .5

Finite Element Configuration Used in Dome Section 


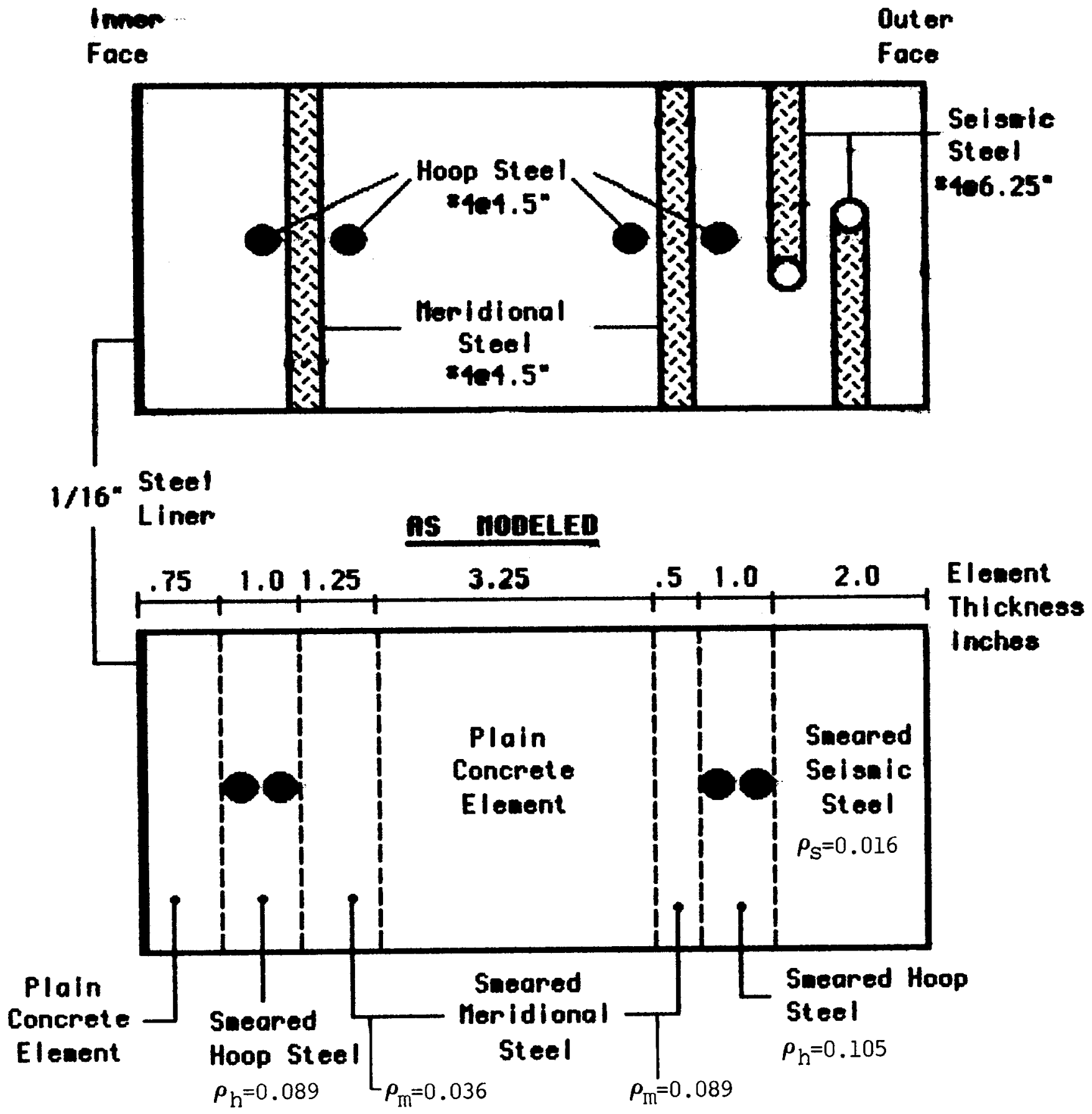

Figure 4.9.6 Cylindrical Elements Between EL 6'-6" and $24^{\prime}-0^{\prime \prime}$ 


\section{AS DESIGUED}

Inner

Face

Outer

Face

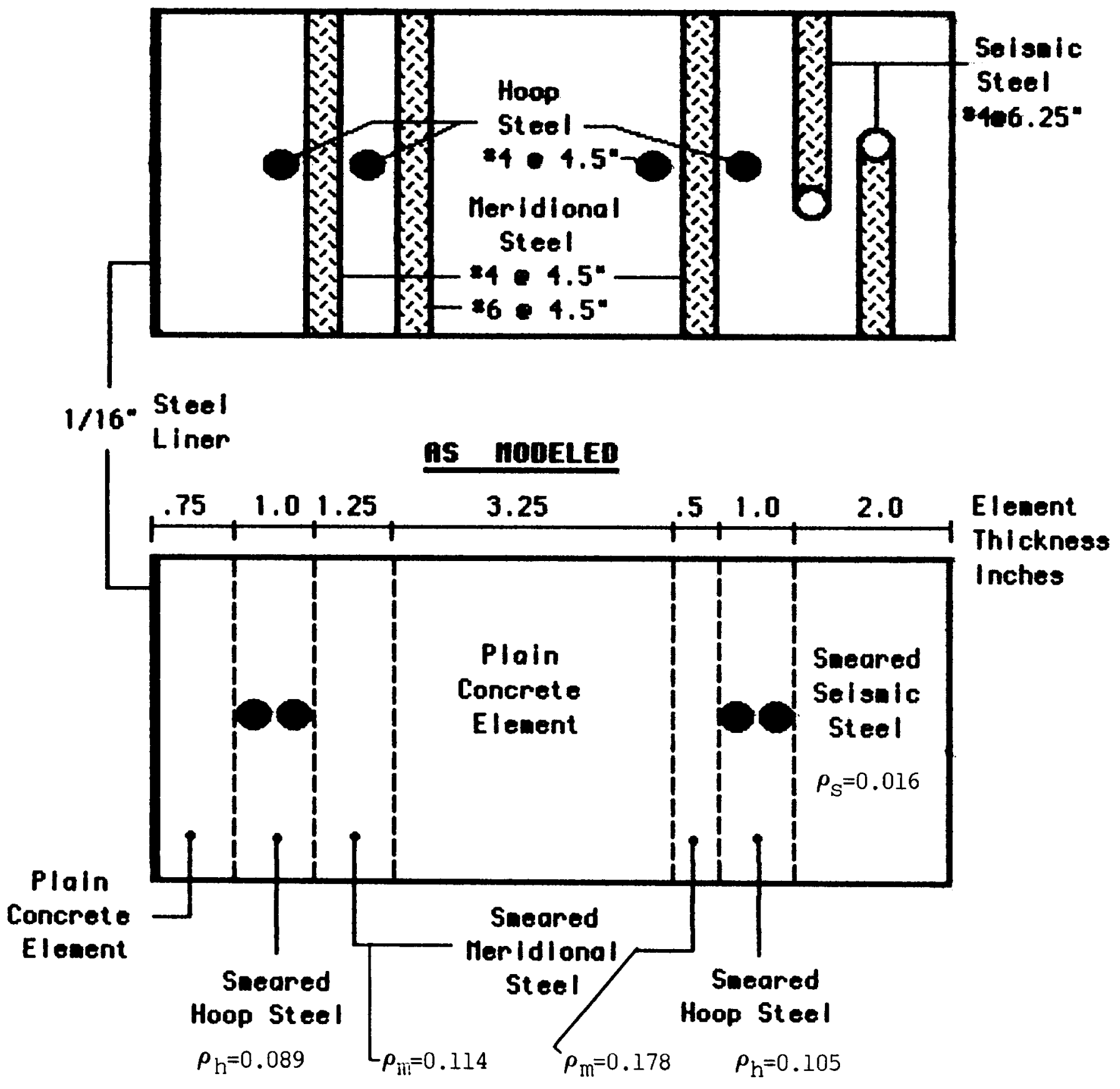

Figure 4.9.7 Cylindrical Elements Between EL 1'-9" and 6'-6" 
AS DESIGHED

Inner

Out er

Face

Face

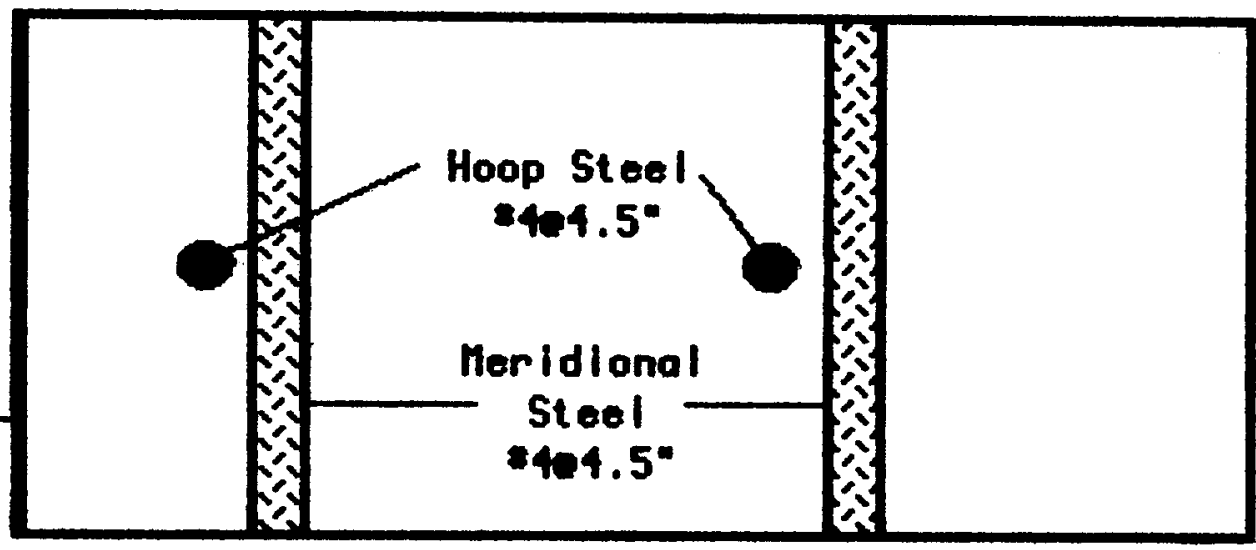

1/12" Steel

AS MODELED

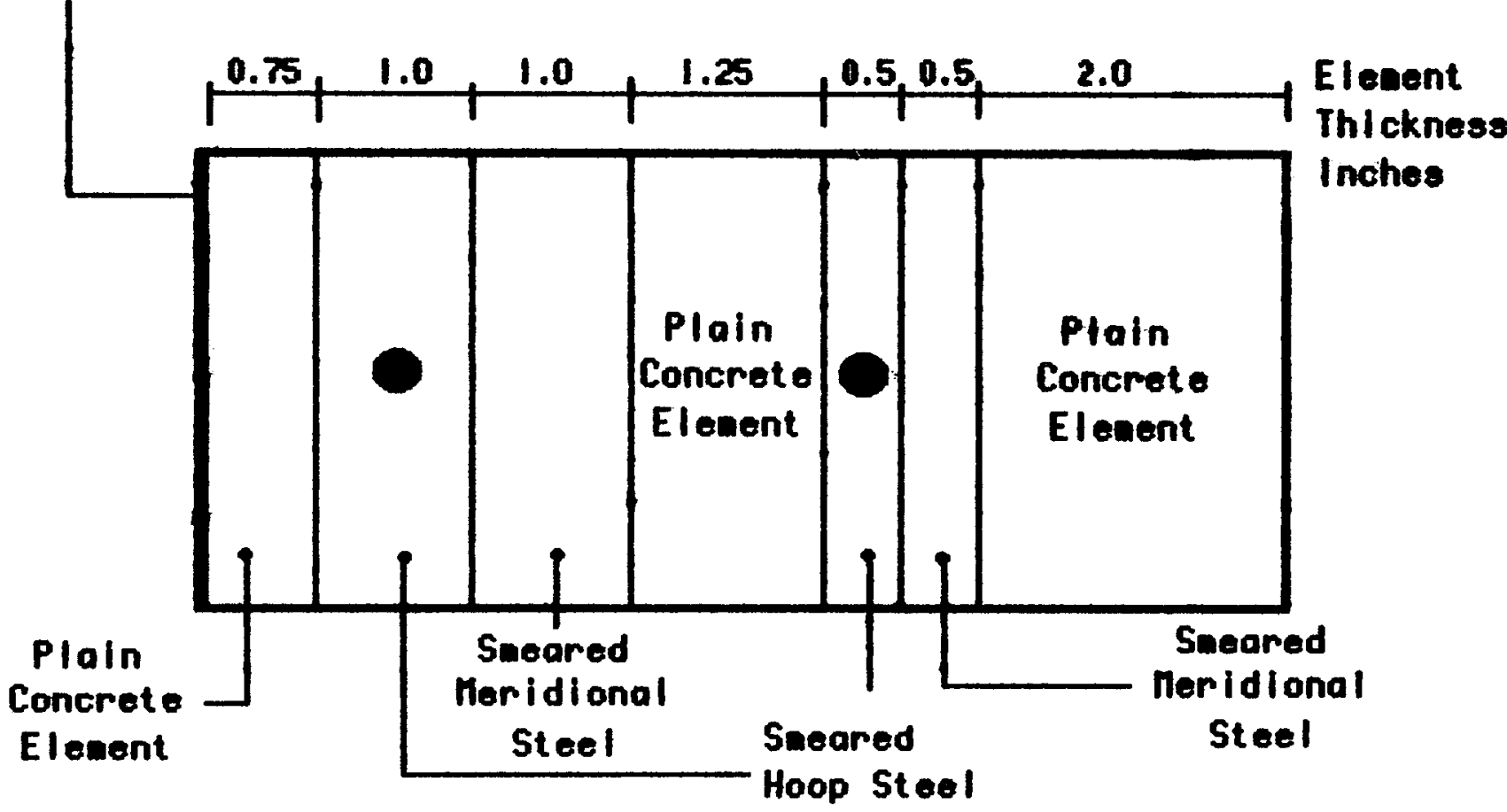

Figure 4.9.8 Element MOdel of Dome Above 11.3 Degrees Azimuth Angle 


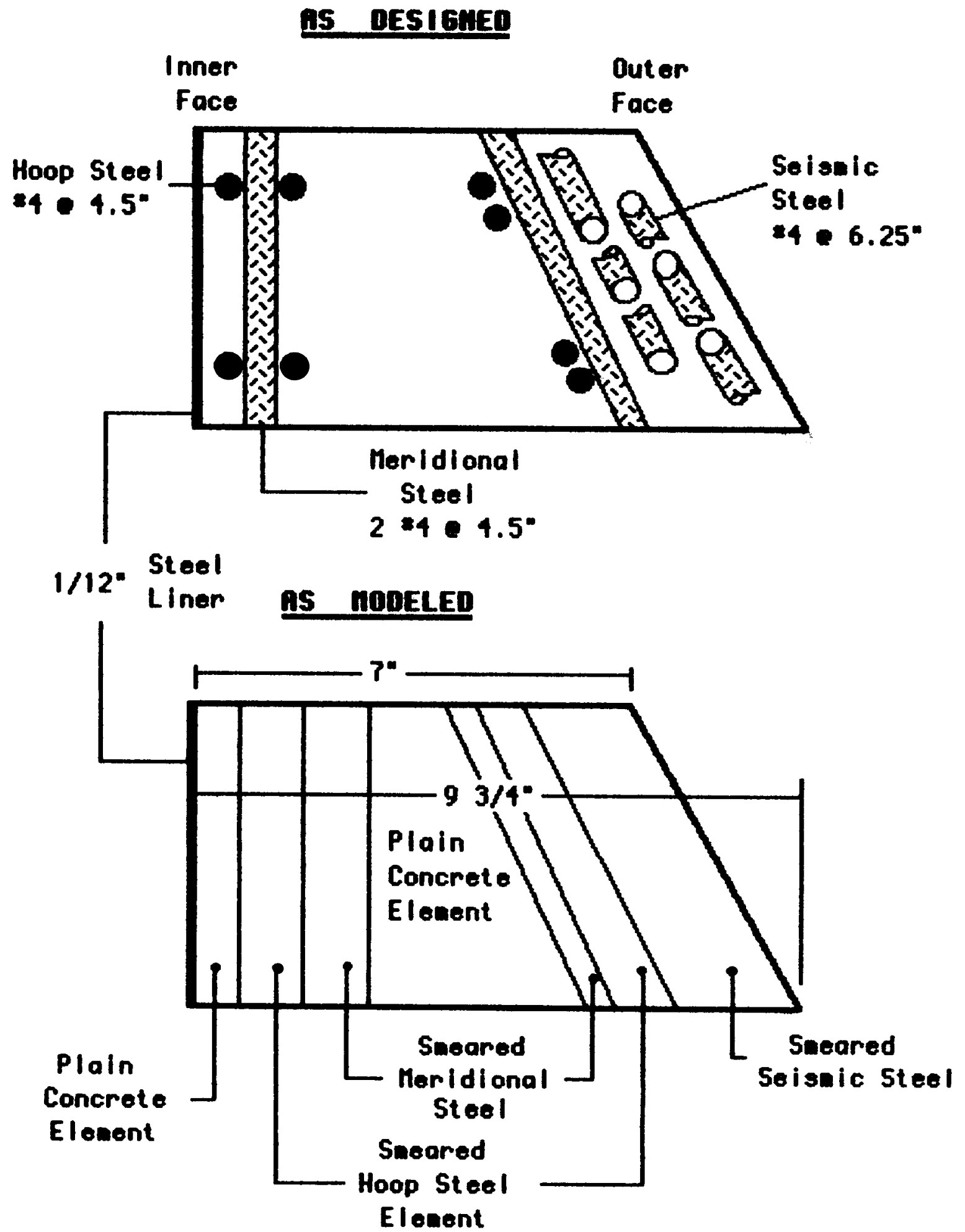

Figure 4.9.9

Transition Elements from Springline to 11.3 Degrees Azimuth Angle 


\section{AS DESIGHED}

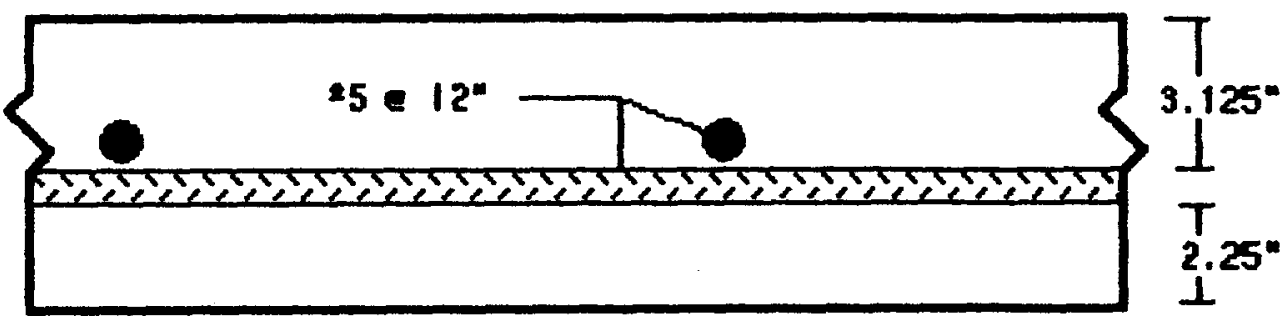

AS MODELED

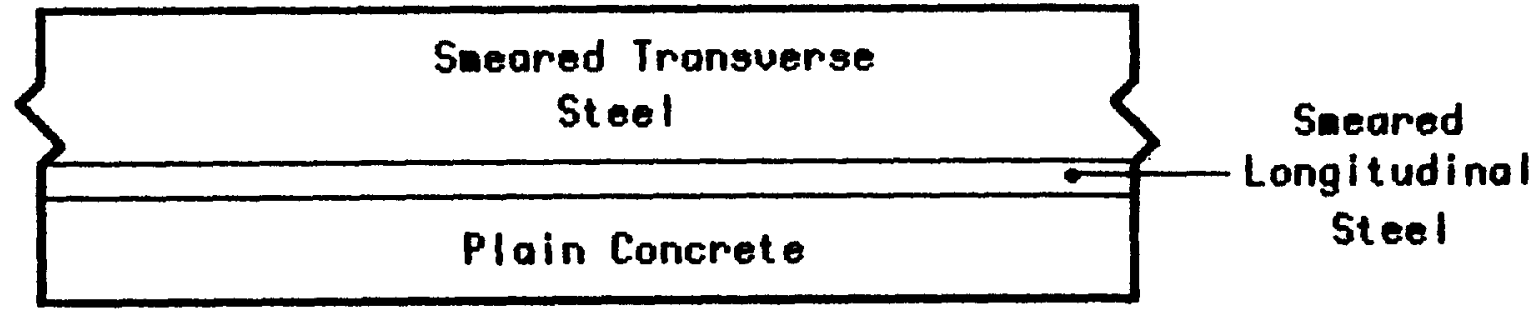

Figure 4.9.10 Elements Through Mudmat 


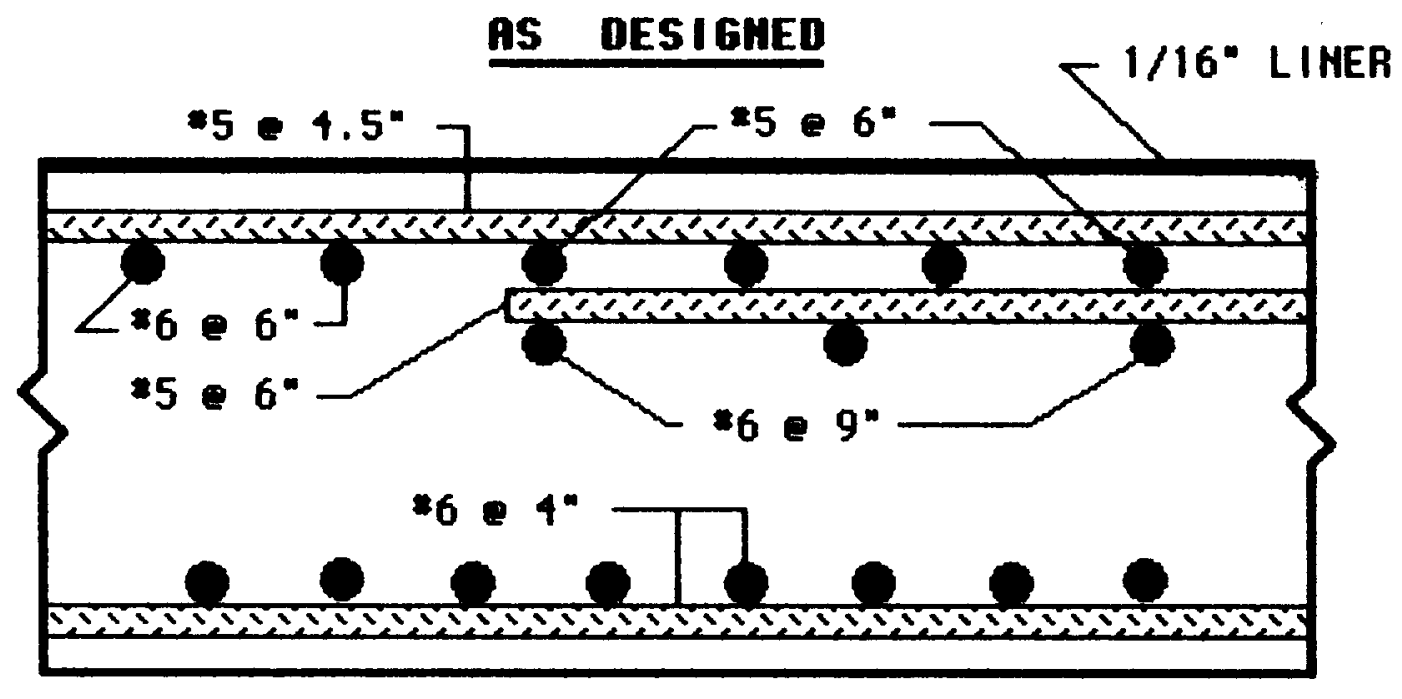

AS MODELED

\begin{tabular}{|c|c|}
\hline Smeared Langitudinal Steel & $2.56^{\prime \prime}$ \\
\hline Smeared Transuerse Steel & $1.38^{\circ}$ \\
\hline $\begin{array}{c}3 \text { Layers of } \\
\text { Plain Concrete }\end{array}$ & 30 \\
& $11.27^{\prime \prime}$ \\
\hline Smeared Transuerse Steel & $10.75^{\prime \prime}$ \\
\hline Smeared Longitudinal Steel & $1.50^{\prime \prime}$ \\
\hline
\end{tabular}

Figure 4.9.11 Finite Elements Through the Basemat 


$$
1
$$




$$
1
$$




$$
\equiv
$$




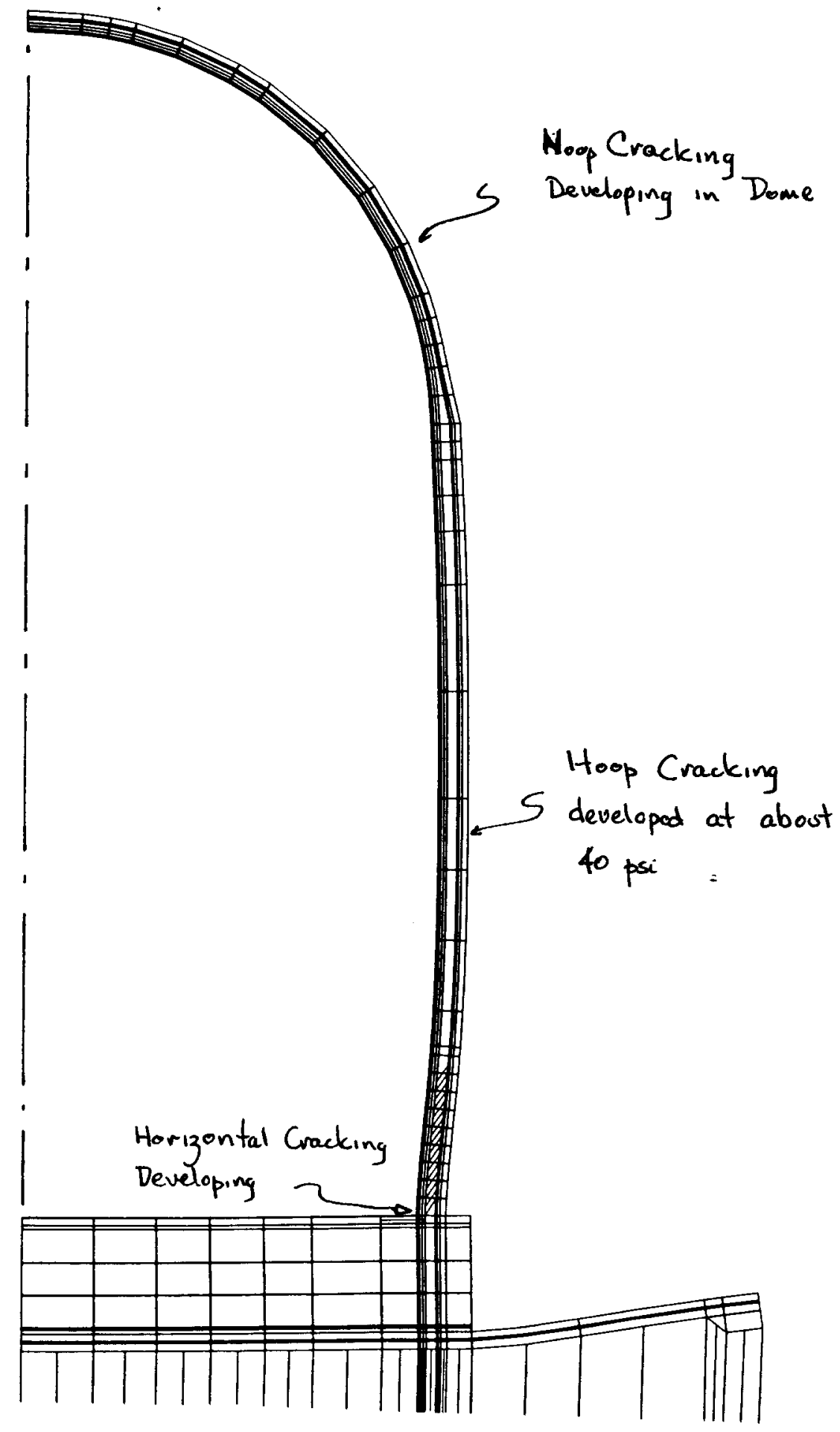

Figure 4.9.15 Deformed Configuration at 50 psi (0.345 Mp)

$-363-$ 


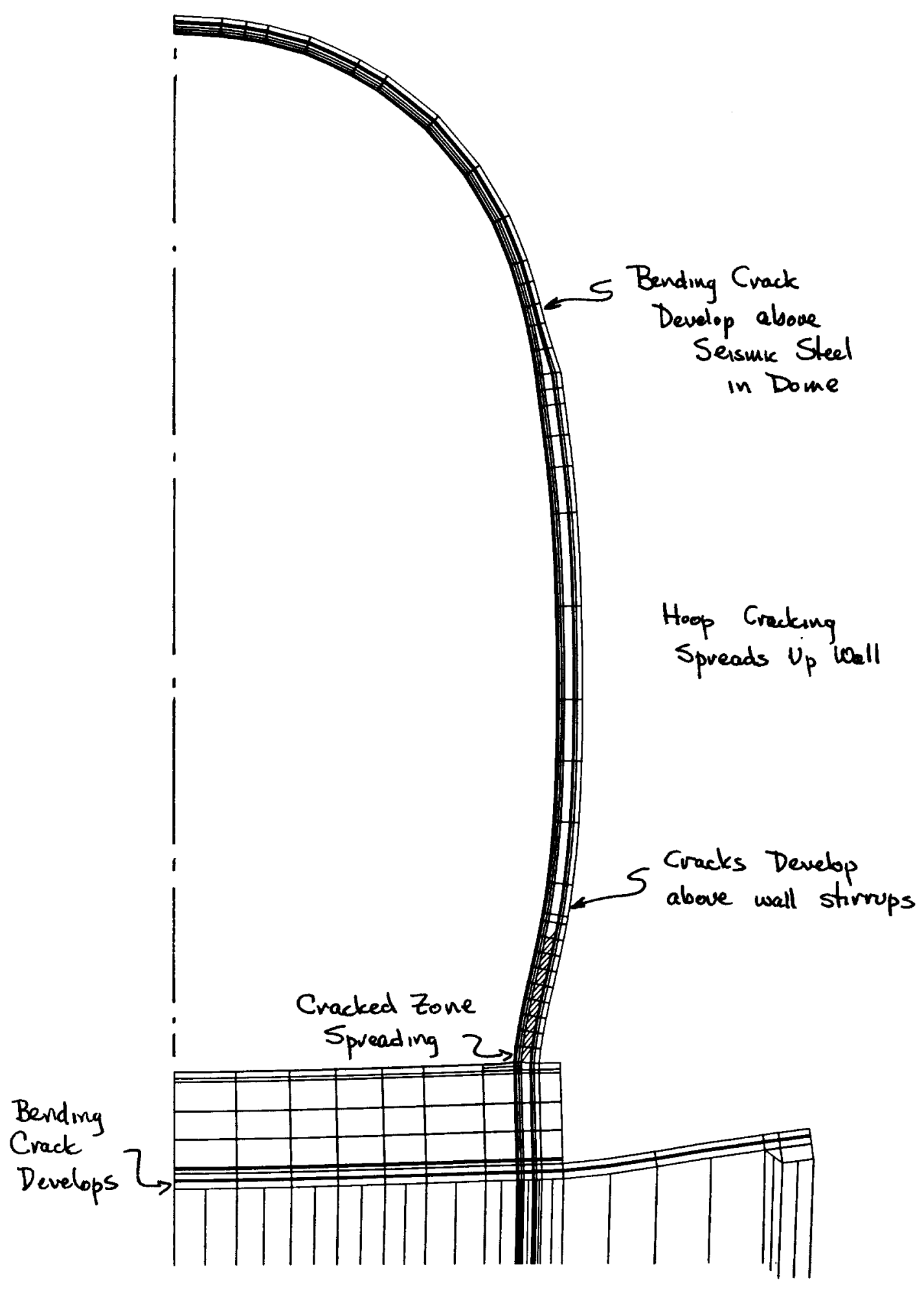

Figure 4.9.16 Deformed Configuration at $70 \mathrm{psi}(0.483 \mathrm{Mpa})$ 


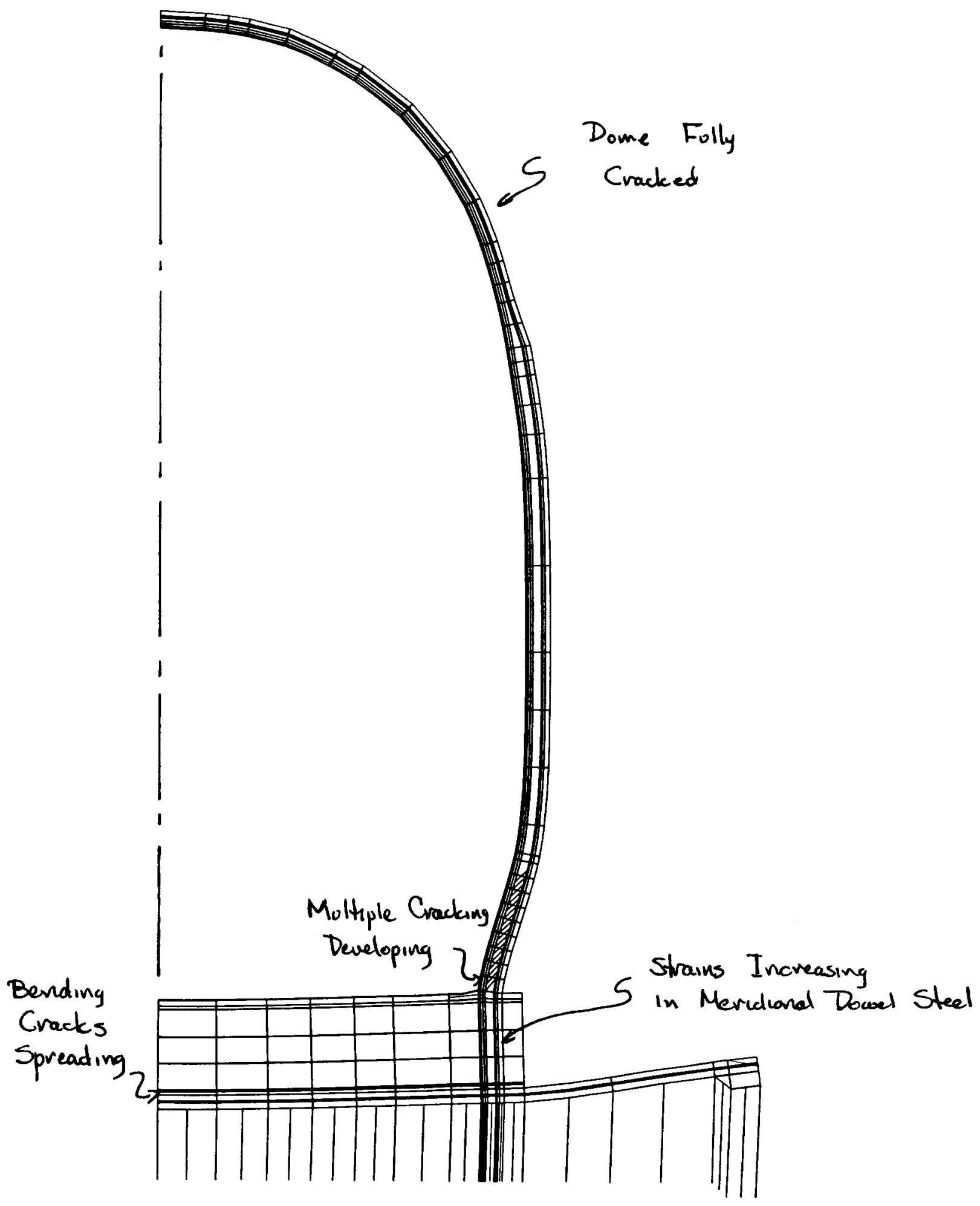

Figure 4.9.17 Deformed Configuration at 80 psi (0.552 Mp)

$-365-$ 


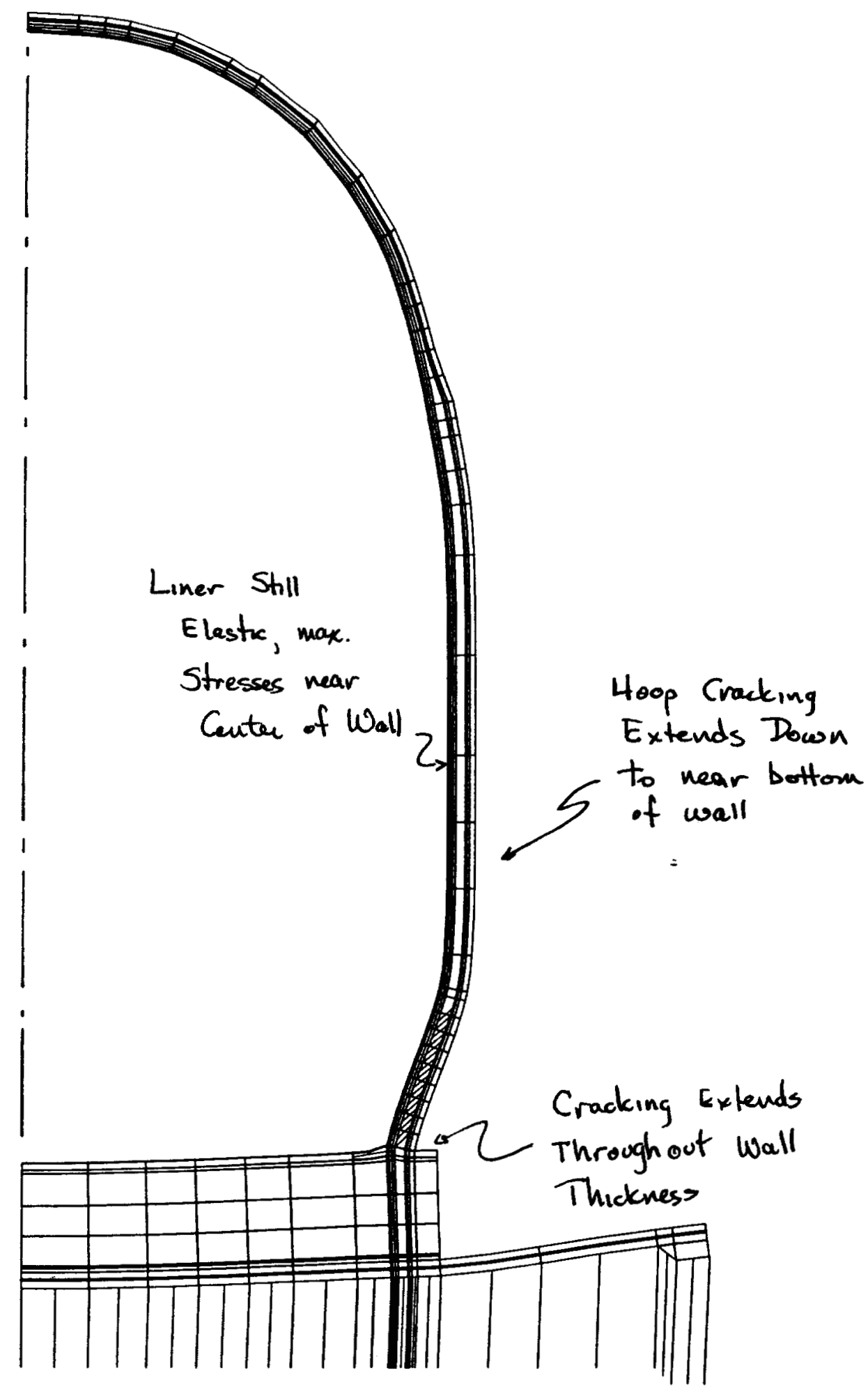

Figure 4.9.18 Deformed Configuration at 90 psi (0.621 Ma)

$-366-$ 


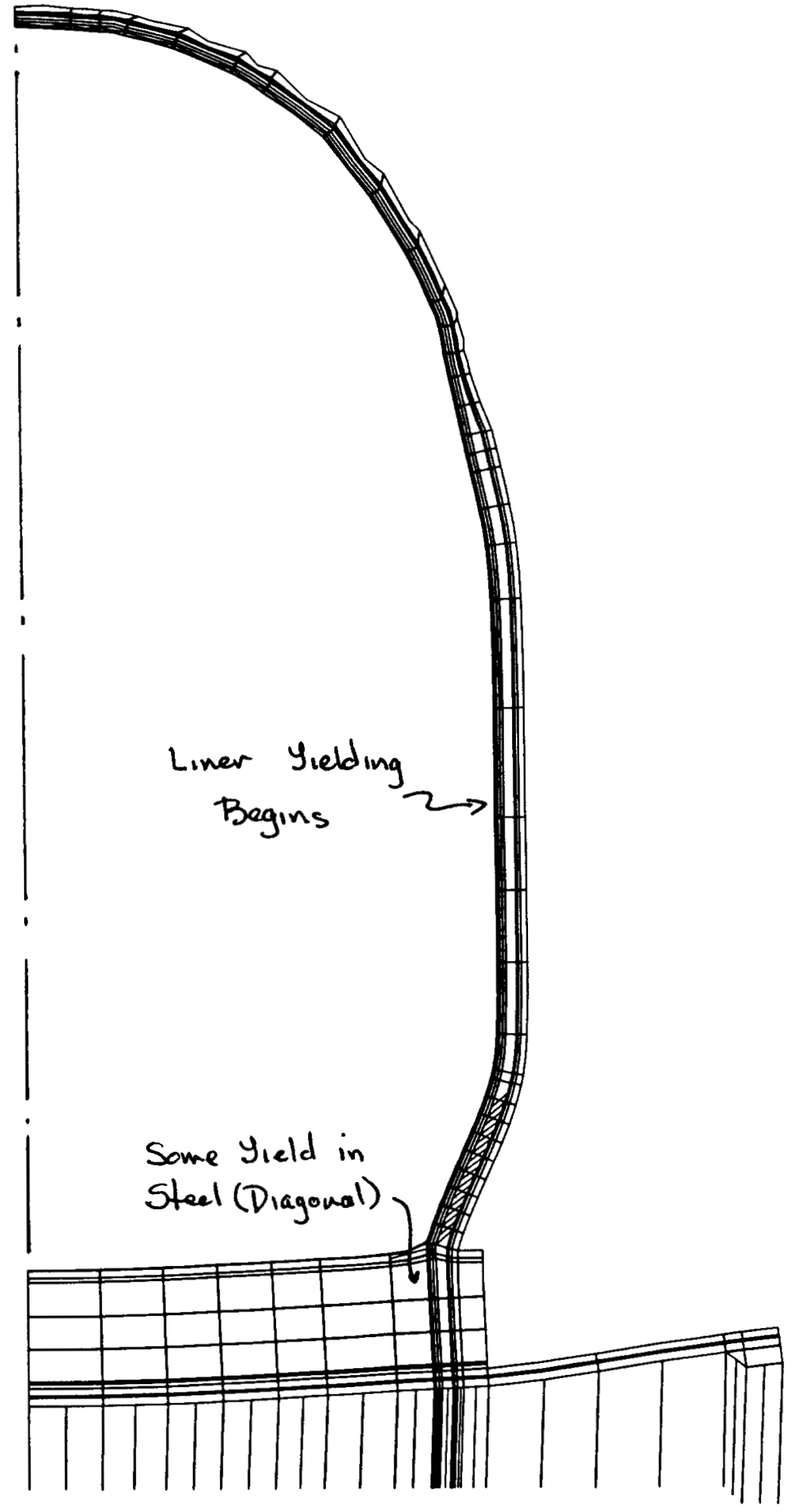

Figure 4.9.19 Deformed Configuration at 104 psi (0.717 Ma)

$-367-$ 


$$
2
$$



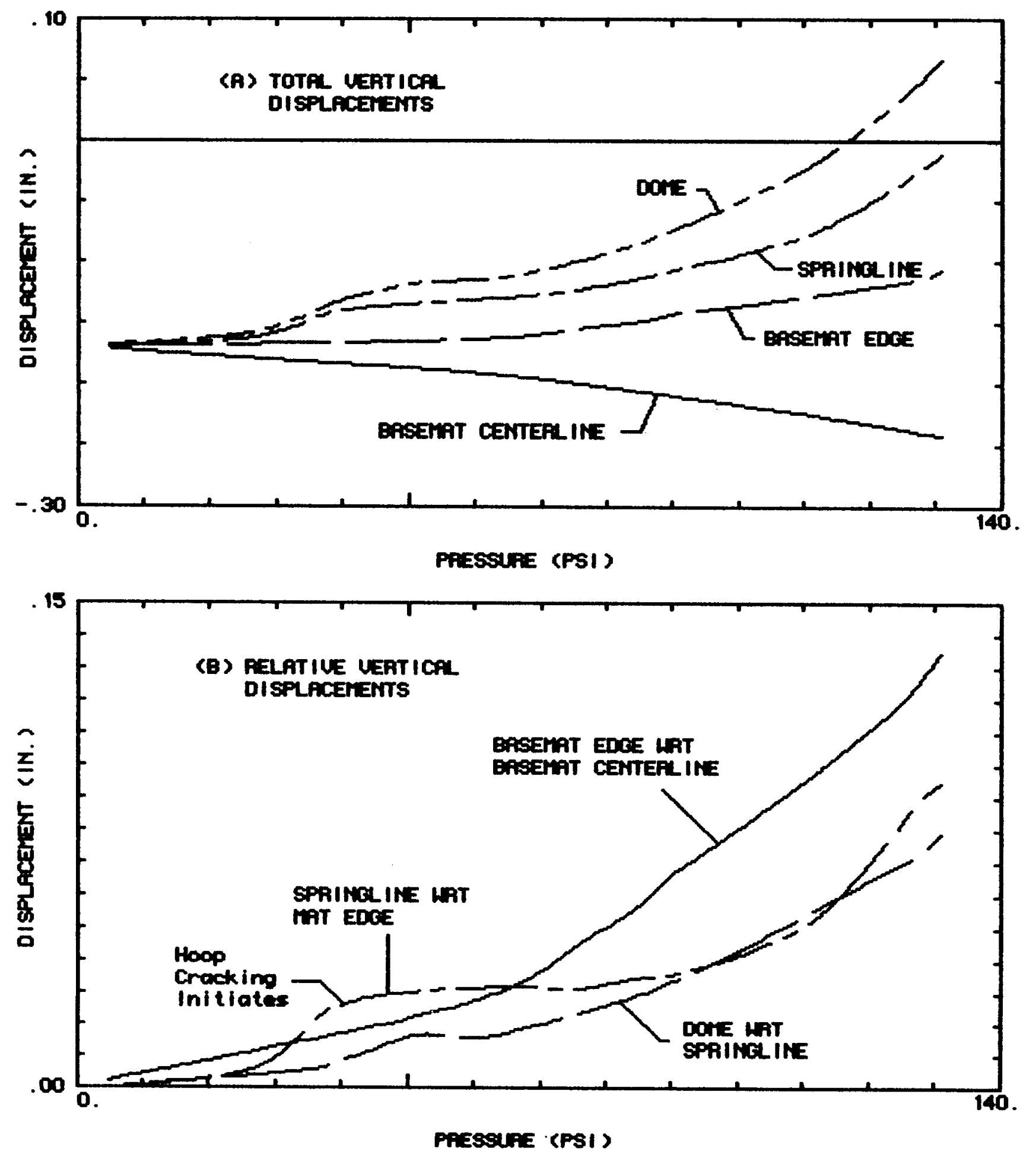

Figure 4.9.21 Computed Vertical Displacements Along Structure 


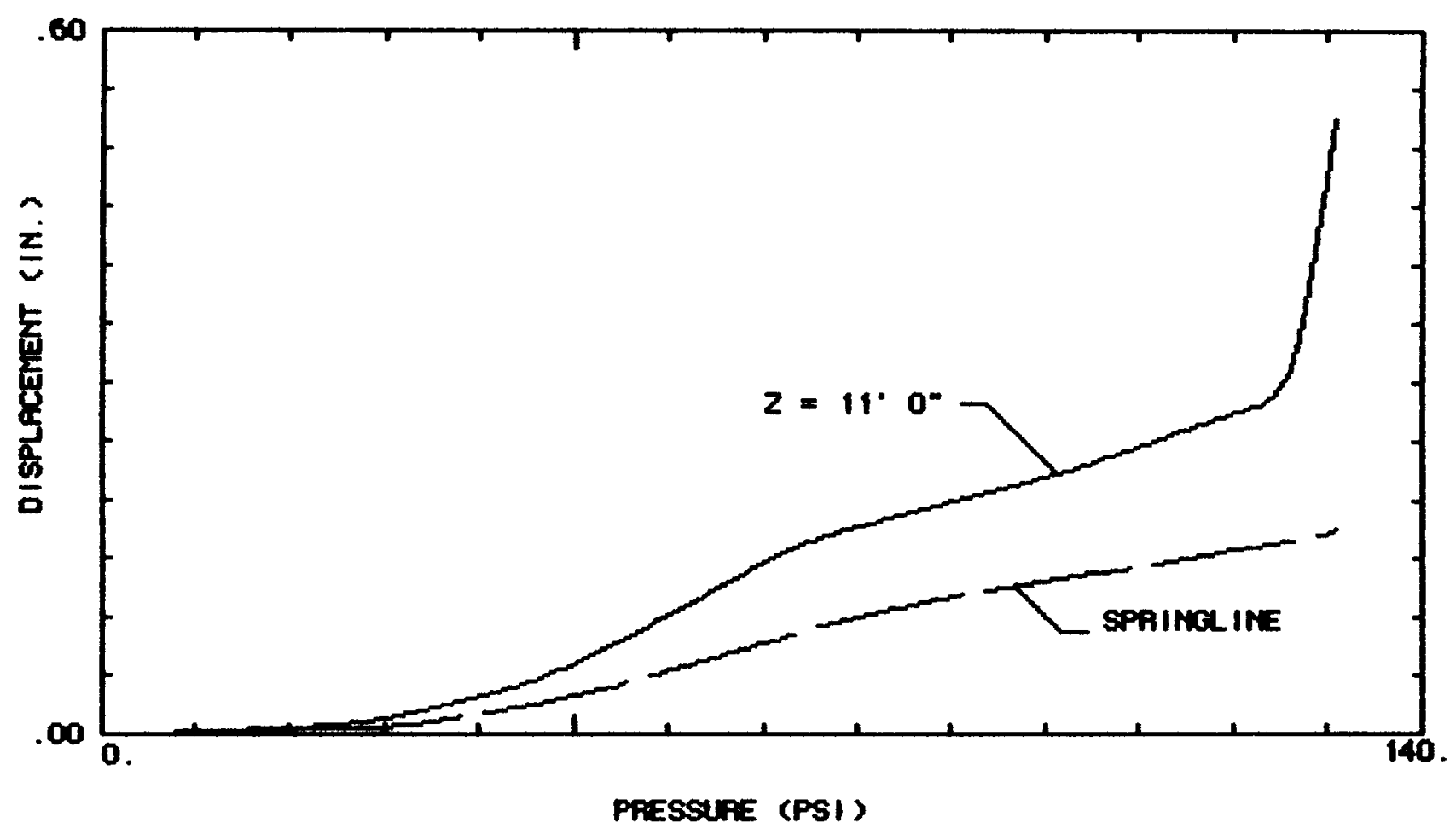

Figure 4.9.22 Radial Displacements Along Cylinder Wall

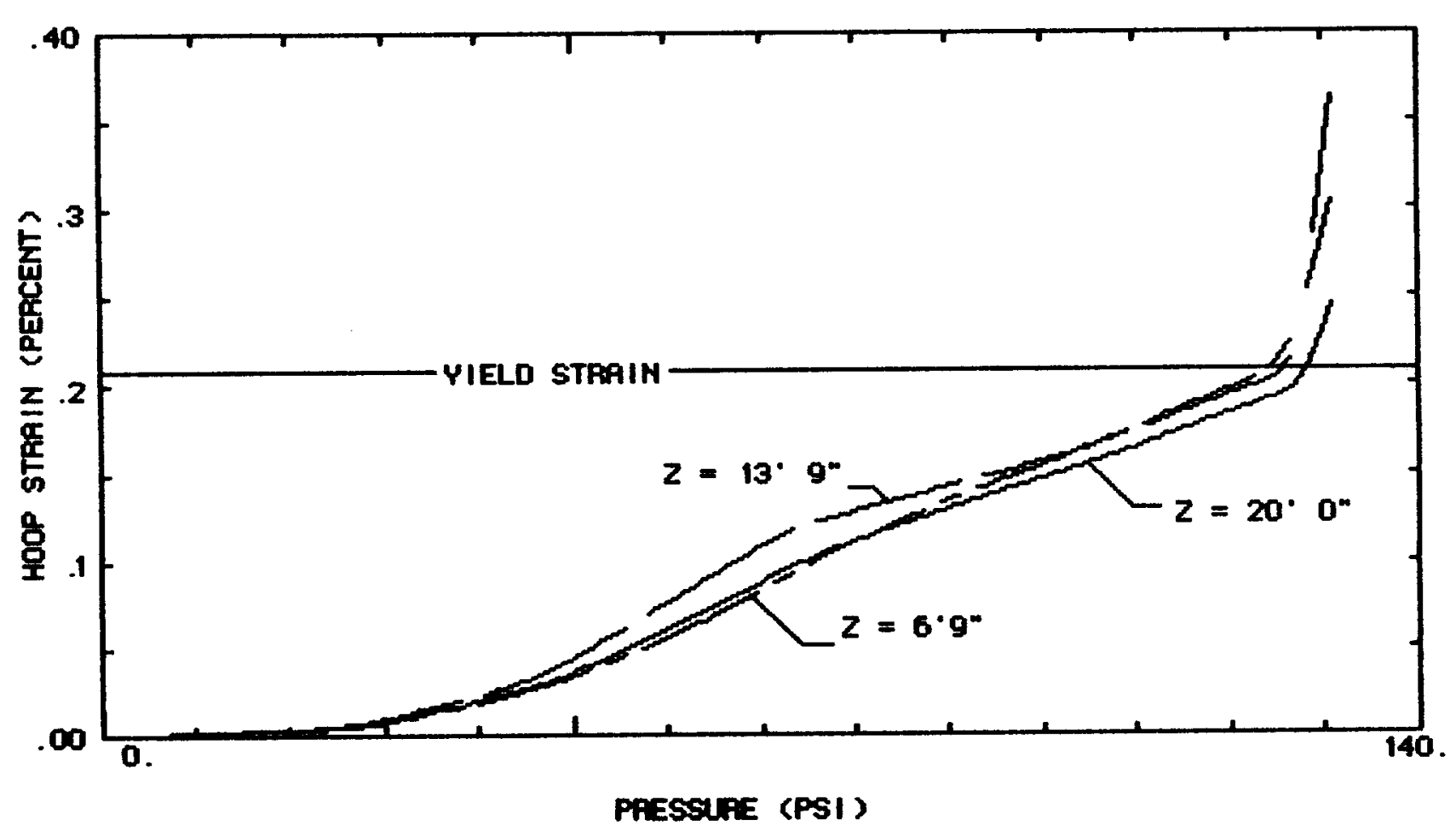

Figure 4.9.23 Hoop Strains Developed in Hoop Rebar 

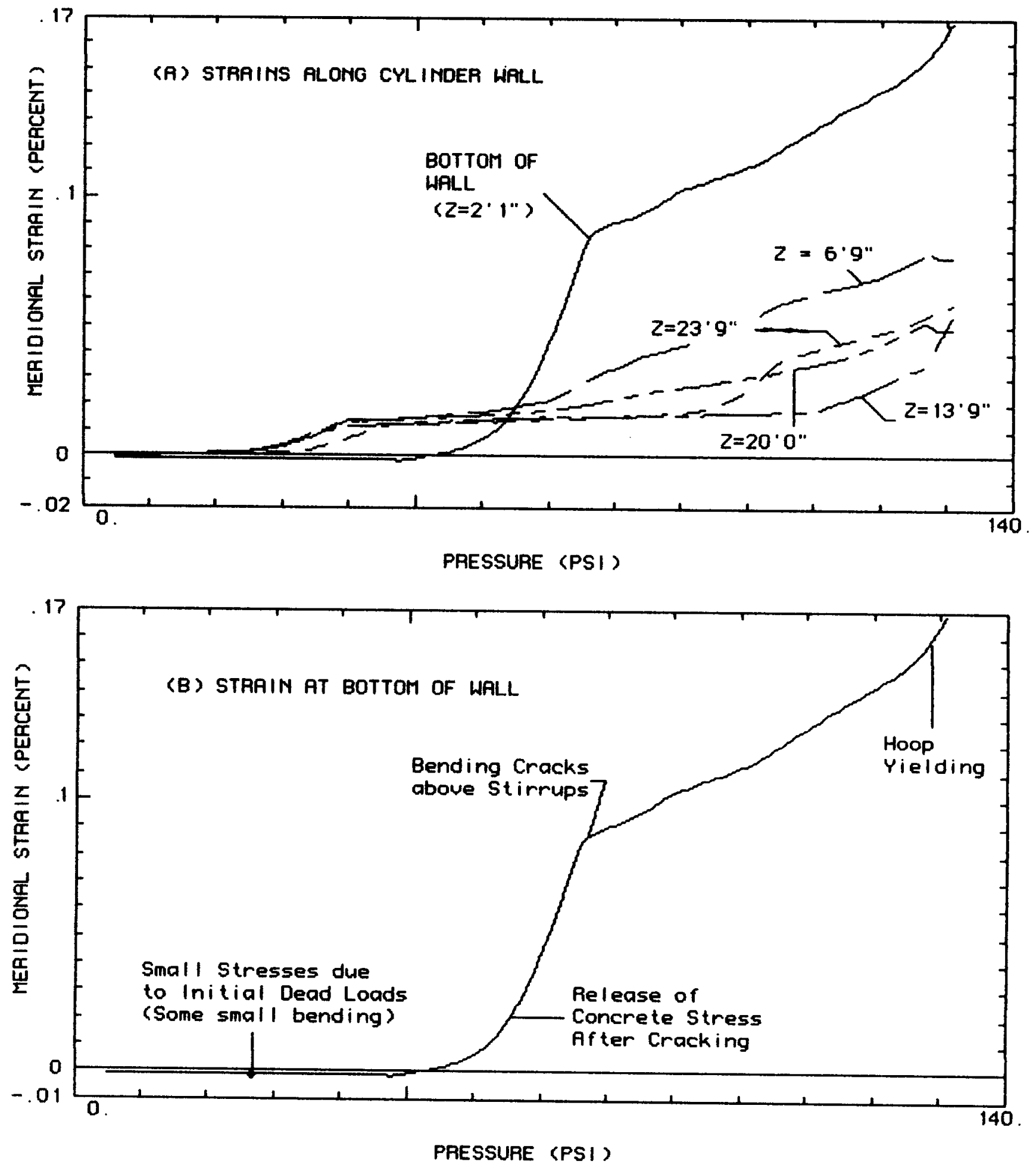

Figure 4.9.24 Strains in Meridional Steel in Layer 5 (Outer Layer) 


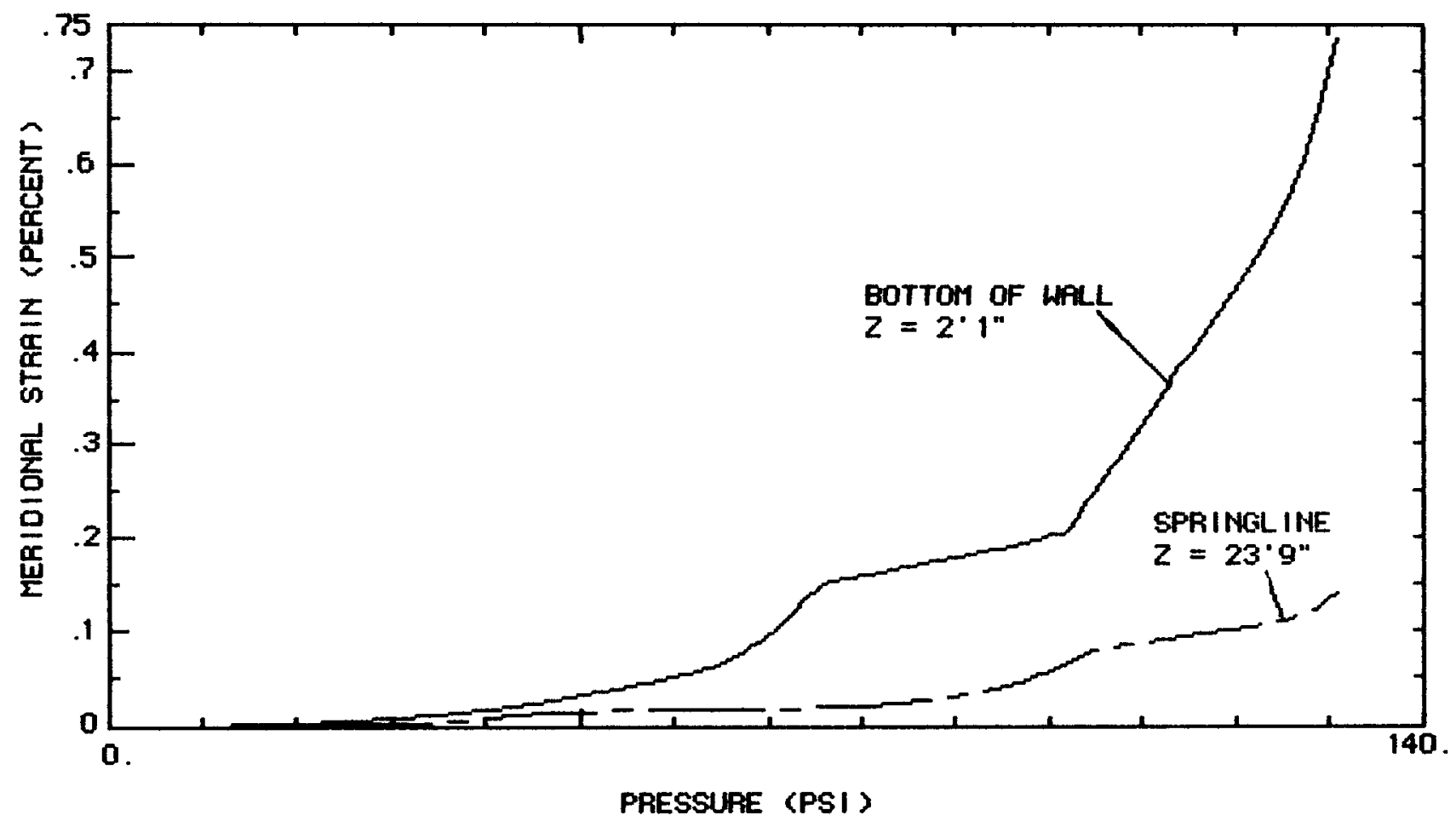

Figure 4.9.25 Strains in Meridional Steel in Layer 2 (Inner Layer) 

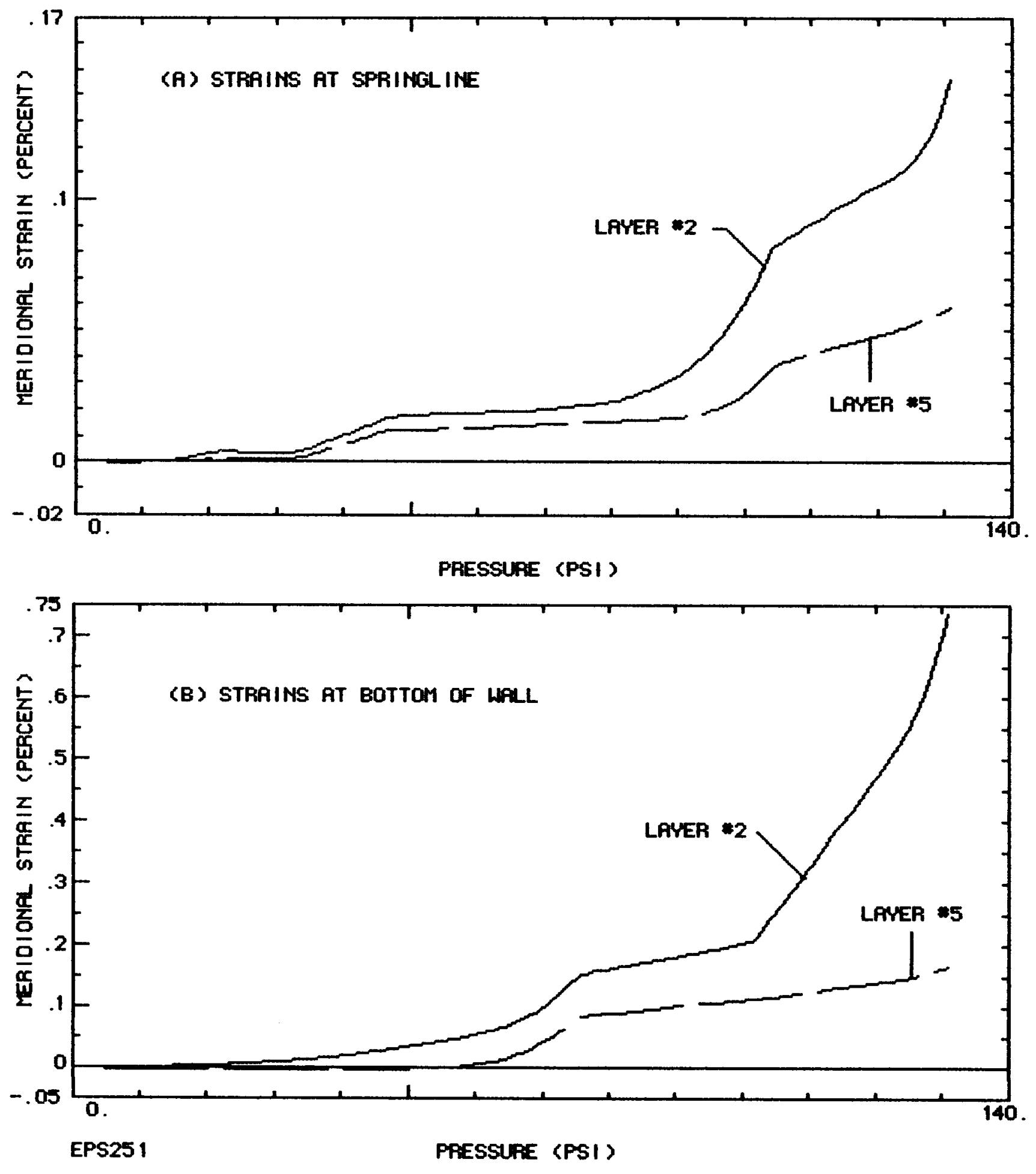

Figure 4.9.26 Comparison of Strains in Meridional Steel 


\subsection{Central Electricity Generating Board}

This section was authored by $S$. Kloosterman, D. Ness, and T. Hunt of Nuclear Design Associates on behalf of the Central Electricity Generating Board, United Kingdom.

\section{4:10.1 Surmary}

The U.S. Nuclear Regulatory Commission has ordered the construction and testing of a 1:6-scale model of a steel lined reinforced concrete containment at Sandia National Laboratories. This report describes the hand calculations and computer analyses which were used to predict the behavior of the model containment at internal pressure loads up to that causing failure. Two computer programs were used for the analyses: the linearelastic PAFEC [4.10.4] and the linear and nonlinear ADINA-TW [4.10.3].

Axisymmetric, plane 2-D and 3-D linear-elastic analyses were carried out to predict the behavior of the containment at internal pressures below those causing concrete cracking.

Nonlinear analysis of the containment was confined to an axisymmetric model which was taken to an internal pressure load of $160 \mathrm{psi}(1.103 \mathrm{MPa})$. At this pressure the analysis terminates due to flexural failure at the wall base junction.

The capability of the containment to retain internal pressure loads is dependent on the extent to which the liner remains leak tight. The difficulty of predicting the actual failure pressure of the containment is discussed together with the effects of the penetrations and associated thickenings. Due to these considerations the pressures obtained from the axisymetric analysis may be regarded as upper bound solutions.

\subsubsection{Introduction}

In the United States a program of model testing has been embarked upon to establish the ultimate failure pressures of existing containments. The 1:6-scale model of a reinforced concrete containment vessel that has been constructed at Sandia National Laboratories (SNL) represents part of this program. The vessel will be pressurized to 15 percent over design pressure, unloaded, and then tested to failure.

An analysis has been performed by NDA to attempt to predict the final outcome of the SNL testing. The analysis initially used hand calculations and finite element models to identify critical factors within the 1:6-scale model such as soil and material properties. A nonlinear axisymetric continuum model was then developed to analyze the structure up to failure. The overall analysis is depicted in Figure 4.10.1.

\subsubsection{Objectives}

Since no functional requirements for the containment structural model at ultimate pressure have been specified, "failure" of the structure is indefinable. However, the term failure within this report is taken to mean the onset of mechanistic behavior and ultimate pressure to be the pressure at which this behavior becomes apparent. The objective of these analyses is 
to predict the progression of cracking and yielding of concrete and stee 1 and to assess the internal gas pressure at the onset of mechanistic behavior.

The objectives of the study described in this report are:

a) to identify the possible modes of failure of the $1: 6$-scale model containment and to determine the associated internal pressures.

b) to identify the mode of failure which gives the minimum Load Factor (K).

c) to gain an understanding of the behavior of the model containment at internal pressures beyond those which produce a linear-elastic response.

d) to provide supporting evidence for the validation of the ADINA-TW nonlinear finite element program.

\subsubsection{Conclusions}

The analysis provides step-by-step information on the behavior of the structure under increasing internal pressure. Significant pressures were identified throughout the analysis.

The results from the nonlinear axisymmetric analysis showed the following:

a) Appreciable cracking of the concrete due to bending at the wall base junction will occur at pressures below the design pressure of the containment.

b) Vertical cracking will commence at mid-height of the wall at a pressure of $50 \mathrm{psi}(0.345 \mathrm{MPa})$ and will spread rapidly through the wall and dome (see figures 4.10 .37 and 4.10 .38 ). In the dome, radial cracking precedes the spread of the vertical cracking from the spring line to the apex of the dome.

c) Horizontal cracking of the concrete due to bending in the wall commences at 58 psi $(0.400 \mathrm{MPa})$. This will cause a loss of axial concrete tensile load carrying capacity causing the cracks to extend locally through the whole wall thickness. Similarly, bending due to the stiffness of the major penetrations and associated thickenings therefore will have an effect on the formation of cracks within the structure.

d) The remainder of the wall will crack in the horizontal direction due to membrane tension in the vertical direction. This will commence at 75 psi $(0.517 \mathrm{MPa})$ at the spring line and slowly progress down the wall.

e) At $82 \mathrm{psi}(0.566 \mathrm{MPa})$ the liner, at mid-cylinder height, will reach initial yield in the hoop direction followed rapidly by hoop yielding over its whole surface.

f) Dome liner yield in the hoop direction will commence at 110 psi $(0.759$ $\mathrm{MPa}$. 
g) The wall liner will reach initial yield in the vertical direction at 115 psi $(0.793 \mathrm{MPa})$ at the spring line and slowly progress down into the wall.

h) The dome liner will begin to yield in the meridional direction at 129 psi $(0.890 \mathrm{MPa})$.

i) Initial yield of the hoop reinforcement at mid-wall will commence at 124 psi $(0.855 \mathrm{MPa})$ with the seismic bar yielding first and all three layers past initial yield by 129 psi $(0.889 \mathrm{MPa})$.

j) At $127 \mathrm{psi}(0.876 \mathrm{MPa})$ the liner at the wall/base junction will yield in the vertical direction due to bending of the wall. The bending will progress to cause the vertical reinforcement to yield at 140 psi $(0.965$ $\mathrm{MPa})$. At $145.5 \mathrm{psi}(1.003 \mathrm{MPa})$ the concrete at the inner corner of the junction will reach 0.2 percent strain in the two principal directions of the element, which will relieve all stresses except for shear. At 159.5 psi (1.100 MPa) crushing will occur at the same level on the outside face of the wall and continue to propagate up to $160 \mathrm{psi}$ (1.103 MPa) when ADINA-TW results indicate flexural failure of the structure at the wall/base junction.

Hand calculations, using a force balancing method, indicate that for membrane areas of the containment barrel, bursting of the cylindrical wall determines the ultimate failure pressure of the containment (see Table 4.10.1). With liner and reinforcement stresses at initial yield (see Figures 4.10.16 and 4.10.17) taken from the test results, it can be shown that the ultimate pressure for an infinitely long cylinder will be 117 psi $(0.806 \mathrm{MPa}), K=2.54$ (see Appendix C).

If material stiffening after yield, equivalent to that incorporated in the ADINA-TW nonlinear analysis, were included in the hand calculation, the Load Factor would increase by 53 percent, the failure pressure becoming 181 psi $(1.23 \mathrm{MPa}), \mathrm{K}=3.9$.

The pressures quoted above are considered to be the upper bound limits for the related conditions. Various geometric and material assumptions as well as analytical methodology limit the accuracy of the results. It is expected that items such as local welding and coupling of rebar will reduce the quoted pressures. Additionally the effect of any imperfections in structural materials which might reduce the quoted pressures cannot be included in the analyses. Development of leaks in the containment which exceed pumping capacity could make it impossible to achieve the expected high pressures required for failure.

In conclusion our prediction is that on the limited analysis carried out, failure will be in line with conclusion ( $j$ ) stated above.

\subsubsection{Failure Modes}

The capacity of the model containment to retain pressure loads is dependent on the liner maintaining a gas seal sufficient to permit continuing pressurization within the capacity of the pumping equipment. Failure of the liner to meet this requirement could be caused by global failure of the associated containment structure. Alternatively it could be due to a local 
failure of the liner and anchorages caused by local concrete cracking or by imperfections in the liner or anchorage materials, e.g., at the wall base junction. It is not considered possible to predict the pressure at which such behavior might occur.

Failure of a section of the containment shell due to membrane tensile loads is preceded by a series of well defined behavioral stages during each of which smooth near linear load/strain characteristics are displayed. The limits of these successive stages, through which the membrane areas of the containment pass under increasing internal pressure, are marked by strain related changes in the material properties of the concrete, liner and reinforcing steel. These limits are:

1) Concrete cracking

2) Liner yield

3) Reinforcement yield

For membrane areas the Load Factor is therefore dependent on the load carrying capacity of the liner and reinforcement steels at strain values beyond those causing their initial yield.

At discontinuities, potential failure could be postulated to occur due to the combined effects of bending, membrane loads and shears. Due to their interaction, assessment of such areas is more difficult than for the membrane areas where only tensile loads occur.

\subsubsection{Method of Analysis}

\subsubsection{Axisymmetric Analysis}

\subsection{Hand Calculations}

Force balancing calculations were employed in areas where tensile membrane failure might occur and where loads on the section could be determined statically; e.g., at the dome apex and in the wall remote from discontinuities. The method uses relatively simple equilibrium equations for thin shells of revolution. Using the stress/strain properties of the concrete, reinforcement and liner material, the method provides an estimate of the internal pressures required to produce successive material cracking and yielding conditions.

The membrane forces due to the internal pressure loads are balanced by the sum of the force changes in the structural materials measured from the zero internal pressure stage. Thus, the change in strain required to reach behavioral limit for the section under consideration can be calculated.

The method assumes that the concrete loses its tensile capacity immediately upon cracking. In reality concrete retains some tensile capacity after cracking, and this effect, termed "tension stiffening" is included in the ADINA-TW nonlinear finite element analysis. 
To demonstrate the application of the force balancing method, calculations for the hoop direction at the mid-height of the wall are included in Appendix C.

Additionally a hand calculation was performed to determine the relative stiffness of the $6 \mathrm{~mm}$ tie bar with respect to the concrete cylinder wall.

\subsection{Computer Analysis}

A plain concrete axisymmetric model with a rigid base, as shown in Figure 4.10.2, was analyzed assuming linear-elastic concrete properties using both the PAFEC and ADINA-TW finite element programs.

The ADINA-TW code is a modified version of ADINA-81 that includes an improved representation of nonlinear concrete properties. The modifications are reported to make the program output more compatible with various test results [4.10.6] and take account of recently published works [4.10.1, $4.10 .2,4.10 .8]$.

Elements representing the reinforcement and liner were then added to the model for further analysis using ADINA-TW as a nonlinear finite element program. The program does not include an axisymmetric plate element and so both reinforcement and liner were modelled as a combination of single noded hoop elements and two noded truss elements. A typical section of the containment wall, actual and as analytically modelled, is shown in Figure 4.10.3. The diagonal reinforcement was modelled as equivalent horizontal and vertical elements assuming equivalent rebar area for these elements.

The soil under the base was modelled as springs with linear-elastic compressive stiffness but with no capacity to accommodate tensile loads. To assess the effects of the soil stiffness on the behavior of the containment, upper and lower bound stiffnesses were investigated, the higher value of $382 \mathrm{ksf} / \mathrm{ft}(60.0 \mathrm{MPa} / \mathrm{m})$ then being used for the further analysis.

A "materially nonlinear only" ADINA-TW option was used for the analysis, i.e., it was assumed that strains and displacements were small enough such that the geometrical effects of changes in positions and shapes of the elements could be neglected. The stiffness matrix was reformed at each load step of the analysis, small load increments being used where rapid behavioral changes were predicted.

\subsubsection{Investigation into the effects of the penetration and associated thickenings}

Asymmetric features of the model containment such as the major penetrations and associated thickenings will produce variations in the uniform stresses of a pure axisymetric structure and could therefore influence the behavior of the structure under increasing internal pressure load.

It must be assumed that the effects of asymetric features will be dissimilar in the elastic and inelastic phases of containment behavior and it is of interest to assess their action throughout the range of pressure loading. 
To estimate the effects of the major penetrations and associated thickenings several linear-elastic models were created and analyzed using the PAFEC finite element program. The models used were:

a) Axisymmetric with the thickenings added as complete bands of elements to the wall of the containment (Figures 4.10 .4 and 4.10 .5 ).

b) A 2-D horizontal slice of $180^{\circ}$ of the containment wall with an axis of symmetry through the center of the plant access penetrations (Figure $4.10 .6)$.

c) A 3-D model of $180^{\circ}$ of the containment wall with symmetry assumed at the level of the penetrations (Figure 4.10.7).

Ideally, a full nonlinear 3-D analytical model should be developed to determine the penetration effects. Initial estimates indicate that this procedure is prohibitively expensive as well as time consuming and thus a partial 3-D model at worst case locations should be used, in spite of the limitations associated with the estimation of boundary conditions and prediction of worst case locations. Therefore, the analysis terminates with the linear analyses summarized above with a judgement made as to the full effects of the penetrations on the failure pressure.

\subsubsection{Material Properties}

The following properties were assumed in the computer analyses.

4.10.7.1 Concrete

4.10.7.1.1 Linear-elastic analysis

The linear-elastic PAFEC analyses used a concrete modulus value of $4350 \mathrm{ksi}$ $(30000 \mathrm{MPa})$, a Poisson's ratio of 0.2 , and a density of $150 \mathrm{lb} / \mathrm{ft}^{3}$ $\left(2400 \mathrm{~kg} / \mathrm{m}^{3}\right)$. The initial ADINA-TW axisymmetric linear analysis, performed as a comparison to PAFEC, used similar properties.

\subsection{Nonlinear analysis}

The concrete properties for use in the nonlinear analysis were assessed from results of tests performed on the concrete of the containment model. The concrete of the containment is generally in a multiaxial stress state and its behavior under increasing load is thus dependent on the stresses in all directions. Representation of behavior under these conditions can only be approximate [4.10.5].

Representation of concrete behavior in the ADINA-TW analyses is required to take account of:

a) failure of the material due to tension and compression

b) post cracking behavior

c) nonlinear stress strain relationships 


\subsection{Concrete Crushing}

The compression failure envelopes (Figure 4.10.8) assumed in the analyses were derived using published test data [4.10.6].

\subsection{Concrete Cracking}

The tensile failure envelope assumed by ADINA-TW is shown in Figure 4.10.9.

Considerable variation exists in results from different types of tensile tests and from results obtained from particular tests. In addition the performance of small test specimens may differ from the performance of a larger structure due to the inability of the specimens to redistribute loads following failure at a local imperfection in the material. Commonly tensile cracking values are taken as between 0.085 and 0.110 times the cylinder strength of the concrete. For these analyses a value of $0.6 \mathrm{ksi}(4.1 \mathrm{MPa})$ $\left(0.10 \mathrm{fc}^{\prime}\right)$ was adopted [4.10.9].

In ADINA-TW it is assumed that a crack is formed in the concrete when the maximum principal tensile stress exceeds the tensile strength of the concrete, the crack forming in a plane perpendicular to the direction of the principal stress. It is assumed that the concrete changes from an isotropic to an orthotropic material with reductions in stiffness normal and parallel to the cracked plane. As load increases, further cracks may occur in planes perpendicular to that fixed by the first crack.

Within section 4.10 horizontal cracking is defined as the result of vertical tensile stresses. Vertical cracking is defined as the result of horizontal stresses and radial cracking is the result of hoop stresses.

\subsection{Post Cracking Properties}

Neglect of post cracking stiffness will give inaccurate representation of the structural behavior of the containment. Therefore the properties of shear retention and tension stiffening are required to model the post cracking behavior of the concrete.

a) Shear retention

After cracking the concrete is still capable of resisting shear forces acting parallel to the plane of the crack due to a combination of aggregate interlock and dowel action of reinforcing bars.

Experimental work [4.10.7] has shown that the retained stiffness depends upon many variables, including the size and shape of the aggregate, the area and diameter of the reinforcement and the tensile strain across the crack. Typically, increasing tensile strain causes an initial loss of stiffness due to crack formation followed by further losses which proceed at a reducing rate. ADINA-TW models the shear retention factor $(\beta)$ as shown in Figure 4.10 .10 .

b) Tensile stiffening

As primary cracking occurs there is a reduction in tensile stiffness as the concrete between the cracks continues to carry some tensile load 
until the eventual breakdown of bond between the reinforcing steel and the concrete. ADINA-TW represents tension stiffening by reducing the concrete tensile stiffness after cracking as the tensile strain increases (Figure 4.10.11).

\subsection{Nonlinear Stress Strain Relationship}

The assumed uniaxial stress-strain relationship is shown in Figure 4.10.12. In the ADINA-TW code this curve is modified for multiaxial conditions by factors $\gamma_{1}$ and $\gamma$ as shown in Figure 4.10.13. The factor $\gamma$ is calculated using the relationship $\gamma=1.4 \gamma_{1}-0.4$ where the ratio of the multiaxial to uniaxial strength $\left(\gamma_{1}\right)$ is derived from the compressive failure envelopes as shown in Figure 4.10.14.

In simple tests [4.10.8] it was shown that Poisson's ratio remains constant until stresses reach approximately 80 percent of the compressive failure stress, after which it begins to increase. ADINA-TW takes account of this effect by using the relationship given in Figure 4.10.15.

\section{$4 \cdot 10.7 .2$ Steel \\ 4.10.7.2.1 Reinforcement}

Reinforcement properties were based on results from tests performed by SNL. The yield stresses and modulus values quoted in the test results were calculated assuming bars of minimum specified area whereas the actual bars tested were of greater area. The values were modified accordingly and the stress-strain curve as shown in Figure 4.10.16 was adopted for the analysis.

\subsection{Liner}

Test results were available for both the $1 / 16$ inch $(1.6 \mathrm{~mm})$ thickness and $1 / 12$ inch $(2.1 \mathrm{~mm})$ thickness plate material. From these results the stressstrain curve as shown in Figure 4.10.17 was derived.

ADINA-TW does not include an axisymmetric plate element and therefore the liner was modelled as a combination of single noded hoop elements and two noded truss elements. In order to represent the stiffness of an actual plate the material stiffness of these elements was increased to allow for Poisson's ratio effects. The factor on the stiffness used was $1 /(1-\nu)$ for both the wall and the dome. This factor is included in Figure 4.10.17.

\subsubsection{Soil}

Test results were available from three plate load tests using 12,18 , and 30 inch $(305,457$, and $762 \mathrm{~mm})$ plates as shown in Figure 4.10.18. Assuming a rigid circular plate and constant bearing stress, a lower bound stiffness value of $26 \mathrm{ksf} / \mathrm{ft}(4.04 \mathrm{MPa} / \mathrm{m})$ was derived for spring elements representing the soil with an upper bound stiffness value of $390 \mathrm{ksf} / \mathrm{ft}(61.26 \mathrm{MPa} / \mathrm{m})$.

It is judged that the final ultimate load will not be significantly influenced by the soil properties. This is due to the thickness of the basemat relative to the domed cylinder structure and to the fact that the 
lower bound stiffness assumed the highly unprobable case of uniform surface soil properties for the whole depth of soil loaded by the containment. The stiffness of $382 \mathrm{ksf} / \mathrm{ft}(60.0 \mathrm{MPa} / \mathrm{m})$ was adopted for the nonlinear analysis.

4.10 .8 Results

4.10.8.1 Axisymetric Analysis

4.10.8.1.1 Hand Calculations

The results from the hand force-balancing calculations are summarized in Table 4.10.1. Barrel bursting due to failure of the hoop reinforcement and liner produces the minimum Load Factor of 2.54. Figure 4.10.19 shows how the section passes through distinct changes in stiffness as strain increases with internal pressure rise.

Hand calculations also indicated that the $6 \mathrm{~mm}$ tie bar at elevation $20^{\prime}-1.5^{\prime \prime}$ $(+6.134 \mathrm{~m})$ has no effect on the overall response of the structure. Further analyses therefore neglect this element.

4.10.8.1.2 Computer Analysis

4.10.8.1.2.1 Linear-Elastic Analysis

A comparison of the stresses and deflections from the PAFEC and ADINA-TW elastic analysis showed that the results of the two analyses were generally in good agreement, thus providing confidence in the accuracy of both codes.

\subsection{Nonlinear Analysis}

Preliminary axisymetric analyses were performed to provide indications of significant events in the behavior of the structure as well as identifying areas sensitive to initial assumptions.

\section{Table 4.10 .1}

Summary of Force-Balancing Hand Calculation Results

Event

\begin{tabular}{|c|c|c|c|c|c|c|}
\hline & \multicolumn{2}{|c|}{ Wall Hoop } & \multicolumn{2}{|c|}{ Wall Vertical } & \multicolumn{2}{|c|}{ Dome } \\
\hline & ps $i$ & $\mathrm{MPa}$ & psi & $\mathrm{MPa}$ & psi & $\mathrm{MPa}$ \\
\hline $\begin{array}{l}1 \mathrm{st} \text { Concrete } \\
\text { Crack }\end{array}$ & 52 & 0.359 & 98 & 0.676 & 74 & 0.510 \\
\hline $\begin{array}{l}\text { Liner Plate } \\
\text { Yield }\end{array}$ & 84 & 0.579 & 108 & 0.743 & 131 & 0.904 \\
\hline Rebar Yield & 117 & 0.806 & 141 & 0.973 & 158 & 1.090 \\
\hline
\end{tabular}

(1) Pressures are calculated using perfectly plastic material properties. See Appendix C for sample calculations. 
A relatively large pressure increment was chosen for the preliminary analyses. The results identified initial concrete cracking and shell yield points which compared well with the hand calculations thus providing confidence in the analytical model up to $124 \mathrm{psi}(0.855 \mathrm{MPa})$.

The final analysis, incorporating information from the preliminary runs, gives detailed results for the entire structure up to and including identification of the failure of the structure. Significant events in the behavior of the containment under increasing pressure load are summarized in Table 4.10.2. A comparison with the results of the hand calculations shows

Table 4.10 .2

Summary of ADINA-TW Nonlinear Axisymmetric Analysis Results

Event

First Concrete Crack at Wall/Base Junction

First Vertical Crack in Cylinder Wall

First Membrane Crack in Dome

First Horizontal Crack in Wall Due to Bending

First Horizontal Crack in Wall Due to Membrane Tension

Initial Yield of Wall Liner in Hoop Direction

Initial Yield of Dome Liner in Hoop Direction

Zero Tensile Retention of Concrete in Hoop Direction

Initial Yield of Liner at Springline in Axial Direction

Initial Yield of Horizontal Reinforcement in Wall

95\% of Wall and Dome Concrete Cracked in Two Directions

Liner Yield at Wall/Base Junction

Initial Yield of Dome Liner in Meridional Direction

Initial Yield of Vertical Reinforcement at Wall/Base Junction

Initial Yield of Vertical Reinforcement at Outer-mid Wall

Initial Yield of Vertical Reinforcement in Dome

Zero Tensile Retention at Inside Corner of Wall/Base Junction

Crushing of Outer Wall at Wall/Base Junction

Flexural Failure of Structure at Wall/Base Junction
Psi $\stackrel{\text { Pressure }}{\mathrm{MPa}}$

20

50

0.138

53

0.345

$53 \quad 0.365$

$58 \quad 0.400$

$75 \quad 0.517$

82

0.565

110

0.758

110

0.758

115

0.793

124

0.855

124

0.855

127

0.876

129

0.889

140

0.965

142

0.979

145

1.000

$145.5 \quad 1.003$

$159.5 \quad 1.100$

160

1.103 
good agreement for events in the membrane areas of the containment. After cracking of the concrete and initial yield of the steel the comparison between the hand and ADINA-TW calculation shows increasing discrepancy. This is due to the fact that the hand calculations assume perfectly plastic materials without increase in stiffness after yielding whereas the computer analysis assumes some stiffening under this condition leading to an increase in the calculated ultimate pressure. For the failure mode of barrel bursting, the hand calculation pressure would increase by 53 percent to 181 psi ( $1.24 \mathrm{MPa})$ for the modified material properties.

The displacement under increasing pressure load of typical nodes of the ADINA-TW model are shown in Figures 4.10 .20 and 4.10 .21 and the displaced shapes of the containment are shown graphically in Figures 4.10 .22 to 4.10.28. At an internal pressure of $129 \mathrm{psi}(0.89 \mathrm{MPa})$ it can be seen that the radial displacements start to increase rapidiy at a height of $16 \mathrm{ft}$ $(4.88 \mathrm{~m})$ above the bottom of the basemat. The start of this increase corresponds with the hoop reinforcement reaching its initial yield value. Because the concrete is extensively cracked the bending stiffness of the section is considerably reduced and there is little capacity for load redistribution to adjoining parts of the structure. As the hoop reinforcement progressively reaches the initial yield point, a significant 'bulge' appears at wall mid-height (Figure 4.10.27).

The ADINA-TW results show cracking of the concrete at the wall base junction, due to bending, occuring from a pressure of $20 \mathrm{psi}(0.138 \mathrm{MPa})$. At design pressure there is appreciable cracking in the area, as shown in Figure 4.10.29. Cracking in the area at the Structural Overpressure Test pressure of 1.15 Pd and at higher pressures is shown in Figures 4.10 .30 to 4.10 .36 .

Note that in Figures 4.10 .29 through 4.10 .36 basemat results are for selected elements only. This is due to machine capacity. File size restricts the amount of output printed and thus certain element results are not available.

Vertical cracking of the concrete wall commences at mid height of the wall, from the inside face, at a pressure of $50 \mathrm{psi}(0.345 \mathrm{MPa})$ and spreads rapidly with further increments of pressure, reaching the bottom of the dome at Structural Overpressure Test pressure, $53 \mathrm{psi}(0.365 \mathrm{MPa})$, as shown in Figure 4.10.37. With further increments of load the cracking spreads towards the apex of the dome as shown in Figure 4.10.38.

Radial cracking at the inside face of the dome, due to a combination of bending and meridional tension spreads from the base towards the apex at the same rate as the vertical cracking (Figure 4.10.38). The radial cracking spreads rapidly and at a pressure of approximately $80 \mathrm{psi}(0.552 \mathrm{MPa}$ ) has extended over the majority of the dome.

Horizontal cracking at the outside face of the wall due to a combination of bending and axial tension commences at $58 \mathrm{psi}(0.400 \mathrm{MPa})$ at approximately $9^{\prime} 2^{\prime \prime}(2.80 \mathrm{~m})$ above the bottom of the basemat. At $70 \mathrm{psi}(0.488 \mathrm{MPa})$ this cracking has extended through much of the section, the loss of the tensile strength of the cracked concrete in resisting membrane loads being significant in extending the depth of the initial cracks (Figure 4.10.39). In the remaining areas of the wall where bending effects are insignificant, 
horizontal cracks occur due to pure membrane tension. These cracks form at a pressure of between $75 \mathrm{psi}(0.517 \mathrm{MPa})$ and $104 \mathrm{psi}(0.718 \mathrm{MPa})$.

Radial cracking at the underside of the base spreads radially from the middle of the structure at a pressure value of $80 \mathrm{psi}(0.552 \mathrm{MPa})$. These cracks are due to high hoop stresses and extend across the entire underside of the basemat by 124 psi $(0.855 \mathrm{MPa})$. Additionally cracking due to bending commences near the containment axis before the pressure reaches $147 \mathrm{psi}$ $(1.104 \mathrm{MPa})$.

Subsequent to the loss of the concrete strength due to cracking, the majority of the pressure load transfers to the internal liner and the various layers of reinforcement. Graphical representation of the steel element stresses relative to increasing pressure are given in Figures 4.10 .40 through 4.10 .44 .

The initial yield of the liner occurs at $82 \mathrm{psi}(0.565 \mathrm{MPa})$ where midcylinder wall yields in the hoop direction. The yield progresses rapidly both upward and downward and the majority of the wall liner is beyond the initial yield stress by 86 psi $(0.593 \mathrm{MPa})$. The high hoop stress continues upward with the dome liner yielding at approximately $110 \mathrm{psi}(0.759 \mathrm{MPa})$.

At this same pressure, the concrete strains in the hoop direction of the wall have reached 0.2 percent. As discussed in Section 4.10.7.1.2.3b the ADINA-TW code allows retention of tensile stresses after cracking with reduction in retention relative to the element strain. At 0.2 percent strain this retention reduces to zero, thus 100 percent of the hoop stress is carried by the liner and hoop reinforcement.

At $115 \mathrm{ps} i(0.793 \mathrm{MPa})$ the axial stress in the liner at the spring line reaches the initial yield value due to a combination of membrane tension and bending. With further pressure increments the affected area of the liner spreads downward into the cylinder wall.

The hoop reinforcement within the cylinder wall, now carrying approximately 80 percent of the load, attains initial yield at $124 \mathrm{psi}(0.855 \mathrm{MPa})$. The inside face reinforcement is the first to yield with all three layers beyond yield by 129 psi $(0.890 \mathrm{MPa})$. This occurs simultaneously with the rapid expansion of the cylinder at mid wall, due to the modified stress-strain behavior of the rebar and liner after yield.

At the later stages of the pressure loading the concrete cracking at the wall base junction, caused by bending at the junction, can be seen to have spread through a substantial depth of the basemat. The strain in the basemat liner adjacent to the corner is shown in Figure 4.10.45. At 127 psi $(0.876 \mathrm{MPa})$ the basemat liner yields adjacent to the junction. At $139 \mathrm{ps} i$ (0.959 MPa) the wall liner yields vertically at a location just above the junction and the inside face vertical reinforcement in the top of the basemat yields. The inside face vertical reinforcement in the wall yields at 142 psi (0.979 $\mathrm{MPa})$.

At a pressure of $145.5 \mathrm{psi}(1.003 \mathrm{MPa})$ the tensile strains in the concrete elements at the inside corner of the wall/base junction exceed 0.2 percent in both principal directions. This is the strain level at which ADINA-TW assumes that tensile retention no longer acts, although shear resistance is 
still available in planes parallel to crack faces. The zone of zero tensile retention extends until, at a pressure of $159.5 \mathrm{psi}$ (1.100 MPa), crushing occurs at the outside face of the wall (see Figure 4.10.35). At 160 psi (1.103 MPa) ADINA-TW shows numerical instability indicating flexural failure at the wall/base junction.

\subsection{Limitations of Results}

Some limitations of the accuracy of the results obtained from the analyses are summarized below:

a) The concrete tensile strength assumed in the ADINA-TW axisymmetric analysis may vary appreciably from that in the concrete of the actual structure. The pressure at which cracking first occurs in the various parts of the structure may therefore also vary from those predicted by the analysis.

However, the analysis is expected to give a good indication of the progression of cracking at various locations within the structure.

b) For the axisymmetric concrete elements, ADINA-TW outputs stress results at nine Gauss points within each element. In addition the output contains a statement as to the state of the concrete at each point, e.g., uncracked or cracked. At locations and pressures where large areas are at the point of cracking small stress variations can make considerable differences to the state of the concrete at each Gauss point. It is important therefore to globally view the presence of such cracking and the associated crack directions rather than to draw conclusions from cracks at individual points.

Stress and strain results for shell elements in and adjacent to cracked concrete elements can show considerable variation dependent on the state of the concrete elements through which they pass. In a similar manner to cracking, the results should be viewed globally.

\subsubsection{Linear-elastic analysis with penetration thickenings}

The ultimate pressure load of $160 \mathrm{psi}(1.103 \mathrm{MPa})$ reported above assumes a purely axisymmetric structure with no penetrations or associated thickenings. The following results give an indication of the effects of the four major penetrations relative to the nonlinear axisymmetric analysis.

\subsection{Axisymmetric Analysis}

The displacements of the axisymmetric PAFEC model with the addition of the penetration thickenings are shown in Figures 4.10 .46 and 4.10 .47 . It can be seen that the effect of the thickenings are nearly symetrical with little appreciable effect caused by the restraint from the base. This is again shown in Figure 4.10.48 where the displacements are compared with the results of the unthickened model.

\subsection{2-D Analys is}

The displacement results from $180^{\circ}$ model, described in Section 4.10.6.2, shows clearly the effect of the penetration thickenings around the 
circumference of the wall (Figure 4.10.49). The thickening with additions both inside and outside of the wall (Hatch B) has a much smaller effect than that with only the outside addition (Hatch $A$ and air lock). This is caused by the centroid of the singularly thickened penetrations being eccentric to that of the intervening shell, this causing bending within the shell due to the membrane tensions.

\subsection{3-D Analysis}

The displacement results from the 3-0 linear analysis are shown in Figures 4.10 .50 and 4.10 .51 . It is seen from the displacements that the effect of the actual hole within the thickened area is insignificant relative to the stiffening effects of the thickenings. Similar to the 2-D analysis, the linear 3-D analysis shows additional bending of the cylinder wall due to the penetration arrangement.

This implies that a more detailed 3-D nonlinear analysis would be required to determine accurately the overall effect of the penetration and their associated thickenings. An estimation of these effects is summarized below.

\subsubsection{Correlation of Results}

The nonlinear axisymetric analysis described in Section 4.10.8.1 determines the ultimate failure pressure for the 1:6-scale model. The calculated pressure of 160 psi ( $1.103 \mathrm{MPa}$ ) assumes a fully continuous, axisymmetric structure, i.e., a containment neglecting all thickenings, penetrations and localized irregularities. Section 4.10.8.2 looks at local effects of the penetrations.

Figures 4.10 .52 and 4.10 .53 compare displacement results of the two analyses within the elastic range of the structure. It is seen in these figures that the preliminary 2-D analysis (Section 4.10.8.2.2) of the $180^{\circ}$ model and the thickened axisymmetric analysis produce results with severe local bending in the region of the penetrations. The 3-D analysis with thickenings and holes reduces of the local bending compared with the unpenetrated case. However bending is significant enough to cause early concrete cracking in the structure (see Figures 4.10 .52 and 4.10 .53 ).

This comparison is made assuming a linear-elastic response of the structure. The nonlinear response can be estimated by using the results of the nonlinear axisymmetric analysis as a guideline (Section 4.10.8.1).

The penetration thickenings will cause concrete cracking in the wall at pressures below those predicted by the nonlinear axisymetric analysis. This cracking will be due to both longitudinal and circumferential bending of the wall. As shown by the nonlinear axisymmetric analysis the loss of tensile capacity in the concrete at the cracks will cause them to extend rapidly through the section. Once this cracking is complete the local bending is relieved and the wall will revert to a simple axisymetric structure and respond similarly to that described within Section 4.10.8.1. 


\section{AXISYMMETRIC ANALYSIS}

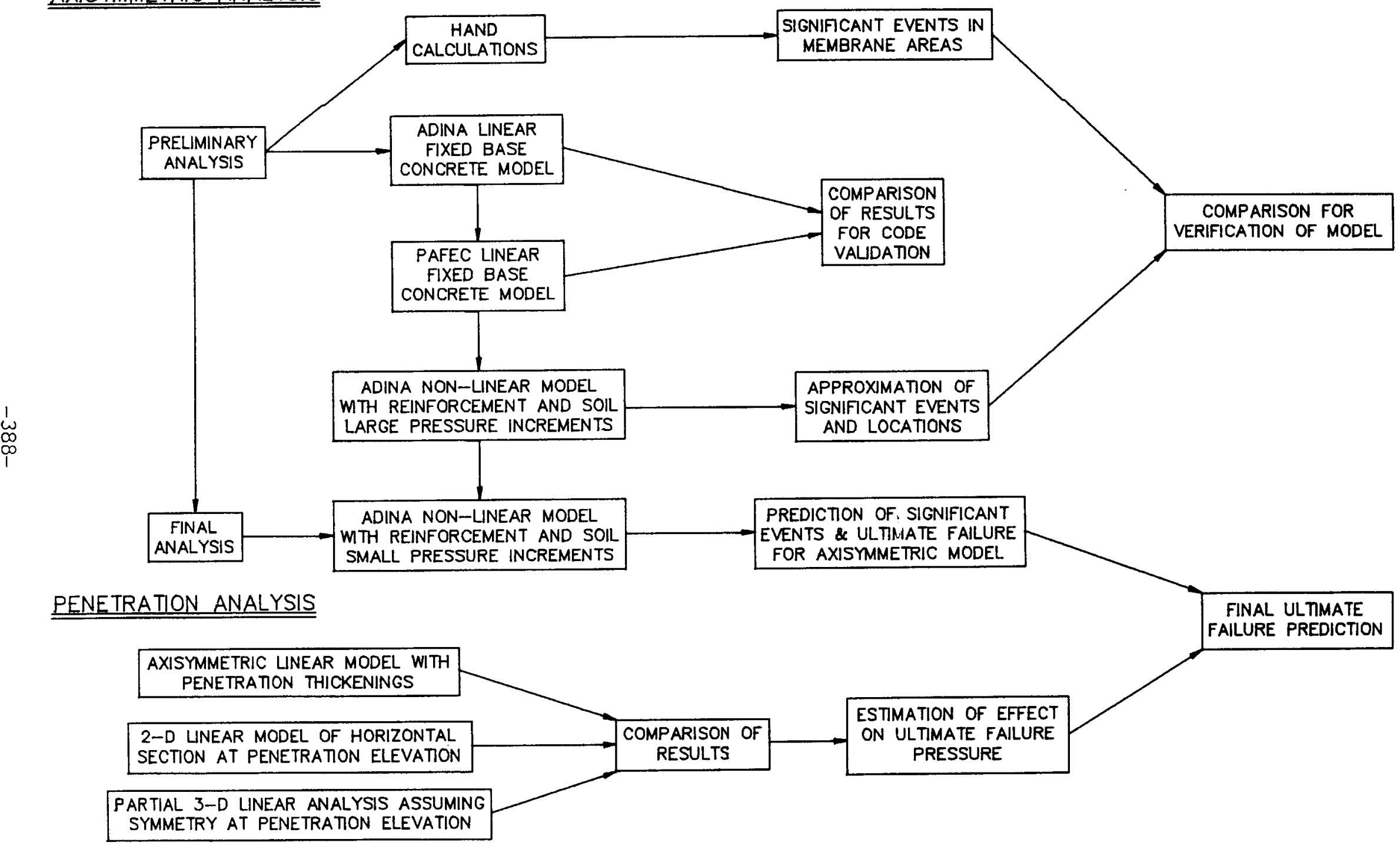

Figure 4.10.1 Summary of 1:6-Scale Model Uitimate Load Analysis 


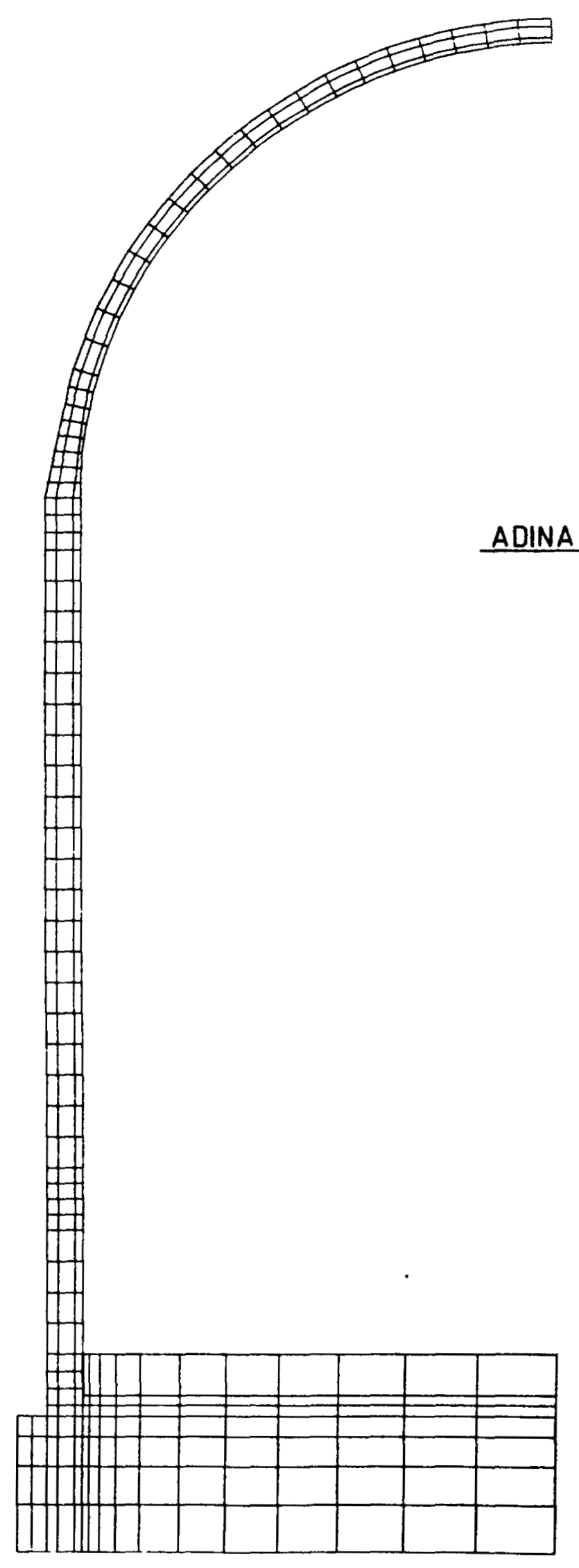

$\mathrm{J}^{\mathrm{z}}$

Figure 4.10.2 ADINA-TW Axisymmetric Model 


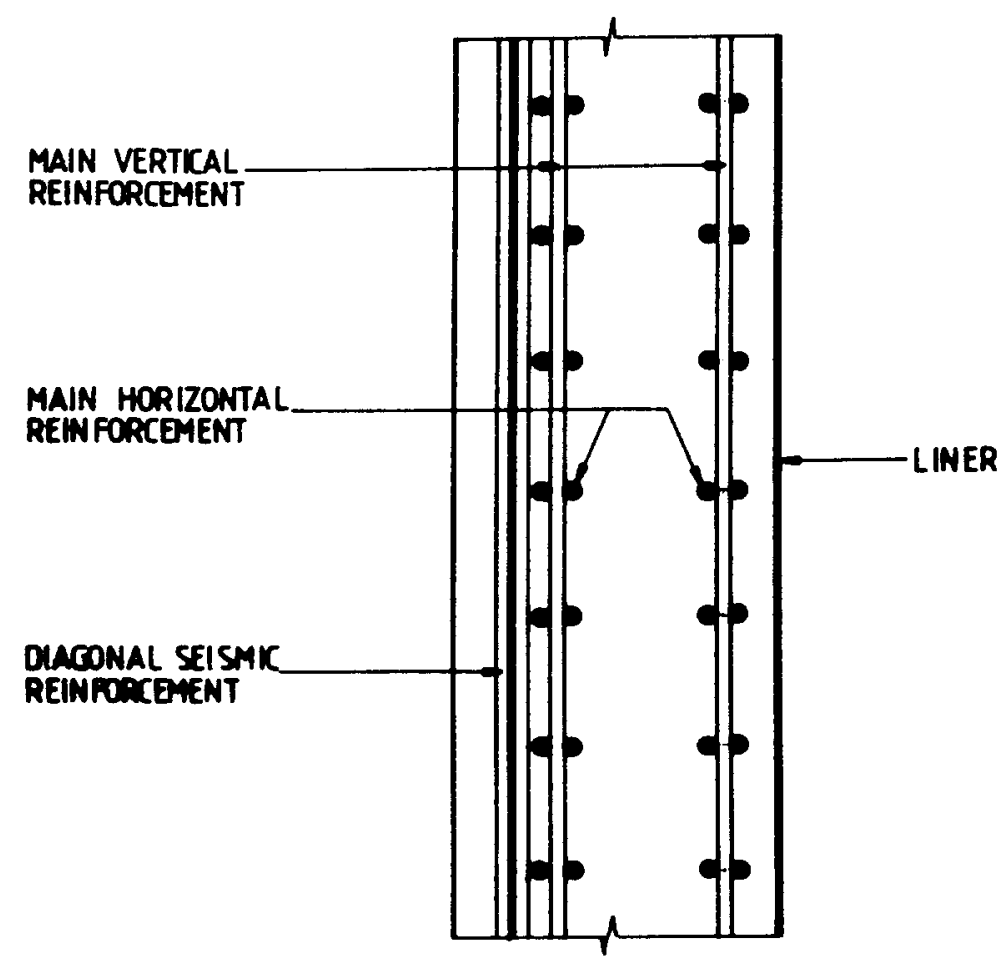

Iypical Section Through Wall

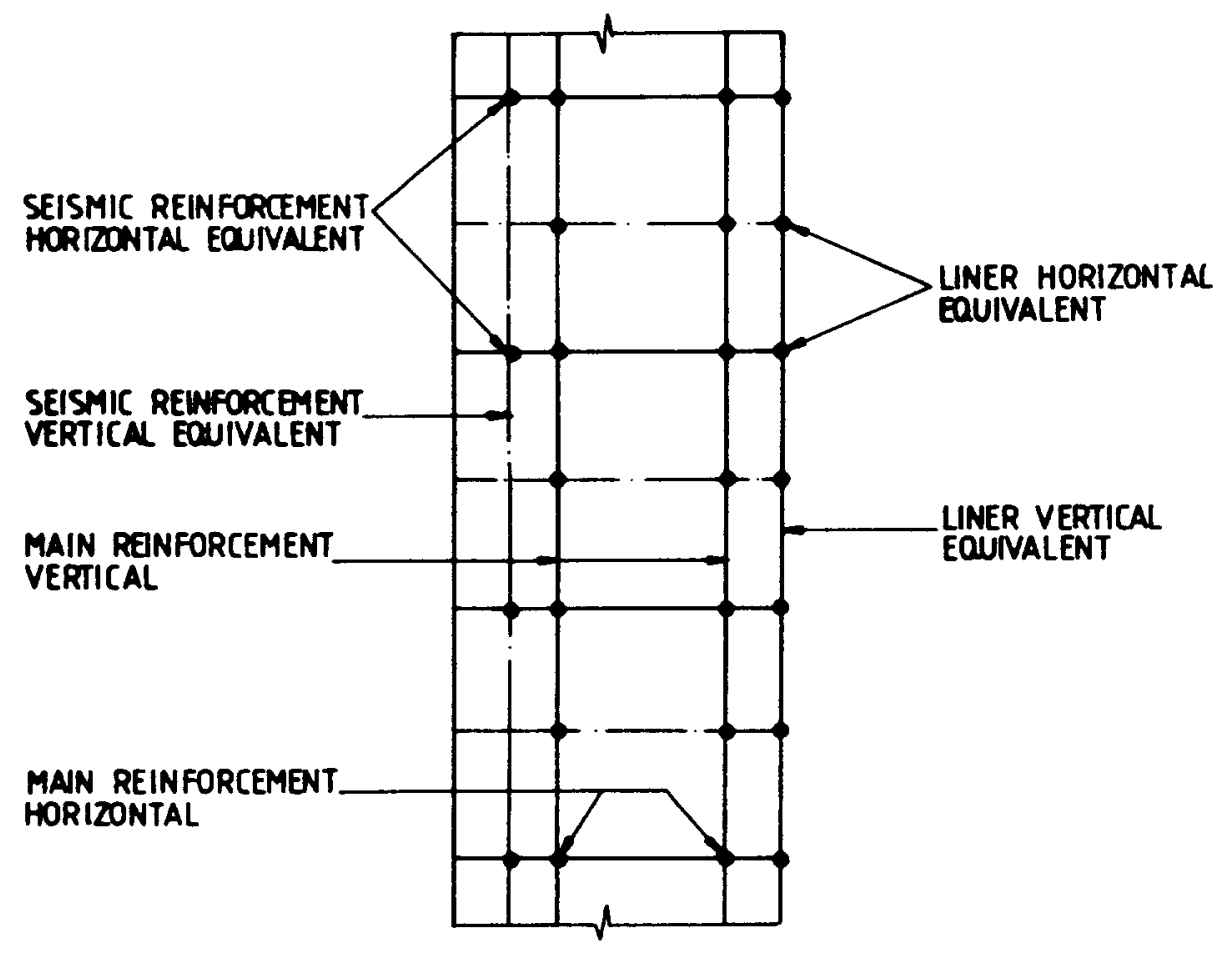

Iypical Section of ADINA Model

Figure 4.10 .3

Wall Reinforcement and Liner - Actual and as Modelled in ADINA-TW 


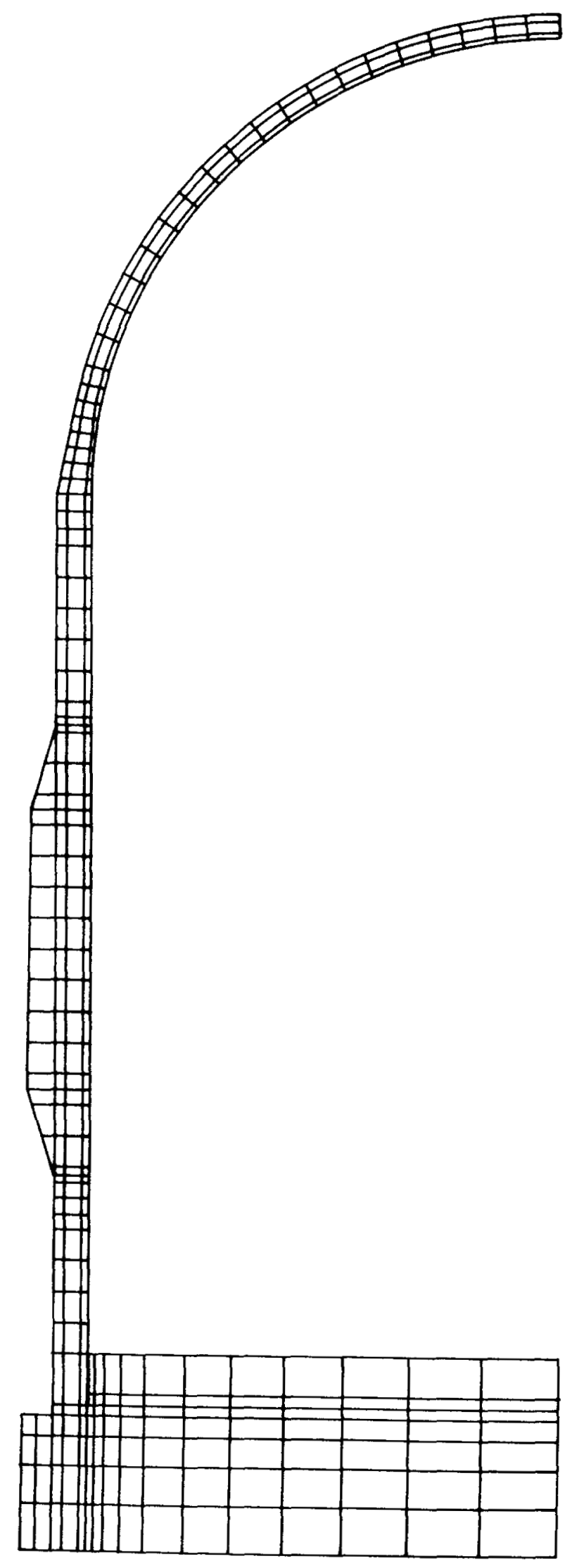

Figure 4.10.4 Axisymmetric Model with Penetration Thickening (Hatch A) 


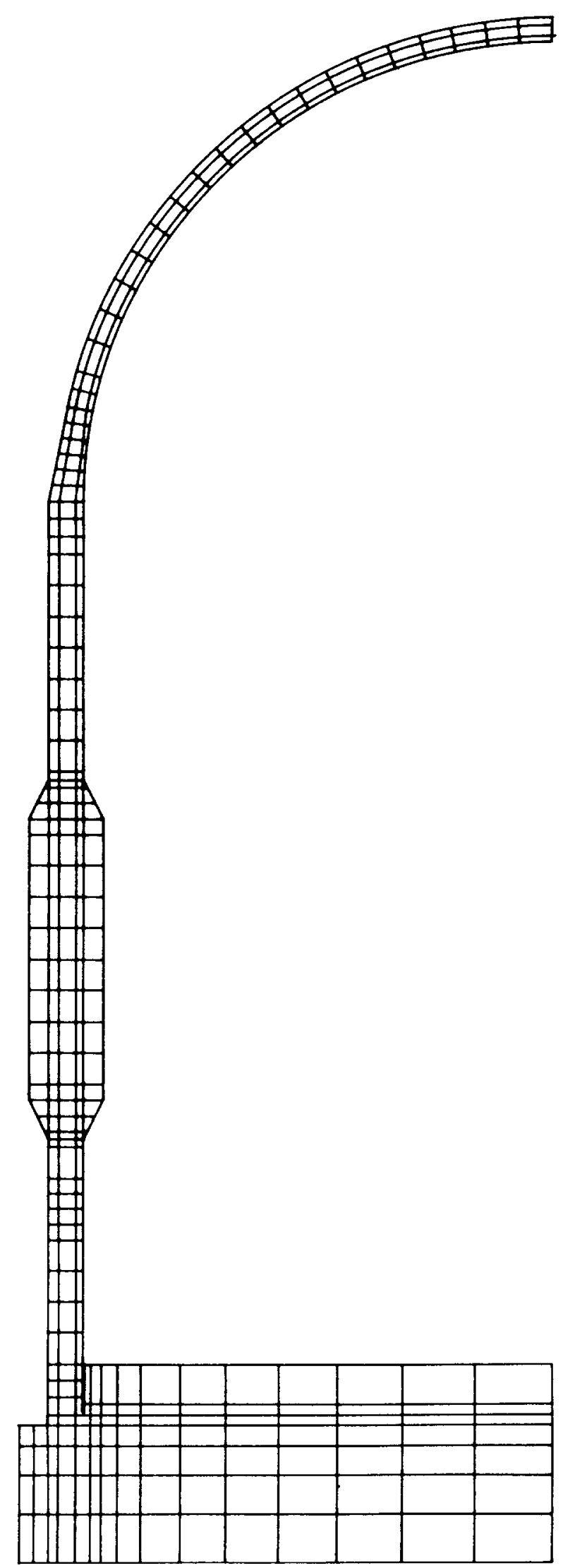

Figure 4.10.5 Axisymmetric Model with Penetration Thickening (Hatch B) $-392-$ 


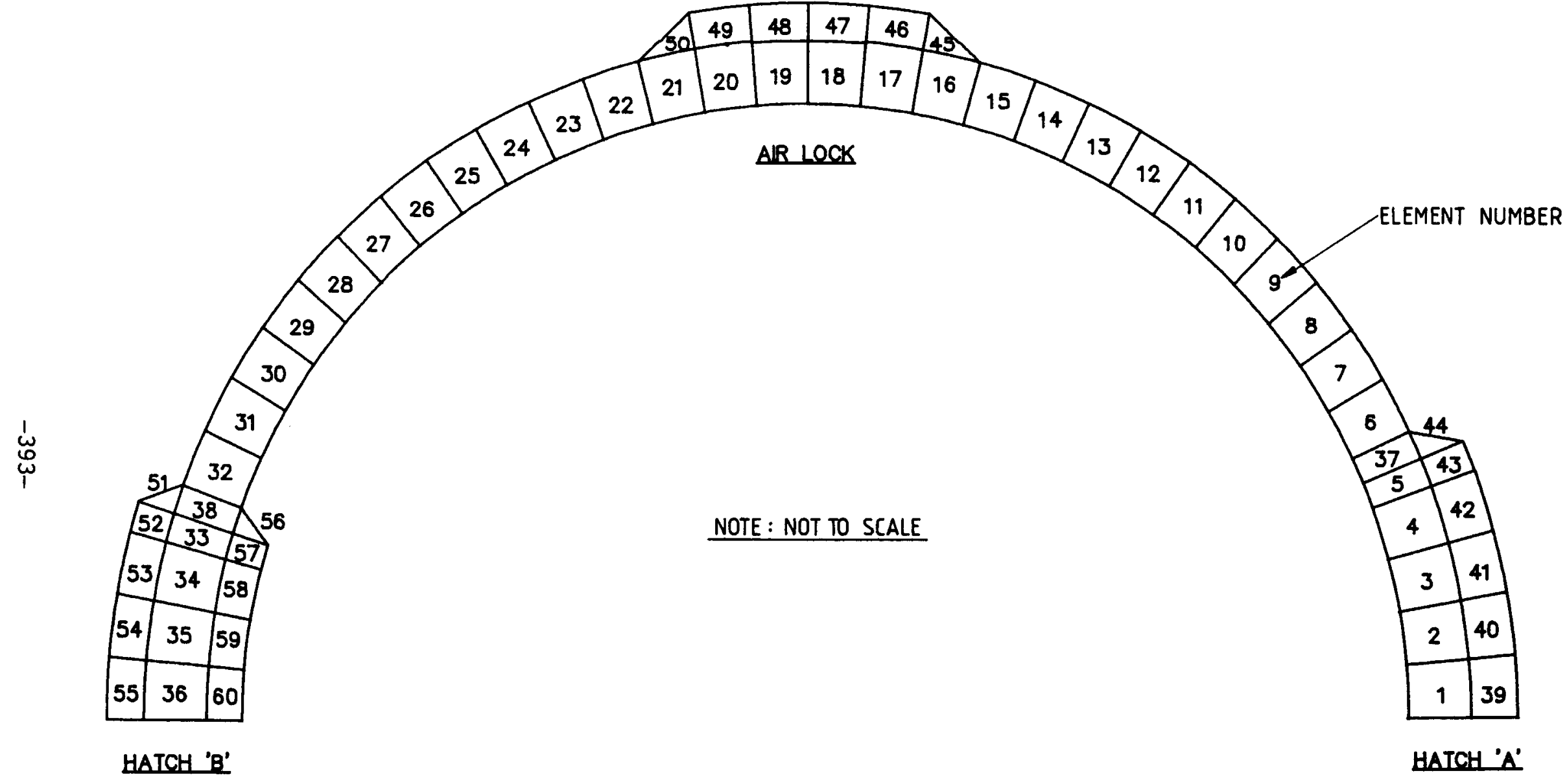

Figure 4.10.6 2-D Linear Elastic Model 


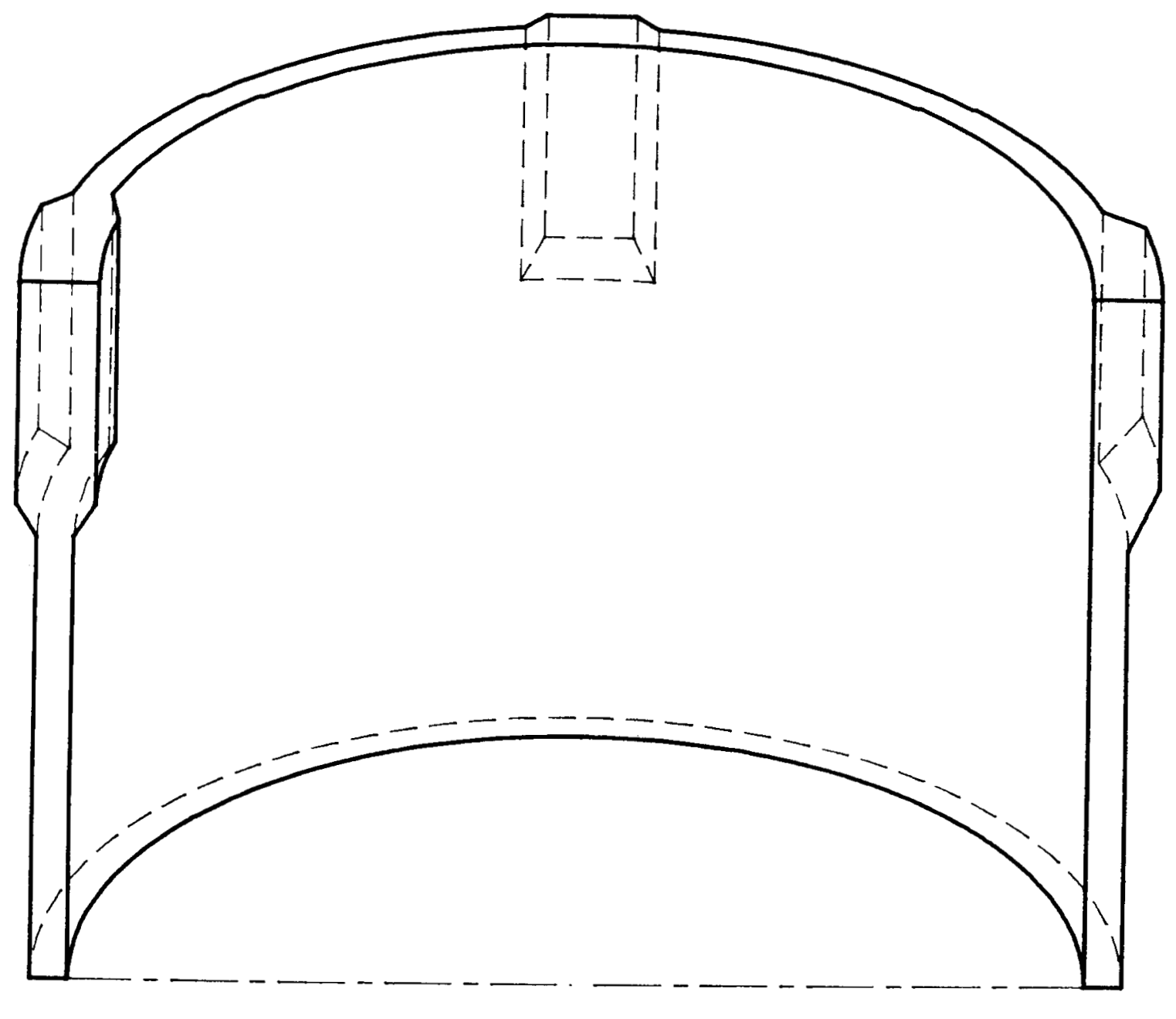

Figure 4.10.7 3-D Linear Elastic Model 


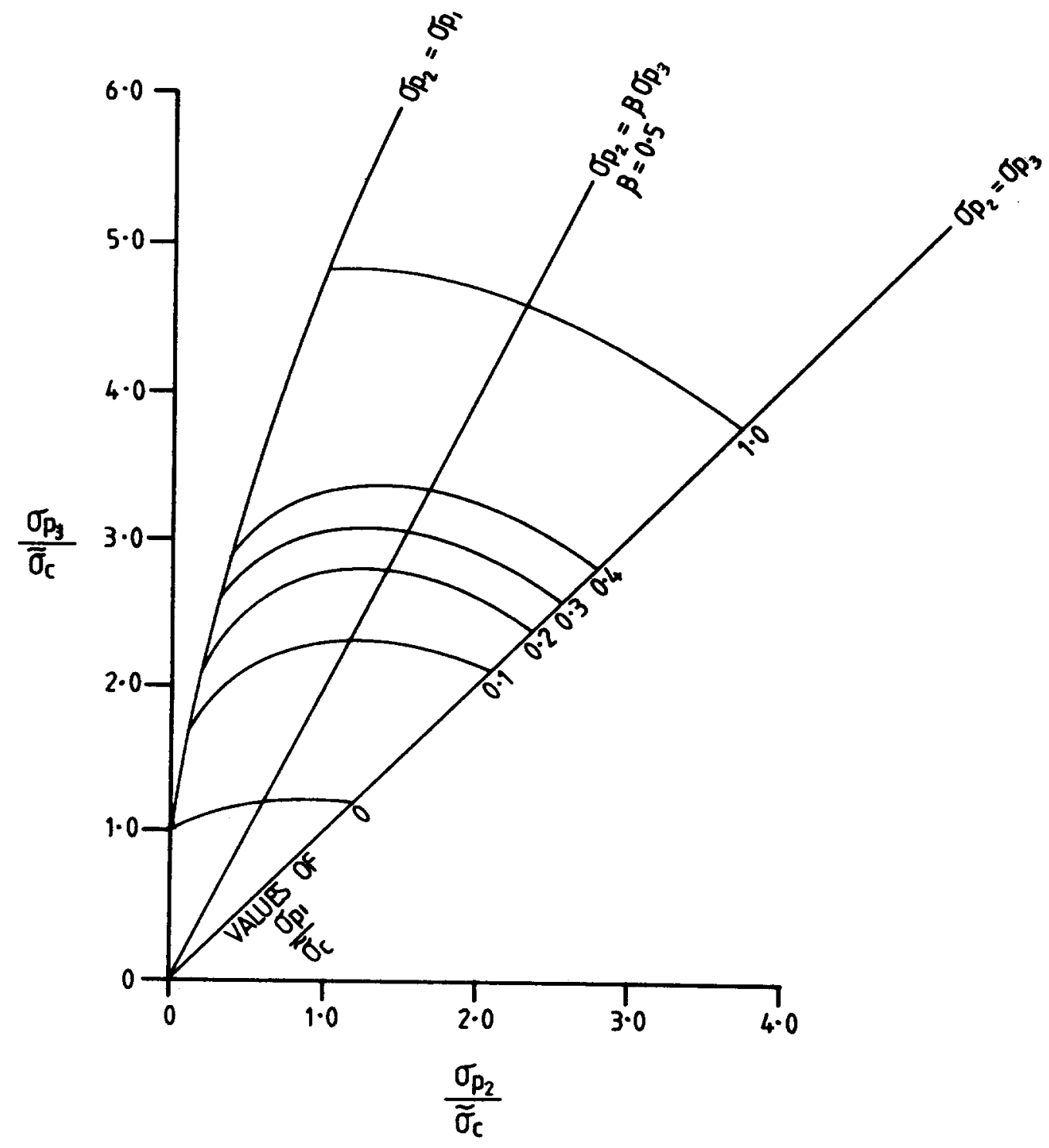

$\begin{array}{ll}\sigma_{p_{1}} \sigma_{p_{2}} \text { AND } \sigma_{p_{3}} & \text { PRINCIPAL STRESSES IN DIRECTIONS } 1,2 \& 3 \\ & \text { WHERE } \sigma_{p_{1}} \geqslant \sigma_{p_{3}} \geqslant \sigma_{p_{3}}\end{array}$

Figure 4.10.8 Triaxial Compressive Failure Envelopes 


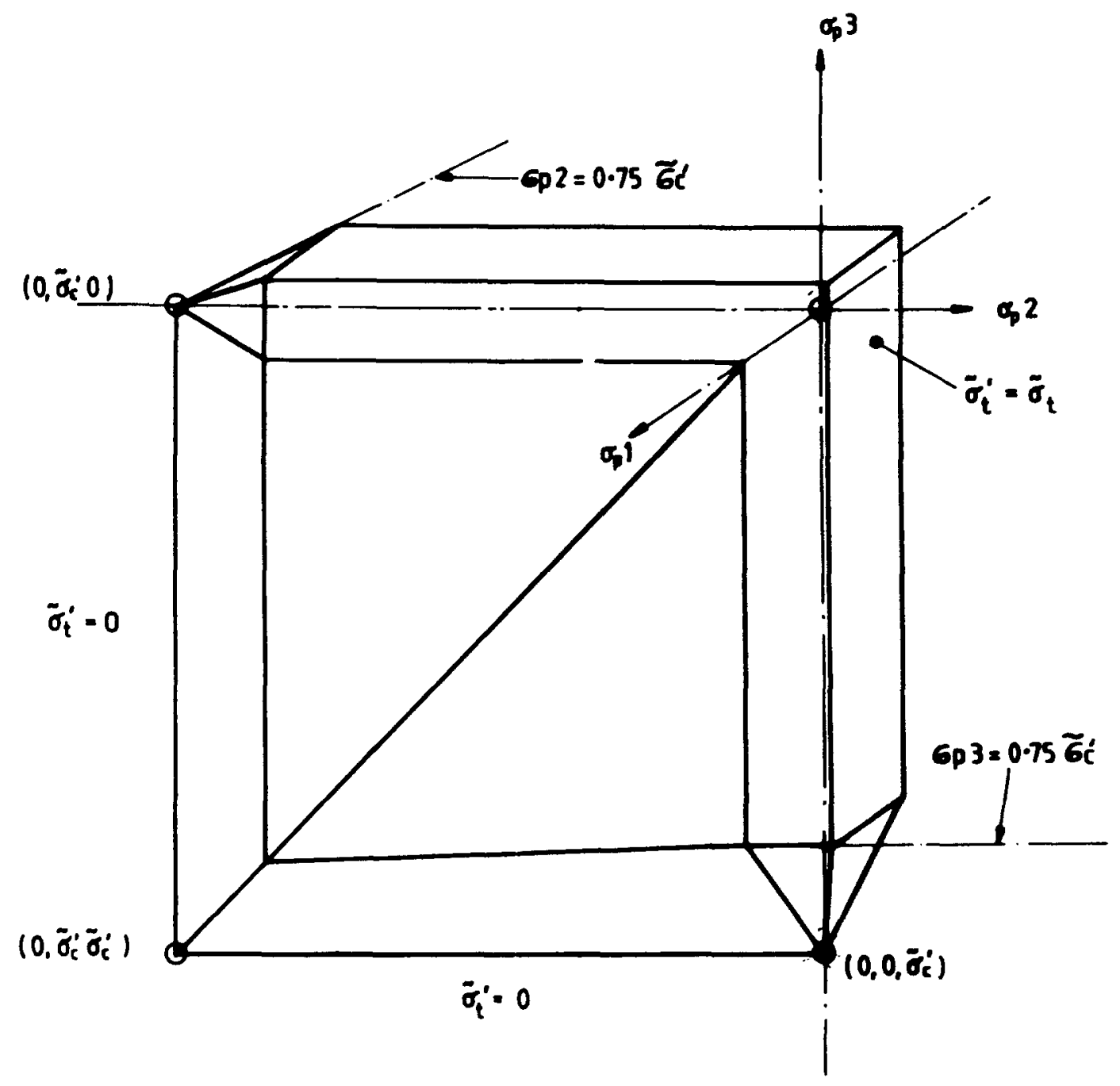

$\tilde{\sigma}_{i}^{\prime}=$ UNIAXIAL MAXIMUM TENSILE STRESS MODIFED FOR MULTUXXIAL CONOTIONS

$\bar{\sigma}_{\text {a }}=$ UNIAXIAL MAXIMUM TENSILE STRESS

* UNIAXINL MAXIMUM COMPRESSIVE STRESS MOOIFIED FOR MULTIAXIAL CONDITIONS

op',ope, op3 PRINCIPAL STRESSES IN DIRECTIONS 1,2 ANO 3

Figure 4.10.9 Triaxial Tensile Failure Envelope 
$B=\frac{G^{\prime}}{G}=\frac{\text { Effective Shear Modulus After Cracking }}{\text { Shear Modulus Prlor to Cracking }}$

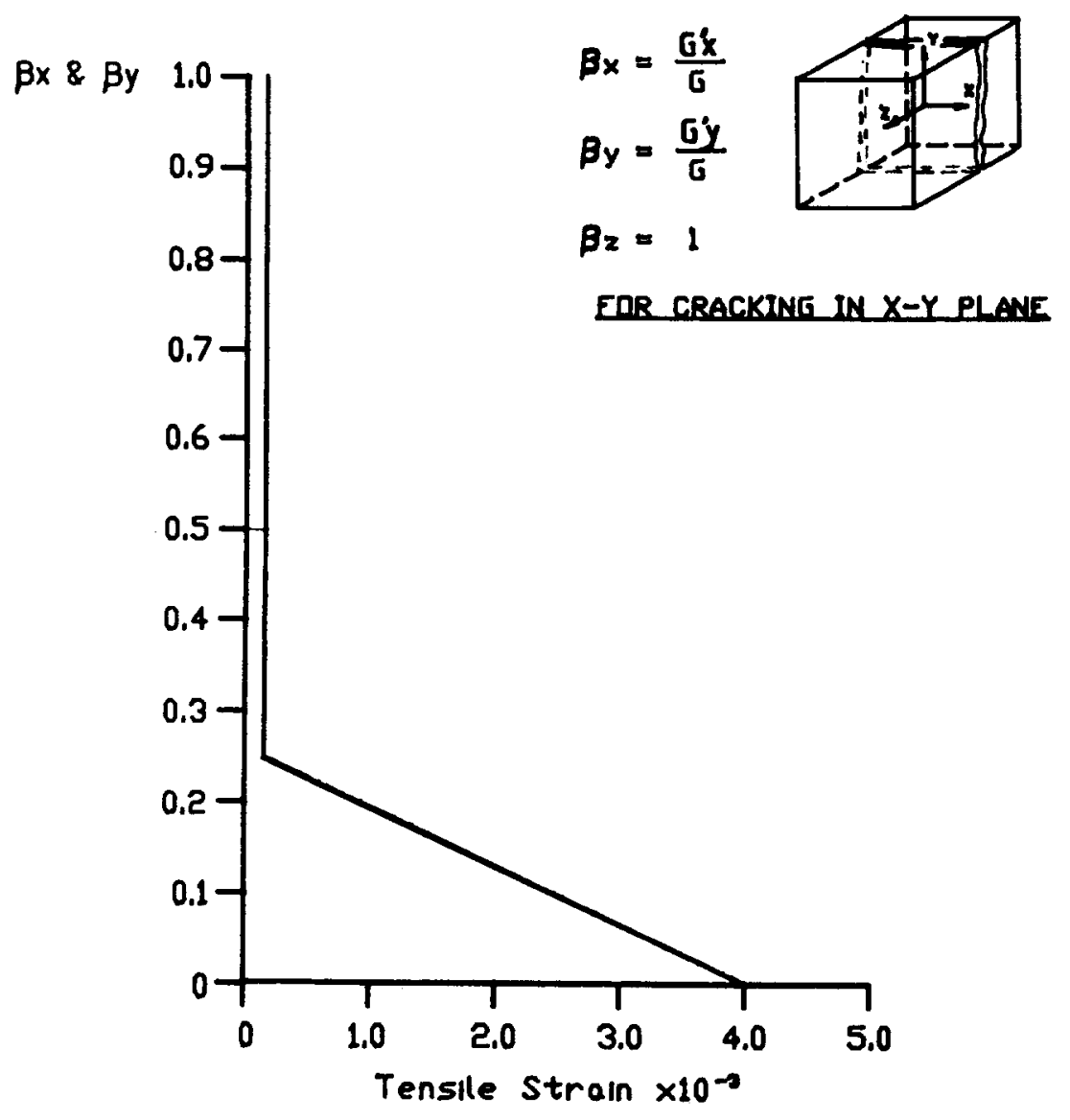

Figure 4.10.10 Shear Retention Factor 


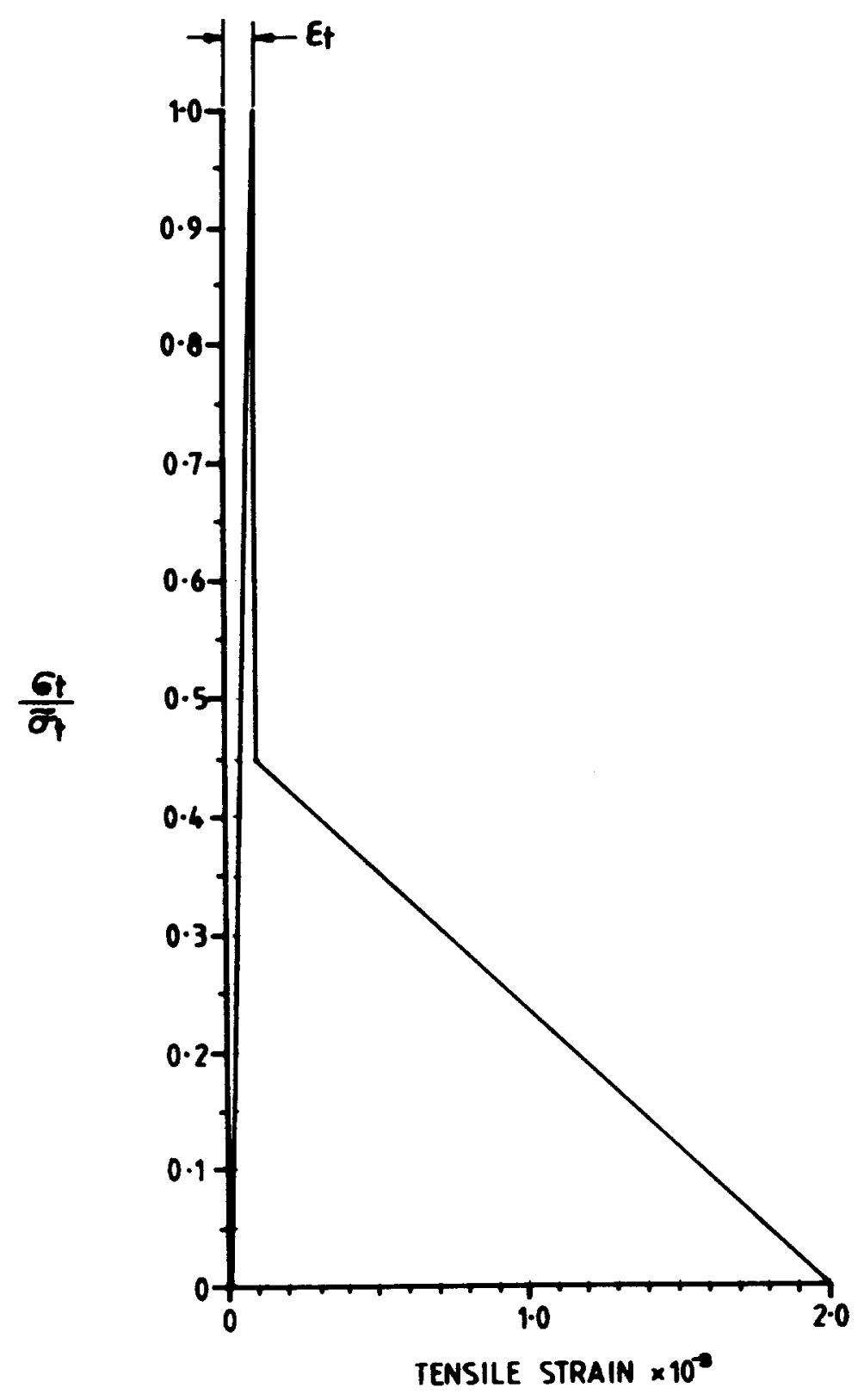

$\sigma_{t}=$ TENSILE STRESS

$\tilde{\sigma}_{+}=$UNIAXIAL MAXIMUM TENSILE STRESS

Figure 4.10.11 Effective Tensile Stiffening 


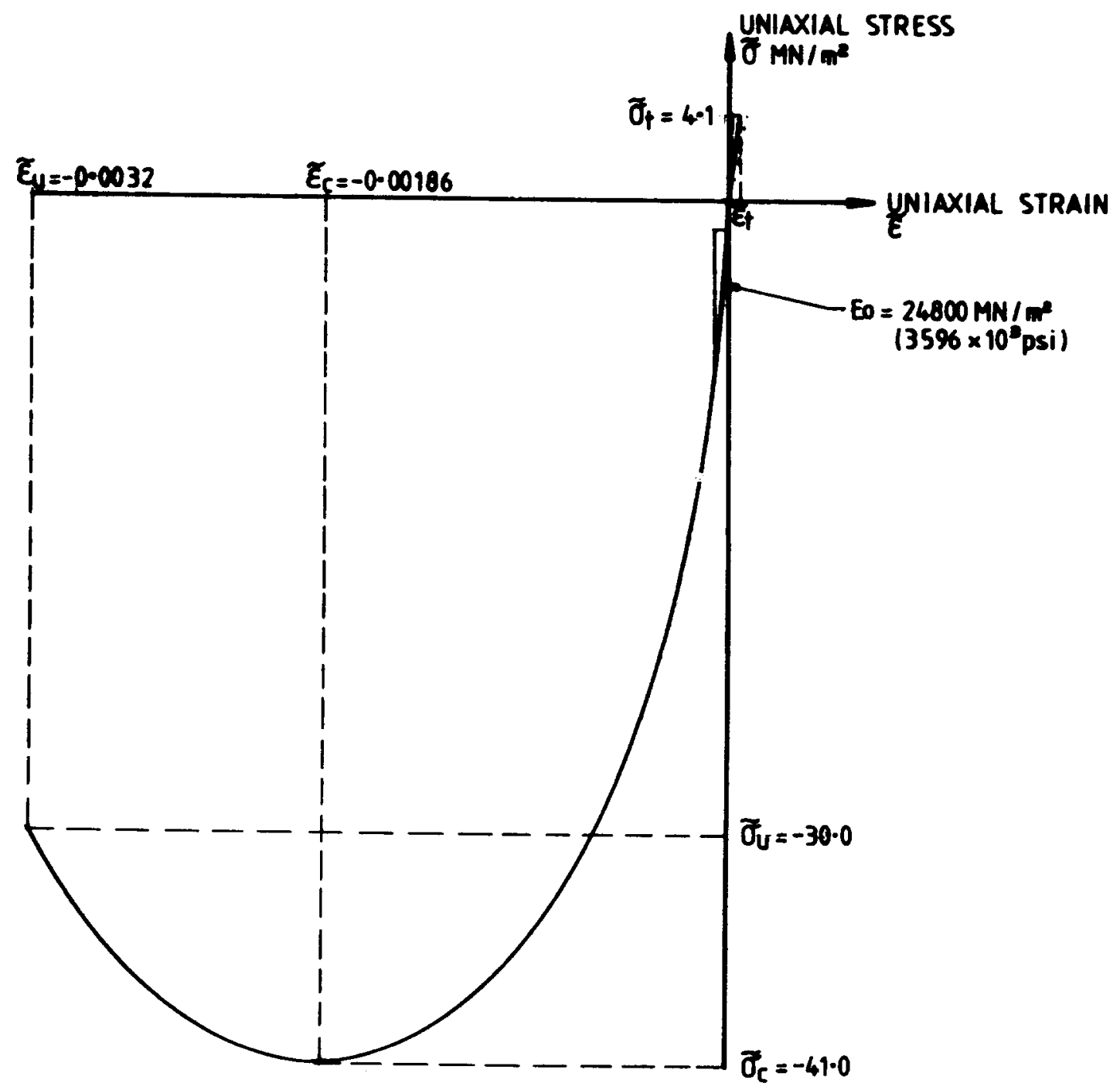

Figure 4.10.12 Uniaxial Stress-strain Relationship for Concrete $-399$ 


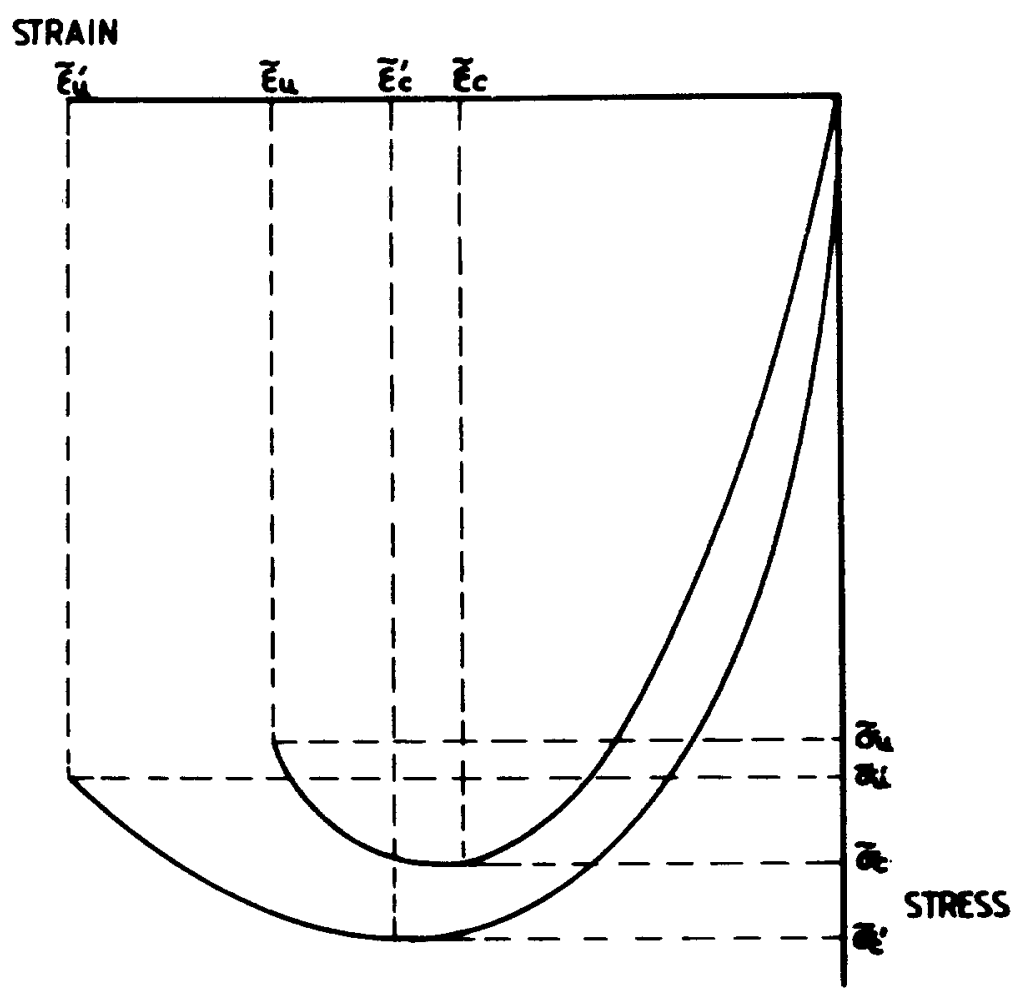

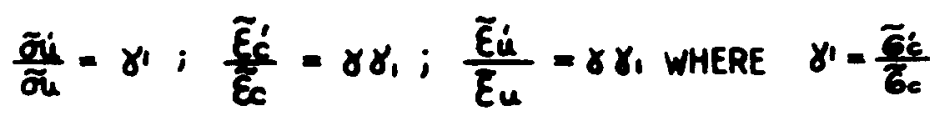

$z_{c}^{\prime}, \bar{\sigma}_{u}, \bar{E}_{\dot{c}}^{\prime} \& \bar{\varepsilon}_{i L}^{\prime}$ ARE THE APPROPRIATE UNIAXIAL PARAMETERS MODIFIED FOR MULTIAXIAL CONDITIONS

Figure 4.10.13 Modification of Uniaxial Stress-strain Relationship for Multiaxial Conditions

$-400$ 


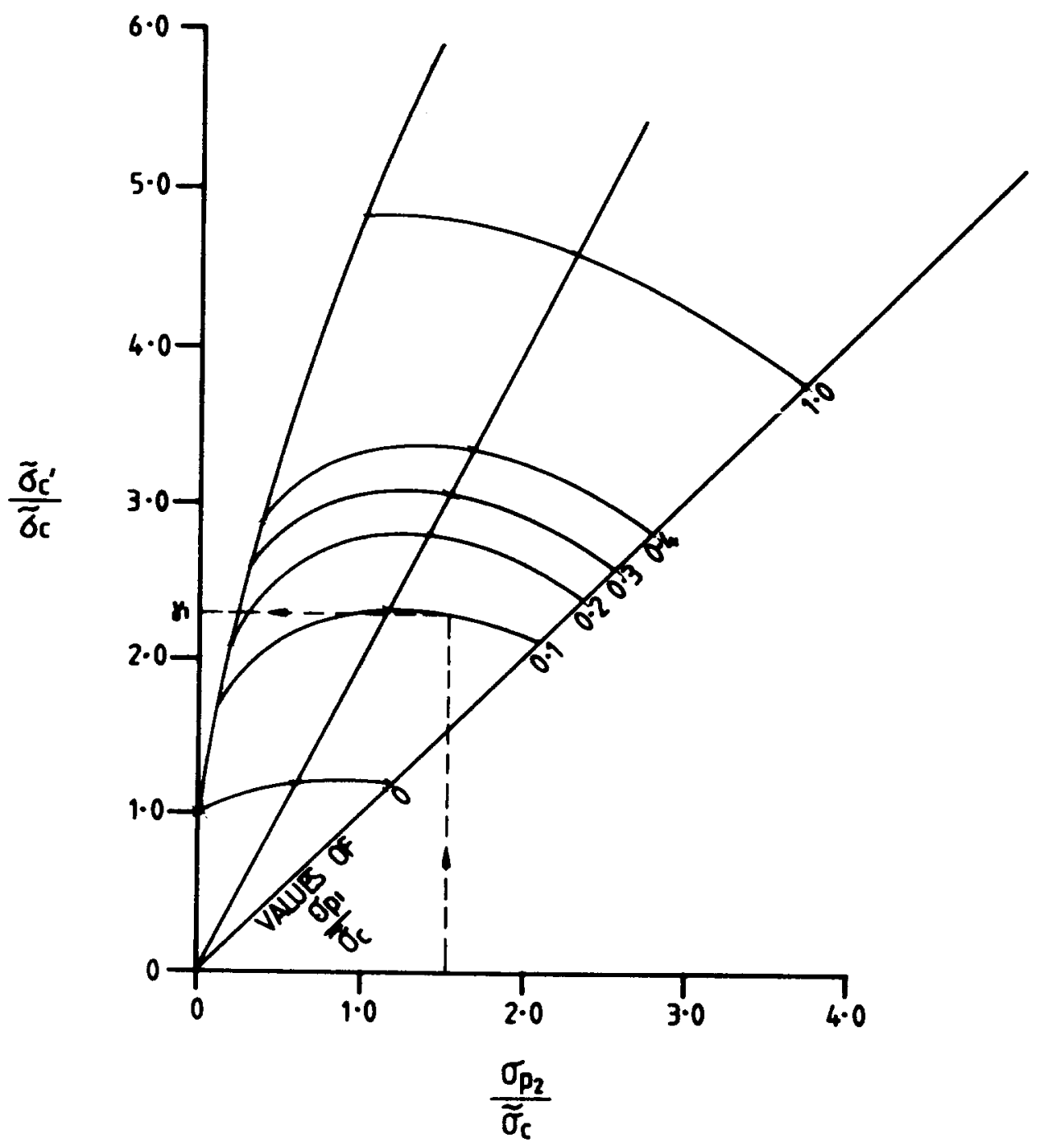

Figure 4.10 .14

Derivation of $\gamma_{1}$ from the Triaxial Compressive Failure Envelope 
$\sigma_{p}=$ ACTUAL COMPRESSIVE PRINCIPAL STRESS

$\tilde{\sigma}_{c}^{\prime}=$ UNIAXIAL MAXIMUM COMPRESSNE STRESS MODIFIED FOR MULTIAXIAL STRESS CONDITIONS

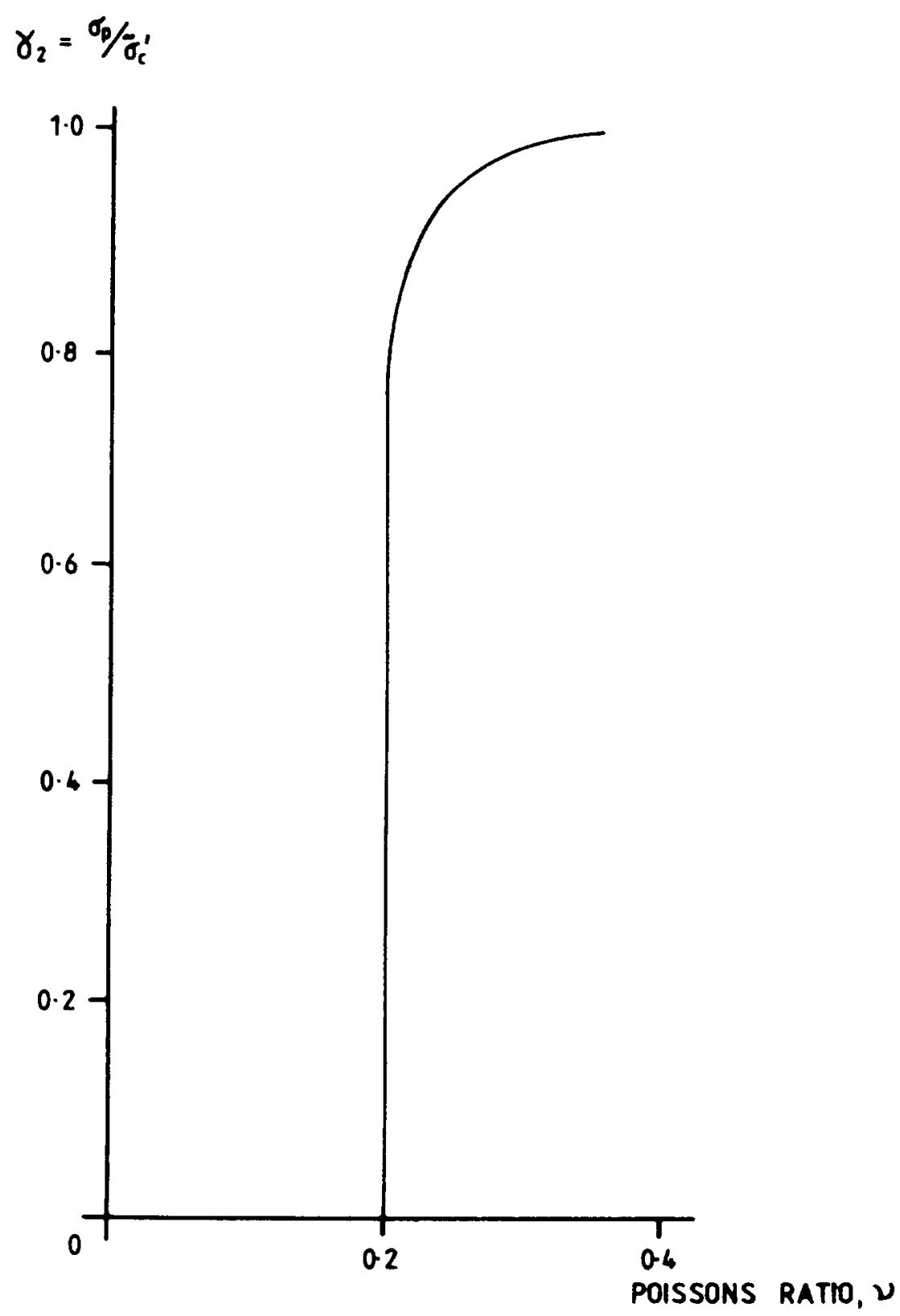

Figure 4.10.15 Poisson's Ratio 


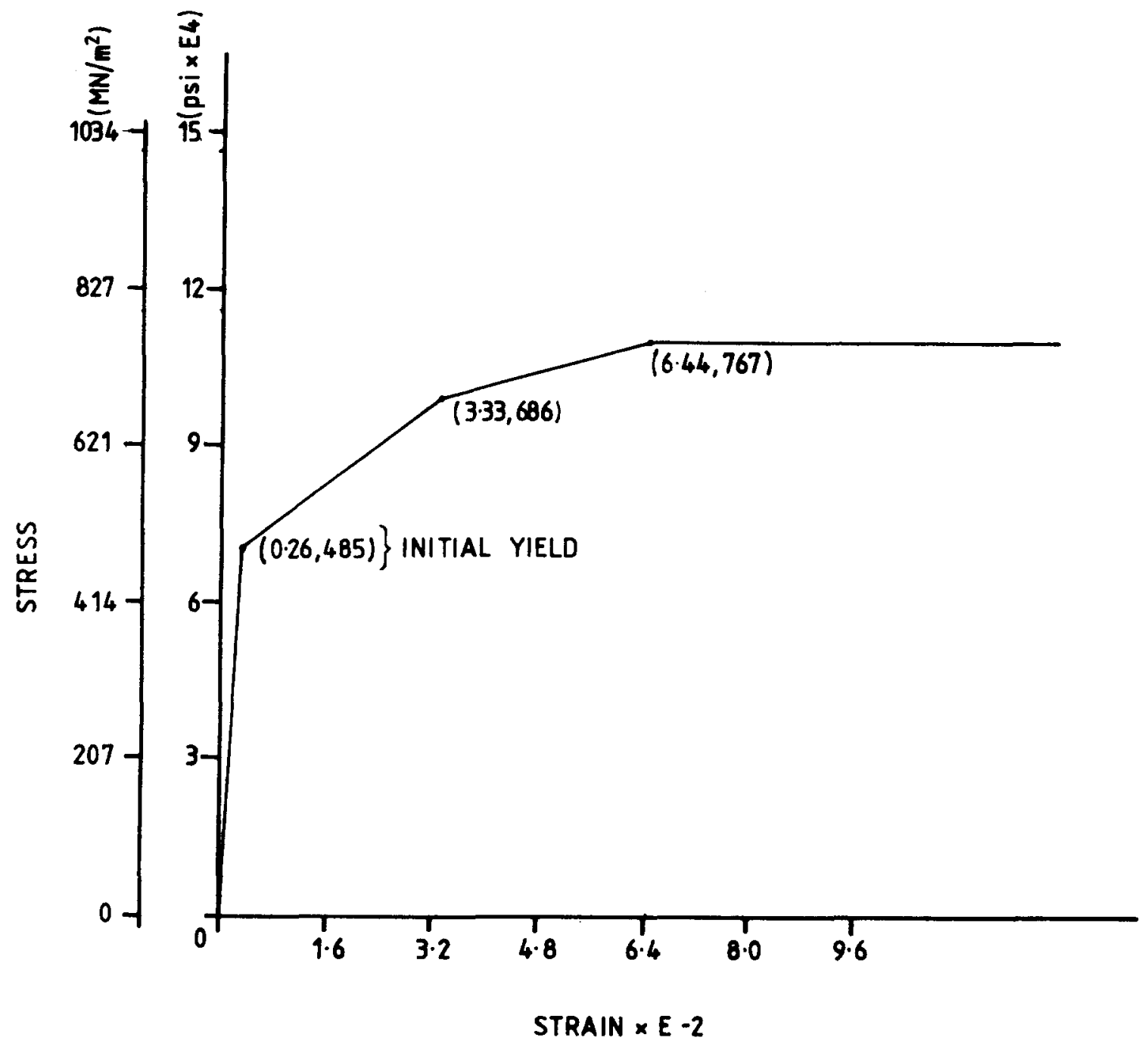

Figure 4.10.16. Stress-strain Relationship for Reinforcenent $-403-$ 


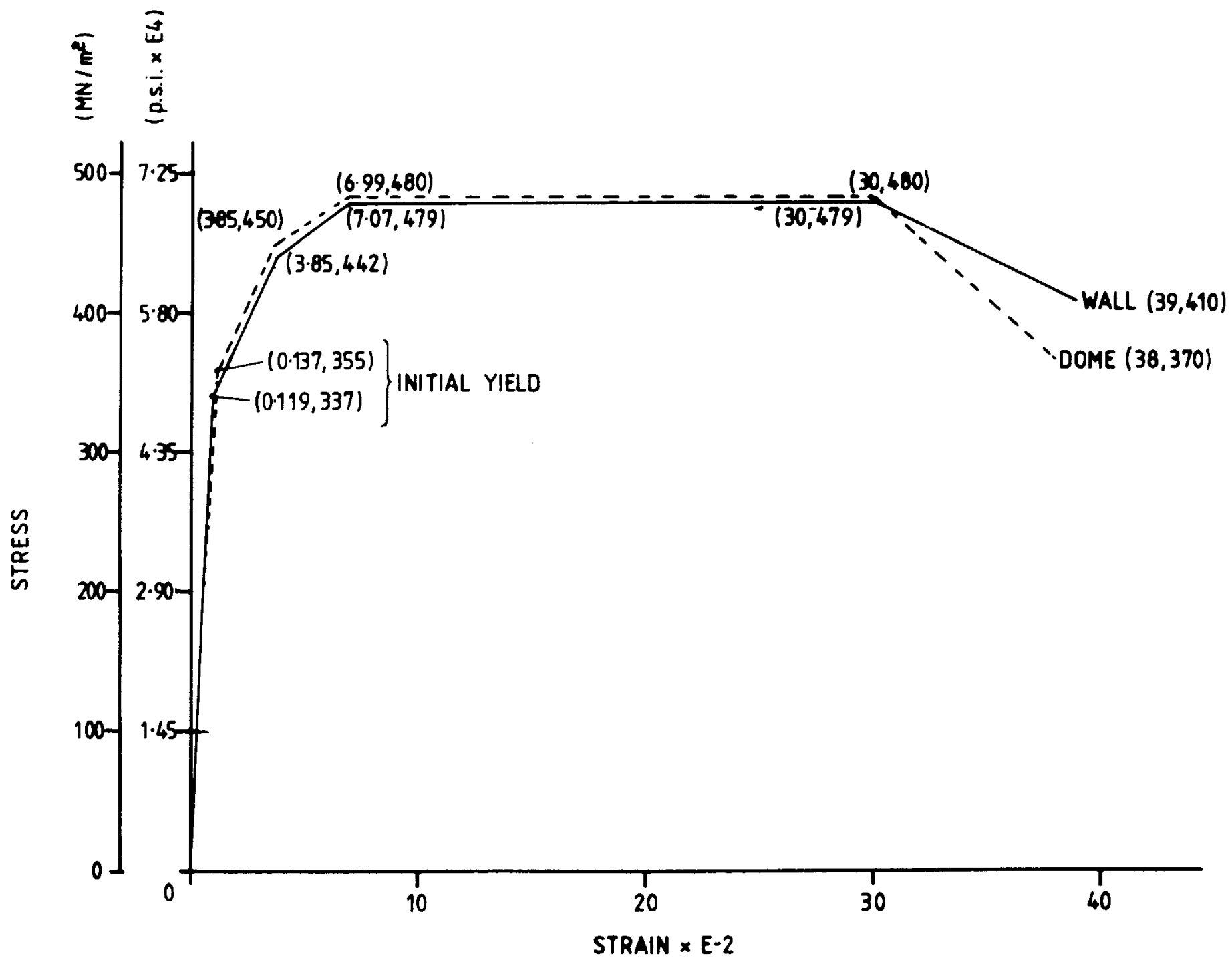

Figure 4.10.17 Stress-strain Relationship for Liner 


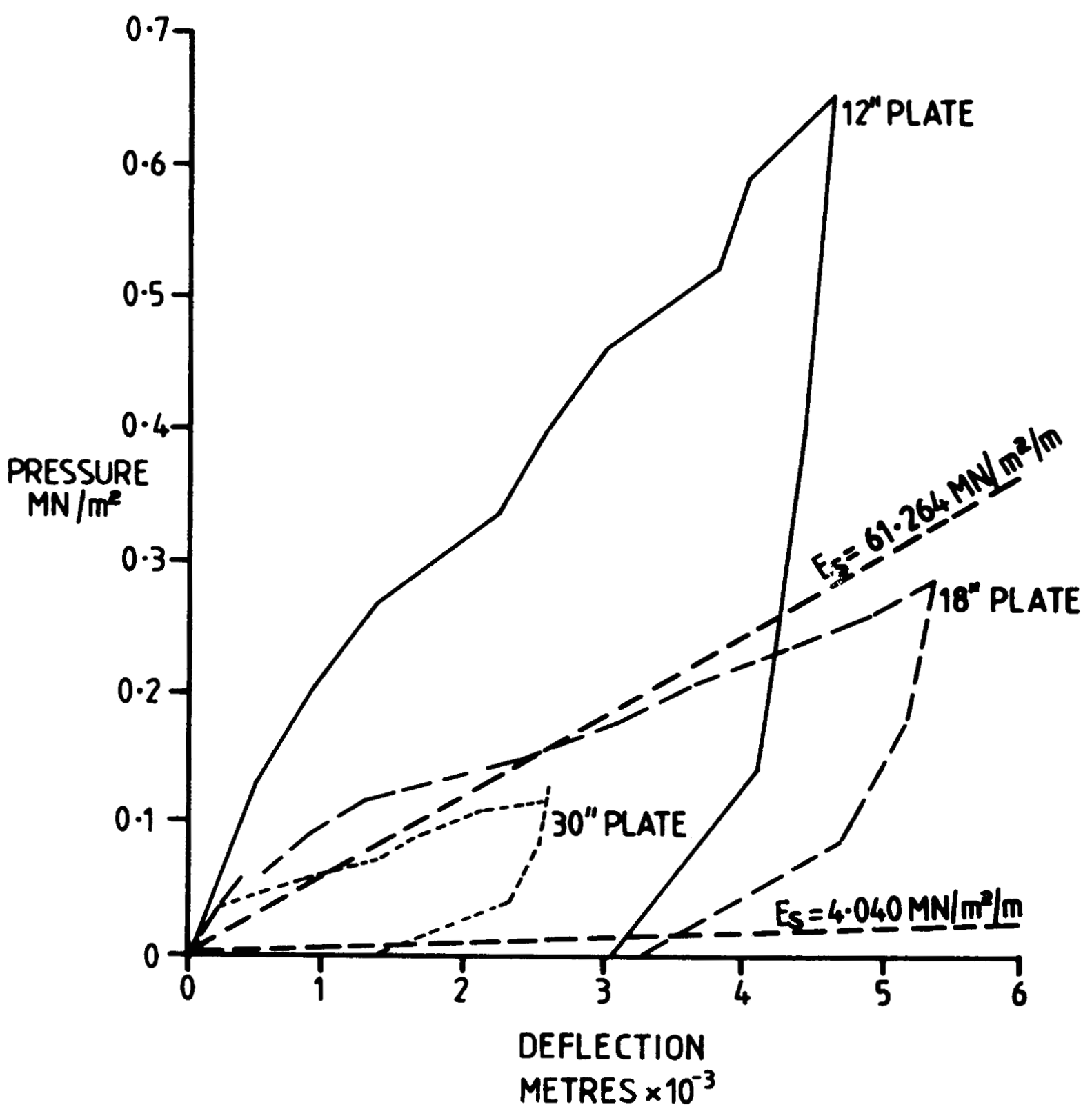

Figure 4.10 .18 Soil Stiffness 


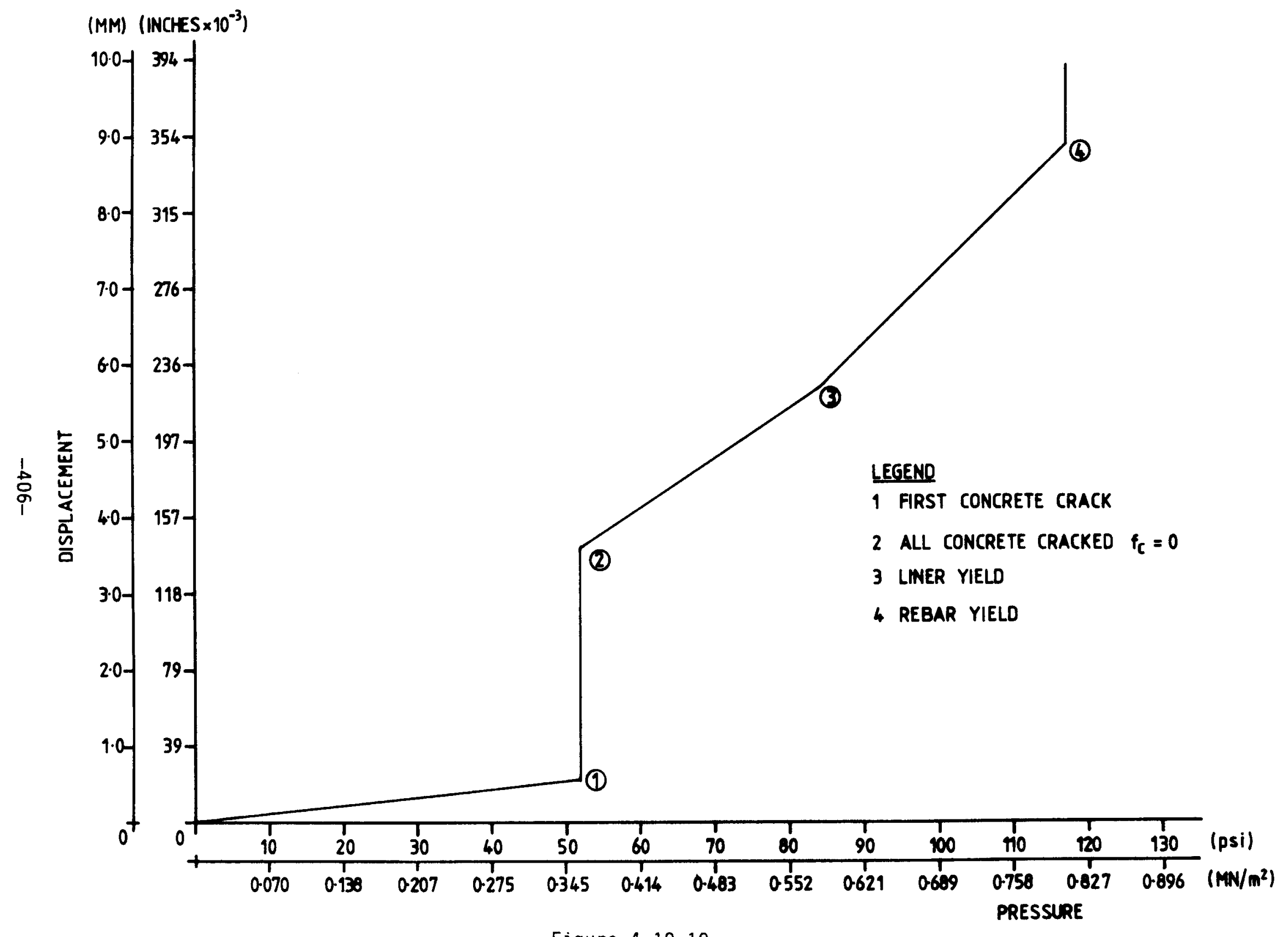

Figure 4.10 .19

Force Balancing Method, Internal Pressure vs Radial Displacement 


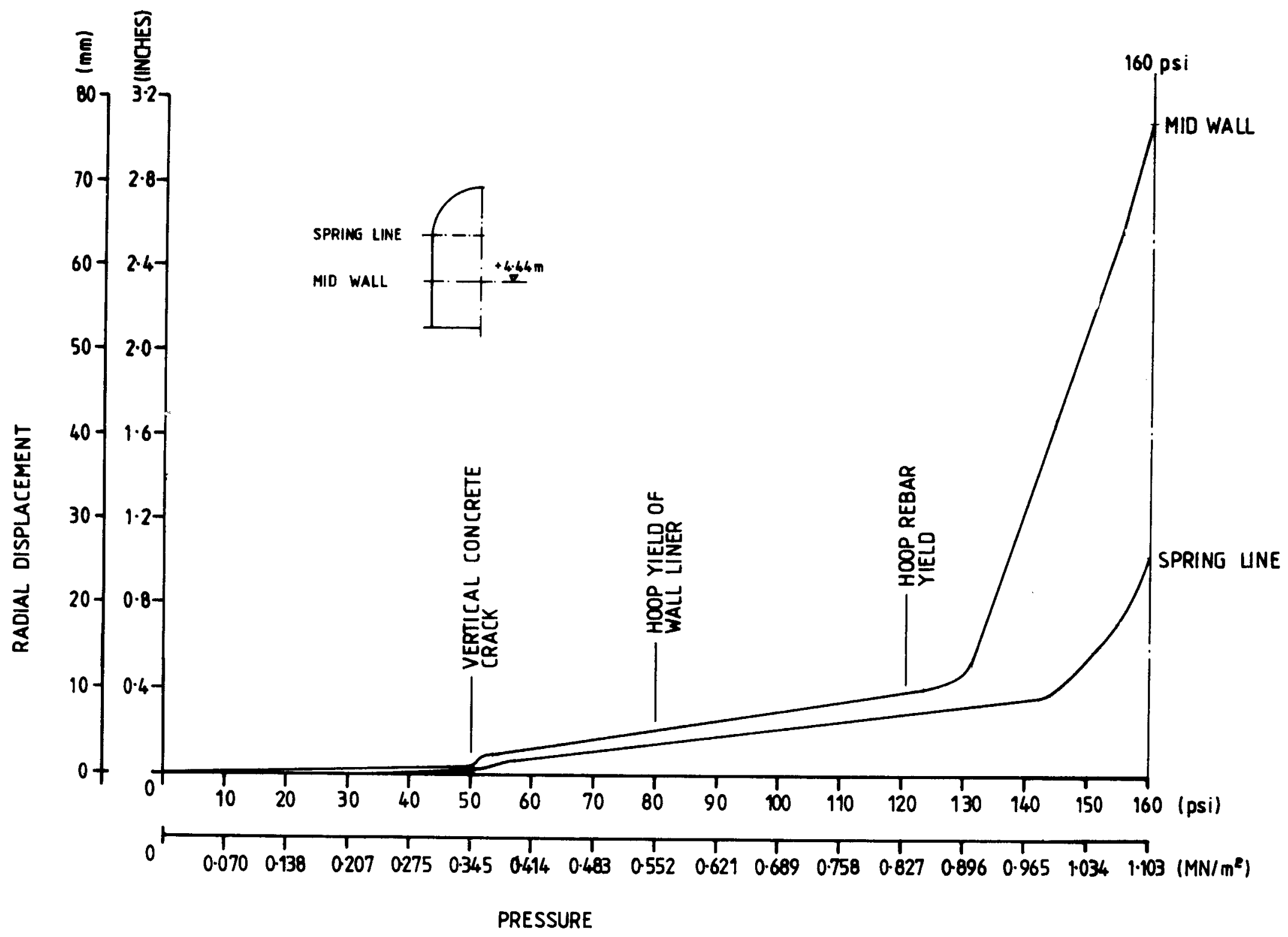

Figure 4.10 .20

Nonlinear Axisymmetric Analysis Displacement Results in Cylinder Wall 


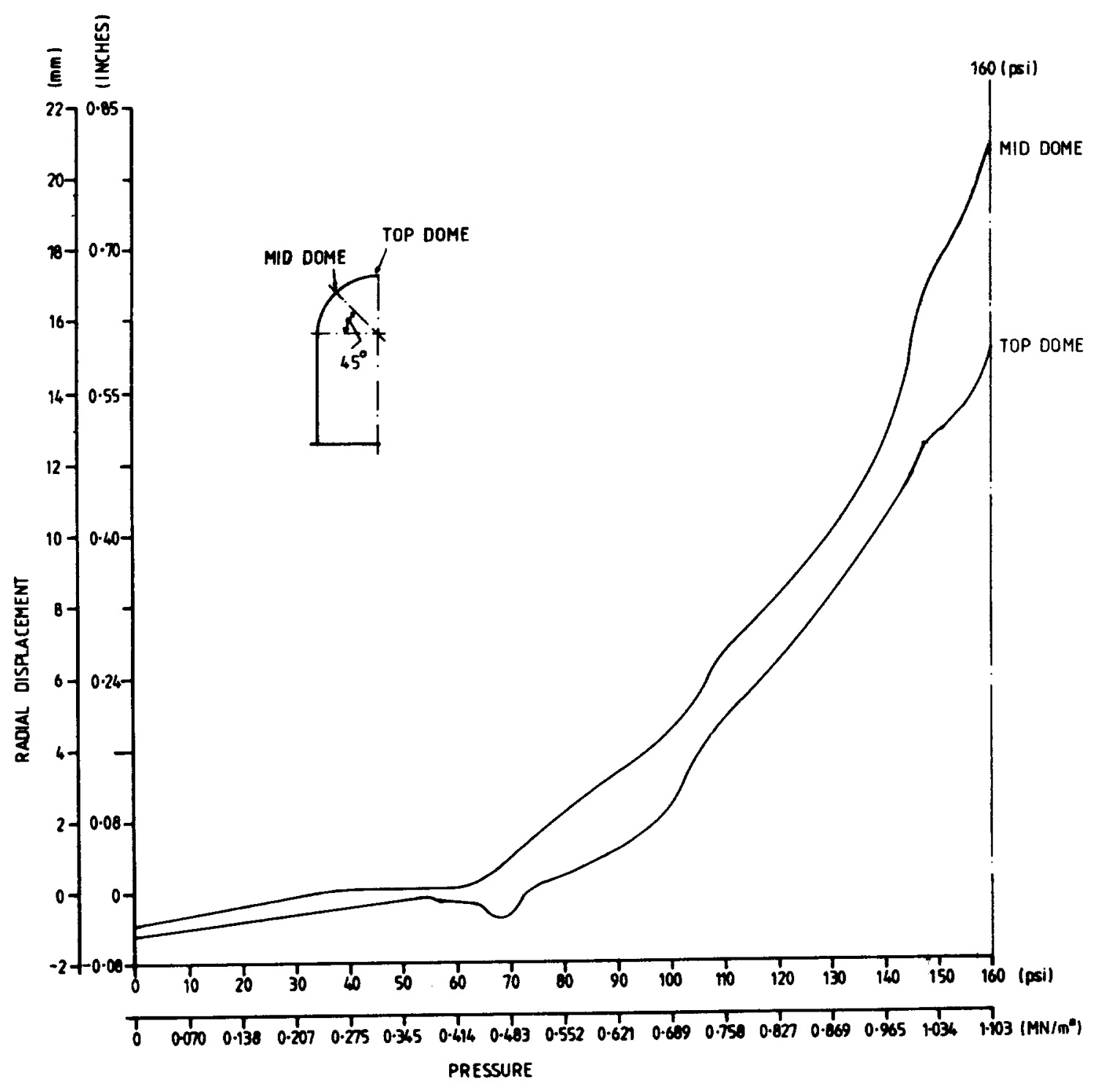

Figure 4.10.21 Nonlinear Axisymmetric Analysis Displacement Results in Dome $-408-$ 


$$
[
$$




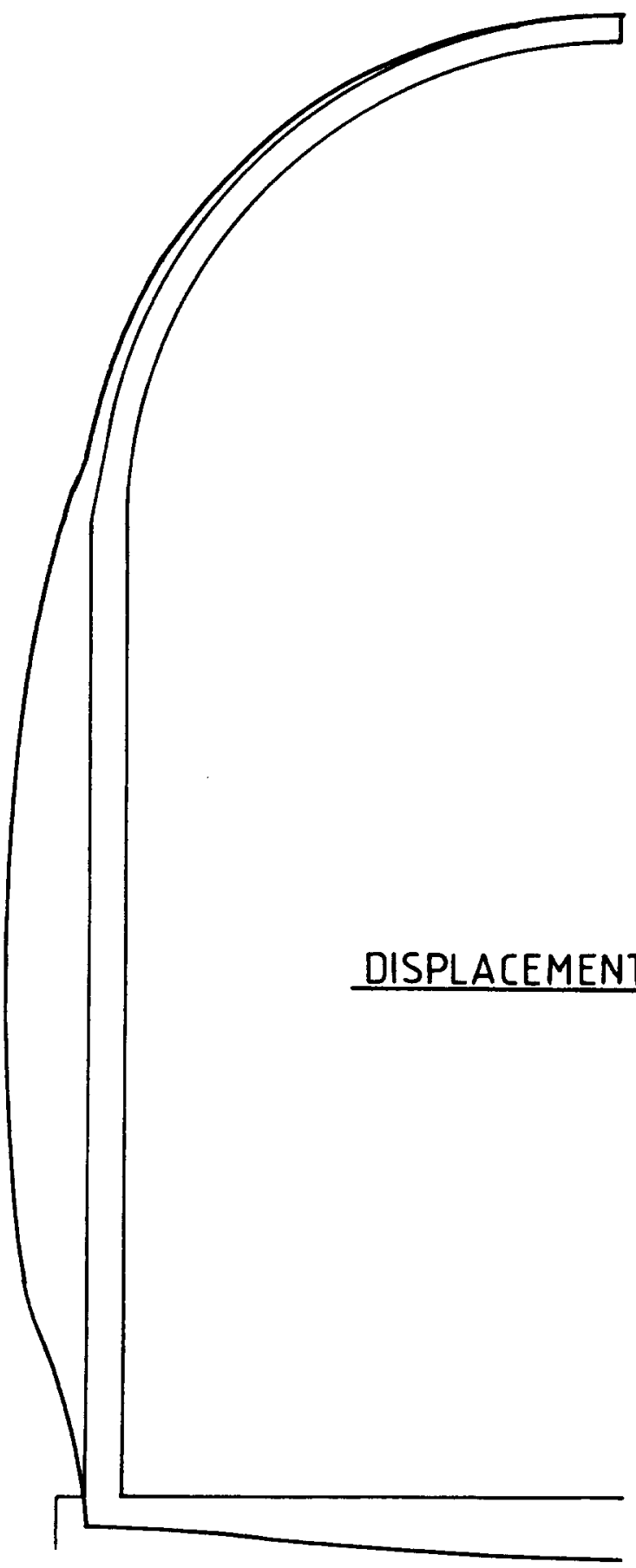

Figure 4.10.23 Displaced Shape at $0.366 \mathrm{MPa}$ (53 psi)

$-410-$ 


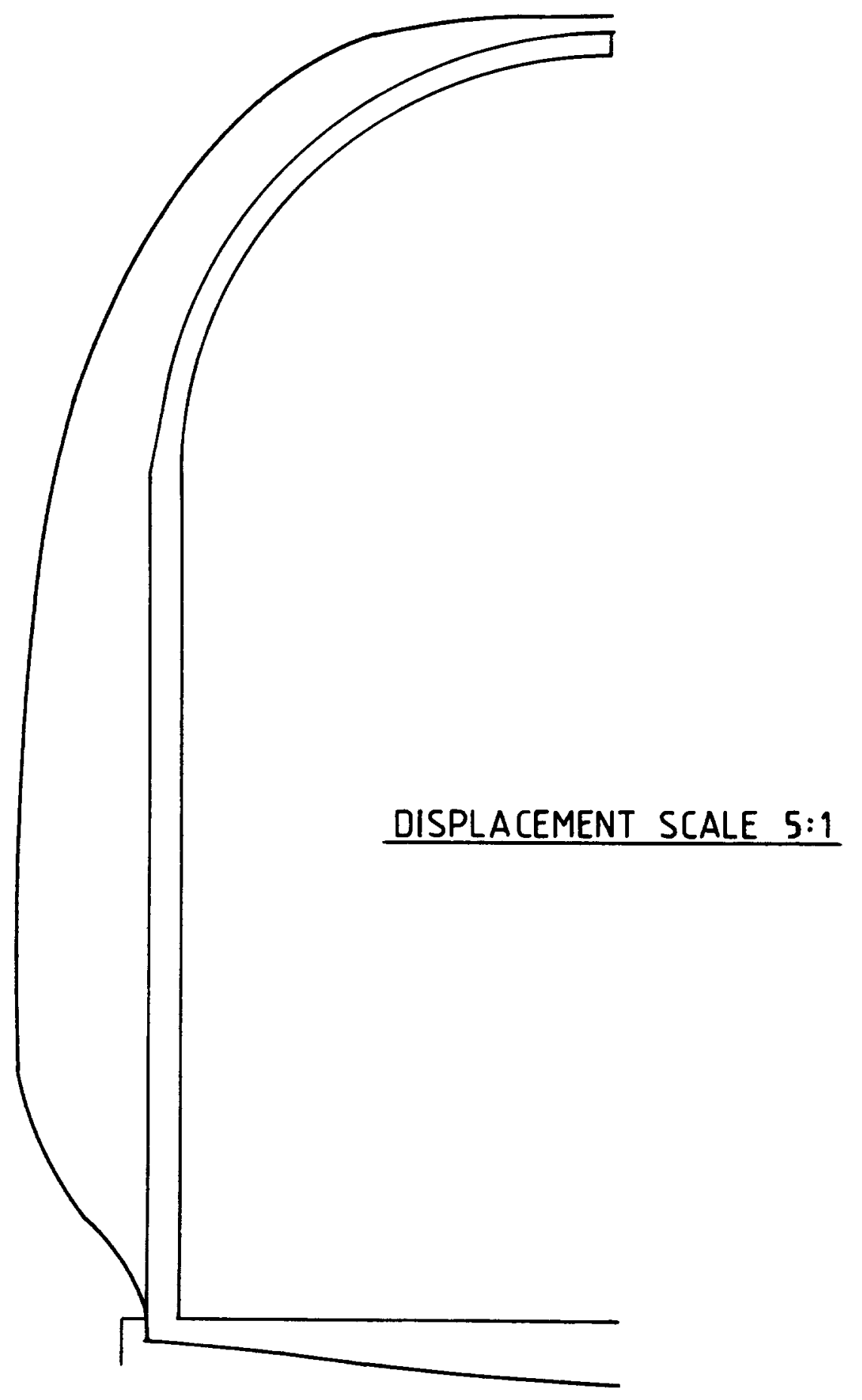

Figure 4.10.24 Displaced Shape at $0.517 \mathrm{MPa}$ (75 psi) $-411-$ 


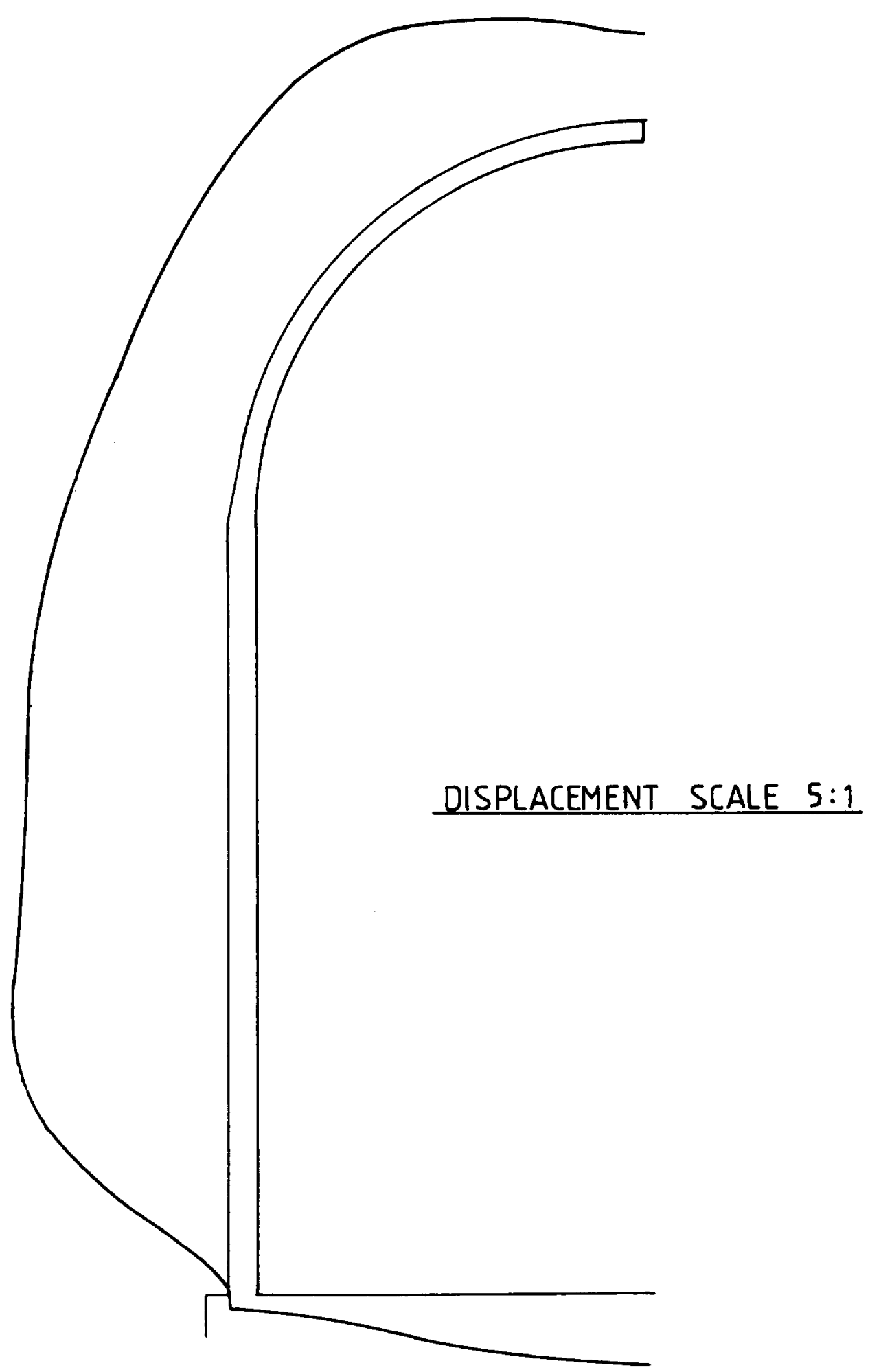

Figure 4.10.25 Displaced Shape at $0.690 \mathrm{MPa}$ (100 psi)

$-412-$ 


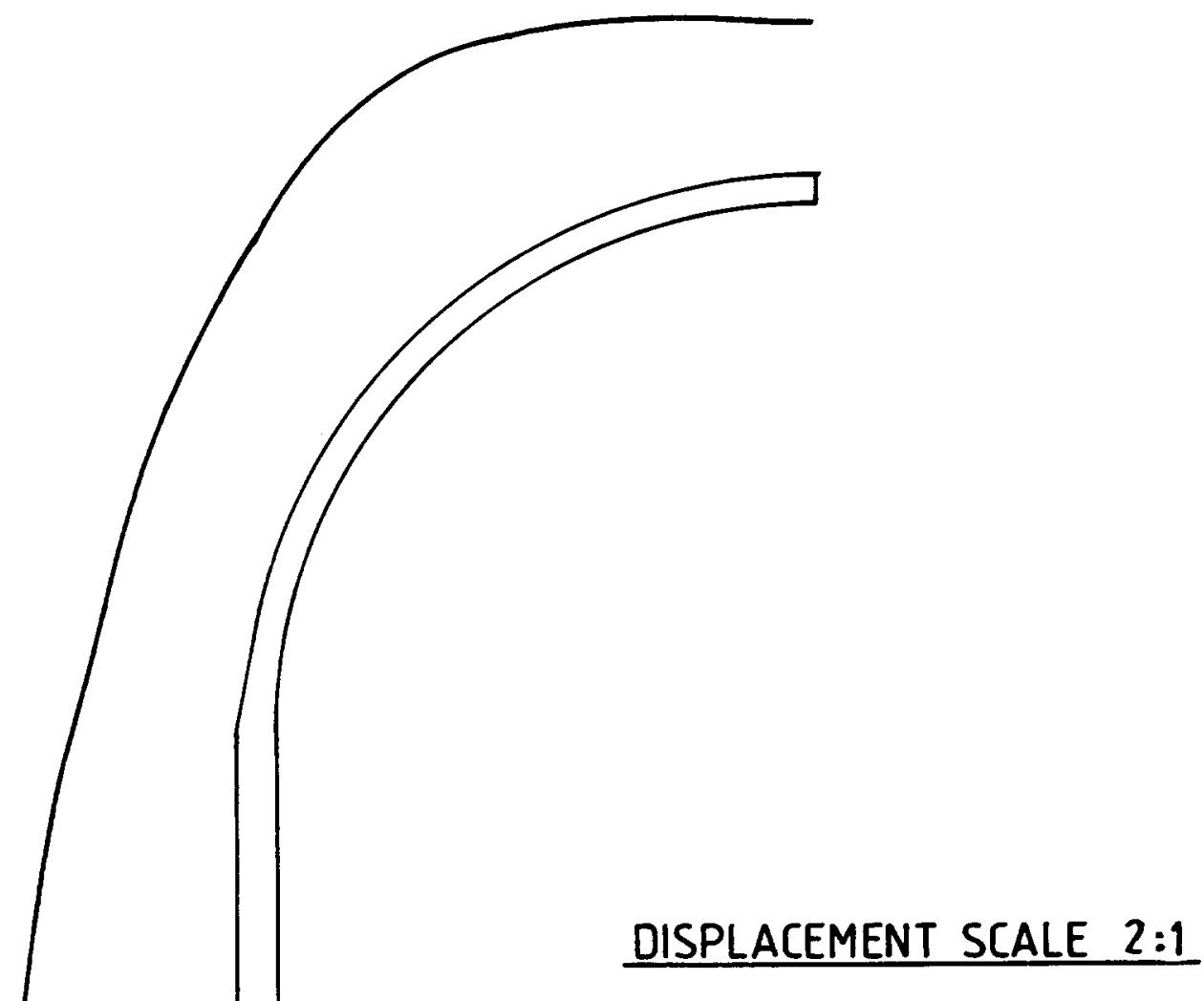

Figure 4.10,26 Displaced Shape at $0.897 \mathrm{MPa}$ (130 psi)

$-413-$ 


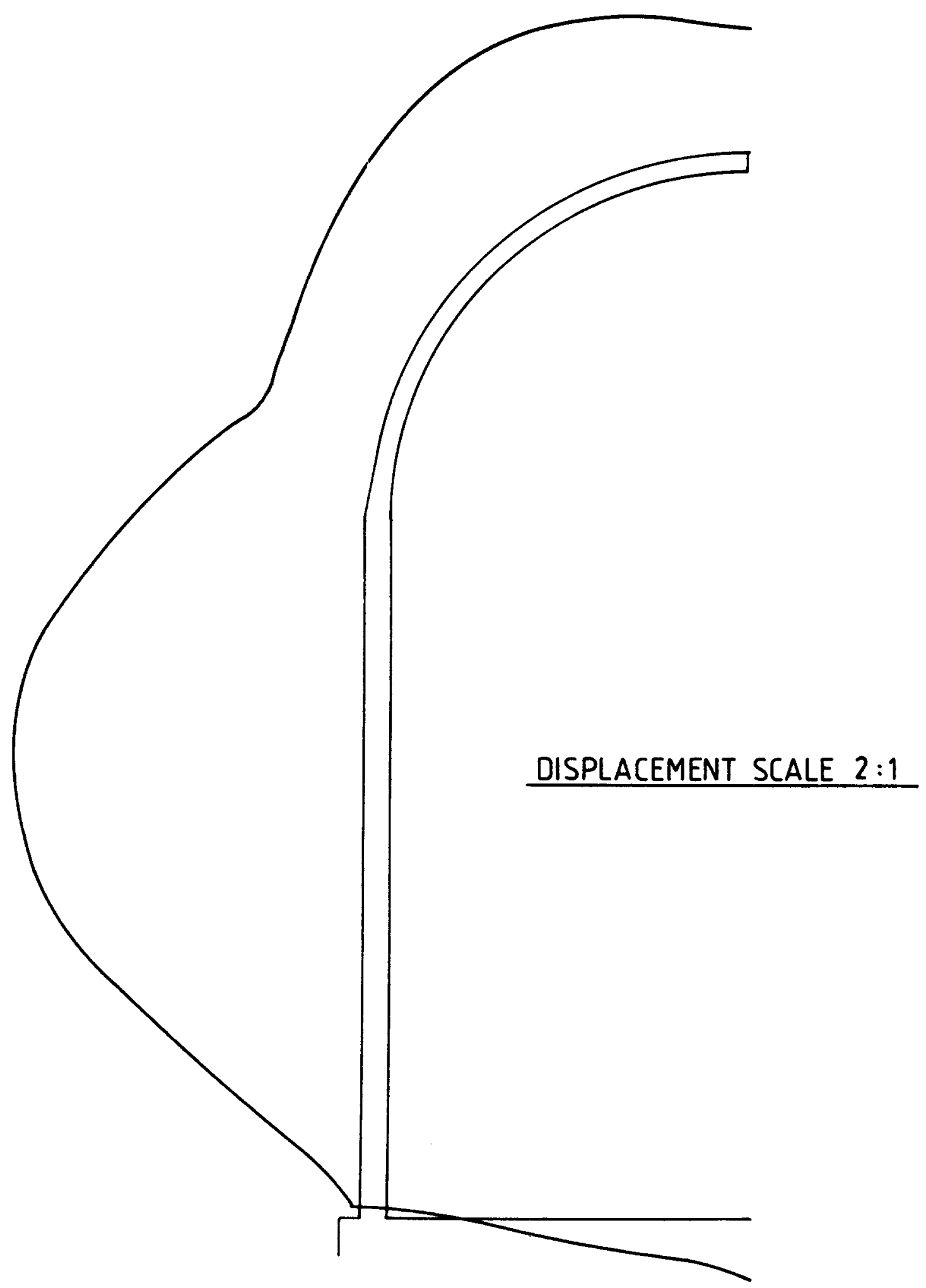

Figure 4.10.27 Displaced Shape at $0.959 \mathrm{MPa}$ (139 psi)

$-414-$ 


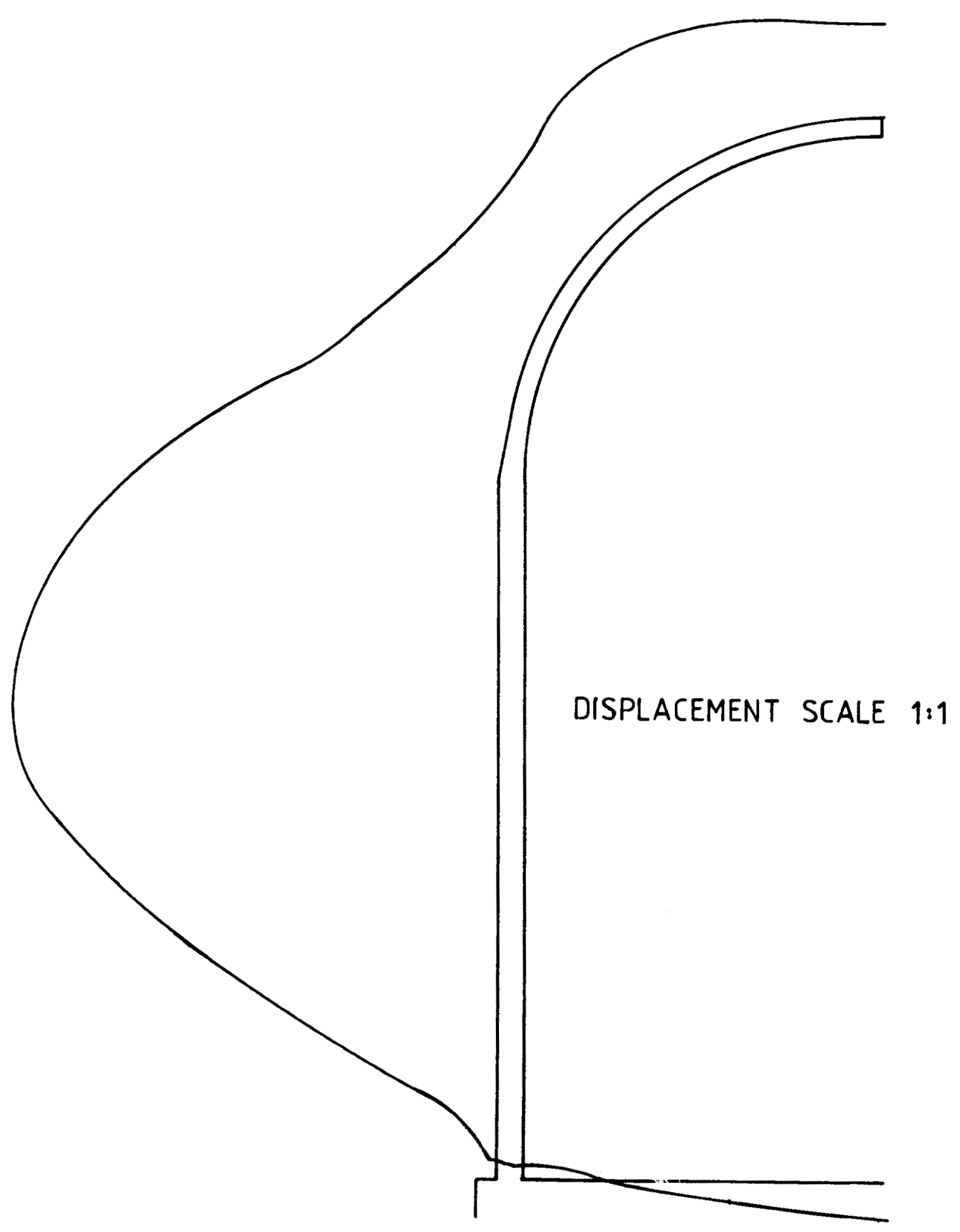

Figure 4.10.28 Displaced Shape at $1.103 \mathrm{MPa}$ (160 psi)

$-415-$ 
DENOTES OUT OF PLANE

CRACKING AND DIRECTION

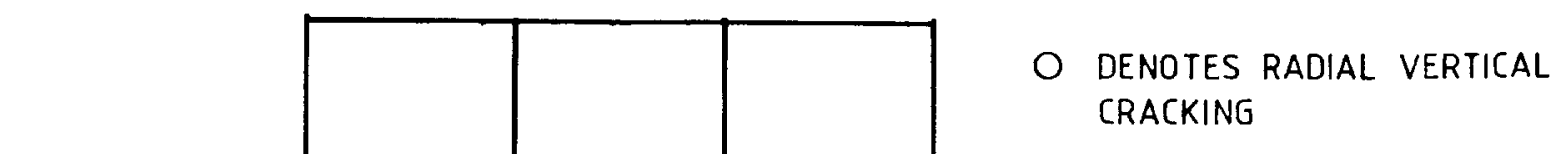




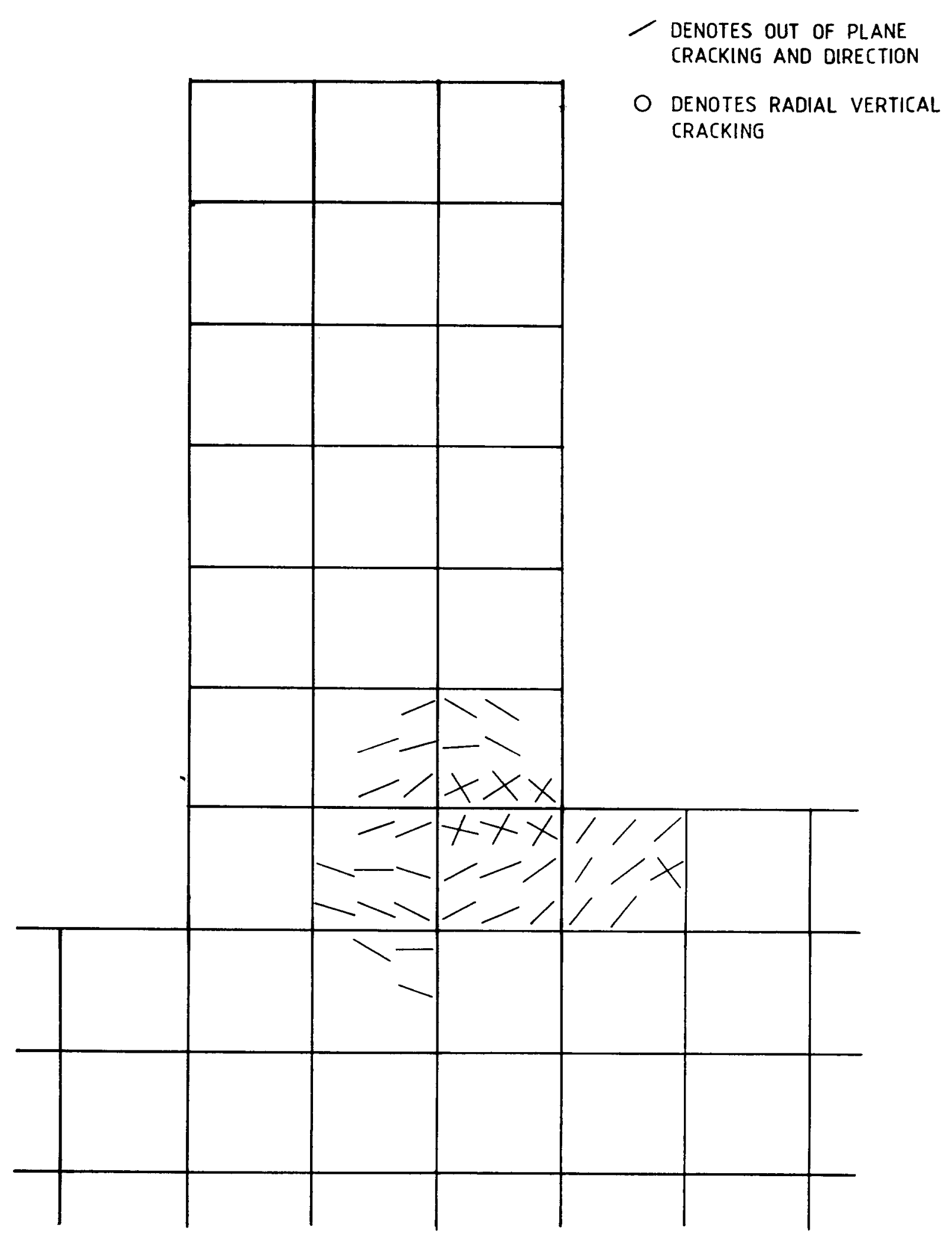

NOT TO SCALE

Figure 4.10.30 Wall/Base Junction Crack Pattern at $0.366 \mathrm{MPa}$ (53 psi) $-417-$ 


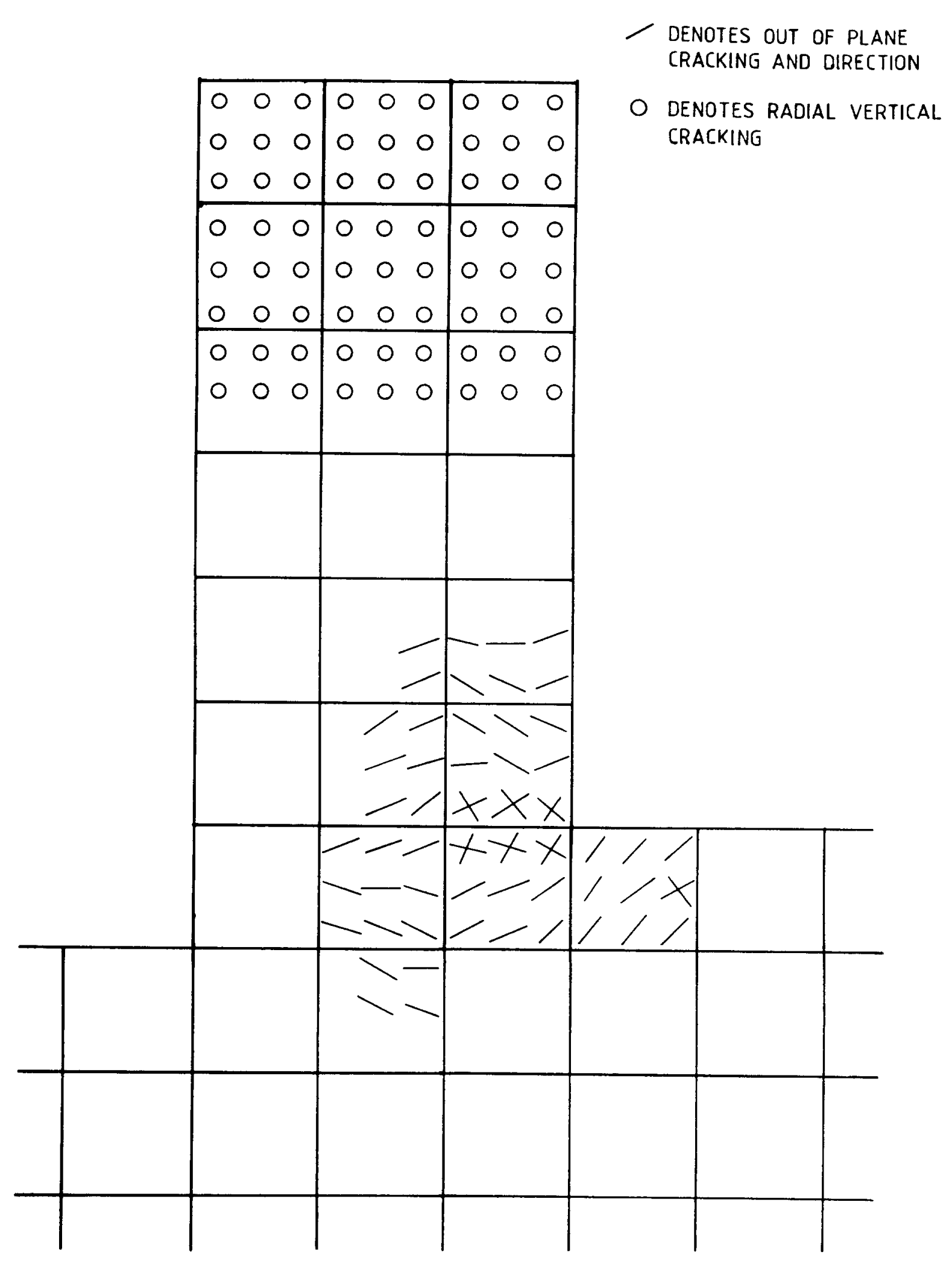

NOT TO SCALE

Figure 4.10.31 Wall/Base Junction Crack Pattern at $0.421 \mathrm{MPa}$ (61 psi) 


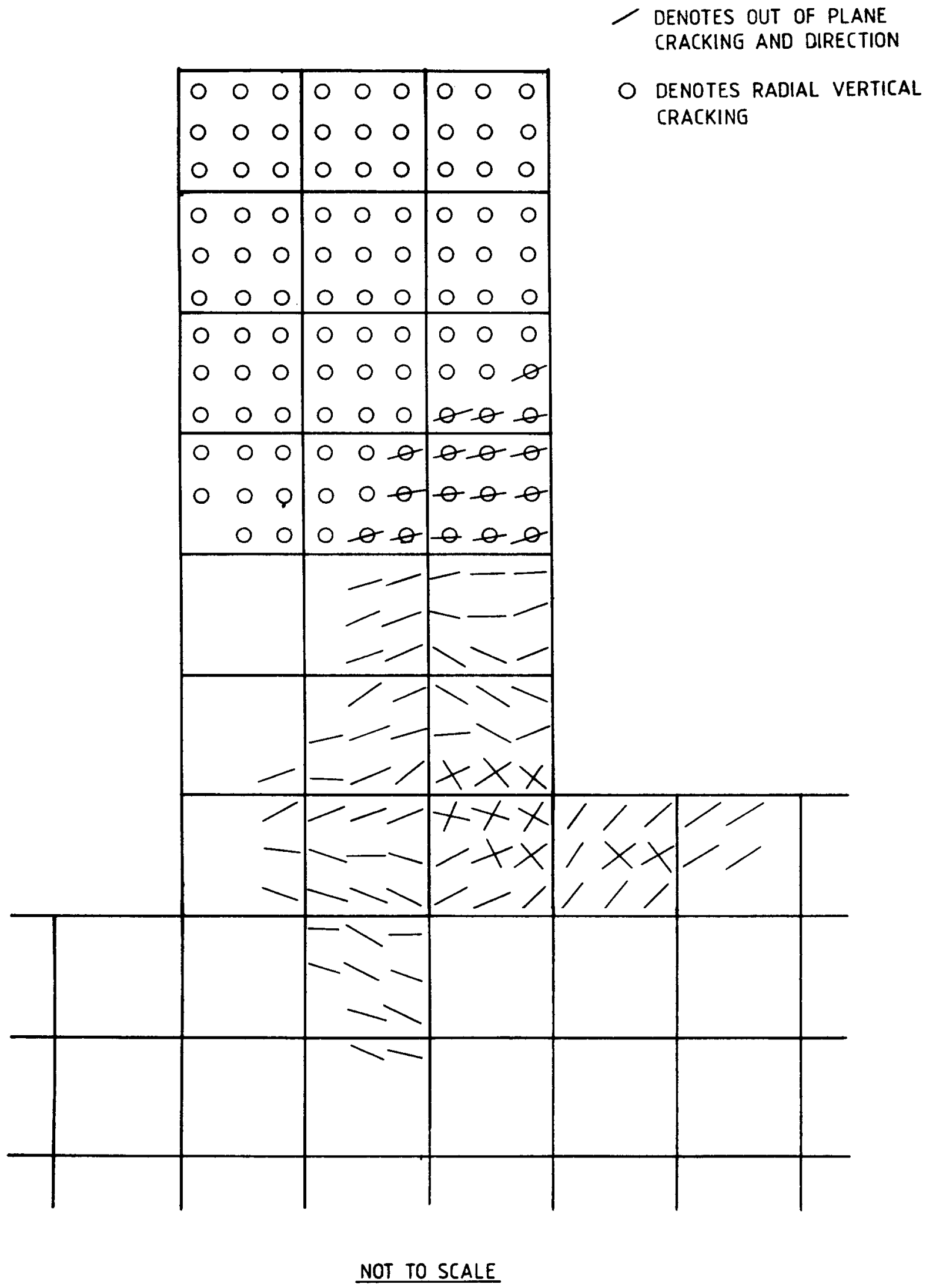

Figure 4.10.32 Wall/Base Junction Crack Pattern at $0.566 \mathrm{MPa}$ (82 psi)

$-419$ 
DENOTES OUT OF PLANE

CRACKING AND DIRECTION

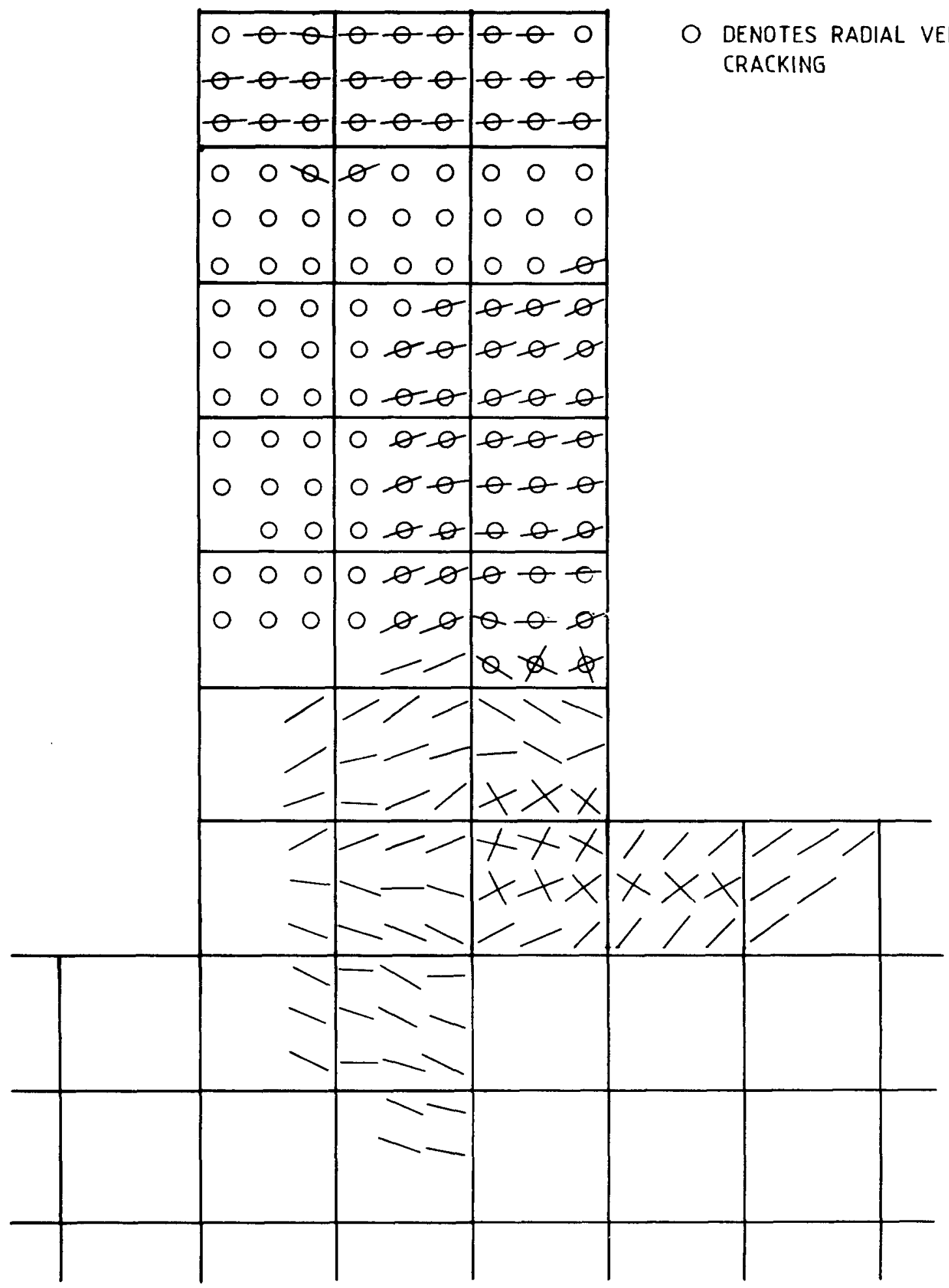

NOT TO SCALE

Figure 4.10.33 Wall/Base Junction Crack Pattern at $0.690 \mathrm{MPa}$ (100 psi) 


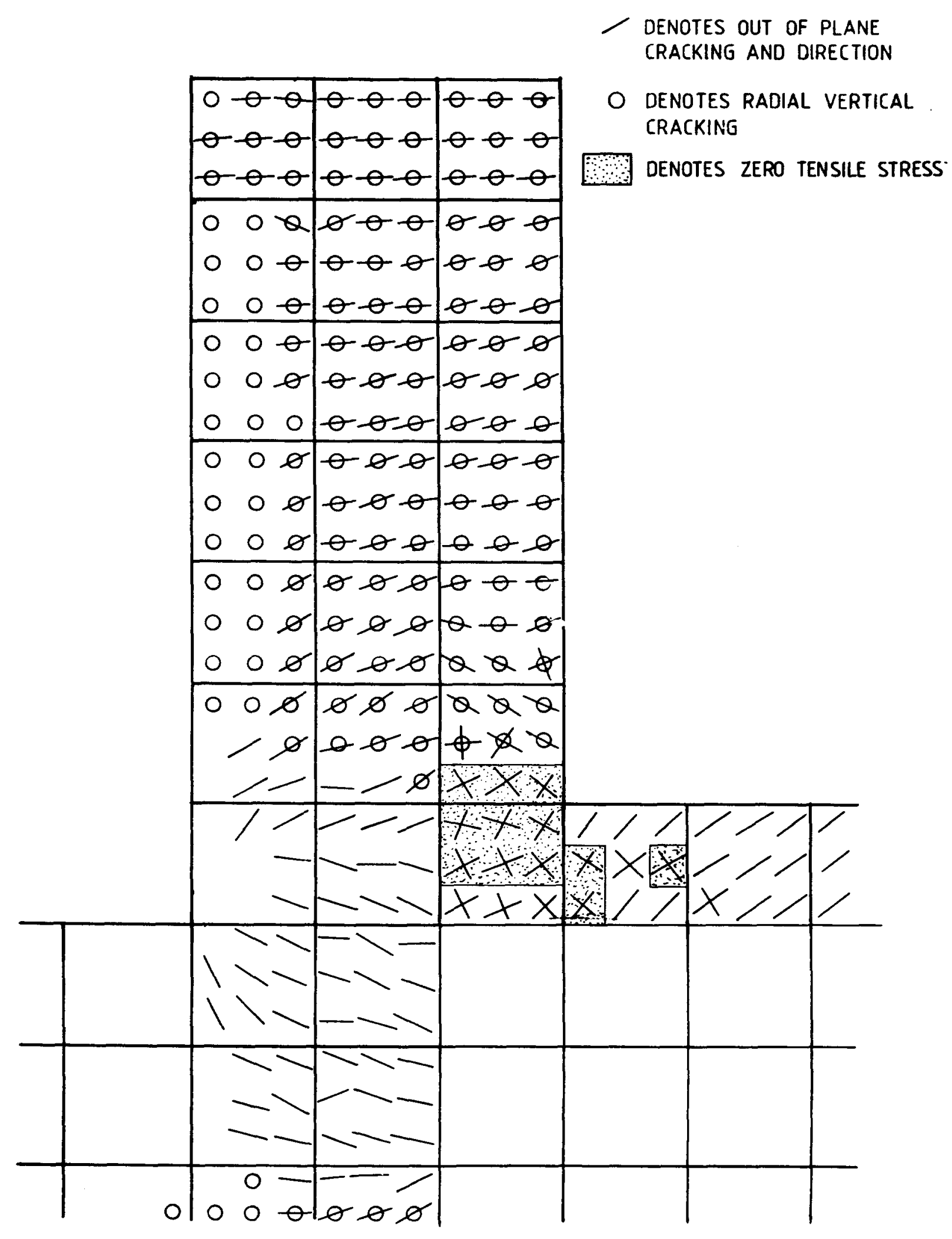

NOT TO SCALE

Figure 4.10.34 Wall/Base Junction Crack Pattern at $1.000 \mathrm{MPa}$ (145 psi) 


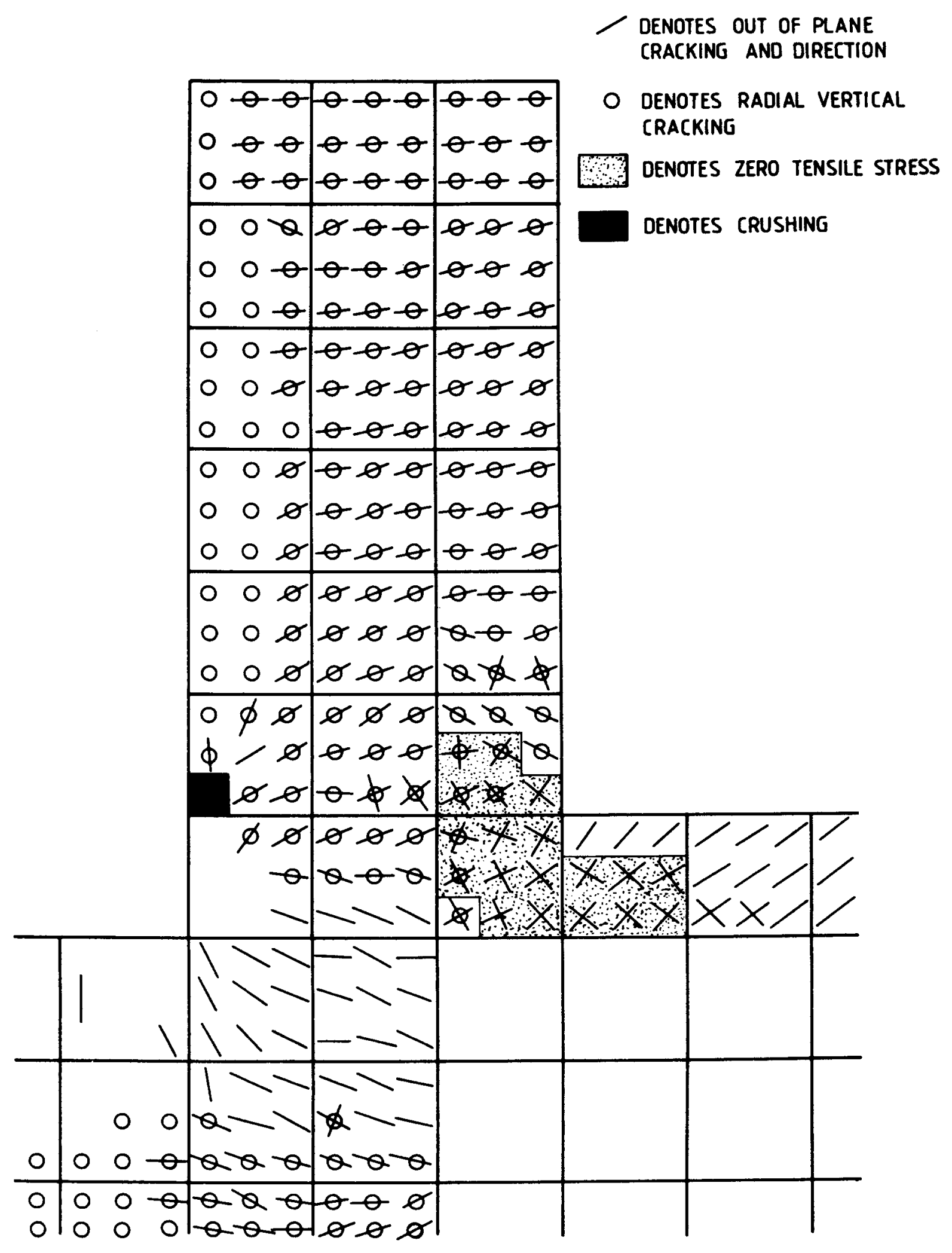

NOT TO SCALE

Figure 4.10.35 Wall/Base Junction Crack Pattern at $1.100 \mathrm{MPa}$ (159.5 psi) 


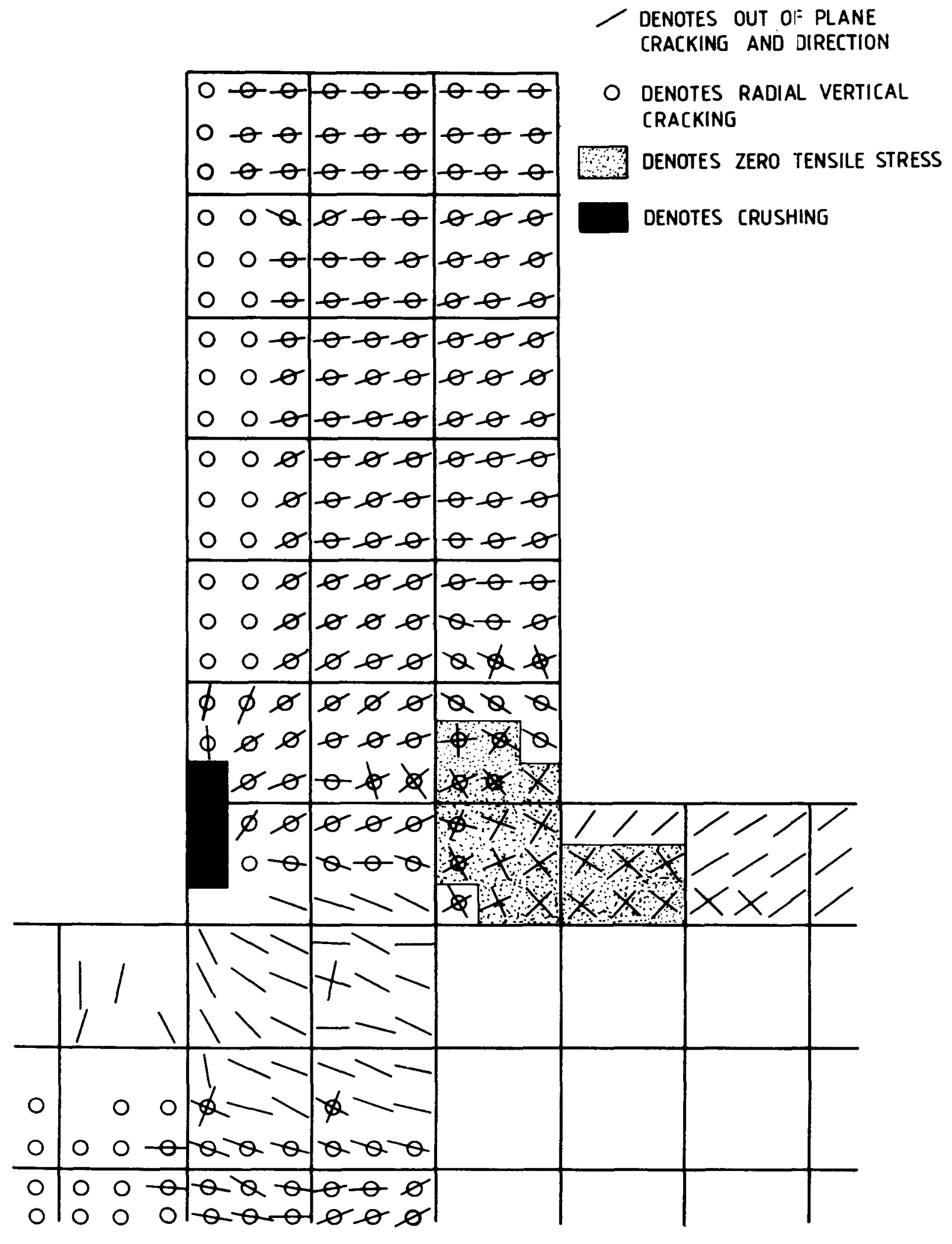

NOT TO SCALE

Figure 4.10.36 Wall/Base Junction Crack Pattern at $1.103 \mathrm{MPa}$ (160 psi) 


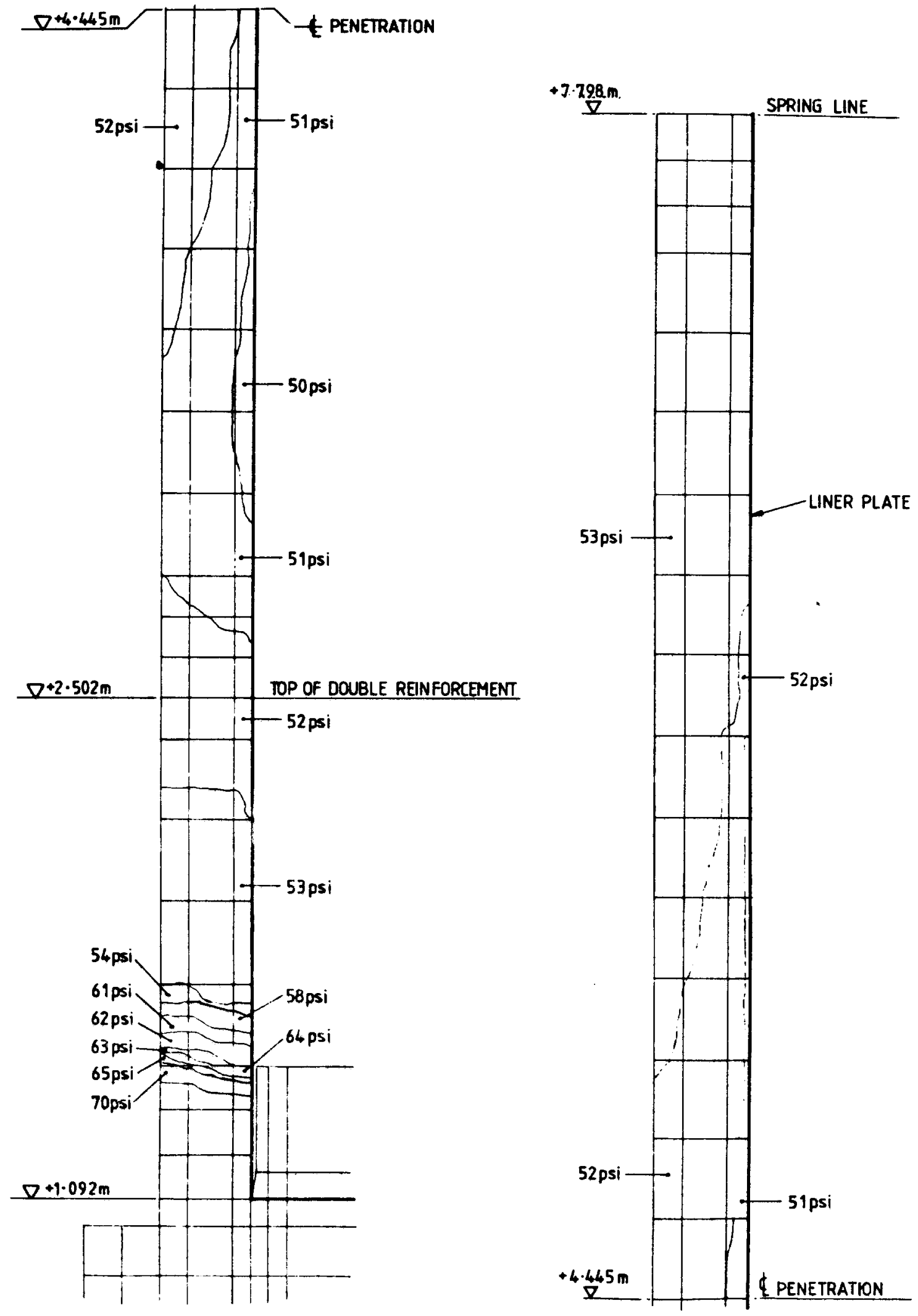

Figure 4.10.37 Progressive Vertical Crack Pattern in Cylindrical Wall 


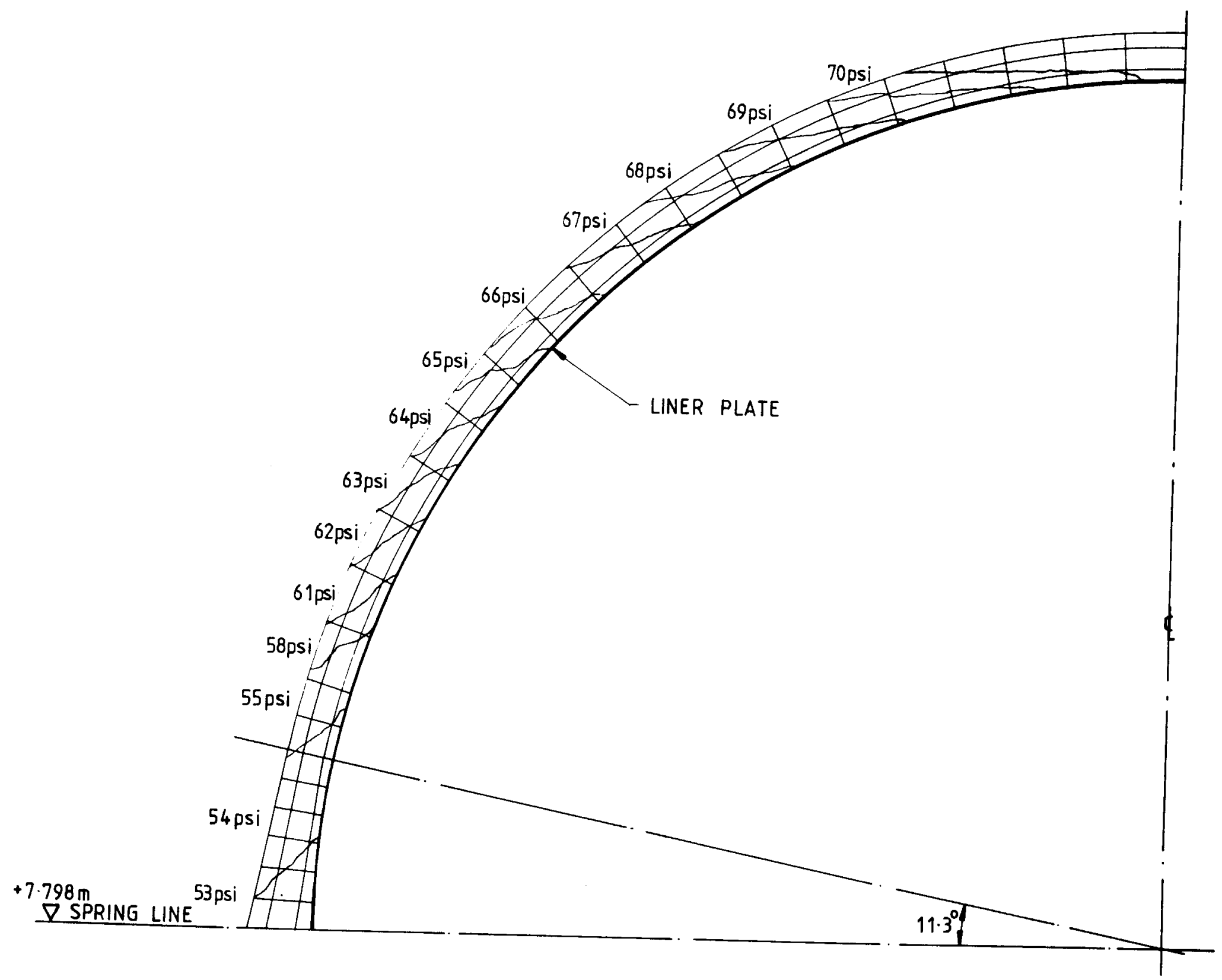

Figure 4.10.38 Progressive Vertical Crack Pattern in Dome 


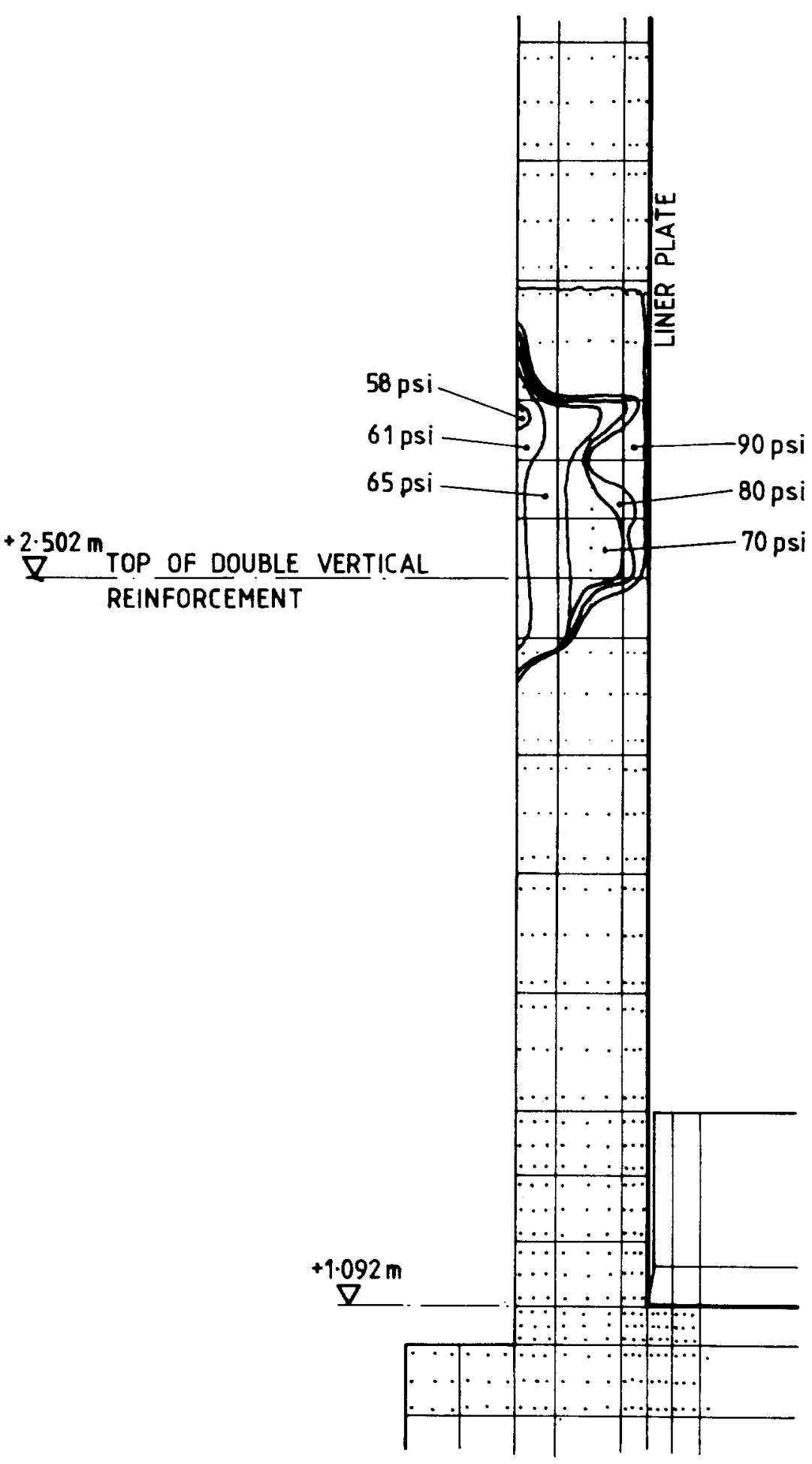

Figure 4.10.39 Progressive Horizontal Crack Pattern $2.5 \mathrm{~m}$ Above Base $-426$ 


\section{LEGEND}

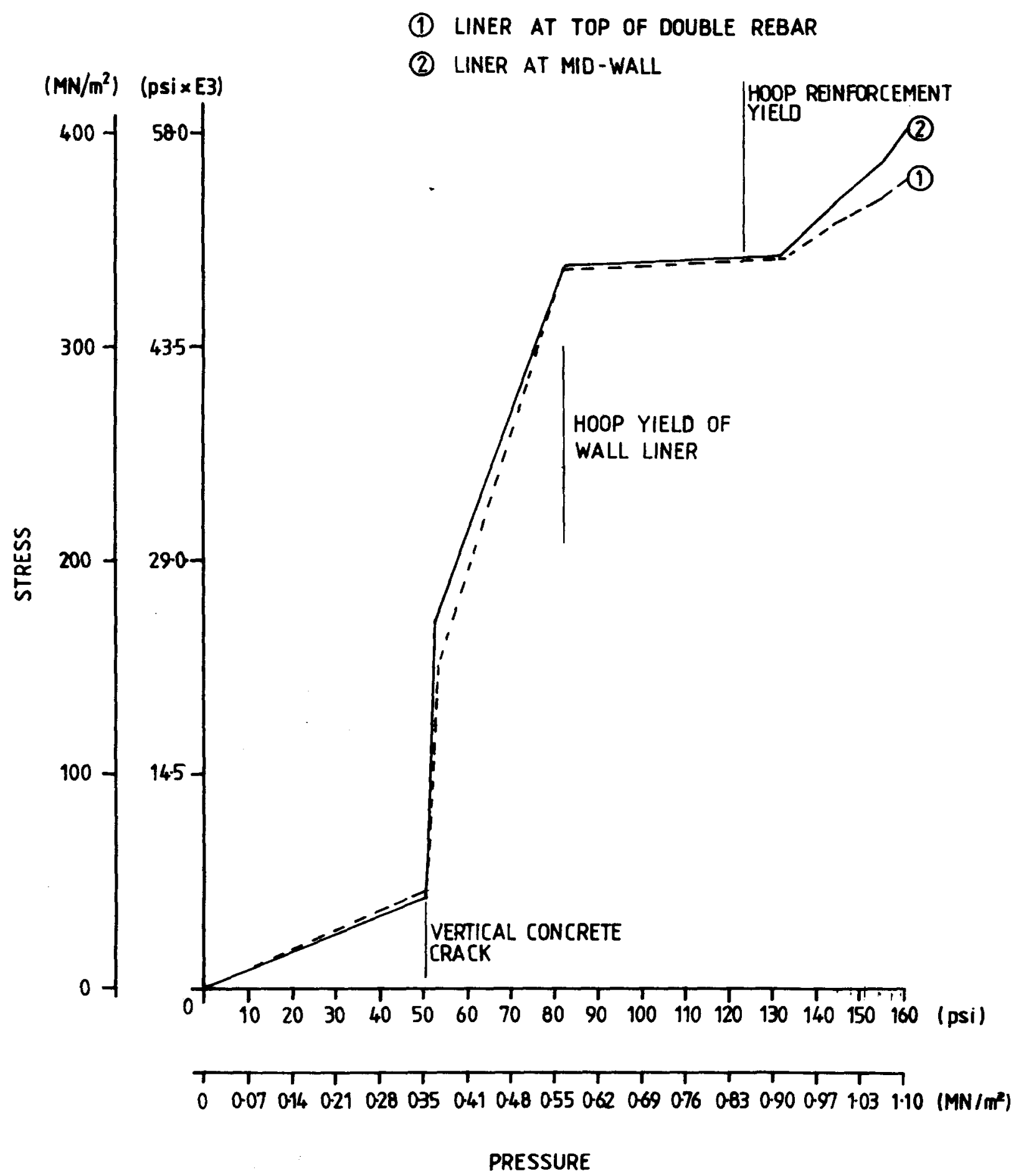

Figure 4.10.40 Wall Liner Hoop Stresses vs Pressure 
$\left(M N / m^{2}\right) \quad(p s i \times E 3)$

\section{LEGEND}

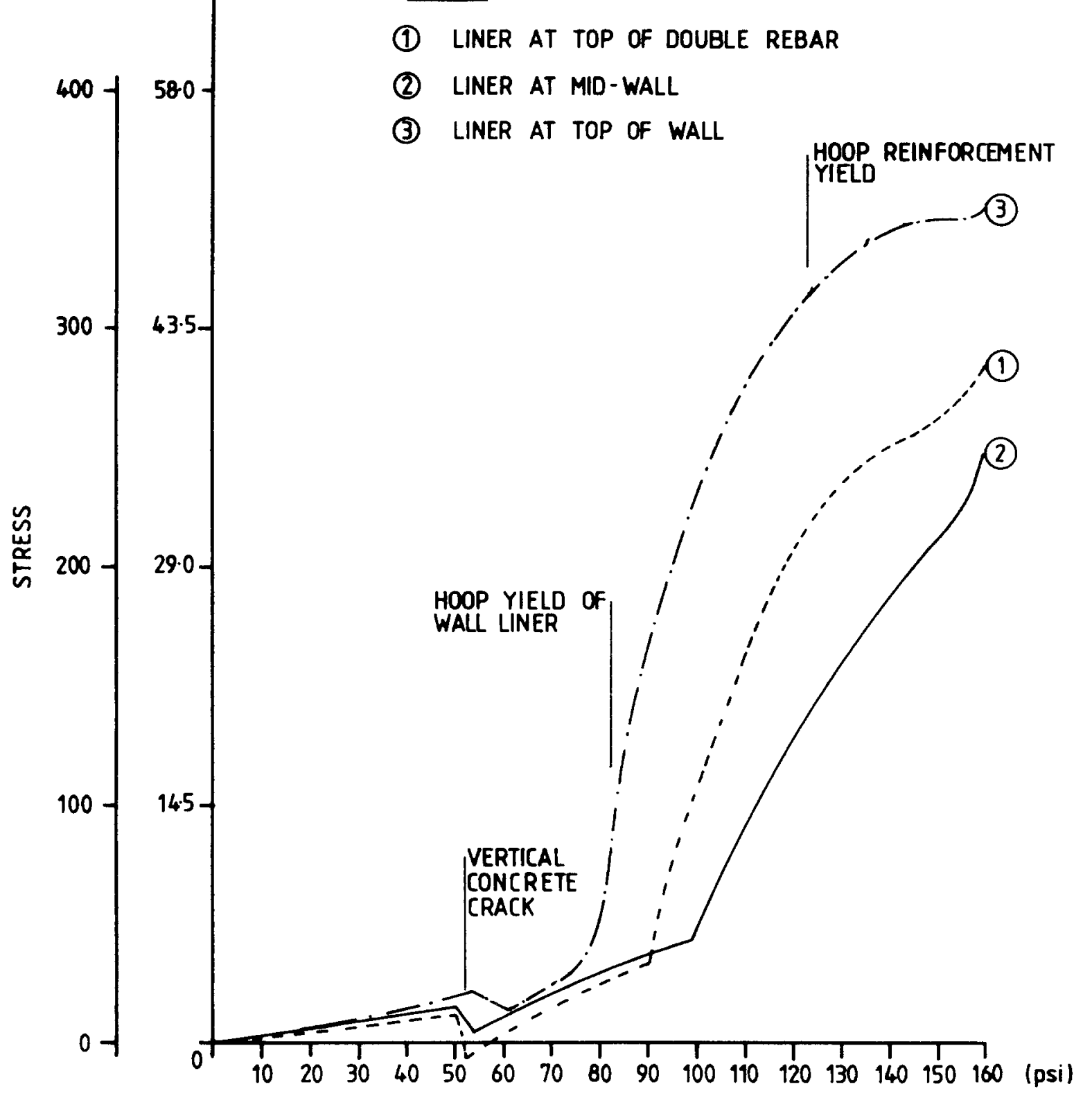

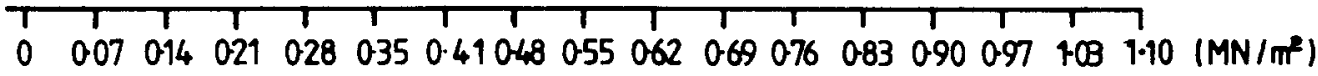

PRESSURE

Figure 4.10.41 Wall Liner Vertical Stresses vs Pressure 


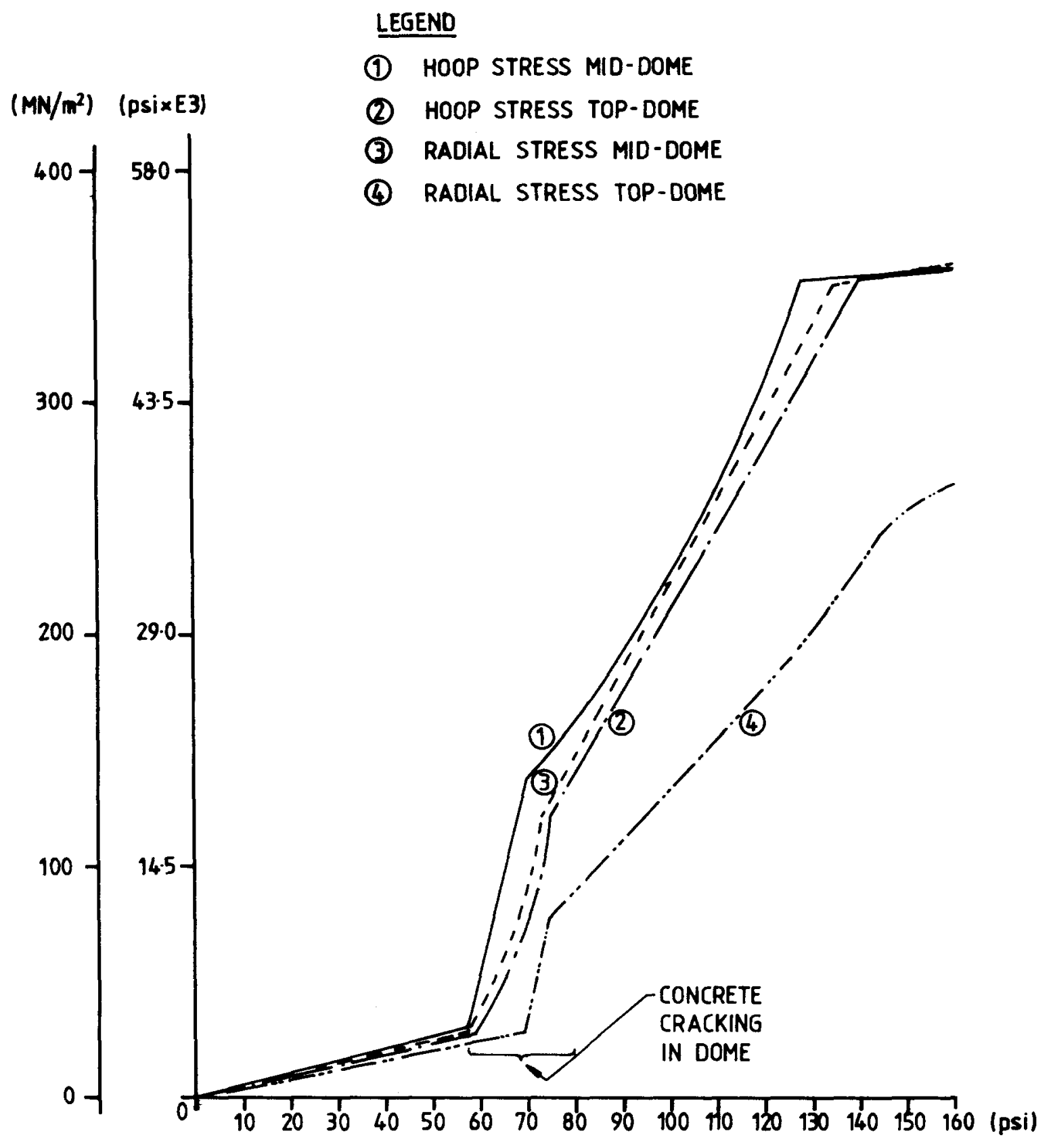

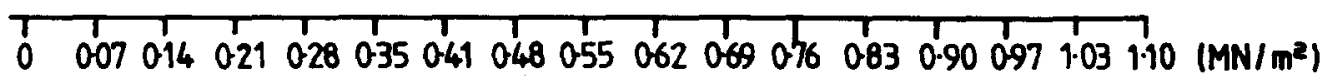
PRESSURE

Figure 4.10.42 Dome Liner Hoop and Radial Stresses vs Pressure 


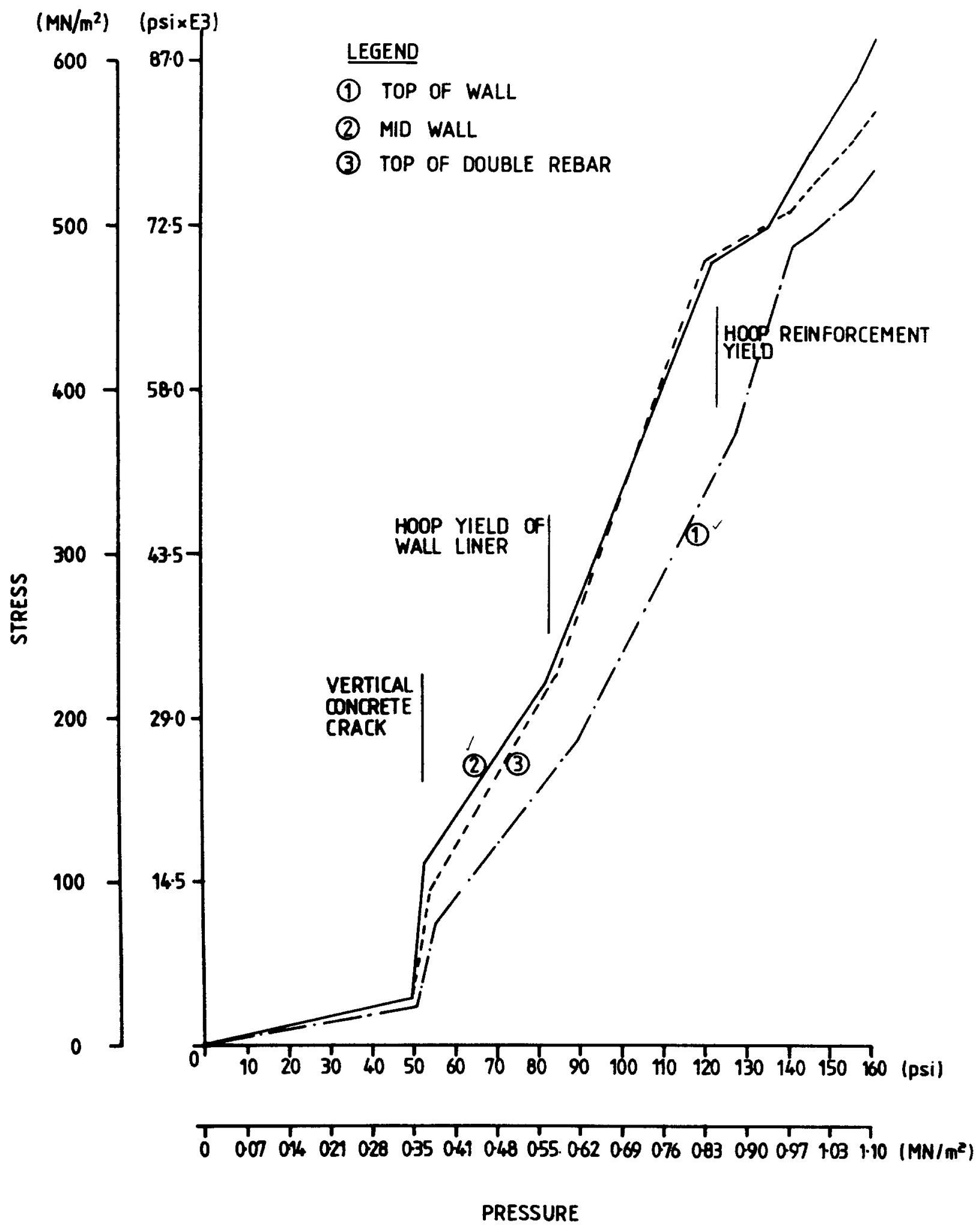

Figure 4.10.43 Rebar Hoop Stresses in Wall vs Pressure 


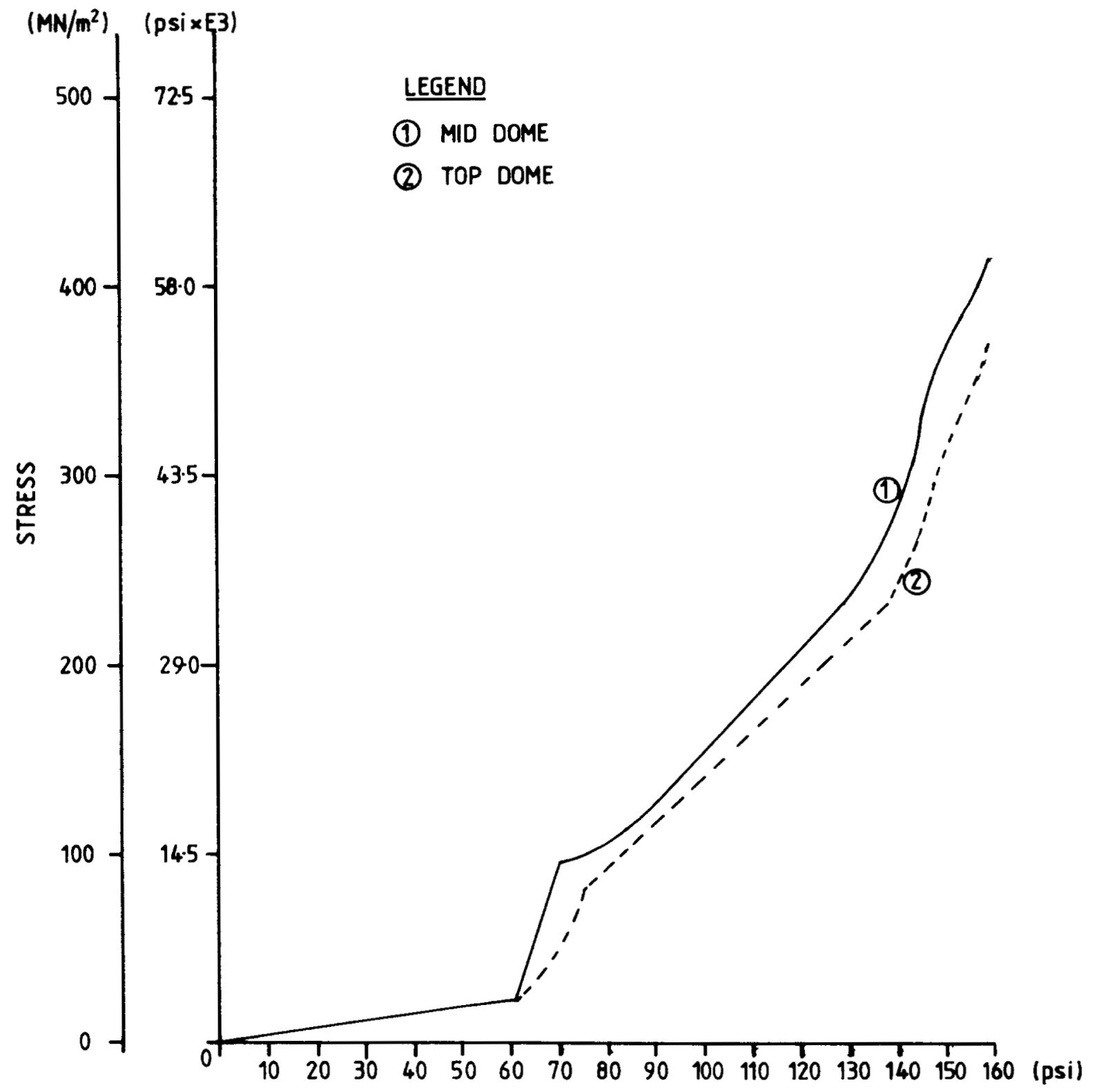

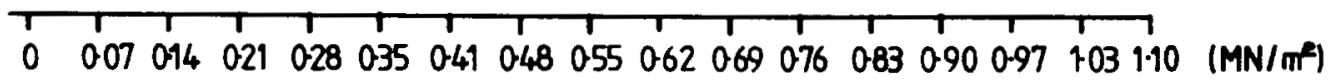

PRESSURE

Figure 4.10.44 Hoop Reinforcement in Dome Stress vs Pressure 


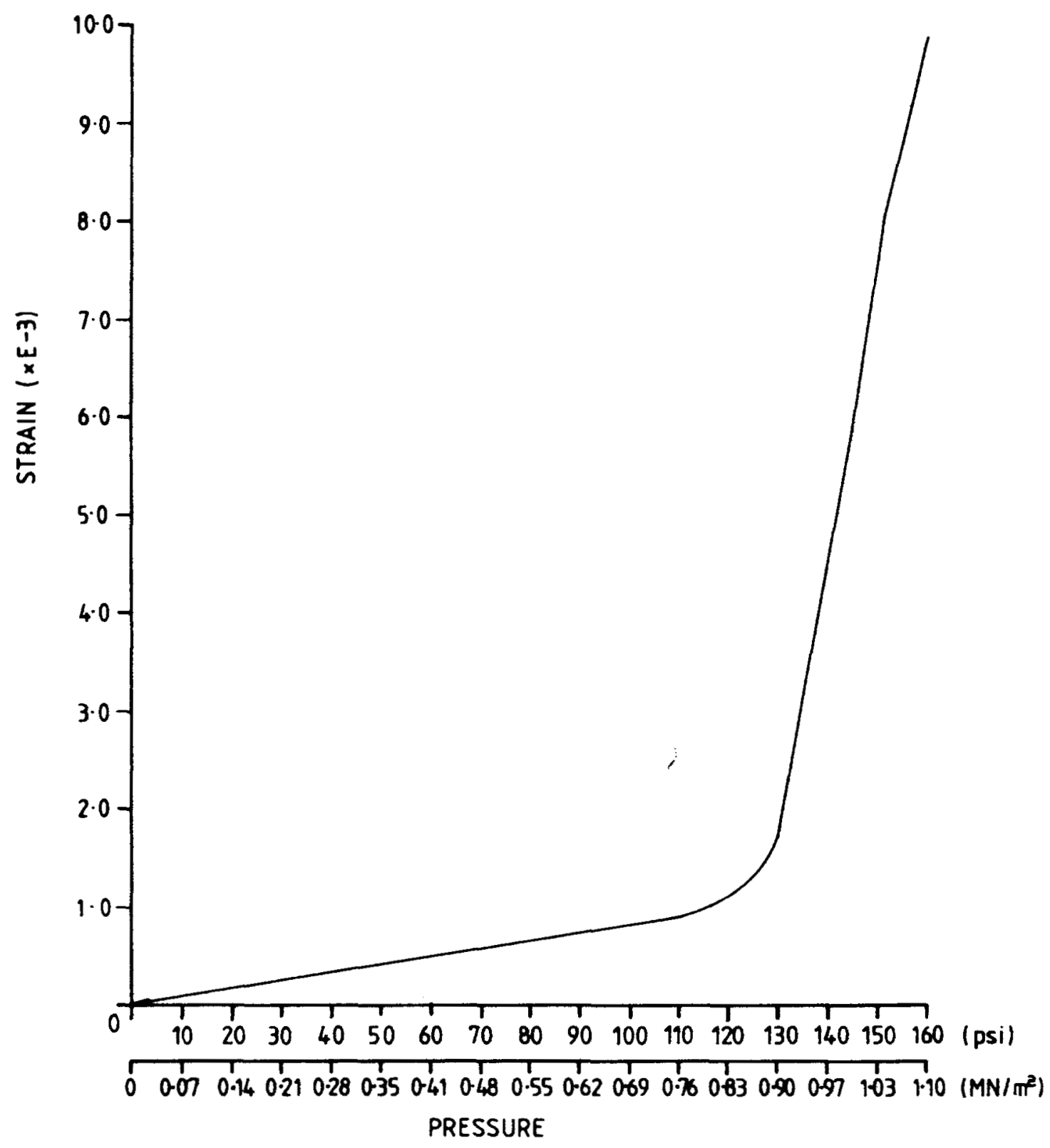

Figure 4.10 .45

Base Liner Plate Radial Strain at Wall/Base Junction vs Pressure 


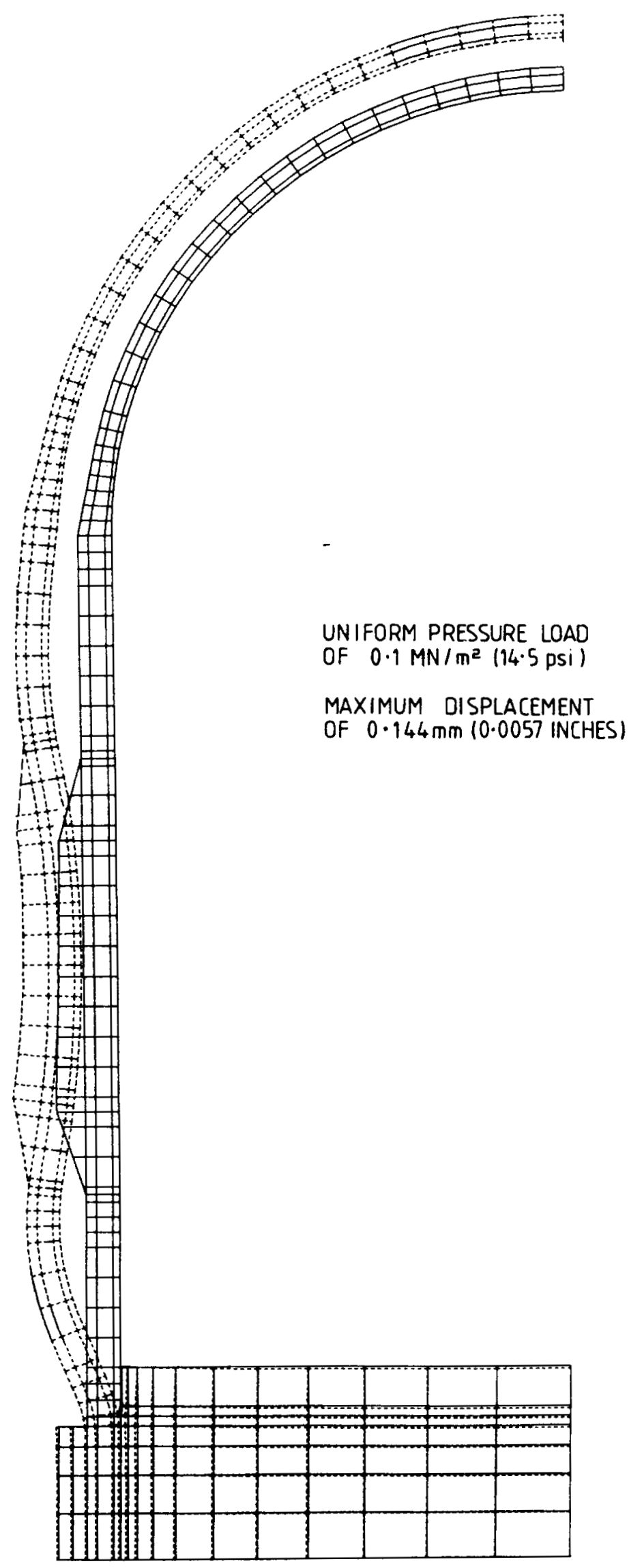

Figure 4.10.46

Displaced Shape of Barrel Thickened Section (Hatch A) at $0.1 \mathrm{MPa}$ (14.5 psi) Linear Elastic Axisymmetric Analysis 


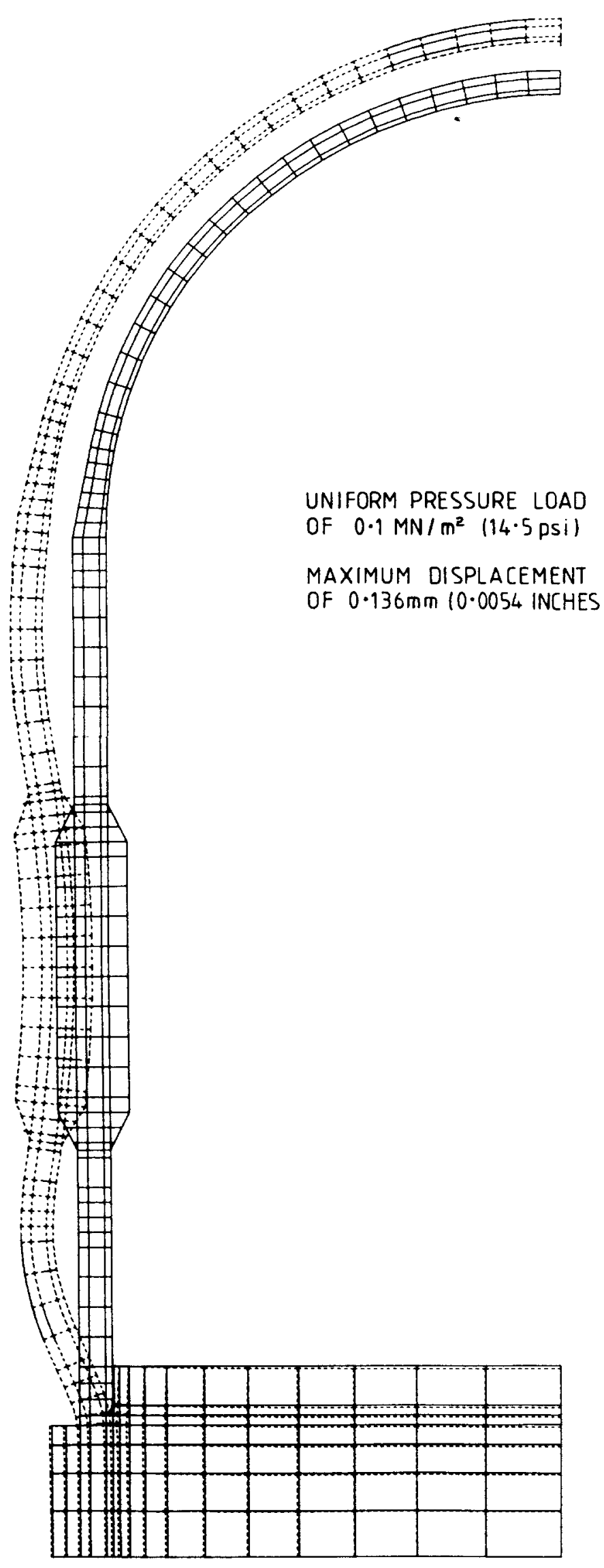

Figure 4.10 .47

Displaced Shape of Barrel Thickened Section (Hatch B) at $0.1 \mathrm{MPa}$ (14.5 psi)

Linear Elastic Axisymmetric Analysis 

(1) - - NO THICKENING
(2) --..-.- OUTSIDE THICKENING
(3) --- - INSIDE AND OUTSIDE THICKENING
(4) HAND CALCULATION OF SIMPLE

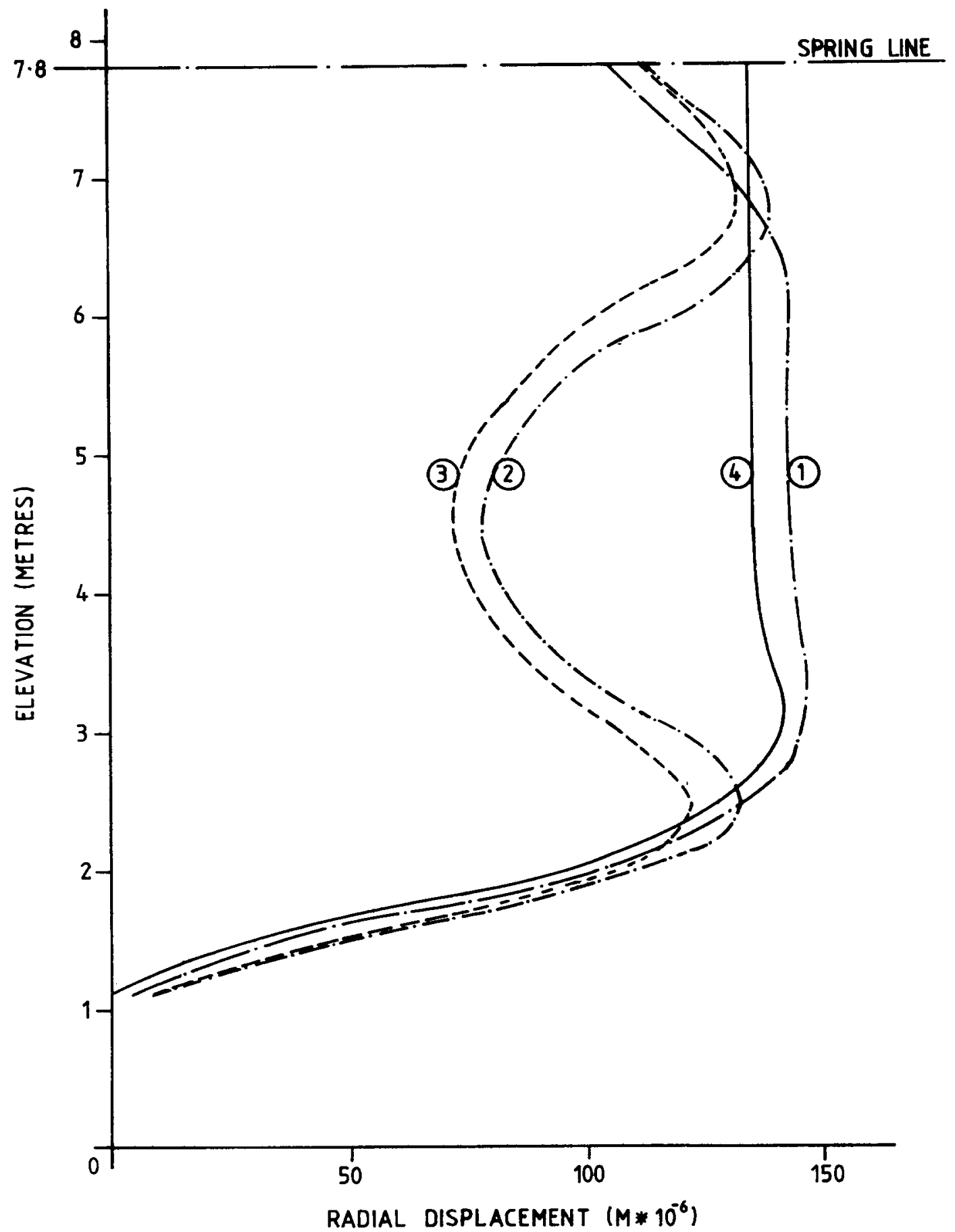

Figure 4.10.48 Comparison of Displacements from an Unthickened Model to Those with Penetration Thickenings 


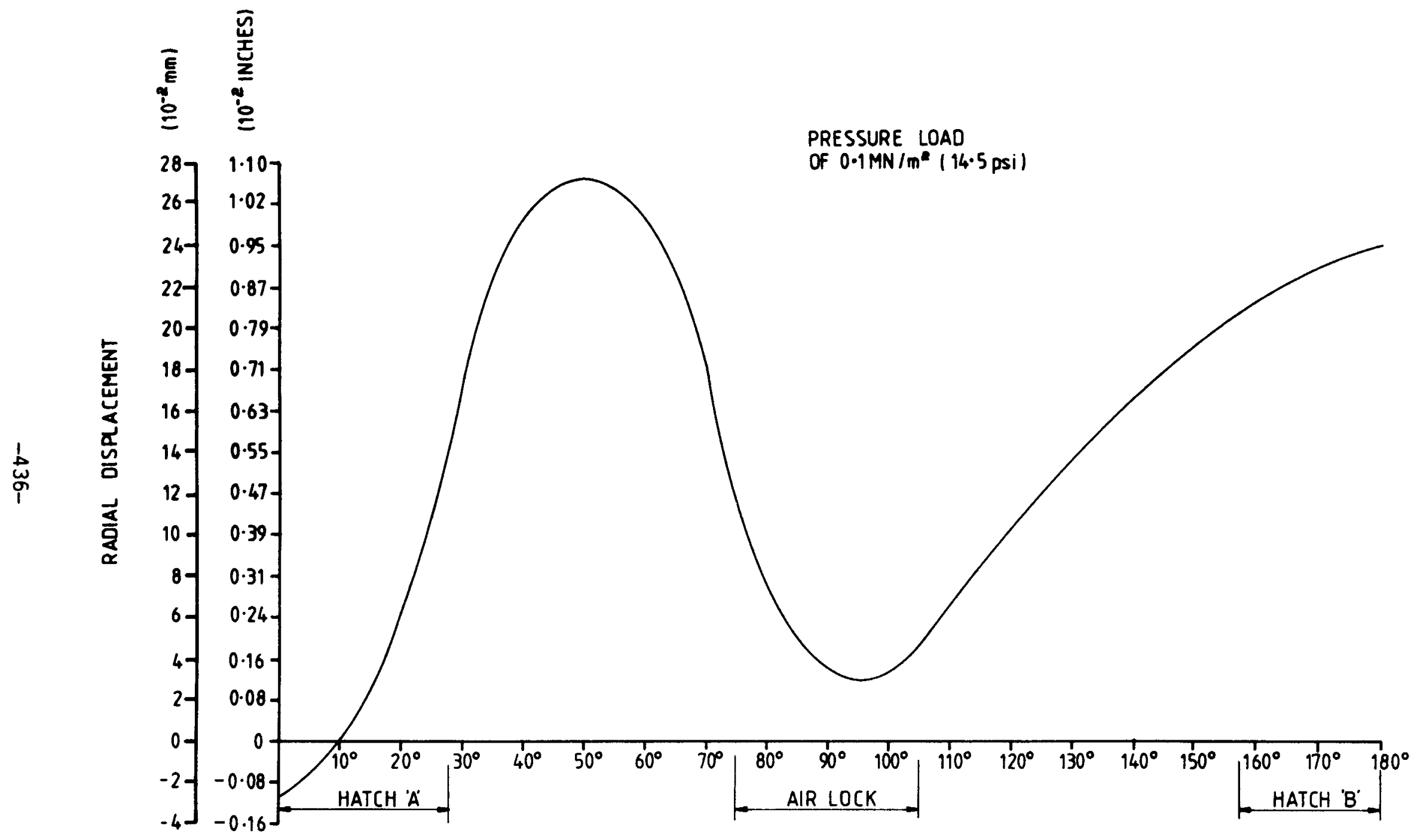

ANGULAR DISTANCE FROM \& HATCH 'A'

Figure 4.10.49 Linear Elastic 2-D Analysis Displacement Results 


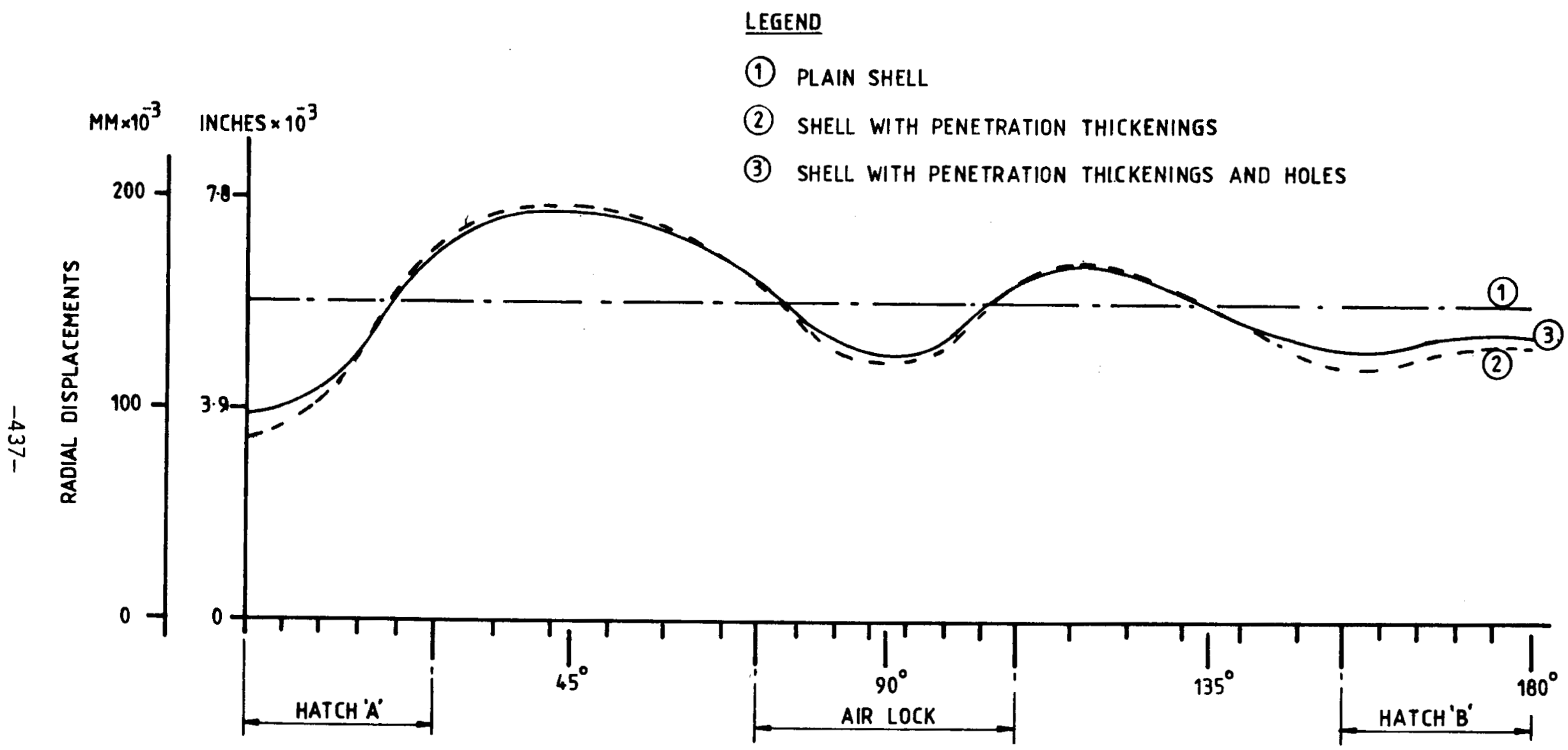

ANGULAR DISTANCE FROM $\$$ HATCH 'A' 


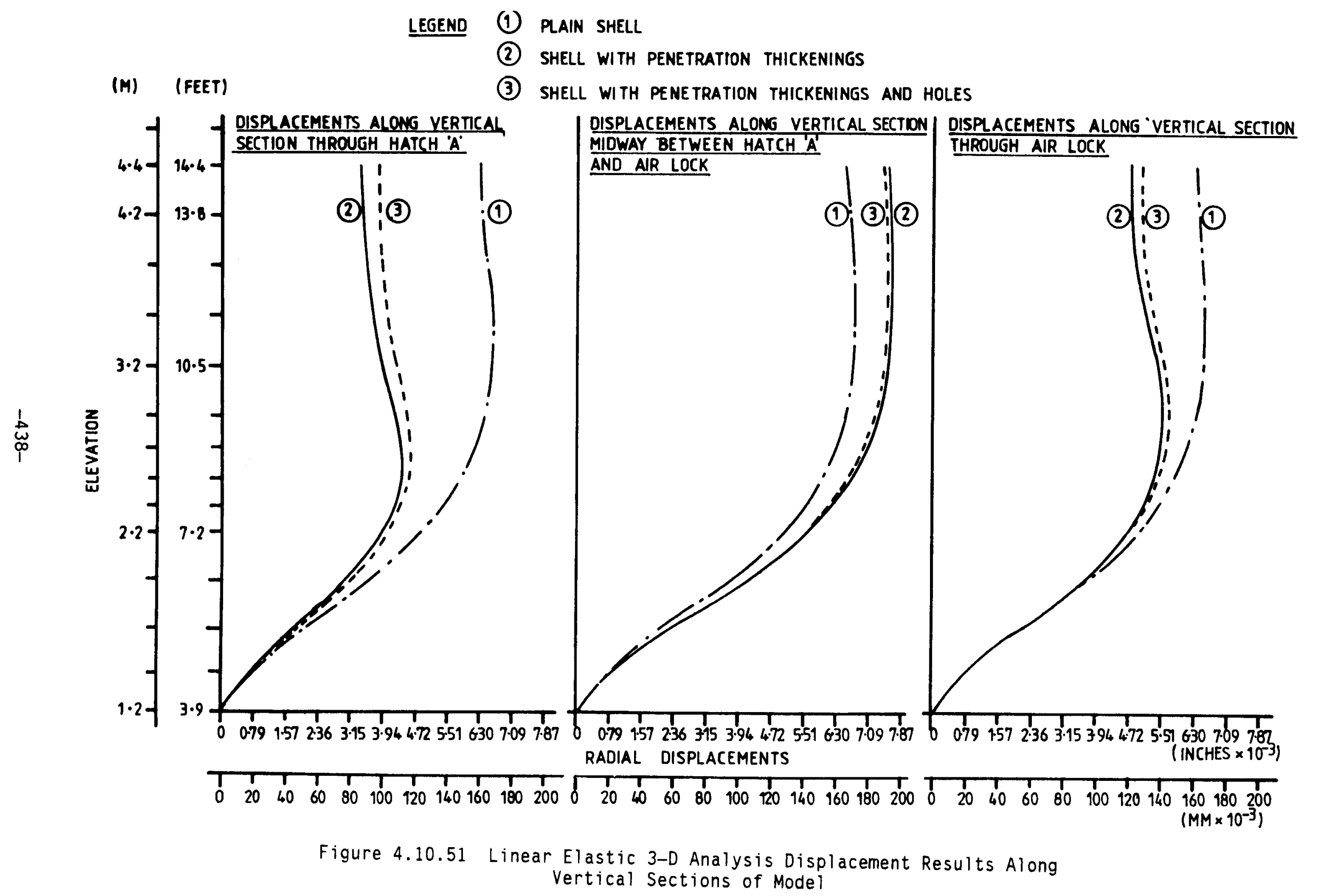




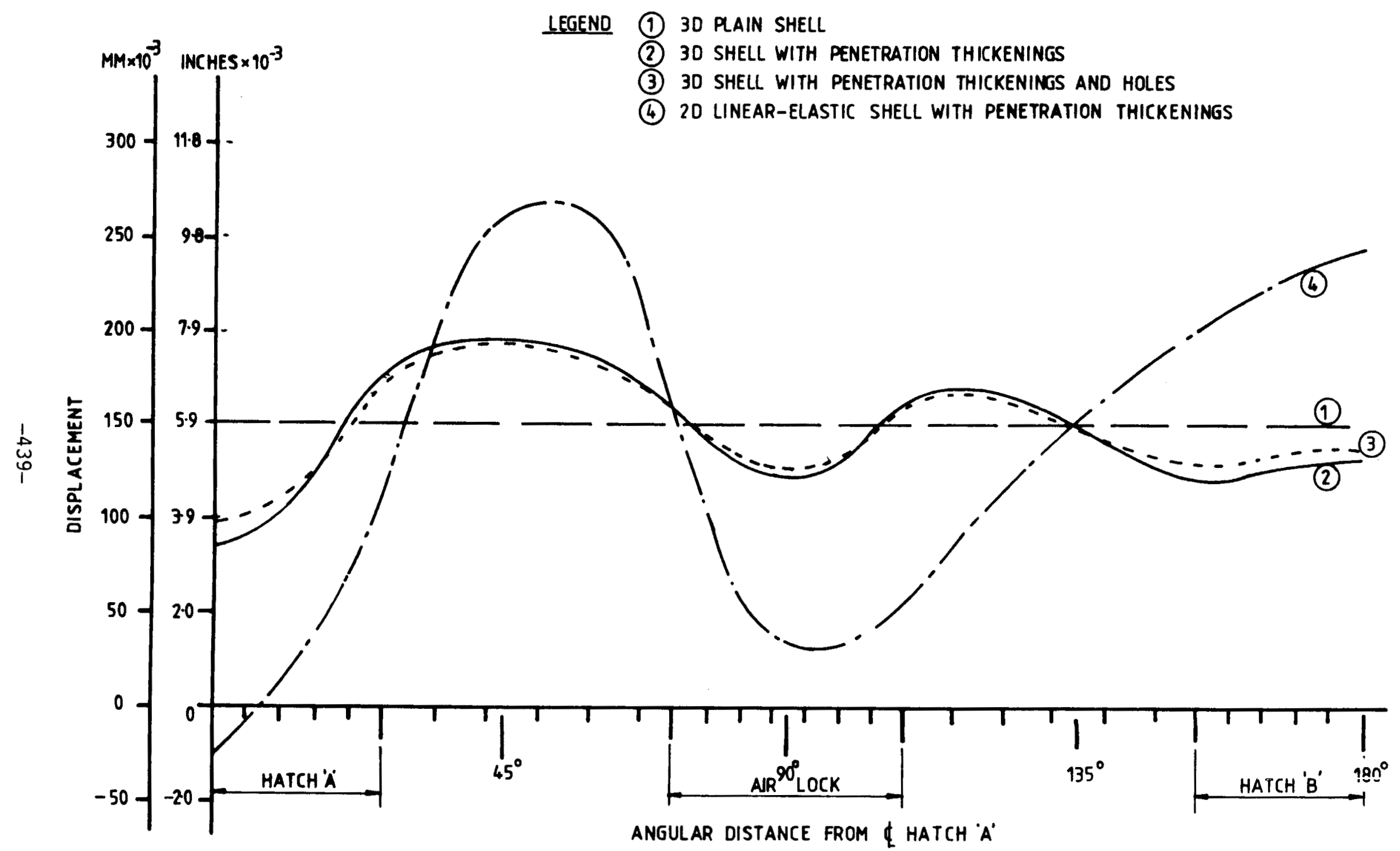

Figure 4.10 .52

Comparison of 3-D Displacements with Linear Elastic 2-D Displacements 


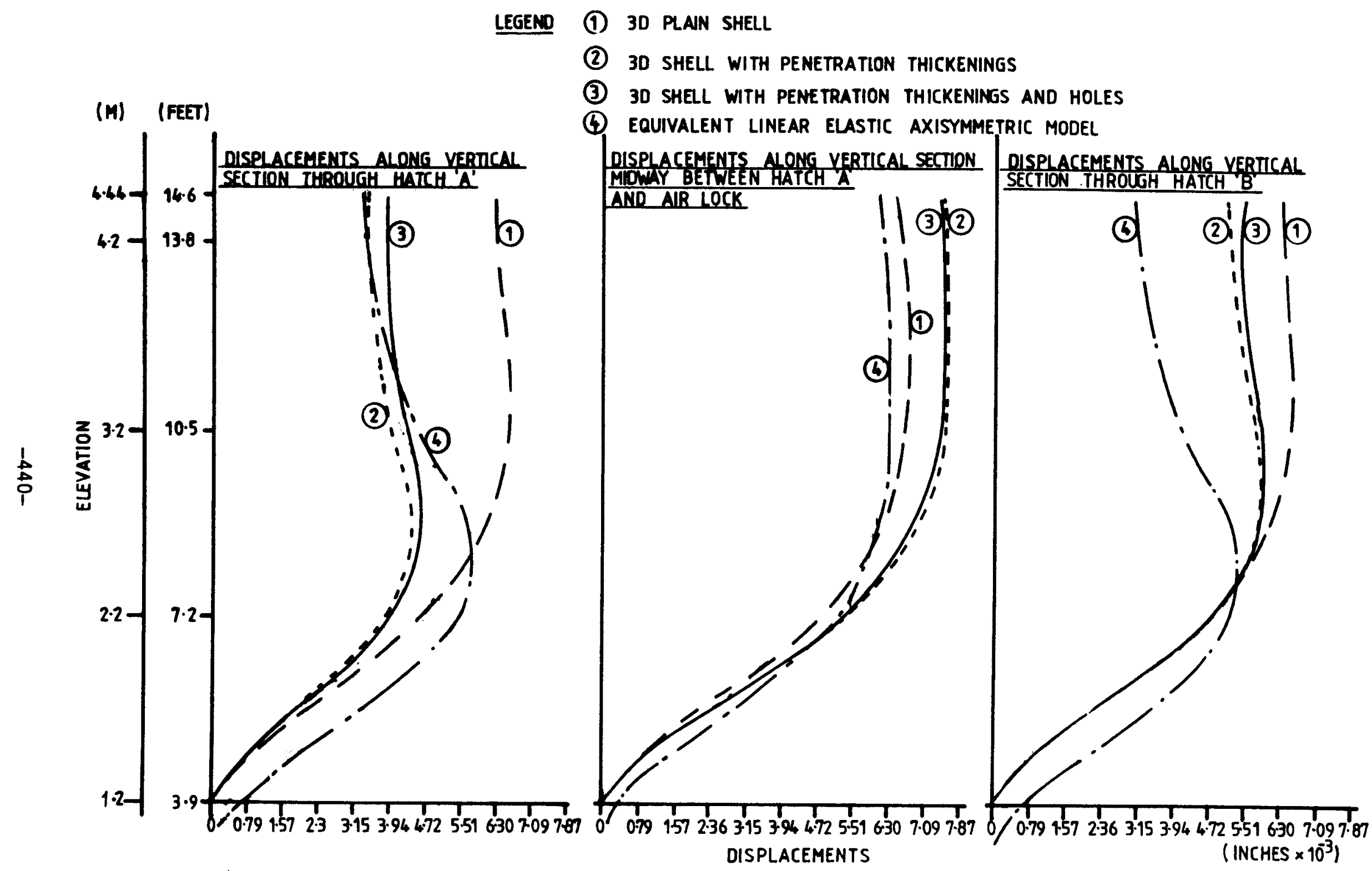

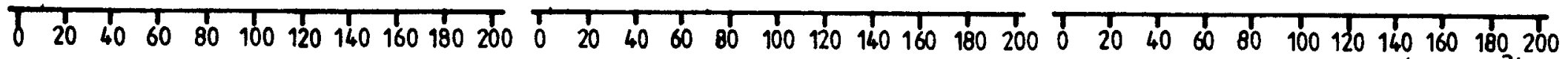

Figure 4.10.53 Comparison of 3-D Displacements with Linear Elastic $\left(M M \times 1 \sigma^{-3}\right)$ Axisymetric Displacements 


\section{CLOSURE}

Pretest analyses for a 1:6-scale reinforced concrete containment model have been described. The analyses were conducted by ten different organizations in the United States and Europe in a round-robin format. The "best estimate" predictions for the failure pressure varied from 116 to 190 psi $(0.800$ to $1.310 \mathrm{MPa})$. Most of the analysts considered the cylinder basemat junction to be the most probable failure location; however, there were wide differences in the mechanism limiting performance. Failure at the basemat cylinder junction was ascribed to liner tearing, shear failure, flexural failure, and crushing. Other limit states that the analysts thought could be realized during testing include (1) the onset of rapidly increasing displacements, (2) liner tearing at penetrations, welds, and other discontinuities, (3) reaching the ultimate strength of splices in the hoop rebar, and (4) reaching the ultimate strength of the basemat rebar. Standard plots, which are presented in Appendix $A$, were generated to facilitate comparisons of the different analyses.

The scale model will be pressurized with nitrogen gas to failure in the spring of 1987 . Over 1000 channels of data will be recorded at discrete pressure intervals. This data will be used to assess the pretest predictions and the analytical methods upon which the predictions are based. A report describing the comparison of analytical and experimental results and evaluations of analytical methods will be published after the test, perhaps in late 1987 or early 1988. 


\section{REFERENCES}

[2.1] VON RIESEMANN, W. A., et al., "U.S. NRC Containment Integrity Programs", Proceedings 8 th Int1. Conf. on Structural Mechanics in Reactor Technology, Brussels, Belgium, August 19-23, 1985, Paper $\mathrm{J} 1 / 2$.

[2.2] HORSCHEL, D. S., "The Design, Fabrication, Testing, and Analyses of Four 1:32-Scale Steel Containment Models", NUREG/CR-3902, SAND842153, Sandia National Laboratories, Albuquerque, NM, to be publ ished.

[2.3] REESE, R. T. and HORSCHEL, D. S., "Design and Fabrication of a 1:8Scale Steel Containment Model", NUREG/CR-3647, SAND84-0048, Sandia National Laboratories, Albuquerque, NM, February 1985.

[2.4] KOENIG, L. N., "Experimental Results for a 1:8-Scale Steel Model Nuclear Power Plant Containment Pressurized to Failure", NUREG/CR4216, SAND85-0790, Sandia National Laboratories, Albuquerque, NM, December 1986.

[2.5] CLAUSS, D. B., "Comparison of Analytical Predictions and Experimental Results for a 1:8-Scale Steel Containment Model Pressurized to Failure", NUREG/CR-4209, SAND85-0679, Sandia National Laboratories, Albuquerque, NM, July 1985.

[2.6] CLAUSS, D. B., "Failure Mechanisms of LWR Steel Containment Buildings Subject to Severe Accident Loadings", Proc. of the Third Workshop on Containment Integrity, NUREG/CP-0076, SAND86-0618, Sandia National Laboratories, Albuquerque, NM, August 1986, pp. 495-510.

[2.7] KOENIG, L. N., "Leakage Potential Through Mechanical Penetrations in a Severe Accident Environment", Proc. of the Third Workshop on Containment Integrity, NUREG/CP-0076, SAND86-0618, Sandia National Laboratories, Albuquerque, NM, August 1986, pp. 557-568.

[2.8] KECK, J. D., "Leak Behavior Through EPAs Under Severe Accident Conditions", Proc. of the Third Workshop on Containment Integrity, NUREG/CP-0076, SAND86-0618, Sandia National Laboratories, Albuquerque, NM, August 1986, pp. 569-580.

[2.9] STEELE, R., Jr., and WATKINS, J. C., "Containment Vent and Purge Valve Test Program Final Report", NUREG/CR-4141, EG\&G Idaho, Inc., Idaho Falls, Idaho, September 1985.

[2.10] CLAUSS, D. B., "An Evaluation of the Leakage Potential of a Personnel Airlock Subject to Severe Accident Loads", Proc. 9th Int]. Conf. on Structural Mechanics in Reactor Technology, Lausanne, Switzerland, August 17-21, 1987, Paper J5/3

[2.11] ADAMS, C. R., "GRAFAID Code User Manual Version 2.0", SAND84-1725, Sandia National Laboratories, Albuquerque, NM, September 1985. 
[3.1] UCCIFERRO, J. J., and HORSCHEL, D. S., "Design and Construction of a Large Reinforced Concrete Containment Model to be Tested to Failure", Proc. 9th Intl. Conf. on Structural Mechanics in Reactor Technology, Lausanne, Switzerland, August 17-21, 1987, Paper J.

[3.2] HORSCHEL, D. S., "Construction of a 1/6-Scale concrete containment Model", proposed for publication in Nuclear Engineering and Design.

[3.3] United Engineers and Constructors, Inc., "1:6 Scale LWR Reinforced Concrete Containment Model for Sandia National Laboratories", Construction Drawings, Drawing Nos. 7847-F-1001 through 7847-F1502, Revised 10/14/85.

[3.4] Chicago Bridge and Iron Company, "264" 0.D. x 22'-0" Length Containment Liner Model for United Engineers and Constr. for Sandia National Laboratories", Construction Drawings, Drawing Nos. 8530711 through 853071-32, Revised 11/21/85.

[3.5] HORSCHEL, D. S., "Design, Construction, and Instrumenting of a $1: 6-$ Scale Reinforced Concrete Containment Building", Sandia National Laboratories, Albuquerque, NM, to be published.

[3.6] "Soil Report", Project Sandia LWR Reinforced Concrete Containment Model, Project NO. $3245 \mathrm{~J} \mathrm{066,} \mathrm{Western} \mathrm{Technologies,} \mathrm{Inc.,}$ Albuquerque, NM, October 1985.

[4.1.1] HIBBITT, H. D., et al., ABAQUS manuals, version 4.5, September 1984 .

[4.1.2] CHEN, A. C. and CHEN, W. F., "Constitutive Relations for Concrete", $\mathrm{J}$. of the Engineering Mechanics Division, ASCE, August 1975, pp. 465-481.

[4.1.3] HORSCHEL, D. S. AND JUNG, J., "Construction and Analys is of a 1/6th-Scale Concrete Containment Model", Proc. of the Third Workshop on Containment Integrity, NUREG/CP-0076, SAND86-0618, Sandia National Laboratories, Albuquerque, NM, August 1986, pp. 407-428.

[4.1.4] AOYAGI, Y. and YAMADA, K., "An Experimental Approach to the Design of Network Reinforcement Against In-Plane Shear in Reinforced Concrete Containments", Proc. 5th Intl. Conf. on Structural Mechanics in Reactor Technology, August 1979, paper J4/7.

[4.1.5] State of the Art Report on Finite Element Analysis of Reinforced Concrete, Task Committee on Finite Element Analysis of Reinforced Concrete Structures, A. H. Nilson, Chairman, ASCE, 1982.

[4.1.6] KRIEG, R. D. and KRIEG, D. B., "Accuracies of Numerical Solution Methods for the Elastic-Perfectly Plastic Model", J. of Pressure Vessel and Piping, Vol. 99, November 1977, pp. 510-515.

[4.1.7] ACI Committee 224, "Cracking of Concrete Members in Direct Tension", ACI Journal, Jan.-Feb. 1986, pp. 3-13. 
[4.2.1] MARCHERTAS, A. H., and KULAK, R. F., "Numerical Modeling of Concrete Under Thermal Loads", Nuclear Engineering and Design, Vol. 68 , No. 2, 1981 , pp. 225-236.

[4.2.2] MARCHERTAS, A. H., and KULAK, R. F., "A Coupled Heat Conduction and Thermal Stress Formulation Using Explicit Integration", ANL-82-47, Argonne National Laboratory, Argonne, IL, June 1982.

[4.2.3] MARCHERTAS, A. H., and KULAK, R. F., "Response Simulation of Concrete Structures to High Temperatures", Proc. of the Workshop on Containment Integrity, Vol. 1, NUREG/CP-0033, SAND82-1659, Sandia National Laboratories, Albuquerque, NM, October 1982, pp. 193-212.

[4.2.4] TAKAHASHI, Y., "Elastic-Plastic Constitutive Modeling of Concrete", ANL-83-23, Argonne National Laboratory, Argonne, IL, March 1983.

[4.2.5] MARCHERTAS, A. H., "Thermo-Mechanical Analysis of Concrete in LMRBR Programs", Nuclear Engineering and Design, Vol. 82, No. 1, 1984, pp. 47-62.

[4.2.6] KULAK, R. F., "A Finite Element Formulation for Fluid-Structure Interaction in Three-Dimensional Space", J. of Pressure Vessel Technology, Vol. 103, No. 2, 1981, pp. 183-190.

[4.2.7] KULAK, R. F., "A Finite Element Quasi-Eulerian Method for ThreeDimensional Fluid-Structure Interactions", Computers and Structures, Vol. 18, No. 2, 1984, pp. 319-332.

[4.2.8] KULAK, R. F., "Three-Dimensional Fluid-Structure Coupling in Transient Analysis", Computers and Structures, Vol. 21, No. 3, 1985, pp. 529-542.

[4.2.9] HSIEH, S. S., TING, E. C., and CHEN, W. F., "A Plastic-Fracture Model for Concrete", Int. J. Solids Structures, Vol. 18, No. 3, 1982 , pp. 181-197.

[4.2.10] BAZANT, Z. P., and OH, B. H., "Crack Band Theory for Fracture of Concrete", Materiaux et Constructions, Vol. 16, No. 93, pp. 155177.

[4.2.11] BELYTSCHKO, T., LIN, J. I., and TSAY, C-S., "Explicit Algorithms

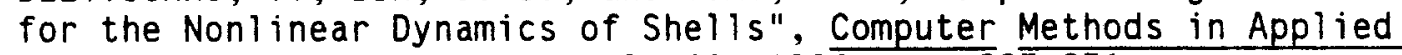

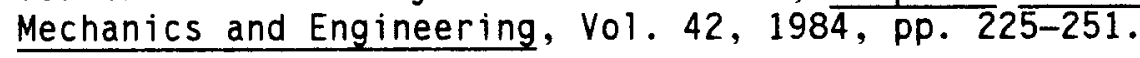

[4.3.1] DUNHAM, R. S., et al., "Methods for Ultimate Load Analysis of Concrete Containments", ANATECH Interim Report to EPRI, EPRI NP4046, RP2172-1, Electric Power Research Institute, Palo Alto, CA, June 1985 .

[4.3.2] DAMERON, R. A., et al., "Methods for Ultimate Load Analysis of Concrete Containments", ANATECH Interim Report to EPRI, Electric Power Research Institute, Palo Alto, CA, to be published by EPRI as proprietary report. 
[4.3.3] HIBBITT, H. D., "ABAQUS-EPGEN - A General Purpose Finite Element Code with Emphasis on Nonlinear Applications", Nuclear Engineering and Design, Vol. $77,1984, \mathrm{pp} .271-298$.

[4.3.4] RASHID, Y. R. and DUNHAM, R. S., "Three-Dimensional Constitutive Model for the Analysis of Concrete Structures", ANATECH Report, ANA-86-0052, July 1986.

[4.3.5] DUNHAM, R. S., RASHID, Y. R., and YUAN, K. A., "Evaluation of Calculational and Material Models for Concrete Containment Structures", Nuclear Engineering and Design, Vol. 90, 1985, pp. 193-208.

[4.3.6] United Engineers, "1:6 Scale LWR Reinforced Concrete Containment Model for Sandia National Laboratories", Construction Drawings, Drawing Nos. 7847-F-1001 through 7847-F-1502, Revised 10/14/85.

[4.3.7] DAMERON, R. A., DUNHAM, R. S., and RASHID, Y. R., "Failure Characteristics of Pipe Penetrations in Concrete Containment", ANATECH Preliminary Report to EPRI ANA-86-0048, Electric Power Research Institute, Palo Alto, CA, Project No. RP2172-1, March 1986.

[4.3.8] MEINHEIT, D. F., et al., "Support Tests for Sandia National Laboratories Design of LWR Reinforced Concrete Containment Model", July 30, 1985 (1:6-Scale Model Welded Stud Test Data provided through private communication with D. S. Horschel, Sandia National Laboratories, April 1986).

[4.3.9] KING, R. T., CANONICO, D. A., and BRINKMAN, C. R., "Elevated Temperature Weldment Behavior as Related to Nuclear Design Criteria", Welding Research Supplement, August 1975, pp. 265s-275s.

[4.3.10] CLAUSS, D. B., Sandia National Laboratories, Private Cormunication to H. T. Tang, EPRI, October 1986.

[4.3.11] SCHULTZ, D. M., et al., "Specimen 3.3 Preliminary Test Results", Private Communication to H. T. Tang, EPRI, October 7, 1986.

[4.4.1] CARRETERO, L., and COMBESCURE A., "Un modele non lineaire de comportement du beton", Report DEMT/SMTS/BAMS/79-034, 1979.

[4.4.2] JAMET, Ph., "Essais triaxiaus sur micro-beton", Technical Note STA/LBR-407, 1983.

[4.4.3] JAMET, Ph., et al., "Perforation of a concrete slab by a missile: finite element approach", Proc. 7th Intl. Conf. on Structural Mechanics in Reactor Technology, Chicago, USA, August 22-25, 1983, Paper $\mathrm{J} 7 / 6$.

[4.4.4] JAMET, Ph., et al., "Calculations of concrete containment tight loss: studies of a reinforced concrete slab with non-uniform thickness", Proc. 7th Intl. Conf. on Structural Mechanics in Reactor Technology, Chicago, USA, August 22-25, 1983, Paper J5/7. 
[4.4.5] NAHAS, G., "Calcul a la ruine des structures en beton arme", Thesis - University of Paris VI (1986).

[4.4.6] ZIENKIEWICZ, 0. C., The finite element method, 3rd Edition MacGraw Hill Book Company.

[4.4.7] Western Technologies Inc., Letter dated October 14th, 1985.

[4.5.1] PAFEC Ltd; Data Preparation User Manual level 6.1, Pafec Limited Strelley Hall, Nottingham, 1984.

[4.5.2] OVE ARUP \& PARTNERS, "Sandia Scale Model Containment Pre-test Analysis", Ref. No. IE86 - 39, July 1986.

[4.5.3] PARK \& PAULEY, Reinforced Concrete Structures, John Wiley \& Sons, 1975.

[4.5.4] CHEN, W. F., Plasticity in Reinforced Concrete, McGraw-Hill Book Company, 1982 .

[4.5.5] GOODSIR, W. J., "The Design of Coupled Frame-Wall Structures for Seismic Actions", PhD Thesis, University of Canterbury, New Zeal and, 1985.

[4.5.6] OVE ARUP \& PARTNERS, "PWR Containment Structure Civil Engineer Support Services, Reliability of Containment Shear Strength", Ref. No. IE 85-165, Dec. 1985.

[4.5.7] CURRIE, T., STUBBS, R. J., CRIPPS, J., MILES, J., "Some Studies of the Response of Pre-stressed Containments for PWR to Earthquakes, Gas Cloud Explosions and Aircraft Crash", Nuclear Engineering and Design, Vol. 96, 1986, pp. 173-182.

[4.6.1] BATHE, K.J., "ADINA. A Finite Element Program for Automatic Dynamic Incremental Nonlinear Analysis", Rev. Dec. 1978, Massachusetts Institute of Technology, Report 82448-1.

[4.6.2] BATHE, K. J., and RAMASWAMY, S., "On Three-Dimensional Nonl inear Analysis of Concrete Structures", Nuclear Engineering and Design, Vol. 52, 1979, pp. 385-409.

[4.6.3] ORSINI, G., "GRAPH78", ENEA-DISP Technical Report (to be published).

[4.7.1] HIBBITT, H. D., KARLSSON, B., and SORENSSEN, P., "ABAQUS User's Manual Version 4.5", HKS, Inc., July 1985.

[4.7.2] GILBERT, R. I., and WARNER, R. F., "Tension Stiffening in Reinforced Concrete Slabs", Proc. of Am. Soc. Civ. Eng., Jnl Strut., Div. $104,1978, \mathrm{p} 18 \overline{85}$.

[4.7.3] Private Communication from D. B. Clauss of Sandia National Laboratories to D. W. Phillips, SRD on $17 / 10 / 86$. 
[4.7.4] HORSCHEL, D. S. AND JUNG, J., "Construction and Analys is of a $1 / 6$ th-Scale Concrete Containment Model", Proc. of the Third Workshop on Containment Integrity, NUREG/CP-0076, SAND86-0618, Sandia National Laboratories, Albuquerque, NM, August 1986, pp. 407-428.

[4.7.5] HARROP, L. P., "Simple Calculations to Predict the Behavior of the Sandia $1 / 6$ Scale Containment Building", Safety and Reliability Directorate of the U.K.A.E.A., November 1986.

[4.8.1] "Soil Report", Project Sandia LWR Reinforced Concrete Containment Model, Project NO. $3245 \mathrm{~J}$ 066, Western Technologies, Inc., Albuquerque, NM, October 1985.

[4.8.2] BATHE, K. J., ADINA (Rev. 1984), Report AE 84-1, ADINA-Engineering, Inc., Watertown, Mass., December 1984.

[4.8.3] OTTOSEN, N. S., "A Failure Criterion for Concrete", J. Eng. Mec., Div. ASCE, Vol. 103, Em 4, August 1977.

[4.8.4] KOLMAR, W., and MEHLHORN, G., "Comparison of shear stiffness formulations for cracked reinforced concrete elements", Proc. of the Intl. Conf. on Computer-Aided Analys is and Design of Concrete Structures, Part I, Split, Yugoslavia, September 1984.

[4.8.5] EIBL, J., NEUROTH, V., and SCHLUETER, F.-H., Test Report, "Reinforced Concrete Elements Subjected to Uniaxial and biaxial Direct Tension", Institut fur Massivbau and Baustofftechnologie, Universtitat Karlsruhe, November 1986.

[4.8.6] PERDIKARIS, P. C., SAID HILMY and WHITE, R. N., "Extensional Stiffness of Precracked R/C Panels", J. Struc. Eng., Vol. 111, No. 3, March 1985.

[4.8.7] ASTM Specification A 516, ASME Boiler and Pressure Vessel Code, Section II, Material Specifications, Part A - Ferrous, 1986.

[4.8.8] ASME Specification SA 106, ASME Boiler and Pressure Vessel Code, Section II, Material Specifications, Part A - Ferrous, 1986.

[4.8.9] ASTM Specification A 36, ASME Boiler and Pressure Vessel Code, Section II, Material Specifications, Part A - Ferrous, 1986.

[4.8.10] BATHE, K. J. and CIMENTO, A. P., "Some Practical Procedures for the Solution of Nonlinear Finite Element Equations", Comp. Meth. Mech. Eng. 22, March 1979.

[4.8.11] Letter by Sandia to Analysts, dated October 17, 1986

[4.8.12] MANJIONE, M. J., "Elevated Temperature Mechanics of Metals", Preprints of the 4 th International Seminar on: Inelastic Analysis and Life Prediction in High Temperature Environment, 7 th SMiRT, August 1983. 
[4.9.1] SHARMA, S., REICH, M., and CHANG, T. Y., "Review of Current Analys is Methodology for Reinforced Concrete Structural

Evaluations", NUREG/CR-3284, Brookhaven National Laboratory, Upton, NY, April 1983.

[4.9.2] SHARMA, S., WANG, Y. K., and REICH, M., "Uitimate Pressure Capacity of Reinforced and Prestressed Concrete Containments", NUREG/CR4149, Brookhaven National Laboratory, Upton, NY, May 1985.

[4.9.3] BATHE, K. J., OZDEMIR, H., and WILSON, E. L., "Static and Dynamic Geometric and Material Nonlinear Analysis", Report No. UC SESM 744, University of California, Berkeley, CA, 1974.

[4.9.4] JUNG, J., "Response of Watts Bar, Main Yankee and Bellefonte Containments to Static Internal Pressurization", Proc. of ANS/ENS Int l. Meeting on LWR Severe Accident Evaluation, Cambridge, MA, September, 1983.

[4.9.5] CHEN, A.C.T. and CHEN, W. F., "Constitutive Relations for Concrete", J. of the Engineering Mechanics Division, ASCE, Vol. 101,1975, pp. 465-481.

[4.10.1] OTTOSEN, N. S., "Constitutive Model for Short Term Loading of Concrete", J. of the Engineering Mechanics Division, ASCE, February 1979 , pp. $1 \overline{27-141 .}$

[4.10.2] KOTSOUOS, M. D., "A Mathematical Description of the Strength Properties of Concrete Under Generalized Stress", Magazine of Concrete Research, Volume 31, No. 108, September 1979, pp. 151-158.

[4.10.3] BATHE, K. J., "ADINA Reference/User Manual", Massachusetts Institute of Technology, September 1981.

[4.10.4] PAFEC DATA PREPARATION USER MANUAL Level 6.1, PAFEC Ltd, Strelley Hall, Nottingham.

[4.10.5] BATHE, K. J., Finite Element Procedures in Engineering Analysis, Prentice Hall, 1982.

[4.10.6] GELDING, J.S., MISTRY, N. S., and WELCH, A. K., "Evaluation of Material Modeis for Reinforced Concrete Structures", Computer and Structures, Vol. 24, No. 2, 1986, pp 225-232.

[4.10.7] CHITNUYANONDH, L., et al., "Effective Tensile Stiffening in Prestressed Concrete Wal1 Segments", Proc. of the 5th Intl. Conf. on Structural Mechanics in Reactor Technology, Berlin, Germany, August 13-17, 1981, Paper J3/4.

[4.10.8] COMITE EURO-INTERNATIONAL DU BETON, "Concrete under multiaxial states of stress. Constitutive equations for practical design", Bulletin d'information, June 1983.

[4.10.9] ASCE/BATHE, Finite Elements in Reinforced Concrete, 1982. 
SNL requested each organization to provide data from their analytical results for standard plots, which were chosen to correspond with areas that were instrumented on the model. Each organization supplied SNL with a magnetic computer tape containing all or some of the standard plots (some of the requested plot data was outside of the scope of some of the organizations analyses). The data was read into a common data base at SNL and results were plotted using the GRAFAID program. Each organization was assigned a unique curve line type as follows:

\section{Curve Line Type}

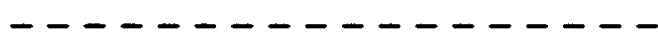

$-\cdot \cdot-\cdot \cdot-\cdot \cdot \cdot \cdot \cdot \cdot \cdot \cdot \cdot \cdot-$
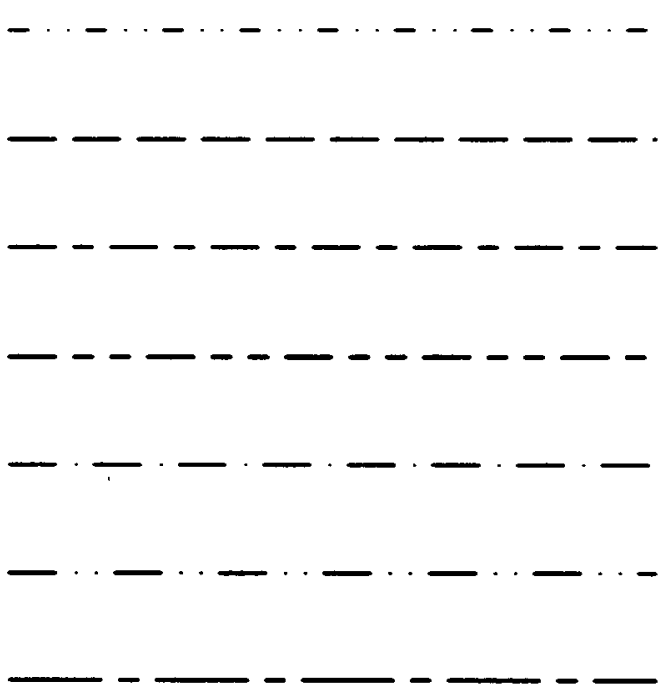

\section{Code Organization}

\section{ANL Argonne National Laboratory}

BNL Brookhaven National Laboratory

CEA Commisariat a L'Energie Atomique

CEGB Central Electricity Generating Board Comitato Nazionale per la ricerca e

ENEA per lo sviluppo dell'Energia Nucleare e delle Energie Alternative

EPRI Electric Power Research Institute

GRS Geselischaft fuer Reaktorsicherheit

NII HM Nuclear Installation Inspectorate

SNL Sandia National Laboratories

S\&RD U.K. Atomic Energy Authority, Safety and Reliability Directorate

Where possible, curves are also labeled with the organization code, although in many cases the results are so similar they are difficult to differentiate.

Generally, the pressure history plots were assigned a range of 0 to 200 ps $i$ (0 to $1.379 \mathrm{MPa}$ ). In some cases, smaller pressure ranges are al so plotted to show certain features of the response such as cracking or general yielding.

A list of the standard plots that were requested appears on the next three pages, followed by the plots themselves. The locations can be interpreted using the Figures and description of the model in Section 3.1. 


\section{Pressure History Plots}

\section{Curve}

No. Plot Quantity (ordinate or y-axis value) ${ }^{1} \quad$ Location $^{2}$

\section{Basemat:}

1 Vertical displacement (uplift)

relative to center of basemat

$$
\begin{aligned}
& z=2^{\prime}-0^{\prime \prime} \\
& r=10^{\prime}-9^{\prime \prime} \\
& \theta=85
\end{aligned}
$$

\begin{tabular}{|c|c|c|}
\hline 2 & Radial displacement of liner & $\begin{array}{l}z=2^{\prime}-2^{\prime \prime} \\
\theta=180\end{array}$ \\
\hline 3 & Radial displacement of liner & $\begin{array}{l}z=4^{\prime}-1 " \\
\theta=180\end{array}$ \\
\hline $\begin{array}{l}4 \\
5\end{array}$ & $\begin{array}{l}\text { Radial displacement of liner } \\
\text { Vertical displacement relative to cylinder base }\end{array}$ & $\begin{array}{l}z=6^{\prime}-0^{\prime \prime} \\
\theta=225\end{array}$ \\
\hline $\begin{array}{l}6 \\
7\end{array}$ & $\begin{array}{l}\text { Radial displacement of liner } \\
\text { Vertical displacement relative to cylinder base }\end{array}$ & $\begin{array}{l}z=11^{\prime}-0^{\prime \prime} \\
\theta=225\end{array}$ \\
\hline $\begin{array}{l}8 \\
9\end{array}$ & $\begin{array}{l}\text { Radial displacement of liner } \\
\text { Vertical displacement relative to cylinder base }\end{array}$ & $\begin{array}{l}z=18^{\prime}-0^{\prime \prime} \\
\theta=225\end{array}$ \\
\hline $\begin{array}{l}10 \\
11\end{array}$ & $\begin{array}{l}\text { Radial displacement of liner } \\
\text { Vertical displacement relative to cylinder base }\end{array}$ & $\begin{array}{l}z=24^{\prime}-0^{\prime \prime} \\
\theta=180\end{array}$ \\
\hline $\begin{array}{l}12 \\
13 \\
14\end{array}$ & $\begin{array}{l}\text { Maximum principal strain on inside liner surface } \\
\text { Axial strain in layer } 5 \text { meridional rebar } \\
\text { Axial strain in layer } 6 \text { hoop rebar }\end{array}$ & $\begin{array}{l}z=6^{\prime}-9^{\prime \prime} \\
\theta=45\end{array}$ \\
\hline $\begin{array}{l}15 \\
16 \\
17\end{array}$ & $\begin{array}{l}\text { Maximum principal strain on inside liner surface } \\
\text { Axial strain in layer } 5 \text { meridional rebar } \\
\text { Axial strain in layer } 6 \text { hoop rebar }\end{array}$ & $\begin{array}{l}z=13^{\prime}-9^{\prime \prime} \\
\theta=45\end{array}$ \\
\hline $\begin{array}{l}18 \\
19 \\
20 \\
21\end{array}$ & $\begin{array}{l}\text { Maximum principal strain on inside liner surface } \\
\text { Axial strain in layer } 5 \text { meridional rebar } \\
\text { Axial strain in layer } 6 \text { hoop rebar } \\
\text { Axial strain in layer } 8 \text { seismic rebar }\end{array}$ & $\begin{array}{l}z=20^{\prime}-0^{\prime \prime} \\
\theta=45\end{array}$ \\
\hline
\end{tabular}

Cyl inder:

1. Abscissa or $x$-axis value is pressure; range from $0-200$ psig (0-13.5 bar)

2. Locations are specified in cylindrical coordinates (base of cylinder is at elevation $\left.z=2^{\prime}-0^{\prime \prime}\right)$. Locations correspond to locations of instrumentation; although circumferential angle is given, in many cases axisymmetric response would be expected. 
Curve

No.

Plot Quantity (ordinate or y-axis value)

Location

22 Maximum principal strain on inside liner surface

23 Axial strain in layer 2 meridional rebar

24 Axial strain in layer 5 meridional rebar

$z=2^{\prime}-0.8^{\prime \prime}$

$\theta=90$

(just above knuckle)

25 Maximum principal strain on inside liner surface

$z=23^{\prime}-11^{\prime \prime}$

26 Axial strain in layer 2 meridional rebar

$\theta=90$

27 Axial strain in layer 5 meridional rebar

Dome :

Vertical displacement of 1 iner at dome apex

$\phi=90$

Vertical displacement of liner relative to springline Horizontal displacement of liner

$\phi=70$

Maximum principal strain on inside liner surface

$\theta=90$

Axial strain in layer 5 meridional rebar

$\theta=90$

Maximum principal strain on inside liner surface

$\phi=37$

Axial strain in layer 5 meridional rebar

\section{Penetrations:}

Horizontal displacement of inside edge of equipment

$z=14^{\prime}-7^{\prime \prime}$

hatch $B$ sleeve at midthickness (top)

$\theta=180$

Radial displacement of liner at $\theta=312.5$

$z=20^{\prime}-1.5^{\prime \prime}$

41 Maximum principal strain on inside liner surface

$z=13^{\prime}-0^{\prime \prime}$

Axial strain in layer 2 meridional rebar

$\theta=158$ 
II. Spatial Plots

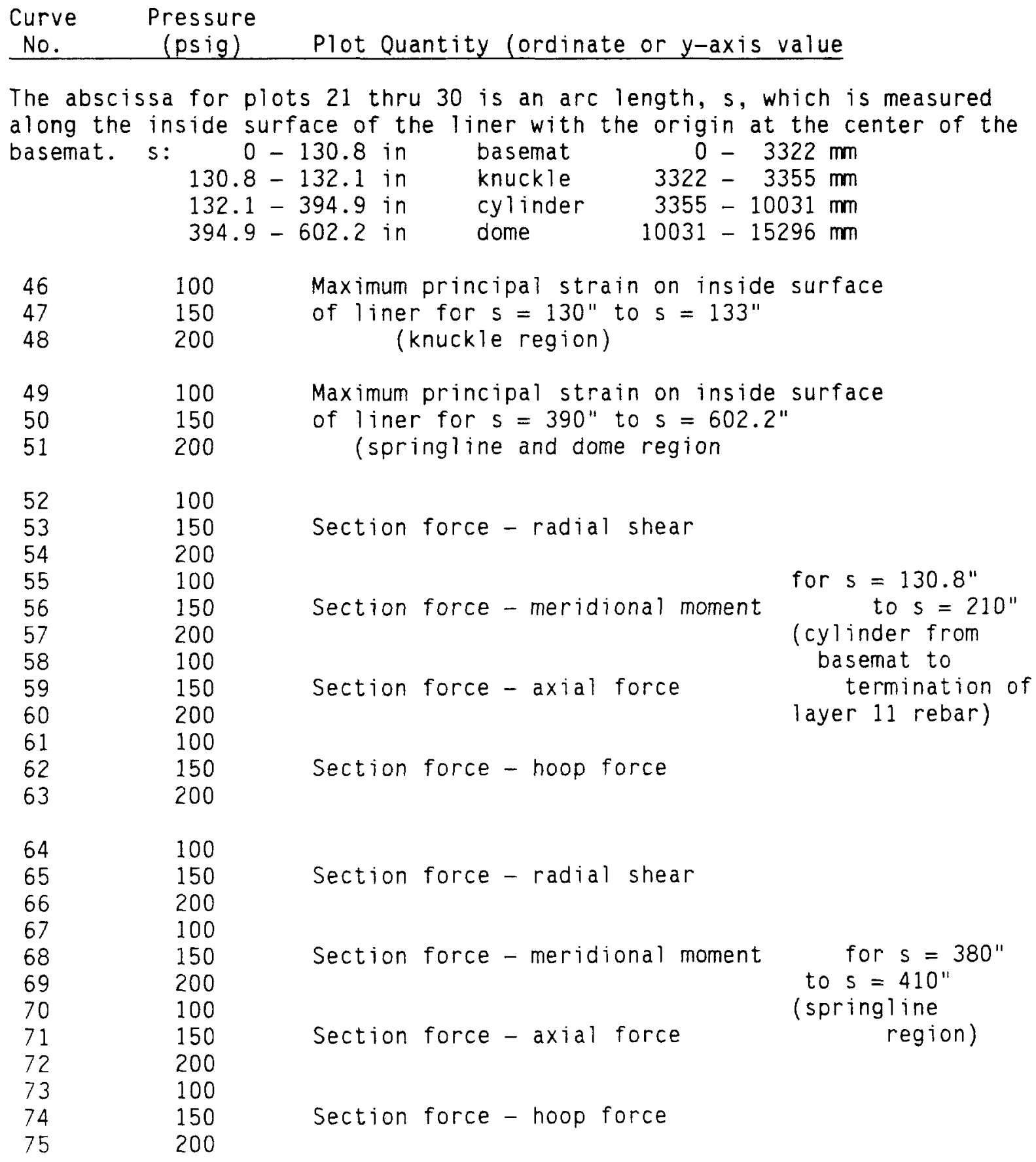

The abscissa is the circumferential angle $\theta$ for plots 31 and 32

$\begin{array}{ll}76 & 100 \\ 77 & 150 \\ 78 & 200 \\ 79 & 100 \\ 80 & 150 \\ 81 & 200\end{array}$

Maximum principal strain on inside liner surface at $z=13^{\prime}-0^{\prime \prime}$ for $\theta=135$ to $\theta=168$ (adjacent to equipment hatch $B$ )

Maximum principal strain on inside liner surface at $z=29^{\prime}-1.5^{\prime \prime}$ for $\theta=270$ to $\theta=312.5$ (adjacent to constrained pipe) 

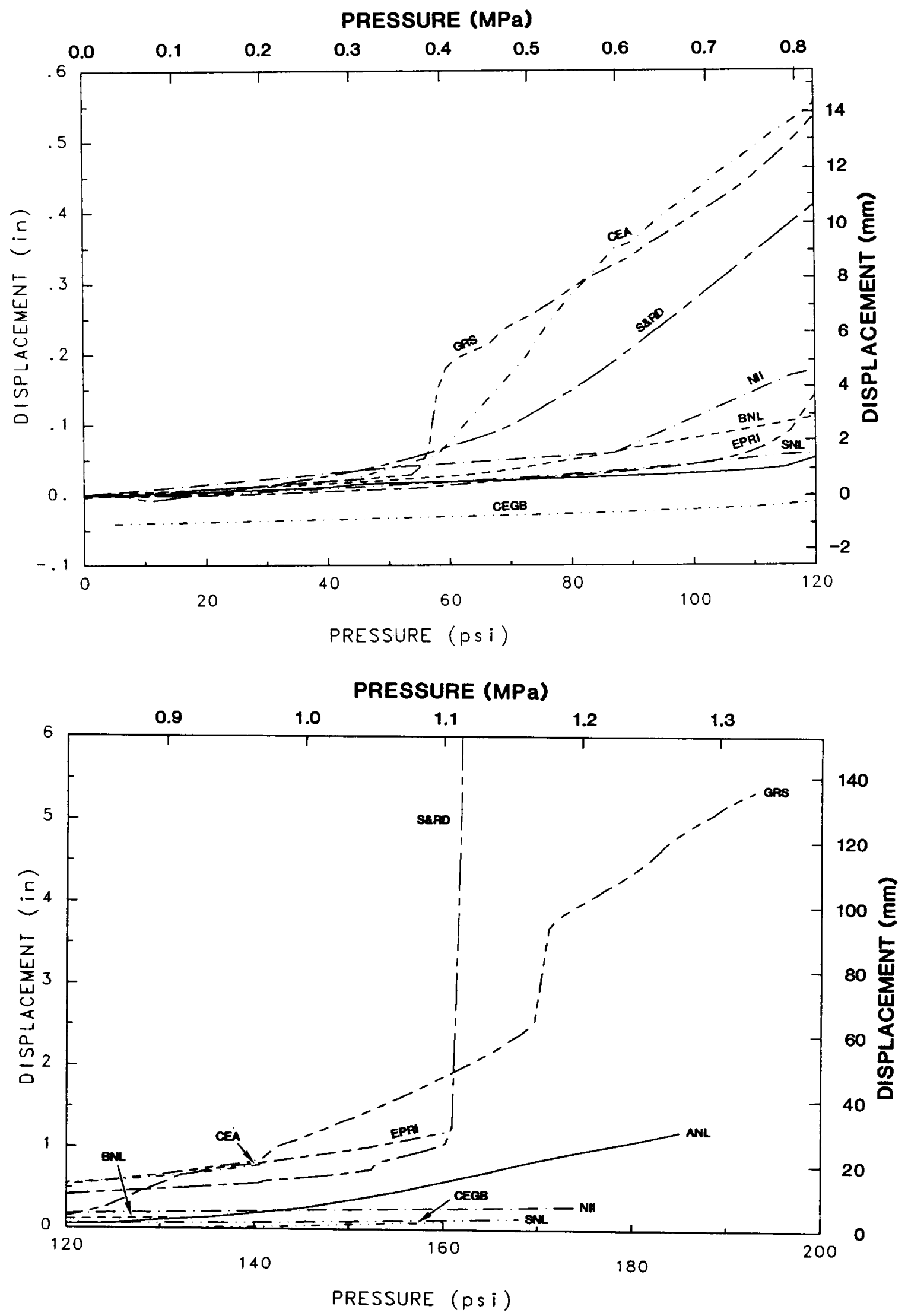

Figure A.1 Vertical displacement (uplift) relative to center of basemat EL $2^{\prime}-0^{\prime \prime}, r=10^{\prime}-9^{\prime \prime}, \theta=85$ 

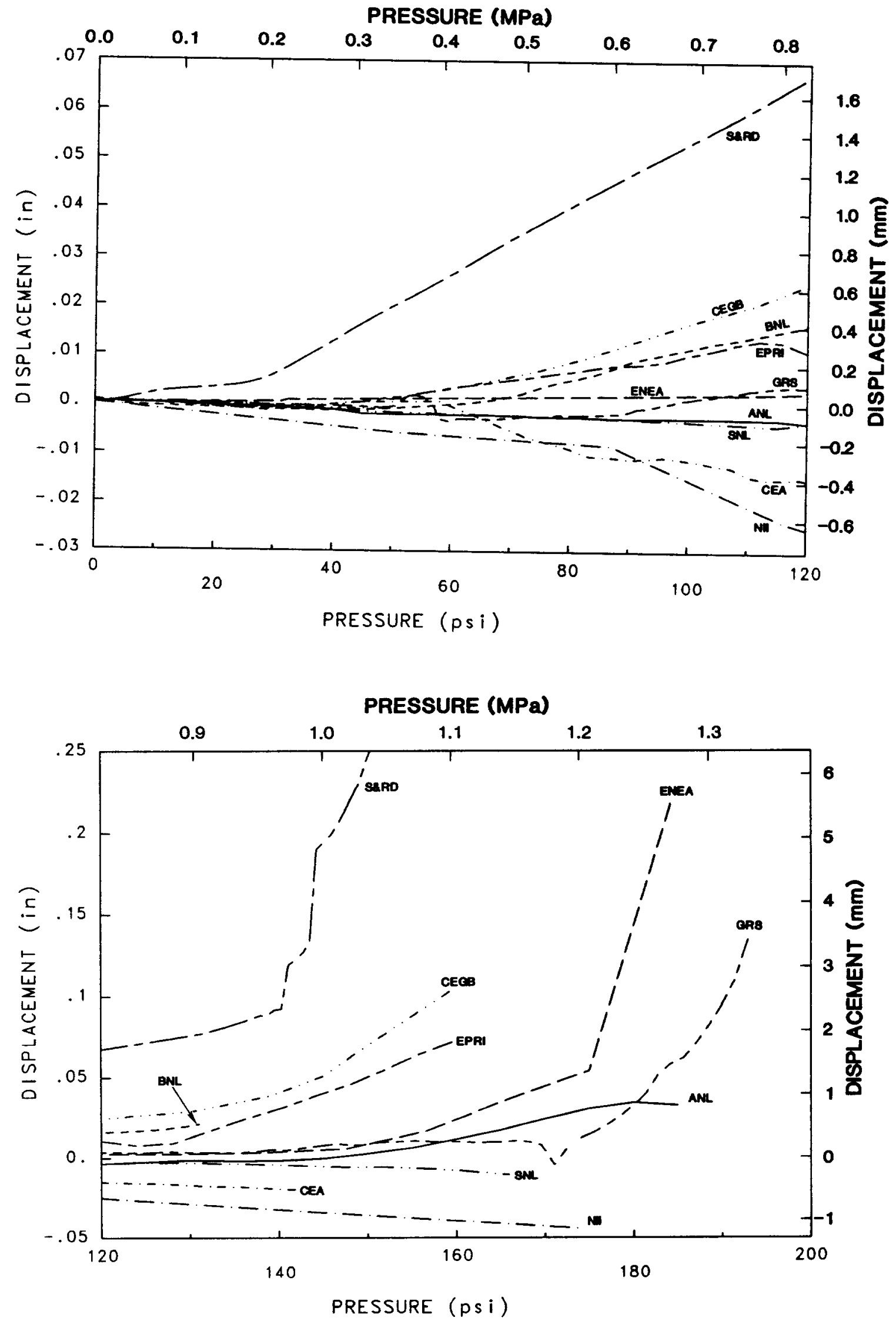

Figure A.2 Radial displacement of liner at EL $2^{\prime}-2^{\prime \prime}, \theta=180$ 

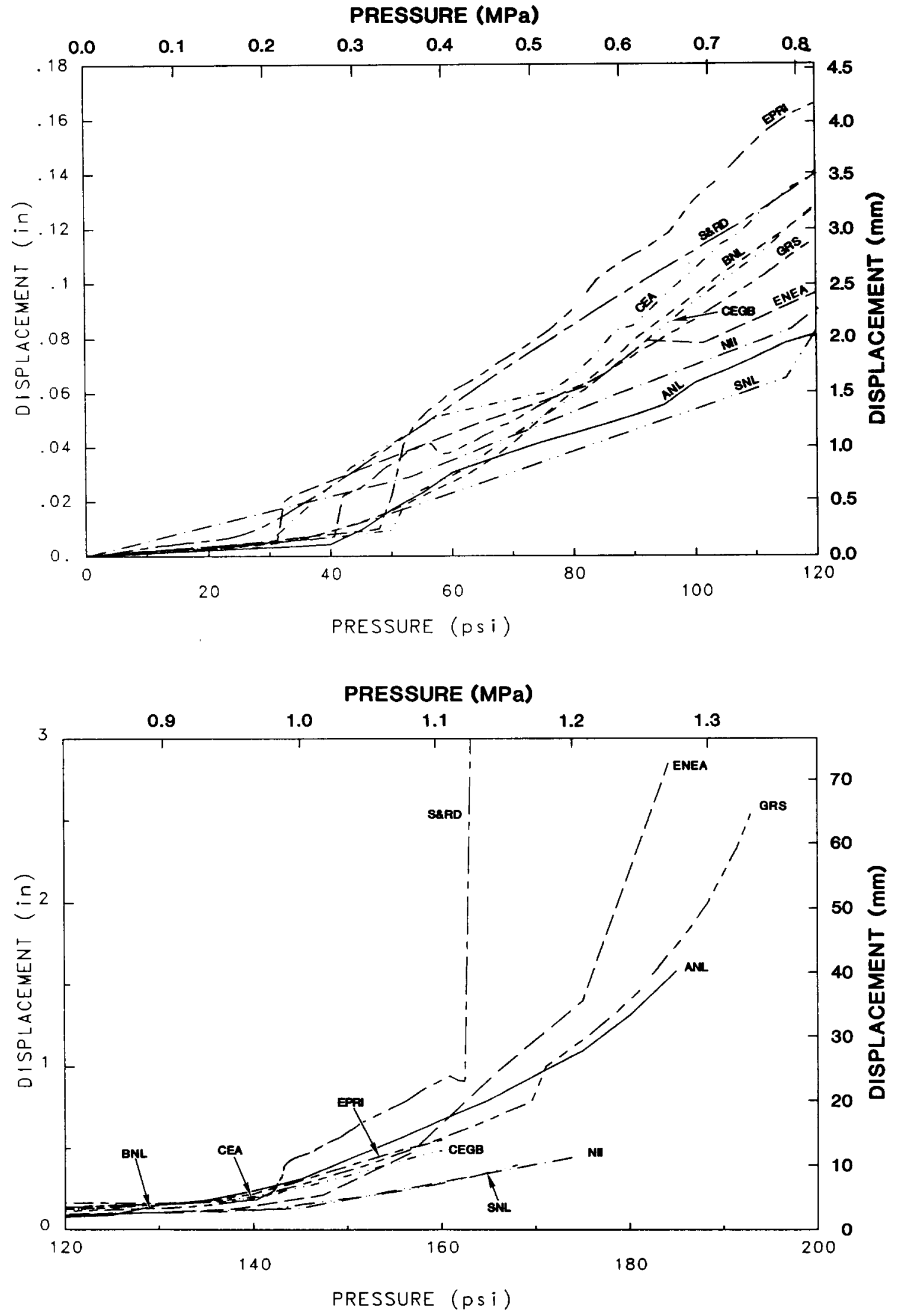

Figure A.3 Radial displacement of liner at EL $4^{\prime}-1{ }^{\prime \prime}, \theta=180$ 
PRESSURE (MPa)
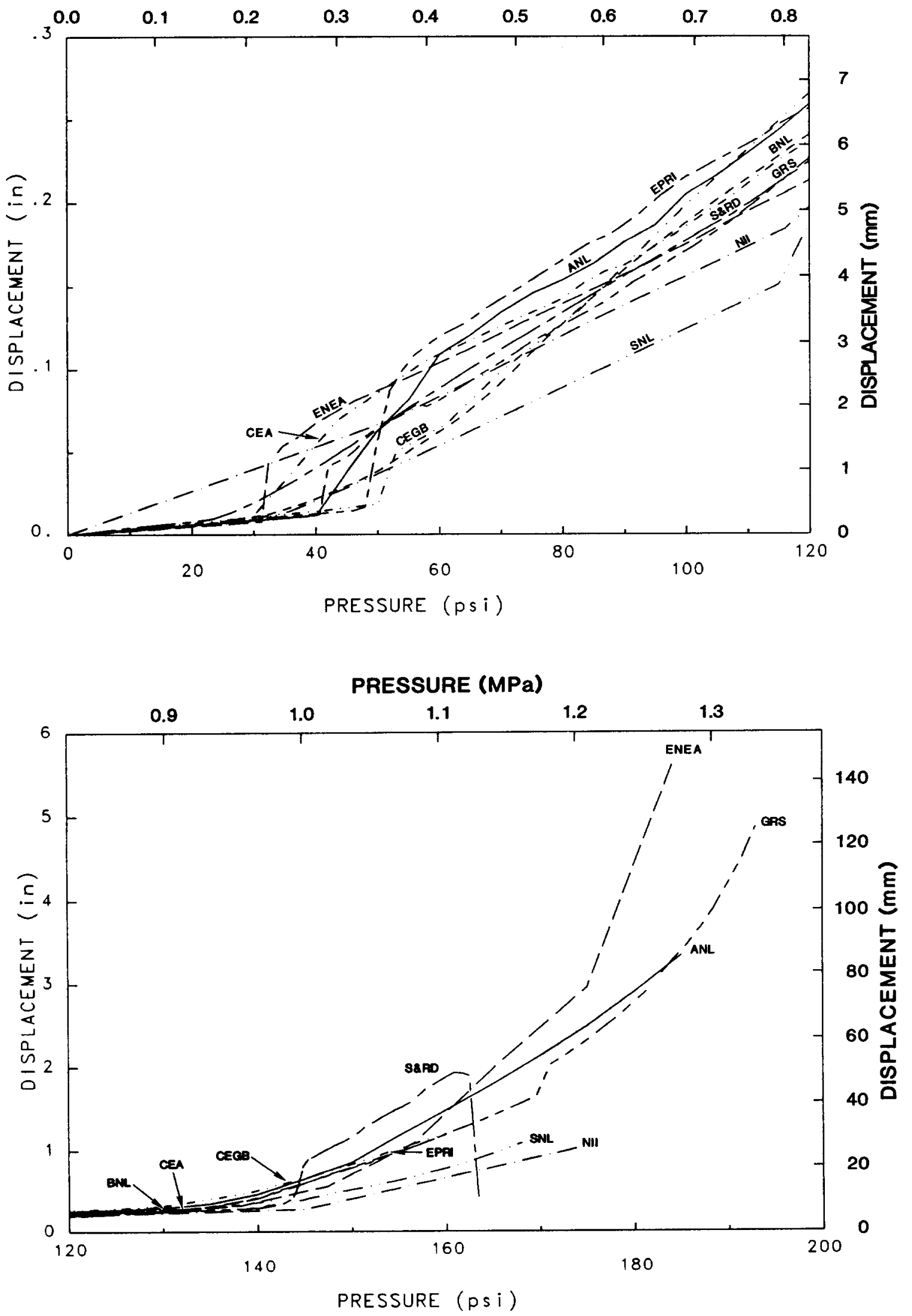

Figure A.4 Radial displacement of liner at EL $6^{\prime}-0^{\prime \prime}, \theta=225$ 

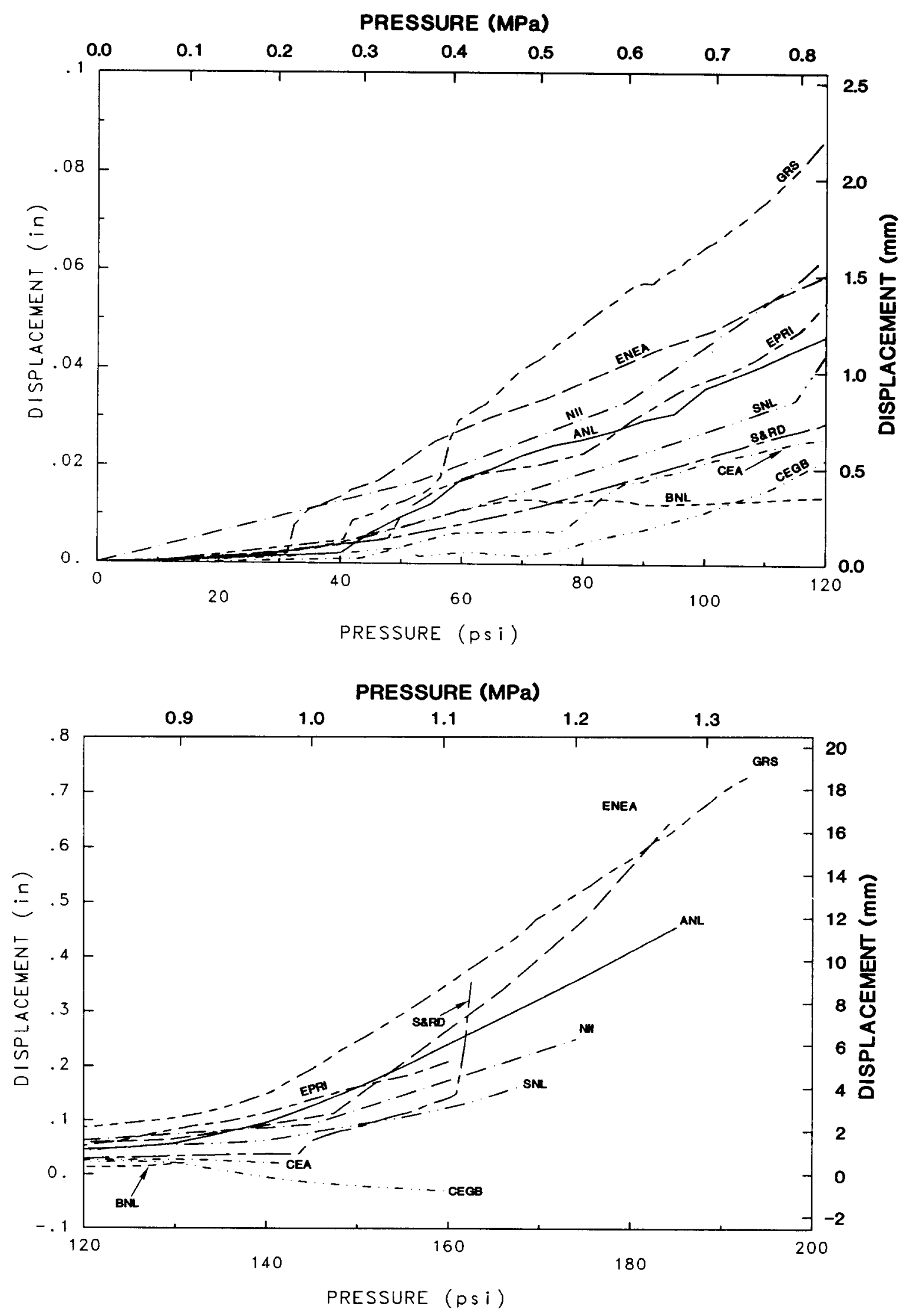

Figure A.5

Vertical displacement relative to cylinder base at $E L 6^{\prime}-0^{\prime \prime}, \theta=225$ 

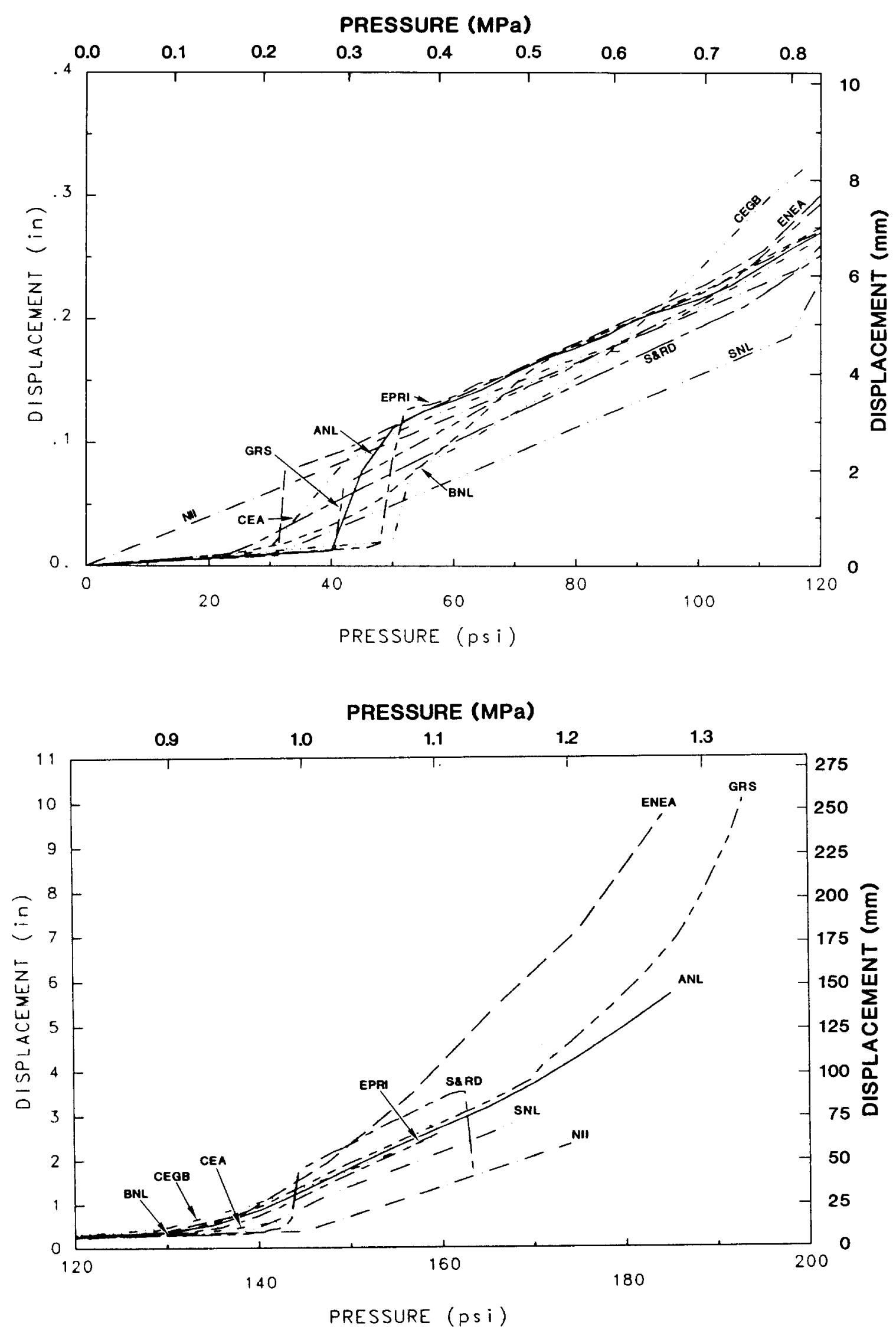

Figure A.6 Radial displacement of liner at EL 11'-0", $\theta=225$ 

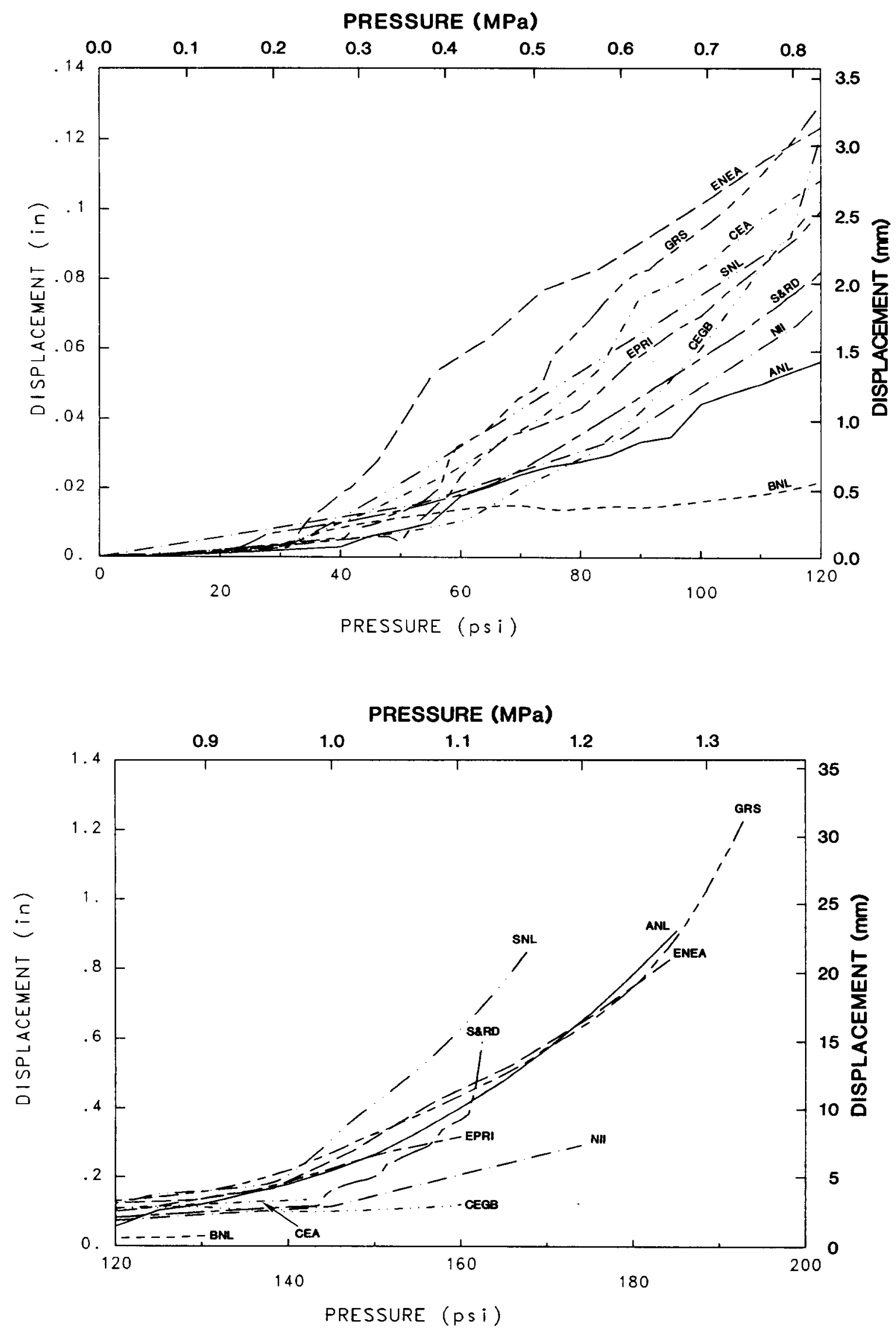

Figure $A .7$

Vertical displacement relative to cylinder base at EL $11^{\prime}-0^{\prime \prime}, \theta=225$ 
PRESSURE (MPa)
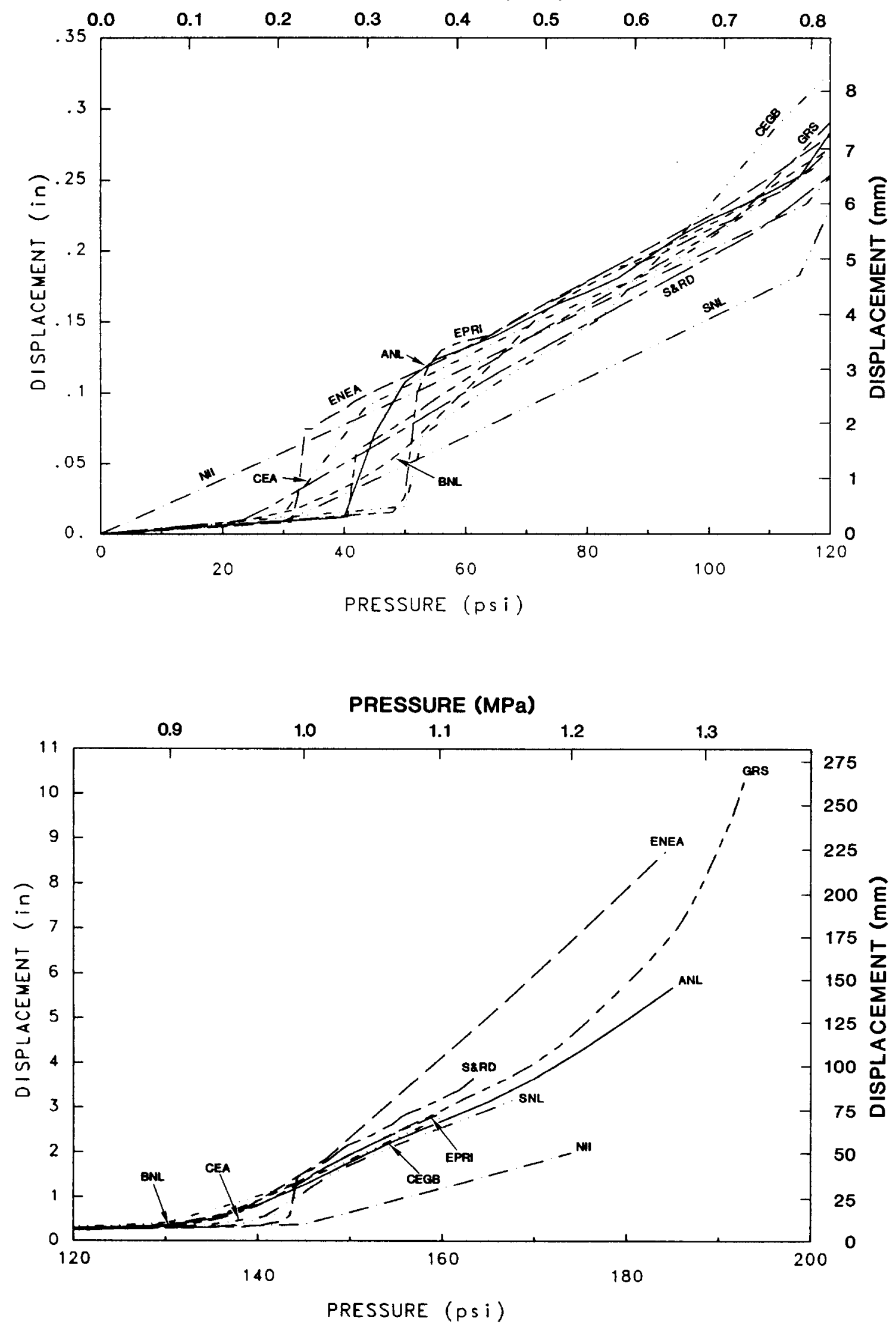

Figure A.8 Radial displacement of liner at EL $18^{\prime}-0^{\prime \prime}, \theta=225$ 


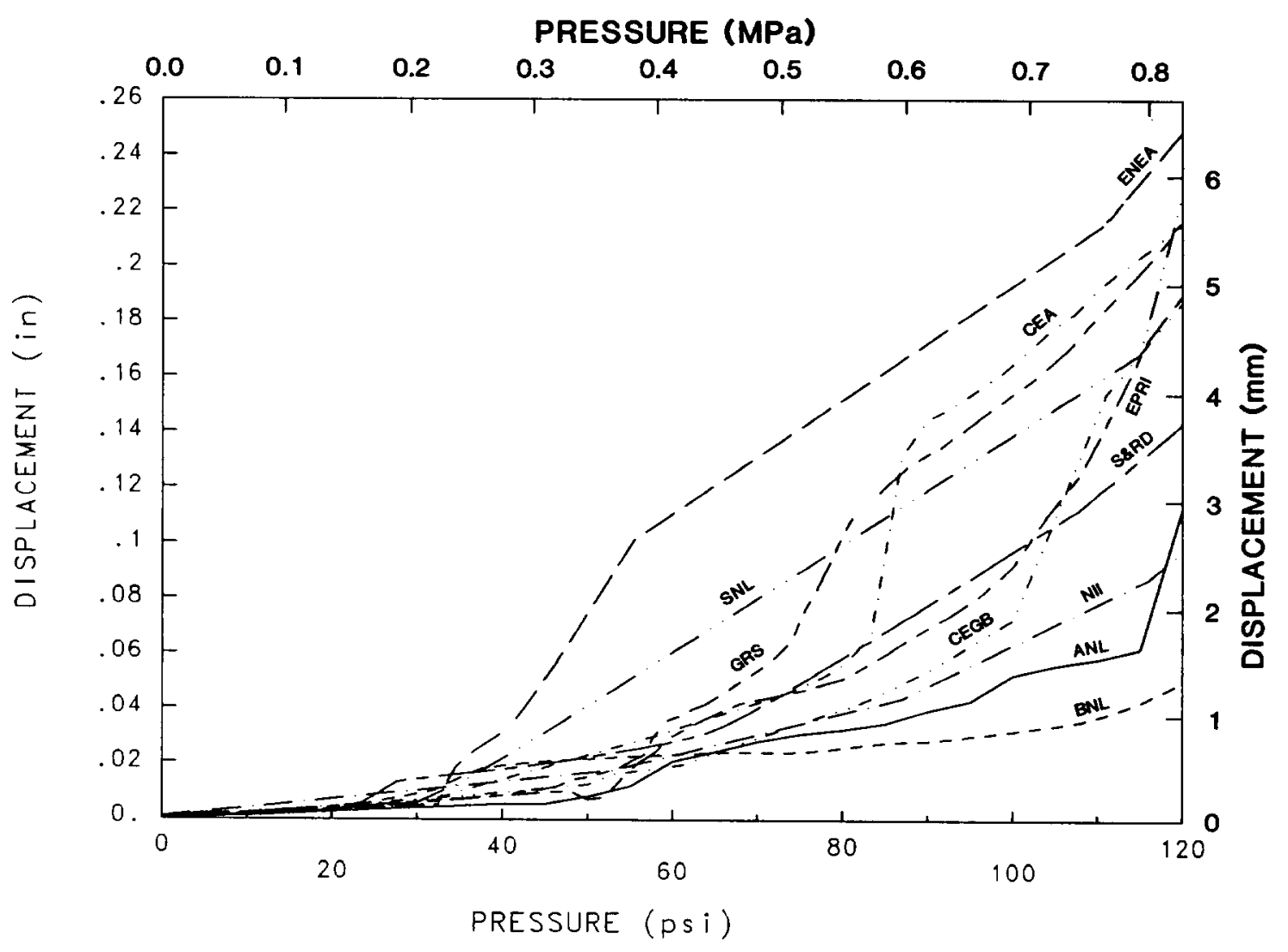

PRESSURE (MPa)

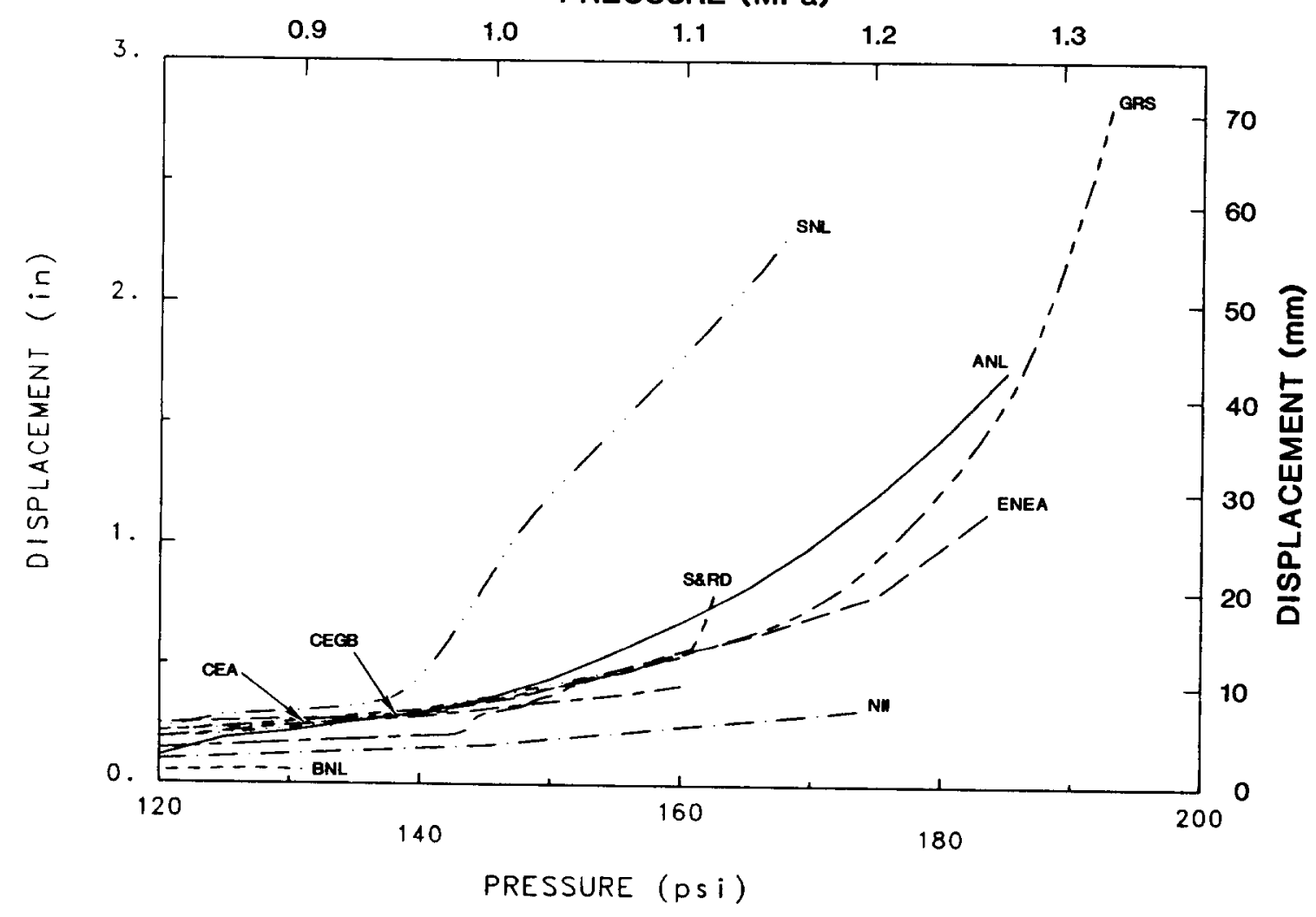

Figure A.9

Vertical displacement relative to cylinder base at EL 18'-0", $\theta=225$ 

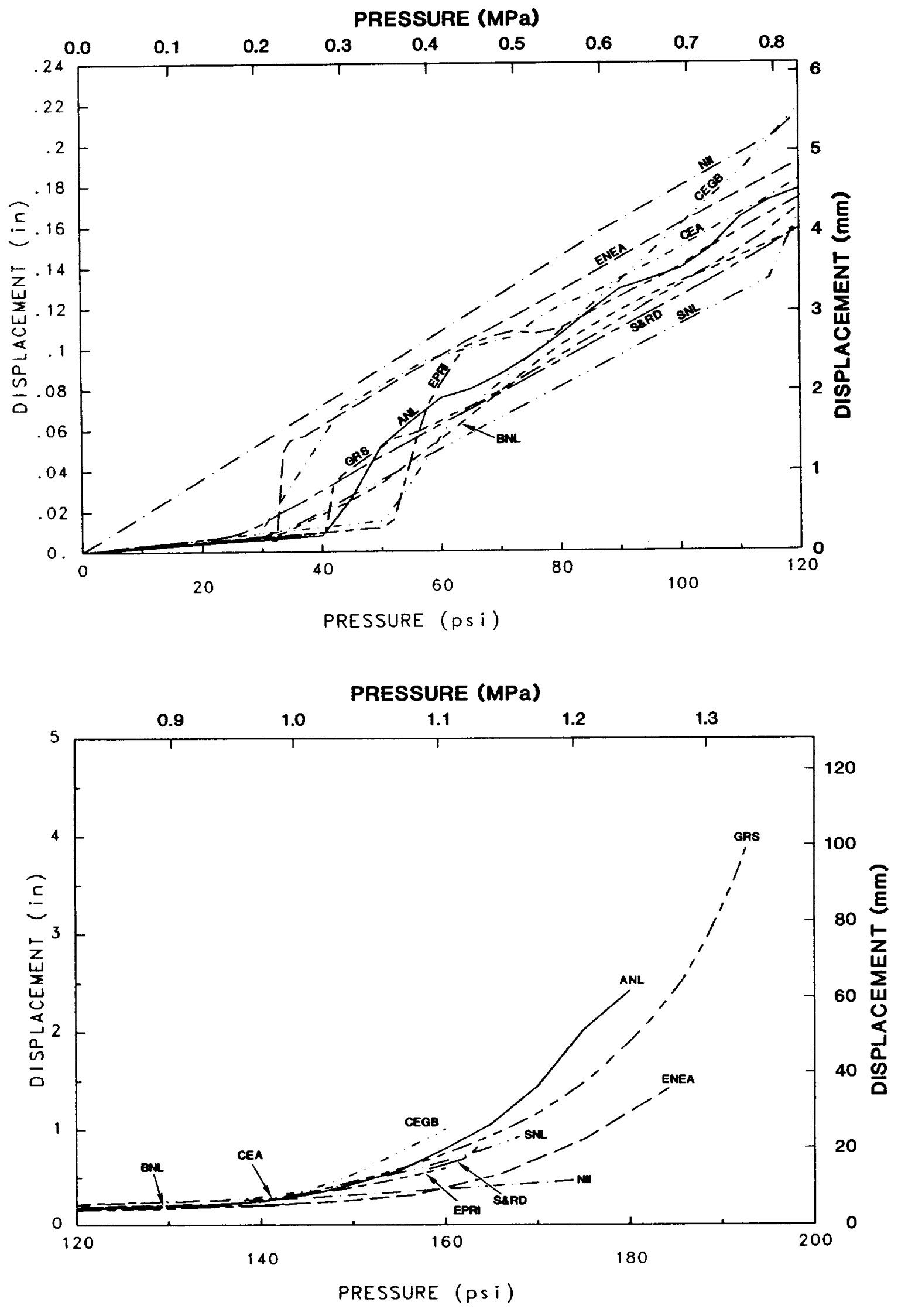

Figure A.10 Radial displacement of liner at EL $24^{\prime}-0^{\prime \prime}, \theta=180$ 

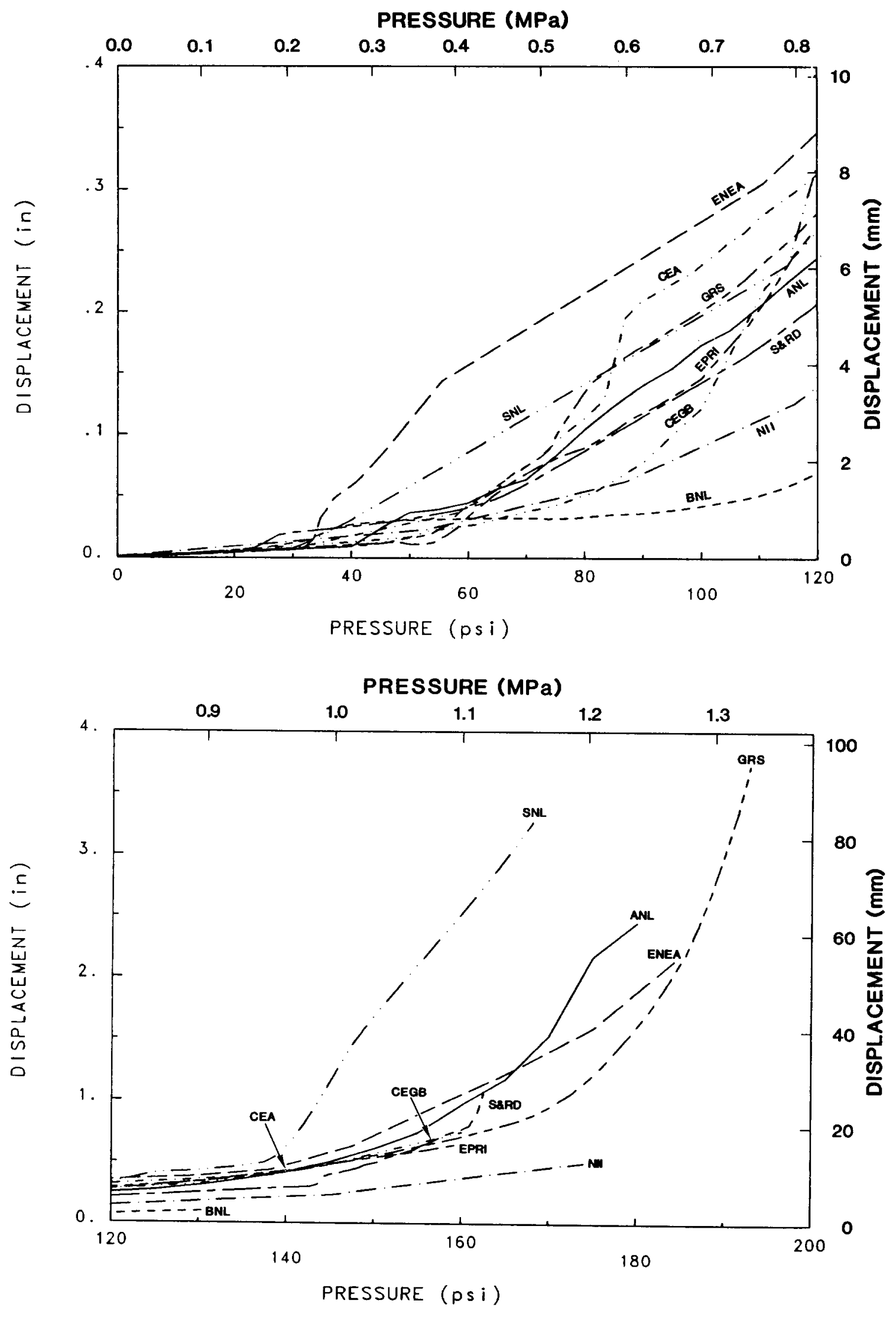

Figure A.11

Vertical displacement relative to cylinder base at EL $24^{\prime}-0^{\prime \prime}, \theta=180$ 

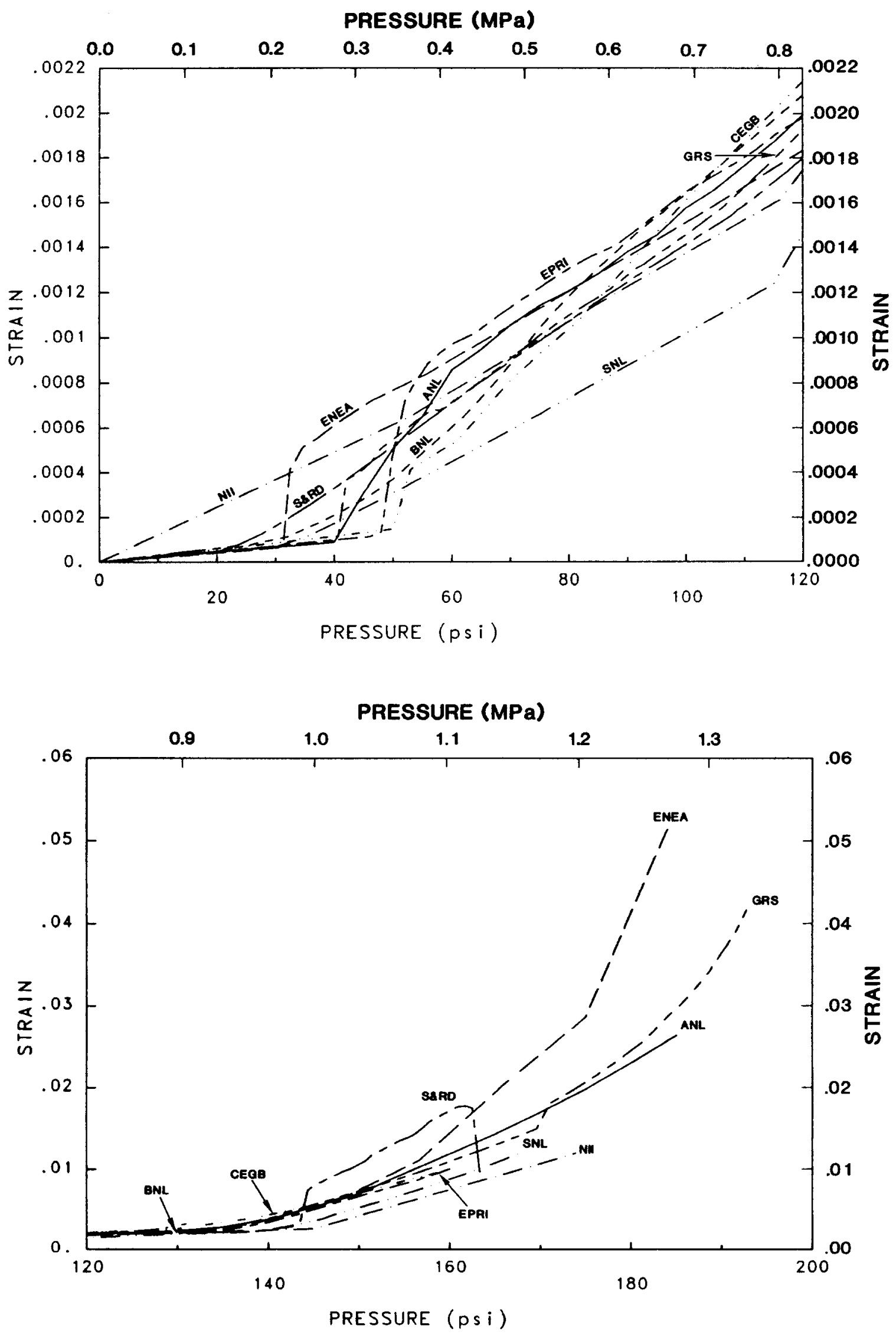

Figure A.12

Maximum principal strain on inside liner surface at $E L 6^{\prime}-9^{\prime \prime}, \theta=45$ 

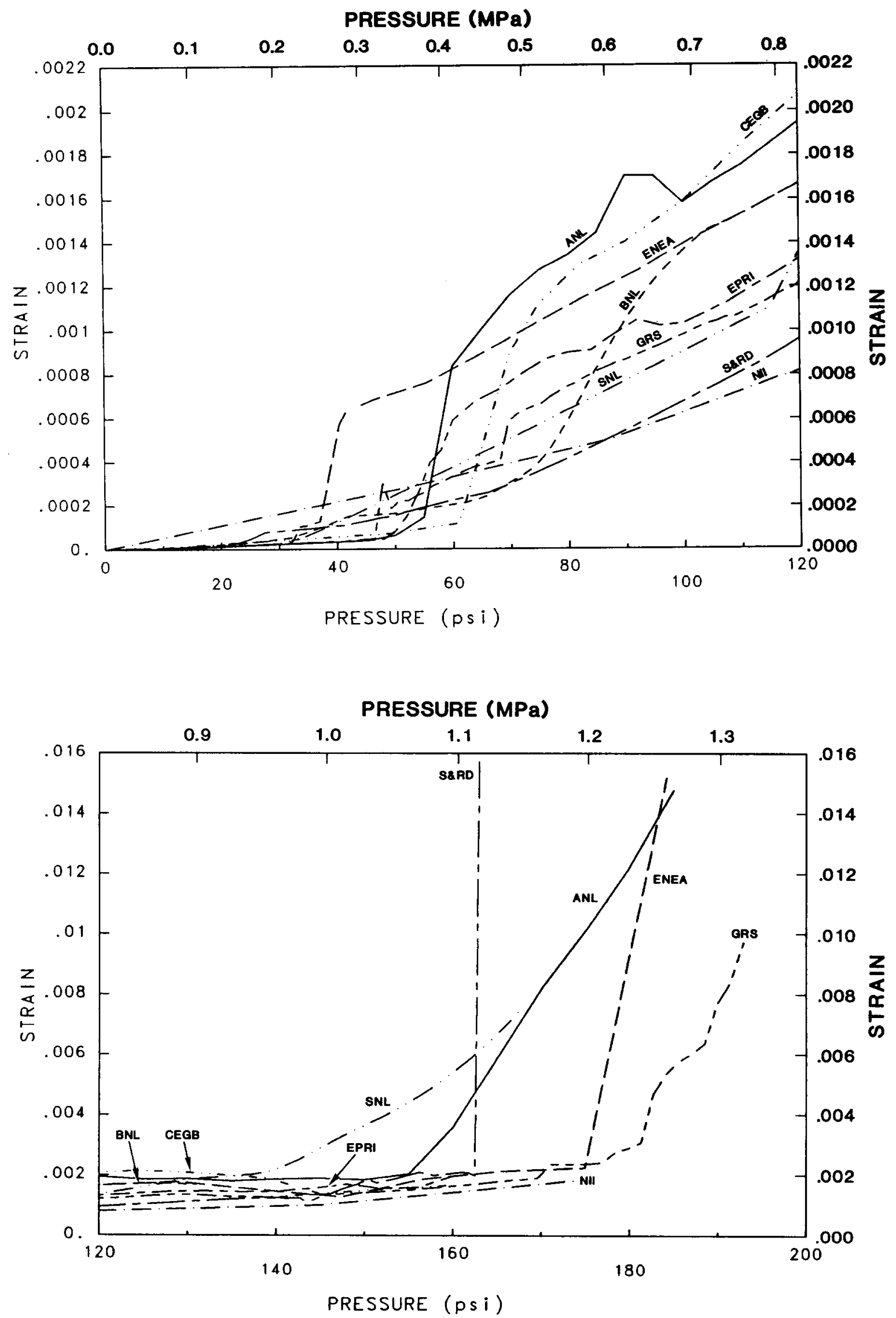

Figure A.13 Axial strain in layer 5 meridional rebar at EL $6^{\prime}-9^{\prime \prime}, \theta=45$ $-465-$ 

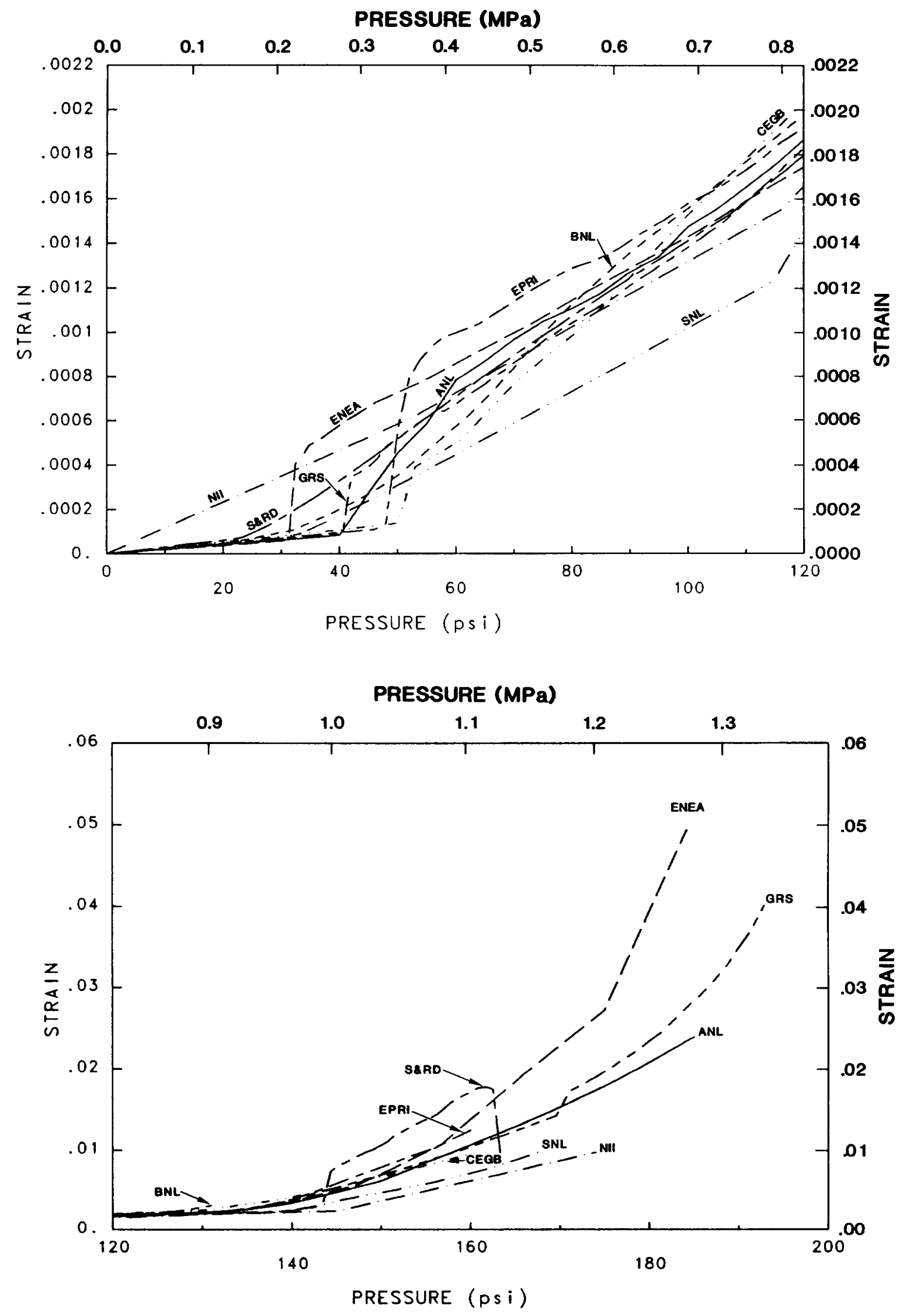

Figure A.14 Axial strain in layer 6 hoop rebar at EL $6^{\prime}-9^{\prime \prime}, \theta=45$ 

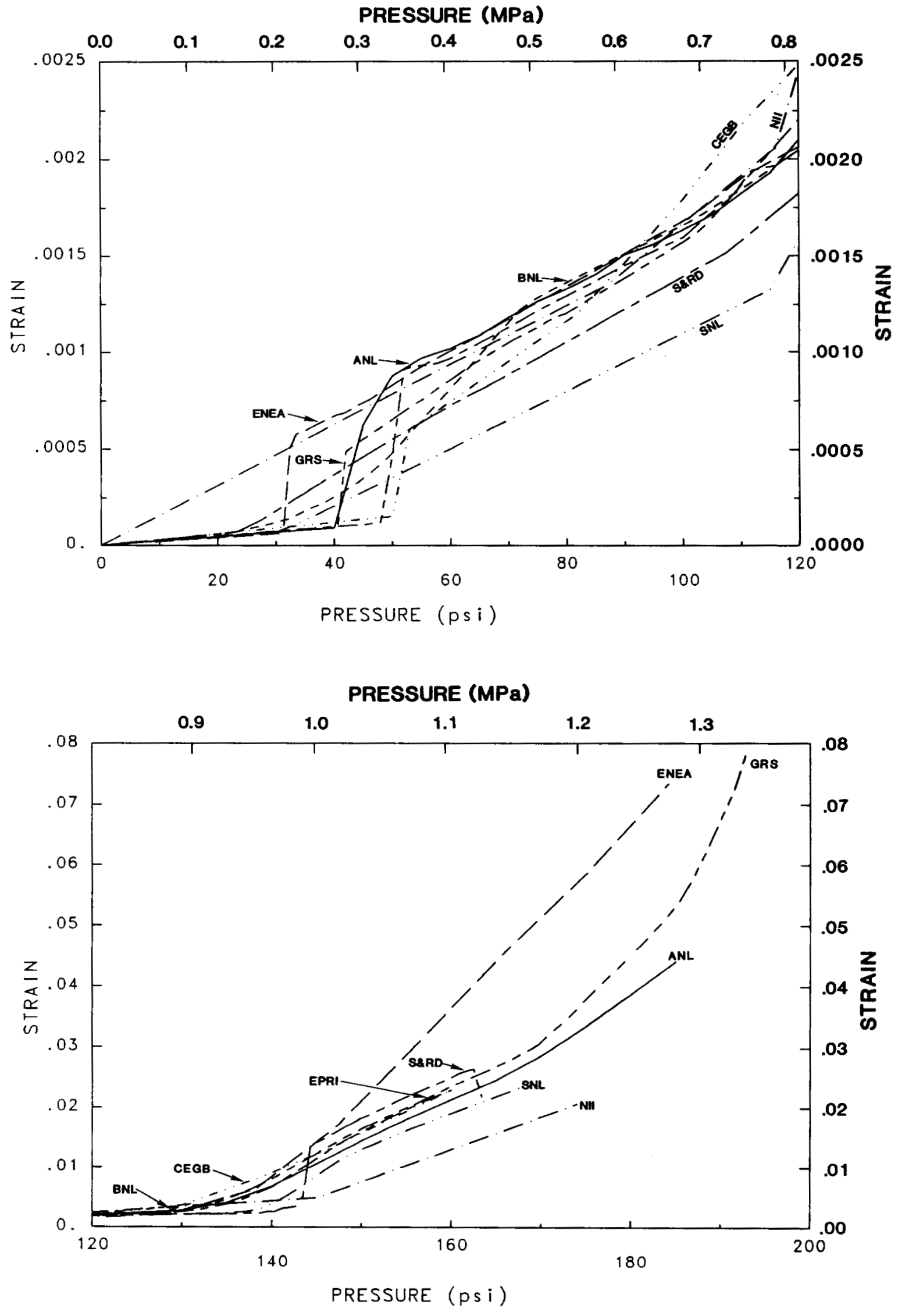

Figure A.15

Maximum principal strain on inside liner surface at EL $13^{\prime}-9^{\prime \prime}, \theta=45$ 
PRESSURE (MPa)
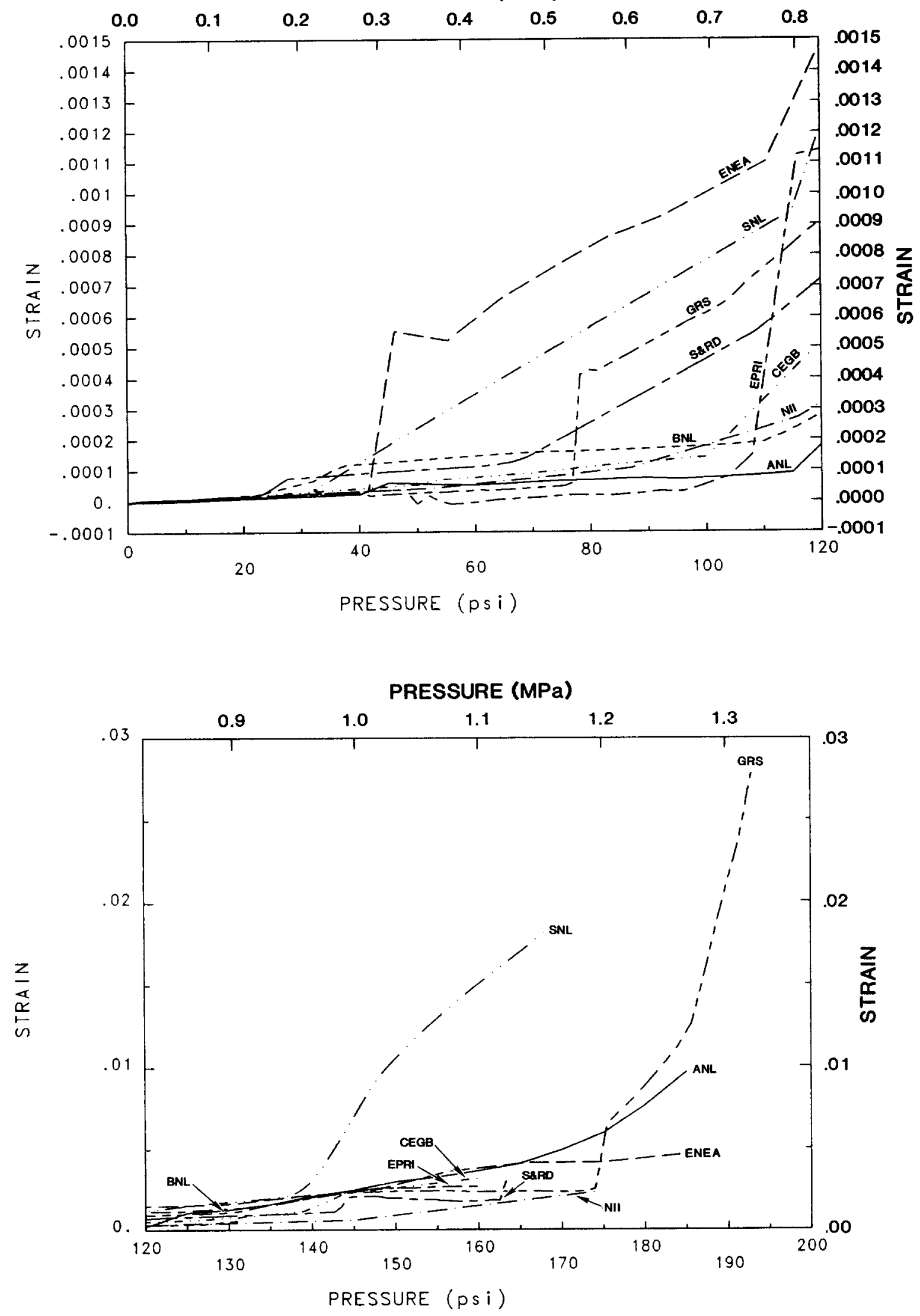

Figure A.16 Axial strain in layer 5 meridional rebar at EL $13^{\prime}-9^{\prime \prime}, \theta=45$ $-468-$ 

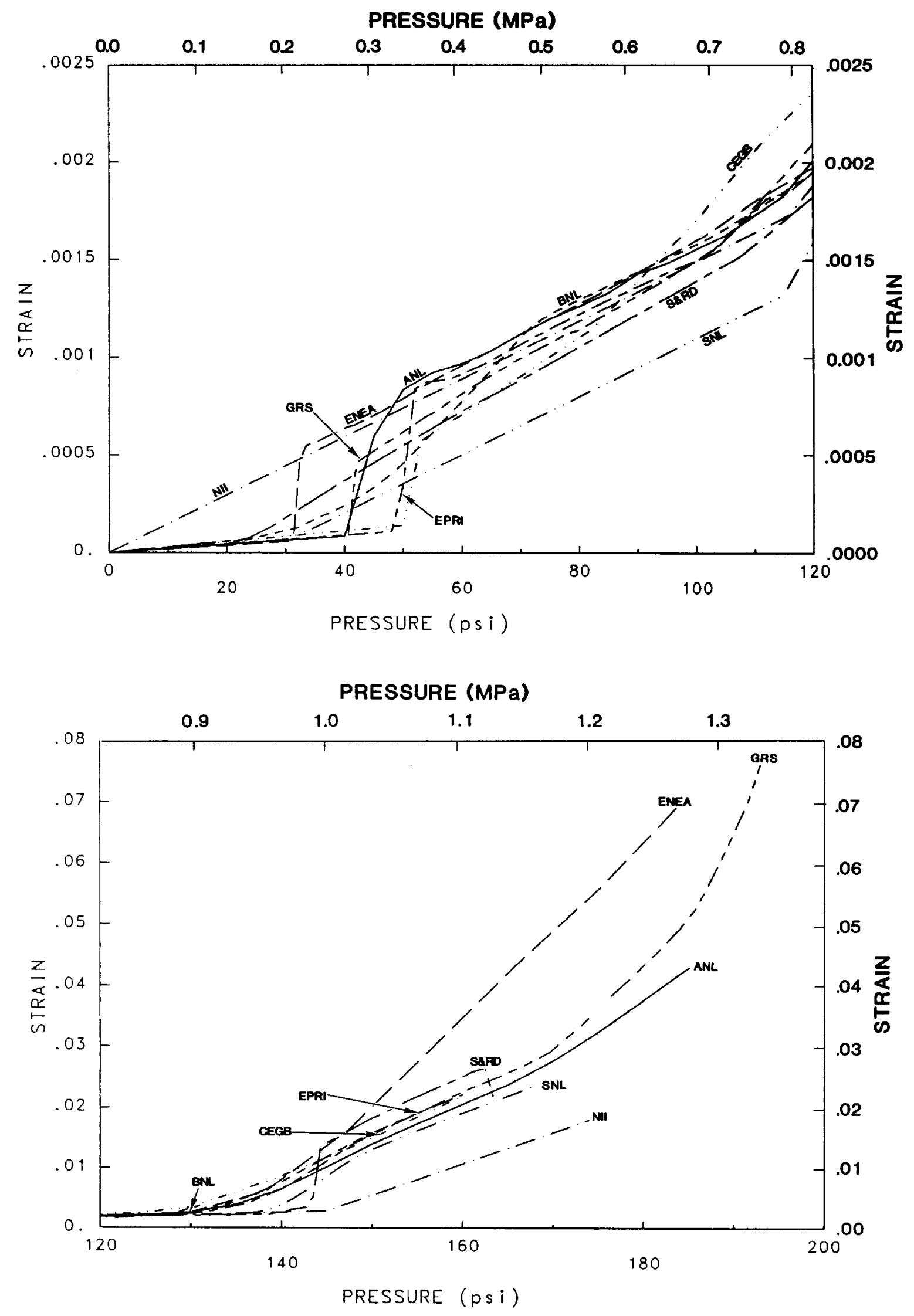

Figure A.17 Axial strain in layer 6 hoop rebar at EL $13^{\prime}-9^{\prime \prime}, \theta=45$ $-469$ 

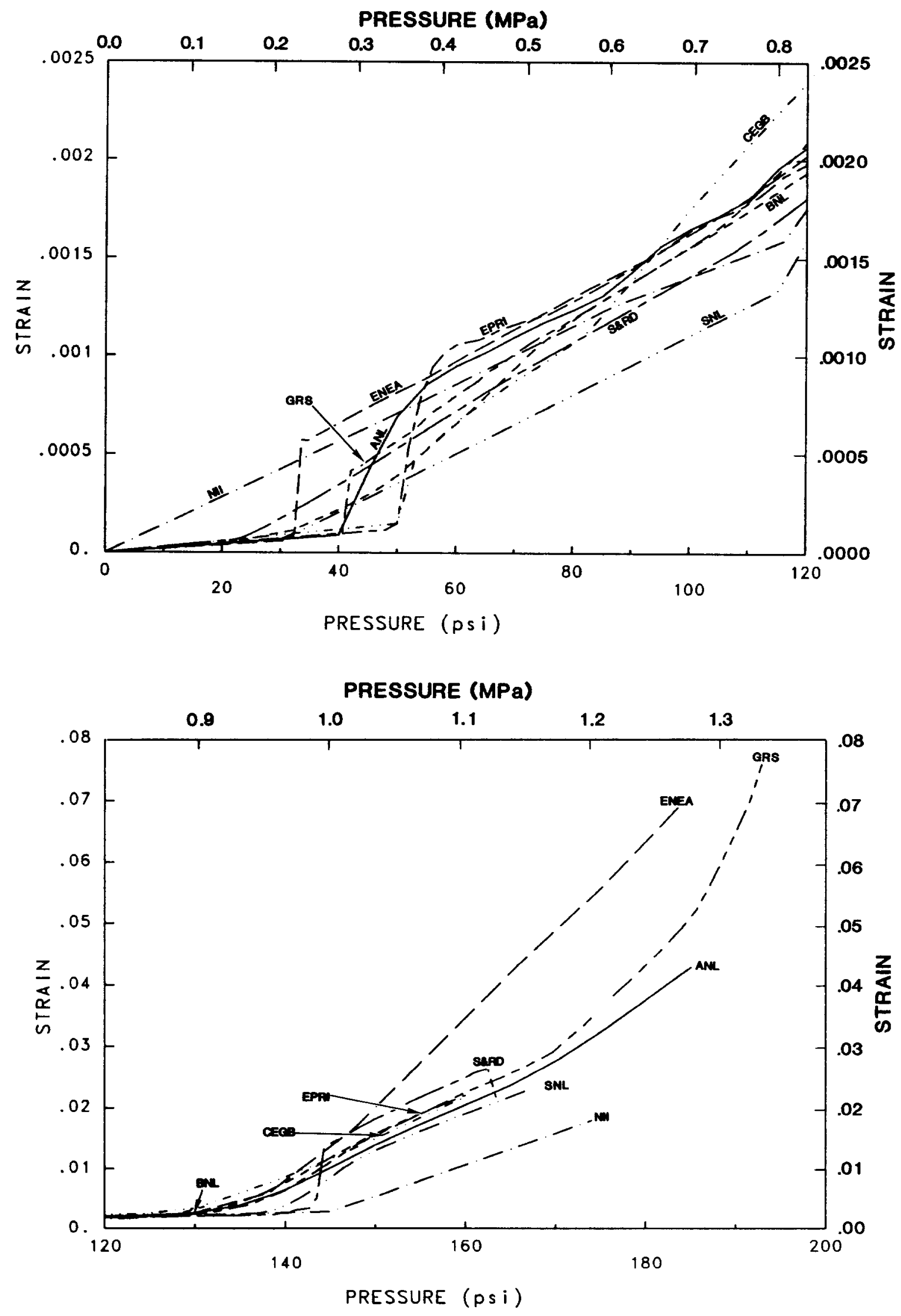

Figure A. 18

Maximum principal strain on inside liner surface at $E L 2^{\prime}-0^{\prime \prime}, \theta=45$ 

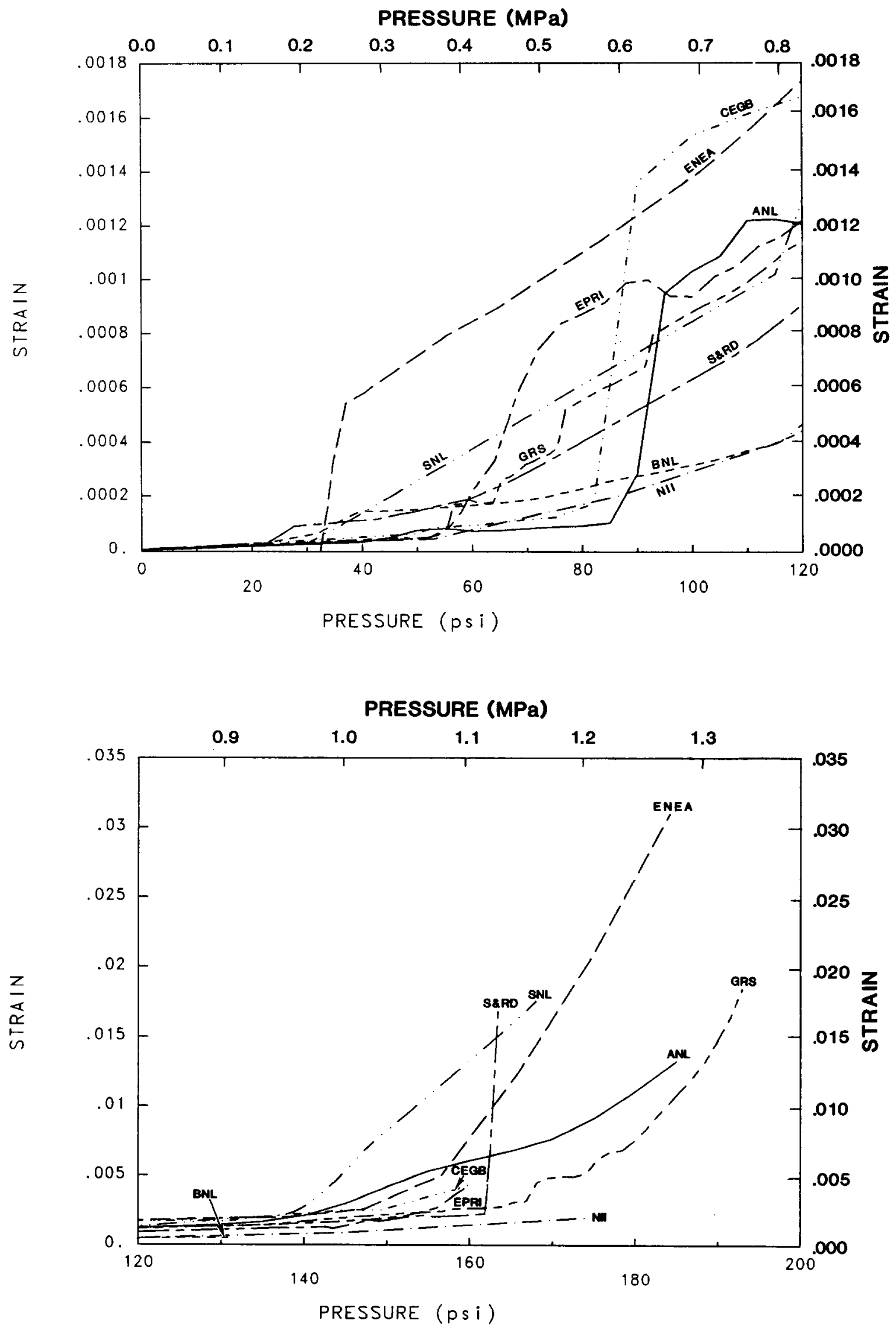

Figure A.19 Axial strain in layer 5 meridional rebar at EL $20^{\prime}-0^{\prime \prime}, \theta=45$ 


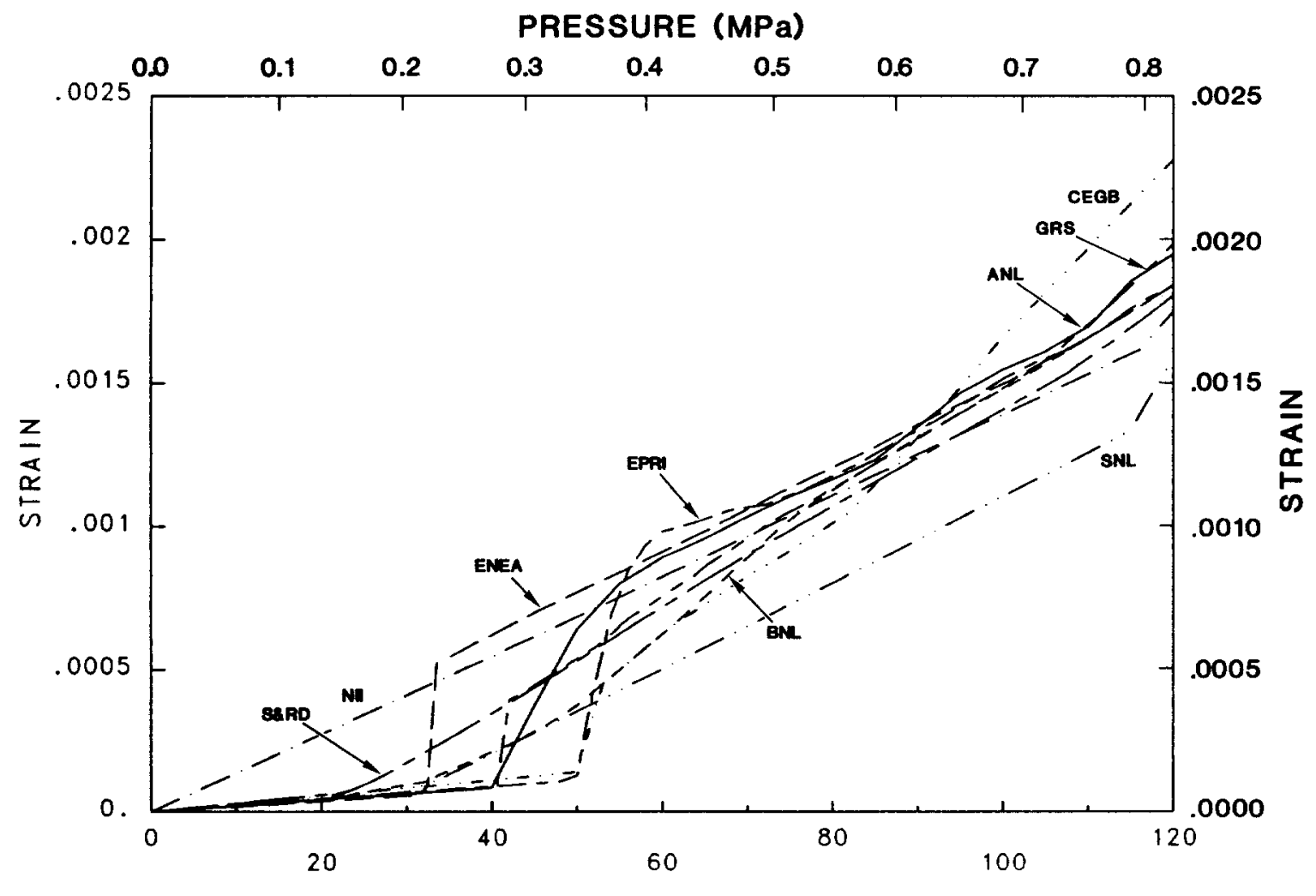

PRESSURE ( $p s i$ )

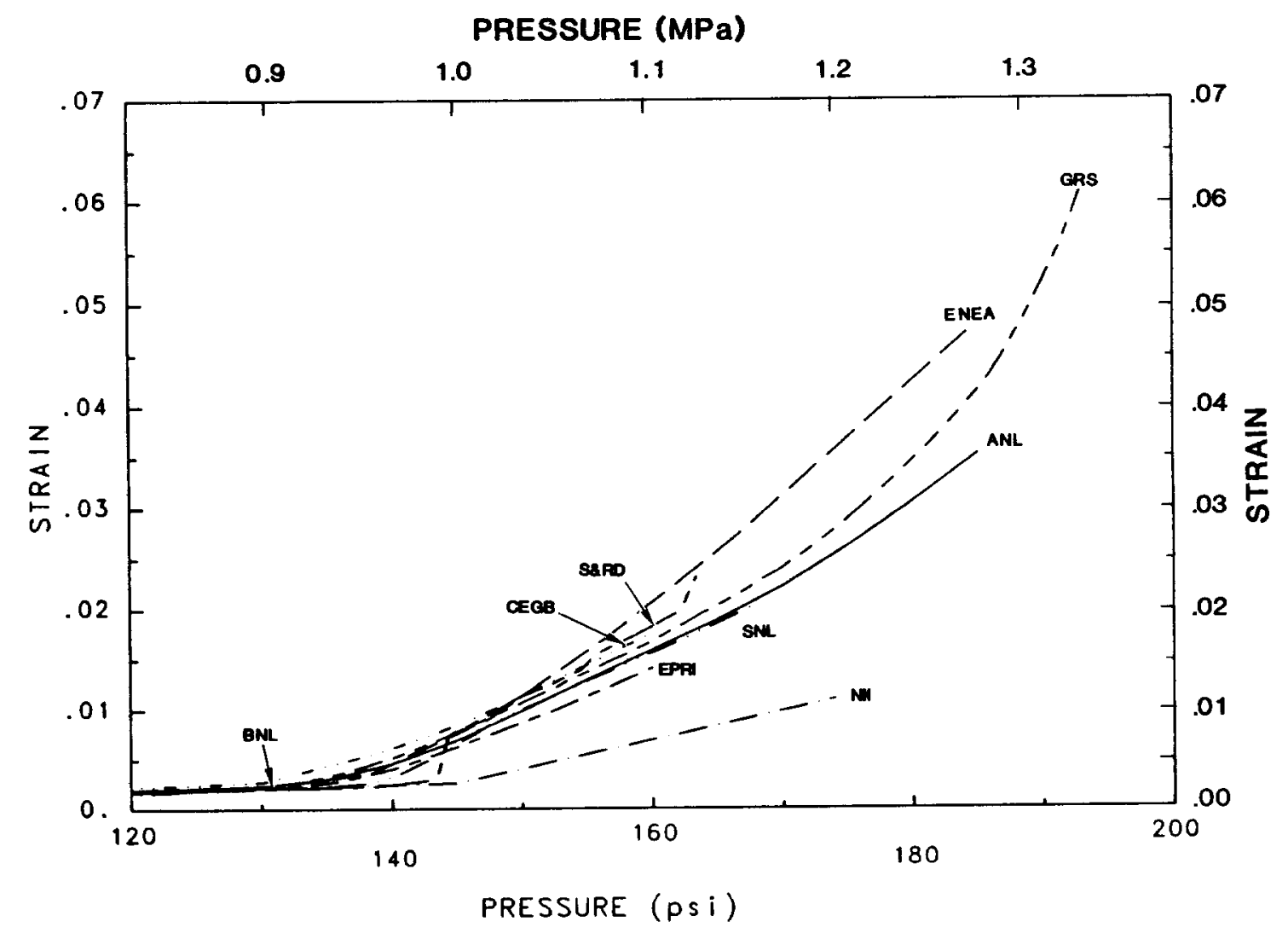

Figure A.20 Axial strain in layer 6 hoop rebar at EL $20^{\prime}-0^{\prime \prime}, \theta=45$ 

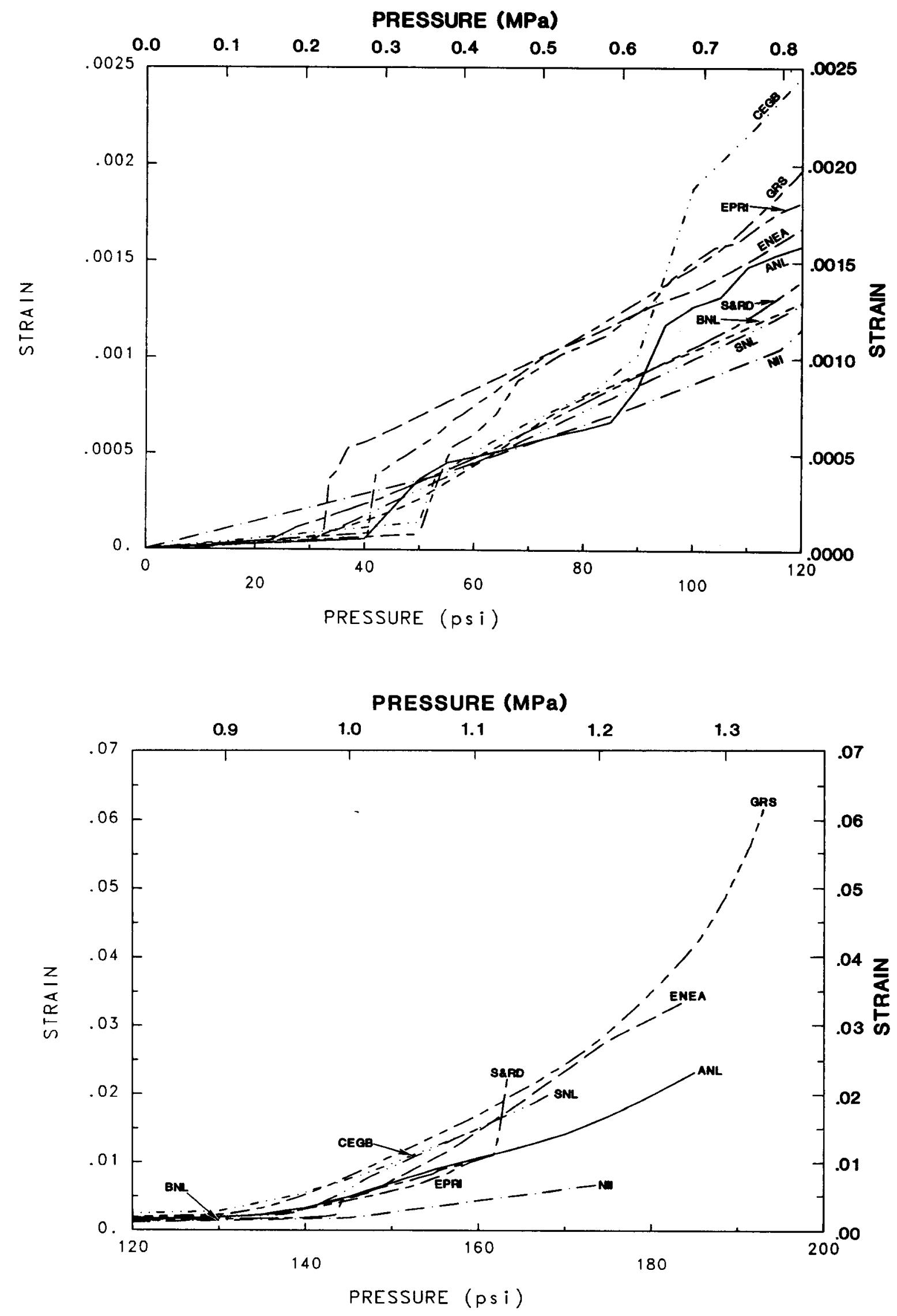

Figure A.21 Axial strain in layer 8 seismic rebar at EL $20^{\prime}-0^{\prime \prime}, \theta=45$ $-473-$ 

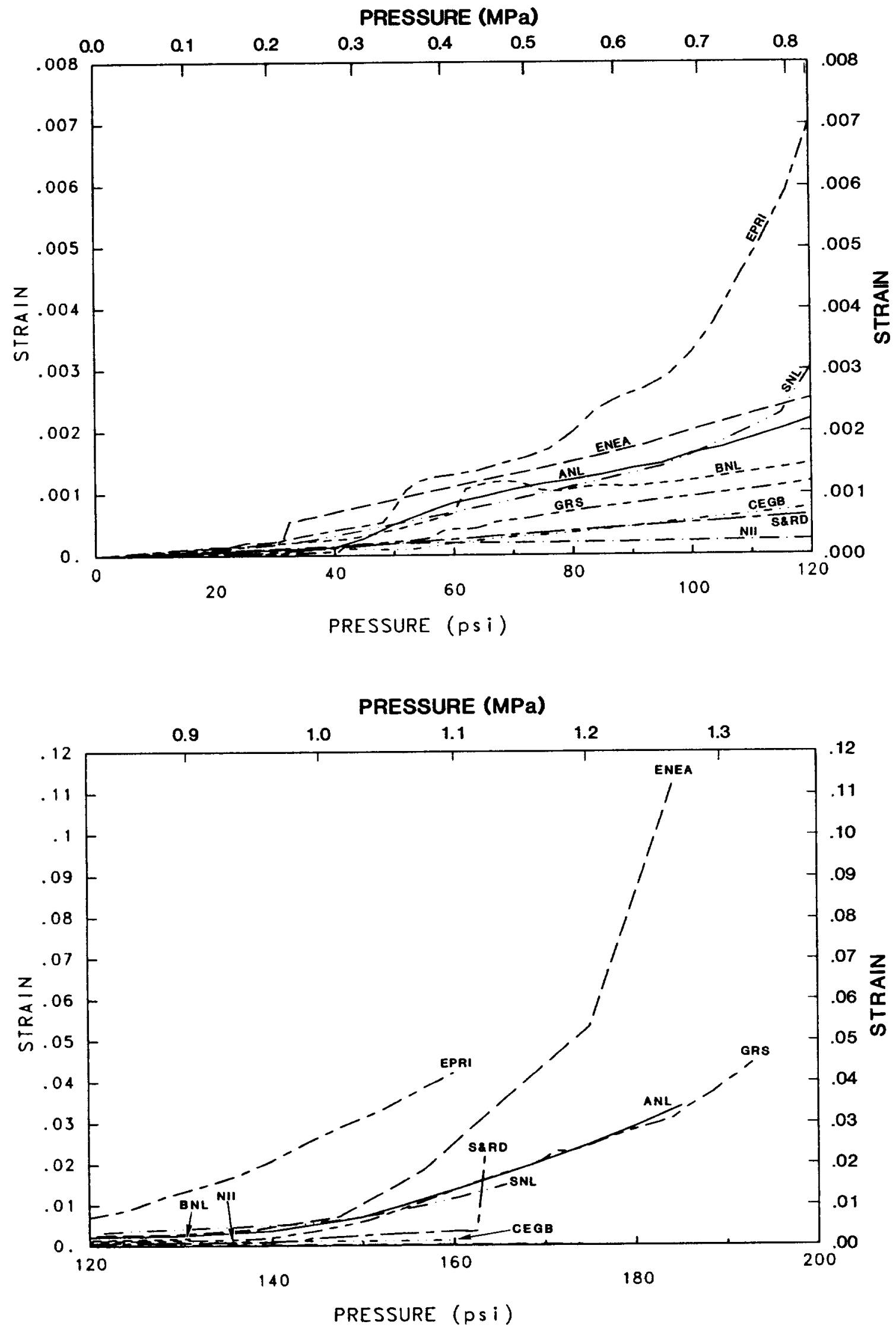

Figure A.22 Maximum principal strain on inside liner surface at EL 2'-0.8", $\theta=90$ (just above knuckle) 

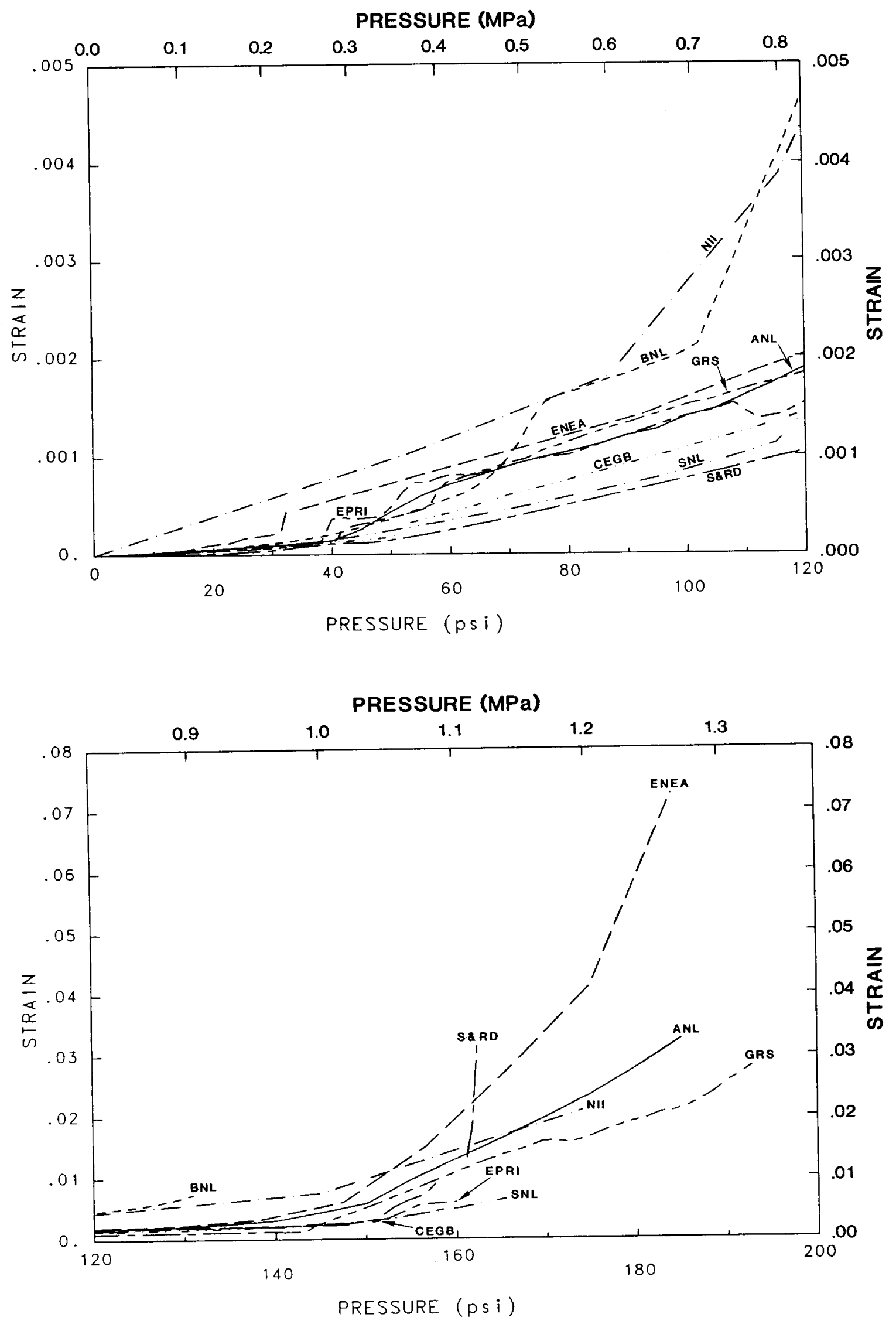

Figure A.23 Axial strain in layer 2 meridional rebar at $E L 2^{\prime}-0.8^{\prime \prime}, \theta=90$ (just above knuckle) 
PRESSURE (MPa)

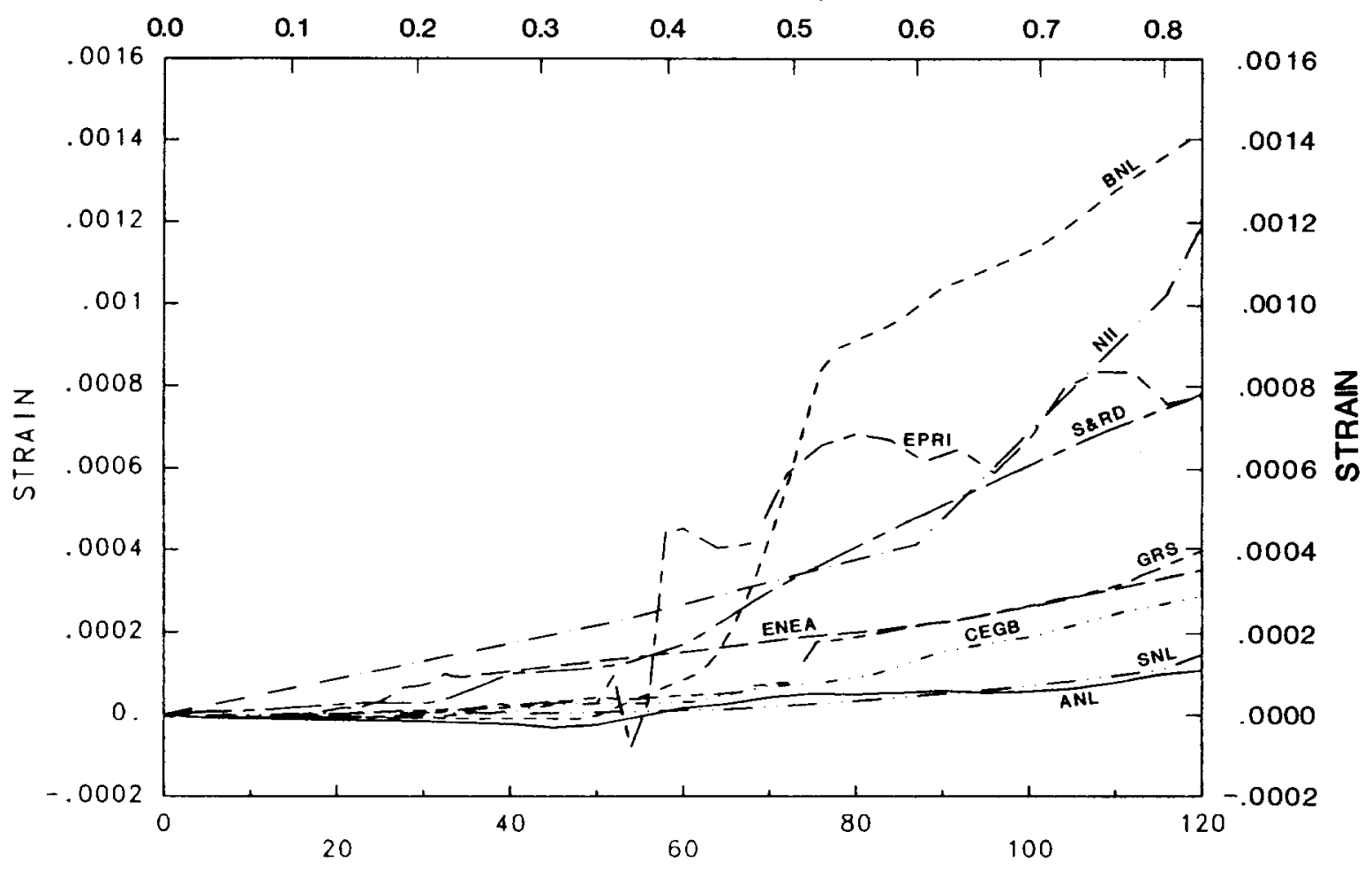

PRESSURE ( $p s i$ )

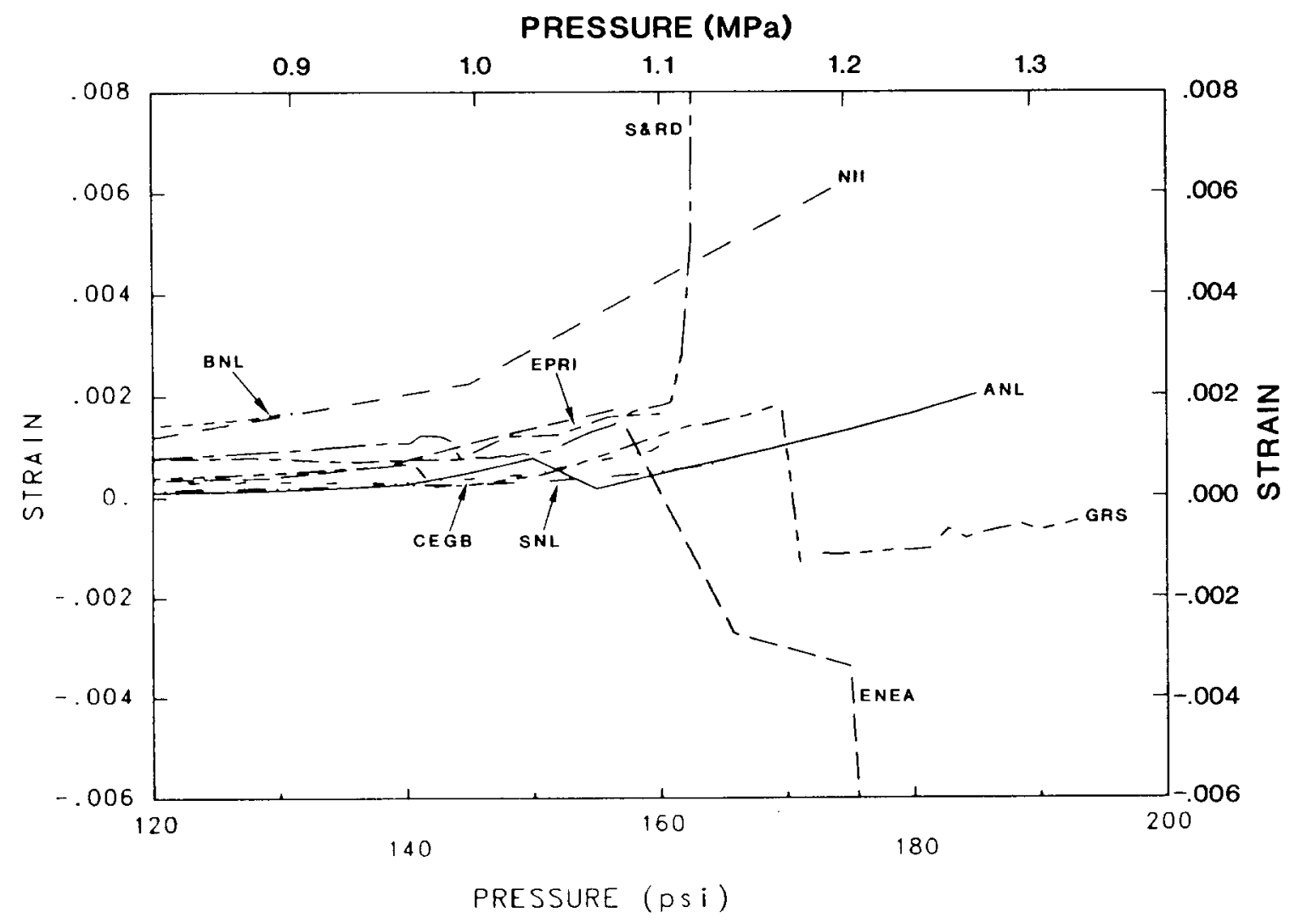

Figure A.24 Axial strain in layer 5 meridional rebar at $E L 2^{\prime}-0.8^{\prime \prime}, \theta=90$ (just above knuckle) 

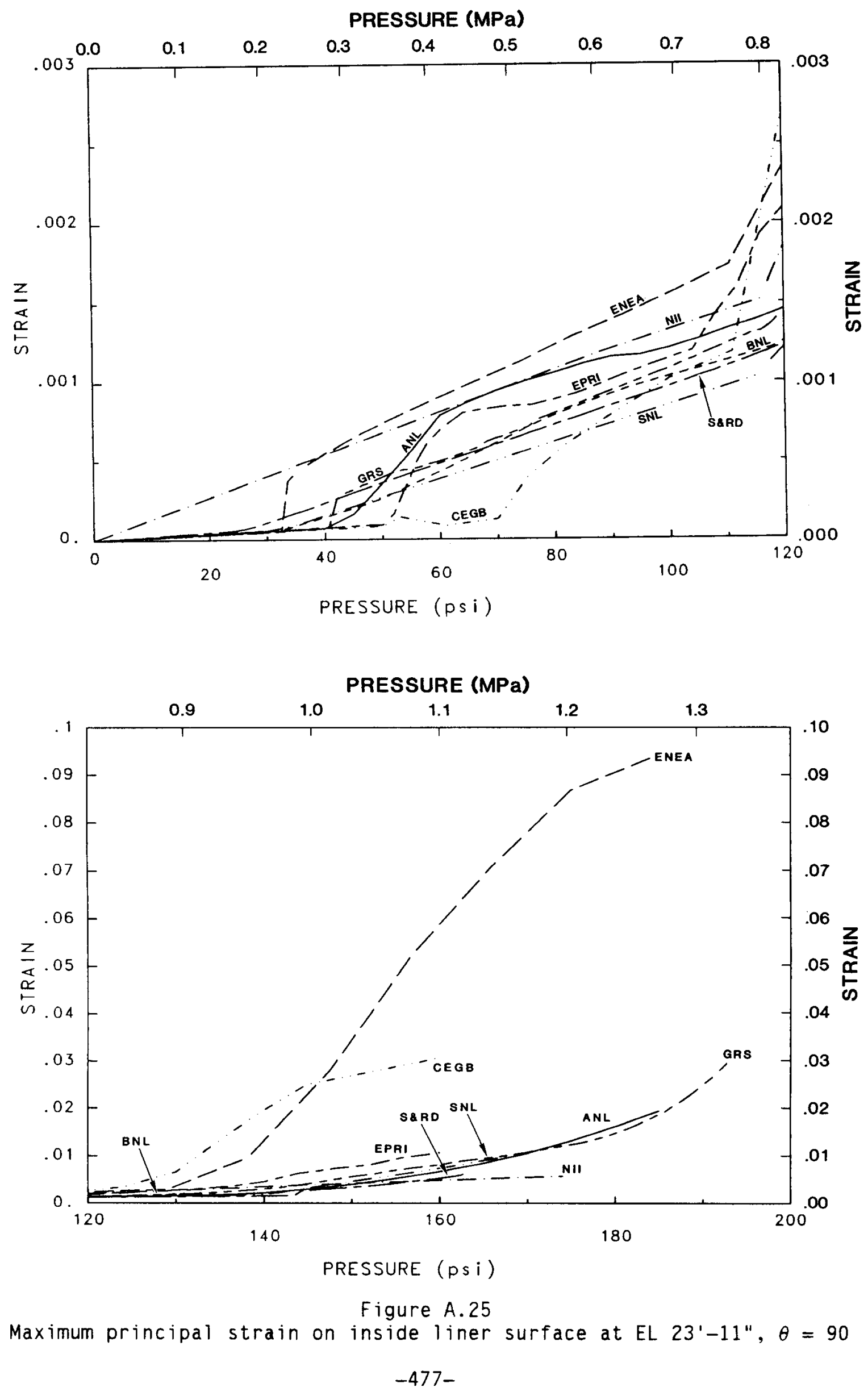

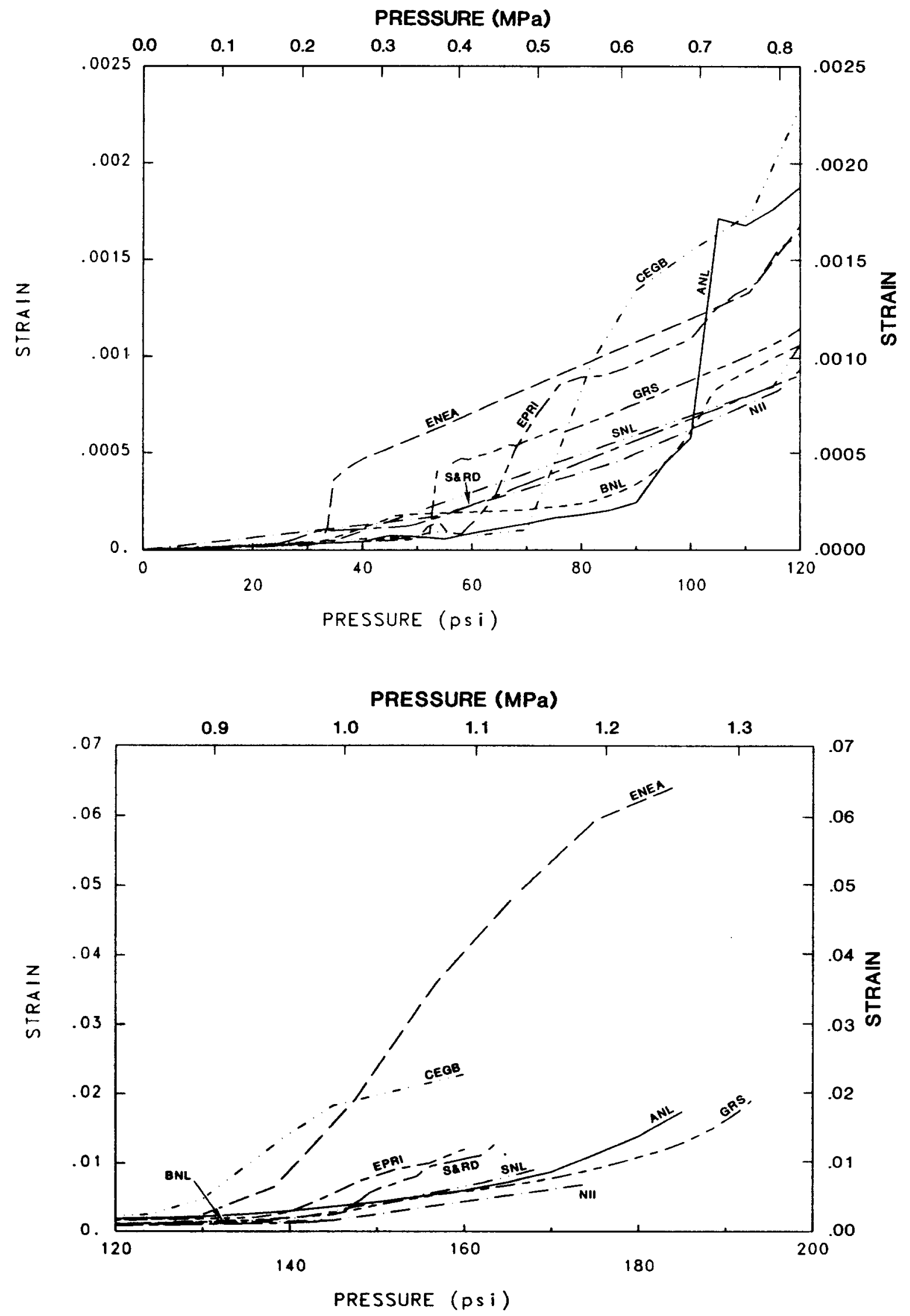

Figure A.26 Axial strain in layer 2 meridional rebar at EL $23^{\prime}-11^{\prime \prime}, \theta=90$ $-478-$ 

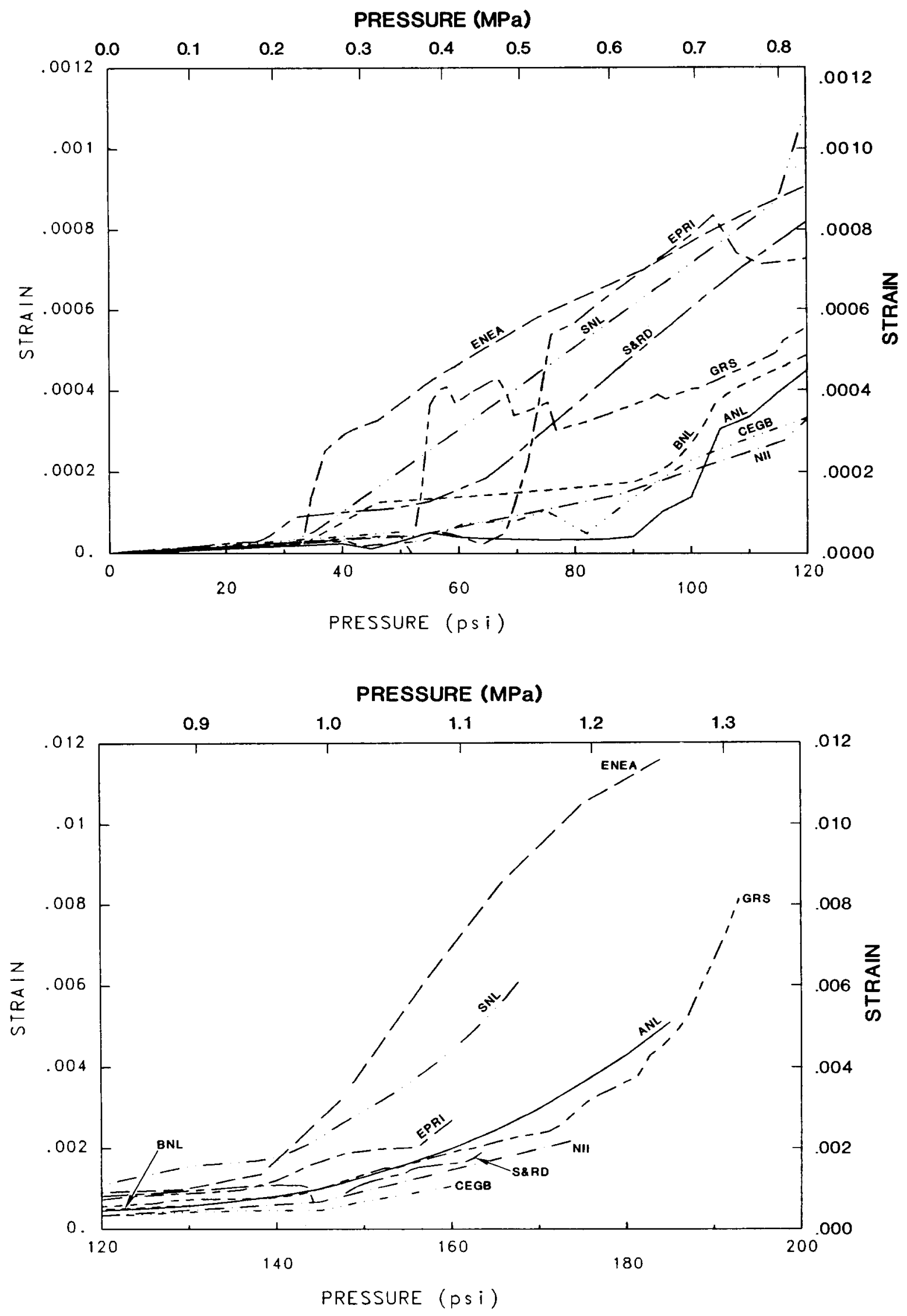

Figure A.27 Axial strain in layer 5 meridional rebar at EL $23^{\prime}-11^{\prime \prime}, \theta=90$ 

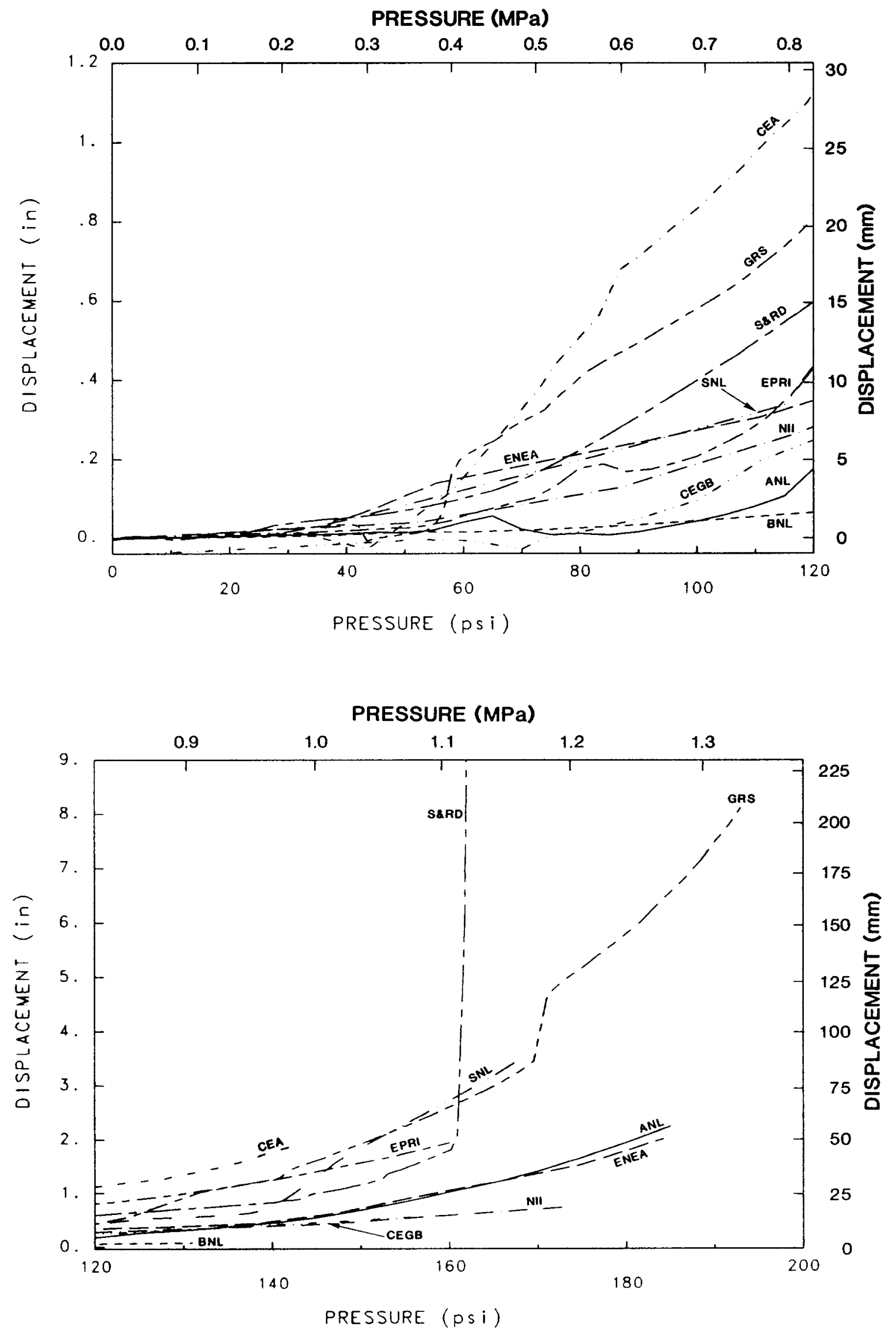

Figure A.28 Vertical displacement of liner at dome apex, $\phi=90$ 

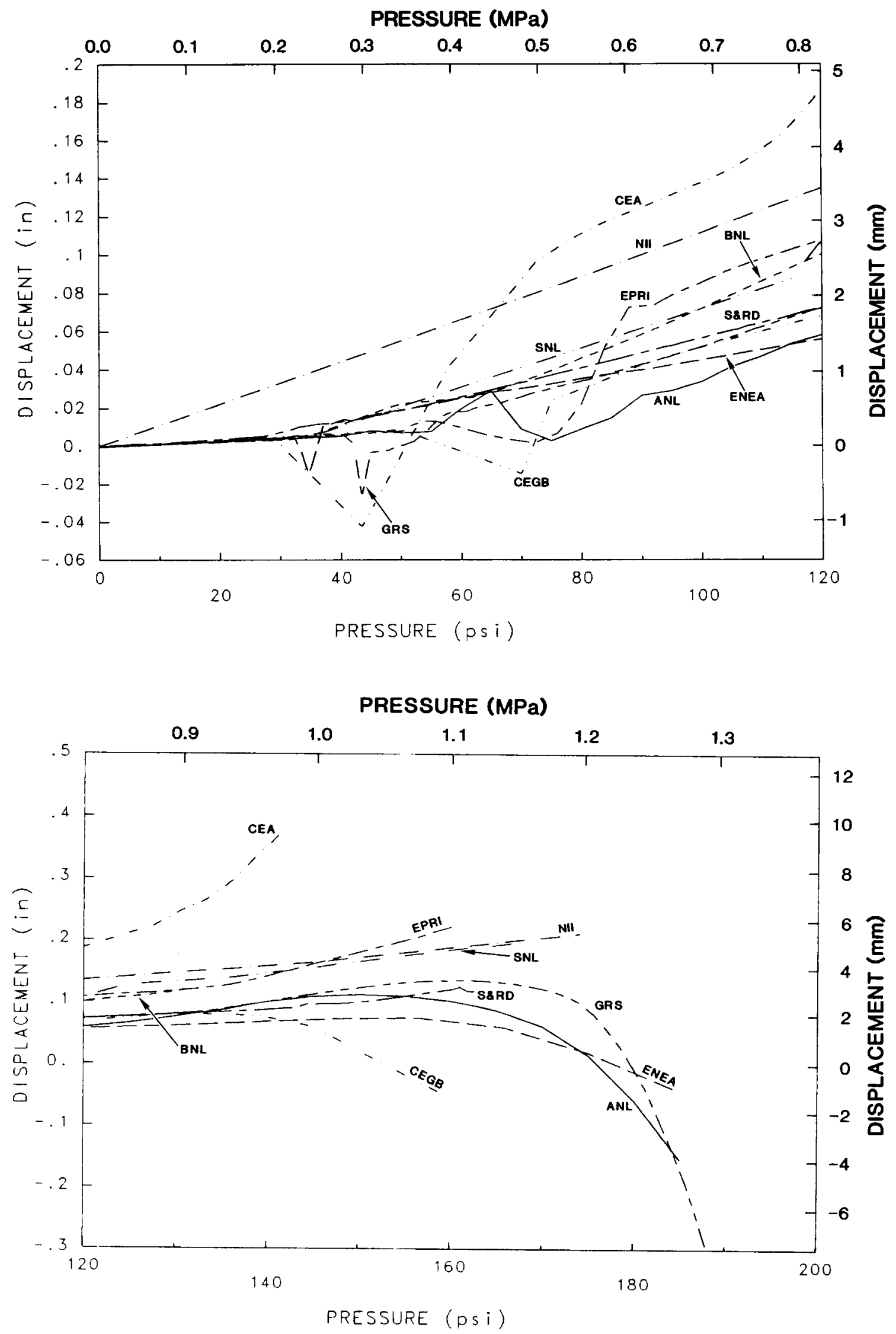

Figure A.29

Vertical displacement of liner relative to springline, $\phi=70, \theta=90$ 

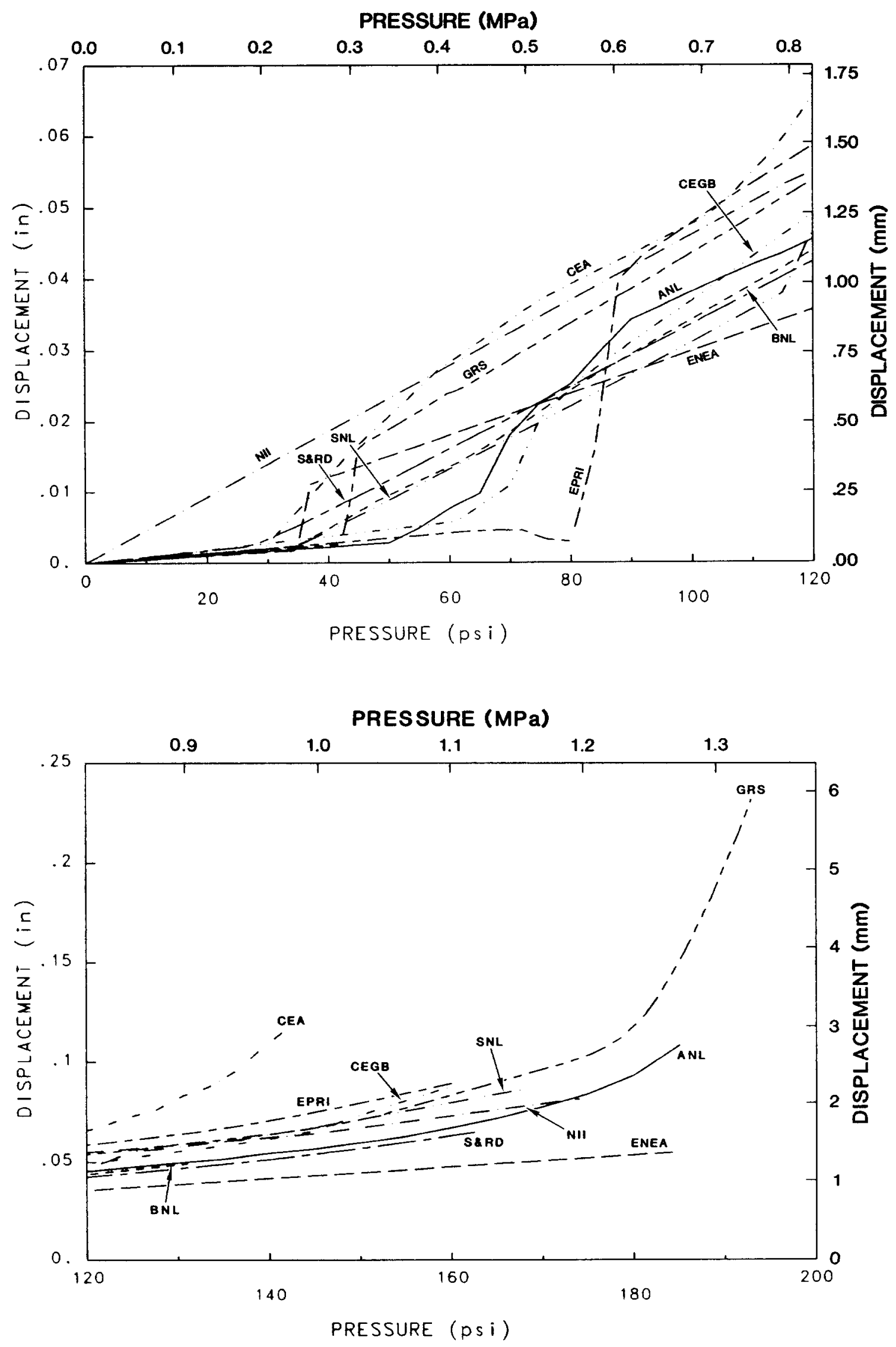

Figure A.30 Horizontal displacement of liner, $\phi=70, \theta=90$ 

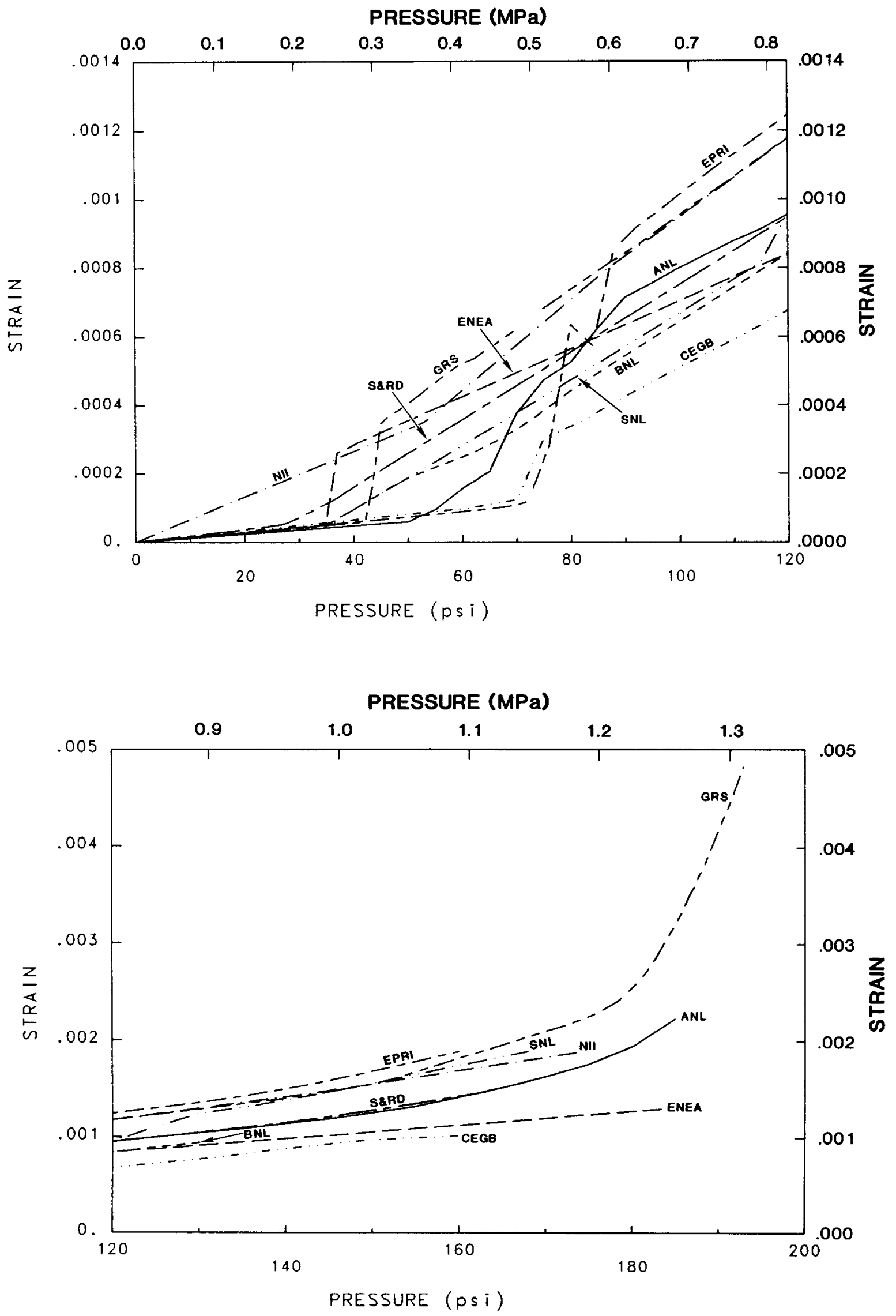

Figure A.31 Maximum principal strain on inside liner surface, $\phi=72, \theta=90$ $-483-$ 
PRESSURE (MPa)
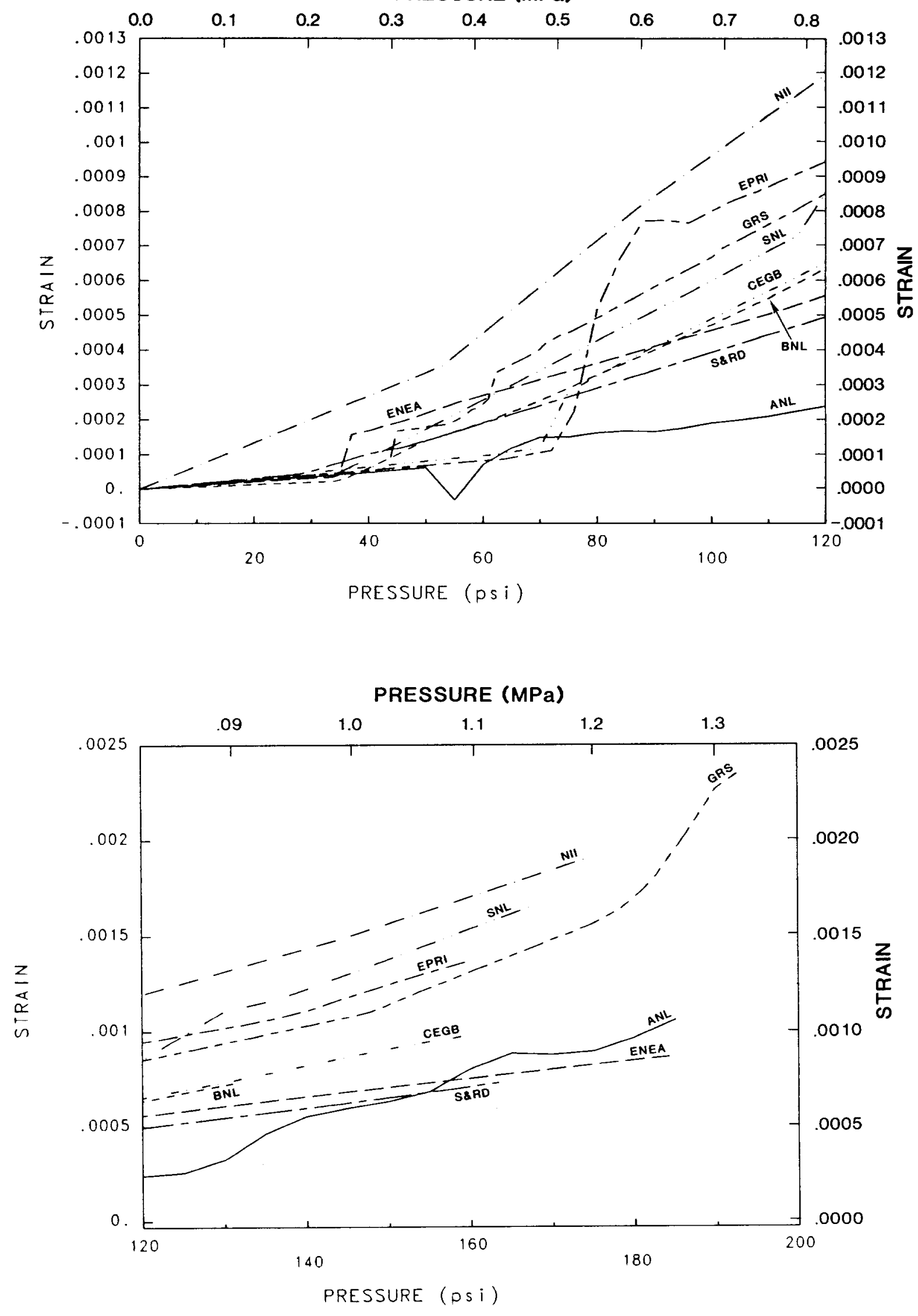

Figure A.32 Axial strain in layer 2 meridional rebar, $\phi=72, \theta=90$ 


\section{PRESSURE (MPa)}

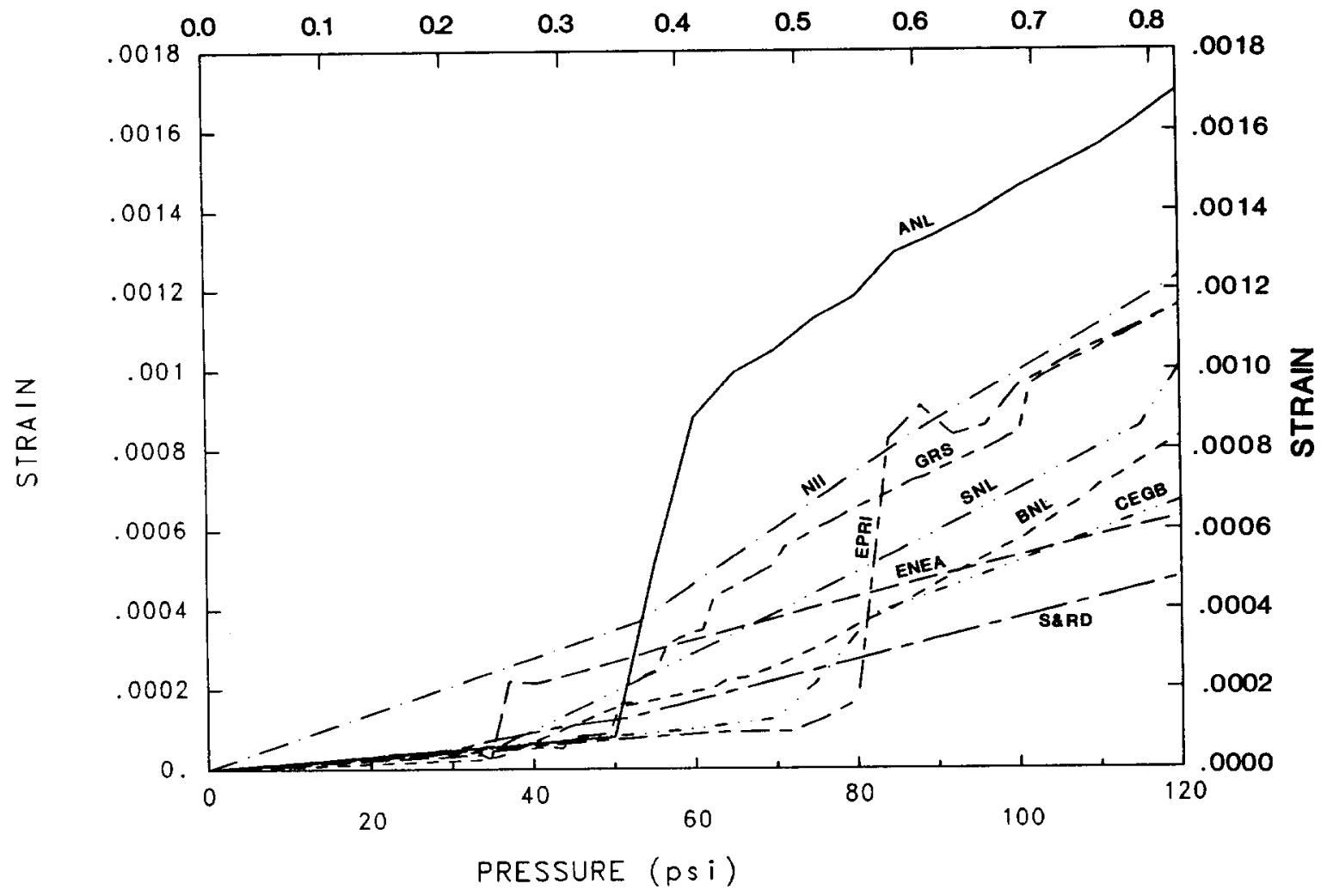

PRESSURE (MPa)

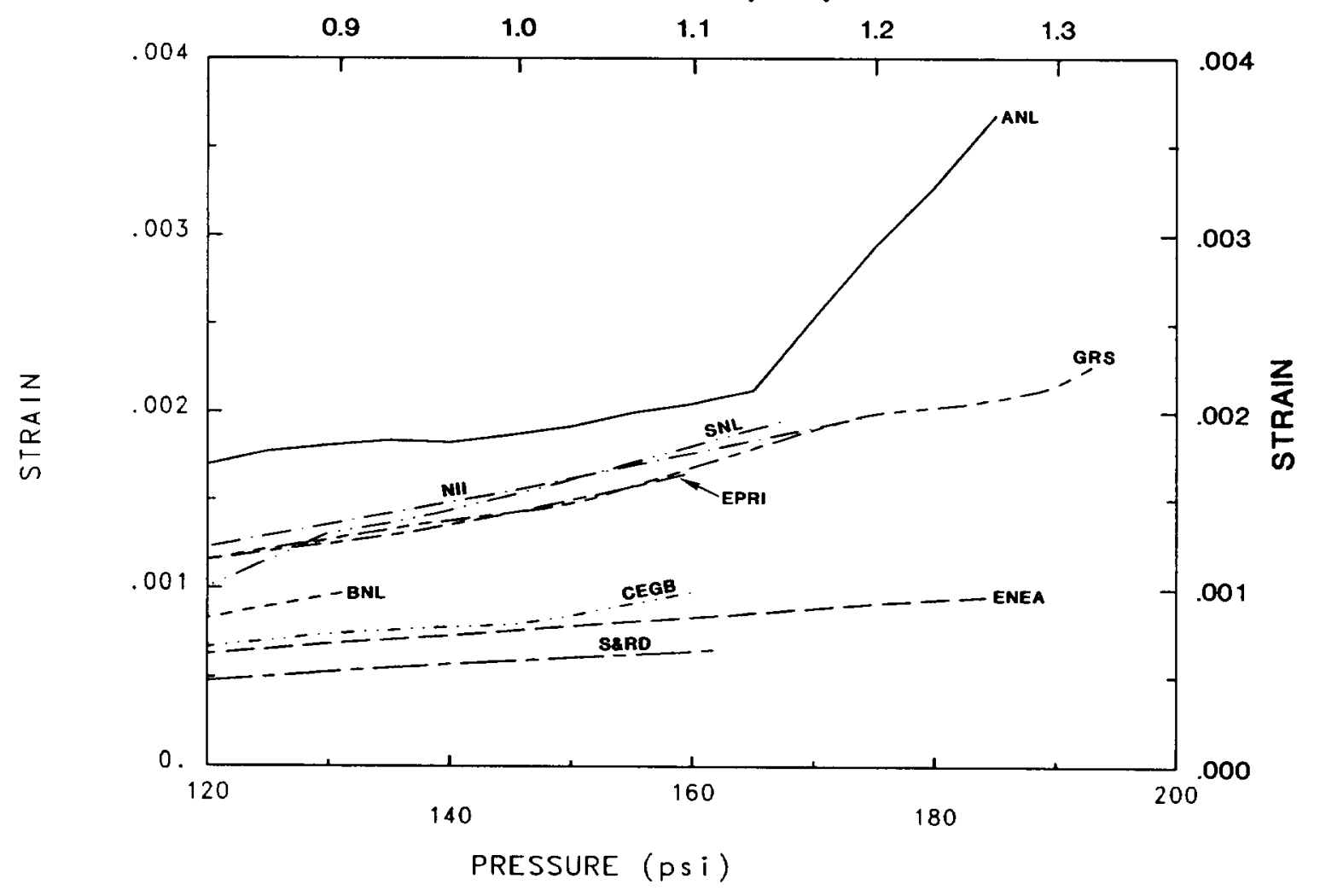

Figure A.33 Axial strain in layer 5 meridional rebar, $\phi=72, \theta=90$ $-485-$ 

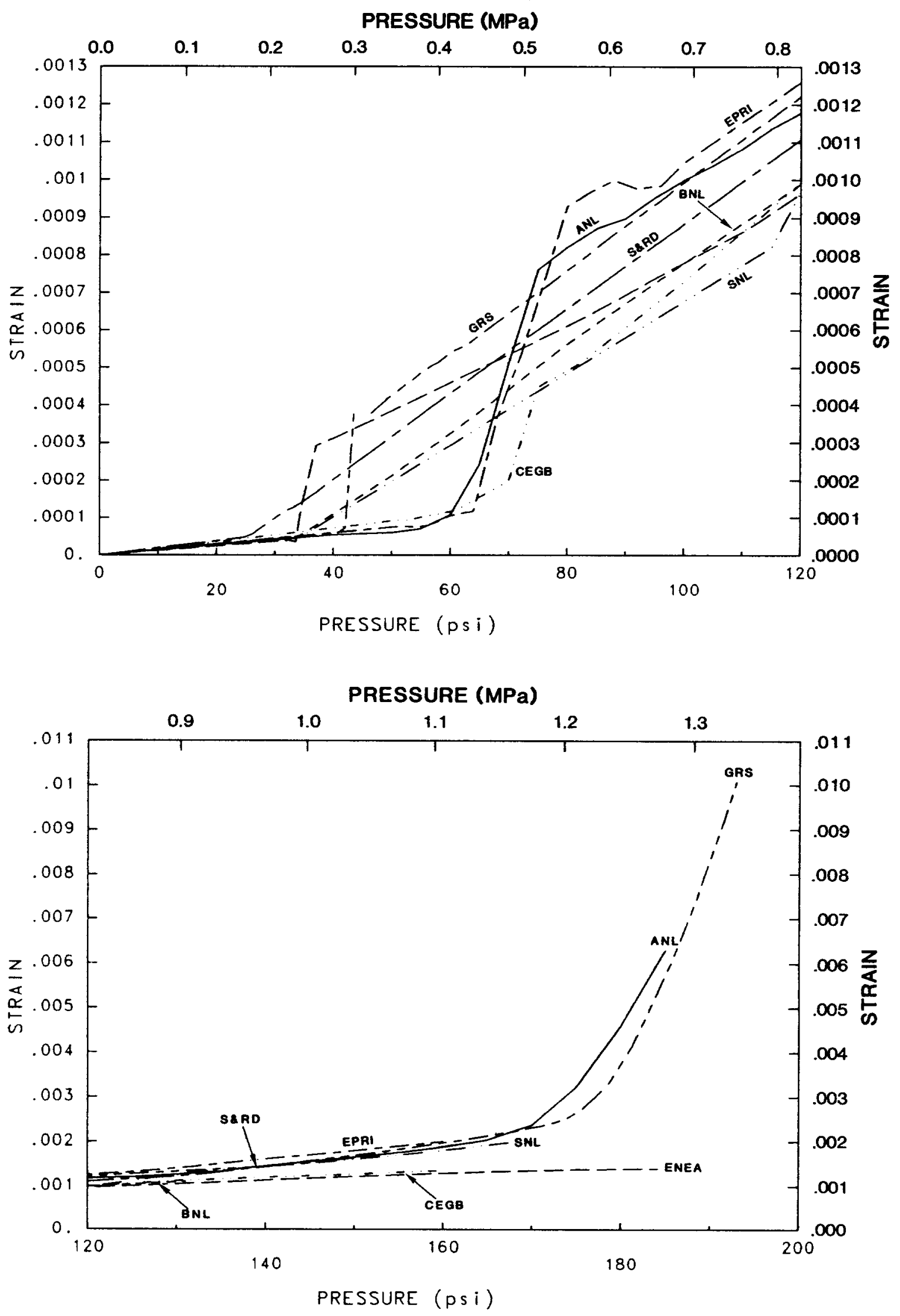

Figure A.34 Maximum principal strain on inside liner surface, $\phi=37, \theta=90$ 
PRESSURE (MPa)
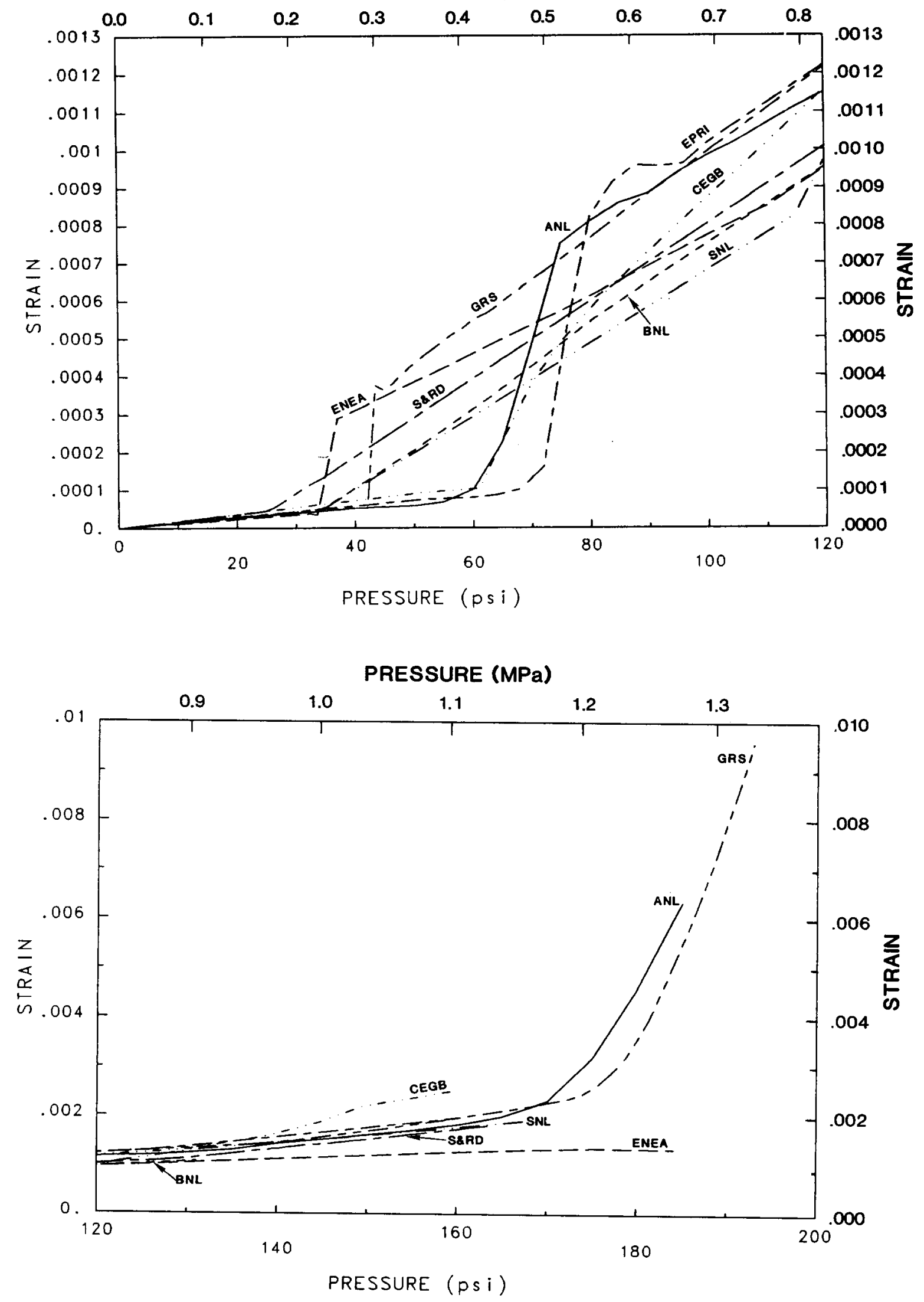

Figure A.35 Axial. strain in layer 4 hoop rebar, $\phi=37, \theta=90$ 

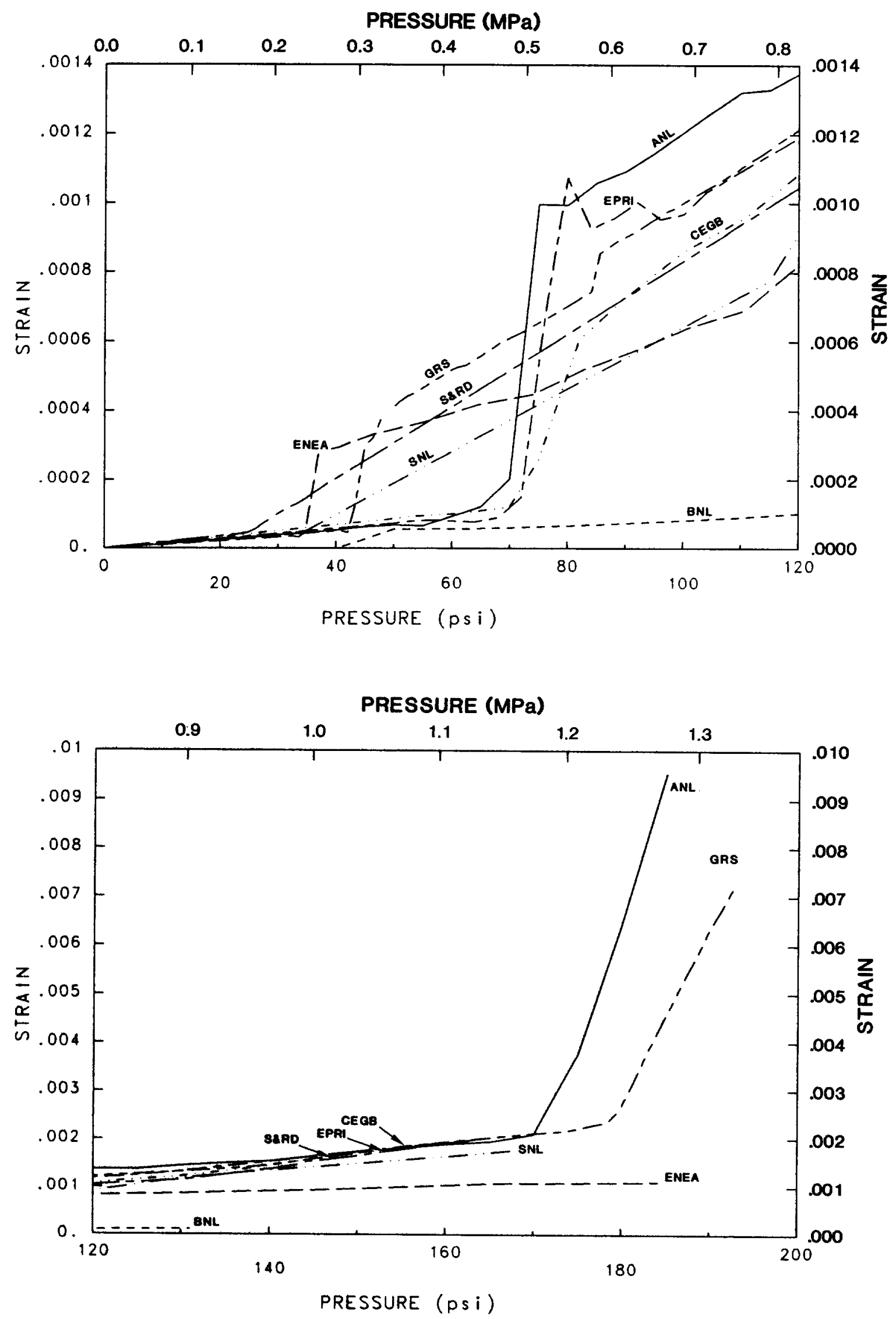

Figure A.36 Axial strain in layer 5 meridional rebar, $\phi=37, \theta=90$ $-488-$ 


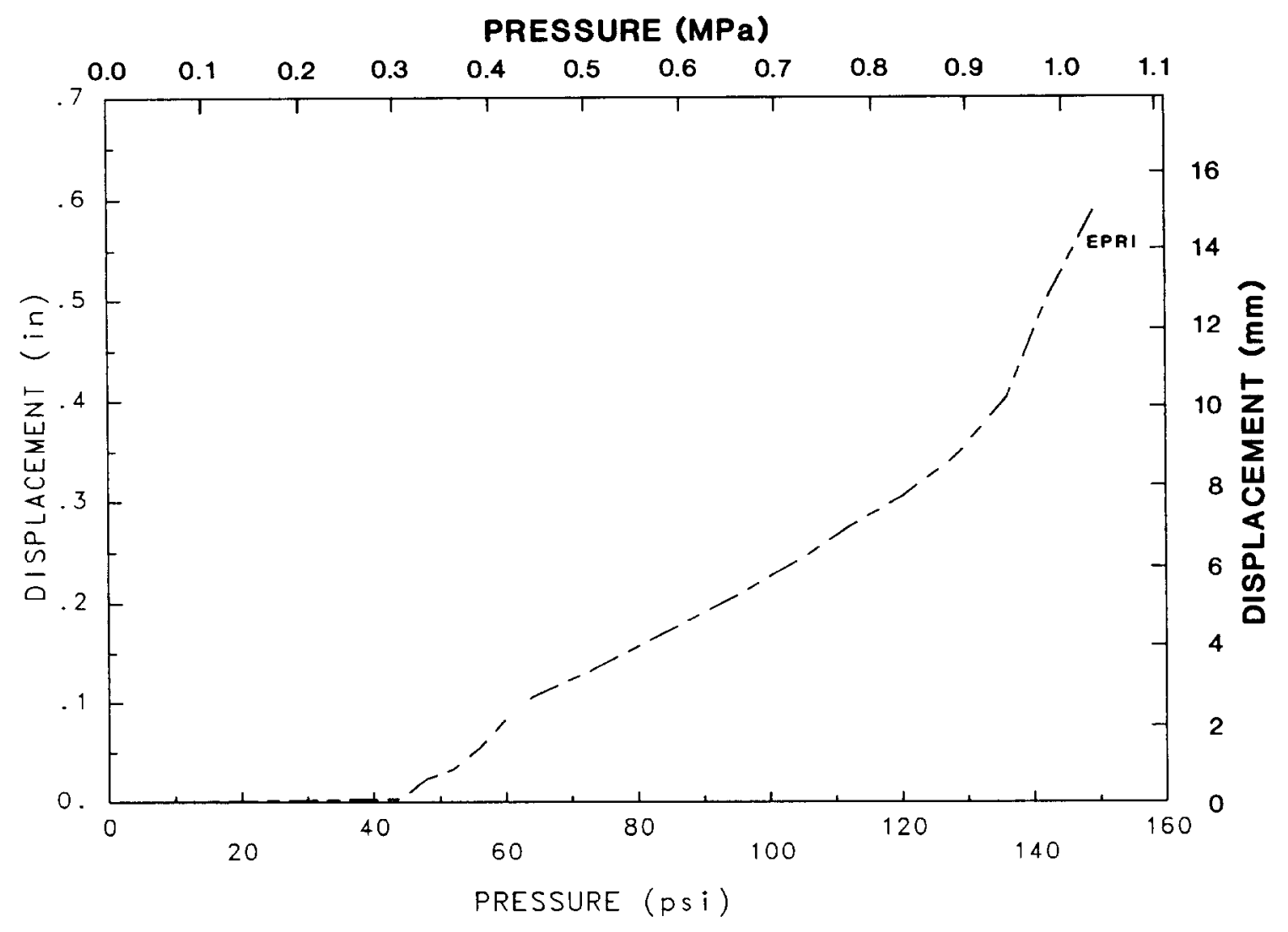

Figure A.37 Horizontal displacement of inside edge of equipment hatch $B$ sleeve at midthickness (top) at EL $14^{\prime}-7^{\prime \prime}, \theta=180$

\section{PRESSURE (MPa)}

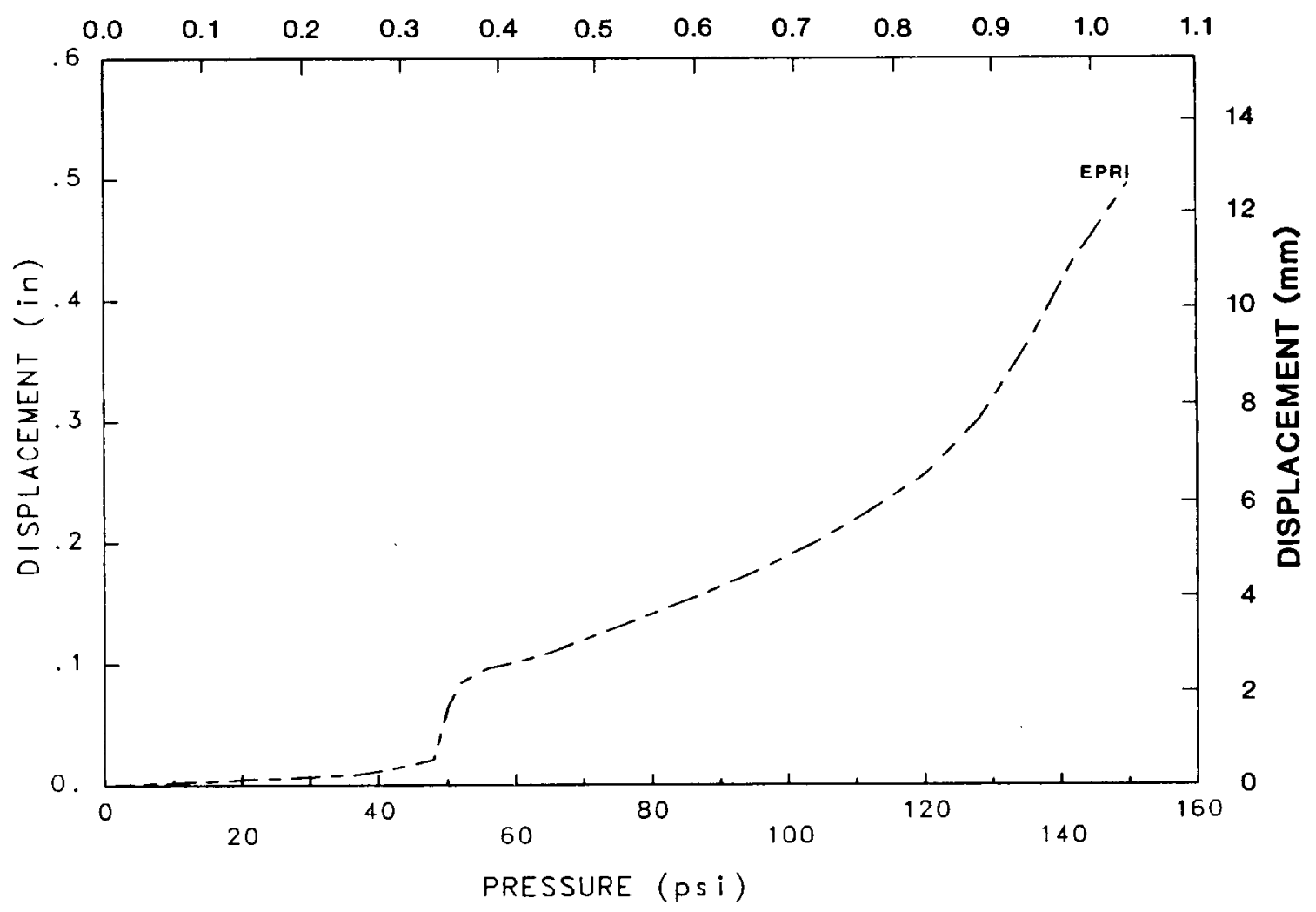

Figure A.38 Radial displacement of liner at EL $20^{\prime}-1.5^{\prime \prime}, \theta=312.5$ (constrained pipe) 


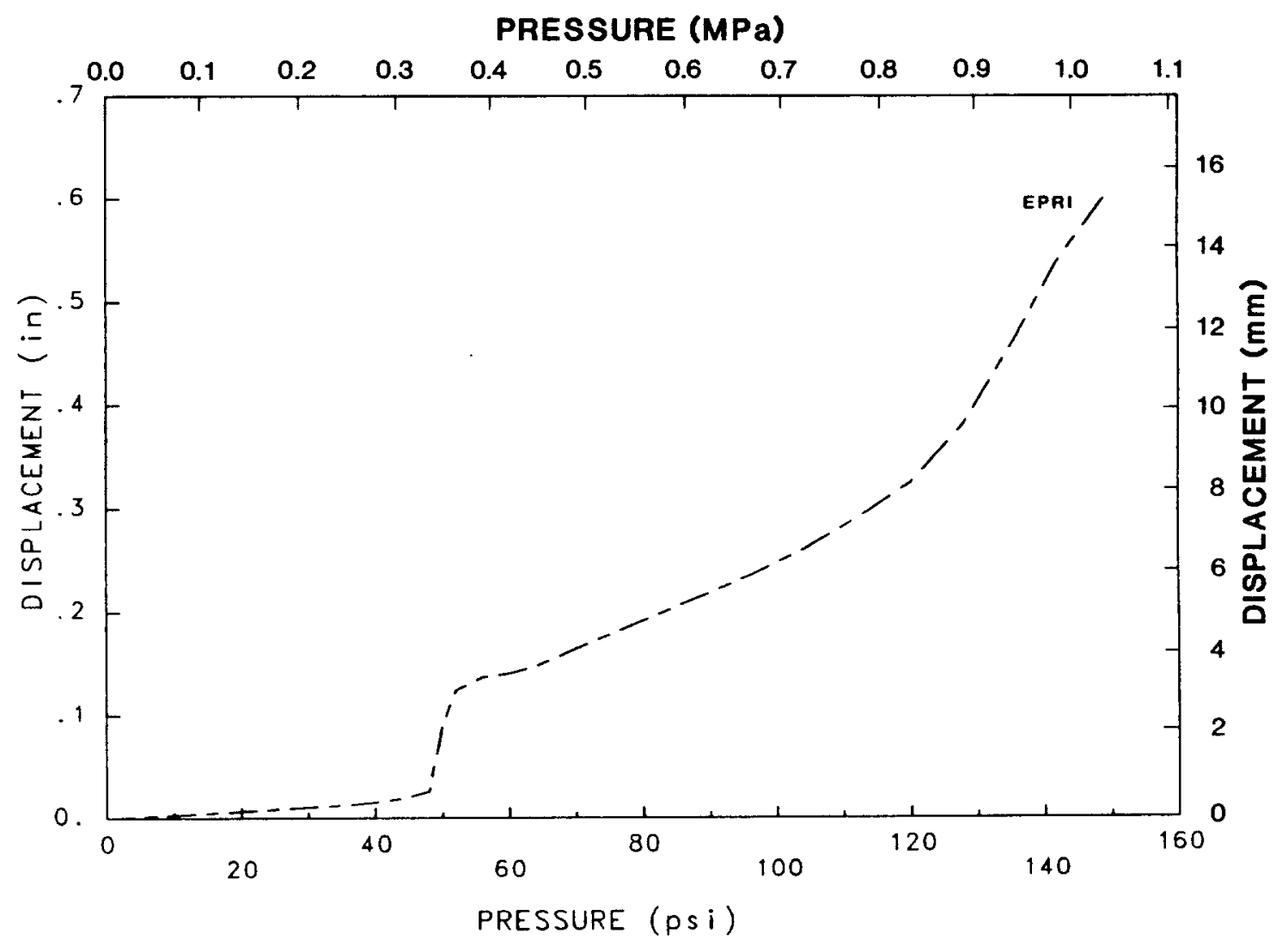

Figure A.39 Radial displacement of liner at EL 20'-1.5", $\theta=305$ (constrained pipe)

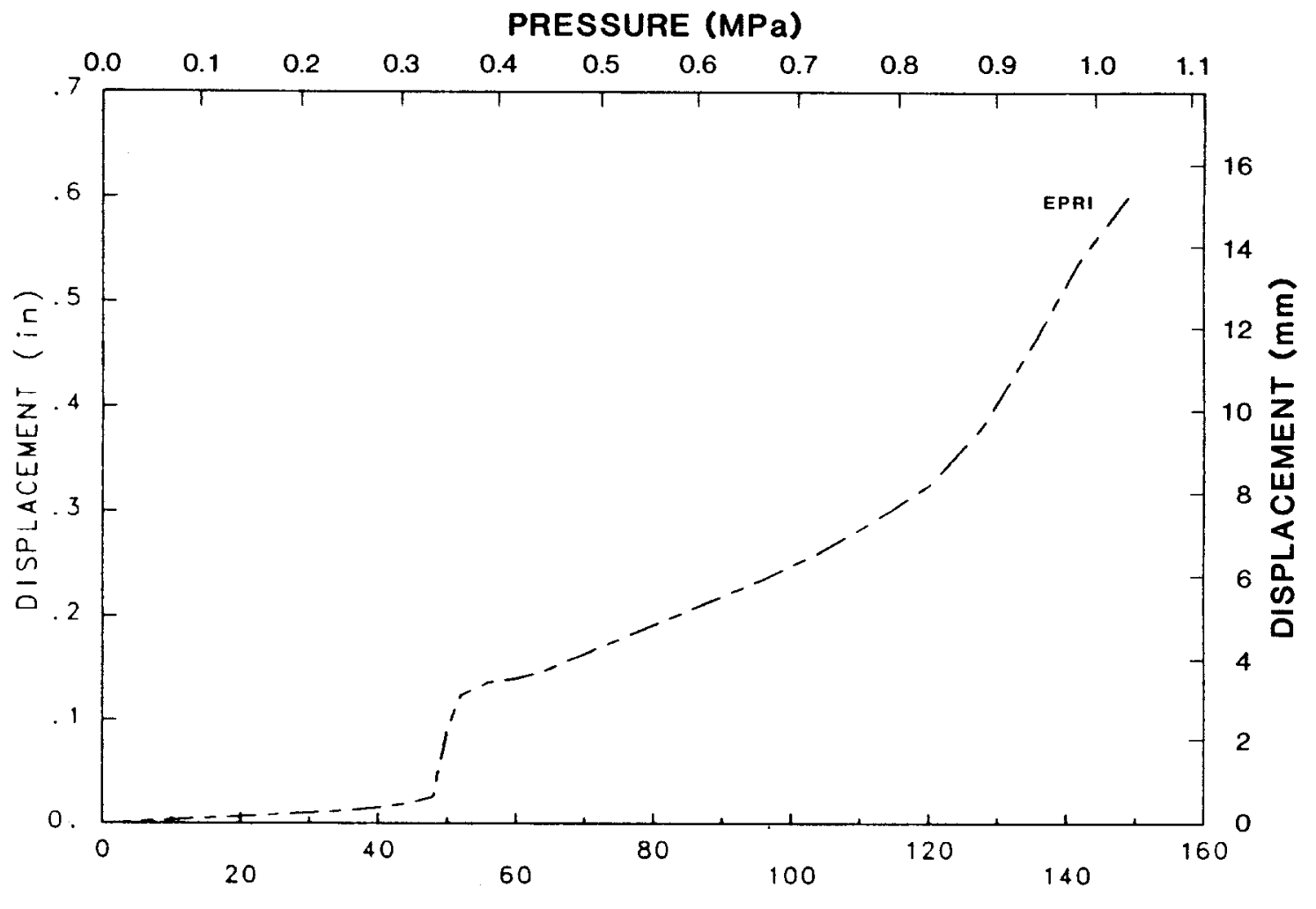

PRESSURE ( $p$ si)

Figure A.40 Radial displacement of liner at EL $20^{\prime}-1.5^{\prime \prime}, \theta=290$ (constrained pipe) 


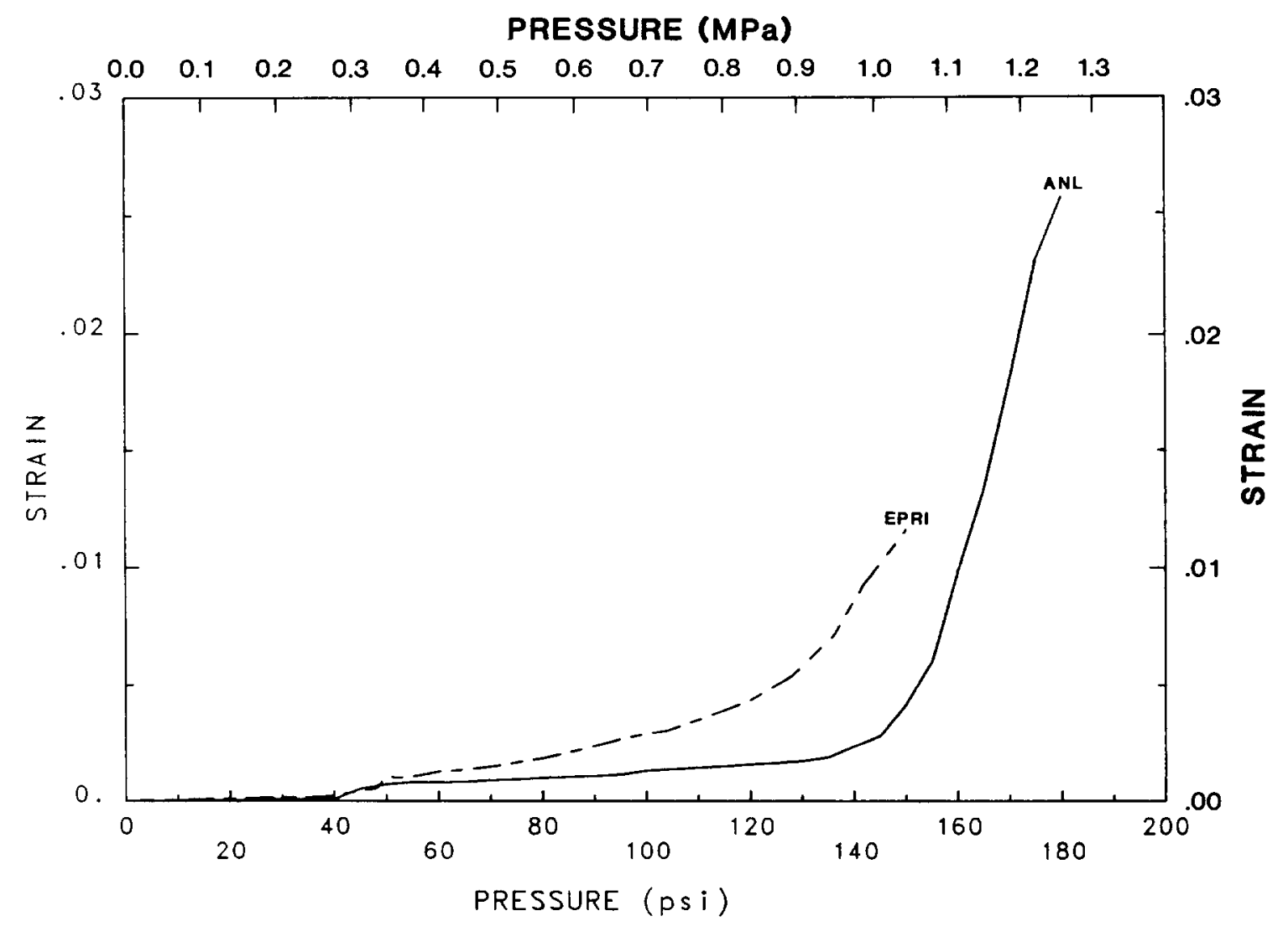

Figure A.41

Maximum principal strain on inside liner surface at EL $13^{\prime}-0^{\prime \prime}, \theta=158$ 


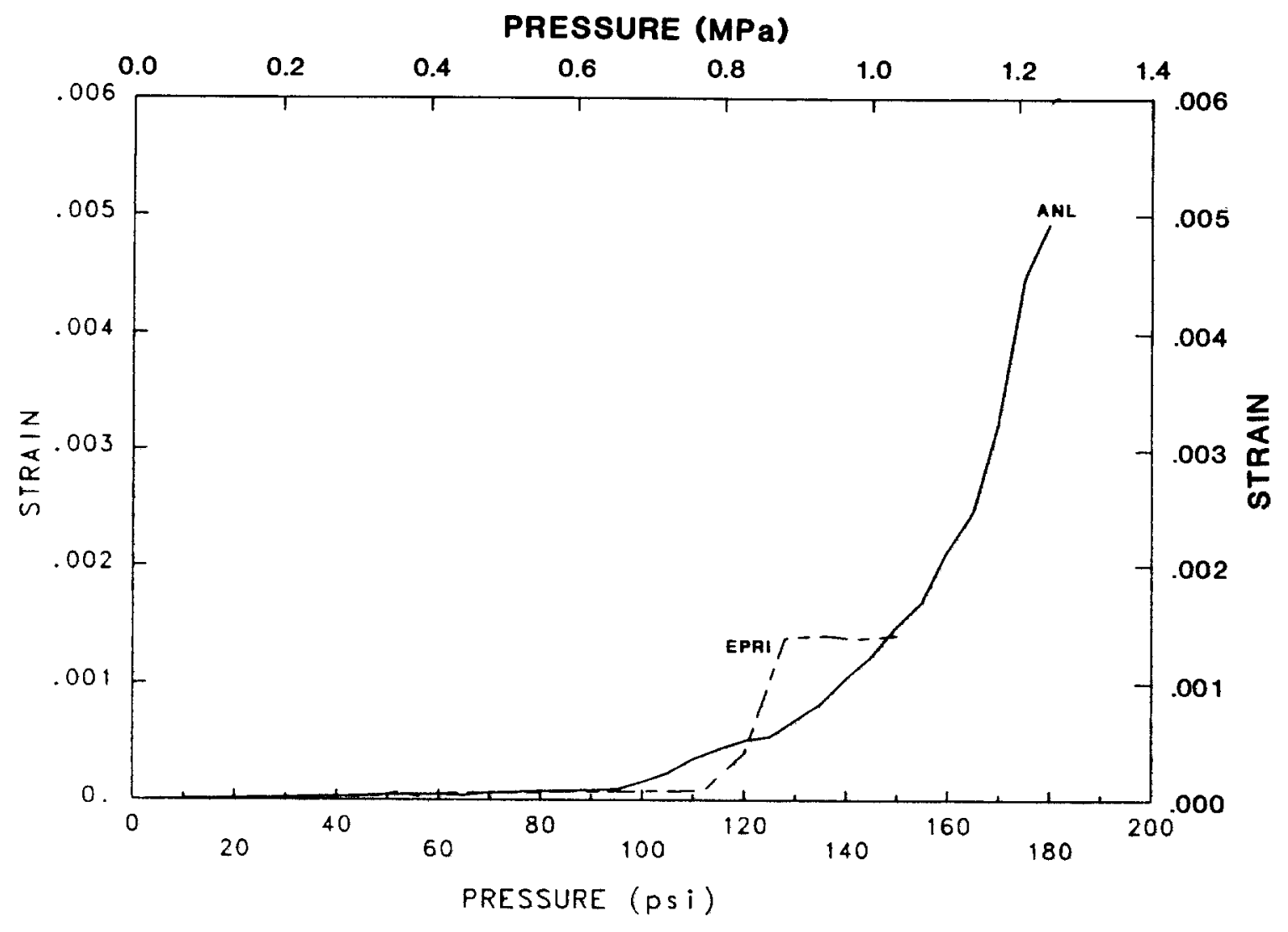

Figure A.42 Axial strain in layer 2 meridional rebar at EL $13^{\prime}-0^{\prime \prime}, \theta=158$

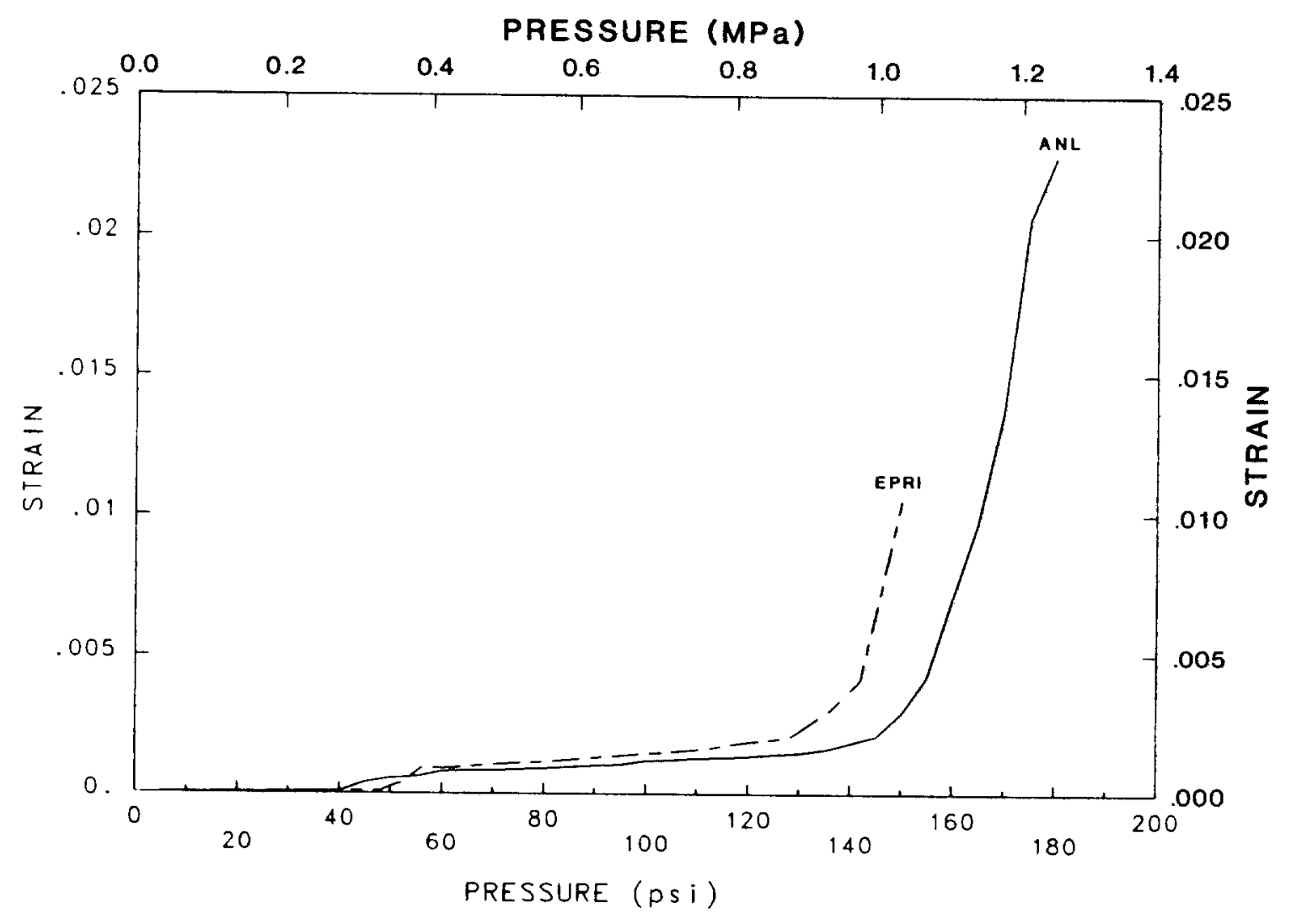

Figure A.43 Axial strain in layer 3 hoop rebar at EL $13^{\prime}-0^{\prime \prime}, \theta=158$ 
PRESSURE (MPa)

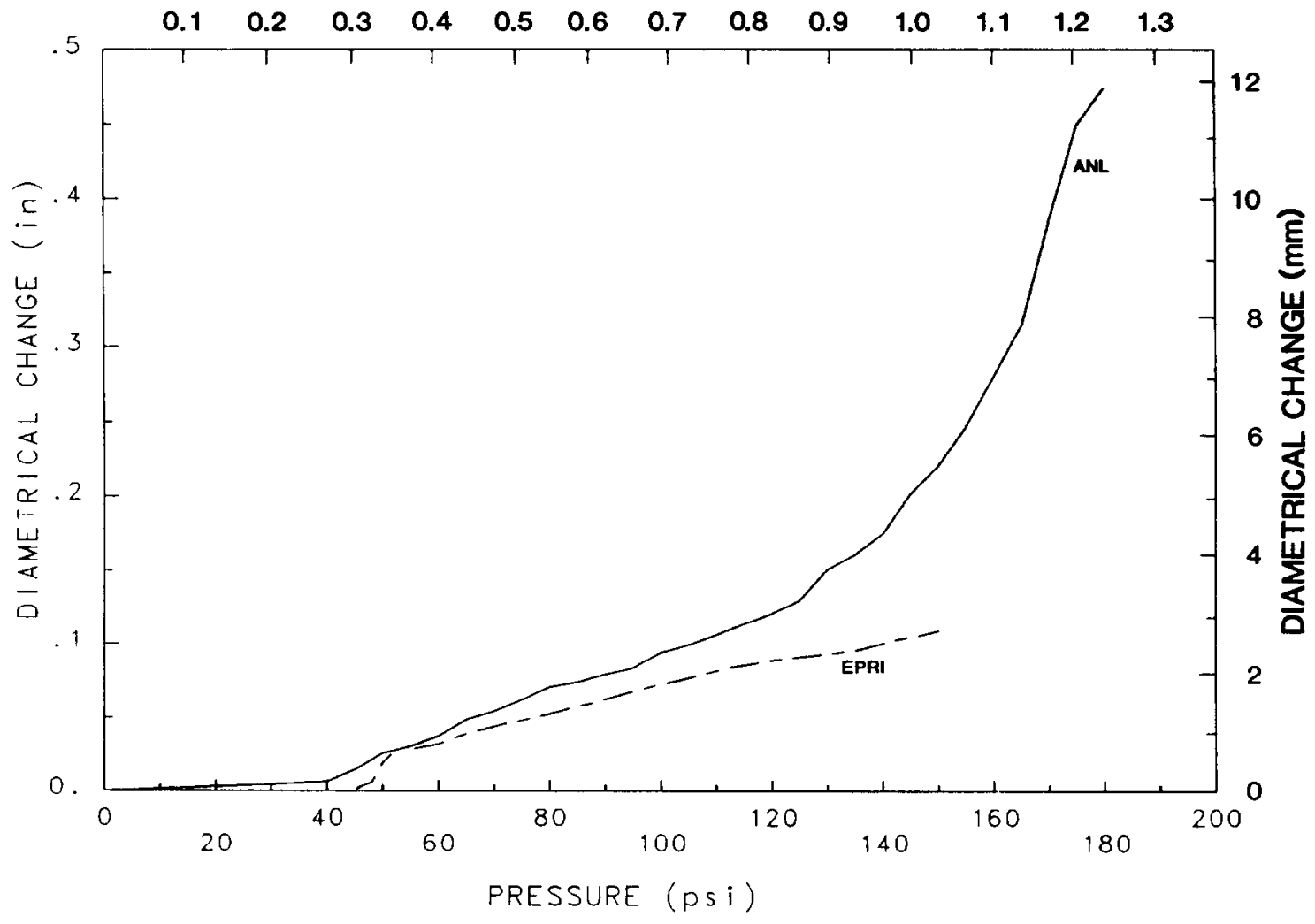

Figure A.44 Change in sleeve horizontal diameter

(Equipment hatch $B$ at mid-thickness of cylinder wall)

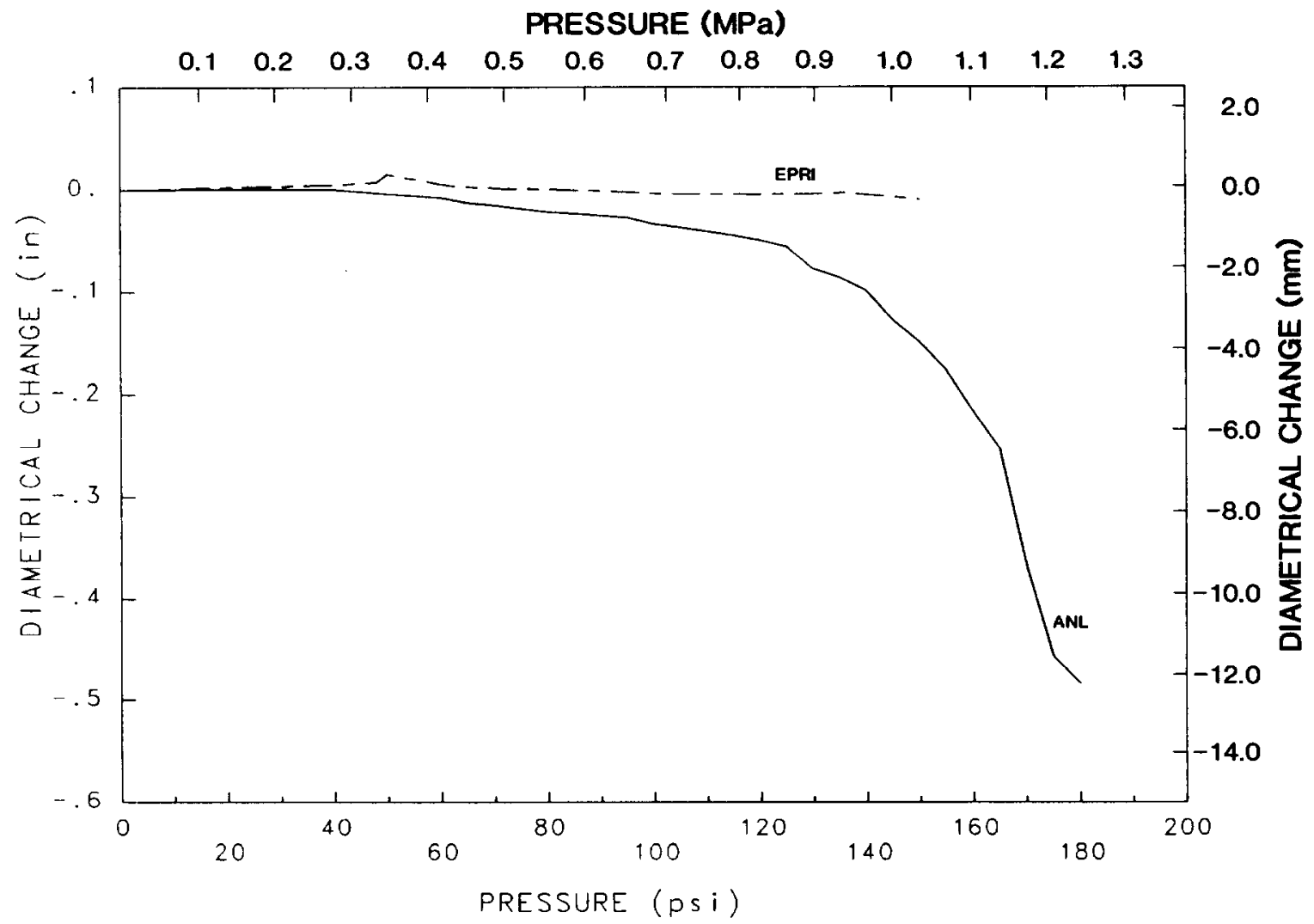

Figure A.45 Change in sleeve vertical diameter (Equipment hatch $B$ at mid-thickness of cylinder wall) 


\section{POSITION (m)}

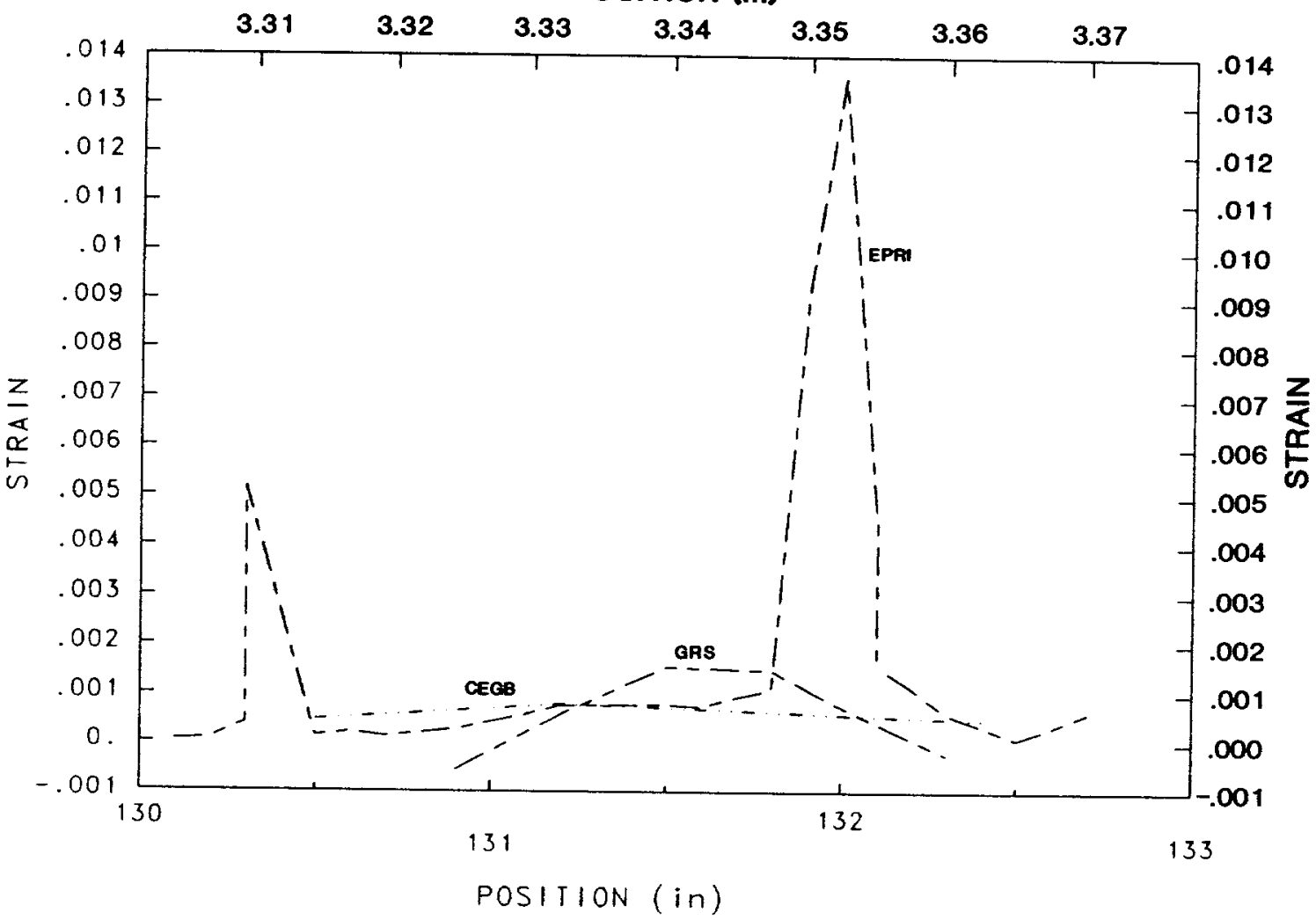

Figure A.46 Maximum principal strain on inside surface of liner vs position (knuckle region) at $100 \mathrm{psi}(0.689 \mathrm{MPa}$ )

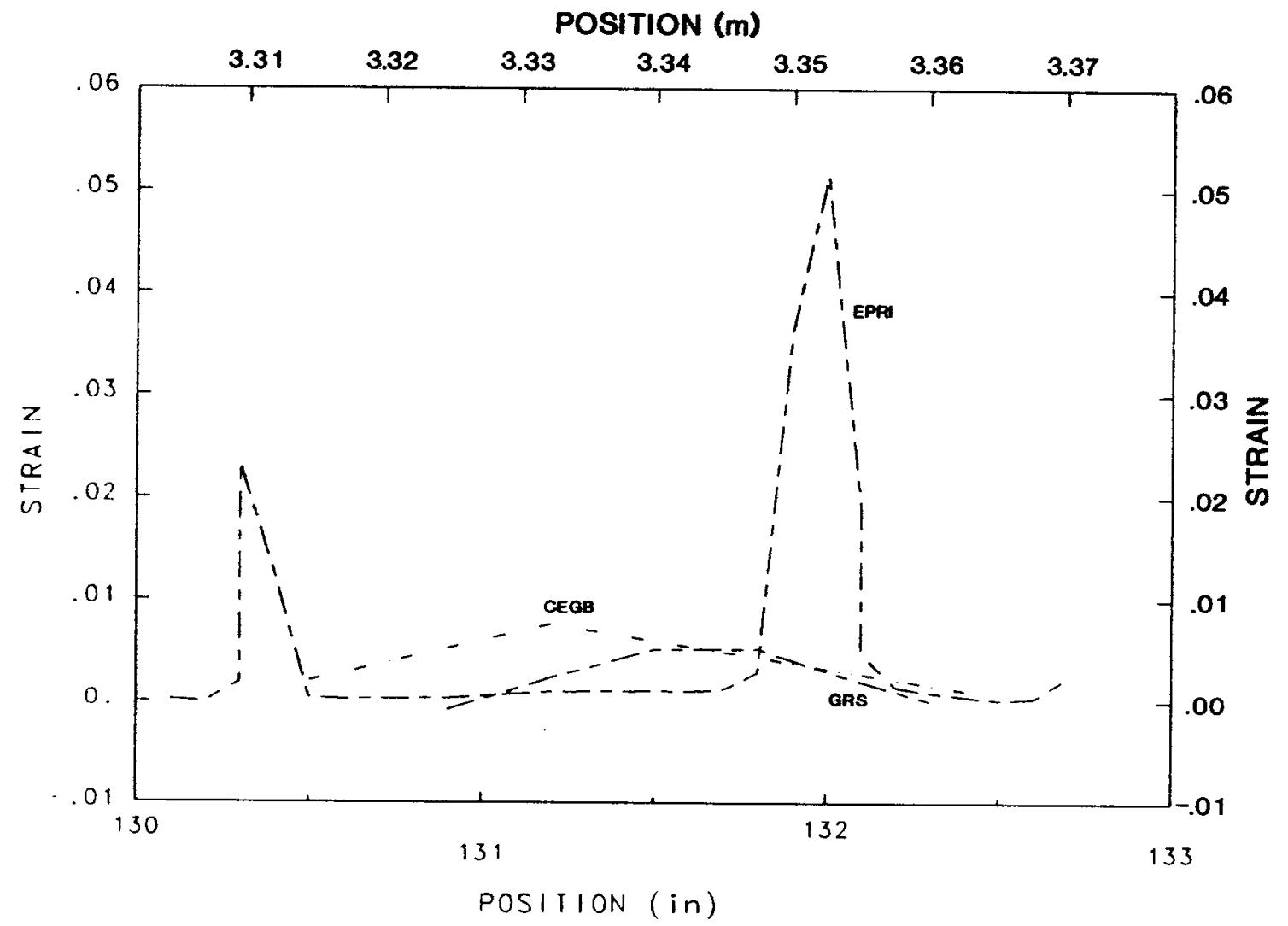

Figure A.47 Maximum principal strain on inside surface of liner vs position (knuckle region) at 150 psi $(1.034 \mathrm{MPa}$ ) 


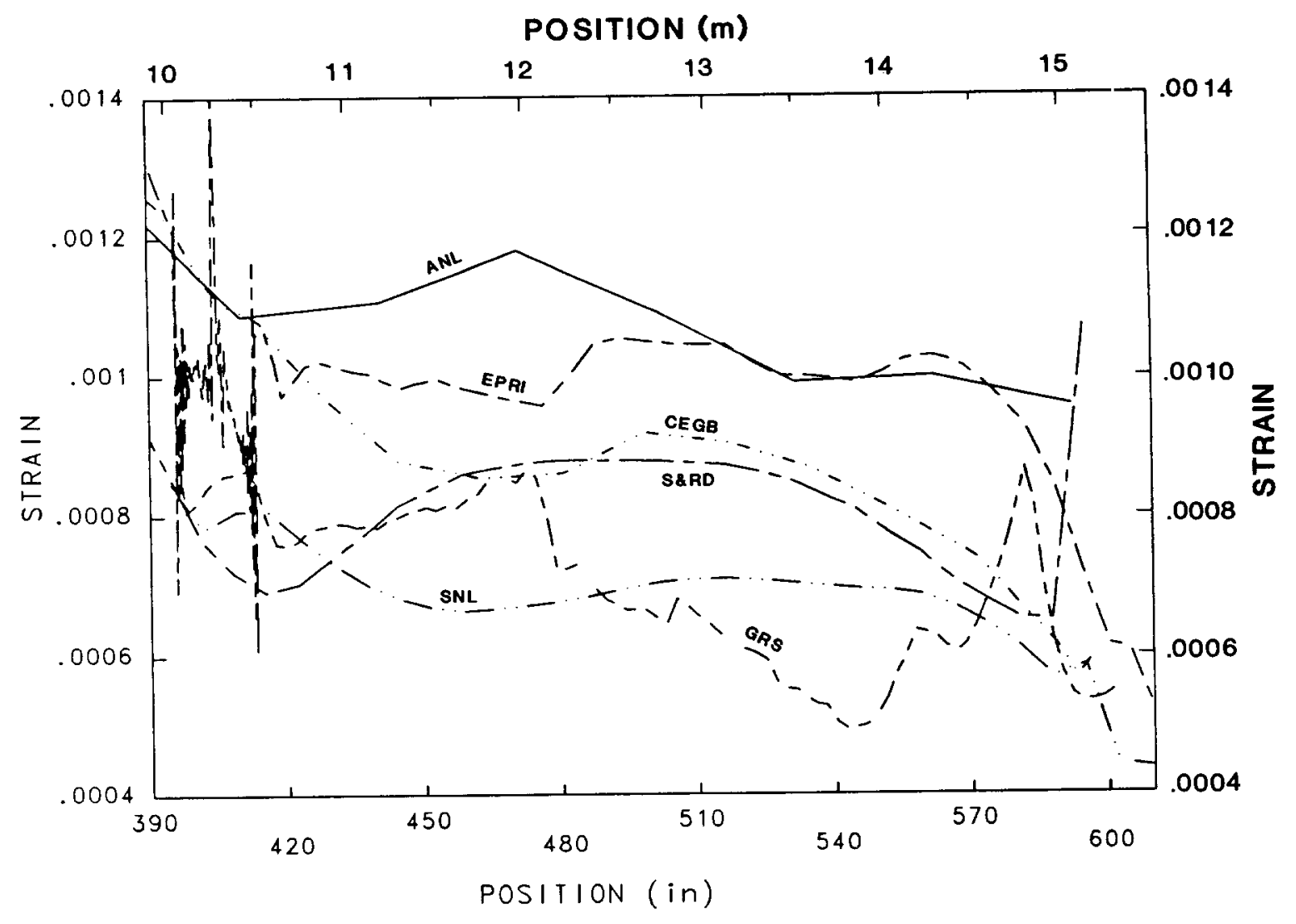

Figure A.49 Maximum principal strain on inside surface of liner vs position (springline and dome region) at $100 \mathrm{psi}(0.689 \mathrm{MPa}$ )

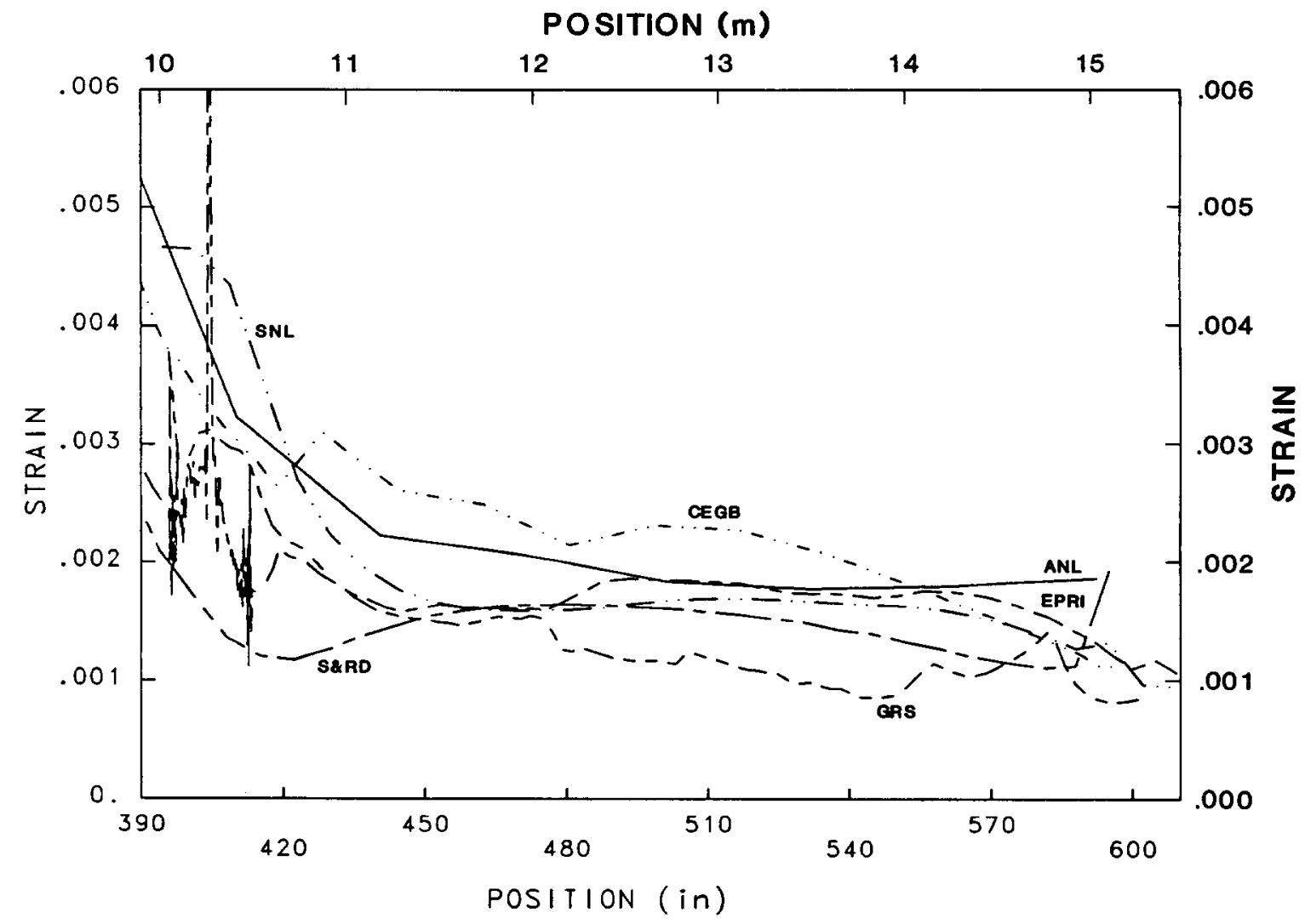

Figure A.50 Maximum principal strain on inside surface of liner vs position (springline and dome region) at $150 \mathrm{psi}$ ( $1.034 \mathrm{MPa}$ ) 


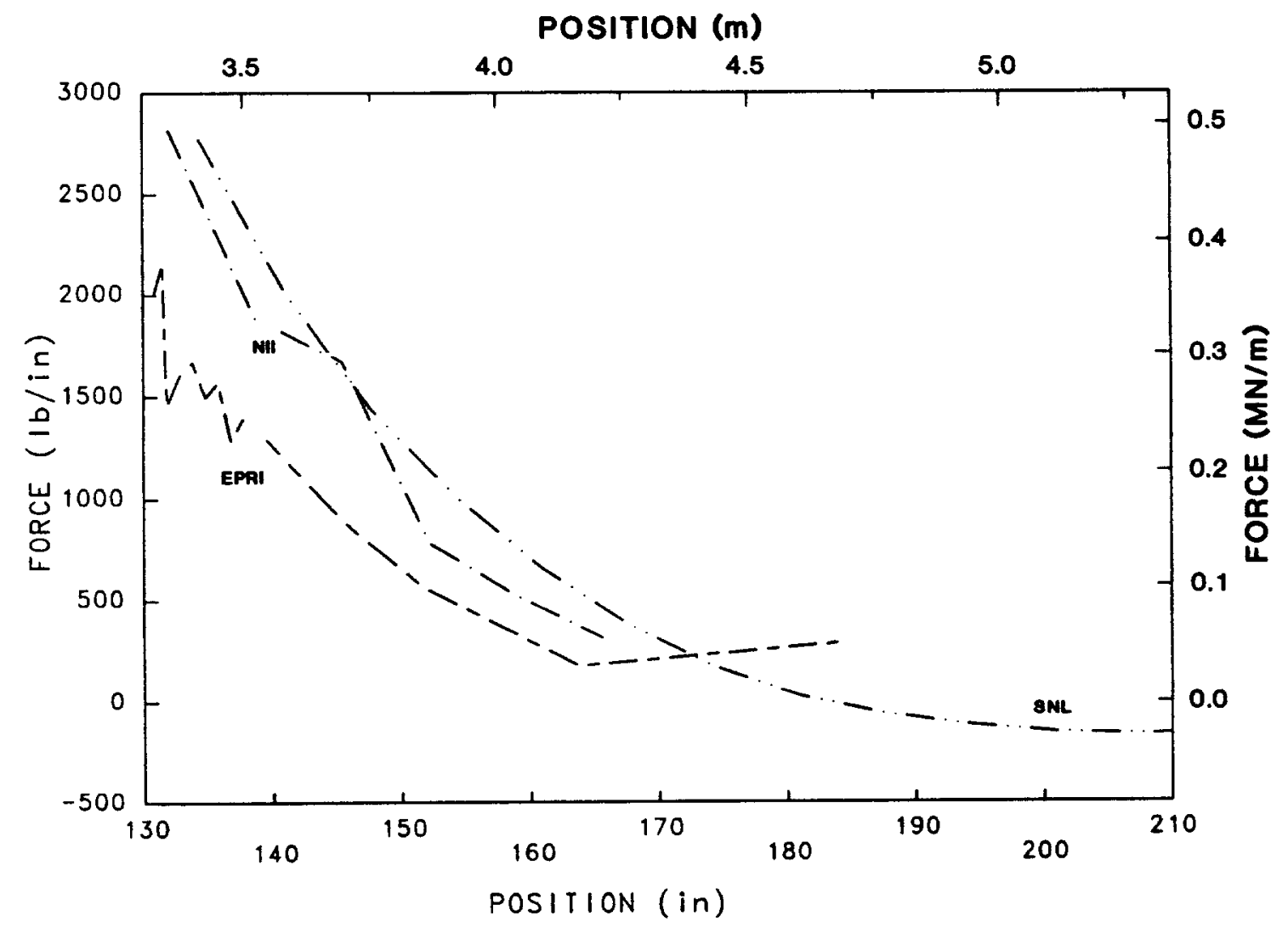

Figure A.52 Radial shear vs pressure (cylinder from basemat to termination of layer 11 rebar) at $100 \mathrm{psi}(0.689 \mathrm{MPa})$

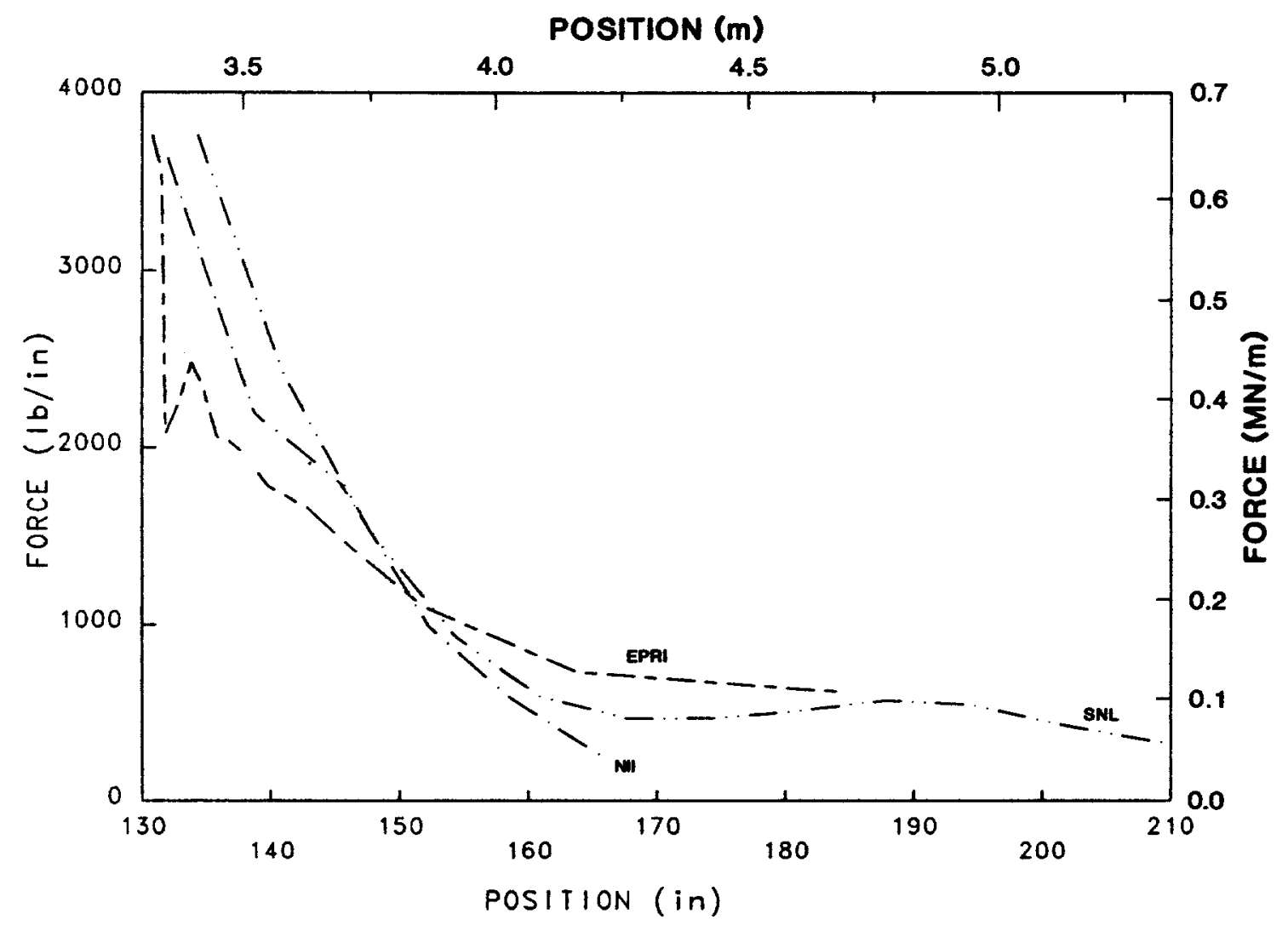

Figure A.53 Radial shear vs pressure (cylinder from basemat to termination of layer 11 rebar) at $150 \mathrm{psi}(1.034 \mathrm{MPa})$ 


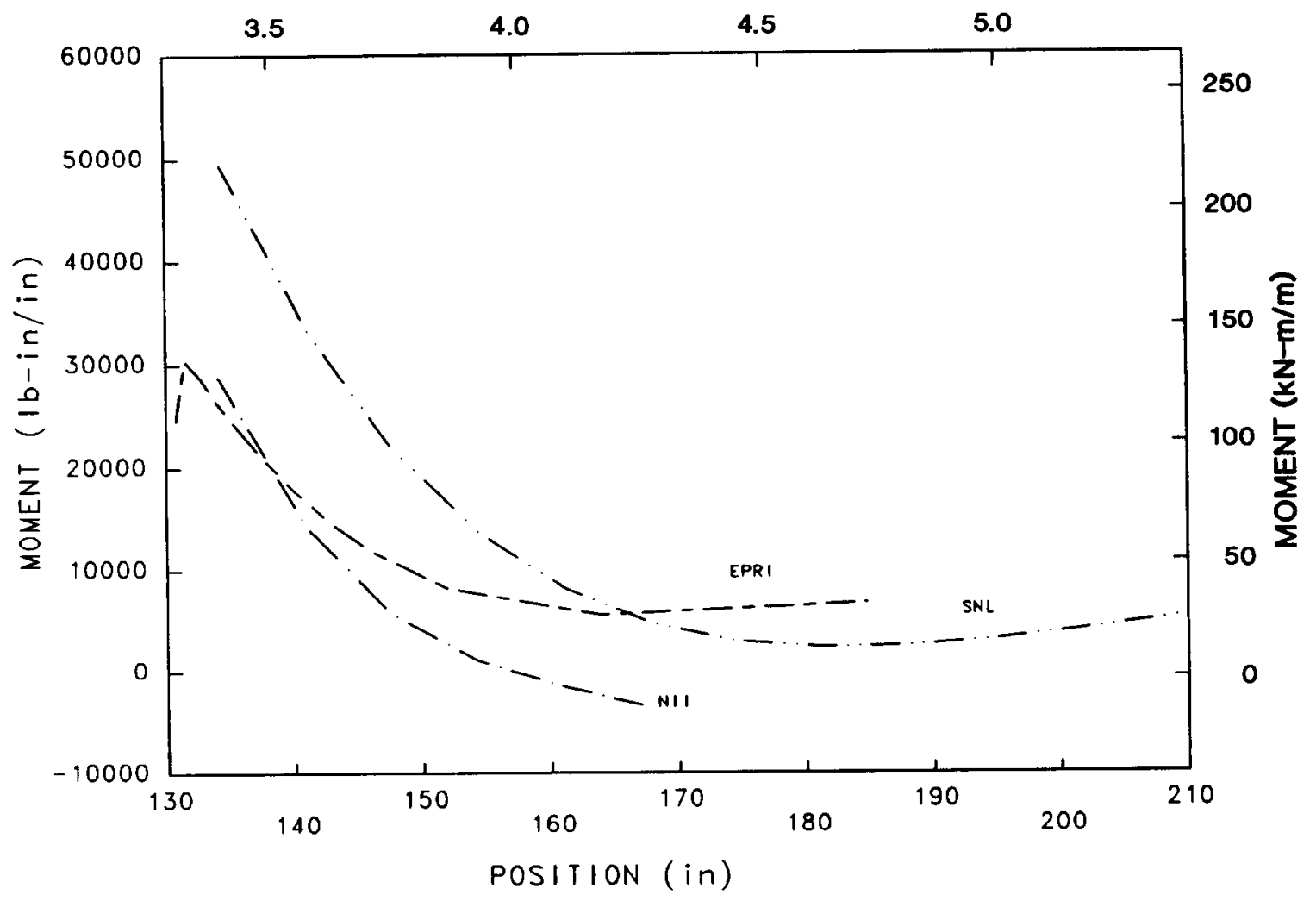

Figure A.55 Meridional moment vs position (cylinder from.basemat to termination of layer 11 rebar) at $100 \mathrm{psi}(0.689 \mathrm{MPa})$

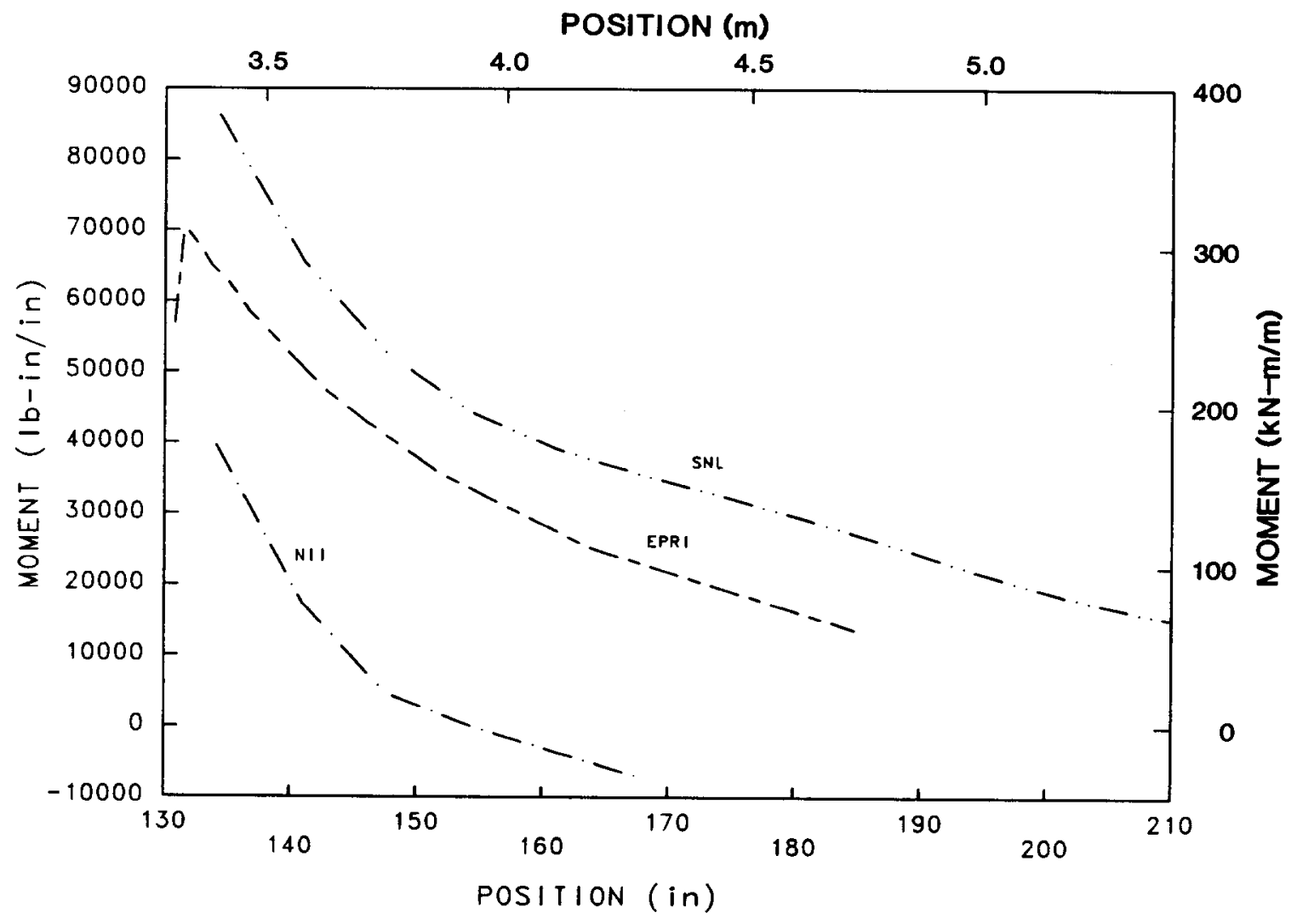

Figure A.56 Meridional moment vs position (cylinder from basemat to termination of layer 11 rebar) at $150 \mathrm{psi}(1.034 \mathrm{MPa})$ 


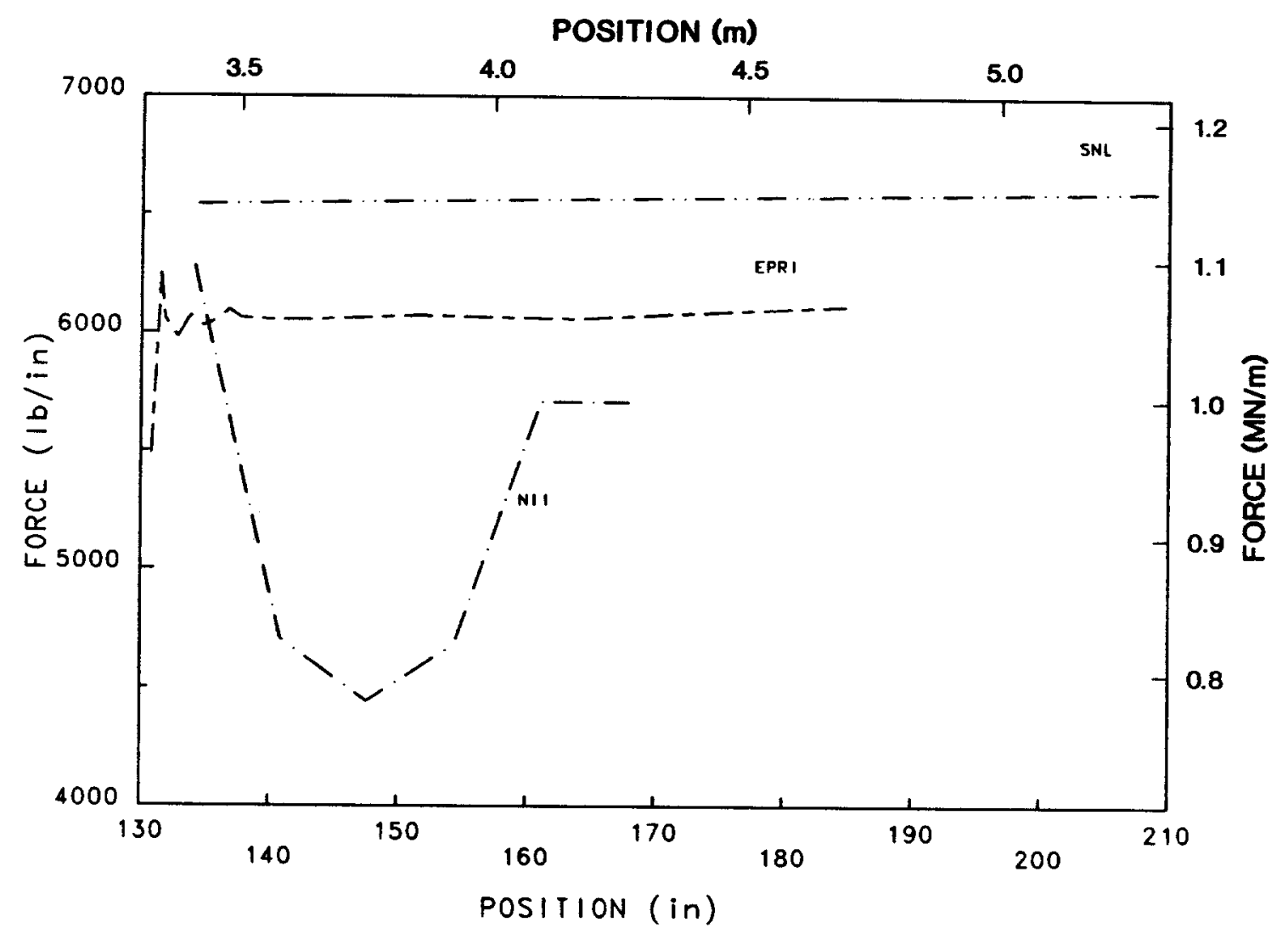

Figure A.58 Axial force vs position (cylinder from basemat to termination of layer 11 rebar) at 100 psi $(0.689 \mathrm{MPa})$

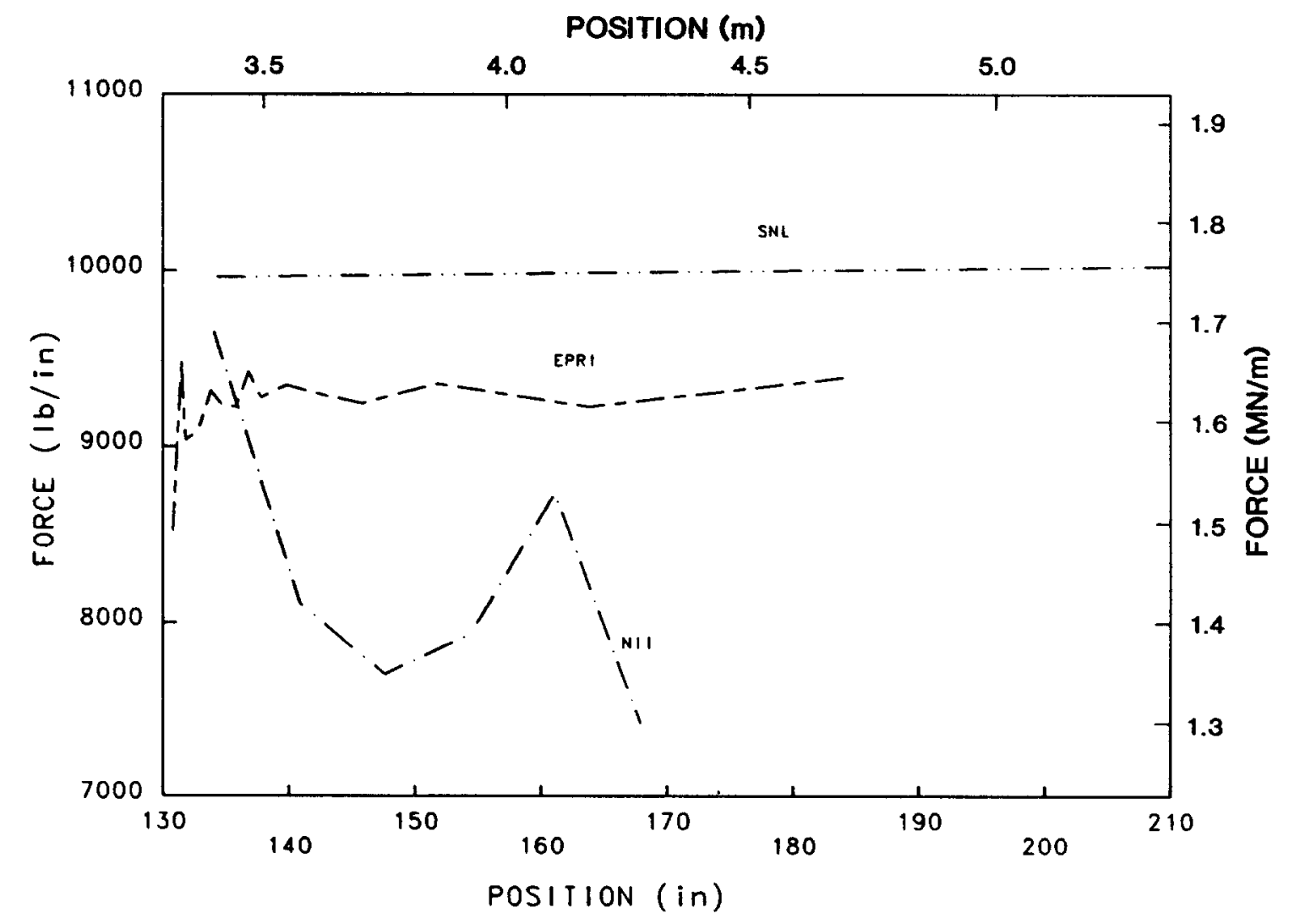

Figure A.59 Axial force vs position (cylinder from basemat to termination of layer 11 rebar) at 150 psi (1.034 MPa) 


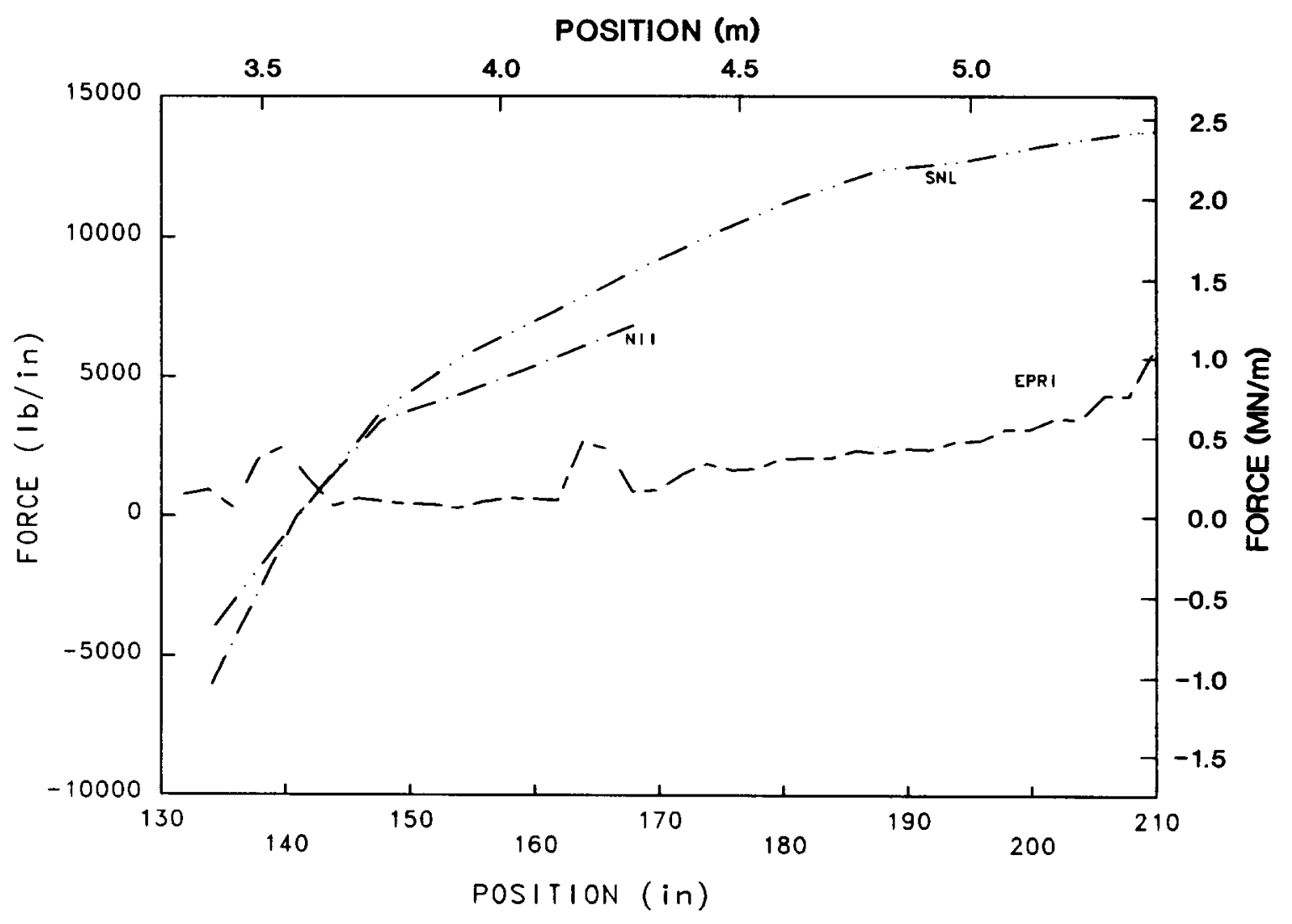

Figure A.61 Hoop force vs position (cylinder from basemat to termination of layer 11 rebar) at $100 \mathrm{psi}(0.689 \mathrm{MPa})$

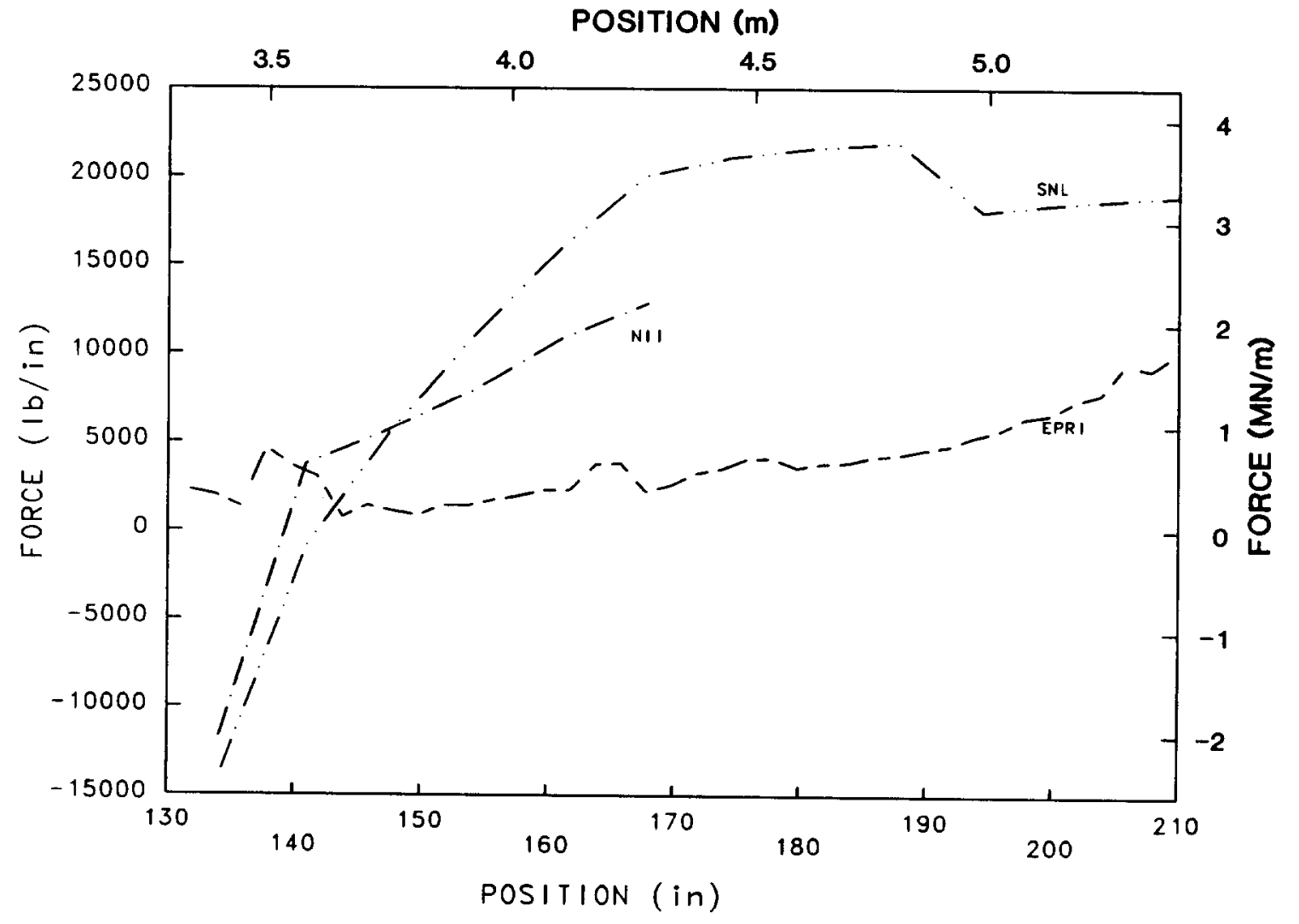

Figure A.62 Hoop force vs position (cylinder from basemat to termination of layer 11 rebar) at $150 \mathrm{psi}(1.034 \mathrm{MPa})$ 


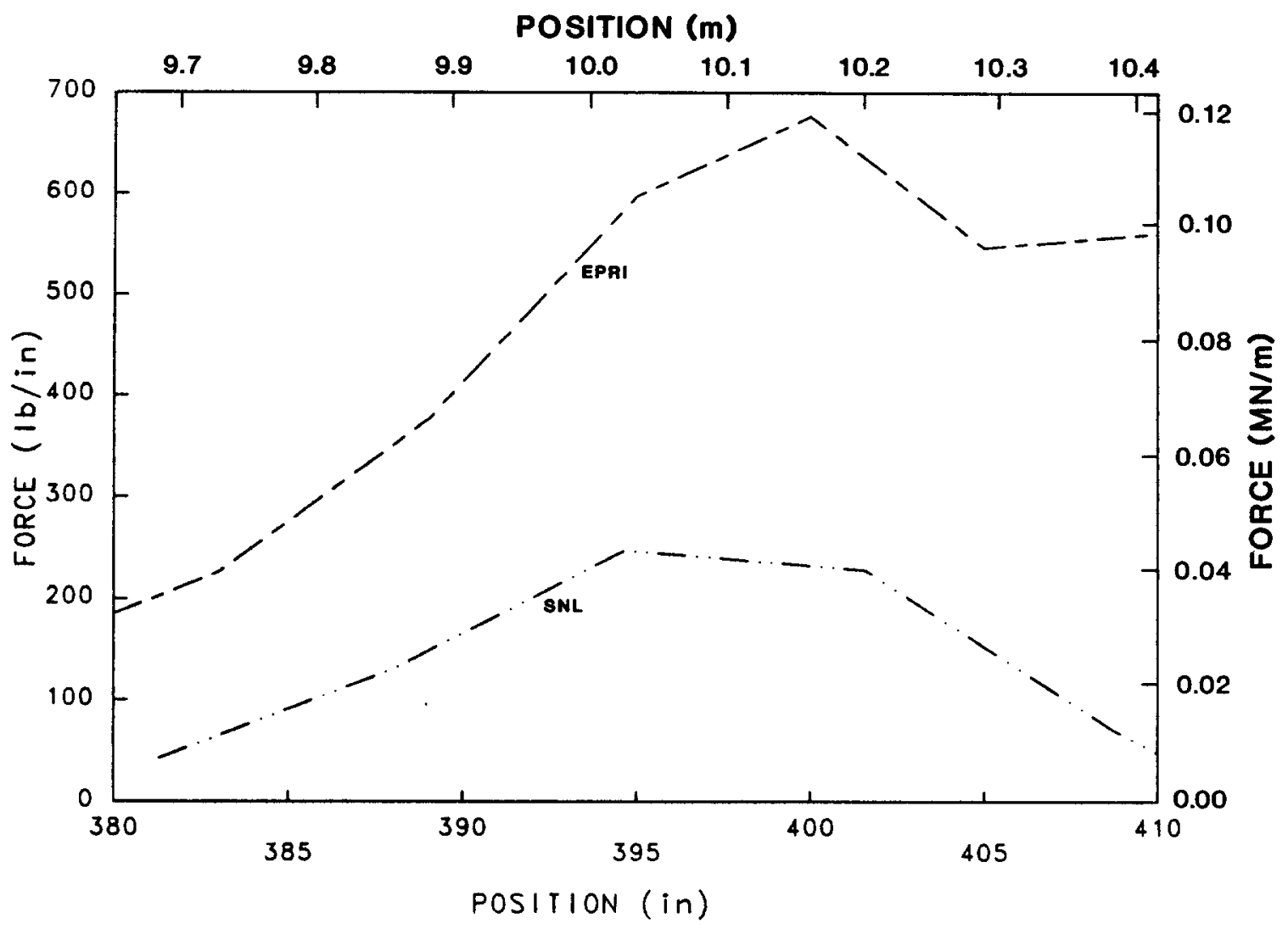

Figure A.64

Radial shear vs position (springline region) at 100 psi (0.689 $\mathrm{MPa}$ )

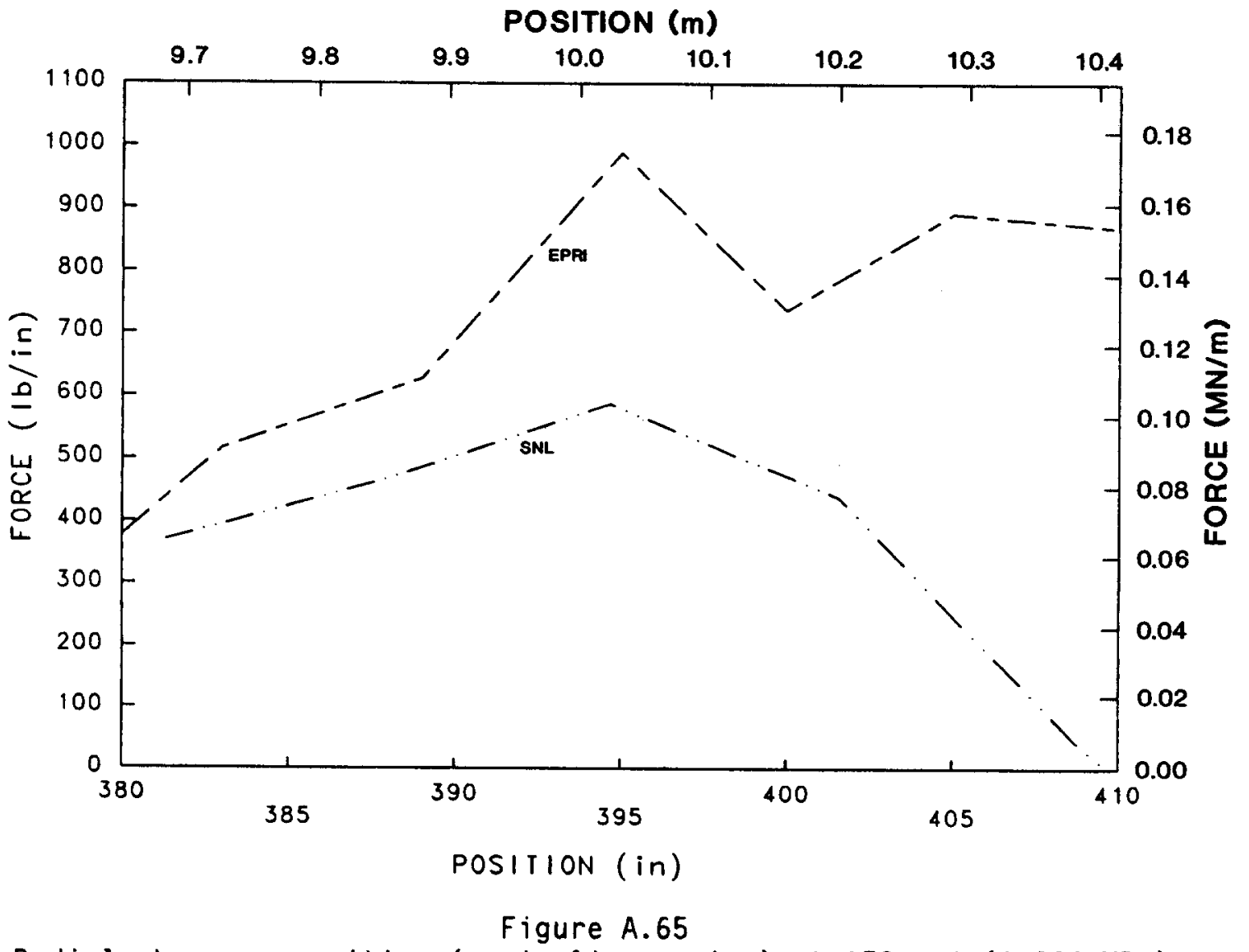

Radial shear vs position (springline region) at 150 psi ( $1.034 \mathrm{MPa}$ ) 


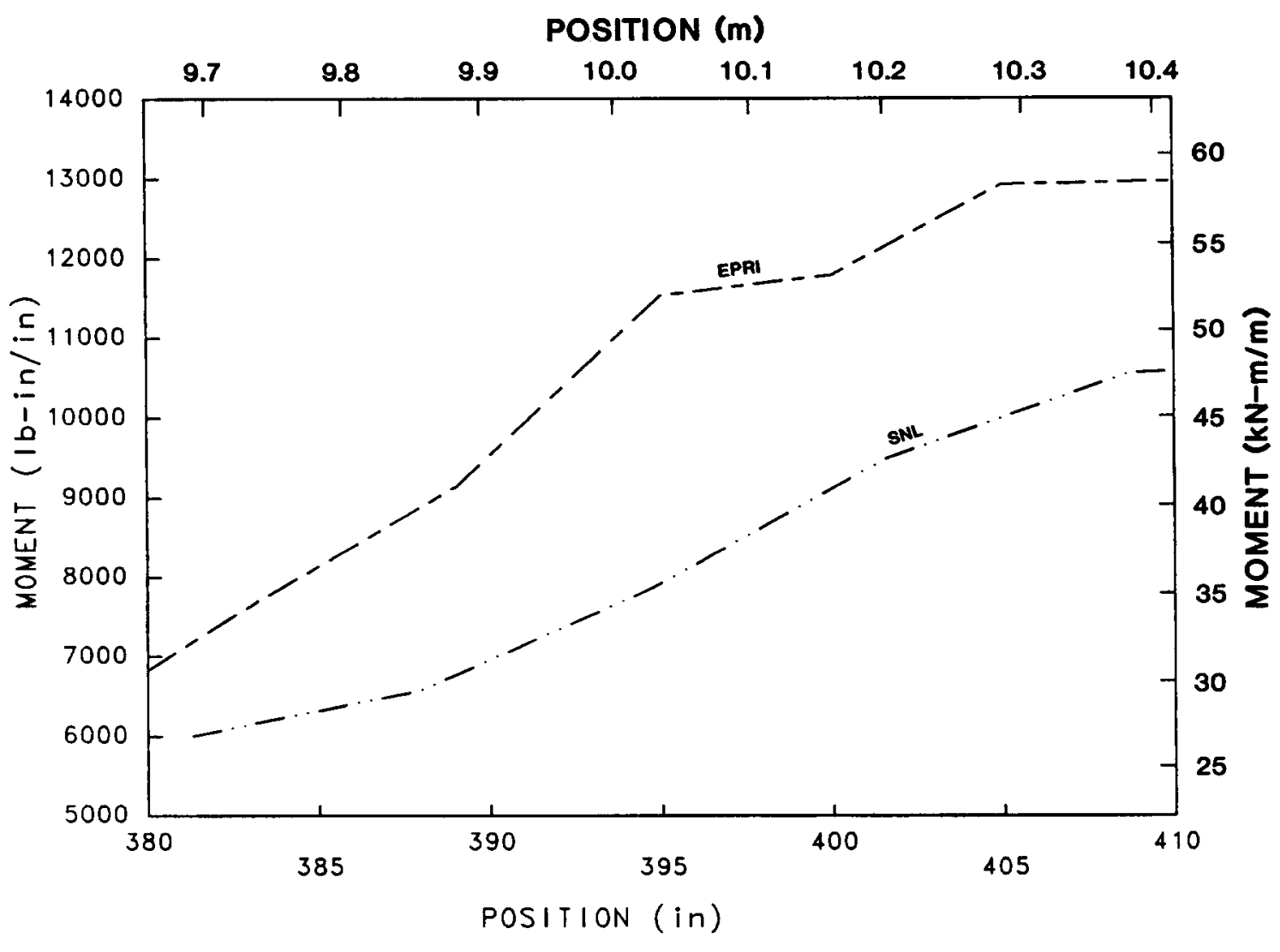

Figure A.67

Meridional moment vs position (springline region) at 100 psi (0.689 $\mathrm{MPa}$ )

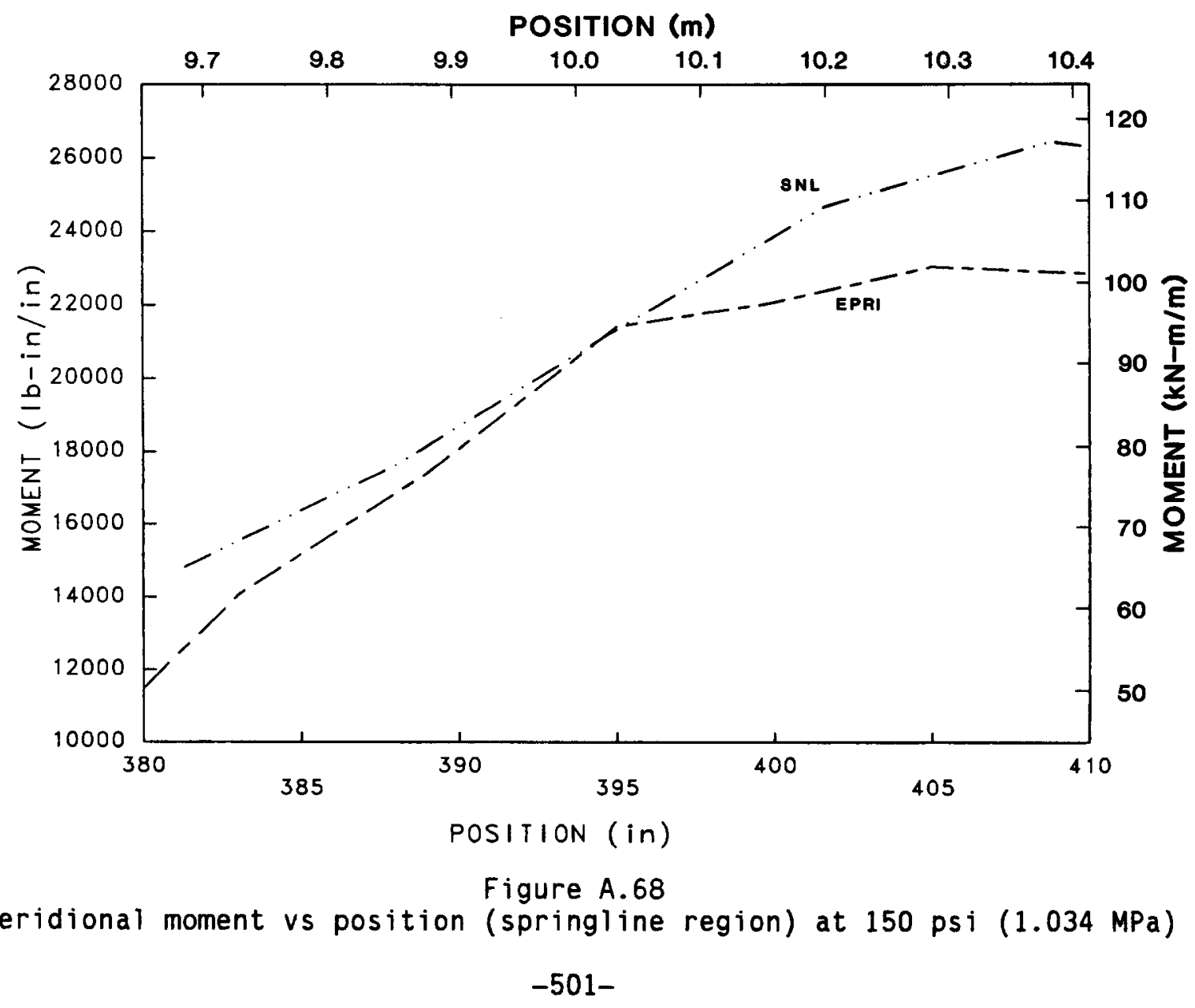




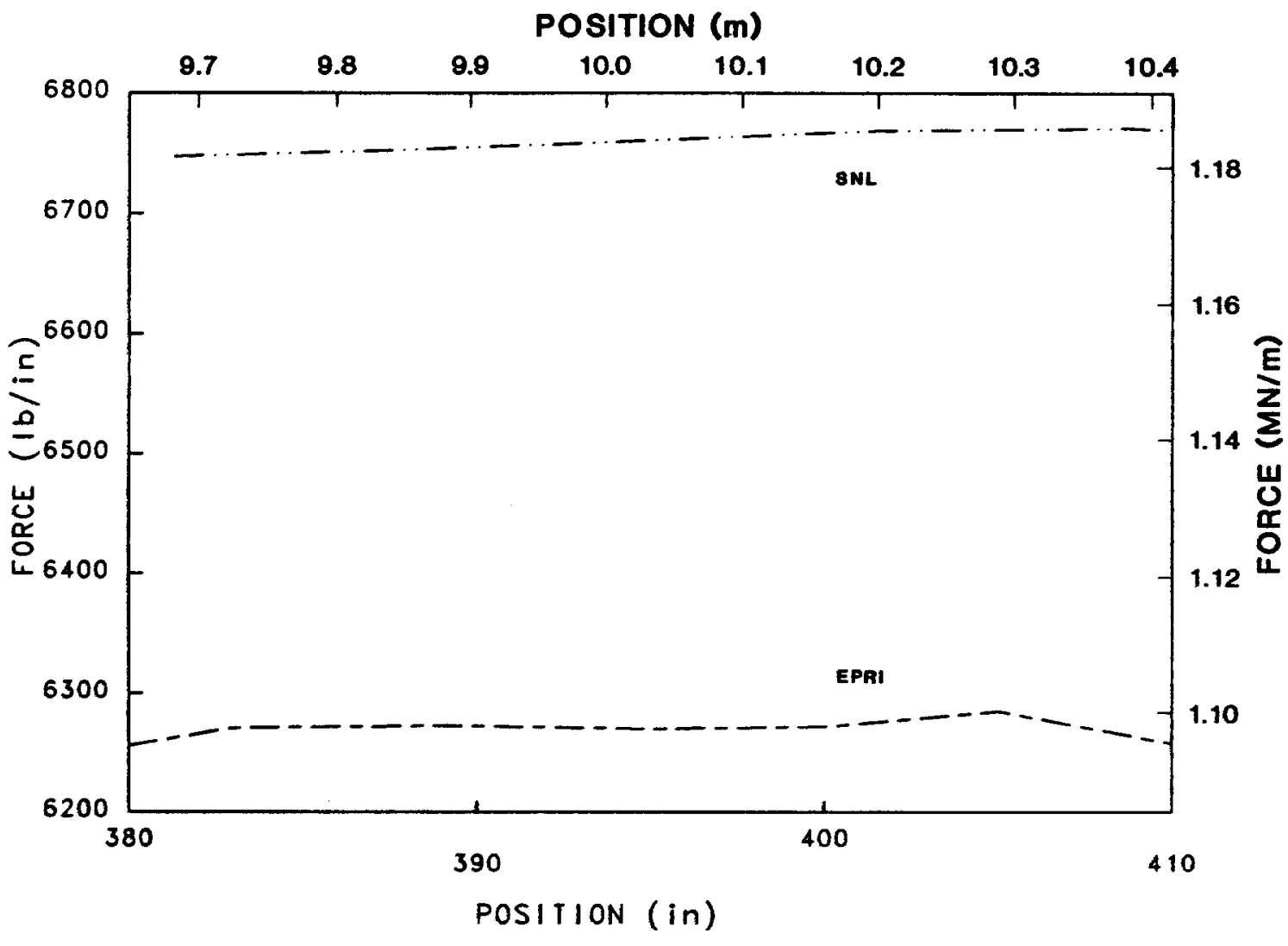

Figure A.70

Axial force vs position (springline region) at $100 \mathrm{psi}$ ( $0.689 \mathrm{MPa}$ )

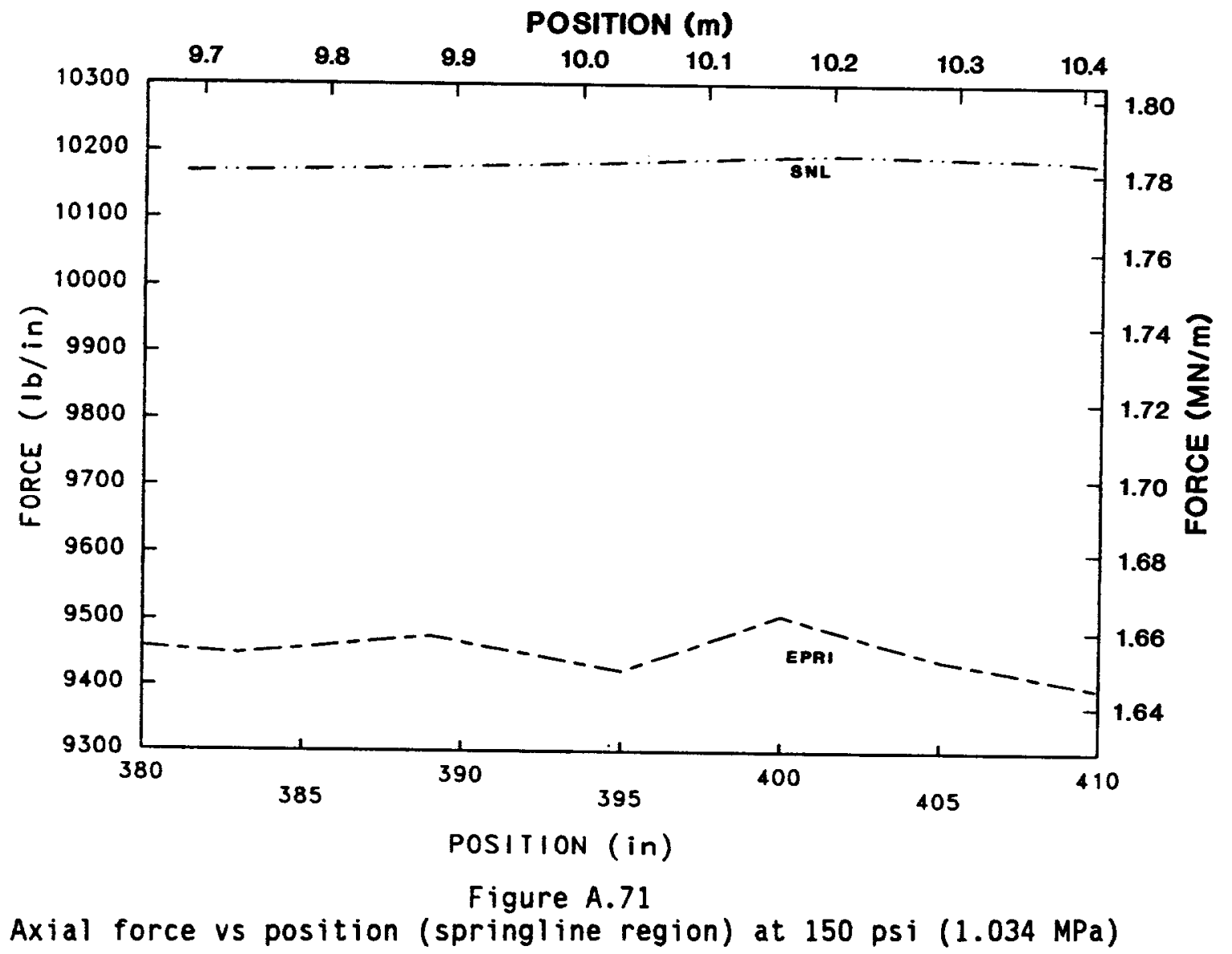




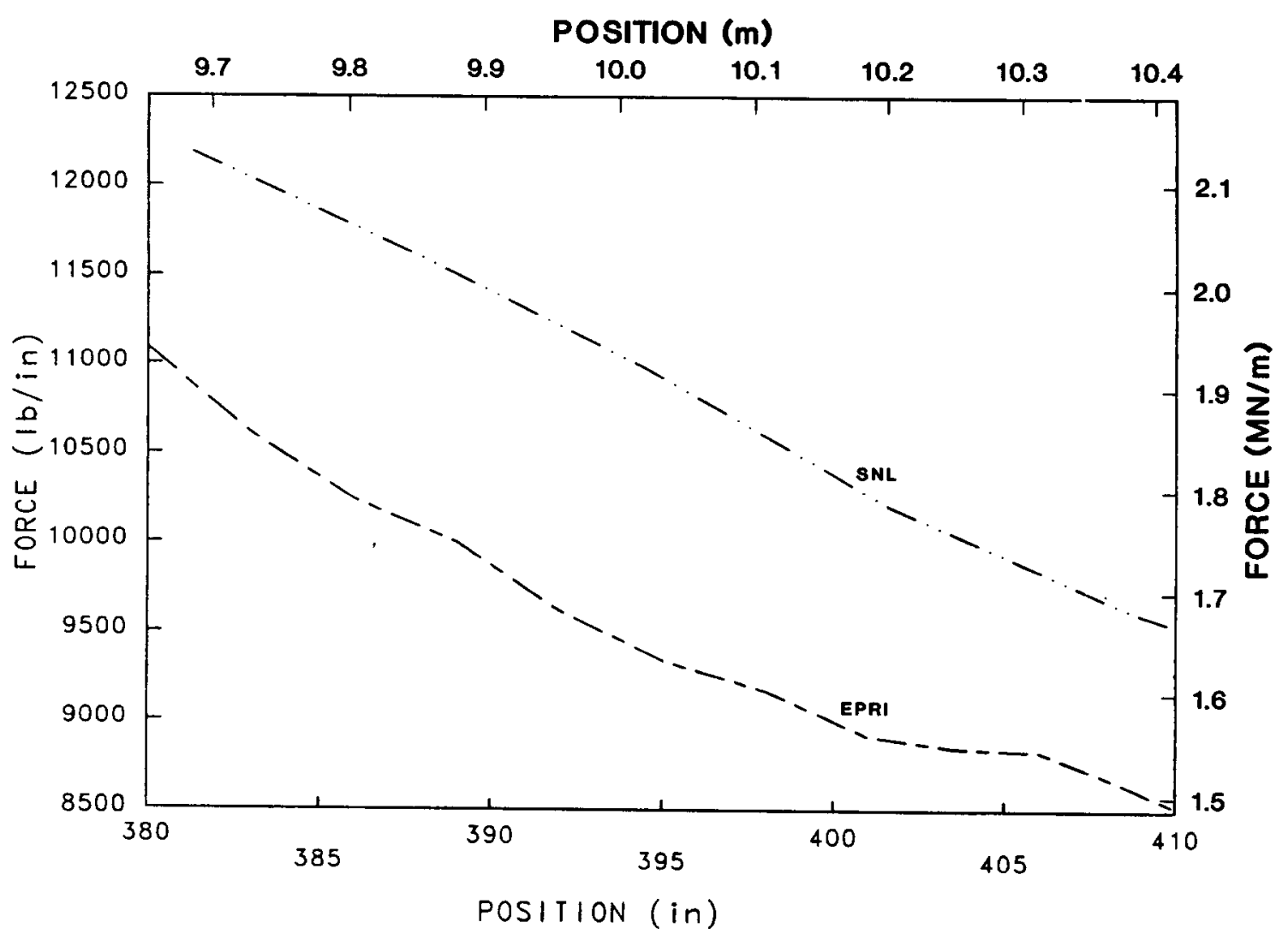

Figure $A .73$

Hoop force vs position (springline region) at $100 \mathrm{psi}(0.689 \mathrm{MPa}$ )

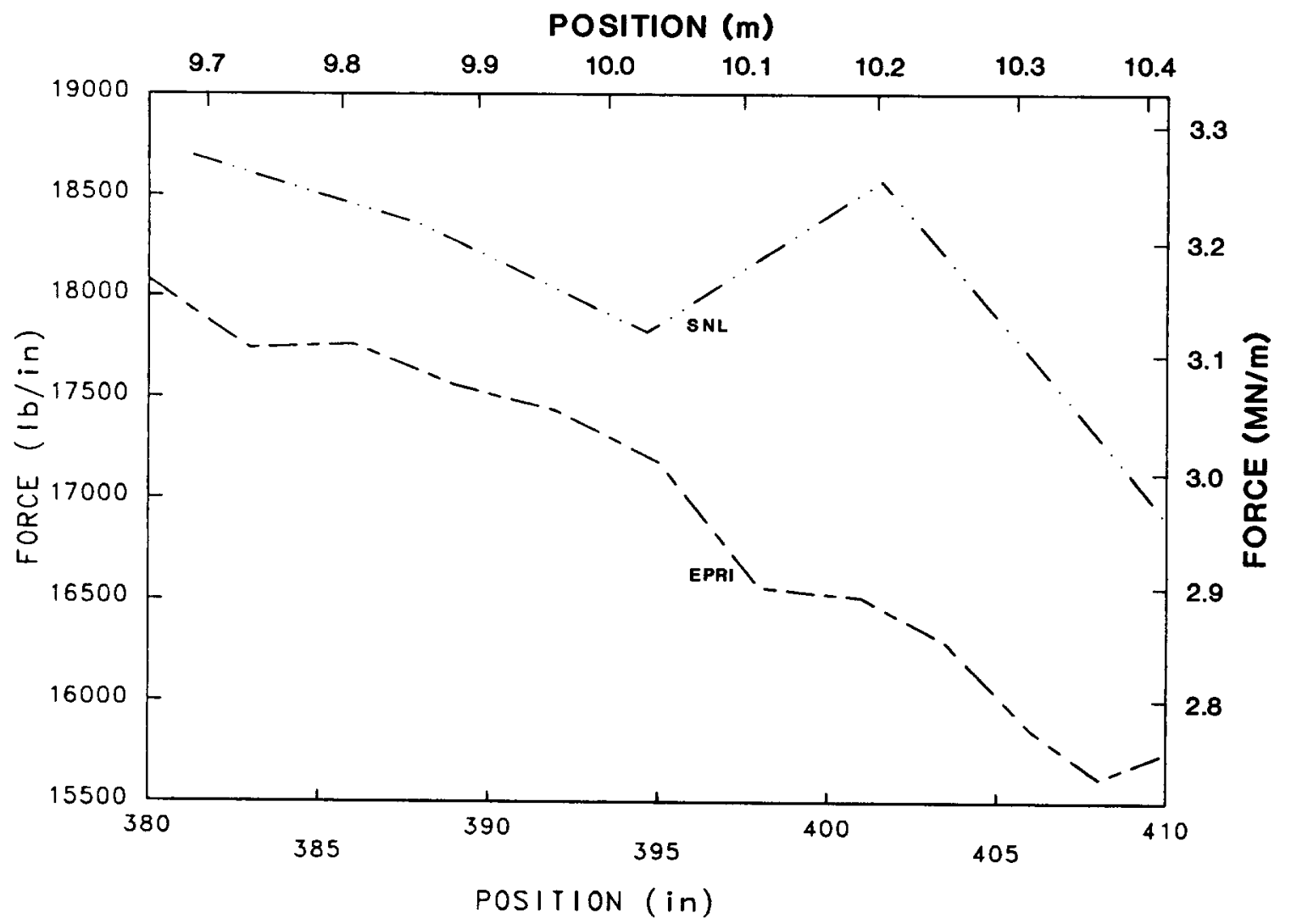

Figure A.74

Hoop force vs position (springline region) at $150 \mathrm{psi}$ (1.034 MPa) 


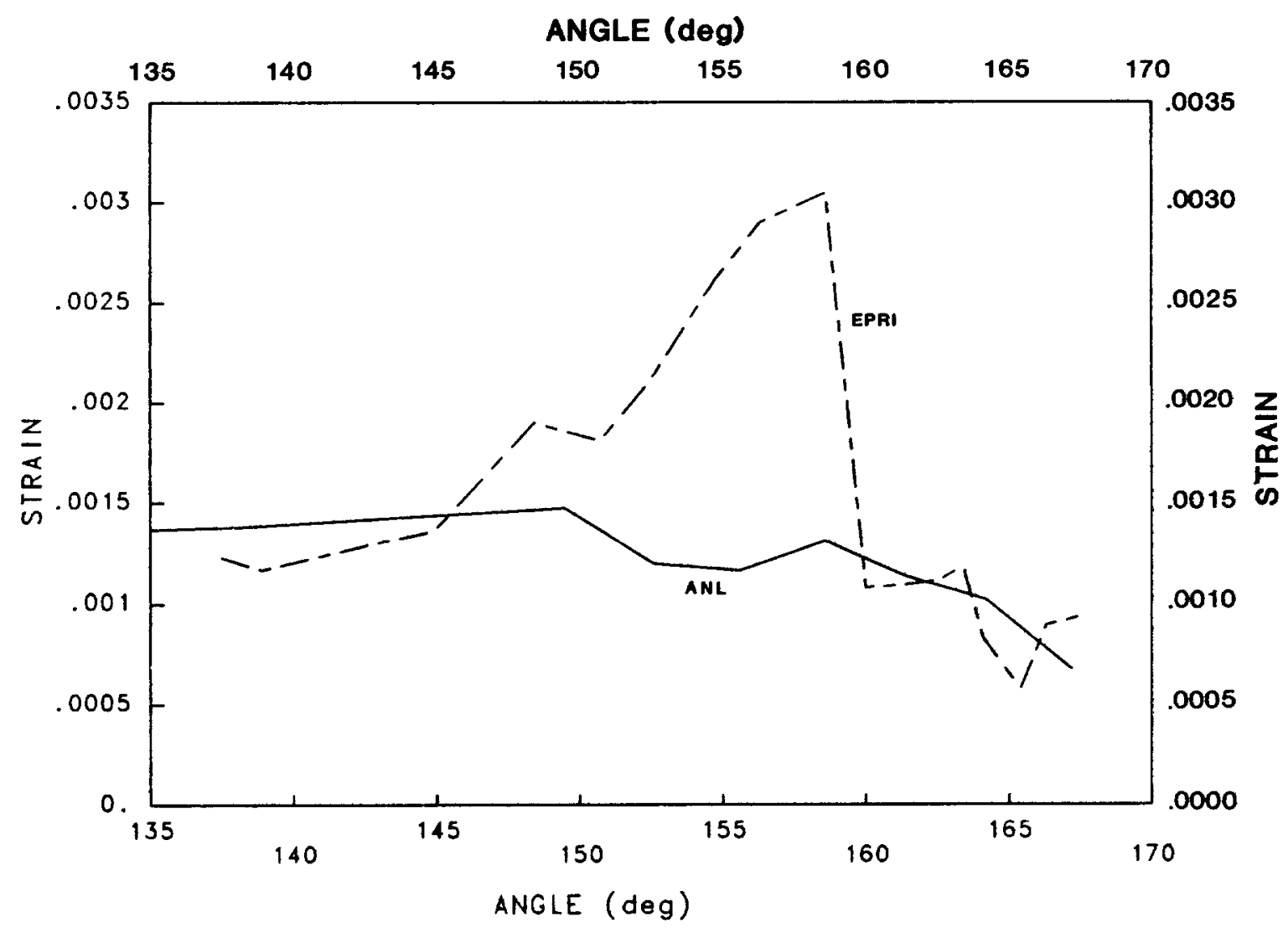

Figure A.76 Maximum principal strain on inside liner surface at EL $13^{\prime}-0^{\prime \prime}$ vs $\theta$ (adjacent to equipment hatch $B$ ) at $100 \mathrm{psi}(0.689 \mathrm{MPa}$ )

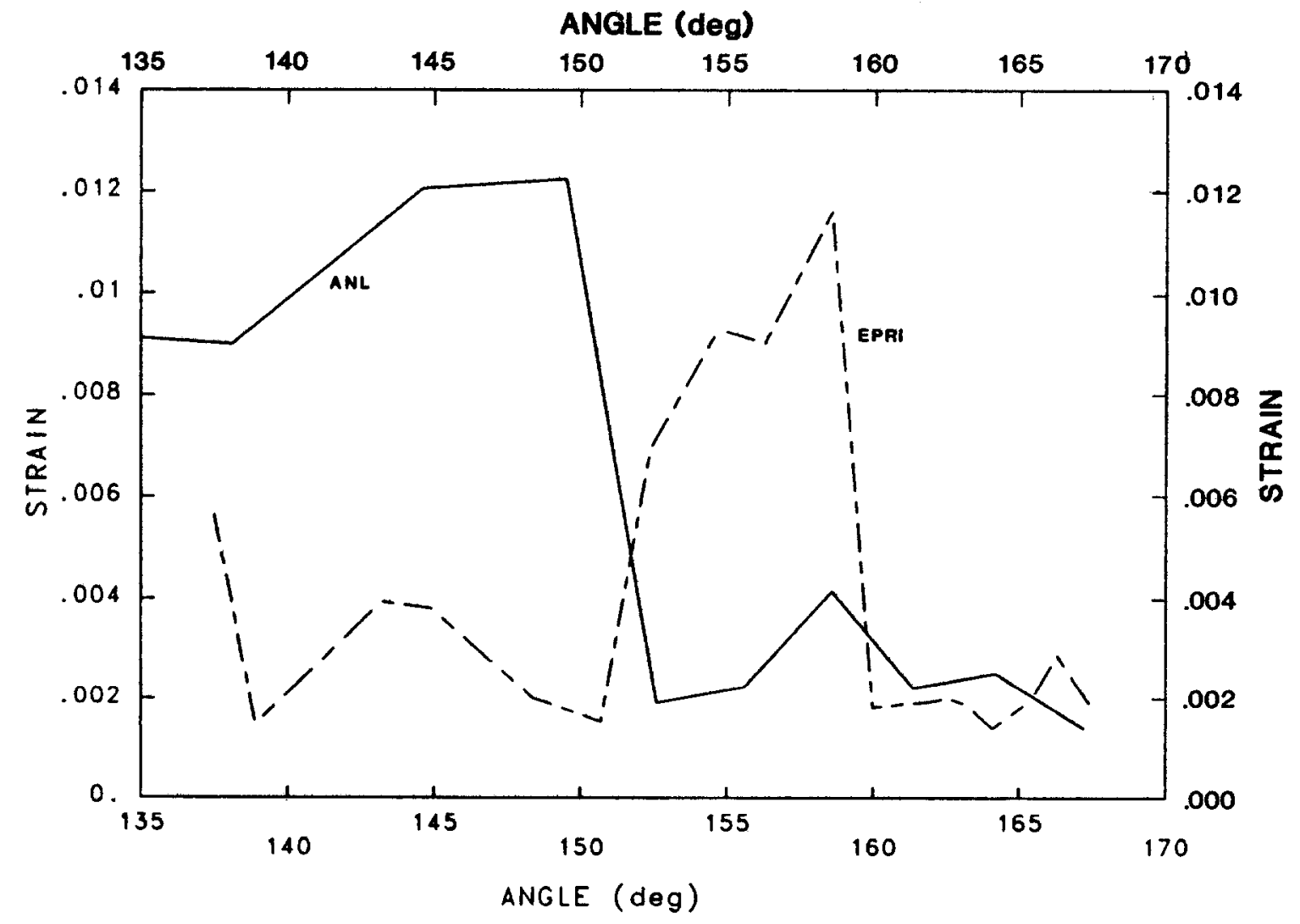

Figure A.77 Maximum principal strain on inside liner surface at EL 13'-0" vs $\theta$ (adjacent to equipment hatch B) at 150 psi (1.034 MPa) 


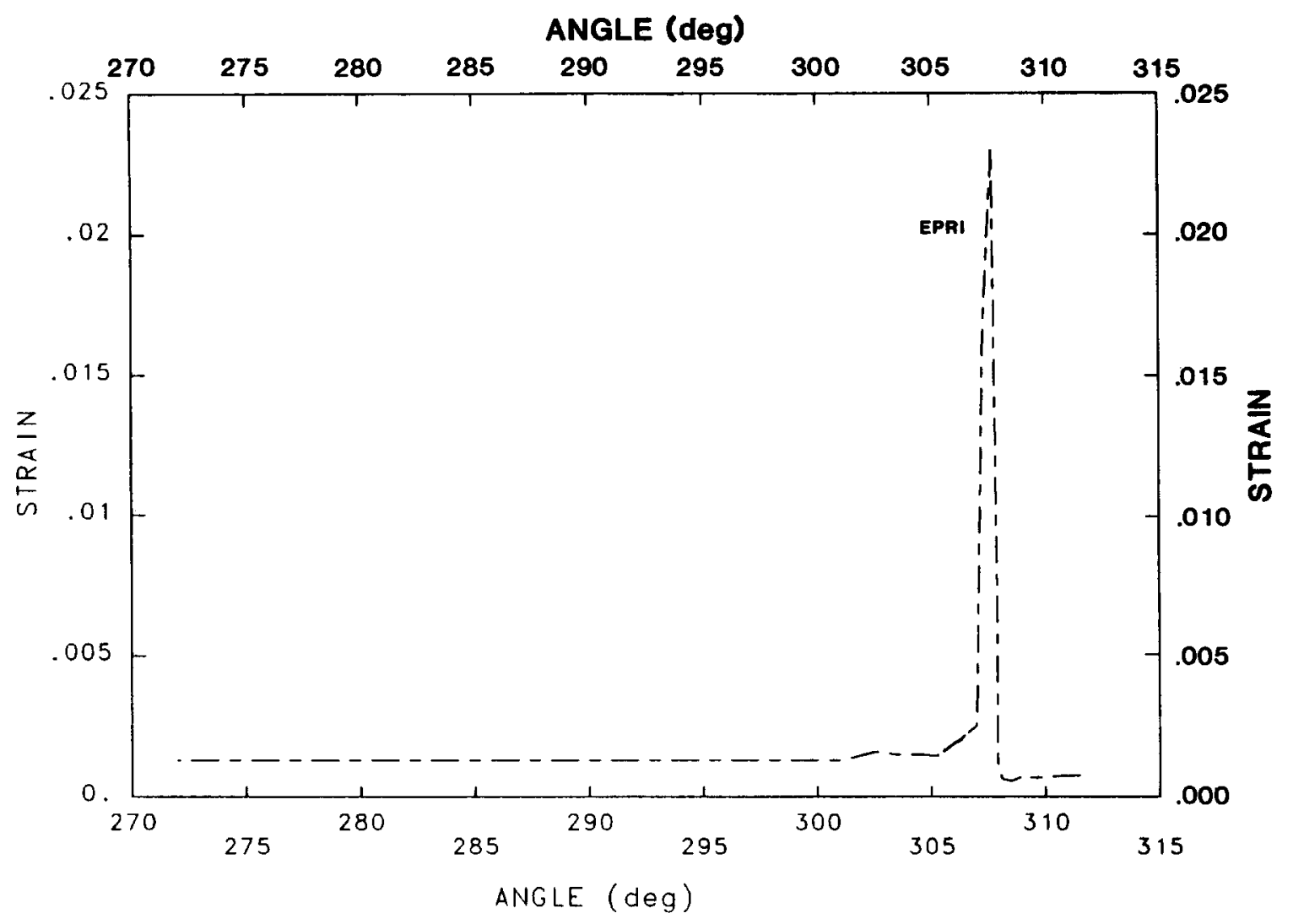

Figure A.79 Maximum principal strain on inside liner surface at EL 29'-1.5" vs $\theta$ (adjacent to constrained pipe) at $100 \mathrm{psi}(0.689 \mathrm{MPa})$

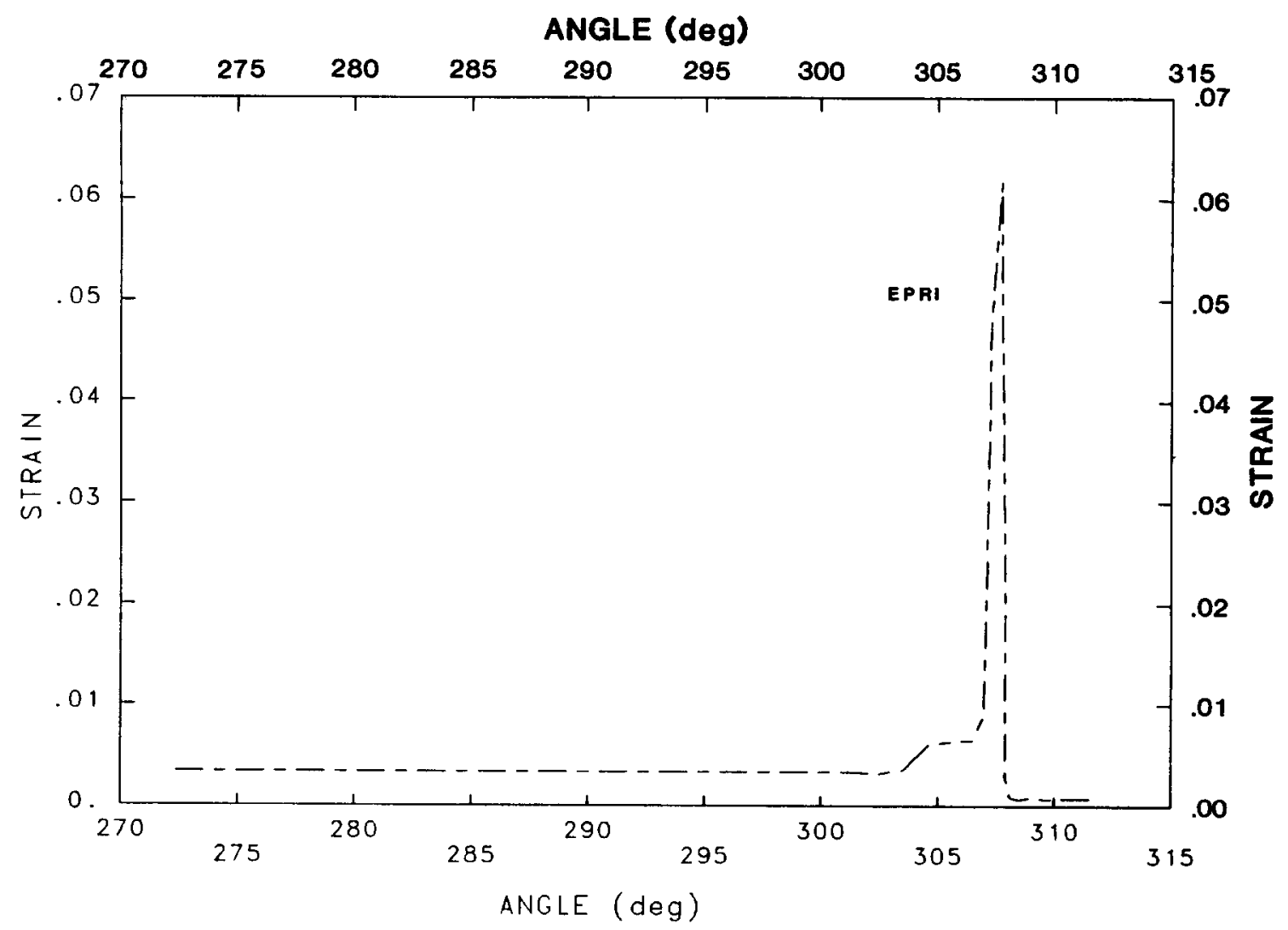

Figure A.80 Maximum principal strain on inside liner surface at EL $29^{\prime}-1.5^{\prime \prime}$ vs $\theta$ (adjacent to constrained pipe) at $150 \mathrm{psi}(1.034 \mathrm{MPa})$ 
APPENDIX B FORCE BALANCING CALCULATIONS (CEGB)

B.1 Example of Force Balancing Calculations

B.1.1 Design Data (Figure B.1)

Design case:- Shell wall with hoop tension

Concrete Properties

Characteristic Cube Strength

Tensile Strength

Young's Modulus of Elasticity

$6.0 \mathrm{ksi}(41.61 \mathrm{MPa})$

$0.6 \mathrm{ksi}(4.16 \mathrm{MPa})$

Reinforcing Steel Properties

Characteristic Yield Stress

Young's Modulus of Elasticity

$3600 \mathrm{ksi}$ (24800 MPa)

Steel Area per $m$ height

$70.5 \mathrm{ksi}(486.0 \mathrm{MPa})$

$26700 \mathrm{ksi}(184000 \mathrm{MPa})$

$6.87 \mathrm{in}^{2}\left(4432 \mathrm{~mm}^{2}\right)$

Liner Steel Properties

Characteristic Yield Stress

Young's Modulus of Elasticity

$49.8 \mathrm{ksi}(343.4 \mathrm{MPa})$

$29200 \mathrm{ksi}(201000 \mathrm{MPa})$

Containment Dimensions

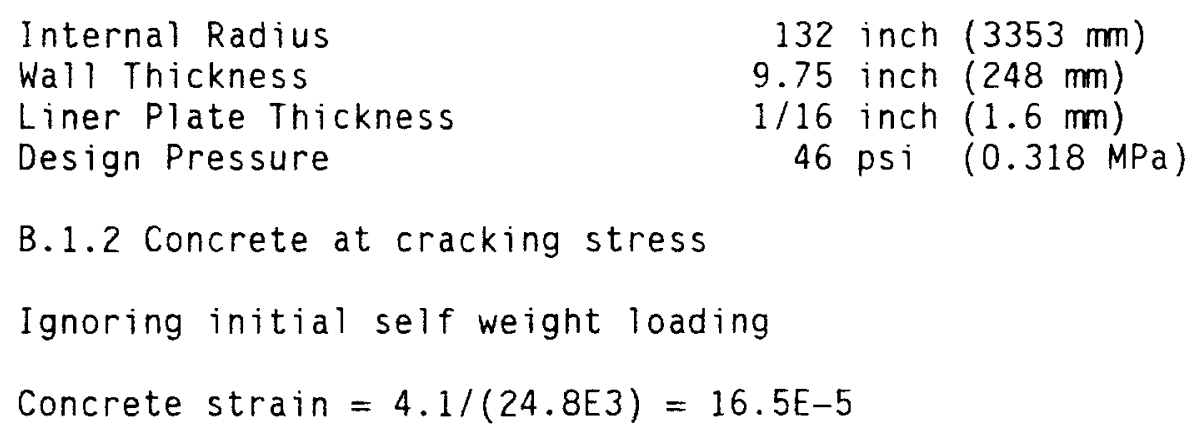

B.1.2 Concrete at cracking stress

Ignoring initial self weight loading

Concrete strain $=4.1 /(24.8 \mathrm{E} 3)=16.5 \mathrm{E}-5$

\begin{tabular}{|c|c|c|c|c|c|}
\hline & \multirow[t]{2}{*}{ Strain } & \multicolumn{2}{|c|}{ Stress } & \multicolumn{2}{|c|}{ Force/Lengtl } \\
\hline & & ks i & $\mathrm{MPa}$ & $\mathrm{lb} / \mathrm{in}$ & $\mathrm{MN} / \mathrm{m}$ \\
\hline $\begin{array}{l}\text { Concrete } \\
\text { Liner } \\
\text { Reinforcement }\end{array}$ & $\begin{array}{l}16.5 E-5 \\
16.5 E-5 \\
16.5 E-5\end{array}$ & $\begin{array}{l}0.59 \\
4.82 \\
4.41\end{array}$ & $\begin{array}{r}4.10 \\
33.23 \\
30.42\end{array}$ & $\begin{array}{r}5806 \\
304 \\
770\end{array}$ & $\begin{array}{l}1.0168 \\
0.0532 \\
0.1348\end{array}$ \\
\hline
\end{tabular}

Therefore internal pressure $=0.359 \mathrm{MPa}(52 \mathrm{psi})$

Radial displacement $=16.5 E-5 \times 3353=0.56 \mathrm{~mm}(.022$ inch $)$ 


\section{B.1.3 Concrete Cracked}

The load carried by the concrete is transferred to the reinforcement and liner.

Additional strain $=1.0168 /(201 E 3 \times 1.6 \times 10 E-3+184 E 3 \times 4432 \times 10 E-6)$ $=89.42 \mathrm{E}-5$

\begin{tabular}{lccccc} 
& Strain & \multicolumn{2}{c}{ Stress } & \multicolumn{2}{c}{ Force/Length } \\
& & $\mathrm{ksi}$ & $\mathrm{MPa}$ & $\mathrm{lb} / \mathrm{in}$ & $\mathrm{MN} / \mathrm{m}$ \\
\hline Liner & $105.92 \mathrm{E}-5$ & 30.9 & 212.90 & 1945 & 0.3406 \\
Reinforcement & $105.92 \mathrm{E}-5$ & 28.3 & 194.89 & 4932 & 0.8638 \\
\hline & & & & Total $=6877$ & 1.2044
\end{tabular}

Radial displacement $=105.92 \mathrm{E}-5 \times 3353=3.55 \mathrm{~mm}(.140$ inch $)$

B.1.4 Liner plate yield

Strain at yield $=343.4 /(201 E 3)=170.85 E-5$

\begin{tabular}{|c|c|c|c|c|c|}
\hline & \multirow[t]{2}{*}{ Strain } & \multicolumn{2}{|c|}{ Stress } & \multicolumn{2}{|c|}{ Force/Length } \\
\hline & & ksi & $\mathrm{MPa}$ & $\mathrm{lb} / \mathrm{in}$ & $M N / m$ \\
\hline Liner & $170.85 E-5$ & 49.8 & 343.40 & 3137 & 0.5494 \\
\hline Reinforcement & $170.85 E-5$ & 45.6 & 314.36 & 7956 & 1.3933 \\
\hline
\end{tabular}

Therefore internal pressure $=0.579 \mathrm{MPa}(84 \mathrm{psi})$

Radial displacement $=170.85 E-5 \times 3353=5.73 \mathrm{~mm}(.226$ in $)$

B.1.5 Reinforcement yield

Strain at yield $=486.0 /(184 E 3)=264.13 E-5$

\begin{tabular}{llrrrr} 
& Strain & \multicolumn{2}{c}{ Stress } & \multicolumn{2}{c}{ Force/Length } \\
& & $\mathrm{ksi}$ & $\mathrm{MPa}$ & $\mathrm{lb} / \mathrm{in}$ & $\mathrm{MN} / \mathrm{m}$ \\
\hline Liner & $264.13 \mathrm{E}-5$ & 49.8 & 343.40 & 3137 & 0.5494 \\
Reinforcement & $264.13 \mathrm{E}-5$ & 40.5 & 486.00 & 12299 & 2.1540 \\
\hline & & & Total $=15436$ & 2.7034
\end{tabular}

Therefore internal pressure $=0.806 \mathrm{MN} / \mathrm{sq} \mathrm{m}(117 \mathrm{psi})$

Radial displacement $=264.13 \mathrm{E}-5 \times 3353=8.86 \mathrm{~mm}(.349 \mathrm{in})$ 


\section{B. 1.6 Summary}

\begin{tabular}{|c|c|c|c|c|c|}
\hline \multirow[t]{2}{*}{ Condition } & \multicolumn{2}{|c|}{ _Pressure } & \multirow{2}{*}{$\frac{P}{P d}$} & \multicolumn{2}{|c|}{$\begin{array}{c}\text { Radial } \\
\text { Displacement }\end{array}$} \\
\hline & $\bar{p} s i$ & $\mathrm{MP} \overline{\mathrm{a}}$ & & inches & mm \\
\hline Concrete at cracking & 52 & 0.359 & 1.13 & .022 & 0.56 \\
\hline Concrete cracked & 52 & 0.359 & 1.13 & .140 & 3.55 \\
\hline Liner plate yield & 84 & 0.579 & 1.83 & .226 & 5.73 \\
\hline Reinforcement yield & 117 & 0.806 & 2.54 & .349 & 8.86 \\
\hline
\end{tabular}



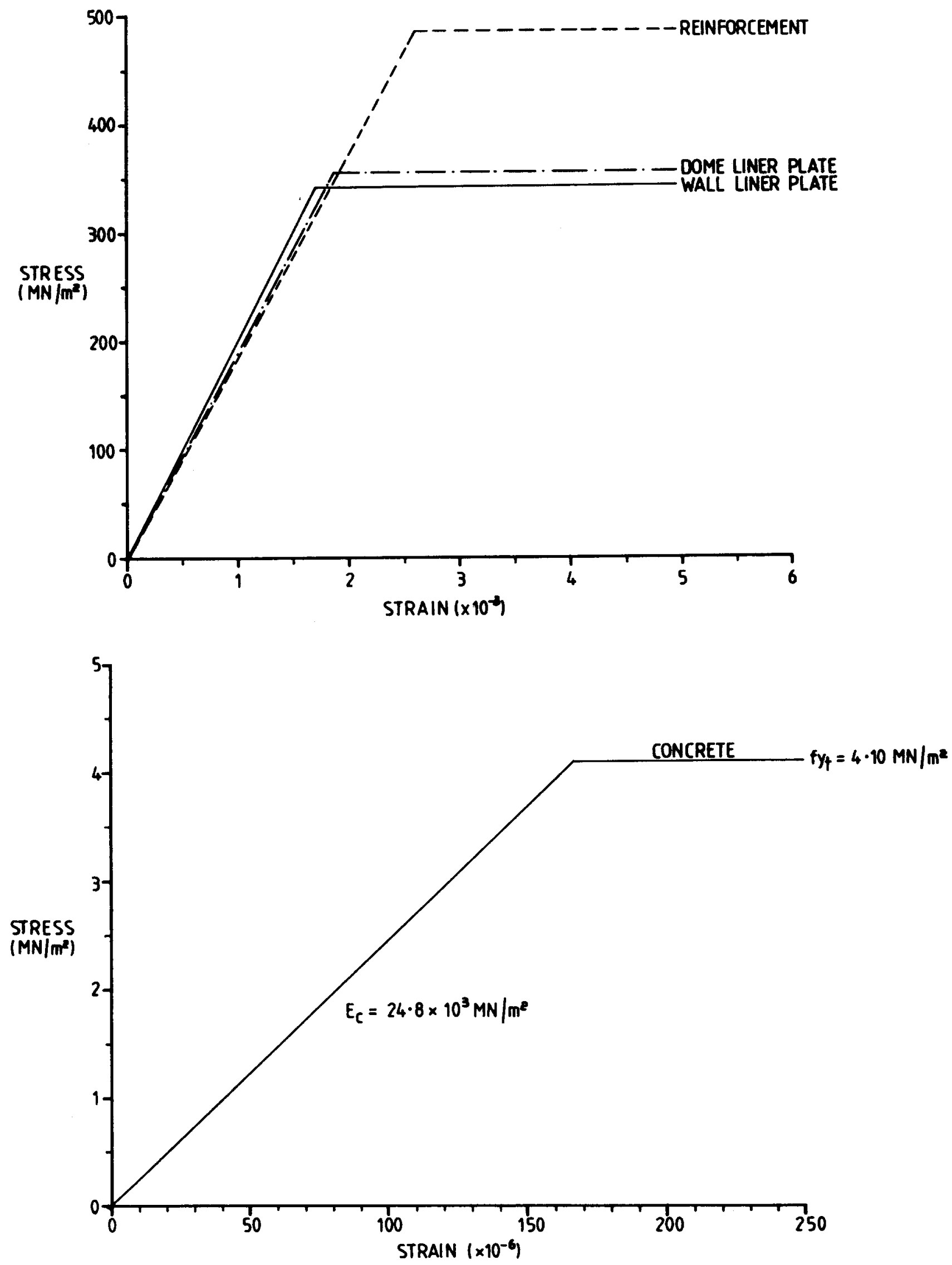

Figure B.1 Assumed Stress-Strain Relationships 
Distribution:

U. S. Government Printing Office Receiving Branch (Attn: NRC Stock) 8610 Cherry Lane Laurel, MD 20707 (500 copies for RI, RD)

US Nuclear Regulatory Commission Engineering Branch 5650 Nicholson Lane Rockville, MD 20852

Attn: J. F. Costello (20 copies) H. L. Graves

US Department of Energy

Office of Nuclear Energy

Mail Stop B-107

NE-540

Washington, DC 20545

Attn: A. Millunzi Bernard J. Rock

D. Giessing ( 3 copies)

CBI NaCon, Inc.

800 Jorie Boulevard

Oak Brook, IL 60521

Attn: Thomas J. Ahl

Wilfred Baker Engineering

218 E. Edgewood Pl.

P. O. Box 6477

San Antonio, TX 78209

Attn: Wilfred E. Baker

William C. Black

2650 Woodside Road

Bethlehem, PA 18017

Wiss, Janney, Elstner Assoc., Inc.

330 Pfingsten Road

Northbrook, IL 60062

Attn: Ted M. Brown

Battelle Columbus Laboratories

505 King Avenue

Columbus, Ohio 43201

Attn: Richard Denning

Bechtel Power Corporation

$12400 \mathrm{E}$. Imperial Highway

Norwalk, CA 90650

Attn: Asadour H. Hadjian
Bechtel Power Corp.

15740 Shady Grove Rd.

Gaithersburg, MD 20877

Attn: T.E. Johnson

K. Y. Lee ( 2 copies)

Dept. of Civil Engineering

University of Illinois

503 W. Michigan

Urbana, IL 61801

Attn: Prof. Mete A. Sozen

Stevenson \& Associates

9217 Midwest Ave.

Cleveland, Ohio 44125

Attn: John D. Stevenson

United Engineers \& Constructors, Inc.

30 S. 17th St.

Philadelphia, PA 19101

Attn: Joseph J. Ucciferro

Electrical Power Research Institute (14 copies)

3412 Hillview Avenue

PO Box 10412

Palo Alto, CA 94304

Attn: H. T. Tang (10), Y. K. Tang Raf Sehgal, J. J. Taylor, W. Loewenstein

School of Civil \& Environ. Engr. Hollister Hall Cornell University Ithaca, NY 14853

Attn: Professor Richard N. White

NUTECH

225 N. Michigan Ave.

16th Floor

Chicago, Illinois 60601

Attn: John Clauss

Iowa State University

Department of Civil Engineering

420 Town Engineering Bldg.

Ames, IA 50011

Attn: L. Greimann

TVA

400 Commerce Ave.

Knoxville, TN 37902

Attn: D. Denton, W9A18 
Los Alamos National Laboratories

PO Box 1663

Mail Stop N576

Los Alamos, NM 87545

Attn: C. Anderson

EQE Inc.

3300 Irvine Aveune

Suite 345

Newport Beach, CA 92660

Attn: M. K. Ravindra

University of Illinois

Dept. of Civil Engineering

Urbana, IL 61801

Attn: C. Siess

EBASCO Services, Inc.

Two World Trade Center

New York, NY 10048

Attn: Robert C. Iotti

EG\&G Idaho

Willow Creek Bldg. W-3

PO Box 1625

Idaho Falls, ID 83415

Attn: B. Barnes, T. L. Bridges

(2 copies)

Sargent \& Lundy Engineers

55 E Monroe St.

Chicago, IL 60603

Attn: A. Walser

P. K. Agrawal (2 copies)

General Electric Company

175 Curtner Ave.

San Jose, CA 95125

Attn: J. E. Love, E. O. Swain, D. K. Henrie, R. Gou (4 copies)

Westinghouse Electric Corp.

Waltz Mill Site

Box 158

Madison, PA 15663

Attn: Vijay K. Sazawal

R. F. Reedy, Inc.

236 N Santa Cruz Ave.

Los Gatos, Ca 95030
Quadrex Corporation

1700 Dell Ave.

Campbell, CA 95008

Attn: Quazi A. Hossain

ANATECH International Corp.

3344 N. Torrey Pines Court

Suite 320

LaJolla, CA 92037

Attn: Y.R. Rashid

Oak Ridge National Laboratory

PO Box $Y$

Oak Ridge, TN 37830

Attn: Steve Hodge

Brookhaven National Laboratory

Building 130

Upton, NY 11973

Attn: C. Hofmeyer, T. Pratt

M. Reich ( 3 copies)

Argonne National Laboratory (12 copies)

9700 South Cass Avenue

Argonne, IL 60439

Attn: J. M. Kennedy (10),

R. F. Kulak,

R. W. Seidensticker

Tennessee Valley Authority 400 Summit Hill Rd.

W9D24C-K

Knoxville, Tennessee 37902

Attn: Nathaniel Foster

University of Wisconsin

Nuclear Engineering Dept.

Madison, WI 53706

Attn: Prof. Michael Corradini

Brookhaven National Laboratory

Building 820M

Upon, NY 11973

Attn: Ted Ginsberg

Dept. of Chemical \& Nuclear Engineering University of California Santa Barbara Santa Barbara, CA 93106

Attn: T. G. Theofanous 
Institut für Mechanik

Universität Innsbruck

Technikerstr. 13

A-6020 Innsbruck

Austria

Attn: Prof. G. I. Schuëller

Nuclear Studies \& Safety Dept.

Ontario Hydro

700 University Avenue

Toronto, Ontario

M5G $1 \times 6$

Canada

Attn: W. J. Penn

University of Alberta

Dept. of Civil Engineering

Edmonton, Alberta

Canada T6G 2G7

Attn: Prof. D. W. Murray

Commissariat a L'Energie Atomique ( 11 copies)

Centre d'Etudes Nucleaires de Saclay

F-91191 Gif-Sur-Yyette Cedex

France

Attn: M. Livolant, P. Jamet (10 copies)

Institut de Protection et de

Surete Nucleaire

Commissariat a l'Energie Atomigue

F-92660 Fontenay-aux-Roses

France

Attn: M. Barbe

Kernforschungszentrum Karlsruhe $\mathrm{GmbH}$

Postfach 3640

D-7500 Karlsruhe

Federal Republic of Germany

Attn: R. Krieg, P. Gast ( 2 copies)

Lehrstuhl fuer Reakordynamik und Reaktorsicherheit

Technische Universitaet Muenchen

D-8046 Garching

Federal Republic of Germany

Attn: Prof. H. Karwat

Staatliche Materialpruefungsanstalt (MPA)

University of Stuttgart

Pfaffenwaldring 32

D-7000 Stuttgart 80 (Vaihingen)

Federal Republic of Germany

Attn: Prof. K.F. Kussmaul
Gesellschaft fuer Reaktorsicherheit (12 copies)

Schwertnergasse 1

D-5000 Köln 1

Federal Republic of Germany

Attn: H. Schulz (10), A. Hoefler,

F. Schleifer

Kraftwerk Union AG

Hammerbacherstr. 12-14

D-8520 Erlangen

Federal Republic of Germany

Attn: $\quad M$. Hintergräber

Ente Nazionale per l'Energia Elettrica

v. le Regina Margherita, 137

Rome

Italy

Attn: Francesco L. Scotto

ISMES

Viale Giulio Cesare 29

I-24100 Bergamo

Italy

Attn: A. Peano

ENEA-DISP

ACO-CIVME

Via Vitaliano Brancati, 48

I-00144 Roma

Italy

Attn: Giuseppe Pino (10 copies)

Nuclear Equipment Design Dept.

Hitachi Works, Hitachi, Ltd.

3-1-1 Saiwai-Cho

Hitachi-Shi

Ibaraki-ken

Japan

Attn: O. Oyamada

Division of Technical Information

Japan Atomic Energy Research Institute

2-2, Uchisaiwai-cho 2-chome

Chiyoda, Tokyo 100

Japan

Attn: Jun-ichi Shimokawa

University of Tokyo

Institute of Industrial Science

22-1, Roppongi 7

Minatu-ku, Tokyo

Japan

Attn: Prof. H. Shibata 


\author{
Civil Engineering Laboratory \\ Central Research Institute of \\ Electric Power Industry \\ 1646 Abiko Abiko-Shi Chiba \\ Japan \\ Attn: Yukio Aoyagi \\ Kajima Corporation \\ No. 1-1, 2-Chome Nishishinjuku \\ Shinjuku-ku \\ Tokyo 160 \\ Japan \\ Attn: K. Umeda \\ Muto Institute of Structural Mechanics \\ Room 3005 Shinjuku Mitsui Building \\ Shinjuku-ku \\ Tokyo, 160 \\ Japan \\ Attn: Tadashi Sugano \\ Nuclear Power Engineering Test Center \\ 6-2, 3-Chome, Toranomor \\ Minatoku \\ Tokyo 105 \\ Japan \\ Attn: Yoshio Tokumaru \\ Japan Atomic Energy Research Inst. \\ Tokai-Mura, Ibaraki-Ken 319-11 \\ Japan \\ Attn: Kunihisa Soda \\ Toshikuni Isozaki (2 copies) \\ Shimizu Construction Co., Ltd. \\ No. 4-17. Etchujima 3-Chome \\ Koto-Ku \\ Tokyo 135 \\ Japan \\ Attn: Toshihiko Ota \\ Shimizu Construction Co., Ltd. \\ No. 18-1, Kyobashi 1-Chome \\ Chuo-ku \\ Tokyo 104 \\ Japan \\ Attn: Toshiaki Fujimori
}

Korea Advanced Energy Research Inst.

P. O. Box 7

Cheong Ryang

Seoul

Korea

Attn: Pilsoon Han

Universidad Politecnica

Escuela Tecnica Superior

de Ingenieros Industriales

Madrid

Spain

Attn: Agustin Alonso

Studsvik Energiteknik $A B$

S-611 82 Nyköping

Sweden

Attn: Kjell O. Johansson

Swedish State Power Board

Nuclear Reactor Safety

S-162 87 Vällingby

Sweden

Attn: Hans Cederberg Per-Eric Ahlström

Ralf Espefaelt ( 3 copies)

Swiss Federal Institute of Technology

Institute of Structural Engineering

ETH-Hoenggerberg, HIL

$\mathrm{CH}-8093$ Zurich

Switzerland

Attn: W. Ammann

Motor-Columbus Consulting Engineers, Inc.

Parkstrasse 27

$\mathrm{CH}-5401$ Baden

Switzerland

Attn: K. Gähler, A. Huber

A. Schopfer (3 copies)

EIR (Swiss Federal Institute for

Reactor Research)

$\mathrm{CH}-5303$ Wuerlingen

Switzerland

Attn: O. Mercier

P. Housemann 
Swiss Federal Nuclear Safety Inspectorate Federal Office of Energy

$\mathrm{CH}-5303$ Wuerenlingen

Switzerland

Attn: S. Chakraborty

Swiss Federal Institute of Technology

Chemin de Bellerive 32

CH-1007 Lausanne

Switzerland

Attn: Prof. F.H. Wittmann

Elektrowatt Ingenieurunternehmung AG

Bellerivestr. 36

$\mathrm{CH}-8022$ Zürich

Switzerland

Attn: John P. Wolf

Institute of Nuclear Energy Research

P. O. Box 3

Lung-Tan

Taiwan 325

Republic of China

Attn: Sen-I Chang

Atomic Energy Establishment

Winfrith

Dorchester Dorset

DT2 8DH

United Kingdom

Attn: Peter Barr

Atomic Energy Authority

Safety and Reliability Directorate

Wigshaw Lane

Culcheth

Warrington WA3 4NE

United Kingdom

Attn: D. W. Phillips (10 copies)

HM Nuclear Installation Inspectorate

St. Peter's House

Stanley Precinct

Bootle L20 3LZ

United Kingdom

Attn: R. J. Stubbs (10 copies)

Northern Illinois University

Mechanical Engineering Dept.

DeKalb, IL 60115

Attn: A. Marchertas
Taylor Woodrow Construction Limited 345 Ruislip Road

Southall, Middlesex

UBI $2 Q X$

United Kingdom

Attn: Carl C. Fleischer

Richard Crowder

Central Electricity Generating Board Barnett Way

Barnwood, Gloucester

GL4 7RS

United Kingdom

Attn: J. Irving

Central Electricity Generating Board

Booths Hall

Chelford Road

Knutsford, Cheshire

WA 16 8QG

United Kingdom

Attn: Carl Lomas (10 copies)

City College of New York

Dept. of Civil Engineering

140 Street and Convent Ave.

New York, NY 10031

Attn: C. Costantino (10 copies)

1520 W. Herrmann (Actg.)

1521 R. D. Krieg

1521 J. R. Weatherby

1523 R. L. Johnson

3141 S. A. Landenberger (5)

3151 W. L. Garner

6400 D. J. McCloskey

6410 N. R. Ortiz

6418 N. R. Ortiz (Actg.)

6419 K. D. Bergeron

6420 J. V. Walker

6440 D. A. Dahlgren

6442 W. A. von Riesemann (25)

6442 D. B. Clauss (5)

6442 D. S. Horschel

6442 D. L. Lambert

6448 D. L. Berry

8024 P. W. Dean 


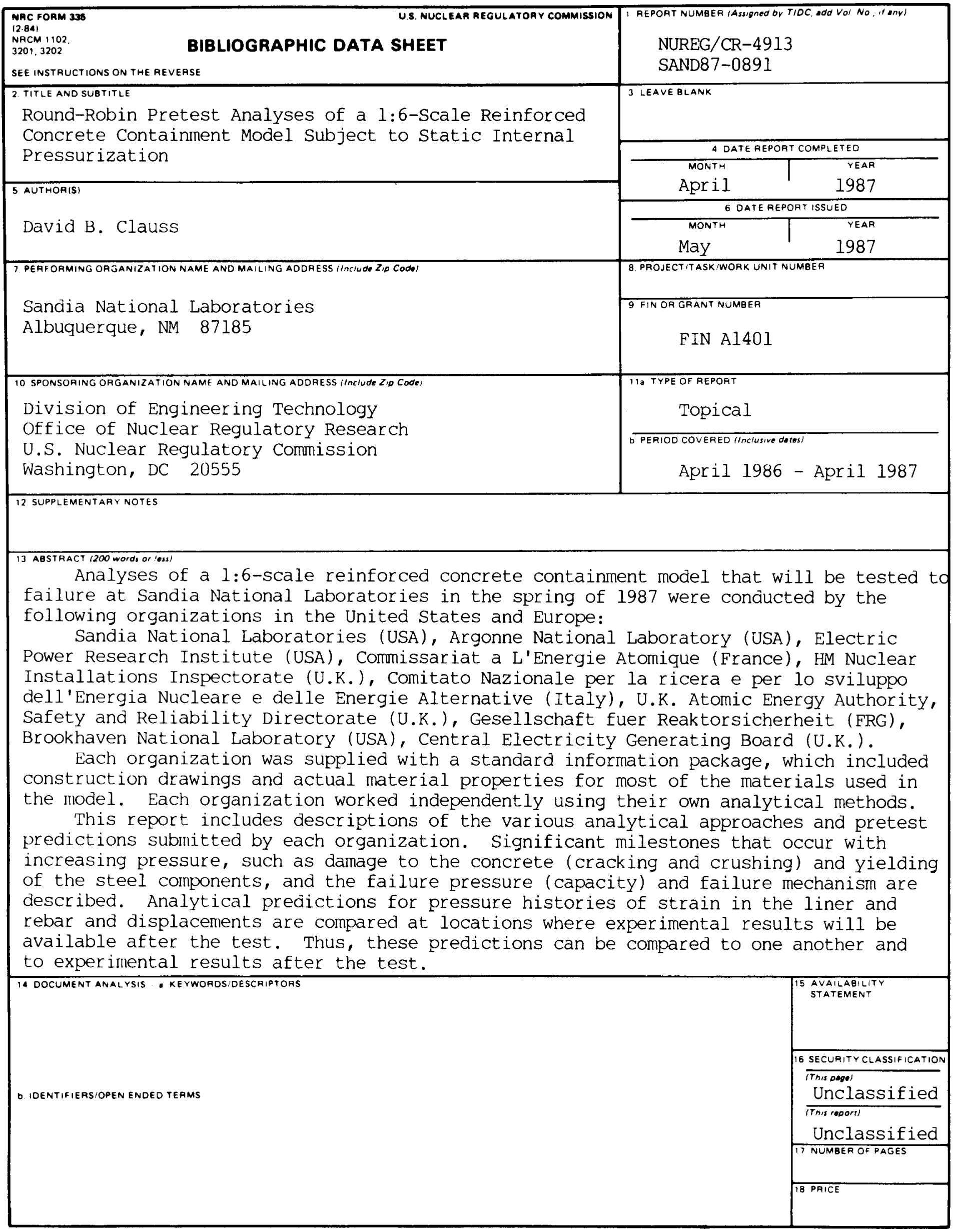

